


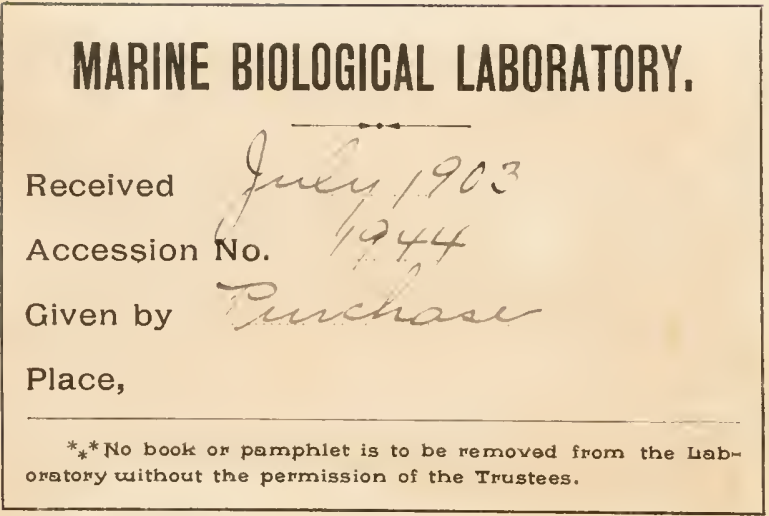




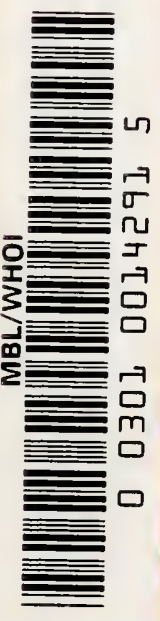



THE

PHYSIOLOGY OF PLANTS

PFEFFER

VOL.. I 
HENRY FROWDE, M.A.

PUBLISHER TO THE UNIVERSITY OF OXFORD

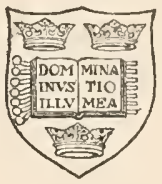

LONDON, EDINBURGH, AND NEIV YORK 
THE

\section{PHYSIOLOGY OF PLANTS}

A TREATISE UPON THE

\section{METABOLISM AND SOURCES OF ENERGY IN PLANTS}

B X

DR. W. PFEFFER

PROFESSOR OF BOTANY IN THE UNIVERSITY OF LEIPZIG

SECOND FULLY REVISED EDITION

TRANSLATED AND EDITED BY

ALFRED J. EWART, D.Sc., Ph.D., F.L.S.

WITH MANY ILLUSTRATIONS

VOLUME I

Oxford

AT THE CLARENDON PRESS

M. D. CCCC 
Oxford

PRINTED AT THE CIARENDON PRESS

BY HORACE HART, M.A.

PRINTER TO THE UNIVERSITY

2393 


\section{PREFACE TO THE FIRST EDITION}

THE following work is not intended as a text-book for beginners, but as a hand-book containing a complete account of the present state of our knowledge concerning the general processes of metabolism and the sources of energy in the plant. Consequently an acquaintance with the principles of anatomy, morphology and physiology, as well as of physics and chemistry, will be assumed on the part of the reader.

The older literature has been referred to only when it forms the basis of our present knowledge. In many places, however, reference has been made to works containing ideas which are now of merely historical interest. Wherever the original literature has been unattainable, the sources of information made use of have been mentioned. In the catalogue of scientific papers, published by the Royal Society of London, a complete account is given of all the existing abstracts, translations, \&c. that have been made of each paper, and by referring to this source the reader can decide whether any other source will be more accessible to him than the one quoted by me. It has not been my purpose to furnish a mere collection of the conclusions and results recorded in these different original publications. On the contrary I have attempted throughout to view the whole critically, and to indicatc what facts may be regarded as definitely established, as well as to point out where only insufficient and incomplete observations are presented to us. If an impulse is thus given to further research, broadening and increasing our knowledge, this book will have fulfilled its most important purpose. Knowing, as I do, its inevitable imperfections and incompleteness, I cannot send it forth with the feeling that all that was originally intended has been accomplished. Two courses only were open to me-either to issue the work as it now stands, and to prepare for a more complete critical account by further research, or to regard the results of many years' labour as studies undertaken for my own instruction, and make no further use of them.

IV. PFEFFER.

TüвINGen, December is, 1 SSo. 


\section{PREFACE TO THE SECOND EDITION}

AFTER much hesitation I have at last resolved to accede to the requests made to me, during several years, to undertake a new edition of my 'Pflanzenphysiologie.' The revision has necessarily been such that what is essentially a new book has been produced. Since the appearance of the first edition, so much work has been done, and our physiological knowledge has been so broadened and deepened, that it needed a full and complete revision of the whole to give a correct idea of our present standpoint. In spite of the changes and additions made, I have been able to retain the general arrangement, which has proved to be an advantageous one in a textbook intended only for those already possessing a knowledge of physiology. Moreover the fundamental principles still remain the same. A comparison between the two editions will show, especially as regards the introductory portion, that the views and ideas, which formed the guiding principles in the first edition have proved correct, and have met with increasingly wide acceptance.

The scope of the book has not been enlarged. As before, it deals only with the fundamental principles of metabolism and the sources of energy, using those terms in the sense defined in the Introduction (Chap. I). This exclusive adherence to important features and general relationships has naturally made it impossible to discuss, or even mention, every detail and individual peculiarity, the significance of many of which is frequently overestimated. Nor can a complete catalogue of all the physiological literature be expected. Nevertheless I have attempted, as far as possible, to deal with the whole of the literature which forms the basis of our general linowledge, and to which its expansion is due. Still I am afraid that, in spite of all my care, much has been forgotten or overlooked, especially since, owing to the pressure of other duties, the time necessary for this work has been obtained with difficulty. This has rendered it impossible for me to study certain problems as deeply as I had wished. Nevertheless, the influence of my own research will be felt in nearly every chapter, although, for the most part, the investigations and experiments made under my direction, or at my instigation, are not expressly mentioned. I have 
often been compelled to represent matters as they are at present understood, although convinced of the insufficiency of the accepted facts and interpretations. In any case, I cannot send forth even this revised edition with the feeling that I have attained what I desired.

Those works which have appeared since the manuscript was finally given to the press have not been noticed in the text. Hence for the first chapter of Vol. I, the references to the literature cease with the beginning of 1896 . Notices of the more important works which have appeared subsequently have, however, been inserted while the book has been in the press.

It is a pleasant duty to record my grateful thanks to Dr. Klemm and Dr. Giessler for their kind assistance in the correction of the proofs.

IV. PFEFFER.

LeIrZIG, September 24, $18_{9 \pi}$. 


\section{PREFACE TO THE ENGLISH EDITION}

ON account of the importance of Professor Pfeffer's new 'Pflanzenphysiologie' it has been considered advisable to produce an immediate translation without waiting for the appearance of the second volume, especially since no translation of the first edition has ever been published. It is, moreover, hoped that a still shorter interval may elapse between the appearance of the second volume and of its translation. The difficulty of the original German has necessitated the exercise of a certain freedom in the process of translation, but an exact interpretation of the original has been given throughout, and all additions, as well as references to the more recent literature, have been inserted in brackets. In those sections, however, of which I had direct personal knowledge, a few slight alterations have been made without any special indication of these being considered necessary. The question of terminology becomes of great importance in the translation of a work which definitely introduces several new principles to botanical physiology, but this has been accomplished practically without the introduction of any new terms. A short foot-note has been added, whenever necessary, explaining the reasons why of two pre-existent terms one has been selected in preference to another.

The whole of the proofs have been submitted to Professor Pfeffer's approval, and in addition they have been subjected to thorough and systematic revision by Professor Reynolds Green. I am under a deep obligation to Professor Green for the valuable aid which he has rendered throughout, and which has materially enhanced whatever merit this book may possess. I must also record my indebtedness to Professor Muirhead and Dr. Church for numerous valuable suggestions and criticisms.

BOtanical Departaent,

ALFRED J. EWART. OXFORD, October, 1899 . 


\title{
CONTENTS
}

\author{
CHAPTER I \\ INTRODUCTION
}

I. General

2. Aim of physiology

3. Nature of irritability .

4. Causal relationship of growth and development

5. Variation and heredity

\section{CHAPTER·II}

\section{Physiological Morphology}

6. Structure and function of plant organs

7. $"$ of protoplasm

8. Origin of plasmatic organs

9. Relationship between nucleus and cytoplasm

I0. Uni- and multi-nucleate cells .

II. Chemistry of the protoplast

\section{CHAPTER III \\ IMBIBITION AND MOLECULAR STRUCTURE}

12. Force of imbibition and swelling of organized bodies . . . . . 70

13. Hypotheses of molecular structure . . . . . . . . . 76

14. Changes of molecular structure due to swelling . $\quad . \quad \ldots \quad . \quad . \quad$. 83

\section{CHAPTER IV}

\section{Mechanism of AbSORPtion and Translocation}

15. General

16. Diosmotic properties of the cell

17. Absorption and excretion

I8. Plasmatic membranes

19. Ingestion and excretion of solid bodies

20. Translocation from cell to cell

21. Diosmotic properties of cuticle and cork

22. Quantitative selective power 
\$23. Nechanism of secretion and excretion

24. Osmotic pressure in the cell . . . . . . . . . . I34

25. Specific powers and activities . . . . . . . . . . . 147

26. Importance of the root system . . . . . . . . . . . . . . 149

27. Absorption of fluids and solids by subaërial organs . . . . . . . 158

28. Importance of the soil . . . . . . . . . . . . 163

\section{CHAPTER V}

The Mechanisit of Gaseous Exchange

29. General

30. Passage of gases through cells and cell-walls

31. Communications of the aeriferous system with the external world . . . I 88

32. Movements and pressure of the internal gases . . . . . . . . 199

\section{CHAPTER VI \\ MOVEMENTS OF WATEK}

33. General view

PART I. Trinsference oF WATFR.

34. Conveyance of water in transpiring plants . . . . . . . . 210

35. Conducting channels . . . . . . . . . . . 213

36. Mechanism of water transport . . . . . . . . . . . . 220

37. Relation between transpiration and absorption of water . . . . . . 228

\section{Part II. Excretion of Water Vapour.}

38. Influence of specific peculiarities on transpiration . . . . . . . 234

39. " external conditions . . . . . . . 245

40. Transpiration under normal conditions . . . . . . . . . 249

Part III. Fxcrfion OF Finil Mater.

41. General view

42. Bleeding of injured plants.

43. Amount and pressure

43. Amount and pressure . . . . . . . . . . . 257

44. Influence of external conditions . . . . . . . . . . . 263

45. Periodicity of bleeding . . . . . . . . . . . . . . . . 264

46. Mechanism of active exudation . . . . . . . . . . . . 268

47. Excretion of water from uninjured plants . . . . . . . . . . 272

48. " from water-pores . . . . . . . . . . . 278

49. " $"$ of nectar . . . . . . . . . 28 I

\section{CHAPTER VII \\ The Food of Plants \\ Part I. General View.}

50. Nutritive metabolism

5I. Circulation of food materials in organic world 
Pakt 11. Assimlation of Carbon Dioxint.

A. Photosynthetic Assimilation.

52. General

53. Structure and properties of the chloroplastid . . . . . . . 312

54. The products of photosynthesis . . . . . . . . . . 317

55. Accumulation of the assimilatory products . . . . . . . . 322

56. Photosynthesis of other carbon compounds . . . . . . . . . . 326

57. Sources and optimal percentage of carbon dioxide . . . . . . . . . 329

58. Influence of the external conditions on photosynthesis . . . . . . 333

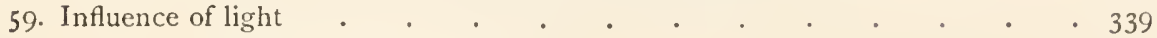

6o. $"$ different rays of spectrum . . . . . . . . 342

61. Theoretical . . . . . . . . . . . . 353

62. Individual and specific peculiarities . . . . . . . . . 356

13. Chemosynthetic Assimilation.

63.

Part III. Absorption of Organic Food.

64. General

65. Special adaptations

66. Nutritive value of different carbon compounds.

67. Special selective power . . . . . . . . . . . . . 387

Part IV. The Assimilation of Nitrogex.

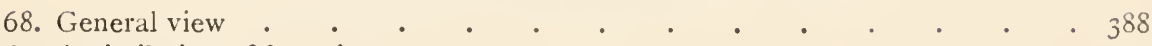

69. Assimilation of free nitrogen . . . . . . . . . . . . . $\quad . \quad 393$

70. Nutritive value of different compounds of nitrogen . $\quad . \quad+\quad . \quad . \quad . \quad 403$

71. Character of processes by which nitrogen is assimilated . . . . . 407

72. Localization of proteid-synthesis . . . . . . . . . . . 409

\section{Part V. The Ash Constituents.}

73. The essential elements

74. Their functions

75. Non-essential ash constituents.

76. Influence of the soil on distribution .

\section{CHAPTER VIII}

\section{Constructive and Destructive Metabolish}

77. General

78. The commoner metabolic products .

79. Constructive and plastic nitrogen compounds

8o. Nitrogenous metabolism .

$8 \mathrm{I}$. ",

82. Carbohydrates and fats

83. Composition of the cell-wall

84. Formation and modification of the cell-wall

85 . Organic acids .

86.

$$
" \text { (continued). }
$$


\$ 87. Glucosides, tannin, and phenols

88. Pigments .

89. Alkaloids, ptomaines, and other poisons .

90. Ethereal oils, resins, \&c.

91. Enzymes

92. External influences.

93. Self-regulation .

\section{CHAPTER IX}

\section{RESPIRATION AND FERMIENTATION}

94. Introductory . . . . . . . . . . . . . . 518

95. Aerobic respiration . . . . . . . . . . . . . 519

96. Products of aerobic respiration . . . . . . . . . . . . . 526

97. Respiratory katabolism in anaerobes . . . . . . . . . 530

98. Sources of energy in anaerobes . . . . . . . . . . . . 532

99. Intramolecular respiration . . . . . . . . . . 536

100. Mechanism and causes of physiological combustion . . . . . . 539

IоI. " " " " $"$ (continued) . . 543

102. Relationship between aerobic and anaerobic respiration . . . . 546

103. General view of certain fermentative activities . . . . . . . 553

104. Influence of the external conditions on respiration . . . . . . $56 \mathrm{I}$

105. Importance of respiration . . . . . . . . . . . . 566

\section{CHAPTER X}

\section{Translocation}

106. Translocation of organic food substances . . . . . . $\quad$. 572

107. " . . . . . . . . . . . . 583

108. Mechanism and causes of translocation . . . . . . . . . . . 587

I09. Special examples . . . . . . . . . . . . . . 592

$\mathrm{INDEX}$. . . . . . . . . . . . 605 


\section{PHYSIOLOGY OF PLANTS}

\section{CHAPTER I.}

INTRODUCTION.

\section{SEctioi i. General.}

ALL terrestrial life follows a continual cycle of development and decay, each individual existence being of limited duration. The largest trees, whirh mav rearh the aoe of more than a thoucand veare are donmed

\section{ERRATUM}

P. 395, 1. I5, for grammes read milligrammes

Pferfer's Physiology of Plants

deprived of nourishment, it finally dies of hunger, while vital activity and growth are only possible in the presence of water and within certain limits of temperature. It is evident that the absence of any one of the necessary conditions must invalidate the remaining ones, so that, if the amount of water is insufficient, or if the temperature sinks too low, the vital activity of the organism is depressed or completely arrested, and sinilarly at a high temperature death ultimately supervenes.

The conditions of life are not identical for all plants, but differ in different cases; no plant, however, can live unless supplied with nourishment and kept at a sufficiently high temperature. Light, calcium, and free oxygen are necessary to most plants, but not to all, and without an appropriate food supply, the continuance of life is in all cases inconceivable. It will be shown subsequently how and by what means plants obtain and make use of their food, but what is at once clear is that nutrient 
material becomes of value to the plant only when subjected to the constructive and destructive metabolism of the living organism. In the course of these processes, the exact nature of which is determined by the vital activity of the organism itself, the substances necessary for growth are elaborated, while, at the same time, kinetic energy is supplied for growth and movement, for internal and external work.

In the absence of such metabolism, vital activity is impossible, and, even in the adult plant, anabolic and katabolic changes are always taking place so long as life remains ${ }^{1}$. In plants as well as in animals a large, indeed often the greater, proportion of the nourishment absorbed serves to supply the energy necessary for the maintenance of life, while a part only is employed in building up the substance and tissues of the organism.

Metabolism and vital activity are mutually interdependent; the energy set free by one metabolic process affords the necessary stimulus to further metabolic activity, just as a fire, by heating the surrounding wood to combustion point, ensures its own continuance.

This correlation and mutual interdependence must also be borne in mind when we attempt to distinguish between chemical and physical processes exhibited by a living plant, and indeed the distinction between these is purely arbitrary. Every chemical change necessarily involves a redistribution of energy, and vice versa; so that any profound study of the phenomena of growth and movement must inevitably include that of the chemical processes with which the former are inextricably associated.

The same general laws hold good for all forms of life, including bacteria. These latter organisms teach us that all the conditions for active life exist in the smallest cell, and that the essential vital processes can be carried on in the entire absence of external differentiation. The development of external members and consequent internal tissue-differentiation is correlated with a more or less complete division of labour and function. Hence Physiology is primarily required to determine the powers and possibilitics of individual organs and cells and their various interactions.

Parts which are no longer living often perform useful functions. Dead cells, air vessels, intercellular spaces, all of which are usually present in the tissues of higher plants, have definite duties to perform, commonly subsidiary to the functions of the living parts, or assisting in the performance of them. Dęad structural units often form the channels for the passage of water and dissolved food materials, while air-spaces permit of gaseous interchange, and allow the circulation of the indispensable oxygen to the innermost tissues. These and similar processes are only of physio-

' [ It must, however, be remembered that dormant life is possible in certain seeds, spores, mosses, \&c., in the total absence of all metabolic and respiratory activities.] 
logical importance so long as the other cells remain alive. The protoplasm is the primary living constituent of the cell; when it dies the cell is dead; and when the cells and tissues die, the plant is dead.

The manifestations of life, when traced back to their ultimate origin, are always found to originate in the protoplasm, an undifferentiated mass of which constitutes the substance of the simplest living elementary organism. Hence one of the tasks of Physiology is to throw light on the manner in which the inherent nature of the protoplasm is responsible for the chemical and physical changes to which it gives rise. From a consideration of the organization of the adult plant, it is evident that each individual protoplast must have a complicated structure: as a matter of fact, the cell-nucleus and the chloroplasts are organs of both general and special importance; vacuoles and cell-walls, though non-living, are also products of vital activity, and of use to the living parts. The protoplasm itself must be made up of elements or bioplasts, and these, though invisible with the highest powers of the microscope, are not of any the less importance on that account. It is as impossible to picture a regular continuance of life otherwise than by the co-operation of different organs and biological elements, as it is to imagine a watch, which could still keep time after the removal of certain of the wheels.

Just as a watch ceases to be a watch when crushed to pieces, though the quality and quantity of the component metals remain the same, so also is the life of any protoplasmic body destroyed when it is crushed, although the resultant mass may contain the same substances, and the same amount of them, as before. This consideration, alone, clearly shows that the most detailed information concerning the substances found in protoplasm will be as insufficient to enable us to elucidate the mystery connected with vital processes, as the most complete chemical knowledge of coal and iron is insufficient to afford an explanation of the steam engine, or of the printing press which the latter may drive.

The chemical nature of the living organism, with its indissolubly connected chemical and mechanical properties, is of much greater importance than that of a machine; for in the changes which take place in the self-regulating protoplasmic mechanism, chemical nature and affinities are, in all cases, of fundamental importance. Hence progress in Physiology necessarily goes hand in hand with progress in Chemistry. From a physiological standpoint it is hardly possible, for example, to overestimate the importance of a complete knowledge of the chemical constitution of the proteids, which take so prominent a part in building up the living protoplast, especially in view of the possibility that each particular species may be characterized by a specific variety of living proteid. This need not however necessarily be the case, for the manner in which the building material is correlated and arranged is of equal 
significance and it is sufficiently obvious that instruments and machines widely differing in shape and function may be constructed from similar masses of iron or brass.

In explaining the relations between cause and effect, Physiology, like every other science: must determine the nature and properties of the different parts affected, as well as the accompanying external factors which may be involved. Both the character of the stimulus to action and the necessary mechanical means by which the resulting phenomenon is effected require to be known, and the more thoroughly a particular phenomenon is investigated, the more numerous are found to be the various related factors which originate and influence it. Nevertheless, an accurate knowledge of the more immediate causes which produce a given result often permits a further and deeper analysis to be made, and may thus afford a satisfactory preliminary explanation of the phenomenon in question, which may serve as a sound basis for further research. Science is based solely on properties and facts ascertained by experiment and observation, that is to say, on a knowledge that under given conditions certain results must necessarily be obtained. The physiologist who determines empirically the part played by each of a number of co-ordinated factors in producing a given phenomenon, does no more than the physicist, who frequently uses a quantity which is capable of being resolved into factors as the starting-point for a research, or the mathematician, who deduces logical conclusions from axioms which he has himself propounded.

We are procceding therefore by a perfectly scientific method when we explain certain movements as being due to the combined action of tissues, or to a disturbance of the relation between the elasticity of the cell-wall and the osmotic pressure which stretches it. Similarly the phenomenon of contractility, or the union of spermatozoid and ovum, may be safely uscd as a sure foundation for further and deeper studies into the nature of life.

Every scientific research, in its groping for ultimate causes, brings us to complex characters and properties, the entities of scholastic philosophy, which we are unable to further resolve. Cohesion, elasticity, gravity, are all regarded as fundamental properties of matter, although it is not impossible that the atom, and hence also its most essential attributes, may be capable of being resolved into still more elementary parts. Physiology, therefore, stands on precisely the same basis as any other science, although it necessarily accepts complex entities and properties as simple ones-as factors, that is to say, which at present cannot be further resolved, since the phenomena of life cannot be referred to the interaction of atoms and different kinds of energy as in the domain of Chemistry and Physics.

It is therefore not surprising that Physiology should be unable to 
explain the specific peculiarities of the organism by reference to its structure and chemical composition. The properties of any chemical combination are necessarily dependent upon the manner in which the individual atoms are linked together, but, even if the latter were known, the ablest chemist would be unable with certainty to prognosticate, although he might make a guess at, the properties of a new compound. The mind of man is as incapable of understanding the ultimate cause of a phenomenon, as it is of grasping the meaning of infinity; so that Newton was fully justified in saying that the seeker after ultimate causes shows thereby that he is not a truly scientific worker.

Everything that goes on in a plant, every movement or alteration of any kind, whether chemical or physical, involves a change of energy just as it does in inorganic matter; either potential energy being converted into kinetic, or kinetic energy into potential. At the same time, the nature of the causal relationship is such, that every vital process in the organism follows as a necessary consequence of its specific disposition and character. Just as an artistic pattern is woven in a fabric by progressively changing certain mechanical arrangements of a weaving machine, so also, in the vital activity of the organism, every change is a sure sign of an alteration in the microcosmic constellations which rule and govern it, independently of whether the change is due to internal causes, or to the operation of external stimuli. If in any given plant the ultimate structure and the sources of its energy, together with all its propertics and characteristics, were as well known as those of a weaving machine or a musical box, then, and then only, should we be able to understand all the phenomena of life as being the natural consequences of given conditions, just as we do in the case of a machine.

For the present, however, we must be content, if we can refer particular functions of the entire organic mechanism with sufficient accuracy to the agencies which initiate or influence them; nor can there be any doubt that precisely the same forces and laws are in operation in the veiled and hidden vital mechanism, as in the rest of nature. Just as we see how a clever mechanic can make the most various worlss of art with the same tools, so also, when we bear in mind the countless combinations into which the commonest natural means and forces may enter, it becomes comprehensible how the wonderful structure and mechanism of the living organism can be attained; indeed, we may even obtain some idea as to how it must have originated. A consideration of the final product alone will never enable us to determine how various and complicated the operations which have given rise to a particular result may be, nor whether they take place simultaneously, or successively.

There is no reason for regarding life as the product of an extraordinary and mystical natural force; it is to be treated simply as a special and 
peculiar manifestation of energy ${ }^{\top}$. He who considers our inability to grasp the essential nature of the vital mechanism to be sufficient ground for assuming the existence of a special vital force, must also permit the aboriginal Australian to endow with magical powers the musical-box or the watch which is totally incomprehensible to him. An intelligent person could without difficulty ultimately gain a clear comprehension of the mechanism of a musical-box or watch, and of the causes determining the particular performance of which it is capable, although it might be quite impossible for him to create such a mechanism, or to procure an historical account of its discovery and perfection. This example may suffice to point out the relationship of biological investigations to the phenomena which they are required to explain. Moreover, since we can only guess at the evolutionary history of the organism, it is only possible for us to deal with the physiological and other properties which it now possesses, and however clearly we may be able to explain the peculiarities of a given plant, as being due to characters and tendencies inherited from its parents, we shall still be unable to determine with certainty the evolutionary origin of that particular species.

In their respective aims and principles, all sciences meet on common ground. When we bear in mind the fact that the division of science into separate subjects is due merely to a convenient mental abstraction, it is easy to realize how worthless is any discussion as to whether Physiology and Astronomy are, for instance, subordinate to, or co-ordinate with, Physics and Chemistry. Each of these subdivisions of science has an equal right to claim independence. Physiology has, more especially, as its ultimate aim, the investigation of the meaning of the elements and forces which are at the disposal of the living organism and their importance in maintaining its vital activitics. This is probably the most difficult and complicated problem which the progress of creation on our earth has given us to solve. It is hardly necessary to point out that no fruitful investigation into the wonderful mechanism of the living organism is possible without a careful study of the simpler relations presented to us, and without the powerful support of Chemistry and Physics.

The chemists and physicists of a hundred years ago had not, and could not have had, the least idea of the telephone or of the various aniline dyes and the various uses to which these have been put during the present century. It is in the same way equally certain that our present knowledge of Physics, Chemistry, and other sciences is insufficient to enable us to realize all the permutations and combinations of the elementary forces and substances which are possible in the living organism, or the very varied

1 On Vital Force see Lotze, Wagner's Handwörterbuch der Physiologic, 18,22, Bd. I; du BoisRaymond, Reden, I867, Bd. II, p. 1. 
uses and purposes which these may subserve. The child, who has just learnt its letters, can hardly be expected to realize or predict the endless variety or combinations of words and sentences which may be constructed from the alphabet.

When therefore we employ the knowledge obtained from other sciences, it must never be forgotten that it is only by direct experimentation with the living organism that we can determine whether a physiological process is actually carried out by it in the way which our present knowledge of Physics and Chemistry renders probable. The reasoning of one who denies the possible ultimate reference of all that takes place in the organism to simple chemical and physical causes is as devoid of true logic as is that of the peasant who, on seeing a locomotive for the first time, argues by analogy that a horse must be concealed inside; and the same deficient logic is involved in the assertion from analogy with Chemistry and Physics that any final explanation of life is possible upon a purely physical and chemical basis alone, for, as is well known, similar, or apparently similar, final results may be attained by very different means.

The methods employed in Physiology are in 110 respect different from those employed in other sciences, and thus, in order to obtain a sufficiently broad comprehension of the phenomena observed, and to permit the establishment of general laws, it is essential that a comparative study of a great variety of different plants should be made. The morphological relationship and the common phylogenetic origin of plants and animals have long been established, and it is only owing to deficient inductive reasoning that there has been so long a delay in the recognition of the important fact, that there are no hard and fast physiological boundaries between the two kingdoms. Essential general relationships exist, not only in the metabolism of plant and animal, but also in the formative power of each. The question as to how far and to what extent psychical phenomena may be recognized in plants and in the lower animals receives in both cases a similar answer ${ }^{1}$. All the fundamental essentials of life are inherent in the undifferentiated protoplast, and even the highest plant or animal is at the earliest stage of its development, as a fertilized ovum, no more than a simple protoplast. No marked morphological differences are perceptible between the fertilized ova of different plants, but nevertheless very marked inherent differences in structure and disposition must exist even at this stage, although they only become perceptible in the process of development. In the protoplast a countless variety of possibilities lies dormant. With progressive development, associated with division of labour, individual functions become more prominent, as a high degree of differentiation is attained. As a general rule, however, when any modification for a special principal function takes place, the processes

${ }^{1}$ See Pfeffer, Die Reizbarkeit der Pflanzen, 1S93, p. 30. 
subservient to this function are less obscured by the other vital processes and functions, and therefore can be more clearly perceived. Consequently the study of such specialized functions is of the utmost importance, and may throw light upon the nature of the vital mechanism. It is of almost equal importance in this respect that we should recognize that, even in unicellular organisms, powers and properties of very different degree of physiological differentiation may be present.

\section{SECTION 2. The Aim of Physiology.}

In the previous pages the aims and purposes of Physiology have been briefly indicated. Regarding them from a gencral point of view, these are to study the nature of all vital phenomena in such a manner, that by referring them to their immediate causes, and subsequently tracing them to their ultimate origin, we may arrive at a complete knowledge of their importance in the life of the organism.

A physiological problem is connected with every manifestation of life. Many phenomena, however, the morphological aspects of which are familar to us, are imperfectly understood from a physiological point of view, and consequently anything like a complete and perfect treatise on Physiology is at present impossible. We cannot, however, leave unmentioned those processes concerning which a certain amount of physiological knowledge has alrcady been obtained, and hence the present volume gives a general account of the sources and means by which plants obtain food and energy, the modifications and changes which the food and energy thus obtained may undergo, and the manifestations of energy which find visible expression in plant life and growth.

It is the province of general Physiology to investigate what are the cssential factors which influence, or actually cause, the definite phenomena that come under our observation, as well as the precise nature of the relationships between them. When once these are clearly established, some idea of the relative importance of the various phenomena may be obtained, which in the absence of this preliminary knowledge is impossible. A textbook of general Physics treats of the properties of steam and electricity, but is not called upon to describe every machine that the wit of man has ever devised ; similarly, it is not, nor can it ever be, the task of general Physiology to describe in detail the peculiarities of single plants or even of special groups of plants. This is the aim of a monographic study. It would consequently be beyond the scope of this book to give a special account of the details of the metabolism of Bacteria, or of the processes of fertilization and development. These and other phenomena will be dealt with only so far as they are connected with, and illustrate, the essential principles of general Physiology.

Since all our physiological knowledge must necessarily be based upon 
information derived from existing species, we are justified in neglecting the problems connected with phylogeny and descent. Neither is it within our province to describe the relations subsisting between the plant and its environment, or its struggle with the variable and varying external conditions presented to it. In order to determine the relative importance of particular factors, the experimental physiologist must work under conditions which he holds in more complete control than he can the varying and multifarious influences operating in nature. The true scientific observer must, however, always submit the results obtained within the narrow walls of the laboratory to the test of nature, our great and inexhaustible mistress and teacher, in order to find out how far the knowledge thus obtained agrees with, and explains, the manifold phenomena which she presents to us.

The sum of our knowledge with regard to the vital economy of the plant, and its relations with, and adaptations to, its dead and living, organic and inorganic surroundings, may be termed plant economy or Bionomy ${ }^{1}$. It is permissible to ignore such relationships when our attention is directed only to the aims and purposes which the plant has in view. Owing to our ignorance of the exact causal relationship of the different phenomena observed, a teleological explanation becomes more and more necessary, and if properly used it is in its way not only perfectly justifiable, but also capable of aiding discovery and stimulating inquiry, when applied to natural phenomena.

It must, however, never be forgotten that the purpose of any given phenomenon can only be determined by an external observer on the basis of the facts which come under his notice. Ideas of purpose being abstract mental conceptions, are not and can never be the direct causes of anything that takes place in the plant. It is therefore always the object of Physiology to investigate the ways and means by which, under certain external conditions, and with varying internal dispositions, some particular final result is produced ${ }^{2}$, and to trace the chain of causes which lead to this result.

The different changes and processes which go on in a living organism must necessarily have a purposeful character. A plant can only live and continue its development when the external conditions are suitable

1 In former times Aristotle, and nowadays Spencer and others, use the term 'Biology' to include the whole of the phenomena with which life confronts us. It seems worth while to retain for the word this general meaning, and to call, as Haeckel does (Generelle Morphologie, 1866, Bd. I, p. 8, Bd. II, p. 236 ; Systematische Phylogenie, 1894, Bd. 1, p. 386 ), the principles of plant economy, to which the name of Biology is sometimes given, Oecology or Bionomy. From an historical point of view, Physiology and Biology are identical terms. Nevertheless, it is justifiable to restrict the term 'Physiology' as applying only to the attempts at a causal explanation of vital phenomena.

${ }^{2}$ On these questions, see B. Lotze, Mikrokosmos, Bd. I; Lange, Geschichte des Materialismus, 2. Aufl., Jd. I, p. 13; Wundt, System der Philosophie, 1869, p. 318. 
to it, just as a fish, adapted only to a life in water, is unable to live in air. Whatever may have been the primary causes of the varied character and dissimilar inherent characteristics of different plants, throughout their genealogical history in successive geological periods, we may feel assured that only such modifications as have been of service to the plant have been retained, and that all purposeless or disadvantageous ones have been gradually eliminated. This simple but comprehensive principle was expressed with perfect clearness by Empedocles more than 2,000 years ago ${ }^{1}$.

\section{SECTION 3. The Nature of Irritability.}

It has already been mentioned that it is in virtue of its inherent disposition and special structure that an organism is able to produce from the same sources of energy all the varied manifestations which are characteristic of its own species and of life in general. In the same way, the kind of work which a machine performs is not affected by the amount, or even by the quality of the coal it consumes, provided only that a sufficient supply of energy is rendered available. The nature of its component parts, and the manner in which they are related to one another, are the all-important factors, and the tension of the same coiled spring may either direct the regular mechanism of a clock or evolve a tune from a musical box. Similarly, the results which the liberation of chemical or physical energy may produce in any given protoplast are entirely dependent upon the inherent specific characters of the latter.

In addition, therefore, to a knowledge of the sources of energy ${ }^{2}$, we require to ascertain the nature and character of the mechanism to which this energy is applied, before we can understand how the causes at work produce the observed mechanical results. In the living organism not only has the nature of its mechanism to be ascertained, but also the processes by which it may obtain energy, for the liberation of energy may produce much more varied results in a living mechanism than in an ordinary machine. Thus an organism may induce, accelerate, inhibit, or modify any given vital process, and indeed in this manner it acts as its own essential regulatory mechanism by causing the different vital processes to be correlated with one another. When we speak of manifestations of irritability as vital processes which involve a liberation of energy ${ }^{3}$, we

1 Lange, 1.c., 1873 , Bd. I, p. 23.

${ }^{2}$ See Pfeffer, Energetik, I 892 , and also vol. ii of this book.

"Ostwald (Ber. d. Sächs. Ges. der Wissenschaften, Leipzig, I 894 , p. 338) speaks of 'liberation of energy' in a more special sense as applying only to what takes place when a new chemical action is induced. An acceleration of a chemical reaction he regards as a katalytic phenomenon. It is frequently impossible to determine to which of these a given physiological reaction is due. Indeed, it is not improbable that these two headings are not broad enough to include all the different means by which physiological reactions are produced. 
thereby definitely imply that we recognize them as the result of the specific character of the organism, and as due to the particular combination of natural forces which it represents ${ }^{1}$.

We are necessarily led to this conclusion by the arguments that induced us to reject the assumption of the existence of a special vital force. Our knowledge of the essential nature of the living mechanism is extremely meagre, and it is hence not surprising that we know very little about the manner in which its manifestations of irritability originate. For this reason it is often doubtful to what degree, and in what respect, a particular vital activity is a manifestation of irritability.

Such phenomena as the bending of a branch by the weight it supports, or the distension of a cell by turgor, are directly due to mechanical forces, but they may nevertheless be indirectly brought about by the operation of an appropriate stimulus, influencing either the turgidity of the cell, or the weight of the branch. The stimulus is a push as it were, to which the organism responds according to its inherent nature and the means at its disposal. Just as a ray of light may induce a man to use his locomotory apparatus and go from darkness to the light of day, so also may light influence the movements or direction of growth of plants; for in plants rooted to the soil the part above ground may bend and grow towards the source of illumination, while in those which are free swimming the entire organism may respond by a movement of translation in the same direction.

Stimuli may be external or internal in origin. In the latter case they are said to be autonomous, and arise from some obscure change in the vital activities of the plant. External stimuli, on the other hand, always act inductively, producing internal changes which may or may not be visibly manifested. They are hence often referred to as inductive stimuli ${ }^{2}$.

When the stimulus is internal in origin, it is usually less apparent than when it is external; in the latter case its intensity may be varied at the will of the observer, and corresponding changes may be noticed in the result produced.

An internal stimulus may, in some cases, be as definite and clearly determinable as an external one. This is the case when a particular reaction can be traced to the production of an enzyme by the organism,

1 From this point of view an exact and scientific comprehension of irritability is possible. Since this is simply a special manifestation of energy, all attempts to prove that the existence of irritability involves properties and forces totally foreign to the inorganic world must necessarily fail. A short account of the progress of our knowledge and idens in this direction will be found given in Die Reizbarkeit der Pflanzen, I $\$_{93}$ (Verhandlung der Gesellschaft d. Naturf. und Aerzte). In the first edition of this book, as well as in Osmot. Untersuch., 1877 , p. 202, the same general principles, as regards the nature of irritable processes, have been previously brought to notice.

2 Sometimes called 'trophic stimuli.' 
or when the shape of a given member can be shown to be partially due to pressures arising automatically during the process of development ${ }^{1}$. In the case of both internal and external stimuli, it is possible to distinguish between thermal, chemical, mechanical, photic, and electric stimuli, according to the character of the opcrating agency.

Manifestations of irritability are a fundamental property of the vital mechanism, and there is perhaps no vital process which is uninfluenced by stimuli, which permit, cause, restrict, or regulate, the particular action in question. Irritability is indeed a general characteristic of all living substance, and is exhibited by the lowest as well as the highest forms. By the development of specific and special forms of irritability, a plant is enabled to react in relation to the external world in the most various ways. Particular manifestations of irritability are also essential in order to regulate and connect the various parts of the vital mechanism, in the developing organism as well as in the adult one. Thus the progress of any given vital activity continually gives rise to stimuli, now accelerating, now inhibiting, the process in question, and so regulating it; while, under particular conditions, either the original activity may be gradually modified, or new activities may be aroused. All phenomena of correlation are brought about in this manner, that is to say, by the operation of regulating stimuli reaching to all parts of the organism. The regulatory mechanism of all complicated machines is similar in character. Thus in the marine engine, the slide- and safety-valves, the eccentrics, and governors, the condensers and feed-pipes, are all automatic in action, and the whole operates more economically and harmoniously than if it were built up of a series of engines, one for each action, all working together, but each one distinct from the others.

Every result which follows stimulation is a manifestation of irritability; it is immaterial from this point of view whether a noticeable movement immediately occurs, or whether there results merely some obscure chemical change, days or weeks after the stimulus has been applied. It is only when perceptible results are produced that irritability can be measured or determined. A moth, flying towards a candle, reacts to the stimulus of light by movement in a particular direction, whereas an attached plant responds by gradually bending towards the source of illumination. In both cases the sign and result of the operation of the given stimulus is the production of a definite movement.

In the most varied and general physiological problems, questions connected with irritability are constantly and continually arising. The clear

1 It is incorrect to denote as the primary cause, either of a reaction, or of the liberation of cnergy for that reaction, what may be really a single link in a chain of causes. According to Clifford, Ilato uses the word 'causc' in sixty-four different senses. See also Sigwart, Logic, I 895 , vol. ii. p. 93 . 
recognition of this fact is of great importance, because until recently the part played by stimuli in all vital phenomena has been overlooked; it must also be remembered, however. that what actually takes place cannot always be definitely referred to the dircct action of the operating stimulus. There can be no doubt, for example, but that the changes of form which are exhibited by plants kept in darkness are a manifestation of irritability, and are due to the absence of light acting as a stimulus, which causes the plant to respond in a manner determined by its inherent nature and the means which it has at its disposal. Every stimulating action of light upon the plant is similar in character. In the same way, when a plant, whose growth has been checked owing to the lack of potassium compounds, is awakened to renewed activity by a supply of the deficient salt, the action is always a stimulating one, whatever may be the nature or equivalency of the changes which the salt at once undergoes in the metabolism of the plant.

Our knowledge of the physiology of irritability is very incomplete. It is, however, possible to realize the general principles which govern it, by reference to the laws ruling mechanical contrivances made by the hand of man. In every machine it is the particular structure and properties which determine whether or not a reaction will be prociuced, and what will be its nature. A finger pressure produces an effect only when it is applied to a particular part of a musical-box, and the same finger pressure may start a steam engine, ring an electric bell, fire a mine, or cause a musical-box to play a tune. In the same way, all plants do not necessarily respond to a given stimulus, nor do they react in a similar manner to the same stimulus. Thus a momentary touch causes the leaflets of Mimosa to fold together, while prolonged contact causes Cuscuta to form haustoria, and the tendrils of Ampelopsis to form suckers.

That different plants should respond differently to the same stimulusas, for example, that one plant should behave geotropically and another ageotropically under the influence of gravity-is not more wonderful or mysterious than that the same propulsive force should drive a locomotive either forwards or backwards, according to the way in which it operates upon the working mechanism.

It is not difficult to realize that there is no essential relation between the stimulus and the result, either in character, or in the amount of energy which each represents. The smallest spark, by igniting a mass of powder, produces an enormous mechanical effect. Similarly, the opening of the steam-pipe supplying an engine always causes the latter to convert the same amount of the energy of the steam into the same kind of motion, and to do relatively the same amount of work. This would still be the case even if the work done in opening the steam-cock were greater than the work which the amount of steanı supplied to the engine would enable it to perform. 
Nor is it necessary that a stimulus should always invoke either an immediate response, or a liberation of all the available energy, as when gunpowder explodes, or when the leaflets of a shaken sensitive plant rapidly fold together. On the contrary, an increase in the intensity of the stimulus is commonly followed by an increase in the energy of the response, just as when an engine is driven more and more rapidly as the steam-cock is gradually opened. Similar purposeful relations exist in the plant between the intensity of the stimulus and the amount of response, as is especially well shown by those movements which increase in rapidity and extent with increasing photic, thermal, or chemical stimulation.

No increase in the response is possible above a certain point, and this is determined by the capabilities of the responding mechanism. Machines may be constructed, which can regulate themselves as plants do, or which may go more and more slowly as the driving force becomes stronger and stronger, and may finally stop when a certain limit is passed. Probably it is a similar action which causes the leaflets of the sensitive plant to close up again if exposed to full sunlight, although when brought from darkness into diffuse daylight they pass from the nyctitropic to the fully expanded condition. The fact that in many cases the paraheliotropic and nyctitropic positions are markedly different, shows that the character as well as the amount of the response may be changed, as soon as the stimulus reaches the necessary intensity. As a general rule, the graphic curve, representing the response to increasing stimulation, rises to a maximum or point of optimal stimulation, beyond which it descends.

Every stimulus must reach a certain minimal intensity in order to produce a perceptible result. Above this point, the effect which the stimulus produces may become noticeable at once, or only after a latent period. Either a rapid or a slow response may accordingly be produced, which may then slowly or quickly pass away again. An analogy is afforded by a clock, which set going by a shake (stimulus), after a given time (latent period) rings an alarm (result). The clock stops as soon as the energy of the coiled mainspring is exhausted. A frozen plant, on the other hand, when restored to vital activity by being thawed, develops its own propulsive energy in a self-regulatory manner, if supplied with nutriment.

It is possible to construct machines in which the same stimulus can produce several different results, directly or indirectly, simultaneously or successively. For the maintenance of the regulatory mechanism of the plant the transmission of stimuli is an absolute necessity, and the electric telegraph affords an illustration of how the effect produced by a stimulus may take place at a distant point.

In every manifestation of irritability, the stimulus and the response must always be clearly distinguished from one another. An irritability, i. e. a power of reacting in a specific manner, is made evident only by the 
production of some definite result. The process of stimulation involves a series of changes due to interaction between the stimulus applied to an organism, and the percipient part affected. A careful distinction must be made between the reception of the stimulus, the series of changes which then take place, and the final result they produce, regardless of whether we are or are not able at present to directly investigate them. In many organs the sensitive region and the responsive tissue are some distance apart; thus the root-tip perceives the stimulus which induces a geotropic or hydrotropic curvature in the regions some distance behind it, while in Drosera the stalk of the tentacle curves, but only the head is sensitive to contact. In all such cases the stimulus must be transmitted, and this transmission of stimuli is the means by which different parts of the plant are linked together, and by which their respective functional activities are correlated with such wonderful accuracy, however widely separated the different parts may be. The conduction of stimuli is probably a very complicated phenomenon, and may take place in a variety of ways. Mimosa pudica, however, affords a case in which the transmission is effected simply by a movement of water. This is probably in correlation with the fact that here the result of the stimulation is a simple physical movement, and it often happens that non-living parts may co-operate with living ones in producing such a manifestation of irritability.

A child who, by pressing a button, causes a musical-box to play a tune, either immediately or after an interval of time, may be quite incapable of understanding the different mechanical contrivances by which the result is produced. If the entire mechanism is hidden from him, he is naturally unable to tell whether the pressure he has applied acts directly upon the musical-box, or indirectly, as by closing an electric circuit, or by setting a clock in motion which at a given moment may cause the alreadywound-up musical-box to play. Whether the mechanism is purely physical, or involves chemical reactions as well, is obviously equally hidden from him, and at the same time the tune which he hears affords not a particle of evidence as to whether the energy which operates on the mechanism of the musical-box is supplied by a falling weight, by hydraulic means, or by steam power. Nor will the most perfect knowledge of the exciting stimulus and the final result which it produces, reveal the complicated series of changes and interactions which may intervene between them. We cannot be certain that the same result is always produced in the same way, and indeed a given stimulus frequently produces different results on different plants, while, on the other hand, a particular response may be produced by different stimuli.

Hence it is easy to understand why we cannot always tell, when the result is different to what was expected, whether this is due to an alteration in the percipient organ or in the processes subsequent to stimulation. Thus, 
although the apex of a root may be fully sensitive, no curvature can take place if no further growth is possible, or if the curving region is imbedded in a gypsum cast. The stimulus is perceived, but no response can be exhibited.

Every plant, and even every single organ, is sensitive to more than one stimulus. In addition to the special functional irritability with which the protoplasts of any organ may be endowed, a more general irritability must also exist, by means of which the processes essential to life are carried on and regulated. Both the general and the special irritability may be affected simultaneously, and thus a multiple response be produced; as when traction applied during a geotropic curvature causes an increased thickness of the cell-walls affected, or when an injury produces an increased respiratory activity or causes streaming movements of the protoplasm in the cells of the curving organ. Similarly, light may induce formative changes in the part illuminated, in addition to the heliotropic curvature which may be induced.

It is clear that a special manifestation of irritability for which a particular organ is especially adapted and intended may be brought into play by many different stimuli. Thus either mechanical, chemical, or photical stimulation may cause the leaflets of Mimosa pudica to fold together, but the more specially adapted the organ or organism, the greater will be the tendency to produce a particular response. It is therefore permissible to speak of 'specific irritability' 1 when we intend to indicate such adaptive preference. An organ or organism could not possibly continue to exist if it responded to all stimuli only in one and the same way.

Thermal, chemical, and photic stimuli may not only all cause in a stem the same kind of curvature, but may simultaneously excite more general irritabilities, and produce other results varying very widely in character. Moreover it is self-evident that a plant which is attached to its substratum can only move towards the source of stimulation by effecting a curvature, as is the case, for example, when it bends towards the incident light.

All organs in which a particular stimulus produces the same definite result are said to be endowed with a special irritability, independently of whether one or more of such irritabilities are present. It is only in this way that we can understand why different plant organs react only to particular stimuli, although they may all have the same powers of movement. Thus one organ may be sensitive to geotropic, heliotropic, and hydrotropic stimuli, another only to geotropic, while a third may only respond to heliotropic stimulation.

Irritability depends as little upon external differentiation as does life itself. Even the lowest plants cannot be considered as inferior to animals,

1 This applies not only to the sense in which the expression is used by Johannes Miiller, but also to its usage in connexion with higher animals. 
either as regards their sensitiveness, or the varied nature of their different irritabilities. Man, for example, is unable directly to perceive the ultraviolet rays which many plants readily detect, and to which they markedly respond.

The excitation of any given irritability by a stimulus may produce either a transitory or a permanent effect. In the former case, as is well illustrated by the sensitive leaflets of Mimosa, as the effect of the stimulus passes away, the self-regulatory mechanism induces the organ to return to its original position of equilibrium; whereas in the latter case a change in the external or internal conditions produces some permanent adaptive alteration, calling forth a new condition of equilibrium suited to the changed conditions ${ }^{1}$. Thus, when a heliotropic curvature is produced, if the direction of the incident light and other external conditions remain constant, the stem adopts the new direction of growth, and as the mechanical tissues differentiate the curvature is rendered permanent.

Similarly, a continuous stimulus produces a continuous effect when a rise of temperature causes the recommencement of growth in a plant which has been in a condition of cold rigor, or increases the rapidity of growth where previously it was slow. The rise of temperature acts only indirectly as a stimulus, arousing or accelerating the processes of growth, which the plant itself regulates, and which are possible only when a supply of energy is available. The direct action of a rise of temperature is shown in the case of a fire, when the heating of the coal to combustion-point incites a renewed liberation of energy; but a marked direct action of heat can, however, hardly be possible in a plant, considering how low is the highest temperature to which it can respond.

The continued stimulating action of warmth is as essential for the maintenance of the life of the plant as a continued light stimulus is necessary to induce and maintain a heliotropic curvature. A progressive diminution in the intensity of light produces a decreasing and finally imperceptible effect, and similarly as the temperature is lowered, so also does the vital activity which is dependent upon it diminish and ultimately cease.

The action of a stimulus is perceptible only during the transition from the previous condition of equilibrium to the new one, and the plant may remain stationary in this new condition of equilibrium, although the stimulus continues to act. Indeed the stimulating action of a certain temperature, and of other agencies as well, is an absolute necessity for the continuance of life. This view was first correctly enunciated by Johannes Müller ${ }^{2}$; and the lack of recognition it has received may be taken as a sign that a clear

1 Transition stages exist between these two types.

${ }^{2}$ J. Müller, Handbuch d. Physiologie d. Menschen, 1844,4 . Aufl., p. 2S. [John Brown Elements of Medicine, London, 17 SS regarded life as the sesult of an aggregate of stimulatory reactions.] 
and comprehensive grasp of the phenomena of stimulation and irritability has not as yet been universally attained.

In the same way potassium salts and compounds of nitrogen are also to be regarded as essential stimuli to vital activity. This stimulating action is especially marked when a plant, impoverished and stunted for want of nitrogen, is incited to rapid development and growth by a supply of saltpetre. Owing to the reciprocity between different parts of the vital mechanism, every vital process involving a chemical change or a transference of energy, radiates kinetic energy in some form or other, which either acts as a stimulus to increased activity or calls fresh activities into existence. Thus, by respiration-that is to say, by the stimulus created by the consumption of oxygen-not only is a supply of energy ensured, but also the irritability of the plant, its power of response to stimuli, is directly or indirectly maintained. In many cases, though not necessarily in all, the stimulating agent may enter into the metabolism of the plant, or take a direct part in the production of the result which it primarily induced ${ }^{\mathbf{1}}$.

This inductive action of a stimulus is by itself insufficient to produce any result, for in every case the organism must have at its disposal the necessary supplies of constructive material and energy.

As development proceeds the continued vital activity of the plant may give rise to a series of progressive disturbances of equilibrium which are continually readjusted, and these may finally result in marked changes of character and disposition, even although normal and constant external conditions are maintained. Such automatic variations are to be regarded as the result of internal stimuli, such as arise in every plant even under perfectly normal and constant external conditions. Reactions taking place in relation to changes in the external conditions are to be regarded as the means by which the inception of an adaptive modification is rendered possible.

In the progress of development a change may take place in the inherent disposition which determines the character of the response to any given stimulus, and this disposition is also liable to modification by the operation of external agencies. Consequently the phenomena to which a particular stimulus will give rise may be affected by antecedent or simultaneous stimulation, influencing or altering the irritability which the first stimulus excites. The intimate correlation of the entire vital mechanism renders it probable that every excitation exercises some effect upon other manifestations of irritability, even though this effect may not always be directly perceptible.

${ }^{1}$ Hüppe (Verhandlungen d. Ges. deutscher Naturf. u. Aerzte, I893, p. 154) is certainly in error in stating, as a general character of a stimulating action, that the operating stimulns supplies the additional energy necessary for the inception of the result produced. See Pfeffer, Jahrbuch f. wiss. Bot., I 89:, Bd. xxvin, p. 239 . 
The same applies to the actual irritability itself, for this is dependent upon particular conditions, and changes as these alter. Below a certain minimum or above a certain maximum temperature irritability ceases to be manifested, and within these limits a change of temperature may so alter the character of a particular irritability as, for example, to cause a negatively heliotropic curvature to be produced instead of a positively heliotropic one.

Speaking generally, it is immaterial whether such a modification of a particular irritability may take place under normal conditions, or be induced by accidental and non-essential external stimuli. The character of the work which a machine is capable of performing may be altered in a similar manner. Thus a change in the position of the revolving cylinder of a musical box causes a different response to be given, and a different tune to be played when the handle is turned.

It is only possible here to give a few examples of the varied and reciprocal interactions of physiological stimuli. Thus mechanical injury causes the primary leaf of Avena to lose for a time its sensibility to heliotropic stimuli. Removal of the terminal bud of a fir-tree induces the nearest lateral branch to assume a marked apogeotropic irritability and become erect; while in many rhizomes illumination causes diageotropic irritability to be converted into geotropic. Again, in certain plants, in order that the periodic movements dependent upon the alternation of day and night may be continued, one of the necessary conditions is that a certain inductive action of gravity shall be allowed to act upon the plant. Moreover, it is very commonly the case that with an increasing intensity of a chemical or other stimulus, the sensibility of the plant diminishes in a corresponding ratio (Weber's law), or becomes modified or completely altered: thus it is well known that many forms of irritability may be weakened or inhibited by the action of ether or chloroform.

Development and vital activity, together with the alterations of irritability to which they may give rise, may be stimulated or modified by factors of very special nature, although they are necessarily dependent upon the maintenance of the essential general conditions: thus, when the young shoots are killed by a spring frost, the more deeply situated reserve buds are so stimulated that they are induced to develop and exchange their passive mode of life for one of marked functional activity. Modifications iiduced in this manner are of the utmost importance to the plant, and often form the means by which a power is acquired of reacting in an appropriate manner to new conditions. When we remember the varied nature of the results which the plant needs, it is hardly surprising to find that such modifications may either increase or dininish a pre-existent irritability, or may even call new irritabilities into existence. Furthermore, it is well known that the powerful stimulation of a particular sensitive organ, or any 
intense concentration of energy directed towards a particular aim. may produce a marked decrease in sensibility to other stimuli.

The change to the new conditions of irritability takes a longer or shorter time; that is to say, a latent period of response always intervenes between a stimulus and the reaction. Accommodation to a change of temperature or to the influence of certain chemical reagents is very rapid, but in many cases, from the length of time which elapses before the end result is produced, it may be concluded that the induction of the new irritable condition takes place but slowly. During this period the after-effect of the original external conditions is being gradually overcome. In case of a reversion to the original conditions, a longer or shorter after-effect will always be perceptible, provided that the new condition of irritability is capable of retrogressive modification, and has not become inherently fixed. An example of the latter is afforded by the permanent bilaterality which is induced in the thallus of Marchantia when the developing gemmae are subjected to unilateral illumination. As a matter of fact, a modification of the inherent characteristics is induced whenever either an internal or an external agency operating upon an organism causes it to assume any properties, however unimportant, which were formerly absent. so that it attains a power of responding differently to special stimuli.

When a plant is again exposed to the conditions under which it previously existed, it may, or may not, revert to exactly the same condition of irritability as it previously exhibited. Such a return to the original condition does actually take place after the leaflets of Mimosa have been caused to close by mechanical stimulation, and the same is also the case when growing seedlings are stimulated, provided that no permanent fixation or alteration has been produced by subsequent growth. When the normal position of the dorsal and ventral surfaces of fern prothalli or of branches of Thuja is reversed, a corresponding change in the bilaterality is induced, but only in the new growths formed under the changed conditions.

From what has already been said, it follows that the result produced by two stimuli acting simultaneously is not simply the sum of the results which they would produce if they acted separately. This is not the case even when the nature of the response is such as to permit of a summation being possible. Whether two stimuli act simultaneously or successively may be of great importance in determining the precise nature of the final result.

We shall be pursuing, therefore, a correct method of investigation if we study the effects produced by a single or a few stimuli under otherwise constant conditions. In the study of irritability we can distinguish between simple and complex stimulation (Induction), or may 
speak of isogenetic or heterogenetic induction ${ }^{1}$. The nature of any given irritability, and hence also the response made to a stimulus, are, as a matter of fact, always influenced by previous stimulation and induction of either general or special character. As in the case of every vital phenomenon, each manifestation of irritability is dependent for its production upon a variety of factors, of which the actual stimulus forms but one.

Both real and imaginary machines afford instances of the manifold nature of the response possible, and of the self-regulatory action of which the different parts are capable. For in many machines, as well as in plants, the different parts of the entire mechanism may act as regulatory stimuli to one another when the whole is at work, so that the ultimate result has a very complex origin.

There can be no doubt that the wonderful correlation existing between the different vital activities is directly due to the complex interactions between the various forms of irritability and the excitatory stimuli.

A knowledge of the essential principles governing the endless variety of possible phenomena of irritability can hardly be obtained by reference to those manifestations which are of peculiar and special character, although the same general principles which have already been established as applying to irritable processes in general, may in these cases also be recognized. The remarkable and complicated phenomena of accommodation are examples of special and peculiar manifestations of irritability, and are in general to be regarded as due to the gradual transition to the new condition of equilibrium which is established by means of the self-regulatory vital activity. Thus it is by means of such accommodatory reactions that plants may gradually be accustomed to doses of poison that originally would have proved fatal, or that a branch may be enabled to resist mechanical strains which would have broken it if suddenly applied. Another example is afforded by the fact that plants which receive a deficient supply of oxygen or nutriment pass after a time into a special condition in which the different vital processes are performed more slowly and economically.

These examples suffice also to show that it is not always immaterial whether the change of conditions takes place instantaneously or only

: These words are used in a different sense from that in which Noll uses them (Heterogene Induction, 1892, p. I4). He fails to realize the general character of heterogenetic induction or the universal importance of stimulatory induction (see Pfeffer, Die Reizbarkeit, \&c., 1893, p. 22). It is neither justifiable nor correct to apply this term only to certain specially noticeable examples of heterogenetic induction, and neglect all the commoner and more general forms of stimulatory induction. Moreover, it is impossible to draw a sharp distinction between the general and special essential conditions, for in any irritable manifestation the action of an additional and unnecessary stimulus becomes essential for the production of the result in its new and modified form. These remarks apply also to the ideas put forward by Herbst, IBiol. Centralbl., I 894 , Bd. xir, p. $73^{2}$. 
gradually. The effect of a blow is different from that of a slowly increasing pressure, not only from a mechanical point of view, but also in respect to its stimulatory action: thus a steel band may break when suddenly bent, although it may undergo a very marked curvature without breaking when the pressure upon it is gradually increased.

It is not surprising that the mechanical or stimulating effect of a high temperature, want of nourishment, \&c., may be resisted for a short time, although a more prolonged action may produce injury or death. Indeed death inevitably supervenes, if the conditions are such that growth and increase are impossible.

As has been already pointed out, every vital manifestation is dependent upon the specific nature of the living organism. and it is the inherent characteristics and peculiarities of the organism which determine whether any response to a given stimulus is possible, and the character that this shall assume. In the same way, results which have been primarily induced by external influences are finally determined by the inherent internal properties of the organism, independently of the fact that the interactions between the external and internal conditions may either have been merely mechanical or of the nature of co-ordinating stimuli.

A particular combination of external conditions is essential for the continued existence and for the vital activity of the plant. Within these physiological limits, all external conditions influence every activity of which the plant is capable in a manner corresponding to their intensity and duration. The same general relationships exist between the causes of a given change and the nature of that change, in every substance, living or dead: thus it is the inherent properties of a piece of iron, or of a machine, which determine whether a physical or chemical agency will produce any effect upon them, and what the action will be. Hence any alteration in the result obtained is an infallible sign that, in some way or other, an alteration of the original conditions of equilibrium has taken place.

In living organisms, and also in the developmental history of our planet, much less has been achieved by sudden, and hence more conspicuous, actions or changes, than by more gradual processes which, although imperceptible at any given moment, are nevertheless able by their continued action to produce final results of altogether disproportionate magnitude.

Cases in which the reaction is rapid or instantaneous, as when the leaflets of the sensitive plant fold together, are of more special character, and such reactions are of less general importance than the numerous slow and gradual regulatory reactions which are constantly occurring. Since stimuli are responsible for the production of the necessary supply of energy, usually solely by calling the self-regulatory mcchanism into action, and as 
they only indirectly govern whatever changes they initiate, it follows that the actual energy of an applied stimulus need neither be great, nor bear any direct relation to the magnitude of the changes induced by its application. A supply of energy must have becn previously accumulated in the responding organ, and the stimulus acts upon this stored energy like a match applied to a barrel of gumpowder. Nor is it necessarily an essential preliminary to a response that the responding organ should be in a labile condition, or be in unstable equilibrium ${ }^{1}$.

Since it is the living organism which forms the subject of all physiological study, the meaning, importance, and action of the factors which constitute the extcrnal world may be most conveniently studied in connexion with the properties and functions of the organism on which they are brought to bear. That is to say, it is wholly incorrect in principle to commence with the external environment, and describe in succession the different physiological reactions produced by each external agency. Probably no external agency is absolutely inactive upon plants, and indeed, with the sole exception of magnetism, every form of energy with which we are acquainted exercises some physiological action or other.

External agencies either act as stimuli producing disproportionate results, or else they originate interactions in which the transfer of energy from agent to organism takes place in definite ratio and equivalent amounts. But it is also possible to regard the external factors and the results they produce from other points of view; for example, from that of the purpose aimed at, the value of the reaction to the plant, the form which the reaction assumes, or the way in which stimulus, reaction, and final result are linked with one another. Since, however, none of these methods introduces any new principles, it is needless to discuss them further.

\section{Section 4. The Causal Relationships of Growth and Development.}

Hitherto we have been considering isolated functions, but the same general principles which govern these apply also to the functional complex which constitutes an organism, in every stage of its development, and hence are applicable to all the processes involved in the successive stages of its ontogenetic progress. The process of development is, indeed, extremely complex, and involves such a linking together of causes and effects, that automatic changes of disposition and correspondingly altered activities are being continually produced and propagated.

That some such causal relationship does actually exist is a necessary postulate, although our present knowledge is insufficient to enable us to directly refer the progress of development, which results in the production 
of an individual of specific shape and character, to the interactions of certain dispositions of which the adult organism in question is the final result. We must, therefore, take as an axiom the principle involved in the statements, that from an acorn nothing but an oak can develop; that the leaf of an oak has become once and for all different from that of a beech; that the root of the beech-tree develops differently from the shoot; and that, in general, the specific shape and nature of the organism are inherited properties, or, in other words, that the characters of the parents will be repeated in their offspring.

Owing to the influence which interaction with the external world exerts, the shape which the plant or any of its parts assumes may vary within certain limits, according to the conditions under which the plant develops. Variation is possible only within certain limits, determined by the extent to which the inherent factors which regulate growth and development may be modified by external agencies. These inherent factors may be termed 'historic or inherited characteristics,' 'specific formative energy,' ' tendency to assume a special shape,' or 'automorphosis' ; but in all cases, provided the continued existence of the organism is possible, the essential features of its inherited characteristics always become manifest whatever the external conditions may be. Individual variations, which may occur under special conditions, are not repeated in the offspring, when these are grown under quite different external environment. This principle is one of general application, although it is especially evident in morphogenetic or formative processes, to which special attention may therefore be paid. Nevertheless, it must be remembered that every morphological variation is an infallible sign that a corresponding modification of the metabolic processes has been initiated. Since every physiological process is dependent upon both internal and external factors, the shape of any organism may be regarded as being due to the interaction of automorphosis and heteromorphosis ${ }^{1}$.

Every local or individual variation due to the influence of a special habitat is the visible sign of a heteromorphic action. In certain plants, endowed with marked powers of reaction and accommodation, the changes thus induced may be so pronounced that, unless various transition stages were known, the extreme forms would be classified as distinct species. It is sufficient here to recall the aquatic and terrestrial forms exhibited by many plants, as well as the peculiar shapes assumed by many fungi and algae when grown in concentrated nutrient media, or by certain fungi when excited to fermentative activity. Moreover, certain algae may be com-

1 To indicate the changes of shape induced by the action of external agencies the term 'heteromorphosis' or 'xenomorphosis' may be employed. Sachs used the term 'mechanumorphosis' in the same general sense, but Herbst (Biol. Centralbl., I 895 , Bd. $x v, p p .37-39$ ) has since used the latter term in a more special sense, as referring to the results produced by pressure and tension. 
pelled to reproduce either entirely asexually or entirely sexually, according to the conditions under which they are cultivated. In such plants, unless the appropriate variations of the external conditions took place, no alternation of sexual and asexual generations would occur. On the other hand, no permanent periodicity could be induced in organisms of this character, even though they encountered an indefinitely prolonged continuance of the alternating changes in the external conditions which induce periodicity, such as the succession of day and night, or the regular alternation of the seasons.

In nature the external conditions are never constant, nor can any precise uniformity be artificially maintained for any length of time. Nevertheless there can be no doubt that very many, and perhaps most plants, would continue to exist and perpetuate their kind under perfectly constant external conditions. For the continued existence of other plants, however, variations in the internal and external conditions may be absolutely essential. Strictly speaking, this is actually the case in those heteroecious parasites which are able to reach their full development, and complete their life-cycle only by migrating from one host to another.

As the result of antagonistic or mutualistic interactions, heteromorphous, or, in other words, formative (morphogenetic), changes of remarkable character are often produced in response to the action of stimuli. The formation of insect or fungal galls by plants forms a good example of such modifications of growth, while in Euphorbia Cyparissias the branches assume a very special and peculiar shape when attacked by a certain aecidium. Lichens afford especially instructive examples of the shape of a compound organism remaining constant, so long as the interacting factors which produce it are unaltered.

It has already been pointed out that, with the exception of those cases in which growth is directly and mechanically restricted-as, for example, when a root is grown in a plaster cast, or penetrates an aperture of given shape-the external influences do not directly give rise to changes in shape and structure, but are merely the agencies which initiate that modification of the vital activity of the plant to which the alteration is due. When such phenomena are indicated by terms derived from the external agencies which induce them (Photomorphosis, Chemomorphosis, Barymorphosis ${ }^{1}$ ), we are no nearer a complete understanding of the chain of internal processes which leads to the final result, than we are when we say that a stem bends towards the light because of its heliotropism.

Among these extremely complicated internal reactions, the counterinfluences of different organs or cells of the plant play a most important part. These influences originate in the plant itself, but nevertheless, so far as the part affected is concerned, they must be regarded as component

\footnotetext{
${ }^{1}$ Sachs, Flora, 1894 , p. 231 ; Herbst, Biol. Centralbl., 1895 , Bd. xv, p. 722.
} 
factors in the environment of the part affected, and hence inust be included in the category of extemal conditions. The influences emanating from living aggregates may be of special character, and produce results possible only to such living agencies.

The entire vital mechanism involves a chain of interactions of an extremely complex character. In fact, in order that all parts may influence one another, and thus render possible a close correlation between the different members, it is absolutely essential for harmonious co-operation that a corresponding readjustment of the entire mechanism shall be produced in response to changes affecting the whole organism or any of its parts. Without such continual readjustment, the continued existence and growth of the organism would be impossible. Since all parts are intimately connected together to form a concrete whole, it follows that any automatic or induced alteration in one organ must influence all the others, although no directly perceptible result may be exhibited by them. In every department of Physiology, cases may be found in which the different component organs of a plant interact with one another in such a manner that marked correlative changes are manifested by all of them, when any one organ is compelled to undergo special modification. Thus, since root and shoot supply particular forms of nutriment to each other, it follows that when the functional activity of the root is depressed, the growth of the stem must be correspondingly retarded. Moreover, since the consumption determines the amount and direction of translocation, a cessation in the consumption of a given substance must finally cause its absorption or production to cease. A whole host of correlative phenomena of this kind are exhibited by plants, and many of them have long been recognized '

Correlating influences are probably continually travelling from one part to another, though they may only be made apparent by the character of the changes induced by modifications of the primary arrangement or conditions. The removal of particular members subtracts from the original aggregate certain of the operating influences originally present, and hence alters the character of the complex mechanism by which automatic correlation is effected. The injury itself may, in addition, directly or indirectly induce a creation of new relationships or modify the original power of reaction. A variety of facts supports these conclusions: for example, the formation of shoots from isolated roots, the development of dormant accessory buds when the young spring shoots are removed, and the production of

1 The term 'correlation' is here used in the general sense in which it was employed by De Candolle and C. Darwin, as including all physiological interactions, whether these find expression as metabolic processes or formative changes. A further subdivision, according to the nature of the causes at work, the character of the chain of intermediate processes, or the final result, is possible, owing to the complicated nature of all correlative phenomena. See, for example, Herbst, Biol. Centralbl., 1895, Brl. v, p. 724 ; Goebel, Flora, 1895 , Lrg.-bd., 1. 195. 
callus over a wound, owing to the activity excited in the meristematic cells adjoining the cut surface. These and similar cases are instances of the pronounced and far-reaching effects produced by stimuli of this rature; for, by the development of dormant buds, reserve food material, hitherto localized in distant parts, may be prepared for transport and gradually removed. Similarly, an injury to the root may produce a perceptible effect upon the most distantly situated stem apices.

In a well-ordered community every individual is of use and service to the whole, and under conditions which necessitate a rearrangement of the functions of the several members, any given official may be compelled to engage in unaccustomed work and perform duties from which he was previously free. Similarly, in the plant community the activity of every cell and of every organ is subservient to the common weal, and may, when necessary, be modified as already indicated so as to fulfil the changed requirements of the whole. Freed from this communal dependence, an isolated part may exhibit latent powers, which were previously but little, even if at all, employed; for in all cases the autonomy of the living cell must necessarily be restricted and under control, so long as it is a subordinate member of the whole.

With progressive development and special adaptation to particular aims and purposes, new powers and properties may be developed or original ones intensified; while others which were present in embryonic life may be weakened or lost in the adult condition. Thus it is that every cell or organ is not necessarily capable of reproducing the entire plant. even though the power of growth is retained; and this is, for cxample, the case with the pollen grain, although it is endowed with marked developmental powers. On the other hand, fragments of roots and leaves, consisting of a few or even single cells, may produce buds, and ultimately new and complete plants. In such cases the inherent reconstructive power, doomed under normal conditions to remain for ever dormant, still persisted, although in the intact organ the cells were adult and adapted to perform special functions. The same considerations apparently apply to the individual cell, for although a formation of a new plant from a single isolated cell has not been observed as yet in the higher multicellular plants, this is obviously due to the difficulty of presenting such a cell with a supply of nutriment of appropriate quality and quantity. No one can doubt that the fertilized ovum has inherent in it the power of building up the entire organism, although it has not been found possible as yet to reproduce the conditions necessary for its development outside the embryo sac. From such negative results no decisive conclusions can be drawn with regard to what potential powers may actually be inherent, for, in every case, these can only become manifest when certain special and specific external conditions are maintained.

The suspended development of the unfertilized ovum is an excellent 
example of the fact that growth and development may be impossible in spite of extremely favourable nutritive conditions. This is also the case during the resting periods exhibited by higher plants, and frequently germination can be induced in the unicellular spores of fungi and algae, only after the lapse of a certain latent period, often amounting to weeks or months. Reawakening may be made evident at once, or after a latent period, by a renewal of the vital activity manifested as growth or in some other form. while at the same time the fact is demonstrated that an indirect or automatic alteration of the internal controlling factors may be possible. Embryonic cells may be regarded as consisting of plastic material, which within certain limits may be induced to grow and develop in different modes and directions, according to the conditions under which they exist. From this point of view, the facts already put forward teach us how various tissue units and organs may be produced from originally similar cells in consequence of the operation of internal causes and the reciprocal influences of the developing parts.

It is owing to the inductive influences to which their position renders them subject, that particular cells, forming part of the primary meristem, or derived from cambial division, elongate markedly and assume the peculiarities which characterize the components of the vascular bundles. That these cells do not in themselves possess an inherent power of always developing in this manner is shown by the fact that if they are exposed by an intentional injury they may develop differently and form callus or other tissue. Moreover, similar internal parenchymatous cells, when exposed peripherally by the removal of the external tissues, may produce a new epidermis either directly, or indirectly by a series of tangential divisions. The thallus of Marchantia affords an instructive example of the inductive action of preexistent parts, for neither in the flattened apical cell, nor in the segments immediately derived from it, is there any fixed dorsi-ventrality. The inherent tendency to dorsi-ventrality is rendered definite and permanent by exposure to light, and in the gemmae the side illuminated always becomes the dorsal surface, even though it may happen in point of fact to be the lower one. Since no reversal of dorsi-ventrality is possible when once induced, it follows that the older parts must forcibly impress a dorsiventrality similar to their own upon each successive new increment, whatever the existing external conditions may be. In the case of fern prothallia, however, the influences radiating from the older parts are not sufficiently powerful to prevent a change in the incidence of the illumination from inducing a reversal of the original dorsi-ventrality on parts formed under such new conditions.

It is therefore the particular aggregate of causes brought to bear at a given moment which determines whether from particular meristematic cells, capable of developing in any direction, a leaf, a flower, or a leafy shoot 
shall arise. The complex influences radiating from the parts already formed exercise upon the developing tissue both a directive (autotrophic) and a formative (automorphic) action. Hence the differences which appear as the tissues become adult do not necessarily indicate that the primary meristems of the stem and root are autonomous, and inherently different from one another. As a matter of fact, a power of forming shoots lies dormant in the root-cells, and indeed, in a few cases, a root apex may be directly modified into the growing-point of a shoot. although, as a general rule, in the normal correlation of the parts this power cannot be exercised.

As growth and development proceed the porver of morphological change is gradually lost, just as clay loses its plasticity when dried and burnt. Even when full powers of growth are retained by certain cells or meristematic cell-complexes, the original freedom may be largely restricted by internal or external inductive action.

All these conclusions are the necessary consequence of the postulate, that every vital process is determined by the interaction between inherent disposition and external environment (Sect. 1); and it is only by a thorough recognition of this fundamental principle, that any sure and certain deductions as to the nature of developmental and formative processes can safely be drawn from our knowledge of the causes controlling them. This caution is the more necessary since conclusions are often formed from facts of morphology and ontogeny which, more especially in regard to phylogenetic problems, do not correspond with the above general principles, and in which the real causative relationship of the different phenomena observed is neglected.

Under the influence of a given aggregation of causes, every part of a plant must necessarily assume a definite and precise form. A change in the internal or external relationships can only modify this form so far as is allowed by the contemporaneous specific characters of the part affected. A cell complex of a primary meristem, which develops to form a leaf only when particular inductive causal relationships are maintained, cannot be regarded as a foliar region, but only as a spot in which is localized a potential and conditional power of leaf-formation. For such a leaf-rudiment does not become a leaf owing to its own inherent and inalienable power of development in that direction, but owing to the directive and formative internal and external influences which would be exerted upon any similar group of meristematic cells occupying the same position, as is instanced by the fact that a change in the external conditions may cause a callus or a shoot to be produced instead of a leaf. In the same way, the region from which either a leaf or a perianth segment may develop according to the external conditions is in reality neither a foliar nor a floral zone, and, although it is convenient in such cases to speak of indifferent regions, in the strict sense of the term no such things really exist. 
Although, therefore, in a particular case a perianth segment may be regarded on phylogenetic grounds as a modified leaf, nevertheless the actual development of the primary meristem tissue may be such as to directly lead to the formation of the perianth segment, without, even transitorily, any of the vestigial inductive conditions coming into play, such as formerly led to the formation of a leaf. Hence it is just as possible that the inductive influences which act upon the rudimentary organ are, at first, such as would lead to the formation of a floral organ, while a secondary change in the nature of the guiding influences at an early stage of development leads to the production of a foliage leaf, as that the reverse process takes place. It is perhaps simpler to regard the early developmental stages as being similar in both cases, the subsequent divergence to form either a foliar or a floral organ being determined by the external and internal influences which act upon the meristematic rudiment.

The correlative and other phenomena just mentioned are merely special examples of the regulatory processes by which the vital mechanism of the entire plant is linked together and adjusted. Without such purposeful regulatory changes the progress of development along particular lines, and according to definite laws, would be impossible ; nor could a harmonious cooperation of the component parts be attained, in the event of a compensatory alteration becoming necessary in correspondence with a change in the external conditions.

Self-regulation is attained in machines as well as in plants by allowing the character and amount of the work done, or the rate at which it is performed, to influence the working mechanism. The same general principle is involved, whether the regulatory mechanism is intended to maintain a condition of equilibrium, or whether progressive or periodically recurring alterations are to be compensated by a corresponding accommodation of the working mechanism to the changed conditions. The governor of a marine engine affords a good example of how the working activity of a machine may bring regulatory processes into play, which tend to maintain a condition of working equilibrium. Speaking in general terms, the needs of a self-regulating mechanism act as the causes inducing a response to the demand. Thus transit of nutriment is regulated by consumption, a tendency to perfect the organism induces isolated shoots to form roots, and the demands for further supporting power may increase the tensile strength of a branch. At the same time these examples show that under normal conditions the potential powers are not exercised to the utmost possible extent, as must, indeed, always be the case when any increased activity may be induced.

The causative relationships of regulatory processes are, in most cases, still rather obscure. It is certain, however, that the very varied regulatory processes which are universally present may be maintained by various means 
or combinations, generally of extremely complex character. In this respect irritability and irritable manifestations are of especial importance.

Usually, either over-accumulation or deficiency, or, in more general terms, any disturbance of equilibrium, acts as a stimulus, while in the regulation of metabolic action the stimulatory influences exerted by mass, quantity, and quality play a most important part. Very often regulatory influences are exerted by enzymes or other chemical substances; and, again, just as the male element not only acts as a stimulus to developmental processes in the ovum, but also influences their formative character, so also is it possible that occasionally, or even perhaps very frequently, a special formative activity may be induced by an accession of living plasmatic particles derived from some other part of the organism.

By means of the plasmatic threads which provide for interprotoplasmic communication from cell to cell, a continuity of the living substance is maintained which is undoubtedly of the highest importance in ensuring the harmonious co-operation of the whole. To what extent other factors, such as a transference of material particles, are of importance in ensuring this harmonious co-operation it is at present impossible to say. When one considers, however, the manner in which the vibrations of a string may be transferred to others at a distance, or how, by means of the telephone, messages and commands may be caused to re-echo far away, it is difficult to see any reason why special stimulating effects may not be transmitted through the nerve-like plasmatic threads by means of vibratory impulses to distant parts. It is, indeed, conceivable that impulses of all kinds, simple and complex, may be transmitted in this manner, although it must at present remain uncertain whether mechanical vibrations, heat-waves, electric currents, \&c., may or may not act as transmitting agents.

\section{SECTION 5. Variation and Heredity.}

In correspondence with the line of treatment we are pursuing, we must restrict ourselves to the discussion of the functions and reactions of existing organisms. The phylogeny of existing plants is also a physiological problem, but one about which only the most fragmentary and disconnected evidence has been collected. For this reason, the fundamental principles which enable us to comprehend the past history and the evolutionary development of existing species must be sought in a knowledge of the vital phenomena which take place under our own eyes in contemporary plant-life. The organisms of the present day are not immutable or unchangeable in character; for, in addition to those alterations which take place during developmental progress, accidental variations may appear, and may be repeated in the offspring, so that, under precisely similar external conditions, the descendants may diverge more or less markedly from the common ancestral type in the 
same or in different directions. Such variations may become permanent and hereditary, either a change of form or an alteration in the products of metabolism taking place ${ }^{1}$.

The production and hereditary transmission of such variations are connected in many ways with the general physiological problems with which we are immediately concerned. When any variation takes place, an alteration in the structure or nature of the protoplast must have previously occurred, provided the variation is not merely a temporarily induced one, but is one capable of hereditary transmission to the offspring. This is true for the lowest as well as the highest plants, and whether the variation is perpetuated by sexual or asexual reproduction. The conclusion that a change of this kind necessarily indicates an alteration in the arrangement or character of the protoplasmic constellation is, indeed, a logical necessity, even though it is impossible to determine exactly how the given variation arises or is induced.

The production of hybrid forms is evidently due to the combination of two different kinds of living substance. There can be no doubt that, if it were possible to interchange the nuclei of two separate and distinct protoplasts, assuming that the strange nuclei and protoplasts could live and grow together, two new organisms would be produced, differing from one another and from the original protoplasts. These special characteristics of the new organisms would be preserved, so long as the union and co-operation between the parts of the new protoplasts were maintained. This would also be the case if, for example, a bacterium existed in intimate and permanent symbiotic union with the protoplast, as a chloroplastid does, and were transmitted from generation to generation in the ovules. It is, as a matter of fact, not inconceivable that the existence of certain species as such depends upon protoplastic or symbiotic unions of similar character to the above. Nor is the possibility excluded that the tiny symbiont might be too small to be visible, or might be unable to continue an independent existence outside of the protoplast. Comparatively recently, lichens were regarded as distinct organisms, although we now know that they are the products of a synthetic union of two distinct plants, and that, by the artificial synthesis of various algae and fungi, new forms, or forms similar to those already known, may be produced with relative ease.

Nevertheless, as is well known, variations capable of hereditary transmission may arise without the help of foreign protoplasm, and certain bacteria afford especially instructive examples of these. Thus in many bacteria, the power of forming either spores or certain metabolic products may be inhibited by a particular mode of treatment, and in some cases this inhibition

1 The literature upon these questions is too voluminous to be quoted. 
is permanent, so that even under normal cultural conditions a reversal never takes place. The variety, thus produced, will hence remain constant in a natural environment, although there always remains the possibility that by the action of other agencies a return to the original condition may be induced. Accidental reversions are, as is well known, by no means uncommon in the higher plants.

It is also possible to induce in certain bacteria variations resembling those mentioned above, but which are not permanent and gradually disappear under normal conditions, though perhaps not until thousands of successive generations have been produced. Since in the case of higher plants this would require thousands of years, no perceptible reversal would be noticed by observations lasting only during the lifetime of a single observer. Hence, in considering this, and similar questions, the rapidly growing, rapidly living, and rapidly reproducing lower forms are of the utmost value.

External conditions act not so much as cirect formative, as indirect inducing agents, and thus produce vital changes, leading to an attainment of new hereditary peculiarities. It is easy to understand why the production of variations of this character should be favoured by subjecting the organism to unusual conditions or requirements, for the disturbances and the unusually labile condition thereby induced may originate changes which reach or even exceed the limit of physiological elasticity, and hence lead ultimately to a permanent modification of the original internal constellation, that is to say, to a variation capable of hereditary transmission. But this takes place only in special cases; all modifications induced by external conditions do not attain hereditary value, but for the most part come and go with varying environment, either in the same plant or from generation to generation.

As concrete examples illustrating these points we may take either a steel spring, in which permanent alteration is only produced when it is bent beyond its limit of elasticity, or a musical-box, the melodious harmony of which is permanently modified when one or more of the pins of the cylinder are bent or broken. Such a change might appear suddenly, and apparently without cause, nor need the same external agency necessarily always produce a similar result. At the same time, it is easy to understand that changes, or conditions preparatory to change, might be induced in the internal arrangement or constellation by continuous use, causing wear and tear, while these changes might gradually or suddenly become manifest by alterations in the musical character of the melody.

Saltatory variations often do appear in organisms, and may arise under precisely similar external conditions in particular individuals only, or may even affect these in different manner or degree. Bacteria afford, however, very good examples of graduated variations affecting all the individuals to a similar extent. Thus, by certain methods, the production of 
poisons or pigment substances may be gradually weakened in successive generations, while, according to the treatment pursued, the new properties may be only temporarily induced, or may be permanently fixed, in the former case gradually disappearing again under normal conditions.

External influences operate only by the changes which they induce in the internal constellation to which the inherent characters of the organism are due. Hence, from what has already been said it follows that exciting causes of internal origin may also produce permanent variations, which are then said to be spontaneous or automatic. As the musical-box serves to illustrate, a change of this kind may take place even when all the vital activities are perfectly normal; but spontaneous variations are more readily induced when an unusual demand is made upon any particular functional activity or activities.

Every peculiarity which is repcated in the offspring, is an hereditary characteristic. Hence it is both illogical and unjustifiable to refuse to pay attention to hereditary metabolic and productive powers, as well as to inherited morphological characters. As is universally the case, whenever a variation may be reproduced without the assistance of the inductive actions of other living elements, some obscure internal change has taken place in the protoplast. This is true, not only for a bacterium, but also for an ovum, for sexual as well as asexual reproduction, since many organisms capable of developing hereditary variations reproduce only asexually.

When however inductive actions are responsible for the production of a given variation, the same developmental progress and final shape must always result as long as the inductive actions are repeated in precisely the same manner. The specific shape of a lichen is due to a definite combination of interacting inductive agencies which always produce the same result. Hence, when a garden variety is propagated by cuttings, it remains uncertain whether or not a cell of the primary meristem, isolated from and uninfluenced by the inductive agencies with which it was formerly surrounded, would or would not reproduce the varietal characters in question, if it were able to form a new plant. Even if such an isolated cell were able to transmit the varietal characters to the plant it produced, we should still have to decide whether or not the transmission of the individual characters took place in a similar manner to that which, on empirical grounds, we conclude to be employed in the egg-cell. Even in an isolated cell a variety of factors demands consideration, and these appear largely to be eliminated in the ovum, for everything that is not absolutely essential for the growth and maintenance of the ovum after fertilization is apparently rejected as far as possible. Since in every single cell the interacting influences of the parts already existent are of importance with regard to the results actually produced, it follows that any special product formed in the plasma or 
cell-sap may exercise a directive influence upon development, and thus cause an individual variation to appear and be maintained.

A detailed discussion of these and related questions would be beyond the scope of this book. It must suffice to indicate the relationships which are of importance in every case in inducing specific shape and character; relationships, morcover, to which sufficient attention is rarely paid in attempts to cxplain the causes of the appearances observed. Whether the reduction of the protoplast to its living essentials increases or diminishes the tendency to variation, and what may be the action of the inductive and directive influences of the living environment, cannot be discussed here. The development of the fertilized egg-cell within the embryo sac is completed under peculiar specific conditions, and it is, perhaps, owing to the influence which these exert, that the vegetative outgrowths which sometimes arise from the wall of the embryo sac of Funkia, Coelobogyne, $\& c$. assume a similar shape and form to the sexually produced embryos.

It would be beyond the scope of this book to do more than indicate briefly a few of the general principles which regulate vital phenomena. Phylogenetic considerations have been purposely neglected, nor has any attention been paid to those theories which are based upon the existence of some presupposed structure in protoplasts, and upon the assumption of a definite division of labour corresponding to this structure. For although we should strive to explain everything by direct reference to the protoplasmic mechanism, our knowledge is as yet quite insufficient to enable us to predict the actual results that will follow as the necessary consequence of the given dispositions. This being the case, while allowing complete freedom of thought to theoretical conceptions, it is essential in every exact research to keep clearly in view the distinction between established facts and theoretical possibilities. When our knowledge of the nature of life is complete it will probably be found that the facts which we know at present form the framework supporting the completed picture.

In a subsequent consideration of the structure and mechanism of the protoplast, the question of heredity will be reopened. So much at least is clear, that every protoplast or part of a protoplast, which has the power of reproducing the entire plant, must contain in itself all that is necessary for the maintenance and development of the species. It does not, however, follow that under actually existing conditions these potential powers are, or can be, exercised.

Starting with the physiological knowledge we already possess, and especially by means of facts derived from the rapidly living lower organisms, it will doubtless be possible to obtain a deeper insight into the causes which originate variations and the manner in which these causes act. With the increased knowledge then at our disposal, we may hope to throw some light upon those variations which, according to the theory of descent, 
must have given rise to the host of species that once existed, or that now exist, for the plants now growing on the surface of the earth are only the twigs and terminal branches of an enormous genealogical tree. In the development and evolutionary history of plants, competition and selection have always played a prominent part in determining whether variations in any given direction were maintained and developed, or suppressed. Whatever part these and other factors may play, the final result is the same; for only those variations which are advantageous to the organism can be permanently retained.

So long as every living organism is found to arise from pre-existent life, the riddle still remains unsolved as to how life first came into existence upon this earth. Nor is it possible for us to say whether the path which leads to the source of life is lost in the obscurity of infinite space, or whether living material was first formed from dead substance on our planet. And however much we may be disposed to believe the latter to be the case, it nevertheless remains uncertain whether the conditions now existing upon our globe are such as to permit a re-creation of life, or whether the necessary conditions were presented only once, and by a special sequence of events, such as we can never hope to reproduce. The particular combinations of causes, to which the creation of life was possibly due, may have existed only as the earth cooled from its original incandescent condition, and perhaps thereby caused certain essential preliminary stages in the production of living substance to arise.

As growth and reproduction go on, the living substance must continually assimilate dead material. The same inherent structure and constellation are, however, retained by the protoplasin in spite of the perpetual change. Nevertheless it is possible that a descendant may not contain a single one of the same atoms that once formed part of a direct ancestor.

Death and destruction, as well as growth and reproduction, are continually taking place. As the resultant of these opposing factors about the same average quantity of living substance is maintained upon the earth so long as the conditions remain constant, but this state of approximate equilibrium will necessarily be disturbed and altered when any alteration takes place in the external conditions ${ }^{1}$.

So long as the earth was in a molten condition, no life such as is now known to us could have existed upon it; - while, on the other hand, the gradual diminution of life, as we proceed towards the Arctic regions, shows us that the present total aggregate of living substance would undergo a corresponding decrease, if similar climatic conditions held their sway over the entire earth.

${ }^{1}$ Preyer, Nat. Wochenschr., 1891, Bd. VI, p. 92. A good criticism by Errera, Rev. Phil., 1891, p. 322. 


\section{CHAPTER II}

PHYSIOLOGICAL, MORPHOLOGY.

\section{Section 6. The Structure and Function of Plant-organs.}

WiTH the exception of certain low organisms, all plants undergo more or less marked morphological differentiation. It is a very suggestive fact that this morphological differentiation develops in close correlation with division of labour, that is to say, with the modification of particular parts to perform particular functions. Thus, in order that a root may properly perform its special duties, it must be differently constituted to a shoot, while a leafy branch must be different in form to one bearing flowers. It does not, however, come within the scope of a textbook of general Physiology to describe the details of the relationship between form and function, and moreover a knowledge of vegetable anatomy and morphology is assumed on the part of the reader ${ }^{1}$.

A differentiation into root and shoot can almost always be recognized. This differentiation is directly due to the fact that nearly all plants pass through their entire life-history permanently fixed to one spot. The organs which absorb inorganic or organic nutriment from the substratum on which the plant grows, and at the same time form an anchoring attachment, have naturally a very different form and structure from the subaerial organs which subserve entirely different functions. Such differentiation may be found even in non-septate plants, as Botrydium and Mucor; though, owing to their simple structure, the differentiation into distinct members is clearly not as marked as it is in higher forms. In these latter the subdivision of the shoot into leaves and branches, together with the large amount of surface which the former present, ensures the maintenance of favourable conditions for the functional activity of the chlorophyll mechanism. These conditions are a sufficiently strong illumination, and an adequate supply of carbonic acid gas. Since, however, the amount of transpiration is increased by the same arrangement, it is easy to understand

${ }^{1}$ In addition to the usual textbooks attention may be called to Goebel, Vergleichende Entwickelungsgeschichte d. Pflanzenorgane, $188_{3}$; Sachs, Vorlesungen iiber Pflanzenphysiologie, I887, 2. Aufl, p. 3 [Eng. Trans. by H. Marshall Ward, Clarendon Press, 1887, p. 3 seq.]. 
that the plant may sacrifice a portion of its power of $\mathrm{CO}_{2}$-assimilation in order to avoid a dangerous loss of water. This is attained by reducing the amount of surface exposed, and may or may not be accompanied by a disappearance of the leaves and an increased development of cuticle, while, at the same time, chlorophyll usually develops in the peripheral and most favourably situated tissues. Such compromises are everywhere necessary in order that the different organs may work harmoniously together, and that the conditions necessary to the life of the organism may be created and maintained.

As is well known, the leaves are much reduced in non-green plants, and this affords distinct evidence that the increased surface presented by ordinary leaves is of value primarily to the chlorophyll mechanism. At the same time, the frequently remarkable development of the perianth segments is in no wise inconsistent with this conclusion, for the perianth segments are leaves specially modified for quite different purposes and functions. Organs of similar origin frequently diverge markedly from one another in habit, form, and function. Thus rhizomes, tubers, corms, \&c. are all modified stems which have adopted a subterranean mode of life for protection against prolonged cold or drought; and similarly foliar organs are made to serve widely different functions in all higher plants. It is sufficient to mention carpels, stamens, floral leaves, prophylls, tendrils, thorns, \&c., to realize how manifold are the functional and structural modifications which a foliar organ may undergo.

Owing to the fact that we select as types the most highly differentiated forms, we may speak of plants as being rudimentary in comparison with these, if they have not yet reached this high degree of development, and, again, of reduced forms which have become simple by degeneration from a higher condition. It is in full accordance with the theory of descent, that an early diverging line of development, such as terminates, for example, in the common toadstool, should at no period show any marked parallelism to the line which terminates in a flowering plant. In all cases the need of the plant to fix itself and absorb nutriment must necessarily lead to the formation of anchoring and absorbing organs, which, it may be noticed, are present even in those fungi which pass their entire existence underground and in darkness. An externally visible segmentation is by no means an essential requirement, as is shown by the existence of simple algae and fungi, which are spherical and unicellular even when adult. Such non-rooted organisms frequently develop cilia or flagellae, as locomotory organs by means of which movements of translocation may take place. Morphological differentiation into vegetative and reproductive organs is also possible in plants which are not fixed to a particular substratum. Since, however, reproductive organs require air, both for the distribution of the spores, \&c., and in order that full development may take place, we find 
that aerial organs are commonly developed by fungi and other plants which can grow in darliness.

The study of comparative morphology reveals all stages of differentiation in form and function; thus, in the development of the plant from the ovum or spore, progressive differentiation takes place, the extent of which varies very much in different plants. The term 'thallus' may be used to represent the least amount of differentiation possible in a multicellular plant, but the distinction is not always definite ${ }^{1}$, for every phanerogamic embryo passes through a thalloid condition. A thalloid form can be differentiated into shoot and root, and it is only in order to indicate the simplicity of the latter in thalloid forms, such as algae, fungi, and hepaticae, that we often speak of rhizoids instead of roots.

Even non-septate plants may exhibit marked morphological differentiation; for example, some species of Caulerpa simulate the form of the vegetative organs of Phanerogams. In plants of considerable sizc, however, the multicellular condition is very important, and indeed cssential to give sufficient strength and rigidity. A large non-septate plant is very liable to be fatally affected by a purely local injury. For these and other reasons, the non-septate condition is impossible for large plants which may be in giant forms as much as 140 metres high, but possible and indeed advantageous for the smaller forms of which the diameter may not exceed $0.001 \mathrm{~mm}$.

The cell-membrane secreted by the protoplasm serves primarily to give rigidity to the plant as a whole, and forms in trees the skeletal framework within which the softer parts are protected from pressure and strain. This solid and permanent envelope, by which the protoplast is enclosed, circumscribes the movement of which the latter is capable, and renders impossible any amoeboid changes of form on the outer surface which is closely adpressed to the cell-wall. The protoplast can increase the size of its dwelling-place, and induce permanent alterations of shape, only so long as it can cause a growth of the cell-wall to take place.

Peculiarities of form, of the mechanism of movement, and of the mode in which nutriment is obtained, arise owing to the permanent fixation to the substratum. The organisms compelled to live fixed to the ground must necessarily differ in many respects from those which are capable of translatory movement. For this reason it is all the more important that full attention should be paid to those plants which can move from place to place, in order that we may estimate more correctly the different phenomena exhibited to us by plants in general, and may also be able to establish the relations between plants and animals.

In the tissues of the higher plants a more or less marked differentiation

${ }^{1}$ Goebel, Vergleichende Entwickelungsgeschichte d. Pflanzenorgane, I $88_{3}$, pF. I27, I II. 
and division of labour takes place, which may be exhibited externally in the morphological characters and peculiarities of the component members. Owing to the functions which its peripheral position allots to it, the epidermis must acquire properties that vary according to the nature of the organ it covers; similarly, the different parts of the vascular bundles have very different tasks to perform. Very important also are dead elements, such as compose the slieletal framework of all the higher plants, and are found in each protective cork layer, while intercellular spaces also have important functions.

When organs and tissue elements are fully developed, and have attained their full anatomical and morphological differentiation, an adult condition is reached, which persists for a longer or shorter period. To permit of continued growth, and to ensure the possibility of reproduction, embryonic tissue must be preserved in certain regions. It is in virtue of the presence of such embryonic tissue at its apex, that the shoot continues to grow in length, while the meristematic cambial ring enables a tree to add every year a new layer of wood. On the other hand, in the leaf, the loss of the primary meristem involves an ultimate cessation of growth.

The life of every adult and functionally active cell is, as far as we know, of limited duration. In every tree, year by year, the older cells die, so that in the stems of trees hundreds of years old, only a certain number of the youngest annual rings contain living elements. It appears as if the protoplast can only continue to exist by unceasing growth and division, and unless such regeneration takes place, it becomes exhausted, and ceases to be functionally active, just as a machine wears out by continued use. A bacterium cell is in a certain sense immortal, so long as it can grow and divide. If its growth and division were mechanically arrested, however, it would in all probability finally die, although all other conditions might be suitable. This is actually the case in the primary meristem of the root and shoot, for these ultimately die ${ }^{1}$ when embedded in a plaster of Paris cast, though they may remain living for some time.

Where, however, adult organs capable of remaining active for a given length of time are required, each organ and each of its component cells must pass through a series of developmental phases, leading gradually to the final form. The questions connected herewith have already been noticed from a physiological standpoint ${ }^{2}$.

Relation of morphology to Physiology. Although our purpose is specially directed to the consideration of functional aspects, it seems, nevertheless, most convenient to adhere to the morphological distinctions and anatomical nomenclature used in the textbooks of Sachs, De Bary, and Strasburger. The characters which determine

\footnotetext{
1 Pfeffer, Druck und Arbeitsleistung, I893, p. 355.

'See Sachs, Flora, I 893, p. 223.
} 
morphological classification are directly evident to the observer, whereas the appearance of an organ does not always afford direct or trustworthy evidence of its functional value. Indeed, an organ may commonly perform more than one function, and in accordance with prevailing external conditions it may result that for a time a particular function is most in evidence, or even the only active one. Hence in a classification according to function, the same organ, or the same cell, would at one time come under one, and at another under a quite different, category.

We may, however, speak from a physiological point of view of assimilatory, translocatory, and mechanical systems, for morphology deals only with the shape, position, \&c. of the functional parts, and it is evident that organs of the same morphological value may have very different tasks to perform, and vice versa.

In the affairs of everyday life similar instances are to be found. Thus, railways would naturally be classified according to the districts through which they run and the companies to which they belong, though the purposes for which the passengers travel and for which the goods are sent, as well as the uses which both afterwards serve, are of the highest importance and interest. The same line of rails may serve at one time for goods and at another for passenger traffic, or even may be used by the trollies on which navvies travel from place to place. Here, just as in a living organism, similar shape and structure does not necessitate an equivalent functional importance.

\section{SECTION 7. Structure of Protoplasm.}

It has previously (Sect. 1) been stated that Physiology must necessarily seek an explanation of all vital processes in the developmental and formative powers of the protoplast. Our knowledge on this point is still in its infancy, and we must be content if we can gain here and there a glimpse into the internal protoplasmic mechanism. Even though our knowledge with regard to the structure of protoplasm were to be enormously increased, we should still see, not the causes and forces which are acting, but only the results which they produce. The most perfect mental picture of the plant or of the protoplast must necessarily fail to reveal the hidden and invisible causes which make it assume its specific form.

In the different sections of this book, the visible processes taking place in the protoplast will be dealt with so far as an accurate physiological knowledge of them has been obtained, and will be traced as far as possible to their ultimate causes and origin.

A complete morphological knowledge of the shape and structure of the protoplast on the part of the reader is assumed ${ }^{1}$. The following

' See O. Hertwig, Die Zelle u. d. Gewebe, IS93; the literature collated by Zimmermann, Beihefte zum Botan. Centralbl., Bd. III, pp. 206, 32 I, 4 ; I ; I 894 , Bd. IV, p. 8 I ; and the numerous recent special works of Strasburger, Rosen, Bovin, Hertwig, Waldeyer, \&c. 
remarks are intended simply to indicate the physiological standpoint, and to call attention to a few of the more general characteristics exhibited by the protoplast. Neither here, nor in the following chapters, can we discuss the numerous speculations and hypothetical deductions which have arisen in connexion with our morphological knowledge of the protoplast, but which are not based on sufficiently sound evidence to satisfy the physiologist.

Above all, it must be remembered that the simplest protoplast is an organism of very complex structure, and that its various activities result from the interactions of its component parts and organs. The particular result which any given cause produces is due to the special nature of the given protoplast. Every plant must therefore necessarily have certain special protoplasmic characteristics which are peculiar to it alone. At the same time, protoplasts of similar origin may temporarily or permanently acquire special properties by a progressive differentiation of labour, and by adaptation to special aims and purposes. Nevertheless, the plant protoplast, so long as it remains living. retains all the general features which characterize a typical vegetable cell.

In order to attain certain ends, the organism forms parts which are not living or capable of life. One such organ is the cell-wall which the protoplast constructs as a protective mantle in which it may live and work; indeed the protoplast living inside its cell-wall may be compared to a snail in its shell. In certain cases, as in Vaucheria, the protoplasmic contents may escape from the cellulose investment as a naked swarmspore, which later may build for itself a new domicile.

In the protoplast, just as in a snail, the internal structure and functional importance of the component parts require to be studied. Within the protoplast are spaces having considerable functional value, which are surrounded by living substance, but whose contents are not living.

Such are the vacuoles, which subserve a variety of functions. They may serve for the storage of reserve food material, while the dissolved substances which they contain give rise to the osmotic properties of the cell, and preserve these properties during growth. As the vacuoles increase in size the cell becomes much larger, but the amount of protoplasm which it contains undergoes no increase, or but little, so that finally it is reduced to a thin primordial utricle or bag closely adpressed to the cell-wall, and containing a single large central vacuole. Vacuoles are laboratories in which food may often be digested or building material prepared for use, while at the same time they are utilized in translocation. Frequently, as is indicated by their different contents, vacuoles may have special functions to perform, and in such cases the relationships to and interchanges with the vacuoles of other protoplasts are of especial importance. 
The body of the protoplast, the protoplasm as we may call it, is built up of organs and elemental structures. The nucleus is an organ of very general importance, and indeed, a separation into nucleoplasm (karyoplasm) and cytoplasm probably occurs in all protoplasts ${ }^{1}$. On the other hand, chromatophores, including chlorophyll corpuscles, are organs of special character, and are absent from fungi. When such special organs are present they may be given the general name of plastids ${ }^{2}$.

Like all living substance the plasmatic organs are of considerable complexity. This is readily perceptible in the resting nucleus, and is admirably shown when the latter divides, while the chromatin fibres, which are then so markedly visible, may also be seen to have a definite structure of their own. Besides the plastids already mentioned, the cytoplasm may contain minute bodies, often in great numbers, which, regardless of their morphological and physiological nature, may be termed microsomes or microsomata. They may be composed in some cases of non-living substance, but in other cases may be minute living plastids ${ }^{3}$.

But few of the organs and structural elements of which the protoplasm is composed are visible even with the highest powers of the microscope; nevertheless we must of necessity conclude on theoretical grounds that all living material is built up of most minute living units. The gradual progress and increase of our knowledge of the visible structure of plants renders the existence of smaller organs and elements certain, although these still remain to be discovered. Indeed it is not impossible that there are organisms, or developmental stages of minute but visible organisms, which the highest powers of the microscope fail to reveal to us.

In a small cell, or one of the organs of such a cell, the component units must necessarily be still smaller, and yet have positive dimensions; while the smaller and more numerous these units are, the more varied and complicated will the possible combinations be. At the same time a relatively greater surface area is correlated with the smaller size. and this is a factor of the utmost importance; for bacteria teach us what remarkable powers are conferred by extreme minuteness, and what extraordinary processes it renders such organisms capable of performing.

Ideas of size abstracted from the visible world will hardly enable us to realize either the infinitely great or the infinitely small, but

1 On schizophyles, see Hegler, Botan. Centralbl., i 895 , Bd. LxIv, p. 203 ; A. Fischer, Jahrb. f. wiss. Bot., I 895 , Bd. Xxvir, p. I50; Nadson, Botan. Centralbl., I 895 , Bd. LxIII, p. 238 , \&c. ; Palla, Jahrb. f. wiss. Bot., I 893 , Bd. xxy, p. 5 I I ; Zimmermann, Morphol. und Physiol. d. Zell. kernes, 1896 , p. I60; A. Fischer, Unters. iber Cyanoph. und Bacterien, 1897, Pp. 6I I 2 I.

2 The term 'plastid' is used in a variety of ways. See Zimmermann, Pflanzenzelle, I887; Wiesner, Elementarstruktur, I $S_{92}$, p. $S_{3}$; Schutt, Peridineen, $18_{95}$, p. 74 .

3 Hanstein, Das Protoplasma, I88o, p. 22. 
nevertheless the structure of the invisibly minute parts must necessarily be such as will correspond with and conform to the structure of those visible to us.

The honeycomb-like structure which Bütschli has discovered in the protoplasm does not exclude the possibility that in this again, if all were magnified a million times, an equally complicated subordinate structure might be detected. To the naked eye, parenchymatous tissue seems to have a finely honeycombed appearance, and in the cells of which this meshwork is composed no one would have imagined that all the complicated structure revealed by the microscope was present. Even if Buitschli's theory should be confirmed, and attain an equal importance to the first discovery of the cellular structure in plants, we are still far from an, exact knowledge of the laws which govern the structural arrangement of living substance. Thus, it is impossible to say definitely whether the honcycomb-like appearance is due to the protoplasm being frothy or vacuolar, only the walls of the vacuoles being living, or whether the vacuoles themselves are also filled with living matter (= enchylema ${ }^{1}$ ).

Perpetual change is necessary in order that life may be maintained, for the formative and constructive powers of the protoplasm could not exist were it not that changes are always taking place in it, which are so correlated as to preserve the same general composition of the whole. Alterations in the form and position of the nucleus, plastids, \&c. are external signs of internal disturbances of the temporary equilibrium. In the process of mitotic cell-division, the nucleus undergoes marked structural modifications, while similar alterations are necessitated in the invisible plasmatic elements, as these grow and divide. A consideration of the microcosm of planets, stars, and systems around us, will cnable us to understand more clearly the nature of the microcosmic protoplast. In our planetary system the same groupings and conjunctions are periodically repeated. Permanent changes and alterations may take place in the motion, the path, or even the condition of heavenly bodies (comets, meteors, \&c.) under the influence of external causes $^{2}$. To the distant observer, Sirius, mighty sun though it be, appears as a fixed and constant star, just as a marching regiment, seen from afar, looks like a single red dot in which the movements of the component parts cannot be distinguished. In the same way, the visible protoplast

1 These few remarks must suffice on this point. For further details, see the original papers by Liitschli, as well as of O. Hertwig, Zelle, I 893 , p. 18 ; Zimmermann, Beihefte zum Bot. Centralbl., 1893 , Bd. III, p. 213 ; Klemm, Jahrb. f. wiss. Bot., 1895, Bd. xxviII, p. 685. On the foamy structure of siliceous jellies, \&cc., Buitschli, Verhandlg. d. Naturw. Med. Vereins zu Heidelberg, 1894, Bd. v, Heft 3. Hertwig also discusses the granular theory of Altmann. See also Puriewitsch, Ber. d. Bot. Ges., I 897 , p. 239.

${ }^{2}$ See Pfeffer, Untersuch. a. d. Bot. Inst. in Tüibingen, I 886 , IBd. II, p. 316. 
appears to be constant and comparatively unchangeable, merely because the elements of which it is built up are invisible to us. Indeed, even if we could see them, that would help us as little towards understanding the vital mechanism, as our being able to distinguish the legs of a marching army would enable us to find out the causes and reasons why the legs are set in motion.

It is an absolute necessity that there should be only a slight degree of cohesion between its component units, in order to permit of those perpetual changes and alterations which the continued life of the protoplast necessitates. The plasma as a whole is viscous or semi-fluid. At the same time it is quite possible that marked cohesion may exist temporarily or even permanently between separate elements. Indeed, the protoplasm is able to increase its own powers of cohesion in fern antherozooids, in the cilia of zoospores, and in the tough external periplasm of Euglenae. In the peripheral layers of the plasmodia of myxomycetes, changes of consistency may be observed similar to those which take place in gelatine when alternately warmed and cooled. Such changes probably play an important part in the vital mechanism, at one time a particular unit or molecule of albumin being in a fluid, at another in a solid condition '. Pfaundler ${ }^{2}$, indeed, explains the peculiar properties of viscous solids (sealing-wax, \&c.) by assuming the occurrence in them of molecular changes of some such character.

The chromosomes, and many other bodies found in the cell, are probably of a more or less gelatinous consistency, but it is difficult to say whether they approach more to the character of fluids than of solids, or vice versa. Owing to the extremely small size of all such bodies, the influence exerted by surface tensions in maintaining their shape is relatively extremely great. Thus a tiny air bubble adhering to the side of a glass vessel filled with water exhibits several more or less marked characteristics of a solid owing to its relatively high surface tension. Similarly, a fine emulsion of any two non-miscible fluids is more viscous and less fluid than either of the two liquids of which it is composed.

The semi-fluid nature of the protoplasm results then in a tendency to assume a shape, or position of equilibrium, determined by similar mechanical properties to those which give a drop of water its tendency to become spherical. When living, this property may be more or less markedly counteracted by other influences. The changes of shape shown by living protoplasm are signs of continuous internal changes, which may often be so short in duration as to produce no perceptible result, while before

${ }_{1}$ For details, see Pfeffer, Zur Kenntniss d. Plasmahaut u. Vacuolen, 1890, p. 253; Bütschli, Unters. über mikroskop. Schäume, 1892, pp. 144-1 7 I ; Hertwig, Zelle, 1893.

${ }^{2}$ Pfaundler, Sitzungsb. d. Wiener Akad., 18 76, Bd. Lxxir, Abth. 2, 1. 253. 
a movenent corresponding to the new conditions of equilibrium has had time to take place, equilibrium may be again altered and a corresponding change in the movement induced. In accordance with this is the fact that free protoplasts or myxomycete plasmodia having a definite shape tend to assume a rounded and globular form when either their vitality or their powers of movement are depressed. The vacuolar structure, which Biitschli considers protoplasm to possess, will certainly be influenced by, and be the direct result of the same general tendency ${ }^{1}$.

It is impossible at present to say what factors and mechanical agencies operate in inducing all the different processes and changes of form of which protoplasm is capable, though it is safe to assume that these are mainly due to local or general chemical actions, and the changes of surface tension, \&c. which result from and accompany them. The power which protoplasm has of retaining the same general structure, in spite of the perpetual changes going on within it, is as yet an unsolved problem, as well as is the reason for the tendency to reject and expel foreign bodies" which it possesses.

The results observed do not form any direct indication of the inducing causes which are at work. Thus it is both illogical and unmethodical to suppose that in the visible formative changes which accompany mitotic celldivision we see the actual internal causes by which cell-division is induced, and for the same reason it is just as impossible to decide which parts are active and which passive. (Cf. Chap. I.) The translocatory movements and general behaviour of dissolved and undissolved excretory or plastic substances, constantly found in the plasma though not essentially part of it, are of considerable physiological interest. Such substances, or the plastic material alone, may be termed food material (Hertwig, Zelle, p. 24)metaplasm ${ }^{3}$, paraplasm (Kupfer), or deutoplasm (van Beneden). It is, however, not always easy to clearly distinguish between plastic substances and excrete products.

\section{Section 8. The Origin of Plasmatic Organs.}

In order that hereditary characteristics may be successively transmitted from one generation to another, it is necessary that not only the protoplast, but also the elementary organs which the latter contains, should be able to divide and reproduce their own kind. Thus, new nuclei and new chromatophores are formed only by the division of pre-existent chromato-

${ }^{1}$ See Klemm, 'Desorganisationserscheinungen,' Jahrb. f. wiss. Bot., Bd. xxvir, Heft 4, I895, p. 70 .

${ }^{2}$ Pfeffer, Aufnahme und Ausgabe ungelöster Körper, I 890, p. I74.

${ }^{3}$ Hanstein, Bot. Zeitung, 1868 , p. 710. 
phores and nuclei; whilst, within the nucleus itself, it is only by growth and division that any increase in the number of the chromosomes is possible. The same automatic mode of multiplication must necessarily also take place in the minute physiogical units of which the plasma is composed.

The protoplast is thus able to retain its specific character, and has also the power to form from living or dead material new organs of secondary, or even primary, importance.

The cell-wall, for example, is a product of plasmatic activity, and is not formed by the growth or division of a pre-existent wall. Other permanent organs, and transitory ones as well, may be produced partly or entirely from living substance. Just as an embryonic tissue may according to circumstances develop different organs, so also may cilia ${ }^{1}$ arise, under certain circumstances, from the ectoplasm of a myxomycete, as new formations not directly inherited from the parent. The membrane which bounds the protoplast externally, and surrounds every vacuole, has a special functional importance as the limiting membrane of the plasma. Vacuoles, also, are organs which may originate where none previously existed, but which, when once formed, may increase in number by division. In certain cases the vacuoles appear necessarily to increase in 2 number by a process of division of pre-existent vacuoles, and similarly the cell-wall persists from generation to generation of yeast-cells, though it may also undoubtedly be formed where none was previously existent. Like the cell-wall, vacuoles may serve a variety of purposes, and under particular conditions may be absent. It is not at all surprising that nonliving material may be interposed between the living substance which regulates the vital mechanism as a whole, and that such non-living substance may have most important and necessary functions to perform.

Moreover it can hardly be doubted that particular ends may be attained by a specific grouping or arrangement of certain living physiological units, while, in the same way, a formation of new and special protoplastic organs is conceivable. Indeed it is not conclusively determined as yet whether nucleoli and centrosomes are preformed organs which reproduce by division, or whether they may appear at one time and disappear at another ${ }^{3}$.

It is quite erroneous to suppose that each particular organ serves

${ }^{1}$ On cilia, see Zimmermann, Beihefte z. Bot. Centralbl., I 894 , Bd. Iv, p. r69; A. Fischer, Jahrb. f. wiss. Bot., I 895, Bd. xxviI, p. ${ }_{5} 6$.

${ }^{2}$ Pfeffer, Zur Kenntniss d. Plasmahaut u. Vacuolen, r8yo, p. 224. See sections 3 and 18 of this book.

${ }^{3}$ See Strasburger, Jahrb. f. wiss. Bot., 1895 , Bd. xxvil, pp. 151,$172 ; 1897$, Bd. xxx, pp. 379 , $3^{8} 7$; R. Hertwig, Centrosom u. CentralspindeI, 1895 , as well as the literature here cited; Zimmermann, Zellkern, I 896 ; Boveri, Zur Physiol. d. Kern- u. Zelltheilung, IS97. 
one function only. Growth, respiration, and nutrition are essential general functions of all living substance, while, at the same time, it is of the highest importance that different parts of the organism should have an inherent power of becoming definitely modified to perform special functions. There is no justification for the supposition ${ }^{1}$ that only certain parts or layers of the protoplast are concerned respectively with metabolism. respiration, or movement, nor is it possible that the nucleus performs a single function only.

In the protoplast, just as in the entire plant, special organs may be devoted mainly to one particular purpose, as, for example, is the case with chloroplastids, leucoplastids, \&c. The power of producing albumen, acids, colouring material, or even oil (Sect. 82) is not, however, necessarily restricted to particular plastids.

The functional importance of pyrenoids, karyoids ${ }^{2}$, nematoplasts ${ }^{3}$, pigment spots, \&c., is not yet clearly understood. It is besides frequently forgotten, in the hasty creation of new special names ${ }^{4}$, that generically similar organs may differ markedly in form, as well as in their contents. The various purposes chloroplastids and vacuoles may subserve afford excellent examples of this ${ }^{5}$. Moreover, organs, or the units of which organs are composed, may take on, temporarily, different arrangements, shapes, or groupings, as is exemplified by vacuoles, by mitotic celldivision, and in the changes of position of chromatophores and microsomes which may take place in the living cell.

From this it is clear that only a morphological nomenclature of the different parts of the protoplast is possible. Hence there is no justification for Strasburger's ${ }^{6}$ distinction between trophoplasm and kinoplasm, as parts of the cytoplasm with distinct and special physiological properties, for the distinction between the two is merely morphological, and is not based upon any known points of physiological difference.

The distinction drawn between hyaloplasm ${ }^{7}$ and granulo- ${ }^{8}$ or spongioplasm ${ }^{9}$ refers only to actual visible appearances and differences of structure.

Hypotheses regarding the ultimate structure of protoplasm. All considerations concerning ultimate protoplasmic structure lead unavoidably to the conclusion that living substance must be built up of excessively minute organs and units, and that

1 This conclusion has actually been put forward by Brass, Biolog. Studien, I88 3 , Heft 1 .

${ }^{2}$ Palla, Ber. d. Bot. Ges., I 894 , p. I 53.

${ }^{3}$ Zimmermann, Beihefte z. Bot. Centralbl., i 893 , Bd. III, p. 2 I 5.

+ See for example Schütt, Die Peridineen, I895, pp. 4I, 8 I, 87, \&c.

5 Crato, Bot. Zeitung, I893, p. I58; Cohn's Beiträge, 1896 , Bd. vil, p. 407 . On gas vacuoles, Klebahn, Flora, I 895 , p. 24 I.

6 Strasburger, Histologische Beiträge, I 892 , Heft 4, p. 60; I893, Heft 5, p. IOI. See also Zacharias, Flora, I895, Erg.-bd., p. 259 ; Jahrbuch f. wiss. Bot., 1897 , Bd. xxx, p. 375.

7 Pfeffer, Osmot. Untersuch., I 877 , p. I23. On the nomenclature, see Pfeffer, Plasmahaut u. Vacuolen, IS9o, p. 188.

8 Strasburger, Zellbildung u. Zelltheilung, I876, 2. Aufl., p. 286.

${ }^{9}$ Nägeli, Theorie d. Gährung, I 879 , p. I54. 
these, iike the living plasmatic organs visible to us, grow and increase, nourish themselves, and reproduce their kind by division. All hypotheses put forward to explain the phenomena of heredity involve an assumption of the existence of such minute living elements; while the same conclusion must also be drawn from comparison and analogy with the perceptible structure of the living protoplast.

The physiological units of Spencer, the gemmules or pangens of I)arwin (and also of de Vries), the idioplasmatic elements of Nägeli, the plasomes of Wiesner, the biophores of Weismann, are all ultimate units of living substance ', but to give a detailed account of all the different hypotheses connected with these terms is beyond the purpose of this text-book. Besides, the general principles recognized as regulating the interactions between the parts visible to us must also apply to the invisible physiological units, to which the general term 'Pangen' will here be applied. 'These are the primary living units, and may be combined into units of higher ordinal value in a variety of ways, while these again unite to form the organs and structural components of the protoplast. These primary units could hardly all be precisely similar, but even if there were only a few distinct types, still the number of possible combinations is illimitable. A concrete example of similar character is afforded by the alphabet, the letters of which can form a vast number of words, while an endless number of thoughts may be expressed by the combination of these into sentences. In the host of carbon compounds known to chemists we have a direct example of the number of combinations which can be made from three or four elements. With regard to the protoplast, however, it is more probable that a large, or even a vast number of pangens or physiological units, all differing from one another, enter into its composition.

Living substance is therefore to be regarded as being built up of pangens. The changes and transformations which go on in the former may affect numerous pangens or single ones only. In certain cases pangens, i. e. portions of the living substance, may be used to form useful though non-living organs.

A pangen would, in general, only be able to nourish itself, grow, and divide, when combined with other pangens to form a unit of protoplasm. A pangen can hardly be a micella, but must be built up of a number of micellae, or perhaps molecules, arranged in a specific manner. Various chemical elements may thus take part in the formation of a pangen, either directly combining to form it, or forming part of the micellae of which it may be constructed. In the pangen, just as in any machine, the manner in which the component parts are linked together is all-important, and although qualitative or quantitative differences may possibly exist between different pangens, these are not necessarily essential. A pangen must resemble living substance in being capable of imbibition and swelling, while its hypothetically complicated structure is in no wise antagonistic to the micellar theory or hypothesis put forward to explain the nature of organized structures ${ }^{2}$.

These few remarks must suffice, and in conclusion attention may be called to

1 A complete account of the different theories is given by Delage, La structure du protoplasma et l'hérédité, 1895 . See also Wiesner, Elementarstruktur, I\$92; Hertwig, Zelle, 1S93, p. 267.

${ }^{2}$ See sect. I3; Pfeffer, Studien z. Energetik, I892, p. ${ }_{5} 8$. 
the general considerations given in Sect. 5, with which each and every hypothesis must agree.

[It is hardly conceivable that each pangen or physiological unit could possess one specific kind of irritability only, and if pangens have inherent in them all the general irritabilities which characterize the protoplast as a whole, the assumption of these hypothetical units brings us no nearer a comprehension of the vital mechanism. It is possible, however, that different pangens might have certain irritabilities more highly developed than others, and the specific irritability of a sensitive organ might be due to the preponderance of one special kind of pangen, or to the combination of several different kinds. ED.]

\section{Section 9. Relations between the Nucleus and Cytoplasma.}

In the protoplast, as in every organism, the course of events is determined by the interactions of its component parts, which mutually support one another and are mutually interdependent. For the correct determination of these internal protoplastic relationships, the same general rules are applicable as were given in Sect. 6 .

It is comparatively easy to discover the function of an organ such as a chloroplast, which subserves mainly one particular purpose; but it is extremely difficult to make out all the manifold relations of nucleus and cytoplasm, upon the maintenance of which the life of the protoplast depends. Though a comprehensive grasp of the inherent character of these relationships has not yet been attained, still a general indication of the questions at issue may be profitable, especially as a clear realization of the meaning of the phenomena observed is, in many cases, conspicuously wanting.

Since it is the co-existence of nucleus and cytoplasm which constitutes the protoplast, the latter ceases to exist as such when its component parts are separated from one another, just as a lichen, formed by the symbiotic union of an alga and a fungus, is no longer a lichen when its two constituents are cultivated apart from one another. The comparison is none the less accurate because in the latter case the component parts are distinct organisms capable of separate existence. Nor is it necessary that all symbiotic organisms should be capable of cultivation when isolated from one another.

When an organism is transferred from favourable conditions to such as render its continued existence impossible, its vital activity gradually decreases, and death unavoidably supervenes, although until it dies indications of functional activity are still perceptible. This is also the case in separated organs which are incapable of continued existence when isolated. Thus a muscle removed from the body is for a time still capable of contraction (Sect. 52); an isolated chlnrophyll body may continue for 
a time to decompose $\mathrm{CO}_{2}$. while in the same way the nucleus and cytoplasm may continue to carry on certain vital actions for some time after they have been separated from one another.

In all such cases, the observed phenomena result as an after effect ${ }^{1}$ of the previous conditions, which were such as to permit of the growth and functional activity of the different parts, as well as of the formation of new functional organs. Any such organs formed by secondary differentiation exhibit a more or less marked independence when isolated, and the duration of the activity of which a separated organ is capable is largely or mainly dependent upon external conditions. It is, indeed, not impossible that under special culture conditions, a chloroplast ${ }^{2}$, or even a nucleus or mass of cytoplasm, might remain living for a very long time, and might possibly be caused to grow and multiply. If it were possible to cultivate isolated organs in appropriate media, we should undoubtedly find that the same general principles previously recognized as controlling the organism as a whole would be equally applicable to its isolated parts.

As yet, no non-nucleated mass of cytoplasm, nor nucleus free from cytoplasm, has ever been found capable of continued growth and life, however suitable all other conditions might be. In every case the isolated nucleus or mass of cytoplasm dies after a time, though in some cases life is retained and certain vital actions continue to be manifested for a few weeks.

The cytoplasm may be regarded as living so long as it can be plasmolyzed, and thus continues to exhibit the same diosmotic peculiarities as the living protoplast does. The power of movement may be retained in non-nucleated cytoplasm; thus, active streaming movements in both higher and lower plants ${ }^{3}$, amoeboid and streaming movement in Amoebae ${ }^{4}$, or a pulsation of the contractile vacuoles of Protista ${ }^{5}$, may still be possible.

Ciliary movements also continue in the absence of a nucleus, and hence non-nucleated fragments of swarm-spores or Protista may continue to exhibit free swimming movements ${ }^{6}$. The existence of specific peculiarities

${ }^{1}$ It is hence incorrect to suppose that an isolated portion of cytoplasm continues to exist and show functional activity only owing to the after effect of the specific nuclear influences originally acting upon it, for the dependence is a mutual one, and each influences and regulates the other when combined together to form the protoplast.

${ }^{2}$ [See Lwart, Assim. Inhib., Journal of Limean Soc., 1895, vol. xxi, p. 424; also Kny, Ber. d. I). Bot. Ges., Sep., $\mathbf{I} \delta_{97}$; and Ewart, Bot. Centralbl., I $S_{97}$, Oct., Bd. Lxxir, No. 9.]

${ }^{3}$ Pfeffer, Zur Kenntniss d. Plasmahaut u. d. Vacuolen, IS9o, p. 279; Hauptfleisch, Jahrbuch f. wiss. Bot., I $89^{2}$, Bd. Xxı v, p. 172 ; Gerasimoff, Ueber die kernlosen Zellen bei einigen Conjugaten, I 892 u. 1896 . On this and other questions, see the reference by Zimmermann, Beiblätter z. But. Centralbl., I 894 , Bd. IV, p. 8 I ; O. Hertwig, Zelle, I $\varepsilon_{93}$, p. 264; Verworn, Allgem. Physiologie, 1895.

+ Hofer, Exp. Unters. üb. d. Einfluss d. Kernes auf d. Protoplasma, 1S89, p. 486.

${ }^{5}$ Hofer, 1.c. For additional literature, see O. Hertwig and Verworn.

- For literature, see O. Hertwig and Verworn. On the cilia of Bacteria, A. Fischer, Jahrh. f. wis. Bot., 1895 , Bd. Xxvil, p. 153. 
with regard to these and other points is only natural, and to be expected. It is, for example, not impossible that the non-nucleated plasma of particular plants may still be able to form a new cell-wall. That a nucleus devoid of cytoplasm soon dies has been determined by Acqua ${ }^{1}$ in the case of the generative nucleus of the pollen-tube, and by

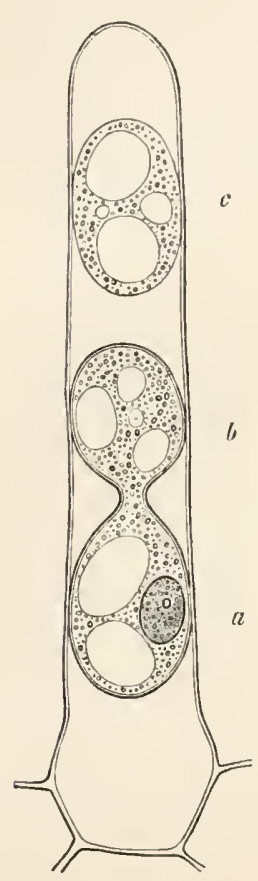

FIG. 1. Root hair of $\mathrm{Cu}$ curbita pepo, plasmolyzed by a ten per cent. solution of grape sugar. After three day's the nucleated portion $(a)$, as well as the portion $(b)$ in connexion with it, have formed a new cell-wall; no such wall has, however, been formed by the non-nucleated isolated portion (c). Verworn ${ }^{2}$ in that of the nucleus of Protista. Demoor ${ }^{3}$ has found that the nuclei of the cells of the staminal hairs of Tradescantia may partly, or entirely, complete the process of mitotic division after the cytoplasm has been killed by chloroform or $\mathrm{CO}_{2}$. This important result shows that nuclear division is possible without the direct help of the cytoplasm. Moreover, division may take place in an atmosphere of hydrogen, i.e. in the absence of oxygen. Under these conditions, and also when streaming movements are arrested by chloroform, no formation of the cell-plate or cellwall takes place, these being products of cytoplasmic activity.

It is clear, therefore, that metabolic processes may continue for a time in isolated parts, while the fact that any streaming movement which may be shown by isolated cytoplasmic masses ceases in the absence of a supply of oxygen shows that respiration must continue in such parts. There can be no doubt that in the presence of oxygen the isolated nucleus also continues to respire.

It is possible, by plasmolyzing a vegetable cell, to separate the protoplasmic contents into nucleated and nonnucleated portions (Fig. r), and by opening such cells these parts may be obtained lying free ${ }^{4}$. Non-nucleated fragments may be directly cut from large infusoria, without employing sugar or saline solutions. In all cases, the nonnucleated fragments ultimately die ${ }^{5}$, but in some of the experiments Klebs performed with algae they remained living for as long as six weeks. Klebs (l.c.) found that, in the case of algae and the leaves of mosses, only the nucleated

1 Acqua, Malpighia, I $\varepsilon_{9}$ I, Bd. v, p. 2 I.

'Verworn, Pfliger's Archiv f. Physiologie, 1892, Bd. LXI, p. I.

s Demoor, Sep.-abdr. aus Archives de Biologie, Bd. Xi1I, pp. 72, 75, \&c., I894. From researches carried out in the Bot. Inst., Leipzig.

${ }^{4}$ Kilebs, Unters. an d. Bot. Inst. zu Tuibingen, I888, Bd. II, p. 552; Klercker, Eine Methode zur Isolirung lebender Protoplasten, I 892 (Kgl. Vetenskaps-Akad. Forhandlingar, Stockholm).

'Schmitz, Festschrift d. Naturforscher-Gesellschaft in Halle, I879, p. 275; Klebs, l.c.; Ilaberland, Beziehungen zwischen Function und Lage des Zellkernes, 1887, p. 83 u. s. w. 
portions could form a new cell-wall, and that they did so soon after being plasmolyzed. Palla ${ }^{1}$ has, however, observed a formation of a cell-wall around non-nucleated fragments of cytoplasm in hairs, pollen-tubes, leaf-cells, and in a few algae. These results are due, as Townsend ${ }^{2}$ has since shown, to the existence of fine, often almost invisible, plasmatic threads, connecting the nucleated and nonnucleated fragments of cytoplasm. No case has as yet been observed in which a non-nucleated mass of cytoplasm has remained capable of secreting a cell-wall around itself, without being in direct connexion with a living nucleus.

The effect of the injury is, to a certain extent, immediately perceptible. Thus, the two halves of a divided amoeba become at once more or less rounded, but in a minute or two pseudopodia are again formed in both halves (Fig. 2, Hofer, 1.c.). The injury may also act as a direct traumatic stimulus, causing instead of

B
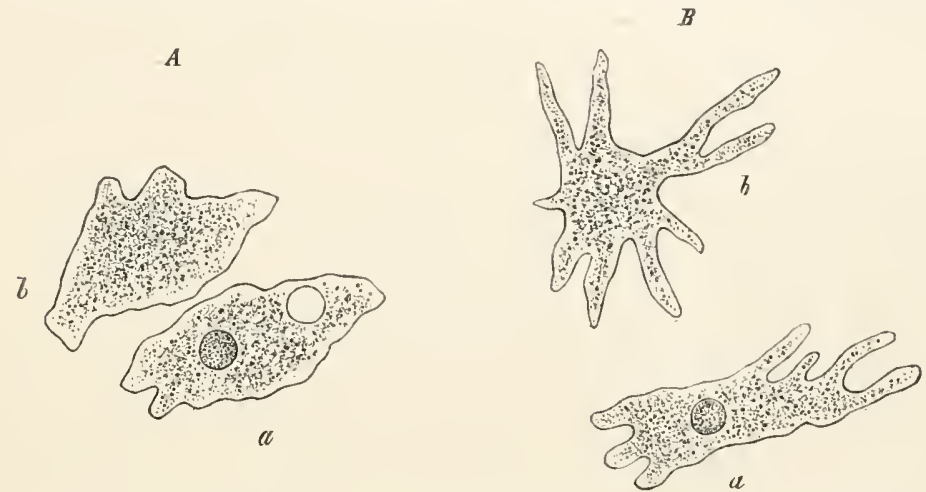

FIg. 2. Amoeba proteus (after Hofer), $A$ immediately, $B$ five minutes after, the separation into two. In $A$ the injury has caused a retraction of the pseudopodia, in $B$ these are formed again in both nucleated and nonnucleated halves.

a retraction of the pseudopodia an acceleration of the ciliary or streaming movements, when such are present. In all cases the vital activity of the nonnucleated portions gradually decreases, thus causing amongst other things, as Hofer found in the non-nucleated amoebae, a weakening of the digestive power of such fragments.

A knowledge of the vital actions of which isolated parts are capable is, though useful and interesting, not of paramount importance. The potential powers of the vital mechanism as a whole are exercised and used in a variety of ways, and many forms of vital activity are possible only to the intact protoplast. Thus, it is certainly incorrect to regard the formation of the cell-wall as being a special function of the nucleus simply because in the absence of the latter no cell-wall can be formed.

Life, growth, and formative changes are possible only when the

1 Palla, Flora, I89o, p. 314. Similar observations by Acqua, Malphigia, Bd. v. See also the reference by Zimmermann, Beihefte z. Bot. Centralbl., I 894 , Bd. Iv, p. $8_{5}$.

2 Townsend, Jahrb. f. wiss. Bot., $18_{97}$, Bd. $x x x$, p. $48_{4}$. 
nucleus and cytoplasm co-operate with each other. The character of the species and the power of continued existence reside neither in the nucleus nor in the cytoplasm, but only in the combination of the two which forms the normal protoplast. Just as a new lichen may be synthesized by changing either the symbiotic fungus or the symbiotic alga, so also might a new species or variety of a plant be prodiced if the nucleus of one plant could be combined successfully with the cytoplasm of another. The combination of a variety of nuclei with the same cytoplasm, or the combination of one nucleus with different cytoplasms, would in every case originate a specifically distinct organism, for a given protoplast must always contain the same component parts, just as a given lichen does. A change in the external conditions may cause a corresponding, and more or less marked, modification of the shape and character of the organism; but such change either affects the nucleus and cytoplasm to a relatively equal extent or does not cause the slightest alteration in their inherent nature.

Until the nuclei and cytoplasms of different plants can be combined together, it is impossible to empirically determine the foregoing problems. Hybrids formed by the union of entire protoplasts derived from different species exhibit characteristics intermediate between their two parents. Since the sperm ${ }^{1}$ conveys to the ovum not only the male pro-nucleus, but also a certain amount of cytoplasm, it is not justifiable to assume that the nuclei alone are of importance in fertilization. Nor has it been conclusively proved that the hereditary characteristics of the parents are transmitted solely by the nucleus. Bover ${ }^{2}$ found that the non-nucleated orum of an Echinoderm, when fertilized by the spcrmatozoid from another species, developed the peculiarities which characterized the latter. This is not, however, a conclusive proof that the nucleus alone is concerned with the transmission of acquired characteristics, for the spermatozoon is a protoplast and introduces cytoplasm as well as a nucleus into the ovum. The facts before us by no means justify the conclusion that the nucleus is the centre of all the vital activities of the protoplast, while the cytoplasm is nutritive and entirely subordinate to it. In no case has an isolated mass of cytoplasm been seen to divide spontaneously. Demoor (1. c.) has, however, shown that the nucleus may divide partially or completely after the cytoplasm has bcen killed, and it is therefore probable that it is the nucleus which initiates cell-division, although in the isolated nucleus complete division may be as impossible as it is in the isolated cytoplasm.

There is no doubt that the nucleus, formerly comparatively neglected,

${ }^{1}$ On spermatozooids and allied literature, see Belajeff, Flora, Ergänzungsband, IN'94. p. 1.

${ }^{2}$ Boveri, Archiv fo Entwickelungsmechanik, 1895 , Bd. II, p. 394; Seeliger, ibid., Is 94 , Bd. I, p. 204 . 
has of late acquired a fictitious importance, probably quite disproportionate to its real functional value ", owing to the peculiar and interesting formative changes which have been found to take place in it. The decreased, or even subordinate importance, commonly attributed to the nucleus subsequently to the discovery of centrosomes ${ }^{2}$, is another example of how our judgement of the functional importance of an organ is influenced by our knowledge of that part of its structure which is visible to us. It is not impossible that there would be no lack of theories put forward ascribing to the cytoplasm predominant importance, if only the remarkable structural peculiarities, which it certainly possesses, were actually visible. However important and interesting the relatively large size of the nucleus in embryonic cells may be this does not afford any conclusive evidence one way or the other ${ }^{3}$. Size is not all-important. The value of an individual to society is hardly at all dependent upon his stature, and indeed, minuteness may endow an organism with great powers. Thus, Bacteria afford a good example of how, owing to their enormous powers of propagation, tiny living organisms are able to produce the most extraordinary results and even to destroy the largest and most highly specialized beings. Moreover, it must be remembered that in fertilization the male and female elements probably mutually stimulate each other, and the most minute quantity of a stimulating substance may induce very marked and farreaching changes.

The result of the division of labour which has taken place in the protoplast is that in certain functions the nucleus, in others the cytoplasm, plays the principal part. For the perfect performance of any function, however, mutual co-operation is necessary; for there can be no doubt but that in every instance each influences and stimulates the other. Even though the nucleus were especially concerned in emitting stimulating impulses, and were in certain cases even comparable with a central nervous system, the above considerations would nevertheless be in no wise vitiated. It is only natural and to be expected that formative changes should be markedly influenced by the nucleus. The action of a stimulus affecting the nucleus and cytoplast, such as that which gives rise, for example, to a formation of galls, will be modified and largely determined by the nature and character of the growing tissues affected. Hence, galls formed by the

1 See also Verworn, Allgemeine Physiologie, I 895, p. ${ }_{4} 86$.

2 For literature on Centrosomes, see p. 40. Also O. Hertwig, Zelle, I 893 , pp. 47-146; Haacke, Biol. Centralbl., I 895 , Bd. Xıv, p. 44; Boveri, Zur Physiologie der Kern- und Zelltheilung, I897; Strasburger, Jahrb. f. wiss. Bot., I $\$_{97}$, Bd. III, p. $3 \$_{7}$. [The aggregation of the nuclear chromatin into a definite number of chromatin filaments appears to be merely a sign of great nuclear activity, and is not characteristic of mitosis only, for it may be seen in actively secreting gland-cells (Huie, 1. c., later).]

${ }^{3}$ On changes of size in the nucleus, see Fr. Schwarz, Cohn's Beiträge zur Biologie, I $892, \mathrm{Bd} . \mathrm{v}$, p. So ; Zacharias, Flora, 1895 , Ergänzungsband, p. 217. 
same insect on different plants might be expected to differ markedly from , one another ${ }^{1}$.

In the protoplast, the nucleus and cytoplasm exhibit a variety of activities, and the inherent connexion and relationship between the two are much more intimate than they are between two distinct but adjacent cells of a tissuc. The nucleus and cytoplasm live in closest symbiosis, and it is possible, and indeed probable, that an exchange of living substance or of physiological units may take place between them ${ }^{2}$. It has not yet been definitely determined whether, or to what extent, any exchange of spindlethreads centrosomes, nucleoli, \&c., may take place between the nucleus and cytoplasm. In certain stages of nuclear division, according to some authors, no definite boundary exists between the nucleus and cytoplasm ${ }^{3}$; the nuclear membrane being absorbed.

Though a knowledge of perceptible results forms the primary and essential basis of scientific research, still we must never fall into the error of supposing that we see the actual operating causes in the movements or changes of shape, \&c., which may accompany, or apparently initiate, the final result (cf. Sect. 8). Identical results may be produced by widely different means; and hence the general resemblance between karyolinetic figures and magnetic lines of force ${ }^{4}$ in no wise indicates that the former are produced in a similar manner to the latter. Although the visible phenomena of nuclear-and cell-division are the result of invisible changes and forces, it still remains of interest and importance to establish if possible accurate comparisons with allied phenomena. It can only be decided by prolonged experimental research whether the occurrence of amitotic cell-division is always an accurate indication that in such cells something is wanting which cells undergoing mitotic division possess ${ }^{5}$. Recent studies have shown that the processes of nuclear and cell-division do not always necessarily follow exactly the same course, and even if no living animal of the

'For a summary of our knowledge on this subject, see Eckstein, Pflanzen-gallen und Gallenthiere, $\mathrm{I} 89 \mathrm{I}$.

${ }^{2}$ [Huie (Quart. Journ. of Micros. Science, $\mathrm{xxxIx}, \mathrm{r} \mathrm{S}_{97}, \mathrm{p} .387$, 'Changes in the cell-organs of Drosera') states that in a stimulated gland-cell a portion of the cytoplasm disappears, and concludes that it is replaced by the nucleus absorbing food material, metalolising it, and excreting it into the plasma. The staining reactions on which these conclusions are based, however, hardly suffice to establish this theory.]

${ }^{3}$ Cf. Zacharias, Flora, Ergänzungsband, 1895 , p. 252; Zimmermann, Beihefte z. Botan. Centralbl., I 894 , Bd. Iv, p. 86, and the litcrature here given. [Also Sargant, Annals of Botany; I 896, p. 445.$]$

- Cf. Errera, Compt. rend. d. l. Soc. Royale d. Botanique de Relgique, i $\delta 9^{\circ}$, Bd. xxix, p. 17.

5 Hegler, Bot. Ccntralbl., I\$ 95 , Bd. Lxiv, p. 203 ; Zimmermann, Beihefte z. Bot. Centralbl., I893, Bd. III, p. 352 ; Strasburger, Histologische Beiträge, I 893 , Heft 5, p. 99 . According to Gerasimoff (Ueber die kernlosen Zellen bei Conjugaten, IS92, p. 9) the application of cold causes mitotic division which has already recommenced to become reversed, the nucleus becoming normal again, whilst subsequent division takes place amitotically. [This is readily explicable if the formation of mitotic figures is simply an indication of great nuclear activity.] 
present day is without a nucleus, it still remains cxtremely probable that no differentiation into nucleoplasm and cytoplasm had as yet taken place in more primitive forms than now exist.

The character of every form of vital activity is detcrmined by the protoplast as a whole, i.e. by the co-operation and correlation between the nucleoplasm and cytoplasm, and it is the union of these entities which determines all the special peculiarities of a given species. If it were possible to cause an isolated nucleus or cytoplasm to continue its life and growth, either of these would probably present us with a new organisn having very different characteristics from those of the original protoplast. The theories of heredity set up by Darwin, Spencer, and Nägeli, in which a part is ascribed to both nucleoplasm and cytoplasm, are just as much in accordance with our present knowledge as are the deductive theories originated by de Vries and Weismann, which are based upon the unproved axiom that it is the nucleus alone which transmits hereditary characters to the offspring.

It is not necessary to discuss here either these hypotheses or the problems of variation and heredity which they are supposed to explain. They have already been dealt with in general terms in connexion with the ultimate structure of the protoplast (Sect. 5). No hypothesis is necessary to support the logical conclusion that, whatever changes take place, cells must always be present somewhere or other which retain in themselves a miniature replica of the characteristics and properties of the entire organism and a potential power of developing and impressing these characteristics upon the products of division. We may denote the plasma of such cells as idioplasm, keimplasm, hereditary or embryonic plasm or substance ', without connecting a particular theory with any of these terms.

Just as the cells of an embryonic tissue may develop in widely different directions, and when adult commonly lose all or most of their embryonic properties, so also may the embryonic plasma be modified or changed in a varicty of ways, or undergo constructive or adaptive modifications which cause it to lose its embryonic nature and reproductive powers partly or entirely. Observations made on plants decisively negative Weismann's theory of heredity, according to which the 'reproductive plasma' concerned in heredity and the preservation of the species, and the 'somatoplasm,' by which the life of the individual is maintained, remain always distinct and separate from each other, even when present in the same protoplast $^{2}$.

1 It may be left an open question as to whether cells capable of forming new tissues (cambium cells, \&c.), but not of forming new entire organisms, are not also to be regarded as embryonic cells.

2 Further details in the works of O. Hertwig, Delage, \&rc. 


\section{Siction 10. Uni- and Multi-nucleate Cells.}

In every cell a nucleus is constantly present, but in many cases more than one, and in some cases a very large number may be found. The latter is always the case in unicellular plants of considerable size, such as the Siphoncae (Multinucleatae), Mucorinae, and Myxomycetes. Unicellular tissue elements may also be multinucleate, as is the case, for example, in those laticiferous tubes which are derived from a single cell. The division of the nucleus is not always necessarily followed by the division of the cell, and it is by the continued division of the nucleus, unaccompanied by corresponding cellular division, that multinucleate cells are produced.

Even when no cellular division occurs, the nuclear segments separate from one another, and distribute themselves regularly throughout the cell so that no large portion of the cytoplasm is without a nucleus associated with it. This is the reason why isolated fragments of the plasma of Vaucheria, Mucor, \&c., can regenerate the entire plant, for since in such plants the nuclei are very small and numerous, an exceedingly small fragment may still contain a nucleus, and hence be capable of regenerating the cntire plant. Whether in such cases the small size of the nuclei is of importance in this respect, or whether this minuteness is connected in some way with the absence of cellular division ${ }^{1}$, is impossible at present to say.

The multinucleate nature of large cells ensures that the necessary and important interactions between the nucleus and the particular portion of cytoplasm with which it is connected do not need to be transmitted through too great an intervening space. In this way the multinucleate cell attains the same ends as do the small uninucleate cells forming a tissue. It is not known what is the utmost distance across which the nucleus and cytoplasm may interact and influence one another, and the distance a stimulus may be transmitted through a living tissue, multicellular and therefore multinucleate also, has no bearing on the point at issuc. In cytoplasmic fragments which are able to secrete a cell-wall owing to their being connected with a nucleated protoplast by a delicate plasmatic thread, we have an actual example of a stimulating influence, affecting a particular function, being transmitted across a distance amounting, in some cases, to several millimetres 2 .

Experiments of a similar character to those made by Townsend may possibly throw further light on these and other related questions. Any large uninucleate cells which may occur in a tissue are generally adult and fully grown, and are hence no longer called upon to exercise all the primitive functions of which a cell is capable. Very possibly, in such large

2 Townsend, Inaugural dissertation, Leipzig, p. 19, 1897 (Jahrb. f. wiss, Bot., I 897 ). 
cells the streaming movements which continually convey new portions of cytoplasm to the close neighbourhood of the nucleus are of considerable importance, as indeed any changes in the form or position of the nucleus itself may also be.

It is, for example, often the case that the nucleus is found in the neighbourhood of the point at which the growth of the cell-wall is most active ${ }^{1}$. This is not, however, always the case, for the nucleus in the root hairs of Trianea bogotensis, probably for mechanical reasons, remains at the broadened base of the root hair, and not at the apex where growth in length is active ${ }^{2}$.

The plasmatic threads which pass through the walls of the contiguous cells of a tissue unite the different protoplasts together, and thus ensure the harmonious co-operation of the whole by allowing each protoplast to communicate with and interact with its neighbours ${ }^{3}$ (Sect. 4). The existence of protoplasmic continuity renders it possible that single elementary organs, as for example sieve-tubes ${ }^{4}$, may exist and show vital activity without possessing a nucleus of their own, while in order that cilia may remain active, a living intracellular connexion must exist between them and the nucleated protoplast ${ }^{5}$.

By this intercellular protoplasmic continuity, the protoplasts of multicellular plants are bound together to form a concrete whole. Hence it is impossible to lay down any hard and fast line of demarcation between multicellular and unicellular multinucleate plants, especially as the plasmatic connexions in multicellular plants show all grades of transition from the finest threads to coarse strands. Indeed, in some cases, as for example in Cladophora, the formation of the transverse partition walls may be partially suppressed under certain conditions. The segmentation of the organism into cells is of importance in many respects, for it ensures a sufficiently firm and rigid structure, and at the same time provides suitable dwelling-places for the soft-bodied protoplasts (Sect. 6). Moreover, local injuries are less dangerous than in large unicellular plants, while the raried forms and contents of the cells directly demonstrate what a marked differentiation of labour is possible between the component cells

1 [Haberlandt, Beziehungen zwischen Function und Lage des Zellkernes, r 887.]

${ }^{2}$ Fig. in Unters. a. d. Bot. Inst. z. Tübingen, r886, Bd. I1, Taf. ii, Fig. 5. See also Pfeffer. I 896 , Ber. d. Sächs. Ges. d. Wiss., p. 505.

${ }^{3}$ On plasmatic threads and their mode of formation, see the reference by Zimmermann, Reihefte z. Bot. Centralbl., I 893 , Bd. II , p. 328 ; A. Meyer, Bot. Zeitg., I 896 , p. I87; Bericht d. Bot. Ges., 1897 , p. 166; Pfeffer, 1896, 1.c., Sect. 20. [Kohl, Bot. Centralbl., Nov. I897; Gardiner, Phil. Trans., I $8 \varsigma_{3}$, p. 817.$]$

4 Zacharias, Flora, i 895 , Ergänzungsband, p. 224.

5 For other examples of extra-cellular protoplasm, see Schütt, Die Peridineen, I 895, p. I 37 ; Sauvageau, Bot. Centralbl., I89o, Bd. xLI, p. 293 ; Mangin, Recherches anatom. s. 1. composés pectiques, 1893 , p. 59 . 
of a multicellular organism. So marked a differcntiation of labour is impossible in unicellular plants, although in very large multinucleate forms, and cspecially in those such as Caulerpa, \&c., which stand on the borderland betwecn uniccllular and multicellular plants, a segmentation into root and shoot, \&c. may take place, even although the rotating cndoplasm travels continually through all parts.

Following the usual nomenclature, we may call each distinct individual protoplast a cell, independently of whether it is surrounded by a cell-wall (dermatoplast) or is naked (gymnoplast). Unicellular protoplasts may, using the nomenclature put forward by Hanstein ${ }^{1}$, be termed monoplasts, multinucleate protoplasts, symplasts. The Siphoneae and other multinucleate protoplasts are to be regarded as unicellular plants, not as non-cellular plants, as suggested by Sachs 2 .

Since the terms cell and protoplast definitely indicate the combination of nucleus and cytoplasm, it seems hardly necessary to introduce the term energid to represent the combination of a nucleus and cytoplasm which forms the living cell unit. To call a protoplast an 'energid 'will not make it any easier to understand its nature and vital mechanism. Sachs seems to state explicitly that in multinucleate cells each energid is to be regarded as a definite whole, even though no visible line of demarcation exists ${ }^{3}$. It is, however, hardly credible that a multinucleate cell is made up of a series of energids, in each of which the living substance remains separate and distinct from its neighbours, and indeed it may be directly seen that the cytoplasm in the neighbourhood of each nucleus is continually changing when streaming takes place, while if the nucleus rotates also, it is continually being associated with new portions of the non-moving ectoplasmic layer. Moreover it is quite certain that the cytoplasm lying between a number of nuclei is influenced by all of them to an extent corresponding to their respective distances from it, so that all these considerations compel us to regard a multinucleate cell as a single unit, both morphologically and physiologically.

[It will be noticed that the discussion of this essentially morphological question from a purely physiological point of view creates considerable confusion. The word 'cell' has such widely different meanings attached to it that it seems inadvisable to use it as a scientific term of restricted application. In the widest possible sense, any differentiated mass of plasma-with or without a cellwall, with one or with many nuclei-will constitute a cell, while a protoplast is formed by the symbiotic union of a single nucleus and a single plasma mass only, and represents the lowest unit capable of separate existence, for that non-nucleated forms exist at the present day is doubtful. A typical vegetable cell will therefore be composed of a cellulose vesicle enclosing a single protoplast. A number of protoplasts may be united together to form a multinucleate coenocyte, which may

1 Hanstein, Bot. Abh. I88o, Bd. Iv, p. 9.

${ }^{2}$ Sachs, Ueber einzellige Pflanzen, Sitzungsb. d. Phys.-Med. Gesellschaft zu Würzburg, i 878.

${ }^{3}$ Sachs, Flora, 1892, p. 57 ; ibid., 1895 , Erg.-bd. p. 406. See also Zimmermann, Beihefte z. Bot. Centralbl., I 893 , Bd. III, p. 207 ; Strasburger, Histologische Beiträge, I 893 , IBd. v, p. ıoS. 
or may not be enclosed by a cell-wall. The cellular segments of Cladophora, Chara, \&c., are therefore coenocytes, and Codium, Neomeris, Caulerpa, \&c., apparently represent less highly specialized evolutionary developments in the same direction, while the body of a typical Myxomycete corresponds to a single coenocytic cell, in the Acrasiae the fusion of the constituent protoplasts being an imperfect one. In such a plant as Neomeris dumetosa each of the rudimentary cell segments, into which the plant is divided, retains its own chloroplastids, nuclei, and the bulk of the plasma, and the same applies to other Siphoneae as well, so that these organisms can no more be regarded as unicellular than a row of sieve-tubes, and indeed a phanerogamic tree, in so far as protoplasmic connexions exist between its component protoplasts, might with equal justice be regarded as a single cell. Moreover, if it is admitted that the reproductive organs form an essential part of the entire plant, then Mucor, Vaucheria, Neomeris, Sc., all when adult become multicellular, for, using the term in its most restricted sense, only those organisms are unicellular which are composed of a single protoplast. The usually accepted distinction between uni- and multicellular plants is an artificial one, and since it was originally founded on a misconception, no importance need now be attached to it. Ed.]

\section{SECTION II. Chemistry of the Protoplast.}

As its structure indicates, the protoplast is a physiological but not a chemical entity, for even if all the substances of which it is composed could be mixed together in the right proportions, we should still fail to create a new living protoplast. Indeed, the life of the protoplast, as well as that of the smallest organ it contains, is destroyed by triturition, and even the smallest physiological units are not unorganized chemical compounds, but are organized bodies having a definite structure.

The most complete chemical knowledge of the substances composing the protoplast (Sect. 1 ) is as little able to make us understand the nature of the living organism, as a knowledge of the chemistry of iron and coal is to enable us to comprehend the mechanism of the steam-engine and the printing-press, if we are unacquainted with them. Nevertheless, a thorough knowledge of the chemistry of the protoplast forms an essential preliminary to all studies upon the nature of the vital mechanism. Indeed, it is hardly possible to over-estimate the physiological importance of a complete knowledge of the chemical nature and properties of the different proteid substances.

Chemical affinities and chemical quality play a more important and complicated role in an organism than in a machine. It is by chemical changes in the protoplast itself that building material is formed, and the necessary energy rendered available; while nourishment, growth, and movement are all directly or indirectly dependent upon chemical affinities and properties, to which also the retention of the same general composition 
by the protoplast, in spite of its unceasing internal changes, is directly due. In the vital mechanism, chemical changes are indissolubly connected with physical changes of either form or energy, and thus chemical problems are necessarily always involved in the phenomena of growth, movement, and indeed all vital manifestations. The term 'constellation,' used with reference to the aggregate of conditions regulating the vital mechanism, includes both the chemical nature and relationships of the component parts, as well as their structural organization and the way in which they are combined together to build up the protoplast. It can hardly be doubted that a difference in the character of the organism may be produced either by a difference in the structure of the protoplast or its component parts, or by a difference in the chemical nature of the latter, or by a combination of chemical and structural differences.

When different lichen forms arise from similar algae and fungi, we have an example in which the component parts are chemically identical, but differ in the way in which they are arranged. Similarly, if the nucleus and cytoplasm of different plants could be combined together the new protoplast would certainly resemble in properties neither of the original protoplasts, nor would the nucleus or cytoplasm of the new combination resemble those of the old combinations from which they were derived, and with which they are chemically identical.

On the other hand, it may with certainty be assumed that the nature and properties of physiological units, as well as of the organs and elements which are formed from them, are largely determined by the chemical character of the component molecules. So that, if from one physiological unit particular molecules could be removed and others substituted, the special inherent characteristics of the unit in question would be altered.

It is impossible at present to determine to what cxtent the peculiarities of different organisms are due to differences in the chemical nature of the component parts of the protoplast, and to what extent they are due to differences in the protoplastic structure, or whether in all cases both chemical and structural differences exist side by side. Frequently differences in the chemical nature of different nuclei \&c., may be detected, but the methods at our disposal are inadequate to enable us to recognize all the chemical differences which may exist between the great variety of proteid substances that undoubtedly take part in the formation of the protoplast. A very slight difference in constitution may be of considerable physiological importance, since thereby the nutritive value of a particular substance may be markedly altered.

The same general principles would be applicable to protoplasts which were not mainly built up of proteid substances, as are those found on the Earth. We have no grounds for supposing that nothing of a vital nature could possibly exist in the absence of all proteid materials, and 
no one can say whether or no under special conditions, such as existed on the earth before it cooled, or such as are found on other planets, life of a totally different character may not have existed, or in the latter case may not still exist. It is not impossible that there may be organisms in which the place of carbon is taken by silicon, or by some other element. Since the number of compounds in which silicon can replace carbon is relatively small, and since we know of no element which can combine with others in the multifarious ways that carbon does, such life would necessarily be of simpler character, and capable of vital manifestations of much less varied character than is the form of life which the protoplast reveals to us. The fact that we know so little even of our sister planets, and the possibility of the existence on them and elsewhere of elements which are new to us, renders vain all such speculation.

Sufficient has, however, been said to show that it is a fundamental error to suppose that protoplasm is a definite chemical entity, or that one particular substance is responsible for the vital powers of the whole.

It has not yet been satisfactorily determined whether, in addition to proteids; other bodies, such as lecithin, cholesterin ${ }^{1}$, fats or carbo-hydrates, may not form directly, or in combination with proteids, an essential part of the living protoplast, for a substance may be essential, even though but little of it be present. On the other hand, the constant presence of certain materials does not afford conclusive evidence on this point, for frequently substances are constantly present which do not form an essential part of living protoplasm.

Our chemical knowledge of the group of bodies known as proteids is as yet very incomplete, and owing to their large and complicated molecules, the variety of possible combinations is much greater than in the group of the carbo-hydrates. It is certain that a very small proportion are as yet known of all the possible proteids and their combinations. Our present knowledge on this point indicates that they are a group in which very trifling stimuli readily produce molecular and other changes, or induce processes of decomposition or polymerization. Hence it is possible that the proteids present in the living cell become disorganized when death takes place, either as the result of contact with substances from which they were separated so long as life was retained², or because they can exist only under very peculiar and special conditions. No chemical analysis of dead plasma can reveal to us, or give us any information about, proteids of this character ; hence, at present a knowledge

1 Kossel (Archiv f. Physiologie, I891, p. I8I) concludes that this union is essential. On Choltsterin in plants, see Schulze, Zeitschr., f. Physiol. Chemie, IS90, Bd. xiv, p. $5^{12}$. On Lecithin, Schultze, Versuchsstat., 1894 , Bd, xLı11, p. 307 ; Stocklasa, Ber. d. Chem. Gesellschaft, 1896, p. 2761 .

${ }^{2}$ Pfeffer, Oxydationsvorgängc in lebenden Zellen, is $859, \mathrm{p} .45 \%$. 
of the precise nature of all the different forms of proteid is impossible. Indeed, the very names of nuclein, albumin, \&c., are simply generic terms for different groups of proteids.

It is the changeable, inconstant, and manifo!d nature of proteid substances, which renders them of great importance, and makes them essential in the maintenance of life. The different varieties of proteid ${ }^{1}$ have widely different physiological properties, for enzymes, poisonous tox-albumins, and even chitin, found in the cell-walls of fungi, all belong to this group.

A number of proteids also take part in the formation of living protoplasm, and indeed, it is possible that in every organism a specific form of proteid of local or general distribution is always present, for there is actual evidence to show that different parts of the cell are characterized by special forms of this material. Thus, the chromatin of the nucleus contains much nuclein, while the spindle-threads of the cytoplasm are different in composition and contain but little nuclein. In dead reserve food material, howerer, nuclein may also be present. A certain difference always exists between reserve food material and organically assimilated substance, for the fo d material becomes of use to the organism, and is partly converted into building material, only after it has undergone certain preparatory changes.

Both macro- and micro-chemistry lead us to conclude that certain differences exist between the proteids of different plants, and other facts point to the same conclusion. Thus it is evident that thermophile bacteria, which can develop at $74^{\circ} \mathrm{C}$., and spores, which, even when moist, can withstand a temperature of $110^{\circ} \mathrm{C}$. for half an hour, cannot possibly contain any formis of proteid which coagulate at the relatively low temperatures fatal to ordinary plants.

Summary of the chemical properties. The mode of treatment applied to proteids in text-books of chemistry is a good illustration of how little is known concerning them. A fairly good account of the better-known proteid substances is given in several text-books of physiological chemistry ${ }^{2}$, in which the important phenomena of solution, crystallization, coagulation, digestion, \&c., are dealt with. Although in these treatises animal proteids are chiefly considered, still all the more

'[The term 'proteid' is here used in a slightly broader sense than is usually customary. Thus, strictly speaking, mucin and chitin are probably not true proteids.]

${ }_{2}$ Neumeister, Lehrbuch d. Physiol. Chemie, $18_{93}$, Bd. I; Hammarsten, ibid., 2. Auf., i $S_{95}$; Bunge, ibid., 3. Auf., 1S95; Hoppe-Seyler, Handb. d. physiologisch-pathologisch chemischen Analyse, 6. Aufl., IS93. A full account of the vegetable proteids is given by Drechsel, under the heading 'Liweisskörper,' in the Handwörterbuch d. Chemie, Bd. III, 1885; also by F. Schwarz, Cohn's Beiträge z. Biologie, Bd. v, ISS7. On the relations between animal and regetable proteids see E. Schulze, 'In wie weit stimmen Pflanzen u. Thierkörper in ihrer Zusammensetzung überein,' Sep.-abdr. aus d. Vierteljahrsschrift d. Naturf.-Ges. in Zürich, $1 S_{94}$; Palladin, Zeitschr. f. Biologie, 1894 , p. I91; Fleurent, Compt. rend., 1893, T. cxvis, p. 790. 
important proteids appear to be common both to animals and plants, and indeed both animal and vegetable protoplasts seem to be composed of essentially similar forms of proteid. The mode in which proteids are obtained, and their importance in metabolism, will be dealt with in subsequent chapters.

The group of proteid substances includes albumins, enzymes, mucin, chondrin, chitin ${ }^{2}$, \&c., bodies differing widely in character and properties, and from which various more or less closely allied substances may be derived. All proteids contain nitrogen, but not all of them contain sulphur or phosphorus. As a general rule, hydrolytic decomposition results firstly in a formation of peptones and allied substances, and finally amides are produced, while frequently, but not always, carbohydrates, as well as leucin, tyrosin, and other aromatic substances ${ }^{2}$, may appear. The latter need not, however, necessarily form an integral part of the molecule of all proteids, although it is to the aromatic radicles that the results given by Millon's test and indeed the principal colour reactions of proteids are due.

Without assuming that these decomposition products form integral parts of the molecule of albumin, it is nevertheless certain that the latter is built up of numerous groups of atoms. Qualitative and quantitative differences in the latter, together with substitution, polymerization, and different arrangements of the component groups, render an endless variety of combinations possible. Certain proteids, such as the vitellins found in aleurone grains, are even crystallizable. Whether the molecule of living proteid is a polymerization product of aspartic aldehyde, or is composed of a chain of cyan-alcohols, united to a benzene nucleus ${ }^{3}$. or has a chemical constitution quite different from either of these, is impossible at present to say ${ }^{4}$, and it is moreover doubtful whether any definite protoplasmic molecule does actually exist.

Among proteid substances three main classes may be recognized, namely, albumins, globulins, and albuminoids ${ }^{5}$. Albumins may unite with various substances, and thus form a number of compound proteids; for example, by adding metaphosphoric acid to albumin a substance closely resembling nuclein is produced $^{6}$, while by the combination of albumin with carbo-hydrates, glucosides,

1 According to Halliburton (Physiol. Chem.) the true vegetable proteids, like those of animals, may be separated into seven classes-albumins, globulins, albuminates, proteoses, peptones, 'coagulated' proteids, such as the gluten of flour, and enzymes. Nuclein, plastin, chitin, mucin, chondrin, \&c., are all albuminoids, differing in varying degrees from true proteids. Nuclein is a highly phosphorized substance, as also is plastin. All mucins are glucosides, i.e. compounds of a proteid with a gum, which latter, by treatment with dilute sulphuric acid, can be hydrated into a reducing but non-fermentable sugar. Enzymes may possibly be nucleo-proteids.]

${ }^{2}$ Cf. Drechsel, 1.c., p. 549 ; S. Schulze, Landwirthsch. Jahrbiicher, 1892 , Bd. Xxi, p. I 2 I ; Malfatti, Bot. Centralbl., I893, Bd. Lv, p. I 52 ; Cohn, Zeitschr. für physiol. Chemie, 1896, Bd. xxiı, p. 153 ; Kossel, ibid., p. 176 ; Hedin, ibid., p. 19 1.

${ }^{3}$ [Lathom, Brit. Med. Journal, vol. i, 1886, p. 629.]

4 Cf. Drechsel, 1.c., pp. 538 and 547 .

${ }^{5}$ See Neumeister, 1. c., p. 33. Also Hammarsten, Zeitschr. für physiol. Chemie, 1894, Bd. x1x, p. 19. On the synthetic production of certain proteids see the literature quoted by Neumeister, Physiol. Chemie, I893, Bd. I, p. 44. Also Kossel, Archiv f. Anat. u. Physiol., Ihysiol. Abtheilung, 1893 , p. 157 .

- For an account of nuclein-and its compounds see the works alrcady quoted, and also Milroy, Zeitschr. f. physiol. Chemie, 1896 , Bd. xxir, p. 307. 
such as mucin, are formed. Nuclein may unite with albumin to form nucleoalbumins, and to this class the plastin of microscopists probably belongs. In nuclein, from 3 to 9.6 per cent. of phosphorus is present; in nucleo-albumin, the proportion is naturally lessened. Similarly, in glucosides the percentage of nitrogen frequently diminishes from the 16 per cent. of typical albumin to as little as 10 per cent.

Mucin, keratin, chitin, \&c., are examples of albuminoids, and are substances which, though obtained principally from animals, are also undoubtedly widely distributed in the vegetable kingdom.

The different forms of proteids, and their combinations and derivatives, are used by the plant in various ways; thus nuclein takes a very prominent part in the formation of the nucleus, nucleo-albumins are often found in the form of food material (legumin, conglutin, \&c.), and the plastin of which the cytoplasm appears to be very largely composed contains less phosphorus than nuclein does, and is hence probably a nucleo-albumin ${ }^{1}$.

Micro-chemical research indicates that the chromatin threads are composed mainly of nucleins rich in phosphorus, and that the nuclear substance in which these threads are embedded is composed of proteids, which are readily dissolved by an acid solution of pepsin ${ }^{2}$.

Nuclein is not digested by pepsin, and only with difficulty by trypsin, and hence these very properties may possibly render it especially useful as a building material or permanent skeletal framework. When nucleo-albumins are used as food material, nuclein may either be obtained directly by the molecule being split into two, or the living substance may itself form the nuclein building material, after the nucleoalbumin food material has been assimilated by it.

Both from a chemical and from a physiological point of view, it seems inadvisable to give special names to characterize all the minute micro-chemical points of difference which exist between the various forms of proteid; for, as is well known, micro-chemical properties may be markedly modified by infiltration with small quantities of other substances. Hence it is only natural that the attempts by F. Schwarz ${ }^{3}$ to replace the general groupings of proteid substances by a series of such special names have met with but little success ${ }^{4}$.

The Changes which accompany Death. When the plasma is killed, it undergoes a variety of chemical and physical changes, indicated by the loss of plasticity, by the separating out of various mineral, proteid or pigment ${ }^{5}$ substances, and by other

${ }^{1}$ Malfatti, Bot. Centralbl., 1893 , Bd. Lv, p. 152. See also F. Schwarz, Cohn's Beiträge, i 892 , Bd. v, p. 126 .

"See Malfatti, Schwarz, \&c., l.c., for the action of ferments on nuclein; Popoff, Zeitschr. f. physiol. Chemie, i\$94, Bd. xvil1, p. 539. See also Zimmermann, Zellkem, 1S96, and Zeitschr. f. Mikroskopie, ז 896 , Bd. xır, p. $45^{8}$.

' F. Schwarz, Cohn's Beiträge z. Biologie, $1 S_{92}, \mathrm{Bd}$. v.

4 See O. Hertwig, Zelle, 1893, p. 37 ; Zimmermann, Beihefte z. Bot. Centralbl., 1893, Bd. 1II, p. 323.

${ }^{3}$ Pfefier, Unters. a. d. Bot. Inst. z. Tübingen, 1886 , Bd. 11, p. 276 . See also Campbell, ibid., p. 569 . 
visible alterations. 'The study of the changes which occur when the protoplasm dies is obviously of the highest importance.

The physical charactcrs of the dead protoplast, and especially its power of absorbing colour-stains in varying degree, give us a valuable suggestion and indication of what may be the probable nature and structure it possessed during life. The absorption of stains can be used, however, only with the utmost caution as a test of the chemical nature of any given part, for absorption is dependent upon physical rather than chemical properties ${ }^{1}$. Thus carbon in the form of a diamond is non-absorbent, but in the form of charcoal has marked absorptive powers, and hence can decolorize coloured fluids. Substances are known, both in technical industry and in science, which may be rendered capable of absorbing pigments by a special mode of preparation, although they retain the same chemical constitution ${ }^{2}$. It is only in the light of these facts that any conclusions can be drawn from the cyanophil or erythrophil character of the nucleus, or from the basophilous or eosinophilous behaviour of the plasma ${ }^{*}$.

Percentage Composition. In any analysis of the entire protoplast, various substances not actually forming part of the living substance are necessarily included, and these are also present in the plasmodium of a Myxomycete. Hence the percentage composition of the latter is as incapable of giving an empirical or percentage formula for the plasma, as is the analysis of a highly protoplasmic tissue from which the cell-walls have been removed. Reinke ${ }^{4}$ found that the plasmodium of Aethalium septicum contains, when fresh, as much water as is found in moderately turgid tissues (Sect. 33).

The plasmodium contains 71.6 per cent. of water, of which 66.7 per cent. may be removed by strong pressure. In soo parts of the air-dry materials were found :

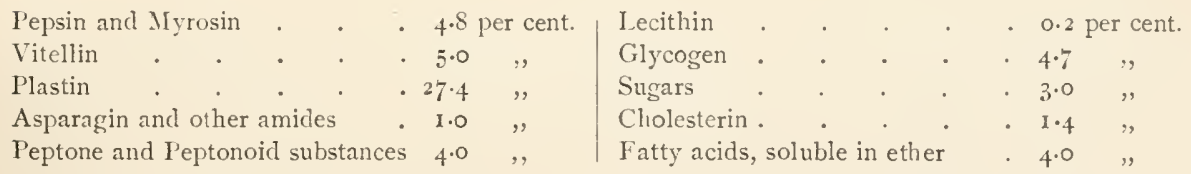

In the remaining 44.5 per cent. a number of organic substances are included, as well as the ash constituents, of which calcium carbonate forms $27 \cdot 7$ per cent. The nucleins, which are not specially mentioned here, comprise in leucocytes, according to Kossel ${ }^{5}, 34 \cdot 26$ per cent. of the entire weight.

The so-called active albumin. It is impossiblc at present to say exactly what changes the proteids of the protoplast undergo on death. Loew and Bokorny have constructed a formula for living proteid, and suppose that vital activity is centered in

1 Ostwald, Lehrbuch der allgem. Chemie, i 89 r, 2. Aufl., Bd. i, p. Iо93.

${ }^{2}$ See Pfeffer, 1. c. ; A. Fischer, Unters. über Cyanophyceae u. Bacterien, 1897, pp. 5, 118.

3 Rosen, Cohn's Beiträge, $\mathrm{x} S_{95}, \mathrm{Bd}$. vir, pp. 225,304 , and the literature there cited. See also Lilienfeld, Centralbl. f. Physiol., I893, Bd. vir, p. 621 ; A. Fischer, 1. c., Heine, Zeitschr. f. physiol. Chemie, I $896, \mathrm{Bd} . \mathrm{xxI}, \mathrm{p} .494$. On the alkaline and acid reactions of protoplasm see Sect. 86.

" Reinke, Unters, a. d. Bot. Lab. in Göttingen, I881, Heft 2, p. 54. See also Hofmeister, Pflanzenzelle, $186_{7}$, p. 2.

5 See O. Hertwig. Die Zelle n. d. Gewebe, I893, p. 17. 
the aldehyde groups which it contains. 'These are, however, speculations to which neither chemistry nor physiology lend support '.

The method of silver-reduction which Loew and Bokorny employed is not, as they stippose, a specific test for aldehydes ${ }^{2}$. In certain cases, the reducing substances are not proteids at all, while the disappearance of the power of reduction after death is frequently merely due to an exosmosis of the substances in question. If by treatment with caffein, antipyrin, ammonium carbonate, aniline dyes, \&c., a dead cell is caused to retain these reducing substances, a granular reductionprecipitate is formed when a solution of silver is added. These granules are called by Loew and Bokorny, 'Proteosomes,' and regarded by them as a sure sign of the presence of active albumin. (See the quoted works of $189 \mathrm{I}$ and 1895.) Since, however, the granular precipitations formed by caffein, \&c., partly retain their reducing powers after being boiled, they can hardly be characterized by that lability and tendency to decomposition which Loew and Bokorny state to be an essential property of their active albumin ${ }^{3}$.

Loew and Bokorny now indeed admit that these reducing substances are commonly present only in the cell-sap, and see in their 'active albumin' not a component of living plasma, as they originally maintained, but simply a substance of importance in metabolism. No further attention need therefore be given to the suggestion that in active albumin we have the directive and decisive agent in originating vital processes. For in every case the inherent nature, structure, and organization of the protoplasm are of paramount importance for all vital phenomena, and the knowledge that particular food substances are necessary for its growth and maintenance, as indeed must necessarily be the case, gives us but little or no deeper insight into its internal mechanism, or into the causes which affect and govern this mechanism.

Since the precipitations which, according to Loew and Bokorny, are a certain test for the presence of active albumin, are apparently not produced in many plants, it is impossible that these reducing substances can have any such general and essential importance as Loew and Bokorny originally supposed. For since it may be readily proved that the caffein and other substances do actually penetrate the plasma and appear in the cell-sap, it follows that the non-formation of any precipitate is due to the absence of the reducing substances. A more direct proof that the active albumin does not form an essential part of the living plasma is afforded by the fact that cells in which the 'active albumin' is kept permanently precipitated by caffein, \&c., remain living and capable of active growth.

The chemical nature of the bodies in question is therefore immaterial to the point at issue. In certain cases, no proteid substance can be detected in the precipitated 'proteosomes,' while similar granular precipitates may be produced

${ }^{1}$ Loew und Bokorny, Die chemischen Kraftquellen im lebenden Protoplasma, I882; Biol. Centralbl., I891, Bd. XI, p. 5 ; Flora, 1896, p. 68, \&c. Cf. Pfeffer, Flora, 1889, p. 46 ; Klemm, Flora, 1892, p. 395, and Ber. d. Bot. Ges., I 892 , p. 237 ; Zimmermann, Beihefte z. Bot. Centralbl., I 893 , Bd. III, p. 323 , and the literature here cited.

${ }^{2}$ See Baumann, l'fliger's Archiv f. Physiol., I882, Bd, xxix, p. 400.

${ }^{3}$ See Correns, Jahrbuch f. wiss. Bot., is94, Bd. xxv1, p. $64 \mathrm{I}$. 
when phloroglucin or tannin is present. 'Thus in capillary tubes, or artificial cells formed by precipitation membranes of tannic acid and gelatine, treatment with caffein, ammonium carbonate, \&c., produces similar granular precipitates, which have the power of reducing a silver solution. (Secs, 16 and 22.) Hence it is impossible to agree with Loew and Bokorny in their assumption that the precipitate always and necessarily results from a polymerization of 'active albumin.'

For a science dependent on facts, and not on dogma, the speculations of L. and B. cannot be regarded as forming even a tenable temporary working hypothesis. Indeed, it is hardly possible that vital activity could be the inalienable property of a single chemical substance, or that all the phenomena of life could result from the changes which a single chemical substance undergoes.

The above objections and criticisms apply only to the incorrect interpretations and conclusions drawn by Loew and Bokorny from the facts observed by them. That particular proteid substances play an important part in the vital mechanism, and that various changes may occur in them at death, are general conclusions attained long ago. Independently of Loew and Bokorny's speculative hypothesis, it has been concluded on other grounds that a continual decomposition of proteid substances is necessary for the maintenance of active life, and that some form of albumin or albumin-compound may continually be produced, the instability of which is the direct cause of the phenomenon of respiration, and of other vital processes as well. (Chaps. VIII and IX.) It is possible that among such physiologically active substances aldehyde groups may play a part, and perhaps many forms of sugar also, for carbohydrates may be combined with and form a constitutional part of the molecule of certain proteid substances, e. g. glucosides, such as mucin. 


\section{CHAPTER III}

\section{IMBIBITION AND MOLECULAR STRUCTURE}

\section{Section 12. The Force of Imbibition, and the Swelling of Organized Bodies.}

IN order that the different vital processes which characterize the living organism may be maintained, water and dissolved substances must penetrate every part of the cell. The cell-wall, protoplasm, and indeed all organized substances have the power of imbibing water and swelling. This imbibed water causes the component particles of organized structures to separate more or less widely from one another and thus brings about an increase in volume of the whole, while the reverse change takes place when the water evaporates. This property is not restricted to organized substances only ${ }^{1}$ : thus gelatine and agar swell in water, collodion swells in ether, and indiarubber in bisulphide of carbon. When the particles of a solid become so widely separated that they pass beyond the spheres of their respective attractive affinities, a condition resembling that of solution is produced, and the organized structure is lost, if any were originally present. It is preferable to restrict the term 'organized' to substances formed by the living organism, and not to apply it to all bodies capable of imbibition and swelling, as was formerly done in connexion with Nägeli's theory ${ }^{2}$.

Since all hypotheses concerning the molecular structure of organized bodies are primarily dependent upon the phenomena of imbibition and swelling which these exhibit, a gencral account of the phenomena in question will be useful, especially as in physical text-books the points of physiological importance are not brought prominently forward. From

1 Viz. Acrylcolloid, Tullens, Am. d. Chemie u. Pharmacie, 1874 , Bd. cLxxi, p. 356. For other examples see Pickering, Centralbl. f. Physiol., I895, Bd. IX, p. 599. The formation of cellulose by chemical synthesis is only a question of time. On the swelling of sphaerocrystals, A. Meyer, Stärkekörner, 1895, p. 108 .

' 1. Aufl., Bd. I, p. I3. See Pfeffer, Studien zur Energetik, $\mathbf{I S 9 2}_{92}$ p. $1_{5} S_{\text {. }}$ 
a general standpoint it is unimportant whether the discrete particles of which the swclling substance is composed are molecules, micellae or other units. Hence for the present no spccial molecular structure need be assumed.

The swelling is dependent not only upon the nature of the substance itself, but upon the character of the fluid absorbed: thus cell-walls swell in water but not in alchohol or turpentine, while gum arabic, in water alone, swells indefinitcly and finally passes into solution, whereas in the presence of a little alcohol the gum arabic absorbs only a certain amount of water, and swells to a limited extent. A variety of intermediate stages are possible between the limited absorption of water which causcs simple swelling, and the unlimited absorption which gives rise to a condition of solution. It is indeed often doubtful whether certain colloid substances do actually dissolve in the fluid throughout which they are distributed ${ }^{1}$.

Limited solubility in a given fluid is due to the latter having a restricted power of taking up the particles of the dissolved substance. Hence imbibition, swelling, and the formation of the so-called solid solutions are all closely related phenomena. Thus when carbon is absorbed by a piece of iron, hydrogen by palladium or spongy platinum, or when mercury is taken up by other metals to form amalgams, the penetration of the foreign element causes a certain change of volume ${ }^{2}$.

The amount of imbibition of which organized bodies are capable varies within wide limits: thus corky membranes hardly swell at all, while the gelatinous envelopes of the Nostocaceae, \&c., according to Nägeli $^{3}$, contain 200 of water to one part of solid substance. The dry walls of wood tracheides can absorb $4^{8}$ to $5^{\mathrm{I}}$ per cent. of water ${ }^{4}$, whereas the amount of water present in the protoplasm varies from 60 to 90 per cent. It is true the latter is semi-fluid, but as is shown by the gelatinous membranes of the Nostocaceae, a solid condition is possible even when the amount of water present is extremely great. The semi-fluid nature of the plasma is of the highest physiological importance, as it renders internal and external alterations and movements more readily possible. In the cell-wall on the other hand a certain degree of rigidity is esscntial, and hence its component particles exhibit a high degree of cohesion, even when the imbibition and swelling are so great that they are relatively widely separated from one another.

${ }^{1}$ Cf. Lehmann, Molecularphysik, I888, Bd. I, p. 526 ; Barns und Schneider, Zeitschr. f. physik. Chemie, I89I, Bd. virr, p. 278 . On Glycogen, \&c., Brucke, Vorles. über Physiol., I88I, 3. Auf., p. 325; and Errera, l'Epiplasme des Ascomycetes, I882, p. 70.

${ }^{2}$ Cf. Van 't Hoff, Zeitschr. f. physik. Chemie, r 890 , Bd. v, p. 325 ; Küster, ibid., 189f, Bd. XIII, P. 445 .

3 Nägreli, Stärkekörner, 1858 , p. $3^{1} 2$; other examples by Hofmeister, Pflanzenzelle, 1867 , p. 214 .

4 Sachs, Arb. d. Bot, Inst, in Wiirzburg, 1879, Bd. II, p. 3I 2. Cf. Sect. 27. 
Increase of volume is not always entirely due to the imbibition of water by the swelling substance, for when a seed swells, the greater part of the water is absorbed osmotically, and accumulates inside the cell. The swelling which ensues may induce a widening of the spaces or canals through which water is conveyed, and the same is also the case in nonliving structures, such as a bath sponge or a dead moss-leaf. When an organized structure dries up, these spaces collapse and disappear, so that no air enters and transparency is retained, while if placed in water again the spaces and canals containing water again appear. It follows that in colloidal bodies the individual particles (molecules or groups of molecules) must be combined together to form a skeletal meshwork ${ }^{1}$; and indeed the reticulate structure visible in protoplasm (Sect. 7), and also in non-living jelly ${ }^{2}$, may be directly due to some such arrangement, which is, moreover, well adapted to attain a high degree of cohesion, when a small amount of substance occupies a large volume.

Only a small portion of the imbibed water is, under such conditions, subject to the molecular influences radiating from the component molecular groups, for the sphere of influence governed by a single molecule or group of molecules is immeasurably small. Hence it is possible to distinguish between the bound molecular imbibition-water or adhesion-water which is in immediate contact with the molecules of the imbibing substance, and the capillary imbibition-water which fills the central parts of the minute interstitial spaces and canals ${ }^{3}$. Since the radius through which molecular influences can be exerted is so excessively small, even a trifling separation of the component molecules or micellae involves the presence of a certain amount of capillary imbibition-water.

The difference between swelling and non-swelling bodies is not due to any peculiarity in the manner in which the water is held, but is caused by their different behaviour when water penetrates them, for the extent to which a substance swells is dependent upon the amount of capillary imbibition-water which can be absorbed ${ }^{4}$.

Cell-walls and other bodies capable of imbibition show every transition from a marked to an imperceptible increase in volume. Gelatinous sub-

${ }^{1}$ Lehmann, Molecularphysik, 1888 , Bd. I, p. $5^{25}$; Nägeli, Theorie d. Gährung, 1879, pp. 102 and 127; Van Bemmellen, Beibl. z. Ann. d. Physik u. Chemie, I889, Bd. XIII, p. 63; Kekuli, Die wiss. Ziele u. Leistungen d. Chemie, I87s, p. 22; von Bemmellen, Die Absorption, 1897.

${ }^{2}$ Bütschli, Unters. über mikros. Schäume, $1 \mathrm{~S}_{92}$, p. 218 ; Gerinnungs-Schäumen in Sphärokrystallen, I894; Úber Structurkunst in nat. quellb. Substanzen, I895 (Sep.-abdr. a. d. Verh. d. Natur. Med. Ver. z. Heidelberg); Über den Bau quellbarer Körper, 1896; Steinbrinck, Ber. d. Bot. Ges., 18y6, p. 29 ; Kolkwitz, ibid., p. 106.

3 Pfeffer, Osmot. Unters., 1877, p. 39 .

"This idea was promulgated in the first edition. See also Schwenderer, Sitzungsb. d. Berliner Akad., 1886, Bcl. xxxiv, p. 590; A. Meyer, Unters. über die Stärkekörner, I895, p. I08; Bot. 'Zeitg., 1896, p. 330 . 
stances and cell-walls when hardened in alcohol lose the power of swelling in water, in this respect behaving like porous bodies the pores of which have become filled with air as the alcohol evaporated. W'hen a pile of glass plates is placed in water, each plate becomes slightly separated from its neighbours as capillary water penetrates between the plates, and the height of the whole pile is slightly increased. For the same reason imbibition causes in a finely porous sphaero-crystal a degree of swelling, the extent of which is determined by the amount of colnesion existing between the radially-arranged acicular crystals of which it is composed.

The amount of swelling, and, indeed, all the phenomena of imbibition, capillarity and absorption are manifestations of the same natural forces as are exhibited in the phenomena of surface-tension? Surface-tension energy, or in other words, the attraction existing between the water and the imbibing substance, causes the former to penetrate between the component particles of the latter, and force them asunder as far as the cohesion existing between the component particles of the given substance will allow. Since, however, the distance through which the energy of surface-tension can act is excessively small 2 , the power of swelling, as well as the expansive force which the swelling may exert, rapidly diminishes as the amount of the imbibed water increases. The first molecules of water are absorbed with extraordinary energy, and undergo considerable compression, but as imbibition progresses the force of absorption rapidly decreases, and further expansion ceases before the films of water which separate the component parts of the imbibing substance from one another have attained a measurable thickness ${ }^{3}$.

The same general relationships hold good, whether the component elements separated by the water of imbibition are molecules, micellae, or groups of micellae, or whether the micellae or micellar groups are themselves capable of swelling.

In addition, chemical union, partial solution, and other factors may be correlated with imbibition phenomena, while from the differences which have been shown to exist between solution and swelling, it follows that in most cases, though not necessarily in all, a sharp distinction may be drawn between the swelling due to simple imbibition and that due to processes of solution ${ }^{4}$. The swelling which substances may undergo in

1 Pfeffer, Studien zur Energetik, I\$92, p. 163 .

2 See for example Winkelmann, Handbuch d. Physik, I891, Bd. I, p. 476 .

3 For the phenomena of swelling and the theoretical conciusions which may be deduced from them, see Nägeli, Stärkekörner, I 858, p. 332 ; Reinke, Bot. Abhandlung von Hanstein, I 879 , Bd. IV, p. 1 ; Rodewald, Landw. Versuchsst., 1894. Bd. XLv, p. 201. For theory of swelling, Reinke, Nach. d. Ges. d. Wiss. z! Göltingen, 1894 , p. 1 .

- A. Meyer (Unters. über die Stärkekörner, 1895 , p. 129 distinguishes in starch between the swelling of imbibition and the swelling due to solution. 
various fluids is in all essential points similar to that occurring in water. Hence the fact that cell-walls do not swell in alcohol or carbon bisulphide merely shows that the attraction existing between the substance of the cell-wall and either fluid, i. e. the force of imbibition, is not strong enough to overcome the resistance to separation offered by the component molecules, or micellae. There is no need for a special explanation of the fact that the amount of swelling in a watery solution is different from what it is in pure water, nor why water and salts are absorbed from a solution in different proportion to that existing in the solution in question, for if the affinity for water is the more marked it is only natural that a correspondingly larger relative amount of water should be absorbed. For example, when dried bladders are placed in a saturated solution of Glaubers salts, the absorption of water causes crystals to be deposited from the solution ${ }^{1}$. It is not, however, always the water which is most actively absorbed, as is shown by the absorption of dyes, salts, \&c. by coagulated egg-albumin or charcoal. Similarly indiarubber absorbs, from a mixture of water and alcohol, only the alcohol, and thus reduces the alcoholic percentage of the mixture. In all such cases the concentration of the watery solution imbibed will alter as the amount absorbed increases, and as the particles of the imbibing substance separate more widely from one another. If the attraction for water is relatively very great, the water imbibed may be either pure or in the form of an extremely dilute solution. Indeed, the water and salts of a saline solution may actually be separated by filtration through semi-permeable membranes capable of imbibition. These phenomena are of the highest physiological importance, for the diosmotic exchanges of the cell are determined and regulated by the power of absorbing water and dissolved substances possessed by the cell and its membranes.

The molecular movements and changes brought about by imbibition must necessarily have important bearings upon the chemical and other processes taking place in organized bodies. In a state of very fine subdivision the sum total of the surface-tensions of the component particles of a substance is very great, and becomes of decisive importance. Thus charcoal or spongy platinum are, in virtue of the enormous amount of surface exposed, capable of marked absorption or of inducing various chemical reactions, while in the diamond or in a piece of solid platinum these powers are reduced almost to vanishing point. Indeed the phenomena of absorption are on the borderland between chemistry and physics, and apparently are the direct results of the same properties and forces which determine the consistency and rigidity of solid bodies. The close study of the phenomena of absorption and surface-tension is of value in many

${ }^{1}$ Ludwig, Zeitschr. f. rationelle Medicin, von Henle u. Pfeufer, I $S_{49}$, Bd. vin, p. I5; Pfeffer, Osmot. Unters., I87\%, p. 40. 
ways, and may indeed have important bearings on the problems of chemical affinity ${ }^{1}$.

In order to overcome the cohesion of the component parts, and thus separate them from one another, great internal force must be exerted in the process of imbibition, and hence the swelling body may exert great pressure against external resistance by means of the surface-tension energy thus brought into play. Thus the expansive powers of swelling wood are well known, while according to Rodewald ${ }^{2}$, a pressure of 2523 atmospheres would be necessary to neutralize the force with which starch imbibes water and thus prevent any swelling. The force of imbibition, and hence also the power of expanding against external pressure, rapidly diminish as the absorption of water goes on. Thus Reinke ${ }^{3}$ found that from a frond of Laminaria saturated with water a trifling pressure squeezes out some water, but that to produce the same result, when the frond contained 63 per cent. of the possible content of water, required a pressure of 16 , and when 48 per cent., of 200 atmospheres.

It is not always the actual swelling force of the imbibing substance which is measured in such experiments, for just as in a bath-sponge any water present in the spaces which may occur between the framework of the swelling substance will be the first to be pressed out, so also in tissues composed of thin-walled turgescent cells, any pressure on the cell will cause water to be squeezed out as soon as the osmotic force with which the turgid cell retains water is overcome 4 . When a dried seed swells, imbibition and osmosis work together, and the force with which seeds absorb water is admirably illustrated by the use of moistened peas to split open skulls required for anatomical purposes. Hales found that peas when swelling in water could lift a weight of 83.5 kilos.

Since the time of Hales, various researches on the swelling of seeds have been carried out, but these have not materially added to our knowledge of either the nature of turgor or the processes involved in swelling ${ }^{5}$.

Within the plant, the cell-walls, protoplast, \&c. are often subjected to considerable pressure, influencing to a certain extent the amount of imbibitory swelling possible. In most cases the pressure is insufficient to cause any decrease of volume, but in many algae the inner layers of the cell-wall swell up and become gelatinous, when the pressure exerted on them by the protoplasm is removed ${ }^{6}$, while

'See Ostwald, Lehrb. d. allgem. Chemie, I891, 2. Aufl., Bd. I, p. 1085. Also Section 28 (Absorption from Soil) and Chap. VIII (Chemical Reactions in the Cell).

${ }^{2}$ Rodewald, Versuchsst., IS94, Bd. XLv, p. 237. On the energy generated in non-swelling bodies by imbibition see Jamin, Compt. rend., I 860, T. L, p. 3 I I.

${ }^{3}$ Reinke, 1. c., p. 54. Similar resẹarches by Liebig, Unters. über die Ursachen d. Saftbewegung im thierischen Organismus, 1848 , p. 5, and Ludwig, Lehrb. d. Physiol. d. Menschen, I 858 , Bd. I, p. 72 .

- Pfeffer, Druck und Arbeitsleistung, 1893, p. 288.

${ }^{5}$ See Detmer, Physiologie der Keimung, ISSo, p. I5; Nobbe, Samenkunde, IS76, p. Ioo; Reinke, l.c. Of the newer literature may be mentioned, Schindler, Wollny's Forschungen auf d. Geb. d. Agriculturphysik, ISSI, Bd. Iv, p. I9t; Schmidt, Versuchsst., IS89, Bd. xxxvı, p. 243; Régnard, Compt. rend. d. I. Soc. de Biologie, ISS9, p. 252 ; Grihaut, ibid., I889, p. 230; Bogdanoff, Versuchsst., I 893 , Bd. XI.II, p. 3 I ; Gain, Bull. d. 1. Soc. Bot. de France, I 894 , T. Xli, p. 490 ; Coupin, Anı. d. Sci. Nat., I 895 , viii॰ Sér., T. II, p. I 29.

- Pfeffer, Osmot. Unters., i $\delta_{77}$, p. 217 . 
even in the protoplasm itself changes of volume may be observed as the result of altered pressure.

The condensation of water on the surface of the molecules of the substance which it penetrates causes a relatively large amount of energy to be liberated in the form of heat when swelling or non-swelling bodies absorb water. This is, however, a purely physical problem ${ }^{\text {. }}$.

\section{SECTION 13. Hypotheses of Molecular Structure.}

All ideas of molecular structure rest on a hypothetical basis, and indeed atoms and molecules are simply convenient mental abstractions and may have no actual existence. Just as atoms and molecules are supposed to be the ultimate units of chemical structure, so also are the micellae supposed to be the ultimate elements of which organized bodies are composed, but we cannot postulate for the latter the same degree of certainty and hypothetical importance that we can for the former, so long as it remains uncertain whether the water which penetrates a substance capable of swelling separates molecules, or aggregates of molecules from one another. The power of swelling may be present in bodies of very different molecular structure, and need not necessarily be always precisely similar in character.

Nevertheless the attempts of Nägeli to establish a hypothesis of the intimate structure of organized bodies based upon the phenomena of growth and swelling, and also upon the optical characteristics exhibited by starchgrains, cell-walls, crystalloids, \&c., still remain of great value, and more especially as his ideas were put forward at a time when physicists and chemists had not as yet paid any attention to the molecular structure of organized bodies. It is true that the mode of growth of starch-grains which Nägeli assumed, and on which his first theoretical conclusions were based, has now been proved erroneous. Nevertheless his clear and precise deductions and conclusions are of all the more value, for in them we have the first conscious attempt to explain the peculiarities of organized bodies by reference to the particular molecular structure to which their several properties are due. The essentials of Nägeli's hypothesis are, in a slightly amplified form, still applicable to all the phenomena observed, and even at the present time afford the most thorough and satisfactory explanation of the molecular processes involved in growth and imbibition.

'See for example I.ehmann. Molecular-Physik, I888, Bd. I, pp. 289,548 ; Ostwald, Lehrb. d. allgem. Chemie, 3891 , 2. AuA., Bd. I, p. 1085; Winkelmann, Handb. d. Physik, 1891, Bd. I, p. 48o. On the sweiling of gelatine, see Wiedemann u. Luideking, Ann. d. Physik, I885, Jid. Xv, p. 5.3. On production of heat by swelling, starch, \&c., Nägreli, Theorie d. Gährung, 1879, p. 1.3.3; Reinke, Bot. Abhandlung von Hanstein, is 79 , Bd. IV, p. 70 ; Rodewald, Versuchsst., I894, Bd. XLv, p. 218. On the condensation of the imbibed water, Nägeli, Stärkekörner, I 858 , p. 53 ; Reinke, 1. c., pp. 61 and 132 . 
According to Nägeli ${ }^{1}$, the ultimate elements of "hich organized bodies are composed, i.e. the minute particles between which water penetrates when swelling takes place, are not molecules, but molecular aggregates, termed by him ' micellae".' These micellae may be built up of molecules, groups of molecules, or be even more complex in nature. They have a definite structure, and like crystals, when broken, the fragments retain the properties of the whole, while the same is also the case when a micella or micellar structure composed of homogeneous micellae increases in size. In a crystalloid, or an artificial cellulose membrane, the miccllae are all of the same kind, but in organized bodies, according to Nägeli, the micellae probably vary in size and quality, while in the protoplasm very heterogeneous elements are certainly aggregated together (Sect. 78). (Cf. Fig. 3.)

The micellae may differ much in shape, while similar and dissimilar micellae may be combined together in all manner of ways. By their behaviour when dried, and the appearances presented under polarized light, \&c., Nägeli was, however, led to conclude that the micellae of organized bodies are in general crystalline. or at least polyhedral, and that in solid bodies the axes of the micellae are arranged in a definite manner, either parallel to one another, or in a radial arrangement like the radial acicular crystals which form a sphaerocrystal. Nägeli later (1879) supposed that the micellae may combine in various ways to form units of higher ordinal value, and concluded that in colloidal substances the micellac unite to form a meshwork in three dimensions.

Why Nägeli should postulate a power of swelling

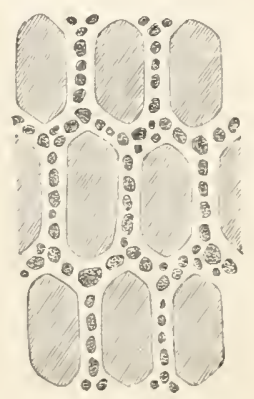

F1G. 3. Schematic representation of molecular structure, according to Nägeli and Schwendener. as a general property of micellac is not easy to see, for the groups of molecules which may form the elemental units of an organized structure capable of swelling may themselves be unable to imbibe water $^{3}$. If the living physiological units are, in correspondence with their

` Nägeli, Die Stärkekörner, I858, p. 322; Ủber die krystallähnlichen Proteinkörper, I862, Bot. Mitth., Bd. I, p. 2 I 7 ; U. d. inneren Bau d. veg. Zellmembran, 1864 , ibid., pp. I and 46 ; Theorie der Gährung, 1879, p. I21; Theorie d. Abstammungslehre, 1884, p. 35 ; Nägeli und Schwendener, Das Mikroskop, 18 77,2 . Aufl, p 532.

${ }^{2}$ Nägeli und Schwendener, Das Mikroskop, 1877 , 1.c., p. 42. . Nägeli at first gave to the micellae the name of molecules, already appropriated in Chemistry. A micella is typically composed of a group of molecules, but may be more or less complicated than this. The term 'Tagma' (Osmot. Unters., 1877 , p. 32) corresponds to Nägeli's micella, and the latter term may therefore be adopted. A molecular combination of fixed and definite character has been termed by Nägeli (Theolie der Gährung, I879, p. 122) a pleon.

${ }^{3}$ Water of crystallization is here disregarded, but it may nevertheless, under particular conditions, be held in a similar manner to that in which imbibed water is held. In general, a distinction can be made between water of crystallization and water of imbibition (constitutional water and water of adhesion). Pfeffer, Osmot. Unters., 1877, p. 35: Nägeli, Theorie d. Gährung, 1879, p. - 29. 
physical structure, to be regarded as micellae, they are undoubtedly micellae which are capable of imbibing water and swelling, for chemical changes take place in these pangenetic units, and chemical substances penetrate to their interior. Indeed an internal molecular change is always possible without the micellar structure being disorganized. This is the case when, for example, a cellulose cell-wall is converted into nitro-cellulose, or the latter reconverted into cellulose.

Other conditions being equal, a greater amount of swelling will become possible as the micellae are broken up into smaller and smaller micellae, and the total micellar surface increased ${ }^{1}$. The amount of swelling is, however, influenced by so many other factors that it must always remain uncertain whether the different amount of water present in the successive layers of cellwalls and starch-grains is merely due to the component micellae varying in size in different layers. Nägeli's ideas as to the progress and causes of striation and stratification are partly based upon erroneous observations, and hence the conclusions which he drew from the optical properties of superposed lamellae containing varying amounts of water lose for the most part their necessary basis of fact ${ }^{2}$.

In the different layers of the starch-grain, amylose and amylodextrin are present in unequal proportions ${ }^{3}$. Similarly it is uncertain whether the layers of the cell-wall are chemically identical. Moreover other factors may modify the power of swelling, such as the union of the micellae to form a skeletal framework, or the occurrence of those infiltrations by which cell-walls are characteristically modified to form cork and cuticle.

Many organized bodies show a different capacity for swelling in one direction than in another, and the velocity with which light is transmitted may differ according to the way in which it traverses the organized substance, being more rapid in one direction than another. From these facts Nägeli drew conclusions upon the mode of arrangement of the micellae, which are not, however, necessarily correct; for the amount of swelling is dependent upon a variety of conditions; while the study of the phenomena of polarization will not reveal the actual shape of the micellae, though it may indicate that in particular cases they are anisotropous, and all arranged in the same manner. On the other hand the isotropy of the protoplasm, does not exclude the possibility of the component micellae being anisotropous and crystalline but heterogeneously arranged, for the optical properties of

1 Nägreli, Stärkekörner, 1858 , pp. 333, 345.

2 See Krabbe, Jahrb. f. wiss. Bot., 1887 , Bd. xvi11, p. 346 ; Correns, ibid., I892, Bd. xxıII, p. 254; Zimmermann, Beilräge zur Morphologie u. Physiologie, $1893, \mathrm{P} .302$; as well as the works here cited.

${ }^{3}$ A. Meyer, Unters. uiber d. Stärkekörner, i $\delta_{95}$, p. 2 . On the behaviour of starch when boiled, 1. c., P. 129 . 
the protoplasm are equally dependent upon the arrangement of its component parts.

It is at present uncertain whether the molecules of both organic and inorganic substances do not very commonly combine in aggregates which may be termed micellae. Thus crystals, in which the component parts are regularly and definitely arranged, are supposed by many authors to be built up of physical units or micellae, although others deny this ${ }^{1}$. The meshwork arrangement postulated for colloid substances might be due, either to a union of micellae, or of molecules to form the bars of the meshwork ${ }^{2}$. In the latter case a piece of such a gelatinous colloid would form a single gigantic micella, just as a large crystal forms a single homogeneous unit by the direct union of the molecules composing it. There is no reason why such molecular unions to form micellae might not be large enough to be visible. Thus the units or crystalline needles of which sphaerocrystals are composed are of measurable size, and yet unite to form a whole which is capable of imbibition.

As the ultimate structural elements of which the protoplast is composed, physiological units are necessarily postulated, and these may be either formed of groups of molecules, i. e. micellae, or of groups of micellae. The micellar hypothesis is especially applicable to the protoplast, and hence it is difficult to see why $W$ iesner ${ }^{3}$ regards the existence of physico-chemical units or micellae as being incompatible with the existence of the physiological units which build up the protoplast. The physiological units, micellae or micellar complexes as the case may be, must unite to form the different organs of which the protoplasm is built up, but this is no reason for regarding the latter as a single micella, or as a micellar or molecular complex of uniform character and forming a concrete whole, for the protoplast is certainly a complicated piece of mechanism built up of a vast number of different parts.

From the above it is clear that we cannot assume that the products of protoplasmic activity are necessarily built up of micellae, though this is

1 Lehmann, Molecularphysik, I889, Bd. II, pp. 276, 379, and the literature there cited; Ostwald, Lehrb. d. allgem. Chemie, I89I, 2. Aufl., Bd. I, pp. 376, 397; Groth, Die Molecular-Beschaffenheit d. Krystalle, I888. On the regular arrangement of minute crystals in fluicls, see Lehrmann, Zeitschr. f. physik. Chemie, I89o, Bd. v, p. 427 .

2 See Sect. 12. Kekulé (Die wiss. Ziele u. Leistungen d. Chemie, I878, p. 22) assumes that in colloids the meshwork is a molecular one. Strasburger (Bau u. Wachsthum d. Zellhaut, I882, p. 224) has attempted to prove that all organized bodies have a similar molecular structural arrangement in the form of a molecular meshwork. From other points of view, however, which have been overlooked by Strasburger, an assumption of the existence of physiological and hence also of physical units is rendered necessary. As has already been mentioned (Sect. I 2), the peculiarities observed in the mode of swelling of given bodies may be produced by a linking together of either molecules or micellae. See also Schwendener, Über Quellung und Doppelbrechung, in Sitzungsb. d. Berliner Akademie, 1887 , Bd. xxxiv, p. 659 .

3 Wiesner, Die Elementarstructur, I8r2. Cf. Pfeffer, Studien z. Energetik, 1892, p. I57, and Sect. 8 of this book. 
probably the case with the cell-wall and starch-grains. Indeed Nägeli first based his micellar hypothesis upon observations made upon starch-grains, cell-walls and crystalloids, and only later extended this view to the protoplasm itself, in which the character and mode of arrangement of the micellae and micellar complexes are especially varied and complicated.

The micellar theory enables us to refer all physiological processes to the domain of molecular phenomena. It is true that all such reasoning is of a speculative and hypothetical character, but nevertheless a clear grasp of the principles of the micellar hypothesis is of great importance, although we may be as yet unable to explain physiological phenomena by direct reference to the form, structure, composition and relationships of the component parts of the living organism. Not only is this the case with regard to the living protoplast, but the same also applies to dead organic structures as well. Thus when a dead cell-wall swells in one case more markedly in one direction than in another, but in another case equally in all directions, we do not know whether the peculiarity is due to a different shape of the component micellae or to a difference in the way in whch they are arranged.

Crystals capable of Imbibition (Crystalloids) ${ }^{1}$. If ordinary crystals are assumed to be built up of crystalline particles, it must also be admitted that the component particles of crystalloids are regularly arranged. Since it has been found possible to obtain the crystalloid proteids in crystalline form outside of the organism, there can be no doubt that these crystalloids are formed, and grow, within a living cell in exactly the same manner as they do in the external world. It belongs therefore to the science of Crystallography to study and explain the structure and properties of crystalloids. The degree of swelling of which a regular crystalloid is capable is constant along the different axes, but different in the different kinds of crystals.

Starch-grains ${ }^{2}$. The mode of swelling and the optical phenomena exhibited by starch-grains indicate that the grain is built up of crystalline and anisotropous elements having their longer axes arranged radially. According to Nägeli, these are crystalline micellae, according to Schimper, and Meyer, minute crystals (Trichites). In both cases, the existence of minute crystalline elements is assumed. Schimper and Meyer, however, use the term sphaerocrystal more generally, and apply it also to those cases in which the component crystalline elements are so small as

${ }^{1}$ Nägeli, Bot. Mitth., I862, Bd. I, p. $21_{7}$; Schimper, Unters, über d. Protein-krystalloide, I873; Lehmann, Molecularphysik, I888, Bd. I, p. 550. On the artificial formation of crystalloids, see Grübler, Über krys. Eiweiss d. Kürbissamens, I $88_{1}$; Drechsel, Journal f. prakt. Chemie, 1879 , Bd. XIX, p. 33I; Ritthausen, l.c., I882, Bd. Xxv, p. I 50 , and the literature there quoted. See also Schimper, Bot. Zeitung, $188_{3}$, p. 152 .

${ }^{2}$ Nägeli, Die Stärkekörner, $185_{5} 8$; Bot. Zeitung, I881, p. $63_{2}$; Schimper, Bot. Zeitung, 1881, p. 233; A. Meyer, Unters. über Stärkekörner, I $95 ., \mathrm{pp} .116$. \&.c., and the literature cited by Meyer in Bot. 7.eitung, 1896. p. 330 . 
to be invisible. As a matter of fact micellae may attain a considerable size, while any crystal may be regarded either as a micella or micellar complex.

Even though the amount of swelling possible is not entirely dependent on the form and size of the component micellae, nevertheless Nägeli's conclusion that the micellae of the starch-grain have their long axes radially arranged still holds good. To this conclusion he was led by the study of the absorption of water by the grain, and by the fact that the most marked swelling takes place in the tangential direction. When boiled, starch swells greatly to form a paste, and probably not only does a disorganization of the micellae ensue, but also a hydrolytic decomposition of the polymeric molecules, which play so important a part throughout the group of carbo-hydrates ${ }^{1}$. The phenomena of imbibition and swelling do not necessarily postulate a growth by intussusception, and the arguments based by Nägeli on the supposed growth of the starch-grains by intussusception fall to the ground, for it appears that starch-grains normally grow by apposition.

Cell-walls. Many of Nägeli's conclusions drawn from the striations shown by cell-walls have proved to be erroneous, but nevertheless the numerous researches conducted on the cell-wall have as yet brought no facts to light which are not in accord with the micellar hypothesis. The power of swelling differently in different directions might be due to the axes of the micellae being of different lengths, or to other causes, such as, for example, to the component micellae being more firmly attached to one another in one direction than in another. Such questions are however not vital, but merely physical problems, which do not further concern us except in so far as they affect vital phenomena. The study of the causes of the torsion, thickening, shortening, splitting, \&c., which may be produced by normal (hygroscopic) swelling, as well as by abnormal and excessive swelling (Steinbrinck's 'Ueberquellung'), is a task for the physicist and not for the physiologist. If these phenomena are due to the particular shape or special arrangement of the component micellae, it follows from the facts observed that one axis of each micella must be perpendicular to, the other two parallel to, the surface of the cellwall, and at the same time often forming an acute angle with the long axis of the cell ${ }^{2}$.

It is difficult to hazard an opinion as to whether the particles, or dermatosomes of Wiesner, into which the cell-wall separates when subjected to certain treatment, such as in the process of so-called carbonization, are really preformed structures, and whether if so, they represent micellae or micellar complexes, for similar phenomena are also exhibited by artificially produced cell-walls ${ }^{3}$.

Optical Characters. Although the optical appearances presented by organized structures under polarized light were previously known, Nägeli was the first to see their real significance, and to found upon them conclusions as to molecular structure.

1 See A. Meyer, 1. c., p. 129.

2 Steinbrinck (Zur Theorie der Flächenquellung, IS9x) gives the literature. See also Nägeli und Schwendener, Mikroskop, I877, 2. Aufl., p. fif $_{4}$; Schwendener, Sitzungsb. d. Berl. Akad., IS87, Bd. xxxiv, p. 659 .

3 Wiesner, Elementarstructur, I 892 ; Pfeffer, Energetik, IS92, 1. 253 ; Correns, Jahrb. f. wiss. Bot., I 894, Bd. xxvi, p. 655 . 
Optical properties, as far as they indicate differences of molecular structure, may attain considerable importance in physics and chemistry ${ }^{1}$. A few remarks must, however, here suffice, a knowledge of the phenomena and causes of double refraction being assumed on the part of the reader, while for further details he is referred to the literature on this subject ${ }^{2}$.

The protoplast, saturated with water, is isotropous, but several of its products, cell-walls, starch-grains, and crystalloids, are anisotropous, and as dead substances retain the same optical properties.

When first formed the cell-wall is either not at all, or only very slightly doubly refractive. Marked anisotropy only appears as it becomes older, and is not always due to precisely the same causes. In the cuticle, as Ambronn ${ }^{3}$ has shown, the anisotropy is caused by an infiltration of doubly refractive waxy substances. When warmed, these melt, and the anisotropy disappears; when cooled, they congeal, and the anisotropy returns. In general, the optical effects are probably due to the anisotropy of the micellae or micellar complexes. Nevertheless, it is always possible that in some cases double refraction is partly or entirely due to differences of tension existing between the successive layers.

Since the smallest fragments of a cell-wall or starch-grain still show double refraction, their anisotropy cannot possibly be due to differences of tension existing only in the intact wall or grain. In many cases the optical effect is hardly, or not at all, altered by applying marked tension or strain, but in other cases, as shown by recent research, such treatment causes a marked alteration of the optical properties ${ }^{4}$. Swelling may also produce either an imperceptible or a very marked optical result. Usually as the swelling increases the double refraction decreases ${ }^{5}$; indeed some bodies, such as softened gelatine, become doubly refractive only when dried.

In cases where double refraction is due to the component elements or micellae, these must be arranged for the most part, or entirely, in a definite manner. Thus in a cylindrical cell one axis of the optical ellipsoid of elasticity is radial, the other two tangential to the surface, and at the same time they form acute or right angles with the main axis of the cell. The shortest optical axis falls in the plane in which the greatest swelling is possible ${ }^{6}$. From this fact, and from the way in which the arrangement of the optical axes corresponds with the direction of the visible markings, it is justifiable to conclude that all these appearances are produced by the same specific internal structure.

1 Cf. Ostwald, Lehrb. d. allgem. Chemie, I 89 I, 2. Auf., Bd. I, 1. $4^{60}$.

2 Ambronn, Einleitung z. Benutzung d. Polarisationsmikroskops, 1892; Nägeli und Schwendener, Mikroskop, 1877, 2. Aufl, p. 299; Schwendener, Sitzungsb. d. Berl. Akad., 1877, p. 659 ; 1889 , p. 233 ; 189o, p. II31; Ebner, Unters. ü. Ursachen d. Anisotropie, 1882, and Sitzungsb. d. Wien. Akad., 1885, Bd. Xcr, Abth. 2, p. 35 ; Correns, in Beiträge z. Morphol., \&c., v. Zimmermann, 1893 , IBd. I, p. 302 . The works by Nägeli are in Bot. Mitth., Kd. I, 1862, p. 183, \&c., and Beiträge z. wiss. Bot., $186_{3}$, Heft 3, p. 1 . In the above works the literature is quoted, including that which seeks to refer all double refraction to unequal tension.

s Ambronn, Ber. d. Bot. Ges., I888, p. 226.

4 Zimmermann, ibid., I884, p. 35 ; Schwendener, l.c., 1887, p. 687, and 1889; Ebner, 1.c.

${ }^{5}$ Schwendener, 1 c., 1887 , p. 695.

- Nägeli und Schwendener, Mikroskop, 1877,2 . Auff., p. 356 , and the literature cited above. 
Other Peculiarities. It might be expected that the physical properties and powers of conduction of organized bodies should be different in the three planes intersecting at right angles, and the little that is known on this point supports this conclusion. The properties of elasticity and rigidity, the conduction of sound ${ }^{1}$, heat, and electricity, have not as yet been investigated in connexion with the molecular structure of organized bodies. The general phenomena connected with elasticity, tissue tensions, heat, light, and electricity will be dealt with later. In this connexion Ambronn's investigations are of great interest ${ }^{2}$, for he has shown that gelatine plates, in which anisotropy has been induced by tensions set up whilst drying, orientate themselves in magnetic and electric fields, just like anisotropous crystals.

\section{SECTION 14. Changes of Molecular Structure due to Swelling.}

In the vital mechanism, constructive and destructive processes are always taking place, as well as a variety of intermediate changes, by which the properties of the protoplast and of its organs may be more or less modified to suit the economy of the whole. Thus in the case of a cell-wall, the modifications it may undergo, leading to the formation of cork cuticle or wood, are of the utmost importance in the economy of the plant in connexion with the necessary supply of water. In some cases, as, for example, when cell-walls or starch-grains are dissolved, the original organization is entirely destroyed, while the material thus obtained becomes directly available to the organism in a new form. Similar results may be produced by the direct action of chemical reagents, but the plasma is extremely susceptible to various injurious agencies, and if the action of any one of these is fatal, a profound structural alteration takes place, such as always accompanies death.

When the protoplasm dies, the non-living organized structures, such as the cell-wall, starch-grains, crystalloids, \&c., which it produced when alive appear to retain their original molecular structure. The optical appearances and the power of swelling, at any rate, remain the same, and certain slight changes of properties ${ }^{3}$ which may take place are probably due to an infiltration of certain constituents of the cell contents. It is nevertheless possible that the cell-wall undergoes changes when the cell dies (independently of the changes due to the death of the plasmatic threads which traverse it), although these have as yet escaped observation. Any changes produced by the action of solvents, or of the chemical substances which led to the death of the plasma, are of course quite different in character, and due to the direct action of the agency in question. Even boiling water may not only

1 On the conduction of sound, see Savart, Annales d. Chimie et Physique, IS29, T. XL, P. I I3, and physical textbooks.

2 Ambronn, Ber. d. Sächs. Ges. d. Wiss., liot., I S91, p. 394.

3 Such as caused by staining or the absorption of dyes. See Nägeli, lot. Zeitung., ISSI, p. 649. 
permanently alter starch-grains, but may also remove from many cell-walls certain constituent substances ${ }^{1}$. Moreover, severe tension may produce mechanical ruptures, as is instanced by the formation of cracks in dried starch-grains, and by the bursting of the outer layers of the cell-wall, when bast fibres swell in dilute sulphuric acid.

External agencies, the application of which causes no permanent injury, may induce temporary alterations of molecular structure, from which a refurn to the normal condition is possible. The intensity necessary to convert a temporary modification into a permanent change is specifically different for each organism, and for each part of an organism. This is well illustrated by the differences which exist between the resistant powers of different plants to desiccation. Thus certain lichens and mosses and many seeds are able to withstand severe and almost complete desiccation, whereas many plants die as soon as the percentage of water present is reduced at all markedly below the normal, and between these two extremes a variety of intermediate stages occur ${ }^{2}$.

For the maintenance of vital activity, a sufficient supply of water is absolutely essential. The presence of water causes a marked alteration in certain of the properties of bodies which are capable of imbibition and swelling; thus the cell-wall when saturated with water is soft and pliable, but, like the plasma, becomes hard and brittle when dry. At the same time other physical properties, such as rigidity, elasticity, ductility, and the power of conduction of heat, electricity, \&c., undergo a more or less pronounced alteration, which increases progressively with the loss of water.

The separation of the micellae from each other by the water of imbibition gives them greater freedom of movement and more marked powers of adjustment. Thus the cell-wall of Caulerpa, or the thallus of Fucus, which when dry are extremely brittle, may safely be strongly bent when they have absorbed a little water, and when fully saturated may be twisted into a spiral without any rupture taking place. Since such torsion does not cause the exudation of any water, it follows that a redistribution of owater must take place in the thallus, a little of the imbibed fluid being transferred from the concave to the convex side. When a dry membrane swells slightly, its power of resistance to stresses and strains increases under normal conditions, but when the swelling is very great, as, for example, when the gelatinous modification takes place, this power, as might be expected, decreases.

1 In Cetraria islandica, many algae, \&c. The changes observed by Jonas Cohn (Jahrb. f. wiss. Bot., I 892 , Bd. XxIV, p. I60) to take place when collenchyma cells are boiled for some time are probably due to a similar action.

${ }^{2}$ Schröder, Bot. Unters. a. Tübingen, Bd. II, Heft I, I 886 . Ewart, On the power of withstanding desiccation in plants, Trans. L. Pool. Biol. Soc., vol. xi, 1897, p. 151 ; 1896 ; and 1894 , p. 234. 
The conditions existing at any given time are of the highest importance in determining the nature and extent of the action of an external agency. Thus most seeds when dried can be heated to $100^{\circ} \mathrm{C}$. for a time without being at all injured thereby, but if saturated with water a temperature of $70^{\circ} \mathrm{C}$. rapidly kills them. In the same way, starch-grains and proteids ${ }^{1}$ must when dry be heated to a much higher temperature in order to destroy their molecular structure than when saturated with imbibed water. A thorough linowledge of these and similar relationships is absolutely essential to enable us to form a correct estimation of all the processes and reactions which take place in the living organism, while in addition these phenomena afford important indications as to the probable molecular structure of organized bodies.

${ }^{1}$ Lewith, Beiträge zur Theorie der Disinfektion, Archiv f. exper. Pathol., r8yo, xxvi, P. 341. 


\section{CHAPTER IV}

THE MECIIANISM OF ABSORPTION AND TRANSLOCATION

\section{SECtion I5. General.}

EVERY plant and every cell must be supplied with not only water, but also other necessary substances, while at the same time certain products of metabolism, such as carbon dioxide, must be excreted. Plants absorb all substances offered to them in soluble form, whether useful or not. The importance and mode of utilization of the absorbed food material will be dealt with later; at present we have to consider the ways and means by which food material is obtained and accumulated, as well as the conditions which influence absorption and translocation. It is clear that in the cell itself only those fluids and dissolved substances which are imbibed by the cell-wall and protoplasm are able to penetrate to the interior of the cell. The cell-wall, saturated with water, is in general much more permeable than the protoplasm, and hence many substances can pass through the cell-wall, but not through the living layer of plasma within. Thus the plasma while living is impermeable to the coloured cell-sap which it may cnclose (beetroot, \&c.), but when the cell is killed, the red sap quickly diosmoscs through the dead and altered plasma, and also through the cell-wall which it was previously unable to reach. In the same way, when a cell is plasmolysed by a coloured saline solution, the latter penetrates the cell-wall but not the plasma, and hence accumulates in the space left vacant between them. A substance imbibed by the cellulose cell-walls may reach the centre of a tissue without having penetrated a single protoplast. It is indeed possible that the water and salts absorbed by the roots pass mainly if not entirely through either the walls of living cells or the walls and cavities of dead wood fibres, \&c., so that only on reaching the crown of a tree do they penetrate the protoplasts of the actively growing tissues localized there. Thus, when watery solutions of indigo-carmine, or aniline-blue are absorbed by the root, or by the cut surface of a stem, they may be traced by the colouration they produce, and can be shown 
to pass throughout the entire plant, although the living protoplasts do not absorb either of these pigments. Hence substances which are absorbed only when they reach the protoplasts of a given tissue, may be largely conveyed thither by the dead elements through which the water current passes, while so long as an active current is maintained, the protoplasts contiguous to the conducting channels may absorb only a fractional amount even of the dissolved substances which are capable of endosmosis. Hence in this way soluble materials may attain a wide distribution without any direct transference from protoplast to protoplast being necessary. The protoplast of a cell in the interior of a tissue draws the substance it requires from the imbibed fluid of the adjacent cell-walls in much the same way as would the protoplast of a cell swimming freely in water. Similar relationships hold good for substances excreted by the cell, independently of whether the excrete products are directly eliminated or are deposited in more or less distantly situated tissues.

When a continuous epidermal layer covers the surface of the plant, absorbed substances must pass through this layer before penetrating to the interior of the plant, but when openings such as stomata and lenticels are present, substances may penetrate without necessarily undergoing immediate absorption. Stomata and lenticels, so widely distributed in the higher plants, are of the utmost importance for gaseous interchange as well as for the excretion of water vapour, and in many plants, locally distributed water stomata permit an excretion of fluid water. Moreover, by means of communicating intercellular spaces, gases may reach the innermost cells and tissues without having been absorbed by any of the more peripherally situated cells or cell-walls. Living or dead elements, in the form of either specially elongated cells, or of vessels produced by the longitudinal union of rows of cells, are largely employed for the rapid transport of water and dissolved substances to distant parts. All such arrangements are of the highest importance, in so far as they render possible a sufficiently rapid transference of the substances required in metabolism, and it is by these arteries, so to speak, that the cells and tissues in which metabolism is active are supplied with food material, the conducting channels being subordinate to the organs they supply. In the higher plants, as well as in the lower ones, absorption and excretion are not, in the true sense, functions of the entire organism, but of individual cells, and it is with this exchange of substance occurring in the individual cell that we are immediately concerned. In attacking this problem, we are at once confronted by all those difficulties which arise when we attempt to solve any problem involving a knowledge of the inherent nature of the vital mechanism.

In order to reach the cell-sap in the interior of a cell, fluids and dissolved substances must pass by diosmosis first through the cell-wall, when one is present, then through the external plasmatic limiting mem- 
brane, thus reaching the plasma, and finally through the internal plasmatic membrane bounding the vacuole. A naked plasma mass or gymnoplast may take in solid, insoluble, or undissolved substance, and thus the plasmodia of Myxomycetes may be observed to ingest particles of sand, spores, oildrops, \&c., and after a time to reject them (Sect. 19). It is owing to the semifluid consistency of protoplasm, and its power of immediately closing a wound or opening made in it, that the ingestion and excretion of solid bodies are possible. Indeed, the protoplasm of a closed cell may either ingest crystals, oildrops, \&c. from the cell-sap or excrete them into it.

Organized structures may even penetrate protoplasts provided with a cell-wall, as, for example, when plants are infested with the hyphac of parasitic fungi or attacked by certain bacteria. When, however, solid substances external to the cell are rendered soluble by the action of excreted acids or ferments, the actual process of absorption is of the normal character, since the substances are imbibed in solution. The roots of both higher and lower plants may actually exert a solvent influence of this nature by means of acid excretions, while the developing embryo, and parasitic and saprophytic plants as well, may excrete solvent enzymes. There can be no doubt that the neighbouring cells of a tissue may affect one another in a similar manner, and possibly in other ways also, while the fine plasmatic threads which connect the different protoplasts with one another render an additional means of communication available for the transference of food material or the conduction of stimuli.

As every active living cell is saturated with imbibed water, and as even those seeds, mosses, and lichens which can withstand desiccation remain dormant until they are supplied with water, the penetration of gases into dry organized structures is of subordinate physiological importance. Indeed, even the walls of dead cells contain more or less imbibed watcr, so long as they are surrounded by living tissues, and it is only in peripherally situated dead tissues that the percentage of imbibed water may be reduced almost to nil. Gases pass in solution through a cell-wall saturated with water by a process of diosmosis, just as they do through a soap bubble, and the same general principles hold good for such gaseous interchange as for the diosmosis of other dissolved substances. The movements of gases through dry organized bodies will be discussed later in giving a special account of the gaseous interchanges.

The diosmotic properties of cell-walls, protoplasts, \&c., are specifically different: thus suberized or cuticularized cell-walls scarcely allow water to pass through them at all, or only with great difficulty. This peculiarity is attained by the cell-wall becoming more or less completely impregnated with fatty, waxy, or resinous substances, and a piece of paper that has been partially or entirely saturated with wax, fat, or oil, gives a fairly 
accurate representation of the nature of cork or cuticle. In practice, paper prepared in this manner is assumed to be impermeable to water, but nevertheless slight quantities of gases may pass through, as well as any other substances that are at all soluble in the impregnating oil. Similarly, a thin film of india-rubber shows that a membrane impermeable to water may nevertheless be penetrated in perceptible amount by carbonic acid and other gases. The impermeability of cork and cuticle is only relative, although it may often be extremely pronounced. This property is of great importance to the aerial organs, which are, as a general rule, covered either by a cuticle or by a corky investment, so that protection is afforded against any excessive and ineffective loss of water, while gaseous interchange, though rendered more difficult, may still take place with sufficient activity to supply all needs. Cells, however, in which the walls are suberized on all sides, and which are thus only with difficulty permeable to water, are, as a general rule, completely dead, probably because the amount of exchange allowed is insufficient for the requirements of the living protoplast. The specific nature of the cell-wall determines whether dissolved substances will reach the protoplasm, and in what relative amount. Almost without exception, however, all substances that can diosmose through the external limiting plasmatic membrane can also pass through the cellwall so long as it is saturated with imbibed water, and also through corky and cuticularized cell-walls, though usually with excessive slowness. On the other hand, very many substances are unable to penetrate the living plasma, although they may readily diosmose through the moist cell-wall. Apparently, however, the diosmotic properties of the plasmatic membranes are liable to modification, frequently of a regulatory character in connexion with the temporary needs of the living organism, so that a particular substance can be absorbed at one time, but not at another. The means by which changes of the diosmotic properties of the plasma may be induced are probably of very special character, and it is conceivable that movements and changes of position of the component micellae of the peripheral plasmatic membrane may modify its diosmotic character. The suberization which particular cell-walls may undergo is a similar change, directly due to the activity of the living protoplast, and induced for the benefit of the organism as a whole.

The character of the cell-wall and of the plasmatic membranes determines whether a given substance will penetrate to the interior of a cell, and any such substance will continue to be absorbed until a condition of equilibrium is reached, when all further absorption ceases. If, however, this condition of equilibrium is continually disturbed, continuity of absorption may result, and in this way, relatively large quantities of a particular substance may be absorbed from an extremely dilute solution. or of two given substances, one may be absorbed in large amount, the 
other scarcely at all. A continuous disturbance of equilibrium may be maintained, if the absorbed substances at once undergo a more or less marked chcmical change or alteration into soluble or insoluble compounds of different character.

The power of determining the relative amounts of absorption (and also of excretion) of particular substances is dependent upon the changes and modifications, in the widest sense, which these undergo when absorbed, and hence upon the vital activity of the organism. Every substance which accumulates in the cell must therefore necessarily enter it in a slightly different form. In many cases, this may actually be observed, and so far no other explanation appears to be necessary; although it is quite possible, that in certain cases the influence of the protoplasm may be such as to cause particular substances to accumulate within the cell in the same form as that in which they entered.

The osmotic properties of the substances present in solution in the cell-sap are such as to cause the protoplast, when in the form of a primordial utricle, to be closely adpressed against the cell-wall, which serves as a support for it, and which it stretches to a certain extent. The preservation of this condition of turgidity by an internal regulatory mechanism is cssential for the continuance of life and growth, and in turgid cells, the osmotic pressure may attain a value of from a few to many atmospheres, whereas in gymnoplasts, the osmotic pressure is only trifling and, indeed, could hardly be otherwise. The substances, to which the osmotic pressure is due, are in general compounds incapable of diosmosis, and a very dilute solution of a non-diosmosing crystalloid body will suffice to maintain a comparatively high osmotic pressure. In an isosmotic saline solution, this pressure is just equilibrated, whereas in a more concentrated solution the protoplast becomes plasmolyzed, and contracts, until a condition of isosmotic equilibrium is again reached.

\section{SECTION 16. The Diosmotic Properties of the Cell.}

Having now taken a general view of the subject, we may pass on to a more detailed account of the osmotic exchanges of turgid cells, whose cellulose walls are readily permeable by water. 'The osmotic properties of the protoplasm are of similar character, whether it is naked, or surrounded by a cell-ivall; hence the general account given will apply to both, and the importance of certain special properties of the cell-wall will be mentioned later (Sect. 21). In order to reach the cell-sap a particle of water, or dissolved substance, must diosmose first through the cell-ivall $(z)$ and the plasmatic membrane $\left(p^{1}\right)$, which is closely applied to it, and finally pass through the internal limiting plasmatic membrane $\left(p^{2}\right)$, which bounds the vacuole (Fig. 4). Any substance dissolved in the cell-sap must 
diosmose outwardly in precisely the reverse direction ${ }^{1}$, in order to be removed from the cell.

The same general relationships hold equally good. whether the diosmotic properties of the plasmatic limiting membranes and of the remaining mass of the plasma are, or are not, constantly of similar character throughout. As a matter of fact, there is no cloubt that the osmotic properties of the plasma and its membranes are by no means alike, nor that they may vary from time to time. Mention will be made (Sect. I 8 ) of the necessity for the existence of these plasmatic limiting membranes, on both internal and external surfaces of the primordial utricle, and of the manner in which these are preserved and retained however the shape of the protoplast may alter. These physiologically important structures may be termed the plasmatic membranes, the peripheral one, bounding the ectoplasm externally, being called the ectoplasmic membrane, the internal one, bounding the endoplasm internally, being called the endoplasmic or vacuolar membrane ${ }^{2}$.

In every case, the limiting membranes determine whether or not a given substance shall be absorbed. It may readily be seen that dyes which do not diosmose, penetrate neither the ectoplasmic, nor the vacuolar membrane, as the case may be; whereas

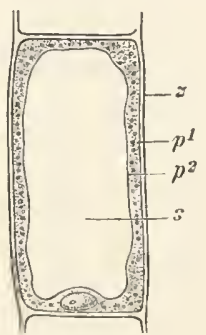

$a$

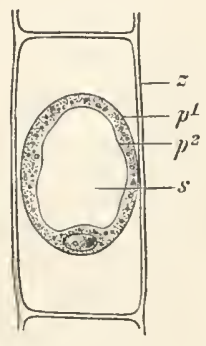

l)

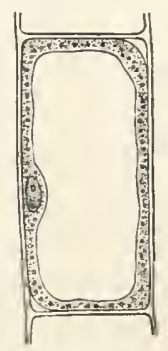

$c$

Fig. +. Cells from the radicle of Zea Mais $(\times 375),(a)$ in water; (b) after plasmolysis in 5 per cent. $\mathrm{KNO}_{3} ;(c)$ in 2.3 per cent. $\mathrm{KNO}_{3}$.

any substance that passes these, rapidly diffuses throughout the mass of the plasma, and either reaches the cell-sap, or escapes from the cell, according to whether the substance in question was within or without the cell.

It is true, that the protoplast is not homogeneous, but the above facts and conclusions are reconcilable with the most varied internal structure. For the present it may be left undecided, whether in certain cases diosmosing substances do not actually penetrate the living structural elements of the plasma, and whether the nucleus, chromatophores, \&c. may be covered permanently, or temporarily, by a limiting membrane having special diosmotic properties (see Sect. I8). If the space between two diosmotic membranes were filled with glass beads and water, dissolved substances would pass through the capillary interstices and spaces, without penetrating the glass beads, which correspond in this analogy to the

${ }^{2}$ See Pfeffer, Zur Kenntniss der Plasmahaut u. d. Vacuolen, i Sgo, p. 188. 
plasmatic elements. If the diosmosing substances do actually penetrate the structural plasmatic elements and are transmitted by them, then the model would have to be altered by filling the space between the two membranes with a mass of gclatinous substance, through which the diosmosing salt must penetrate, in order to pass from the one membrane to the other. In both cases, however, the general principles are similar, for in the latter, as in the plasma, we have to deal with a spongy framework impregnated with water, through which soluble crystalloids diffuse with the same rapidity as they do in water ${ }^{\text {? }}$. Even though the transmission of a diosmosing substance through the mass of the plasma were mainly due to diosmosis, nevertheless the distances to be traversed are very small, and more or less pronounced movements of the plasma may accelerate the transference of substance from one part to another.

Many soluble crystalloid substances cannot diffuse through plasmatic membranes, and hence the elements or micellae of which the latter are composed must be closely associated together, leaving relatively narrow micellar interstices, and this conclusion necessarily holds good, whatever the substance or structure of the plasmatic membrane may be. Like the rest of the protoplast, the membranes which cover it are of a viscous consistency, and may be stretched considerably without being ruptured. For diosmosis, i.e. for diffusion through a separating membrane ${ }^{2}$, it is immaterial whether the membranes in question are solid or fluid in nature, and all the essential characteristics of imbibition and diosmosis are fulfilled even when a substance diosmoses merely because it is soluble in the substance of the separating membrane.

The cell is therefore an osmotic system, composed of a series of membranes, one outside the other; the outermost, or cell-wall, affording rigidity to the whole, and permitting the existence of powerful internal osmotic pressure, which would otherwise unavoidably rupture a body of such feeble consistency and rigidity as the naked protoplast.

The diosmosis of crystalloids through this system is of similar character to that through an animal membrane such as the wall of the bladder. Most soluble substances readily diosmose through the cell-wall, but many crystalloids are unable to diffuse through the plasmatic membranes, or to penetrate to the plasma. The greater permeability of the cell-wall is shown by the fact, that substances dissolved in the cell-sap pass outwards as soon as the plasma is killed. Thus the water, in which cherries or clean slices of beetroot are boiled, only becomes red, when the high

1 Voigtländer, Zeitschr. f. physik. Chemie, I889, Bd. III, p. $3^{16}$. The first experiments on Osmosis were performed by Graham, 1862 . Later literature by Voigtländer. See Ostwald, Allgem. Chemie, 189 I, 2. $\Lambda$ ufl, Bd. v, p. 687.

2 See llinkelmann, Handb. d. Physik, I89ז, Bd. I, p. 6r 8. 
temperature has caused the death of the plasmatic films which surround the cell-sap, and prevent the pigment substances dissolved in the sap from escaping. The fact that the cell-wall cnclosing a living cell is readily permeable, becomes directly manifest when plasmolysis is produced by means of sugar, or saline solutions coloured with indigo-carmine, anilineblue, or the red sap of cherries or beetroot, for the coloured fluid appears in the space formed between the cell-wall and the contracted protoplast. The fact, that the protoplast has been caused to contract, shows that the plasmolysing substance has penetrated the cell-wall, but cannot pass through the protoplasm, with which it now comes into contact. If no subsequent increase in volume of the protoplast takes place, it follows, that no perceptible amount of the plasmolytically active substance diosmoses through the plasmatic membranes, for should even small traces of the plasmolysing osmotic substance slowly penetrate through the plasma to the cell-sap, a gradual rise of the internal osmotic pressure would ensue, causing an increase in volume of the contracted protoplast, and ultimately complete removal of plasmolysis.

As a general rule, substances, which can diosmose through the plasmatic membrane, can also pass through the cell-wall saturated with imbibed water. Hence a substance can often pass through the cell-wall but not through the plasma, whereas one which can diosmose outwardly through the plasma and its membranes, is usully not prevented by the cellwall from escaping to the exterior. The plasmatic membrane adpressed to the cell-wall, decides whether a substance may penetrate the plasma, and thus together with the vacuolar membrane determines whether penetration to the cell-sap is possible. Further, the plasmatic membranes confer upon the cell the important property of being able to retain dissolved substances, which might otherwise diffuse outwardly and be lost.

As a matter of fact, and as is shown in the processes of nutrition, numerous substances are absorbed by the protoplast, and many different waste products are excreted, while very large a mounts of nutritive substances must be absorbed from relatively extremely dilute solutions to supply the plant with the various mineral constituents it requires. When dealing with processes of such intricate and obscure character, an experimental investigation of the final results may be instituted, but it is only possible to directly follow the process of absorption, when its path or termination is marked by a colouration, precipitation, or some visible reaction.

In this respect, the behaviour of the cell with regard to certain aniline dyes is especially instructive. These are absorbed by the protoplast from very dilute solutions and deposited in the cell, in a short time accumulating there to a marked extent ${ }^{1}$. Here we are dealing 
with substances, with which normally the plant does not come into contact, which are not used in metabolism, and which yet penetrate to the cell-sap without any vital activity being involved. Further, it is not every pigment, nor all the compounds that a pigment substance may form, which may be thus absorbed and passively secreted '. This subject is dealt with in greater detail later (Sect. 22), but it may be mentioned that, for example, methyl-blue accumulates in some cases in dissolved form in the cell-sap (roots of Lemma minor, root hairs of Trianea bogotensis), while in others, a blue precipitate is formed (Spirogyra, roots of Azolla). This reaction begins in a few minutes in a 0.001 to 0.0005 per cent. solution of the pigment substance, and rapidly leads to a marked accumulation in the cell-sap. The minute traces of methyl-blue diosmosing through the plasma are not sufficient in amount to cause any perceptible colouration of the latter. In the plasma of the root hairs of Trianea, however, methyl-violet and cyanin accumulate in sufficient amount, using solutions of similar dilution, to cause the appearance of a perceptible violet tinge ${ }^{2}$, and hence, in such cases, the actual passage of the pigment through the plasma is directly visible. If the root hairs are placed in pure water as soon as the absorption of the dye has commenced, the colouration of the plasma gradually disappears, as the dye passes inwards to accumulate in the cell-sap. The above-mentioned clyes penetrate all living cells, but it is only where a certain amount of passive secretion takes place, that the pigment substance accumulates in perceptible amount. Such accumulation is only possible when the dye is converted, as rapidly as it is absorbed, into a modification, which is unable to diosmose through the plasma membranes, or escape from the cell. The precise character of the still soluble combination in which pigment accumulates in the cell-sap of Trianea, Lemna, Elodea, \&c., is as yet unknown. The blue precipitate which methyl-blue produces in the cell-sap of Spirogyrer and Azolla, is a compound of tannic acid and methyl-blue, and if this compound is presented to the cell in solution it is found to be incapable of diosmosing through the plasmatic membranes. That the diosmotic properties of the different soluble compounds of any given substance may markedly differ, can be directly demonstrated by experimentation with artificial precipitation membranes ${ }^{3}$. It may also be mentioned that the protoplast is

1 In passive or indirect secretion, substance presented to the cell is absorbed and accumulated by means of the formation of non-diosmosing compounds. In active or direct secretion the substance is produced by the cell itself, and may either accumulate or be excreted by it. The two processes are not always perfectly distinct.]

${ }^{2}$ For further details see Pfeffer, 1.c., p. 247. On the staining of the living nucleus, Campbell, Unters. a. d. Bot. Inst. z. Tübingen, r888, Bd. Ir, p. 569 ; Lauterborn, Über Bau u. Kerntheilung d. Diatomeen, 1893 ; Palla, Jahrb. f. wiss. Bot., 1893, Bd. Xxv, p. 535. See also Pfeffer, 1. c., p. 273 . On the changes in the mode of pigment absorption coincident with death, see Pfeffer, 1. c., p. 276 .

"See for example Walden, Zeitschr. f. physik. Chemie, 1892 , Bd, x, p. 699 . 
unable to absorb commercial aniline-blue or nigrosin even when placed in relatively concentrated solutions.

The colouration produced does not give any indication of the nature of the compound formed within the cell, any more than the detection of glucose or nitrates in the dead cell enables us to determine in what form these substances were present in the living cell. Hence we have no justification for supposing that soluble substances found in the dead cell, such as glucose or nitrates, cannot diosmose through the plasma, or if an absorption of these substances can be proved to occur, that they are retained in the cell, because the plasma permits them to diffuse inwards but not outwards. When substances presented to the cell are absorbed only because they have undergone, as frequently happens, an extra-cellular modification rendering them capable of diosmosis, it is only to these modified products, and not to the original substances, that a capacity for absorption can be ascribed.

Such preparatory changes are easy to determine, and perhaps only take place in immediate contact with the plasmatic membrane. In order to investigate the diosmotic powers of a given substance, and to find out whether it diosmoses directly without undergoing any preparatory modification, it is usually advisable to determine whether, and to what extent, exosmosis is possible; for if polysaccharides or proteids, for example, appear in the surrounding water, it is hardly possible that they can have been formed by extracellular synthesis or condensation from other exosmosing substances. As a matter of fact, very many substances may exosmose from living cells, and this is especially the case in fungi and bacteria, from which substances may be excreted which can only with great difficulty diosmose through parchment, such as peptones, albuminous substances, and enzymes, which are also probably proteid in nature ${ }^{1}$. Puriewitsch has observed an exosmosis of albuminous substance from the living endosperm of Zea MTais and Phocnix, and from tubers of the Dahlia, \&c., while he found that asparagin may be extracted by water from the living cotyledons of Lupinus. Similarly, Hanstein and Puriewitsch ${ }^{2}$ found that an exosmosis of glucose was possible from the living endosperm of

1 On the excretion of enzymes see Sect.91. Albuminous substances and peptones are frequently transferred from the living cells of fungi, bacteria, and yeast to the flud in which they lie. It is, however, important to remember that such transference also takes place when the cells die. For the influence of external conditions on such excretion see Sect. $1 \%$. From the above, it may be concluded that albuminous substances and peptones may be absorbed by the living cell without undergoing any previous change or decomposition. Certain organisms, having no power of producing proteolytic enzymes, may be directly nourished with albuminous substances or peptone. See Beyerinck. L'Aliment protogène, I 89 r, pp. 3 and I 8 (Sep.-abdr. aus Archiv. Néerlandaises, T. XxIV); alsu Sects. $66,91,108$.

${ }^{2}$ Hanstein, Flora, I894, Erg.-bel., p. 419 ; Puriewitsch, Ber. d. Bot. Ges., I896, p. 205. See Sects. 93, 109. 
Zea Mais, as well as of a polysaccharide, probably cane-sugar, which is also one of the sugars excreted by nectaries.

These illustrations may suffice to indicate that protoplasts can directly absorb and excrete a variety of substances of both simple and complex molecular constitution. There is, therefore, no occasion to doubt that the numerous nutritive or waste substances, absorbed or excreted by the protoplast, diosmose directly without undergoing any preparatory change, unless there are definite reasons to the contrary. Even fats and fatty acids may be directly absorbed by living cells, and probably wander from cell to cell in the form of a fine emulsion.

Certain substances penetrate every living protoplast that has been examined, but it is quite certain that more detailed and minute experimentation will show that qualitative and quantitative differences exist between the respective diosmotic properties of such substances; and, moreover, the diosmotic properties of the protoplast may apparently become modified in the progress of development in response to special external conditions.

With regard to aniline dyes, acids, and alkalies, differences have been recorded in the quantities absorbed by different cells. According to Heidenheim ${ }^{1}$, however, the epithelial cells of the kidney can absorb and excrete indigo-carmine, which is a pigment incapable of penetrating living plant cells. By plasmolytic and other methods, differences in the power and rapidity with which substances are absorbed can be detected with greater or less accuracy.

Absorption of dyes, and the precipitations caused by these and other substances. In the work previously quoted ${ }^{2}$, and in the studies connected therewith ${ }^{3}$, a detailed account of the absorption of aniline dyes will be found. In Sect. 22 these are again mentioned, in so far as they demonstrate the selective powers of the living cell and its powers of passive secretion. If plants of Lemna minor are allowed to float on a 0.0005 per cent. solution of methyl-blue, the blue colouration soon observed in the roots is a visible indication that colouring material is absorbed by them, and accumulated in the cell-sap. If we employ a leafy shoot of Elodea the absorption of the dye is demonstrated by the marked colouration assumed by the leaves, and by the gradual decolourization of the coloured solution in which the leaves are immersed. Provided that the coloured solutions employed are sufficiently dilute, normal growth continues, and a poisonous influence is only exerted when the colouring material can penetrate the plasma to a sufficient extent. Hence a large amount of the dye may accumulate in the cell-sap without

1 Hermann, Handb. d. Physiologie, ${ }_{188}$, Bd. v, Abtheilung I, p. $34^{6}$. Cf. Pfeffer, Unters. a. d. I3ot. Inst. z. Tüibingen, 1886, Bd. II, p. 270.

2 Pfeffer, l. c.; also Plasmahaut u. Vacuolen, I 890, p. 285.

3 Wieler, Jahrb. f. wiss. Bot., I888, Bd. xix, p. 119 ; Wortmann und Stilling, Anilinfarbstoffe als Antiseptica, I 890 , p. 47 ; Overton, Bot. Centralbl., I89o, Bd. XI.IV, 1). 6 ; and the works already cited on p. 94, note 1. In animals see Kowalevsky, Biol. Centralbl., I $\$ 89$, Bd. Ix, p. 33; Feist, Physiol. Centralbl, 1890 , IBd. IV, 1. 324, \&c. 
producing any injurious effect, provided that it is in the form of a non-diosmosing compound.

The different forms in which the dye accumulates, as well as the varying results which the same pigments produce on different plants, indicate that the substances which render accumulation possible are not always identical. Of those substances, which cause a deposition of pigment in the cell-sap, the best known are Tannin and Phloroglucin. Other substances may also be active, but their nature is as yet obscure. In some cases two or more such substances may be present in the cell (Pfeffer, 1. c., pp. I9I, 273), and by their combined action determine whether the absorbed dye shall accumulate in the cell in soluble, or insoluble, form '. When a pigment is precipitated inside the cell, it may also remove other substances present in the cell-sap, either mechanically or in combined form. Treatment with caffein, antipyrin or ammonium carbonate causes any tannin present in the cell-sap to be precipitated, and the presence in the cell of other substances, of which phloroglucin is the only one known ${ }^{2}$, may cause similar precipitation when tissues are treated with the above re-agents. Since these substances, which may be said to be the agents of passive secretion, vary both as regards the nature and the number of them present in the cell, it is clear, that the above reaction may, or may not, form a direct test of the extent to which the cell in question can passively secrete aniline dyes. It is easy also to understand, that it may be possible for one plant to absorb Methyl-blue and also Bismarck-brown, while another only absorbs the former ${ }^{3}$.

The precipitates formed when certain cells are treated with caffein are undoubtedly insoluble compounds produced by the union of the reagent with substances present in the cell. Whether the precipitation caused by ammonium carbonate is of similar nature is doubtful, for precipitates may also be formed by neutralization or crystallization. On treatment with water, the precipitates produced by ammonium carbonate, caffein, \&c. re-dissolve, but this merely indicates that the reagents in question may be readily removed by water from the living cell. A similar diosmotic separation of the absorbed substance from that with which it combines may take place either normally, or only under special conditions, when the living cells absorb aniline dyes (Sect. 22). As a general rule, these reactions take place only in the cell-sap, but it need not surprise us if we find them occurring in the plasma in certain cases ${ }^{4}$, indeed, as a matter of fact, certain aniline dyes do actually accumulate in the plasma. Even when in certain cases the precipitate is perhaps found to consist of a double phosphate of ammonium and magnesium, or of proteid compounds, the essential features of the phenomenon will still remain the same.

1 See Pfeffer, l.c., pp. 232, 245; Lehmann, Zeitschr. f. physik. Chemie, I894, Bd. XIv, p. I57.

2 Loew u. Bokorny, Bot. Zeitung, I887, p. 849 ; Flora, Ergänzungsband, I892, p. II7, \&c.; Klemm, Ber. d. Bot. Ges., I892, p. 237 ; Flora, I892, p. 400 ; Bot. Centralbl., I894, Bd. Lvir, p. I97; Zimmermann, Beihefte z. Bot. Centralbl., I893, Bd. III, p. 324; further literature here quoted. On the precipitates which Darwin found Am. carbonate might produce, see Kilercker, Studien ì. Gerbstoffvacuolen, I8S8 (Tübinger Dissertation); Pfeffer, Unters. a. d. Bot. Inst. z. Tuibingen, 1886, Bd. 11, p. 239, and Flora, 1889, p. 52.

${ }^{3}$ Klemm, Flora, I892, p. $4^{12}$; Pfeffer, Unters. a. d. Bot. Inst. z. Tübingen, l.c., pp. 191, 273.

"See Klemm, Hlora, 1892, p. fos; Zimmermann, 1. c. 
The existence of proteids in the precipitates formed in the cell-sap has not as yet been demonstrated, but it is extremely probable that they are actually present. When experiments are conducted under conditions physically similar to those existing within the plant, as for example, in artificial tannin-gelatine cells, or in capillary tubes containing tannin and closed by a tannin-gelatine precipitationmembrane, it is found that irrigation with solutions of aniline dyes, caffein, ammonium carbonate, \&c. produces similar precipitations ${ }^{1}$ to those which these re-agents induce in living cells ${ }^{2}$. In both cases the precipitates have the same character and reactions, and show the same power of reducing silver salts, which Loew and Bokorny have supposed to be a satisfactory test for active albumin (Sect. I I).

Colouration and precipitation tests for diosmotic absorption. Free acids (acetic, tartaric, phosphoric, \&c.), as well as carbonic acid, and the caustic alkalies, readily and rapidly penetrate the living protoplast. This can be easily demonstrated by allowing very dilute acid or alkaline solutions to act upon cells containing blue or red cell-sap (floral leaves of Pulmonaria or Rosa, sections of beet-root, or staminal hairs of Tradescantia). The presence of a cuticle renders penetration more difficult, but otherwise a change of blue to red colouration or red to blue, as the case may be, soon takes place, and as a rule the original colour returns when the tissue is well washed with water, while, if the reagents are sufficiently dilute, and the action not too prolonged, the cells are uninjured and remain living. Solutions of Iodine and Mercuric chloride also rapidly and readily penetrate the cell, but exercise a very pronounced injurious and poisonous effect ${ }^{3}$.

The absorption of Ammonium carbonate, Caffein, Antipyrin, \&c. is indicated by the formation of a precipitate within the cell, without any injurious effect being necessarily produced. In many plants the rapid penetration of Hydrogen-peroxide is made evident by the colourations or decolourizations produced within the cells ${ }^{4}$, and similarly dissolved oxygen also readily diosmoses through typical cell-walls.

Chemical methods. Absorption and passive secretion may in many cases be estimated or determined by macro- or micro-chemical methods. The formation of starch in chloroplastids or leucoplastids may serve as an indication of the absorption of various sugars and certain other substances (Glycerine, etc.), (Sect. 54), while in other plants a supply of sugar leads to an accumulation of glucose ${ }^{5}$ or other carbohydrates. Similarly in many plants nitrates ${ }^{6}$ may accumulate to such an extent that the dried substance burns like touch-paper. Other plants again store up large accumulations of phosphates, sulphates, \&c. ${ }^{7}$ inside living cells.

Plasmolytic tests. When the contracted protoplast slowly re-expands after

1 [The formation of these precipitates is usually included among the phenomena of 'aggregation,' but it is better to restrict the latter term to the formative changes occurring in the protoplast, since the one is a vital but the other a purely physical phenomenon.]

${ }^{2}$ Cf. Klercker, Klemm, and Pfeffer, 1. c.

3 Pfeffer, Osmot. Unters., I 877 , p. 140.

- Pfeffer, Zur Kenntniss d. Oxydationsrorgänge, 1889 .

${ }^{5}$ Schimper, Bot. Zeitung, $188_{5}$, pp. 743, 758 ; Puriewitsch, Ber. d. Bot. Ges., 1896, p. 206.

- Wolf, Landw. Versuchsst., I864, Bd. v1, p. 220 ; Frank, Ber. d. Bot. Ges., 1887, p. $47^{2}$; Schimper, Bot. Zeitung, 1888, p. 121. Further literature sect. 70.

7 Schimper, Bot. Zeitung, 1888, 1.c. 
plasmolysis, this must be due either to a gradual absorption of the plasmolysing salt, or to an internal production of osmotically active substances, and the latter does actually occur when Fungi ${ }^{1}$ are placed in concentrated solutions. If the gradual return to the normal condition of turgidity takes place only when the cell is plasmolysed by solutions of particular substances, it is evident that the reexpansion of the protoplast is due to its being more or less permeable to the substance in question. The researches of de Vries $^{2}$, Wieler ${ }^{3}$, Klebs ${ }^{4}$, Janse ${ }^{5}$, Overton ${ }^{6}$, and others, have been carried out from this standpoint, and it has been found that many. of both higher and lower plants absorb during an entire day no perceptible amount of $\mathrm{KNO}_{3}, \mathrm{NaCl}$, cane sugar, \&c., but that all grades of transition may be found, leading ultimately to plants, whose protoplasts are readily penetrated by these substances. This is shown especially well by Janse's researches and by Fischer's ${ }^{7}$ work on Bacteria. In the latter case, perhaps owing to the relatively large surface exposed, the absorption of the plasmolysing salt and the re-expansion of the protoplast are extremely rapid. The bacterial protoplast appears to be readily permeable not only by $\mathrm{KNO}_{3}, \mathrm{NaCl}$, and cane sugar, but also by other substances, and this property, permitting as it does a rapid accommodation to any change in the concentration of the external medium, must be of great importance to these organisms. 'The plasmodia of Mycetozoa also appear to be readily permeable by many salts, including $\mathrm{Ca} \mathrm{SO}_{4}$ and the amido-acid, Asparagin ${ }^{8}$.

As de Vries and Klebs have shown, glycerine and urea are substances which can penetrate all protoplasts, and often with marked rapidity. Here also all degrees of permeability are exhibited, for the bud scales of Begonia manicata allow these substances to penetrate only with difficulty. Overton has recently determined, by means of the plasmolytic method, the extent of the diosmosis of which many different substances are capable.

Exosmosis. The occurrence even of slight exosmosis of the soluble substances which the cell contains, and to which the preservation of turgor is due, would render it impossible for any alga or other aquatic plant to remain turgid, or to retain its soluble reserve-food-material, while even from a beet-root a large part of its sugar would be extracted by the surrounding damp soil. In other cases, however, plants or parts of plants are specially adapted for exosmosis, and may either be constantly able to excrete sugar or other substances, or may only do so under special conditions (cf. Sects. 93, I09). Hence, it is not surprising that in different researches the same results are not always obtained.

${ }^{1}$ See Pfeffer, Druck u. Arbeitsleistungen, 1893 , pp. 304, $4^{28}$.

${ }^{2}$ De Vries, Sur la perméab. d. protopl. d. betteraves rouges, is 7 I (Sep.-abdr. aus Archiv. Néerland., 187 , T. vI); Jahrb. f. wiss. Bot., $188_{4}$, Bd. Xiv, p. $44^{1}$; Bot. Zeitung, 1888 , p. 229 , and 1889 , p. 309 .

3 Wieler, Ber. d. Bot. Ges., 1887 , Bd. v, p. 375 .

4 Klebs, Unters. a. d. Bot. Inst. z. Tübingen, i 888 , Bd. II, p. 540.

5 Janse, Mededeelingen d. Kon. Akad. v. Wetenschappen, Amsterdam, 1888, p. 332.

6 Overton, Osmot. Eigenschaften, 1895 (Sep.-abdr. a. Vierteljahrsschr. d. Naturf.-Ges. z. Zürich, Bd. XI) ; Zeitschr. f. physik. Chemie, 1897 , Bd. xxı1, p. 189.

7 A. Fischer, Jahrb. f. wiss. Bot., I 895, Rd, Xxvir, p. I50.

- Pfeffer, Plasmahaut u. Vacuolen, 1890, p. 220. 
According to Czapek the roots of Phanerogams generally excrete small traces of substances, one of which is acid potassium phosphate ${ }^{1}$, but since the amount excreted is extremely small, it is not surprising that macrochemical investigations have frequently yielded negative results ${ }^{2}$. The older positive results are for the most part indecisive, since sufficient precautions were not taken to ensure that the excreted substances were not derived from dead or dying cells, as was probably the case with regard to the sugar found by Boussingault and Detmer (l. c.) in the water in which fruits had lain.

Fats. The results obtained by R. H. Schmidt ${ }^{3}$ establish the fact that fatty substances, and especially fluid fatty acids, can penetrate the living cell with comparative ease. The presence of only a minute amount of a fatty acid renders possible a fairly rapid absorption of neutral oil, such as olive oil, which the cell normally absorbs only slowly and in trifling amount. To demonstrate the absorption of fat, a strip of filter paper impregnated with the fatty substance may be inserted in a longitudinal incision of about $\mathrm{I} \mathrm{cm}$. length made in the young etiolated stem of a seedling of Pisum satizum. If the fat has been coloured with alkanna, it can be traced into the intercellular spaces, and after a few hours perceptible absorption has occurred, leading in one to two days to a marked accumulation within the neighbouring cells. At first the fat is present in the plasma in so fine a state of division, that in some cases it only becomes visible when the action of reagents causes the minute droplets to coalesce with one another. This takes place normally, as a general rule, as the quantity present increases, and later still, fat globules appear to a greater or less extent in the cell-sap.

The fat is apparently absorbed as such, and hence it is probable that oils also are directly transferred from cell to cell. The appearance and accumulation of fatty material follow the same course in nature, for when fat undergoes translocation, free fatty acids appear to be always present (Sect. 82). Even plants which are normally poor in oily substances have also the power of absorbing fat, while those fungi, which may be nourished by neutral fats, must apparently be able to split the fat into the fatty acid and glycerine of which it is composed. Fungal hyphae, growing on solid cacao-butter, absorb and extract nutriment from it, and the protoplasts of higher plants can absorb it when the temperature is high enough $\left(30^{\circ} \mathrm{C}\right)$ to keep it in a fluid condition.

The means by which the particles of oily substance are enabled to penetrate the cell-wall saturated with water, and to pass through it, as well as through the plasmatic membranes, have not yet been determined. Oil is, however, able to pass in the form of a fine einulsion through animal membranes impregnated with soap or bile, and probably in plants also the process is one of emulsification. Dead

1 Czapek, Jahrb. f. wiss. Bot., I896, Bd. xxix, p. $3^{21}$.

2 Lit.: Hofmeister, Pflanzenzelle, 1867, p. 4; J. Boussingault, Agron., Chim. agric. et Physiol., I874, vol. v, p. 309 ; Pfeffer, Landw. Jahrb., I876, Bd. v, p. I 25 ; Schulze und Umlauft, l.c., I 876 , Bd. v, p. 828 ; Detmer, Forsch. a. d. Geb. d. Agriculturphysik, I879, Bd. II, p. 372 ; van Tieghem et Bonnier, Bull. d. l. Soc. Bot. d. France, is8o, vol. xxvii, p. 116.

${ }^{3}$ R. H. Schmidt, Flora, I891, p. 300. See Pfeffer, Aufnahme u. Ausgabe ungrelöster Körper, 1890, p. 179. The literature is quoted in these works. 
plant membranes saturated with pure water do not seem to allow any of the fats or fatty acids with which they may be brought in contact to pass through them. The emulsification theory is supported by the fact that the fatty acids, as well as the neutral fats containing fatty acids, are readily emulsified by means of sodium phosphate and other reagents, and this would explain the importance of the presence of fatty acids for the translocation of solid fats or liquid oil ${ }^{1}$. It is possible that the supposed solubility of fats in egg albumen, and in concentrated sugar solution ${ }^{2}$, may be merely due to the production of a very fine emulsion.

Wax, Balsams, and Ethereal oils are apparently not formed merely by changes taking place external to the living cell, but probably are frequently, or even usually produced by the protoplast, and subsequently pass through its substance, and through the cell-wall to reach the regions where they are deposited ${ }^{3}$, or as in the case of the essential oils which give flowers their perfume, they pass to the surface, and are then slowly given off to the surrounding air. On the other hand, as the cuticle develops and becomes impregnated with wax, the imbibed water with which it was originally saturated is gradually expelled.

\section{Section 17. Special Account of the Processes of Absorption and Excretion.}

The general laws of diosmosis are directly applicable in determining the exchanges which take place between the organism and the external world. Every substance which can diosmose through the different cellular membranes penetrates the protoplast, independently of whether it may be useful, unnecessary, or even poisonous ${ }^{4}$. All observed facts are in harmony with this law, although it is not always easy actually to determine the conditions under which a particular result is obtained. Thus, not only is the quality of the diosmosing substance of importance, but the fact that the diosmotic properties of the plasmatic membrane may vary must be taken into consideration, and moreover a substance may be changed and rendered fit for diosmosis by intracellular or extracellular processes of more or less uncertain character ${ }^{5}$. It must also be remembered that the plasmatic membrane is a living and dependent organ, by means of which the protoplast

'Hermann, Handbuch d. Physiol., Bd. v, pp. Iz 8 , 29I ; Bunge, Lehrb. d. physiol. Chemie, I894, 3. Aufl., p. 176; Quincke, Pflüger's Archiv f. Physiol., i 879 , p. I36.

Pacht, Centralbl. f. Physiol., is 88 , Bd. 11, p. 688.

3 Pfeffer, 1.c., p. 179 , and the lit. there cited.

4 This is demonstrated by the marked amount of unnecessary ash constituents which may be taken in, as well as directly by the absorption of aniline dyes. The older researches by Saussure (Rech. chim. s. 1. Végétation, ISo4, p. 247), Vogel (Jour. f. prak. Chemie, I842, 13d. xxv, p. 209), Trinchinetti (Bot. Zeitung, I $S_{45}$, p. III) - see the literature given by Deherain, Ann. d. sci. nat., 1867 , v. sér., T. v111, p. 180)-are for us of little importance, for only the absorption by the entire plant was determined, and in part the methods employed caused many of the protoplasts to die.

${ }_{5}^{5}$ See Pfeffer, Zur Kenntniss d. Plasmahaut u. Vacuolen, I $\delta 90,1$. 279, and Unters. a. d. Bot. Inst. z. Tübingen, r $8 \& 6$, Bid. 11: p. 299. 
regulates its intercourse with the external world, and hence, as might be expected, the plasmatic membranes of different plants exhibit certain specific differences and peculiarities. Apparently it is by temporary or permanent alterations in the specific nature of the plasmatic membrane that an absorption (or excretion) of a particular substance may be temporarily or permanently permitted or prevented. There can be no doubt that the physiological properties of the living ectoplasmic membrane are capable of much more marked alteration or modification than are those of the dead cell-wall immediately enclosing it. This is especially the case, owing to the fact that the component micellae of the plasmatic membrane may be loosened from their neighbours and carried away to the internal plasmatic layers. Many facts indicate that frequently a change in the diosmotic properties of the living cell is the means by which a particular result is produced, although at present this statement is incapable of absolute proof. It is always possible that a change in the diosmotic properties of the plasmatic membranes may affect only one particular substance, while, with regard to all others, the protoplast may appear to have the same diosmotic properties as before. No difference of osmotic properties can be detected in most cases between the living protoplast and the isolated vacuolar membrane (Sects. 22, 108), for all dyes which are capable or incapable of diosmosing through the protoplast are capable or incapable, as the case may be, of passing through the vacuolar membrane also. Free acids and alkalies give similar results, and as far as plasmolytic methods may be trusted, the same is true for $\mathrm{KNO}_{3}, \mathrm{NaCl}$, and many other substances ${ }^{1}$.

Imbibition and diosmosis are always dependent upon the nature of the membranes or lamellae to be traversed, no matter whether these are composed of solid or fluid substance. Hence infiltration of the lamellae by another substance may markedly affect their diosmotic properties. The cuticle affords a striking example of the utilization of this peculiarity by the plant.

In treating of the absorption of fats and oils, we have seen that the infiltration of the separating membrane with another substance (phosphate of sodium, \&c.) renders a passage through the membrane possible, and finely emulsified oil may pass through the cell-wall without any actual solution being necessary, while slight mechanical pressure may suffice to squeeze oil drops and solid bodies of measurable diameter through the plasmatic membrane, which closes behind them, just as a film of oil closes over a needle which has passed through it. Hence those substances which

1 Pfeffer, l. c., where it is also shown that the action of chloroform, or a deficiency of oxygen, \&c., does not prevent the absorption of aniline dyes. For these results attention only is paid to those cases in which the plasmatic membranes are still living and plastic. Puriewitsch and Czapek have recently shown that treatment with chloroform may produce a depressant effect on diosmosis (Sect. 108). 
are incapable of diosmosing find no point of entry or exit, either during or after the passage of the oil drop or solid particle. There can be no doubt that the molecules, or molecular complexes, of dissolved substances penetrate between the component elements (micellae) of the plasmatic membranes, and diosmose only when the imbibitory force with which the substances in question are attracted is sufficiently powerful to overcome those molecular forces which may be operating antagonistically (Sect. I6).

The relationships just indicated are of perfectly general application. The quality of the separating membrane, whether fluid or solid in nature, and the size of the molecules of the diosmosing substance, are of importance in determining the possibility of diosmosis as well as its character . Nevertheless, as the living organism shows, the relative size of the diosmosing molecules in relation to that of the micellar interstices through which they pass is by no means all-important ${ }^{2}$. Thus, the same protoplast. which is impermeable to $\mathrm{NaCl}$ or $\mathrm{KNO}_{3}$, may allow the larger molecules of methyl-blue, of albuminous substances, and perhaps also of other colloids to penetrate freely. This selective peculiarity, which is of the utmost importance to the plant, may be connected in some way with the fact, indicated by the ready penetration of solid particles, that the passage through the plasmatic membranes involves only a trifling expenditure of energy.

The conditions which regulate absorption and diosmosis have already been indicated when dealing with the phenomena of imbibition and swelling (Sect. I2). It is possible that the molecular forces, which are here at work, may induce molecular decompositions or other chemical changes, and this may indeed frequently form an essential part of the process of diosmosis. Thus it is possible, for example, that $\mathrm{KNO}_{3}$ is only enabled to penetrate into the cell by being split up into its component ions, or that cane sugar is absorbed by the plasmatic membrane as a monosaccharide, and is recondensed into saccharose on excretion into the cell-sap.

When chemical union takes place a substance may very readily be transmitted through a solid non-porous membrane. This would be the case with nitric acid, for example, supposing that on one side of a dry cellulose membrane nitrification took place, while on the other side nitro-

1 The problem has already been investigated from this point of view in Osmot. Unters., 1877 , and also in Unters. a. d. Bot. Inst. z. Tübingen, I 886 , Bd. II, p. 301 . Direct proofs by experiments with artificial precipitation membranes have also been brought forward. More recently other authors have come to the same conclusion: Tamman, Zeitschr. f. physik. Chemie, IS92, Bd. $x$, p. 25. ; Walden, ibid., p. 699 ; Fünfstiick, Ber. d. Bot. Ges., I 893 , Generalvers., p. So ; Overton, Über osmot. Eigensch., I $\mathrm{S}_{55}$ (Sep.-abdr. a. Vierteljahrsschr. d. Naturf.-Ges. in Zürich, Jahrb. 40). On the influence of the character of the membrane: Raoul, Zeitschr. f. physik. Chemie, IS95, Bd. XvIr, p. 737.

2 [That is, absorption does not necessarily run parallel with the rapidity of diffusion, or with the osmotic power, of a given substance, both of which are dependent upon the size of the molecules of the dissolved substance.] 
cellulose was continually reduced. Moreover, molecules of sodium may be transmitted through glass by a process of electrolysis, and it is possible that the weak electric currents circulating in living cells ${ }^{1}$ may under particular conditions, and in virtue of their continuous action, exercise an important influence upon translocatory processes. Moreover, the nature of the plasma is such as to render it possible that a substance may combine chemically with the plasmatic elements, thus being transmitted internally, and then set free again. When vacuoles are emptied non-diosmosing substances do actually pass through the protoplasm ${ }^{2}$.

By these or other means non-diosmosing and even insoluble bodies are transferred by the vital activity of the protoplast through its substance and membranes in either direction. In the case of substances capable of diosmosis a physical transference is all that is necessary, though where a production of diosmosing compounds must first take place this is only possible with the assistance of the living organism. To maintain a continuous supply, the protoplast alters or modifies the diosmosing substances as fast as they are absorbed; and hence all the different diosmotic processes are originated and correlated by the vital activity of the living organism ${ }^{3}$.

In general, the direction in which a substance is absorbed is dependent upon the maintenance of a certain potential energy of absorption, and though it is possible to imagine a plasmatic membrane as having the power of temporarily or permanently allowing a substance to diosmose in one direction only, still no such case has as yet been proved to exist ${ }^{4}$. Apparently the nature of the solution with which it is in contact has a distinct influence upon the nature and powers of the plasmatic membrane, and it is thus quite possible that differences arising from such causes may exist between the ectoplasmic and the vacuolar membranes. Localized differences between the different regions of a plasmatic membrane may serve to permit of the escape of a particular substance at a given spot, or to allow a transference to a particular neighbouring cell to be possible.

The actual final results are not only dependent upon the diosmosis, but are also influenced by all the varied conditions and circumstances which modify and direct diosmosis, or render it possible. From what has been already said, it is at once evident why it should often be extremely difficult to determine all the factors at work in producing a given result.

${ }^{1}$ Haacke, Flora, 1892, p. 455. Walden (Zeitschr. f. physik. Chemie, I $99^{2}, \mathrm{Bd} . \mathrm{x}, \mathrm{p} . \mathrm{fI} S$ ) has shown that the permeability of a membrane by a compound, and by the ions into which the compound may be resolved, is not the same, as Ostwald (ibid., I89o, Bd. vi, p. 69) suggested.

${ }^{2}$ Pfeffer, Vacuolenhant, I 890, p. $2 S_{3}$.

3 Pfeffer, ibid., IS90, p. 290 ; Studien zur Energetik, I892, p. 268.

- For details see Pfeffer, Plasmahaut u. Vacuolen, IS9o, p. 2SS, and Overton, Über osmot. Eigenschaften, \&c., I 895 , p. 26 (Sep.-abdr. a. d. Vierteljahrsschr. d. Naturf.-Ges. in Zürich). In the first-named work it is shown that Janse assumed the existence of permeability in one direction on insufficient grounds. 
The special properties of the plasmatic membrane render it possible that a dissolved colloid substance may pass through it, but not through the water-saturated cell-wall, although the micellar interstices of the plasmatic membrane are certainly much narrower than those of the latter, and although in the cell-wall the micellar interstices probably form minute canals, across which the molecular influences radiating from the micellar walls are unable to extend.

In dealing with the selective powers (Sect. 22), still further instances will be brought forward to show how external agencies may by inducing metabolic changes, directly or indirectly, cause the diosmosis of a particular substance. From this point of view, the peculiarities which Nägeli ${ }^{1}$ observed in the yeast of alcoholic fermentation are worthy of further investigation. Nägeli found that peptone and albunin are excreted by yeast plants in an alkaline medium, but that, in an acid medium, albumin alone is excreted, and that only when fermentation is active. It is possible that in this case the existence of the solvent for albumin becomes of importance by extracting albumin from the protoplast, or rendering the diosmosis of the former through the cell-wall possible ${ }^{2}$. The manner in which tartrate of iron acts in favouring the nutrition of algae with sugar is quite uncertain, though Klebs ${ }^{3}$ suggested that it may possibly aid in the absorption of sugar.

Diosmosis. This term applies to the diffusion whieh may take place through a solid or fluid membrane. The nature of these phenomena as well as the imbibitory processes to which they are directly due have already been described, in so far as they affect, or are related to physiological processes (Sect. 12). A more complete physical account is unnecessary here, since a full and rational description of the phenomena of osmosis is now given in all physical text-books ${ }^{4}$.

Reasons have already been advanced for the conclusion that in semi-permeable membranes the interstices filled with water can have only a relatively very smal diameter (Sect. 16). When a particle of dissolved material passes through the axis of an interstitial canal, only the margins of which are influenced by the molecular forces radiating from its walls, we can speak of capillary diosmosis, as contrasted with molecular diosmosis, in correspondence with the distinction already pointed out between capillary and molecular imbibition (Sect. I z). Probably both forms of diosmosis take place simultaneously through cell-wall, although the small diameter of the capillary spaces, together with the relative rigidity of the substance of the wall, allows the large molecules or molecular complexes of colloid substances to diosmose only with difficulty, or not at all. By the diosmotic

1 Theorie d. Gährung, 1879 , pp. 79 and 1 $_{5}$. See also Gayon et Dubourg, Compt. rend., r 886, T. C11, p. 978 . Sufficient attention has not been paid to the possibility of the proteid substances being partly or entirely derived from dead cells.

${ }^{2}$ Nägeli, Sitzungsb. d. Bair. Akad., I878, p. 169.

3 Klebs, Unters. a. d. Bot. Inst. z. Tüibingen, I 886, Bd. II, p. 544.

- Ostwald, Lehrb. d. allgem. Chemie, I 891 , 2. Auf., Bd. I, pp. 651, 674; Winkelmann, Handb. d. Physik, 1891, Bd. I, p. 6r8. Also Pfeffer, Osmot. Unters., I877. 
removal of the substances produced, a feeble chemical reaction may finally be brought to completion, but an account of this phenomenon and of osmotic pressure will be given later (Sects. 22 and 93).

Precipitation membranes. For the study of osmotic processes, the precipitation membranes which Traube ${ }^{1}$ first employed are of great importance. These demonstrate how a limiting precipitation-membrane may be formed and maintained where two fluids, or a fluid and a solid, come into contact. The precipitation-membranes formed by tannic acid and gelatine or mucilage form especially favourable experimental material. Thus, if the end of a glass rod, on which a little mucilage has been allowed to dry, be immersed in a two per cent. solution of tannin, after a short time a transparent continuous membranous film is formed around the dissolving mucilage. This membrane is kept tense by the osmotic nature of the mucilaginous contents, and is forced to increase in surface area by the stretching to which it is subjected. To obtain marked growth, a gelatinous gum should be employed containing from ten to fifteen per cent. of sugar. If in addition the gum

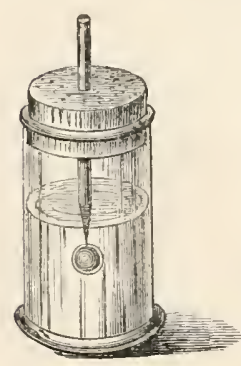

FiG. 5. contains a little indigo-carmine, or aniline-blue, it may be observed that these substances do not diosmose through the separating membrane. The solid American mouth-glue ${ }^{2}$ may be conveniently employed for this purpose, but sometimes the addition of a little gelatine is necessary. The experiment represented in Fig. 5 is of even more general utility and simplicity. The cylinder contains a two to five per cent. solution of potassium. ferrocyanide, and the capillary end of the glass tube contains a drop of a solution of cupric sulphate. The other end of the glass tube is closed by means of the finger, and the capillary end is immersed in the fluid contained in the cylinder, so that the column of fluid inside the tube is slightly higher than that outside. If a crystal of sulphate of copper is thrown into a solution of ferrocyanide of potassium, a similar precipitation-membrane is formed. (On the production of membranes for diosmotic researches, see Sect. 22.)

Moreover, as Traube has shown, an osmotic membrane may be formed by the mere contact of a solution with pure water, provided a certain amount of precipitation is thereby induced. This is actually the case when a concentrated solution of tannin containing dissolved tannic-acid-gelatine is employed, for when diluted the latter is precipitated, and hence when the solution mentioned is in contact with water, a precipitation-membrane is formed between the two. The works already quoted $^{3}$ give evidence to show that the specific permeability varies in different membranes. According to Traube, infiltrations of the membrane may modify its permeability, and Pringsheim ${ }^{4}$, from results obtained with gelatinous membranes,

1 Traube, Archiv f. Anat. u. Physiol., $186_{7}$, p. $8_{7}$; Bot. Zeitung, $18_{75}$, p. $5^{6}$. See Pfeffer, 1. c., p. I r.

${ }^{2}$ [A soluble tasteless gum used upon the adherent flaps of envelopes, \&c.]

3 See p. 103 , note $I$.

4 l'ringsheim, Jahrb. f. wiss. Bot., 1 s95, Br. xxvit, p. 1. 
concluded that permeability may also be modified by the conditions under which the membrane is formed.

Precipitation-membranes of tannic acid with gelatine or mucilage grow and increase in size with perfect regularity, provided no disturbing influences are present. When the solution of tannin is replaced by water, the conditions necessary for growth are removed, so that, as the osmotic pressure continues to increase, the delicate separating membrane soon ruptures. The surface growth of membranes formed by the precipitation of ferrocyanide of copper or prussianblue can only continue for a short time, and to a limited extent. As soon as further growth ceases, the increasing internal osmotic pressure soon ruptures the cell. The fluid, which then exudes, becomes immediately invested by a new precipitation-membrane, and the repetition of this process may result in the production of the most curious figures ${ }^{1}$.

\section{SECTION I8. The Plasmatic Membranes.}

The general principles previously laid down as governing the exchanges of substance taking place between the protoplast and the external world would still hold good, even though no differences existed between the diosmotic properties of the general mass of the protoplast and the limiting membranes which enclose it; if, for example, the entire thickness of the primordial utricle behaved like a single very thick plasmatic membrane. There are, however, weighty reasons for concluding that the deductions arrived at in Sect. 16 are correct; but only a short account of these reasons can be given here ${ }^{2}$.

The question at issue would be definitely answered in the manner already indicated, if it were found that substances, commonly present or artificially introduced, diffused through the central mass of the plasma, but did not appear in the vacuoles or in the water outside, proving that they are incapable of diosmosing through the plasmatic membranes. As a matter of fact, a variety of observations make it probable that such is actually the case in the living protoplast, but no absolutely convincing proof has as yet been brought forward. When, however, the plasmatic membrane is caused to assume a condition of rigor by treatment with very dilute hydrochloric acid, its original diosmotic properties are usually at first retained, and a dye, which is unable to penetrate the plasmatic membrane, will, if it finds entry through a slit in the latter, rapidly diffuse through the enclosed central mass of the dead plasma.

1 The objections which Sachs (Lehrb., 4. Auf., i $88_{7}$, p. 645) raises against Traube are based on the production of these eruptions. In the production of the so-called myelin forms, similar eruptive actions probably also take part. See Briicke, Sitzungsb. d. Wien. Akad., I8 79 , Bd. Lxxix, Abth. iii, April.

${ }^{2}$ Details by Pfeffer, Zur Kennt. d. Plasmahaut u. d. Vacuolen, 1890, pp. 224,244 . For an account of the nomenclature employed, see the same work, p. ISS, and also Sect. 8 of this book. 
The difference in behaviour between the two, when dead, indicates the existence of a certain amount of differentiation between the plasma and the membrane which encloses it, and the fact, that by sudden plasmolysis the vacuolar membrane may be actually separated, points in the same direction. Moreorer, the thinnest plasmatic films, such as surround the distended vacuoles formed by isolated fragments of plasma (see Fig. 6), retain their original diosmotic properties.

It has also been indicated (Sect. I6) that the existence of a plasmatic membrane does not necessitate any special structure in the central plasma, vacuolar or otherwise. In every case, we ultimately require to determine what are the paths along which a substance diffuses through the plasma, and whether penetration is possible in the case of certain plasmatic organs or elemental units ${ }^{1}$. Unfortunately, structural appearances seen under the microscope afford no conclusive evidence as to the diosmotic

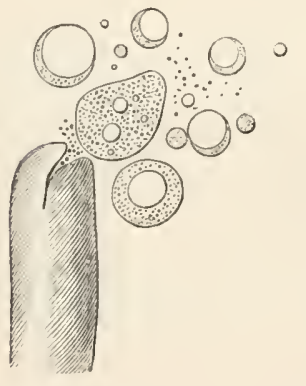

FIG. 6. The formation of vacuoles in fragments of plasma immersed in water, which have been forced by pressure from a young root hair of Hydrocharis morsus-ranae $(\times 4.50)$. properties of the parts examined. The ultimate structure must, however, in all cases, whether a plasmatic membrane be present or not, be such as to afford an explanation of all the facts brought to light by experiment. The fact that the diosmotic properties of the vacuolar membrane are preserved, however rapidly it may be increasing in surface area, proves that it never becomes discontinuous. This fact, however, forms no argument against the existence of a plasmatic membrane, for in artificial precipitation-membranes continuity is maintained, even when growth is extremely rapid.

All the evidence goes to show that the internal masses of plasma, when necessary, can take on in any exposed peripheral layer the function and character of a plasmatic limiting membrane. The latter hence is not, as de Vries and his pupils concluded on insufficient grounds, an organ, which, like the nucleus, can only be derived from its own kind ${ }^{2}$. Every mass of watery fluid present in the protoplasm must be surrounded by a vacuolar membrane to form a larger or smaller vacuole, while masses of plasma which have escaped from the cell, also become clothed by a plasmatic membrane, and form large vacuolar bubbles in water, but not in plasmolysing solutions. From what has just been said above, it follows that a plasmatic membrane must be inmediately formed on the freshly exposed surface as it comes into contact, not with plasma, but with other media, and

1 See Bütschli, Unters. über mikroskop. Schäume, $189^{2}$, p. 150.

${ }^{2}$ Pfeffer, $1890,1 . c .$, p. 224 ; Klebs, Bot. Zeitung, 1 890, P. 550, and 1891 , p. 343 ; Buitschli, 1. c. (Mikr. Schäume), $189^{2}$, p. 146 , and the literature here cited; also Sect. 8 of this book. 
especially water. Nevertheless, the determining causes cannot at present be precisely defined, and it is hardly probable that the plasmatic membrane is simply the direct expression of the physical surface tension, which is necessarily always present. The latter may be, however, of decisive importance in the formation of the membrane by means of the molecular forces exerted by it, while, at the same time, contact with the medium may cause the substances in the peripheral film to be precipitated in an insoluble form, which redissolves when returned to the interior of the plasma. Since a membrane is formed on every isolated fragment of plasma, even when its vital activity is reduced to the lowest ebb, it seems as if the actual exposure of the peripheral film induces the formation of the plasmatic membrane, without involving anything of the nature of a stimulatory process or stimulatory reaction. If this be the case, the actual plasmatic membrane will in general be of such minimal size as to be incapable of measurement, and theoretically, a single or double molecular layer is sufficient for the maintenance of all the diosmotic properties possessed by the plasmatic membrane.

A hyaloplasmic border of measurable thickness can hardly be identical with the plasmatic membrane throughout its whole extent, although the mere existence of such a border, since it indicates that its internal surface has the power of repelling granules, shows that a certain similarity must exist between its characters and those of a plasmatic membrane. The peripheral hyaloplasmic layer, however, as has already been pointed out (Sect. 7), must be of great service to the organism, owing to its power of forming solid and permanent skeletal products.

The medium with which the plasma is in contact will in general exert some influence upon the formation of the plasmatic membrane, if the latter originates in the manner described. It is not known, however, whether the limiting membranes, formed when in contact with water, oil, saline solutions, or air ${ }^{1}$, differ in their osmotic properties, and if so to what extent. These and similar problems are of importance in connexion with the maintenance of the identity of the nucleus, chromatophores ${ }^{2}$, and other plasmatic organs, as well as with regard to plasmatic fragments of a myxomycete, which have been ingested by the plasmodium of another species and yet remain distinct. The latter case leads to the important and still unsolved problem as to why it is that only plasmodia of the same species fuse with one another ${ }^{3}$, a problem which is also of the utmost importance in connexion with sexual and other phenomena.

1 On gas vacuoles, see Klebahn, Flora, 1895, p. 241 (Phycochromaceae); Engelmann, Zool. Anzeiger, 1878 , p. $15^{2}$; Hermann, Handb. f. Physiol., 187 s, Bd. I, p. $34^{8}$ (Protozoa).

${ }^{2}$ Pfeffer, I 890 , l. c., p. $25^{2}$.

${ }^{3}$ Čelakovský, Flora, I 892 ; Errg.-bd., I892, p. 212. 
Although the exact chemical constitution of the plasmatic membrane is not known, there can be no doubt that it is largely composed of proteid substances. This is indicated by the rigor caused by dilute acids, mercuric chloride, iodine, \&c., and so far as any determination is possible, the membrane, when fixed by such treatment, gives the reactions characteristic of certain proteid substances. As the result of the action of very dilute solutions of metallic salts, \&c., the plasmatic membrane becomes more and more permeable, without any interruption of its continuity ensuing ${ }^{1}$, i. e., it undergoes changes, which might be explained as being due to the coagulation or fixation of the proteid substances it contains. It is, of course, possible that other substances may be present as well, but from the characteristics already given it is evident that the diosmotic membrane cannot be a film of oil covering the protoplast as Quincke ${ }^{2}$ was led to suppose by experiments made upon other objects. Such a view is further contradicted by a variety of different physiological experiences, as, for example, by the fact that diosmosis is not dependent upon the solubility of the diosmosing substance in oil.

Historical. Nägeli was the first to call attention to the importance of the peculiar osmotic properties which are possessed by the cell, and more especially the protoplast. Then Traube's discovery of semi-permeable precipitation-membranes ${ }^{3}$ formed the basis for a correct interpretation of osmosis in general ${ }^{4}$, and hence also of the osmotic properties of the cell. The relationships between the various processes involved in the absorption of substances by the plant, including the conditions under which accumulation and translocation take place, and the influences exercised thereon by osmotic pressure, have been investigated by Pfeffer, as previously described ${ }^{5}$. The absorption of certain aniline dyes by living cells enables many points to be more clearly demonstrated and accurately determined ${ }^{6}$, and also renders clear the fact that a functional division of labour may exist in the protoplast in regard to the processes involved in diosmosis. Formerly this possibility was overlooked, the protoplast being regarded as behaving uniformly at all points, but neither the existence of such physiological division of labour, nor the presence

1 Pfeffer, Osmot. Unters., I877, p. I41 ; Plasmahaut u. Vacuolen, I89o, p. 241 ; de Vries, Jahrb. f. wiss. Bot., 1885 , Bd. XVI, pp. 508,529 .

${ }^{2}$ Quincke, Ann. d. Phys. u. Chemie, I888, N. F., Bd. Xxxv, p. 630 ; seq. u. I 894, N. F., Bd. LIII, p. 625. Jy this later work, the matter has not been altered in any way (see Pfeffer, l.c., p. 246), and that a slight solubility in oil should be shown by a single diosmosing substance has no general importance. [E. Orerton (Ueber die allgemeinen osmotischen Eigenschaften d. Zelle, Vierteljahrsschrift d. Naturf.-Ges. in Zürich, XLIV, I899, p. 110) concludes that cholesterin or a cholesterin ether impregnates the plasmatic membrane and mainly determines its diosmotic properties and its permeability.]

${ }^{3}$ [A semi-permeable membrane is one which is penetrated by water or any other fluids which it can imbibe, but not by all substances which may be dissolved in the latter.]

${ }^{4}$ Nägeli, l'flanzenphysiol. Unters., I $5_{55}$, I, pp. I-35. See also Pfeffer, Plasmahaut, \&cc., pp. 242 and 316.

${ }^{5}$ Pfeffer, Landw. Jahrb., I 876 , Bd. v, p. 87 ; Osmot. Unters., I 877 .

${ }^{6}$ Pfeffer, Unters. a. d. Bot. Inst. z. Tübingen, i 886 , Bd. II, p. I 79. A summary, and additional ubservations, in Plasmahaut u. Vacuolen, 1890. 
of a plasmatic membrane, disturb in any way the general principles by which absorption and diosmosis are regulated.

\section{SECTION 19. The Ingestion and Excretion of Solid Bodies.}

In virtue of its physical properties, the protoplast has the power of allowing a solid body to pass through it, without forming any passage through which non-diosmosing substances might enter. The plasmodia of Myxomycetes, and other gymnoplasts exhibiting amoeboid movements, make extensive use of this power, and thus particles of indigo placed near a plasmodium are soon taken in to a considerable extent (Fig. 7). All sorts of substances, including oil-drops and small living organisms, may be ingested, and, indeed, it seems probable that the readiness with which solid bodies are taken in by the plasmodia of Chondrioderma or Aethalium depends indeed solely upon mechanical factors. The solid particles are carried by streaming movements to the interior, and may in part penetrate the vacuoles, while from time to time they are ejected again, so that in clear water a plasmodium may free itself from all foreign particles in from one to two days?'

Similarly, crystals and other solid bodies naturally produced or introduced artificially, may be transferred in dermatoplasts from the protoplasm to the cell-sap, or in the reverse direction, but the cell-wall prevents any escape externally, although a passage through the plasma may be possible. Organisms which bore through the cell-wall can penetrate the protoplasm without difficulty, and other facts show that, were it not for the presence of the cell-wall, solid particles could be taken into

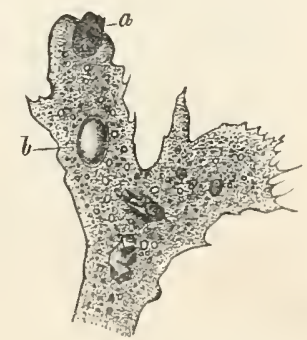

FIG. 7. Portion of a plasmodium of Chondrioderma dif. forme. A foreign body is being ingested at $a$, while internally various ingested particles are present, including an oil-drop, $b$. $(\times 200)$ the cell with comparative ease. Indeed, oil appears to be absorbed in the form of minute droplets (Sect. I6), and the processes, by means of which gymnoplasts ingest their nutritive material in greater or less degree, apparently attain, under certain conditions, considerable importance in dermatoplasts. At the same time, a power of throwing out useless substances or excreta is equally necessary to a gymnoplast, and the mode of nutrition of a Myxomycete is therefore essentially that of an animal. In the higher Protozoa, ingestion and excretion occur at a certain localized region, and ultimately this becomes a well-defined oral aperture.

1 Details by Pfeffer, Aufnahme u. Ausgabe ungelöster Körper, IS9o, p. I50; Čelakorský, Flora, 1892, Erg.-bd., p. 182. The older literature is given here, and also an account of the culture and treatment of Myxomycetes. Of the more recent literature concerning Amocha, the work by Dantec, Ann. d. l'Inst. Pasteur, $1 S_{90}$, iv, p. 756 , and $1 S_{91}, v$, p. I70, may be mentioned. 
In Myxomycetes, amocboid movements suffice to mechanically introduce and expel foreign particles from the substance of the protoplasm. The same is also the case in protoplasts enclosed by a cell-wall, and since, in such cells, the protoplasm is relatively small in amount, all unused solid substances usually collect in the cell-sap. These include calcium oxalate, as well as dead fragments of protoplasm. Hence it follows that there must be a definite reason for other solid particles remaining permanently imbedded in the plasma. This applies not only to actual protoplastic organs, but also to certain algae which live symbiotically in the bodies of many Infusoria, Radiolaria, \&c., but which, when they are ingested by a plasmodium, are always rejected sooner or later. Starch grains and chlorophyll grains, when ingested, meet with the same fate; so that, as soon as starch grains are freed from the chromatoplasts which have produced them, they will almost certainly be transferred by the plasma to the cell-sap. Moreover, there is little doubt that stimulatory influences may come into play, causing certain substances to be ingested with greater avidity than others, as, for example, by means of chemotactic attraction. In such cases, the organism is evidently able to directly select the food material it prefers ${ }^{1}$.

On the other hand, small organisms, by means of their own activity, aided or unaided by an attractive stimulus, are able to penetrate protoplasts and thus to be ingested. This takes place when Bacteria, fungal hyphae, Myxamoebae, Plasmodiophora, \&c., penetrate plant-cells. In these and other cases, such as the process of fertilization by means of a pollen tube, the penetrating body must first make its way through the cell-wall. (See Sect. 65.)

Further research is needed to determine the means by which the necessary intimate approximation is assured, as well as the causes inducing both active penetration by foreign bodies, and their passive ingestion. The mechanism involved may, moreover, be of complicated character, and may in certain cases even involve special stimulatory reactions. We are here dealing with important physiological relationships, of which only a few have received a causal explanation, and these will be mentioned later. We may however state in illustration, that the antherozooid of a fern is attracted to the ovum by the chemical attraction exerted by malic acid derived from the contents of the archegonium, and thus antherozooids may penetrate the archegonia of widely different species; they fuse, however, only with the ova of their own species.

\section{SECtion 20. Translocation from cell to cell.}

We have already seen that the fundamental problems connected with the exchange of substance are to be studied in relation to the mechanism

${ }^{1}$ Examples of such processes are given by de Bary, Pilze, $188_{4}$, p. 481 , $8 \mathrm{c}$. The same phenomena are shown by leucocytes, and these may also pass through membranes, walls of capillaries, \&c. 
of the individual cell. Nevertheless, in tissues various ways and means are employed to attain a complete and adequate distribution of necessary materials. Thus substances are frequently transported over considerable distances through dead tissue elements, or through cell-walls, and when thus transmitted from cell to cell they may reach their goal rapidly, or only after a prolonged journey. Further details of the processes involved and their utility to the plant will be given later (Chap. X), and at the same time the causes inducing and accelerating translocation will be discussed, as well as the restriction of the translocation currents to special paths, as physiological division of labour becomes more pronounced. At this point we are only concerned with the general principles involved in the exchanges between adjacent cells.

When a substance passes out of one protoplast, penetrates the intervening cell-wall, and is absorbed by an adjacent protoplast, an operation takes place whose general principles have already been recognized in connexion with diosmotic exchange. This is also the case when the possibility of such exchange and the production of the necessary conditions are due to interacting influences between neighbouring protoplasts. In addition to this, however, we have to consider how, and to what extent, the plasmatic connexions which usually, and perhaps always, connect the contiguous living cells of a tissue to one another may be utilized in translocation. These living plasmatic connexions ${ }^{1}$ usually pass as fine threads through tiny pores or canals in

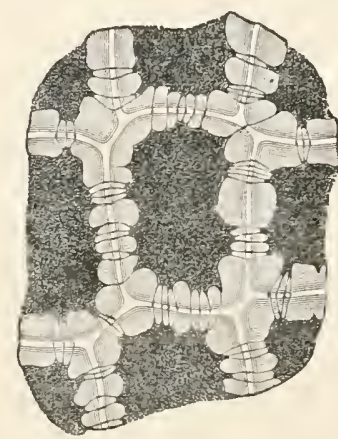

FIG. 8. From the bark of Rhamnus Frangula fixed with I, caused to swell by treatment with $\mathrm{H}_{2} \mathrm{SO}_{4}$ and stained with aniline-blue. This brings the plasmatic connexions plainly to view. $\left(\times 5^{80}\right.$. $)$ the cell-wall, but in some cases, as in sieve tubes, they take the form of coarse and relatively thick strands.

From general physiological considerations the attainment and maintenance of harmonious co-operation throughout the plant by the intercommunication of stimuli renders the existence of living continuity so absolutely necessary, that had it not already been discovered its presence must have been assumed, for in no other way could the observed phenomena have been explained. It is, moreover, possible that the protoplasmic communications may aid in the transfer of substances from cell to cell, and even that in special cases their primary function may be to

${ }^{1}$ Kienitz.Gerloff, Bot. Zeitung, I891, p. I; 1893 , p. $3^{6}$; Zimmermann, Summary in Beihefte z. Bot. Centralbl., IS93, Bd. III, p. 328 ; and the main literature. See also Wahrlich, Bot. Centralbl., I893, Bd. Lv, p. 368, and Czapek, Sitzungsb. d. Wien. Akad., 1 $\varepsilon_{97}$, Bd. Cvi, Abth. I, p. 155 . 
act as translocatory channels for nutritive and plastic materials. The plasmatic threads may also serve for the transmission of living material or of stimulating substances from one part to another, the threads here acting as the channels along which stimulatory influences flow.

In order to attain a rapid power of translocation, these plasmatic threads are not absolutely essential, and they have nothing at all to do with the actual entry of substances from the external world, or with the final exit of excrete metabolic products, and yet both excretion and absorption can take place to an adequate extent. In the germination of $Z c a$ mais, the store of reserve food material is absorbed by the superposed scutellum as rapidly as it becomes soluble. so that in this case relatively rapid translocation is possible without the aid of any interprotoplasmic connexion. It is therefore clear that substances may be directly translocated in internal tissues from cell to cell through the intervening cellulosewalls as rapidly as the limits of safety and economical transport will allow. When such repeated transference from cell to cell takes place through separating walls, the length of the series through which the current is passing has no influence upon the rapidity with which substances are transferred from any cell to those immediately adjacent to it, for each cell has its own special potential energy of absorption, determined by its specific powers of passive secretion. (See Sects. 22 and 108).

Cells can absorb a variety of substances, including colloids, while oils may pass unaltered through the cell-wall and plasma membrane (Sects. 16-19). Other examples of rapid absorption and accumulation of substances have already been given (Sects. I6 and 22). Aniline dyes also may be observed to be transferred with normal rapidity from cell to cell without the aid of interprotoplasmic connexions.

Diosmotic transmission is not only sufficient for all necessary transference, but is actually employed in translocation, although it is possible that the plasmatic connexions may play an important accessory part. The latter is certainly the case in sieve tubes (Sect. 106). though it does not necessarily follow that the finer plasmatic connexions are of equal importance. In these, in spite of their minute sectional area, transference is possible by the aid of pressure or as a vital process, and, indeed, it is even possible for living plasmatic masses to penetrate through cell-walls in which no pre-existent openings are present (Sect. I9). It can hardly be doubted that for particular purposes, and to attain special ends, plasmatic elements or masses may pass from one cell to another. There are, however, no observations in favour of the view that such transference is of importance in the translocation of nutritive or excretory substances from one cell to another. Were the latter the case, the fineness of the threads would render necessary extremely active streaming currents directed both towards the interior and the exterior of the cell; and to 
such, as well as to any passive diffusion through their substance, the relatively trifling total sectional areas of the threads would interpose a marked hindrance ${ }^{?}$.

Certain minor disadvantages are often the inevitable accompaniment of any special modification for the performance of a particular function. It might in some cases be essential that particular substances should be directly transferred from the plasma of one protoplast to that of another, and for this, the existence of plasmatic connexions would be of great value, although their minute diameter necessitates the expenditure of a large amount of energy to secure such transference. Thus in rhizoids, algal filaments, and indeed in all cases in which the outer walls of a chain of cells are readily permeable, there is an ever-present danger that substances which can diosmose through the cell-wall may pass out and be lost in the surrounding water or damp soil. The same risk is involved even when the protoplasts only allow substances to pass through that portion of the cell-wall where the two cells come into contact. In these and in similar cases we require to determine how, and by what means, translocation is carried on without loss. No such danger usually exists in the interior of tissues, where, indeed, substances appear to wander to a large extent externally to the protoplasts, but even here a restriction to special paths is possible, as will be shown later (Sect. 108).

Our knowledge of the mechanism of translocation, and especially of the importance of the plasmatic connexions, is still very incomplete. With regard to the latter, no decisive experiments have as yet been performed, although according to certain authors ${ }^{2}$, translocation takes place, mainly, or entirely, through the connecting plasmatic threads. Such views, however, cannot claim to be based upon a careful consideration of all the facts observed. Since the thinnest threads will suffice to maintain living continuity, the coarse thick connexions observed in the case of the sieve tubes may indicate that the plasmatic strands are here mainly employed in the transference of substances from one segment of the sieve tube to another.

With morphological relationships we are not concerned, nor is it necessary to discuss whether the threads are of secondary origin or are present from the commencement, persisting during cell-division between the nodules of cellulose which unite to form the cell-plate or dividing wall ${ }^{3}$. That a secondary formation of connecting threads may be possible is indicated by the fact that in certain cases the living protoplasm has been shown to be capable of boring its way through cell-walls.

1 Pfeffer, Studien z. Energetik, I 892, p. 272.

2 'Thus Kienitz-Gerloff (see Zimmermann, 1.c., p. 331). Kienitz-Gerloff gives an account of the views held with regard to the transmission of stimuli by the plasmatic threads, 1. c., I893, p. 49. Cf. Pfeffer, Ber. d. Sächs. Ges. d. W'iss., 1896, p. 505 .

s Zimmermann, 1.c., p. 330. 


\section{SECTION 21. Diosmotic Properties of Cuticle and Cork.}

While it is necessary that wherever rapid transference is of importance, permeable cell-walls must be retained, it is, on the other hand, in the highest degree essential that plants should be able to produce on their exterior cell-walls, which permit water and other substances to pass through them only with difficulty, or even not at all. It is by such means, as illustrated by the formation of cuticle and cork, that exchanges with the external world, such as those involved in transpiration and absorption of water, are limited both in locality and extent to actual requirements; while in case of need, internal tissues may be isolated by special impermeable investing layers. Details of these adaptations will be given in connexion with the functions to which they are subservient (Sects. 30, 38 ), but their importance depends mainly upon the diosmotic properties of the cork and cuticle, of which a short account must be given here. It is clear that, as the cell-wall becomes more and more impermeable, the existence of the enclosed protoplast will be more and more difficult, and will ultimately become impossible. Hence, as is well known, cork-cells are always dead, if the walls are completely suberized; and those tissues die, which are isolated by the development of a cork layer beneath them. Epidermal cells, however, can remain alive, because communication with the tissue beneath remains undisturbed; although by the cuticularization of the outer wall the transpiration and absorption of the protoplasts are markedly diminished.

The physical properties of cork are indicated by its technical uses, and it is these same properties which give cork its biological importance. The function of the cuticle covering aerial parts is similar in character. When well developed it opposes a marked hindrance to the passage of water or water vapour, but all grades of transition may be shown to the readily permeable cuticle of submerged aquatic plants, which, as is well known, rapidly wither when exposed to the air. The permeability of cork also varies, as is shown by the widely different powers of imbibition possessed by various kinds of cork ${ }^{i}$.

That cork is not completely impermeable to water is at once shown by the fact that it swells somewhat in water and shrinks again on drying. Indeed in every case the impermeability to water gradually increases as development progresses, until under favourable conditions the cuticle may be so thick as to allow only the merest trace of water to pass through it; while cork may form a series of layers, through which practically no water is either exhaled or absorbed (Sects. 30, 38).

Tissue paper acquires similar properties when it is impregnated more or less thoroughly with wax, fat, or resinous materials, and leaves covered 
with a film of excreted wax cannot be wetted, nor so long as this is the case will any water be absorbed by them.

The increased impermeability of the cuticle acquired under conditions in which transpiration is very active, and the formation and regeneration of the cuticle on surfaces exposed externally, are evident signs of its regulatory and adaptive development ${ }^{\text {? }}$. In no less degree cork is formed in direct correlation with the developmental progress and needs of the aerial organs, \&c., and it is developed over areas exposed either in the normal progress of events (viz. leaf-fall) or by accidental injury .

As the permeability to water decreases so also does the permeability to dissolved substances diminish, so that the cuticle may be almost impermeable both to water and to dissolved salts. At the same time it is possible that the permeability to water may be diminished more markedly than to other substances. Thus films of india rubber or of waxed paper allow alcohol, carbon dioxide, and oxygen to pass slowly through, but are almost completely impermeable to water. Similarly a cuticle, through which feeble transpiration is possible, may not allow the traces of $\mathrm{CO}_{2}$ present in the air to diffuse through with sufficient rapidity to permit a formation of starch in the assimilating cells immediately beneath (Sect. 57), but may permit sufficiently rapid gaseous exchanges for the continuance of respiration (Sects. 29, 30), for by means of the bacterium method it may be readily observed that oxygen passes with comparative ease through the thin cuticle of an assimilating epidermal hair of Cucurbita ${ }^{2}$. Changes of permeability may be produced by impregnation with other substances ${ }^{3}$ or by alterations in the quality of the membrane (see Sect. I4). Thus by the death of the non-suberized layers of the bark, an increased protection against transpiration is afforded, while in producing that diminution of permeability which old wood fibres undergo, the formation of gummy substances appears to be of considerable importance. It is, however, impossible at present to determine precisely why functionally active tracheae only allow air to diffuse through them with difficulty (Sect. 32).

The rate of diffusion is inversely proportional to the thickness of the membrane, and hence the thick gelatinous or slimy coverings of many algae and certain roots may exercise a distinct protective influence, although they allow some substances to pass through them with comparative ease, as is shown by the readiness with which plasmolysis may be induced ${ }^{4}$. Enclosing membranes of this character interpose a marked hindrance to transpiration as they dry. Gelatinous membranes have a special tendency to retain

1 Kohl, Transpiration, Iss6, p. I13; Tittmann, Jahrb. f. wiss. Bot., 1896, Bd. xxx, p. II6. See Sect. 38 .

[Ewart, Journ. Linn. Soc. Bot., Vol. xxxi, p. 366.]

3 On the influence of silicification, see Kohl, Kalksalze u. Kieselsäure i. d. Pflanze, 1889, p. 228.

4 See Schilling, Flora, I 894 , P. 35 I; Walliczek, Jahrb. f. wiss. Bot., I893, Bd. Xxv, p. 273. 
certain substances, and it is possible that their permeability may be markedly affected by a formation of precipitation membranes, when salts of calcium or iron come into contact with them, and form definite compounds. It is in virtue of some such action, that when a bladder containing a solution of ferric chloride is immersed in hard tap-water, none of the iron salt diffuses through the membrane.

The possession of a power of swelling, or of allowing transpiration to proceed, shows that no cork or cuticle is absolutely impermeable to water. Thus when $\mathrm{KNO}_{3}$ or $\mathrm{K}_{2} \mathrm{SO}_{4}$ is placed upon a moistened and wetted area of the thin cork layer covering potatoes, or of the thick cuticle covering the leaves of Ilex, Prunus Laurocerasus, \&c., a little water is very slowly absorbed by the salt from the leaf or potato ${ }^{1}$. Since the energy exerted by a saturated solution of $\mathrm{KNO}_{3}$ in drawing water through a cuticular or corky membrane corresponds to a pressure of about seventy atmospheres, it is easy to understand why various authors have been unable to obtain any perceptible filtration of water through thin lamellae of cork by the aid of an air-pump ?

The osmotic passage of salts may be indicated by the production of plasmolysis and by observing the rapidity with which the salts pass, while from the amount of plasmolysis produced in a given time, it may be shown that the cuticle of exposed aerial parts is far less readily permeable than that of submerged parts. Experiments with separated multicellular hairs show that the salt penetrates with much greater rapidity through the uncuticularized transverse walls. The same thing is indicated when the change of colouration is followed, which dilute ammonia produces in the coloured cell-sap of the staminal hairs of Tradescantia, or in that of the leaf tentacles of Drosera ${ }^{3}$. It is not necessary to mention in detail different researches, in which by other methods the passage of certain salts through the cuticle of leaves, $\& c$. has been demonstrated ${ }^{*}$. The absorption of water, which causes certain pollen grains to burst, will be referred to later (Sect. 22) ${ }^{5}$, and no detailed description will be given here of the formation, distribution, or special peculiarities of the cork, cuticle, or cuticular layers of different plants ${ }^{6}$.

${ }^{1}$ Buisgen (Honigthau, IS91, p. 26), in the objections raised by him, has apparently failed to realize the meaning of the wetting, or the enormous energy which osmotic forces can exert. See Pfeffer, Studien z. Energetik, 1892 , p. 266. On wetting, Lehmann, Mol.-physik, ISS9, Bd. Ir, p. 106, \&c.

${ }^{2}$ Hofmeister, Pflanzenzelle, 1867 , p. ${ }_{3} 8$; Eder, Sitzungsb. d. Wien. Akad., 1873, Bd. Lxxı1, Abth. I, p. 258 ; Zacharias, Bot. Zeit ung, is 89 , p. 644.

${ }^{3}$ Pfeffer, Osmot. Unters., I 877 , p. I 99 . In connexion with the absorption of aniline dyes, see Pfeffer, Unters. a. d. Bot. Inst. z. Tübingen, I 886, Bd. I1, p. 201.

' Boussingault, Agron., Chim. agr. et Physiol., i 878 , Vol. vi, p. $3^{6} 4$; Wille, Cohn's Beiträge, I $88_{7}$, Bd. XIv, p. $3^{1} 4$; Bürgerstein, Übersicht über d. Unters. u. d. Wasseraufnahme d. Blätter, I 891 (Sep.-abdr. a. d. Jahresb. d. Leopoldstädter Gymn. in Wien).

${ }^{3}$ On the protective sheaths of vascular tissues, \&c., see Schwendener, Die Schutz-Scheiden u. ihre Verstärkungen, IS82, p. 6.

${ }^{6}$ See de Bary, Vergleichende Anat., 1877 , pp. 77, 114 ; Zimmermann, Pflanzenzelle, I887, p. I17; Mikrotechnik, I892, p. 146 ; Tschirch, Pflanzenanatomie, 1889 , p. 117; E. Weiss, Beiträge z. Kennt. d. Korkbildung, 1890 (Denkschr. d. Regensburger Bot. Ges.). On the duration of the 
According to the researches mentioned, the glycerine compounds of stearic, palmitic, phloric and suberic acids are the most important impregnating substances. Some of these compounds are insoluble in chloroform, ether, \&c., but are saponified by potash '. The double refraction of the cuticle is due to the presence of these substances (Sect. I3), which have, it may be noticed, different melting-points.

\section{Section 22. Quantitative Selective Power.}

Every plant and every cell has definite specific needs, to satisfy which certain substances are absorbed in large amount, whilc of others little or no use is made. Thus most plants are unable to directly utilize atmospheric nitrogen, nor is the carbonic acid gas of the air of any value to those without chlorophyll, but all aerobic plants consume oxygen in large quantities. In the same way the substances presented to a plant in a nutrient solution are absorbed in direct proportion to the amounts used and required in metabolism, so that a substance present in abundance may not be diminished in quantity, while the last traces may be absorbed of another substance present in small amount. Both higher and lower plants possess this selective power, which is dependent upon and is regulated in a specific manner by the vital activity of the plant itself, independently of whether the substances absorbed are essential or non-essential to its well-being.

It is quite immaterial whether the selected substances are cmployed as building material or stored up as food reserves, or whether they are returned to the external medium in changed form as waste metabolic products for which the plant has no further use. Thus in a confined space a plant will absorb the last traces of oxygen present. although the whole of this is finally exhaled again in the form of carbonic acid; while of the sugars which a fungus or yeast-cell extracts from a culture fluid, large quantities are returned to the surrounding media in the form of carbonic acid gas, oxalic acid, and alcohol.

This selective power is made manifest by changes taking place in the composition of the surrounding medium, as well as by the retention and accumulation of particular substances within the plant. In the latter case, the results produced by selective action are well shown when the ash constituents accumulated by the plant are contrastcd in quality and amount with the mineral constituents present in the nutrient solution with which the plant is supplied. It can then be seen that from ordinary tap-water, containing mere traces of salts, plants may collect large quantities of nonvolatilizable mineral constituents, and moreover that these constituents are

life of cork cells, see Koppen, Das Verhalten d. Rinde unserer Laubbäume, 1889, p. 480 (Nova Acta d. Leopoldin. Akad.).

${ }^{1}$ Gilson, Bot. Centralbl., I891, Bd. Xlv, p. I I ; Fluickiger, ibid., I892, Bd. L, p. 90 ; Wisselingh, ibid., 1895 , Bd. Lxir, p. 234 . 
absorbed in proportions altogether different from those obtaining in the water in question. Similarly the large quantities of organic material, which green plants produce, form an indication of the extent to which plants manage to ultimately accumulate relatively enormous quantities of a substance presented in excessive dilution, as is the case with the carbonic acid gas of the air.

This selective and accumulative power may be very clearly demonstrated by means of certain aniline dyes (see Sect. I6), because the colouration enables the process of absorption to be followed, and the region where accumulation takes place to be determined. As has already been described, methyl-blue penetrates all plant-cells, but accumulates by passive secretion only when it combines with substances present in the cell-sap to form either a coloured precipitate, or a non-exosmosing, though soluble and coloured compound. The latter takes place in the roots of Lemna minor, for in the epidermal cells, the dye may reach a concentration of one per cent. in from one to three days, although the external fluid contained only $0.000 \mathrm{I}$ per cent. of methyl-blue. Hence, in this time as much dye is carried to the cell-sap and retained there, as is present in one thousand times the quantity of external fluid ${ }^{1}$.

Even when the water contains in $100,000,000$ parts only one part of methyl-blue, the cells of Lemna accumulate the pigment substance, if only sufficient quantities of the solution are employed, or if the percentage of the dye present is kept constant. It is true that each individual cell absorbs the dye in trifling absolute amount, for a single cell weighs less than $0.001 \mathrm{mgr}$, but a mass of cells weighing I mgr. may passively secrete as much of the dye as is present in a litre of the dilute solution, so that Ioo litres of the latter contain as much dye as $100 \mathrm{mgr}$. weight of cells can absorb and accumulate.

In a similar manner plants can obtain the potassium, phosphoric acid, and other substances, which they require, from extremely dilute solutions, provided either that fresh fluid is frequently supplied, or that the percentage of salts present is continually being readjusted and kept constant. These substances and the aniline dyes mentioned, as well as other bodies, may be completely removed ${ }^{2}$ from a limited amount of a dilute solution. In the same way living plants enclosed in a confined space may absorb every trace of oxygen from the air surrounding them.

This power of accumulating particular substances is shown not only by plants, but in all cases in which the absorbed substances are fixed as rapidly as they are taken in. Thus potash withdraws carbonic acid from the air

1 Pfeffer, Unters. a. d. Bot. Inst. z. Tübingen, i $\$ 86$, Bd. II, p. I $9 \$$.

2 For examples see Versuchsstat., I865, Bd. viı, p. 93, and Beitr. d. Sächs. Ges. d. Wiss., Leipzig, I 875 , i, p. 76 ; Nobbe und Siegert, Versuchsstat., I $86_{4}$, Bd. vi, p. 42. Fungi may devour all the sugar present in a nutrient solution. On the protection of certain substances, when others preferable to the plant are present, see Sect. 67 . 
even more rapidly than does an equal surface area of a leaf, assimilating in sunlight; while in a drop of tannic acid, surrounded by a precipitation membranc formed by the solution of gelatine in which the drop is floating, methyl-blue is absorbed and accumulated, for preciscly the same reason as in a living cell containing tannic acid in solution in the cell-sap.

In order to demonstrate the mode of accumulation of substances in a cell, the precipitation of copper by zinc may be employed. In a glass cylinder, the ends of which are covered by parchment or linen, a roll of zinc is inclosed, and after being filled with water the whole is immersed in a very dilute solution of sulphate of copper (Fig. 6). The appearance of zinc sulphate in the outer fluid shows that one of the decomposition productions of sulphuric acid diffuses outwardly.

On the method of using aniline dye to demonstrate passive secretion, see p. 94 . In a watery solution of methyl violet of the strength I : I $00,000,000$, living cells soon become distinctly coloured, and before long are injuriously affected. Hence, it is easy to understand why still more poisonous metallic salts may exercise a fatal effect even when much more dilute solutions are employed. Nägeli ${ }^{1}$ found the poisonous character of ordinary distilled water to be due to its containing a trace of copper or other metals, and proved that one part of copper in $1,000,000,000$ parts of water sufficed to kill a Spirogyra filament. With a limited amount of the watery solution, the poisonous effect decreases as the number of plants immersed increases, for as the total quantity of the metallic salt is now distributed over a larger

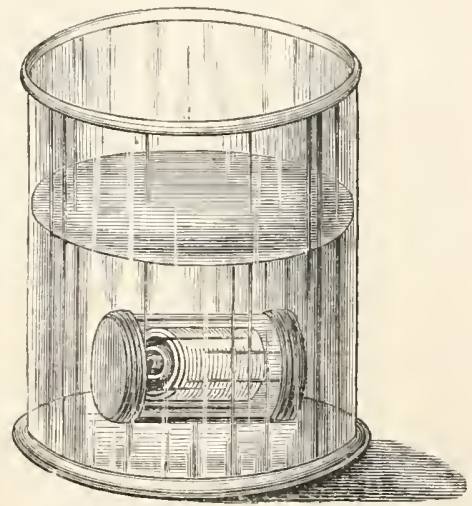

FIG. 9. number of cells, the lethal limit may no longer be reached in any single cell. If the poisonous effect produced by such dilute solutions were independent of the duration of the period of absorption (which is, however, not the case), and were dependent only upon the final accumulation of a certain quantity of the poisonous substance, then by using sufficiently large quantities of fluid, any solution of a poisonous substance, however mucl diluted, must ultimately prove fatal.

The cleansing and purifying power of earth, \&c. is the natural consequence of its absorptive properties, properties which play a very important part in Nature. Similarly, it is owing to the absorbent powers of glass that bottles or flasks which have once contained solutions of copper salts or aniline dyes will, even after repeated washing with water, gradually convert the pure water with which they may be filled into a weak and poisonous solution of the substances in question. Plants

1 Nägeli, Oligodynamische Erscheinungen in lebenden Zellen, I $S_{93}$ (I)enkschr. d. Sihweiz. Naturf.-Ges., Bd. xxxili). On the differences in the manner of death, according to whether the poisonous action is rapid or slow, see Israel, Archiv f. Path. Anat., 1 S97, Bd. CXLv11, p. 293. 
submerged in distilled water in bottles which once contained methyl-blue, or violet, will become distinctly coloured, and this affords a useful illustration of the amount of care which must be taken in physiological experiments to insure absolute purity and cleanliness. Owing to this pronounced power of selective absorption and passive secretion, it becomes increasingly difficult to insure the entire absenee of all iron, or of potassium, or phosphoric acid, \&c., when a small number of plants are grown upon a large quantity of culture fluid.

The special diosmotic properties of the plant and of the substances absorbed, together with the changes which the penetrating substances may undergo internally or externally to the cell, suffice to explain the attainment of this physiological selective capacity. The diosmotic nature of a substance determines in the first instance whether it can penetrate the plant, either reaching the interior of the cell, or not passing beyond the cell-wall, and for the outward passage of a substance, diosmotic relationships are of equal importance. In either case, if the substance can pass from cell to cell, it will continue to be absorbed (or evolved), until a condition of osmotic equilibrium is reached. This is never attained if the substance is continually being altered or removed; and in this manner the most minute traces of a salt present in a dilute solution may be absorbed.

Within the plant, accumulation of a substance is due to its undergoing metabolic alteration as rapidly as it is absorbed, while the substances which the plant rejects are removed by the surrounding medium. The evolution of carbonic acid gas formed in a plant or of alcohol produced in a yeast-cell continues as long as the accumulation of these bodies in the surrounding air or fluid is prevented. "The excrete diosmosing substances formed in a tissue-cell move away from a certain centre of repulsion towards the excreting surface or region, translocation being due to diffusion, imbibition, and diosmosis. Translocation of substances produced within the plant from one region to another is due primarily to the fact that at the latter point the diosmosing substances are being altered or used in metabolism. The changes which the translocated substances undergo may be of far-reaching nature or of only trifling degree, for it is sufficient that an insoluble combination should be precipitated either inside or outside the cell, or again that a soluble but non-diosmosing compound should be formed in the cell-sap. Even when no accumulation takes place, a plant or even a single cell may serve as an attractive centre for a diosmosing substance, for if the latter is converted into a different, but still diosmotic compound, which in turn diosmoses from the cell, a continual current will be thus maintained, the one substance passing into the cell. the other away from it. Under appropriate conditions and in certain cases, of which examples have already been 
given, only a portion of the product may diosmose from the cell, while the rest is retained.

That the formation of insoluble compounds is frequently the cause inducing an accumulation of particular substances, is well illustrated by the formation and growth of cell-walls, starch grains, crystals of calcium oxalate, \&c. The dissolved substances (sugars, salts of organic acids, potassium nitrate, \&c.) which are contained in the cell and which do not diosmose from it, must be present in a non-diosmosing form, and must have passed through the plasmatic membrane as diosmosing compounds. The fact that the reactions for nitrates or reducing-sugar may be obtained on testing dead cells, does not necessarily indicate that these substances are actually present in this form in the living cell (Sect. 16), for the nondiosmosing compounds may be stable only under the conditions presented during its life ${ }^{1}$.

With regard to non-essential substances, which are occasionally absorbed and secreted in large amount, the same general principles hold good. In the case of the aniline dyes, it has been definitely proved that their passive secretion by the living cell is due to the formation of non-diosmosing compounds as absorption continues. This must also be the case with the soluble sodium compounds present in the plant. whereas silica, which is often very abundant, is commonly deposited in an insoluble form impregnating the cell-wall.

All accumulation or excretion, dependent upon diosmotic exchange, is governed by the above laws, independently of the precise manner in which diosmosis is temporarily or permanently produced or rendered possible. The passage through the cell-membranes is due to the action of the same molecular forces which operate in the production of all diosmotic exchange. This is still the case, when the accumulation of a substance within a cell is duc to the fact that the plasmatic membrane allows particular molecules colliding against it to pass through only in one direction, so that they enter the cell but cannot escape from it (Sect. I6).

An accumulation might also take place by vitalistic means, either by the aid of plasmatic movements, or by some other manifestation of energy directed to this end, the molecules of the given substance being forcibly transferred through the protoplasmic substance and membranes (Sect. 16). It is true that there is at present no evidence pointing to the existence of any such power, or indicating that the plasmatic membrane may be permeable to certain substances in one direction only. Nevertheless, it seems quite rational to suppose that among its varied powers, the living organism is able in particular cases to employ special means 
to attain a given purpose, and by changes or processes of some such character as described above, to cause substances to accumulate in isolated cells, or to induce translocation across isotonically osmotic tissue aggregates.

In all cases, osmotic exchanges are correlated and regulated by the vital activity of the organism. Both the formation of the plasmatic membranes, as well as any modification of their diosmotic properties which may arise later, are the work of the living organism, which also initiates those intra- or extra-cellular changes to which the continuance of exosmosis or endosmosis is to be ascribed, and similarly the substances which render the passive secretion of aniline dyes or other substances possible are products of the metabolism of the absorbent organism.

By the employment of these means singly or in combination, the plant is enabled to fulfil all demands made upon it. We shall frequently have occasion to point out, in dealing with the phenomena of translocation and metabolism, how the processes involved are connected with and regulated by the osmotic properties and powers of passive secretion, \&c. possessed by the regions or tissues concerned. The intimate correlation existing throughout the entire plant is such as to warrant the assumption that the osmotic properties utilized by the organism may be influenced and modified in the most various ways by the operation of external agencies upon the plant, as well as by ontogenetic development.

The power of the cell to retain certain substances and reject others is of fundamental importance. Unless the final products of metabolism $\left(\mathrm{CO}_{2}\right.$, alcohol, \&c.) are continually removed, the vital activity of the plant soon ceases. Moreover, by continued diosmotic removal of a product, a reaction, which is at first trifling or imperceptible in amount, may frequently, owing to the influence of mass in chemical reactions, be continued to completion.

This again can be demonstrated by means of the accumulation of methylblue in a cell of Azolla or Limna, for the stain is permanently retained while the cell remains in pure water, but if the plant is placed in a large quantity of a 0.01 per cent. solution of citric acid, the colouring substance is gradually withdrawn from the cell and in one to a few days has entirely disappeared '. On penetration, the acid decomposes a fraction of the tannate of methylblue, and could do no more were it not that the citric acid salt produced is continually being diosmotically removed by the comparatively infinite volume of water outside. As this process continues, the complete removal of the pigment substance is finally assured, while the tannin remains behind in the cell, which is capable therefore of again accumulating

1 Ifeffer, Unters. a. d. Bot. Inst. z. Tübingen, 1886 , Bd. II, p. 286. The best material to use is Lenna minor. 
methyl-blue. Moreover, the plant itself may produce an acid and remove the dye, as is actually the case in Spirogyra, while any external agencies which induce a formation of acid will also lead to an excretion of the absorbed pigment substance.

In similar circumstances a weaker acid may completely decompose the salt of a stronger acid, provided the partial reaction is rendered progressive by the continued removal of one of the products of the reaction, by diosmotic or other means. This is in complete accordance with chemical laws, and reactions of this character play a most important part in metabolic processes and in the regulation of metabolic activity ! Since actions of this character may be combined with other factors or form part of a stimulatory chain, they may be utilized by the organism in the most varied manner to attain very different ends. Moreover, the living cell is a complex of organs or separate laboratories, so to speak, in which special powers and structural peculiarities are inherent, while the minute size of the whole and of the parts is of great importance for the continuance of a reaction, since it induces and favours both exchange in general and the removal of any given product in particular (Sects. I 2, 93). Bearing in mind the extremely large surface area of a bacterium or yeast-cell relatively to its bulk, no especially active diosmosis is necessary to permit of the marked exchanges which take place during fermentative activity (Sect. 102). The activity of diosmotic exchange may however be influenced by physical factors to a considerable extent. Thus the greater the difference in concentration between the fluids separated by the diosmotic membrane, the more rapid imbibition and diosmosis will be, for it is by this difference of potential that diffusion and diosmosis are induced and regulated.

In order to allow rapid diosmotic exchange it is therefore of great importance that the substance after passing through the membrane shall either be carried further into the interior of the cell during endosmosis, or removed by the surrounding fluid during exosmosis. This is more rapidly attained by moving currents than by unaided diffusion, for the latter does not suffice to satisfy the requirements of the cell when food-materials must be extracted from a large quantity of air or from a dilute solution. Common salt is a substance capable of rapid diffusion, but by diffusion alone it requires a period of 319 days to transfer a milligramme of salt from a 10 per cent. solution through a distance of $1 \mathrm{~mm}$. To attain the same result in the case of egg-albumin, a period of about

1 Pfeffer, Osmot. Unters., 1877, p. 163 ; Unters. a. d. Bot. Inst. z. Tübingen, 1888, p. 293 ; Zur Kenntniss d. Oxydationsvorgänge, $1889, p_{0}{ }_{4} 63$ (cf. Sect. 93). For chemistry see Ostwald, Grundriss d. allgem. Chemie, 1890 , 2. Aufl., p. 316 , and Loth. Meyer, Die mod. Theorien d. Chemie, I $88_{4}$, 5. Aufl, p. 482. Here a comparison between the combining avidities of different acids is given (pp. 508,517 ). 
fourteen years would be necessary ${ }^{1}$. Hence the accumulation and passive secretion of methyl-blue would be extremely slow if the external fluid were completely at rest. As a matter of fact, however, the currents set up by changes of temperature, or by mechanical vibrations, are under normal conditions sufficient to provide a continual supply of fluid without any great expenditure of energy on the part of the plant being necessary. Both in air and water (including the water of the soil), the operation of a variety of natural causes ensures a continual and complete adnixture of the whole, and continually adjusts differences of composition between adjacent tracts of air or water. Without this continuous readjustment, green leaves would be unable to withdraw carbonic acid from the surrounding ocean of air with sufficient rapidity, nor would the roots obtain a sufficiency of phosphates or nitrates from the pure spring water in which they may be submerged.

Differences of temperature, together with automatic or induced plasmatic movements, suffice to procure continuous admixture within the cell, and the water currents induced by transpiration are of the utmost importance in plants which grow above ground. These water currents convey water and dissolved substances over long distances, and even to the summit of a tree, mainly through dead tissue-elements (Chap. X). Currents passing in a particular direction hasten the transference of substances from cell to cell, but nevertheless cannot compel any non-diosmosing material to penetrate the protoplasm.

In the above sense all movements are of importance. The streaming of plasma is a directly visible movement, by which conspicuous admixture and transference is possible within the cell. Indeed in some cases it may be essential for the maintenance of sufficiently rapid exchange, although in general plasma-streaming is not absolutely essential, for in many plants it is not perceptibly active, even along the translocatory channels ${ }^{2}$. Other

1 Stephan, Sitzungsb. d. Wien. Akad., I879, Bd. Lxxix, Abth. 2, p. 214. See also Ostwald, Lehrb. d. allgem. Chemie, I 89 I, 2nd ed., Vol. I, p. 697, and Winkelmann, Handb. d. Physik, I $\$_{91}$, Bd. 1, p. 604. On connected physiological questions, see Pfeffer, Energetik, I892, p. 268. Since diffusion is as rapid in soft gelatine as in water, for purposes of demonstration glass cylinders may be partly filled with gelatine, and a colourcd solution, such as indigo, poured over it, the diffusion of the latter being directly visible and readily observed. If phenolphtalein and sodium carbonate (or hydrochloric acid) be added to the gelatine, the diffusion of hydrochloric acid (or sodium carbonate) may be followed (Ostwald, l.c., p. 68). Agar and gelatinous citric acid may be used for the same purpose. On the slow diffusion of certain aniline dyes, see Pfeffer, 1.c., I 886, Bd. II, p. 302.

2 The general importance of all mechanical admixing movement was first brought into promi. nence by Pfeffer (Unters. a. d. Bot. Inst. 2. Tübingen, I886, Bd. II, p. 3I4, and Energetik, p. 270\%. De Vries regarded plasma-streaming as a necessary factor in translocation (Bot. Zeitung, I 885, P I ). In many cases, however, no marked streaming movements are normally perceptible, though as the result of injury, \&c., streaming may commence (Hauptfleisch, Jahrb. f. wiss. Bot., 1892, Bd. xxiv, p. 173). On the absence of streaming in the sieve tubes, see Strasburger, Bau u. Verrichtung d. Leitungsbahnen, 1891 , p. 363 . Protoplasmic connexions have been dealt with in Sect. 20. See also Czapek, Sitzungsb. d. Wien. Akad., I897, Abth. I, Bd. Cvi, p. 155. 
factors which are always at work causing mechanical admixture inside the cell are in themselves sufficiently active to convey a penetrating particle from one end of the cell to the other with adequate rapidity, and indeed as a general rule transference within the cell involves no marked delay. It is moreover important to note, that in the passage along translocation tissues (composed of elongated cells), the number of walls and total thickness of the cellulose membranes to be crossed is relatively small. The passage through the cell-wall, when unassisted by water currents passing in one direction, is markedly assisted by mechanical mixing movements both inside and outside the cell.

Historical. Mülder ${ }^{1}$ was probably the first to explain the selective power as being due to the co-operation of diosmosis and chemical metamorphosis. A complete account, of which the general features still hold good, was first given by Schulz-Fleeth ${ }^{2}$. Greater accuracy was only attained as the diosmotic properties of the cell began to be understood. In connexion with nutritive processes, Pfeffer developed the principles governing the selective and excretory powers, in the works already quoted. That plants absorb substances in other proportions than those in which they may be present in the nutrient fluid presented to the plant was, it is true, already made known by de Saussure ${ }^{3}$ and others. Considering the standpoint of natural science at that time, it is not surprising that the controlling factors should have been incorrectly interpreted, and thus in older writings the erroneous statement is found, that currents of water are necessary to introduce the substances which the plant accumulates ${ }^{4}$.

Selection of the ash constituents. Recognition of the accumulation of ash constituents in the plant, and of the unequal proportions present in plants growing in the same soil, first called attention to the selective capacities of plants. An extended series of examples is afforded by numerous ash analyses ${ }^{5}$, while instances of changes of composition induced by plants in nutrient solutions will be found in the literature of water-culture given later (Sect. 73 '.

As an example, the following analyses by Goedechens, of algae obtained from the west coast of Scotland, are given ${ }^{6}$. The total ash is given in percentage of dry substance, the single constituents in percentage of the ash itself.

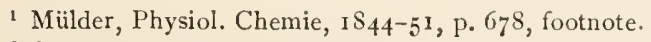

${ }^{2}$ Schulz-Fleeth, Der rationelle Ackerbau, I $3_{3}^{6}$, p. I 24 ; Rochleder, Chemie u. Physiol. d. Pflanzen, I $85^{8}$, p. I 37 , also gives a correct account.

3 Saussure, Rech. chim., I 804 , p. 24 S; Trinchinetti, Bot. Zeitung, I845, p. I I 1.

- Thus Woodward, Phil. Trans., 1699, Vol. xxI, pp. 221. 253; Senebier, Physiol. Veget., I 800 , Vol. III, p. 28 ; de Candolle, Pflanzenphysiol., translated into German by Roper, I833, Vol. I, p. 62.

3 E. Wolff, Aschenanal. v. landw. Producten, pt. I, I $\$ 7$ I ; pt. 2, I 88 . Numerous analyses are given by Liebig, Die Chemie in Anwend. auf Agric. u. Physiol., 1876, 9. Aufl., p. 522; J. König, Chemie d. menschl. Nahr.- u. Genussmittel, I 889,3 ed.

- Goedechens, Ann. d. Chemie u. Pharmacie. See Wolff, I. c., I 87 I, p. 130. 


\begin{tabular}{|c|c|c|c|c|}
\hline & $\begin{array}{l}\text { Fucus } \\
\text { vesiculosus. }\end{array}$ & $\begin{array}{l}\text { Ascophylllum } \\
\text { nodosum. }\end{array}$ & $\begin{array}{l}\text { lincus } \\
\text { serralus. }\end{array}$ & $\begin{array}{c}\text { Laminaria } \\
\text { digitata. }\end{array}$ \\
\hline Total Ash per cent. & $1.3 \cdot 89$ & 14.51 & 13.89 & 18.64 \\
\hline $\mathrm{K}, \mathrm{O}$ & 15.23 & 10.07 & 4.51 & 22.40 \\
\hline $\mathrm{Na}_{2} \mathrm{O}$ & 24.54 & 26.59 & $3 \mathrm{I} \cdot 37$ & 24.09 \\
\hline $\mathrm{CaO}$ & $9 \cdot 78$ & 12.80 & 16.36 & I 1.86 \\
\hline $\mathrm{MIgO}$ & $7 \cdot 16$ & 10.93 & II. 66 & $7 \cdot 44$ \\
\hline $\mathrm{Fe}_{2} \mathrm{O}_{3}$ & 0.33 & 0.29 & 0.34 & 0.62 \\
\hline $\mathrm{P}_{2} \mathrm{O}_{3}$ & $I \cdot 36$ & $1.5^{2}$ & $4 \cdot 40$ & $2 \cdot 56$ \\
\hline $\mathrm{SO}_{3}{ }^{\circ}$ & 28.16 & 26.69 & 21.06 & $13 \cdot 26$ \\
\hline $\mathrm{SiO}_{2}$ & $I \cdot 35$ & 1.20 & 0.43 & $1 \cdot 56$ \\
\hline $\mathrm{Cl}$ & 15.24 & 12.24 & II. 39 & $17 \cdot 23$ \\
\hline I & 0.31 & $0.4^{6}$ & $\mathrm{I} \cdot \mathrm{I} 3$ & 3.08 \\
\hline
\end{tabular}

In these analyses the relatively great accumulation of iodine may be noted, since sea-water contains less than $0.00,0001$ per cent. of iodides ${ }^{1}$.

Forchhammer ${ }^{2}$ found only a trace of manganese in the ferric oxide obtained from about twenty pounds of sea-water. The ash of the marine alga Padina Pavonia contains, however, as much as 8.I9 per cent. of manganese (total ash, 34.75 per cent.). In Sects. 73 and 75 mention is made of the fact that, in addition to manganese and silica, other non-essential elements may accumulate in certain plants to a marked extent.

The following analyses, made by Gorup-Besanez (Wolff, l.c., p. I33), of unattached floating plants of Trapa natans, show how ash constituents may be absorbed in percentage amounts differing from those in the water in which the plant is growing, and they also show how the percentage composition varies in different stages of development and in various parts of the plant, not only as regards the total amount of ash, but also as regards its percentage composition. The percentage of ash to dry substance and the percentage amounts of the individual constituents present in the ash are tabulated. Whole plants were taken for analysis from the same locality, one set in May and another in June. Pericarps also were analyzed, but these had unfortunately been immersed in water since the previous year. Ten thousand parts of the water contained 0.8044 of ash, the percentage composition of which is given beneath.

\begin{tabular}{|c|c|c|c|c|c|c|c|c|c|}
\hline & Pure Ash per cent. & $\mathrm{K}_{2} \mathrm{O}$ & $\mathrm{Na}_{2} \mathrm{O}$ & $\mathrm{CaO}$ & $\mathrm{MgO}$ & $\mathrm{Fe}_{2} \mathrm{O}_{3}$ & $\mathrm{SO}_{3}$ & $\mathrm{SiO}_{2}$ & $\mathrm{Cl}$ \\
\hline$[9$ & & 6.89 & I. 41 & 14.0 & $7 \cdot 56$ & $29 \cdot 62$ & $2 \cdot 73$ & 28.66 & 0.65 \\
\hline June & 13.69 & 6.06 & 2.71 & 17.65 & $5 \cdot 1$ & 23.40 & $2.5^{2}$ & $27 \cdot 34$ & 0.46 \\
\hline Pericarps & $7 \cdot 75$ & $1 \cdot 26$ & 0.63 & $9 \cdot 7^{8}$ & $0.9 \mathrm{I}$ & 68.60 & $3.9^{2}$ & 4.84 & 0.41 \\
\hline Water & & 9.08 & $9 \cdot 22$ & $4^{2 \cdot} \cdot 44$ & 18.09 & I.1 2 & 17.03 & 1.90 & I. Is \\
\hline
\end{tabular}

De Saussure ${ }^{3}$ long ago had recognized that the composition of the ash of a particular plant might be markedly influenced by the nature of the soil on which it was growing. As is shown by water cultures, the degree of concentration of the whole fluid, or even of one constituent, may produce perceptible and often very

1 Liebig, l. c., pp. 73,525 .

2 Forchhammer, Ann. d. Phys. u. Chemie, I 855 , Bd. xcv, p. 84.

3 Saussure, Rech. chim., 1804, Tables d'incinérations, No. 67 seq. See also Wolff. 
marked results ${ }^{1}$. Similar effects are produced by all factors which influence the growth and activity of the plant. The influence of illumination upon the composition of the ash has been investigated by R. Weber ${ }^{2}$, \&c. Analyses of the ash of healthy and unhealthy plants are given by Wolff, and also comparative analyses of the ash of parasites and of their host plants.

Relation between the water and salts absorbed. When a watery solution is absorbed, the water and salts are as a general rule taken up in a different ratio to that existing in the external medium. The absorption of a salt is determined primarily by its diosmotic properties and by the selective power of the plant under observation. At the same time, an inwardly directed current of water may accelerate absorption, and in such cases it is always possible to arrange conditions in which either the salt or the water shall be absorbed in greater relative proportion than in the solution presented to the plant. Thus, by diminishing or stopping transpiration, it is always possible to cause relatively more salt to be absorbed than water.

De Saussure ${ }^{3}$ and other investigators ${ }^{4}$ employed very dilute solutions and always used transpiring plants for experimentation; hence they concluded that plants absorbed their nutriment in extremely dilute form. The so-called 'de Saussure's law', which has been accepted more especially in text-books of agricultural chemistry, is merely a special case, and not a general law applicable to absorption under all conditions. Later researches by W. Wolff with transpiring plants, and by Knop and Biedermann with swelling seeds, have moreover shown that when solutions of appropriate strength are used, certain salts are absorbed in greater relative amount than water ${ }^{5}$.

\section{SECTION 23. The Mechanism of Secretion and Excretion.}

Excretion must always accompany vital activity, owing to the continued production of katabolic substances whose removal is necessary. The chief excreta are water and carbon dioxide, and, in green assimilating plants, oxygen; but in many cases, and especially in fermentative organisms, compounds such as alcohol and various organic acids, as well as other substances, may be excreted. The eliminated products may either result from the plant's own secretory activity, or, in the case of Myxomycetes, may be solid particles which have been previously ingested (Sect. I9).

In addition to the uses mentioned in Sect. 20 and Chap. $X$, many secretory products attain special importance in a variety of ways. It is

1 See Wolff, Versuchsstation, I 865 , Bd. vir, p. I93, and 1868, Bd. x, p. 3 ; Nobbe, ibid., I87o, Bd. xiIr, p. $3_{3} 3$, \&c. On the infuence of chalky soils, see Malaguti et Durocher, Ann. d. sc. nat., I 858 , iv. sér., vol. ix, p. 230 ; Flèche et Grandeau. Ann. d. chim. et d. physique, r874, v. sér., vol. v, p. 354 .

2 R. Weber, Versuchsstat., I $\$ 75$, Bd. xviıI, p. $3^{6}$

3 Saussure, Rech. chim., $180_{4}$, p. 247 .

* A. Trinchinetti, Bot. Zeitung, I $8_{45}$, p. I I ; Schlossberger, Ann. d. Chemie t. Pharmacie, $18_{52}$, Bd. Ixxxi, p. I 72 ; Hert, ibid., I 854 , Bd. Lxxxix, p. 334 .

5 W. Wolff, Versuchsstat., I 864 , Bd. vi, p. 203 , and $\mathrm{r} 865$, Bd. viI, p. I93. See also Knop, ibid., I859, Bd. I, p. I94, and I864, Bd. vi, p. 8 I ; also Biedermann, 1. c., I867, Bd. 1x, p. 3 I 2. 
sufficient here to mention the ferments, acids, \&cc., by the solvent action of which substances are rendered available for absorption, or by which, as in parasitism, penetration into another organism is rendered possible. Wax, ethereal oils, resin, and gelatinous envelopes ${ }^{1}$ are all secretions of the protoplast, which are directly excreted as such, or attain their final composition by extra-cellular metamorphosis. In this sense the cell-wall itself may be regarded as a secretory product excreted by the protoplast (Sect. 84).

Secretory products frequently undergo physiologically important changes when excreted by the cell. When a plant renders a nutrient fluid alkaline, substances such as earthy phosphates, \&c., which are soluble only in an acid solution, are precipitated, while the excretion of oxalic acid, which is of frequent occurrence in fungi, causes a precipitation of calcium oxalate when a soluble calcium salt is present. On the other hand, the nature of the secretory products is influenced and regulated in a variety of ways by internal and external agencies, as is the case with regard to the production of oxalic acid (Sect. 86) and of enzymes (Sect. 9I), while in various carnivorous phanerogams (Sect. 55) definite stimuli induce or accelerate the secretion of enzymes and free acid $^{2}$.

All that has becn said about the causes and mechanism of the exchange holds good for the special case, in which the substances excreted are secretory products. As is indicated by the recovery from plasmolysis, salts which are present in the fluid imbibed by the cell-wall, but which cannot penetrate the protoplast, will diffuse outwards until equilibrium is reached, when a plant is removed from a nutrient or plasmolysing solution to pure water ${ }^{3}$.

When a plant is actively transpiring, the continual introduction of new traces of salts by the water current must lead to a certain accumulation of these salts, since backward diffusion is but slow. Nobbe and Siegert 4 actually found that patches of saline incrustation, mainly of potassium chloride, were formed on the stem and leaves of buckwheat, and to a less extent on those of barley, when the plants were grown on a watery solution containing I per cent. of inorganic salts (see Sect. 38). The soluble incrustations which may sometimes be seen on plants growing on saline soil are probably of similar origin, although other factors, including perhaps

1 Klebs, Unters. a. d. Bot. Inst. z. Tübingen, I 886 , Bd. II, p. 404; Hauptfleisch, Zellmembrane in Hitilgallerte d. Desmidiaceen, I 888.

${ }^{2}$ On the acid secretion of certain hairs see Stahl, Pflanzen u. Schnecken, I88S, p. $4^{1}$.

3 This has been observed by Knop, Versuchsst., I $\$ 60$, p. S6, and W. Wolff (1. c., 1864, Bd. vi, p. 230 ), in plants transferred from nutrient solutions to water. Similar researches, partly however on plants in which the protoplasts had been killed by immersal in over-concentrated solutions, are given by M. Macaire, Ann. d. Chemie u. Pharm., I883, Bd. virl, p. 789 ; Unger, Über den Einfluss des Bodens, \&c., I8 86 , p. 147 ; E. Walser, Ann. d. sci. nat., 1840, ii. sér., T. Xiv, p. Ior; Wiegmann und Polstorff, Über d. anorg. Bestandtheile d. Pfanzen, I8 $4_{2}^{2}$, p. 49 ; Cauvet, Ann. d. sci. nat., I861, iv. sér., T. Xv, p. 320.

t Nobbe und Siegert, Versuchsst., I864, Id. vr, p. 37 ; Nobhe, ibid., I864, Bd. Ix, p. 479. 
a power of active secretion, may come into play in certain cases ${ }^{1}$. The deposition of calcareous scales on the leaves of many Saxifrages and other plants $^{2}$ shows how transpiration may lead to an excretion and external deposition of insoluble substances. Transpiration may also aid in the deposition of silica, which is, however, ultimately dependent upon other factors, and which takes place equally well in diatoms living submerged in water, although no transpiration is here possible.

All secretions, whether produced by entire members or particular glands (nectaries, hairs, \&c.), are products of the vital activity of the plant. The excreted substances may have been absorbed by the plants as such, or may be products of its own secretory activity. In both cases, the conditions necessary for diosmosis and exosmosis may be generated by the plant itself, or the transference of the excreta may take place in other ways-as, for example, when a substance which cannot penetrate the protoplast passes from the root to the leaves, where it is excreted entirely in the imbibition water saturating the cell-walls. The study of the excreta still affords unexhausted means of attacking these problems. Thus, special factors must render excretion possible in nectaries from which an abundance of sugar escapes, or in fungal cells which evolve considerable quantities of acids, for these substances may be present within the cell in a more dilute solution than is excreted.

All protoplasmic secretions which appear externally and are lost to the plant, or which can take no further part in metabolism, are to be regarded as excretory substances. Various secretory products, however, when excreted by the protoplast, may remain in more or less permanent connexion with the organism and attain considerable functional value in its economy. The extra-cellular changes which take place in such substances are also influenced by the relationship existing between mass and chemical reaction: thus the tannate of methyl-blue is decomposed outside as well as inside the cell, provided the least trace of a diosmosing compound is formed by the acid with which it is brought into contact (see Sect. 22). Similarly potassium nitrate, when absorbed, is gradually completely converted into a salt of an organic acid, the traces of nitric acid set free being immediately absorbed by the protoplast. To enable a portion only of a compound to be absorbed, the action of an intermediary agency is not always necessary, provided that the component parts of the compound are only loosely associated together. Thus when calcium

${ }^{1}$ Volkens, Flora d. aegypt. Wiiste, I $88_{7}$, p. 27 ; Ber. d. Bot. Ges., I $88_{7}$, p. 434; Marloth, ibid., p. 32 I. Older literature : de Candolle, Physiol, trans. into German by Roper, 1833, p. 206 ; Meyer, Pflanzenphysiol, , I8 38 , ii, p. 530.

${ }^{2}$ Unger, Sitzungsb. d. Wien. Akad., I861, Bd. xiı11, p. 5 I4 ; Volkens, Ber. d. Bot. Ges. I884, p. 334; Kohl, Kalksalze u. Kieselsäure in d. Pflanze, 1889, p. 99 ; Anatomical details in de Bary, Comp. Anat. p. 123. 
bi-carbonate is absorbed by a green plant, the chalk forms an extracellular deposit while the liberated carbon dioxide is assimilated. Similarly sodium bi-carbonate $\left(\mathrm{NaHCO}_{3}\right)$ may lose when presented to the plant as much as 70 per cent. of its carbonic acid. The sodium carbonate produced $\left(\mathrm{Na}_{2} \mathrm{CO}_{3}\right)$ does not penetrate within the cells, but remains in the external fluid which it turns markedly alkaline ${ }^{1}$.

Alkaline and acid reactions may be produced by substances directly excreted by the plant. Thus fungi may form and excrete acids or ammonia when fed solely upon proteids (Chap. VIII). A few phanerogams evolve trimethylamine, while Chara and certain algae give off alkaline secretions in pure water, which may be detected by the colour-reaction of phenolpthalein, or by the decomposition of Prussian-blue previously deposited upon the cell-wall ${ }^{2}$. It is hence not surprising that in water-cultures of phanerogams the fluid may either become alkaline ${ }^{3}$, or acid, according to its composition. In certain researches by Rautenberg and Kuhn ${ }^{4}$, the presence of a large amount of ammonium chloride caused so marked a production of acid that maize and kidney-bean seedlings soon died. Moreover, according to Biedermann, when seeds swell in neutral solutions of chlorides of the alkalies or alkaline earths, the external fluid becomes alkaline, while according to Knop, when the seeds are in water containing calcium sulphate, the calcium of the salt is absorbed in somewhat greater amount than is the acid ${ }^{5}$.

Calcareous Incrustations. The excretion of chalk by the plant may take place in a variety of ways. Hence the incrustations found especially well marked on certain fresh-water and marine algae, and upon leaves and stems of Potamogeton and other water plants, as well as on fungi and the roots of land plants, need not necessarily have the same origin ${ }^{6}$. In many mosses (Eucladium verticillatum, Trichostomum tophaceum, \&c.) growing in water rich in bi-carbonate of lime, the incrustation is formed but little more actively than it is on dead objects, the living plant hence playing an almost passive part ${ }^{7}$. The incrustations on algae are apparently mainly due to the activity of the plant itself, for under similar

${ }^{1}$ Hassak, Unters. a. d. Bot. Inst. z. Tübingen, I $\$ 88$, Bd. II, p. 47 I.

${ }^{2}$ Klebs, Unters. a. d. I'ot. Inst. z. Tübingen, I $\$ 86$, Bd. I , p. 340 ; Hassak, ibid., p. 476.

${ }^{3}$ Knop, Ann. d. Chem. u. P'harm., IS62, Bd. CXXI, p. $3^{\mathrm{I}} 3$. On alkalinity in sand cultures, see Boussingault, Agron., Chim. agr., \&c., i 860 , T. I, pp. 273, 279 ; Stutzer, Versuchsst., 1878 , Bd. xxi, p. III. It is not impossiblc that here bacterial activity may produce the results observed.

4 Rautenberg und Kuhn, Versuchsst., I864, Bd. vi, p. 35S. Cf. Dworzak und Knop, Ber. d. Sächs. Ges. d. Wiss., Leipzig, 1875 , i, p. 74 ; Wagner, Versuchsst., 187 I, Bd. xirr, p. 221 . Under different conditions, Czapek (Jahrbuch. f. wiss. Bot., IS96, Bd. xxıx, p. 23) found no secretion of acil to be caused by the presence of sal-ammoniac, nor does it necessarily follow that the roots of all plants will have this power. See Sect. 28.

${ }_{5}^{5}$ Biedermann, Versuchsst., I $\$ 6_{7}$, Bd. 1X, p. 3 I2 ; Knop, Versuchsst., I $86_{4}$, Bd. vi, p. 81 .

${ }^{6}$ For literature see Kohl, Kalksalze u. Kieselsäure in der Pflanze, I889, p. 99. Of more recent researches cf. Cramer, Über die verticillirten Siphoneen Neomeris u. Bornetella, ISgo, p. 9; [Church, Annals of Botany, vol. ix, I895, p. 602 ]. With the chalk, calcium oxalate \&c. may be associated.

${ }^{7}$ Cf. Unger, Mer. d. Wien. Akad., 1861, ii, p. 509. 
conditions it is only particular species that become covered by a deposit of chalk. Since, moreover, all algae assimilate, the removal of the carbonic acid cannot be the sole and primary cause of the deposition, especially in forms such as Corallina, in which the chalk impregnates the plant throughout, or in Melobesia, which grows in depths where the illumination is relatively feeble. Hassak has indeed shown that in light Chara plants become incrusted with chalk only in water which contains calcium sulphate or chloride'. This takes place by means of an alkaline secretion, which however only produces an actual deposition when the carbonic acid is assimilated by the plant, for carbonic acid gradually dissolves away the chalky incrustation in water which is not saturated with calcium bicarbonate. Herein ties the necessity for illumination which alone, when calcium bicarbonate is present, must lead to a deposition of chalk, although an incrustation is formed upon the plant only when certain other factors of definite and specific character enter into play ${ }^{2}$. In the presence of calcium salts or of a saturated solution of the bicarbonate, an alkaline excretion will suffice to cause a precipitation of chalk, without any $\mathrm{CO}_{2}$-assimilation being necessary. It is perhaps in this manner that the incrustations on fungi and roots are formed.

It is in complete accordance with this external origin that the deposition of chalk may be not merely external, but may also take place in the substance of the cell-wall, especially when the outer layers of the latter are gelatinous ${ }^{3}$. Nevertheless, the possibility remains that both the calcium pectate ${ }^{4}$, and the traces of chalk, which frequently impregnate cell-walls in the interior of the plant, may have a similar metabolic origin. The calcium carbonate found in plasmodia, in cells of the seeds of Celtis, \&c., is of internal origin, and the chalk found in the hollow leaf scales of the rhizome of Lathraea may also be a secretory product ${ }^{5}$. The same is the case with the scaly deposit formed on Saxifrages, \&c., although here also similar neighbouring plants, in which the drops of water excreted by the water stomata on the leaf-teeth dry up daily, show no such chalky deposit.

These deposits, incrustations and infiltrations are of importance to the plant in so far as they confer protection and increase rigidity ${ }^{f}$, while from a geological standpoint they are essential for the formation of certain rocks, and indeed may lead to the production of chalk rocks from calcium sulphate ${ }^{7}$.

${ }^{1}$ Hassak, Unters. a. d. Bot. Inst. z. Tübingen, I 8s8, Bd. Il, p. $4^{6} 5$.

${ }^{2}$ Raspail, Nouveau système d. chim. organique, I833, p. 32 I. Cf. Meyer, Physiol., 1837 , Bd. 1, p. 126 ; Hanstein, Sitzungsb. d. Niederrh. Ges., Mai, 1878; Berthold, Jahrb. f. wiss. Bot., I882, Bd. xill, p. 710. Pringsheim (ibid, I858, Bd. I, p. I33) regards the remoral of $\mathrm{CO}_{2}$ as the sole cause, while Bischoff (Die kryp. Gew. Deutschlands, I\&2\&, i. Lief., p. $2 \mathrm{I}$ ), and also Payen (Mém. prés. p. div. savants, $\left.18_{4} 6, \mathrm{~T} .1 \mathrm{x}, \mathrm{p} . \jmath^{8}\right)$ suppose, on insufficient grounds, that the incrustation is excreted by the plant.

${ }^{3}$ See Leitgeb, Sitzungsb. d. Wien. Akad, 1887 , Bd. Xcvi, i, p. 2 I ; [Church, Ann. of Bot., 1895 , ix, p. 602].

${ }^{4}$ Melnikoff, Unters. über d. Vork. d. kohlens. Kalks in Pflanzen, Bonner Diss., 1877, p. 17. See also Kohl, 1.c.; Giesenhagen, Flora, 1890, p. 2, and Ber. d. Bot. Ges., 189I, p. 74 ; Zimmermann, ibid., I 891 , p. 17 . See Mangin, Compt. rend., $18_{92}$, T. Cxv, p. 260 ; Rech. anat. s. l. composés pectiques, $1893, \mathrm{P} \cdot 4^{8}$.

${ }^{5}$ Kohl, l.c.; Scherffel, Mitth. a. d. Bot. Inst. z. Graz, I888, Heft 2, p. 209.

'Stahl, Pflanzen u. Schnecken, I $\$ 88$, p. 7o.

${ }^{7}$ Credner, Elemente der Geologie, 189 I 7 . Aufl., pp. 266, 270. 
The incrustations of ferric oxide, formed on many algae, bacteria, \&c., present us with similar problems as to the causes of their origin ". In certain cases, the oxidation of ferrous salts to ferric oxide may render energy available for metabolism (Sects. 63 and 96 ).

\section{SECTION 24. Osmotic Pressure in the Cell.}

The non-diosmosing substances dissolved in the cell-sap exert in every case an osmotic pressure determined by definite physical laws, and this in ordinary turgescent cells usually averages from five to ten atmospheres. By this pressure, directed in the first instance against the semi-permeable vacuolar membrane, the viscous protoplasm, which always forms a complete and continuous enclosing envelope, is pressed against and brought into the closest contact with the cell-wall (Fig. IO). The cell-wall reacts correspondingly with an equal but internally directed pressure. The intensity

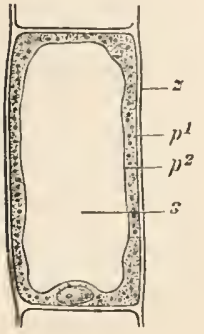

$n$

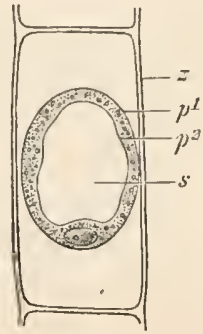

6

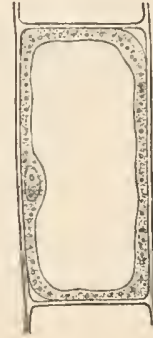

c
Fig. 10. From the root of Zea Mazs, $(a)$ in water ; $(b)$ plasmolysed in 5 per cent. $\mathrm{KNO}_{3} ;(c)$ slight plasmolysis in $2 \cdot 3$ per cent. $\mathrm{KNO}_{3}$. of the osmotic pressure and the degree of cohesion of the cell-wall determine to what extent the latter will be stretched or whether it will be ruptured-as happens, for example, when various pollen grains are placed in pure water ${ }^{2}$. It is by means of this tension, to which the name of turgor is given ${ }^{3}$, that thin-walled cells are stretched and stiffened, just as a bladder or india-rubber balloon stiffens when forcibly injected with water or air ${ }^{\star}$. Hence plants collapse and wither when by plasmolysis or by transpiration so much water is removed from the cells that the osmotic tension and the turgid condition connected therewith are partly or entirely removed.

Without the resistance offered to extension by the cell-wall, no marked osmotic pressure could be attained, for naked vacuolated masses of protoplasm are stretched and ultimately ruptured by a relatively trifling internal pressure. Fluid lamellac, however, when pressed against a rigid

${ }_{1}$ Hanstein, Sitzungsb. d. Niederrh. Ges., May, 1878; Winogradsky, Bot. Zeitung, 1888, p. 261 ; Molisch, Die Pflanze u. ihre Beziehung zum Eisen, 1892 , pp. I8, 30, 60.

2 See Lidforss, Jahrb. f. wiss. Bot., I 896, Bd. xxix, p. 1.

3 This tension is for the most part due to osmotic energy, and hence it is admissible to designate the total hydrostatic tension, regardless of its origin, as 'turgor.' Cf. Pfeffer, Plasmahaut u. Vacuolen, 1890, p. 297.

- Cf. Nägeli und Schwendener, Mikroskop, 1877, 2. Aufl, p. to4. 
support, can withstand almost any intensity of pressure. Hence an extremely high osmotic pressure may be maintained so long as the dissolved substances do not diosmose through the semi-permeable plasma. A working model of the relationships existing in the cell is presented when a semi-permeable precipitation membrane is formed within, and adpressed to, an enclosing cell of porous clay, for the latter resembles the cell-wall in so far as it allows crystalloid substances to diosmose through with relative ease. It is not the porous clay cell (or cell-wall), but the precipitation membrane (or plasmatic membrane) which is of primary importance with regard to the production and maintenance of the osmotic pressure.

The pressure exerted against the clay cell remains unchanged; even if a second smaller precipitation membrane is formed within the first and floats freely in the central fluid. For, provided the membranes are semipermeable, if the osmotic pressure is higher inside the inner membrane than outside of it, this membrane will grow until sufficient water is absorbed to bring the inner cell to the same osmotic level as the outer, equilibrium then being again restored throughout the system. The living cell is actually such a system of two plasmatic membranes, one enclosing the other (Sect. I 8 and Fig. Io), nor does it make any essential difference whether the plasma forms an external layer only, or also crosses the vacuole in the form of strands. It is also evident that the appearance of numerous vacuoles in the plasma will not alter the turgid pressure, while the presence of a welldefined vacuole simply adds an additional membrane to the osmotic system.

All substances present in solution in the plasma exert an equivalent osmotic force, but if such dissolved substances are absent the power of swelling inherent in the plasma must resist the pressure which the osmotic tension exerts upon it. A similar relationship would exist in the porous cell just described, if the space between the two precipitationmembranes were filled with gelatine free from all saline admixture. For the gelatine would diminish in volume until the imbibitory swelling force, which rapidly increases as the amount of imbibed water decreases, is just equal to the osmotic pressure which the inner precipitation-membrane (like the vacuolar membrane) exerts against the gelatine. When plasmolysis is produced the same general relationship is maintained, for now the resistance of the cell-wall is replaced by the osmotic pressure which the plasmolysing fluid exerts upon the ectoplasmic membrane.

The semi-fluid nature of the protoplasm does not presuppose that it has no power of swelling, and this power may counteract the osmotic pressure exerted upon it. It is probable, however, that dissolved substances are present in the plasma, and these may aid, or even be of primary importance, in maintaining internal osmotic equilibrium, so that the protoplasmic power of swelling may not be called into play to counterbalance the pressure 
exerted upon it ${ }^{1}$. Though such an arrangement appears to be advantageous, the facts at our disposal do not suffice to form a definite opinion. Very concentrated solutions of albuminous or other colloid substances may be present in the plasma, but their osmotic energy is extremely feeble. Colloid substances do actually exhibit transitional stages between the swollen and the dissolved condition (Chap. III). However that may be, the semifluid nature of the plasma renders possible rapid adjustment to differences of pressure. This is directly shown by plasmolysis, in which phenomenon, provided the cell-wall allows the saline solution to pass readily through, the plasma contracts until a condition of equilibrium is reached (Fig. 10), the cell-sap giving up water until its osmotic concentration is equal to that of the fluid outside. The osmotic pressure is proportional to the degree of concentration, but the force of imbibition increases in geometric proportion as the amount of water present decreases (Sect. I2). Hence, if the respective decreases in the volume of the plasma and cell-sap were known, it would be possible to determine whether it is the osmotic energy of the plasma, or its power of swelling, which resists the pressure exerted upon it. Every vacuole must alter its size, when necessary, until its contents become isosmotic with the rest of the cell, and hence it is possible by observing these changes of volume to determine the precise spot where an increased or decreased hydrostatic pressure originates ${ }^{2}$.

The semi-fluid plasma can interpose no appreciable hindrance to the full transmission of the internal osmotic pressure to the cell-wall outside. Nor can the force of turgor be perceptibly raised above the osmotic pressure of the cell-sap by any active contraction of the plasma, while owing to the loose arrangement of its component parts, the tendency of the stretched plasma to passive contraction cannot possibly exercise any appreciable effect in increasing the internal hydrostatic pressure.

The pressure of the cell-sap against the plasma and cell-wall will be partially antagonized, according to definite physical laws, by the centrally directed pressure which every curved limiting membrane exerts. Since the tangential pressure existing in the peripheral membrane is not precisely known, this centripetal force cannot be exactly determined. Nevertheless in cells of ordinary size it is of trifling importance in comparison to the osmotic pressure, for even with a radius of $0.01 \mathrm{~mm}$. it cannot exceed 0.03 to 0.06 atmospheres. In vacuoles of $0.0005 \mathrm{~mm}$. radius the centripetal pressure would, it is true, reach $I \cdot 5$ to 3 atmospheres, and hence without a correspondingly increased internal pressure such vacuoles could not be maintained or even formed. Similarly the vacuoles which may be artificially produced in a plasmodium by means of asparagin, gradually become

1 Pfeffer, Osmot. Unters., 1877 , p. I 70 ; Plasmahaut u. Vacuolen, 1890, p. 294.

${ }^{2}$ Pfeffer, Osmot. Unters., 1877, p. 180 seq. ; Plasmahaut u. Vacuolen, 1890, pp. $296,322$. 
smaller and ultimately disappear as this substance diosmoses away. In the same way the gas of gas-vacuoles must be under compression ${ }^{1}$.

From what has been said, it follows that in plasmodia or other soft gymnoplasts, no highly osmotic substances can be present in solution. Although the presence of highly osmotic solutions is, hence, not an essential feature of the protoplasmic constitution, nevertheless the possibility remains, that in other cases marked osmotic forces may be developed in the plasma, in correspondence with the fact that the osmotic powers of the cellsap may be only trifling or very strong, according to the ends which are to be attained and in adaptation to the surrounding relationships ${ }^{2}$. It is always possible, for example, that in the plasma of Myxomycetes concentrated solutions of feebly osmotic substances may be present. Except for the maintenance of vacuoles, the existence of marked osmotic pressure is not in general essential, although in dermatoplasts turgidity is an essential condition for normal growth, and hence for the maintenance of the life of the organism. Turgidity also serves to make soft tissues firm and rigid, and thus enables work to be done against considerable external resistance.

The internal hydrostatic pressure will be highest when the plant is lying in water, and when the cell-walls are permeated with pure water, for if they are imbibed with a saline solution, the pressure of turgor will be decreased by the corresponding osmotic equivalent, while, when the solution is sufficiently concentrated, water is withdrawn from the protoplast until plasmolysis occurs. A solution which is just able to remove the turgidity of the cell is one which has the same osmotic value as the cell-sap has. To produce the same effect by solutions of various substances, these must have the same osmotic value, and hence by observing the degree of concentration of different solutions necessary to produce incipient plasmolysis, the osmotic value of the different substances used may be estimated. This method does not determine the absolute osmotic pressure, but the relative values thus obtained will enable such determinations to be made for all the substances under observation as soon as the absolute value is determined for any one of them.

For non-diosmosing substances, the osmotic pressure is independent of the nature of the semi-permeable membrane. Hence physical measurements made by artificial means give accurate values for the pressure exerted when in contact with the plasmatic membrane. According to such physical estimations a one per cent. (volume) solution of cane-sugar at $15^{\circ} \mathrm{C}$. develops a pressure of 0.69 atmospheres, a one per cent. solution of $\mathrm{KNO}_{3}, 3.5$, and a similar solution of $\mathrm{NaCl}, 4 \cdot \mathrm{I} 6$ atmospheres (Table, p. I+6).

1 Klebahn, Flora, IS95, p. 24 I (for Phycochromaceae); Engelmann, Zool. Anzeiger, I 878 , p. I 52 , and in Hermann's Handb. d. Physiol., i 879 , Bd. I, p. $34^{8}$.

${ }^{2}$ Turgor and osmotic pressure are not necessarily dependent upon the existence of vacuoles, as Went (Jahrbuch f. wiss. Bot., I888, Bd. xix, p. 350), for example, erroneously assumes. 
Osmotic pressure is a function of the number of molecules present in a unit of volume. The reason for the absence of a precise correspondence in certain cases between the ratio of volume to gaseous pressure and that of osmotic pressure to molecular weight, is that the number of dissolved molecules present in a unit of volume may be more than the molecular weight of the dissolved substance would indicate, owing to dissociation having taken place in the process of solution. In colloid substances, the relationship of molecular weight to unit of volume is such as to generate only a relatively trifling osmotic pressure, and this in the case of a one per cent. solution of gum-arabic amounts to 0.085 of an atmosphere, which is not a fortieth part of the pressure generated by a solution of $\mathrm{KNO}_{3}$ of the same concentration.

The earlier researches with bladders, parchment, \&c., necessarily gave relatively high osmotic pressures for colloid substances, since these diosmose but slowly or not at all ${ }^{1}$, and hence yield nearly the maximal possible osmotic pressure for such substances. With membranes of this character the maximal pressure can never be reached, and it falls more and more below its real value as diosmosis is increasingly active.

Just as is the case with gases, the osmotic pressure increases with the number of molecules present in a unit of volume. With highly concentrated solutions certain deviations occur, but nevertheless from a physiological point of view, and under the conditions usually existing, the osmotic pressure may be assumed to be proportionate to the degree of concentration of the given substance.

Similarly, the osmotic pressure alters with the temperature, according to the same laws that influence gaseous pressure. Since by a rise of temperature of $15^{\circ} \mathrm{C}$. the pressure is only raised from 100 to 105.5 , it is evident that temperature can never exercise any marked direct effect upon turgor in plants. A plasmolytic condition of equilibrium is for example not perceptibly modified by a change of temperature ${ }^{2}$.

From what has been said, it follows that, provided the active substances do not diosmose, the thickness or quality of the film of plasma, and its permeability to water, influence the time necessary for the restoration of equilibrium, but have no effect upon the pressure ultimately produced.

${ }^{1}$ Pfeffer, Osmot. Unters., $18 \% 7, \mathrm{p} .73$.

${ }^{2}$ See Ostwald, Allgem. Chem., i 89 I, 2. Aufl, Bd. I, pp. 659, 669; Pfeffer, Plasmahaut u. Vacuolen, $1890, \mathrm{p} .307$. The reactive power of the organism is such, that a change of temperature may act as a stimulus and give rise to a different result, an alteration taking place in either the osmotically active substances, or in the diosmotic properties of the protoplast. Nevertheless, the physical fundamentals mentioned above hold good here also, as well as in those cases in which the cell develops an active pumping (or sucking) force (Sect. $4^{6}$ ), and thus decreases (or increases) the osmotic pressure, according to the amount of water driven out (or sucked in). In every case, it is only possible to determine the work done by osmotic pressure, and made use of by the plant, by direct observation. No further remarks can be made here, since we are concerned only with the exposition of certain fundamental principles (Krabbe, Jahrb. f. wiss. Bot., 16y6, Bd. xxix, p. 447). 
This needs to be emphasized with especial clearness, since the attempt has frequently been made to explain a decrease of turgidity as being due to an increased and more rapid power of filtration through the plasma ${ }^{1}$.

The existence of a high osmotic pressure in turgid cells is made evident by the extremely energetic manifestations of which they are capable. These latter may be measured with fair accuracy by equilibrating them against measurable forms of energy. Thus, having found by the plasmolytic method the degree of concentration of an isosmotic (isotonic) saline solution, the turgid force of the cell is at once given, if the osmotic pressure of the isosmotic saline solution is known.

By results obtained in this manner, it is found that the pressure of turgor in land and fresh-water plants is usually equivalent to from 1.5 to 3.0 per cent. $\mathrm{KNO}_{3}$, i. e. equals a pressure of five to eleven atmospheres ${ }^{2}$. Even in starved and emaciated cells, the turgidity does not usually fall below one per cent. $\mathrm{KNO}_{3}$, i. e. $3 \cdot 5$ atmospheres ${ }^{3}$.

Frequently, the turgidity rises far above the average limits given, especially in cells, where dissolved and osmotically active reserve materials accumulate. Thus the pressure of turgor in the bulb of the onion (Allizm cepa), or better still the beet-root, may be equivalent, or more than equivalent, to five to six per cent. of $\mathrm{KNO}_{3}$ (twenty-five to thirty per cent. of cane-sugar), i.e. to a pressure of fifteen to twenty-one atmospheres ${ }^{4}$. As the reserve materials are removed, the internal hydrostatic pressure decreases, hence the turgor differs according to the stage of development, and under the action of various external conditions. Since during the growth and stretching of the cell-wall, turgidity remains fairly constant, a regulatory and correlating adjustment must continually go on, and in the same manner more or less marked differences may be maintained in neighbouring cells with different osmotic and other properties ${ }^{5}$.

All cells, which can accommodate themselves to concentrated nutrient solutions, must be able to increase the amount of osmotic substances which they contain. This is attained either by a direct absorption of the salts present in the external medium (as occurs in Bacteria), or by a correspondingly increased production of osmotically active substances. It is almost entirely by the latter means that Aspergillus niger, Pcnicillium

1 Pfeffer, I 890,1 . c., p. 302.

${ }^{2}$ For examples see de Vries, Jahrb. f. wiss. Bot., I88 4 , Bd. XIv, p. 427 ; Stange, Bot. Zeitung, I 892 , p. 277 ; J. M. Janse, Permeabilität des Protoplasmas, ISSS (Sep.-abdr. a. d. Verh. d. Akad. d. Wiss. zu Amsterdam).

${ }^{3}$ Stange, l. c., p. 396 ; Copeland, Einfluss von Licht u. Temperatur auf d. Turgor, I 896, p. 52.

${ }^{4}$ See de Vries, Sur la perméab. du protoplasma d. betteraves rouges, I 8 I , P. 7 (Sep.-abdr. a. Archiv. Néerland., T. VI), and Unters. über die mechanischen Ursachen d. Zellstreckung, IS 77 ; Copeland, l. c.

${ }^{5}$ See Pfeffer, Druck u. Arbeitsleistungen, I\$93, p. 297. The importance of these and other relationships will be dealt with in the chapters directly concerning them. 
glaucum, \&c., attain their power of growing on concentrated solutions, for they are able to remain turgid, even when the osmotic concentration of the cell-sap is equivalent to thirty-eight per cent. of $\mathrm{NaNO}_{3}$ (=forty-five per cent. of $\mathrm{KNO}_{3}=1.57$ atmospheres) ${ }^{1}$. Certain powers of accommodation are exhibited by other plants also, but only to a relatively trifling extent. Since the normal turgid pressure in the cells of marine algae is in general similar to that in terrestrial plants, it follows that in comparison with the latter the osmotic value of the cell-sap must be higher by about three per cent. of $\mathrm{NaCl}\left(3.7 \text { per cent. of } \mathrm{KNO}_{3}\right)^{2}$.

It is hardly surprising that, if plants which have become accommodated to a concentrated solution are suddenly placed in pure water, the increased internal pressure may be so great as to cause their cells to burst, for, in the case of Aspergillus, this pressure may amount to as much as 160 atmospheres. The strength of the cell-wall develops correspondingly to the demands made upon it, and the small size and diameter of the cell aid markedly in the attainment of the necessary resistant powers, for these increase as the radius decreases ${ }^{3}$. Many special features still require explanation, as, for example, how a bivalved diatom retains its form in spite of the internal hydrostatic pressure ${ }^{4}$. It is clear, morcover, that the turgidity produced by the storage of soluble materials will depend upon whether these are osmotically active or not.

The actual turgidity of the cell is due to the sum of the osmotic pressures exerted by the different substances present in the cell-sap. Hence it may happen that in one cell fifty to seventy per cent. of the osmotic energy is due to a substance, which is entirely absent from another cell. From the table on p. 146 it can be seen that the hydrostatic pressure is usually equivalent to that of solutions of $\mathrm{NaCl}, \mathrm{KNO}_{3}$ concentration of from one to five per cent., or to that of similar solutions of the organic acids (malic, tartaric, citric, oxalic) or their salts, which are so widely distributed in plants; whereas three to five times as much cane-sugar would need to be present to produce the same result; and in a cell filled with a thick solution of gum-arabic, or other colloid substance, the osmotic pressure would perhaps not be greater than that of a one per cent. solution of $\mathrm{KNO}_{3}$.

The fluid obtained by pressure gives a fairly accurate representation of the composition of the cell-sap, especially when the protoplast is

1 Esehenhagen, Einfluss verseh. Concent. auf Schimmelpilze, Leipziger Diss., 1889. Further literature: K. Bruhne, Beitr. z. Physiol. u. Morph. d. nied. Organismen von Zopf, 1894, Heft 4, p. I (Fungi); B. Stange, Bot. Zeitung, I892, p. 277 (Ihanerogams); A. Richter, Flora, I892, p. 9 (Algae); A. Fiseher, Jahrb. f. wiss. Bot., i 895, Bd. Xxvir, p. 15 I (Bacteria). See Sect. 73.

${ }^{2}$ See also Oltmanns, Sitzungsb. d. Berl. Akad., I891, x, p. 201.

${ }^{3}$ Pfeffer, Die period. Bewegungen der Blattorgane, I875, p. 114; Nägeli und Schwendener, Mikroskop, 18 77,2 . Aufl., p. $\mathbf{1}^{12}$.

+ See O. Miiller, Ber. d. Bot. Ges., r $88_{9}, 1$. 74 . 
relatively attenuated. Judging by this method, more than half the osmotic pressure of the beet-root has been found to be due to cane-sugar, and a similar proportion of that of the onion to glucose, which latter may be responsible for as much as cighty per cent. of the pressure of turgor in rose petals. In the leaf-stalks of Gunnera scabra about fifty-four per cent. is due to $\mathrm{KCl}$, and in the pith of the shoot apex of Hclianthus tuberosus about forty-one per cent. is due to $\mathrm{KNO}_{3}{ }^{1}$. In the leaf-stalk of Rhcum, oxalic acid is of primary importance (accounting for sixty-two per cent.). The same is also the case in Oxalis, while many Crassulaceae accumulate large amounts of malic acid ${ }^{2}$.

High turgidity is a necessary result of the accumulation of soluble osmotic reserve-materials, but it is of subordinate importance only. In other cases, as for example in growing cells, the maintenance and regulation of turgor are of the utmost importance, and for this purpose the plant appears preferably to employ organic acids, although sometimes other substances, and even inorganic salts, may be utilized.

The production of organic acids appears to be especially favourable for the maintenance of turgor, for although during the oxidation of dextrose to citric acid the osmotic pressure remains the same, when dextrose is oxidized to malic or oxalic acid, an increased osmotic pressure is generated, while at the same time energy is liberated ${ }^{3}$.

The union of the acid with an alkaline base still further increases the osmotic pressure, which, however, is very slightly if at all modified by combination with an alkaline earth.

In the economy of the plant, according to the character which metabolism assumes, it is of great importance that substances of lower or of higher osmotic powers may be produced from the same nutrient material, and a knowledge of the osmotic powers of secreting cells will aid us in establishing conclusions as to the causes of passive, or even active, secretion.

In an insoluble form (as starch, oil, or proteid), reserve-material may be stored up without causing any increase of osmotic pressure, and even by the mere condensation of two molecules of a monosaccharide to one of a disaccharide (cane-sugar), the osmotic pressure may be reduced to one half of what it previously was.

If the sugar, as it penetrates the cell, forms a glucoside by combining with some substance already present, no increase in the number of

${ }^{1}$ De Vries, Jahrb. f. wiss. Bot., 1884 , Bd. xrv, p. 589 . De Vries himself has corrected his original supposition (Bot. Zeitung, $18 \% 9, \mathrm{p}, 848$ ) that the organic acids were of primary importance in maintaining turgidity.

${ }^{2}$ De Vries, I.c., p. 58I ; G. Kraus, Stoffwechsel d. Crassulaccen, I886, p. 4. See also the literature on absorbed soluble substances, Sects. 22 and Io9. Hansen (Mitth. a. d. zool. Station zu Neapel, I893, Bd. xi, p. ${ }_{25}$ S) gives an analysis of the cell-sap of tralonia utricularis.

${ }^{3}$ Pfeffer, Studien z. Energetik, I 892 , p. 197. 
molecules, or of the osmotic pressure, will be caused there through such absorption and passive secretion ${ }^{2}$. The fact that the turgor rapidly sinks, as the glucose is removed from the bulb scales of Allium cepa, indicates the absence of any such mode of storage in this plant.

As a general rule, the osmotic pressure of a mixture will be the sum of the partial pressures exerted by its individual constituents ${ }^{2}$. So long as the number of molecules, or free ions, remains the same, the osmotic pressure will be unaltered, whatever interchanges may take place. By means of the osmotic coefficients given in the table on p. 146, it may be shown that the same pressure is generated in a mixture of potassium chloride and magnesium sulphate, no matter whether the chlorine is combined with magnesium or with potassium ${ }^{3}$. These approximate coefficients, though not precisely accurate, are sufficiently so to determine the osmotic value of a mixture from a physiological point of view, if the amounts present of sugars, organic or inorganic acids, alkalies, or alkaline earths are known; indeed de Vries has estimated the hydrostatic pressure exerted by the different groups of substances present in the cell-sap ${ }^{4}$.

From the table it may also be seen that the isosmatic coefficient of an acid increases by unity for every atom of an alkali it combines with, but remains unchanged when united with an alkaline earth, and hence the replacement of potassium by magnesium causes the osmotic pressure to fall.

In order to understand the causes which regulate turgidity, all the factors at work must be known, for in the service of the plant, turgidity is employed and regulated in the most various ways, as will be seen when the individual vital functions are dealt with in detail ${ }^{5}$. The regulation of turgor which accompanies growth, and which also occurs when plants are transferred to more concentrated or more dilute solutions, has already been incidentally mentioned. This is attained by an absorption or a metabolic production of osmotic substances, or by both taking place together, until

1 Pfeffer, Unters. a. d. Bot. Inst. z. Tübingen, 1886, Bd. II, p. 309. Compensatory adjustments are always possible.

3 This is not in all cases precisely true, owing to the influences (combination, dissociation, \&c.) which the constituents of a solution may exert upon one another. See for example Tamman, Zeitschr. f. physik. Chemie, I $\$ 92$, Bd. IX, p. IoS. Nevertheless, in most cases the first statement holds good, as is shown by the rates of diffusion of the individual components remaining the same (see Ostwald, Allgcm. Chemie, 1892, 2. Auf., Bd. I, p. 692), and also by osmotic determinations (Pfeffer, Osmot. Unters., I877, p. 67 ; de Vries, Jahrb. f. wiss. Bot., 1884, Bd. xiv, p. $4^{80}$ ).

3 This is the case with all mixtures of salts, proviced that no precipitate is formed, nor any of the new products removed by diosmosis from the cell. Dehérain, by estimating the freezing-point of expressed sap, has found that in young seedlings the osmotic pressure lies between 4.8 and 9.8 atmospheres (Compt. rend., i 896, T. xxiı, p. 898).

- De Vries, l. c., p. 54T.

' For a general account see Pfeffer, Druck u. Arbeitsleistungen, $1 \S_{93}, \mathrm{pp} .296,{ }_{22} 8$; Studien $z$. Energetik, $18_{92}, \mathrm{pp} .237,248$. 
the difference of potential between the fluids inside and outside the cell is equilibrated. Rapid variations of turgidity may be produced in the same manner, and these probably often occur, but can be detected only when they induce reactions which are themselves visible, as for example when the anther-filaments of Cynareae shorten on stimulation, owing to a sudden diminution of turgidity allowing the stretched and elastic cellulose membranes to contract. To permit a rapid reaction, a rapid filtration of water out of the cells is all that is required. If this is assured, then under particular conditions the same result must always be produced, independently of whether the fall of osmotic pressure is due to the removal of a salt, or to a chemical change, or to some other means. Various chemical changes are possible, which, without any precipitation being produced, may lead to a sudden diminution of the osmotic pressure, while a power of returning to the original condition of equilibrium is in every case possible and indeed necessary ${ }^{1}$.

A reduction of turgidity due to the vital activity of the plant itself must finally lead to a contraction of the protoplast similar to that caused by plasmolysis, and produced in the same way as when a diminution in the amount of substance dissolved in a vacuole causes it to become smaller. Examples of such contractions are afforded during the process of conjugation in Spirogyra, and also when cell-formation is accompanied by rejuvenescence.

Osmotic energy, it must be clearly borne in mind, is simply the mechanical means employed by the plant to attain various ends, and made use of in developmental processes, as well as in various reactions to stimulation. Any change taking place in the osmotic properties of either the cell-sap or plasma must cause a corresponding alteration in the tension of the stretched cell-wall and be followed by a readjustment of the entire osmotic system.

Historical. Nägeli was not only the first to recognize the extreme importance of the diosmotic peculiarities of the cell, but he also clearly understood the causes of turgidity, and recognized that further plasmolysis ceases as soon as the external fluid is isosmotic with the cell-sap 2. De Vries first used the term 'plasmolysis,' and the unexpectedly high values, which osmotic pressure was found capable of attaining, were shown by Pfeffer to be the natural consequences of the physical agencies at work $^{3}$. By de Vries the relative osmotic values of different substances were

1 See Pfeffer, Plasmahaut u. Vacuolen, I 890, p. 320 , and Studien z. Energetik, I 892.

${ }^{2}$ Nägeli, Pflanzenphysiol. Unters., I855, I, p. 2I. For details see Pfeffer, Plasmanant u. Vacuolen, I 890, p. 3 I6. Here it is also mentioned that Dutrochet was the first to use saline solutions to remove turgor.

${ }^{3}$ Pfeffer, Period. Beweg., I\$75, p. II 1 ; Physiol. Unters., I\$73, p. II9; de Vries, Unters. über d. mechan. Ursachen d. Zellstreckung, i 877 ; Pfeffer, Osmot. Unters., is 77 . 
determined, and the general relationship established between molecular weight and osmotic value. The knowledge of the fact, that in the absence of all exosmosis the membrane exercises no effect whatever upon osmotic pressure, first enabled an exact determination of the osmotic pressure in the cell to be made from physical data $^{1}$.

The physics of osmotic pressure. Physiology has to deal with osmotic pressure as a fact, which cannot be altered in the least by theoretical conclusions as to its origin. The whole phenomenon is most clearly and thoroughly explained by the theory advanced by van 't Hoff on the basis of Pfeffer's researches ${ }^{2}$. According to this theory, osmotic pressure arises in the same way that gaseous pressure does. In a closed semi-permeable cell, the molecules of a dissolved substance exert upon the limiting membrane a pressure similar to that which, according to Mariotte's law and Avogadro's hypothesis, the colliding molecules of a gas exercise upon the walls of the space in which they are confined. A cell filled with $\mathrm{CO}_{2}$ and $\mathrm{H}$, through the enclosing membrane of which the $\mathrm{CO}_{2}$ cannot diffuse but the $\mathrm{H}$ can, might represent a turgid cell; the stretching is due to the non-diosmosing $\mathrm{CO}_{2}$ alone, while the $\mathrm{H}$ does not diosmose, because the cell is supposed to be immersed in an atmosphere of this gas. One gramme-molecule of gas (i. e. of $\mathrm{CO}_{2}, 44$ grm.) occupies a space of 22.4 litres at $760 \mathrm{~m} . \mathrm{m}$. pressure and $\circ \mathrm{C}$., and hence exerts a pressure of 22.4 atm. when reduced to I litre. The same pressure must be exerted by a solution of $342 \mathrm{grm}$. of cane sugar in I litre of water. Hence, for a solution of I grm. of cane sugar in $100 \mathrm{c.cm}$. the pressure will be at $\circ^{\circ} \mathrm{C}$, $0.655 \mathrm{~atm}$., and at I $5^{\circ} \mathrm{C}$. $0.69 \mathrm{~atm}$. Pfeffer, by direct measurement, obtained values at this temperature of from 0.62 to $0.7 \mathrm{I}$ atm. Hence it follows that osmotic values may be calculated directly from physical data with perfect safety and accuracy.

The osmotic pressure is naturally dependent upon the amount of water present, and can indeed be regarded as the mechanical result of the endosmosis or exosmosis of water. Osmotic pressure is a function of the number of molecules, or of ions, in the unit of volume, and just as with gases, any variations from the laws regulating the relationships between molecular weight and gaseous or osmotic pressure, unavoidably postulate the occurrence of more or less marked molecular association or dissociation ${ }^{3}$. Steam pressure, electric conductivity, and atomic heat show similar molecular relationships.

The methods employed to determine osmotic pressure are given in the original work by Pfeffer, and reference may also be made to physical and chemical sources ${ }^{4}$.

1 Pfeffer, Plasmahaut u. Vacuolen, 1890, p. 302 ; Ostwald, Allgem. Chemie, 1891, 2. Aufl., Bd. I, p. 661. L. Meyer's contradictions (Sitzungsb. d. Berl. Akad., 1891, p. 993) rest upon an erroneous basis, as van 't Hoff (Zeitschr. f. physik. Chem., I 892 , Bd. $1 \mathrm{x}, \mathrm{p} .447$ ) has shown. Pfeffer (Osmot Unters.) assumed that the nature of the semi-permeable membrane influcnced the osmotic pressure, and hence the conclusions made with regard to the determination of osmotic pressure by reference to isosmotic values were logically correct, although it was also concluded that variations in the plasmatic membrane could have no marked influence upon the turgid pressure.

${ }^{2}$ See Ostwald, Allgcm. Chemie, $18_{91}$ r, 2. Aufl., Bd. I, 1. 67r. See also Pfeffer, Vacuolenhaut, I: 90, p. 318 .

${ }^{3}$ Cf. van 't Hoff, Ber. d. Chem. Ges., 1894 , p. 19.

${ }_{4}^{4}$ Ostwald, 1.c., p. 656; Winkelmann, Physik, I 891, 13d. I, p. 624. Incidentally (Plasmahaut u. Vacuolen, r 890 , p. 3 Io) it has been pointed out that, for physical experiments, a simpler and more 
Here also will be found the essentials concerning the formerly much discussed osmotic equivalent, and the sub-maximal osmotic powers of diosmosing substances.

Physiological estimations. By removing the turgor and determining the weight necessary to stretch a tissue to its original length, the work done in the living plant by osmotic energy may be calculated, while the osmotic value of a substance may be found by determining the concentration necessary to remove turgidity. For various reasons only approximately accurate results are obtained by these methods, but it was the high osmotic pressures found to exist in the plant which afforded the stimulus to the physical researches mentioned ${ }^{1}$.

Isosmotic determinations. The estimations of the osmotic value of different substances have for the most part been made by de Vries, who employed for this purpose epidermal cells with coloured sap, obtained from the leaf sheath of Curcuma rubricaulis, from leaves of Tradescentia discolor, and from the petiolar scales of Begonia manicata. Tradescentia discolor is especially useful, since it can always be procured. In the leaves of this plant, the cells of the upper epidermis, lying over and near the midrib, have comparatively the same turgidity (about 0.12 equivalent value ${ }^{2}=\mathrm{I} \cdot 2$ per cent. solution by volume of $\mathrm{KNO}_{3}$ ). If preparations are placed in beakers containing 5 to $10 \mathrm{c} . \mathrm{cm}$. of fluid, a condition of equilibrium is reached in from $\frac{1}{2}$ to $I \frac{1}{2}$ hours. By determining the degree of concentration necessary to cause most of the cells to show incipient plasmolysis (Fig. Io, p. I 16), the osmotic values of non-diosmosing and non-injurious substances may be obtained to within 0.01 to 0.02 equivalents $\left(=0.1\right.$ to 0.2 per cent. $\mathrm{KNO}_{3}$ ) of their actual value.

At the commencement of plasmolysis, the osmotic pressure of the external fluid is slightly higher than that of the turgid cell, even when the cell-wall does not undergo any elastic contraction. If a marked contraction occurs, the decrease in volume must be determined before an accurate estimation of the original turgid pressure is possible. Temporary variations of turgidity, due to transitory reactions, cannot be detected by the plasmolytic method ${ }^{3}$.

Changes of size and form, due to the action of the internal hydrostatic pressure, may be used to determine the value of the latter: thus in split stems, curvatures take place owing to the unequal tissue-tensions, and de Vries, by finding the degree of concentration of a plasmolysing fluid necessary to bring about a subsequent straightening, has been able to make a series of osmotic determinations (1. c., p. $4 \delta_{4}$ ). It is not necessary to make any mention of other physiological methods, or of the physical apparatus which may be employed for this purpose ${ }^{4}$.

readily attainable apparatus would be advantageous. It was with a similar apparatus to that employed by Pfeffer that Ladenburg (Ber. d. Chem. Ges., I889, p. 1225) and Adie (Beibl. z. Ann. d. physik. Chem., I89I, Bd. xv, p. 749 ) worked. Certain technical details are given by Wralden (Zeitschr. f. physik. Chem., 1892, Bd. x, p. ¡oo).

${ }^{1}$ See Pfeffer, Osmot. Unters., I877, I'reface. On such pressure determinations, cf. Pfeffer, Physiol. Unters., 1873, p. 122, and Period. Beweg., 1575, pp. 155, 111 ; Plasmahaut u. Vacuolen, 1870, p. 309 ; de Vries, Mechan. Unters. über Zellstreckung, I877, p. II 8 , and Jahrb. f. wiss. Bot., 1884 , Bd. X1V, p. 529. In these works additional references will be found.

${ }^{2}$ [Turgidity is measured in osmotic equivalents of $\mathrm{KNO}_{3}$, a decinormal solution (10.1 grm. in a litre of water) being taken as the unit of value.]

3 Pfeffer, Energetik, 1892, p. 228.

* Hamburger (Zeitschr. f. physik. Chemie, I89o, Bd, v1, p. 319) made use of the red bloodPFEFFER 
Osmotic values. In column III of the table below the molecular weight of each substance is given, and in column VI the weight which, when dissolved in a litre of water, produces a solution isosmotic with a decinormal solution of $\mathrm{KNO}_{3}$ (Io. $\mathrm{x}$ grm. in a litre of water). This contains nearly $\mathrm{x}$ per cent. of $\mathrm{KNO}_{3}$, and hence the approximate degree of concentration, necessary for a given solution to be isosmotic with a I per cent. solution of $\mathrm{KNO}$, can at once be seen. Column V gives the relative osmotic values, taking a I.OI per cent. $\mathrm{KNO}_{3}$ solution as unity.

\begin{tabular}{|c|c|c|c|c|c|c|c|c|}
\hline \multirow{3}{*}{ Substance. } & II & III & & 1V & $\mathrm{V}$ & WI & & VII \\
\hline & \multirow[t]{2}{*}{ Formula. } & \multirow{2}{*}{$\begin{array}{l}\text { Mole- } \\
\text { cular } \\
\text { weight. }\end{array}$} & \multicolumn{2}{|c|}{$\begin{array}{l}\text { Isosmotic } \\
\text { coefficient. }\end{array}$} & \multirow{2}{*}{$\begin{array}{l}\text { Value of a } \\
\text { 1 per cent. } \\
\text { solution in } \\
\text { relation to } \\
\text { 1.Or per } \\
\text { cent. KNO } \\
\text { solution. }\end{array}$} & \multirow{2}{*}{$\begin{array}{c}\text { Concen- } \\
\text { tration } \\
\text { isosmotic } \\
\text { with a } \\
\text { deci- } \\
\text { normal } \\
\text { solution } \\
\text { of } \mathrm{KNO}_{3} \text {. }\end{array}$} & \multicolumn{2}{|c|}{$\begin{array}{l}\text { Osmotic pressure } \\
\text { of } \mathrm{I} \text { grm. in } \\
100 \mathrm{cc} \text {. solution. }\end{array}$} \\
\hline & & & Found. & $\begin{array}{l}\text { In round } \\
\text { numbers. }\end{array}$ & & & Atmosp. & $\mathrm{Cm} \cdot \mathrm{Hg}$. \\
\hline Cane sugar & $\mathrm{C}_{12} \mathrm{H}_{2 i} \mathrm{O}_{11}$ & $34^{2}$ & 1.88 & 2 & 0.195 & $5 \cdot 13$ & 0.69 & $52 \cdot 4$ \\
\hline Dextrose and laevulose & $\mathrm{C}_{6} \mathrm{H}_{12} \mathrm{O}_{6}$ & I So & 1.88 & 2 & 0.37 & $2 \cdot 70$ & $I \cdot 25$ & $99 \cdot 5$ \\
\hline Glycerine & $\mathrm{C}_{3} \mathrm{H}_{8} \mathrm{O}_{3}$ & 92 & $1 \cdot 78$ & 2 & 0.73 & $\mathrm{I} \cdot 39$ & $2 \cdot 5+$ & $193 \cdot 3$ \\
\hline Citric acid & $\mathrm{C}_{6} \mathrm{H}_{8} \mathrm{O}_{7}$ & $19^{2}$ & 2.02 & 2 & $0 \cdot 35$ & 2.88 & 1.23 & $93 \cdot 3$ \\
\hline Tartaric acid & $\mathrm{C}_{4} \mathrm{H}_{6} \mathrm{O}_{6}$ & 150 & $2 \cdot 02$ & 2 & 0.44 & $2 \cdot 25$ & $1 \cdot 57$ & 119.4 \\
\hline Malic acid & $\mathrm{C}_{4} \mathrm{H}_{6} \mathrm{O}_{5}$ & I 34 & $1.9^{8}$ & 2 & 0.50 & 2.01 & $1 \cdot 76$ & I $33 \cdot 7$ \\
\hline Oxalic acid & $\mathrm{C}_{2} \mathrm{H}_{2} \mathrm{O}_{4}$ & 90 & & 2 & $0.7 t$ & $\mathrm{I} \cdot 35$ & 2.62 & $199 \cdot 0$ \\
\hline Potassium nitrate & $\mathrm{KNO}_{3}$ & IOI & $3 \cdot 0$ & 3 & 0.99 & I.OI & $3 \cdot 50$ & $266 \cdot 0$ \\
\hline Sodium nitrate & $\mathrm{NaNO}_{3}$ & $\delta_{5}$ & $3 \cdot 0$ & 3 & I. I 8 & 0.85 & $+\cdot 16$ & 316.1 \\
\hline Potassium chloride & $\mathrm{KCl}$ & $7+5$ & $3 \cdot 0$ & 3 & $1 \cdot 34$ & 0.74 & +77 & $363 \cdot 0$ \\
\hline Sodium chloride & $\mathrm{NaCl}$ & $5^{8 \cdot 5}$ & $3 \cdot 0$ & 3 & $I \cdot 7 \mathrm{I}$ & $0.5^{8}$ & 6.09 & $4^{6} 3_{3} \cdot 2$ \\
\hline Ammonium chloride & $\mathrm{NH}_{4} \mathrm{Cl}$ & $53 \cdot 5$ & $3 \cdot 0$ & 3 & $\mathrm{I} \cdot 87$ & 0.53 & 6.67 & $506 \cdot 3$ \\
\hline Potassium bicitrate & $\mathrm{KH}_{2} \mathrm{C}_{6} \mathrm{H}_{5} \mathrm{O}_{7}$ & I 30 & $3 \cdot 05$ & 3 & 0.77 & $I \cdot 30$ & $2 \cdot 7^{2}$ & $206 \cdot 7$ \\
\hline Potassium oxalate & $\mathrm{K}_{2} \mathrm{C}_{2} \mathrm{O}_{4}$ & 166 & $3 \cdot 93$ & 4 & 0.80 & $1 \cdot 2+$ & 2.85 & $216 \cdot 7$ \\
\hline Potassium sulphate & $\mathrm{K}_{2} \mathrm{SO}_{4}$ & I 74 & $3 \cdot 90$ & 4 & 0.77 & $1 \cdot 30$ & $2 \cdot 7^{2}$ & $206 \cdot 7$ \\
\hline Acid potassium phosphate & $\mathrm{K}_{2} \mathrm{HPO}_{4}$ & 174 & $3 \cdot 96$ & 4 & 0.77 & $1 \cdot 30$ & $2 \cdot 7^{2}$ & $206 \cdot 7$ \\
\hline Basic potassium tartrate & $\mathrm{K}_{2} \mathrm{C}_{4} \mathrm{H}_{4} \mathrm{O}_{6}$ & 226 & 3.99 & 4 & 0.59 & 1.69 & 2.09 & I $59 \cdot 0$ \\
\hline Basic potassium malate & $\mathrm{K}_{2} \mathrm{C}_{4} \mathrm{H}_{4} \mathrm{O}_{5}$ & 210 & $4 \cdot I I$ & + & 0.63 & I.57 & 2.25 & 171.1 \\
\hline Potassium citrate & $\mathrm{K}_{2} \mathrm{HC}_{6} \mathrm{H}_{5} \mathrm{O}_{7}$ & 268 & 4.08 & 4 & 0.50 & 2.01 & $1 \cdot 75$ & $133 \cdot 6$ \\
\hline Basic potassium citrate & $\mathrm{K}_{3} \mathrm{C}_{6} \mathrm{H}_{3} \mathrm{O}_{7}$ & 306 & $5 \cdot 01$ & 5 & 0.54 & I. $8_{4}$ & $I \cdot 9^{2}$ & $146 \cdot 0$ \\
\hline Magnesium malate & $\mathrm{MgC}_{4} \mathrm{H}_{4} \mathrm{O}_{5}$ & I 56 & 1.88 & 2 & 0.43 & $2 \cdot 35$ & $1 \cdot 51$ & I I $4 \cdot 8$ \\
\hline Magnesium sulphate & 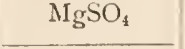 & I 20 & $\mathrm{I} \cdot 96$ & 2 & $0.5^{6}$ & $\mathrm{I} \cdot \mathrm{SO}$ & $1 \cdot 93$ & $149 \cdot 2$ \\
\hline Magnesium citrate & $\mathrm{Mg}_{3}\left(\mathrm{C}_{6} \mathrm{H}_{5} \mathrm{O}_{7}\right)_{2}$ & $45^{\circ}$ & $3 \cdot 88$ & 4 & 0.30 & $3 \cdot 37$ & 1.05 & $79 \cdot 7$ \\
\hline Magnesium chloride & $\mathrm{MgCl}_{2}$ & 95 & $4 \cdot 33$ & 4 & $I \cdot 40$ & 0.71 & 4.99 & $37^{8} \cdot 4$ \\
\hline Calcium chloride & $\mathrm{CaCl}_{2}$ & I I I & $4 \cdot 33$ & 4 & $I \cdot 20$ & $0.8_{3}$ & $4 \cdot 26$ & $323 \cdot 6$ \\
\hline $\begin{array}{l}\text { Gum-arabic } \\
\text { Dextrin }\end{array}$ & & & & & & $\begin{array}{l}4^{I} \cdot 32 \\
16 \cdot 18\end{array}$ & $\begin{array}{l}0.08_{5} \\
0.218\end{array}$ & $\begin{array}{r}6.5 \\
\text { I } 6.6\end{array}$ \\
\hline
\end{tabular}

corpuscles. See also Koppen (ibid., 1895, Bd. Xv1, p. 261). Wladimiroff attempted to found a method upon certain peculiarities shown by bacteria (ilid., I $\$ 9 \mathrm{I}$, Bd. vil, p. 528), which is, however, unreliable (A. Fischer, Jahrb. f. wiss. Bot., 1895, Bd. xxv11, p. I53).

For physical methods see Ostwald, Lehrb. d. allgem. Chemie, Iigr, 2. Aufl, Bd. 1, p. 666. By means of certain definite relationships existing between vapour tension and osmotic pressure, the average turgidity existing in a tissue could be calculated in terms of osmotic value from data of vapour tension. 
Owing to the occasional occurrence of dissociation, a constant relationship does not always exist between the osmotic values of substances and their molecular weights. Thus, using the coefficients given in column IV, the weight of cane-sugar (VI) isotonic with the saltpetre unit may be obtained by multiplying the molecular weight of the cane sugar by $\frac{3}{2}$. It is true that the vapour tensions, electric conductivities, \&c., of different substances indicate that the relationships existing between the latter cannot always be represented by definite coefficients in the form of whole numbers. Nevertheless, the empirical values (column IV) do not markedly differ from the round numbers found by experiment, at least for the substances given. For physiological purposes approximate values only are necessary, and hence in columns V-VII the values obtained from these coefficients in relation to saltpetre are given. From the considerations already mentioned ${ }^{3}$, it follows that the usage of these coefficients, and the corresponding osmotic grouping of different substances which they render possible, are of great importance from a physiological point of view.

From the estimated pressure of cane-sugar (p. 146), and from the relative values given in column VI, the osmotic pressure exerted by solutions containing $\mathrm{I} \mathrm{grm}$. in $100 \mathrm{c.cm}$. of water is calculated in atmospheres and in centimetres of Mercury. The pressures obtained by Pfeffer for gum-arabic and dextrin are also added, and from them the values given in column VI for these substances have been calculated.

\section{Section 25. Specific Powers and Activities.}

In all plants which exhibit functional division of labour, the different members are concerned in varying degrees with absorption and excretion. Such activity is in general determined and regulated by the arrangements and factors already described, i.e. chiefly by the specific needs and metabolic activities of the given part, by the readiness with which gases, as well as fluid and dissolved substances, diffuse towards the interior or the exterior of the plant, and by the quality and composition of the surrounding medium. It is obvious that the sub-aërial parts will be chiefly of importance for gaseous exchange, including under this head all volatile materials, while water and dissolved substances must be absorbed mainly by the subterranean or submerged organs. Different members when in contact with water do not necessarily absorb substances in equal amount, for absorption is influenced both by diosmotic properties and by specific metabolic activity. Hence in the cells of a Confervoid filament, provided they are not functionally

1 The table is compiled from the results obtained by de Vries (Jahrb. f. wiss. Bot., $188_{4}$, Bd. XIv, p. 536; Bot Zeitung, I 888 , p. 229). The pressures exerted are giren from the values calculated by Pfeffer. 
precisely similar, quantitative differences at least must exist in their absorptive and excretory powers, and even in the submerged non-septate Caulerpa a differentiation of this character exists between the root and shoot parts, while in young non-septate Mucor plants the differences existing between the sub-aërial hyphac and the subterranean mycelium are still more marked.

Under normal conditions, the functional powers of which a plant is capable are not all exercised, or at least not to the full possible extent. Thus, aquatic organs are able to absorb gases directly from the air, while sub-aërial organs can absorb water and dissolved substances to a greater or less extent. Probably this power is not entirely absent from any leaf or young stem, provided that, if necessary, wetting is rendered certain by the removal of any waxy layer present. Nevertheless the cuticle and cork commonly so diminish the power of absorbing fluids, that even under the most favourable experimental conditions the loss of water by transpiration cannot be made good by absorption through these layers, nor can a sufficient supply of nutrient material be absorbed through cuticularized epidermis.

The members of a plant must necessarily be so constructed as to be able under normal conditions to satisfy their own special needs and the general requirements of the entire organism. Full attention must therefore be paid to the importance and function of each organ, to the source and utilization of water and nutrient material, as well as to the nature of the surrounding medium, and the external influences which operate upon the organ in question. All these factors together enable us to form a correct general estimate of the demands made upon the functional activities of any given organ. From considerations of this kind, it is readily explicable that in sub-aërial organs the necessity of protection against excessive transpiration causes the absorptive power for water and salts to decrease almost to nil, while the parts growing in water or soil are incapable of a sub-aërial existence either entirely or as far as young absorptive parts are concerned, owing to the readiness with which they lose water by evaporation. In general, when a particular purpose is in view, the other needs of the organ, as well as the general conditions for existence, must be taken into consideration, and hence the existence of a certain correlation of growth between the root and shoot is essential. The former, as it develops more fully, affords a firmer support and acquires an increased power of absorption. Moreover, as has already been mentioned in more general terms (Sect. 6), members of unequal morphological value may be entrusted with similar physiological functions and vice versa.

The different parts of an organ are not however of equal functional importance. Those parts on which cork and cuticle are best developed are the least permeable to water and dissolved salts, as well as to gases and 
water-vapour. Hence as a general rule, the older parts of the root are of only trifling importance for the absorption of water and salts, while the root hairs, which die off further behind, markedly increase the absorptive power of the younger parts. Wherever an exchange of water and salts is necessary (Sect. 2I), the cuticle remains permeable, but in other cases the permeability for water and salts, and also for gases and water-vapour, is more or less markedly diminished by the impregnation of the cuticle with waxy and other substances. This is generally the case in the sub-aërial parts, in which, however, at least in higher plants, the stomata and lenticels afford open passages for gaseous exchange and for the exit of water-vapour.

Absorption and excretion are directly or indirectly influenced by all agencies which awake special activities in the plant, or divert pre-existent ones into new paths. The progress of development brings about modifications in the quality of the absorptive membranes, or in the vital activities of the plant or its members, or even causes certain organs to be exposed to changed conditions of environment. In regard to the latter, it is sufficient to recall the fact that the cotyledons of Ricinus and Pinus at first absorb nourishment from the endosperm and later on function as green foliage leaves, and that the young leaves of Butomus and Nymphaea are at first immersed in water and subsequently float on the surface or rise above it. It is not necessary to depict the various other changes which the progress of development may bring about, or to enumerate the plants which are able to live on land as well as in water, or those which after a parasitic stage continue their life cycle as saprophytes.

It does not belong to general physiology, but to special biology, to describe the varied adaptations by which individual plants ensure for themselvcs an adequate supply of nutriment and water, or the means by which the necessary exchanges with the external world are maintained. Hence only a few features of general importance can be dealt with here, and since parasites and saprophytes may be more appropriately discussed in connexion with the absorption of organic nutriment, no special mention need now be made of them.

\section{SECTION 26. The Importance of the Root-system.}

The differentiation into root and shoot is accompanied by a marked division of labour. Thus in terrestrial plants the roots usually fix the plant to the soil and absorb water and nutritive material, while in order to satisfy the greater demands made upon it as the developing shoot system above ground increases in size, the root system must grow and become stronger, so as to provide a firmer attachment and to render possible an increased 
absorption of water and salts. The subterranean absorptive and anchoring root-systems of both the higher and lower plants develop according to these principles, and indeed the same laws govern the development of the absorptive organs of fungi, organisms which can only absorb their nutriment from organic substances. The many and varied specific peculiarities presented by different root-systems cannot be discussed or even indicated here, but instead it must suffice to give an account of the typical root-system of one of the higher plants.

The formation of lateral roots commences shortly after the radicle penetrates the soil ${ }^{1}$. These increase in length so as to cover a large area, while by means of tertiary branches of limited growth, which grow out in all directions, a very thorough absorption of moisture becomes possible ${ }^{2}$. Root-hairs arise from the young roots of most terrestrial plants, and burrow between the particles of earth, so that in this way the extent of absorptive surface may be markedly increased, often as much as from five to twelve times ${ }^{3}$. These root-hairs come into very close connexion with the earthy particles, which often become partially imbedded in their walls, and may cause the root-hairs to assume very peculiar shapes. Frequently, it is impossible to remove all of the particles without injuring the root-hair ${ }^{4}$ (Fig. II).

The parts of the root are only of importance for absorption during a limited period, for as the well-known change of colour indicates, when the root grows older and deep-seated cork ${ }^{5}$ is formed, the cortex, epidermis and root-hairs die and peel off, while at the same time the absorptive power almost entirely disappears. Since, moreover, the smaller absorptive roots usually die after having persisted for a certain length of time, it is only by continued growth and by the formation of new roots that an adequate functional activity of the root-system is maintained, so that as the area of ground covered becomes larger, the extent of absorptive surface also increases. It is owing to this close union between the root-hairs and the soil particles that when roots are carefully lifted from the soil, the latter remains attached wherever root-hairs are present ${ }^{6}$. Thus in the seedling of Sinapis shown

1 The absorption of water by seeds, and their swelling, cannot be entered into. The seed-coats in many cases only allow water to penctrate with difficulty, so that when incisions are made in them, the seeds swell much more rapidly. In other cascs, water penetrates mainly or more rapidly at certain points. See Detmer, Physiologie des Keimungsprocesses, I 880 , and the literature quoted in Sect. I 2. On the existence of special regions for the absorption of water, see Mattirolo and Buscaloni, liot. Zeitg., I890, p. 397 ; Haberlandt, Physiol. Anat., I884, p. 3I3. On the way in which germinating seedlings become attached, see Kklebs, Unters. a. d. Bot. Inst. z. Tübingen, I 885 , I, p. 537, \&c.

2 Th. Hartig, Anat. u. Physiol. d. Holzpflanzen, 1878, p. 251 ; Resa, Úber die Periode der Wurzelbildung, Bonner Dissertation, I877; Frank, Lehrb., I 892 , Bd. I, p. 3 I6.

${ }^{3} \mathrm{Fr}$. Schwarz, Unters. a. d. Bot. Inst. z. Tübingen, i $8 s_{3}$, Bd. 1, p. I 40.

4 See F. Schwarz, 1. c., pp. 142, I79.

${ }^{5}$ Höveler, Bot. Zeitung, 1877, p. 785 .

- Treviranus, Physiologie, 1838 , Bd. II, p. I13. By imbedding in gelatine to which formic 
in Fig. 12 this covering is present all over the root, with the exception of the extreme apex where no root-hairs are present, whereas in the older root-system of Triticum shown in Fig. 13 the soil remains attached only to the younger parts to which root-hairs are now restricted.

From the above it is clear why plants wither much sooner when only the older parts are submerged, than when the younger parts are in contact with water. This was first observed by de la Baisse ${ }^{1}$, who passed the younger roots through the tube
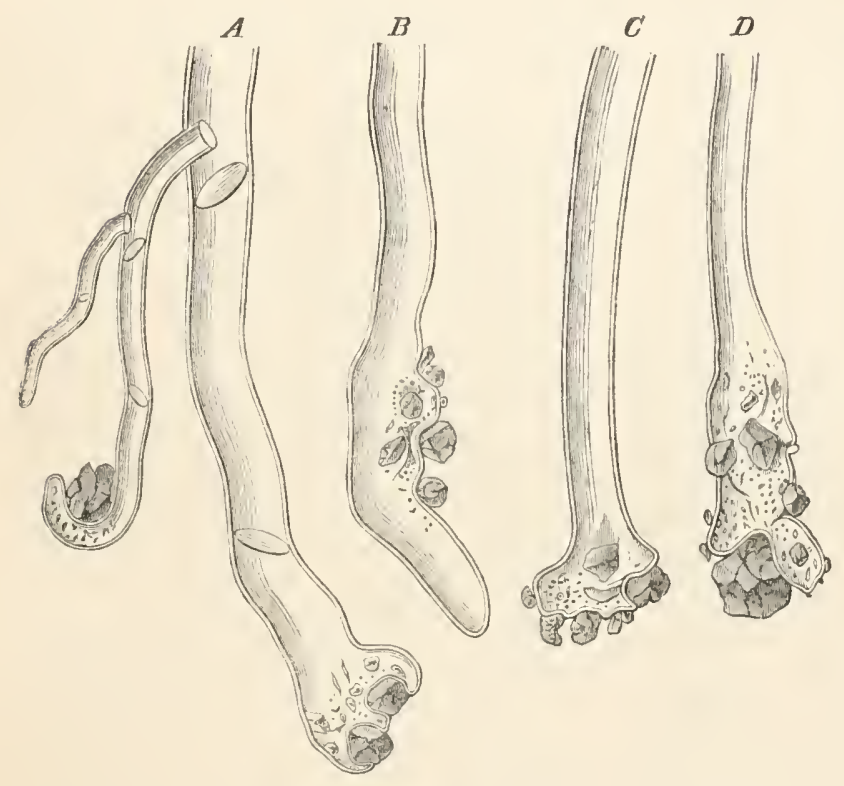

FIG. 11. A, rhizoid of Polytrichum juniperinum; $B$, of Marchantia polymorpla $\left(\times 240 . C_{1}\right.$ root-hair of Poa annua; and D of Draba verna $(\times 320$ ! The preparations are obtained by shaking gently in water.

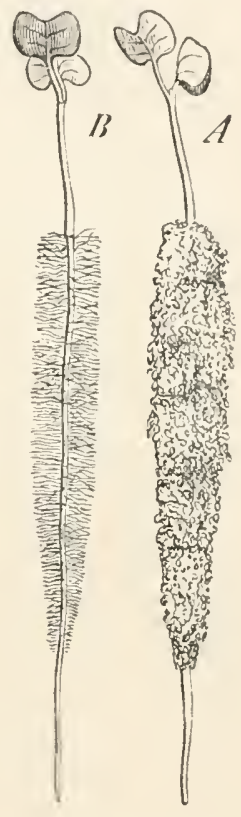

FIG. 12. Seedlings of Sinapis alba grown in sand. $A$ has been gently shaken to remove the superfluous sand particles. In $B$, the sand particles have been removed by gentle agitation in water. (After Sachs.)

of a filter-funnel, and closed the mouth of the tube, so that, on filling the funnel, only the older roots were in contact with water. By means of aniline dyes capable of absorption, it may be shown that, even after several days, no perceptible amount penetrates the older parts of the root, whereas in the younger parts, the

aldehyde has been added, permanent preparations suitable for demonstration may be made. For such purposes it is best to use roots grown in emery powder.

1 De la Baisse, in Duhamel, Naturgesch. d. Bäume, $176_{5}$, Bd. II, p. 148. Similar researches on horse-radish roots are given by Senebier (Physiol. végét., ISoo, T. I, P. 3II) and by Meyen (Physiol., is 38 , Bd. II, p. I 8 ). 
dye is rapidly absorbed ${ }^{1}$. By similar experiments, as well as by the lessened rapidity with which plasmolysis is produced, it may be seen that in Confervae,

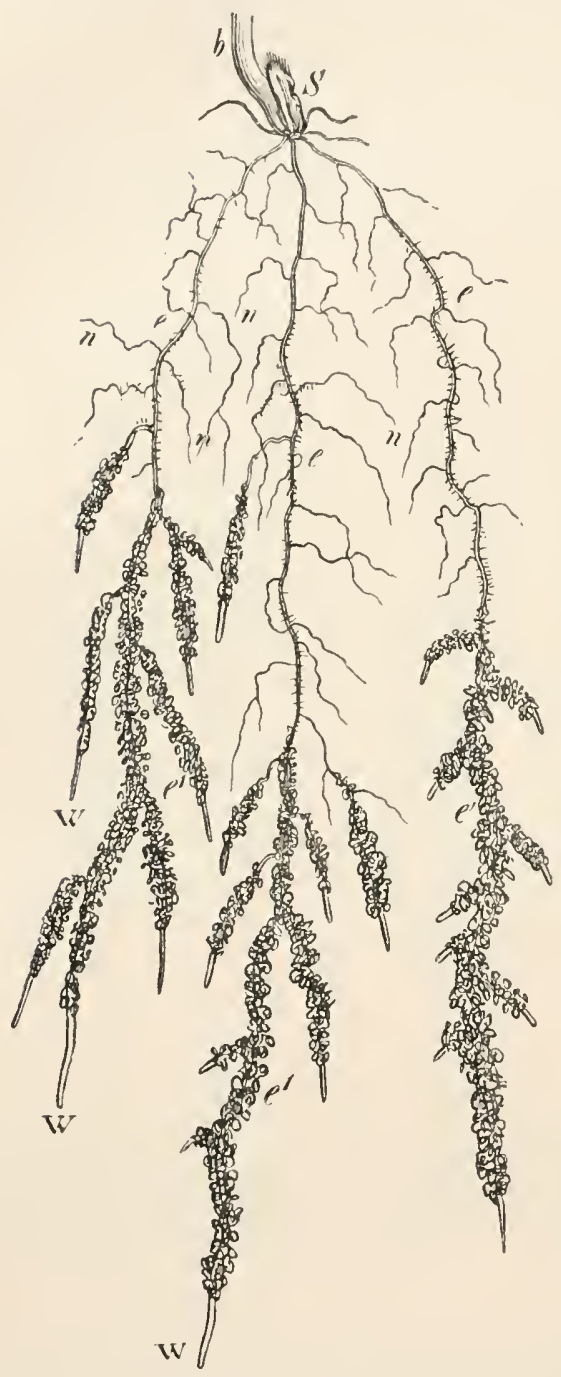

FIG. 13. Seedlings of Triticume vulgare grown in garden humus. After shaking, the earth adheres only to the portions bearing root-hairs, $e^{\prime}$. At $e$ no root-hairs are present and the lateral roots have in part died away. (After Sachs.) rhizoids of mosses, \&c., as a general rule the permeability of the cell-wall decreases as it grows older. The supposition, originally put forward by Grew, and supported by de Candolle ${ }^{2}$, that the root-cap acted like a sponge and absorbed the major part of the water and salts which the plant requires, is incorrect, and its falsity has been experimentally proved by Ohlert ${ }^{3}$.

Specific types of root-system are presented by different plants. Thus many plants have a poorly developed root-system, which soon ceases to grow, while other plants continue to acquire new territory for a long period of time, and ultimately produce very large rootsystems. In other plants again, such as the Clover or the red Fir, the tap-root buries itself very deeply and hence absorbs nutriment from the deeper layers of the soil, into which plants with more horizontal root-systems do not penetrate. The latter, however, in the Pine and the Poplar, cover a large surface area, as is immediately obvious when the roots of the Poplar appear projecting above the surface of the ground.

Very often, it is true, plants are forced to accommodate their root-systems to the conditions existing in the soil, and hence no constant relation exists between the sub-aërial and the underground organs of

1 Pfeffer, Unters. a. d. Bot. Inst. z. Tüibingen, I886, Bd. II, p. 201.

${ }^{2}$ De Candolle, Organographie végét., 1827 , T. I, p. 260. [Hence the old term 'spongioles.']

${ }^{3}$ Ohlert, Linnaea, $18_{37}$, Bd. xi, p. 62 r. On roots without a root-cap, see Waage, Ber. d. Bot. Ges., I 89 I, p. 132 . 
a given plant. The relative development of the root-system varies widely in different species, and not infrequently an extremely marked development of the root-system is characteristic of plants which do not develop far above the surface of the ground. This is, for example, the case in many mosses and fungi, while, as is well known, the mycelium of a mushroom encompasses a very large area of soil.

To indicate the marked development the root-systen may attain in a short period of time, Nobbe's ${ }^{1}$ comparative observations upon the Pine (Pinus sylvestris, flat root-system) and upon the Fir (Pinus abies, deep root-system) may be mentioned. The measurements were made on one-year-old seedlings grown in sand moistened with nutrient solution. It was found that the total length of the roots formed in one year was, in the pine, 12 metres, in the fir, 2 metres, with a total surface area of $20,5{ }^{1} 5 \mathrm{sq}$. mm. and $4, \mathrm{r} 39 \mathrm{sq}$. mm. respectively. 'The roots of the pine in six months develop throughout a volume of soil, represented by a reversed cone 80 to $90 \mathrm{~cm}$. high, and with almost 2,000 sq. $\mathrm{cm}$. surface. It is hence easy to understand why the pine is able to grow in poor soil, and in spite of the superficial distribution of its roots is nevertheless adequately fixed and anchored to the ground.

In rapidly growing plants, an even more marked development may be attained during a single summer's vegetative activity. Thus, Nobbe estimates the total length of the root-systems of ripe cereals at from 500 to $600 \mathrm{~m}$; S. Clark, for a large water-melon, at 25 kilometres, so that such a plant may easily have directly drained a cubic metre of earth. It may also be mentioned that, in deep and loose sandy soil, the roots of Barley and Mustard penetrate to a depth of I metre, those of the perennial Clover and of Lathyrus sylvestris to from 2 to 3 metres. Schumacher has constructed tables giving the comparative dry weights of the root and shoot systems of different plants ${ }^{2}$.

External conditions may, as is well known, markedly modify the development of the root-system, and frequently, owing to the impossibility of further penetration, the roots are restricted to particular layers of soil. Thus when a tap-root meets a rock, it branches out over the impenetrable surface and forms a flat root-system. Besides these mechanical agencies, the growth and character of the root-system may be influenced by injuries, and directly or indirectly in a variety of other ways. Moreover, all those correlative influences come into play in the root, which arise from its relationships to the parts above ground, and from the linked and interacting

1 Nobbe, Versuchsst., I875, Bd. xvili, p. 279.

${ }^{2}$ Of the literature may be quoted: Fraas, Wurzelleben der Culturpflanzen, 1870 ; Hellriegel, Jahresb. d. Agr.-Chem., I864, p. Io7 ; W. Schuhmacher, ibid., I\$67, p. 83 ; Nobbe, Versuchsst., I872, Bd. Xv, p. 39r ; Thiel, Landw. Centralbl., I87o, Ir, p. 249, and the figures from the "Wandtafeln' of Nathusius, iv. Ser., I875; H. Miiller, Landw. Jahrb., 1875, IV, p. 399; Resa, Über die Periode d. Wurzelbildung, I 877 ; Höveler, Jahrb. f. wiss. Bot., I 892 , Bd. xxiv, p. 296 ; Frank, Lehrb., I892, Bd. I, p. 306 ; Gain, Ann. d. sci. nat., I 894 , vii. sér., T. xx, p. 63. 
functional activities of the root and shoot systems. The manner in which external conditions affect growth will first be dealt with subsequently, and at the same time it will be shown that a modification of growth is often the result of the action of different and varying factors, and that these commonly cannot be clearly distinguished from one another. In addition to the direct and indirect mechanical effects already noticed, the growth and development of the roots and all underground parts are dependent upon the amount of water present in the soil, while the branching throughout the soil is influenced by the amount and character of the food material, as well as by the quality and concentration of the water of the soil, by the aeration of the soil, by its temperature, and in certain cases by the penetration of light. The power of reaction and accommodation to these and other conditions is naturally of the utmost importance, and it is well known that very many plants grow, and find all the conditions necessary to their existence, in soils and media of widely different character.

The resistance of an ordinary soil and even of stiff clay is in general easily overcome by the roots, but when the resistance offered is considerable, the plastic root apices bend to one side, and by continued growth in length may creep round the obstaclc. If no lateral curvature is possible, the turgid and swollen root apex may burrow its way through soft tufa (solidified volcanic mud) or even through the tissues of a living plant ${ }^{1}$.

In potted plants the centrifugally growing roots apply themselves closely to the sides of the pot with which they come in contact, and thus form against it a closely felted mass of roots. In spite of this, such plants have subservient to them a much smaller area of soil than others growing in the open, and hence old pot plants, which have fallen off in vigour, may be strengthened by removal to a larger pot, or by giving them a supply of nutrient salts ${ }^{2}$. Such plants also become stronger if the roots are allowed to grow through the hole at the bottom of the pot into the soil beneath, and this method of cultivation is often well adapted to obtain strong plants for experimental purposes.

Since growth is retarded in dry soils, the root-system develops more markedly in the moister layers, to which young roots are attracted by their hydrotropism. It is the action of moisture which induces the development of roots on the rhizophores of Sclaginclla, and no doubt it is for a similar reason that aërial roots branch vigorously when they penctrate the ground ${ }^{3}$. In some cases, plants which grow well in water-

1 Details by Pfeffer, Druck und Arbeitsleistungen, I 893, p. $3^{62}$; Peirie, Bot. Zeitg., I 894, p. I69. On the rhizoids of mosses, see Haberlandt, Jahrb. f. wiss. Bot, I886, Bd. Xvı1, p. 476 ; Höveler, ibid., 1892 , Bd. xxiv, p. 296 . On the penetration by fungi, \&c., see Sect. 65 .

${ }^{2}$ Lindley, Theory of Gardening, $1 \$_{42}$, p. 189 ; Sachs, Flora, $189^{2}$, p. I 73 ; C. Kraus, Forsch. a. d. Geb. d. Agr.-Physik, I894, Bd. Xvir, p. 55.

${ }^{3}$ Pfeffer, Arb. aus Wiirzburg, 1871 , Bd. I, p. 97. See Schimper, Bot. Centralbl., I $88_{4}$, Bd. xvit, p. $2 \delta_{5}$, and Bot. Mitth. a. d. Tropen, I 888 , Heft 2. 
cultures develop badly or not at all in soil over-saturated with water, and this is probably due to insufficient aeration, that is to a deficiency of oxygen ${ }^{1}$.

In water-cultures, there is necessarily a certain concentration at which the optimal development of the root-system takes place, for in too dilute a solution active growth is impossible, while, when the concentration is excessive, growth is inhibited and finally ceases. Nobbe ${ }^{2}$ found that, with Barley and Buckwheat, the best development of the root-system was produced in a nutrient solution containing from one-half to two parts of inorganic salt per thousand of water, while in a solution of ten per thousand, the lateral roots were unable to continue their development.

For similar reasons, too high or too low a percentage of dissolved salts in the soil must hinder development, and hence in a very poor soil the root-system develops badly ${ }^{3}$. In producing such results other special actions apparently come into play: thus roots which grow through alternating layers of sand and earth branch more markedly in the latter. This can only be due to a local stimulus exercised either by the nutriment as a whole, or by certain special substances, for the contact with solid substances and the presence of water act in the layers of sand also, as well as in a layer of humus poor in nutrient salts, in which the branching is similar to that in sand. That specific chemical stimuli may influence growth is well known, and indeed without such a stimulus the seeds of Orobanche cannot germinate (cf. Sect. 64). It is obviously of the utmost advantage in the economy of the plant that a richer soil should induce a better development of the root-system.

Nobbe ${ }^{4}$ performed similar researches with Maize and Clover, employing layers of the same soil placed in a box with and without previous saturation with nutrient material. In the soil with less nutriment, the development and branching of the root-system was less marked. The same was observed by Thiel and by Höveler in alternating layers of sand and humus. The absence of a single essential nutrient substance apparently brings about a similar result, and this is shown by certain of Frank's researches, in which one portion of the root-system of the same plant (Maize and Peas) grew in soil containing nitrates, the other in soil in which no nitrates were present ".

1 Cf. Waeker, Jahrb. f. wiss. Bot., I 898 , Bd. xxxı, p. 7 I.

${ }^{2}$ Nobbe, Versuchsst., I864, Bd. vi, p. 22 ; Wieler, Bot. Zeitg., I889, p. 550. In some cases, roots which pass from the soil into water become markedly elongated, as Duhamel first noticed (Naturgesch. d. Bäume, 1764, Bd. I, p. 107).

${ }^{3}$ Cf. Fr. Schwarz, Zeitschr. f. Forst- u. Jagdwesen, I892, p. 89 (for Pinus sylvestris). Kinight (Phil. Trans., i 8 I I, p. 21 I) long ago observed that roots grow more strongly in good soil.

4 Nobbe, Versuchsst., 1862, Bd. IV, p. 217 , and 1869, Bd. x, p. 94 . Similar experiments by Stohmann, Jahresb. d. Agr.-Chemie, I 868-9, p. 242.

5 Thiel, quoted by Sachs, Exp. Physiol., 1865, p. 17 8 ; Höveler, Jahrb. f. wiss. Bot., I892, Bd. xxıv, p. 294 ; A. B. Frank, Bot. Zeitg., I 893 , p. I 53. 
The formation of root-hairs is also influenced by the external conditions. The most marked development of root-hair takes place on roots grown in air saturated with water-vapour, or in moderately dry soil ${ }^{1}$. The roots of Zea mays, Cucurbita pepo, Acorus calamus, form root-hairs in soil, but in a normal water-culture they may be entirely or partly absent. A lack of moisture, as well as too high concentration of the water in the soil and too great mechanical resistance, hinder the formation of root-hairs ${ }^{2}$. Aërial roots form root-hairs at the points of contact with soil or damp walls because more moisture is present here, and not owing to the absence of light, or to the action of a contact-stimulus, at least in those cases which have been closely investigated ${ }^{3}$. The further growth of the root-hairs is influenced by contact, as might be expected.

We shall see later (Sect. 65) that the mycorhizae, formed on the roots of forest trees by symbiotic union with fungal hyphae, act in a similar manner to root-hairs, and it is possible that by means of such mycorhizae, especially when endophytic, the older roots may remain capable of absorbing water and dissolved substances.

The character of a root-system is the result of the interaction between its inherent specific peculiarities and the external conditions under which its development takes place. It is owing to the latter that the root or rhizomes of the same plant penetrate more deeply in loose soil than in tough clayey loam, and that in its lateral development the root-system does not maintain the same distance from the surface at all points. This distance is mainly influenced by the aeration and distribution of water, but temperature and illumination are also of importance. An exact determination of the precise influence of each different factor is extremely difficult, for abundant aeration retards nitrification, while other agencies, including putrefactive changes, may alter the conditions existing in the soil.

The shape and position of the root-system must obviously be adapted as far as possible to the external conditions, and thus in cereals the main development of the root-system occurs in the same layers of the soil, whether the seeds were sown near the surface or buried deep in the ground ${ }^{4}$ (Fig. I4), while when a rhizome is planted at some distance below the surface, the new increments are directed upwards until the normal depth

1 Observed long ago by Unger, Anatomie, 1855, p. 309. For details see Fr. Schwarz, Unters. a. d. Bot. Inst. z. Tïbingen, $188_{3}$, Bd. 1, p. 135, where the literature is given.

${ }^{2}$ Nobbe, Versuchsst., I862, Bd. IV, p. 217 , and I 868 , Bd. x, p. 94; Pfeffer, Druck und Arbeitsleistung, I 893 .

${ }^{3}$ See Fr. Schwarz, 1.c., p. 148 . Cf. also Went, Bot. Centralbl., 1894 , Bd. Lix, p. 367 . [In the aerial roots of Vanilla aromatica, moisture is essential for the formation of the root-hairs; darkness and contact accelerate, while light and dryness retard it : Ewart, Contact Irritability, Ann. d. Jard. bot. de Buitenzorg, 1898 , p. 236 .]

4 Literature: G. Kraus, Forsch. a. d. Geb. der Agr.-Physik, I889, Bd. XII, p. 259 ; 1894 , Bd. xvir, p. 35 ; 1896 , Bd. Xix, p. I7 ; Kossowitsch, ibid., IS94, Bd. XVII, p. I04. 
is attained, when further growth is horizontal. Nevertheless, a grain of wheat which is sown at too great a depth, even if it germinates, may not be able to reach the surface, or if it does, it will suffer from a certain disadvantage as compared with seedlings which germinated at a more favourable depth. It is moreover easy to see why the roots may develop badly or die when they are buried too deep in transplanting, or when soil is heaped around the base of the stem. Practical experience tells us that deeply rooting plants can more readily accommodate themselves to a superficial development of the root-system, than the reverse.

The power of adaptation is of the highest biological importance, and may be so marked that typical land-plants can be cultivated in water (Sect. 73), and may even accommodate themselves to the changed conditions, when the roots grown in soil are suddenly brought into water or vice versa. It is easy to understand that such a sudden change may often produce transitory ill effects, and that when transferred from water to soil, the plants may wither because the roots and root-hairs are not in proper contact with the particles of soil. On the other hand, when placed in

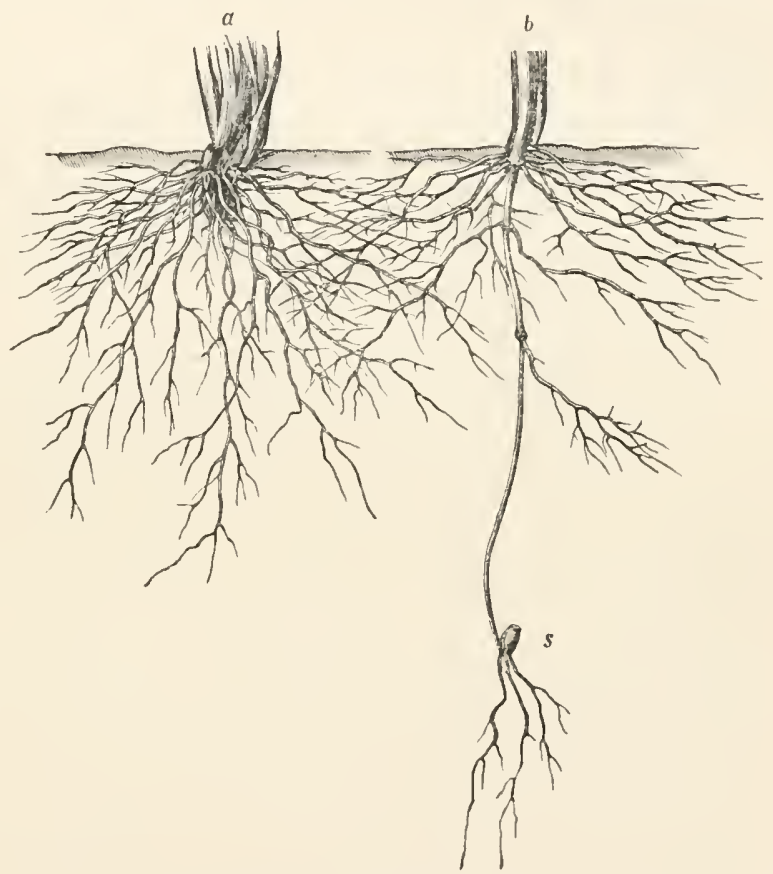

FIG. 14. Hordeum vulgare. $a$ sown on the surface; $b$ deeply buried; $s=$ the fruit grain. About one-third natural size.

a nutrient solution, the root-hairs and root may have been injured by the removal from the soil. For these and similar reasons, a number of the roots die off after the transference has bcen made, and are gradually replaced by new growths. The roots of seedlings grown in earth or sawdust are not perceptibly injured when placed in water, and even older roots may without doubt accommodate themselves to an aquatic life ${ }^{1}$. Indeed, if the transference were sufficiently gradual, this would

1 Literature: Sachs, Versuchsst., I860, Bd. II, p. 13 ; Knop, Versuchsst., 1863, Bd. v, p. 96; Knop und W. Wolff, 1.c., I 865 , Bd. viI, p. 345. On the anatomical differences between water- and 
probably be possible in all cases, for then the injurious effects of a sudden change might be avoided.

\section{SECTION 27. Absorption of Fluids and Solids by the Sub-aërial Organs.}

The sub-aërial organs of land-plants are specially concerned with gaseous exchange, and under normal conditions have little or nothing to do with the absorption of water as rain or dew, the latter being in general only of importance to the plant as the sources from which the moisture of the soil is derived. As a matter of fact various means are provided for carrying away rain as quickly as possible from the leaves, such as their form. position, movements, and other properties ${ }^{1}$, while so long as the surface of the leaf is not wetted, no absorption of water is possible. On the leaves of Nelumbium, $N y^{\prime} m p h a c a$, \&c., drops of water roll about like globules of mercury on a glass plate without wetting or adhering to any part. This power of remaining unwetted is probably for the purpose of maintaining an unimpaired rate of gaseous exchange, and a fall of rain does not as a general rule block up the stomata, nor does any water pass through them even when completely immersed.

The function of the cuticularized epidermis of aërial parts is to check transpiration, and hence it can hardly have any marked powers of absorption. If therefore the sub-aërial parts readily absorb water, the plant must either be able to withstand temporary desiccation, or must possess arrangements by which in periods of drought the excessive loss of water can by other means be guarded against, or it must grow in a very moist habitat. All these conditions are found in nature. Among our own plants many mosses and lichens are known which can dry up without being injured, and which, when moistened with water in the form of rain or dew, become at once turgid again and resume the vital activity which was interrupted by desiccation ${ }^{2}$. Thicse mosses and lichens which grow upon bare rocks must indeed obtain all their water in the form of rain and dew,

earth-roots, cf. P'erseke (Formänderungen der W'urzeln in Erde u. in Wasser, I $8_{77}$, p. 45 ). Roothairs may assume abnormal shapes when suddenly transferred to a new medium. See F. Schwarz, Unters. a. d. Bot. Inst. z. Tül,ingen, 1883 , Bd. I, p. 182 ; Wortmann, Bot. Zeitung, 1889, p. 279 ; Wieler, ibid., p. $55^{\circ}$.

${ }^{1}$ Cf. $3^{8}, 39$; Stahl, Regenfall und Blattgestalt., 1893 .

2 Schioder, Unters. a. d. Bot. Inst. z. Tübingen, Bd. II, I left I, 1886. On the special adaptations for the storage of water found in many Musci, cf. Goebel, Pflanzenbiol. Schilderungen, 1889, p. 27 ; Flora, 1893 , p. 423 ; Keeble, Annals of Botany, 1895, Bd. IX, p. 59 ; Jungner, Bot. Centralbl., 1895, Bd. Lxi, p. 434. On Hymenophyllaceae, Giesenhagen, Flora, I 890, p. 455. [The dried living cells of such plants contain not air but a more or less perfect vacuum. Hence when moistened they become almost immediately filled with water and turgid, which would otherwise be impossible. (Cf. Kamerling, Lot. Centralbl., Bd. LxxiI, 1897 , p. 49.) Plants of Dicranum scoparium and Cladonia rangiferina, after being kept air-dry for three months, showed an immediate resumption of respiration when moistened, and a power of $\mathrm{CO}_{2}$-assimilation at once or after a short latent period: Ewart, Trans. Liverpool Biol. Soc., vol. xi, I 897 , p. i 52 .] 
while the ash constituents, which the rock may not be able to supply, must be obtained from the dust particles of the air, which are retained by the closely growing tufts and suffice for the needs of the plant when its growth is slow ${ }^{1}$. Direct experiments show that not only the rhizoids but also the leaves of mosses, and the entire thallus of lichens and hepatics, are able to absorb dissolved substances.

In warmer climes there are numerous epiphytes, which without being compelled to withstand continually recurring desiccation, obtain by means of special arrangements, and owing to the heavy rainfall, sufficient water for all their needs, at the same time receiving the necessary nutrient salts entirely or partially from the air ${ }^{2}$, since they have no direct connexion with the soil.

The white velamen found on the aërial roots of many orchids and aroids is of great functional value in this respect ${ }^{3}$, for, as the change in colour indicates, the dead cells of the velamen absorb the water reaching them in the form of rain or dew, while the inner portions of the root utilize the water thus obtained, absorbing it just as a radicle absorbs water from a wet sponge into which it may have grown.

In the cases of other epiphytes, such as Oncidimm altissimmm (Orchid), Anthurimm Higgclii (Aroid), Asplenium scrratum (Fern), the aerial roots form large tangled masses, in which organic débris accumulates often to so great an extent as to form a total bulk of half a cubic metre. In this manner a mass of humus is collected, by means of which the plant is able to obtain water, as well as nutritive material from rain and dew. In Aspleninm nidns-aris and some Bromelias the same end is attained by the leaves becoming arranged like a filter funnel, and in certain of the latter, in which the water is collected mainly in these leaf-funnels, the bases of the leaves are able to absorb water and dissolved substances, and indeed, according to Schimper, may supply the plant with sufficient water for all requirements. In Tillandsia usneoides, whose thin stem and narrow leaves are found hanging freely from branches or even iron beams, the capillary spaces beneath the scale-like hairs suck in dew and rain-water (Schimper), for at these points water can easily be absorbed and transferred to the interior, a power which is absent from the rest of the epidermis.

Sub-aerrial organs are utilized for the absorption of water and dissolved substances to an equally marked extent in certain carnivorous phanerogams,

1 In fallen snow a large quantity of dust is present, even on mountains, and this is left behind when the snow melts. Reinsch's conclusion (Chem. Centralbl, is $\delta_{71}$, p. 520 ) that higher plants obtain most of their potassium and phosphoric acid from the air is, howerer, erroneous.

${ }^{2}$ Literature: Schimper, Bot. Centralbl., $188_{4}$, Bd. xvil, p. 192, and Bot. Mitth. a. d. Tropen, 1888, Heft 2; Goebel, Pflanzenbiol. Schilderungen, 1889, p. 202; Haberlanit, Bot. Tropenreise, 1893, p. 172; Went, Ann. du Jard. bot. de Buitenzorg, I 894 , T. XII, p. I.

${ }_{3}$ For the structure of these cf. Leitgeb, Denkschr. d. Wiener Akad, 1864, Bd. xxv, p. I79; Meinecke, Flora, 1894 , p. I33. 
(Sect. 65) in which leaves or parts of leaves may be specially modified to form absorptive organs ${ }^{\mathrm{t}}$.

The leaves of land-plants are never completely incapable of absorbing water, provided that they can be wetted (Sect. 21 ), and hence in any given case it is simply a question as to the extent to which this power has been developed and increased, or how far it is employed under the given conditions. In typical land-plants, the leaves have no practical importance as absorptive organs for fluids, owing to their relative impermeability and to the causes previously mentioned. A little of the water collected in the leaf-sheaths of Dipsacus, Umbelliferae, \&c., may be absorbed, although these plants do not normally require any supply of water from

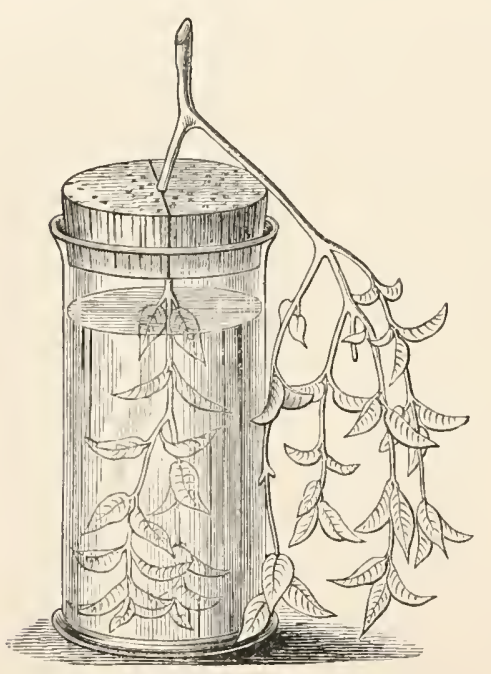

FIG. 1.5 .

this source. It must moreover always be remembered that the properties of the cuticle, including its permeability to water, may be markedly modified by the cultural conditions (Sect. 2I).

The gradual recovery of a flaccid leaf, when the lamina is immersed in water, shows directly that water has been absorbed through the outer surface. If, as in Fig. 15, half of a leafy branch is immersed in water, the withering of the leaves exposed to air may be prevented, as was first observed by Marriotte and Hales ${ }^{2}$. In other plants, the absorption of water only suffices to slightly delay the withering of the exposed portion (Wiesner). By weighing this apparatus (Fig. 15 ), the amount of water transpired, and hence the approximate amount absorbed, may be found. Boussingault ${ }^{3}$ has shown that the latter is not always trifling, and that the surfaces of the branches allow but little water to penetrate, while water may be absorbed by leaves which possess few or no stomata. These results have been confirmed by other observers, and according to Wiesner's researches, even the leaves of Sedum Fabaria can absorb a little water ${ }^{4}$.

1 On the absorption of water by the trumpets of Sarracenia, cf. Wiesner, quoted by Bïgerstein, Wasseraufnahme d. Pflanzen, 1891, p. 28 . On the pitchers of Dischidia raftesiana, see Treub, Ann. d. Jard. bot. d. Buitenzorg, I882, T. II, ]. 32 ; [Groom, Ann. of Bot., I893, vol. vii, p. 223 ; Scolt and Sargent, ibid., p. 243].

${ }^{2}$ Mariotte, Euvres d. Mariotte, I 717 , p. 133 ; Hales, Statics, 1748, p. 78 .

${ }^{3}$ Boussingault, Agron., Chim. agric., \&c., 1878 , T. vi, p. 364 .

4 A complcte bibliography is given by Bürgerstein, Ubersicht der Unters. iiber die Wasseraufnahme der Pflanzen durch die Oberfläche d. Blätter, 1891 (Sep.-abdr. a. d. 27. Jahresb. d. Leopoldstädter Obergymnasiums in Wien). Of the special works may be mentioned: Wiesner, Sitzungsb. d. Wiener Akad., 1882, Bd. Lxxxvi, p. 24I ; Kny, Ber. d. Bot. Ges., I886, p. xxxvi; Wille, Cohn's 
A simple experiment of Boussingault's suffices to demonstrate that salts may pass through the cuticle. If a drop of a very dilute solution of $\mathrm{KNO}_{3}, \mathrm{Ca} \mathrm{SO}$, \& . is placed on a beech or other leaf, tiny crystals form on this spot when rapid evaporation is allowed, but not when the leaf is covered with a watch-glass, so that the drop evaporates slowly and the salt has time to penetrate. Hence, small amounts of dissolved substances carried down by rain or dew may be directly absorbed by the foliage leaves. For further details see the literature referred to, in which will be found discussions as to whether hairs and other organs are adapted to retain water or lead it away. It may be mentioned that frequently the cuticle over the veins is especially permeable to water.

Condensation of worter vapour. All plants must be supplied with fluid water, for even in air completely saturated with water-vapour full turgidity cannot be restored to a flaccid tissue. Pfeffer found that a moss (Catharinea undulata) had not absorbed the full amount of water necessary to produce normal turgidity, after being kept at a constant temperature for twelve days in a saturated atmosphere, in which the cell-walls soon swelled and hence caused the shrivelled leaf to expand. If, however, a formation of dew is induced by changes of temperature, complete turgidity may be attained in from two to four days. This result was obtained both with plants rooted in a little soil and with others suspended freely, for the soil only becomes sufficiently moist when dew is formed in abundance. The formation of dew which takes place in ordinary plant-houses does not suffice for this, for freely hanging epiphytic orchids gradually decrease in weight, if left unwatered ${ }^{1}$.

Air-dried plants are, however, able to condense a certain amount of water when placed in air saturated with moisture, and this is indicated by the twisting of the beaks of Erodium gruimum, or of the awns of Stipa pemata, which are used as hygrometers, and by the increased pliability of the thallus of Laminaria or of Lichens. This condensation of watervapour in the substance of the cell-wall is only possible by means of the enormous surface-tension energy brought to bear (Sect. I2), and as the amount of water absorbed increases, this force rapidly decreases, so that further absorption takes place more and more slowly. R. Hartig found that wood-shavings kept at a constant temperature in saturated air were still increasing in weight after fifty-seven days. Sachs found that the maximal swelling was attained much more rapidly, but this was probably

Beitı̈̈ge zu Biol., 1887, Bd. Iv, p. 310 ; Chimielewsky, Bot. Centralbl., I 889 , Bd. xxxvı1I, p. 790 ; Ganong, Bot. Centralbl., 1894, Bd. L.1x, p. 180 . On the absorption of water bys water-pores, see Habcrlandt, Sitzungsb. d. Wiener Akad., 1894, Bd. cur, Abth. i, p. 502 ; 1895 , Bd. c1v, Abth. i, pp. $9^{6,110 .}$ Cf. also Sect. $4^{8}$.

${ }_{1}$ Unger, Sitzungsb. d. Wien. Akad., I864, Ld. xxv, p. 179; Duchartre, Compt. rend., I869, T. Lxvil, p. 773 . 
owing to a formation of dew, for which trifling differences of temperature suffice $^{1}$.

Similarly salts deliquesce in saturated air, although dilute solutions, such as the cell-sap, condense water-vapour with extreme slowness. Plants of Reamuria hirtella, Tamarix mannifera, \&c., are covered with a saline incrustation during the day-time, but nevertheless the condensation of water-vapour is certainly insufficient to supply all the water the plant requires. The hygroscopic saline incrustations of many desert plants may induce an abundant formation of dew, but it has yet to be discovered what are the precise means by which they make use of the water thus obtained, for in order to draw water from such concentrated solutions, the cell-sap would apparently need to attain a still higher osmotic concentration. It has yet to be determined whether this is attained by regulatory adaptation, or whether perhaps the impermeability of the cuticle is such as to afford a protection against highly plasmolytic saline solutions ${ }^{2}$.

Since the presence of salt lowers vapour-tension, water-vapour must necessarily be transferred from pure water to a condensing saline solution, when both are enclosed in a chamber filled with saturated air. Such distillation takes place extremely slowly, for the potential differences which originate it are but slight. Thus at $20^{\circ} \mathrm{C}$, the presence of I per cent. of $\mathrm{KNO}_{3}$ lowers the vapour-tension of water only by about $0.1 \mathrm{~mm} .^{3}$ In the plant, the attractions for water which the cell-wall and cell-sap exert always set themselves in equilibrium, and hence the cell-walls are in that condition of imbibition which lowers the vapour-tension of water by the same amount as does the particular degree of concentration of the cell-sap 4. The osmotic energy of a solution, and hence also of a plant, may accordingly be determined by means of its vapour tension (Sect. 24).

If a I per cent. $\mathrm{KNO}_{3}$ solution is raised from $20^{\circ} \mathrm{C}$. to $20.2^{\circ} \mathrm{C}$, the depression

1 R. Hartig, Unters. a. d. Forstbot. Inst. z. München, I882, Bd. II, p. 17 ; Sachs, Arb. aus Wiirzburg, I $879, \mathrm{Bd} .1 \mathrm{I}, \mathrm{p} .309$. In other observations upon the absorption of moisture from the air, sufficient care has not been taken to exclude all dew-formation. Wilhelm, Bot. Jahresb., I $8_{3}$, p. 39 (seeds); Detmer, Beiträge 2. Theorie d. Wurzeldruckes, 1877 , p. 18 (lichens); Kerner, Pflanzenleben, $\mathbf{1} 88_{7}$, Bd. I, p. 201 (lichens and mosses ; Borzi, Acqua in rapporto alla vegetazione, \&c., Auszug a. d. Acten d. internat. bot. Congresses, 1892. See also H. Dixon and J. Joly, Phil. Trans., 1895, vol. clixxui, p. 575 .

${ }_{2}$ Volkens, Flora d. ägypt. Wüiste, I887, p. 27 ; Ber. d. Bot. Ges., IS87, p. 434 ; Marloth, ibid., p. 321. See also Sect. 23. According to Marloth (1. c.), the saline incrustation contains $17 \cdot 2$ per cent. $\mathrm{Na} \mathrm{NO}_{3} ; 12$ per cent. $\mathrm{MgSO}_{4} 7 \mathrm{H}_{2} \mathrm{O} ; 5.5$ per cent. $\mathrm{NaCl}$; and 51.9 per cent. $\mathrm{CaCO}_{3}$. Vulkens' observation that the glands are not plasmolyzed is of little importance (Ber. d. Bot. Ges., I 887 , p. 43t). Plasmolysis may be prevented by various means; cf. Pfeffer, Druck u. Arbeitsleistung, 1S94, p. 307. In regard to nectaries see Sect. 49 .

${ }^{3}$ See Ostwald, Allgem. Chemie, I891, 2. Auf., Bd. I, p. 707 .

- See Pfeffer, Studien zur Energetik, I892, p. 258 . On swelling, see Sect. 12. Maxwell (Theorie der Wärme, 1878, p. 326) has shown how and why the concave menisci in capillaries lower the vapour tension, and cause a condensation of water vapour; a capillary of $0.00 \mathrm{r} \mathrm{mm}$. bore lowers the vapour tension by about one-thousandth part. 
of the vapour-tension is not merely nullified, but undergoes a rise of about $0.24 \mathrm{~mm}$. Hence, this trifling difference of temperature will suffice to cause a I per cent. solution of $\mathrm{KNO}_{3}$ to give off water-vapour in a saturated atmosphere. For the same reason a very slight warming is sufficient to render transpiration possible in saturated air, while a trifling difference of temperature will cause a formation of dew upon the colder body. Movements of the air will be of material assistance in accelerating such accumulative processes.

\section{SECTION 28. Importance and Properties of the Soil.}

Substances are taken in by the plant in essentially the same manner whether the absorptive organs are immersed in water or in moist soil, for only water and dissolved substances are capable of absorption, and in all cases the quantitative selective power is regulated by the diosmotic properties of the plant and the character of its metabolism. The poverty of the vegetation of sandy soils is an indication that an admixture with organic material, as in loam or humus, is of marked advantage for the growth and nourishment even of those plants which may be cultivated in sand or water, and which do not extract any organic nutriment from the soil, but only water and inorganic salts.

The properties of humus soils are of the utmost biological importance, but it is the task of text-books on agriculture to describe them fully ${ }^{1}$. We are at present concerned only with the importance of the soil as the source of inorganic nutriment, and the question whether certain plants may obtain a portion or the whole of their organic nutriment from humus will be discussed later (Sects. 64 and 67).

The soil is of use and advantage to the plant not only as the source from which its necessary nutriment is drawn, but also because (I) it enables the roots to obtain a firm hold and thus to support the erect aërial organs (cf. Sect. 26); (2) all the organs buried in the soil come into contact with air, as well as water and the substances dissolved in the latter; (3) useful nutritive materials, obtained from the rain or by manuring, or rendered soluble by the weathering of the soil, are retained owing to the absorptive power of the latter, and are presented to the plant in the most suitable form, namely in an exceedingly dilute solution.

The absorptive power of the soil has also a marked effect upon the form in which the nutritive materials are presented to the plant, for by this means particular salts may be decomposed, retained, or removed in some way or other, and thus many injurious effects prevented, including those due to over-concentration. When plants are grown in water-cultures,

1 A. Meyer, Lehrb. d. Agriculturchemie, 1895 , Bd. I1 ; R. Sachsse, ibid., 1888; Detmer, Die naturwiss. Grundlagen d. landw. Bodenkunde, 18;6, \&c. 
or sand free from humus, the culture media frequently become markedly alkaline, whereas in ordinary soil no such injurious change occurs. The presence of humus is also of importance, inasmuch as it tends to maintain more equable temperatures, and thus protect the perennial parts buricd in the soil.

A fruitful soil is a conglomeration of mineral detritus and organic remains. Agriculturists distinguish a series of soils, according to the texture, the fineness of division, the relative amounts of coarse and fine particles, and the relation between the organic and inorganic constituents.

Without entering into the detailed characteristics of different soils, or the methods by which the value of a particular soil is determined, we

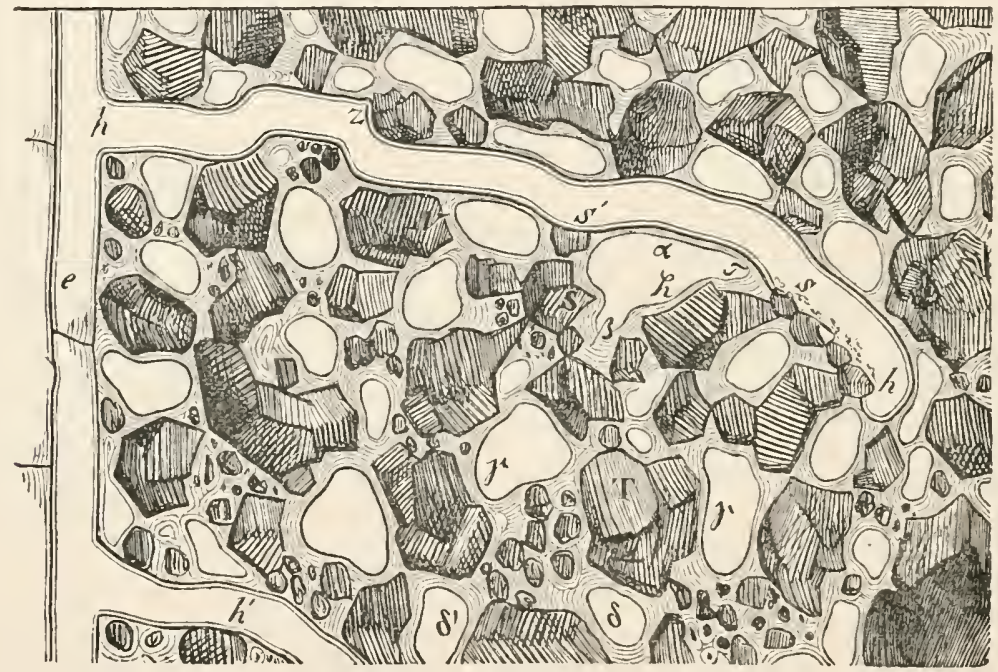

FIG. 16. From the root-epidermis (piliferous layer) the root-hairs $h$ and $h$ arise. The solid soil particles are darkly shaded $(T$, the air spaces $(\delta, \gamma, \varepsilon c$ ) are white, the adherent water is indicated by concentric lines. (After Sachs.)

may turn to the consideration of a typical humus soil, containing a certain amount of organic material. Such a soil retains a large quantity of water. Air-drying gradually removes a large amount of the water sucked in by the soil, but even during hot dry summer days, a humus soil still retains a certain percentage, although this may be so firmly held as to be unavailable for the plant's use. It is only in an over-saturated soil that the spaces between the soil particles are almost all filled with water, for air is usually present in abundance, especially in the larger interspaces (Fig. 16).

It is easy to see why the growth of many terrestrial plants is injuriously affected when the soil is over-saturated with water, for the aeration of the soil is then imperfect and the roots are insufficiently supplied with oxygen, while decompositions induced or aided by certain organisms 
(anaerobic bacteria, \&c.), which then develop in abundance, may directly or indirectly injure the root-system or retard its development.

In a normal soil, permeated throughout by moisture, the water is held by capillarity, just as it would be in a system of capillaries containing alternating columns of air and water. A very thin film of water is retained with great tenacity by the molecular forces exerted on the wetted surface of each soil-particle, and the same is also the case with regard to the water absorbed by any organized bodies capable of swelling, which may be present in the soil. The elongating roots and root-hairs push themselves between the soil-particles, as do also the rhizoids and nutritive hyphae of cryptogamic plants, and indeed all growing parts which are buried in the soil. Each growing apex follows the path of least resistance, and hence its course is often extremely sinuous, but when once penetration is assured, roots may exert considerable lateral pressure as they increase in thickness. In Fig. I6, it may be seen that the root-hair comes in contact with air $(a)$ at one part, and with water at another, but even at (a) the cell-wall will remain fully permeated with water, since the air spaces are in general saturated with water-vapour, and since a thin film of water will be present as a general rule on the outer surface of the root-hair, thus preventing direct contact between it and the surrounding air. A similar film is also interposed wherever the root-hairs touch soil-particles $\left(s, s^{\prime}\right)$, and hence even at those points an absorption of water and dissolved substances into the interior of the cell is still possible. This is also the case when, as represented in Fig. II (p. ${ }_{15} \mathrm{I}$ ), the root-hairs or rhizoids have grown around and partially enclosed soil-particles, causing the root-hairs to assume remarkable shapes, and rendering a separation of the particles impossible without injuring the latter.

The water absorbed by the root is replaced by fresh water from the surrounding zones, and these again draw upon regions further removed, so that in this way, as was first shown by Schulz-Fleeth ${ }^{1}$, plants may draw upon tracts of soil into which no portion of the root-system actually penetrates. As the percentage of water present in the soil decreases, the plant absorbs its necessary supply with greater and greater difficulty, not only because the water continually decreases, but also because the particles of fluid are more and more firmly held, as the soil dries. Hence a condition is ultimately reached, in which the plant is unable to withdraw from the soil the moisture still present in it; and just as the plant is unable to obtain by condensation from a saturated atmosphere sufficient water for all its requirements, so also is a dry soil unable by the condensation of water-vapour to become sufficiently moist to supply the plant with fluid water. It is, however, easy to understand that a plant 
should remain turgescent in a soil from which no water can be squeezcd by even marked pressurc.

An important property of soil is its power of withdrawing many inorganic and organic bodics from their solutions, so that liquid manurc, when filtered through a sufficiently thick layer of soil, passes away as an almost colourless fluid, retaining only traces of certain inorganic and organic substances which were previously present in abundance. Numerous researches have been made with regard to the absorptive powers of different kinds of soils, more especially for inorganic salts, and it has been found that compounds of potassium, ammonium, sodium, calcium, magnesium, phosphoric acid, and commonly silicic acid also, are retained by an ordinary soil, while sulphuric, nitric, and hydrochloric acids are absorbed cither to a very slight extent, or not at all. The above mentioned alkalics and alkaline earths are retained by the soil when presented to it in the form of oxides, and also when they reach the soil as more or less soluble salts. In the last case, a double decomposition takes place in the soil, so that when a sulphate, nitrate, or chloride of an alkali is added, in gencral a corresponding salt of calcium, or else of another alkaline base, passes away in solution, while alkaline phosphates may be directly retained by the soil without any such change. The order of absorptive power for the oxides and salts of alkalies and alkaline earth is with most soils as follows: potassium, ammonium, magnesium, sodium, calcium. From this it is easy to see that when a solution of common salt is added to an ordinary soil, it will be for the most part a calcium salt that passes away in solution, and hence usually only a trifling absorption of calcium salts is possible. Nevertheless the result obtained depends largely upon the quality of the soil, and also upon the nature and quantity of the salt added. Thus under certain circumstances, magnesium may be replaced and driven out of the soil by calcium.

In addition to the above, many other substances, such as tannin, dyes, \&c., may be absorbed, while the energetic absorption of metallic salts, of which humus is capable, enables it very largely to render these innocuous. Gases also may undergo perceptible condensation in humus soil, but it is obvious that in all cases absorption must cease when the saturation point is reached. In ordinary farmed land, however, the maximal accumulation of food substances hardly ever occurs, and the power of absorption depends almost entirely on the amount of humus which soils contain, for pure sandy and gravelly soils have only feeble absorptive powers.

From a physiological standpoint, it is of subordinate interest whether absorption is duc to chemical or physical fixation, but on the other hand it is of the utmost importance that absorption is never permanent and absolute, so that the repcated addition of water continually removes traces of 
the absorbed substances. IVater containing carbonic acid is a more active solvent than pure water, while dilute hydrochloric or nitric acid are still more energetic in their action and may remove from a soil the whole of the substances it has absorbed. Similarly certain saline solutions, by inducing double decompositions, render particular constituents of the soil soluble. The water in the ground is always a very dilute saline solution, as is cvidenced by the composition of drainage water, and its percentage of dissolved salts is increased by the aid of carbonic acid gas derived from respiration and from organic decompositions in the soil. In this solution, not merely are substances present which have been absorbed, but also others derived from the mineral soil particles. As the latter oxidize or decompose, aided by the action of carbonic acid, or saline solutions, various substances arc rendered available for the plant's use, either passing directly into solution, or being first absorbed and retained by the soil. The same takes place with those ash constituents set free by the decomposition of organic remains. The slow but unceasing decomposition of humus is of great importance, for with the aid of micro-organisms traces of nitrates are continually produced, so that a supply of this important nitrogenous compound is always available, and at the same time the loss of the valuable nitrates by drainage from the soil is reduced to a minimum.

The water from a fertile soil always contains the substances which the plant requires in the form of a dilute solution, and when a small quantity of any one of these is absorbed, a corresponding amount passes from the absorbed into the freely soluble condition. It is not merely the water and portions of soil, with which the roots are in direct contact, that are of service to the plant, for the disturbance of equilibrium caused by the absorption of any substance induces diffusion towards the absorbing organ, while the currents of water flowing to the roots convey fresh supplies of saline substances. Hence, widely separated areas may be utilized by the plant, provided the character of the soil and the percentage of water present interpose no obstacles. Terrestrial plants are therefore supplied with soluble nutriment in much the same manner as are aquatic plants by the very dilute solutions in which they grow. As the result of the changes which inorganic nutritive materials may undergo in connexion with absorption, the latter may always be prescnted to the plant in the same form, whatever salts are added to the soil.

Absorbed substances are usually distributed only very slowly by currents of water in the earth, so that some time elapses before the localized differences due to absorption are equalized in a normal garden-soil. Hence it is important that the branching root-system shall grow towards the uncxhausted areas of soil, and come into close contact with fresh particles of earth. When the root absorbs nutriment, the differences of equilibrium thercby induced will be rapidly readjusted across short distances, with the 
aid of the absorbed substances held by the soil (Sect. 22). Moreover, roots may exert a direct solvent action upon the particles of soil with which they are in contact, by means of the carbonic acid produced by respiration. Czapek finds that roots for the most part do not evolve any perceptible amounts of other free acids, but that they are able to excrete acid potassium phosphate, which may exercise a solvent action either directly or by inducing double decompositions with chlorides or nitrates.

In such cases, it must be directly determined whether or not a particular root may not always, or under special conditions only, be able to excrete free acids for solvent purposes. Various fungi can indeed evolve large quantities of organic acids, and the production of acid depends largely upon the way in which they are nourished, as well as upon other conditions, for the amount of acid produced may be increased by continuous neutralization (Sect. 86). The energetic corroding powers of lichens are probably due to the excretion of free acid, and it is well known that other secretory products, such as enzymes, are frequently employed to convert insoluble substances into soluble ones which can be absorbed, or to enable a parasitic or saprophytic organism to penetrate into the interior of living or dead bodies (Sect. 65). Similar secretory powers have been developed by the roots of many parasitic and saprophytic phanerogams, and hence it would not be surprising to find that certain typical flowering plants are able to excrete fixed acids in considerable amount, and thus render soluble the mineral constituents which they require.

There is no doubt that the characteristics of the root-system, as just described, have not in all cases the same importance, for in a water-culture irregular currents constantly occur, and these suffice to bring fresh supplies - of dissolved material continually into contact with the roots. According to the external conditions, a plant will be either sufficiently provided in this manner, or will require to obtain a greater or less amount of its nutriment by the active spoliatory means above described.

The retention of reater. A general account of the causes leading to the retention of water by the soil has already been given, and it is not intended here to give a detailed account of all the factors of importance in this respect, such as the quality, consistency, or fineness of division of the soil. Various other circumstances influence the actual amount of water present in the soil. Thus, when free fluid water is present near to the surface, the soil immediately above will be fully saturated with capillary water (= full capacity), whereas, when the level of the free water of the soil is lower, there will be little or no capillary water in the surface layers (= lowest or absolute capacity) ${ }^{\prime}$. When fully saturated and all superfluous water allowed to drain away, sandy soils contain least, humus soils most, water.

1 Cf. A. Mayer, Agriculturchemie, I895, 4. Auf., Bd. II, p. I35. 
Meister ${ }^{1}$ found that 1000 grammes of a sandy soil absorbed 304 c.c. of water, while the same weight of a turfy soil took up 1052 c.c., hence containing 45.4 vol. per cent. in one case, and 63.7 in the other. Other soils showed intermediate values between these two extremes. The amount of water which a soil can retain bears no relation to the amount which plants are able to withdraw from it, for when air-dry, different soils retain very varied amounts of water, which the plant is in general unable to absorb to any appreciable extent. A rough generalization of the quantities of water available for the plant's use in different soils when saturated with water is given by Sachs' ${ }^{2}$ experiments. In these the amounts of water still present when plants begin to wither, were compared with the quantity necessary to saturate the soil. A young tolacco plant began to wither when the water was reduced to 12.3 per cent., and the soil, when saturated, contained 46 per cent. by weight of water, so that in such a saturated soil 33.7 per cent. of water was available for use. In a clay soil this amount was $52 \cdot \mathrm{I}-S=44 \cdot \mathrm{I}$ per cent, in coarse quartz sand $20 \cdot 8-\mathrm{I} \cdot 5=\mathrm{I} 9 \cdot 3$ per cent., according to corresponding experiments performed with similar tobacco plants, the amounts $I 2 \cdot 3,8$, and $I \cdot 5$ per cent. being the approximate percentages of water held in the air-dry condition by the soils under examination. These results also show that a plant cannot reduce the amount of water to so low a percentage in a soil rich in humus as in a sandy one, but nevertheless when the soils are saturated, more water is available for the plant's use in the former, owing to its power of retaining water being very much more marked than it is in a sandy soil.

Empirical results show that the soil is unable to supply the plant with water by the condensation of water vapour (cf. Sect. 27), and therefore plants slowly die if the leaves are freely exposed while the roots and soil are kept in saturated air. The stunted growth which Sachs ${ }^{3}$ observed during his experiments under such conditions was probably rendered possible by the formation of dew upon the soil and roots.

Absorption by the soil. The power of soils to retain dissolved substances was originally discovered by Gazzeri. Amplified and extended observations were then made by Th. Way, and at a later date Liebig called attention to the importance of this property in the economy of nature ${ }^{4}$. Since then various researches have been performed to determine the actual processes and causes of absorption ${ }^{5}$.

The question as to the mode of absorption can only be touched upon. Liebig and others were inclined to regard the process as a physical one, whereas Rautenberg, A. Beyer and others supposed it to be chemical in nature. Discussion on this point is largely purposeless, since the boundary between chemistry and physics is an arbitrary one, and moreover varies according to the standpoint from which the subject under discussion is regarded. Chemical proçesses do certainly come into play, when, for example, potassium chloride is added to a soil, and the potassium is retained while calcium chloride passes away in solution. Other substances, however,

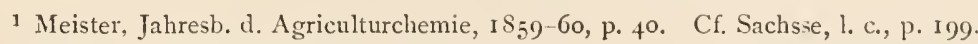

${ }^{2}$ Sachs, Versuchisst., I 859 , Bd. I, p. 234.

${ }^{3}$ Sachs, 1.c., p. ${ }^{36}$. Cf. A. Mayer, 1. c., Bd. 11, p. 131; Sachsse, 1.c., p. 209.

- See Versuchsst., 1873 , Bd. Xvi, p. 56 ; Way, Jour. of the Royal Agric. Soc., 1850, vol. xi, p. $3^{\mathrm{I}} 3$, and vol. xv, p. 91 ; Liebig, Ann. d. Chem. u. Pharm., I $858, \mathrm{Bd} . \mathrm{Cv}, \mathrm{p}$. Iog.

${ }_{5}^{5}$ Details in the quoted works of A. Mayer, Sachsse, \&c. See also van Bemmelen, Versuchsst., I 879, Bd. XxiII, p. $265 ; 1888$, Bd. xxxv, p. 69 ; and Die Absorption, I897. 
such as dyes and pigments, may be mechanically retained by the soil without undergoing any perceptible change, just as dyes are absorbed by charcoal or coagulated egg albumin. According to various researches, including quite recent ones, there can be no doubt that the alkalies and alkaline earths are fixed by the soil in the form of silicates. When all the hydrosilicates present in the soil are destroyed by boiling with hydrochloric acid, no further fixation of alkalies, \&c. is possible, nor can an alkali drive out an alkaline earth, whereas caustic alkalies and alkaline carbonates are still absorbed, since they form insoluble compounds with the silica or siliceous acid present in the soil.

Although it is usually by the formation of silicates that the above bases are fixed, nevertheless other absorptive processes take place in a very different manner. Thus many dyes, tannic acid, and other bodies which do not form silicates, and do not enter into combination with them, are retained by the soil (cf. Sect. I2); hence it does not necessarily follow that the whole of the alkalies or alkaline earths absorbed are held in the form of silicates. It is indeed possible that a formation of insoluble phosphates may take place, and that alumina or ferric oxide when present may fix a certain amount of ammonia. Moreover, the humus acids may aid in the process of absorption by forming insoluble compounds with different saline and other substances.

For purposes of demonstration, an inverted cylindrical bell-jar, $250 \mathrm{~mm}$. high, having a basal opening through which a tube passes, may be employed. This is filled with garden soil resting upon a layer of sand and cotton wool, and if a little alizarin solution, reddened by the presence of a trace of ammonia, is added, it filters through as an almost colourless fluid, whereas, through a similar depth of sand and cotton wool, the solution soon passes unaltered owing to the feeble absorptive power of the latter.

The nutritive solution in the soil. The free water of the soil contains on the average only trifling quantities of organic and inorganic substances, as is shown by analyzing spring- or drain-water, which contains as a general rule from 0.01 to 0.03 per cent. of solid substances. Exceptionally, as for example in saline regions, the water of the soil reaches a high degree of concentration. Direct researches have shown that only traces of the absorbed substances are dissolved by water, and Peters found that to extract one part of potassium 28,000 to 36,600 parts of water are necessary, while Bretschneider found that it required 51,612 parts of water to dissolve one part of phosphoric acid ${ }^{1}$. It is of considerable importance in the economy of the soil that traces only of substances which cannot be retained should be present at a given time, for in this way the loss of saltpetre is largely avoided, although in fertile soils it is continualiy being formed from ammonia and insoluble nitrogenous compounds (Sect. 63).

The nature of the soil and of the substances absorbed has naturally a marked influence upon the concentration and composition of the water it contains, and by means of saline solutions, or by the action of carbonic acid, the solution of certain

1 J'cters, Versuchsst., I $\$ 60,13$ d. II, P. 135; Bretschneider, Jahresb. d. Agriculturchemie, I 865 , 1. 22. For further details on this and following points, see Sachsse, 1. c., p. IS2; $\Lambda$. Mayer, 1. c. 
substances is favoured and accelerated ${ }^{1}$. Carbonic acid is formed in abundance by oxidation and decomposition in a soil rich in humus, and hence the air permeating it contains more carbon dioxide than does atmospheric air. In an ordinary soil the very variable percentage of this gas will average from 0.2 to $\mathrm{I} \cdot \mathrm{O}$ per cent. at a depth of $\mathrm{I}$ metre, and at 6 metres may rise to as much as 8 per cent., or in certain soils even higher than this ${ }^{2}$. Along with this increased amount of carbon dioxide, which is in itself injurious to ordinary aerobic plants, the amount of oxygen present decreases, but not below i 3.6 per cent. in Fleck's researches (see Sachsse, 1. c.) on the air present in a garden soil. Nevertheless, in dense soils or masses of mud where the aeration may be very imperfect, and especially when micro-organisms are present, the amount of oxygen may decrease so markedly that anaerobic bacteria are able to develop. In such media, roots are unable to grow unless they contain sufficiently large air canals to supply them with the necessary oxygen, and even then the decomposition products produced in sour soil frequently exert an injurious or fatal effect upon roots growing in it.

Solvent action of roots, rhizoids, Erc. The solvent action of roots is admirably indicated by the natural etchings frequently found upon calcareous stones lying in the soil ; such etchings may be readily obtained by causing roots to grow upon polished white or black slabs of marble ${ }^{3}$ (Fig. I 7). Sachs recommends that a marble plate should be placed in a pot filled with earth or sawdust, and that seedlings should be grown in it, so that the roots come into contact with the marble plate and spread out horizontally over its surface. The same result can also be readily obtained by placing the young

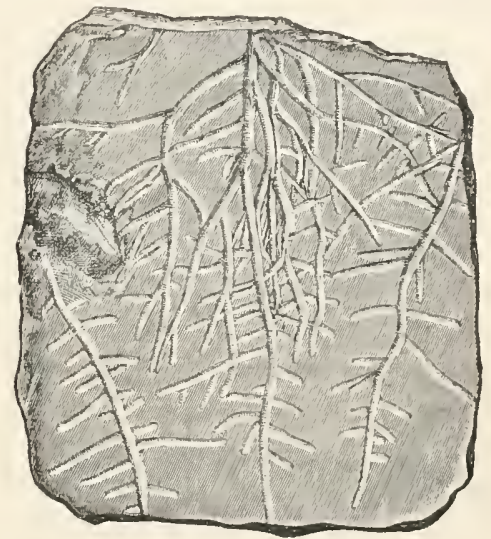

FIG. 1\%. A natural root etching found upon a piece of Solenhofer slate. (Nat. size) radicle of Pisum, Phaseolus, \&c., when 5 to ro $\mathrm{cm}$. long, upon the polished upper surface of the slab, covering it with a few layers of wet filter-paper, and placing a glass plate over the whole to keep the root adpressed to the slab. The slab may be placed in a glass dish under a bell-jar, with the end of the filter-paper dipping into a nutrient solution which thus moistens the root (Sect. 73). The projecting plumule grows upwards, and after two to six weeks the roots can be seen to have formed a roughened etching, or even distinct furrows upon the plate. After a still longer period, much deeper furrows may be produced, such as often occur in nature.

In a similar manner Sachs produced etchings on plates of dolomite, magnesian,

1 Cf. Sachsse, l.c., p. I 8 I, and the literature there given.

${ }^{2}$ Sachsse, l.c., p. I 42 ; Ebermayer, Forsch. a. d. Geb. d. Agriculturphysik, I89o, Bd. XIII, p. I 5.

${ }^{3}$ First correctly interpreted by Liebig, Ann. d. Chem. u. Pharm., I 858 , Bd. Cv, p. I 39 . De Candolle (Physiol., 1833, T. I, p. r86) had already called attention to the corrosions of rocks due to lichens. After the death of the root, the pattern may apparently continue for a time to be etched more deeply by the action of putrefactive products and of the organisms then present in abundance. Also Sachs, Bot. Zeitung, 1860 , p. i i $;$. 
and oolitic limestones. When plates of gypsum are used, the course of the roots is represented by raised lines, since the roots partially protect the gypsum from the solvent action of the water in the soil. If, however, plaster of Paris and powdered chalk are made into a paste and poured upon a glass plate, the roots form similar etchings to those formed on marble upon the shining surface thus produced. Czapek' ${ }^{1}$ used calcium phosphate, aluminium phosphate, \&c., instead of chalk, in order to determine the behaviour of the roots towards these substances. He found that they exercise no effect upon aluminium phosphate, which is corroded by all inorganic acids, and by oxalic, formic, tartaric, malic, citric, and butyric acids, but is not attacked by carbonic, acetic, or propionic acids.

According to these and other researches of Czapek's, it appears that, with the exception of carbonic acid, the roots of land-plants excrete no free acids. The permanent reddening " produced by many roots when pressed upon litmus-paper is, as a general rule, due to acid phosphate of potassium $\left(\mathrm{KH}_{2} \mathrm{PO}_{4}\right)$, which is usually given off in minute traces from young roots, or to a similar acid phosphate. The reddening of a neutral watery litmus solution induced by living roots usually disappears on boiling, and can be used, together with the decolourization of red phenolphtalein solution, to demonstrate the excretion of carbonic acid by them.

Certain proofs of the excretion of other free acids by the roots have never been brought forward, for free acetic acid (Oudemans and Rauwenhoff, l.c.) and butyric acid (Boussingault) are, according to Czapek, not present in the normal root secretions, and although Czapek finds formic acid to be commonly present, as stated by Goebel, it is always combined with bases ${ }^{3}$.

As a matter of fact all the solvent actions hitherto observed can be brought about by the action of carbonic acid. The sharpness of the outline formed upon marble is readily explicable when we remember that the most energetic action of the $\mathrm{CO}_{2}$ takes place at the point of contact, and that by the continuous removal of the soluble products a marked result can finally be produced. Thus a welldefined etching may be produced when a small glass jar covered with printed parchment and filled with water impregnated with carbonic acid is inverted upon a marble slab, if the water in the jar is continually renewed. The parchment may be covered with writing in wax, and to produce a more rapid reaction a dilute solution of hydrochloric acid may be used, while by means of the apparatus given in Fig. 18 , it may be shown how the soluble products formed by the exosmosing acid may be diosmotically removed and transferred to the interior of the cell ${ }^{4}$.

1 Czapek, Jahrb. f. wiss. Bot., I 896, Bd. xxIx, p. $3^{21 .}$

2 Becquerel, Archiv. de Bot., 1833 , T. I, p. 400 ; Oudemans u. Rauwenhoff, Linnaea, 1859-60, Bd. Xxx, p. 220 ; Molisch, Sitzungsb. d. Wiener Akad., I887, Bd. Xcv1, Abth. i, p. Io5 ; Czapek, 1. c. From certain unproved statements of Moldenhawer (Beiträge z. Anat. d. Pflanzen, I 8 I 2, p. 3I 2) as to the solvent actions of roots, Sprengel (Die Lehre vom Diinger, 1839, p. 23 ) assumed that they were able to excrete fixed acids. See also de Candolle, Physiol., I833, T. 1, p. I86; Liebig, Ann. d. Chem. u. Pharm., i $\$_{5} 8, \mathrm{Bd}$. cv, p. I 39.

${ }^{3}$ Boussingault, Die Landwirthschaft, 2. Aufl., i 85 I, Bd. 1, p. 24; Goebel, Pflanzenbiol. Schilderungen, I $89 \mathrm{I}$, Bd. II, p. 2 I I.

4 First employed for demonstration purposes by Zöller, 1pon Liebig's recommendation : Versuchsst., $186_{3}$, Bd. v, p. $45^{\circ}$ 
The absence of any solvent action upon plates of aluminium phosphate shows that at no period of its development are any strong fixed acids excreted by the root, nor can any free hydrochloric acid be formed by the interaction of the monopotassium phosphate with metallic chlorides ${ }^{1}$. Similarly the carbonic acid excreted by the roots is unable to liberate any free acid from chlorides, nitrates, sulphates, \&c., for were only a trace set free, as this was repeatedly fixed and removed, the continuance of the process would finally lead to a marked result (cf. Sect. 22). An example of such action is afforded by oxalic acid and by the weaker tartaric acid as well, for if sodium chloride or potassium nitrate are added to solutions of these acids, they attack pieces of marble more energetically than they did before ${ }^{2}$. Actions of this character assuredly occur in nature, when, as in fungi, organic acids are excreted, and at the same time the other necessary conditions are fulfilled. With or without such aid, fungi exert very pronounced solvent actions by the aid of acid secretions, as, for example, when lichens eat into the rocks on which they grow ${ }^{3}$. It is not yet precisely determined whether those algae, which can bore into chalky rocks, do so by means of the excretion of carbonic acid, or of other acids also ${ }^{4}$. It is moreover possible, though details cannot be given here, that organisms can at the same time form chalky deposits, and dissolve chalky rocks; so that it is not surprising that, according to Rosen ${ }^{5}$, a plasmodium of Aethalium septicum, although it contains chalk, may corrode a marble plate.

Since fungi excrete acids under special cultural and nutritive conditions, while under different circumstances they produce alkalies,

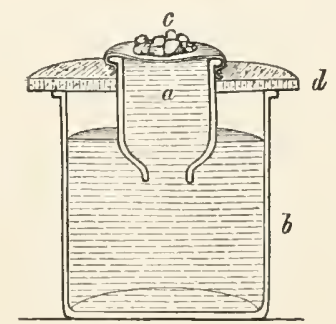

Fig. 18. The glass cylinder ( $a$ ) is covered with parchment $(c)$ and is filled with very dilute hydrochloric acid, as is also the outer vessel $(b)$. If pieces of marble are placed on $c$, in an hour or so the presence of calcium in the outer fluid may be demonstrated by the addition of oxalic acid and ammonia. Optical section, one-ffth natural size.) it appears possible that higher plants may, under special conditions, be able to excrete free acids other than carbonic acid. A few observations already mentioned (Sect. 22) do indeed favour this conclusion, while it must not be forgotten that a production of acid may, as is the case in fungi, take place in a regulatory manner, in correspondence with the demands made upon the organism. A variety of different acids may be excreted by fungi. According to

${ }^{1}$ Maby, Chem. Centralbl., i 978 , p. 56 ; Czapek, Jahrb. f. wiss. Bot., I 896 , Bd. xxix, p. $3^{6} 5$.

${ }^{2}$ Emmerling, Ber. d. Chem. Ges., I 877 , Bd. x, p. 650 ; Versuchsst., I884, Bd. xxx, p. I09. This results from the respective affinities of the different acids present, on which see L. Aleyer, Die mod. Theorien d. Chem., $188_{4}, 5$. Aufl., p. 482 , and Sect. 22. On the proofs of the presence of free $\mathrm{HCl}$, \&c. in such cases, see Salkowshi, Ber. d. Chem. Ges., Ref. $189^{2}$, p. 343 ; Detmer, Bot. Zeitung, I $88_{4}$, p. 79 .

3 Bachmann, Ber. d. Bot. Ges., I 89o, p. I4I ; x892, p. 35 ; Fünfstiick, Beiträge z. wiss. Bot., I895, Bd. I, p. I 57 . On fungi, cf. Zopf, Die Pilze, $1890, \mathrm{p} .185$. That by means of this acid-secretion, fungi can bore rapidly through plates of chalk to which they are chemotactically attracted has been shown by Lind's researches carried out in the Bot. Inst., Leipzig.

${ }^{4}$ Cohn, Jahresb. d. Schles. Ges. f. vaterl. Cultur, $x 893$, p. I 9 , and the literature there cited ; also C. Schröter u. O. Kirchner, Vegetation des Bodensees, $I_{9} 97$.

${ }^{5}$ Quoted by Cohn, 1.c., p. 22. 
Czapek, the acid reaction of the roots of Hyacinthus orientalis is due to a compound of oxalic acid (probably the acid potassium salt).

No other secretory products are excreted in perceptible amount by the roots of terrestrial plants ${ }^{1}$, and since a soil rich in humus would neutralize and nullify small quantities of poisonous secretions, there is all the less reason to assume that any injurious effect can be exerted upon the growth and development of neighbouring plants in this manner ${ }^{2}$. Although the solvent action of the roots of higher green plants is not as energetic as it was formerly supposed to be, it is nevertheless by no means of trifling importance, although Liebig's ${ }^{3}$ conclusion that terrestrial plants absorb all their ash constituents from the soil by means of the solvent action of the roots, is certainly incorrect. It is indeed certain that, in a rich soil, and with a sufficiently active circulation of water, the roots could absorb all the nourishment they require without the necessity of any intimate union with the particles of soil, whereas in a poor and dry soil a close application and approximation of the root-hairs to the soil particles is of the utmost importance.

As a general rule, the plant is able to obtain possession of substances absorbed and retained by the soil, whereas insoluble and non-decomposable compounds are on the other hand unavailable for use, so that a plant may die for want of potassium, phosphorus or nitrogen, in a soil which is found to contain large quantitics of these substances on analysis. The constituents extracted from a soil by dilute acid or water do not afford an accurate representation of its fertility, for such analyses do not take into consideration the substances which may be gradually rendered available by slow decomposition and disintegration ${ }^{4}$.

In order to obtain food materials, many plants exert very energetic solvent and disintegratory action, and thus lichens are able to create for themselves a suitable habitat on bare rocks, and to obtain a supply of nutriment from them (cf. Sect. 27). The rhizoids of mosses and lichens slowly push their way between the sandy particles which their own activity has loosened, and the commingling of these particles of dust with the dead

${ }^{1}$ In contradiction to Mfolisch's assumption (Sitzungsb. d. Wiener Akad., I887, Bd. xcvi, p. 84 ) Czapek finds (Jahrb. f. wiss. Bot., I $896, \mathrm{Bd}, \mathrm{xx} I x, \mathrm{p} .374$ ) that in the root-secretions of Pbanerogams enzymes are not present at all, or not in perceptible amount. On the supposed oxidizing secretions, see Pfeffer, Oxydationsvorgänge, I $88_{9}$, p. 406, and Höveler, Jahrb. f. wiss. Bot., I 892 , Bd. xxıv,

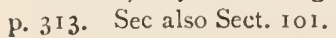

${ }_{2}$ This conclusion, which formerly found common acceptance, has long been proved to be erroneous. For literature see Mohl, Veg. Zelle, $18 \mathfrak{j} \mathbf{1}$, p. 95. Cf. on fungi, \&c., Sect. $9^{2}$.

${ }^{3}$ Liebig, Ann. d. Chem. u. Pharm., I 858 , Bd. Cv, p. I $3^{8} ;$ Die Chemie in Anwend. a. Agric. u. Physiol., 1876, p. I 20. Details on these points by A. Mayer, Agr.-Chem., 1895, 4. Aufl., Bd. II, p. 103 .

* Details in the quoted works of agricultural chemistry. (Of the latcr researches, sec König u. Haselhoff, Landw. Jahrb., I 894 , Bd. xxirI, p. 1009. 
remains of the plants themselves produces a little soil, poor in character though it must be. It can, however, afford lodgement for plants less modest in their demands than lichens, and these continue the work of their predecessors, until finally, perhaps after frequent changes, an abundant vegetation grows from a fruitful humus soil as a monument to the first settlers upon the original barren rocks. On volcanic lavas, and upon the débris from alpine avalanches, the gradual progress of the new vegetation may frequently be followed to its final luxuriance from its modest commencement by mosses and lichens ${ }^{1}$.

Provided a supply of moisture is assured, eren phanerogamic plants are able to take part in the work of colonization on pure sand, or pulverized stones, and to aid in the creation of a rich and fertile soil. No detailed account is possible here of this perpetual creation and change, or of the unceasing organic circulation upon our planet, in which the plant-world plays so important a part (cf. Sect. 51). It may however be mentioned that micro-organisms play a most important part in the changes and decompositions which take place in the soil, and that by the oxidizing activity of certain bacteria, nitrogenous compounds may give rise to the potassium nitrate or saltpetre, which most of the higher plants prefer as a source of nitrogen. Attention must also be paid to the mechanical actions of roots in binding together loose sand and in disintegrating rocks, while animals such as earthworms may aid in various ways in the production of a fruitful soil\%.

${ }^{1}$ Cf. Humboldt, Reisen in d. Aequinoctialgegenden, I, p. 143; Göppert, Flora, I860, p. 161 ; Senft, ibid., I860, p. I93; Pfeffer, Jahrb. d. Schweizer Alpenclubs, I867-68, iv. Jahrgang, p. 462, and Bryeograph. Stud. a. d. rhätisch. Alpen, I87o, p. I35 (Sep.-abdr. a. d. Denkschr. d. Schweiz. Naturf.-Ges.). [The gradual return of vegetation to the island of Krakatoa near Java, which was completely covered by lava and tuff more than twenty-five years ago, illustrates the same process admirably. Here the first plants to appear were bacteria and lowly organized algae (Cy nophycene, $\&$ c.), owing probably to climatic conditions, \&c. ; now large trees are growing by the shore, and many phanerogamic shrubs, \&c. more inland.]

${ }^{2}$ Ch. Darwin, The Formation of Mould by Earthworms, ISsz; Wollny, Forsch. a. d. Geb. d. Agr.-Physik, I8go, Bd. xiII, p. $3^{8 r .}$ 


\section{CHAPTER V}

THE MECHANISM OF GASEOUS EXCHANGE

\section{SECtion 29. General.}

IT is well known that gaseous substances play a most important part in metabolism: thus, in aerobic plants a sufficient supply of oxygen must be ensured, and the carbon dioxide produced by respiration must be removed. Green plants, when illuminated, decompose large quantities of carbonic acid gas and produce free oxygen, although of the former gas traces only are present in the atmosphere. Many plants, especially ferment-organisms, produce besides carbon dioxide, other gases, such as hydrogen, sulphuretted hydrogen, and carburetted hydrogen or marsh gas. Neutral gases, such as hydrogen or nitrogen, also penetrate the plant by means of its air spaces, and permeate the living cells as well, even when not utilized in metabolism.

In a few cases gas-vacuoles may be present (cf. Sect. 22), but otherwise turgid cells always contain gases in dissolved form, the solution of the latter being a necessary consequence for their passage through the cell-wall saturated with imbibed water and penetration to the interior of the cell. The gaseous molecules are absorbed by the layer of imbibition-water against which they collide, and diosmose through to the interior in a dissolved condition. Any internal accumulation necessarily leads to an exosmosis of the gas produced, which is given off to the external air in gaseous form by the over-saturated imbibition water on the free surface of the cell.

The mode of absorption is essentially similar when the cell is surrounded by a layer of water, which absorbs fresh supplies of oxygen or carbonic acid from the air, as the cells which consume these gases extract them from the fluid in which they lie. The outward passage of internally produced gases does not usually lead to any formation of air bubbles upon the free surface of the cell, which only occurs when the rate of excretion is so rapid that the water becomes locally over-saturated. Owing to the high internal osmotic pressure, super-saturation is hardly possible within the cell, and hence certain special factors must be responsible for the formation and maintenance of gas-vacuoles.

In a turgid cell the entry or exit of gas differs from that of liquid 
bodies, only in the fact that the absorbed or evolved gas is first brought into solution or liberated on the outer surface of the cell-wall. Hence, the gaseous exchanges in an air-space surrounded on all sides by turgid cells take place entirely diosmotically, as is the case also for aeriferous cells and vessels, to enter which the gaseous particles must pass through an absorbent cellulose membrane.

Diosmotic absorption is essential in all turgid cells, and the acriferous system is simply for the purpose of rapidly distributing gaseous products throughout the interior of the plant, so that the slower diosmotic transference from cell to cell may be only necessary over short distances. Similarly, certain well-arranged channels serve to convey water to the immediate neighbourhood of the transpiring cells and tissues. In correspondence with the purpose which it subserves, we find, as a general rule, that the formation of a more or less marked acriferous system of intercellular spaces accompanies all marked tissue differentiation. Even in submerged aquatic plants a well-developed but closed aeriferous system is present, although the final exit and entry of gases can take place only by diosmotic means. Stomata and lenticels are usually present in terrestrial plants, and through these open channels gases may pass directly into the interior of the plant.

The existence of such open channels becomes more and more important as the impermeability to gases of the cuticle and cork increases. Moreover, by means of the system of communicating intercellular spaces, gases are brought directly into contact with very large areas of readily permeable cell-walls. Usually, as the impermeability of the cuticle and cork to water increases, they become also less permeable to gases and dissolved substances, and hence when a thick and relatively impermeable cuticle is developed for protection agairst transpiration, gaseous exchange is rendered more or less difficult, though not perhaps to the same relative extent. It is generally of the utmost importance that sufficient oxygen for the continuance of respiration and vital activity may still reach the living cells, when,. in order to lower the rate of transpiration, the stomata close and are no longer available for the entry or exit of gases. The closing of the stomata also causes the production of organic substance to be markedly diminished, for the traces of carbon dioxide present in the air are unable to diffuse with sufficient rapidity through the cuticle to the chloroplastids lying beneath it. For the general good of the plant it is, however, unavoidable that particular functions should sometimes be unfavourably affected, and thus a regulatory protection against excessive transpiration cannot be exercised without at the same time causing the rate of gaseous exchange to be diminished (Sect. 27). Even in those mosses which can withstand drought, their vital activity gradually diminishes, and ultimately ceases when the percentage of water falls below a certain limit. 
The same general principles are of importance in gaseous exchange as in the exchange of other substances, and hence attention has already been incidentally paid to gases as well as to solids and liquids (Chap. IV). It has moreover been shown that carbon dioxide and oxygen, as well as nitrogen and other gases, can pass with relative ease through cell-walls saturated with water, and can also diosmose through the protoplast within.

The readiness with which air currents are induced usually ensures a sufficiently rapid removal of gaseous products and a continual presentation of fresh supplies, so that an assimilating leaf may in a relatively short period of time obtain possession of all the carbon present as carbon dioxide in a large volume of air (Sect. 57). No doubt similar streaming and admixing currents tend to maintain uniformity of composition in the air of the aeriferous system, and thus enable localized differences to be evenly distributed and continually readjusted. The special peculiarities developed in conncxion with the powcr of gaseous exchange cannot be mentioned in detail, but a general idea of the activity of individual organs in gaseous interchanges may be ostained by the consideration of the general factors regulating exchange of all kinds (Sects. 25-27), allowing for the fact that gases are especially favoured in so far as they can be absorbed not only by sub-aërial organs, but also by those immersed in water. As a matter of fact, an aquatic plant may be able to obtain all the gases it requires when it is in a moist atmosphere, and many amphibious aquatic plants are compelled, according to circumstances, to live at one time submerged in water, at another time exposed to air.

The amount of transpiration possible affords on the whole a correct indication of the rapidity with which gaseous exchanges can take place, for both gases and water vapour are largely dependent for entry and exit upon the prescnce of open channels, as well as upon the nature of the epidermal and other limiting membranes. The permeability of the cell-walls to gases and water-vapour usually rises and falls concomitantly with the permeability to water as such, and hence the cuticles of submerged plants and of mossleaves allow carbon dioxide, oxygen, \&c. to pass through with ease, whereas in terrestrial plants the impregnation of the cuticular membrane with wax produces a marked diminution of permeability to these and other gases. It is, however, an important physical fact that cuticularization appears to render the walls more impermeable to water and water-vapour than to carbon dioxide, oxycyen, \&c. (Sects. 21 and 30 ).

All that has been said is directly applicable to cell-walls infiltrated with water, with which we are mainly concerned in turgid plants. As the cell-wall drics, its permeability to gases decreases, and to a greater relative extent in unaltered cellulose walls saturated with water of imbibition than in strongly cuticularized ones (Sect. 30). Just as is also the case with soft gelatine, no pores filled with air appear in the substance of the cell-wall 
on drying, and hence no direct filtration of gas is possible through such a membrane when dry. The diosmotic passage of gases through a membrane is determined by their solubility in the fluid it has imbibed, and hence it follows that as the percentage of water present becomes greater, the permeability to soluble gases increases. Gases are, however, transferred much more slowly by diosmotic means than by movements in mass, and hence they can penetrate and pass through a plate of gypsum much more rapidly when its pores are open than when the plate is moistened and the pores filled with water. For the same reason, when water is allowed to block the stomata or intercellular air channels, a marked hindrance is interposed to rapid gaseous exchange, and hence it is of great importance to the plant that any such blocking of the points of entry and the neighbouring passages should be avoided as far as possible.

The stomata and aeriferous system are indeed admirably adapted for, and of the utmost importance in, gaseous exchange, and usually only the sub-aerial organs are provided with open channels for the entry or exit of gases. The stomata continue to serve as openings for the passage of gases when a leaf immersed in water retains an adhering film of air, giving it a silvery appearance, for by means of this film, dissolved gases are obtained from the water in gaseous form and directly transferred to the plant without any such arrangements being necessary as are exhibited by the respiratory organs of fishes. Submerged leaves may under such circumstances obtain an abundance of carbon dioxide, but when the leaf is actually wetted and the stomata closed by water, the amount absorbed is not sufficient to permit any formation of starch, just as would be the case in similar leaves exposed to air if the stomata were closed and the cuticle thick and impermeable (Sect. 5\%). On the other hand, submerged aquatic plants can absorb sufficient amounts of all the gases they require, including carbonic acid gas, in the absence of any intermediary air film, and indeed nonvolatile dissolved substances can only be absorbed by parts directly wetted by water ${ }^{1}$.

From what has just been said, it is clear that direct absorption takes place through the peripheral walls, and that where open gaseous channels are present, they aid in gaseous exchange to an extent determined by the specific peculiarities of the plant and its organs, as well as by the conditions under which these exist ${ }^{2}$. It has already been mentioned that a sub-aërial

1 Merget (Compt. rend., 1877, T. Lxxiv, pp. 376, 959) erroneously supposed that the presence of a thin film of air was always necessary. Cf. also Devaux, Ann. d. sci. nat., ISS9, vii. sér., T. $1 x$, p. 40 .

2 The double power of gaseous exchange through open channels and through cell-membranes was perhaps first correctly recognized by Dutrochet (Ann. d. sci. nat., IS32, T. $\mathrm{xxV}, \mathrm{p} .242$ ). Garreau gave similar correct interpretations in various later works. Merget's cunclusion (Compt. rend., 1877 , T. Lxxxiv, p. 376 that gases penetrate almost entirely by means of the stomata, and 
leaf camnot as a general rule absorb sufficient carbon dioxide when its stomata are closed, and that when its cuticle is highly impermeable it may even be unable to obtain the full supply of oxygen needed for respiration ${ }^{\mathrm{T}}$. The cliaracters of the cork and cuticle differ according to the stage of development, and are subject to modification in correspondence with varying external conditions (Sects. 21 and $3^{8}$ ). The diameter of the stomatal pores is moreover changeable, and all passage of gases or water-vapour through them ceases as soon as a falling off in the supply of water causes the stomata to close. Many other factors also influence both the direct and the diosmotic transference of gases, and thus the streaming movements originated by differences of pressure are mainly of importance for gaseous exchange in open air-channels. Moreover, the relative development of the intercellular aeriferous system will naturally largely determine the functional importance of the stomata.

An account of the very varied kinds of aeriferous systems which may be met with cannot be given here ${ }^{2}$, but a few details concerning the rapidity of gaseous transference through them will be mentioned later. It is obvious that large and well-developed intercellular channels, such as are found in many aquatic plants, will render rapid admixture possible, while when the intercommunicating air-spaces are feebly developed or absent, localized differences in the pressure and composition of the enclosed air may be maintained. According therefore to the degree of development of the intercommunicating channels, as well as to other circumstances, the stomata and lenticels will either be of merely localized importance or will furnish gaseous supplies for distant organs. Thus, in an actively assimilating leaf, carbon dioxide is immediately absorbed by the green cells which border upon the stomata (Sect. 57), whereas the well-developed aeriferous system of Nymphaed, Typha, Equisetum, and of swamp plants in general, is undoubtedly for the purpose of transferring the necessary oxygen to the rhizomes and roots. A similar functional importance attaches to the erect 'breathing' roots which frequently arise above the mud of a mangrove swamp ${ }^{3}$, for otherwise roots growing in mud containing anaerobic bacteria could obtain no oxygen. Even in a stiff clay soil a sufficiency of

Barthélemy's contradictory assumption (ibid., p. 663) that the stomata are only of subordinate importance in gaseous exchange, do not require any special criticism.

1 See Mangin, Compt. rend., 1887 , T. cv, p. 879 .

${ }^{2}$ Cf. Haberlandt, Physiol. Pflanzenanat., I 896,2 . Aufl, p. 375.

${ }^{3}$ Cf. Jost, Bot. Zeitung, I 887 , p. $60 \mathrm{I}$; Schenck, Flora, I 889 , p. 83 ; Karsten, Mangrove Vegetation, Bibliotheca Botanica, I89I, P. 55 ; Goebel, Ber. d. Bot. Ges., I886, p. 249, and Pflanzenbiol. Schilderungen, 1893 , II, p. 25.5 . [Wieler (Jahrb. f. wiss. Bot., Bd. xxxiI, p. 503) states that the 'pneumathodes' dcscribed by Jost on palm roots are not breathing organs, but are formed, owing to the direct action of water inducing localized hypertrophy. The intercellular spaces are plugged, and arc only permeable to water under prcssure. That special breathing organs do, however, exist on the roots of many plants can hardly be doubted.] 
oxygen is frequently not directly attainable by the roots, and similarly the roots of plants grown in water-cultures must obtain a portion of their oxygen from the sub-aërial organs, while the roots of seedlings of Vicia $f a b a$, Pisum, \&c., appear able when necessary to obtain a sufficient supply of oxygen solely through these channels ${ }^{1}$.

The diffusion of air is not prevented by the thin transverse diaphragms which cross the air canals of sone aquatic plants, although these cause the transference to become somewhat slower. Such diaphragms give rigidity to the plant, and prevent the entrance of mud, \&c., when the older parts die and rot away, or when the rhizomes are accidentally wounded.

In spite of its importance in gaseous exchange, the development of the aeriferous system is not to be regarded solely from this standpoint, for intercellular spaces, however formed, usually become filled with air, just as dead cells do. Apparently the large central air-spaces present in the stems of grasses, umbellifers, \&c., are not produced specially for purposes of gaseous exchange, but rather for economy of construction, for a hollow cylinder with binding pieces at intervals (the nodes) afforcls a maximum of strength and rigidity with a minimum of constructive material. In aquatic plants again, air-spaces are of importance as floats to support the plant and keep it erect.

It is therefore hardly to be expected that the degree of development of the aeriferous system in a tissue should precisely correspond to its gaseous requirements, although intercellular spaces filled with air always appear in the tissues at an early stage in the development of the primary meristem. The younger portions of the apical meristem obtain all the oxygen and other substances which they require by diosmotic transference through several layers of cells, and in these and similar extremely active tissues very narrow intercellular spaces suffice to satisfy the demands for oxygen made by extremely energetic respiration. Bearing in mind that traces only of carbonic acid are present in the air, it is easy to understand why the aeriferous system should in general be well developed in chlorophyllous tissues.

It is therefore distinctly advantageous not only that in strongly illuminated leaves the aeriferous system should become more marked than in those exposed to feeble light, but also that in amphibious plants the intercellular system should frequently attain its most marked development when the plants are completely submerged ${ }^{2}$. Nevertheless, the results produced by the external conditions are not in this or any other case to be regarded solely from a teleological standpoint; and intercellular spaces may serve other purposes, as, for example, when they form secretory

1 See Pfeffer, Druck u. Arbeitsleistung, I 893 , p. 245.

2 Stahl, Über d. Einfluss d. sonnigen u. schattigen Standorts auf d. Ausbildung d. Laubblätter, IS83, p. I7; Schenk, Biol. d. Wassergewächse, ISS6, and Jahrb. f. wiss. Bot., ISS9, Bd. Xx, p. 526 ; Goebel, Pflanzenbiol. Schilderungen, 1893 , Bd. II, p. 255 , and the literature here quoted. 
reservoirs. The partial or complete filling of dead cells and vessels with air is not usually for the purpose of ensuring more active aeration, for no open communication exists between these aeriferous tissue-elements and the actual intercellular aeriferous system.

Under normal conditions the aeriferous system is kept free from water, as is indeed necessary for its continued functional activity. The nonwetting of the leaf, and the various contrivances by which rain and dew are led away from it, ensure that the stomata shall not be occluded by water (Sect. 27). When transpiration is active the intercellular spaces always contain air. and it is only when the sap accumulates to excess that they become partially injected with water ${ }^{1}$. In this case, and also when they have been artificially injected, the water disappears from the intercellular spaces ${ }^{2}$ soon after transpiration commences. No noticeable negative pressure is, however, produced in intercellular spaces which are in open communication with the external air, whereas in vessels the loss of water produces a negative pressure tending to suck it in again when the supply is more abundant (Chap. VI).

Submerged plants are always in a condition of maximal turgidity, and the positive pressure generated by the liberation of oxygen during the assimilation of carbonic dioxide (Sect. 32) tends to keep the aeriferous system open and filled with air. Thus the bladders of Fucus remain tense even when subjected to considerable pressure, and they may burst, owing to the removal of the external hydrostatic pressure, when the plant is suddenly brought to the surface ${ }^{3}$. Other factors must also influence the formation of intercellular spaces, since these appear sooner or later in the primary meristem, and to a more or less marked extent in organs free from chlorophyll.

As the external appearance indicates, the volume which the air-spaces occupy varies very much. In the leaves of most terrestrial plants air-spaces form $\frac{1}{4}$ to $\frac{1}{5}$ of the entire volume. Unger ${ }^{4}$ found a maximal amount of $7 \mathrm{I} \cdot 3$ per cent. by volume of air in the leaves of the floating Pistia texensis, and a minimal value of 3.5 per cent. in the fleshy leaves of Begonia hydrocotylifolia. In wood the amount of air varies inversely with the amount of water present, as shown by the facts given in Chapter VI ${ }^{5}$.

1 Cf. on root-pressure, Sect. 41. Additional examples by Westermaier, Sitzungsb. d. Berl. Akad., 1884 , p. I 107 ; Jönsson, Botaniska Notisera, 1892, p. 252.

2 Moll, Unters, iiber Tropfenausscheidung u. Injection d. Blätter, I8So, p. 7 I (Sep.-abdr. aus Verslagen en Mededeelingen d. Akad. d. Wiss., Amsterdam). Also Barthélemy, Ann. d. sci. nat., 1874 , v. sér., T. xıx, p. I67.

3 Berthold, Mitth. a. d. Zool. Stat, in Neapel, I8S2, Bd. III, p. 431. On gas vacuoles, see Sect. 22 .

4 Unger, Sitzungsb. d. Wien. Akad., I 854, Bd. xI, p. 367. See also Stahl, Über d. Einfluss d. sonnigen u. schattigen Standorts auf d. Ausbildung d. Laubblätter, $188_{3}$, p. I 8 ; Aubert, Rev. gén. de Botanique, $I_{92}$, T. IV, p. 276 .

${ }^{5}$ Estimations by Sachs, Úber d. Porosität d. Tannenholzes, 187\%, p. Io; Dufour, Arb. aus Wïzburg, 1884 , Bd. 11, p. 37 ; Pappenheim, Bot. Centralbl., I892, Bd. xLix, p. 36. 


\section{Section 30. The Passage of Gases through Cells and Cell-walls.}

Except in the case of primordial cells, gases must pass through cellwalls to reach the living protoplast. The permeability of the cell-wall is dependent upon its thickness and specific quality, and also upon whether it is dry or saturated with water. In living turgid cells the walls are entirely or partially saturated, and hence the consideration of the gaseous exchanges through membranes containing water of imbibition is of the utmost importance. Amongst such the cork and cuticle are included. although these retain less and less water as the impregnation with waxy substances becomes more and more complete (Sect. 2I).

As a general rule the loss of water renders gaseous exchange increasingly difficult; Wiesner found that completely dried cellulose walls were quite impermeable to gases, while in cuticularized, suberized, and lignified cell-walls the permeability merely underwent a pronounced decrease on drying ${ }^{1}$. Probably absolute impermeability is never reached, for the negative pressure originated in parenchymatous cells by drying disappears after a time. W'iesner's results may therefore be regarded as coinciding with those of Lictzmann ${ }^{2}$, who found that only a marked diminution of the permeability took place in cellulose and other membranes under the conditions mentioned.

No attention need be paid to the contradictory older researches, since the different results obtained might have been due to a formation of minute slits or to other causes. It is, however, quite possible that in different cell-walls specific differences exist, or that the permeability to gases may be increased by a formation of pores or fissures during drying, for under certain conditions a gelatine film cracks as it dries, though not when dried after fixation in alcohol. Further critical rescarches are required to decide whether, as different workers state, the walls of the tracheae do actually become more permeable to gases as they dry ${ }^{3}$.

The cell-wall therefore behaves similarly to a film of gelatine, whose permeability to gases rapidly decreases as it dries. A plate of nonswelling gypsum behaves very differently, for as it dries its pores fill with air, and hence gases diffuse in mass and with much greater rapidity than was the case at first. It is apparently owing to an impregnation with

1 Wiesner u. Molisch, Sitzungsb. d. Wien. Akad., r\$89, Bd. Xcvir, Abth. i, p. 7r2. [Kamerling (Zur Biol. u. Physiol. d. Zellmembran, Bot. Centralbl., IS97. Bd. Lxxir, p. 49) finds that in the interior of living dried moss-cells a vacuum is maintained for an almost indefinite length of time.]

${ }^{2}$ Lietzmann, Flora, I8s7, p. 3.39 .

${ }^{3}$ Of the literature given by Lietzmann and Wiesner, may be mentioned here: Barthélemy, Ann.

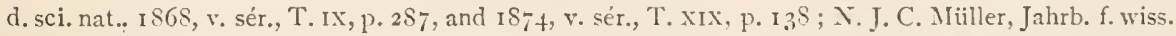
Bot., I869-70, Bd. vir. p. I75. On tracheal walls, see Wiesner, Sitzungsb. d. Wien. Akad., i879. Bd. Lxxix, Abth. i, p. 33 (sep.); Drude, Studien über d. Cons.-methode d. Holzes, ISS9; Strasburger, Über Bau u. Vorrichtungen d. Leitungsbahnen, ISgr, p. $7 \mathrm{Ir}$. 
fatty material that the permeability of the cork and cuticle decreases to a relatively less extent on drying, but it has not as yet been determined what are the means by which lignified walls attain similar properties.

Except in the layers of bark, dry cell-walls hardly ever occur in the living plant. Cell-walls are, however, not always in a condition of maximal saturation, but at the commencement of withering, the amount of water present even in the walls bounding the aeriferous canals is not sufficiently reduced to perceptibly affect the diosmotic gaseous exchange (cf. Sect. 12 and Chap. VI). It is also doubtful whether the already small amount of water which the cuticular layers of the living epidermis contain, is temporarily still further reduced to any great extent when transpiration is very active. In any case, such changes are more likely to be concerned in the regulation of transpiration than of gaseous exchange, and the same importance probably attaches to the drying of gelatinous investments. Gases and water-vapour pass but slowly through the bark, provided no open pores are present, while as shown by mosses, if no protecting corky or cuticular layers are present the influence of the decreased amount of water held by the walls does not suffice under normal conditions to prevent further and complete drying, although the last traces of water are held with great tenacity. Walls saturated with water, which alone need now be considered, behave just as a moist gelatine layer or fixed film of water would do. This is also the case when the water is partly or entirely replaced by fatty substances, though here, as in the case of an india-rubber film, only gases soluble in the water of imbibition or in the substance of the wall can pass through it. The main principles which regulate the diosmosis of dissolved substances in general apply also to this diosmotic transference, and determine the positive or negative osmotic pressures due to the presence or absence of particular gases. An increased partial pressure causes an increased absorption by the saturated walls, and hence acts similarly to an increase in the concentration of a watery solution. The same change occurs when the partial pressure is raised by mechanical compression of the external air, except that the increased external mechanical pressure must be taken into consideration, as is also necessary in dealing with turgid pressures. It is clear, however, that no additional channels for gaseous absorption are opened by such mechanical pressure, nor are any open passages formed by driving out the fluid filling the micellar interstices of the cell-wall. Hence a diosmosing gas will be transferred in a given direction only when its density or partial pressure is greater on one side of a membrane than on the other, no matter whether the difference is due to the presence of a higher percentage of the gas on that side or to mechanical compression ${ }^{1}$. When the passage of the gas is sufficiently

1 Wiesner apparently (1.c.) supposes a difference to exist between an increased partial pressure produced by a different admixture of the component gases and that caused by compression. This is 
rapid, gas bubbles may appear on the submerged surfacc of a diosmotic membrane.

All the experimental evidence shows that the more soluble carbonic acid diffuses much more rapidly than oxygen does through cell-walls saturated with water, and that oxygen diosmoses more rapidly than nitrogen, which is still less soluble. This is admirably indicated by the rapid collapse of a moist bladder filled with carbonic acid gas and surrounded by air. Devaux's ' researches upon diosmosis through entire cell-layers of living aquatic plants gave rates of transference precisely similar to those for water-lamellae, and probably the same would hold good for all completely saturated membrancs capable of pronounced imbibition.

Gases are unable to diffuse at the same rate through a cuticularized membrane impregnated with wax as through unaltered cellulose, for the rate of transference depends upon the nature of the imbibed substances. It appears, however, that carbon dioxide diffuses more rapidly through the cuticle, or through a lamella of india-rubber, than oxygen does, and the latter more rapidly than nitrogen, although no decisive conclusion can be made from the researches mentioned ${ }^{2}$.

The impregnation of the cuticle and cork markedly decreases their permeability for gases, and hence in general the more pronounced the impregnation and the consequent depression of transpiration, the slower the diosmosis of gases will be (cf. Sect. 21.) The relationships are similar to those shown when a piece of paper is impregnated with fat, and although comparative experiments have not as yet been made, there can be no doubt that in the cuticle also the permeability for gases decreases less rapidly than that for water, as the impregnation becomes more and more complete. It is not impossible that in this way a membrane may become totally impermeable to water, and yet allow gases to pass through to a considerablc extent, as is actually the case with a film of india-rubber.

In any case, it is of great importance that oxygen shall be able to diosmose to a sufficient extent even through a strongly developed cuticle (Sect. 29). This is shown by the fact that when the basal ends of hairs of Tradescantia, Momordica, Urtica, \&c., arc imbedded in vaseline, the protoplasm may continue to show fully active streaming movements, for streaming is dependent upon respiration, and in this case the whole of the oxygen necessary for respiration must pass through the cuticle ${ }^{3}$.

certainly an error, although it is quite possible that by compression the pressure may be insufficiently increased to catse perceptible gaseous diosmosis under the given experimental conditions.

1 Devaux, Ann. d. sci. nat., I889, vii. sér., T. $\mathrm{Ix}$, p. 63 .

2 Wiesner, I879, l.c., p. 704; Mangin, Ann. d. sci. nat., I887, T. crv, p. 1809 ; N. J. C. Miiller, Jahrb. f. wiss. Bot., $1869-70$, Bd. vir, p. I69; Barthélemy, Ann. d. sci. nat., I 874 , v. sér., T. xix, p. I38. Cf. Winkelmann, Physik, I891, Bd. I, p. 657 ; Kayser, Ann. d. Physik u. Chenie, I891, Bd. XLIII, p. 544 .

${ }^{3}$ [This proof is only satisfactory when streaming continues under such conditions for prolonged 
Normal respiration continues in many leaves if the surface bearing stomata is smeared with vascline, but in other cases oxygen diffuses inwardly in insufficient quantity to allow full respiration to continue under such circumstances. The same will usually be the case when tissues are completely surrounded by relatively impermeable cork layers ${ }^{1}$.

It is easy to understand why the closure of the stomata, eren in leaves with hardly any cuticle, may suffice to prevent any production of starch, for owing to the exccedingly minute percentage of carbon dioxide present in ordinary air, the assimilation of this gas is active only when gaseous exchange is extremely rapid (Sect. 57). Starch may appear, however, in leaves with blocked stomata in an atmosphere containing 20 to 40 per cent. of carbon dioxide. Blackman ${ }^{2}$ has indeed shown definitely that this gas diffuses in appreciable amount through the thick cuticle of leaves of Nerium oleander and Prumus laurocerasus in such an atmosphere. Similarly, when the surface bearing stomata is smeared with vaseline, the carbon dioxide produced by respiration diffuses outwards more and more rapidly through the cuticle as it accumulates, whereas at first it escaped almost entirely through the stomata. From these considerations, as well as from the large amount which can be assimilated when the stomata are open, it follows that the cell-walls bordering upon the intercellular spaces must be readily permeable to gases.

The feebly cuticularized epidermis of aquatic plants is readily permeable to water, and it allows gases also to diosmose with ease. The formation of bubbles which accompanies the assimilation of carbon dioxide (Sect. 32) demonstrates directly that large amounts of this gas diffuse into the interior even when the surrounding water contains but little ${ }^{3}$.

periods of time, for the necessary energy might be derived from intramolecular respiration, and as a matter of fact, certain aerobic plants (Chara, Nitclla, \&rc.) continue to show rotation for long periods of time (one or more weeks) in the absence of free oxygen (cf. Ewart, Journ. Linn. Soc. Bot., Vol. xxxi, I $\$ 96$, p. 42 , and Kühne, Zeitschr. f. Biol., Bd. Xxxvi, p. I, ISg\$). By means of the bacterium method it can, however, be shown that oxygen diosmoses outwardly through the cuticularized walls of an assimilating hair-cell lying in water, but more rapidly through the uncuticularized cnd-walls, and the difference becomcs increasingly marked as the cuticularization is more complete (cf. Ewart, 1. c., p. 365). Diosmosis will be cqually possible in air, provided that the properties of the cuticular film are unaltered by immersion and that its water-percentage remains constant.]

1 On the permeability of cork for gascs, see Lietzmann, Flora, i $88_{7}, \mathrm{p} .376$; Wiesner und Molisch, Sitzungsb. d. Wien. Akad., I\$\$9, Bd. xcvirI, Abth. i, p. 678; Wiesner, ibid., I 879. Bd. Ixxix, Abth. i, p. 4 (Sep..

2 Blackman, Phıl. Trans., I 895 , Vol. clxxxvi, p. 556; 4 nnals of Botany, 1895, Vol. Ix, p. 164. Hence it was that Boussingault found that $\mathrm{CO}_{2}$-assimilation took place in atmospheres containing a large percentage of carbon dioxide, although the stomata of the leaves had been closed by vaseline (Agron., Chim. agric., \&c., I 868 , T. Iv, p. 375. See also Garreau, Ann. d. sci. nat., I $\$ 49$, iii. sẻr., T. xIII, p. 343).

3 The amount of a gas absorbed by a given rolume of water depends upon the solubility as well as the partial pressure of the former. Hence at $15^{\circ} \mathrm{C}$. water contains, when fully saturated, $63.8 \mathrm{~N}$, $34.0 \mathrm{O}, 2.2 \mathrm{CO}_{2}$, whercas the air above contains $21 \mathrm{O}, 79 \mathrm{~N}, 0.04 \mathrm{CO}_{2}$. A litre of such water 
The existence of this ready permeability is shown also by Devaux's researches, in which absorption was induced by exhausting the air of the intercellular spaces, and it is not surprising to find that the result was the same whether the plants were immersed in water or surrounded by saturated air ${ }^{1}$.

It is hardly surprising that in many plants special combinations may occur, which may however without difficulty be resolved into their component factors; the essential principles regulating these have already been given. Thus in the outer wall of the epidermis the layers from without inwards are less and less cuticularized, so that diffusion must go on with increasing readiness in this direction, as a gas passes inwards. The temperature also exercises a certain influence upon gaseous diosmosis, but according to Mangin this is not very marked $\%$

Nomenclature. The passage of dissolved gaseous particles through membranes may be termed diosmosis, as such passage is in the case of other dissolved substances. When the gas passes in gaseous form through the pores of the dividing wall (Graham's diffusion and effusion ${ }^{3}$ ), it is preferable to speak of filtration when the gas is caused to stream through the pores of the membrane by being under compression on the one side of it, and of gaseous diffusion (interdiffusion) when the movement is the result of the different composition of the air on the two surfaces of the partition wall. When filtration takes place through long narrow canals, as in intercellular spaces, we may speak of capillary streaming (Graham's transpiration).

Filtration and gaseous diffusion take place through the stomata, lenticels, \&c., and their rapidity varies inversely as the square root of the density of the gas concerned. The rapidly diosmosing carbon dioxide diffuses more slowly than oxygen or nitrogen, but these differences almost disappear as the influence which the dividing membrane exerts becomes more prominent." We may speak of the diosmosis of gases through membranes, independently of whether mere solution, or chemical combinations, or other agencies, take part in the process. The formula for the passage through a film of paper, as obtained by Exner's researches, is represented by $\frac{C}{\sqrt{d}}$, where $C$ is the coefficient of absorption, and $d$ the density of the gas ${ }^{4}$.

contains at $\tilde{\sigma}^{\circ} \mathrm{C}$. about $2 \mathrm{I} \cdot 5$, at $20^{\circ} \mathrm{C}$. about $\mathbf{I} 6.7 \mathrm{c.cm}$. of gas. Frequently water is not fully saturated, and in the depths of the ocean only two-thirds to one-half, or less, of the possible amount of oxygen may be present. Cf. Bunsen, Gasometrische Methoden, I877; Zacharias, Die Thier-u. Pflanzenwelt d. Süsswassers, I891, p. I5; Devaux, 1.c., p. 53; Goebel, Pflanzenbiol. Stud., I\$93. 2. Th., p. 248; Huifner, Archiv f. Anat. u. Physiol., I 897 , p. I 5 (Physiol. Abth.).

1 Since in air the exosmosing gas is more rapidly removed, the diosmotic transference might take place somewhat more rapidly in air than in water. Cf. Wiesner, IS89, 1. c., p. 70 5 .

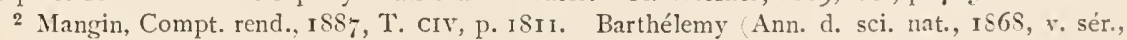
T. $\mathrm{sx}, \mathrm{p} .287$ ) found that the influence of the temperature was much more pronounced.

${ }^{3}$ Graham, Ann. d. Phys. u. Chemie, IS63, Bd. CXx, p. 4IS. For the physical relationships the reader is referred to physical textbooks, such as Winkelmann, Handb. d. Physik, IS9r, Bd. I, p. 640 ; Reis, Lehrb. d. Physik, I893, 8. Aufl., p. 235.

${ }^{4}$ Cf. Winkelmann, 1.c. p. 651 , and the literature there cited. Similar relationships have been found for india-rubber. 
From these co-operating factors (absorption and gaseous diffusion) it follows that the less soluble hydrogen will pass through more rapidly than the heavier oxygen gas, and Devaux found this to be the case during diffusion through layers of turgid cells ${ }^{1}$. Obriously the processes of diffusion, filtration, \&c., may co-operate in various other ways. It is, moreover, easy to understand that in the streaming of gases through capillaries precisely the same relationships will not be found to exist in rigid capillaries ${ }^{2}$ as in the intercellular capillaries of tissues, the diameter and dimensions of which may be altered by pressure.

Methods. A detailed account of all the different researches and experimental methods employed can hardly be given. In some cases portions of the upper epidermis of a leaf free from stomata were employed (N. J. C. Müller, Lietzmann, Mangin), in others entire leaves were used (Wiesner, Blackman). The object was fixed by cement, or by screwing up the two halves of a tube across which it was stretched. A difference of pressure was then created between the two sides by evacuation or compression, or a different gas led into one end. The rapidity of the diosmosis or diffusion may be determined either

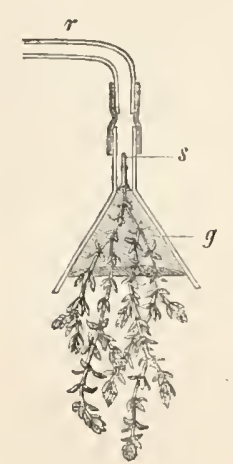

FIG. I9. by noticing the differences of level, \&c., on the two sides, or the percentage composition of the gas present on either or both sides may be estimated. To prevent rupture, Nüller used a plate of gypsum as a support, while Lietzmann and Wiesner attained the same end by using small fragments only. When the isolated epidermis is employed, the diosmotic transference takes place through a layer of cells, just as when a unilamellar leaf of a moss (Mnium) or of a fern (Hymenophyllum) is used, or even when an ivy leaf is employed, for in the last case gases pass readily through the stomata and intercellular spaces to the upper epidermis, which is free from them. In certain researches the stomata were closed by smearing with fat (Garreau, Boussingault, Stahl, Blackman) or with gelatine (Mangin).

In Devaux's experiments with submerged water plants (Elodea, Ceratophyllum, \&c.) the arrangement shown in Fig. I9 was used. The plants were so imbedded in gelatine in the filter funnel, that by evacuation at $r$, gases were obtained which have diffused through the exposed outer epidermal layers, and have passed through the aeriferous canals to the cut surface of the stem $(s)$ projecting above the gelatine. Devaux mentions the extent to which errors, due to the existence of gases in the plant previous to the commencement of the experiment, and to the evolution of the carbon dioxide produced by respiration, may be avoided or adjusted.

\section{Section 31. The Communications of the Aeriferous System with the External World.}

The aeriferous system communicates with the external world, neglecting accidental fissures in the peripheral tissue layers, mainly by means of the

1 Devaux, Ann. d. sci. nat., I $\$ 89$, vii. sér., T. 1X, p. 95 ; also Wiesner u. Molisch, 1. c., p. 7 I 3 .

2 Wiesner und Molisch, l. c., p. 707. 
stomata in the epidermis, and of intercellular passages through lenticels or through spongy cork in the periderm. These are not found in all plants, but become necessary whenever gaseous exchange is rendered difficult by the presence of comparatively impermeable external walls developed as a protection against excessive transpiration (Sects. 27, 29).

According to Klebahn ', intercellular air-channels are commonly present in the periderm, though they are often feebly developed. It is by a localized production of spongy cork that the lenticels found on branches, stems, and roots are formed, and wherever stomata were originally present, the lenticels commonly appear beneath them, so that the aeriferous system remains provided with a point of exit at the same spot.

Stomata are found almost without exception upon sub-aërial organs only, and in greatest abundance upon the green leaves ${ }^{2}$. This is apparently because they are partly for the purpose of cnabling the traces of carbon dioxide present in the air to be fully utilized, for, as has already been shown, when the stomata are closed the absorption of this gas is commonly insufficient to permit any formation of starch, whereas oxygen in most cases still penetrates in sufficient quantity for all requirements. Hence it is that stomata are scarce or absent in organs or plants with but little or no chlorophyll ${ }^{3}$. The presence of stomata is, however, apparently advantageous when marked surface development (as in petals) induces the formation of a relatively impermeable cuticle as a protection against transpiration.

To give a detailed account of the presence and distribution of the stomata is beyond our purpose ${ }^{4}$. It is well known that in leaves frequently one surface, usually the under one, is more richly provided with stomata than the other, and this surface may indeed be the only one which bears them. In all cases the well-developed intercellular space-system ensures that carbon dioxide shall be conveyed in sufficient amount to the more highly chlorophyllous cells of the upper surface, and the importance of these tiny but numerous openings in gaseous exchange is shown by the fact

1 Klebahn, Die Rindenporen, $188_{4}$ (Sep.-abdr. a. d. Jenaischen Zeitschr. f. Naturwiss., Bd. x). On lenti-cells, see de Bary, Comp. Anatom., IS77, p. $575 ;$ Haberlandt, Physiol. Pflanzenanat., 1896 , 2. Aufl, p. 407. On the spongy cork of aeriferous breathing-roots, see the literature given in Sect. 29 ; also A. Weisse, Ber. d. Pot. Ges., IS97, p. 303. [A. Wieler, Die Function d. Pneumathoden u. d. Aerenchymes, Jahrb. f. wiss. Bot., i $S 98$, Bd. xxxi , p. 503.]

2 In Musci, stomata are present only on the spore capsule. On the development of the peculiar stomata of the Marchantiaceae, see Leitgeb, Sitzungsb. d. Wien. Akad, ISSo, Bd. Lxxxi, Abth. i, p. 40 .

3 Johow, Jahrb. f. wiss. Bot., $1 S_{9}$, Bd. xx, p. 506. On the occurrence of stomata on subterranean organs, see Hohnfeldt, Bot. Jahresb., 185o, Bd. I, p. $4^{S}$; Kohl, Transpiration d. Ptlanzen, 1886 , p. 26.

4 A general account is given by de Bary, Comp. Anat., 1877 ; Haberlandt, I. c. ; Tschirch, Pflanzen-anat., $18 S 9$, p. 43I. Also A. Weiss, Sitzungsb. d. Wien. Akad., I8go, Bd. xcrx, Abth. i, p. 307 ; Wagner, ibid., 1 S92, Bd. Cr, Abth. i, p. 5 I 3. 
that when they are closed the assimilation of carbon dioxide almost entirely ceases.

The character and position of the points of exit are such as at once to indicate that they have been developed for purposes of acration. Thus stomata are usually absent in submerged plants, and in parts growing normally beneath the surface of the water ${ }^{1}$, whereas on those organs which rise above the water, and on the upper surfaces of floating leaves, they may be as abundant as in terestrial plants. Moreover, by the arrangements for leading away water and other adaptive modifications, any danger that the capillary pores may suck in water and become blocked is usually avoided ${ }^{2}$. Under ordinary circumstances neither heavy falls of rain or dew, nor even temporary immersion in water, cause any such occlusion of the stomata.

Just as intercellular spaces may serve special purposes, acting, for example, as secretory reservoirs, so also may the stomata subserve functions foreign to their original purpose. Thus water-stomata serve for the excretion of water and watery solutions, while the sparsely scattcred stomata which may be present on submerged plants have lost their primitive importance as gaseous channels. Many stomata never become fully developed, or they may barely open, or may soon permanently close ${ }^{3}$. Occasionally resinous masses may block the stomata in the leaves of the Coniferae ${ }^{4}$, and it is perhaps partly owing to the adherence of particles of soot that Coniferae and other evergreen plants with erect leaves, or with leaves bearing stomata on their upper surfaces, grow badly in the neighbourhood of towns and factories.

The width of the stomatal aperture varies according to the external conditions, and especially important is the fact, which Amici first observed, but which Mohl first definitely established, that as a general rule the stomata pores narrow as turgidity decreases, and become completely closed as soon as any signs of withering are manifest, or even earlier ${ }^{5}$. In this way the evaporation of water is rendered increasingly difficult, and as far as possible a fatal loss of water is avoided, although at the expense of the function

1 For literature see Kohl, Transpiration d. Pflanzen, I\$86, p. 26 ; Sauvaugreau, Compt. rend., 18 o, T. III, p. 313 ; Goebel, Pflanzenbiol. Schilderungen, 1893, 2. Th., p. 2 +o.

2 Cf. Tschirch, Anat., ISS9, p. 438 ; Volkens, Ber. d. Hot. Ges., 1 890, p. 120 ; Kerner, Pflanzenleben, 1887, p. 266. On the draining away of water, see Sect. 27 and the literature there given.

${ }^{3}$ De Bary, Comp. Anat., 1887, 1. 54. Czeck's data (Bot. Zeitung, p. 805) are not applicable to the point at issue, according to Schwendener (Monatsb. d. Berl. Akad,, 1881, p. 866). See also Kohl, Transpiration d. Pflanzen, 1886, p. 27. On the closure by cellular outgrowths, see Molisch, Sitzungsb. d. Wien. Akad., 1888 , Bd. Xcril, Abth. i, p. 298. According to Stahl, the stomata of various evergreens are closed in winter (Bot. Zeitung, 1894, p. 126). A similar closure commonly accompanies the assumption of the autumnal colouration by the leaves.

+ Thomas, Jahrb. f. wiss. Bot., $1865-6$, Bd. Iv, p. 28 ; Wilhelm, Ber. d. But. Ges., 1883, p. 325.

5 Mohl, Bot. Zeitung, 1856 , p. 697 ; confirmed by Leitgeb, Mitth. d. Bot. Inst. in Graz, เ886, p. 125 ; Schwendener, Monatsb. d. Merl. Akad., I88ı, p. 833 , \&c. 
of $\mathrm{CO}_{2}$-assimilation, which is necessarily diminished almost to nil. The formation of a cuticle and the regulatory action of the stomata is essentially intended for the retention of the necessary amount of water, and it is in view of this function that the special adaptive modifications are to be regarded (cf. Sect. $3^{8}$ ).

It is hardly surprising that in a few cases true stomata, as well as water stomata and the peculiar stomata of Marchantia, close only partially or not at all when the plant becomes flaccid. According to de Bary, this is the case in the stomata on the leaves of Kaulfussia, and according to Schwendener in those of Cynosurus echinatus and a few other grasses ${ }^{1}$. Schwendener's conclusion that nonclosing stomata were the rule in aquatic plants has not been found absolutely correct, although Stahl found that frequently in cases of plants of marshy habitats (Alisma plantago, Acorus calamus, \&c.) the stomata remain open in flaccid leaves, which is a property exhibited also by many woody plants which prefer a wet soil (Salix, Alnus), and by other plants as well ${ }^{2}$.

The classical researches of Mohl have shown that the width of a stoma varies according to the turgidity of its guard-cells, and the same author has proved that stomata which open in water may be caused to close by the plasmolyzing action of a sugar solution (1.c., p. 702). The movements and changes of form of the guard-cells due to these variations of turgidity have been more closely studied by Schwendener and his successors, but Mohl was the first to prove that in the isolated stomatal apparatus an increase of turgidity causes a widening of the stoma, a decrease leading to a narrowing of the aperture, and ultimately to complcte closure. By means of comparative experiments, in which, when necessary, all the cells in the epidermis surrounding the guard-cells were ruptured, it was found that the antagonistic action of the neighbouring epidermal cells might more or less markedly influence the movements of the guard-cells, so that in extreme cases, instead of the attempted opening, a closure of the stoma might be produced.

Schwendener (1. c., I 88 I, p. 853), Haberlandt (l. c.), Schäfer (1. c., p. 204) recognized the influence exerted by the neighbouring cells, but did not attach much importance to it, whereas Mohl (1. c.) and Leitgeb arrived in certain cases at a contrary opinion. Thus both the latter saw that the removal or rupture of the surrounding epidermal cells caused the stomata of many plants to open more widely, while similar treatment caused stomata to open which had partially or entirely closed owing to the immersion of the leaves in water. This closure in water was seen by $\mathrm{Mohl}^{3}$ to take place in various grasses, and also in Amaryllis formosissima,

1 De Bary, Comp. Anat., $187 \tau$, p. $5^{8}$; Schwendener, Sitzungsb. d. Berl. Akad., I889, p. 69.

${ }^{2}$ See Kohl, Transpiration d. Pflanzen, 18S6, p. 25 ; Haberlandt, Flora, IS87, p. 100 ; Schaefer, Jahrb. f. wiss. Bot., I8SS, Bd. XIx, p. 196; Stahl, Bot. Zeitung, IS94, pp. 123, 137. [Rosenberg (Ueber die Transp. d. Halophyten, Kongl. Vetenskap. Akad. Förhandlingar, IS97, No. 9, p. 531) finds that numerous Haluphytes are able to close their stomata.]

${ }^{3}$ Mohl, l.c. Confirmatory results by Unger, Sitzungsb. d. Wien, Akad., i 857 , Bd. XXY. 1\% + 68 ; 
whereas in the case of our native orchids, and a few other plants, wetting with water caused the stomata to open more widely. Since the isolated guard-cells always react in the same manner, these differences can only result from the opposing action of the surrounding tissue, and, as Mohl found, this is frequently very feeble in character. Hence arise the contradictory results obtained by different workers, which, however, differ quantitatively only; and are perhaps partially due to the influence exerted by the cultural conditions. Mohl observed that when flaccid leaves of Amaryllis formosissima with closed stomata are placed in water, the stomata open at first and become fully expanded in about five minutes, then narrowing and ultimately closing again. This is owing to the antagonistic action of the guard-cells and the surrounding epidermal cells, and to the unequal rapidity with which these absorb water. Stahl ${ }^{1}$ observed that in the drooping leaves of certain plants the stomata remain open in air saturated with moisture, though when transpiration is permitted they completely close, but it has yet to be determined whether this is due to the antagonistic action of the epidermal cells or to other causes.

From the above researches it appears that as a general rule it is the neighbouring epidermal cells which act antagonistically to the guard-cells, but it is not surprising to find that apparently in many cases some influence is exerted by the union of the epidernis with the tissues beneath (see also Mohl, l. c., pp. 703, 7I7). According to Benecke ${ }^{2}$, when subsidiary guard-cells are present, these are of importance in shielding the guard-cells proper from pressure and tension of external origin.

The mechanism of the opening movements. The movements of the stomatal apparatus caused by changes of turgidity are due to its form and structural relationships. This was originally recognized by Mohl, but the relationships actually existing were first made clear by Schwendener. From his researches and those of his pupils ${ }^{3}$, it appears that precisely the same mechanism is not employed in all cases to produce the opening movement which follows a rise of turgidity. It must, however, suffice to indicate a few only of these relationships.

In most cases the opening is probably due to the concave surface of the guard-cell (d, Fig. 20) being less extensible than the convex one (e), and hence elongating less as the turgidity increases. The same result is produced when air is forced into an india-rubber tube having one side thicker than the other. By employing two such pieces of tubing, a model may be constructed in which, when the india-rubber walls of the 'guard-cells' are subjected to increased tension, they separate from one another and open the 'stoma.' This result is attained in the guard-cells by an increased thickness of the less tensile walls, as can be seen in the transverse section of the stoma of Helleborus shown in Fig. 20. In this particular case it is also

N. J. C. Miiller, Jahrb. f. wiss. Bot., 1872 , Bd. v1I1, p. 75 ; Leitgeb, Mitth. d. Bot. Inst. in Graz, 1886 , p. 125, \& c.

1 Stahl, Bot. Zeitung, 1894 , p. 121.

${ }^{2}$ Benecke, Bot. Zeitung, IS92, p. 538 .

${ }^{3}$ Schwendener, Monatsb. d. Berl. Akad., I881, p. 833 ; Sitzungsb. d. Berl. Akad., 1889, p. 65 (Gramineae and Cyperaceae). A concise résumé is given by Haberlandt, Physiol. Anat., 1896, 2. Auf., p. 396. Also Haberlandt, Jahrb. f. wiss. Bot., I 886, Bd. XvIl, p. 461 (Mosses), and Flora, 1887 , p. Io6; Schäfer, Jahrb. f. wiss. Bot., I88s, Pd. xix, p. 200. 
shown how the sectional outline alters as the stoma opens, and how the inner part of the stomatal aperture widens, but not the outer.

In Graminae and Cyperaceae the apposed ends of the guard-cells are thinwalled ( $a$, Fig. $2 \mathrm{I})$, while the edges in contact are thick and rigid (b, Fig. $2 \mathrm{I})$. An increase of turgidity causes the end portions to expand and push the rigid lips of the stoma apart from one another. Even the stomata of different moss capsules do not all act in precisely the same manner. 'Thus, according to Haberlandt, in Mnium cuspidatum the guardcells are stretched and elliptical in the direction $e-d$, as in Fig. 20 , but become rounded as the turgidity increases, and thus cause the stoma to open. Further peculiarities are to be found in the literature quoted, especially that by Haberlandt and Schäfer.

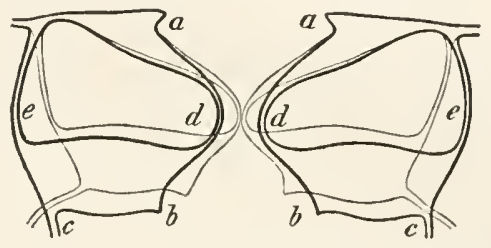

FIG. 20. Stoma of Helleborus spec. in the open and closed condition. (After Schwendener.)

Even stomata with essentially similar opening mechanisms show various differences as regards the changes of form and position produced by varying turgidity, but these are for the most part of merely accessory importance. Thus in many stomata which open by the method first given, no widening of the internal aperture of the stoma occurs, as it does in Hellehorus. Schwendener gives various examples of the antagonistic action of the epidermal cells, but in other cases the guard-cells are loosely attached to the neighbouring cells, so that full freedom of movement is assured.

When those parts of concave walls which touch one another are less thickened, this appears to be of advantage in securing a more complete closure; at the same time the neighbouring parts of the wall are highly cuticularized and strongly thickened, to ensure an adequate protection against transpiration. If the concave edge were too thin and
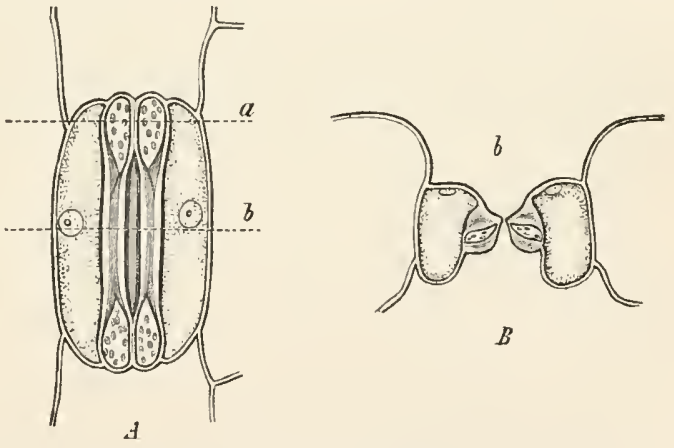

B

FIG. 2I. Stoma of Avena sativa. $A$, in face view; $B$, a translerse section i hrough $b\left(\times_{5} u(y)\right.$.

extensible it would bulge out as the turgidity of the guard-cells increased, and thus partly nullify their action in opening the stoma. The thinness of a portion of the convex outer surface is apparently in order to favour diosmotic exchange with neighbouring cells.

External agcncies which influence turgor will alter the width of the stomatal openings to a greater or less extent, though since different plants and different cells have different powers of reaction, it is not to be expected that the same result should in every case be produced by the same stimulus. The effects produced by changes of illumination indicate this. Thus, 
as Mohl observed, light generally causes an opening or widening of the stomata, while darkness induces narrowing or closure $^{1}$, whereas Leitgeb found that in certain plants the stomata open more widely in darkness. Kohl mentions that the stomata in the leaf of Trianea bogotensis rapidly open more widely when exposed to light, and hence are suitable for purposes of demonstration.

Changes of temperature are much less potent than changes in illumination, for in most cases the former do not markedly affect the width of the stomatal apertures ${ }^{2}$. In certain plants a change of temperature has been found to produce similar effects to those produced by light ${ }^{3}$. Mechanical disturbances appear to be inoperative, while the closure Müller found to be caused by induction shocks is due, according to Leitgeb, to the guard-cells being injured or killed ${ }^{4}$.

These and similar reactions consequent upon changes of turgidity are not necessarily of great biological significance. The widening of the stomata which strong illumination causes in a turgid plant may be of importance in allowing an increased amount of carbon dioxide to be available for assimilation. On the other hand, the closure of the stomata caused by wetting or by darkness may be of use, in some cases at any rate, by preventing the capillary stomatal pores from being occluded by rain or dew .

Owing to the reactive powers of the guard-cells, the width of the stoma is unavoidably subject to certain variations under normal vegetative conditions. So long, however, as the plant remains fully turgid, the stomata are opened most widely during the day, but for the most part close partially during the night, as has been shown by different investigators ${ }^{5}$.

It can hardly be doubted that the opening and closing of the stomata are due to variations of turgidity induced by the light or heat rays within the living cells, although the precise manner in which illumination influences turgor is not yet known. According to the nature of the plant and to the external circumstances, darkness may apparently cause either an increase or a diminution of the turgidity of the guard-cells and epidermal cells. In a few of the cases investigated by Leitgeb (l. c., p. 175) the closure of the stomata at night seems to be caused by the more markedly increased turgidity of the surrounding epidermal cells. Frequently, according to N. J. C. Müller (1. c., p. 80) and Schwendener (1.c., I889, p. 7r), the

1 Schwendener, Monatsb. d. Berl. Akad., 1881, p. 862 ; Kohl, Transpiration d. Pflanzen, 1886, p. 36 ; Leitgeb, Mitth. d. Bot. Inst. zu Graz, 1886, pp. 137, 182 ; Schäfer, Jahrb. f. wiss. Bot., 1888, Bd. XIx, p. 196 ; Stahl, Bot. Zeitung, I894, p. 124 ; Schellenberg, ibid., 1896, p. 175 ; Stahl, ibid., 1897, p. 73. [F. Darwin, Observations on Stomata, Phil. Trans., June 16, 1898.]

${ }_{2}$ Schwendener, l. c., p. $\delta 6_{3}$; Kohl, l. c., p. ${ }_{3} \delta$; Leitgeb, l.c., pp. ${ }_{134},{ }_{3} 3$.

s Czech, Bot. Zeitung, 1869, p. 805 ; N. J. C. Müller, Jahrb. f. wiss. Bot., 1872, Bd. vili, p. 90 ; Eberdt, Transpiration d. Pflanzen, 1889 , p. 52.

- Leitgeb, 1. c., pp. 144, 145 ; N. J. C. Müller, 1.c., p. 96.

3 Unger, Sitzungsb. d. Wien. Akad., $186 \mathrm{r}$, Bd. Xl1v, p. 335 ; Czech, Bot. Zeitung, 1869, p. 804 ; Leitgeb, l. c., p. 149. 
hydrostatic pressure in the guard-cells is more marked than that of the other epidermal cells, but Pfeffer could find no perceptible difference between them in Amaryllis formosissima and Tradescantia discolor.

Turgidity and its regulation are in all cases dependent upon the amount of material available for osmotic purposes. Hence the formation of organic substance by the assimilatory activity of the chloroplastids may play a prominent part in inducing the movements of the guard-cells. Nevertheless, the movements caused by light are not due solely to the accumulation of soluble assimilatory products, as Mohl (l.c., p. 7I 7) and Kohl (l.c., p. 39) suppose, for in well-nourished plants light causes the stomata to open even in an atmosphere free from carbon dioxide. Light acts here as a stimulus causing movement, just as it does in other movements induced by stimuli. It is only owing to the insufficient development of the specific reactive power that stomata with non-chlorophyllous guard-cells ${ }^{1}$, and indeed a few in which chlorophyll is present, sometimes do not perceptibly react to a change in the illumination.

It is not, however, surprising that the guard-cells should react more energetically after previous exposure to light (Mohl, 1.c., p. 7 I 6), or that they should be independent of the accumulation of assimilated products; external influences, such as light, frequently exercise inductive actions of a tonic character which persist for a time (Leitgeb, l.c., p. x 29). When a stoma is abnormally wide open the amount of insoluble reserve-food-material in the guard-cells decreases, but this merely corresponds with the usual phenomena of turgor regulation.

The opening of the stomata in every case increases the rapidity of gaseous exchange, which is, however, dependent upon other circumstances as well, such as the length of the capillary channels and the position of the stomata. In sunken stomata, and especially when the channel leading to the pore passage is narrow, gaseous diffusion is rendered more difficult. The stomata are always, it is true, exceedingly narrow openings, since the diameter of the pore passage rarely surpasses $0.03 \mathrm{~mm}$., and its area c.0046 sq. mm. ${ }^{2}$

This is, however, compensated by their great abundance, for commonly 100 to 300 stomata may be present on I sq. mm. of leaf surface, and in a few cases as many as 700 . Taken as a whole, the gaseous exchanges through the lenticels are less in amount, for these are very much fewer in number, although a lenticel transmits more gas through its numerous intercellular canals than does a single stomatal pore. Moreover, even in young

1 Kohl, 1.c., p. 39. The guard-cells in etiolated plants may, according to Leitgeb (1.c., p. 175), be widely open. [It must, however, be remembered that etiolated chloroplastids are capable of weak $\mathrm{CO}_{2}$-assimilation in the absence of all chlorophyll (cf.Sect. $5^{2}$ ). In leaves in which the chloroplastids of the guard-cells had been bleached or rendered permanently inactive by the prolonged action of intense light (Ewart, Ann. of Bot., I 897 , Yol. XI, p. 475), the stomata were fuund in all cases to be closed, but this might be simply owing to the guard-cells having been injured, for when the exposure is intense and prolonged, the death of the entire cell soon follows that of the chloroplastids.]

${ }^{2}$ For anatomical details, see de Bary, Comp. Anat., 1877, p. 39; Mohl, 1.c.; Weiss, Jahrb. f. wiss. Bot., $186_{5}-6,13 d .1$, p. $181,8 c$. 
stems with a chlorophyllous cortex, the porous cork through which air passes to the periderm layers has not also to provide for the transference of the traces of carbon dioxide to actively assimilating cells, as is the case in the leaf.

Above all things, it depends upon the development of the aeriferous system whether the gaseous exchanges through stomata and lenticels are mainly of local importance, or are of service to distant parts as well. As a matter of fact, when the aeriferous system is well developed, a slight pressure suffices to drive air through a long piece of stem until it emerges through the stomatal pores of the leaf (Sect. 32). On the other hand, differences of pressure become equalized only slowly in the plants of the Crassulaceae, whereas, according to Devaux, gases circulate more readily in cucumbers, apples, potatoes, and beetroots ${ }^{1}$.

Even in plants with well-developed aeriferous systems, gases are not directly conveyed to all the cells, but must travel in solution for a short distance to reach them. It is only by means of diosmosis that an exchange is possible with the tracheides and tracheal vessels which are more or less completely filled with air, for these are not in open communication with the aeriferous system. The intercellular spaces of the medullary rays, however, extend near to or touch the tracheae, and usually communicate with the external air by channels of porous cork ${ }^{2}$.

That the stomata place the aeriferous system in direct and open communication with the external air may be proved by microscopical observation as well as by direct experiments, such as those conducted by Dutrochet, and later by RaffenauDelile, Unger, Sachs, \&c. The principle of these experiments is essentially the same, though various modifications have been adopted, for in all cases air or some other gas is driven through leaves or leaf twigs, and the exit of bubbles from the leaf or cut surface of the stem or petiole observed under water ${ }^{3}$. The arrangements shown in Figs. 22 and 23 are well adapted to demonstrate the transference of gases through the intercellular spaces and stomata. In Fig. 22 the petiole of the leaf, $d$, passes into the glass cylinder $g$, which is two-thirds filled with water. If necessary, air-tight connexions are ensured by means of gelatine or cocoa-butter. On placing the tube $c$ in connexion with an air pump, as soon as the pressure in $g$ is lowered to a certain point a stream of air-bubbles comes from the cut surface of the petiole immersed in water. In Fig. 23 the leaf is inclosed in the glass cylinder

${ }^{1}$ Bonnier, Rev. gén. de Bot., I 893 , T. v, p. II I ; Devaux, ibid., I 89 I, T. III, p. 49, and Ann. d. sci. nat., I89I, vii. sér., T. xıv, p. 309 .

${ }^{2}$ Amici, Ann. d. sci. nat., I 824 , T. II, p. 241 ; Sachs, Über Porosität d. Holzes, I877, p. 4 ; Russow, Bot. Centralbl., I $\$ 8_{3}$, Bd. X111, p. 366 ; Strasburger, Bau u. Verrichtung d. Leitungsbahnen, I891, p. 710, \&c.; de Bary, Comp. Anat., I877, p. 33 S ; Russow, 1.c.; Strasburger, 1.c., p. 480; Herbst, Bot. Centralbi., I894, Bd. Lv1I, p. 4 I 2. See also de Vries, Bot. Zeitung, I 886, p. 788.

${ }^{3}$ Dutrochet, Ann. d. sci. nat., I $83_{2}$, T. Xxv, p. 248 , and Mém. p. servir à l'histoire d. végétaux, Bruxelles, I 837 , p. I 72 ; Raffenau-Delile, Aun. d. sci. nat., I 8 I I, ii. sér., T. xvi, p. 328 ; Unger, Sitzungsb. d. Wien. Akad., I 857 , Bd. xxv, p. $46 \mathrm{r}$; Sachs, Fxperimentalplyysiol., I865, p. ${ }_{52} 2$. 
$g$, to which the glass cylinder $f$ containing water is fitted. By pouring mercury into $J$, or by means of water pressure, the air in $g$ may be compressed and thus driven through the petiole. The cut surface of the petiole may be observed by means of a lens or low-power microscope, and as v. Höhnel ' has shown, the gas bubbles may be seen to pass out from the intercellular spaces of the pith and cortex even under moderate pressure only. In the leaves of Nymphaea, Funkia, Calla aethiopica, Arum maculatum, Rumex, \&c., a pressure of one-eighth to one-fifth of an atmosphere is sufficient, and hence air can be driven through the leaves by means of the lungs. Even when the lamina of Nelumbium speciosum is immersed in water, bubbles of air may pass out from it. If when it is exposed to air, one blows through the petiole, the currents of air escaping from the stomata cause

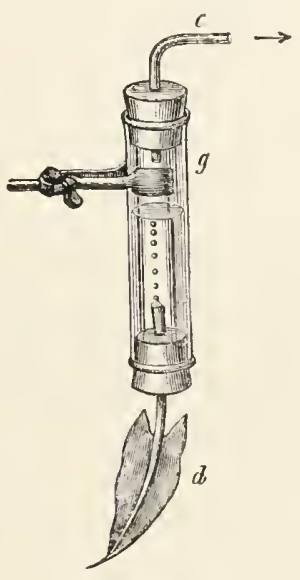

FiG. 22.

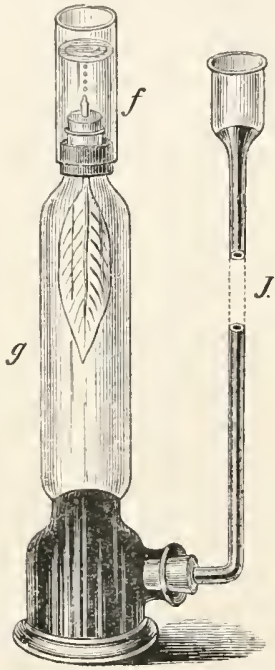

FIG. 23.

the mobile drops of water on its upper surface to roll to and fro, as was first observed by Raffenau-Delile.

If the stomata are occluded by water, usually no air-bubbles can be driven through them by a pressure of two to five times greater intensity. This is because the water filling the stomatal pore is held with considerable force, owing to the narrowness of the opening ${ }^{2}$. That the stomata are still open is shown by the fact that water may be gradually drawn into the intercellular spaces of the mesophyll, causing the leaf to become a darker green colour ${ }^{3}$. As might be expected, the widening of the stomata allows streams of gas to pass through them more rapidly ${ }^{4}$; but it is, however, not always immaterial whether the stream of gas is caused by

1 V. Höhnel, Jahrb. f. wiss. Bot., I879, Bd. Xi1, p. 52. A similar convenient apparatus was employed by Strasburger, IS9I, l.c., p. 7 IS.

2 A thread of water occupying a capillary tube $0.01 \mathrm{~mm}$. diameter is only driven out by a pressure of about $22 \mathrm{~cm}$. of mercury. Cf. Nägeli u. Schwendener, Mikroskop, 1877,2 . Auf., p. 366 .

${ }^{3}$ Dutrochet, Mémoires, Bruxelles, i 837, p. I 72 . Similar experiments by Unger and Sachs.

* See N. J. C. Muiller, Jahrb. f. wiss. Bot., I 872 , Bd. vill, p. Iо3. 
a negative or a positive pressure in the intercellular system, for in the former case, according to Barthélemy ${ }^{1}$, the stomata on the leaves of Nymphaea alba, Ranunculus, Ficaria, \&c., close, although it may be easily proved by means of the apparatus already described that this does not always occur.

Porous cork. By means of the apparatus already described (Figs. 22 and 23 ) the direct passage of gases through lenticels may be demonstrated. Twigs are preferable, one end of which is closed by an india-rubber cap, so that the passage of gases through the opened vessels is prevented. On subjecting the twig to pressure, air-bubbles will be driven out from the intercellular spaces on the cut surface immersed in water. With a pressure of three-quarters to one atmosphere, air-bubbles . may stream from certain of the open ends of the vessels, and this can be observed without interruption if the bark is removed from the immersed portion of the twig. Air is apparently conveyed to the tracheae by the intercellular spaces of the medullary rays, and thence passes diosmotically through the walls of the vessels, but it is only when the pressure is relatively high that the diosmotic transference is sufficiently rapid to cause an actual current of air towards the open mouth of the vessel. It is also possible by driving in air at the cut surface to cause streams of gas to pass out through the lenticels ${ }^{2}$. The cessation of the stream of bubbles when the lenticels are saturated with water shows that the gases pass directly through the open intercellular canals of these organs.

Even when distinct lenticels are absent, as in Vitis, Lonicera, \&c., gases may, according to Klebahn, still diffuse through the intercellular spaces of the spongy cork occurring in the periderm, and also between the suberinized endodermal cells. The details of the formation and distribution of porous cork are given by Klebahn along with a critical account of the previous literature. According to his own experiments, porous cork, when once formed, functions permanently as an open gaseous channel. In many plants, however, the size of the intercellular spaces alters in the growth of successive years, and it is probably owing to the altered rates of diffusion thereby caused that many authors have concluded that lenticels close in winter. Klebahn has, however, shown that even in winter a pressure of 5 to $6 \mathrm{~cm}$. of mercury causes bubbles of gas to be driven through the lenticels (Klebahn, r 884 , p. $5^{6} 3$ ). In order to regulate the loss of water by evaporation, it would be of distinct advantage if the changes of form which the dead cells undergo as they dry were such as to close more or less completely the intercellular spaces of the periderm, but this is a point to determine which detailed experimental investigation is needed ${ }^{3}$.

1 Barthélemy, Ann. d. sci. nat., I\$74, v. sér., T. xix, p. I 5o. See also Wiesner, Sitzungsb. d. Wien. Akad., I879, Bd. Lxxix, p. $3^{S}$ (Sep.). N. J. C. Müller's researches (Jahrb. f. wiss. Bot., 1869-70, Bd. VII, p. 161) leave undetermined the causes to which the different effects produced according to the direction of the gaseous current are due.

${ }^{2}$ Experiments by Stahl, Bot. Zeitung, 1873, p. 6r3; G. Haberlandt, Sitzungsb. d. Wien. Akad., I875, .Jd. LxxiI (July session); Klebahn, Ber. d. Bot. Ges., I883, p. I 13, and Jenaische Zeitschr. f. Nat.-wiss., 1884 , p. $5^{62}$; Hales, Statics, I 748, p. 91, and Pl. vii, Fig. $3^{2}$. Von Höbnel was the first to prove that air bubbles pass out of the vessels when the pressure is high (Jahrb. f. wiss. Bot., I 879 , Bd. XII, p. 49'. Details by Strasburger, Bau u. Verrichtung d. Leitungsbahnen, I89I, p. 7 I6.

${ }^{3}$ Cf. Klebahn, 1. c., I884, p. 574 ; Devaux, Ann. d. sci. nat., I 891 , vii. sér., T. XIv, p. 340. 


\section{SECTION 32. The Movements and Pressure of the Internal Gases.}

In the living plant various causes induce a continual disturbance of equilibrium, and not only respiration, but also the assimilation of carbon dioxide acts in this manner, as indeed does every metabolic process involving construction or consumption. It is, moreover, the disappearance of water from tissue-elements which leads to the formation of air-spaces in them, and the pressure and composition of the enclosed air depends on the one hand upon the rapidity with which the water is removed, and on the other upon the rate at which the gaseous exchanges proceed, while the maintenance of any constant difference of pressure or composition necessitates the existence of a definite relationship between the interchanges which restore equilibrium.

Differences in percentage composition may cause differences of pressure to arise, and vice versa, for just as a bladder filled with carbon dioxide collapses when in contact with air, so a negative pressure may be caused in an enclosed intercellular space in which a large quantity of rapidly diosmosing carbon dioxide is present. When, however, gaseous diosmosis is induced by negative pressure, oxygen penetrates in greater abundance from the air than nitrogen does, and indeed all the facts observed coincide with the general principles already given (Sect. 30 ). Thus, when the aeriferous system is well developed and in direct connexion with the external world, the pressure and composition of the enclosed gas differs but little from that of the surrounding air. In terrestrial plants with a strongly developed cuticle, we find, however, greater differences in the composition of the enclosed air than in submerged plants with more permeable epidermal layers, and obviously more or less marked differences may exist between the intercellular air of roots, stems, and leaves. The air of the tracheae may be under pronounced negative pressure, as is always possible in air-spaces which are not in direct communication with one another or with the external world, whereas at the same time the air of the intercellular spaces may be slightly compressed.

The external conditions not only influence the pressure and movements of the enclosed air indirectly by the effect they produce on respiration, the assimilation of carbon dioxide, transpiration, the width of the stomatal pores, \&c., but their effect is felt directly in various ways which are purely physical. Such are the bending and shaking of branches due to the wind, \&c., also the variations of barometric pressure and of temperature. All movements of diffusion, however induced, are of the highest importance in accelerating gaseous exchange, and a current of gas in any given direction will continue so long as the difference of potential which induces it is maintained.

Of the normal metabolic processes, the assimilation of carbon dioxide usually tends to induce a positive pressure in the intercellular air, respiration 
a negative one. Hence, when a cut is made in the stem of an uninjured, submerged, green aquatic plant which is well illuminated, bubbles of gas ooze out and continue to stream from the cut surface in slow or rapid succession so long as light reaches the plant. A similar positive pressure is exhibited when terrestrial plants are immersed in water, although so long as they are in air, and their stomata are open, no perceptible difference of pressure can be developed. This positive pressure is mainly due to the decomposition of the carbon dioxide in the green illuminated parts, and to the excretion of part of the liberated oxygen into the intercellular spaces, whence it diosmoses outwards less rapidly than the more soluble carbon dioxide does. Hence the pressure falls in darkness, for then the production of oxygen ceases ; indeed, a slight negative pressure is usually produced, provided no disturbing influences intervene, owing to the carbon dioxide formed by respiration diffusing more rapidly than oxygen. It is mainly in this manner that differences are produced in the enclosed air of submerged aquatic plants, according to whether they are illuminated or not. During the day the percentage of oxygen on the whole increases, the enclosed gas containing more oxygen than the air does. In darkness the reverse takes place, and the minimal percentage of carbon dioxide present during the day increases. The composition of the gas present in the chlorophyll-containing organs of land plants changes in a similar manner, while owing to the presence of an impermeable cuticle, more marked differences than are found in submerged aquatic plants may arise if the stomata are closed. The intercellular air always contains more or less nitrogen, for the gaseous exchange is perfectly general, and hence under normal conditions pure oxygen or pure carbon dioxide is never present within the plant.

A negative pressure is created without the aid of any special vital activity when the water which living or dead tissue-elements contain is withdrawn from them by transpiration. How perfect a vacuum will be produced, and how long the internal negative pressure will be maintained, will depend upon the rapidity with which air penetrates the enclosing membranes ${ }^{1}$. The shrivelling of thin-walled tissues when dried is partly the result of the negative pressure created within the cells, which is frequently very marked. Thus in vessels and tracheides the internal pressure may often fall to one-third or one-fourth of that of the atmosphere. It is not yet, however, quite certain whether the negative pressure in the tracheae, \&c. is due simply to the loss of water by transpiration, or whether in addition to this, other factors aid in exhausting the air within the vessels.

The existence of internal negative pressure in the tracheae plays a most

1 [Kamerling finds that the cells of certain moss-leaves, Sclaginella lepidophylla, \&c., are impermeable to air when dry, and hence within the cell a vacuum may be maintained for an indefinite length of time (Bot. Centralbl., I 897 , Bd. Lxxil, p. 53).] 
important part in the transport of water, but it is nevertheless probable that a certain negative pressure, partly due to the evaporation of water, may arise in intercellular spaces, for these also may suck in water to a certain extent (Sect. 29). It has, however, not yet been determined whether the intercellular spaces aid by any such means in the absorption of water. The marked negative pressure of the intercellular air which Goebel ${ }^{1}$ observed on cloudy days in the leaf-stalks of Nymphaea, Nelumbium, Colocasia, \&c., might have been produced in various ways, for independently of the action of darkness, when the intercellular air is rich in oxygen, the more rapid diosmosis of the respiratory carbon dioxide suffices to induce pronounced negative pressure. It is probably mainly in the latter manner that the slight negative pressures observed in the intercellular spaces of tubers ${ }^{2}$, \&c., and of submerged water plants, are produced in the absence of any transpiration. Detailed investigations are necessary in such cases to determine the precise means by which the intercellular spaces become permanently filled with air.

The existence of rarefied air in plants was first shown by experiments performed by Hales ${ }^{3}$. If the base of a leafy twig is immersed in water, as shown in Fig. 24, and the cut side branch, $b$, is attached by an indiarubber connexion to the glass tube $a$, filled with air and having its lower end in water or mercury, the latter rises in the tube $a$, showing that air has been drawn in through the cut surface

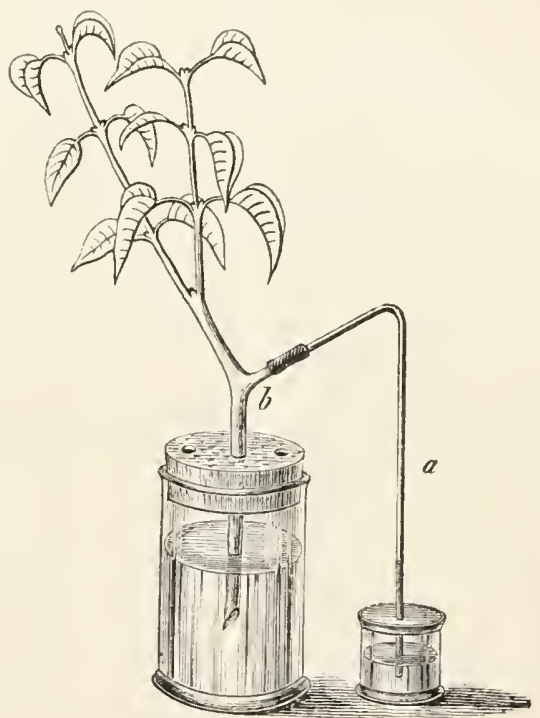

F1G. 24 . of the side branch. In these and similar experiments the mercury may be raised to a height of $3-5 \mathrm{~cm}$., although the result produced is chiefly or entirely due to the rarefication existing in the intercellular air. By using barked twigs, or by inserting manometers in the wood of trees, much higher negative pressures are usually obtained ${ }^{4}$.

1 Goebel, Pflanzenbiol. Schilderungen, 1S93, 2. Th., p. 25 I.

${ }^{2}$ Devanx, Ann. d. sci. nat., IS9I, vii. sér., T. xrv, p. 393. On the feeble development of the intercellular spaces in certain tubers, see Sect. $3 \mathrm{I}$.

3 Hales, Statics, I $7 \Varangle$ S, p. 90 . Similar experiments by Meyer, Physiol., I 838 , Bd. 1I, p. 73 ; Sachs, Experimentalphysiol., 1865, p. 26I ; Barthélemy, Ann. d. sci. nat., I874, v. sér., T. xix, p. 150; Bonnier, Rev. gén. de Bot., IS93, T. v, p. I3, \&c.

* See Th. Hartig, Bot. Zeitung, IS6r, p. IS ; Böhm, Versuchsst., rS7 heim, Bot. Centralbl., I892, Bd. xisx, p. 2 ; Bonnier, 1893 , 1. c. 
When transpiration is active, drops of water alternate with bubbles of rarified air in the vessels, and hence when one end of a tracheal vessel is opened the air it contains contracts until it is at the atmospheric pressure, and the contents are pressed towards the closed end of the vessel (or tracheidal cell). If a twig or leaf-stalk is cut under mercury, the latter is sucked into the opened vessels, and partially fills them. This method was first employed by v. Höhnel ${ }^{1}$, who observed the entry of mercury into many of the vessels when transpiring woody and herbaceous plants were used, and frequently found the vessels injected with mercury for a distance of 50 to $60 \mathrm{~cm}$. from the cut surface. Similar results may be obtained by employing oil, melted cocoa-butter, or paraffin coloured with alkanna or lamp-black.

The fact that mercury is sucked in indicates the existence of a marked internal negative pressure, for to overcome the capillary depression of mercury in, for example, the rather narrow vessels of Aesculus hippocastamum of 25 to $30 \mu$ bore, a pressure of 30 to 40 centimetres is needed. As soon, however, as this resistance is overcome and the mercury enters, any further rise in the vessels will depend, just as in a glass tube, upon the volume occupied by the rarefied air in their interior, and therefore also upon the length of the vessels. Hence the height to which the mercury rises, even when the capillary depression is allowed for, does not form an accurate measure of the rarefication of the internal air, as v. Höhnel erroneously supposed. For the lengths of the tracheal segments differ, and in different plants the tracheae vary from a few centimetres to as much as 3 metres in length ${ }^{2}$. A correct estimation of the negative pressure may, however, be obtained by observing the decrease in volume which the contracting air bubbles undergo when the vessels are opened, and from Schwendener's ' researches it appears that the pressure of the enclosed air in the tracheae may be as low as one-third to one-fourth of the atmospheric pressure ( 25 to 19 centimetres of mercury), while similar values have been obtained by Pappenheim ${ }^{4}$ for the tracheides of Coniferae. A complete vacuum is never formed, as Scheit supposed, and this could hardly be expected, since gases diosmose through the walls of the vessels (Sect. 30). Correspondingly rarefied air to that in the dead vessels apparently exists in the other non-living elements of the xylem, although owing to their shortness and narrowness mercury is unable to penetrate them ${ }^{5}$. The existence of a negative pressure may, however, be shown by means of coloured water, which penetrates further when the vessel is opened directly under the coloured solution than when it is at first exposed to air. Haberlandt

1 V. Höhnel, Über d. negativen Druck in d. Gefässluft; Strasburger, Dissertation, I 876 , and Jahrb. f. wiss. Bnt., I879, Bd. XII, p. 77. Also Strasburger, Bau u. Verrichtung d. Leitungsbahnen, I 89 r, p. 7 I 2 ; Schwendener, Sitzungsb. d. Berl. Akad., I 893 , Bd.XL, p. $\$_{42}$. V. Höhnel (F. Haberlandt's wissenschaft.-prakt. Unters, a. d. Gcb. d. Pflanzenbaues, I8 87, Bd. 11, p. I 22 ) and others have also employed coloured solutions. In this case, owing to the capillary attraction exerted, the vessels are injected for a greater distance than when mercury is used.

2 Adler, Unters. iiber d. Längenausdehnung d. Gefässräume, Jenaer Dissert., I892, and Bot. Centralbl., I 892 , Bd. L1I, p. I 28 ; Strasburger, Über das Saftsteigen, s 893 , p. 50.

${ }^{3}$ Schwendener, Sitzungsb. d. Berl. Akad., 1893, Bd. xL, p. 844 .

- Pappenheim, Bot. Centralbl., I892, Bd. Xlıx, p. I61.

${ }^{5}$ Scheit, Jenaische Zcitschr. f. Nat.-wiss., I 885 , N. F., Bd. x1r, p. 678; Kamerling, Flora, I 897 , Ergänzungsband, p. I r. On the existence of living vessels, see Lange, Flora, I 891, p. 52. 
has shown that a negative pressure exists in the elements of the central cylinder of a $\operatorname{moss}{ }^{1}$.

These gaseous tensions arise, as v. Höhnel has shown, in direct connexion with the changing amounts of water present. Hence, when fully saturated with water, no negative pressure exists in vessels or tracheides (as the manometer also shows, Fig. 24, p. 20I), whereas it appears as soon as transpiration commences, and is most marked when the latter is fully active. As the water in the chain of air and water columns (Jamin's chain) which fill the vessels increases again, so does the negative pressure of the enclosed air decrease ${ }^{2}$, and hence such negative pressure often disappears during the night in herbaceous plants whose vessels may become filled with water. In woody plants as well, as v. Höhnel has shown, the negative pressure gradually disappears when transpiration ceases.

The rarefication of the enclosed air which results from the removal of water is only very gradually neutralized, as v. Höhnel recognized ( $1879, \mathrm{p} .76$ ), owing to the slowness witl which gases diosmose through lignified walls (Sect. 2I, 30). It is still uncertain whether the daily variations in the percentage of water act directly, as $\mathrm{v}$. Höhnel supposed, or whether other factors co-operate in maintaining a negative pressure within the vessels. Obviously the pressure must finally become uniform, unless the difference of potential is continually restored, and hence it is easy to understand that in leafless trees during winter the negative pressure in the air of the wood vessels is reduced to a minimum or to nil ${ }^{3}$. Bearing in mind the different factors which influence or induce the movement of water, it is not surprising to find that the pressure may differ in neighbouring vessels, though it is approximately the same at different levels of the same tree ${ }^{4}$. In the root the negative pressure is frequently less marked than in the stem 5 .

Isolated twigs, after being exposed to air for a time, again show a negative pressure when a new surface is exposed near to the old one. This is partly due to the reopening of tracheides which have gradually become closed, and partly owing to the opening of others hitherto intact. A closure rendering possible the re-establishment of a negative pressure in opened vessels may take place by means of an exudation of slimy material, or by the growth of bacteria on the cut surface, or by other means as well ${ }^{6}$.

The positive pressure of the intercellular air is due to the excretion into the intercellular air-spaces of a portion of the oxygen produced by the decomposition of

1 Haberlandt, Jahrb. f. wiss. Bot., I $\$ 86$, Bd. Xvir, p. 416.

${ }^{2}$ V. Höhnel, Jahrb. f. wiss. Bot., 1879 , Bd. X11, p. 121. On the contents of the vessels, cf. Böhm, Bot. Zeitung, IS79, p. 255 ; v. Höhnel, I 879, 1. c., p. I21 ; Volkens, Jahrb. d. bot. Gart. in Berlin, 1883, Bd. 11, p. I81; Schwendener, Sitzungsb. d. Berl. Akad., I886, p. 566; Strasburger, Bau u. Verrichtung d. Leitungsbahnen, I $S_{91}$, pp. $68_{5}, 695$, and Über das Saftsteigen, $1 S_{93}$, p. 55 .

${ }^{3}$ V. Höhnel, 1.c., p. I I5; Strasburger, 1891, l.c., p. 715. Contrary to the negative results of v. Höhnel, Böhm (Ber. d. Bot. Ges., 1889, Generalvers., p. $5^{2}$ ) found a certain negative pressure to exist in winter, and hence it is possible that exceptions may exist.

4 Schwendener, Sitzungsb. d. Berl. Akad., 1892, p. 923; IS93, p. S44; Strasburger, Über d. Saftsteigen, $x 8_{93}$, p. $5^{6}$.

${ }^{3}$ Strasburger, Bau u. Verrichtung d. Leitungsbahnen, $x S_{91}$, p. 715.

${ }^{6}$ V. Höhnel, 1. c., 18 6 , p. 20, and Bot. Zeitung, 1879, p. 320; Strasburger, 1891, 1. c., p. 7I 4. 
carbon dioxide. The continuance of this process causes a more or less rapid stream of bubbles to emerge from the cut stem of illuminated submerged plants with welldeveloped intercellular systems (Elodea, Myriophyllum, Ceratophyllum, \&c.) (Fig. 25), and the number of these bubbles affords a measure of the amount of carbon dioxide assimilated (Sect. 52). That the stream of bubbles is due to the production of oxygen is shown by the following facts: (I) it rises and falls within certain limits as the intensity of the illumination increases or diminishes, and ceases almost immediately in darkness; (2) it stops without the plant being injured when all free carbon dioxide is removed by the addition of lime-water ${ }^{1}$. Similarly, insolation produces no continuous stream of bubbles unless the function of photosynthetic assimilation can be exercised ${ }^{2}$, although a rise of temperature may cause a few bubbles to escape

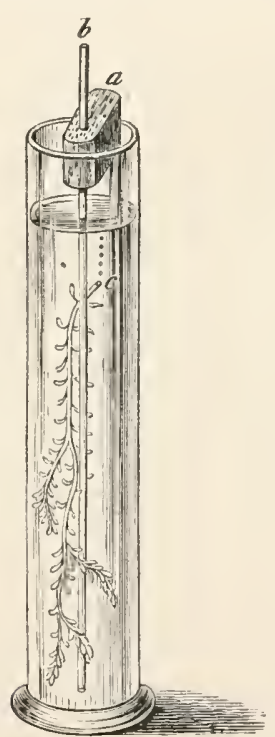

Fig. 25. The glass rod $(b)$, to which the plant is attached by a thread, passes through the $\operatorname{cork}(a)$, by which it is held firmly.

for a moment or two. It is, however, always possible that by a rise of temperature under certain conditions a stream of bubbles may be produced in quite a different way, for in the phenomenon of thermo-diffusion discovered by Dufour and studied by Feddersen ${ }^{3}$ a current of gas may flow from the warmer to the colder side of a separating membrane. Moreover, in a closed porous clay cell or bladder a distinct pressure, increasing as the temperature rises, is produced when the enclosed air is saturated with moisture, but the surrounding air remains dry ${ }^{4}$. Similarly, when a plant containing air rich in oxygen is brought into water rich in carbon dioxide, it is easy to see why a stream of bubbles may continue to escape for a time in darkness ${ }^{5}$. It is also evident that the bubbles, under normal conditions, will not be composed of pure oxygen, but that their composition will depend upon the intensity of the production of oxygen, upon the percentage amounts of gas dissolved in the water, as well as upon many other factors.

It is true that the production of oxygen rarely gives rise to any very great pressure in intact acquatic plants, but nevertheless it is sufficient to force out bubbles of gas against a water-pressure of 20 to $30 \mathrm{~cm}$., if the cut surface of the stem is at that depth below the surface when the plant is upright ${ }^{6}$. No detailed investigations have as yet been made as to the manner in which the high internal gaseous pressures found in the Fucaceae, \&c. are produced (Sect. 29).

'F. Schwarz, Unters. a. d. Bot. Inst. z. Tübingen, I 88 I, Bd. I, p. 97.

2 An exception to the contrary is given by N. J. C. Müller, Bot. Unters., i 876, Bd. I, p. $3^{80 .}$

3 Details by Naumann, Allgem. Chem., I877, p. 26 I.

4 For an explanation of this phenomenon, see Kundt, Ann d. Ihysik u. Chemie, I $8_{77}$, N. F., Bd. Il, p. I 7 .

3 Van Tieghem, Ann. d. sci. nat., I 868, v. ser., T. Ix, p. 269 ; Lecoq, Compt. rend., I867, T. Lxv, p. III , and I869, Bd. Lxix, p. 531 ; N. J. C. Muiller, Jahrb. f. wiss. Bot., I873-4, Bd. Ix, p. 37 ; Devaux, Ann. d. sci. nat., I889, vii. sér., T. IX, p. 138 .

${ }^{6}$ Lechartier, Ann. d. sci. nat., I 867 , v. sér., T. vilı, p. $3^{6}+$ 
Other gaseous currents in plants. I)isregarding the irregular gaseous currents caused by bending or swaying movements, changes of temperature, \&c., there appear to be other external agencies which induce streams of gas in particular directions wherever these are readily produced. The currents induced in this manner may, according to Raffenau-Delile ", become so strong in Nelumbium speciosum on bright days that the gas streaming out of the stomata causes the drops of water on the leaf to run to and fro. According to Merget ${ }^{2}$, when a hot body is held near a leaf of Nelumbium, a current of air passes inwards from the surface of the le.r. The same takes place in a dead leaf, and it is powerful enough to overcome a pressure equal to that of a column of $\mathrm{I}$ to 3 centimetres of water. This relatively trifling difference of pressure suffices in Nelumbium or Nymphaea to maintain a current of air in the plant through the rhizome from one leaf to another".

The precise manner in which gaseous currents of this character are set up is not yet clear, and hence it must remain uncertain whether the necessary energy is derived from a difference of temperature, or whether a difference of potential is maintained by processes connected with transpiration, or whether other causes or combinations of causes come into play. A trifling propulsive force will not suffice to produce any strong gaseous currents through the stomata and intercellular spaces when the resistance offered is great, and still less when the entry of gases takes place by diosmotic means (Sect. 30).

Composition of the enclosed gases. Here the results obtained correspond i general with what might be expected on theoretical grounds (p. 99). Thus the composition may vary more or less markedly from the normal percentage, while the combined action of certain factors may cause the indifferent gas nitrogen to be present in different relative amount to that in the air. Under normal conditions the enclosed gas is never composed of pure nitrogen, as some authors have erroneously supposed $^{4}$. A detailed consideration of the observed percentage compositions is hardly necessary, since external conditions may modify the amounts present. Moreover, in obtaining the air for analysis, an admixture with larger or smaller quantities of absorbed or dissolved gases may frequently occur. For similar reasons the percentage composition of the dissolved gas varies, but maintains a certain correspondence with that of the intercellular air. A few further remarks will be made later (Sect. 96), and the reasons for the non-formation of gas vacuoles in the interior of a turgid cell have already been given (Sec. 29).

In intact plants of Elodea, Ceratophyllum, \&c., owing to the ease with which diosmotic exchange takes place, the enclosed air differs but little in composition from that of the atmosphere during day and night ${ }^{5}$. Nevertheless, when exposed

1 Raffenau-Delile, Ann. d. sci. nat, $1 \S_{4}$ I, ii. sér., T. xvi, p. 328 .

${ }^{2}$ Merget, Compt. rend., I8 73 , T. Lxxvir, p. $1469 ; 1874$, T. Lxxvir, p. $88_{4}$.

${ }^{3}$ Barthélemy, Ann. d. sci. nat., I874, v. sér., T. XIX, p. 152. Cf. also Lechartier, ibid., 1867, v. sér., T. vili, p. $3^{6} 4$.

- This is stated by E. Schulze (Lehrb. d. Chem. f. Landw., $18 \overline{3} 3$, I, p. 58 ) to be the case in grass haulms and other hollow stems, and by Barthélemy (Ann. d. sci. nat., I 874 , v. sér., T. XIX, p. I67) in Pontederia; but these results are contrary to those of all other invcstigators.

${ }^{6}$ Devaux, Ann. d. sci. nat., I889, vii. sér., T. 1x, p. 99. As the stream of bubbles continues, the escaping air becomes gradually richer in oxygen, for reasons that are easy to see (cf. Sect. 52 ). 
to light, the oxygen perceptibly increases in amount. A similar increase is perceptible in the seed-pods of Pisum and Colutea when exposed to light ${ }^{1}$. In the bladders of Fucaceae, \&c., the amount of oxygen present may increase much more markedly, even to as much as $3^{6}$ per cent. ${ }^{2}$, while in spite of the presence of open stomata an abundance of oxygen may accumulate in the leaves of plants belonging to the Crassulaceae (Aubert, I 892, 1. c., p. 275).

When respiration alone is possible, the amount of oxygen present decreases to a greater or less extent, while the percentage of carbon dioxide increases. Thus Devaux found (1. c., I $89 \mathrm{I}$, p. 352) in the intercellular air of the roots of Daucas carota 77.3 to 89 per cent. nitrogen; 0.4 to 10.6 per cent. oxygen; $\mathbf{I} \cdot 4$ to 17.8 per cent. carbon dioxide. Similar numbers have been found for bulbs, tubers, \&c. Hence it is easy to see that the amount of oxygen present may not suffice to satisfy all requirements when the respiratory activity is unusually pronounced. Even in the roots of Nuphar luteum, in spite of their well-developed intercellular system, the oxygen may, according to Dutrochet ${ }^{3}$, sink as low as 8 per cent.

The air enclosed in the vessels and other tissue-elements of the wood, according to Kruticki, does not differ in composition very greatly from that of the atmosphere, except that in winter it is richer in carbon dioxide and poorer in oxygen. Faivre and Dupré, however, obtained precisely opposite results as regards the air in the vessels of Morus and Vitis ${ }^{4}$. These apparent contradictions can, however, be reconciled with one another, for in all probability when the air in the vessels becomes rarefied a gas will at first collect which is relatively rich in both oxygen and carbon dioxide.

1 Observed first by Ingenhousz, Versuche mit Pflanzen, I788, Bd, II, p. 58. Of the works carried out on these and other plants, the following may be mentiored : Saussure, Ann. d. chim. et phys., I82I, T. X1X, p. I50; Calvert et Ferrand, Ann. d. sci. nat., I 844 , iii. sér., T. III, p. 377 ; Gardner, Froriep's Neue Notizen, 1846 , Bd. xxxix, p. 323 ; Erdmann, Jahresb. d. Chem., I 855 , p. 727 ; Baudrimont, Compt. rend., I855, T. xLI, p. I78; Martin, ibid., 1866, T. LxiI, p. 737 ; Saintpierre et Magnien, ibid., 1876, T. Lxxxi11, p. 490 ; Joulin, Bot. Centralbl., IS8 I, Bd. v, p. Io 2 ; Peyrou, ibid., I89I, Bd. Xlv, p. 217 ; Devaux, Ann. d. sci. nat., I891, vii. sér., T. x1v, p. 297 ; Aubert, Rev. gén. d. Bot., 1892 , T. Iv, p. 275.

2 Aimé, Ann. d. sci. nat., $18_{4}$ I, iii. sér., T. II, p. 536 ; Wille, Bot. Jahresb., I889, p. 226.

3 Dutrochet, Mémoires, \&c., Bruxelles, 1837 , p. 175.

4 Kruticki, Bot. Centralbl., r $\$ 89$, Bd. Xxxıx, p. 30; Faivre et Dupré, Ann. d. sci. nat., IS66, v. sér., T. VI, p. 366. Bischoff's statement that the air of the vessels is rich in oxygen (De vera vasorum plantarum structura et functione commentatio, I $829, \mathrm{p} .8 \mathrm{I}$ ) is without value, owing to the faulty experimental methods employed. 


\section{CHAPTER VI}

THE MOVEMENTS OF WATER

\section{SEction 33. General view.}

A PLANT can only live when it obtains a sufficient supply of water, with which all its parts are saturated, and which in turgescent tissues generally forms 60 to 90 per cent. of the entire weight. The amount of water present may vary greatly, and a decrease is indicated by flaccidity and withering, which if not checked is ultimately followed in most cases by death. Seeds, mosses, lichens, \&c., which are able to withstand desiccation, are first awakened to new activity when they are supplied with water, and become turgid. Variations of turgidity are always induced by the gain and loss of water, and in terrestrial plants water must pass from the subterranean root-system to the transpiring organs in order to compensate for the loss by transpiration. The amount of water which passes in this manner through a terrestrial plant is in general very much greater than the amount present at any given moment, while in comparison with the former the amount of water which escapes when a vine stem bleeds is but trifling, as is also the amount which is used in metabolism as a source of oxygen and hydrogen.

The ways and means by which water is absorbed have already been discussed (Sects. 25-27), and we are therefore at present concerned only with the phenomena connected with the transport of water from one part to another. The water-current is mainly employed to make good the loss by transpiration, a function which, as has already been indicated in connexion with the movements of gases (Chap. V), is more or less markedly limited and regulated by the structural relationships and inherent peculiarities exhibited by particular plants. A certain limitation is necessary in order to maintain the proper relationship between the loss and the supply. Where the climate and habitat are such that temporary periods of drought occur, plants which are killed by desiccation can only survive the unfavourable periods by economizing as far as possible their store of water. The various means by which this end is attained cannot be discussed here ; nevertheless, it is well known to how great an extent the adaption to a dry 
climate may alter the shape and structure of individual plants. and even the general habit of the whole vegetation.

There is a certain optimal supply of water for terrestrial plants at which growth and development are most active. When submerged, many plants are incapable of any development at all, and very frequently the complete saturation of the soil with water exercises a retarding influence upon vegetative activity. Moreover, the infiltration of the intercellular spaces with water is disadvantageous to most terrestrial as well as to most aquatic plants, so that it is only in those of the latter which have no air-spaces that the vegetative conditions are most favourable when they contain the highest possible amount of water. Transpiration and the water-currents which it induces are of importance in various ways, particularly in accelerating the passage of the salts absorbed from the soil up to the summit of a tall tree.

Each cell strives to absorb water until it is fully turgid, and hence a stream will be originated in this manner which will extend from the absorbing to the transpiring organs. In a multicellular fungal hypha this water passes from cell to cell, but special channels become necessary when water must be carried to distant organs to supply that which is lost by active transpiration. Numerous researches have shown us clearly that where differentiation of tissue and division of labour exist, the xylem portions of the fibro-rascular bundles are entrusted with this function. How and by what means the water is so easily raised and so rapidly transferred to the summits of the tallest trees has not as yet been satisfactorily explained. It is certain, however, that the water is not merely driven upwards from the roots or base of the stem by the root-pressure acting like a force pump, but that the removal of water from the conducting channels exerts a force transmitted backwards as far as the absorbing organs, causing in these a corresponding entry of water (Sects. 34,35 ).

It is only when transpiration is feeble and a large amount of water has accumulated that fluid exudes from a decapitated root-stock or from the end of a cut branch. Such 'bleeding' is shown by cut stems of vines, beeches, \&c., in spring before the leaves unfold, and in summer also if transpiration is prevented. The bleeding of a dccapitated stump ceases after a time, while if a plant is cut while actively transpiring, the surface of its attached stump may at first absorb water.

In the process of bleeding, water or a watery solution exudes from the vessels and tracheides, and the amount lost in this manner may finally far exceed the joint volume of the root-system and stump of the stem. The water escapes from the cut surface because here the least resistance to its exit is interposed. The continuity of the stream, as well as the pressure which is indicated if a manometer is attached to the stump of the stem, shows that forces are active in the interior of the stem, which drive the water into the cavities of the vessels or other spaces, and so create internal positive pressure. 
The bleeding- or exudation-pressure ${ }^{1}$ which not only roots, but stems also, are able to generatc, is usually less than that of one atmosphere, and hence this pressure of exudation, even were it shown by all plants, which is not the case, would be quite insufficient to explain the raising of water to the summits of lofty trees. Moreover, it is just when the greatest quantities of water are being carried upwards that no active pressure of exudation is manifested. Similarly, herbaccous plants can fully supply themselves with water without the aid of any 'bleeding-pressure,' although during damp nights water frequently accumulates in them to a sufficient extent to render an exudation of water possible. This is the case in Impatiens, and in certain Aroids and Grasses, as is shown by the escape of drops of water from the leaf-teeth or other points, for this is dependent upon an overaccumulation of the water driven forcibly upwards. All excretion of water as such is not, however, necessarily connected with the existence of a pressure of exudation, for fluid may still be excreted from nectaries when the plant is suffering from drought. In this case the soluble constituents of the nectar cause the excretion of the water, which, just as in plasmolysis, must necessarily escape when a permeable tissue is in contact on one surface with an osmotically active substance. The exudation-pressure is, however, due to the water being driven in one direction by the direct action of living cells. The details of this process, as well as the relations between the exudation-pressure and the supply of water, will be discussed in the different sections of this chapter.

Vegetables, fruits, and succulent tissues in general contain 70 to 90 per cent. of water, and in certain very fleshy and watery fruits as much as 95 per cent. may be present. In turgid cells the cell-sap does not usually contain more than 3 to 6 per cent. of solids, while the protoplasm generally comprises io to 30 per cent. (Sect. II). Soft cell-walls probably contain a similar amount of water, while lignified walls when fully saturated hold about 50 per cent. (Sect. I2). As trees grow older the percentage of dead cells increases, and these, according to circumstances, may be filled with air or water to a varying extent. Nevertheless, even in periods of drought, the amount of water present in trees does not readily fall below 30 per cent., and may at times rise to as much as 70 per cent.

Numerous data and comparisons of the amounts of water present in different plants and different tissues are given by Ebermayer, Physiol. Chemie der Pflanzen, I 882, p. 2 ; J. König, Chemie der Nahrungs- und Genussmittel, r889, Th. I, p. 64 I. In these works, and in the treatises quoted therein, determinations are given of the changes in the percentage of water as plants and organs grow older. Cf. also Aubert, Ann. d. sci. nat., I 892 , vii. sér., T. Xv'1, p. 59.

\footnotetext{
${ }^{1}$ [ For a definition of this term see p. 254.]
} 


\section{PART I.}

\section{SECTION 34. The Conveyance of Water in Transpiring Plants.}

In a simple mould fungus transference of water from cell to cell suffices for all requirements, whereas in more highly developed terrestrial plants a special conducting system is necessary in order that water may be conveyed in sufficient amount and with sufficient rapidity to replace the loss by transpiration, which is frequently very pronounced (Sect. 33). Water travels very slowly from place to place so long as it depends upon transference from cell to cell in a turgid tissue. Thus cylinders of living pith or strips of cortex having their lower ends dipping in water begin to wither at a height of from 5 to I $_{5}$ centimetres, even when transpiration is only moderately active ${ }^{1}$.

This special transference of water is entrusted to the xylem of the fibrovascular strands, whose branches convey water along good conducting channels to every part, so that only a narrow stretch of the less active intermediate connective tissue need be traversed to replace the water given off by a transpiring epidermal cell of a leaf or stem. The final transference is therefore similar to that in Penicillium, in which the water passes from cell to cell in order to travel from the absorbing mycelium to the transpiring conidium.

In both cases it is the difference of potential due to the loss of water which induces the conveyance of fresh supplies to the transpiring parts. When a mycelium absorbs water from the substratum, equilibrium is restored by a flow of water towards the point from which it has been removed, and a similar stream follows any localized removal of water, so that in the fibrovascular conducting system an upward current is maintained towards the transpiring leaves; this occurs also even when the water is withdrawn from an isolated fibrovascular strand without the help of the surrounding parenchyma.

It is not yet clear how this active raising and transference takes place in the conducting tissue. The parenchyma abutting upon the conducting strands withdraws water from them in a similar manner to that in which the roots absorb water from the soil, and in both cases currents are induced towards the absorbing organ by a backwardly transmitted sucking-force. The latter, whether special conducting channels are present or not, reaches to the root and to the soil outside, from which it withdraws water. Hence, as transpiration continues, water is sucked in through the cut surface of

1 Thus: Westermaier, Ber. d. Bot. Ges., I88 3 , p. 37 ; Sitzungsb. d. Berl. Akad., I884, Bd. XLviII, p. I110; Jahrb. f. wiss. Bot., I88, Bd. xv, p. 627. On Laminaria see Sachs, Arb. d. Bot. Inst. in Würzburg, 1879 , Bd. II, p. 315 . 
a twig immersed in water, and in the arrangement shown in Fig. 26 this causes a gradual rise of the mercury in the arm $a^{1}$.

The mercury is seldom raised to a height of more than 10 to $30 \mathrm{~cm}$., especially in the case of herbaceous plants, for at that pressure air is in most cases sucked in through the surface of the branch; if, however, the outer layers are more impermeable to air, the mercury may be raised still higher. Thus Th. Hartig observed a negative pressure equal to $76 \mathrm{~cm}$. of mercury when he inserted manometers into holes bored as far as the alburnum of the wood cylinder. A similar sucking-force can be traced right down to the roots when transpiration is active. The amount of water present increases towards the roots, and the negative pressure as a rule steadily decreases, but at the same time, by means of a series of manometers, an irregular rise and fall of the sucking-force can be observed, especially in trees, which is similar to that noticed with regard to the internal gaseous tensions (Sect. 32). The causes which induce or influence these variations are similar in both cases ${ }^{2}$.

These results, the essential principles of which were correctly interpreted by Hales, prove that the upward current of water in a transpiring plant is not due to any pumping action of the roots or stem, such as produces an exudation-pressure. The latter is reached only when the plant is fully saturated with water, and hence at first the cut surface of a stem or root sucks in water, and then after a shorter or longer interval begins to bleed. Moreover, this exudation-pressure is not attained in many plants, and even where it is present, it is usually insufficient to raise the water to the summit

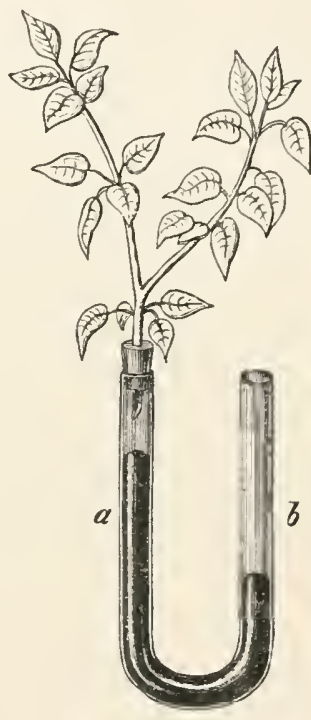

FiG. 26. of a tree, while, as a general rule, the amount of water which escapes from a cut stem is less than would have been transpired by the upper leafy portion ${ }^{3}$. By this backwardly transmitted suction the water in the roots is drawn into the vascular conducting channels, and at the same time the conditions necessary for fresh absorption from the soil outside are created by the

${ }^{1}$ Hales, Statics, I 748, pp. 26, ${ }_{4} 8$, \&c.; Meyer, Pflanzenphysiol., I $\$_{3} 8$, Bd. II, p. 7o; Th. Hartig, Bot. Zeitung, I $86 \mathbf{I}, \mathrm{p} . \mathbf{1}_{7}$, and I $86_{3}, \mathrm{p} . \mathbf{2 8 0}$. On the corrections necessary see v. Höhnel. On the negative pressure of the tracheal air, 1876, p. 6 , see also Unger, Sitzungsb. d. Wien. Akad., I864, Bd. Xliv, p. S (Sep.); Böhm, Ber. d. Bot. Ges., ISS9, Generalvers, p. 53, and Bot. Centralbl., I89o, Bd. Xli1, p. 234; Strasburger, Leitungsbahnen, IS9r, p. 782 ; Vines, Ann. of Bot., I896, Vol. X, p. 292.

${ }^{2}$ Cf. Schwendener, Sitzungsb. d. Berl. Akad., 1896 , Bd. xxxıv, p. $58_{3}$.

${ }^{3}$ Examples by Hofmeister, Flora, IS62, p. 107 ; Sachs, Lehrb., 3. Aufl., p. 598, and Arb. d. Bot. Inst. in Wiirzburg, $18_{73}, \mathrm{Bd}$. I, p. 28 . 
agency of the cortex and by means of the root-hairs. No one-sided pumping action of the cortical cells is necessary for such absorption, though if any does actually occur, it naturally aids and accelerates the latter. Water is absorbed almost solely by the younger portions of the root, but nevertheless the total absorbent surface is so great as to render only a slow transference through the cortical cells necessary (Sect. 26).

That the consumption and removal of water by an organ should directly cause a fresh supply to be drawn in is a highly advantageous arrangement, and indeed is necessary for the essential self-regulation exhibited by every functional activity. Thus according to their respective transpiratory activities, one leaf will obtain a large supply of water, another little or none, while if necessary, the cortex may withdraw water from the vascular system at any point of its course. The conducting channels are moreover capable of conveying water in the opposite direction, as is shown by the fact that twigs remain fresh when placed upside down with their distal ends in water, and that a small tree, the stem of which has been cut through, may be provided with water by means of a branch which has coalesced with another tree ${ }^{1}$. As a matter of fact, the water-current seems to pass with equal rapidity in either direction, for the slight delay which is generally observed is satisfactorily explained by the fact that the current in the downward direction has frequently a longer course to travel through the vascular bundles in order to reach a given point, than was the case with the ascending stream ${ }^{2}$.

From what has been said above, it is evident that a sufficiently dry soil will withdraw water from a turgid root, and if water is supplied through the leaves or twigs, a current may pass from these to the dry soil (cf. Sect. 28).

Without any interchange of water with the surrounding medium, a transport of fluid may take place within the plant, corresponding to the attracting forces at work at a given point. Thus water may be withdrawn from a potato suspended in air by the shoots which it may develop ${ }^{3}$, while a plant of Sempervionm hanging freely in a glass cylinder has been observed to grow in length apically for a long time by means of watcr extracted from its older parts ${ }^{4}$.

Transpiration removes water in the first instance from the cell-wall, but in each cell equilibrium is rapidly restored between the imbibitory and osmotic attractions for

1 Hales, Statics, 1748 , p. 77 ; Duhamel, Naturgesch. d. Bäume, 1765, Bd. II, p. 240 ; Cotta, Naturbeobachtungen übcr d. Bewegung d. Saftes, I 806, p. 22 ; Unger, Sitzungsb. d. Wien. Akad., ı 868, Bd. LvıI, Abth. i, p. 7 (Sep.); Strasburger, Leitungsbahnen, IS9 I, p. 583 . [In large banyan trees with numerous secondary stems, this phenomenon may occur naturally, for as the older central trunks die away, it may occasionally happen that in certain of the horizontal branches the original direction of the current of sap becomes reversed over a certain distance.]

2 Cf. Strasburger, l. c., p. $5^{8} 3$.

3 Nägeli, Sitzungsb. d. Bairischen Akad., r\$6 I, r, p. 249.

${ }^{4}$ Cf. de Candolle, Pflanzenphysiol., ${ }_{1833}$, Bd. I, p. I76, and Treviranus, Physiol., 1835, Bd. 1, p. 5 II. 
water, so that the diminution of turgidity forms a direct indication of the force with which the cell endeavours to absorb water ${ }^{1}$. The cells bordering upon the waterbearing xylem act by means of the energy thus developed, and in plants well supplied with water, the xylem elements retain their fluid contents with but feeble energy, for a slight fall of turgidity in the epidermis suffices to give rise to an outwardly directed current of water. The attraction of water is obviously dependent not upon the absolute hydrostatic pressure, but upon its relative intensity as compared with that of the regions from which water is withdrawn. Nevertheless, it is selfevident that when a tissue loses water, the cells with feeble osmotic energy will soon collapse, while the more highly osmotic ones will remain turgid for a longer time.

\section{SECTION 35. The Conducting Channels.}

That water is transferred mainly through the xylem has been confirmed by numerous experiments made since the classical ones by Hales ${ }^{2}$. Thus if the continuity of the pith and cortex be interrupted, but the wood left intact, the leaves remain turgid even when transpiration is active. If, however, the wood cylinder is cut through, but the continuity of the cortex and pith retained, withering begins almost as soon as it does in the leaves of a separated branch; the same thing occurs if a cut branch is allowed to obtain water only by means of immersed strips of cortex or bark, even when the latter, as in the lime-tree, contains a large amount of bast fibres or collenchyma. Hence it follows that none of these tissue elements are capable of rapid transference of water ${ }^{3}$. Similarly, the large amount of fundamental parenchyma which herbaceous plants contain cannot convey water with sufficient rapidity to prevent withering, whereas a few thin fibrovascular bundles may supply all the water required.

Since the conductivity of the wood is lost in the inner duramen layers, the water passes in trees only through a greater or less number of the outer annual rings of wood, and not through the central core. In oaks, cherry-trees, pines, \&c., a moderately deep ringing incision in the wood suffices, by interrupting the continuity of the splint-wood, to cause the withering and death of the parts above, whereas the leafy parts remain fiesh and turgid after a similar incision in the beech, birch, and other splint-wood trees, in which the older annual rings retain their alburnum character and can convey water in sufficient amount * Even in such splint-wood the power of conducting water decreases to a certain extent with increasing age ${ }^{5}$, but in palms and in

1 Sect. 27 , and Pfeffer, Studien z. Energctik, 1892, p. 258.

${ }^{2}$ Hales, Statics, $1_{74} 8$, pp. 76,8 I, \&c. ; Duhamel, Naturgesch. d. Bäume, $\mathbf{1}_{7} 6_{5}$, Bd. II, p. 234 ; Knight, Phil.Trans., ISOI , 1I, p. 234 , \&c. Further literature by Strasburger, Leitungsbahnen, IS9I, p. 5 I 5 .

${ }^{3}$ Cf. J. Cohn, Jahrb. f. wiss. Bot., I892, Bd. Xxiv, p. I 72 .

4 Knight, Phil. Trans., I Sor, 11, p. 349; Th. Hartig, Bot. Zeitung, I 865 , p. 268 ; R. Hartig, Ber. d. Bot. Ges., ISS8, p. 222 ; Strasburger, Leitungsbahnen, I89I, p. 515 and the literature here quoted.

${ }^{5}$ R. Hartig, 1.c. ; Wieler, Jahrb. f. wiss. Bot., I 888 , Bd. xIx, p. 82, and Ber. d. Bot. Ges., I 888. p. 406 ; Strasburger, 1.c., p. 592 . 
certain monocotyledons which do not undergo any secondary increase in thickness, the same vascular bundles must in part preserve their conductivity for a very long time. No detailed investigations have as yet been made to determine whether, and to what extent, a compulsory demand may prolong the conductivity of the alburnum and hinder its conversion into duramen.

The individual elements of the xylem are of unequal functional value, and hence on this account alone it might be anticipated that the spring and autumn xylem would neither be concerned to the same extent, nor apparently in the same manner, with the transference of water ${ }^{1}$.

From what has already been said, it appears that the tracheae and tracheides are the most important agents in the conveyance of water, but it has yet to be determined whether and how far the wood-fibres ${ }^{2}$ and other tissue-elements aid in the process. Physiological transitional forms may possibly exist between wood-fibres, tracheides, and tracheae, for which conclusion there is distinct morphological evidence. It is possible that some of these elements serve more for the conduction, others for the storage of water. The activity of conduction may apparently be temporarily inhibited or permanently suppressed at an early period in individual conducting elements.

Similar results are obtained by other means, and especially by the use of coloured solutions, for the colouration which the parts of the wood undergo serves to indicate the path of the transpiration current. The dyes are actually carried along by the latter, as is shown by the fact that when transpiration is suppressed, indigo-carmine, eosin, or anilineblue are absorbed only with extreme slowness, whereas in a transpiring plant they ascend with relative rapidity in those parts of the wood which have already been recognized as forming the paths by which the water travels. The dye gradually diffuses from the conducting channels to neighbouring non-conducting tissues and tissue-elements. Nevertheless, the experimental results obtained, if critically interpreted, leave no doubt that the dyes are at first carried with the water along the tracheides and tracheae. This is also the case with those dyes which neither injure nor penetrate living cells (indigo-carmine, aniline-blue, \&c.), and in such cases the water current must pass through dead elements, as far as the dissolved dye can be traced.

It must be remembered that all tissues can absorb water to a greater or less extent, and can surrender it again to neighbouring parts. It is the feeble conducting power of the parenchyma, which necessitates the formation of special conducting channels (Sect. 33), and the readiness

' Cf. Strasburger, l. c., p. $59^{2}$; Schwendener, Sitzungsb. d. Berl. Akad., i 992 , Bd. Xliv, p. 927. and the literature there given.

${ }^{2}$ Cf. Strasburger, Über d. Saftsteigen, 1893 , p. 25 ; Schwendener, I. c., I892, p. 930. 
with which the water is transferred in such channels causes almost the whole supply to pass through them, for the upward suction is propagated extremely slowly in badly conducting tissues. Thus when, for example, the youngest annual ring conducts water best, the transpirationcurrent will pass mainly through this layer, but if the continuity of the younger wood is destroyed, the older alburnum layers may still be able to supply all the water required, the acceleration of the current through these channels being apparently attained by an increase in the motive power. Such increase would be produced by a diminution in the amount of water present in the transpiring cells and the tissues connected with them, for in this way an increased potential energy of absorption and a more pronounced suction-force would be generated.

An increased propulsive force becomes necessary when the sectional area of active conducting tissue undergoes any diminution, for the current must be so accelerated that the same amount of water shall pass through in a given time. A local narrowing, as, for example, in a water-tap, only exercises a slight influence upon the amount of water that issues forth, whereas the interpolation of a long narrow tube produces a pronounced effect. Similarly, it is of great importance in the conduction of water whether a long or a short distance needs to be traversed in channels which convey it with less freedom.

These relationships and their attendant consequences must all be considered in discussing the problems connected with the movements of water in plants. As a matter of fact, the resistance to its passage varies at different points, while at each point of connexion of different systems of vascular bundles, such as those of the stem and leaf, the structural relationships are such as to indicate that the transference of water must take place to a certain extent in a different manner from that which is characteristic of either system. When the continuity of the younger wood is interrupted by a saw-cut, sufficient water may still pass through the older alburnum layers, but it does not necessarily follow that the same would be the case if, by removing a long cylinder of the young wood, the water were forced to travel for a considerable distance through the older layers. All our knowledge of the poorly conducting tissues shows that under normal conditions the amount of water which they can transfer in vitue of their relative sectional area does not suffice for the needs of the plant, although in transpiring leaves the conductivity of the parenchyma cells is sufficient for all normal requirements. Similarly in the root, owing to its enormous absorptive surface, the slow transference through the parenchyma conveys an abundance of water from the soil to the conducting channels.

As their function demands, the fibrovascular strands, including the tracheal elements, form a connected system, composed of a series of longitudinal conducting channels. Owing to the lack of ressels in the rudi- 
mentary vascular bundles of mosses, sufficient water cannot be transferred through the stem to prevent the drying up which active transpiration tends to produce ${ }^{1}$. In submerged phanerogams, in which no rapid transference of water is necessary, the tracheal elements are rudimentary or few in number, whereas in twining plants they are developed to a very marked extent, for here large quantities of water must be conveyed through thin stems for considerable distances ${ }^{2}$.

Within certain limits restricted by the specific powers of the organism, the development of the conducting system is favoured when an increased demand is made upon it, and although no completely satisfactory and conclusive experiments have as yet been performed, still the results obtained by Kohl, by Hartig, and by Jost ${ }^{3}$, tend to show that increased transpiration induces a more marked development of the wood cylinder. The fact that the woody elements are unequally developed in the terrestrial and aquatic forms of amphibious plants corresponds with this conclusion ${ }^{4}$. A discussion of anatomical details and specific peculiarities is hardly necessary here ${ }^{5}$, and hence no description need be given of the modes of union between different vascular bundles, between successive annual rings, between the primary and secondary wood of the root, between graft and stock, between parasite and host plant ${ }^{6}$. Nor can the transition tissue be described by which the transference of water between the vascular bundles and the surrounding parenchyma is rendered possible ${ }^{7}$.

The diosmotic properties of the cell-walls contiguous to the vascular bundles are always of great importance, and frequently the pits on the tracheal walls will form points at which the transference of water is especially easy. Nevertheless it must not be forgotten that the other parts of the wall are permeable also, and when the pits are few in number less of the water may pass through them than through the remaining parts of the wall (cf. Sects. 20 and 2I). It is, for example, by no means certain

I Cf. Haberlandt, Jahrb. f. wiss. Bot., I886, Bd. xvil, p. 374 ; Vaizey, Ann. of Bot., I887, Vol. I, p. 147 .

2 Westermaier u. Ambronn, Flora, I88 I, p. 4I7; H. Schenck, Beiträge z. Biol. d. Lianen, I 893, Bd. II, p. 6 .

${ }^{3}$ Kohl, Transpiration d. Pfanzen, I886, p. Ir6. Cf. also Wieler, Bot. Zeitung, I889, p. 549; R. Hartig, Ber. d. Bot. Ges., I888, p. 224 ; Bot. Zeitung, I892, p. I76 ; Jost, Bot. Zeitung, I89I, p. $546 ;$ I 893, p. 90 .

4 Haberlandt, Physiol. Anat., IS96, 2. Aufl., p. 277 ; Constantin, Ann. d. sci. nat., I 884 , vi. sér., T. XIX, p. 287 ; Schenck, Ber. d. Bot. Ges., I 885 , p. 481 .

5 Details by de Bary, Comp. Anat., r 877 ; Haberlandt, Physiol. Anat., I896; Strasburger, Bau u. Verricht. d. Leitungsbahnen, IS9I.

- Details by Gnentzsch, Flora, I888, p. 309 ; Strasburger, 1. c., I891, and Saftsteigen, 1893, p. 22 ; Schwendener, Sitzungsb. d. Berl. Akad., I 892, Bd. Xı.Iv, p. 928 ; Jahn, Bot. Centralbl., I 894, Bd. LIx, p. 360 ; Strasburger, I89 I, 1. c., p. 503. Cf. Pierce, Ann. of Bot., I 893, Vol. vil, p. 29 I.

7 In addition to the general works quoted above, cf, upon roots Siedler, Cohn's Beiträge, I 887 , Bd. v, p. 405 . 
whether the presence of well-developed bordered pits in the wood of Coniferae is intended more for mechanical support than for purposes of exchange, or whether these pits subserve quite different functions ${ }^{1}$.

It is one of the aims of physiological science to determine the functional importance of these and other arrangements, but any attempt to directly deduce the functional importance of an organ from its visible structure frequently leads to one-sided or erroneous conclusions. For example, Godlewski (Sect. 36) has attempted to use the anatomical arrangement of the wood elements as an argument in support of his vitalistic theory of the ascent of water, whereas Strasburger with equal force contends that the arrangement indicates the absence of any such vital action. Moreover it must be remembered that the structural arrangement is such as to permit of the transference of water in both directions.

The lateral connexions in the vascular bundles also allow water to pass along oblique paths, as has been shown by various researches performed subsequently to those of Hales and Duhamel ${ }^{2}$. Thus if two sawcuts are made one above the other on opposite sides of twigs of the oak or fir, each passing the centre of the stem, sufficient water still reaches the leaves to keep them turgid. By using coloured solutions the curved paths which the water follows may be made visible. If however, as in Ficus elastica, lateral communications are imperfectly developed, cutting in this manner may cause the leaves to become flaccid ${ }^{3}$.

The elongation of the conducting elements usually causes the transference of water to take place preferably in the longitudinal direction, as is shown by appropriate researches with coloured solutions ${ }^{4}$. For these and other reasons the transference in the oblique direction is somewhat more difficult, and hence the leaves wither when a large number of alternating cuts are made one above the other on an oak branch.

Experiments with coloured solutions. To determine the channels by which water travels, coloured solutions have been employed since the time of Magnol (1709) and de la Baisse (1733). Later still substances were employed by Unger, Rauwenhoff, \&c., whose presence could be detected by means of reagents ${ }^{5}$. Un-

1 Russow, Bot. Centralbl., I883, Bd. XIII, p. 134; Schwendener, l. c., I892, p. 938; Strasburger, 1. c., $189^{2}$, pp. 473,$768 ; 1893$, pp. $25,82,8$ c.

${ }^{2}$ Lit. by Strasburger, Bat u. Verricht. d. Leitungsbahnen, I $\delta_{91}$, p. 595. On herbaceous plants cf. Haberlandt, Physiol. Anat., i 896, p. 322.

${ }^{3}$ Strasburger, Saftsteigen, 1893, p. 34.

4 Th. Hartig, Bot. Zeitung, 1853, p. 3 I 3 ; Wieler, Forst- u. Jagdzeitung, 189I, p. 278 ; Strasburger, l. c., I891 ; K. E. F. Schmidt, Abhandlungen d. Naturf.-Ges. z. Halle, 1893, Bd. XIx, p. 85. Other experiments in this direction are given by Wiesner, Unters. über d. Bewegung d. Imbibitionswasser, I 875 , p. 10, \&c. (Sep.-abdr. a. Sitzungsb. d. Wien. Akad., I875, Bd. Lxxil, Abth. i).

${ }_{5}^{5}$ Cf. Treviranus, Physiol., I 835 , Bd. I, p. 285 ; Mohl, Zelle, I851, p. 73 ; Sachs, Gesch. d. Bot., 1875 , p. 522 ; Strasburger, Bau u. Verricht. d. Leitungsbahnen, 1891, p. 555. 
fortunately, the inaccurate use of these important methods has often led to erroneous conclusions. Above all it must be borne in mind that the dye, like water, diffuses in all possible directions from the conducting elements, so that ultimately those cell-walls and cells will be most deeply coloured which absorb and accumulate the colouring material, even though such parts may not function at all in the conduction of water ${ }^{1}$. Before any conclusions can be made, the dependence of the distribution of the colouring material upon the water-current which carries it must be established, and the tissue-elements in which the dye first appears must in all cases be determined. For purposes of demonstration, white flowers of Crocus, Iris, Lilium, Primula sinensis, \&c., may be advantageously employed. When the cut stalks of the flowers or inflorescences are placed in a deep blue solution of indigo-carmine, the first appearance of the coloured fluid in the fibrovascular strands may be observed and its gradual progress followed as transpiration continues. If twigs are used, the progress of the dye can be traced by making a series of transverse sections. For researches with trees, the oak or lime will serve, and the use of eosin or indigo-carmine is to be recommended, whereas methyl-blue, fuchsin, and a few other dyes are less suitable, for reasons given later ${ }^{2}$.

That the dye is actually carried along with the water-current is shown by the fact that in strongly transpiring plants it usually travels 0.5 to 3 metres forward in an hour, whereas without any transpiration it travels barely $\mathrm{I} \mathrm{cm}$. in the same time. In every case the plant must be previously saturated with water, and cut stems or stalks must be kept at least half to one hour in water before use, since otherwise the negative pressure in the opened vessels would cause the coloured solutions to be sucked in quite independently of the transpiration current $^{3}$. Entire plants are not so well adapted for such experiments, for the dye passes with much greater difficulty through the living epidermal and cortical cells of the root than water does. Thus water can pass directly through the cells, whereas bodies which cannot diosmose through the protoplasts, such as indigocarmine, aniline-blue, or nigrosin, travel only through the cell-walls. Hence, owing to the relatively small sectional area of these, such substances are transferred with comparative slowness ${ }^{4}$, and even dyes which penetrate the protoplast travel more slowly than water does. Moreover, the dyes which can be absorbed, eosin included, exercise in general a poisonous influence even when very dilute, and this accelerates the transference of the dye, whereas other causes (passive secretion, \&c.) tend to retard its passage. Coloured solutions first appear in the tracheae and tracheides of the alburnum, whence diffusion to the surrounding tissue-elements soon occurs. This result is best obtained with indigo-carmine, which is not absorbed or secreted to any noticeable extent, and the use of a deeply-coloured saturated solution makes

${ }^{1}$ For examples see Sachs, Arb. d. Bot. Inst. in Wïrzburg, i875, Bd. II, p. 150. Cf. also Schwendener, Sitzungsb. d. Berl. Akad., I 892 , Bd. XLIT, p. 925.

${ }^{2}$ See Strasburger, 1.c., pp. 551, 566 .

${ }^{3}$ Cf. Sect. 32 ; also Sachs, Arb. d. Bot. Inst. in Würzburg, 1878 , Bd. 11, p. I57 ; Strasburger, 1. c., I $89 \mathrm{I}, \mathrm{p} .589$.

+ Cf. on this and the following, Sects. 15, I6, \&c., where Pfeffer's researches with dyes are given (Unters. a. d. Bot. Inst. z. Tuibingen, I 886 , Bd. $1 \mathrm{I}$, p. 268). Wieler (Jahrb. f. wiss. Bot., I $888, \mathrm{Bd}$. XIX, p. 119) has recently employed entire plants. 
the recognition of its first appearance easier. Strasburger mainly employed eosin, but this pigment is retained by the tracheal walls, and, indeed, by all lignified membranes".

As might be expected, experiments with dyes show that in certain tracheae and tracheides water travels more rapidly than in others ${ }^{2}$, but in every case the experiments with dyes only indicate that water travels rapidly in certain tissueelements. The possibility, however, always remains that in other elements which the dye cannot penetrate water may also travel, though more slowly. Living parenchyma, which is impermeable to a particular dye, might appear in such researches to be quite inactive, and yet be really the seat of a rapid transference of water. The results already given show, however, that the latter is not actually the case. By other experiments, such as by injecting the vessels with gelatine, the latter have been proved to be the active agents in the transport of water.

The importance of the experiments with dyes is not lessened by the fact that the water travels more rapidly than the dye. This phenomenon can be observed when filter-paper is dipped into solutions of aniline dyes, and it is the necessary consequence of the retention (cf. Sect. 28) of a certain amount of the dye, so that the solution passes unchanged only when the saturation point of the wetted material is reached. Hence experiments with dyes do not give the full rapidity of the watercurrent. Nevertheless, when indigo-carmine or eosin is used the difference is not very marked, and not much greater than when lithium is employed, the latter not being perceptibly retained by the lignified walls. Owing to their much slower rates of transference, methyl-blue, fuchsin, \&c. are less adapted for such researches ${ }^{3}$.

Rapidity of the zuater-current. The appearance of salts of Lithium, readily recognizable by spectroscopic examination, was utilized by Sachs as a test for the rapidity of water-transference in the conducting channels of a transpiring plant ${ }^{4}$.

By watering the earth around potted plants with a $\mathrm{I}$ to 3 per cent. solution of Lithium nitrate, a rapidity of transference of $0 \cdot 18$ to $2 \cdot$ I metres per hour was observed. Similar values were given by most of the plants used by Strasburger, although he placed cut stalks in the solution and for the most part used a dye, eosin, as the indicator ${ }^{5}$. In certain plants in which the water-currents are rapid, a transference through as much as 6 metres was observed in an hour, with normal transpiration (Bryonia, Cucurbita). These results were observed after the cut stems had remained for a time in water, for if the negative pressure exhibited immediately after the stem

1 The faulty methods and errors of Bokorny (Jahrb. f. wiss. Bot., I890, Bd. xxI, p. 496) have been sufficiently exposed by Hansen (Flora, I 890 , p. 270 ) and Strasburger (Leitungsbahnen, $189 \mathrm{I}$, p. 557 ).

${ }_{2}$ Wieler, Jahrb. f. wiss. Bot., IS88, Bd. xix, p. 566 ; Strasburger, I $\$ 91$, 1. c., p. 566 .

${ }^{3}$ Literature : Lehmann, Molecularphysik, I888, Bd. I, p. 573; Goppelsröder, Über Capillaranalyse, I889; Sachs, Arb. d. Bot. Inst. in Wiirzburg, I878, Bd. II, p. I57; Strasburger, Bau u. Verricht. d. Leitungsbahnen, IS9I, p. 550.

4 Sachs, Arb. d. Bot. Inst. in Wüirzburg, 1878 , Bd. II, p. I 48 . Lithium was first used by $\mathrm{M}^{\mathrm{c}} \mathrm{Nab}$, Trans. of the Bot. Soc. of Edinburgh, I87 I, Vol. XI, p. 45, and Trans. of the R. Irish Acad., I $87_{4}$, p. 343 ; later by Pfitzer (Jahrb. f. wiss. Bot., I $87 \%$, Bd. xI, p. I77). Pfitzer used salts of thallium, $\mathrm{M}^{\mathrm{c} N a b}$ of caesium as well. On the poisonous properties of lithium, and its distribution in the plant, see Gaunersdorffer, Versuchsst., i 887 , Bd. xxxiv, p. I 7 I.

${ }^{5}$ Strasburger, Bau u. Verricht. d. Leitungsbahnen, ז 89 I, p. $5 \$ 8$. 
was cut was allowed to aid in sucking in the solution employed, the transference of the dissolved substance took place twice as rapidly ${ }^{1}$.

As rapid a current as this may therefore occur in at least some of the wood elements, and no doubt when transpiration is very active and the general conditions are favourable, the rapidity might become still more marked. As it is, it is extremely rapid in comparison with protoplasmic streaming or rotation. In the different conducting elements water will certainly not travel with exactly the same speed, while any narrowing or broadening of the conducting channels will undoubtedly influence the maximal as well as the mean rapidity of the water-current. It is impossible to determine the mean for the water-current until the precise sectional area concerned is known. Since, however, the conducting area in the vascular bundles of a sunflower is not very great, the water must travel with great mean rapidity in order to convey upwards the 0.865 kilos ( $\mathrm{I}_{4}^{1} \mathrm{lb}$. or I pint) of water which Hales found a sunflower could exhale in twelve hours in the form of water-vapour ${ }^{2}$. In the stem of a beech-tree the water must travel, according to the calculation of Schwendener, at a daily rate of 2 metres in order to counterbalance the average loss by transpiration during the summèr months.

\section{SECTION 36. The Mechanism of Water-transport.}

How and by what means the water is so rapidly transferred even to the summits of the tallest trees has not as yet been satisfactorily explained. It has unfortunately not even been determined whether the aid of living cells is quite unnecessary, although if this were so, the problem would be much restricted-and would become almost entirely a physical one.

Böhm has shown indeed that sufficient water may be transferred through a dead piece of stem to keep the upper living leafy portion fresh and turgid for a few days. Strasburger ${ }^{3}$ has obtained similar results by killing portions of the stems of living trees, \&c., often for a distance of as much as I 2 metres (nearly to feet), by heat or by watering with poisonous substances. In his interpretation of these facts, however, he does not mention the closely allied question, as to whether the assistance of living cells is not necessary to maintain the power of the dead tissue to conduct water for any length of time ${ }^{4}$. The gradual disappearance of the conductivity for water may result from the loss of such cells, and need not

1 Examples by Pfitzer, 1.c., p. 213 ; Strasburger, 1. c. Cf. Sachs, 1. c., p. I 7 I.

${ }^{2}$ Hales, Statics, $1_{74} 8$, pp. 3 , Io. Cf. also Sachs, l. c., p. I53.

${ }^{3}$ Böhm, Ber. d. Bot. Ges., 18S9, Generalvers., p. 55 ; $1 S_{92}^{2}$, p. 622 ; 1593 , p. 203 . Hartig gave in ${ }_{1} S_{53}$ (Bot. Zeitung, p. 313) similar observations made upon stems killed by the absorption of solutions of iron salts. Strasburger, Bau u. Verricht. d. Leitungsbahnen, IS9I, p. 645 ; Über das Saftsteigen, 1893 , p. I1 .

† For other views see Schwendener, Sitzungsb. d. Berl. Akad., 1892, Bd. xLiv, p. $93^{2}$. 
necessarily be due either to any blocking of the conducting channels or to changes taking place in them. If the loss of conductivity is due to the absence of living cells, injection with water should temporarily restore it, and indeed Strasburger ${ }^{-1}$ has found that shoots killed in various ways and dried have their conductivity restored for a time after such injection. It is necessary also to determine whether the loss of conducting power in the duramen is owing to the blocking of its tracheal channels ${ }^{2}$, or is due to the gradual death of all the living cells it contains.

On the other hand, no conclusive proof has been brought forward to show that living cells aid in the transference of water through the conducting channels when these are once established ${ }^{3}$. The apparent absence of any other explanation cannot be regarded as a proof that vital actions aid in the transport of water, for when dealing with complicated phenomena of this kind, it is quite possible that certain co-operating factors or conditions may be overlooked or insufficiently considered. What now appears inexplicable or incomprehensible might be easily understood were our knowledge more complete. It seems probable indeed that water may perform its entire journey in dead tissueelements. The fact that dyes which do not penetrate the living cells rapidly ascend with the transpiration-current points to this conclusion, especially when the dye is one which, like indigo-carmine, does not injure the living cells in any way. Along with the dye, the water which carries it must also travel upwards through dead vessels, without entering any living cells which might pump it upwards to a higher level. Even if the maintenance or production of the conditions necessary for the conduction of water is due to the living cells injecting water into the vessels and thus exercising a pumping action, the fact still remains that the actual transference takes place almost entirely through dead tissue-elements.

The facts already given suffice to show that water travels in the tracheal elements, and largely in their lumina. This is admirably illustrated by the results of injection with a substance solidifying on cooling. If a shoot is injected from the cut surface upwards for a short distance with fluid gelatine, cocoa-butter, or paraffin, the leaves fade soon after the injected fluid solidifies, and the withering is as rapid when the cut surface is immersed in water as when it is exposed to $\mathrm{air}^{4}$. Water cannot be

1 Strasburger, 1. c., 1891 , p. 657 .

${ }^{2}$ On the blocking of the trachene see Wieler, Biol. Centralbl., 1893 , Bd. xirl, p. 586.

$s$ The behaviour at low temperatures (cf. Schwendener, Sitzungsb. d. Berl. Akad., 1892, Bd. XI.Iv, p. 945) is no proof of this, for the diminished conductive porrer of the tracheal channels appears to be explicable solely from physical causes. Cf. Sect. 37 .

- Elfving (Bot. Zeitung, I882, p. 7I4) and Vesque (Ann. d. sci. nat., I884, vi. sér., T. Xix, p. I88) used cocoa-butter; Scheit (Bot.Zeitung, $188_{4}$, p. 201) gelatine. More critical experiments were carried out by Errera (Bot. Zeitung, I886, p. I6́) with gelatine. Confirmatory results have 
transferred through the substance of the tracheal walls with sufficient rapidity, over even comparatively short distances, to supply transpiring leaves with the water they require, and hence in stems injected with gelatine, \&c., dyes pass upwards with extreme slowness ${ }^{1}$.

As Kohl ${ }^{2}$ has shown, withering ensues when the lumina of the tracheae are partially closed by the pressure of a compression screw, while when this is removed the flow of water becomes normal again. It must be borne in mind that fluid gelatine does not hinder the transit of water, and hence the normal current of water recommences if the solidified injected gelatine is melted by raising the temperature to about $30^{\circ}-32^{\circ} \mathrm{C}$. Disregarding the intercellular spaces, which do not concern us here, the gelatine penetrates into and blocks up the cavities of the tracheae, the tracheides, and eventually the bast fibres as well. Hence it follows that all the other tissue-elements together, living or dead, are unable to convey the water which the plant requires; at the same time the supposition put forward by Sachs ${ }^{3}$ that the water travels most rapidly in the lignified walls of the wood-tracheae is decisively negatived, for the pressure and injection experiments show that the excessive conductivity which Sachs' theory presupposes is not actually possessed by the walls of the wood vessels. A most marked conductivity would moreover be necessary, for a trifling difference of potential suffices to induce a flow of water, so that a very slight force must overcome the enormous resistance to filtration interposed by a length of 20 metres or more of lignified cellulose. For these and other reasons it is impossible that the water could travel in the substance of the walls with sufficient rapidity to supply the requirements of transpiration, although the force of imbibition (Sect. I2) would suffice to raise the water higher than the summit of the tallest tree ${ }^{4}$.

The tracheal channels are occupied in transpiring plants by a chain of water-columns and air-bubbles (Jamin's chain) ${ }^{5}$, and the water can either

been obtained by Strasburger (Ban u. Verricht. d. Leitungsbahnen, 1891, p. 541) with gelatine, and by Dixon and Joly Ann. of Bot., I 895 , Vol. Ix, p. 403) with gelatine and paraffin.

1 Dixon and Joly (1. c.) have also showed that after injection with paraffin, water does actually travel upwards through the tracheal walls, but only very slowly.

${ }^{2}$ Liohl, Bot. Zeitung, $188_{5}$, p. 522, and Transpiration d. Pflanzen, 18S6, p. I1 ; F. Darwin and Phillips, Proc. of the Camb. Phil. Soc., I886, Vol. v, p. $3^{6} 4$; Strasburger, 1. c., p. 603. As Russow has shown (Bot. Centralbl., $188_{3}$, Bd. xıI, p. 99 ; cf. also Godlewski, Jahrb. f. wiss. Bot., $188_{4}$, Bd. xv, p. 628, the lumina are not, as a general rule, closed when the stem is sharply bent. Dufour (Arb. d. Bot. Inst. in Würzburg, ISS 4 , Bd. III, p. 4I) erroneonsly concluded from such experiments that he had established a proof of the imbibition theory.

3 Sachs, Arb. d. Bot. Inst. in Wïrzburg, 1878 , Bd. II, pp. I 48 , 291 ; Vorles. iiber Pflanzenphysiol., 1887 , 2. Aufl, p. 201 .

+ See Godlewski, Jahrb. f. wiss. Bot., $188_{4}$, Bd. xv, p. 602 ; Schwendener, Sitzungsb. d. Berl. Akad., I886, Bd. xxxıv, p. 591 ; Pfeffer, Studien zur Energetik, 1892, p. ${ }_{2} 5^{\text {s. }}$

"From the literature quoted in Sect. $3^{2}$, it may be seen that this Jamin's chain persists even when but little water is present. 
pass upwards by a forward movement of the entire chain of air and water, or the chain remaining stationary, individual particles of water may wander round the air-bubbles from water-column to water-column ${ }^{1}$. A trifling difference of pressure does indeed suffice to cause a movement of the entire chain ${ }^{2}$, but nevertheless the main current of water cannot be produced in this manner, for since air diosmoses only slowly through the moist tracheal walls (Sect. 32), the bubbles would collect at the upper ends of the tracheae and tracheides and thus interpose a marked resistance to the passage of water, while whenever the tracheae and tracheides are only of moderate length, as is often the case, this resistance will be interposed at very many points.

As a matter of fact various observations show that the air-bubbles, although they may vary in size, remain at rest even when a current of water may be flowing past them ${ }^{2}$. It is, however, impossible as yet to say whether the water passes from column to column through the tracheal walls or through the film of water (adhesion-water) which surrounds each airbubble. We do not even know what thickness this film of water attains, or what resistance it interposes to any movement in mass. A full discussion of these and related questions is impossible here ${ }^{3}$, but it may be mentioned that, under the given conditions, the thickness of the film may be such that the greater portion is beyond the influence of the energetic attraction exerted by the surface molecules of the organized membrane which it touches. Moreover, one of the surprising results due to surface tension is that in a very thin film of fluid a rapid movement may be produced by a corresponding difference of potential.

As the distance between the water-columns increases, the transit of water will apparently become more and more difficult, and hence withering commences when the amount of free water present in the wood (Sect. 33) sinks below a certain limit. It is by no means immaterial whether the water passes the same length of air as short bubbles with the watercolumns close together, or as long bubbles with them far apart, but further research is still needed in this direction. In the wood of transpiring trees fully supplied with water, Schwendener ${ }^{4}$ found that the water-columns were from $0 . I$ to $0.5 \mathrm{~mm}$. long, whereas the interposed bubbles of rarified air may be as much as $1 \mathrm{~mm}$. in length.

For purposes of simplicity we have confined ourselves to the con-

1 That the transference across the air-bubbles could take place rapidly enongh by evaporation and condensation is impossible. Cf. Dixon and Joly, Ann. of Bot., 1895 , Vol. Ix, p. 4 I 9.

2 Observations and literature by Strasburger, Bau u. Verricht. d. Leitungsbahnen, $\mathbf{r} \& 9 \mathrm{I}$, and Über das Saftsteigen, I 893 , p. So; Schwendener, Sitzungsb. d. Berl. Akad., Bd. xxxiv, p. इ9I, and $189^{2}$, Bd. Xliv, p. 920 ; Askenasy, Über das Saftsteigen, $18_{93}$, p. I7 Sep.-abdr. a. d. Verhandlungen d. Naturf. Vereins in Heidelberg).

${ }^{3}$ [See Kamerling, Oberflächenspannung u. Cohäsion, Bot. Centralbl., Bd. Lxxı11, I898, p. 470.]

4 Schwendener, Sitzungsb. d. Berl. Akad., I886, Bd. xxxiv, p. $56_{7} ; 1893$, Bd. XI., p. 842 . 
sideration of the movements of water in longitudinal rows of tracheae and tracheides, and have neglected the possibility of any current flowing obliquely or transversely. The same general principles which regulate the former are, however, at work here also, and hence no special account need be given of the details concerning the transverse convection of water. It is also impossible at present to say whether the water-columns in neighbouring tracheae correspond to one another, and by a transference of water through the separating walls establish a continuous network of waterchannels throughout the xylem as a whole. We do not know, moreover, whether movements or alterations of size of the air-bubbles play any part in the transference of water.

A satisfactory explanation of the means by which the transpirationcurrent is maintained has not yet been brought forward. If no vital actions take part in it, then it is obvious that we have only an incomplete knowledge of the causes at work, and of the relationships of the different factors concerned. A column of water in a suitable capillary tube, in the form of a Jamin's chain, may attain a height of 142 metres, which is as much as is necessary for the highest tree. The necessary capillary and other conditions are not, however, fulfilled in a column of tracheae, and taking into account all the conditions actually existing, no greater height than 50 metres could be attained in this manner ${ }^{1}$.

Dixon and Joly, as well as Askenasy ${ }^{2}$, have lately attempted a purely physical explanation of this problem. They point out that, owing to the high cohesion of water, the breaking strain for a continuous column may be more than 50 atmospheres when no lateral twisting or displacement is possible. The suction exerted by the leaves is supposed to be transmitted backwards as far as the roots through cohering columns of water. More attention must, however, be paid to the conditions actually existing in the plant, before any such explanation can be accepted. Without attempting a critical discussion of the views of these authors, it may be pointed out that the walls of the tracheae can absorb or give off water with equal readiness, and that the negative pressure exerted by a continuous water-column would tend to cause an inward and downward stream of water in the upper parts at least. Besides, the presence of air-bubbles forms one of the conditions which render the rupture of a water-column readily possible, and the valuable experiments of Dixon and $\mathrm{Joly}^{3}$ do not afford conclusive proof that the water-columns in

1 Schwendener, Sitzungsb. d. Berl. Akad., 1892, Bd. xulv, p. 916; 1893 , Bd. xL, p. 835 ; Steinbrinck, Ber. d. Bot. Ges., 1894, p. 127.

${ }^{2}$ Dixon and Joly, Phil. Trans., 1895 , Vol. Clxxxvi, p. $5^{63}$; Proc. of the Irish Acad., 1896, Vol. III, p. 767 ; also Ann. of Bot., 1896, Vol. x, p. 630; Askenasy, Über d. Saftsteigen, I 895 (Sep.-abdr. a. d. Verhandlungen d. Naturh.-med. Vereins in Heidelberg); also 1896.

${ }^{3}$ Dixon and Joly, Ann. of Bot., is 895 , Vol. Ix, p. 403 . 
the tracheal elements do actually offer great resistance to mechanical rupture.

On the other hand, the gaseous tensions existing in the tracheae can hardly be of primary importance in producing the water-current, if this is still shown when the influence of any negative pressure is entirely nullified, as in vacuo. That this is the case is indicated by the fact that neither a diminution nor an increase in the external pressure diminishes or increases the rapidity of the water-current ${ }^{2}$. Hence, although the negative pressure existing in the tracheal channels must be of importance in regulating the flow of water, it cannot be the actual cause of it ${ }^{2}$. It must be remembered, however, that the tracheae become completely filled with water when the supply is sufficiently abundant, even when the plants are in a vacuum, and the presence of air-bubbles, which must be dissolved away, acts merely as a temporary hindrance to complete saturation. In herbaceous plants the tracheae and tracheides may occasionally be completely filled with water in the ordinary course of events ${ }^{3}$, and it would be well to learn whether a similar filling of the tracheae may take place as far as the summit of a lofty tree, or whether continuous water-columns of this length cannot exist, and if so, whether the formation of a chain of columns of water and air is a necessity in tall plants.

It is possible that in this short sketch important experiments and facts have been overlooked, while unimportant details have been made unnecessarily prominent. This is, however, unavoidable as matters are at present, and whatever the final solution of the problem may be, the causes at work must necessarily be such that the conducting channels become filled to a certain extent with water, and that by a disturbance of equilibrium a corresponding flow of water is induced. It is, however, impossible to tell from these simple essential conditions whether the transference of water takes place with or without the active aid of living cells ${ }^{4}$.

Historical. Many facts concerning the transpiratory current were established by Grew, Malpighi, Mariotte, Woodward, and others, but it was the masterly researches of Stephen Hales ${ }^{5}$ which laid the foundation of our present knowledge.

${ }^{1}$ Strasburger, Bau u. Verricht. d. Leitungsbahnen, I 891 , p. 793 ; Über das Saftsteigen, I 893 , p. 64 ; Dixon and Joly, Phil. Trans., i 895 , Vol. CLxxxvi, p. 564.

${ }^{2}$ Variations in the amount of air present are shown almost solely in the alburnum wood: Hartig, Ber. d. Bot. Ges., I888, p. 223 , and the literature given in Sect. 37. On the relative amounts of air and water present in wood, see also Sachs, Vorles, uiber P'flanzenphysiol., 1887, 2. Aufl., p. 2 I 9.

${ }^{3}$ Cf. p. 220 and Sect. 32 ; also Schwendener, Sitzungsb. d. Berl. Akad., I 892, Bd. xLiv, p. 93I ; Strasburger, l. c., I 893, p. 25.

- [H. H. Dixon (Proc. R. Jrish Soc., I898, Vol. Iv, pp. 618, 627) concludes that the latter is the case, whereas Schukowsky and Wottschal (Nat. Hist. Soc. of Moscow, Dec. 1897) consider that the ascent of water is a purely physical phenomenon. Wottschal indeed states that the movements of water in a column of sand are similar to those in the stem of a tree. Cf. Bot. Centralbl., Bd. Lxxvir, is99, p. 337.]

"Hales, Statics, 174\$; cf. Sachs, History of Botany (Gamsey and Balfour', I \$86, p. :00; PFEFFFR 
Hales proved that the water travels in the wood, and that the transference of water takes place in transpiring plants by means of an upward sucking action, and not by a vis a tergo. The general principles regulating the movements of water were thus early established, and since then repeated attempts have been made to explain the mechanism as well as the causes of the transpiration-current ${ }^{1}$. Some authors saw a sufficient explanation in capillary or osmotic forces, both of which were regarded as insufficient by others, while explanations were not wanting in which a vital action was postulated. The problem is still unsolved, in spite of all the advances made and the deeper insight which has been gained ${ }^{2}$.

Sachs $(1878)$ regarded the water as being transferred by imbibition through the tracheal walls, without considering any vital action necessary, but this 'imbibition theory' is now untenable (p. 2rg). Godlewski, on the other hand, considered the pumping action of living cells to be necessary, but was unable to prove this hypothesis ${ }^{3}$. Hence no discussion of his theoretical conclusions is necessary, nor of those of Janse and Westermaier ${ }^{*}$, as to the way in which living cells may act in raising water. Schwendener did not support any particular theory, but apparently was inclined on theoretical grounds to regard a vital action as necessary. By the clearness of his physical explanations he has, however, aided much towards a precise definition of the problem at issue. That Hartig's so-called 'gas-pressure' theory did not suffice to explain the raising of water in trees had already been shown by Godlewski ${ }^{5}$.

We owe various important experiments to Böhm ${ }^{6}$, who was, however, less fortunate in his interpretations of the facts observed, and who finally came to regard a simple capillary ascent as a sufficient explanation. Böhm showed that water may be transferred through a dead piece of stem for a considerable distance. Strasburger extended these experiments, and obtained many important results, without however being able to give a satisfactory explanation of the problem. The why and wherefore is not explained by the conclusion that the distribution of the air, water, \&c. is such as to produce a condition of equilibrium, any disturbance of which causes a corresponding flow of water. Nor do the important researches of I ixon and Joly, or those of Askensasy, afford a completely satisfactory explanation.

Filtration under pressure. In the process of normal bleeding, water is forced

Treviranus, Physiol, ${ }_{1} 8_{35}$, Bil. I, p. 300. [Neliemiah Grew, Anatomy of Plants, 1682, pp. 79, 123.]

1 Cf. Mohl, Grundz. d. Anat. u. Pliysiol., 185 1, P. 70 ; Meyen, Pflanzenphysiol., I8 38 , Bd. II, pp. 50, 55, \&c.; Treviranus, Physiol., 1835 , Bd. 1, p. $28_{4}$; de Candolle, Physiol., $18_{33}$, T. I, p. 79.

${ }^{2}$ Cf. also the litcrature references in the Annals of Botany, 1896, Vol. $x$, p. 630 .

${ }^{3}$ Godlewski, Jalırb. f. wiss. Bot., I $88 \mathrm{f}, \mathrm{Bd}, \mathrm{xv}$, p. 602. [A similar explanation was given two hundred years ago by Nehemiah Gicw, 1. c., p. 126.]

* Janse, Jahrb. f. wiss. Bot., 1887 , Bd. xvirI, p. 68 ; Westermaier, Ber. d. Bot. Ges., $188_{3}$, p. $37 \mathrm{r}$, and Silzungsb. d. Berl. Akad., I $88_{5}$, Bd. XLVIII, p. I10 5 .

${ }^{5}$ Godlewski, l.c., p. $5 s_{3}$. There also $($ p. 627 ) the untenable hypotheses of Scheit are dealt with. R. Hattig, Die Gasdrucktheorie, $188_{3}$; Schwendener, Sitzungsb. d. Berl. Akad., 1886, I893.

${ }^{6}$ Böhm, Ber. d. Bot. ('es., IS89, Generalvers., p. 46; I892, p. 692; I893, p. 203, \&c. A critical discussion of Böhm's views is given by Godlewsli, l. c., p. 57 I : Schwendener, Sitzungsb. d. Berl. Akad., 1892, p. $93^{\text {f. }}$ 
out under pressure (Sect. 42), and owing to the saturation of the tracheal elements with water, the conditions are not quite the same as when transpiration is active. In the former case the chain of air and water becomes more and more reduced, and may ultimately consist of water only, so that the water-current has less resistance offered to it, and is hence accelerated, as may be directly shown when a trachea is opened at both ends, and the chain of air and water driven out by a continuous current of fluid.

Observations made on filtration under pressure can only be extended to the transpiration-current with extreme caution. Water filters but slowly through living cells under pressure, so that any water forced into a piece of stem passes almost entirely through the wood, and especially the tracheae, to appear on the other cut surface. Water filters through the wood more slowly across the vascular elements, and the power of filtration diminishes still more in the duramen (Sect. 35) ${ }^{\mathrm{I}}$. The amount of water filtering through a cut surface gradually diminishes, owing to the occlusion of the channels by suspended particles and by slimy growths, which may be derived partly from bacteria and partly from the escaped contents of opened cells, while a formation of tyloses may also aid in blocking the tracheal channels. The occlusion is, however, mainly at the cut surface, for the removal of a thin section markedly increases the rate at which water is absorbed ${ }^{2}$. It is, perhaps, owing to the fact that no solid particles enter them, that intact tracheal elements are able to serve as good conducting channels for a long time, provided that they undergo no special modifications.

The rule of transmission of water by simple filtration under pressure is rapid, and thus when a piece of stem from a plant saturated with water is removed and suspended vertically, a certain, and under appropriate conditions a considerable, amount of water exudes from the lower cut surface ${ }^{3}$. Water continues to be exuded until the menisci formed in the tracheae on the upper surface are able to support the columns of water suspended from them. If a drop of water is placed on the upper surface, the equilibrium is disturbed, and a corresponding drop appears on the under surface. This phenomenon, which has been frequently misunderstood, was first correctly explained by Godlewski *

${ }^{1}$ See Sachs, Arb. d. Bot. Inst. in Wïrzburg, I879, Bd. II, p. 285 ; Strasburger, Bau u. Verricht. d. Leitungsbahnen, I $8_{9}$ I, p. 8 I9; Nägeli u. Schwendener, Mikroskop, I 8 7 7 , 2. Aut., p. 385 ; Unger, Sitzungsb. d. Wien. Akad., I868, Bd. Lvirr, Abth. i.

${ }^{2}$ Hales, Statics, 1748, p. 71 ; Brïcke, Ann. d. Physik. u. Chemie, 1844, Bd. Lxin, p. 187; Sachs, Arb. d. Bot. Inst. in Wiirzburg, I879, Bd. II, p. 300; v. Höhnel, Bot. Zeitung, I879, p. 302 ; Strasburger, 1.c., I891, p. 564; Wieler, Cohn's Beiträge zur Biologie, i S9.3, Bd, vi, p. I 49 ; Biol. Centralbl., I 893 , Bd. xıII, p. 58.4 .

${ }^{3}$ Observations on various plants have been made by Gaudichaud, Porteau, and Schimper. Cf. the literature given by Strasburger, 1891, 1. c., p. 823 .

+ Godlewski, Jahrb. f. wiss. Bot., I $\$ S_{4}$, Bd. $x v$, p. $5 S S$; Schwendencr, Sitzungsb. d. Berl. Akad., 1886, Bd. xxxiv, p. $5^{82}$. 


\section{SECTION 37. The Relation between Transpiration and the Absorption of Water.}

The ratio between the amounts of water transpired and absorbed is not constant but varies from time to time as external conditions alter. When the loss by transpiration becomes relatively greater, the percentage of water in the plant decreases. If the loss is so marked that the turgidity of the living cells falls below a certain limit, the leaves droop and the plant becomes flaccid. An increase in the amount of water absorbed will cause the cells to become turgid again, provided that the loss of water has not been so great as to injure them fatally. As absorption continues, the amount of water present increases until a certain level is reached at which the gain and loss just counterpoise one another, and the percentage of water will remain constant at this level so long as the external and internal conditions are unaltered. The rate of transpiration is markedly influenced by external conditions, as well as by the character of the transpiring organs. At the same time the plant can absorb more water from a humid than from a dry soil, and hence in nature the percentage of water which a plant contains must be continually undergoing slight variations, though these are gencrally insufficient to produce any immediately perceptible external result.

The various methods by which excessive transpiration is prevented (Sect. $38-40$ ) are of the utmost biological importance when the supply of water is limited. Certain plants, such as Sempervioum, many of the Cactaceae, and others also transpire so slowly that it may be months before cut branches lose sufficient water to kill them. Such forms can hence exist in dry climates or regions, even though they may be without any supply of water for prolonged periods of time. Plants, which rapidly wither when cut, soon dic under such conditions unless they are able to survive desiccation, as is the case in certain Mosses, Lichens, and Protophyta. The power of accumulating a store of water and of existing upon this supply for a certain time is of great biological importance in the members of the Crassulaceac and various bulbous and tuberous plants, for they are thereby enabled to survive periods of drought ${ }^{1}$. Indeed, in many cases special tissues for water-storage may be developed, as, for example, in the leaves of Peperomia, and these when necessary supply water to the green tissues and keep them turgid ${ }^{2}$. The causes which regulate the movements and distribution of water throughout the plant as a whole are also responsible

${ }^{1}$ For examples see Haberlandt, Physiol. Anat., I896, 2. Aufl., p. 347 ; Volkens, Flora d. ägypt. Wüste, I 887 , p. $5_{2}$; Goebel, Pflanzenbiol. Schildermgen, I 889,1 , p. 25.

2 Westermaier, Jahrb. f. wiss. Bot., I $88_{4}$, Bd. XIV, p. 43 ; IIaberlandt, 1. c. ; Volkens, 1. c., \&c. Pfitzer (Jahrb. fo wiss. Bot., $1872, \mathrm{Bd}$. vili, p. I6) was the first to recognize the importance of water-tissues. Every tissue can function, to a greater or less extent, in the manner indicated. On collenchyma, sec C. Mïller, Ber. d. Bot. Ges., 1890, p. I64. 
for the localized transference of water which occurs in such cases as these. The cells in which the osmotic energy is weakest are naturally the first to lose their turgidity and collapse (Sect. 34), while in some cases the water-storage cells undergo an elastic contraction and so are able to give off water and yet remain filled with fluid. Similarly, it is by maintaining a continual difference of osmotic potential that a cut shoot is able to grow at the expense of the older parts, which shrivel up as water is withdrawn from them (Sect. 34). Owing to the rigidity of their walls, tracheides and tracheae do not shrivel or collapse when they lose their watery contents. Water is readily withdrawn from dead cells, such as the vascular elements just mentioned, but as the loss of water continues the contained air becomes more and more rarified, so that a correspondingly increasing resistance is offered to any further removal (Sect. 34).

We have already discussed the means by which plants obtain water from the soil through their roots, and in some cases from other sources also by special sub-aërial organs (Sects. 25-27). It has already been mentioned that plants can absorb the required water more easily from very moist soils than from comparatively dry ones; indeed a very dry soil may actually withdraw water from the plant ${ }^{1}$. Hence a gradual decrease in the amount of water present in the soil will ultimately cause a plant to become flaccid if transpiration continues. On the other hand, plants which have become flaccid during a hot day recover during the night, not because the soil becomes more moist, but because the cooler and damper night air causes the rate of transpiration to diminish.

The amount of water which a plant contains naturally varies as the extemal conditions alter, and as a general rule the highest possible accumulation takes place when transpiration is prevented and when a high exudation-pressure is reached. The absorption of water is dependent in various ways upon the external conditions, just as transpiration is. Thus the presence of saline solutions in the soil must diminish the absorption of water to an extent corresponding with their osmotic equivalency. A regulatory compensation may however be possible within certain limits (Sect. 24), and it has yet to be determined whether the osmotic energy of the root-cells does or docs not increase in a soil which is always poor in water, or from which water is withdrawn only with difficulty.

The dependence of absorption upon the temperature is shown by the fact that tobacco plants in pots begin to flag as soon as the temperature of the moist soil falls to from $2^{\circ} \mathrm{C}$. to $4^{\circ} \mathrm{C}$, and become stiff and turgid again when the temperature is raised and the roots actively absorb water ${ }^{2}$.

${ }^{1}$ Cf. Sect. 34. Burgerstein (Materialien zu ciner Monographie d. Transpiration, i $88_{9}, 2$. Th., p. 5o) gives a summary of the experiments performed by Sachs, Heinrich, \&c., on the influence of the percentage of water in the soil upon absorption.

${ }^{2}$ Sachs, Bot. Zeitung, I860, p. 124. 
Direct experiments by Vesque, Kohl, Eberdt, and Kosaroff ${ }^{1}$ have shown that the absorption of water by roots, and also by cut stems, diminishes as the temperature falls. Nevertheless a perceptible absorption of water may still occur at or even below zero. Thus Kosaroff found that a flaccid plant might absorb sufficient water from soil frozen at -3 to $-4^{\circ} \mathrm{C}$. to become turgid again in air saturated with water vapour, or even to counterpoise feeble transpiration. This is of course possible only in plants whose roots are not injured by such exposure (Chelidonium, Sinapis, Chrysanthemum indicum). At -3 to $-4^{\circ} \mathrm{C}$. the whole of the water present in the soil or in the plant is not frozen, so that from a purely physical point of view it is easy to understand that a root of Salix or Chrysanthemum may absorb water at $-3 \mathrm{C}^{\circ}$. or $-4^{\circ} \mathrm{C}$. from a block of ice into which it is frozen.

A low temperature retards the passage of water through the living root-cells and vascular tissue. This is a necessary physical consequence of the low temperature ${ }^{2}$; indeed in the vascular tissue it appears to be due to physical causes alone, for it has been observed that a transpiring plant of Passiflora cocrulea or of Lonicera sempervirens, whose roots were kept in moist soil at $15^{\circ} \mathrm{C}$. to $20^{\circ} \mathrm{C}$., remained turgid, although the stem was cooled to $0^{\circ} \mathrm{C}$. for a length of $70 \mathrm{~cm} .{ }^{3}$, but became flaccid when the temperature of the stem was lowered to $-1 \cdot 5^{\circ} \mathrm{C}$. to $-3^{\circ} \mathrm{C}$. Dixon and Joly ${ }^{4}$, however, have observed a slight movement of water in tracheae at a temperature of $-5^{\circ} \mathrm{C}$.

The vital activity of the intermediary cortical and epidermal cells appears to be of importance in the transference of water to the tracheal channels. This is indicated by the disproportionate influence exerted by temperatures approaching or falling beneath zero, but more conclusive evidence is afforded by the fact that the presence of a high percentage of carbon dioxide in the gases of the soil or in a culture fluid causes a diminution in the rate of absorption. while if the surrounding oxygen be replaced by a neutral gas a similar but less marked result is produced ${ }^{5}$. The same agencies will cause a slowing or cessation of bleeding, and the changes induced in both cases are somewhat closely related.

Evergreens transpire much less actively in winter, partly because the

1 Vesque, Ann. d. sci. nat., I876, vi. sér., T. IV, p. 89 , and 1878 , vi. sér., T. vi, p. 169 ; Kohl, Transp. d. Pflanzen, ISS6, p. 63; Eberdt, ibid., I8S9, p. 6I; Kosaroff, Einfluss versch. Factoren auf d. Wasseraufnahme, Leipziger Dissertation, 1897 .

${ }^{2}$ According to Dimitrievicz (Haberland's wiss. prakt. Unters., 1875, Bd. 1, p. 75) and Reinke (Hanstein, Bot. Abhandlungen, I 879 , Bd. IV, p. 83 ), the swelling of seeds takes place more slowly at a low temperature. Detmer's experiments upon the rapidity with which wood absorbs water at different temperatures are less conclusive (Beiträge zur Theorie d. Wurzeldrucks, $1 \delta_{77}$, p. ${ }_{3} \delta$ ).

${ }^{3}$ Kosaroff, l.c.

- Dixon and Joly, Anuals of Botany, 1895 , Vol. IX, p. $4^{16}$.

"Kosaroff, 1.c. W. Wolff (Jahresb. d. Agriculturchemie, 1870-2, p. 134) found the passage of a stream of carbonic acid gas through a nutrient solution acted in a similar manner. 
temperature is low, and partly because the stomata are closed. Hence the roots are able to absorb sufficient water to balance the loss by transpiration, as was first noticed by Hales, and confirmed by Duhamel by observations upon a branch of an evergreen oak grafted on a deciduous one ${ }^{1}$. If during winter branches of a plant growing in the open air are led into a greenhouse they develop transpiring leaves, and this affords an additional proof that in spite of the low temperature a large quantity of water may be absorbed by the roots and transported through the stem ${ }^{2}$. Other plants, however, are unable to extract sufficient water from a frozen soil, so that they become flaccid and are ultimately injured when the external conditions are such that the subaërial parts transpire actively. Hence Kihlmann ${ }^{3}$ is probably correct in his interpretation of the meaning of the protective modifications against excessive transpiration which are shown by many northern plants, although they grow in moist soil or swampy habitats. By these means the plant protects itself against fatal or injurious loss of water when the temperature of the soil is low and absorption is difficult.

In the normal progress of development the conductivity of a tracheal element (Sect. 35) gradually diminishes as time goes on, as does the absorptive power of any particular region of a root. Similarly the permeability of a cut surface of a stem gradually diminishes when kept in water, so that after a few days the leaves begin to droop. This fact was well known to Hales ${ }^{4}$; it is due to the blocking of the opened tracheae with mucilage, bacterial zooglocae, \&c., while in addition changes may take place in the conducting tissue-elements near the cut surface, rendering them less efficient for water-transport.

When a stem is cut across, air is drawn into the opened tracheae and tracheides owing to their internal negative pressure, and hence the absorption of water is rendered more difficult. In herbaceous plants the lessened rate at which water is then absorbed is sufficient to cause pronounced flaccidity, even though the cut stem is immediately placed in water. If the section is made under water the latter is drawn into the tracheae instead of air,

1 Hales, Statics, $174^{8}$, p. 29 ; Duhamel, De l'exploitation des bois, 1764 , T. I, p. 337 .

${ }^{2}$ Experiments of this kind were performed by Duhamel (Naturgesch. d. Bäume, I $76_{5}$, Bd. II, p. 219) and Knight (Treviranus, Reiträge zur Pflanzenphysiol., isi1, p. 120) on vines, by Mustel (cf. de Candolle, Physiol. of Plants, T. I, p. 426) upon other woody plants.

${ }^{3}$ Kihlmann, Pflanzenbiol. Studien aus Russisch-Lappland, I \$9o, p. 10.4; Stenström, Flora, I 895 , p. 153. [In a swampy soil the deficiency of oxygen may render absorption more difficult, and Nilsson even concludes that the xerophytic habit may be directly clue to the poverty of the soil (cf. Bot. Centralbl., Bd. Lxxvi, p. 9), for in richer s sil the xerophytic character may disappear. It is also possible that, as in the case of mangrove vegetation, the xerophytic adaptation may aid in avoiding an over-accumulation of injurious saline and other substances present in the soil and conreyed to the leaves by the transpiration current. Cf. also Rosenberg, Utber die Transpiration der Halophyten, Konigl. Vetenskaps-Akad. Förhandlingar, 1897, No. 9, p. 531.]

${ }^{4}$ Hales, Statics, 1748 , p. I 8 . Cf. also Unger, Studien z. Kenntniss d. Saftlaufes in d. Pflanzen, I864, p. 3 (Sep.-abdr. aus Sitzungsb. d. Wien. Akad., Bd. L). 
and no flagging occurs. Similarly, if a fresh cut is made under water 5 to $6 \mathrm{~cm}$. above the one injected with air, the new surface absorbs water at a sufficient rate to replace what is being lost by transpiration. The same result may be produced by forcing water under pressure into the stem. Thus the pressure of a column of mercury $20-40 \mathrm{~cm}$. high generally suffices to make flaccid shoots turgid again (Fig. 27), and also restores to them the power of absorbing water against negative pressure. If the experiment is performed with the apparatus figured on p. 2II (Fig. 26) the mercury will be raised in the arm $a$ and supported there ${ }^{1}$.

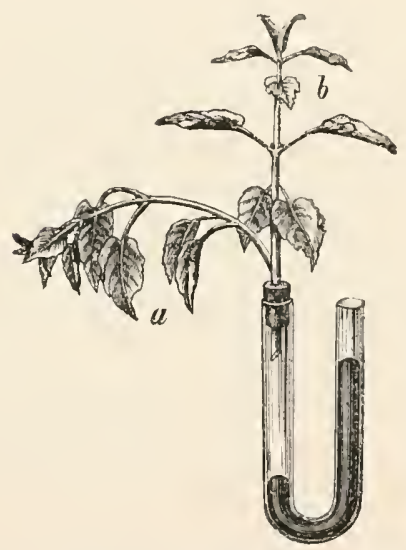

F1G. 27. The flaccid shoot of Impatiens parvi: flora has passed in one hour from the position $(a)$ to that of $(b)$, owing to the pressure of the mercury forcing water into it.

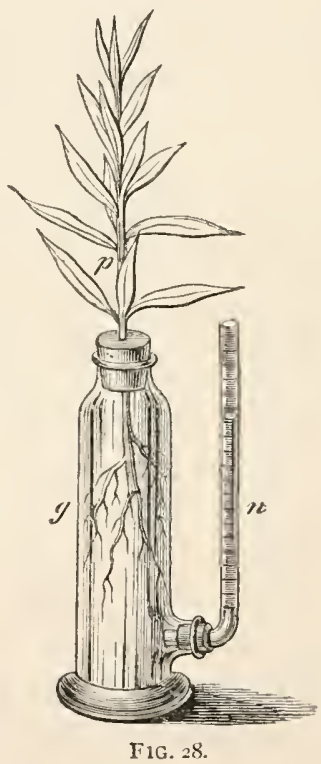

To show the relationship between transpiration and the absorption of zeater the simple apparatus depicted in Fig. 28 may be employed. A cut stem or young rooted plant is fixed so that the root system or lower end of the stem is immersed in the water which fills the air-tight cylinder $g$. The amount of water absorbed is registered by the scale on the tube $n$, and by placing the entire apparatus on a balance the weight lost by transpiration can be determined. A somewhat different arrangement may be used for more exact experiments (cf. Sect. 38 ). By such means it has been shown from the time of Hales onwards that under constant conditions the amounts of water absorbed and exhaled correspond ${ }^{2}$, and that any change of conditions tending to produce an increased rate of transpiration

1 After Sachs (1870) had called attention to this phenomenon, de Vries studied it more closely (Arb. d. Bot. Inst. in Wuirzburg, 1873, Bd. I, p. 287). The explanation given above is due to v. Höhnel (Haberlandt's wiss, prakt. Unters., 1877, Bd. II, p. I 29). See also Strasburger, Bau u. Verricht. d. Leitungsbahnen, i S91, p. 680.

${ }^{2}$ Hales, Statics, 1748 , p. Is. Later, Unger, Sitzungsb. d. Wien. Akad., I861, Bd. XLIV, p. 360 ; 
ultimately induces more rapid absorption, until a new condition of equilibrium is reached which corresponds to the changed conditions, so that the amounts absorbed and exhaled again correspond. The effects of changes of temperature are complicated by the changes of volume which they cause. Thus when the plant is warmed water may be driven out of it by the expansion of the air enclosed in the tracheae. This takes place when fairly moist wood is warmed, so that the specific gravity of the wood is lessened and it may eventually float in warm water, though it sinks when first immersed ${ }^{1}$.

The amount of water which trees contain is liable to variation, just as is the case in herbaceous plants. Th. Hartig, and more especially R. Hartig, have shown that, as might be expected, the wood usually contains more water in summer than in winter, the minimum commonly occurring in autumn, the maximum in spring. The data obtained by Geleznow and Duhamel agree with these results? In the latter case the accumulation of water causes a considerable internal pressure to be developed, while when the leaves expand the amount of water which the stem contains undergoes a marked diminution. In deciduous trees the spring maximum is more marked than in evergreens, as might naturally be expected, since the latter begin to transpire actively immediately the external conditions become favourable. It is hardly surprising that the curves obtained for different plants should not be precisely similar, and that variations may be shown in different years. During a prolonged rainy period the wood will undoubtedly contain more than the normal amount of water, while under special conditions a daily variation may become perceptible.

The energy with which water is absorbed is not necessarily directly dependent upon the amount of water which the plant contains. We need not therefore give a detailed account of the variations in the amount of water observed in the upper and lower portions of the trunks of trees, \&c. (see 'Th. Hartig, 1. c.). These variations occur for the most part in the alburnum, and only to a slight degree in the duramen. The latter normally contains but little water, and the amount alters only slightly even when transpiration is very active (Sect. 35) . Hartig found that at a point ro metres from the ground, the amount of water present in the stem of a beech, varied from $4 \mathrm{I}$ to 85 per cent. (the total area of cell-spaces being 100).

Rauwenhoff, Archives néerlandaises, ı 868, T. III, p. $3^{18}$; J. Boussingault, Agron., Chim. agr., \&c., I 878 , Vol. vi, p. 3 I ; Vesque, Kohl, Eberdt, in the literature quoted on p. 212.

1 This phenomenon was first observed by Dalibard, Duhamel, \&c., and more closely studied by Sachs (Bot. Zeitung, 1860 , p. 253 ; Arb. d. Bot. Inst. in Würzburg, I879, Bd. II, P. 317). A correct explanation was first given by IIofmeister (Flora, I861, p. Ior).

2 Th. Hartig, Bot. Zeitung, I868, p. I 7 ; I 558, p. 329 ; R. Hartig, Lehrb. d. Anat. u. Physiol., I891, ṕ. 202; Unters. aus d. forstbot. Inst. zu Miinchen, I882, Il, p. 20; 1883, III, 1. 47 ; Holz d. Nadelwaldbäume, I 885 , p. 95 ; Geleznow, Mélang. biologiques tirés du Bulletin. d. l'Académie. d. St.-Pétersbourg, I872, T. Ix, p. 667; Ann. d. sci. nat., I 876, vi. sér., T.1II, p. 344; Dubamel, Dc l'exploitation des bois, 1764, p. 476 . 


\section{PAR'T II.}

\section{THE EACRETION OF WATER VAPOUR 1 .}

\section{SECTION 38. The Influence of Specific Peculiarities upon Transpiration.}

All sub-aërial organs give off water-vapour to a greater or less extent, and indeed it could hardly be otherwise in organs which are adapted for rapid gaseous exchange with the surrounding air. It is, however, necessary that, according to the conditions under which the plant exists, transpiration may be so regulated and controlled that turgidity can be maintained, for in plants which are sensitive to drought the percentage of water must never fall below a certain minimum (Sects. $25,27,33$ ).

Many plants are compelled to use the little water they can obtain in the most economical manner possible, and in such cases adaptations to protect them from excessive transpiration are most markedly developed. Indeed, the special shape and structure of typical xerophilous plants have mainly this importance, for in order that they may cope with the conditions under which they exist, the surface-area is reduced as far as possible, although this places the plant at a disadvantage in other ways. Thus the regulatory diminution of transpiration which becomes necessary when the supply of water is limited involves a hindrance to gaseous exchange, and thus prevents the full functional activity of the chlorophyll-apparatus from being exercised (Sects. 29 and 30).

To insure the harmonious co-operation of the whole, mutual concessions of the most varied character are everywhere necessary, so that, for the general good, peculiarities are often developed which may to a certain extent hinder particular functional activities. This is the case with the factor of transpiration with which a terrestrial plant has to reckon, and it makes certain sacrifices in order that a purposeful regulatory control of this function may be possible, although such modification frequently causes the death of plants which have been transported to unsuitable habitats. Transpiration is not a mere useless or even burdensome physical necessity, but on the contrary it is of considerable physiological importance when properly regulated ${ }^{2}$.

Thus the rapid distribution of dissolved substances is in a large measure due to the transpiration current (Sects. 22 and 35), and it is

1 Burgerstein has given a complete summary of the literature (Materialien zu einer Monographie d. Transp. d. Pflanzen, $188_{7}$, Th. i, and $188_{9}$, Th. ii, Sep.-abdr. a. d. Verhandlungen d. Zool.-bot. Ges. in Wien).

2 Cf. Stahl, Bot. Zeitung, 1894 , p. 14r. The importance of transpiration was shown by Bonssingault, Die Landw. in ihrer Beziehung zur Chemie u. Physik, $188_{4}$, Bd. I, p. 20. 
probable that trees and even herbs would be unable to obtain the required ash constituents in sufficient amount from the dilute solutions present in the soil by the slow process of diffusion. Transpiration probably aids gaseous exchange, and may also-serve to prevent plants exposed to the sun from being overheated. It is morcover, not impossible that transpiration and the processes connected with it exert influences upon growth and development which may be of the lighest importance. How far this is true is doubtful as yet, but, on these grounds alone, it is possible that many plants may be quite unable to live and develop when transpiration is prevented.

No general conclusions can be drawn with regard to trees, \&c., from the fact that transpiration is unnecessary in submerged plants, whether amphibious or purely aquatic, for the conditions under which they live are totally different. Decisive experiments upon these questions have not as yet been made, nor have trees been grown under conditions where transpiration is impossible. Even in moist climates this function suffices to very conspicuously aid in the transference of the constituents of the ash to the leaves, \&c.' Schlösing ' found that tobacco plants grown under bell-jars, so that but little transpiration was possible, did not grow as well as others in the open. Though a variety of other factors enter into play here, such experiments suffice to show that transpiration favours the absorption of the constituents of the ash ${ }^{3}$. It is not yet known whether the plant accumulates more than it needs when transpiration is active, and whether the necessary minimum can be absorbed without the aid of transpiration. When the solution of any salt present in the soil is comparatively concentrated, transpiration may actually cause it to accumulate to an injurious extent (Sect. 23).

Since transpiration and the processes connected with it indirectly favour growth and development, there must be a certain optimal rate for each plant, which will vary somewhat according to the moistness of the soil and other external conditions. An excessive evolution of water-vapour causes a diminution of turgidity, and hence a lessened rapidity of growth. This conclusion is supported by comparative observations made upon plants grown in atmospheres of varying humidity, and in soils containing different percentages of water ${ }^{4}$.

1 Haberlandt, Sitzungsb. d. Wien. Akad., I 892, Bd. CI, Abth. i, p. Sog. Cf. also Stahl, 1.c.; Burgerstein, Ber. d. Bot. Ges., I 897 , p. I55; Giltay, Jahrb. f. wiss. Bot., $18_{97}$, Bd. xxx, p. 61 5 ; Wiesner, Anı. d. Jard. bot. d. Buitenzorg, 1897 , T. xiv, p. 277 ; [Haberlandt, Jahrb. f. wiss. Bot., xxxi, I897, p. 273; Giltay, 1.c., I898, xxxı1, p. 453].

${ }^{2}$ Schlösing, Ann. d. sci. nat., I869, v. sér., T. x, p. 366 . Cf. also Kohl, Transpiration, 1886 , p. II 3 , and Ebermayer, Bot. Jahresb., $188_{4}$, p. 8.

${ }^{3}$ [Wollny (Unters. über den Einfluss d. Luftfeuchtigkeit auf Wachsthum (Inaug. Diss.), Halle, 1898 ; cf. Bot. Centralbl., Bd. Lxxvi, p. 249) finds that in moist air the dry weight and total amount of salts become greater than in dry air. These results are probably due to the necessary protection against transpiration in dry air retarding the gaseous exchange, and thus also carbon dioxide assimilation and growth.]

* Tschaplowitz, Bot. Zeitung, I $88_{3}$, p. 353 ; Gain, Ann. d. sci. nat., I $\$_{94}$, v. sér., T. xx, p. I35 ; Fittbogen, Versuchsst., 187o, Bd. XIII, p. I09; Avédissian, Bot. Centralbl., I 896, Bd. Lxviı, p. 379. 
Transpiration is influenced by the same external conditions as the evaporation of water in general. It varies, however, very much in different plants, and in different parts of the same plant under similar external conditions. This is because the formation and escape of water-vapour is largely dependent upon the specific peculiarities of the transpiring organs. In the first place the plant employs relatively impermeable layers, such as cork and cuticle, to restrict to a greater or less extent the evaporation of water; while, on the other hand, the loss of water by evaporation is favoured by the existence of open channels to the exterior, of which the stomata are the most important. Since, however, the stomata close in time of need, the plant is able to regulate its transpiratory activity to a greater or less extent.

These structural arrangements and the adaptative modifications which they may undergo have already been described in connexion with the exchange of gases (Chap. V), where reasons have been given why any check on transpiration also diminishes the rapidity of gaseous exchange. A gaseous particle in order to escape from a cell must pass in dissolved form to the outer surface of the cell-wall, and thence into the gaseous condition if the partial pressure (or the degree of saturation) of the air outside is sufficiently low, and the principle is the same whether the gas or water-vapour is evolved from the free surface of a leaf or from the cells which line its intercellular spaces. The former may be termed cuticular, and the latter diastomatic or intracellular transpiration ${ }^{1}$, since in the second case the water-vapour finds exit through the open stomata and lenticels to the external air. When stomata are present, a certain specific but variable relationship exists between the amounts of water lost by diastomatic and cuticular transpiration. As the cuticle becomes more and more impermeable, diastomatic transpiration increases in importance. On the other hand, it diminishes as the apertures of the stomata narrow, and ceases when they are entirely closed.

How far the transpiration can be reduced to a very low ebb if necessary, depends therefore upon the relative impermeability of the cuticle. In members of the Cactaceae, Crassulaceae, and in certain Orchids, the cuticle is extremely impermeable, so that when there is any deficiency of water; or even when the plants are suspended in dry air, they may remain comparatively fresh for weeks and shrivel but little if at all, whereas when the sap is abundant the stomata open and are available for rapid gaseous exchange. Less xerophilous plants, such as comprise most of our native flora, do not possess so impermeable a cuticular layer, and hence they continue to lose water even when the stomata are closed,

${ }^{1}$ [Either of these terms is preferable to that of 'stomatal transpiration,' since the water-vapour merely escapes through the stomatal pore, and is not erolved from the stoma itself, as the latter term would indicate.] 
and rapidly become flaccid when water is not supplied to them. Nevertheless the properties of the cuticle of the leaves and all sub-aërial parts are such as to oppose a marked hindrance to transpiration, whercas in submerged plants, roots, \&c., where the conditions of life demand the cxistence of a readily permeable epidermis, no such check to transpiration is interposed. Consequently when they are exposed to the air such parts rapidly dry and shrivel. Even in the leaves of our native terrestrial plants a very large proportion, often indeed the greater amount, of the water is lost by diastomatic (intracellular) transpiration. Hence so long as the stomata are open, the leaf surface on which they are most abundant transpires most actively. In the leaves of the oak and the beech, and still more in those of the ivy, the cuticular transpiration is very much less in amount than the diastomatic, and hence the closure of the stomata causes in all these plants a diminution of transpiration which is frequently extremely pronounced. When transpiration is excessive in relation to absorption, the stomata close for the most part before any actual flaccidity has been established. It is only in plants which under normal conditions never suffer from the want of water, that the stomata do not close when subjected to artificial drought.

We may turn our attention first to the leaves, since owing to their large surface-area and the numerous stomata which they contain, they evolve the largest quantities of water-vapour. The young twigs and branches do not usually give off more than $\frac{1}{5}$ to $\frac{1}{30}$ of the total amount of water-vapour transpired ${ }^{1}$, and as layers of cork and bark are formed, their powers of transpiration are still further reduced. Since comparatively little water-vapour escapes through the lenticels, the lack of any regulatory power of opening and closing them is of trifling importance ${ }^{2}$ (Sect. $3 \mathrm{I}$ ).

In xerophilous plants the reduction in the relative amount of transpiring surface is produced in various ways. The leaves may become small or may be absent, or they may assume a thick and fleshy character (Semperivioum, \&c.), while in the absence of leaves, the flattened stems or branches which take their place (Ruscus, \&c.) may be of marked thickness (Cactaceae) ${ }^{3}$. A piece of the stem of Echinocactus would transpire 300 times as much water if it were in the form of thin leaf lamellae. It is the relative amount of water lost which is of importance, and hence it is desirable to measure transpiration in terms of the mass or weight of the entire plant.

${ }^{1}$ Hales, Statics, $174^{8}$, p. $5^{80}$; Guettard, Histoire d. l'Acad. royale, $174^{8}$, p. $5^{80}$; Hartig, Bot. Zeitung, I 863, p. 260; Wiesner u. Pacher, Oesterr. Bot. Zeitschr., 1875, Nr. 5 ; Eder, Sitzungsb. d. Wien. Akad., I $8_{75}$, Bd. LxxiI, Abth. i, p. 267 ; Burgerstein, $188_{9}$, II, p. I 4 ; Kny, Ber. d. Bot. Ges, 1895, p. 374 .

${ }^{2}$ Haberlandt, Beiträge z. Kenntniss d. Lenticellen, i 875 , p. I7 (Sep.-abdr. a. d. Sitzungsb. d. Wien. Akad., Bd. LxxiI, Abth. i.

${ }^{3}$ Cf. Kerncr, Pflanzenleben, I $88_{7}$, Bd. I, p. 302.

* Noll, Flora, i 89.3 . p. 35.5 
The rate or intensity of transpiration is found by estimating the amount of water given off from a unit of transpiring surface in a given time. The transpiratory intensity is sometimes greater in a turgid succulent leaf than it is in an ordinary thin one ${ }^{1}$, but in comparison with their respective bulk or weight, the transpiration is very much greater in the latter case than in the former. Moreover the great reduction of the cuticular transpiration enables xerophilous plants to reduce the loss of water to a minimum when necessary. The intensity of the cuticular transpiration of young roots, \&c. is on the other hand so pronounced that they are undoubtedly frequently able to transpire more actively than thin leaves with numerous stomata.

Any increase in the surface-area due to the formation of outgrowths tends to favour the rate of evaporation, but nevertheless the presence of strongly cuticularized scales or overlapping hairs may exercise a certain check upon transpiration ${ }^{2}$. A covering of dead hairs always lessens transpiration, but this means is only employed to a limited extent by plants. In certain cases leaves are able, by rolling themselves up, to diminish the amount of surface exposed, and thus to check the rate of transpiration when necessary ${ }^{3}$. The denseness of the foliage, the phyllotaxis of the leaves, and their position with regard to the incident rays of light are also of importance. The sun's action may be tempered by the assumption of a vertical position, and the active paraheliotropic movements which certain leaves or leaflets can execute may be of importance from this point of view ${ }^{4}$. As the amount of water present decreases, transpiration becomes less and less active, and ceases when the air-dried parts contain only hygroscopic water.

So long as the plant remains turgid, the physical action of an increasing concentration of the cell-sap does not diminish the rate of transpiration to any marked extent ${ }^{5}$. Any change in the osmotic energy causes a corresponding change in the amount of water imbibed by the cell-wall, and hence affects the rate of evaporation from the latter (Sect. 27), but the possible

1 For examples see Aubert, Ann. d. sci. nat., I 892, vii. sér., T. xvr. p. So; also Burgerstein, l. c., II, P. 24 .

${ }^{2}$ For literature see Burgerstein, 1. c., Ir, P. 457 ; Kerner, Pflanzenleben, I887, Bd. I, p. 289. On chalky and saline incrustations see Sect. 23.

${ }^{3}$ See Burgerstein, Ir, p. 60 ; Kerner, l. c., p. I34; Kihlmann, Pflanzenbiol. Studien, 189o, p. 105. The heating action of sunlight will hardly be decreased to any appreciable extent by the admixture of less diathermanous ethereal oils with the vapour evolved. Literature: Burgerstein, II, p. 457 .

- Burgerstein, 1. c., II, p. 260 . [This is by no meaus always the case. Thus in Albizza safonaria, Calliandra haematocephala, Dalhergia linga, and Cassia montana, from the distribution of the stomata and the character of the paraheliotropic position which the leaflets assume, it follows that the rate of transpiration ean hardly be cliecked, and may even be increased if the stomata remain open. It is only in such plants as Mimosa, Robinia, Acacia, \&c., that transpiration is maskedly checked when the leaflets fold together with their stomatic surfaces apposed in assuming the paraheliotropic position (Ewart, The Effects of Tropical Insolation, Annals of Botany, 1897 . Pp. 455-9).]

${ }^{3}$ Śre Aubert, $A n n$. d. sci. nat., $189^{2}$, vii. sér.: T. xvr, p. 64. 
differences of osmotic energy correspond to comparatively small differences of vapour tension, and hence exercise but little effect upon transpiration ${ }^{1}$. The latter maly, however, be markedly diminished if the leaves become coated by an investing gelatinous covering which dries and forms a more or less impermeable film on their outer surfaces (Sects. 23, 30). The film covering the so-called 'varnished' leaves may act in this manner, as also do the waxy films found on the epidermis of many plants.

During development various adaptative modifications and peculiarities arise by means of which transpiration may be regulated according to the external conditions and the necessities of the plant. Thus, neglecting the effects of the increase in the bulk of the leaf, the development of a cuticle causes a diminution in its transpiratory powers, whereas the concomitant cevelopment and opening of the stomata acts in the opposite manner, until with the autumnal closure all diastomatic transpiration ceases. Hence, under similar external conditions, a transpiratory maximum occurs in each plant at a particular stage of development, and the curve of transpiratory activity may frequently exhibit secondary maxima ${ }^{2}$.

As is well known, the supply of water has a great influence on growth and development, and a moderate deficiency of water causes transpiring plants to develop peculiarities which tend to the more perfect utilization of the supply and also to the limitation of the loss by transpiration. The entire shape, the slow growth, and the small size of those plants which grow in dry habitats, are all produced in response to the special conditions under which they exist. Even the shedding of a number of the leaves, which occasionally occurs during a summer drought, may be of biological importance as a last attempt to avoid irretrievable injury.

The cuticle is, as a general rule, more strongly developed when there is a scarcity of water, or even when the supply is abundant but transpiration active, than when a damp atmosphere or the absence of sunlight reduces the latter to a minimum. The occurrence of wax impregnating the cuticle is much less conspicuous in leaves grown in moist air or in those which are submerged, even in the cases of plants whose leaves are normally covered by a waxy bloom ${ }^{3}$. Changes of this kind are not, however, always due

1 The presence of a solution of tannic acid cannot diminish the rate of transpiration more markcdly than any other saline solution of equivalent osmotic value. For this supposed action of tannic acid, see the literature given by Burgerstein, 1. c., II, p. 63 .

${ }^{2}$ For leaves see v. Höhnel, Wollny's Forschungen auf d. Geb. d. Agriculturphysik, 1878, Bd.

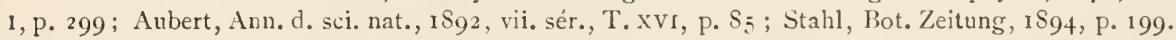
Further literature: Guettard, Histoire d. l'Acad, royale de Paris, 1748 , p. 579 , and 1749 , p. $29^{2}$; Fleischmann, Versuchsst., 1867 , Bd. IX, p. 182 ; Vesque, Ann. d. sci. nat., 1877, vi. sér., T. IV, p. 89 ; N. J. C. Miiller, Bot. Unters., I877, Bd. I, p. 155 ; Bonnier et Mangin, Ann. d. sci. nat., 1884 , ri. sér., T. Xvir, p. 295 (Fungi). For further literature see Burgerstein, 1. c., II, p. 24.

${ }^{3}$ Kohl, Transpiration, 1886 , p. II 3 ; Tittmann, Jahrb. f. wiss. Bot., I\$ 86 , Bd. xxx, p. I16. Cf. also Burgerstein, 1. c., II, pp. 45,61 , and Sect. 21 . 
to tran piration alone, for other influences may frequently come into play, while the peculiaritics observed may be due to the action of a combination of different factors ${ }^{1}$.

The external conditions act as a stimulus which causes the plant to accommodate itself to them as far as its specific powers allow, and in the majority of cases its possible range of accommodation is not sufficient to allow it to develop indifferently either on land or submerged in water, or in both dry and wet habitats. During its development the plant adapts itself as far as possible to the conditions under which it is growing, but nevertheless even when adult a certain amount of accommodation is still possible, though the permanent changes which can be induced are comparatively trifling in extent. Transient and rapid changes, such as the movements of the stomata, \&c., serve to modify the transpiration according to the conditions cxisting at the moment, and thus to exercise a certain regulatory control. The vital activity of the plant is therefore of decisive importance in determining the activity of transpiration ${ }^{2}$, while the warmth which the plant itself produces may also have some influence upon this function. The actual evaporation of water is a purely physical phenomenon, dependent in a plant, as in a dead body, upon the physical properties of the body in question, and upon the external conditions. These properties, however, alter when the plant dies, and the evaporation of water is then usually accelerated, as is indicated by the more rapid drying of dead parts, and as direct experiments have proved ${ }^{3}$. Moreover, it must not be forgotten that the permeability of the cork and cuticle may be altered when a plant is killed, especially if heat is employed for that purpose.

Water evaporates more rapidly from a free surface, or from wet filter-paper, than from a leaf. Unger found from numerous researches that under similar conditions I.4 to 6.9 times more water evaporated from a free surface of water than from a similar area of ordinary green leaves ${ }^{4}$. Plants such as Cacti probably often exhale a hundred times less vapour than would a similar area of water. In ordinary leaves the amount of internal free surface is very great, but the intercellular spaces are always filled with damp air. Diffusion takes place only slowly through the narrow stomatal pores, and hence the most markedly developed

${ }^{1}$ On light and shacle leaves, Stahl, Einfluss d. sonnigen u. schattigen Standorts a. d. Laubblätter, I $88_{3}$, p. I9: Eberdt, Ber. d. Bot. Ges., I 888, p. 37 I ; Stenström, Flora, I 895 , P. I 3 I ; Génean de Lamarlièrc, Rev. gén. d. Bot., I 892 , T. $1 \mathrm{~V}, \mathrm{p} .48 \mathrm{I}$, and the literature here quoted.

${ }^{2}$ [Only in this sense can transpiration be regarded as a vital process. Cf. H. Dixon, Proc. R. Irish Soc., I 898 , Vol. I, pp. 61 $8,627$.

${ }^{3}$ Mohl, Bot. Zeitung, I $8_{47}$, p. 323 ; Nägeli, Sitzungsb. d. Bair. Akad., 1861, Bd. I, p. 262 ; Just. Cohn's Beiträge z. Biol., 1875 , Bd. I, p. 24.

- Cf. Knop, Versuchsst., 1864 , Bd. vi, p. 250 ; Baranetzky, Bot. Zeitung, 18 $7^{2}$, p. 62 , footnotc. On the evaporation of watcr from the soil see Sachsse, Agriculturchenie, isss. p. 203; Alessandri, 13ot. Jahresh., 1SSS. p. 74. 
intercellular system is barely able to raise the evaporating power of a leaf to the same level as that of a free surface of water.

Methods. To demonstrate transpiration, we may employ the cobalt reaction ${ }^{1}$, or any other hygrometric test. Quantitative estimations may be made either by determining the loss of weight, or by observing the amount of water absorbed. The latter is an indirect method, and is only approximately correct at any given moment. Woodward determined the transpiration by weighing the plant from time to time; Mariotte collected the exhaled water vapour as did Guettard, while Hales measured also the amount of water absorbed ${ }^{2}$. These three methods have undergone various minor modifications in the hands of later investigators.

The balance shown in Fig. 29 may be used to measure the weight of water lost by transpiration ${ }^{3}$. When a plant in a pot is used, the pot must be placed in a vessel of lead or glass with a perforated cover, through which the stem may project into the air ${ }^{4}$ When a plant growing in water is employed (Fig. 28, p. 232), it must be fixed in the glass vessel by means of an airtight cork, or the water may be covered with a layer of oil. This latter method was used by Unger, but can hardly be recommended ${ }^{5}$.

If the apparatus by which the amount of water absorbed is measured, be weighed from time to time, it is found that under

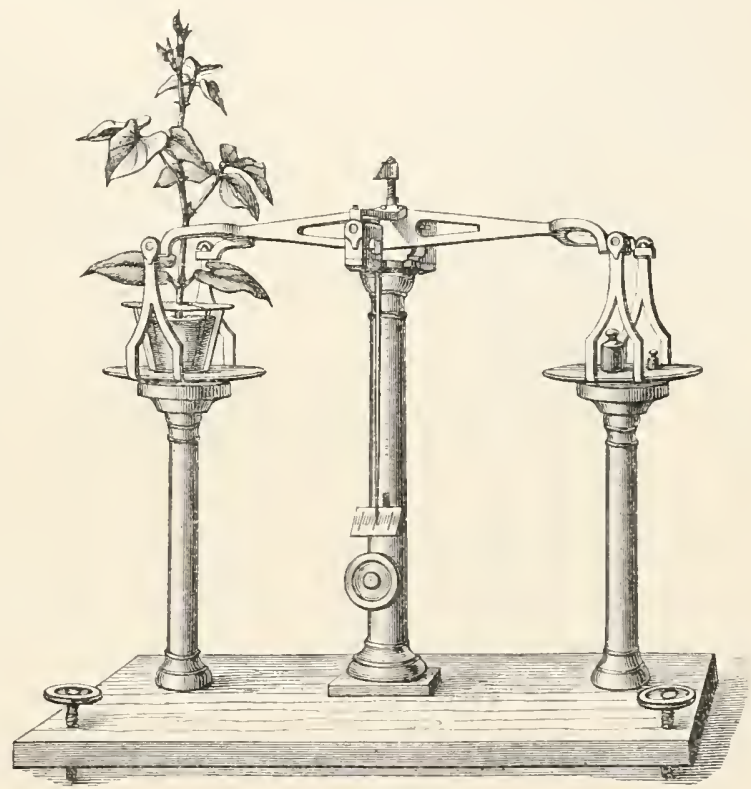

F1G. 20. normal conditions the absorption of water affords a sufficiently accurate indication of the amount transpired. The apparatus shown in Fig. 30 is adapted for more delicate measurements, since the narrow tube $(a)$ renders a slight amount of

1 [Paper impregnated with cobalt chloride is blue when dry but turns rose-pink as it absorbs water, various shades of colouration indicating the relative degree of saturation.]

${ }_{2}$ Unger, Sitzungsb. d. Wien. Akad., I 861, Bd. xlrv, p. 206 ; Sachs, Experimentalphysiol., IS65, p. 231 ; Woodward, Mhil. Trans., I699, Vol. xxı, Nr. 253, p. I98; Mariotte, CEuvres de Mariotte, 1717 , p. 135 ; Guettard, Hist. d. l'Acad. roy. de Paris, $174^{8}$, p. 57 s.

3 Extremely good balances of this kind, weighing up to 5 kilos, may be obtained from Kern u. Sohn in Ebingen, Württemberg.

4 Cf. v. Höhnel, Transp.-grösse forstl. Gewächse, I 879, p. 4 .

5 Unger, Sitzungsb. d. Wien. Akad., IS6I, Bd. XLIT, p. 362. The changes in weight caused by respiration, assimilation, or by the excretion of ethereal oils or other substances, are so trifling as to be negligible in comparison with the loss in weight due to transpiration. The transpired water is found to be nearly pure when collected. Cf. the older literature given by Treviranus, Physiol., Bd. I, P. 493 . 
absorption clearly visible. Since the tube $(a)$ is horizontal, no alteration in the waterpressure is produced as transpiration continues, while by opening a tap or clamp at $b$ fresh water can be allowed to flow into $a$ and fill it when nearly emptied ${ }^{I}$.

When a transpiring plant is covered by a bell-jar, the dew that condenses upon the inner surface of the latter affords direct evidence that the plant is exhaling water-vapour. If a vessel containing anhydrous calcium chloride is placed under the bell-jar, the increase in weight of the deliquescent substance forms a measure of the transpiratory activity of the objects employed, such as single leaves or even different surfaces of the same leaf. The cobalt-paper method first eniployed by Stahl is of great use for such comparative determinations ${ }^{2}$.

For a description of the automatically registering apparatus used by Vesque, Eder, Krutizsky, Marey, and

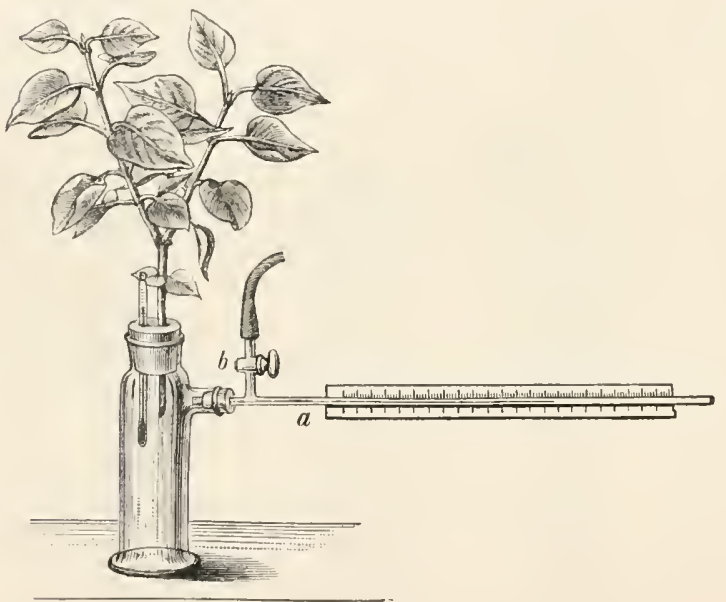

F1G. 30. Anderson, the reader is referred to their original works ${ }^{3}$. It is not difficult to cause a beam- or, better, a springbalance to register automatically any change in weight, while by means of a float used in conjunction with a simple mechanical arrangement the absorption of water in a potometer may be graphically registered 4

'The surface area of a leaf is most easily determined by preparing a piece of paper of corresponding outline, and finding the weight of a unit of area of the latter. The outline of the leaf may be traced upon the paper in pencil, or obtained photographically by using ferrous or albumenized paper. The use of a planimeter is hardly necessary, while the determination of the area by means of a glass plate divided into squares is neither so accurate or convenient as is the method of weighing ${ }^{5}$.

${ }_{1}$ Potometers of this kind were used by Vesque, Ann. d. sci. nat., i 868 , vi. sér., T. vi, p. I 83 ; Moll, Archiv. Néerlandaises, I $88_{4}$, T. Xvin ; Bonnier et Mangin, Ann. d. sci. nat., $188_{4}$, vi. sér., 'T. xVII, p. 288 ; Kohl, Transpiration, 1886, p. 6r; Eberdt, ibid., 1889, p. 8.

${ }^{2}$ Stahl, Bot. Zeitung, I 894 , p. I I 8 . Merget (Compt. 1end., 1878, T. Lxxviri, p. 293) used a similar paper prepared with chlorides of iron and palladium.

3 Vesque, Ann. d. sci. nat., I868, vi. sér., T. vi, p. 186 ; Eder, Unters. iiber d. Aussch. von Wasserdampf, 1875, p. 106 ; Sep.-abdr. aus Sitzungsb. d. Wien. Akad., Bd. Lxxil, Abth. i; Krutizsky, Bot. Zeitung, 1878 , p. 161 ; Marey, Méthode graphique, $18_{7} 8$, p. 276 ; Anderson, Minnesota Bot. Studies, I894, p. I 77 .

1 See Langendorff, Physiol. Graphik, $1 \$_{91}$. Several forms of apparatus for registering growth will be described later.

${ }^{3}$ The former method was used by Haberlandt (Wissensch. prakt. Unters. a. d. Gebiete d. Pflanzenbaues, 1877 , Bd. II, p. 140), the latter by Hales (Statics, 1748, p. 2) and Unger (Sitzungsb. d. Wien. Akad., 186r, Bd. XLII, P. 195). 
The cuticle and stomata. The unequal transpiratory activities of the different surfaces of a leaf may be admirably demonstrated by means of pieces of filter-paper saturated with a $\mathrm{I}$ to 5 per cent. solution of cobalt chloride and then completely dried. If a piece is laid upon each side of the leaf, and the whole is brought betwecn two glass plates, as in Fig. $3 \mathrm{I}$, the paper on the stomatic surface may redden in from a few seconds to a minute or two, while on the upper surface even of thin leaves, if free from stomata (Tropaeolum, Sparmannia, Populus, Pyrus), a reddening appears only after 3 to 60 minutes. When the cuticle is strongly developed (Ficus elastica, Begonia, Hedera, \&c.), it may take as long as from a few hours to two days before the trifling amount of water required to redden the paper is transpired through the cuticle $^{1}$. If the cobalt paper is placed beneath a tiny bell-jar attached hermetically by means of fat to a cuticular leaf-surface free from stomata, no water can reach the paper except by cuticular transpiration. The fact that a positive result is always ultimately produced shows that, as has already been said (Sect. 2 I), the most strongly developed cuticle is not quite, though almost impermeable. It is hardly surprising, however, that no transpiration can be detected through such a cuticle when less delicate methods are employed ${ }^{2}$.

By using leaves, and comparing the amounts of water transpired when the stomata are open and when they are closed, it may also be shown by the cobalt method that diastomatic transpiration is always pronounced in terrestrial plants, and that when the cuticle is very impermeable almost all the transpired water-vapour escapes through the stomata (Sect. 3I). When the

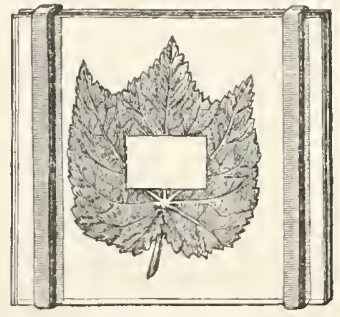

F1G. 3I. stomata are closed, it frequently happens that no perceptible difference is observed between the permeability of the cuticle of the two surfaces.

The high importance of diastomatic transpiration was first proved by Guettard, Bonnet (I 754), Eder (1. c.), and Boussingault, who prevented all transpiration from the surface bearing stomata by smearing it with fat or cement, or by placing the leaves with these surfaces in contact with one another ${ }^{3}$. Garreau's results ${ }^{4}$ correspond with those already given. In his numerous experiments the arrangement shown in Fig. 32 was adopted. Each of the bell-jars cemented to the upper and the under surface of the leaf contains a ressel of calcium chloride, and the respective increase

1 Stahl, Bot. Zeitung, 1894, p. 119; ibid., 1897, p. 99. [Rosenberg (1. c. on p. 231) states that many Halophytes transpire more actively from the upper surfaces of the leaves than from the lower, although the former contain fewer stomata than the latter.]

${ }_{2}$ Thus cf. Eder, Unters. iiber die Aussch. v. Wasserdampf, I875, p. 102 (Sep.-abdr. a. d. Sitzungsb. d. Wien. Akad., Bcl. Lxxis); Garreau, Ann. d. sci. nat, $18_{49}$, iii. sér., T. xiı, p. 336. Further literature by Burgerstein.

${ }^{3}$ Guettard, Hist. d. l'Acad. roy. de Paris, 1748 , p. 579 ; 1749 , p. 292 ; Boussingault, Agron., Chim. agric., \&c., i 878 , T. vi, p. 353. Further literature by Burgerstein, II, p. 18. Cf. also Stahl, Bot. Zeitung, I 894 , p. I 29.

* Garreau, Ann. d. sci. nat., I849, iii. sér., T. xiII, p. 336. Cf. also von Höhnel, Wollny's Forsch. auf d. Geb. d. Agriculturphysik, 1878 , Bd. I, p. 320. A similar method was employed by Unger (Sitzungsb. d. Wien. Akad., I861, Bd. xl.rv, p. 327), and by Risler (Archiv d. sciences phys. et nat. de Genève, I 87 I, T. XLII, p. 236 ). [By employing two leaves, and fixing one of them with its ventral surface upwards, the error due to the different relative positions of the absorbing and transpiring surfaces in the two halves of the apparatus may be partially compensated for.]

R 2 
in weight of the two vessels indicates the amount of water exhaled from the upper and under surfaces of the leaf. The tubes $m$ and $m^{\prime}$ are closed with oil, and serve at the same time as manometers. Of the numerous experiments made by these means, the following examples may be given :-

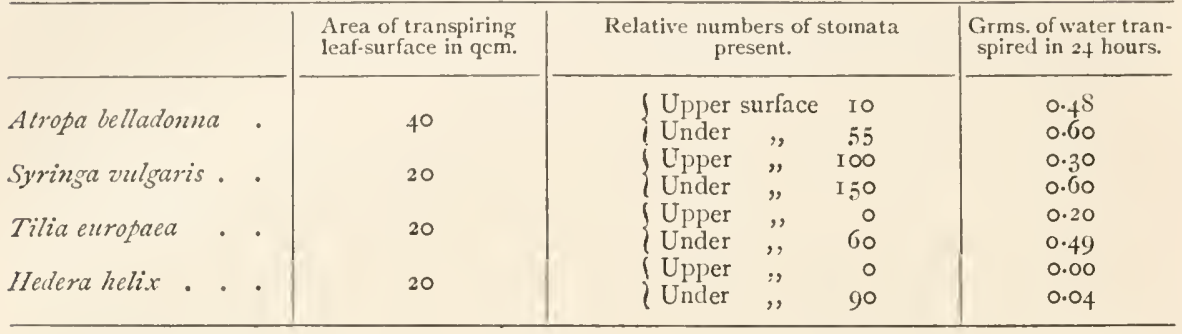

Transpiration becomes much more active if the cuticle is removed, as experiments with apples and cactus stems have shown ${ }^{1}$, but the exposed surface soon

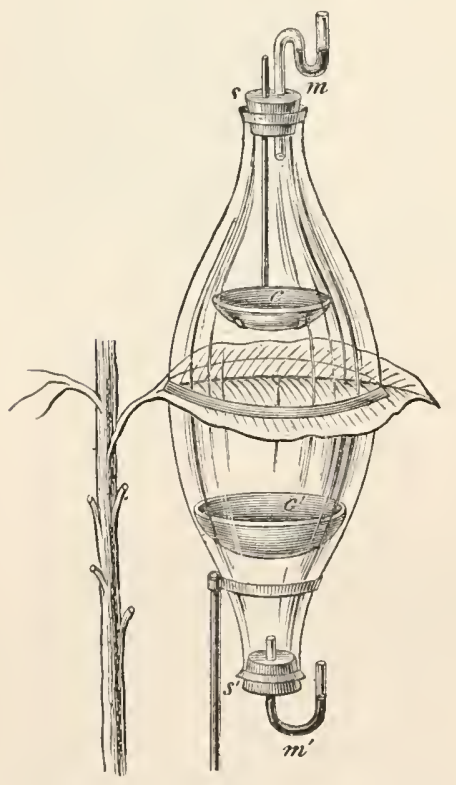

IIIG. 32 . dries, and other changes occur which cause the rate of transpiration to gradually decrease again.

The removal of any waxy covering the leaf may possess also favours transpiration ${ }^{2}$, and at the same time the surface can be more readily wetted with water. IVater-vapour is able to pass through a thin layer of fat, though only very slowly ${ }^{3}$; indeed Boussingault showed long ago that smearing a leaf with fat does not entirely stop transpiration (l. c., p. 357).

Why it is that syringing the leaves with water markedly increases the transpiratory activity in some cases but not in others, must be left unanswered. It is, however, easy to understand why a decrease in the rate of transpiration on one side of the leaf may frequently, though not always, cause the transpiratory activity to increase on the other ${ }^{4}$.

It is not desirable to give an account here of the transpiration of different plants or plant organs, and Burgerstein gives a summary of all that is known on this point. Even in many subterranean organs protective adaptions against transpiration are found, such as the corky covering of rhizomes and tubers, or the cuticular skin of bulbs.

1 Boussingault, Agron., Chim. agric., \&c., 1878, T. vi, p. 349; Nägeli, Sitzungsb. d. Münch. Akad., I861, I, p. 238 ; Just, Cohn's Beiträge, I875, Bd. I, p. II ; Aubert, Ann. d. sci. nat., I892, vir, T. xvi, p. 76. Cf. de Candolle, Physiol., T. I, p. 90.

${ }^{2}$ Garreau, Ann. d. sci. nat., 1849 , iii. sér., T. XIII, p. $3^{22}$; Haberlandt, Wiss. prakt. Unters. a. d. Geb. d. Pflanzenbaues, I8 77 , Bd. II, p. I 56 , and the literature given by Burgerstein, 1. c., II, p. 17 .

${ }^{3}$ Laspeyres, Ann. d. Physik u. Chemie, 1878, N. F., Bd. II, p. $47^{8}$.

4 Unger, Sitzungsb. d. Wien. Akad., I86I, Bd. Xliv, p. 340; Kohl, Transpiration, I886, p. I5. 


\section{SECTION 39. The Influence of External Conditions upon Transpiration.}

The external conditions affect transpiration both directly and indirectly, and hence the effect produced by them is by no means necessarily the same as it would be in the case of evaporation from a free surface of water. A change in the conditions may alter the characters of the transpiring organ; and when the external conditions are such as to favour very rapid evaporation, the stomata may close, and hence the rate of transpiration markedly decrease. Some such arrangement is essential in order that a purposeful regulation of this function may be possible. Attention must be paid not merely to the direct physical action of the external conditions, but also to their indirect influences, for only by these means can a clear comprehension be obtained of the causes which produce any ascertained result. In studying the simplest reaction, all the factors influencing it must be taken into consideration, though this is often far from easy, and therefore frequently neglected. The task is made all the more difficult by the fact that alterations in the external conditions may not only cause transitory variations changing as the conditions alter, but may also produce permanent accommodatory modifications in the plant or its parts.

A general account has been given of the ways and means by which transpiration may be influenced, and of the power which the plant possesses of reacting to changed conditions (Sect. 38 ). It now remains to describe the general effects produced by changes in the external conditions, special attention being paid to the more permanent of these and to the effects produced by the transitory variations they exhibit.

Hunidity of the air. It has been known from the time of Hales that as the moistness of the air increases, transpiration decreases ${ }^{1}$. As the air becomes drier the transpiration does not necessarily increase correspondingly, for as soon as the water present in the plant begins to decrease the transpiration-curve falls. A plant is able to transpire even in a saturated atmosphere, provided that it is warmer than the surrounding air. The respiratory activity always tends to warm the plant slightly, and hence renders a certain amount of transpiration possible in saturated air $^{2}$, so that the'spadix of an aroid, owing to its comparatively high temperature, transpires actively, and causes water to condense on the wall of a bell-jar placed over it ${ }^{3}$. In most cases the heat thus produced

${ }_{1}^{1}$ Experiments by Unger, Sitzungsb. d. Wien. Akad., 1861, Bd. xurv, P. 203. Further literature: Burgerstein, II, p. 45; Eberdt, Transpiration, 1889, p. 34. The influence of the supply of water, and of the moistness of the soil, may be judged from Sects. 37 and 38 .

${ }_{2}$ The literature is given by Burgerstein, II, p. 45, but is for the most part very inconclusive.

${ }^{3}$ Cf. Vol. Ir, The Production of Heat; also G. Kraus, Blïthenwärme bei Arum italicum, $188_{4}, \mathrm{p} .54$. 
is but slight in amount, as is also the transpiration dependent upon it; but nevertheless only a very slightly higher temperature is necessary to enable distinct transpiration to proceed in a saturated atmosphere, while on the other hand a very slightly lower temperature than that of the surrounding saturated air is sufficient to cause water to be condensed upon the plant as upon any other cold body (Sect. 27). Any radiant heat absorbed by the plant will naturally affect the rate of transpiration, and the loss of heat by radiation will have the opposite effect. For these and other reasons the amount of water transpired by the plant in a saturated atmosphere can hardly be used to determine the amount of heat produced by it, as was proposed by Sachs ${ }^{1}$. It has already been shown (Sect. 27) that the vapour tension is but little affected by the condition of turgidity, so that even in a flaccid plant a very slight difference of temperature above that of the surrounding saturated air suffices to cause water to be evaporated.

The temperature of the plant and of the air exercise in every case a direct influence upon transpiration, just as the temperature does upon evaporation in general. In addition, several indirect effects are produced, for the turgidity of the tissues, the movements and absorption of water, the width of the stomata, are all influenced by the temperature. Even at temperatures below zero, plants are still able to transpire perceptibly; Burgerstein detected feeble transpiration in leafy branches of Taxus baccata at $-10.7^{\circ} \mathrm{C}$., while Wiesner and Pacher obtained similar results with leafless branches of the horse-chestnut at a temperature which was always below zero, and occasionally fell as low as $-13^{\circ} \mathrm{C} .{ }^{2}$ The rate of transpiration must be different during, and just after, a change of temperature from what it becomes subsequently, for the air acquires the new temperature more rapidly than the plant, and a rise of temperature will cause the saturated air within the plant to expand, and hence will temporarily accelerate the escape of water-vapour. Moreover, a change in the temperature of the air influences the rate of transpiration much more markedly than it does the absorption of water ${ }^{3}$.

The visible ray's of the spectrum, so far as they are absorbed and converted into thermal vibrations, must influence transpiration according to the heating effect they produce. Many other influences which light exercises are not without effect upon transpiration, for light affects the width of the stomata and the daily changes in the tissue tensions, while the assimilation of carbon-dioxide accelerates gaseous exchange and thus favours transpiration. As the resultant of these factors, it appears that as a general rule exposure to light causes transpiration to increase, while

1 Sachs, Sitzungsb. d. Wien. Akad., 1857 , Bd. xxvi, p. 326.

${ }^{2}$ Burgerstein, Oesterreich. Bot. Zeitung, 1875 , No. 6 ; Wiesner u. Pacher, ibid., 1875 , Nr. 5 ; Kohl, Transpiration, 1886 , p. 75 ; Eberdt, ibid., 1889 , p. $4^{2}$; Burgerstein, 1. c., II, p. $4^{2}$.

3 Vesque, Ann. d. sci. nat., 1878 , vi. sér., T. vi, p. IS9; also Eberdt, 1. c. 
darkness causes it to diminish. The difference is not generally very great, however, so long as the illumination is feeble, but in plants whose stomata close in darkness it may in certain cases become considerable'. The heating effect exercised by direct sunlight increases very greatly the rate of transpiration so long as the plant is turgescent. Light also eventually influences the shape and development of plants to a very pronounced extent. It is to this action, and especially to the different degree of development of the cuticle, that etiolated plants, and those growing in the shade, are able under similar conditions to transpire more actively than others which have been grown from the first in strong light.

The researches of Daubeny and Miquel left little doubt that transpiration is increased by exposure to diffuse light, and this fact has been made certain by Baranetzky, Wiesner, Hellriegel, Kohl, v. Tieghem, Eberdt, \&c. ${ }^{2}$ The increase has not only been observed in green and etiolated plants, but Bonnier and Mangin found it occur also in fungi. It cannot be therefore due merely to the opening of the stomata caused by light ${ }^{3}$.

As examples, a few of Wiesner's ${ }^{4}$ results are given below; in these the amount of transpiration was determined by weighing.

The small maize plants used had their roots attached to soil, while the cut stems of the inflorescences or flower-stalks were immersed in water covered by a layer of oil. From what has already been said, it is not surprising to find that transpiration does not immediately attain a constant rate, and that the variations produced by changes of illumination are not always precisely similar, and do not correspond entirely with the results obtained by other investigators with different plants ${ }^{5}$. In the following table Wiesner gives the amounts of water exhaled per hour from roo sq. $\mathrm{cm}$. of surface.

\begin{tabular}{|c|c|c|c|c|}
\hline & & In darkness. & In diffuse daylight. & In sunlight. \\
\hline Plants of Zea Mays (etiolated). & & $106 \mathrm{mgr}$. & II $2 \mathrm{mgr}$. & $290 \mathrm{mgr}$. \\
\hline Flower of Spartizm jumcezm " & - & $97 \quad$, & I I $4 \quad$, & $7^{85} \quad$, \\
\hline " Malva arborea & . & $\begin{array}{l}04, ", \\
23\end{array}$ & $\begin{array}{l}09 \\
28 \quad, "\end{array}$ & $\begin{aligned} 17+ & , \\
70 & , "\end{aligned}$ \\
\hline
\end{tabular}

The more active transpiration observed in the green maize plants exposed to sunlight is apparently due to the large amount of light they absorb exercising a pronounced heating effect upon them. V. Tieghem ${ }^{6}$ speaks of this increased transpiration as chioro-vaporization, but from this term it must not be supposed

I Stahl, Bot. Zeitung, 1894 , p. I 25 . Cf. Sect. 3 r.

2 See the literature by Burgerstein, I1, p. 34, and Eberdt, Transp. d. Pflanze, I889, p. 4; Daubeny, Phil. Trans., I $\delta_{3} 6$, I, p. I 59 ; Miquel, Ann. d. sci. nat., I $\delta_{39}$, ii. sér., T. XI, p. 43.

3 Bonnier et Mangin, Ann. d. sci. nat., I $88_{4}$, vi. sér., T. xvir, p. 301.

4 Wiesner, Über d. Einfluss d. Lichtes u. d. strahl. Wärme a. d. Transpiration, I876, p. 2 I (Sep.-abdr. aus Sitzungsb. d. Wien. Akad., Bd. Lxxiv, Abth. i). Kohl (1.c., p. I5) finds, in opposition to Wiesner, that the stomata of etiolated maize plants are entirely or partly open.

${ }^{5}$ For literature see Burgerstein, rl, p. 43 I.

- Van Tieghem, Bull. d. 1. Soc. Bot. de France, I 886, p. 88 . On the importance of the red dye in absorbing heat, see Stahl, Ann. d. Jard. bot. d. Buitenzorg, I 896, T. xilr, p. I 48 . Cf. Sect. 88 . 
that the chloroplastids are organs developed especially for the purpose of increasing the transpiratory activity during illumination.

Wiesner (l.c.) found that when green plants were exposed to light of various colours, the most marked increase in the transpiration was caused by the rays which were most freely absorbed, and Comes ${ }^{1}$ has confirmed these results by using coloured flowers. Since a large part of the radiant energy which the chloroplastids absorb is converted into potential chemical energy in the assimilation of carbonic acid, it follows that assimilation must diminish the rate of transpiration to a certain extent. The decrease can, however, be but slight, and hence it is doubtful whether it caused the increased transpiration which Deherain and Jumelle ${ }^{2}$ observed, when, owing to the absence of carbon dioxide, no assimilation was possible. Indeed Kohl, as well as $\mathbf{E}$. and J. Verschaffeldt, obtained the very opposite result under such conditions ${ }^{3}$. It must be borne in mind that the direct or indirect effects due to the absence of carbon dioxide may easily outweigh the slightly increased heating effect produced by the cessation of photosynthetic assimilation.

Air-curyents and mechanical vibrations. It is well known how remarkably the wind accelerates evaporation, and hence transpiration also ${ }^{4}$. This is primarily due to the continual removal of the water-vapour as fast as it is formed, a marked difference of potential being thus maintained. Mechanical movements of the branches, \&c. caused by a strong wind accelerate the diffusion currents of the intercellular air, and hence more especially favour diastomatic transpiration. All mechanical vibrations must act in a similar manner, but in some cases shaking causes the tissue tensions to diminish, and the plants to become flaccid, while the changes of volume thus produced influence the gaseous interchanges in the intercellular spaces. When turgid plants are vigorously shaken, the rate of transpiration is increased to a certain extent, but the action is rather a complex one, and hence it is not surprising that the effects produced differ in detail, and that in some cases the rise in the rate of transpiration is transitory, in others permanent. Shaking does not appear to cause a closure of the stomata (Sect. 3I), but nevertheless it may possibly induce a narrowing of the apertures, while the increased transpiratory activity may call the regulatory mechanism into play.

The variations in the atmospheric pressure are in general too slight to influence the intercellular diffusion of gases to any great extent. The

${ }^{1}$ Comes, Bot. Centralbl., I880, p. I 21. The other literature is given by Burgerstein, II, p. 39, but is frequently inconclusive.

2 Déhérain, Ann. d. scị. nat., I876, vi. sér., T. IV, p. I77; Jumelle, Rev. gén. de bot., I889, T. I, p. 37 ; I 890 , T. II, p. 417 ; I891, T. III, p. 241 ; Kohl, Transp., 1886 , p. 44; E. u. J. Verschaffeldt, Dodonea, I89o, Jahrg. II, p. $3^{2} 4$. See also Burgerstein, II, p. 434.

${ }^{3}$ For literature see Burgerstein, II, p. 46; Eberdt, Transpiration, I889, p. 78 ; Stahl, Bot. Zeitung, 1897 , p. 100.

4 Baranetzky, Bot. Zeitung, 1872, p. 89 ; Kohl, Transpiration, I886, p. 86; Eberdt, ibid., I 889, p. 68 . 
barometric pressure does not affect the evaporation of water to so great an extent as might be thought, at least within the limits at which terrestrial plants grow, and even on lofty mountains the influence which the low pressure exerts upon transpiration is not very marked ${ }^{1}$.

Dissolied substances. Concentrated solutions diminish the rate of transpiration, just as a dry soil does (Sect. 37), and even normal nutrient solutions exercise a slight retarding action. According to Burgerstein a slight effect is produced even when the percentage of salts present is less than O.I per cent., but the effect in this case is probably not a direct physical one, but an indirect one, causing an alteration in the plant's transpiratory powers. Plants appear to react to many substances in this manner, as is shown by various researches, especially those of Burgerstein ${ }^{2}$, who found that the presence of slight amounts of certain substances might cause a moderate rise or fall in the transpiratory activity.

The effects which small traces of certain substances may exercise upon transpiration will presumably be still produced when they are absorbed from the soil. Nevertheless, for reasons that are easy to see, the results produced by the addition of various substances to the soil are not always the same, and are not sufficiently definite to enable any clear general laws to be established. Hence it is only necessary to mention that, as Sachs ${ }^{3}$ has shown, and as Burgerstein (1.c.) has since established more in detail, transpiration is decreased by the addition of small quantities of tartaric, oxalic, nitric, or carbonic acid to the soil, whereas it is increased by alkalies such as potash, soda, or ammonia. This result may be produced without any injury to the plant, although the 0.15 to 0.3 per cent. solutions which Burgerstein used must often have been deleterious. The details are given in Burgerstein's papers, and from these it appears that the effect which a salt may exercise upon transpiration when added to a nutrient solution in which a plant is growing, may be different to that produced when a similar quantity of the same salt is offered to another plant cultivated in distilled water.

\section{SECTION 40. Transpiration under Normal Conditions.}

In a condition of nature the transpiratory activity of a plant undergoes marked alterations as the external conditions change, and, morcover, the transpiratory power alters as development continues, for the relative amount of transpiring surface may be increased, while the nature and power of

1 The incorrect opinions of certain authors are referred to by Burgerstein, II, p. 49.

${ }^{2}$ Burgerstein, Unters. iiber die Beziehung d. Nährstoffe zur Transpiration, I. Reihe, ${ }_{1} \delta_{7} 6$ (Sitzungsb. d. Wien. Akad., Bd. Lxxır, Abth. i); II. Reihe, I 878 (ibid., Bd. Lxxvir, Abth. i); and Materialien zu einer Monog. d. Transp., ISS8, it, p. $5^{\text {I. }}$

${ }^{3}$ Sachs, Versuchsst., i $8_{59}$, Bd. I, p. 203 . For additional literature see Burgerstein, I 879 , p. 5 I. 
reaction of the transpiring organs may be modified. In correspondence with the climatic conditions, a yearly periodicity can generally be recognized in the transpiration even of evergreens, the maximum naturally occurring during summer. Even in the coldest winter transpiration does not entirely cease, though it becomes comparatively trifing in amount. According to Guettard ${ }^{1}$, a cypress-tree lost more water in six summer days than during the whole of the winter. The amount of water transpired by a plant during successive days is subject to considerable variation; for many reasons also much more water is transpired during a bright day than during the night, while a heavy fall of dew may cause transpiration to be reduced to a minimum ${ }^{2}$.

Even although the external conditions were kept perfectly constant, the progress of development would still cause marked changes in the transpiratory powers of the plant. Under constant conditions, however, transpiration varies but little in the course of a day, or during shorter intervals. Apparently, therefore, the process exhibits no marked periodicity independently of the external conditions; indeed various authors have been unable to detect any independent periodicity at all, although Eberdt has observed the occurrence of slight variations in the rate of transpiration under constant conditions ${ }^{3}$. As a matter of fact, even when the external conditions are perfectly constant, movements may occur, as well as changes of the tissue tensions and of root-pressure, \&c., and all of these may exert a more or less marked influence upon transpiration. It is possible that in certain plants variations in the width of the stomata may be produced as the result of certain of the various automatic or induced changes continually occurring in plants.

The amount of water which many turgid plants can transpire under favourable conditions is very great. On bright summer days very commonly J to to c.c. of water may be given off per 24 hours from a single square centimetre of leaf-surface. Some plants, on the other hand, under similar conditions exhale only $\frac{1}{10}$ th of this amount ${ }^{4}$. When a very large surfacearea is exposed, the total amount of transpiration may be very great: Hales found that a sunflower having a total area of leaf-surface of about

1 Guettard, Hist. d. l'Acad. roy., 1749, p. 291. On the transpiration of conifers in winter, see Hartig, Bot. Zeitung, 1861, p. 20.

${ }^{2}$ Observed by Hales and also Unger, Sitzungsb. d. Wien. Akad., I86I, Bd. xLIv, p. 214 ; F. Haberlandt, Wiss. prakt. Unters., 1877 , Bd. 11 , p. I51; J. Boussinga ult, Agron., Chim. agric., \&c., I 878 , T. vi, p. 299 , \&c.

${ }^{3}$ Baranetzky, Bot. Zeitung, $187^{2}$, p. 107 ; Barthélemy, Compt. rend., I 873 , T. LxxviI, p. 108 I ; Eder, Sitzungsb. d. Wien. Akad, I 875 , Bd. Lxxir, Abth. i, p. 374 ; Eberdt, Transpiration, 1889, p. 93. Cf. also Burgerstein, II, p. 53.

4 Fr. Iaberlandt, Wiss. prakt. Unters. a. d. Geb. d. Pflanzenbaues, I $S_{77}$, Bd. II, p. I46 ; G. Haberlandt, Sitzungsb. d. Wien. Akad., I892, Bd. Cr, Abth. i, p. 807 ; v. Höhnel, Transp. d. forstl. Gewächse, 1879 (Sep.-abdr. a. Mitth. a. d. forstl. Versuchswesen Oesterreichs), and Forsch. a. d. Geb. d. Agriculturphysik, I88 I, Iv, p. $43^{8}$; Aubert, Ann. d. sci. nat., I892, vii. sér., T. xvi, p. So ; Burgerstein, Material. zu einer Monog. d. Transpiration, I889, II, p. 55, \&cc. 
9 sq. m. ( $10 \frac{2}{3}$ sq. yds.) lost 0.85 kilogrammes ( 1 pint) of water during a single dry day. During a very hot day large trees may lose more than 400 kilogrammes, but during a rainy day at times not more than a few kilogrammes. V. Höhnel has calculated that a birch-tree with about 200,000 leaves transpired 300 to 400 kilogrammes of water during a single hot day, while for a iI5-year-old beech he estimated the average amount of water transpired daily between June I and Sept. I to be 75 kilogrammes. The amount of water given off from 400 to 600 similar trees occupying an area of one hectare ( $2 \frac{1}{2}$ acres) from June I to Dec. $I$ is, according to v. Höhnel, from $2 \cdot 4$ to 3.5 million kilogrammes.

In making these calculations, v. Höhnel attempted to allow for the fact that the conditions are not equally favourable for the different parts of the plant, and that a tree surrounded by others will naturally transpire less actively than when by itself in the open. The estimations made by F. Haberlandt are apparently somewhat too high, because these factors were not sufficiently taken into consideration. Haberlandt found that oat-plants covering one hectare transpired $2,277,760 \mathrm{kgs}$. during a single vegetative period, while barley plants covering a similar area exhaled I,236,7 10 kgs. of water. He also calculated that the amount of water transpired by a single plant during its development was, in the case of maize I $4 \mathrm{kgs}$., hemp $27 \mathrm{kgs}$., sunflower $27 \mathrm{kgs}$, the periods of development being 173 days, 140 days, and 140 days respectively. These amounts of water correspond to a rainfall of $227 \cdot 8$ to $123 \cdot 7$ millimetres.

The annual amount of rainfall, which in Germany is about $600 \mathrm{~mm}$. or $23 \frac{1}{2}$ inches, and in England is about 28 inches, is thus seen to be greater than the amount of water which can be lost by transpiration, especially since during winter the latter is reduced to a minimum. Some such relationship must naturally exist when the whole of a country is taken into consideration, although the direct evaporation from the soil is by no means inconsiderable. In the neighbourhood of rivers, or where the ground-water is abundant, more water may be transpired over a small area with very luxuriant vegetation than the annual rainfall furnishes. These are all matters which are of the utmost importance in the economy of nature, but they come more within the scope of a textbook of agriculture, and hence no comparison need be made here between the amounts of water-vapour given off by soil when bare and when covered with vegetation ${ }^{1}$.

1 See for example Sachsse, Agriculturchemie, 1888 , p. 427; Wollny, Forsch. a. d. Geb. d. Agriculturphysik, I881, Bd. Iv, p. 85 ; Alessandri, Jahresb. d. Bot., 1888, 1, p. 74 . 


\section{PART III.}

THE EXCRETION OF WATER IN THE FORM OF FLUID.

\section{SECTION +I. General View.}

The power of excreting water in the form of fluid is widely distributed throughout the vegetable kingdom, and it may either take place from the intact plant or may occur only after injury, as in the bleeding of cut stems. In the former case the water escapes through stomata or other openings, but both phenomena are closely related to one another, for it is owing to the activity of living cells that water is forced into the vessels, intercellular spaces, \&c., from which it escapes in the fluid condition at the point where least resistance is offered to its exit. It may happen that the watersecreting cells lie on the surface of the plant and are directly exposed, in which case special conducting channels and excretory pores are no longer necessary and may be absent. Frequently again in leaves and flowers special groups of epidermal cells, or single epidermal hairs, may have the power of excreting watery solutions. The same power is also possessed by the hyphac of various unicellular and nulticellular fungi.

The process is in all cases due to active exudation from living cells, just as is the case in the glands of animals, and it is not always produced in the same manner. The excretion of water, except in nectaries, is probably for the most part not dependent upon the presence of dissolved substances outside the cells, but is due to internal causes originated by the vital activity of the living protoplasts, the process being one of active excretion, active exudation, or filtration under pressure. If the escape of water is however due to the presence of osmotic substances outside the cell, then we may speak of an osmotic withdrawal of water or of plasmolytic excretion. The latter occurs in nectaries, for there the sugar present outside the cells acts in the same way as a piece of sugar placed upon a freshly cut surface of a beet-root or potato. The nectaries continue therefore to excrete water even when the plant is flaccid, a fact of considerable biological importance, whereas bleeding and the excretion from water-pores take place only when the plant is fully turgid, for unless this is the case no active exudation is possible.

The actual excretion of water does not give any indication of the causes which induce it. It is, however, certain that a dissolved substance exerts the same equivalent osmotic action under all circumstances, so that any one-sided accumulation of such substances will cause a flow of water in a definite direction, provided that the cellular and other arrangements interpose no insurmountable obstacle. No doubt the excretion may be 
partly plasmolytic, and partly active, and it is in many cases still uncertain which of the two processes is at work, or when both are active, which is of the greater importance. Nevertheless, as a general rule, bleeding, as well as the excretion of water from water-pores, \&c., is an active secretory process, whereas the excretion of water in nectaries is due to plasmolytic action. In both cases the osmotic powers of living cells are involved, for in producing the plasmolytic excretion of water the living plant creates the necessary conditions, i. e. it produces and excretes the osmotic substances which induce an outward flow of water.

The exudation of water is simply a special example of the varied processes of excretion and absorption without which living cells could not continue to exist (cf. Sect. 23). Wherever particular parts are endowed with a special secretory activity, we may term them glands, whether we are dealing with a single cell (hairs, \&c.), or with organs of complex structure. A distinction can be made between digestive glands, sugar-glands or nectaries, and water-glands, the 'Emissaria' of Moll and the 'Hydathodes' of Haberlandt being simply water-glands ${ }^{1}$. Nectaries may also be regarded as water-glands, if we pay attention only to the fact that they can excrete water, though the actual withdrawal of water is due to the plasmolytic action of the sugar which the cells of the nectary excrete. Water-glands may be further classified in a variety of ways, either according to their shape or morphological nature, or according to the position of the secreting cells or of the exit channels. Burgerstein uses the special term 'Guttation' when the water escapes through water-pores ${ }^{2}$.

The phenomenon of bleeding may be dealt with first, for it has been the most deeply studied. An explanation of the causes which induce bleeding will give the key to a comprehension of the active secretion from uninjured organs.

\section{SECTION 42. The Bleeding of Injured Plants.}

By bleeding or weeping is meant the exudation of water under pressure from injured regions, such as may take place, for example, when stems, roots, branches, or even leaves are cut across, or when a hole is bored into the trunk of a tree. The phenomenon of bleeding has been known from very ancient times in the cases of the vine and maple. When holes are bored into the stem of the latter in spring-time, large quantities of sap gradually flow out; if the stems or branches of the vine are cut, water exudes from them and

1 Moll, Unters. uiber Tropfenausscheidung u. Injection, ISSo (Sep.-abdr. a. Meded. d. Konig. Akad. d. Wetenschappen 2, Bd. xv); Haberlandt, Sitzungsb. d. Wien. Akad., 1894, Bd. cur, Abth. i, p. 494. All organs capable of excreting water can also absorb it when a deficiency exists in the plant, just as the root may be caused to excrete water instead of absorbing it (cf. Sect. 34).

2 Burgerstein, Material. zu einer Monog. d. Transpiration, I889, I, p. 5 . 
drips away. The water is pressed out with a certain force, as may be shown by fixing a manometer tube to the decapitated stem, or to a hole bored into a tree trunk, and noticing the height of the column of water or mercury which can be supported by it (Fig. 33, p. 2.56). The pressure of exudation ${ }^{1}$ is often considerable.

Bleeding may be shown by many plants provided they are sufficiently saturated with water. If the accumulation of the latter is due to the absence of transpiration, water may commence to flow immediately the stem is cut across. This is the reason why a vine-stem bleeds when injured in spring before the buds have opened. When transpiration is active, however, the amount of water present in the stem diminishes (Sect. 34), so that in summer the stump of a branch may absorb water at first for a time, and often in large amount before any exudation begins. The latter never takes place so long as transpiration is active, but only when the absorbed water is allowed to accumulate.

The fact that bleeding is possible in summer when transpiration is prevented was first recognized by Hofmeister, while the phenomenon of bleeding was first observed by Ray, and studied more in detail by Hales. The latter observers used trees only, but Hofmeister showed that bleeding was possible from herbaceous plants as well ${ }^{2}$. These authors and others who followed them considered the active exudation of water to be due to the pressure caused by the absorptive activity of the roots, and positive results were indeed first obtained with root-stocks and with single roots or even root apices ${ }^{3}$. Many aërial stems and other organs, however, possess a similar power of bleeding, as was first shown by Pitra, and confirmed later by C. Kraus and Wieler ${ }^{4}$. Whenever water is driven into the vessels or intercellular spaces by the active living cells of the stem or even of leaves, a pressure sufficient to cause a more or less marked exudation of water will be produced ${ }^{5}$.

If leafy branches of Pinus sylvestris, Qucrous robur, Prunus cerasus, \&c.,

1 [The term 'root-pressure' is obviously incorrect as applied to this general phenomenon, while 'bleeding-prcssure' suggests an incorrect analogy with the arterial pressure of animals. 'Sappressure,' on the other hand, is extremely vague, whereas 'exudation-pressure' or 'pressure of exudation ' seems to meet all rcquirements, and may therefore replace 'root-pressure.' The latter term may, howcrer, still be retained to indicate the osmotic pressure of absorption which may be generated in the root when immersed in water or in a dilute nutrient solution. Root-pressure is therefore a physical, not a vital phenomenon.]

${ }^{2}$ Hofmeister, Flora, ${ }_{1} \delta_{5} S$, p. I ; Ber. d. Sächs. Ges. d. Wiss. zu Leipzig, I $8_{57}$, Bd. IX, p. I 49 ; Kay, Hist. plantar., 1686 , Vol. I, p. 8 ; Hales, Statics, 1748 .

${ }^{3}$ Researches of this kind were made by Dutrochet, Mémoires, Bruxelles, I837, p. 201 ; by Dassen, F. Froriep's Neue Notizen, I $8_{4} 6$, N. F., Bd. Xxxix, p. I33; and also by Hofmeister, C. Kraus, \& c. [Cf. H. S. Chamberlain, Bull. d. l'université de Genève, T. II, I897, P. I.]

${ }^{4}$ Pitra, Jahrb. f. wiss. Bot., I $S_{77}$, Bd. Xr, p. 437 ; C. Kraus, Flora, I882, p. 2 ; I883, p. 2 ; Forsch. a. d. Geb. d.Agricultu physik, 1887 , Bd. X, p. 67 ; Wieler, Cohn's Beiträge, I 893 , Bd. vi, p. I. this fact.

5 Briicke (Ann. d. Physil u. Chemie, $18_{44}$, Bd. LXir, pp. 203, 206) first called attention to 
are immersed in water so that only the cut end projects, the latter begins after a time to bleed. The aid of the leaves is, however, unnecessary, for branches denuded of them may also exude water if the corky layers are removed, or if the wood is partially exposed so that water can be absorbed, while at the same time its escape through the cut ends of the immersed twigs is prevented by a covering of varnish, or by means of india-rubber caps. In this case the active cells which absorb water and force it into the vessels evidently form part of the living stem. Such cells are also present in leaves, as the positive results of a few experiments show. Hence, in certain cases, the leaves may aid in producing the exudationpressure exhibited when experiments are carried out in this manner. A similar action has also been observed in the stems of herbaceous plants, in the peduncles of flowers, or inflorescences, in rhizomes, bulbs, \&c. A visible example is afforded when pieces of young grass stems are buried in wet sand, for drops of water soon exude from the projecting upper end of the grass haulm.

The roots undoubtedly excite the most active exudation of water, but that is simply because they are"the best absorptive organs the plant possesses. Nevertheless, the root-stock does not always bleed more actively than do portions of the stem, and, indeed, in several plants an active exudation of water has been observed only from isolated pieces of the stem. No decisive conclusion can, however, be drawn from experiments of this kind as to what may be the precise part played by any given organ in the intact plant. It is, however, at once evident that the different parts of a plant co-operate to very unequal extents in producing the phenomenon in question, while the parts played by the individual cells of an organ differ very widely from one another.

The exudation of water cannot always be produced in the same manner, or by the same means. The power of bleeding differs frequently at different periods of development, and in certain cases is induced only by the action of special external conditions (Sect. 44). The contradictory opinions often expressed concerning the bleeding power of a particular plant are due to this fact, as well as to individual peculiarities, while for the most part the observations relate only to the roots, or stumps of the stem ${ }^{1}$. Most of the higher plants are apparently capable of an active exudation of water either always or only at certain times. Nevertheless, the same genus may contain bleeding as well as non-bleeding species, and hence too much importance must not be attached to the fact that most of the Coniferae experimented with can bleed but little or not at all. It is, moreover, not surprising that mosses also can bleed, for here the same cell activities come into play as are

\footnotetext{
1 For details see Wieler, 1. c.: P. I3.
} 
exhibited in the excretion of water by fungal hyphae. Since Wieler (1.c., p. 2$)^{1}$ gives a list of plants which exhibit bleeding, it is sufficient to mention that in addition to the vine and maple, the following plants are suitable for the study of this phenomenon: Dahlia variabilis, Impaticns sultani, Ricinus communis, Calla acthiopica, Zea mays, Nicotiana tabacum.

The power of generating an exudation-pressure cannot be an essential factor in the transference of water, for it is absent from many herbs and trees, and is never shown so long as transpiration is active (Sect. 33). It is, however, possible that the living cells of the root may have a tendency to transfer water towards the vessels in a 'vitalistic' manner, but at present no definite conclusion can be made (Sect. $3^{6}$ ). The accumulation of sap brought about by the action of the forces which tend to produce an exudationpressure is certainly of use to the plant, especially in spring-time. Thus the retarded unfolding of the buds can be hastened by forcing

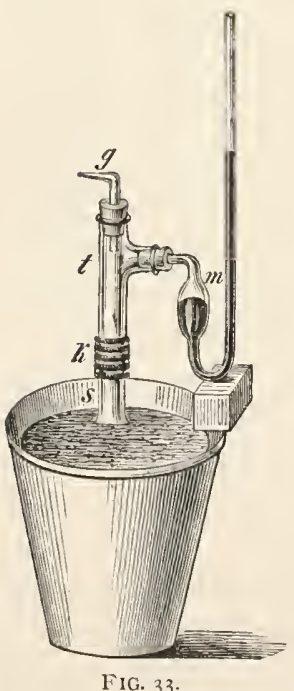

FIG. 33 . water into cut branches ${ }^{2}$. This can only take place to a definite extent, for beyond certain limits the forcible injection of water retards growth (cf. Sect. 47).

The apparatus given in Fig. 33 may be used to measure the pressure of exudation. The stump of the stem of a plant grown in earth or water is fixed by a firm india-rubber connexion to a glass tubc, $t$, to which a mercury manorneter, $m$, is attached by an india-rubber tube. The tube, $t$, is filled with water and closed by an india-rubber cork through which another tube $g$ passes. The capillary end of $g$ is closed by heating, so that no air remains in the apparatus. By pushing $g$ downwards the mercury may be caused more rapidly to reach the final height which the exudation-pressure can support. Instead of a tube at $g$ a glass tap may be used, as in Fig. 34, and the amount of fluid excreted can be measured directly if the escape of the displaced air is possible through the cork $a$. By means of an air-tight india-rubber cork a manometer or drainage-tube may be fixed in a hole bored into a tree trunk. Schwendener ${ }^{3}$ uscd plugs with lateral holes at their apices.

An automatic registration is possible by allowing the changes of position of a burette float to be marked on a rotating drum as the escaping sap raises the float upwards (Baranetzky; cf. supra, Sect. $3^{8}$ ). The sap may also be collected in tubes arranged as in Fig. 35, so that at stated intervals the cylinder moves and a new vessel comes under the drainage-tube. The rotation may be caused by an

${ }^{1}$ [Sce also Molisch, Ann. d. Jard. bot. de Buitenzorg, $2^{\text {̀̀me }}$ supplément, IS9S, p. 23 ; Figdor, Sitzungsl). d. Wien. Akad., May 20, ISy8.]

${ }^{2}$ Böhm, Sitzungsb. d. Wien. Akad., ISø3, Bd. Xlvil, Abth. i, p. I2; Sachs, Experimentalphysiol., 1865 , p. 242; Strasburger, Bau u. Verricht. d. Leitungsbahnen, $18_{91} 1$, p. $S_{43}$. [Exhibited especially well by the flower buds of Magnolia.]

${ }^{3}$ Sichwendener, Sitzungsb. d. Berl. Akad., 1S86, p. $5^{5} 3$. 
electro-magnet together with a clock-work arrangement which produces electrical contact at intervals. A photographic registration is also possible, and may be advantageous, as also the use of a Brudon's spring manometer to register either the positive exudation-pressure or the negative sucking force (Vines) '.

\section{Section 43. Amount of Water Exuded and the Pressure of Exudation.}

If saturated with water a plant begins to bleed the moment it is decapitated; otherwise the cxudation of water commences after a longer or shorter interval, and gradually rises to a maximum 2 . After one to two days the flow from a stump connected with the roots begins to decrease, and in many plants ceases after four to seven days. Others bleed for a longer

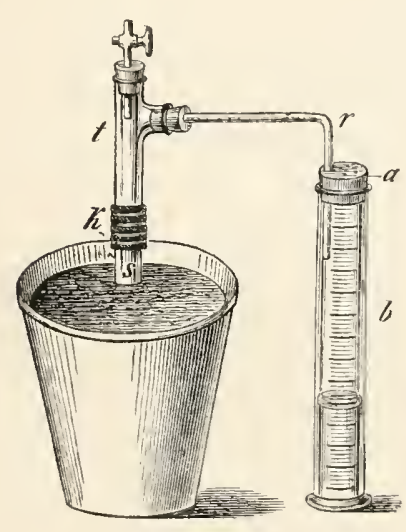

FIG. 34 .

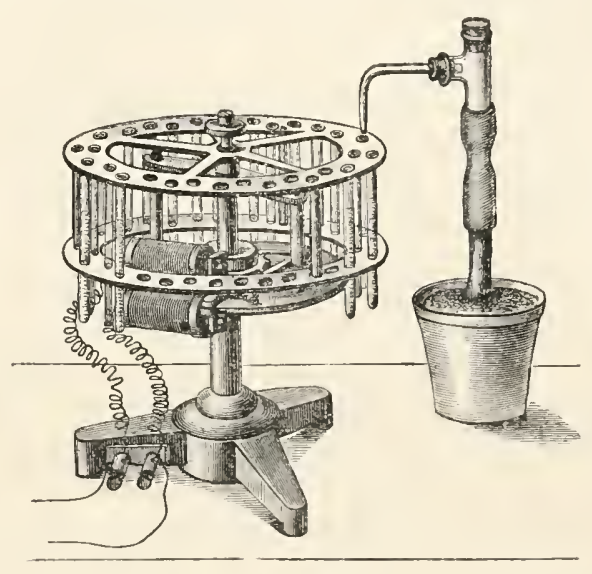

FIG. 35.

time, however; according to Hartig a woody plant may continue to exude water for a month, and the decapitated flowering axis of Agave for as long as five months (Humboldt) ${ }^{3}$. Independently of the fact that the roots ultimately become sickly, the decrease of the flow is due to the vessels in the neighbourhood of the wound becoming gradually blocked (Sect. $3^{6}$ ), and hence by exposing a fresh surface the escape of water may be caused to recommence, as was first observed by Duhamel ${ }^{4}$.

Cut shoots only begin to exude water after they have been immersed

1 Baranetzky, Unters. ïber d. Periodicität d. Blutens, I 873 , pp. 19, 23 (Sep.-abdr. a. d. Abhandlungen d. Naturf.-Ges. zu Halle, Bd. xuI ). The apparatus given in Fig. 35 is prepared by the instrument-maker Albrecht, in Tiibingen. On photographic registration, see Langendorff, Physiol. Graphik, 1891 , p. 90 ; Vines, Annals of Botany, 1896 , Vol. x, p. 291. [The negative pressures observed by Vines were due to physical imbibition on dead branches combined with evaporation.]

${ }^{2}$ Baranetzky, Unters. ïber d. Periodicität d. Blutens, Abhandlungen d. Naturf.-Ges. zu Halle, I 873 , Bd. XIII, p. 30 ; Brosig, Die Lehre v. d. Wurzelkraft, 1876, p. 24.

${ }_{3}$ Quoted by Meyen, Pflanzenphysiol., i 838 , Bd. II, p. 85 ; Th. Hartig, Bot. Zeitung, I 862 , p. 89.

4 Duhamel, Naturgesch. d. Bäume, I; 64 , Bd. I, p. 89 . 
in water for some time, and often only after one or two days (Pitra, Wieler). This is probably due to the fecble absorptive powers of the leaves and branches, for when young branches are used, the bleeding generally commences sooner, and it may eren appear almost immediately if they are injected with water (Wieler). It is quite possible that in other cases also the conditions necessary to cause bleeding develop gradually, for in many inactive root-stumps an exudation of water may be induced by special treatment. Bleeding is, however, a vital phenomenon, and is not produced by an cvolution of gas due to internal decomposition, as Böhm ${ }^{1}$ supposed, although an internal production of gas might easily cause drops of water to be forced out.

The amount of water exuded varies very much, and it is easy to understand why cut branches often bleed but little. When the process is active, the amount of fluid that escapes soon surpasses the total volume of the absorbing root-system in many cases. Hofmeister found that a root-stock of Urtica wrens excreted 1I,260 cubic millimetres of fluid in $2 \frac{1}{2}$ days, whercas the volume of the entire root-system was only 1,450 cubic millimetres.

Instances of active bleeding. Wieler ${ }^{2}$ collected from a hole bored in a twelveyear-old birch 36 of sap litres in 7 days, i. e. about 5 litres per day, while Clark obtained from other species 6.8 kilogrammes in I day. Canstein gives the daily amount escaping from a vine as from to to 950 cubic centimetres or more, and according to Humboldt the greatest flow in one day from a decapitated flowering axis of Agave was 7.5 litres ( $=375$ cubic inches), while during the whole time of bleeding ( 4 to 5 months), 995 litres ( $=50,000$ cubic inches) of sap escaped. From a cut stem of Musanga, $0.7 \mathrm{I}$ litres of sap were forced out per hour during the night-time, according to Lecomte.

The sap exudes from the xylem, and especially from the tracheae and tracheides ${ }^{3}$. Bleeding thercfore corresponds closely with filtration under pressure, and in both cases, according to circumstances, the escaping fluid may or may not be mingled with air-bubbles (cf. Sect. 36). These are, however, usually absent, especially when the tracheal elements are completely filled with water, although air-bubbles may appear at first if the opened tracheal elements contain chains of air-bubbles and watercolumns ${ }^{4}$.

1 Jöhm, Bot. Zeitung, 188o, p. 34 .

2 Wieler, Cohn's Beiträge, I\$93, Bd. VI, p. II 4. See also Hofmeister, Detmer, \&c. ; Meyen, Pflanzenphysiol., 1838 , Bd. II, p. 85 ; Lecomte, Compt. rend., 1894 , T. cxix, p. I8r. [Molisch (Ann. d. Jard. bot. d. Buitenzorg, II. suppl., I 898, p. 23) collected nearly 8 litres in eleven hours from a single bore-hole in the stem of a liana (Conocephalus).]

${ }^{3}$ Schwendener, Sitzungsb. d. Berl. Akad., 1886, p. 575; I892, p. 531 ; Strasburger, Leitungsbahnen, 1891 , p. 859 ; also Brücke, Ann. d. Physik u. Chemie, 1844, Bd. Lxin, p. I8r, \&c.

4 Literature : Unger, Sitzungsb. d. Wien. Akad., 1857, Bd. xxv, p. 442; Hofmeister, Flora, I 862, pp. 102, II 3; Baranetzky, 1. c., 1873, p. 29; Strasburger, 1. c., p. $8_{47}$. No attention need be 
It is apparently owing to their position and structural peculiarities that the active living cells can force water into the tracheae. Were they also able to force water into the intercellular spaces, the latter would become filled with fluid, as is the case when water is forced in by pressure, or when internal water-glands are actively secreting (Sect. 48). The pressures actually produced in the active cells are usually insufficient to inject the intercellular spaces with water to any marked extent, if at all; but it has yet to be determined whether the intercellular spaces still remain filled with air when a high exudation-pressure is attained. It is still uncertain what part is played by the secretory activity of waterglands and by other arrangements in preventing the injection of the intercellular system with water (cf. Sects. 29 and 47). Water-glands afford examples of localized secretory activity by which water may be forced under pressure through inactive parenchyma cells, and hence it is not surprising that occasionally the wood-parenchyma or pith may bleed when a stem is cut across ${ }^{1}$.

The exudation-pressure, as registered by a manometer, corresponds in many plants, including several trees, only to a water-column of a few centimetres in height, but in a very few cases may reach 1 to 2 atmospheres.

Wieler (1.c., p. 122) gives a summary of the observations made by Hales, Hofmeister, Horvath, Clark, and himself. The pressures vary very much. In Morus albus $=\mathrm{I} \cdot 2 \mathrm{~cm}$. of mercury; in Fraxinus excelsior, $2 \cdot \mathrm{I} \mathrm{cm}$.; Ricinus communis, $33.4 \mathrm{~cm}$.; Uriica dioica, $46 \mathrm{~cm}$. Hales noticed a pressure of $107 \mathrm{~cm}$. in Vitis vinifera, and a similar pressure is shown by Cucurbita pepo. Clark gives $192 \mathrm{~cm}$. for Betula lenta, and Wieler observed the pressure rise above $139 \mathrm{~cm}$. in Betula alba.

According to Pitra, the exudation-pressure of cut branches immersed in water may be greater or smaller than that shown by the stump of the stem connected with the roots. The highest pressures observed by him on cut leafy branches were: Prunus cerasus, I I.6 cm.; Pinus sylvestris, II.4 cm.; Betula, $7.5 \mathrm{~cm}$. of mercury. Pitra also made comparative experiments upon the bleeding pressure of the rootsystem and of the sub-aërial organs of the same individual.

The pressure registered by a manometer does not afford an exact measure of the energy manifested by the individual active cells, although it gives approximately the exudation-pressure of the plant as a whole, for to produce any manometric pressure the water pumped onwards by the active cells must filter through inactive cells and tissues, and thus overcome a certain internal resistance. If the mercury column is artificially raised,

paid here to the possibility of a formation of bubbles of gas, owing to processes of decomposition being induced.

${ }^{1}$ Cf. C. Kraus, Forsch. a. d. Geb. d. Agriculturphysik, ISS8, Bd. X, pp. I2, 24. 
it soon sinks again, showing that a backward filtration has taken place against this internal resistance.

The absence of any exudation of water from the intact surfaces of stems and leaves is due to the fact that their cuticular and corky investments, as well as their structure, make them permeable with much greater difficulty than the roots, and the permeability of the latter prevents the pressure being raised to an extent sufficient to drive water through the subaërial parts, if these possess no water-glands. Without doubt, however, the different parts of the root are not equally active or equally permeable, and hence a local circulation of water may take place in an intact plant, passing cither from one branch of the root to another, or even transferring water from one part of the stem to another independently of the transpiration-current ${ }^{1}$. No conclusive experiments have as yet been made to determine whether movements of water produced in this way do actually occur in plants which are not transpiring, and if so, to what extent.

A similar circulation of water may possibly be exhibited by water-plants, but the experiments which Unger supposed to afford satisfactory proof have been shown by Wieler to be inconclusive, while Sauvageau and Strasburger have not supported their conclusions by experiment ${ }^{2}$.

As a general rule a lower exudation-pressure is exhibited in manometers placed near the summit of a tree, but the pressure does not decrease at all in proportion to the height. Frequently, indeed, manometers inserted at the same level, or close to another, register widely different pressures. This was shown first by Hales, and has since been corroborated by Brïcke and others ${ }^{3}$. The explanation is that in the different parts of the root and stem, water may be forced into the vessels by the neighbouring living cells, and that a certain amount of time is required to adjust differences of pressure thus produced, so that the water-pressure in a plant is not necessarily distributed as it would be in a glass tube. Hence, it is not surprising to find that when one of the lower branches of a vine is cut away the manometric pressure registered by an upper one sinks to a relatively small extent, and comparatively slowly. Similarly, when an erect vine stem is placed in a horizontal position, a manometer attached to it is affected very much less than one would be if it were

${ }^{1}$ Cf. Pfeffer, Studien z. Energetik, I892, p. 264.

2 Unger, Sitzungsb. d. Wien. Akad., IS6I, Bd. XuIv, p. 367 ; Wieler, 1. c., p. I 2 ; Hochrentiner,

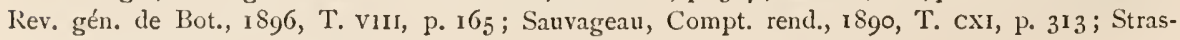
burger, Leitungsbahnen, 1891, p. 930.

s Ifales, Statics, I748, p. 67 ; Brücke, Ann. d. Physik u. Chemie, 1844, Bd. LxIII, p. I95; Hofmeister, Flora, 1858 , p. 3 , and 1862 , p. 117 ; Horvath, 1. c., p. 44 ; N. J. C. Müller, Bot. Unters., 1877 , Bd. I, pp. 47, 269; Th. Hartig, Bot. Zeitung, 1863, p. 281, and in Luft-, Boden- u. Pflanzenkunde, 1877, 1). 268 ; Schwendener, Sitzungsh. d. Berl. Akad., I 856, P. 583. 
connected with a glass tube filled with water whose position was similarly changed ${ }^{1}$. Under certain conditions an injury may cause a marked fall in the pressure registered by a manometer, as Horvath observed on cutting off a latcral root of Helianthus annuus ${ }^{2}$.

The amount of fluid that escapes is dependent upon the relation between the resistance offered by the channels through which water is driven, and the forces which push it onwards. It is therefore the resultant of two varying factors, and hence a high exudation-pressure does not necessarily produce a copious flow. The highest manometric pressure is reached when the driving force is just counterbalanced, and a condition of equilibrium attained. The exudation-pressure is only dependent within certain limits upon the rapidity with which water is driven inwards and onwards through the plant, for as the pressure increases, water is forced into the vessels less and less rapidly, and hence, as Hales first showed, the higher the column to be supported the more slowly the mercury rises in the manometer. As the pressure upon the cut surface increases, the amount of fluid escaping must therefore necessarily decrease ${ }^{3}$, provided that the plant calls no compensating regulatory mechanism into play. The apparatus given in Fig. 33 may be used for an experiment of this kind, by making the open arm of the manometer of the required height and collecting the mercury as it flows over. If the manometer bulb $(m)$ is sufficiently large, it takes some time before the water exuded from the stem begins to interfere with the action of the instrument. Detmer has shown that the rate of flow increases when the atmospheric pressure diminishes ${ }^{4}$.

Since pieces of the stem are capable of originating active exudation, it is impossible to predict how the escape of sap will be influenced by the length of stem left attached to the roots. Detmer found in Ricimus no difference in the amount of sap excreted from plants decapitated near the base and others decapitated higher up, but Baranetzky, in his experiments with Ricimus and Helianthus ammus ${ }^{5}$, observed that the flow was more conspicuous from the plant with the longer piece of stem. No conclusions can be drawn from the unequal amounts of fluid that have been observed to escape through manometers placed at different levels ${ }^{6}$,

1 See Hofmeister, Flora, 1862 , p. 117 .

${ }^{2}$ Similar observations are given by Th. Hartig, Bot. Zeitung, 186.3, p. 281 .

3 As Sachs found (Lehrb., 1874 , 4. Aufl., p. 658). [According to Chamberlain (Bull. d. Lab. d. Genève, 1897 , T. II, p. 330), the plants respond by accelerating the flow. Chamberlain has not, however, kept the direct action and the physiological reactions clear and distinct from one another.]

${ }^{4}$ Detmer, in Schenk u. Lïrssen, Mitth. a. d. Gesammtgeb. d. Bot., I 874 , pp. 439, 453; Wieler, Cohn's Beiträge, IS93, Bd. vi, p. I 7

${ }^{5}$ Detmer, Beiträge z. Theorie d. Wurzeldruckes, 18 ; 7 , 1) 28 ; Baranetzky, Abh. d. Naturf.Ges. z. Halle, I 873 , Bd. xiri, p. 52.

${ }^{6}$ Cf. Unger, Sitzungsb. d. Wien. Akad., $1 s_{57}, \mathrm{Bd} . \mathrm{xxv}, \mathrm{p} .447$. 
for it is impossible to tell how far the results recorded have been influenced by the exudation-pressure, by the characters of the boring, by transpiration, \&c. It has, however, been definitely established that in spring the power of exuding water travels slowly up the stem, so that sap escapes from a hole made at the base sooner than from one made at a higher level ${ }^{1}$. When transpiration becomes active, the escape of water usually ccases first in the upper borings.

Quality of the escaping sap. In many plants the sap almost resembles springwater, but in other cases trifling or even large quantities of organic substances are dissolved in it. The sap of the maple and the birch contains, in addition to small quantities of organic acids and proteids, so much sugar as to repay extraction, while the sap may be fermented to form an alcoholic beverage. In the sap of Acer platanoides Schröder found I.I 5 to 3.4 per cent. of cane sugar, which is not far short of the amount present in the sap of Acer saccharinum, estimated by Clark at 3.57 per cent. $^{2}$ On the other hand, tobacco and potato plants, sunflowers, vines, and indeed most plants, yield a very dilute and watery sap. Since these plants in some cases generate a higher bleeding-pressure than the maple does, there appears to be no direct relation between the osmotic concentration of the sap and the cxudation-pressure ${ }^{3}$. Any accumulation of organic substances in the waterchannels will render possible a rapid transference of reserve food-material to the transpiring organs (Sect. 106).

The composition of the sap is not constant, and may change as bleeding continues, owing to the large amounts of dissolved material which may be lost by the plant, even although a regulatory formation of fresh sugar may be excited; still, when bleeding is active, the sap gradually becomes poorer and poorer in the latter constituent ${ }^{4}$, although the percentage of sugar often undergoes irregular variations of indeterminate origin. Even in the watery sap of the sunflower, Ulbricht was able to detect a still further dilution after prolonged bleeding ${ }^{5}$, and in other experiments also a fall in the specific gravity of the sap has been noted. A change may also occur after a time in the acid or alkaline reaction of the escaping sap ${ }^{6}$.

Observations upon the sap are recorded by Knight, Beiträge z. Physiol. v. Treviranus, I8II, p. I62; Biot, Compt. rend., I841, T. XII, p. 357 ; Unger, Sitzungsb. d. Wien. Akad., ז 857 , Bd. xxı, p. 445 ; Peckolt, Jahresb. d. Chemie, I862, p. 89; Berger, Jahresb. d. Agriculturch., I 867, p. I09; Neubauer, Jahresb. d. Botanik, I 874, p. 854 ; Rotondi u. Ghizzoni, Jahresb. d. Botanik, 1879, II, p. 366 ;

1 Such olservations have been made by Knight (Treviranus, Beiträge z. Pflanzenphysiol., 18 I I, p. 257); Briicke(1. c., p. 83); Schröder (Versuchsst., 1871, Bd. xıv, p. I20); Horvath (Beiträge, \&c., I 877, p. 55).

${ }^{2}$ Schröder, Versuchsst., 1871 , Bd. xiv, p. 118 (also for Betula); also Jahrb. f. wiss. Bot., 1869-70, Bd. vir, p. 261 ; Clark, Flora, I875, p. 509.

${ }^{3}$ See Wieler, Cohn's Beiträge, 1893 , Bd. vi, p. ${ }_{5} 8$.

- See also A. Fischer, Jahrb. f. wiss. Bot., I890, Bd. xxi1, p. 156.

5 Ulbricht, Versuchsst., 1865 , Bd. vi, p. 468 ; 1866, Bd. viI, p. 185.

- C. Kraus, Ber. d. Bot. Ges., I 884 , p. 115 ; Forsch. a. d. Geb. d. Agriculturphysik, I888, Bd. x, p. 6, \&c. 
Ravizza, ibid., I 888 , I, p. 68; Hornberger, Bot. Centralbl., i 888, Bd. xxxiI, p. 227 ; A. Fischer, Jahrb. f. wiss. Bot., 1890, Bd. xxil, p. 73; Wieler, Cohn's Beiträge, I $\delta 93$, Bd. vi, p. 158 .

\section{SECTION 44. The Influence of External Conditions.}

Like all vital phenomena, bleeding is markedly dependent upon external conditions, and thus both the flow of sap and the exudationpressure diminish as transpiration becomes more active. A dry soil exerts a similar sucking action, and may indeed ultimately induce a backward flow of the ascending sap ${ }^{1}$.

Saline solutions, again, by withdrawing water from the plant, may cause bleeding to diminish or cease ${ }^{2}$. Wieler observed that in a variety of plants the escape of water completely ceased when I per cent. of potassium nitrate or 2 per cent. of glycerine was added to the nutrient solution in which they were grown, but that after a time the exudation of water recommenced. As the salt is slowly absorbed, the plasmolytic action of the concentrated solution is neutralized, while any temporary injurious action of the sudden change will soon disappear as the plant gradually accommodates itself to the altered conditions. In the process of accommodation transitory variations may occur, the origin of which is quite uncertain. When an injured plant which has been subject to drought for some time is watered, it may at first bleed very actively before it assumes the rate of flow corresponding to the new conditions. Similarly, by suddenly increasing the height of the column of mercury which the bleeding-pressure supports, the latter is caused to fall at first below the new level, which corresponds to the changed conditions ${ }^{3}$.

Temperature. When the temperature is too low, bleeding ceases, even although an absorption of water may still be possible (Sect. 37). Many European plants bleed perceptibly even at $0^{\circ} \mathrm{C}$., but in Ricinus, according to Wieler, an exudation of water is only possible above $I^{\circ}$ to $3^{\circ} \mathrm{C}$, and in Cucurbita melo not below $7^{\circ}$ to $9^{\circ} \mathrm{C}$, according to Detmer. The flow rapidly increases as the temperature rises, but it is uncertain whether the rise continues until the temperature becomes dangerously high, or whether it falls again after reaching a certain optimum. It is, moreover, doubtful whether the same relation exists between the pressure and the amount of flow at all temperatures.

${ }^{1}$ Experiments of this kind by Hales, Hofmeister, Flora, $1 \delta_{5}^{8}$, p. 6 ; Detmer, Beiträge, $18_{77}$, p. 34; Baranetzky, 1. c., p. 31 ; Brosig, Die Lehre von d. Wurzelkraft, Breslau, 1876, p. 25.

2 Wieler, Cohn's Beiträge z. Biol., I $\delta_{93}$, Bd. vi, p. 52 ; Brosig, l. c., p. 25 ; Detmer, Mitth. a. d. Gesammtgeb. d. Bot. v. Schenk u. Lürssen, 1874, Bd. I, p. $45^{2}$.

${ }^{3}$ Cf. Wieler, l.e., p. 49. Gain (Rev. gén., I 895 , T. I1I, p. 80) did not observe any such temporary rise; Wieler, 1. c., p. 126. 
In what has been said so far only the activity shown after a given temperature has persisted for a time has been considered, for a change of temperature produces a direct physical effect upon the rate of flow, owing to the altered volume of the enclosed gases (Sect. 37). A sudden rise or fall of temperature may thus temporarily affect the height of the manometer, owing to the acceleration or retardation of the flow of water which is involved. Bleeding at constant temperatures cannot be due to any such transitory actions as these, as Matteuci and Sarrabat erroneously concluded. Similarly, Hofmeister incorrectly supposed that a continuous exudation of water can be maintained by the pressures and changes of volume due to alterations in the tissue tensions ${ }^{1}$.

Light and Gravity'. Light apparently exercises some influence upon the escape of water, as is indicated by the daily periodicity of the latter; but gravity appears to be without effect, for Wieler found that the rate of flow from a plant was the same in an inverted as in a normal position.

Oxygen and Chloroform. In the absence of oxygen the exudation of water ceases, although the cells may remain turgid. This is the case at any rate in the grass seedlings examined by Wieler, for the escape of water ceased as soon as the air was replaced by hydrogen. That the exudation of water is a vital phenomenon is shown by the fact that it ceases when the plant is anaesthetized by chloroform, as Wieler proved by placing the roots of scedlings, or of older plants from water cultures, in a dilute solution of chloroform in water. The experiments are not always conclusive, since if the chloroform is too strong the plant is readily injured, or may be killed ${ }^{2}$.

\section{SECTION 45. The Periodicity of Bleeding.}

The power of bleeding probably varies at different stages of ontogenetic development, and like other vital phenomena may be subject to periodic alterations. The older observations rendered the existence of a yearly periodicity probable, especially in trees, but this was first definitely ascertaincd by Wicler ${ }^{3}$, who by using plants growing in pots was able to obtain constant external conditions throughout the year. According to these observations, most hibernating trees lose all power of exuding

I Sarrabat, quoted by Dutrochet, Mémoires, I $\$_{37}$, p. 199; Matteuci, cf. Hofmeister, Flora, 1S62, pp. IоI, I 7. G. Kraus and Detmer made similar conclusions (cf. Wieler, l.c., p. 152).

2 Wieler, 1. c., pp. 65,69 .

` Wieler, Cohn's Beiträge, I $\$ 93$, Bd. vi, p. 72 , and Tharander, Forstl. Jahrb., I $\$ 93$, Bd. XlıII, 1. I 56 . The older literature (Ray, Hofmeister, Hartig, \&c.) is given by Wieler. 
water for a certain period during winter. Under normal conditions $V$ itis vinifera was usually unable to bleed in January, and Acer platanoides in November, whereas plants of Betula alba for the most part did not bleed in November and December, Ampelopsis quinquefolia not from November to April, Populus canadensis not from August to May. The maximal power of bleeding occurs in spring, and presumably the exudation of water is most active when the pressure causing it is greatest, although it is by no means easy to construct a curve from the relative amounts of sap which escape from a plant at different times of the year.

This periodicity is without doubt simply a direct expression of the internal changes which are repeated every year in hibernating trees, and since we are dealing with a single function only, it does not follow that a stoppage of growth must necessarily cause a disappearance of the power of exuding water. The plant continues to respire throughout the entire year, though not always at the same rate, and in the same way it can respond to an injury, although the amount of response possible is not always the same. It is possible that plants may exist which are always capable of bleeding, and indeed some of the above-mentioned plants can exude water when their winter sleep is deepest. According to Wieler, the highest exudation-pressure does not always exist at the time when the buds are expanding and new roots are being formed ${ }^{1}$. The fact that the highest pressure is exhibited in spring (Sect. 42) shows that the phenomenon is of considerable importance in the plant's economy, especially at this period of the year.

When a plant is transferred to a country where the seasons are different, or to the tropics, where the seasonal differences may be but slight, the periodicity under discussion will probably ultimately become modified, and in the plants of a moist tropical climate we may safely conclude that the power of exuding water will either be entirely absent or present all the year round. When an exudation of water is excited in winter, it does not necessarily follow that the winter rest will be broken and that the buds will commence to expand; Wieler was able indeed to induce bleeding during the resting period without causing any recommencement of growth. The external agencies by which an exudation of water may be excited do not produce the same result in all plants and under all circumstances, as indeed we might expect, since each plant has its own specific power of reacting to a particular stimulus. A peculiar combination of conditions occurring in nature may undoubtedly sometimes enable plants to bleed at an unusual time.

Wicler awakened a power of bleeding in various woody plants during the resting period by decapitating them and subsequently repeatedly 
warming the pots in which they were growing for twelve hours to $37^{\circ} \mathrm{C}$. to $39^{\circ} \mathrm{C}$. When once induced, the exudation of water may persist under the original conditions. Some plants were induced to bleed by keeping the roots in saline solutions for some time, and then replacing them in water or in a normal nutricnt solution. Morcover, Scheit and Wieler were also able to cause an escape of water from certain plants by creating a vacuum over the cut stem.

Under normal conditions of growth, and especially when transpiration is active, the relationships are usually such that no positive exudation-pressure can be produced. This is commonly the case in trees in summer time, whereas in herbs the necessary accumulation of water frequently takes place, especially during the night. As the lcaves unfold on a tree, and transpiration becomes increasingly active, the bleeding-pressure gradually disappears, and as the process of foliation continues, the daily variations indicated by a manometer attached to the tree become more and more marked ${ }^{1}$.

Both the pressure and the rate of flow show more or less marked variations during short intervals of time, and these may occur in decapitated stems or roots, even when the external conditions are kept perfectly constant and no transpiration is permitted. These are apparently partly due to the daily periodicity, and partly to oscillations of short duration ('automatic variations'), such as usually accompany any vital manifestation, and are especially well shown in the processes of growth and of movement. A certain genetic relationship probably exists between all such variations, for they are simply the cxternal signs of a changed internal activity. The connexion is, however, obscure as yet, and since our knowledge of the causes which induce a periodicity in the power of bleeding is insufficient to render any satisfactory explanation possible, it will be better to discuss the general problems of periodicity in plant life in connexion with the phenomena of growth and movement. It is not even known in a single case whether the pressure and the amount of flow vary correspondingly, and although it is probable that the rate of flow increases as the pressure rises, frequently no precise relationship appears to exist betwcen the two ${ }^{2}$.

The daily periodicity was first recognized by Hofmeister, and was more closely studied by Baranetzky, Detmer, Brosig, and Wieler ${ }^{3}$. According to these authors

1 Cf. Hales, Statics, 1748 , pp. 68 , 73, \&c. ; Brücke, Ann. d. Physik u. Chemie, 1844 , Bd. LxiII, p. 193; Hofmeister, Flora, 1858 , p. 6; Th. Hartig, Bot. Zeitung, 1861, p. 17. Brücke found that during prolonged experiments on a vine the daily variations decreased, probably because the wood became less readily permeablc, and hence allowed differences of pressure to be manifested only slowly and with difficulty.

2 Wieler, l.c., p. 146; Hofmeister, Flora, 1862, p. 114. Pitra (1.c.) was unable to detect any daily variation in cut twigs.

${ }^{3}$ Hofmeister, Flora, 1862 , p. 106 ; Baranetzky, Unters. über d. Period. d. Blutens, in Abhandlungen d. Naturf.-Ges. zu Halle, 1873 , Bd. xir 1, p. 3 ; Detmer, Beiträge z. Theorie d. W'urzeldruckes, 
a decided daily periodicity cannot be detected in all cases, and it is even doubtful whether the maximum for a given plant always occurs at the same time. Baranetzky did indeed find that the maximum flow occurred at a specific time-in some cases in the morning, in others in the afternoon-and that, like the growth-periodicity, each specific bleeding-periodicity was comparatively constant. Nevertheless, many exceptions to this are known, and Wieler has even found that the bleeding-curves for two different individuals of Almus glutinosa may exhibit entirely different periodicities (1. c., p. I 36$)$.

It is possible that the periodicity in bleeding is induced by the periodicity in certain of the vital processes to which the exudation of water is due, such as, for example, the power of reacting to an injury; hence the bleeding-periodicity may vary as the causes alter which induce it. It is at the same time possible that the daily periodicity of bleeding, like the daily periodicity of growth and movement, may be induced by the periodic alternation of night and day influencing internal . pressure and the turgidity of the tissues. When dealing with the phenomena of growth and movement, a more detailed account will be given of the manner in which the rhythmic repetition of particular external conditions may cause a pronounced periodicity to be induced, owing to the continual summation of the new stimuli with the after-effects of the previous ones. A pendulum may be caused to swing to and fro with considerable amplitude by regularly applying a very feeble force at any given point of its course. When the force is no longer applied, the pendulum continues to swing for a time, the oscillations become less and less marked, but the same interval still intervenes between each, i. e. an induced periodicity may gradually die away again, but its time limit remains constant.

According to Baranetzky the daily periodicity in the exudation of water is actually induced by the alternation of night and day, and hence it is not at first shown by young plants, but gradually makes its appearance if they develop under normal conditions. Baranetzky found that an alteration in the periods of illumination caused the daily periodicity to change; Brosig (1.c., p. 35) remarked that in a certain plant no such effect was produced; Baranetzky again has shown that in many cases no daily periodicity at all is exlibited. It is, however, only natural that such exceptions should exist, when we consider that no two plants are specifically identical. A bleeding-periodicity may be induced in a transpiring plant, although no actual exudation of water occurs during the period of induction, hence it is probable that in such cases a periodicity has been induced in certain other allied vital activities upon which bleeding depends. Moreover, it was in the root that a daily bleedingperiodicity was first observed and measured, although the root is not directly exposed to the periodic alternation of daylight and darkness.

1877, p. $4^{1}$; Brosig, Die Lehre von d. Wurzelkraft, 1876, p. 30; Wieler, 1.c., p. 129, where details are given. 


\section{SECTION 46. The Mechanism of Active Exurlation.}

An active exudation of water is only possible when living cells force water with sufficient energy into the tracheal elements or into the intercellular spaces, and the character of the processes involved may be easily understood by reference to the structure and properties of such living cells. Thus, in the accompanying schematic diagram (Fig. $3^{6}$ ), if water is forced in sufficient amount into the air space $(h)$ by the surrounding cells, fluid will escape when the air space is opened, while if a manometer is placed in connexion with it, the mercury will be driven upwards until the backward filtration caused by the increasing pressure just counterbalances the excretory activity of the living cells, so that a condition of equilibrium is

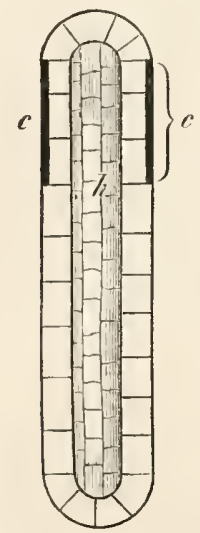

FIG. 36 . reached. The pressure will necessarily be less marked if a number of the cells bordering upon the air-space are inactive, for less water is pumped in a given time into the latter, and a backward filtration through the inactive cells may readily occur. When water can undergo backward filtration through certain cells more readily than others, an internal circulation of water may be produced (Sect. 43). The attainment of a high bleeding-pressure is favoured when the inactive cells (the zone $c$ in Fig. $3^{6}$ ) are comparatively inipermeable to water. As a general rule, however, the resulting bleeding-pressure will hardly attain a height corresponding to the intensity with which the most active cells excrete water.

To produce an exudation-pressure it is not necessary that the active cells must border upon the tracheae or the spaces in which the water accumulates, although such a position is obviously advantageous. The living cells of the wood, medullary rays, and wood parenchyma are actually able to excrete water, for both stems and roots may exude it after their cortex has been removed '. It is, however, not yet certain whether the epidermal cells of the root which primarily absorb water are themselves able to exert an exudation-pressure, or indeed whether they aid at all in producing it.

The tracheal elements, as the normal conducting channels, are easily able to transfer the water forced into them to points far removed where exit is possible. The exudation-pressure of water-glands is produced in a manner essentially similar to that of ordinary bleeding, no matter whether

1 See the experiments on the bleeding of barked twigs, Sect. 42. That the roots of Richardia acthiofica continue to bleed after the cortex has been removed is certain. Wicler, Cohn's Beiträge, 1893 , Bd. vi, p. $4^{6}$. 
the cells which surround the intercellular space into which water is excreted are themselves active, or whether water simply filters through them. In the pitcher of Nepenthes the water is excreted into an external cavity.

All such excretion of water is due to the activity of living cells, and need not necessarily be always produced in the same manner (Sect. 4I). Besides this active excretion under pressure, a passive or plasmolytic excretion of water is possible. In the former case the escape of water from the cell is due to the internal character and activity of the protoplast, while in the latter case the water is withdrawn from the cells by the osmotic attraction of external substances ${ }^{1}$, as occurs, for example, in nectaries (Sect. 49). Indeed, provided the cell-walls are permeable to water, the presence of sugar or any other soluble and highly osmotic substance on the outer surface of a cell must necessarily cause water to be withdrawn until a condition of equilibrium is restored. So long as the osmotically active substance remains outside, water will continue to be excreted even after the plant becomes flaccid; moreover, evaporation will tend to concentrate the external solution, and hence to exert a greater osmotic attraction upon the diminishing percentage of water still contained in the cells with which the osmotic solution is in contact.

This is therefore a process which is regulated by the purely physical laws of osmosis and osmotic action. Hence a trifling difference of concentration in the fluids touching the opposite sides of a cell, or of a plate of tissue composed of living cells, is sufficient to induce a filtration of water even against marked pressure. A difference of concentration equivalent to a C. I per cent. solution of potassium nitrate corresponds to the pressure of a column of mercury 27 centimetres high, while a difference of I per cent. will drive water through the cells with a force equivalent to a pressure of $3 \cdot 5$ atmospheres (cf. table, p. 146).

In nectaries the secretion and excretion of sugar is undoubtedly of primary importance, the excretion of water following as a necessary consequence; bleeding, on the other hand, is due to an intracellular secretion under pressure by special active cells. This must necessarily be the case whenever pure water or an extremely dilute solution is excreted, for otherwise no marked bleeding-pressure could be attained. The bleeding-pressure of a vine stem often exceeds that of one atmosphere, although the sap holds hardly any more dissolved substance than river water does ${ }^{2}$.

A plasmolytic excretion of water can continue only so long as the higher concentration of the extracellular fluid is maintained, and hence

${ }^{1}$ See Pfeffer, Studien z. Energetik, 1892, p. 265.

${ }^{2}$ Unger (Sitzungsb. d. Wien. Akad., $18_{57}, \mathrm{Bd} . \mathrm{xxv}, \mathrm{p} .444$ ) found that the specific gravity of the sap bleeding from a vine may fall as low as 0.0001 . A saltpetre solution of the same specific gravity would contain about 0.016 per cent. of salt. The detailed composition of the sap obtained by bleeding is given by Ravizza, Bot. Jahresb., is8s, p. 68 . 
the conditions necessary for such an excretion can only be maintained with difficulty in the neighbourhood of cells which actively excrete water. The transitory effect of the application of a concentrated solution to a plant is shown by the fact that an active exudation of water is only temporarily diminished or stopped by placing the root system in a solution of potassium nitrate (Sect. 44). Bearing in mind this behaviour, it is easy to see why Wieler ${ }^{1}$ was unable to induce any bleeding from inactive decapitated plants by filling the tube attached to the stump of the stem with solutions of saltpetre of from 0.1 to 1 per cent. concentration.

On these grounds we may with comparative certainty conclude that even when the escaping sap is rich in dissolved materials (Sect. 43), the exudationpressure is still produced by the excretion of water under pressure from the active cells, and that the sugar present in the sap is a food material in process of translocation, and is not formed in order to excite any plasmolytic excretion of water. Dissolved substances will naturally influence the exudation of water according to their distribution and osmotic energy, and it is possible that in certain cases both an active and a plasmolytic excretion of water may co-operate in producing the exudation-pressure.

The means by which intracellular activity is able to cause an active excretion of water have not yet been discovered, and since the same end may be attained in various ways, it is impossible to make any theoretical deductions as to what the nature of the process must be ${ }^{2}$. The escape of water from the active cells may be produced in a variety of ways, and may also be possible in one direction only. Thus a particular distribution of the osmotically active cell-contents may be maintained, or changes in the pressure exerted upon the enclosed cell-sap may occur, due to pulsations or active contractions. Alterations in the internal pressure are of common occurrence, and these might exercise a pumping action in a particular direction under certain special conditions; but it does not follow that the bleeding-pressure is necessarily produced in this manner, nor is it of necessity coupled with the existence of pulsating vacuoles. Moreover, no exudationpressure would be produced even by these means if backward filtration were possible, as is the case when water is excreted into the intercellular spaces of a stimulated staminal filament of one of the Cynareae, or into those of a stimulated pulvinus of Mimosa.

When an active exudation of water is artificially induced, the excreting cells have to be aroused by the action of an external stimulus. This stimulating action is shown very markedly by the glands on the leaf of Dionaea muscipula, which are caused to excrete an abundance of water (along with pepsin and a little acid) by certain chemical stimuli, and which return

2 Cf. Pfeffer. Energetik, 1892, 1) 265 , and Osmot. Unters., I 877, p. 223 ; also Wieler, 1. c., p. I51. 
to the inactive condition when these are removed (Sect. 65). Similarly, in Drosera and other carnivorous plants particular stimuli may excite or accelerate the excretion of fluid. Even in these cases the mechanism of the secretory process has not yet been precisely determined; nevertheless, it is certain that in Drosera and Dionaea we have to deal with an active excretion of water. It must, however, be remembered that the escape of water is in all cases due directly or indirectly to the activity of living cells, for even in the passive or plasmolytic excretion of water the osmotic substances concerned are produced by living cells. The functional activity thus excited persists as long as a higher osmotic concentration is maintained externally to a permeable cell or tissue. An active excretion of water may, however, be stopped by the action of chloroform or by the absence of oxygen.

When turgidity falls below a certain limit no perceptible excretion of water is possible so long as the cell absorbs water with greater rapidity than it excretes it. The fact that in flaccid plants the nectaries still continue to excrete water shows that the process is not one of active excretion under pressure, but is purely passive, the distribution of water being determined by the osmotic concentrations of the fluids outside and inside the cells.

Dutrochet erroneously supposed that bleeding was due to osmotic action, whereas Hofmeister's interpretation was correct, in so far as he recognized the active filtration and exudation of water into the interior of the plant as the immediate cause of bleeding ${ }^{1}$. The attempts of Hofmeister and other authors to refer the pressure of exudation to the vital activity of special cells were not very successful, owing to the fact that the osmotic relationships were not properly understood at the time they wrote, and to their imagining that the explanation of the osmotic powers of the cell, and of the transmission of water in a particular direction, was to be found in the properties of the cell-wall. Hence the apparatus ${ }^{2}$ employed by Hofmeister and others to demonstrate the process of 'bleeding' does not represent at all accurately what actually occurs in the plant. Nor was any clear distinction made between an intracellular activity independent of exosmosis, and the action of an extracellular substance causing a withdrawal of water from the cell. A full account of these problems is given in Pfeffer's Osmotische Untersuchungen, 1877, p. 223, where the principal views with regard to them have been brought into agreement with our present knowledge. It has been shown more recently by Pfeffer that the character of the plasmatic membrane has no influence upon the osmotic pressure, so long as no exosmosis is possible, and hence a change in the osmotic properties of the plasmatic membrane will hardly suffice to cause water to be driven from the cell in a definite direction ${ }^{3}$.

1 Dutrochet, Mém. d. végétaux et d. animaux, Bruxelles, 1837,p. 202; Hofmeister, Flora, i 8 5, p. $8 ; 1862$, p. $14^{2}$.

${ }_{2}$ Hoffmann, Ann. d. Phys. u. Chem., Bd. Cxvir, p. $26_{4}$; Sachs, Experimentalphysiol., $186_{5}$, p. 207 ; Detmer, Beiträge z. Theorie d. Wurzeldruckes, 1877, p. 21 . Cf. the summary gisen by Pfeffer, Energetik, 1892 , p. 265 .

3 Pfeffer, Zur Kenntniss d. Plasmahaut u. Vacuolen, I 890, p. 302 (cf. Sect. ${ }^{2} 4$ ). 
Neither a low nor a high osmotic pressure can drive water through a semi-permeable membrane in one direction only. Hence a twofold physical error is involved in the attempt to explain the forcible transmission of water in a particular direction as being simply due to its escape in the direction where least resistance is offered to filtration, as the turgidity of the cell increases '. As soon as the full hydrostatic pressure is reached, there is perfect equilibrium on all sides with regard to the entry and exit of water, while before that time, at every point where an exchange was possible, the inward current of water must have been greater than the outward.

Nor can any difference in character between the two opposite cellulose walls of a cell cause water to be actively forced in a particular direction, although as soon as any difference of potential has been created, the permeability of the cell-wall becomes of importance in determining the direction and character of the ensuing movement of water. Indeed, appropriate differences existing between the enclosing cellulose envelopes of the cells may easily cause the current to take place in a definite direction, and thus lead to an excretion of water. The cell-wall, however, plays even then a purely passive part, and is only indirectly of use as an aid to the active exudation of water. From these considerations it follows that the conditions which create and maintain bleeding are not due to the existence of pits, pores, or permeable areas in the walls either of the active cells or of the tracheae into which the water is forced. The fact that an exudation of water is not always possible shows that a special anatomical arrangement and structure is necessary to produce it, but these can hardly be the actual exciting causes. Moreover, as has already been mentioned, the water may be carried equally well in the opposite direction by the conducting elements. The erroneous suppositions of certain authors that changes of temperature or of the tissue tensions may be the primary agencies in inducing an active exudation of water have already been discussed (Sect. 44).

\section{SECTION 47. The Excretion of Water from Uninjured Plants.}

Many uninjured plants and plant organs are able to excrete water in a fluid condition, and just as in the case of its exudation from cut surfaces, the phenomenon is due to the activity of living cells, by which water is driven out at certain points from the intact plant. We are brought directly into contact with this excretory activity when the water-excreting cells are on the surface of the plant, as is the case in nectaries, as well as in certain hairs and fungal hyphae. When, however, the cells which excrete water are internal in position, the only evidence of their functional activity is afforded by the water which escapes by means of more or less specialized channels, or through groups of passive cells. This latter case, therefore, closely corresponds with the phenomenon of bleeding as regards the manner in which the water escapes; in part, at least, the excretion of water through water- 
pores, \&c. is due to the production of a high exudation-pressure. Under these circumstances the channels or intercellular spaces through which the water flows, as well as the internal or external point of exit, have a purely passive function to perform in conducting away the excreted water. The function of the channels is the same whether the water is passively excretcd, or whether it is driven onwards by the pressure due to the pumping activity of special water-excreting cells. The cells which excrete the water may be near to or far removed from the spot where it ultimately appears, as may also be the case in normal bleeding. The character of the conducting channcls, as well as of the pores by which it escapes, if any exist, may markedly influence the rate at which an escape of water is possible, but nevertheless the cause of its escape in all cases is directly or indirectly the excretory activity of living cells. The sinking of such cells in a pit-like depression is the first step towards the formation of a water-gland with a well-defined duct or exit passage. At first the secreting cells may linc the entire space thus enclosed, and only later become restricted to its base.

These considerations suffice to make it clear that no sharp distinction can be drawn between the excretion of water from injurcd and from uninjured organs, for in both cases the water escapes from special points only because of the pressure of exudation which forces it onwards. Indeed, in certain cases the normal progress of development may cause external parts to be ruptured or thrown off, and so produce points at which an active outward filtration of water is possible. The drops of water which appear on the buds of the hormbeam and other trees are produced by the internal exudation-pressure driving water through the scar formed when last year's leaf was disarticulated ${ }^{1}$. In many leaves again the openings through which water can escape are produced by rupture, and in the same way waterstomata may be mechanically enlarged or widened (Sect. 48).

The mode in which water may escape through open channels to the exterior has already been discussed. The excretion of water through the water-stomata often found on leaves is due in some cases to a generally distributed hydrostatic pressure, but in others to an active excretion of water from special cells bordering upon the cavity beneath the waterstoma. The excretion of water from typical nectaries, on the other hand, is plasmolytic in nature, but as a decp-seated position, such as occurs in many nectaries, is also possible, the fluid may cscape to the exterior by a pore or duct. In water-pores and nectaries two different types of cxcretory mechanisms are exhibited, as will be made still more clcar by the special account of each given later (Sects. 48 and 49). It is not necessary to discuss the distinctive means by which every excretion of

1 Strasburger, Bau u. Verricht. d. Leitungsbahnen, i $\delta_{9}$ I, p. $\delta_{4}$ r. I Iere also the related literature is quoted (Th. Hartig. Anat. u. Physiol, d. Holzpflanzen. i $8 ; 8, p \cdot 34 \pi, \& c$.). 
water is rendered possible, and indeed our knowledge of the cell mechanisms at work is in many cases quite incomplete.

It is never easy to say whether we are dealing with an active or a passive excretion of water; moreover a combination of the two is always possible, even in the case of nectaries, in which the plasmolytic excretion of water is most marked, and indeed appears to be sufficient for all requirements. On the other hand, the process of excretion may be primarily an active one, although the excreted fluid may contain large amounts of dissolved substances, and the extracellular dissolved substances must in all cases exercise an effect proportionate to their osmotic energy. A very dilute solution may possess an osmotic energy corresponding to a statical pressure which is by no means inconsiderable (cf. Sect. 46), although it may be insufficient to produce any marked exudation-pressure (as is the case, for example, in T itis). When no such comparison is possible, or when, as is often the case, the water is driven forth with only relatively feeble energy, special researches are necessary to determine whether the flow of water is produced directly by the vital activity of living cells, or is caused by the presence of a dilute or concentrated solution of osmotic substances outside the cell. It must clearly be understood that an osmotic pressure acts in a precisely similar way to an equivalent mechanical pressure in causing a filtration of water through the cell-walls or tissues, so that there is no essential difference between a flow of water caused by a change in either the mechanical or the osmotic pressures acting upon a cell.

When a copious excretion of water is stopped by a slight decrease of turgidity, we may conclude with comparative certainty that the phenomenon was due to an active excretion of water by living cells. This conclusion does not, however, necessarily hold good for those cases in which the water is forced out with only feeble energy, or in which the water slowly oozes through walls which are only permeable with difficulty, for it is then possible that a slight increase in the rate of transpiration may have induced a decrease in the turgidity, and at the same time have caused the dilute extracellular saline solution to dry up, so that it ceases to exert any plasmolytic action in withdrawing water. In such a case the decreased turgidity and the cessation of the plasmolytic action would only be accidentally correlated with one another, so that the stoppage of the flow of water would by no means indicate that the process was one of active exudation. Moreover, as a dilute solution evaporates and becomes more concentrated, its osmotic energy increases enormously, so that the flow of water would increase although the internal conditions remained the same. The external solution rapidly dries up as soon as water in only small quantities can be withdrawn from the contiguous cells, and when this occurs the salts may not be deposited on the surface of the plant, owing to the fact that the cell-wall can imbibe small amounts of saline 
substances. No flow of water will become apparent externally when the cells which are actively or passively excreting water are deeply seated, and the surrounding inactive tissue absorbs the water again as fast as it is excreted.

Bearing in mind all these possibilitics it is impossible to say whether the drops of water which appear upon the sporangiophore of Pilobolus, when it is kept in a saturated atmosphere, are formed by the active or the plasmolytic excretion of water (Fig. 37). As a matter of fact a drop of the fluid leaves a crystalline deposit when allowed to dry upon a cover slip. It is therefore possible that the excretion of water is plasmolytic in this case, for any osmotic substance must always tend to withdraw water from a fully turgid cell with which it is in contact.

It is, however, probable that the excretion of water into the pitcher of Nepenthes is an active process although the flow may continue when the plant is not fully turgid. On the other hand, the small amount of material dissolved in the pitcher-fluid is quite sufficient to support a higher column of water than would suffice to fill the pitcher; still even in a saturated atmosphere the pitchers are never completely filled ${ }^{1}$. Apparently either a backward filtration must be possible. or else the same constant level must be maintained by a regulatory influence upon the excretory activity exerted by the pressure. In Dioncrea and other carnivorous plants the excretion of water is influenced by or dependent upon external stimuli. As we have already seen (Sect. 46) the power of bleeding may be similarly aroused by certain external agencies.

The hairs both of carnivorous and other plants can often excrete water. Those which occur on the leaves and stems of Cicr arictimun, Circaca intctiana, Epilobinm

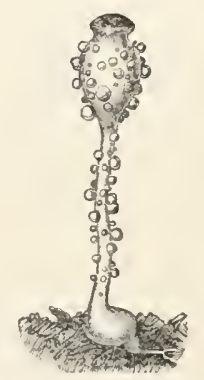

Fig. 37. Pilobolus crystallinus $(\times 10)$. hirsutum, \&c., pour out an acid fluid, while in a saturated atmosphere drops of water appear commonls to be excreted by most hairs and by the rhizoids of Marchantia, \&c. ${ }^{2}$ Since these drops of fluid always contain dissolved substances (Sect. 28) it is impossible to say whether they are produced by intracellular excretion under pressure, although this appears probable. On the other hand, in glandular hairs which cxcrete a sugary fluid the process is probably similar to that which occurs in nectaries. The excretion of water from Mucorineac, as well as from Penicillinm and other filamentous fungi, is probably similar to that exhibited by Pilo-

1 W'unsclımann, Über die Gattung Nepenthes, $187^{2}$, pp. 25, 31 (Composition of the pitcherfluid; Filling of pitchers on cut branches). ( $f$. also on the excretion of water in Cephalotus and Sarracenia, Goebel, Pflanzenbiol. Schilderungen, i 893 , Th. ii. p. 170; IS91, p. I 10.

${ }^{2}$ Stahl, Pflanzen u. Schnecken, I 888, p. 42; de Candolle, Pflanzenphysiol., i 8.3. Vol. I, p. 190. On the secretory activity of the hairs of Fuchsia globosa see Gardiner, l'roceedings of the Camb. Phil. Soc., $188_{4}$, Vol, v, p. 39 ; Molisch. Sitzungsb. d. Wion. Akad.. 1S87. Mrd. Xcri. Abth. i, p. 103; Crapek, Jahrb. f. wiss. Bot., 1896 . Bd. xxix, p. $32 \mathrm{i}$. 
bolus ${ }^{1}$. The pseudo-parenchymatous mycelia of many fungi often excrete large quantities of fluid, which in certain cases at least contains very little dissolved substances. Merulius lacrymans, several species of Polyporus, Nyctalis, \&c., the growing ends of the mycelial strands of Hypoxylon carpophilum, and the scelerotia of certain species of Coprimus and Peziza all have this power. The sugary fluid which may escape from the conidium stage of Claviceps purpurea is probably excreted in a similar manner to that in nectaries.

The power of excreting water appears often to be present in subterranean organs as well, as is no doubt the case in root-hairs; according to Darwin, the scale-leaves of Lathraea squamaria also excrete water in abundance ${ }^{2}$. When the exudation-pressure becomes very pronounced, the roots may in certain parts excrete water instead of absorbing it, and in a similar manner a circulation of water may be produced in submerged plants (Sect. 46). A tendency of this nature must always exist when two neighbouring roots are kept immersed in solutions of different osmotic strength.

The excretion of water may serve a variety of purposes, such as to bring a substance secreted by the cell into a position where its purpose may be fulfilled, or where its full power may be exercised. Herein lies the importance of the excretion of water in nectaries and in camivorous plants, \&c., for in the former case the excretion of sugar is accelerated, while in the latter an enzyme is contained in the escaping water, which also facilitates the absorption of the products of digestion. In the same way the excretion of water from rhizoids and root-hairs may enable a special solvent influence to be exercised in some cases upon the living or dead substratum, and may aid in the penetration of fungal hyphae into living organisms or organized structures; indeed it may first make such penetration possible. Again, the pollen grain germinates in the fluid excreted by the stigma, and a similar process fills the micropylar orifice with a solution of the substances which attract the pollen tube to its proper destination. In some plants, as for example Corsinia marchantioides ${ }^{3}$, the antherozooids reach the archegonium by means of the water which the plant itself excretes, although in other cases dewdrops appear to serve the same function. It is possible that the water which the roots may excrete may serve in some cases to soften the earth and enable the growing roots

${ }^{1}$ On the excretion from Fungi see Zopf, Die Pilze, I S9O, p. I 86 ; Brefeld, Unters, tiber Schimmelpilze, I 881 , Ileft 4, p. 68 ; Schmitz, Linnaen, 1 $8_{43}$, Bd. Xvir, p. 472 ; W'ieler, Colın's Beiträge, 1893 , Bd. VI, P. 16 .

2 Darwin, liewegungsvermögen, 188 I, p. 71 ; Haberlandt, Jahrb. f. wiss. Bot., I $\$ 97$, Bd. xxx, P. 510 [Goebel, Über d. biologische Bedeutung d. Blatthöhlen bei Tossia u. Lathraca, Flora, I.XXxi11, Heft .3, I897; Groom, Annals of Botany, Vol. xi, I $\left.897 \cdot \mathrm{p} .3_{3} \mathrm{~S}_{5}\right]$.

${ }^{3}$ Leitgeb, Flora, 1885 , p. 3.30 . 
and rhizomes to penetrate it more casily. In some cases, however, the excretion of water is an unavoidable accompaniment to more important phenomena, as for example the contraction of a protoplast which is going to form spores, or the escape of water from the stimulated cells of a contractile tissue.

The function of the water-stomata is to get rid of superfluous water, and so to avoid any pronounced injection of the intercellular spaces with fluid, such as a high exudation-pressure might cause ${ }^{1}$. When the latter is maintained for some time, however, the intercellular spaces gradually become filled with water in spite of the presence of water-stomata, as Moll found (1.c., p. 73); under normal conditions they generally remain filled with air or are only temporarily and partially injected with water. The means by which the intercellular system is kept free from water have already been mentioned (Sects. 43 and 46 ), and, morcover, the backward filtration through the root, which may occur when the cxudation-pressure is very high, acts as a regulatory check upon the over accumulation of water.

The amount of water which the water-stomata excrete is in general not sufficiently great to accelerate the transpiratory current ${ }^{2}$ to any particular extent; even in moist climates this stream is sufficiently strong to provide an adequate supply of the nutrient salts required (cf. Sect. $3^{8}$ ). Nor can the excretion of water serve as a general means by which excreted products may be thrown off, for it may happen that the functional activity of the water-stomata, \&c., is never called into play. The fact that calcareous deposits are formed only on certain plants shows that their appearance is due to some specific peculiarity of the plants in question (Sect. 23).

Since the regions where water exudes must be readily permeable, they are naturally also better able to absorb water than the relatively impermeable epidermis, when an external supply is presented to the water-excreting organs of a flaccid plant ${ }^{3}$.

A formation of dewdrops by the condensation of water is not the same thing as an excretion of water by the plant, and a fertile source of error lies in the fact that drops of water may be caused to collect upon and fall from the leaf by purely physical agencies. Arendt ${ }^{4}$ has shown that with a particular kind of surface a capillary ascent of water upon the stems and leaf-stalks is possible, and so by spreading along the veins a regular series of drops of water may collect and fall from the tips of hanging leaves. This takes place in Leonurus cardiuca,

1 Moll, Unters. über Tropfenausscheidung u. Injection, 1880, pp. 81, 101 ; Gardiner, Proceedings of the Camb. Phil. Soc., 1884, Vol. v, p. $4^{2}$; Haberlandt, Sitzungsb. d. Wien. Akad., I895, Vol. CIr, Abth. i, p. 11 I.

${ }^{2}$ Cf. Haberlandt, 1.c., p. 111 ; Burgerstein, Ber. d. Bot. Ges., 1897, p. 155 . See also Sect. 38.

${ }^{3}$ Cf. Sect. 27. This is therefore by no means a specific peculiarity of certain plants, as IIaberlandt supposes (Sitzungsb. d. Wien. Akad., 1894, Bd. Cur, Abth. i, p. 494). In the same way such absorbent organs as the roots are able to excrete water under certain conditions.

${ }^{4}$ Arendt, Flora, 1843. P. 152; Stahl, Regenfall u. Blattgestalt, 1893, p. I1 2. 
Ballota nigra, Urtica dioica, $\mathrm{Nc}$., even when the water has only to be raised to a height of a few centimetres; in such cases it is easy by using a coloured solution to make out the path which the water follows. The same physical and structural peculiarities are concerned in this phenomenon, as render possible the rapid drainage of rain-water from leaves.

\section{Section 48. The Excretion from Water-Pores.}

The leaves of many higher plants are able to excrete water in the liquid form by means of special water-pores when in a saturated atmosphere and when fully turgid. As a general rule the drops of water form directly over the water-pores, which are usually situated upon the leaf-teeth (Fig. $3^{8}$ ) (Impaticns, Fuchsia, Tropaeolnm, I'itis, Salix, \&c.), but may occur in other situations, as for cxample, on or near the tip of the leaf (in aroids and grasses). When the pores are abundant and active, water may actually drip from the leaf. The filtration through

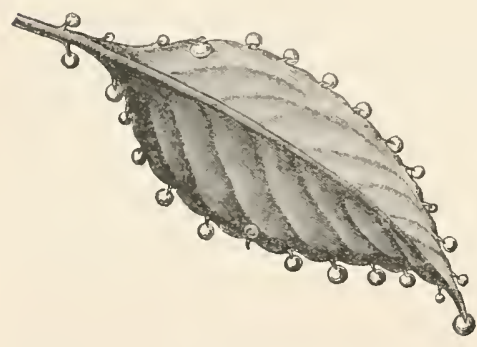

FIG. $3^{8}$. Leaf with secreting water-pores. the external point of exit is usually only passive, the cells which generate the pressure of exudation being usually more decply seated. Hence the forcible injection of water induces a more rapid flow through the water-stomata, while in the day time the excretion of water usually ceases, and cven during the night it only occurs when a sufficiently high exudationpressure is reached ${ }^{1}$.

As might be expected, water-glands also exist in which there is an excretion of water by the cells which line the cavity immediately beneath the water-stoma, or in which we find only an acceleration of the outward filtration of water due to the internal pressure of exudation. This is the case, according to Haberlandt, in certain more or less superficially situated water-glands (Conocephalus, Ficus), which yicld a very watery fluid, and which therefore very probably cxcrete water by means of the internal activity of the cells of the water-gland. On the other hand, deep-seated nectaries also exist in which the water is plasmolytically excreted.

1 The experiments of Moll (Unters. über Tropfenausscheidung u. Injcction von Blättern, I88o, Sup.-abdr. a. Vcrslagen en Meded. d. Kon. Akad. Amsterdam; prel. comm. Bot. Zeitung, 1880, 1. 49) were followed by those of Volkens (Jahrb. d. Bot. Gartens in Berlin, I883, Bd. I1, p. I66) and Gardiner (Proc. Camb. Phil. Soc., I884, Bd. v, p. 35). A summary of the cases in which these and other authors have detected a power of bleeding is given by Wieler, Cohn's Beiträge, I893, Bd. VI, p. 16. See also Haberlandt (Sitzungsb. d. Wien. Akad., I894, Bd. c1Il, Abth. i, p. 489, and 1895 , Bd. civ, $A$ bth. i, p. 55 ; Jahrb. f. wiss. Bot., 1897 , Bd. xxx, p. 511 ). That an excretion of water might be caused by forcing water' into the plant was noticed by de Bary in 1869 (Bot. Zeitung, p. 883), and by Prantl (Flora, 1872, p. $3^{81}$ ). 
The excretion of water from water-pores is a typical process of filtration under pressure, for as Moll has shown, no water escapes from a cut branch however well supplied it may be, whereas the flow commences as soon as water is forcibly injected. Similarly, Haberlandt found that ordinary water-stomata continued to excrete after the epithelial tissue through which filtration occurs liad been killed by poison, whereas in the glands of Conocephalus such treatment caused a cessation of the flow, for in them the excretion of water is independent of the general internal hydrostatic pressure ${ }^{1}$.

The fact that the flow ceases as soon as a branch is cut off is a sufficient proof that the excretion of water is due to the general exudationpressure, but if no water is allowed to escape from the cut surface of the stem the water-stomata may remain functionally active so long as the cut branch can maintain a sufficiently high internal hydrostatic pressure. Cut stems may indeed in certain cases continue to excrete water, but it does not follow that the process is independent of the general internal pressure, so that the conclusions which Gardiner drew as to the active excretion of water by the epithelial cells of certain water-pores are not necessarily correct ${ }^{2}$.

As regards the shape and anatomical structure ${ }^{3}$ of the water-excreting organs, it must suffice to say that for the most part they are in the form of the so-called 'water-pores,' i. e. modified widely open stomata, beneath which a respiratory cavity persists. 'This becomes filled with water driven into it by filtration through the intermediary cells which are usually directly connected with a subjacent vascular bundle. The intermediate tissue has somewhat the form of an epithelium, between the cells of which small or large intercellular spaces occur, and these may aid in the escape of water ${ }^{4}$. Moll observed an escape of water coloured with dye from the waterpores of Phytolacca decandra when it was forced into the plant by pressure, but the

${ }^{1}$ Haberlandt, l. c., 1894, p. $513 ; 1895$, pp. $63,81,107$. [Spanjer denies that water is ever actively secreted through glandular hydathodes, and states that the escape of water is always due to filtration under the generally distributed internal hydrostatic pressure (Bot. Zeitung, I 898, Nos. 3 and 4). It is worthy of notice that the latter is very marked in Conocephalus. No final decision is, however, as yet possible. Cf. also 1. c., pp. I $77,24 \mathrm{I}, 315$.

? Gardiner, l.c.p. If; cf.also C. Kraus, Studien a.d.Geb.d. Agriculturphysik, I882, Bd.v, p. 435 .

${ }^{3}$ For details see the above works. Cf. also de Bary, Anat., I877, pp. 54, 391 ; Haberlandt. Physiol. Anat., I896, 2. Aufl, P. 415 ; Nestler, Unters, iiber die sog. Wasserspalten, I $89+$ (Sep.-abdr. a. Nova Acta d. Leopoldina); Nestler, Sitzungsb. d. Wien. Akad, 1896, Bd. cV, Abth. i, p. 52 I. [Max v. Minden, Beitr. z. anat. u. physiol. Kenntniss Wassersecemierender Organe, Bibl. IBot. I899. Heft 46, pp. I-7I.]

${ }^{4}$ Haberlandt (1.c., 1895 , p. I07) makes a physical error in supposing that the high osmotic picssure existing in the epithelial cells will hinder the filtration of water through them. 13oth the osmotic absorption and the excretion of water are simply processes of filtration under pressure, and the one may replace the other. Moreover, the facts observed do not justify Iaberlandt's conclusion (1.c., I 895 , p. I10) that the stimulating action of a high internal hydrostatic pressure is necessary in order to cause an excretion of water from glands which have an intracellular excretory activity of their own, for the stoppage of all excretion of water is a mechanical necessity when the amount present falls below a certain limit. 
dye probably passed, not through the epithelial cells, but through the spaces between them. In Aroids the space beneath the water-stoma takes the form of a long intracellular canal that runs parallel to the marginal bundle, and thus presents a large surface through which water may filter. According to Haberlandt (1. c., I 895, p. 89) stomata may function as water-pores in young grass leaves, but in old ones it has hitherto been supposed that the water-pores are merely cracks which appear in the epidermis. It is, moreover, obvious that water may be driven through ordinary stomata whenever the intercellular spaces are injected with water (Moll).

Since the excretion of water from water-pores is largely or entirely dependent upon the internal hydrostatic pressure, it will be influenced by the process of development, and by external agencies, to an extent corresponding to the effect they produce upon the general activity of exudation. The excretion of water is dependent upon the amount present, but no critical experiments have been performed to determine the influence which external agencies may exert ${ }^{1}$.

Duchartie and Unger ${ }^{2}$ observed that the excretion of water from Aroids decreases when the plant is exposed to light, but this is probably because the rate

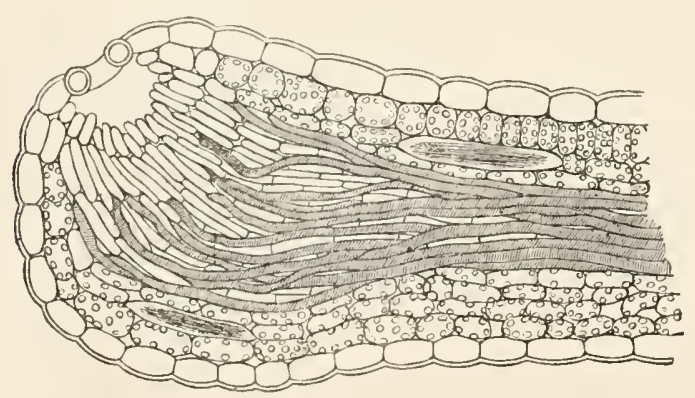

FıG. 39. Fuchsia hybrida (hort. Longitudinal section through water-pore. $(\times 100)$. of transpiration increases, and similar objections may be made to the results obtained by Gardiner (1.c., p. 42), as well as to the observations made upon the daily variations in the amount of water present in the pitchers of Nepenthes, which are supposed to be due to the action of light ${ }^{3}$. A few cases have, however, already been mentioned (Sect. 47) in which the excretion of water

is undoubtedly excited by an external stimulus.

The permeability of the cells through which the water filters is naturally also of importance in determining whether any excretion of water is possible, as well as the rate at which it may occur. It must, therefore, be determined in each case by empirical investigations whether the water-pores of old leaves cease to excrete water because the activity of the cells which generate the exudation-pressure decreases, or because the epithelial cells offer more resistance to the passage of water through them. Moreover, it is only in certain plants that even a maximal hydrostatic pressure is able to cause drops of water to fall from the leaves. The most active excretion of water as yet observed occurs in certain Aroids; thus Musset saw as much as eighty-five drops fall from the tip of the leaf of Colocasia esculenta in one

${ }^{1}$ See Volkens, l.c., p. I72; de la Rue, Bot. Zeitung, 1866, p. 317 ; Duchartre, Ann. d. sci. nat., I859, iv. sér., T. XII, p. 267; Schmidt, Linnaca, 1831, Bd. vi, p. 65.

Unger, Sitzungsb. d. Wien. Akad., I $8_{5} 8$, Bd. xxvil, p. 15.

${ }^{3}$ Korthals, \&c., quoted by Wunschmann, Über die Gattung Nepenthes, I\&72, p. 28; Goebel, Pflanzenbiol. Schilderungen, 1891, Th, ii, p. 160; 1893, Th. iii, p. 168 (Sarracenia). 
minute, while from the leaf of Colocasia antiquorum Duchartre counted twenty-five to twenty-six drops per minute, and collected from the latter plant 22.6 grams of fluid in a single night ${ }^{1}$. Although as a general rule the drops collect slowly, in Colocasia Musset noticed that they appeared suddenly, and might be thrown to a distance of Io centimetres, but Munting ( 1672 ) was probably exaggerating when he stated that he had seen a fine stream of water, like a fountain, springing from the leaves of Aroids ${ }^{2}$, although it is not impossible that such a result might be produced if water were forced into the plant under strong pressture. At any rate it is possible by such means to increase very considerably the rate at which water is excreted, and so to drive large quantities of fluid through the plant in a comparatively short time:".

The fluid excreted from the water-stomata has been found to have a composition similar to the sap obtained from bleeding plants, except that it is more diluted (Sect. 43), since it contains only 0.001 to 0.05 per cent. of dissolved substances '. The fluid from the pitchers of Nepenthes is not quite so dilute, and contains, according to Völcker, $0.8_{5}$ to 0.92 per cent. of solids ${ }^{5}$. On the secretion of enzymes, Sc. by Nepenthes and other carnivorous plants, see Sect. 65 .

\section{SECTION 49. The Excretion of Nectar.}

A nectary may be produced at any point where the cells can excrete sugar, provided the cell-walls are permeable to water, and that the osmotic action of the extracellular sugar is thus able to withdraw water from them (Sects. 46, 47). An artificial nectary may be formed by placing a little sugar in a hollow on the cut surface of a beetroot or a potato, when water is extracted from the tissues until the sugar all passes into solution, and so long as the bectroot or potato contains plenty of water, the size of the drop may remain constant in spite of continuous evaporation (Fig. 40, $n$ ), or it may increase considerably in a saturated

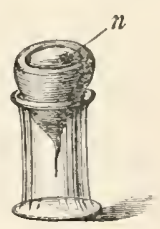

FIG. to. atmosphere. Just as in ordinary plasmolysis, scarcely anything but water is withdrawn from the cell, so that when a bectroot is used for this experiment, the sugar solution remains uncoloured so long as all the cells with which it is in contact remain living.

1 Musset, Compt. rend., $18_{5}$, T. LXI, p. $68_{3}$; Duchartre, 1.c., p. 250 ; Cormu, Compt. rend., I 897, T. cxiv, p. 666.

${ }^{2}$ Reference in Flora, $18_{37}$, p. 717 .

${ }^{3}$ Sachs, Vorlesungen iiber Pllanzenphysiol., I 887 , 2. Aut., p. 259.

" Unger, Sitzungsb. d. Wien. Akad., $1 \$_{5} \delta$, Pd. Xxvin, p. 19; Duchartre, 1. c., p. 24I, and the literature given by Burgerstein (Naterialicn, \&c., I 889 , II, p. 409); also Haberlandt, 1. c., I 895 , p. 62 ; Völcker, quoted by Wunschmann, Über die Gattung Nepenthes, IS 72, p. 25.

${ }_{5}^{5}$ [Koorders (Ann. d. Jard. bot. d. Buitenzorg, T. XIv, IS97, pp. 355, 449) finds that the fluid present in the 'water-calyces' of many tropical flowers contains from 0.3 to 0.7 of sulids, the greater part of which usually consists of organic substances, probably chiefly sugar. The fluid may be acid or alkaline, and is apparently intermediate in character betwcen that excreted by hydathodes and that by nectaries.] 
A plasmolytic excretion of water is still possible long after bleeding and all active excretion of water have ceased owing to a diminution of turgidity. If nectaries excreted water only in this latter manner, they would dry up just when they were most likely to be useful to the plant, for during the period of vegetative activity most plants are not sufficiently turgid to be able to bleed. As it is the excretion of nectar may continue however active transpiration may be, which is a fact of great biological importance. Even when the plant is quite flaccid, fluid nectar may still be present, and Wilson has shown that the feebly active nectaries on the leaves of Prumus lauroccrasus may continue to excrete nectar if liept in moist air, although the leafy branches may have lost more than one-fourth of the amount of water normally present ${ }^{1}$.

When transpiration is very active, the extracellular solution gradually concentrates as the plant loses more and more water, and ultimately dries up, as may readily be observed in many extrafloral nectaries. Indeed the nectar is always more concentrated during periods of drought, and crystals of sugar may even be found in it at such times, when it may contain over 100 per cent. of sugar; the usual proportion is only 30 to 10 per cent., which may sink to less than I per cent. when the plant is fully turgid and when no transpiration is allowed ${ }^{2}$. In this last case the amount of fluid excreted is considerable, and drops of nectar may even overflow and fall from the nectary.

The nectary can apparently excrete as much water as is necessary in this plasmolytic manner; indeed under normal vegetative conditions no active filtration is possible as a general rule, so that it is doubtful whether the latter ever takes part in the process, for when the plant is rich in water, any active filtration which might then be possible would simply cause the plant to waste still more sugar by increasing the rate at which nectar escapes from the overflowing glands.

The absence of any active intracellular power of excreting water is definitely proved in those cases in which the flow of water stops as soon as all the extracellular sugar is removed by rinsing out the nectary with water, and recommences when a little sugar is replaced upon them. Instances are afforded by the floral nectaries of Fritillaria imperialis, Accr pseudoplatanus, and the petiolar nectaries of Prumus lamocerasus (Wilson, 1.c.). The excretion of water by the nectaries cannot be stopped in this manner if the plant itself is able to replace the sugar that has been washed away, and in such cases the reappearance of the sugar shows that an excretion of this substance is possible. The power of repeatedly excreting

1 Unger, Flora, $18_{44}$, p. 707 Nectaries on the petiole of Acacia); Wilson, Unters. a. d. liot. Inst. 1. Tüibingen, I $S S_{1}$, Bd. I, p. 8 .

${ }^{2}$ Sec Bomier, l. e.. p. S.s, and other works yuoted later. 
sugar or other substances is exhibited by certain plants only. and may be modified in the progress of development as well as by the external conditions. A single thorough rinsing with water may suffice to stop completely the excretion of nectar from the large nectaries at the base of the perianth of fully opened Fritillaria flowers, whereas in quite young flowers the washing with water must be repeated two to four times, and in Pmms lamrocerasus five to six times in the course of one to a few days to produce the same result. It appears that in certain cases fresh sugar is continually excreted, for Schimper ${ }^{1}$ found that the excretion of nectar from the petiolar nectaries of Cassia neglecta took place continually, although they were daily rinsed with water for eight days. Such a plant may lose a large amount of sugar if the nectar is continually washed away by rain or removed by animals, but this is simply in correspondence with the general law that an increased demand causes an increased production.

The secretion and excretion of an osmotically active substance (sugar) is the only vital process which takes part in the formation of nectar. This sugar is usually secreted by the gland-cells of the nectary, but in many cases a certain amount of sugar is produced by a metamorphosis of the cell-wall, possibly due to some vital action exerted by the plasma within. This latter process usually seems to occur only once, when the secretory activity first commences; and since all nectaries can apparently excrete sugar, it seems probable that this metamorphosis of the cell-wall is not for the purpose of providing extracellular sugar, but is of primary importance in rendering the excretion of nectar more easy, by increasing the permeability of the partially metamorphosed cuticular walls. The cuticle may, however, be readily permeable at the outset (Sects. 2 I and 27). and hence it is not surprising to find that in other nectaries and vater-glands it may persist unaltered.

The general principles regulating the excretion of sugar by nectaries have already been discussed (Chap. IV). It is, however, not precisely known how the glandular cells are able to excrete sugar, though when once this occurs, the subsequent processes are comparatively simple and easy to understand. Other points also are not quite clear; it has yet to be determined how the protoplasts of the gland-cells manage to avoid being injured by the relatively enormous osmotic forces which act upon them when the nectar becomes highly concentrated (see Sect. 23), and moreover it is not easy to see why the sugar is retained in the nectary instead of being sucked backwards into the tissues. There must be some hindrance or other to the backward filtration of sugar through the glandcells; and perhaps the continual excretion of sugar may prevent any backward flow, for when this excretion ceases and the nectar dries up, 
no sugar is left behind. This may be observed on many nectaries which have been functionally active for a long time (Wilson, 1.c., p. 13), whereas others still remain filled if the flower falls off before its tissues are dead. Eren when the sugar is ultimately reabsorbed and used in metabolism, there is no reason to suppose, as Bonnier ${ }^{1}$ does, that the nectar is to be regarded mainly as a store of reserve food-material, for a more wasteful and dangerous mode of storing reserve food could hardly be conceived. Besides, it is well known that by means of this food the attraction of insects is secured, which either ensure cross fertilization, or protect the plant from foes, or are of use to it in other ways.

The works here quoted ${ }^{2}$ give a full account of the form, structure, and distribution of both floral and extra-floral nectaries, and of the glandular sugar-secreting cells which form an essential part of each nectary. The nature and structure of an active nectary must in every case be such that the conditions already laid down can be fulfilled. 'The glandular secreting cells are often small and possess dense contents ; they are usually superficial in position, but may be more deeply situated with ducts leading to the exterior. In a compound nectary of this type the glandular areas may abut so closely upon one another as to form a continuous layer, although the ducts may remain distinct. It is only in certain cases that the cuticle is thrown off more or less completely, while it is easy to understand that all the starch the secreting cells contain may partially or entirely disappear when sugar is actively excreted. A supply of water and of food-material is necessary in order that a continuation of the secretory activity of the nectary may be possible.

Mistorical. No distinction was made between the excretion of water by active exudation and by nectaries until Pfeffer's Osmotische Untersuchungen appeared ( 1877 , p. 232). The ideas there put forward have been confirmed by Wilson's researches. The doubts thrown by certain authors upon the occurrence of any plasmolytic excretion of water can only be maintained by ignoring the physical and physiological principles involved, and by neglecting to distinguish between the excretion of the sugar and of the water, that is, between the cause and the result ${ }^{3}$.

Composition ${ }^{4}$. In addition to sugar, only small quantities of other substances are present in the nectar, but their existence is indicated by the odour, taste, or acid reaction of the nectar from certain plants. Minute traces of nitrogenous sulsstances may be present, although von Planta could not detect any in the nectars he examined. 'The sugar may occur in the form of dextrose, laevulose, saccharose, or even mannitose (Fraximus, Sambucus), while dextrin or other carbohydrates

1 Bonnicr, Ann. d. sci. nat., 1878 , vi. sér., T. vil1, p. 199.

2 De Bary, Anat., 1877, p. 93 ; Kerner, Yflanzenleben, 1891 , Bd. 1, p. 168; Reinke, Jahrb. f. wiss. Bot., 18;6, Bd. x, p. 119 ; Bonnier, Ann. d. sci. nat., I878, vi. sér., T. vı11, p. I ; Poulscn, Bot. Centralbl., ISSi, Bd. vi, p. 7 ; Grassmann, Flora, I884, p. 13 ; Stadler, Beiträge z. Kennt. d. Nectarien, 1886 ; Schimper, 1. c.; Acton, Annals of Botany, IS8S-9, Vol. 11, 1. 53 ; Wettstein, Bot. Jahresb., ISSs, p. 528 , \&c.; Schniewind-Thies, Beiträge z. Kennt. d. Scptalnectarien, 1897.

s Büsgen, IIonigthau, ISg1, 1. 31. Cf. Pfeffer, linergetik, 1892, p. $26 \%$, and Sects. 46 and 47 .

- Sec von Planta, Zeitschr. f. physiol. Clemie, ISS6, Bd. x, p. 227 ; Bonnier, 1. c., p. 84 , and the literature quoted therein. 
which form mucilaginous solutions may also be present, as in the nectar from the flower of Pinguicula alpina, in which, according to Stadler (l.c., p. 73', no actual sugar is present. This observation however requires confirmation. No cane-sugar is present in certain nectars (for instance that of Protea mellifera, according to von Planta), whereas in other cases most of the sugar is present in this form. In the nectar from the flowers of Hoya carnosa, $87 \cdot 4$ per cent. of the sugar is cane-sugar. According to Bonnier, the maximum amount of cane-sugar is found when the secretion is most active, so that saccharose apparently is in part inverted after it has been excreted, and hence it arises that the greater part of the sugar present in the nectar may be in the form of dextrose. In any case, however, cane-sugar is nearly always found in the nectar, as Braconnot showed in $184^{1}{ }^{1}$; and though it does not follow that this is the only form of sugar which can be excreted, it is certain that saccharose does actually diosmose as such through the protoplast (Sect. I6). In the works already mentioned, data are given concerning the varying amount of water present in the nectar.

The infuence of the cxternal conditions. As is often the case, the secretion of nectar commences at a certain stage of development, which differs in different plants, and ceases after a longer or shorter period of activity ${ }^{2}$. The general vegetative conditions, as might be expected, also exercise a more or less marked influence upon the excretion of nectar, which is diminished in starved plants, owing to all the available food being preferably employed in internal metabolism. Apparently this was the reason why Schimper found that the functional activity of the nectaries of Cassia neglecta ceased in a few days when the plants were kept in darkness, or in an atmosphere deprived of carbon dioxide, whereas the secretion continued when the leaf was exposed to light and the nectaries only were kept in darkness ${ }^{3}$.

In certain cases light may also exercise an obscure but apparently direct effect as well. Wilson ${ }^{4}$ found that illumination causes the excretion of nectar to commence in the stipular nectaries of Ticia faba, even in an atmosphere free from carbon dioxide, whereas in darkness the excretory activity is not developed, or even ceases if it has begun, although a plentiful supply of food may be available. Similarly, it can hardly be due to the want of food that the floral nectaries of Eranthis hiemalis do not secrete when

${ }^{1}$ Cf. also E. Schulze und Frankfurt, Zeitschr. f physiol. Chemie, 1895 , Bd. xx, p. 532. [P. Knuth (Über den Nachweis ron Nektarien auf chemischem Wege, Bot. Centralbl., Bd. Lxxvi, 1 S98, p. ; 6 ) uses the precipitation of indigo from orthonitrophenylpropiolic acid in the presence of grape sugar as a test for the nectariferous character of the gland-cells of supposed nectaries, but it is hardly possible that this method can apply to all cases, for a reducing sugar need not necessarily be present.]

${ }^{2}$ For actual data, see Bonnier, l. c., p. 192; Schimper, l. c., p. 72.

3 Schimper, 1.c., p. 75. On the influence of the soil, see Bonnier, Bot. Centralbl., Beihefte, IS94, Bd. Iv, p. $4^{19}$.

\& Wrilson, l.c., p. I2. The dependence of the secretion on light in this plant and in the flowers of Lobelia erinus was first discovered by Darwin (Cross and S lffertilization, IS $77, \mathrm{p} .388$ ). 
exposed to strong diffuse light. In certain other flowers (Fritillaria, Helleborus) the secretory activity is developed in darkness, as is also the case in the foliar nectaries of Prmms laurocerasus and Cassia neglecta. A special investigation is therefore necessary in each case to determine whether light influences the secretion of nectar directly or only indirectly.

The functional activity of the nectaries can only be developed at a certain temperature, but when it has once commenced, certain nectaries may continue to excrete at a temperature below that necessary for the inception of their secretory activity. Wilson observed that the foliar nectaries of Prumus laurocerasus continued to excrete nectar at from i to $5{ }^{\circ} \mathrm{C}$.

Honcy-dew, $\mathcal{G} c$. It is not necessary to discuss all the cases in which sugar may be normally or pathologically excreted. The formation of honey-dew is a pathological phenomenon, and usually, but not always, as Büsgen supposed, aphides are the exciting cause. Bonnier has shown that honey-dew may appear when no aphides are present, and it then frequently oozes out through the stomata ${ }^{1}$. The formation of honey-dew is favoured when cool nights follow warm and dry days; Bonnier was able indeed to cause a production of honey-dew by keeping in a saturated atmosphere branches which had been immersed in water. The honey-dew may be formed in such abundance, especially in damp weather, that it may drip from the trees, as is the case in the so-called rain-trees of the tropics (Caesalpinia pluviosa, Calliandra Sammin).

1 Bonnier, Rev. gén. d. Bot., $1 \varepsilon_{96}$, T. vrir, p. 22 ; Büsgen. Der Honigthau, I $\varepsilon_{91}$, and here the related literature will also be found.

${ }^{2}$ Cf. Unger, Sitzungsb. d. Wien. Akad., 1857, Bd. $x \times v, p .450$; Boussingault, Agron., Chim. agric., \&c., 1874, T. V, 1. 3.3; Nusset, Bot. Jahresb., 1879, p. 222 ; Biisgen, 1. c., p. 26 ; Dyer, Bot. Jahresb., $1878, p \cdot 326$. 


\section{CHAPTER VII \\ TIIE FOOD OF PLANTS}

\section{PART I}

GENERAL, VIEW

\section{Section 50. Nutritive Metabolism.}

A SEED or a spore contains but a small portion of the food material which the plant will require during the course of its existence, and hence an additional supply of nutriment must be obtained from without. The amount of food material required may be very great in comparison with that contained in the original germ, as when an oak develops from an acorn, or when a few fungal spores produce in a few days a mycelium many thousand times heavier than their original weight. The percentage composition of a dried plant is such as to indicate that it is carbon and its compounds, i.e. organic substances, which are of primary importance to it.

As a general rule nutrient material becomes of use to the plant only after it has undergone various chemical changes, frequently extremely complex in character, which are included under the head of metabolism. The plant is able to assimilate absorbed food materials into its own living substance, and so to obtain a continual supply of plastic material, while at the same time various destructive processes of metabolism afford the supply of energy necessary for continued existence. In the plant, just as in the animal, both constructive and destructive metabolism are always active, while the fact that a portion of the food must always be used to provide the necessary supply of energy, prevents a plant ever containing the whole of the material which has been absorbed in the process of development. Indeed, it is of the utmost importance that plants should be able to excrete those metabolic products of which no further use can be made.

The fact that a fungus or a higher plant may grow when fed with sugar or glycerine shows that it is able to form from these substances all 
the numerous carbon compounds which take part in metabolism, and by the oxidation of which a supply of energy is obtained. Even when a cell or organ becomes adult a continuance of metabolism is essential so long as life remains, although as a matter of fact the energetic metabolic changes which may still proceed are mainly directed towards the liberation of an adequate supply of energy. Indeed, only a small portion of the organic food-supply may be used as constructive material even by an organ which is growing rapidly, for without the continual supply of energy which the katabolic processes afford. the vital mechanism must come to a standstill as certainly as a steam-engine must cease working when the fires are extinguished. Hence, both animals and plants die of hunger as soon as all the reserve supplies which can be made available as food are consumed, and no further supply of energy or food can be obtained. Though dependent for its existence upon its internal metabolism, the plant or the protoplast can exercise a certain regulatory control over its own metabolic activity.

Nutrition involves chemical metamorphoses of extremely varied character, and hence a few general remarks are necessary upon the nature of the changes which the food material may undergo. Nutrient substances are frequently rendered capable of absorption by extracellular agencies (as in digestion, \&c.), while stored food, by undergoing intracellular metamorphosis, may be transformed into a mobile product which can be translocated to the points where it is required. Moreover, chloroplastids are able to produce sugar or other food material by the remarkable synthetic metabolism of which they are capable under appropriate conditions, and the sugar thus produced plays precisely the same part in the general metabolism of the plant as it would if it were absorbed directly from without. Similarly, all plants appear to be able to form proteid substances, although these may be entirely absent from the food material. The production of proteids by synthesis is a step towards the formation of living protoplasm, and hence is to be regarded as the means by which the latter is constructed and nourished. It is, however, frequently impossible to distinguish clearly between those changes which serve to provide energy and those which render the nutrient material available for use. Indeed, it is probable that the two processes are inextricably associated, owing to the intimate relationships which exist between all vital processes, and their mutual dependence upon one another.

Under the heading of 'metabolism' all those chemical changes are included which are produced by the organism and are of service to it, no matter whether the changes are intracellular or extracellular in origin, or what may be the nature of the causes to which they may be due, or of the means by which they may be performed. The processes occurring in a living organism cannot possibly be expressed in the form of a chemical 
equation, for the result which the energy liberated by a chemical change may produce is entirely dependent upon the inherent nature of the vital mechanism, as well as upon the character, mode of action, and point of application of the impelling force. Similarly, a food substance may be used in various ways, for in different parts of the plant, and even in the same cell, a supply of sugar may be converted into a variety of different carbohydrates, or may be consumed in respiration, or may serve to form the cell-wall, or to produce proteid substance, or may be excreted in the form of nectar in order to attract insects. Again, a change in the internal or external conditions may cause a food substance which has been kept in reserve for a long time to be drawn into the metabolism of the plant, and either to be consumed or used for constructive or other purposes.

It is possible, in a general sense, to distinguish three classes of metabolic products from one another. These are (I) building material, formative or constructive substances; (2) plastic or trophic substances; and (3) aplastic, non-trophic substances. Plastic substances are those which either at once, or after being stored for a time as reserve food, are drawn into metabolism and serve as nutrient material. Aplastic substances are such as take no further part in metabolism, so that they include the substances which form the permanent structural framework of the plant, although we may regard them from a special point of view as building or constructive material $^{1}$.

Many substances take no further part in the exclusively vital processes of metabolism, although they have special and definite functions to perform. Thus enzymes may render other substances soluble or prepare them for use, while tannins, ethereal oils, alkaloid poisons, \&c., are of greater or less importance as protective or attractive agencies. Substances of this kind, which subserve certain specific ends, are produced only at certain times or only in limited amount, and thus can be readily distinguished from those metabolic products which form the inevitable accompaniment to all vital activity, and which are continually being produced so long as any metabolism is possible (Sect. 77). The carbonic acid of respiration and the alcohol produced by fermentation are metabolic products of this character, and in order that the conditions necessary for continued existence may be maintained, such products must be excreted and removed. Erery product which the plant excretes is not necessarily uscless, for the enzymes, the ethereal oils, the nectar, \&c., are of service to the plant for the most part only when excreted. The same is the case when a plastic substance is excreted from one cell in order that it may be absorbed by another, and

${ }^{1}$ [It is perhaps simpler and less confusing to speak solely of (1) plastic substances which can be used in metabolism, and (2) aplastic substances which, under normal circumstances, can undergo no further metabolic change. Hence an excreted plastic substance is still classified as a plastic product.] 
this is a process which plays a very important part in translocation, and indeed in all metabolic exchanges.

No sharp and clear distinction can therefore be drawn between the different classes of metabolic products, for the same substance may frequently be employed in different ways, and incleed the same food particle may serve a variety of purposes during the progress of development or as the external conditions alter. Thus a cell-wall which has hitherto formed part of the mechanical supporting tissue may be transformed into soluble plastic material, and in many of the component parts of the protoplast interchanges and exchanges of substance are probably of continual occurrence. Similarly, the sugar present in the nectar as an aplastic product may be drawn into metabolism again, and in a starving plant the same fate often befalls substances which under normal conditions would have remained intact (Sect. 93), while even such a pronouncedly excretory product as carbonic acid may be reassimilated when the chloroplastids are functionally active.

It is possible to distinguish in metabolism between the processes of 'assimilation' or anabolism and those of 'dissimilation' or katabolism. The term 'assimilation' is sometimes restricted to those processes of constructive metabolism which lead to the formation of organized structures, but it is better to use it as corresponding to the more general term anabolism, and as also including those processes which terminate in the production of plastic substances. Thus the formation of carbohydrates in chloroplastids is a process of assimilation, as is also the formation of proteids by synthesis, and the production of various organic substances by certain fungi from formic or acetic acids. Dissimilation includes all liatabolic changes taking place in the contrary direction, and it is easy to see that the processes of assimilation and dissimilation are often inextricably connected ${ }^{1}$.

The term 'assimilation' was used in practically the same sense as above by Bischoff and Schleiden, and this corresponds to its usage in animal physiology. 'The restriction of the term of assimilation by Sachs to the production of organic material in chloroplastids is therefore incorrect, both from historical and physiological standpoints. The latter is merely a special form of assimilation, and may be termed carbon dioxide assimilation, or more shortly $\mathrm{CO}_{2}$-assimilation 2 .

${ }^{1}$ Still further distinctions may be made. Thus Roux distinguishes between auto-assimilation and the assimilation of foreign substances (Ergcb. d. Anat. u. Entwick, herausg. v. Merkel u. Bonnet, I $89^{2}$, Bd. Ii, p. 430).

${ }^{2}$ Bischoff, Handbuch d. Bot. Term. u. Syst., IS 33 , Bd. I, p. I3; Schleiden, Grundziige d. wiss. Bot., I $8_{45}$, 2. Aufl., Bd. I, p. 278 ; also Nägeli, Sitzungsb. d. Mïnch. Akad., I 879 , p. $2 S_{4}$; Sachs, Experimentalphysiol., I $6_{5}$, p. IS. [The term Carbon-assimilation occurs in the original German, but its use is hardly to be recommended since the corresponding term Nitrogen-assimilation has 
These distinctions are based upon physiological characteristics and purposes, which latter are frequently attained in widely different ways; thus, assimilatory products may be produced by dissociation as well as by synthesis; for example, carbohydrates may be formed either by synthesis from water and carbonic acid, or by the self-decomposition of the protoplasm, or by the complete metamorphosis of proteids. Indeed, plastic substances or special constructive materials are'very often produced by the decomposition of more complex molecules (Chap. VIII).

It is of the highest importance to obtain a thorough knowledge of the nature and causes of the chemical changes and processes occurring in the living organism, and we may use the terms synthesis (anabolism) and analysis (katabolism) in the same sense as the chemist does, so long as we are dealing with purely chemical processes, if we remember that dissimilation and assimilation are terms which have a physiological meaning, and hence do not quite correspond to synthesis and analysis, for an assimilatory product may be produced either by analysis or by synthesis, that is, by progressive or retrogressive chemical metamorphosis.

Constructive and destructive chemical changes are necessarily of constant occurrence, both in constructive metabolism and in the processes by which a supply of energy is obtained. Every chemical decomposition affords a supply of energy to the plant, and whenever any synthesis converts a certain amount of kinetic into potential energy, the former must be derived from processes of decomposition or fermentation, such as lead to the production of carbonic acid, alcohol, \&c. ${ }^{1}$ Hence, during the progress of development various synthetic products appear in greater or less a mount, together with those derived from the unceasing dissimilation. In an adult plant very much less constructive assimilation is necessary, and in cells which merely require a supply of energy, all such synthetic processes may under special nutritive conditions be absent.

Metabolism is possible only when a supply of appropriate food material is assured, but all plants do not require the same food, and the difference is especially marked with regard to carbon compounds. Thus many fungi are able to grow when sugar is the sole organic substance supplied to them, if the other elements they require are presented in appropriate form as inorganic salts, while in a few cases the sugar may be replaced by formic acid. Certain fungi, however, are unable to form proteids by synthesis when

already been employed to indicate the assimilation of free nitrogen by certain bacteria, \&x. The term carbon dioxide assimilation is moreover the more comprehensive one, and includes the two allied subordinate processes of carbon-assimilation and oxygen-excretion, which processes are probably separated from one another by distinct intervals of time and place, howerer minute these latter may be.]

${ }^{1}$ See Pfeffer, Studien z. Energetik, I $\$ q^{2}$. 
nitrogen is presented to them in the form of ammonium nitrate, and they must therefore be supplied with peptone or albumin; on the other hand a few organisms are actually able to assimilate free nitrogen (Sect. 68). The fact that growth is possible under these circumstances shows that all the substances necessary for constructive metabolism can be produced, and that a supply of energy is also obtained. A fungus, which can grow with formic acid as its sole organic food, decomposes a portion of this nutriment in respiratory and other processes, and so obtains the energy necessary for the synthesis of carbohydrates, fats, and even proteids from formic acid, water, and simple inorganic salts. The nitrite and nitrate bacteria can actually commence their synthetic metabolism with carbon dioxide and water, just as a green plant does, the necessary supply of energy being obtained by the oxidation of ammonia to nitrous or to nitric acid (Sect. 63). It is not impossible that organisms may be discovered which obtain the energy necessary for the assimilation of carbon dioxide by the decomposition of organic substances, as is indeed actually the case in those fungi which can be fed with formic acid, for it is immaterial whether the energy is obtained from the same compound that is assimilated or from another one. In all cases every manifestation of energy of which the plant is capable is dependent upon chemical changes of some kind or other'.

With the exception of the nitrite and nitrate bacteria, no organisms are known which can utilize chemical energy in the production of organic food from carbon dioxide. Green plants, however, possess a mechanism by means of which the energy of the rays of light can be employed for this purpose, while the purple water-bacteria possess a compound assimilatory pigment by means of which not only light but also dark heat rays may be utilized in the assimilation of carbonic acid (Sect. 52), and it is incleed possible that organisms may be discovered which are able to make use of other forms of radiant energy for the assimilation of carbon from carbon dioxide. Indeed, in certain cases electrical energy may be of service, for organic and nutritive substances may be artificially produced by synthesis under the influence of electrical forces. It is possible, therefore, to distinguish between photosynthesis, thermosynthesis, chemosynthesis, elcctrosynthesis. \&c., according to the source from which the energy for synthesis is obtained.

Owing to their power of manufacturing organic food, green plants can develop in water or in pure sand, provided the necessary inorganic salts are supplied to them, whereas a fungus can only grow on such a medium when sugar is added. Fungi, and other plants without chlorophyll (with the exceptions mentioned above), either obtain their organic food from dead

1 For details see Pfeffer, Studien 2. Energetik, I 892. 
organic matter, as saprophytes, or from living organisms, on which they are parasitic. Certain plants, however, obtain a portion of their organic food from the external world, while the rest is formed from carbonic acid and water; and many plants when adult obtain all their nutriment in this manner, whereas when young the whole of their organic food was absorbed from without (Sect.64). There can be no doubt that we may ultimately succeed in completely and satisfactorily replacing the sugar which the green plant itself produces by introducing appropriate artificial food (Sect. 55).

However important the photosynthetic production of organic material may be in the nutrition of the green plant and in the balance of nature, it is simply a special mode of obtaining organic nutriment, and the nonchlorophyllous plant uses the sugar which it obtains from without in just the same manner as the plant which can manufacture sugar for itself. Moreover, the power of producing sugar is limited to the chloroplastids, so that the plasma of a cell containing chlorophyll, and all the nonchlorophyllous cells of the shoot and of the root, live upon the sugar supplied to them in the same way as saprophytic or parasitic fungi, which absorb the substances constructed from the sugar produced by green plants.

An animal is not always feeding, nor is a plant occupied continuously in obtaining food, although, as in every organism, life involves unceasing metabolism and a continuous liberation of energy, dependent for the most part upon the processes of respiration which persist during day and night in both animals and plants. In green plants, however, in the daytime respiration is largely masked by the assimilation of carbon dioxide, which is much more active under normal conditions than is the former. The total amount of carbon assimilated must naturally be greater than that consumed in respiration, in order that a green plant which obtains all its organic food by its own assimilatory activity may be able to grow and increase in dry weight. Not merely the organic food of all plants is derived from this source, but the flesh and blood of animals is of similar origin, for carbon dioxide and water, which are the ultimate products of decomposition, are synthesized again by green plants by means of radiant energy from the sun.

The photosynthetic assimilation in the chloroplastid only provides the organic food, which in green and non-green plants, and in animals also, has the same function to perform. Whatever its source may be it provides plastic and constructive material, and at the same time a supply of potential energy. In all essential fcatures, therefore, the metabolism of plants resembles that of animals, for in both cases it is based upon chemical changes involving a liberation or redistribution of energy ; and plants, like animals, must sacrifice a large portion, and often almost the whole, of their nutriment in order to provide a sufficient supply of kinetic energy. In 
relation to their respective weights, the respiratory activity is often much greater in plants than it is in a mammal, although in the former no constant body temperature has to be maintained (Sect. 95). The mere fact that so many non-green organisms exist should suffice to show that the metabolism in plants is not essentially different to that in animals, although this is an error which is still frequently made .

No definite line of demarcation can be drawn between animals and plants, either from a physiological or a morphological standpoint, for both have a common origin, and it is only in the higher forms that highly specialized structures and peculiar physiological properties have been acquired. Hence it is naturally in the lower forms that the resemblances and points of similarity are most marked, but all embryonic protoplasts are similarly constructed and constituted. At the same time, in plants as well as in animals, special substances may be formed, either for definite purposes or as the unavoidable by-products of a slightly different metabolism. Cellulose is a product of this nature, and is very largely employed by plants as a mechanical and supporting framework; in animals it occurs so rarely that its presence was formerly erroneously supposed to indicate the vegetable nature of an organism. There is indeed less difference between the metabolic products of certain plants and animals than exists between those of some species of bacteria, fungi, and other plants. Little is known concerning the metabolism of the lowest animals, but it is probably very similar to that of plants, and, as time goes on, more and more of the substances supposed to be characteristic of animal metabolism are being discovered in plants. It is possible that many of the lower animals can form proteids by synthesis, while plants exist which have not this property, and which must therefore obtain their proteid food from other plants or animals. However the proteids may be obtained, they are subjected to various changes in the course of metabolism, and indeed with certain forms of nutriment a fungus may be only able to grow by completely decomposing the proteids supplied to it (cf. Sects. 77.80, 64, 68).

In the progress of their phylogenetic development most plants have become adapted to a permanently non-motile existence, and are hence unable to seek their food in the way that most animals do, although various forms exist which occupy an intermediate position as regards their mode of nutrition. Animals commonly swallow solid food, whereas plants always absorb it in solution; but this is by no means an essential point of difference, for the solid food is digested and made soluble in the stomach of an animal in a manner similar to that in which the pitcher of a carnivorous plant digests a piece of meat. Many fungi are also able to excrete 
enzymcs, by means of which extracellular digestion is possible, and certain animals, on the other hand, such as the Tapc-worms and many Infusoria, absorb nutrimcnt in solution through the outer body wall. In Protozoa undigested fragments are thrown out again through a permanent aperture (mouth or anus) or through a temporary opcning made in the body wall, and the same occurs when the plasmodium of a Myxomycete excretes undigested solid particles. No such cxcretion is nccessary in typical plants, owing to the fact that the whole of the food is absorbed in solution, while the final excretory products of metabolism arc for the most part volatile or soluble, and hence arc able to escape from the enclosed plant cells to the surrounding air or water.

Higher plants exhibit a large amount of differentiation of labour as regards the parts which the various organs play in obtaining nutriment. In terrestrial plants the roots which attach them to the soil absorb the nutritive substances prescnt in the latter, while the leaves are mainly concerncd in the absorption of gases from the air. In order to obtain food, a certain amount of energy must be expended even in plants which do not hunt down their prey as animals do, for the development of absorptive organs and the penetration of the root into the soil involve a certain expenditure of energy. The same occurs when enzymes are secreted; indeed the plant frequently sacrifices a large proportion of its food in order to obtain the energy necessary to construct the essential plastic and constructive materials.

Carbon compounds provide both energy and materials for growth, so that it is imperativc that an adequate supply of the appropriate organic food shall always be at the plant's disposal. In addition to carbon, hydrogen, oxygen, and nitrogen, plants contain small traccs of certain other essential elements, which form part of the ash left bchind on burning. Thesc are potassium, magnesium, phosphorus, sulphur, iron, and, except in Fungi and certain Algae, calcium also. These ash constituents are always prescnt not only in a picce of wood, but in every cell and every particle of protoplasm. Silicon, sodium, and other elements may also occur, and although these are not necessary to the plant, they may occasionally be present in great abundance. Much more than the necessary minimum of the essential elements is commonly absorbed, and hence the ash may form as much as 20 per cent. of the dry weight in a few cases, although the presence of 2 to + per cent. by weight of ash suffices for all requirements. Only very small amounts of sulphur and iron are neccssary, but nevertheless, in the absence of these minute quantities the vital activity of the plant comes to a standstill, just as a watch stops when the tiniest wheel is rcmoved which forms an essential part of its working mechanism.

The analysis of the ash constituents gives no indication as to the form in which the mincral elcments are retaincd by the living organism, but it is 
at least certain that the ash constituents, as well as nitrogen, become of importance only when actually assimilated. Sulphur takes part in the formation of the molecule of proteid compounds, and many remarkable phosphorized proteids exist. A plant may contain large quantities of uncombined nitrates or ammonium salts, and frequently the elements of the ash may in part be present in the form of inorganic compounds. Many of the organic metabolic products contain neither nitrogen nor any ash constituent, for in the processes of metabolism not only carbon, but also nitrogen and the mineral constituents, may be dissociated again as inorganic compounds from the organic substances with which they were united.

It is one of the aims of physiology to trace all the changes which an absorbed food material may undergo, and the first thing necessary is to determine empirically which are the essential elements, and which compounds of them form most suitable food. From this point of view it is permissible to treat separately of the ash constituents as a group apart, for most plants absorb them in inorganic form, or are at least able to do so if necessary. Nitrogen also may be absorbed either in the form of an inorganic salt or of an organic compound.

The very unequal nutritive values of different carbon compounds show that every molecular combination is not of equal value in the nutrition of a particular plant. The inorganic salts absorbed are for the most part highly oxidized, and hence the energy necessary for their assimilation by the protoplasm must be derived from the oxidation of pre-cxistent carbon compounds by respiration, or from intracellular molecular decompositions which involve a liberation of energy. Carbon compounds do not, however, furnish the sole source of energy in all cases, for the energy necessary for the synthesis of proteids from carbon dioxide, water, \&c. by the nitrite and nitrate bacteria, is obtained by the oxidation of ammonia to nitrous acid, or of nitrous to nitric acid, while certain other bacteria obtain encrgy by the oxidation of sulphur or sulphuretted hydrogen.

It is extremely difficult to determine precisely what part an element plays in the metabolism of a plant, for it may be utilized in a variety of ways; indeed it is not always easy to say to what extent a particular substance serves as food material at all. If every element which is of use to the plant is regarded as having a nutritive function, then both the non-essential sodium and silicon must be admitted as forming part of the food of plants, for these elements are certainly not entirely useless. Such carbon compounds must also be included as remain intact under normal conditions, and are consumed only when the plant is starved. Nutritive substances may be replaced by others of this character; but we understand by an essential clement one without a certain minimum of which the plant is unable to exist. 


\section{SECTION 51. The Circulation of Food Materials in the Organic World.}

In the entire organic world destruction and reconstruction proceed simultaneously and without cessation, and in order to supply the energy necessary for the maintenance of life upon the earth, enormous masses of organic material must be daily decomposed into carbon dioxide and water. Living organisms aid in the destruction of dead ones, and such exceedingly minute forms as bacteria are especially adapted for this purpose, owing to their rapid powers of multiplication and their intense metabolic activity. Processes of oxidation also play an important part in the decomposition of organic remains, and though these and the vitalistic aids to disorganization often seem of trifling importance, nevertheless, owing to the continuity of their action, much more may be accomplished by them than by sudden and violent, but local, conflagrations, as, for example, the burning of a forest. Slow decomposition ultimately results, after many changes, in the production of the same final products as those of ordinary combustion, namely, carbon dioxide, water, inorganic salts of nitrogen, and residual ash.

Unless certain plants had the power of regenerating organic substance from these ultimate products of decomposition. the supply of organic material would gradually be consumed, and all terrestrial life would cease. This power is practically restricted to green plants, so that both animals and plants derive their energy either directly or indirectly from the sun's rays, which are converted into chemical energy by the agency of the chlorophyllapparatus. When a piece of coal is burnt, a portion of the sun's radiant energy is liberated which was stored up in the form of potential energy many years ago, so that every steam-engine is directly or indirectly driven by the sun.

The absolute amount of energy thus liberated or stored up cannot be precisely calculated, since the processes of decomposition and reconstruction in the living organism are frequently interrupted by converse changes; moreover, both construction and destruction occur simultaneously in every meadow, forest, or pond. The fact that life has persisted for many ages shows, however, that in spite of local or temporary variations, an approximate balance has, on the whole, been maintained, for had the processes of destruction been the least degree more active than those of reconstruction, the total amount of organic material must ultimately have gradually decreased, until all further continuance of life became impossible. This is a logical conclusion of more decisive value than any observations made during the limited period allotted to man. The latter, however, do actually show that the average percentage amounts of oxygen and carbon dioxide present in the air remain approximately constant. 
The amounts of organic material which are decomposed and reconstructed in the progress of a year are relatively enormous; thus mankind produces I,200 million kilogrammes of carbon dioxide daily, taking the population of the earth as I,500 millions, and the amount daily evolved by a single man as from 800 to 900 grammes. The whole of the rest of the animal kingdom without doubt decompose much more organic material than mankind does, as do also the countless host of parasitic or saprophytic plants. By burning coal, man restores large quantities of carbon dioxide to the air, the carbon of which had been stored by the plants of past ages. Supposing that the 460,000 million kilogrammes of coal ${ }^{1}$ annually burnt contain only 75 per cent. of carbon, still this gives a yearly addition of $1,265,000$ million kilogrammes of carbon dioxide to the air.

The atmosphere contains from 2,000 to 3,000 billion kilogrammes of carbon dioxide, although the average amount present is only $0.0_{3}$ to $\mathrm{c} . \mathrm{O}_{4}$ per cent. In comparison with these numbers the amount of carbonic acid produced annually is a very appreciable quantity, and the percentage in the air remains constant, because green plants are just able to decompose this annual addition. The importance of the assimilation of carbon dioxide can therefore hardly be overestimated, for not only does it keep the percentage of carbon dioxide well beneath the limit at which it becomes poisonous, but it also renders possible a continual reconstruction of organic substance from the ultimate products of decomposition. In Europe the harvest from a hectare averages about 6,700 to 7,800 kilogrammes, so that the total yield from all the fields and meadows of Germany would contain about 13,000 billion kilogrammes of carbon, to obtain which, 50,000 billion kilogrammes of carbon dioxide must be decomposed 2. Ebermayer has calculated that a hectare ( $2 \frac{1}{2}$ acres) of forest requires annually about I I,coo kilogrammes of carbon dioxide, and hence that the total forests of Bavaria must have assimilated about 29,000 million kilogrammes of this gas in one year, and set free about 20,000 million kilogrammes of oxygen, while the total amount of carbon dioxide produced by the fires and respiration of the Bavarian people was less than half the amount required.

The production of organic material is still more active in tropical climates when the external conditions are favourable, but, at the same time, the more rapid decomposition and disintegration render a certain compensatory adjustment possible ${ }^{3}$. Local differences in the rate of production and decomposition naturally do not perceptibly influence the general

1 Credner, Elemente d. Geologie, $1 \varepsilon_{91}, 7$. Aufl., p. $4^{6} 4$.

2 Ad. Meyer, Versuchsst., 1892 , Bd. XL, p. 205 ; Ebenmayer, Sitzungsb. d. Bair. Akad., $188_{5}$, Bd. $\mathrm{xr}$, p. $3 \circ 3$.

${ }^{3}$ Cf. Lwart, Ann. d. Jard. Bot. d. Buitenzorg, 2. suppl., IS98, p. S9. 
average, but the fact that the most important end-product of decomposition is a gas $\left(\mathrm{CO}_{2}\right)$ is of great value in ensuring a rapid transference of carbon from one locality to another, while at the same time the mobile nature of water and its ready evaporation are factors of great importance in maintaining the balance of nature.

Plants are able to assimilate the ash constituents and compounds of nitrogen only by means of the energy derived from the combustion of the organic substance which is produced by means of solar radiation. Certain organisms are even able to assimilate free nitrogen by means of chemical energy, while on the other hand the decomposition of the synthesized nitrogenous products may render available a larger or smaller supply of kinetic energy; and indeed a decomposition of nitrogenous organic compounds which may or may not involve a liberation of free nitrogen is an absolute necessity in the balance of nature. On the other hand, the re-combination of free nitrogen (Sect. 68) can be brought about by certain organisms and by electrical discharges. The chemical energy contained in the organic or inorganic substances produced by the partial or complete decomposition of organized bodies is in all cases derived from the work done by the absorbed sunlight. Similarly, it is stored solar energy which a nitrite bacterium derives from the oxidation of ammonia, and which enables it to build up organic substance from carbon dioxide and water ${ }^{1}$. This is the case again when ammonium nitrite formed in the air by electric discharges is oxidized, for the energy of the lightning comes indirectly from the sun. If a sufficient supply of oxidizable inorganic compounds was deposited as the earth cooled, the oxidation of these might have afforded the energy necessary for the existence of the first and most primitive organisms, so that these need not necessarily have been at the outset furnished with the power of assimilating carbon dioxide (cf. Sect. 5).

The perpetual changes on the surface of the earth are by no means entirely due to the existence of life upon it. Indeed, a knowledge of the changes to which inorganic nature is subject is of the utmost importance for a clear comprehension of vital phenomena; but the former is more the province of the chemist, the physicist, and the astronomer than of the physiologist, though it must be remembered that these are convenient limitations established by man, and not by nature, and that a comprehensive view of the entire cosmic scheme is possible only by disregarding any such artificial distinctions.

Stationary chemical and physical equilibrium may be maintained for longer or shorter periods both in animate and inanimate nature. Thus in a living oak tree, a particle of carbon may be retained as a fixed constituent

1 Pfeffer, Studien z. Energetik, ISy2, p. 206. 
of a cell-wall for more than a thousand years, and millions of years may elapse before the carbon of a particle of coal is converted into carbon dioxide, or before the carbonic acid of a limestone rock is set free again. Similarly, atoms of oxygen, nitrogen, phosphorus, \&c., may be withdrawn from circulation for a longer or shorter period in some form or other which is inaccessible for the time being. In this respect climatic and telluric factors are of especial importance, and these are largely dependent upon cosmic relationships, and especially upon the radiant energy emitted by the sun. It is these agencies which determine the distribution of heat and of light over the surface of the globe, thus creating over certain areas the essential general conditions for the existence of living beings, of which the organisms containing chlorophyll, by converting radiant into chemical energy, supply the propulsive force for the entire organic world.

Sudden cruptions of energy, producing catastrophic changes, influence the surface of our planet less than docs the gradual wear and tear of ages, and similarly, in the organic world much more is done by slow and gradual actions extcnded over long periods of time than by sudden and rapid alterations. The development of a plant involves a gradual but incessant process of construction, and it is by their unceasing activity, aided by their minuteness, that bacteria are able to decompose such vast quantities of organic substance in the progress of a year. Similarly, it must have taken millions of years to produce the enormous deposits of organic material which have decomposed to form the coal-beds.

The changes and decompositions which occur in dead substances are of great importance to the organic world, and thus the carbon of coal can be assimilated by a green organism only when it has been converted into carbon dioxide, either by burning or by more gradual oxidation, such as may occur in nature when oxide of iron is present. Organic substances are continually undergoing decomposition, and such products as carbon monoxide, carburettcd hydrogen, \&c., may by oxidation be converted into substances which can be again assimilated.

The weathering and decomposition of rocks takes place mainly by mcchanical and chemical means, but living organisms may also assist in the process, and it is they alone which produce the admixture with organic materials that constitutes a humus soil. In the weathering of silicious rocks carbonic acid is usually fixed and removed for the time being, but above a certain temperature silicic acid drives out carbonic. Hence, when the earth was still hot, the air must have contained much more carbon dioxide than it does now, and this excess was probably deposited in the carbon of the coal deposits. The first organisms that appeared on the surface of the earth probably had a more abundant supply of carbon dioxide than now exists, and were under different conditions in other respects also (Sect. 57). This antagonism between carbonic and silicic acids is of 
the utmost importance in the formation of rocks and in determining the configuration of the surface of the globe, for both substances are universally present, and the decomposition and reconstruction of silicious and carboniferous rocks are processes which are perpetually recurring.

The distribution of diffusible and transportable substances is a purely physical problem, as is also the continual readjustment of differences of potential. From this standpoint it is of the highest importance that oxygen and carbon dioxide should be gases which are readily and rapidly distributed throughout the atmosphere. Water, on the other hand, will wash out all the saline constituents of a sandy soil, and hence arises the great importance of the absorptive and retentive powers of a soil rich in humus (Sect. 28). When the covering of vegetation disappears from a localized area, the unavoidable trifling loss of nitrogenous compounds and ash constituents is counterbalanced by fresh supplies derived from the water of the soil, from rain and dust, and from the weathering of rocky fragments, so that by means of the absorptive powers of the humus the nourishment necessary to permit of the reappearance of vegetation is retained for a time. These agencies are insufficient, however, to make up for the large quantities of ash constituents and nitrogenous compounds which are removed from the soil by the annual harvest, and hence gradual impoverishment ensues unless the constituents which are removed are replaced by means of a supply of appropriate manure.

By the weathering of rock, and of the rocky particles in the soil, a continual supply of the essential ash constituents may be provided for for a very long, but not for an indefinite, time, and indeed every river annually carries a certain amount of the soluble saline constituents of the soil to the sea. The gradual sinking and rising of land which is always occurring, is perhaps quite sufficient to counterbalance this loss, for the soil of a new continent rising from the floor of the ocean may contain all the constituents removed by rivers from pre-existent continents and carried to the sea. The nitrogen of the nitrogenous compounds washed away from the soil may return to dry land in the form of volatile compounds, such as ammonia and oxides of nitrogen, or may be set free as atmospheric nitrogen. Our knowledge of life and the conditions of existence in the depths of the ocean is very incomplete ${ }^{1}$; we do not know whether all the organic food for deep-sea life is derived from the surface, or whether certain of the deep-sea organisms can produce, by means of chemical energy, the organic material required by themselves and by the organisms which may prey upon them, or whether organisms

1 For the lowest depths at which Bacteria have been found, see Dieudonné, Biol. Centralbl., I 895, p. 108; C. Schröter, Vegetation des Bodensees, I 896, p. 16. 
may not exist which are able to liberate free oxygen by means of energy derived from chemical decompositions, and which may thus enable aerobic organisms to exist in the depths of the ocean.

\section{PART II}

THE ASSIMILATION OF CARBON DIOXIDE

\section{A. Photosynthetic Assimilation ${ }^{1}$.}

\section{SECTION 52. General.}

It is the chlorophyll-containing parts and organs which have this special power of producing organic substance from carbon dioxide and water, by means of the energy of radiant light, a process which is accompanied by an excretion of free oxygen. For the correct interpretation of this process Engelmann's ${ }^{2}$ observation that purple bacteria have a similar power of photosynthetically assimilating carbon dioxide is of great importance, although the feeble assimilatory activity of these organisms cannot be of much importance in the balance of nature.

Since chlorophyllous plants obtain all their organic food from the synthesis of carbon dioxide and water, they are able to grow in pure sand if supplied with the other essential elements in the form of appropriate inorganic compounds. Thus maize, barley, buckwheat, beans, and very many other plants grow admirably under such cultural conditions, in which the large amount of organic materials, ultimately far surpassing the weight of the seed, are obtained by the photosynthetic assimilation of carbon dioxide. The entire organic substance of the harvest taken from a field, or of a tree removed from a forest, is derived from the same source, for

' [The term 'photosynthctic assimilation' is a perfectly general one, and would include the assimilation of other compounds by the aid of light, should any such processes be discovered in the future. The photosynthetic assimilation of carbonic acid may be termed carbon dioxide assimilation. 'Carbon-assimilation' is obviously incorrect, for in analogy with the term 'nitrogen-assimilation' it would indicate that carbon could be directly assimilated. The uncouth term 'photosyntax' is quite unnecessary, and moreover has been erroneously used to indicate all cases of carbon dioxide assimilation, although the occurrence of a power of chemosynthetic assimilation of carbonic acid in certain liacteria was already well-known.]

${ }^{2}$ Engelmann, Bot. Zeitung, i 888 , p. 66r. [The chromophyll of purple bacteria appears to be a compound pigment, from which chlorophyll and a pinkish-red dye may be extracted.] 
such plants may not absorb any organic nutriment even from a soil rich in humus (Sects. 50, 51, and 64). Indeed green plants produce much more organic material than can possibly be harvested, for a large part is decomposed again in respiration, or is lost by the fall of old lcaves or dead parts.

The assimilation of carbonic acid is possible only by means of the radiant energy of the sun, and it ceases instantly in darkness, so that only those chlorophyll bodies are functionally active which are exposed to light, and hence chloroplastids are usually found only in the subaërial organs, and especially in the leaves. An adequate supply of carbon dioxide and an optimal intensity of light are neccssary for the full functional activity of the chloroplastids. The atmosphere contains mere traces of carbon dioxide, and hence various adaptations have arisen by means of which rapid gaseous exchange is rendered possible, for almost the whole of the vast quantity of this gas which a green leaf may assimilate comes directly from the air. The same applies to an aquatic plant, for the water usually contains but little carbon dioxide, though relatively more than the air.

In a confined space the supply of carbonic acid is extremely limited, and is soon used up, while every morning the respiratory carbon dioxide which has accumulated during the night suffices to allow the chloroplastids to exercise their normal functional activity for a short time. If, however, the exhaled carbon dioxide is continually absorbed by a solution of potash, the dry weight of the plant gradually decreases just as it would do if kept continually in darkness.

Since respiration necessarily accompanies all vital activity it must also continue without cessation in all cells which are assimilating carbonic acid. This is shown by the fact that growth and streaming movements are still exhibited when a green cell is exposed to light in an atmospherc of hydrogen. It is possible to prove directly that both green and nongreen cells evolve carbon dioxide acid without cessation, both in the light and in darkness (Chap. IX), although the chlorophyllous cell when exposed to light may, as the resultant of the two processes of respiration and assimilation, excrete oxygen and absorb carbon dioxide from the surrounding air. In darkness green plants respire undisturbedly, and hence there must be a certain intensity of illumination at which the composition of the surrounding air remains unchanged.

From what has been said above it is evident that the assimilation of carbonic acid is simply a special means by which a supply of organic food is ensured, and that this food has the same importance in the metabolism of the plant as has similar organic food absorbed from without. This organic food, from whatcver source it is derived, must be distributed to all parts which consume it, and hence the starch-grains which may appear in the chloroplastids of many plants when assimilation is active, 
disappear again when the latter is feeble or absent. Starch is therefore one of the products of carbon dioxide assimilation, but it is quite uncertain what are the precise changes which lead to its formation (Sect. 54), and indeed no clear insight into the processes involved in the assimilation of carbonic acid has as yet been obtained. It is not impossible that the synthesis does not always take place in precisely the same manner in all plants, and it may be very different in purple bacteria and in normal chlorophyllous plants. (Cf. Sect. 6I.)

The assimilation of carbon dioxide is a vital function, the chloroplastids being living mechanisms specially adapted for this purpose, and capable of exercising their functional activity only when all the essential component parts are intact. The coloured pigment chlorophyll simply forms a cooperating member of this mechanism, and indeed the assimilation of carbon dioxide may be possible when no chlorophyll is present. Colourless chloroplastids, it is true, show no power of assimilating carbon dioxide, but the yellow etiolin-corpuscles formed by most green plants when grown in darkness may exhibit a faint power of carrying out this process. The full assimilatory powers are, however, exhibited only by normal green chloroplastids $^{1}$, and hence the importance of the chlorophyll pigment seems merely to be due to the fact that by means of it a very pronounced absorption of the radiant energy of light is possible.

By means of the bacterium-method, Engelmann (Bot. Zeitung, I 88 I, p. 445) observed in an isolated case that the etiolated chloroplastids of a plant grown in darkness showed a faint evolution of oxygen when exposed to light. A possible explanation, however, was that minute traces of chlorophyll were present, though difficult of detection by direct observation, for as a matter of fact, many plants form an abundance of chlorophyll in darkness. Ewart (Journ. of Linn. Soc., I 897, Vol. xxxI, p. 573) has, however, conclusively proved that in the absence of all traces of chlorophyll, etiolated chloroplastids may show a faint power of carbon dioxide assimilation. After a prolonged sojourn in darkness this power is lost, evidently owing to some functional derangement, since the same amount of etiolin may be present as before. Etiolin (xanthophyll) is therefore an assimilatory pigment, though not nearly so efficient as is chlorophyll. Kohl (Ber. d. Bot. Ges., Bd. $\mathrm{xv}, \mathrm{I} 897$, p. II I) supposes that the secondary assimilatory maximum in the blue region of the spectrum is due to the absorptive activity of the xanthophyll and carotin present in all chloroplastids.

Chlorophyll when isolated is as little able to effect any assimilation of carbon dioxide ${ }^{2}$ as when it forms part of a dead chloroplastid, and it is

${ }^{1}$ Cf. Boussingault, Ann. d. sci. nat., I864, v. sér., T. I, p. 315 , and r 869 , v. sér., T. x, p. 337 ; Engelmann, Bot. Zeitung, 1887, p. 419 .

2 Regnault's contradictory results are incorrect (Compt. rend., I $s_{5}$, T. CI, p. I293). See Jodin 
possible by various agencies to inhibit the power of carbon dioxide assimilation for a longer or shorter time without perceptibly affecting the shape or colour of the chloroplastids (Sect. $5^{8}$ ). Hence it is evident that the mere presence of chlorophyll in the cytoplasm will not necessarily confer a power of assimilating carbon dioxide upon it, for the process can only proceed when the proper functional relationship exists between the two ${ }^{1}$. Hence it is not impossible that normal green chloroplastids may be found which never develop any power of assimilating carbon dioxide, and in all cases the assimilatory activity of a chloroplastid is not dependent solely upon the amount of chlorophyll which it contains.

The chloroplastids are often coloured red or brown by an admixture with other pigments, but the latter do not hinder the process of carbon dioxide assimilation, and may indeed be of importance in rendering certain rays of light available for use (Sect. 60), although when combined with the plasma of a non-chlorophyllous chromatophore they confer upon it no power of photosynthetic assimilation. Thus red and yellow chromatophores in which no chlorophyll is present, as well as colourless leucoplastids, cannot assimilate carbon dioxide, and the purple bacteria are able to assimilate apparently only because they contain a certain amount of chlorophyll, for other coloured bacteria in which no chlorophyll is present have not this power $^{2}$. It is, however, not impossible that colourless organisms may exist which are capable of assimilating carbon dioxide when exposed to light or to heat rays; indeed we are acquainted with certain colourless bacteria which are actually able to assimilate carbon dioxide by means of chemical energy (Sects. 50 and 63).

No living chlorophyllous chromatophore has as yet been observed which permanently exhibits an entire incapacity for photosynthetic assimilation, and Ewart has shown that an inherent power of carbon dioxide assimilation resides in all the chloroplastids which Dehnecke supposed were permanently inactive. Thus the green starch-bearing plastids in the stem of Pellionia and other plants are able to assimilate carbon dioxide when the starch is partially or entirely removed ${ }^{3}$. Similarly the chloroplastids of Euphrasia officinalis exhibit an active power of carbon dioxide assimilation, but are readily rendered inactive by injurious external agencies (Sect. 58). It was apparently owing to a condition of temporary inactivity having

(ibid., r 886, T. cII, p. 264), Pringsheim (Ber. d. Bot. Ges., I886, p. lxxxvi), and Beyerinck (Bot. Zeitung, $\left.1890, \mathrm{p} \cdot 74^{2}\right)$.

${ }^{1}$ [The cells of certain phanerogams may contain diffuse chlorophyll, and yet be able to assimilate (Ewart, Journal Linn. Soc., Vol. xxxi, pp. 449, 569). We are probably dealing here with undifferentiated chlorophyll corpuscles.]

${ }^{2}$ Ewart, Annals of Botany, XI, I $\$_{97}$, p. 486 ; Journal Linn. Soc., Vol. xxxili, I 897 , pp. I 23,147 .

${ }^{3}$ Dehnecke, Über nicht assimilirende Chlorophyllkörper, Bonn, IS80, p. 45; Ewart, Assim. Inhib., Journ. of I.inn. Soc., 1896, Xxxi, p. 436. 
been induced in the plants employed by Bonnier that he was able to detect no evolution of oxygen from them ${ }^{1}$. Other green parasites, such as the mistletoe ${ }^{2}$, actively decompose carbon dioxide, and even Neottia nidus avis, when exposed to bright light, gives off slightly more oxygen than it consumes, although it contains only very little chlorophyll ${ }^{3}$. A distinct power of photosynthetic assimilation is exhibited by the brownish and yellowish chloroplastids of Cuscuta cephalanti and C. europaea (Ewart, l.c., p. 448), and the active photosynthesis of which red and brown algae are capable has long been known ${ }^{4}$. All the lower animals which contain definite chloroplastids can assimilate carbon dioxide when exposed to light, and hence it is unnecessary to discuss whether these are symbiotic algae or an actual part of the animal itself ${ }^{5}$.

The photosynthetic assimilation of carbon dioxide is possible only in the presence of cllorophyll or of etiolin, and hence non-green plants or parts of plants exhale approximately the same amount of carbon dioxide in the light as in darkness, as was first shown by Senebier and de Saussure (Sect. 104). This applies not only to fungi and to roots, but also to leaves which have become colourless and chlorotic owing to a deficiency of iron ${ }^{6}$.

It is easy to show by means of the bacterium-method that in each single cell or mass of cytoplasm the powcr of evolving oxygen in the light is directly dependent upon the presence of chlorophyll. On the other hand, the chloroplastids can form starch in the darkness if supplied with sugar, and this leucoplastic function may be exercised in the entire absence of all chlorophyll (Sect. 55).

The actual assimilation of carbon dioxide probably talies place entirely in the chloroplastid, for by means of the delicate bacterium-method it may be shown that isolated chloroplastids occasionally continue to evolve oxygen in the light for a few hours, if placed in an isosmotic sugar solution ?. An isolated chlorophyll body may therefore, like a separated

1 Bonnier, Compt. rend., I891, T. CXIII, p. I074. Cf. Ewart, 1.c., p. 446.

${ }^{2}$ Luck, Ann. d. Chem. u. Pharm., i $8_{51}$, Bd. Lxxvin, p. 85.

${ }^{3}$ Drude, Biol. v. Monotropa u. Neottia, I873, p. I8. Cf. Wiesner, Flora, 1874, p. 73.

* Poiret, de Candolle, Physiol. d. Plantes, T. II, p. 703; Daubeny, Phil. Trans., 1836, Pt. i, p. 153 .

${ }^{5}$ [Both may be possible; thus the yellow cells of Radiolaria and the chloroplastids of Hydra viridis (Zoochloranthellae, Beyerinck) seem to be symbiotic algae (Brandt, Monatsber. d. Berl. Akad., I88 1 , p. 388 ), whereas in Vorticella campanula the chlorophyll is diffuse, and forms part of the animal's substance, and in the brown flagellate infusorian known as Pcridinium (Ceratium) tripos the diffuse assimilatory pigment seems likewise to form part of the body of the animal.] Cf, also Bïtschli, Protozoen, 1887-9, Bd. 111, p. 1473; Dantec, Ann. d. l'Inst. Pasteur, 1892, T. vi, p. 190, and the literature here quoted. See also Scct. 65.

${ }^{6}$ Pfeffer, Physiol., x. Aufl., Bd. I, p. I85; Zimmermann, Beiträge z. Morph. u. Physiol., 1893, p. 30 .

7 Engelmann, Bot. Zeitung. I88 I, p. 446; Haberlandt, Function u. Lage d. Zellkernes, 1887, p. I18; Ewart, Journ, of Linn. Soc., 1896, Vol. xxxi, p. 423. Observations made upon the chloro- 
muscle, continue its functional activity for some time after it has been removed from its normal habitat. The evolution of oxygen from the isolated chlorophyll body is always feeble, and soon ceases, which is hardly surprising when we bear in mind the fact that the chloroplastids when intact in the cell may be rendered inactive by various external agencies ${ }^{1}$. The positive results quoted do not however negative the possibility that the assimilatory activity of the chloroplastid is aided and sustained by the relationships which exist between it and the surrounding plasma, though it may be shown by means of the bacterium-method that in Spirogyra oxygen is evolved only from the regions of the cell-wall upon which the chlorophyll-band abuts, and hence presumably is produced only in the latter ${ }^{2}$.

Certain red bacteria (Monas okeni, M. vinosa; Clathrocystis roseopersicina; Bacterinm photometricum), which Engelmann supposed to be without chlorophyll, show a very weak evolution of oxygen when exposed to light, but this suffices to allow these aerobic organisms to grow in the absence of any external supply of free oxygen, if they are illuminated $"$.

That oxygen is evolved from the chlorophyll bodies has been proved by means of the bacterium method, and hence it is generally concluded that the decomposition of carbon dioxide and the production of organic substance are also localized in them. It does not, however, necessarily follow that the oxygen evolved is directly derived from the decomposition of carbon dioxide, for Ewart has shown that certain coloured bacteria can absorb oxygen in marked amount, and evolve it again when its partial pressure is reduced, in some cases in sufficient quantity to keep aerobic bacteria in movement for as long as twelve hours *. In this case, however, the evolution of oxygen is a physical phenomenon, and continues equally well in the absence of light, whereas the evolution of oxygen from green organisms ceases almost immediately in darkness.

Engelmann was unable to detect any chlorophyll in the purple bacteria, but Bütschli observed that Chromatium okenii, when treated with alcohol, turns green, and Ewart has recently brought forward evidence to show that these purple bacteria

phyll organs of Hydra viridis are inconclusive, for here we are probably dealing with symbiotic algae. [See also Beyerinck, Bot. Zeitung, 1890 , pp. 745, 784 ; Kny, Ber. d. D. Bot. Ges., Bd. xv, 1897 , p. 388 ; Ewart, Bot. Centralbl., 1897, Bd. Lxvir, p. I09; Kny, ibid., Lxxin, p. $7_{26}$; Ewart, 1.c., June, I 898, p. 33.]

1 Ewart, Journ. of Linn. Soc., Vol. xxxi, p. 425. Klebs' (Unters. a. d. Bot. Inst. z. Tübingen, I888, Bd. II, p. 555) observations upon the power of forming starch in chloroplastids contained in non-nucleated masses of cytoplasm pointed in the same direction.

${ }^{2}$ Cf. Engelmann, Die Erscheinung der Sauerstoffausscheidung chromophyllhaltiger Zellen, I 894, Figs. 7, 8, I 2 (Sep.-abdr. a. d. Verh. d. Amsterd. Akad.)

Engelmann, Bot. Zeitung, 1888, p. 663 ; Ewart, Journ. of Linn. Soc., Vol. xxxiri, p. 151 ; Annals of Botany, Vol. XI, p. $4^{\$ 6}$.

${ }^{t}$ Ewart, Journ. of Linn. Soc., Xxxin, p. 123. 
actually contain a compound chromophyll, which may be separated into chlorophyll and a pinkish-red pigment by treatment with alcohol ${ }^{1}$. The displacement of the assimilatory maximum to the infra-red is therefore apparently due to the red pigment acting as a sensitisor.

According to Buitschli the bacterio-purpurin is restricted to the outer layer of the bacterial plasma ${ }^{2}$, and the only bacteria in which the pigment appears to be definitely associated with the plasma are those green and red bacteria which contain chlorophyll and have a distinct power of carbon dioxide assimilation. In all other bacteria the pigment appears to be an excretion, and in very many cases it is easy to see that the bacteria are colourless so long as they remain living, while it is only in certain cases that the bacterial pigment has the peculiar property of being able to occlude oxygen. In Sfirillum rubrum, however, the pigment appears to form part of the bacterial plasma, and Engelmann includes S. rubrum among the list of the red assimilating bacteria, although Ewart was unable to detect any evolution of oxygen from this form, nor indeed from any other bacterium than those already mentioned.

Historical. Priestley, the discoverer of oxygen, was the first to recognize that green plants purify air rendered foul by the respiration of animals, and Ingenhousz showed that this took place in light only, and that in darkness plants, like animals, gave off carbonic acid gas (fixed air). Ingenhousz did not, however, make it clear that the exhalation of oxygen (dephlogisticated air) was accompanied by a corresponding decomposition of carbon dioxide. This discovery was reserved for Senebier, who in his first work, and still more clearly in the later ones, established the fact that organic substance is produced from carbonic acid gas and water, while oxygen is excreted ${ }^{3}$. Senebier's experiments were by no means perfect, and it was the masterly researches of Th. de Saussure ${ }^{4}$ which first clearly

1 Ewart, Annals of Botany, I 897 , Vol. XI, p. 486 . A green pigment exhibiting red fluorescence and the characteristic bands of chlorophyll may be extracted by means of alcohol and benzene, not only from pure cultures of Bacterium photometricum, but also from Chromatium okenii and C. vinosa. Cf. also Biitschli, Über den Bau d. Bacterien, I 890, p. 9. Even though Engelmann did not work with pure cultures, the results observed could hardly have been produced by the presence of green Bacteria (cf. Winogradsky, Beiträge z. Morphol. u. Physiol. d. Bacter., I888, Heft i, p. 56). Engelmann's results have since been confirmed by Ewart (Journ. of Linn. Soc., XxxIII, I 897 , p. I 5 I). Elfving supposes that the red Saccharomyces glutinis can assimilate carbon dioxide (Stud. ii. Einwirkung d. Lichtes auf Pilze, I890, p. I7), but Ewart was unable to detect any evolution of oxygen from a red yeast isolated from the air.

${ }^{2}$ [Fischer states (Unters. uiber Bact. u. Cyanophy., Jena, I897, p. 120) that the pigment is uniformly distributed in Chromatium.]

${ }^{3}$ Friestley, Phil. Trans., 1772, Vol. Lx11, pp. I68, 193. Cf. Sachs, Gesch. d. Bot., I875, p. 53 I. Bonnet's observations (Unters. ii. d. Nutzen d. Blätter, Arnold, 1762, p. 14) are without value, for he considered the evolution of bubbles from green plants exposed to light under water to be a purely physical phenomenon. The older ideas as to the origin of plant-food are given by Sachs, l.c., p. 495 ; Ingenhousz, Experiments on Plants, 1779 ; Senebier, Mémoires physicochimiques, 1782 ; Recherches s. l. lumière solaire, I783; Physiol. végét., I 800 , T. III, P. I84; T. IV, Pp. 37, 165. As Pringsheim (Über Chlorophyllfunction u. Lichtwirkung i. d. Pflanze, I882, p. 26, \&c.) has shown, Hansen (Arb. d. Bot. Inst. in Würzburg, I882, Bd. II, p. 560) unjustly depreciates the value of Senebier's researches.

* Th. de Saussure, Rech. chim. s. l. végétation, 1804 . 
established the essential features of the process of carbon dioxide assimilation, and broadened and deepened our knowledge of this phenomenon. De Saussure proved decisively that the assimilation of carbon dioxide provides the green plant with organic food, and that the power of respiration which all living plants exhibit is a totally different phenomenon. Respiration and carbon dioxide assimilation were not, however, always kept distinct, although Dutrochet and Meyen regarded them as being separate processes quite independent of one another (see Sects. 50, 95). The 'humus theory' in vogue at the time prevented de Saussure from recognizing the universal importance of photosynthetic assimilation, and it was not until this theory had been upset that the importance of carbon dioxide assimilation in the economy of nature was fully recognized (see Sect. 95).

When Senebier, de Saussure, and Ingenhousz had clearly established the fact that all green parts can decompose carbonic acid gas, whereas all uncoloured parts and the coloured non-chlorophyllous petals of flowers, \&c. have not this power, it became possible to establish the dependence of carbon dioxide assimilation on the presence of chlorophyll, and to explain the possession of this power by red foliage leaves ${ }^{1}$ as being due to the presence of chlorophyll in them. De Saussure probably held this opinion, although his excessive caution prevented him from definitely expressing it. Dutrochet ${ }^{2}$, however, regarded the power of carbon dioxide assimilation as being directly dependent upon the presence of chlorophyll, and from this time onwards the same teaching has been repeated in all the better text-books. Cloez ${ }^{3}$ showed subsequently that coloured leaves are only able to assimilate carbon dioxide by means of the chlorophyll which they contain.

Senebier, de Saussure, and de Candolle discussed more or less fully the further changes which the organic food thus obtained must undergo in metabolism ${ }^{4}$, and as soon as it was certain that all organic food was obtained in this manner, it became evident that the assimilatory products must undergo most intricate and complicated metamorphoses, in order to produce the different complex substances which the plant contains. Various hypotheses have been put forward concerning the primary products of carbon dioxide assimilation (Pringsheim, 1. c., p. 67). Mohl, Unger, and Boussingault concluded that these were carbohydrates, but it was Sachs who first showed that the starch which appears in chloroplastids exposed to light is formed by the assimilation of carbon dioxide ${ }^{5}$. The importance of Sachs' researches is in no wise affected by the fact that starch is not always

1 Shown first by Senebier and Saussure. Further researches by Corenwinder, Compt. rend., $186_{3}$, T. LVII, p. 268.

${ }^{2}$ Dutrochet, Mémoires, \&c., Bruxelles, I 837 , p. I 86 . Thus Mohl, I85 I ; Unger, I855, \&c. Cf. Pringsheim, 1. c., pp. 26, 45, \&c.

${ }^{3}$ Cloez, Compt. rend., I $86_{3}$, T. LVIl, p. 834 ; Anı. d. sci. nat., $186_{3}$, iv. sér., T. xx, p. 184.

${ }^{4}$ Senebier, Physiol. végét., I80o, T. IV, p. $16_{5}$; de Candolle, Physiol. (Röper', 1833 , T. I, pp. 117, I39, I7o, \&c.

${ }_{5}$ Mohl, Grundzüge d. Anat. u. Physiol., $18_{5} \mathrm{I}$, p. 45. Unger (ibid., I $\delta_{55}$, p. 265) gives chemical equations to explain the production of carbohydrates and free oxygen. Sachs, Bot. Zeitung, I 862, p. 368 ; 1864, p. 288 . Mohl (Vermischte Schriften, I845, p. 355 ; Bot. Zeitung, I 855, p. I 15 ) and Nägeli (Die Stärkekömer, ${ }_{1} \delta_{5} \delta$, p. 398 ) do not bring the appearance of starch into genetic relationship with the assimilation of carbonic acid. 
formed, and that it is certainly not the primary product of carbon dioxide assimilation, as to the details of which we are still completely in the dark (cf. Sect. 54).

Methods. It can easily be shown that bubbles of gas rich in oxygen escape from the cut stems of illuminated aquatic plants, such as Elodea, Ceratophyllum, Potamogeton (Sect. 32), but the leaves of terrestrial plants are less suited for this purpose (Sect. 57). Either the apparatus shown in Fig. 25 may be used, or the gases may be collected by means of the simple arrangement shown in Fig. 4r, where the bubbles collect in the filter funnel $t$, and may be shown to be rich in oxygen by direct analysis or by opening the tap $h$, and holding a glowing match in the stream of issuing gas. It is easy to show that the rate at which the bubbles are produced is directly dependent upon the intensity of the illumination, and

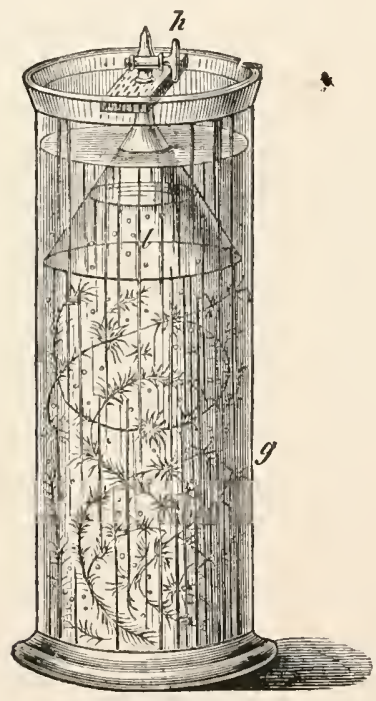

FIG. 4 I.

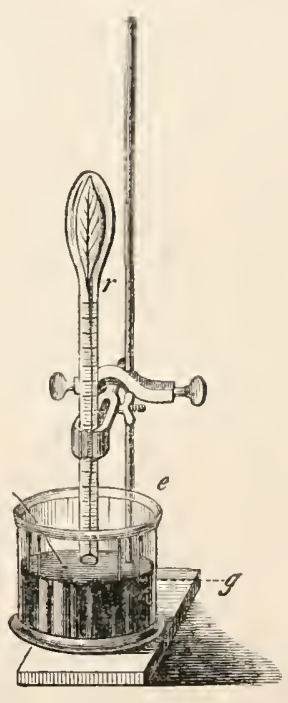

FIG. 42 .

Kohl, by counting the number of bubbles evolved and measuring the diameter of each, has been able to apply this method to determine the relative effects of the various rays of the spectrum (Sect. 60) ${ }^{1}$. By projecting the apparatus upon a screen the formation of the bubbles may be demonstrated to a large audience, and it may be shown that they stop directly lime-water is added (Sect. 32). Masses of green algae may also be used, for these slowly sink in darkness, but in the light they are raised to the surface by the adhering bubbles of evolved oxygen.

Since the time of Ingenhousz, analyses of the surrounding air have been made in order to determine the gaseous changes incident to the assimilation of carbon dioxide, and a simple apparatus, well adapted for a variety of experiments of this kind, is shown in Fig. 42. A leaf attached to a platinum wire is introduced into

${ }^{1}$ Kohl, Ber. d. Bot. Ges., r 897 , p. III. The bubble-counting method was first employed by Dutrochet (Mémoires, \&c., Bruxelles, I837, p. I82) and Sachs (Bot. Zeitung, I864, p. 363). 
the expanded end of a calibrated tube $r$, and by means of an india-rubber pipe air is sucked out of $r$ until the mercury is at the required height. A measured quantity of pure carbon dioxide is then introduced. After exposure to light the leaf is withdrawn by means of the platinum thread, and by analyzing the residual gas it can be found how much carbon dioxide has been decomposed and how much oxygen produced. By a control experiment carried on in darkness the amount of carbon dioxide produced by respiration can be determined.

Various other methods may be employed, but no account can be given here of these nor of the methods of gas analysis. Large volumes of gas may be analyzed rapidly and with sufficient accuracy by Hempel's method, while for small samples of gas Bonnier and Mangin's apparatus may be used; the latter in its newest form is extremely serviceable. Kreusler has described an apparatus by means of which a small but almost constant quantity of carbon dioxide may be maintained in an enclosed volume of air surrounding an assimilating plant ${ }^{1}$.

Engelmann introduced an admirable and irreplaceable physiological method by employing the movement of certain aerobic bacteria as a test for the presence of oxygen ${ }^{2}$. Single cells, algal filaments, sections of leaves, \&c. are placed in a fluid containing aerobic bacteria, and after covering the preparation with a coverslip, the latter is ringed with vaselin-paraffin or paraffin-wax. In darkness the enclosed oxygen is soon exhausted and the bacteria come to rest; as soon as light is admitted the bacteria in the neighbourhood of the assimilating cells begin to move, and are chemotactically attracted in the same manner as they would be by a bubble of air or oxygen (Fig. 43). By this excessively delicate reaction the billionth part of a milligramme of oxygen can be detected, and hence

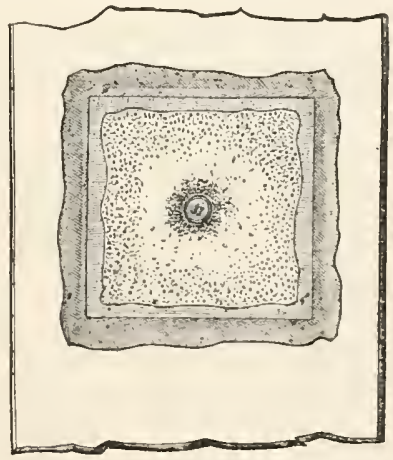

FIG. 43 . In the centre is an assimilating alga. also the mere trace of free oxygen which escapes from the objects examined, so that the assimilatory activity of single cells, or parts of cells, may be tested. This physiological method can only be used as a test for the evolution or non-evolution of oxygen, though it may be applied in a variety of ways $^{3}$ (cf. Sects. 58, 60). The employment of appropriate bacteria is obviously essential, and these are to be found among the forms usually grouped under the general term 'Bacterium termo.' Bacillus liquefaciens v. vulgaris, Beyerinck, is a suitable form, and may be isolated from the integuments of peas, and cultivated on Agar-agar. Material from young cultures may be added either to water, sugar

1 Hempel, Gasanalytische Methoden, 2. Aufl., I 890 ; Bonnier et Mangin, Rev. générale. I 89 I, T. 1II, p. 97. Cf. also Stich, Flora, I89I, p. 7. A method of obtaining small samples of the air from a closed receiver is given by Richards, Annals of Botany, I896, Vol. x, p. 534; Kreusler, Landw. Jahrb., I 885 , Bd. xiv, p. 913.

${ }^{2}$ Engelmann, Bot. Zeitung, 1881 , p. 442 ; $188_{3}$, p. 4 ; 1886 , p. 49; $188_{7}$, p. 102; Die Entstehungsweise d. Sauerstoffausscheidung, I 894 Sep.-abdr. a. d. Verh. d. Amsterd. Akad.).

3 On the macroscopically visible results, cf. Beyerinck, Bot. Zeitung, 189o, p. 743 . 
solution, or 0.2 to 0.5 per cent. solution of neutral meat extract. For prolonged experiments water or dilute sugar-solution is preferable, for in meat extract the bacterial products soon exercise an injurious effect upon the cell or tissue examined ${ }^{1}$. The forms known as Spirillum ( $S$. undula and temue) may also be used, but these are so exceedingly sensitive that unless the utmost care is taken there is considerable danger of experimental error. Spirillum is attracted only by a feeble supply of oxygen, and is repelled wherever a high partial pressure of this gas exists.

As a test for carbon dioxide assimilation Beyerinck has employed the phosphorescence of certain bacteria, which is dependent upon a supply of free oxygen. The glowing of phosphorus, the conversion of haemoglobin into oxyhaemoglobin, and the oxidation of reduced indigo carmine to indigo blue, have been used for the same purpose ${ }^{2}$. In every case all free oxygen must be removed at the outset from the object employed for the experiment.

\section{SECtion 53. The Structure and Properties of the Chloroplastid.}

The chlorophyll bodies belong to the group of plasmatic organs or plastids (Sect. 7 and 8), known as chromatophores ${ }^{3}$, which are derived

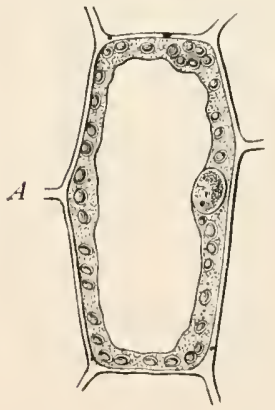

Fig. 44. (A) Cell from leaf of Vallisueria spiralis $(\times 450) ;(B)$ and $(C)$ are highly magnified chloroplastids from the leaf of Selaginella martensit, $(B)$ in surface view, $(C)$ in optical median section. The oval bodies in the interior are starch grains.

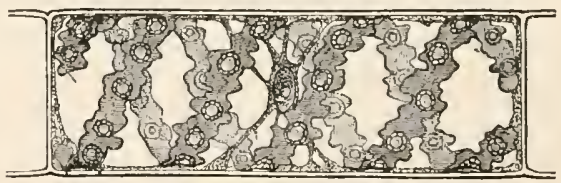

FiG. 45. Spirogyra spec. $(\times \mathbf{I} 80)$.

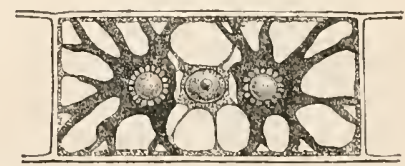

F1G. +6. Zygnema cruciatum ( $\times 800$ )

only from their like. Hence they increase only by the division of preexistent plastids, and may subsequently differentiate into chloroplastids, chromoplastids, or leucoplastids ${ }^{4}$, as the case may be, while the colourless

1 Cf. Pfeffer, Unters. a. d. Bot. Inst. z. Tübingen, 1888, Bd. 1I, p. 589; Ewart, Bot. Centralbl., 1897 , Bd. Lxxir, No. 9 .

2 Beyerinck, Bot. Zeitung, I89o, p. 744; Boussingault, Ann. d. sci. nat., 1869 , v. sér., T. $x$, p. 330 (Phosphorus); Hoppe-Seyler, Zeitschr. f. physiol. Chem., 1879 , Bd. 11, p. 425; Engelmann, Pfüger's Archiv f. Physiol., IS88, Bd. XLII, p. 186 (reduced haemoglobin); Beyerinck, Bot. Zeitung, 1890 , p. $74^{2}$ (reduced indigo).

${ }^{3}$ [This term is here applied to all plastids which have the power of becoming pigmented if oertain conditions are fulfilled. Hence chromatophores may at times be colourless.]

* For details see Zimmermann, Pflanzenzelle, 1887 , p. 45, and the collected literature given by 
leucoplastids formed in darkness may become green chloroplastids when exposed to light. These again may be converted into differently coloured chromatophores, as for example in the ripening of a fruit; they then lose the power of assimilating carbon dioxide, although the mere presence of another pigment does not render the latter process impossible as long as any chlorophyll is retained ${ }^{1}$. Indeed, among Algae the association of the chlorophyll with a brown or red pigment is a normal phenomenon. Thus it is permissible to term all chromoplastids which contain chlorophyll. chloroplastids, on account of their special functional importance, and in case of need the terms rhodoplastid, phaeoplastid, \&c. can also be used.

The shape of a chloroplastid may be markedly affected by the nature of the cell in which it lies ${ }^{2}$, but the Conjugatae render evident the fact that specific differences may also exist between different chlorophyll bodies. The external resemblance between the chloroplastids of all higher plants does not necessarily indicate that they are completely identical, and it is quite possible that the chloroplastid of a pine tree could not possibly exist in the protoplasm of an oak, for chloroplastids are dependent for their growth and maintenance upon the existence of certain specific relationships with the surrounding plasma, just as is the case also with the nucleus and all plasmatic organs ${ }^{3}$. This also applies to those Algae which live symbiotically in the bodies of certain animals, and which from a physiological standpoint bear a similar relationship to the animal cell which contains them, as do chloroplastids to their parent cell (Sect. 65).

The presence of chromatophores does not necessitate a formation of chloroplastids, for in non-chlorophyllous phanerogams the former may occur but not the latter, and in Fungi all organs of the nature of either chloroplasts or chromoplasts seem to be absent. Chlorophyll is apparently never uniformly distributed throughout the cytoplasm even in the lowest unicellular organisms ${ }^{4}$. Chromatophores may serve a variety of purposes, and it is of great importance to notice that chromatophores which are unable to turn

Zimmermann in Beibl. z. Bot. Centralbl., I 894 , Bd. Iv, p. 90 ; Schimper, Jahrb. f. wiss. Bot., I885, Bd. xvi, p. 1. Ewart (Journ. of Linn. Soc., xxxi, 1896, pp. 390, 427, 438, 449-452, 569, 573-576) has shown that in adult cells no reformation of the chloroplastids is possible when these have been killed, but it is possible that in embryonic cells masses of plasma may directly differentiate into chloroplastids, as for example when a root meristem becomes converted into a green shoot.

1 Ewart, l.c., pp. 390, 437, 448, 45 I.

2 On the occurrence of differently shaped chloroplastids in the same plant, see Zimmermann, Pflanzenzelle, 1887, p. 48 ; Haberlandt, Flora, 1888, p. 291.

${ }^{3}$ Cf. Sects. 4, 7, 8, and Pfeffer, Aufnahme u. Ausgabe ungelöster Körper, 189o, p. 174 ; Celakovsky, Flora, 1892, Ergänzungsband, 1. 224.

" On Cyanophyceae, \&c., cf. Zimmermann, 1894, 1.c., p. 97. On the supposed peripheral distribution of the Bacterio-purpurin in red Bacteria, see Sect. 52 ; A. Fischer, Unters. ü. Cyanoph. u. Bact., I 897, pp. 25, 119 . Diffuse masses of cytoplasm tinged with chlorophyll may also occur normally in the cells of certain phanerogams, without the power of carbon dioxide assinilation being lost. See Ewart, 1. c., pp. 449, 569; Wiesner, Pringsh. Jahrb., Bd. vili, 1872, p. 575. 
green, as well as etiolated chloroplastids, have the power of forming starch from sugar or other suitable nutriment ${ }^{2}$. The formation of starch grains appears, as far as is known, to be possible only when suitable chromatophores are present ${ }^{2}$. The starch grains are generally found in the interior of the chloroplastid, but they may grow until they protrude from it, so that ultimatcly the coloured or uncoloured starch-forming plastid may simply form a small adhering cap-like body attached to the starch grain (Fig. 47, and Zimmermann, I 894, 1.c., p. 92).

It must be remembered that the chloroplastids are plasmatic organs, and hence necessarily of complicated structure (Sects. 7-II). Even the
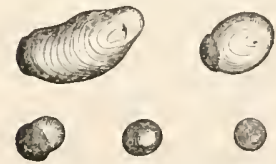

FiG. 47. Chloroplastids with larger and smaller starch grains from the stem of Pellionia Daveanana $(\times 400)$. visible structure of the chloroplastid has not yet bcen fully studicd, but it appears, according to Meycr and Schimper, that the chlorophyll is contained in vacuolar spaces in a colourless protoplasmic stroma, although it is not yet certain whether the chlorophyll is simply dissolved in an oil impregnating the plastid or is attached in some special manner to the living plasma ${ }^{3}$. It is also doubtful whether, as Hansen ${ }^{4}$ supposes, the accessory red and brown pigments, which are soluble in water, colour the ground substance of the plastid. In any case all researches upon the influence of the accessory pigments upon the assimilation of carbon dioxide must take into account this possibility (Sect. 60).

The existence of a certain division of labour in chloroplastids may safely be inferred, and it is possible that pyrenoids are the visible outcome of such functional differentiation, although it is still doubtful whether the pyrenoids found in the large chlorophyll bodies of certain Algae and a few lower plants are functioning elementary organs, or are simply composed of reserve material ${ }^{5}$. The direct protoplasmic connexion with the nucleus which they usually exhibit may point to either conclusion, and, moreover, starch may be formed in other regions of the chloroplastid as well. This stroma-starch seems to be more readily used up than the pyrenoid-starch, and hence the latter behaves more as reserve food-material.

${ }^{1}$ Schimper, Bot.Zeitung, I88o, p. $88_{1}$; Böhm, ibid., I883, p. 36 ; Laurent, Bull. d. 1. Soc. Roy. d. Bot. d. Belgiquc, I888, T. xxvi, p. 243; Saposchnikoff, Ber. d. Bot. Ges., 1889, p. 259 ; Zimmermann, Beiträgc z. Morph. u. Physiol., I893, pp. 29, 89. [II. Winkler, Unters. iiber die Stärkebildung in den verschiedenartigen Chromatophoren, Jahrb. f. wiss. Bot., I89 $\$$, Bd. xxxir, p. $525 \cdot]$

2 A. Meyer, Stärkekörner, 1895 , p. 159; Zimmermann, 1894, 1.c., p. 92 . Telzung (quoted by Zimmermann) states that starch occurs in certain fungi, and moreover no chromatophores or special plastids are necessary for the formation of cellulose.

${ }^{3}$ Cf. Zimmermann, I894, 1. c., p. 90 ; Zimmermann, Zelle, $188_{7}$, p. 59 ; Reinke, Bot. Zeitung, 1886 , p. 169 ; Hansen, Fartstoffe d. Chlorophylls, 1889, p. 86, and Stoffbildung bei den Meeresalgcn, 1893, p. 292 (Sep.-abdr. a. d. Mitth. a. d. Zool. Station zu Neapel, Bd. xi).

4 Hansen, 1893 , l. c., p. 301. Cf. Reinke, Bot. Zeitung, I886, p. I8 I.

${ }^{5}$ For lit. see Zimmermann, I 894 , 1. c., p. 93 ; Klebs, Bot. Zeitung, 1891, p. 793 ; Chimilewsky, Bot. Centralbl., I 897 , Bd. Lxix, p. 277 . 
Chromatophores are built up of a variety of substances, mainly proteid in nature ${ }^{1}$, and in chloroplastids a little chlorophyll is always present, but even when the colour is a decp green it does not exceed O.I per cent. ${ }^{2}$ Yellow pigments from the carotin group seem never to be absent, even from etiolated chloroplastids or from those of red and brown seaweeds ${ }^{3}$. Many of these pigments are soluble in water, but chlorophyll and carotin are exceptional in this respect, so that treatment with hot water suffices to render the presence of chlorophyl immediatcly evident ${ }^{4}$. Except in etiolated chloroplastids, chlorophyll is present in all cases where carbon dioxide assimilation is possible.

Although the conspicuous colour of chlorophyll naturally first attracted attention to the chloroplastids, it is simply an accessory part of the latter, and attains physiological importance only in connexion with the living plastid (p. 304), for the chemical properties of the chlorophyll alone do not suffice to explain the problem of carbon dioxide assimilation, although they must have important bearings upon it. It is not, however, our purpose to give a detailed account of the chemical and optical properties of chlorophyll, and a reference to the literature of the subject must suffice ${ }^{5}$. In Sect. 60 those optical properties which are of importance in connexion with the assimilation of carbon dioxide will be mentioned.

The isolated chlorophyll pigment ${ }^{6}$ contains nitrogen but no iron ${ }^{7}$. According

${ }^{1}$ Cf. Sect. 11 ; also Zimmermann, Zelle, $188_{7}$, 1. 6o, and Beihefte z. Bot. Centralbl., 1 894 , p. 90 , whcre the work of Zacharias and Schwarz is also mentioned. Oil is apparently never absent, and in certain cases is especially abundant. It may serve as a solvent for the chlorophyll. Zimmermann, Zelle, p. 61 ; Schmitz, Chromatophoren d. Algen, 1882, p. 60 ; Engelmann, Bot. Zeitung, 1883, p. 2 I ; Schimper, Jahrb. f. wiss. Bot., 1885 , Bd. xvi, p. 188 ; A. Meyer, Bot. Zeitung, 1885, p. 433. On the presence of Cholesterin, see Reinke, Ber. d. Bot. Ges., 1885 , p. lvi ; Hansen, Farbstoffe d. Chlorophylls, 1889, p. 60.

2 Tschirch, Pflanzenanat., I, I 889 , p. $57^{\circ}$

${ }^{3}$ Found in Florideae by Rosanoff (Mém. d. l'Acad. d. l. Soc. Imp. d. Cherbourg, 1867, T. xill, p. 202); in Fucaceae by Millardet (see G. Kraus, Zur Kenntniss d. Chlorophyllfarbstoffe, $1 \boldsymbol{i}_{2}$, p. 106). Cf. Hansen, Stoffbildung b. d. Meeresalgen, 1893, pp. 263, 292, \&c. A similar red colour is assumed by certain plants when observed through solutions of fuchsin or potassium permanganate (Hansen, 1.c., I 893, p. 299; Noll, Flora, 1893, p. 27). On the simultaneous presence of yellow pigments, cf. Monteverde, Acta horti Petropolitani, 1893, Vol. xıII, p. 176. On Neottia, cf. Lindt, Bot. Zeitung, $188_{5}$, p. 825 .

${ }^{*}$ Cf. Reinkc, Bot. Zeitung, 1886, p. 177 .

${ }^{5}$ Literature: G. Kraus, Zur Kenntniss d. Chlorophyllfarbstoffe, $187^{2}$; A. Meyer, Das Chlorophyllkorn, 1883 ; Tschirch, Unters. ii. das Chlorophyllkorn, $188_{4}$; IHansen, Farbstoffe des Clilorophylls, 1889; Monteverde, Das Absorptionsspektrum des Chlorophylls, I $\$_{93}$ (Sep.-abdr. a. Acta horti Petropolit., Vol. XIrI), and on Protochlorophyll, I S94 (ibid.); Schunck u. Marchlewsk, Ann. d. Chemie, I894, Bd. cclxxvili, p. 329, and Annals of Botany, I $889-90$, Vol. III, p. 65 ; Marchlcwski, Die Chemie d. Chlorophylls, 1895 ; Tschirch, Ber. d. lot. Ges., 1895, p. 76.

${ }^{6}$ [Nehemiah Grew (Anatomy of Plants, $1682, \mathrm{p} .273$ ) extracted green and yellow pigments from leaves by means of oil, and apparently also observed the fluorescence of chlorophyll.]

${ }^{7}$ Molisch, Die Pflanze in ihrer Beziehung zum Eisen, $1892, \mathrm{p} .8_{5}$. On the necessity of iron for the formation of chlorophyll, see Sect. 74 . 
to Schunk and Marchlewski (l. c.) definite products, chlorophyllan and alkali-chlorophyll respectively, may be obtained from chlorophyll by treatment with acids and alkalies. The granular or acicular brown deposits of 'Hypochlorin' observed by Pringsheim were produced in green tissues when they were treated with hydrochloric acid, and according to Meyer and Tschirch ${ }^{1}$ these deposits were composed mainly of chlorophyllan.

Marchlewski is convinced that the chlorophyll isolated from the most various plants is in all cases identical, but nevertheless various compounds of chlorophyll may exist in the living chloroplastid, while it is not even known whether chlorophyll exists in the chloroplastid in dissolved form, and whether it is chemically or mechanically united with the latter. The fact that the spectrum of a living chloroplastid agrees with that of isolated chlorophyll, allowing for the disturbance caused by the solvent medium, does not afford conclusive evidence one way or the other ${ }^{2}$. The trifling spectroscopic differences which extracts from different plants may exhibit are not necessarily due to the presence of specifically distinct forms of chlorophyll, but may be due to impurities, to decomposition products, or to the influence of the solvent medium. There is apparently a chemical relationship between chlorophyll and haemoglobin, for both, according to Schunck and Marchlewski, are derivatives of Pyrrol, and exhibit also certain spectroscopical similarities ${ }^{3}$.

Yellow pigments. The yellowish-red pigments are by no means all identical chemically or spectroscopically, but they all seem to belong to the group of carotins. The typical carotin pigment appears to be present in all chloroplastids, whether green or differently coloured, and many of the supposed varieties are probably only impure extracts. It is impossible to say at present whether this is universally the case, or whether etiolin, protochlorophyll, haemotochrome, \&c., are special forms of carotin or carotin compounds. It is still doubtful whether chlorophyll is formed directly from one or other of these yellow pigments ${ }^{4}$. This would only be possible by a profound chemical change, for carotin is a hydrocarbon (Marchlewski, 1.c., p. 73), whereas chlorophyll is a differently constituted nitrogencontaining body, so that if any one of the yellow pigments is converted into chlorophyll simply by contact with air, it can hardly be a carotin compound. The yellow pigments are usually obtained by agitating an alcoholic extract of leaves with dilute potash, and according to Molisch yellow crystals may be obtained from green tissues by similar treatment ${ }^{5}$.

1 Cf. Hansen, 1. c., 1889, p. $3^{8 .}$

${ }^{2}$ Marchlewski, I895, l.c., p. 63. Monteverde (1.c., 1893 , p. I76) and Etard (Compt. rend., 1895 , T. cxx, p. 275) assume that many varieties of chlorophyll exist. G. Kraus, 1872, l. c., p. 47 ; Hansen, 1. c., 1879, p. 77, and 1893, l.c., p. 294; Reinke, Bot. Zeitung, 1886, p. 196; Monteverde, l. c., 1893, p. 134 .

${ }^{3}$ Schunck und Marchlewski, 1.c., 1894 , p. 288 ; Tschirch, 1.c., 1895, p. 92 ; Nencki, Mém. d. sci. biol. d. l'Inst. Imp. d. Méd. d. St.-Pétersbourg, 1897 , T. v, p. 254.

${ }^{4}$ Hansen, 1. c., 1889, p. 59; Monteverde, 1.c., I 893, p. 186; Schrötter-Kristelli, Bot. Centralbl., 1894, Bd. LXI, p. 33 ; Marchlewski, 1895, l. c., p. 7 ; Molisch, Ber. d. Bot. Ges., 1896, p. 18 ; Tschirch, ibid., I 896 , p. 85 ; Zopf, Biol. Centralbl., 1895 , Bd. xv, p. 418 . Cf. Wiesner, Entstehung d. Chlorophylls, I 877 , p. 25 .

${ }^{5}$ Molisch, Ber. d. Bot. Ges., I 896, p. Is. 
Other pigments. Molisch has recently isolated the phycoerithrin of red seaweeds and the phycocyanin of the Cyanophyceae, and has shown them to be crystallizable proteids. It is possible that the phycophaein of brown seaweeds and other chloroplastic pigments which are soluble in water may also be proteid in nature ${ }^{1}$.

\section{Section 54. The Products of the Assimilation of Carbon Dioxide.}

It is at present impossible to say what the first-formed product in the assimilation of carbon dioxide may be; moreover, it may never accumulate to any extent, but may imnicdiately undergo further modification in various directions, although the first stage of carbon dioxide assimilation is probably identical in all green plants. It is impossible, however, to say whether the same path is followed in all cases until sugar is produced, or whether similarity is exhibited only as far as the production of some simple body, from which, according to circumstances, sugar, oil, starch, proteids, or other visible products may be formed. Many facts indicate that a general agreement is shown in the early production of a carbohydrate, but nevertheless various assimilatory products may appear in a cell, owing to the more or less marked modifications which this carbohydrate, probably sugar, may subsequently undergo.

Physiology can only deal with the visible products of assimilation, and these have precisely the same nutritive value and metabolic importance as similar food supplies obtained from the external world. It is, however, an extremely difficult task to discover what is the precise manner in which this nutriment is produced by the functional activity of the chloroplastid, and it seems improbable that proteid synthesis should be necessarily connected with the assimilation of carbonic acid. On the other hand, carbohydrates may be formed by the decomposition of proteids, and hence it is not impossible that the sugar which appears in the chloroplastid may be derived from proteids of antecedent origin. Similarly, oil may be formed from starch, as well as starch from oil, but it is not certain whether these and other processes can be carried on by the chloroplastid itself without the aid of the external cytoplasm. Thus, if the chloroplastid were unable to form starch from anything but sugar, then starch could only be formed from glycerine if the cytoplasm previously converted this substance into sugar. In any case the insoluble starch must necessarily be formed at the expense of some soluble product, and since many chlorophyllous and non-chlorophyllous plastids can form starch grains when supplied with sugar (Sects. 53, 55), it is obvious that starch can hardly be the primary product of carbon dioxide assimilation.

1 Molisch, Bot. Zeitung, I894, p. I8 r. See also Zimmermann, Beihefte z. liot. Centralbl., I 894 , Bd. IV, p. 9I ; Molisch, Bot. Zeitung, I895, p. I3I ; Hansen, ibid., I884, p. 649; Reinke, ibid., I886, p. I 77 ; Schiitt, ilid., I887, p. 259 . 
The above conclusions are all supported by direct observations and experiments. Thus starch appears as an assimilatory product in most but not in all plants, either soon after assimilation commences, or not until a larger or smaller amount of sugar has accumulated, while in some cases no starch is produced, however much sugar may be present (Sect. 5.5). Instead of starch a certain amount of oil may be formed in some plants, and in many leaves a marked production of proteid substances accompanies carbon dioxide assimilation (Sect. 72). All these substances are produced by the latter process, and are continually removed as plastic or nutritive material to the different parts of the plant. Starch, sugar,

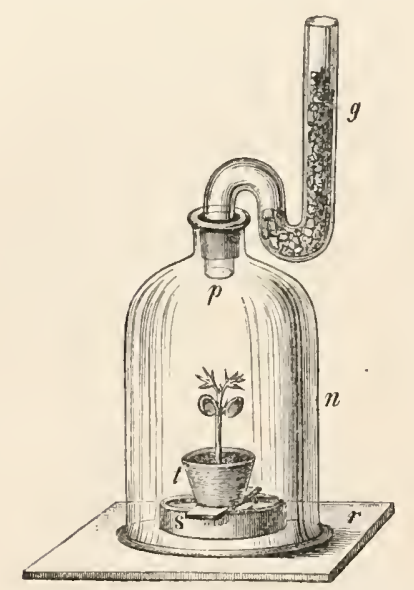

FIG. 48. Apparatus for growing plants $\mathrm{n}$ an atinosphere free from carbon dioxide. The bell-jar $(n)$ is air-tight and is fixed upon the glass plate $(r)$, the tube $(g)$ contains pumice-stone moistened with potash to prevent the entrance of $\mathrm{CO}_{2}$ from without. The vessel $(s)$ also contains potash solution to remove the $\mathrm{CO}_{2}$ given off from the soil or from the plant. It may in some cases be advisable to enclose a vessel containing solid potash or $\mathrm{CaCl}_{2}$ in order that sufficient transpiration may be possible. and the other assimilatory products disappear when carbon dioxide assimilation is suspended, and reappear when it is resumed. This fact was first observed and correctly interpreted by Sachs ${ }^{1}$, who showed that after twenty-four hours' darkness chlorophyllous cells were in most cases devoid of starch, and that the latter soon reappeared in light, but only when carbonic acid gas was present (Pfeffer, Godlewski). The fact that in an atmosphere free from carbon dioxide starch disappears in light or darkness (Fig. $4^{8}$ ) shows that the assimilatory products are translocated from the leaf not only at night, but also in the daytime ${ }^{2}$.

Only those chloroplastids which are directly illuminated assimilate carbon dioxide and produce starch. Hence none of the latter will appear in portions of a leaf which are artificially darkened, so that by covering part of its blade with a stencil plate or a sheet of tinfoil, and subsequently treating the leaf with iodine after removing the chlorophyll, a name may be printed on the leaf in bluish-black letters upon a yellow ground (Fig. 49).

Similarly, the formation of sugar may be shown to be dependent upon the assimilation of carbon dioxide, as may be also the formation of plastic proteid material in the leaf ${ }^{2}$. In order that assimilation may continue

1 Sachs, Bot. Zeitung, 1862 , p. 368, and 1864 , p. 289 ; Pfeffer, Monatsb. d. Berl. Akad., 1873 , p. $7 S_{4}$; Godlewski, Hlora, I $S_{73}$, p. $3 S_{2}$; also Morgen, Pot. Zeitung, $1 \delta_{77}$, pp. 553 , \&c. [See also Costerus, Ann. d. Jard. bot. de Buitenzorg, T. xII, 1894, p. 73.]

2 The fact that proteids are formed in the leaf does not necessarily show that they are produced in the chloroplastids, or that they are primary assimilatory products, by whose decomposition carbohydrates are formed; nor is our knowledge of pyrenoids and crystalloids sufficient to answer this question. Literature: Schmitz, Chromatophoren d. Algen, I\$\$2, p. I50; A. Meyer, Bot. 
unchecked, its products must be continually removed, for any excessive accumulation may cause further assimilation to cease (Sect. 55). As a matter of fact the sugar, which is probably glucose, does not under normal conditions accumulate beyond a certain percentage, being then either removed or converted into starch or proteid, or the glucose may be converted into saccharose and thus the osmotic concentration decreased, for the inhibitory influence is mainly dependent upon the osmotic concentration. Hence a marked accumulation of insoluble or feebly osmotic assimilatory products is possible without injury to the power of carbon dioxide assimilation ${ }^{2}$.

Carbohydrates; Glucosides. The presence and distribution of starch in a leaf can be macroscopically determined by simple treatment with a solution of iodine in chloral hydrate, or by staining with an iodine solution after immersing the leaf in boiling water and subsequently extracting the chlorophyll with alcolool ${ }^{2}$. By careful treatment even very minute starch grains can be detected. Starch may appear in most chloroplastids, but it is not formed in those of Allium cepa ${ }^{3}$, even when assimilation is active and an abundance of sugar accumulates; under such conditions starch appears, however, in the chloroplastids of Musa, Hemerocallis fulva, Muscari moschatum, and many other plants in which a moderate accumulation of sugar does not suffice to induce a deposition of starch $^{4}$ (Sect. 55).

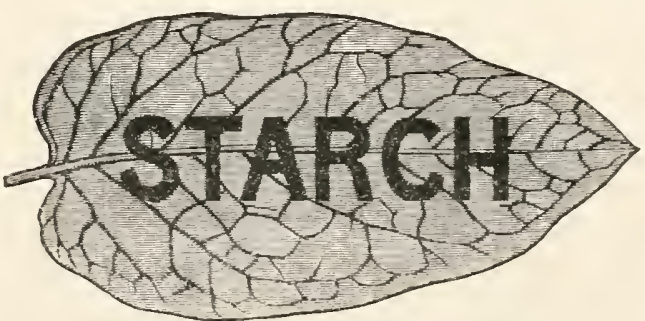

FIG. 49. Leaf coloured with iodine after the method described. A larger or smaller amount of sugar will always accompany starch, and in Allium the assimilatory products are stored up entirely in the form of sugar.

The sugar is commonly a reducing one, usually dextrose or laevulose, but probably all forms of sugar which are translocated or stored may also be formed as direct products of carbon dioxide assimilation. Thus saccharose has often been detected, mannite occurs in the leaves of certain Oleaceae, and sinistrin in those of

Zeitung, IS85, p. 420 ; Chrapowicki, Bot. Centralbl., I889, Bd. xxxIx, p. 352 ; Schimper, Flora, I89o, p. 260 ; Saposchnikoff, Bot. Centralbl., IS95, Bd. LXIII, p. 246.

${ }_{1}$ Cf. Lwart, Journ. Linn, Soc., Vol. xxx I, I896, pp. 429, 435 .

2 Sachs, Arb. d. Bot. Inst. in Würzburg, IS\&4, Bd. III, p. 3; Flora, 1S62, p. I66; Böhm, Sitzungsb. d. Wien. Akad., I 857 , Bd. xxII, p. 500. Cf. Zimmermann, Bot. Mikrotechnik, IS92, p. 221.

${ }^{3}$ Böhm, Sitzungsb. d. Wien. Akad., I $8_{57}$, Bd. xxı, p. 500 ; Sachs, Experimentalphysiol., 1865 , p. 326. Starch may, however, appear in the guard-cells of the stomata and in the bundle-sheaths of the leaves. Sachs, 1.c.; A. Meyer, Bot. Zeitung, ISS 5 , P. $45^{6}$; Rendle, Annals of Botany, ISSs, Vol. II, p. I6I.

Literature : A. Meyer, Bot. Zeitung, $\mathrm{IS}_{5}, \mathrm{pp} .45 \mathrm{I}, 467$, \&c.; Schimper, ibid., p. 786 ; Nadson, Bot. Centralbl., I89o, Bd. Xl.II, p. 50; Godlewski, Flora, IS77, p. 216 . [A little starch may apparently be deposited in the tissues of the bulb of Allium cepa in the immediate neighbourhood of young growing shoots.] 
Yucca filamentosa, but whether glucosides may appear as direct products of photosynthesis is doubtful '. Westermaier incorrectly supposed tannin to be an assimilatory product, and the pentoses do not appear to have any such direct origin 2 . The same doubt applies to the imperfectly known paranylon found in Euglena and other lower organisms, nor can anything be said as to the so-called cyanophycin or the very problematic fucosan of Hansteen ${ }^{3}$.

Oil. In higher plants oil has not as yet been proved to be a direct product of carbon dioxide assimilation, for the oil-drops in the chloroplastids of Strelitzia and other Monocotyledons do not disappear even during a prolonged sojourn in darkness ${ }^{4}$. Similarly, it has still to be proved whether the oil, often present in abundance in Vaucheria sessilis, \&c., and in many Phaeopliyceae and Florideae, is a direct product of photosynthetic assimilation ${ }^{5}$.

Chlorophyll. That chlorophyll is not a product of carbon dioxide assimilation, as Gerland and Sachsse supposed, is shown by the fact that it remains present when no assimilation is possible, and that etiolated plants turn green when exposed to light in an atmosphere deprived of all carbon dioxide ${ }^{6}$. The same also applies to chlorophyllan (hypochlorin), which Pringsheim erroneously supposed to be the

1 Dextrose and laevulose: A. Meyer, 1.c., pp. $467,480,487$, \&c.; Schimper, 1. c., p. 779. Saccharose: Brown and Morris, Journ. of Chem. Soc., I 893 , p. 660 ; Perrey, Compt. rend., I 882 , T. Xc1v, p. II24. Mannite : cf. A. Meyer, Bot. Zeitung, I 886, p. $145 ;$ I $88_{5}$, p. 467 (sinistrin), but according to Brown und Morris (1.c., p. 660) inulin is formed in Iucca, and not sinistrin. Glucosides : Brunner und Chuard, Ber. d. Chem. Ges., i 886, p. 609, but on insufficient grounds.

2 Westermaier, Sitzungsb. d. Berl. Akad., I $8_{5}$, p. $70_{5}$. Cf. G. Kraus, Grundlinien z. Physiol. d. Gerbstoffs, I8S9, p. 46. Pentoses: Brown and Morris, l.c., p. I62; de Chalmont, Ber. d. Chem. Ges., 1894 , p. 2722 .

3 Paramylon: Schimper, Jahrb. f. wiss. Bot., i $\$ 85$, Bd.xvi, p. I99; Schmitz, ibid., I $\$ 84$, Bd. xv, p. I I I ; Klebs, Unters. a. d. Bot. Inst. z. Tüibingen, I $88_{3}$, Bd. I, p. 272 . Cyanophycin: Palla, Jahrb. f. wiss. Bot., I 89.3 , Bd. xxv, p. 554; Hansteen, ibid., I892, Bd. xxiv, p. 346. [Crato (Ber. d. Bot. Ges, 1893 , p. 236) has shown that the supposed ' fucosan ' granules are really vacuoles (= 'physodes') containing phloroglucin, and stain red with vanillin and $\mathrm{HCl}$. Similar 'physodes' are present in abundance in the cells of many Phaeophyceae, sometimes giving the osmic acid, sometimes the vanillin reaction, and occasionally both. The osmic acid reaction is not a conclusive test for the presence of fat or oil, while the phloroglucin may be an accidental by-product, and form but a portien of the contents of the 'physodes.' Prolonged treatment with $\mathrm{H} \mathrm{Cl}$ converts the readily disintegrating vacuolar physodes of Ascophyllum into a fairly stable solid form (Church).]

4 Holle, Flora, I877, p. I33; Wakker, Jahrb. f. wiss. Bot., I888, Bd. xix, p. 474. Briosi's conclusion (Bot. Zeitung, 1873, p. 529) that oil is the prinary product of assimilation in $M / u s a$ cannot possibly be correct, and Ewart (Annals of Botany, Vol. xI, rS97, p. 446) has shown that in Iloya fraterna oil-drops appear in the cells only when an abundance of starch has been formed, and disappear before the starch does. It is evident that the utmost caution is necessary in drawing conclusions from data of this kind.

"Vaucheria, \&c.: Borodin, Bot. Zeitung, IS 78, p. 498; Sclımitz, Chromatophoren der Algen,

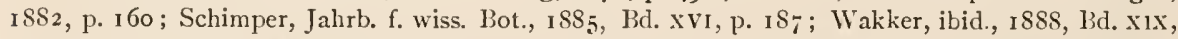
p. 474. On starch in Taucheria, see Walz, Jahrb. f. wiss. Bot., I $866-7$, Bd. v, p. I 29 ; on glucose, Schimper, Bot. Zeitung, 1885, p. 779. Algae: Hansen, Mitth. a. d. Zool. Station zu Neapel, 1893 , Bd. XI, pp. 276,283 ; Bruns, Hlora, I $S_{94}$, Erg.-bd., p. I59. On the occasional presence of 'Floridaean starch,' see Hansen, l. c., p. $28_{5}$; Golenkin, Algologische Notizen, I894, p. 4 ; and Bruns, 1. c., p. I77.

6 Gerland, Ann. d. Chemie u. Physik, I871, Bd. Cxl11, p. 6ro, and r873, Bd. Cxlvir, p. 99; Sachsse, Chemie u. Physiol. d. Farbstoffe, \&c., I877, p. 6i ; Phytochem. Unters., $1880, \mathbf{1}$, p. 3. Similar suppositions are also quoted by Pringsheim, Chlorophyllfunction u. Lichtwirkung, i $\$ \$ 2, \mathrm{p} .36$. 
primary assimilatory product, for Hillburg showed that the quantity of 'hypochlorin' present does not decrease in an atmosphere free from carbon dioxide?

The almost instantaneous stoppage and recommencement of the movement of the bacteria employed as a test for oxygen shows that its evolution commences immediately the green parts are exposed to light, and ceases as soon as the illumination fails. The entire process probably takes place extremely rapidly, for G. Kraus ${ }^{2}$ found that a perceptible amount of starch appeared in a starchless Spirogyra filament after five minutes' exposure to light. In other plants starch is not formed until some hours have elapsed, but this is almost certainly because it is commonly deposited only when the primary products of assimilation have accumulated to a certain extent.

It is not known at what stage in the assimilation of carbon dioxide the oxygen is set free, although various facts show that oxygen and not ozone is liberated. No ozone or hydrogen peroxide is present in the interior of the cell, and Pringsheim's supposition that the oxygen is excreted in the form of a compound which decomposes outside or on the surface of the cell, is certainly erroneous. The liberated oxygen diosmoses rapidly to all parts where a lower partial pressure exists, and hence escapes from the cell before any marked accumulation or condensation is possible ${ }^{3}$.

In the assimilation of carbon dioxide, allowing for the gaseous exchanges due to respiration, slightly more oxygen appears to be produced than would be the case if the following simple equation represented the actual process: $6 \mathrm{CO}_{2}+6 \mathrm{H}_{2} \mathrm{O}=6 \mathrm{O}_{2}+\mathrm{C}_{6} \mathrm{H}_{12} \mathrm{O}_{6}$ (glucose). This does not, however, indicate that the primary products of assimilation are not carbohydrates, for a similar excess of oxygen would appear if a little organic acid continually appeared in metabolism and was decomposed in light with an evolution of oxygen (Sect. 56). Even the first visible product of assimilation does not remain intact, but may continually be converted into other substances; thus Saposchnikoff ${ }^{4}$ found that only $64-87$ per cent. of the assimilated carbon dioxide appears in the leaves of the sunflower in the form of carbohydrates.

Boussingault and others have shown that the assimilation of carbonic acid gas usually causes but little change of volume in the enclosed air, but sometimes a very marked difference may be produced. Bonnier and Mangin, and other investigators,

1 Pringsheim, Jahrb. f. wiss. Bot., I 879-81, Bd. XIr, p. 288 ; Pfeffer, Physiol., I. Ausg., Bd. r, p. 195.

${ }^{2}$ G. Kraus, Jahrb. f. wiss. Bot., I $869-70$, Bd. vir, p. 511 ; Famintzin (Jahrb. f. wiss. Bot., I 867-8, Bd. VI, p. 34) carried out similar researches with Spirogyra at a still earlier date.

${ }^{3}$ [Pringsheim, Sitzungsb. d. Akad. d. Wiss. zu Berlin, I $88_{7}$, Über d. Abhängigkeit d. Assim. grüner Zellen u. Sauerstoffathmung. Cf. Pfeffer, Oxydationsvorgänge in lebenden Zellen, I889, pp. 149,479 , and Ewart, Journ. of Linn. Soc., Vol. XxxI, I 891, p. $4^{18}$. An actively assinilating green cell, if suddenly killed, may evolve faint traces of oxygen for a short time after death, but this is merely oxygen dissolved in the cell-sap, \&c.; see Ewart, l.c., I897, p. I46.] In Sect. Ior an account is given of the power of occluding oxygen possessed by certain bacteria.

* Saposchnikoff, Ber. d. Bot. Ges., I890, p. 241 . On similar researches by Menze, see the eriticism by A. Neyer in Bot. Zeitung, is88, p. 465 . 
have shown that a larger volume of oxygen is produced than the volume of carbon dioxide which is assimilated, but since in respiration the reverse takes place the result is that the air of the receiver retains an approximately constant volume ${ }^{1}$. Thus, according to Bonnier and Mangin, the total volume of the air is unaltered by assimilating ivy leaves, although for every soo cc. of carbon dioxide decomposed, ro8 cc. of oxygen are evolved. This is because the concomitant respiration produces $100 \mathrm{cc}$. of the former gas for every $86 \mathrm{cc}$. of oxygen absorbed (cf. Sect. 96).

All researches have shown that oxygen is the sole gaseous product of carbon dioxide assimilation, but any gases previously present in the plant may escape along with it. This probably explains the increase in the percentage of nitrogen observed by de Saussure in the surrounding air. The gas-bubbles evolved from water plants during the assimilation of carbon dioxide contain less and less nitrogen if we prevent the access of fresh supplies to the water, and hence to the plant also, while that there is no evolution of carbon monoxide, or of gaseous hydrocarbons, has been shown by Cloez and Corenwinder ${ }^{2}$.

\section{SECTION 55. Effect of the Accumulation of the Assimilatory Products (Carbohydrates).}

The assimilatory products are usually transferred by intermediate cells to the neighbouring vascular bundles, and travel in the phloem, as well as in the bundle sheaths and the parenchyma of the veins of the leaves, by which paths carbohydrates, proteids, \&c. may be carried considerable distances ${ }^{3}$. Translocation of any substance is regulated by the amount required for consumption or storage, and as soon as these cease the assimilatory products accumulate in the conducting channels and in the assimilating cells. As this occurs the assimilatory activity gradually decreases and ultimately ceases (Sect. 93).

Boussingault ${ }^{4}$ incidentally observed that the power of assimilating carbon dioxide gradually decreased in an actively assimilating branch

1 Boussingault, Agron., Chim. agric., \&cc., I868, T. Iv, p. 286 ; I 864 , T. Ir , p. 378 ; Pfeffer, Arb. d. Bot. Inst. in Wiirzburg, I87 I, Bd. I, p. 36 ; Godlewski, ibid., I873, Bd. I, p. 343 ; Holle, Flora, 1877, p. I87; Bonnier et Mangin, Ann. d. sci. nat., I886, vii. sér., T. III, p. I, where the methods by which the respiration may be estimated are given. Jumelle, Rev. gén. d. Bot., I $89^{2}$, T. Iv, p. 6 I (I.ichens); Bastit, ibid., 1891, T. III, p. 521 (Mosses). On Crassulaceae, see Sect. 56. On the ultimate changes produced as growth continues, see Th. Schloesing. Compt. rend., I 893 , T. cxvir, p. $75^{6}$.

2 Saussure, Rech. chim., I 804, p. 42 ; Cloez et Gratiolet, Ann. d. chim. et d. phys., I 85 I , iii. sér., T. $x x x i 1$, p. 57. See also Boussingault, Agron., Chim. agric., \&c., I864, T. III, p. 27 I; Cloez, Ann. d. sci. nat., I863, iv. sér., T. xx, p. I 80 ; Corenwinder, Compt. rend., I865, T. Lx, p. I 20.

${ }^{3}$ Schimper, Bot. Zeitung, I885, p 756 ; A. Fischer, Jahrb. f. wiss. Bot., 189 I, Bd. XXII, p. 79 ; Haberlandt, Physiol. Anat., $188_{4}$, p. I 84 , and Ber. d. Bot. Ges., I886, p. 206 . On the translocating substances, see Brown and Morris, Journ. of Chem. Soc., I893, p. 671. Cf. Chap. x.

4 Boussingault, Agron., Chim. agric., \&c., I868, T. Iv, p. 3 I 2 ; Saposchnikoff, Ber. d. Bot. Ges., I89o, p. 238; I891, p. 298; I893, p. 391; Bot. Centralbl., I895, Bd. Lxiri, p. 246; Ewart, Journ. of Linn. Suc., I $896, \mathrm{Vol}$. Xxxi, p. 429 . On the slow disappearance of starch in separated leaves, cf. Sachs, Arb. d. Bot. Inst. in Wïrzburg, I884, Bd. III, p. II ; Saposchnikoff, 1. c., I 890, p. 235. On the influence of ringing upon translocation, see Cuboni, Bot. Centralbl.. I $8_{5}$. Bd. Xxir. p. 4 s. 
when removed from the parent plant, and Saposchnikoff showed by more detailed observations that the assimilatory products (sugar, starch and proteids) cannot increase beyond a certain amount in the leaf of Vitis. In all regulatory processes, any over-accumulation not merely acts mechanically, but also exerts an inhibitory stimulating action upon the chloroplastid, rendering it temporarily inactive, as was directly shown by Ewart's observations, and this condition may persist for a time even though the assimilatory products are partially remored (cf. Sect. 58 ).

Under normal conditions no marked effect upon the assimilatory activity is noticeable. for the continuous removal of the assimilatory products ensures that no excessive accumulation occurs during the day, while during a warm night the starch or sugar disappears entirely or almost entirely from the leaves of most plants, so that at sunrise they contain much less carbohydrate than at nightfall. The translocation continues during both day and night, and moreover exhibits a certain periodicity ${ }^{1}$. Starch disappears only when translocation is more active than carbon dioxide assimilation, and the activity of translocation is directly or indirectly influenced by various external conditions. Any agency which affects the rate of consumption of the assimilatory products will also affect the translocatory activity, and hence may indirectly influence the assimilatory activity of the chloroplastid. In every case, therefore, it is necessary to determine precisely the various factors concerned in producing the result observed, and since all plants do not necessarily react in precisely the same manner, it is not surprising to find that the presence of common salt causes the percentage of starch to increase in some plants but to diminish in others, evidently because the salt influences the translocatory and assimilatory activities to relatively different extents. Temperature, oxygen, amount of water, \&c., exert the same general effect as they do upon all other vital activities ${ }^{2}$.

The assimilatory activity cannot be determined by estimating the increase in the dry weight of the leaf, for any increase simply represents the extent to which photosynthetic assimilation is more active than translocation and respiration. The latter causes the consumption of from $\frac{1}{1} \frac{1}{0}$ to $\frac{1}{40}$ of the organic material which a leaf produces under optimal illumination ${ }^{3}$. Hence it is only when assimilation is very active that carbohydrates

' Sachs, Arb. d. Bot. Inst. in Wïrzburg, I $88_{4}$, Bd. III, p. 7 ; Miller-Thurgau, Landw. Jahrb., 1885 , Bd. xiv, p. So9; Saposchnikoff, Ber. d. Bot. Ges., 189o, p. 235 ; Costerus, Ann. d. Jard. bot. d. Buitenzorg, I894, Bd. XII, p. 73. Cf. also Mer, Beihefte z. Bot. Centralbl., I 89 r, Bd. I, p. 184. [Costerus (l.c.) has shown that in most tropical plants the starch is translocated most actively during the early morning hours.]

2 Pfeffer, Unters. a. d. Bot. Inst. z. Tübingen, 1886 , Bd. II, p. 291 ; Nadson, Bot. Centralb]., 1890, Bd. XLI1, p. $4^{8}$; Lesage, Compt. rend., $18_{91}$, T. CXII, pp. 672, 891 ; Klebs, Bot. Zeitung, I 891 , p. 809 ; Schimper, Indo-Malayische Strandflora, 1891, p. 26; Stange, Bot. Zeitung, r892, p. 394; Richter, Flora, 1892 , p. 55 ; Stahl, Bot. Zeitung, I894, P. I33. Effect of oxygen: Wortmann, Bot. Zeitung, r $\S_{90}$, p. 662 .

${ }^{3}$ Kreusler, Jandw. Jahrb., 1 885 , Bd. xrr, p. 952. (Cf. Sect. 95.) 
accumulate in sufficient amount to reach the limit inhibitory to further assimilation of carbon dioxide'.

Kreusler estimated that, allowing for the respiratory activity, the leaves of Rubus fruticosus in an atmosphere containing 0.3 per cent. of carbonic acid gas decomposed in one hour per square metre of leaf-surface 2.5 grammes of carbon dioxide (=1.54 grm. starch) when exposed to an intensity of electric light corresponding to diffuse daylight. Such determinations afford a more accurate measure of the intensity of carbon dioxide assimilation than do estimations of the increase in the dry weight of the leaf, for the loss by translocation can only be calculated with approximate accuracy.

Sachs, however, made various estimations in the latter manner by halving a leaf, exposing one-half only to light, and then finding the difference in dry weight between the two. It was thus found that the amount assimilated in one hour per square metre of leaf-surface was, for Helianthus annuus i.8 gramme, and for Cucurbita pepo I.5 gramme. Hence a vigorous sunflower with $\mathbf{1} 45$ leaves $\left(=1.5\right.$ sq. metre of leaf-surface) would obtain $3^{6}$ granmes of organic material in fifteen hours of daylight, while a plant of Cucurbita, with i 16 leaves totalling $7 \cdot 3 \mathrm{sq}$. metre surface area, would gain 185 grammes. This productive activity is necessary in order to provide the material accumulated by a sunflower in the course of a single season, for this may amount to 2 kilos. In trees, owing to the enormous leaf-surface, the total amount assimilated must obviously be much greater, for a small tree of Aesculus hippocastanum, 8.5 metres high, possessed about 8,000 leaves with a total of $320 \mathrm{sq}$. metres of leaf-surface.

It is immaterial for metabolic purposes whence the organic food is derived, but, nevertheless, it does not follow that a leaf will be nourished as well by food conveyed from other leaves as by that which it produces itself, for the stoppage of the normal activity creates abnormal conditions which may act injuriously upon the leaf and ultimately cause its death. Thus leaves exposed to light in an atmosphere devoid of carbon dioxide fall ill after a time, lose the power of photosynthetic assimilation, and sooner or later die $^{2}$. Leaves developed from the commencement in darkness remain living for a longer period, but in this case also the weak power of carbon dioxide assimilation, which a well nourished etiolated leaf may acquire, is ultimately lost, as is also the power of turning green when exposed to light (Ewart, 1.c., p. 554). The leaves of many plants are, however, comparatively resistant and may develop to a marked extent in darkness as well as in an atmosphere free from carbon dioxide, proving that the growth of the leaf is not necessarily dependent upon a supply of auto-assimilatory products ${ }^{3}$.

1 Ewart, Journ. Linn. Soc., Vol. xxxi, 1896, pp. 430-38.

"Vochting, Bot. Zeitung, I891, p. II 3 . See also Ewart, Journ. Linn. Soc., Vol. xxxı, p. 567 ; Jost, Pringsh. Jahrb., 1897, 13d. xxvir, p. 403 .

${ }^{3}$ S. H. Vines, Arb. d. Bot. Inst. in Wiirzburg, 1878 , Bil. 11, p. IIt; Sachs, 1. c., $188_{4}$, 
It does not, however, necessarily follow that the auto-assimilatory products can be entirely replaced by an external supply of organic food, for conversely many saprophytic or parasitic plants, which normally obtain all their organic food from without, can be artificially nourished only with difficulty or not at all. Here, as in all cases, it is not merely the quality of the nutriment that is important, but also the form in which it is presented to the plant, the possibility of its absorbtion by the latter, and many other features as well.

Many plants dependent under normal conditions upon auto-assimilation have been artificially nourished to a certain stage of development (cf. Sects. 64, I09), and green leaves may even form starch from an external supply of sugar. This power of depositing starch, when the percentage of sugar reaches a certain limit, is possessed by etiolated chloroplastids, as well as by many non-chlorophyllous chromatophores (Sects. 53, 54). All chromatophores have not this power, and certain chloroplastids never contain starch, perhaps because solvent enzymes are present which dissolve the starch as fast as it is deposited (cf. Sects. 54 and 93).

Starch seems in general to be formed from dextrose and laevulose, and a supply of cane-sugar or glycerine does not always cause it to appear. Such substances as mannite and galactose cause a deposition of starch only in those plants in which these sugars are produced by photosynthetic assimilation ${ }^{1}$, while all other substances, including various organic acids, have hitherto given negative results. The formation of starch in such cases is dependent partly upon the rate at which the proffered substance is absorbed and partly upon many other relationships, so that further research in this direction may considerably modify the views at present held.

Historical. Bohm first showed that chloroplastids formed starch when artificially supplied with sugar, and these results have been considerably extended by A. Meyer, Schimper, Klebs, Laurent, \&c. ${ }^{2}$

Methods. Leaves of Phaseolus, Nicotiana, and Mosses, or Algae, such as Spirogyra, which have been kept in darkness or in a medium deprived of carbon dioxide until all starch has disappeared, may be placed in a 10 to 20 per. cent. solution of dextrose, when starch appears in the chloroplastids of algae and mosses in a single day; in other plants this may not take place till after several days, but in all it may ultimately accumulate to a considerable extent. Allium cepa, however, does not

Bd. 1 II, p. 5 ; Jost, Jahrb. f. wiss. Bot., I 897 , Bd. xxv11, p. 477 ; MacDongal, Journ. Linn. Soc., i 886 , Vol. $\mathrm{xxxi}, \mathrm{p} \cdot 5^{26}$.

1 A. Meyer (1.c., p. 130) states that starch is formed from mannite in the Olcaccae, from galactose in Sileneae, from dulcite in Euonymus curopaeus.

${ }_{2}$ Böhm, Bot. Zeitung, $188_{3}$, p. 35; Bot. Centralbl., I 889 , Bd. xxxvir, p. 200 ; A. Meyer, Bot. Zeitung, I 886, p. 8 I ; Schimper, ibid.. I 885 , p. 737 ; Klebs, Unters. a. d. Bot. Inst. z. Tübingen, I 886 , Bd. II, p. 538 , and Bot. Zeitung, $1 \$_{91}$, p. 512 ; Laurent, Bull.d.1.Soc. Bot. d. Belgique, I888, T. xxvi, p. 243 ; Bokorny, Ber. d. Bot. Ges., I888, p. 116 ; Versuchsst., I 889 , Bd. xxxvi, p. 240 ; Saposchnikoff, Ber. d. Bot. Ges., I 889 , p. 259 ; Acton, Bot. Centralbl., I89o, Bd. XL1v, p. 224 ; Nadson, ibid., I89o, Bd. Xlıl, p. $4^{8}$; Bokorny, Biol. Centralbl., I897, Bd. xviı, p. I. 
form any starch under these conditions (Schimper, l. c., p. 787). There is a certain optimal concentration at which the formation of starch is most active. Laurent observed that potato shoots formed starch when supplied with from 2.5 to 15 per cent. solutions of dextrose, but not when fed with a solution of 20 per cent. strength, although many plants can form plenty of starch at this degree of concentration ', and indeed in Iris, Galanthus, \&c., starch is formed only when the concentration is as high or higher than this. If sufficient sugar is present in the cell a further concentration produced by plasmolysis with potassium nitrate may cause a formation of starch, a fact which was observed by Böhm, but wrongly interpreted by him (1. c., 1889, p. 200). In many plants the fact that a strong solution of sugar causes permanent plasmolysis shows that very little sugar is absorbed, so that the percentages inside and outside of the cell are not necessarily the same; hence the different specific absorptive powers of different cells exercise an important influence in producing the result observed.

According to A. Meyer, the chromatophores of Cacalia suaveolens may form starch when supplied with glycerine. Acton and Nadson obtained similar positive results with most of the leaves examined, and confirmed Klebs' statement that many Algae form starch from glycerine more readily than from cane-sugar. Meyer states that cane-sugar is absorbed by the leaves of Beta without undergoing inversion. On the rapid diosmosis of glycerine see Sects. 16 and 17 .

Laurent found that malic and citric acids, peptone, acetone, various amides and glucosides, were all incapable of inducing any formation of starch. Bokorny? observed that starchless Spirogyra filaments formed starch in dilute solutions of formaldehyde, of methyl alcohol, and of sodium oxymethyl sulphonate (which readily decomposes and produces formaldehyde), though only when exposed to light, but sufficient precautions do not seem to have been taken to guard against the production of starch by assimilation of carbon dioxide. Even if the observations are correct it does not follow that formaldehyde is the primary product of carbon dioxide assimilation, nor can this importance be attached to sugar or glycerine simply because starch may be formed from them. It has yet to be determined in each specific case whether the starch is formed directly, or only after the substance absorbed has undergone preparatory alterations. Certain fungi are able to derive all their organic food, including carbohydrates, from formic acid and from oxymethyls ${ }^{3}$, a fact which is of considerable importance in this connexion.

\section{SECTION 56. May other Carbon Compounds be Photosynthetically Assimilated?}

With the exception of carbon dioxide, no carbon compounds are known which can be photosynthetically assimilated. Thus neither carbon

1 The minima, optima, and maxima in various plants are given by Schimper, Saposchnikoff, \&c.

${ }^{2}$ l. c., 1888 and 1889 ; also Ber. d. Bot. Ges., 1891, p. 103 . On the negative results with formaldehyde and its poisonous properties, cf. Bokorny, 1.c., I 889, p. $23^{6}$; Laurent, 1.c., 1888 , p. 17. On Trioxymethyl, cf. A. Meyer, 1.c., x 886 , p. 137.

${ }^{3}$ Reinke, Unters, a. d. Bot. Lab. in Göttingen, $188_{3}$, Heft 3,1 ' 33 , and ibid., 'Methylal as fungal food.' 
monoxide nor marsh gas $\left(\mathrm{CH}_{4}\right)$ can be assimilated either alone or in the presence of carbon dioxide'. Carbon monoxide acts as a violent poison only to those organisms which contain haemoglobin, and in all other cases it behaves almost as a neutral gas, so that it is very much less poisonous to plants than the dioxide.

In plants of the Crassulaceae and various other green succulents, the free organic acids (malic, isomalic, and oxalic) produced during the night are decomposed in light with an evolution of oxygen, and a production of carbohydrates (starch, \&c.) ${ }^{2}$. Apparently the organic acid undergoes further oxidation under the action of light, the carbon dioxide produced being immediately assimilated, for in non-chlorophyllous plants the organic acid produced at night-time may undergo similar oxidation, although as a general rule the amount of acid formed is comparatively small, and hence the daily variations in the acidity of the tissues are but slight or imperceptible. It is of great importance to fleshy plants that as little carbon dioxide should be lost during the night as possible, and that it should be preserved in the form of non-volatile organic acids for assimilation during daytime, for in these plants gaseous exchange is difficult and a sufficient supply of carbon dioxide is hard to obtain ${ }^{3}$.

Malic, oxalic acids, \&c. are actually decomposed with an evolution of carbon dioxide when light acts upon them in the presence of certain substances, and living plant-tissues appear to act in a similar manner in accelerating such decomposition ${ }^{4}$, for chlorophyllous plants may evolve oxygen at the expense of organic acids which surround the plant in solution, or which have been injected into it ${ }^{5}$.

1 Saussure, Rech. chim., ISo4, p. 208; Boussingault, Agron., Chim. agric., \&c., I868, T. Iv, p. 300 ; Stutzer, Ber. d. Chem. Ges., I 876 , Bd. IX, p. 1570 ; Just, Forsch. a. d. Geb. d. Agriculturphys., 1882, Bd. v, p. 79. On $\mathrm{CH}_{4}$, Boussingault, l. c., p. 300 .

${ }^{2}$ Literature : Ad. Meyer, Versuchsst., I 875 , Bd. xviII, p. $410 ; 1878$, Bd. xxi, p. $277 ; 188_{4}$, Bd. $x x x$, p. 217 ; $188_{7}$, Bd. Xxxıv, p. 127 ; Die Sauerstoffabscheidung fleischiger Pflanzen, I8 76 ; G. Kraus, Über die Wasservertheilung in der Pflanze, IV, $188_{+}$, and Stoffwechsel bei den Crassulaceen, I 886 (Sep.-abdr. a. d. Abhandlungen d. Nat.-Ges. z. Halle, Bd. xvi); H. de Vries, Periodicität im Säuregehalt d. Fettpflanzen, $188_{+}$(Sep.-abdr. a. Meded.d. Akad. in Amsterdam); Warburg, Unters. a. d. Bot. Inst. z. Tübingen, I 886, Bd. II, p. 53; Aubert, Rev. gén. d. Bot., I892, Bd. IV, p. 203 , and Ann. d. sci. nat., I $89^{2}$, vii. sér., T. xvi, p. I ; Purjewicz, Bot. Centralbl., I 894 , Bd. Lvirı, p. 368.

${ }^{3}$ Cf. Warburg, l. c.; A. Meyer, l. c., I 887 , p. I to.

* Becquerel, La lumière, I868, T. II, p. 60 ; Ar. Nüller, Einfluss d. Lichtes auf Wasser, I8 7 , p. 25 ; Ad. Mayer, 1. c., I878, p. 321 ; de Vries, l.c., I884, p. 53 ; Purjewicz, 1.c., I894, p. 37 I ; Wehmer, Bot. Zeitung, I891 , p. 322 ; Richandson, Ber. d. Chem. Ges., I 894 , Ref. p. 496 . A solution containing $\mathrm{Fe}_{2} \mathrm{Cl}_{6}$ and oxalic acid evolves $\mathrm{CO}_{2}$ actively in sunlight. Cf. also Ostwald, Lehrb. d. allgem. Chemie, I893, Bd. II, p. 1031 .

5 Warburg, l. c., I886, p. I12; Mangin, Compt. rend., I889, T. cIx, p. 7 I6. Schmöger (Ber. d. Chem. Ges., IS79, Bd. XII, p. 373) has shown that the increase in the dry weight, which Stutzer (Versuchsst., 1877, Bd. xxi, p.93) observed in plants fed with organic acids, is due to the assimilation of $\mathrm{CO}_{2}$ derived from these acids. Since the decomposition of the acid takes place only under certain conditions, negative results may be oblained in some cases. See Ad. Mayer, Versuchsst., $\mathrm{I} 88_{4}, \mathrm{Bd} . \mathrm{xxx}, \mathrm{p} .226$. Wehmer, indeed, finds that the formation of citric acid in Citromyces is not 
Chemical decompositions of this nature as well as those metabolic changes which are induced directly or indirectly by the action of light (including therefore the formation of chlorophyll), are to be distinguished from the synthetic processes for whose performance light supplies the necessary energy. It is possible that in addition to the assimilation of carbon dioxide other photosynthetic processes may be discovered, and it has not even been tried whether the chlorophyll apparatus is perhaps able to assimilate by photosynthesis such compounds of carbon as $\mathrm{CO} \mathrm{Cl}_{2}, \mathrm{COS}$, $\mathrm{CO}\left(\mathrm{NH}_{2}\right)$, \&c.

Heyne $^{1}$ was the first to observe that the leaves of Bryophyllum calycinum acquired an acid taste during the night, and Link found that the same occurred in certain Crassulaceae. In 1875 Ad. Mayer studied these daily variations more closely and showed that the diminution in acidity was accompanied by an evolution of oxygen. G. Kraus proved the general nature of the phenomenon, and Warburg showed that a marked production of free organic acids might occur not only in plants belonging to the Crassulaceae, Cactaceae, Mesembryanthemum, \&c., but also in many thin leaves possessing a strongly developed cuticle, thus indicating the probable importance of the phenomenon. The relation between the gaseous exchanges and the variations in the acidity have recently been reinvestigated in detail by Aubert.

Plants of the Crassulaceae produce isomalic acid, of the Cactaceae malic acid and Mesembryanthemum oxalic acid (Aubert). Since the acid is formed by oxidation, a plant placed in darkness does not evolve any carbon dioxide until a certain amount of organic acid has accumulated, when the amounts of oxygen absorbed and of carbon dioxide exhaled assume their normal relative proportions. This fact was known to de Saussure, but it is not due to any absorption of carbonic acid, for a succulent plant in an atmosphere deprived of carbonic acid gas still evolves oxygen in abundance when exposed to light ${ }^{2}$. The fact that the accumulation of acid is definitely limited shows that it is not caused by a deficiency of oxygen, but is due to the specific nature of the plant in question, as may be shown by direct experiments ${ }^{3}$.

External conditions influence the accumulation of acid, a high temperature or illumination causing the acidity to diminish. Thus the percentage of acidity decreases to about the same extent when the temperature of a darkened plant is raised from $15^{\circ} \mathrm{C}$ to $45^{\circ} \mathrm{C}^{4}$ (de Vries, Warburg) as when it is exposed to daylight, the difference being that carbon dioxide is exhaled instead of oxygen ;

perceptibly affected by light (Beiträge z. Kenntniss einheimischer Pilze, $18_{93}$, I, p. $4^{8}$ ). Similarly the acidity of dead plants or of expressed sap is not markedly altered by exposure to light (C. Kraus, 1.c., I886, p. 40 ; A. Mayer, Versuchsst., $188_{7}$, Bd. xxxiv, p. 13 I $)$.

${ }_{1}$ Heyne, Jahrb. d. Gewächskunde v. Sprengel, Schrader u. Link, IS19, Heft 2, p. 7o. Cf. G. Kraus, 1.c., $188_{4}$, p. I4.

${ }^{2}$ Saussure, Rech. chim., ISo4, p. 64. C.. Warburg, 1. c., p. 99. See Ad. Mayer, I 87 S, 1.c., p. 284 , \&c.; Aubert, Rev. gén., 1. c., p. 275. On the absorption of $\mathrm{CO}_{2}$, cf. Sect. $9^{6}$.

${ }^{3}$ See Warburg, l.c., p. 85, and Aubert, Ann. d. sci. nat., 1.c., p. 39.

- [Gerber (Ann. d. sci. nat., IV, I897, pp. I-280) states that in fleshy fruits citric and tartaric acids decompose at $30^{\circ} \mathrm{C}$, malic at $15^{\circ} \mathrm{C}$.] 
while in both cases the volume of gas in the receiver increases proportionately to the amount of organic acid decomposed '

Although no absolute proof has been given, it can hardly be doubted that this special production of oxygen takes place at the expense of the accumulated organic acids. Decisive evidence as to whether the acid in the cell-sap may be oxidized to carbon dioxide without the aid of the chloroplastids, might be obtained by inducing in then a condition of assimilatory inhibition, or by exposing an etherized plant to light. The more active decomposing action exerted by the less refrangible rays affords no conclusive evidence one way or the other ${ }^{2}$, but even if the chloroplastids do actually produce carbohydrates directly from organic acids, still this does not support Liebig's" contention that the formation of organic acids is the first step in the assimilation of carbon dioxide.

The accumulation of acid may be very pronounced, and thus Kraus (1. c. I884, p. I9) found that I cubic centimetre of the sap of Bryophyllum, expressed from a plant kept in darkness, neutralized 5.5 cubic centimetres of a c.OOI per cent. solution of sodium hydrate, whereas after being exposed to light I cc. of the sap neutralized only $0.45 \mathrm{cc}$. of the soda solution. According to Mayer (1. c., I878, p. 287) 28 grammes of acid leaves of Bryophyllum when exposed to light may produce $40 \mathrm{cc}$. of oxygen, derived solely from the organic acid which they contain. General summaries are given by Warburg (1. c., p. I 44), and by Aubert (Rev. gén., l. c., p. 422).

\section{SECTION 57. The Sources of Carbon Dioxide and the Optimal Percentage for Assimilation.}

The structural arrangements have already been described by means of which the plant is able to obtain a sufficient supply of carbon dioxide in spite of the small amount present in the surrounding air, and it is owing to these special adaptations that a leaf or green stem absorbs directly from the atmosphere ${ }^{4}$ almost the whole of the carbon dioxide which it assimilates. The amount obtained from the roots and deeper parts of the stem is insufficient to cause any perceptible production of starch, as Moll found when the stem and leaves of a plant were kept in an atmosphere free from carbon dioxide, but its roots were in a humus soil. Minute quantities of dissolved gas may, however, reach the leaves from the roots, and de Saussure $^{5}$ showed that traces of oxygen may be produced when no other

1 For examples of the changes occurring in the composition of the enclosed air under varying conditions, see Aubert, 1. c., pp. 443, 559 .

${ }^{2}$ G. Krans, l.c., I 884, p. $20 ; 1886$, p. 43 , according to whom the opposite results given by other authors are not applicable to the point at issue.

${ }^{3}$ Liebig, Die organ. Chem. in Anwend. auf Agric. u. Physiol., 1840, p. 26, and $18 ; 6$, pp. $1_{7}, 3^{1}$.

4 Recognized by Saussure, Rech. chim., 1804, p. $5^{1}$.

${ }^{5}$ Saussure, 1.c., pp. 112, 122 ; Boussingault, Agron., Chim. agric., \&c., I868, T. IV, p. 294. 
source of carbonic acid gas is available. Indeed the amount derived from the roots may, when transpiration is active, be sufficient to prevent the chloroplastids in the more deeply situated tissues at the base of a green stem from losing the power of assimilating carbon dioxide when exposed to light for prolongcd periods in an atmosphere free from this gas ${ }^{1}$.

Even a feebly developed cuticle is usually so impermeable that the leaves can absorb practically no carbonic acid gas from the surrounding air when the stomata are closed, so that any closure of the latter induced by excessive transpiration causes assimilation almost entirely to cease. Hence the formation of starch is in most cases entirely suppressed under such circumstances, as it is also when the leaf-surface bearing stomata is smeared with fat, whereas if only a portion of the leaf is so smeared, starch appears for the most part or entirely in the unsmeared regions". Blackman has shown that hardly any carbonic acid gas penetrates through the non-stomatic surface of a leaf, whereas when assimilation is active the stomatic surface robs the air drawn slowly over it of almost all its carbon dioxide ${ }^{3}$.

Air currents continually bring fresh supplies to the leaves, and hence large quantities can be assimilated, although but 0.03 to 0.04 per cent. of this gas is present in the atmosphere ${ }^{4}$, so that 4,000 litres of air contain the 1,250 cubic centimetres (about 2.5 grammes) of carbon dioxide that a surface of one square metre of actively assimilating leaves may decompose per hour (Sect. 55). A comparative research showed that a similar area of a 7.5 per cent. solution of soda absorbed carbon dioxide with five to six times greater avidity when air was drawn slowly over it.

Currents of water are of the utmost importance in providing aquatic plants with continual supplies of dissolved carbonic acid. Owing to the marked solubility of the latter a given volume of water can hold a higher percentage than is present in the supernatant air, while still more may be held in the form of dissolved carbonates and bicarbonates ${ }^{5}$. Indeed, according to Hassak, a green plant is not only able to obtain carbon

1 Ewart, Journ. Linn. Soc. Bot., I896, xxxi, p. 569 .

"Stahl, Bot. Zeitung, I894, p. I29. Moll (Landw. Jahrb., I877, Bd. vi, 1).343) obtained a similar result by exposing a portion of a leaf to light in a $\mathrm{CO}_{2}$-free atmosphere, the other portion being in an atmosphere containing this gas.

${ }_{3}$ Blackmann, Phil. Trans., 1895 , Vol. cxxvi, p. $55^{6}$; Annals of Botany, I895, Vol. $\mathrm{x}, \mathrm{p} .164$. When the percentage of $\mathrm{CO}_{2}$ is high, more passes through the cuticle, and to this fact the remarkable exceptions noticed by Boussingault are due (Agron., \&c., I 868, T. IV, p. 359). Cf. also Stahl, l.c., p. 132. Researches on the removal of carbon dioxide from air drawn slowly over assimilating leaves were carried out by Boussingault, Die Landw., I $8_{5}$ I, Bd. I, p. 40 ; Vogel und Witwer, Abhandlungen d. Münch. Akad., I $8_{5^{2}}$, Bd. vi, p. 267 ; Corenwinder, Ann. d. chim. el d. phys., I $8_{5} 8$, iii. sér., T. LIV, p. 321 .

4 Cf. Sachsse, Agriculturchemic, I 888, p. Iо.

${ }_{5}^{5}$ See Sachsse, Agriculturchcmie, I 888, p. 9. Cf. also Fr. 1)arwin and P'c1tz, Proc. Camb. P'hil. Suc., 1896 , Vol. Ix, p. 76 . 
dioxide from Calcium bicarbonate $\left(\mathrm{Ca} 2\left(\mathrm{HCO}_{3}\right)\right.$, but can also make use of 70 per cent. of the carbon dioxide contained in sodium bicarbonate ${ }^{1}$ $\left(\mathrm{Na} \mathrm{HCO}_{3}\right)$.

When the stomata of land-plants are occluded by capillary water, the rate of gaseous exchange. undergoes a very marlied diminution, and hence sub-aërial leaves wetted by water assimilate but little carbonic acid gas when immersed, although so long as the adherence of an intermediary film of air is shown by their silvery appearance, rapid gaseous exchange is still possible, and hence such leaves may continue to assimilate fairly actively under water ${ }^{2}$ (Sect. 29).

The bubbles of gas evolved from assimilating aquatic plants (Sect. 32) are not pure oxygen but contain larger or smaller amounts of nitrogen and carbon dioxide, the amount of oxygen varying from 25 to as much as $9^{8}$ per cent.. ${ }^{3}$, when the evolution has continued for some time and no fresh nitrogen has been allowed access to the plant (Sect. 54). The number of bubbles also depends upon the diosmosis of oxygen into the intercellular spaces, where it accumulates together with other gases, and hence the evolution of gas bubbles does not form an accurate indication of the actual amount of oxygen produced. Indeed, when but little oxygen is formed, it may entirely dissolve in the surrounding water without any bubbles appearing. Nevertheless an approximate measure of the activity of assimilation may be obtained in this manner, and the accuracy of the method largely depends upon the way in which it is applied ${ }^{4}$.

The activity of assimilation is increased by the addition of carbon dioxide to the air, and hence it follows that a well illuminated chloroplastid does not normally obtain as much of this gas as it is capable of decomposing. An excess of carbon dioxide exerts a poisonous influence upon the plant ${ }^{5}$, and hence there is a certain optimal percentage at which assimilation is most active, and this varies not only in different plants, but also according to the external conditions, for both the intensity of the illumination and the rapidity of gaseous exchange must influence the assimilatory curve. Thus when the stomata are closed, assimilation may be active only when the percentage of carbon dioxide present in the external air is abnormally high, whereas under normal conditions a slight increase in the percentage

1 Hassak, Unters. a. d. Bot. Inst. z. Tiibingen, I888, Bd. I1, p. 47 I ; Weyl, Silzungsb. d. Phys.-Med. Ges. in Erlangen, I88I, I. Aug. Cf. Sect. 23.

${ }^{2}$ Böhm, Sitzungsb. d. Wien. Akad., I872, Bd. Lvi, Abth. i, p. I69; Nagamatsz, Arb. d. Bot. Inst. in Wïrzburg, I887, Bd. III, p. 389. [Bonnier Compt. rend., CxxVI, I898, p. IooI) has actually succeeded in developing plants of Mimosa fudica under water.]

${ }^{3}$ See de Candolle, Phanzenphysiol., I8 33 , Hd. I, p. Io2; Daubeny, Phil. Trans., I $S_{36} 6$, Pt. i, p. I57; Cloez et Gratiolet, Ann. d. chim. et d. phys., I8 5 I, iii. sér., T. XxxII, p. 5 I ; N. J. C. Müiller, Jahrb. f. wiss. Bot., I867-8, Bd. vi, pp. $4^{8}+$, \&c.

${ }^{4}$ Cf. Pfeffer, Arb. d. Bot. Inst. in Würzburg, I $8_{7}$ I , Bd. I, p. $5^{2}$; keinke, Bot. Zcitung, I $88_{4}$, p. 24 .

${ }^{5}$ Grischow, Unters. über d. Athmung, Iồ 9 , p. 33 ; Boussingault, Agron., S.c., I868, T. IV: pp. 286, \&c. 
of this gas is sufficient to produce the maximal activity of carbon dioxide assimilation.

Kreusler ', by using the constant illumination of the electric light and maintaining a constant percentage of carbonic acid gas, estimated for the leaves of Rubus, Carpinus, Tropaeolum, \&c., the approximate assimilatory activity in atmospheres containing varying amounts of carbon dioxide. The middle column of the following table indicates how much more of this gas was present than in ordinary air.

$\begin{array}{ccc}\text { Amount of } \mathrm{CO}_{2} \text {. } & \text { Relative assimilatory activity. } \\ \text { Percentage. } & \text { In air }=\mathrm{I} . & \text { In air }=100 . \\ 0.03 & \mathrm{I} & \mathrm{100} \\ 0.06 & 2 & 127 \\ 0.1 \mathrm{I} & 3.5 & 185 \\ 0.56 & \mathrm{I} 7 & 209 \\ 7.26 & 220 & 230 \\ 14.52 & 440 & 266(?)\end{array}$

The optimal percentage appears to lie at about 10 per cent. Godlewski ${ }^{2}$ obtained similar results, while, as might be expected, under strong illumination the optimal percentage is somewhat higher. It is probable that during the period of coal-formation the air contained more carbon dioxide than it does now, and the luxuriant vegetation of this epoch was perhaps partly due to this fact (Sect. 5 I). The optimal percentage for growth is not the same in all cases; Montemartini found that plants grew best in air containing 4 per cent. of carbon dioxide, while de Saussure found that the growth of peas was retarded in the presence of 8 per cent. of this gas; results obtained by other observers show that green plants are ultimately injured by an atmosphere containing 4 to 15 per cent. of this gas, in which at first assimilation is most active ${ }^{3}$. Under normal conditions the carbon dioxide hardly ever accumulates to an injurious or fatal extent in the air, whereas in soil and putrefying water this may often occur ${ }^{4}$. It is not the percentage amount of carbon dioxide but its partial pressure that is all important, and Boussingault and Böhm have shown that when mixed with an indifferent gas under pressure a small percentage may act injuriously ${ }^{5}$.

1 Kreusler, Landw. Jahrb., I 885 , Bd. xiv, p. 95 I.

${ }^{2}$ Godlewski, Arb. d. Rot. Inst. in Wiirzburg, I873, Bd. 1, p. 343. Schutzenberger (Compt. rend., 1873 , T. Lxxvil, p. 272) obtained similar results with water-plants.

$s$ Montemartini, quoted by Lopriore, Jahrb. f. wiss. Bot., I 895 , Bd. xxvir, p. 539; Saussure, Rech.chim., I 804 , p. 29; Davy, Elements of Agric. Chem., I $\delta_{2}$ I, 3 rd ed., p. 205 ; de Vries, Landw. Jahrb., I879, Bd.viII, p.4 ${ }^{\mathrm{I}} 7$. See also the literature given by Lopriore, 1.c., p. $57 \mathrm{I}$; Mangin, Compt. rend., I $896, \mathrm{~T}$. $\mathrm{Cxx} 11, \mathrm{p} .747$, and Étude s. l. végétation d. s. rapports avec l'aération du sol, I896.

4 On the carbonic acid of the soil cf. Sect. 28 . Influence on the growth of roots: Jentys, Bull. d. l'Acad. d. sci. d. Cracovie, July, 1892 .

"Boussingault, Agron., \&c., I 868, T. Iv, p. 286; Böhm, Über Bildung von Sauerstoff durch grïne Landpflanzen, I $\delta_{72}$, p. I $\delta$ (Sep.-abdr. a. Sitzungsb. d. Wien. Akad., Bd. Lxvi, Abth. i). Cf. also N. J. C. Müller, Bot. Unters., I8 76 , Bd. I, p. 353 . 


\section{SECTION 58 . The Influence of External Conditions on the Assimilation of Carbon Dioxide.}

The assimilatory activity being a vital process, is liable to be influenced in various ways by the external conditions, even although the illumination and the supply of carbonic acid gas remain constant. Indeed the formation and development of the chloroplastids themselves are possible only when certain essential conditions are fulfilled, while when adult their assimilatory powers may be directly or indirectly modified or altered. In investigating such alterations it must be remembered that various other changes may occur at the same time, and that the prolonged action of any agency may ultimately produce a different effect to that caused by it when first applied (cf. Chap. I). It is, however, only possible to give a very condensed account of these problems, as well as of the conditions necessary for the development of the chloroplastids.

Conditions necessary for the development of the chloroplastids. It is the inherent nature of the embryonic chromatophore which determines in what direction it will develop, and whether it has a tendency to become a chloroplastid (Sect. 53). The latter turns green, as a general rule, only when exposed to light, for in darkness yellow etiolin corpuscles are formed, and these even in weak light rapidly produce chlorophyll and turn green, provided that they have not lost this power through being kept too long in darkness ${ }^{1}$. Certain plants, however, form green and functionally active chloroplasts in the absence of light, as is the case in the cotyledons of seedlings of various Coniferae (Pinus sylvestris, picea, \&c.), although the winter buds of these plants and the seedlings of Gingko biloba form no chlorophyll in darkness, and seedlings of Larix and Thuja turn green in parts only ${ }^{\circ}$.

The formation of chlorophyll is therefore not necessarily connected with the presence of light, and its non-formation in darkness may be due to disturbances of nutrition or to pathological conditions, for the chloroplastids of many plants become pale or discoloured after a few days' darkness, whereas those of others, such as plants belonging to the Cactaceae

1 Literature: Sachs, Flora, IS62, p. I62, and Bot. Zeitung, IS62, p. 366 ; Wiesner, Entstehung d. Chlorophylls, IS 87 , p. 86 ; Batalin, Bot. Zeitung, 1871, p. 677 ; Monteverde, Acta Horti Petropolitani, I89+, Bd. xIII, p. 215 . On intermittent illumination, cf. Mikosch u. Stöhr, Sitzungsb. d. Wien. Akad., ISSo, Bd. LXxx11, Abth. i, p. 629. On final loss of power of turning green, Detmer, Landw. Jahrb., I882, Bd. X1: p. 224. See also Ewart, Journ. Linn. Soc. Bot., Vol. xxxı, I896, pp. $560,8 \mathrm{c}$.

2 Sachs, Lotos, I859, and Flora, I864, p. 505 ; Mohl, Bot. Zeitung, I861, p. 258 . Schmidt's objections (Einige Wirkungen d. Lichtes auf Pflanzen, IS7o, p. I8) are without value. In Ferns, Mosses, Chara, \&c., light does not seem to be essential for the formation of chlorophyll. See Schimper, Jahrb. f. wiss. Bot., I $\$_{5}$, Bd. Xvi, p. I59, and the literature there given; also Ewart, 1.c., pp. 555, 563, \&c.: Frank, Die natïrliche wagrechte Richtung von Pflanzentheilen, 1870, p. 27 ; Molisch; Oest. Bot. Zeitschr., 1889, Nr. 3 ; Wiesner, Entstehung d. Chlorophylls, I877, p. I I 7 . 
and Coniferac, as well as those of Eloder, Chara, \&c., are more resistant and may remain green for more than a month in darkness ${ }^{1}$. When the supply of nutriment becomes deficient colourless plastids are formed instead of etiolin corpuscles, but most scedlings and bulbous plants are able in darkness to form yellow etiolin corpuscles which exhibit a faint but distinct power of assimilating carbon dioxide (Heliunthus, Cucurbita, Allium, Beta, \&c.). In other cases, as in Zca May's, the etiolin corpuscles, though deep yellow in colour, have no such power, and in all cases this power of assimilation is lost in continued darkness after a shorter or longer period ${ }^{2}$.

Plants turn green most rapidly in light of moderate intensity, and various observations indicate that in the living chloroplastid the decomposition and reconstruction of chlorophyll continue without cessation, especially in strong light ". No direct conclusion as to the behaviour of chlorophyll under normal conditions can be drawn from the blanching of solutions of chlorophyll, or of chloroplastids when exposed to intense sunlight. Pringsheim was able to completely destroy the chlorophyll in chloroplastids by means of concentrated sunlight, but the fact that they remained colourless showed that the power of regenerating chlorophyll had also been lost, if it previously existed ${ }^{4}$. A single day's exposure to full sunlight may, however, cause the leaves of Pisonia alba and of some species of Sclaginella to lose all green colour and become pale yellow or almost white. The chloroplastids, however, retain the power of starch-formation, and in feeble light the green colour may gradually be restored. Various other plants of a smaller degree of sensitiveness show similar but less marked reactions to sunlight ${ }^{5}$.

All the visible rays of the spectrum may excite the formation of chlorophyll, but according to Reinke the maximal effect is produced by those between Fraunhofer's lines $B$ and $D$, while IViesner has shown that in strong light the more refrangible rays are most effective ${ }^{6}$.

1 Literature: Sachs, Flora, I 862 , p. 2 I 8; Bot.Zeitung, 1864, p. 290; Wiesner, Úber die Beziehung d. Lichtes z. Chlorophyll, i 874, p. 49 (Sep.-abdr. a. Sitzungsb. d. Wien. Akad., Bd. Lxix, Abth. i); Frank, Bot. Centralbl., I $8 S_{2}$, Bd. X, p. 230 ; Reinke, Sitzungsb. d. Berl. Akad., I 893, p. 528 ; Ewart, Journ. Linn. Soc., Vol. xxxi, i 896, pp. 564,573 .

${ }^{2}$ Ewart, Journ. Linn. Soc. Bot., 1 896 , Vol. XXxi, pp. $556,561,564,8 \mathrm{c}$.

${ }^{3}$ Sachs, Flora, I862, p. 214 ; Famintzin, Jahrb. f. wiss. Bot., I $867-8$, Bd. vi, p. 47 ; Wiesner, Die natürlichen Einrichtungen zum Schutze d. Chlorophylls, 18 76, p. 21 iSep.-abdr. d. Festschr. d. 7ool.-Bot. Ges. in Wien); Haberlandt, Unters. üher die Winterfärbung ausd. Blätter, I $\delta 6$, p. Iо (Sep.-abdr. a. d. Sitzungsb. d. Wien. Akad., Bd. Lxxir, Abth. i); Schimper, Jahrb. f. wiss. Bot., I $88_{5}$, Bd. Xvi, p. $16_{5}$; Reinke, Bot. Zeitung, I885, p. I33. Ewart (Annals of Botany, Vol. xII, I $898, \mathrm{p} .392$ ) has calculated that a chloroplastid of Elodea may produce, during a day's exposure to bright light, as much as four to five times the amount of chlorophyll it originally contained.

${ }^{4}$ Pringsheim, Jahrb. f, wiss. Bot., I879-8I, Bd.xiI, p. 345. Even after a year no regeneration of chlorophyll took place in chloroplastids of Chara decolorized by prolonged exposure to sunlight (Ewart, Journ. Linn. Soc., Vol. Xxxr, p. 573), whereas in Elodea they may become green again (Ewart, Annals of Botany, I 898 , Vol. XiI, P. 392).

${ }^{5}$ Ewart, Annals of Botany, I897, Vol. XI, p. 442; Wiesner, Sitzungsh. d. kais. Akad. d. Wiss. in Wien, $1894 \cdot$ p. 305.

${ }^{6}$ Reinke.Sitzungsh. d. Berl. Akad., I $\$_{93}$, p. 5,36. The observations of Fardiner and Guillemin 
Tomperature. A low temperature at which slow, growth is possible is* often insufficient for the formation of chlorophyll, so that, as Sachs observed, plants developed at the minimal temperature for growth do not turn green, although the chloroplastids become differentiated, and, according to Elfving, light causes them to become a deeper yellow ${ }^{1}$. The winter browning of Conifers and other plants is caused by the combined action of light and a low temperature, and is due to a partial decomposition of the chlorophyll accompanied by a formation of brown, yellow, or red pigments, and frequently by a partial disorganization of the chloroplastids. In some cases the browning takes place only when the chlorophyll-containing cells are fatally affected, as in Ilex, \&c., but in most Conifers the chloroplastids may recover, turn green, and become functionally active when restored to normal conditions of temperature ${ }^{2}$.

Oxygen: Food supply. The formation of chlorophyll seems to be in general comparatively easily inhibited, for Correns ${ }^{3}$ has shown that a partial pressure of oxygen, at which growth and heliotropic curvature are still possible, does not suffice for the formation of chlorophyll. Unfavourable nutritive conditions may frequently prevent the chloroplastids partially or entirely from turning green, and this is perhaps why the absence of iron produces chlorosis and prevents the normal differentiation of the chloroplastids (Sect. 74).

On the other hand, a self-regulatory development of chlorophyll occurs when a stem of Cuscuta becomes a deeper green if deprived of a hostplant ${ }^{4}$, or when a shoot grown from a colourless root develops green chloroplastids on exposure to light.

Permanent and transitory inhibition. The chloroplastids frequently lose their assimilatory function in the normal progress of development, as when they become converted into red or brown chromoplasts in a ripening fruit, or when the chlorophyll disappears as the leaves acquire their autumnal

are given by Reinke on p. 529. Sachs, Bot. Zeitung, 1864, p. 353 ; Wiesner, Entstehung d. Chlorophylls, I877, p. 39; Die heliotrop. Erscheinungen im Pflanzenreich, I878, p. I94, footnote.

1 Sachs, Flora, $186_{4}, \mathrm{p} \cdot 497$. Wiesner has shown that there is an optimum temperature for the formation of chlorophyll (Entstehung d. Chlorophylls, i $\delta_{77}$, p. 95). Elfving, Arb. d. Bot. Inst. in Wïrzburg, 1880 , Bd. I, p. 495 .

${ }^{2}$ Mohl, Vermischte Schriften, I 845, p. 375 . Details by Schimper, Jahrb. f. wiss. Bot., I $\$ 8_{5}$, Bd. xvı, p. I66; G. Haberlandt, Über die Winterfärbung ausdauernder Blätter, I876, p. Io (Sitzungsb. d. Wien. Akad., Bd. Lxxir, Abth. i); Ewart, Journ. Linn. Soc., I896, Vol. xxxi, p. 390. [H. Winkler (Jahrb. f. wiss. Bot., I $S_{9} 8, \mathrm{Bd}$. $\mathrm{Xxx}_{11}, \mathrm{p} .538$ ) has shown that such discoloured chloro. plastids may acquire the power of starch formation (if supplied with sugar) before they turn green or become capable of carbon dioxide assimilation.]

${ }^{3}$ Correns, Flora, $\mathrm{IS}_{92}$, p. 14 . On the retarding influence of an accumulation of carbon dioxide on the formation of chlorophyll cf. Böhm, Sitzungsb. d. Wien. Akad., I8 73 , p. I4. [Microscopical preparations of etiolated leaves of Elodea, if thinly ringed with vaseline, may show slow rotation, but are unable to turn green. Cf. Ewart, Journ. Linn. Soc., i 896 , Vol. xxxi, p. 566.]

- Pierce, Annals of Botany, r 894 , Vol. viri, p. 81. On the influence of the food-supply on the turning green of seedlings, see Palladin, Ber. d. Bot. Ges., I $89 \mathrm{I}$, P. 229. 
colouration '. In such cases, as well as when the chloroplastid is completely bleached by intense light, the power of assimilating carbon dioxide is irrevocably lost, whereas the chloroplastids of Conifers which have become brown in winter may turn green and recover the power of assimilation in spring (Ewart, l.c., p. 390).

A transitory inhibition of the power of photosynthetic assimilation may be produced, as Ewart has shown, by the action of the most varied injurious agencies, which in more concentrated form ultimately cause death ${ }^{2}$. The stoppage of the chlorophyll-function is to a certain extent a precursor of death, but if the action of the injurious agency is not too prolonged recovery may occur after a longer or shorter latent period. Chloroplastids may be perfectly normal in colour and in external appearance when in this inactive condition, and hence some obscure change in their internal constitution or assimilatory mechanism must have been produced. In certain cases rapid recovery is possible, but in others a prolonged period intervenes before the normal conditions can be restored. Similar latent periods of recovery are exhibited when plasma-streaming, irritability, growth, \&c., have been inhibited by the prolonged action of depressing external agencies.

A weakening and ultimate cessation of the power of assimilating carbon dioxide may be produced by exposure to extremes of temperature or to intense sunlight, by the action of poisons such as carbonic acid, ether, chloroform, antipyrin, \&c., by partial asphyxiation, and by the accumulation of the assimilatory products (Ewart, l.c.). Thus Ewart found that leaves of Ilex, Buxus, Prumus, \&c., exposed for a short time only to a temperature falling from $0^{\circ} \mathrm{C}$ to $-4^{\circ} \mathrm{C}$ or $-6^{\circ} \mathrm{C}$ immediately recommenced to assimilate at a normal temperature, whereas when exposed to the same low temperature for a few days the chloroplastids became completely inactive, and were able, if they remained living, to evolve oxygen in the light only after being kept for from one to twenty-four hours at a normal temperature. It has not been found possible to induce a permanent inhibition of this function without either killing the chloroplastids or destroying the chlorophyll, nor have any normal chloroplastids been as yet observed which have permanently lost all power of photosynthetic assimilation (Sect. 52 ). The weakening in the power of assimilation which Boussingault noticed in leaves kept in an excess of carbon dioxide for some time is simply a special example of assimilatory inhibition, and the cessation of the chlorophyll-function in certain mosses and lichens, observed by Jumelle and apparently supposed by him to be a permanent change, is another example of the same phenomenon ${ }^{3}$.

${ }^{1}$ Kreusler, Landw. Jahrb., I 885, Bd.xIr, p.9.5 ; Engelmann, Bot. Zeitung, I 881 , p. $44^{6}$; Ewart, Journ. Linn. Soc., I 896 , Vol.Xxxi, p. 437. On changes in the shape of the chloroplastids caused by external agencies, cf. Klebs, Unters. a. d. But. Inst. z. Tübingen, $188_{3}$, Bd.1, p. 268 ; Schimper, Jahrb.f. wiss. Bot., I 885 , Bd. LXV1, 1). I94; Richter, Flora, 1892 , p. 55 ; Ewart, 1.c., Pp. $449,45^{1}, 5^{65}, 5^{67}, 569$.

2 Ewart, Journ. Linn. Suc., 1896 , Vol. xxxi, p. $364 ; 1897$, p. 554 . An abstract of the above papers is given by Pfeffer in Ber. d. Sächs. Ges. d. Wiss., $1896, \mathrm{p} . \mathbf{3}^{\mathrm{I}} \mathbf{1}$.

3 Boussingault, Agron., \&c., I 868 , T. 1V, p. 287 ; Pringsheim, Sitzungsb. d. Berl. Akad., 188 ${ }_{i}$; 1. 768. Cf. also Engelmann, Bot. Zeitung, 18ss, p. 717 ; Jumelle, Rev. gén. d. Bot., T. 1v, p. 263 . 
Owing to reactions of this character, as well as to the more or less marked powers of accommodation which all plants possess, it is often difficult or impossible to establish any accurate numerical relationship between the external conditions and the changeable vital activities of the plant, for at a minimal or a maximal temperature the assimilation of carbon dioxide, the processes of growth, \&c., continue feebly for a time, but ultimately cease. The following data are all estimations made under the immediate action of the changed conditions, and hence are only approximately accurate. Within certain favourable limits, however, the chloroplastid rapidly accommodates itself to altered conditions.

Infucnce of the extcrnal conditions-Temperature. The curve of assimilation rises as the temperature increases, and remains fairly constant at an optimum approximating to that for growth, as is shown in a special case in Fig. 50. Above this optimum the assimilatory curve falls again, whereas the respiratory curve continually rises (Fig. 50 and Sect. I I4). The difference between the gain of organic substance by photosynthesis and its loss by respiration hence rapidly dimi-

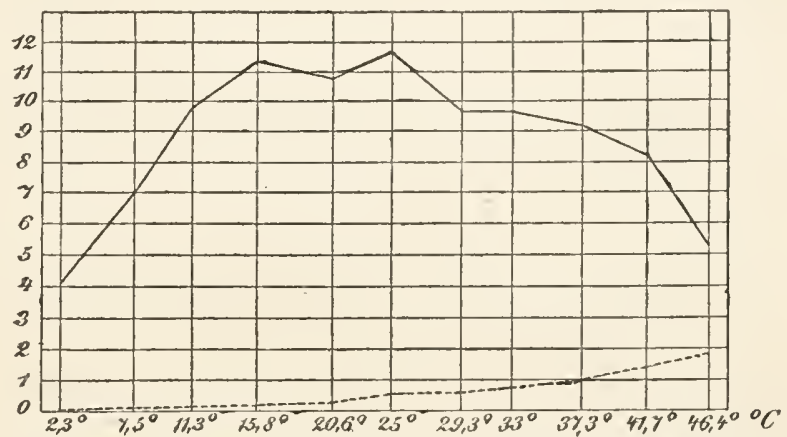

FIG. 50. Assimilation and respiration in a leafy branch of Rubus fruticosus at different temperatures (after Kreusler). When assimilating, the branch is surrounded by air containing 0.3 per cent. $\mathrm{CO}_{2}$, and is illuminated by electric light (=bright diffuse daylight). By adding the height of the respiratory curve to that of the assimilatory one the actual amount of $\mathrm{CO}_{2}$ assimilated is obtained.

… $\mathrm{CO}_{2}$, produced by respiration $\}$ per hour per sq. metre of leaf-surface.

nishes as the temperature rises beyond the optimum, and although in the case given in Fig. 50 assimilation surpasses respiration even to the last, it is often the case that leaves with abundant chlorophyll under brilliant illumination may produce more carbon dioxide at or above $40^{\circ} \mathrm{C}$. than they can decompose (Kreusler, 1.c., 1890, p. 663). If the plants are exposed for some time, the same result may be produced at an even lower temperature. Thus Ewart found under such circumstances that at $37^{\circ} \mathrm{C}$. to $38^{\circ} \mathrm{C}$. he could not detect by the bacterium method any evolution of oxygen from the chlorophyllous cells and tissues of Aspidium, Mimosa, Chara, Elodea, Selaginella, Oxalis, Cystoptcris, Metzgeria, Orthotrichnm, Parmclia, Dicranum, Brynm, \&c., even under optimal illumination. It is very probable that prolonged exposure to a high temperature not only exercises a depressing effect upon the assimilatory powers of the chloroplastids, but also acts as an accelerating stimulus and produces an abnormal increase in the respiratory activity (Sect. 104). The true curve of assimilation can only be obtained by 
taking the respiratory activity into consideration, as was done by Kreusler ; hence the earlier researches do not give the true optimum or the actual relationship between assimilation and temperature ${ }^{1}$.

The assimilatory curve is not the same in all plants, for it is evident that the algae of a snow-flora must be able to assimilate carbon dioxide at very low temperatures, since they may pass through their entire life-history at a temperature which hardly ever rises much above zero ${ }^{2}$. Active and resistant chloroplastids are actually capable of assimilating carbon dioxide for a time at zero or even a few degrees below it, though when the exposure is more prolonged this power is gradually lost even in comparatively resistant plants. Ewart has shown that the immediate effect of a low temperature is sufficient to cause all evolution of oxygen to cease at from $4^{\circ} \mathrm{C}$. to $8^{\circ} \mathrm{C}$. in tropical plants, and at from $0^{\circ} \mathrm{C}$. to $2^{\circ} \mathrm{C}$. in warm-temperate, sub-tropical, and water-plants, while in cool-temperate, arctic and alpine forms, assimilation may cease only when the plants are completely frozen ".

Amount of zeater. A slight diminution of turgidity sufficient to close the stomata will render the absorption of carbon dioxide extremely difficult, and hence may markedly diminish the assimilatory activity of a sub-aërial leaf, whereas in lichens and in the leaves of mosses, where stomata are absent, such a slight decrease produces but little effect upon the assimilation of carbon dioxide, and this process ceases only when the loss of water is very great $^{4}$. Similarly, dilute saline solutions seem to decrease the power of assimilation but little, provided that they exert no poisonous influence upon the chloroplastids, and even a plasmolysed cell may be able to assimilate more or less actively. A solution of cane-sugar of over 40 per cent. strength completely arrests the assimilation of carbon dioxide, and prolonged immersion in a 30 per cent. to 35 per cent. solution of cane-sugar may render the chloroplastids of Elodea, Catharinea, Bryum, Orthotrichum temporarily

${ }^{1}$ Kreusler, Landw. Jahrb., I887, Bd. Xvi, p. 711 ; 1888 , Bd. XviI, p. 161 ; I890, Bd. XIx, p. 649 ; Heinrich, Versuchsst., I88I, Bd. xıII, p. I36; Böhm, Sitzungsb. d. Wien. Akad., I873, Bd. Ixvil, Abth. i; Schützenberger u. Quinquaud, Compt. rend., I873, T. LxxviI, p. 272 ; Prianischnikow, Bot. Jahrb., I876, p. 897. No importance can be attached to Fauncopret's attempt (Compt. rend., 1864, T. LVIII, p. 334) to represent the relation between assimilation and temperature by a simple equation.

${ }^{2}$ Cf. Ewart, Annals of Botany, 1898 , Vol. xir, p. 377 .

3 Boussingault, Ann. d. sci. nat., 1869, v. sér., T. x, p. 336; Kreusler, 1.c., 1888, p. 163; Ewart, l.c., pp. $3^{8} 9,8 \mathrm{c}$. [Jumelle observed that a few conifers and lichens were able apparently to feebly assimilate $\mathrm{CO}_{2}$ at $-40^{\circ} \mathrm{C}$. (Rev. gén., I892, T. IV, p. 263). Since, however, all respiration ceases at $-10^{\circ} \mathrm{C}$. to $-12^{\circ} \mathrm{C}$, it is manifestly impossible that any assimilation of carbon dioxide can take place at $-40^{\circ} \mathrm{C}$, for $\mathrm{CO}_{2}$-assimilation is a vital process involving protoplasmic activity. Ewart has, indeed, shown that the changes in the composition of the air of the receiver noticed by Jumelle do not necessarily indicate that any carbon dioxide had been assimilated at the temperature in question (Ewart, l.c., p. 402).]

${ }^{4}$ Cf. Sect. 58. Gasometric experiments by Boussingault, Agron., \&cc., I868, T. IV, p. 317 ; Kreusler, Landw. Jahrb., I $88_{5}$, Bd. XIv, p. 95i. Mosses, \&rc.: Jumelle, Rev. gén. d. Bot., I 892 , T. IV, pp. 168, 318 ; Bastit, ibid., I891, T. III, p. 521 . 
or permanently inactive (Ewart, 1.c., p. 434). This is perhaps why certain investigators have failed to detect any power of assimilating carbon dioxide in plasmolysed plants ${ }^{\text {. }}$.

Chloroform-Ether. The direct and indirect effects of any agency need carefully to be distinguished from one another; thus by prolonged anaesthetization a chloroplastid may be rendered temporarily inactive, and hence it is not always clear whether the action of chloroform and ether is a direct or indirect one. Fr. Schwarz and Pringsheim found that in strong watery solutions of chloroform and ether assimilation soon ceased, but that no recovery was possible owing to the plants having been fatally injured, whereas Bonnier and Mangin showed that in an atmosphere containing a measured quantity of ether, the assimilation of carbon dioxide was directly inhibited, although respiration continued unchecked. By exposing plants for prolonged periods of time to dilute ether vapour Ewart was able to render the chloroplastids temporarily inactive ${ }^{2}$.

Oxygen. In the absence of free external oxygen a normal chloroplastid will continue to assimilate in an atmosphere of hydrogen containing a little carbon dioxide. Even if no oxygen was originally present it may be shown by the bacterium method that it is evolved as soon as light is admitted. Beyerinck found that plants immersed in a solution of reduced indigo evolved oxygen and caused the solution to turn blue when they were exposed to light. Prolonged asphyxiation caused by a deficiency of oxygen may, however, ultimately induce a condition of temporary or permanent inanition in the chloroplastids during which they lose the power of assimilating carbon dioxide ${ }^{3}$; this was what Boussingault termed 'asphyxie.'

\section{SECTION 59. The Influence of Light.}

Even the feeblest illumination renders the chloroplastids able to decompose carbon dioxide, so that slightly less is given off than in absolute darkness, but oxygen is evolved only when more carbon dioxide is decomposed than is produced by respiration. Hence, even the delicate bacterium method

1 The formation of starch alone is naturally not a sufficient test for the presence of a power of $\mathrm{CO}_{2}$. assimilation (Sect. 55), and hence Meissner's results are inconclusive (Bot. Centralbl., 1894, Bd. Lx, p. 206), as are also those given by Weyl (Sitzungsb. d. Phys.-Med. Soc. zu Erlangen, 1. Aug., 1881 .

${ }^{2} \mathrm{Cl}$. Bernard's observations left it uncertain whether the action of the chloroform was a direct or indirect one (Leçons sur les phénomènes d. l. vie, $1878, \mathrm{p} .278$ ), and Ewart (Ann. of Bot. 1898, Vol. XII, p. 416) has recently shown that the result produced differs according to the duration of the exposure. Fr. Schwarz, Unters. a. d. Bot. Inst. z. Tübingen, I881, Bd. I, p. I02; Pringsheim, Sitzungsb. d. Akad. d. Wiss. zu Berlin, 188 $8_{\gamma}$, Über die Abhängigkeit d. Assim. gr. Zellen von Sauerstoffathmung; Bonnier et Mangin, Ann. d. sci. nat., vii. sér., T. III, 1886, p. 14; Jumelle, Rev. gén. d. Bot., I $89^{2}$, T. II, p. 430 ; Ewart, Journ. Linn. Soc., Xxxi, I896, p. 408.

${ }^{3}$ Pringsheim, 1.c.; Ewart, l.c., pp. 403, 41 8, \&c.; Beyerinck, Bot. Zeitung, 189o, p. $74^{2}$. 
is unable to detect an extremely fecble assimilation of carbon dioxide, such as occurs in moonlight, which is about $\frac{1}{600,000}$ the intensity of sunlight ', whereas in twilight an evolution of oxygen may readily be detected from a chlorophyllous cell by this method.

Respiration and assimilation about balance one another when highly chlorophyllous organs are exposed to light of $\frac{1}{10}$ th to $\frac{1}{40}$ th the intensity of bright diffuse daylight (Sect. 55), but even then an evolution of oxygen may be detected by means of the bacterium method from the more strongly illuminated cells or chloroplastids. Similarly an actively assimilating leaf or green alga may evolve traces of carbon dioxide even when exposed to bright light ${ }^{2}$, nor is it surprising that plants may turn green or react heliotropically when exposed to an intensity of light insufficient to cause any actual evolution of oxygen.

It is well known that green plants are unable to assimilate sufficiently actively in deep shade, and the same is the case with plants kept in a dark room. Even when placed at an exposed window they receive only the light from one-half of the sky, instead of the whole as they do when growing in the open. The light rapidly decreases towards the interior of the room, so that if the window is $2 \mathrm{~m}$. high and $1.5 \mathrm{~m}$. broad, the plant receives at a distance of $0.5 \mathrm{~m}$. only 0.3 , and at a distance of $2 \mathrm{~m}$. only 0.08 of the diffuse daylight it would receive in the open (Detlefsen). Sachs found that a seedling of Tropacolum majus exposed to seven hours' morning light in a west window grew badly, but ultimately increased slightly in dry weight ${ }^{3}$.

The photosynthetic activity increases proportionately to the intensity of the light, as has been repeatedly shown since the first experiments by Wolkoff. There is, however, a limit to the increase, and according to Reinke this is about reached in direct sunlight; above this intensity the number of gas bubbles evolved by Elodea remained about the same even in sixty times concentrated sunlight, and an injurious effect was not exerted until a much higher intensity had been reached, when a diminution

${ }^{1}$ Boussingault, Ann. d. sci. nat., 1869, v. sér., T. $\mathrm{x}, \mathrm{p} .335$. On the northern nights, cf. Curtel, Rev.gén. d. Bot., IS90, T. II, p. I2. [Although no compensation for respiration is possible by the bacterium method, still the latter forms a more certain test for the absence or presence of assimilation than do the methods of gas analysis, for the most highly chlorophyllous phanerogamic leaf contains a large number of non-green cells which respire without cessation. The bacterium method will detect the minutest evolution of oxygen from each individual cell (see Ewart, l.c., pp. $37 \mathrm{I}, 3 \mathrm{So}$ ). Moreover oxygen may be evolved at one part of a cell although it is absorbed at another.]

${ }^{2}$ Cf. Garreau, Ann. d. sci. nat., I85I, T. Xvi, p. 280 ; Blackman, Phil. Trans., r895, Vol.cLxxxvi, pp. 540, 557. [A microscopical method for demonstrating the simultaneous evolution of traces of oxygen and carbon dioxide from green algal filaments exposed to light, by means of hanging drops, onc of an alkaline solution of phenophthalein, the other of water containing not more than a few score Bacterizm termo, in an atmosphere of hydrogen or of nitrogen, is given by Ewart, Journ. Linn. Soc., xxxı1, 1897, pp. 126, 128, \&c.]

${ }^{3}$ Sachs, Experimentalphysiol., $186_{5}$, p. 2 r ; Detlefsen, Arb. d. Bot. Inst. in Würzburg, $188_{4}$, Bd. III, p. $S 8$. 
in the rate at which the bubbles escaped became perceptible ${ }^{1}$. It does not, however, follow that the chloroplastids had reached their maximal activity, for the limited supply of carbon dioxide might have prevented any further increase in the intensity of the light from accelerating the photosynthetic activity beyond a certain limit. On the other hand it is possible that in Elodec and other plants the curve of assimilation falls again if the illumination is increased beyond a certain point, even when a full and adequate supply of carbon dioxide is assured, and this result will certainly be produced by prolonged exposure to intense light, owing to the inactive condition which may be induced in the chloroplastids (Sect. $5^{8}$ ). In any case the intensity of the light which actually reaches the chloroplastids is very much less than that falling upon the outer surface of the plant, and it is very doubtful whether the optimal intensity of illumination for the chloroplastid ever approaches anywhere near to that of strong direct sunlight. Every plant has its own specific requirements and powers of resistance, and it is well known that many shade-plants are unable to withstand continual exposure even to only moderately strong sunlight ${ }^{2}$.

Wolkoff experimented at various distances from a strongly illuminated sheet of ground glass, whereas Reinke exposed the plants he was observing to sunlight concentrated by means of a lens. Both these authors and V. Tieghem also used the bubble-counting method, which is apparently more convenient for such comparative researches than direct gas analysis, and Reinke (1. c., p. 698) criticizes the results obtained in this manner by N. J. C. Müller and Famintzin. Kreusler also found a proportionate relationship to exist between the activity of assimilation and the intensity of the illumination. 'This applies not only to white but to coloured light, as is shown by bubble-counting experiments conducted behind coloured media, and also by Engelmann's researches (Sect. 60) ${ }^{3}$.

Pringsheim's protective theory. No mathematically exact relation can be expected between the photosynthetic activity and the intensity of light, for as the light increases other influences may be exerted, which directly or indirectly modify

1 This result may have been due to the continuous rise of temperature in the water exposed to intense sunlight causing the gases of the intercellular spaces to expand, and hence inducing a stream of bubbles. If plants of Elodea are exposed to concentrated sunlight in water containing ether or chloroform, a stream of bubbles may still continue to escape after the plants have been fatally injured, and it is obvious that no accurate results can be obtained by the bubble-counting mcthod unless the surrounding medium is kept at a perfectly constant temperature. Ewart (Annals of Botany, I 898 , XiI, p. $3_{4}$ ) has shown that living chloroplastids of Elodea and Chara are completely bleached by five to ten minutes' exposure in cold water to sunlight of eight- to ten-times-concentrated photochemical intensity, and that at this intensity of illumination the assimilation of carbon dioxide immediately or almost immediately ceases (p. 393).]

${ }^{2}$ See Ewart, Annals of Botany, I $S_{97}$, p. 440 (Effects of Tropical Insolation).

3 Wolkoff, Jahrb. f. wiss. Bot., I866-7, Bd. v, p. I; Reinke, Bot. Zeitung, I883, p. 7т3; v. Tieghem, Compt. rend., I869, T. Lxix, p. $49^{2}$; N. C. Müller, Bot. Unters., I $87^{2}$, Bd. I, pp. 3 , 374; Famintzin, Ann. d. sci. nat., I8so, vi. sér., T. x, p. 67 ; Kreusler, Landw. Jahrb., 188 5 , Bd. xiv, p. $95^{2}$. 
the assimilatory powers of the chloroplastids. In opposition to Pringsheim's ${ }^{1}$ supposition, all the experimental evidence tends to show that light exercises but little or no influence upon respiration so long as no injurious effect is produced (Sects. ro4 and 56). The fact that intense light may cause a bleaching of the chloroplastids in the presence of oxygen, even if it is accompanied by an increased production of carbonic acid gas, hardly affords safe or direct evidence as to the behaviour under normal conditions. From the erroneous hypothesis that light accelerates respiration ${ }^{2}$, Pringsheim developed the theory that chlorophyll acts as a protection against light, and thus allows the decomposition of carbon dioxide to become more prominent in green cells than in colourless ones, for he supposed that light might induce photosynthesis in colourless cells as well if only the rays which accelerate respiration were extinguished. This theory hardly accords, however, with the large amount of chlorophyll present in shade-loving plants, or with the fact that in very feeble light respiration overpowers assimilation; moreover we should expect to find the most active assimilation occurring in light which had passed through a solution of chlorophyll or through a green leaf, whereas in such light colourless protoplasts and leucoplastids respire with undiminished intensity and show no traces of an evolution of oxygen. Similarly, if such green light is sufficiently concentrated, it may be shown by means of the bacterium method that oxygen is evolved only from the chlorophyll bands of Spirogyra or Mesocarpus, and not from the colourless plasma ${ }^{3}$. These facts so completely deprive Pringsheim's theory of all scientific foundation that further discussion of it is unnecessary.

\section{Section 60. The Influence of the Different Rays of the Spectrum.}

Only the visible light-rays take part in the assimilation of carbon dioxide, except in purple bacteria, in which assimilation is most active in the neighbouring but invisible infra-red rays. A correct knowledge of the assimilatory effect of the different regions of the spectrum can only be obtained by determining the amounts of carbon dioxide decomposed by the superficial chloroplastids, for the more deeply seated ones receive light of altogether different composition to that which falls upon the outer surface. The former gives the primary curve of assimilation which Engelmann * obtained by means of the bacterium method, whereas the estimation of the total assimilation yields the secondary curve. The primary curve p. lxxxiv.

1 Pringsheim, Jahrb. f. wiss. Bot., 1879-8I, Bd. xIr, p. 374; Ber. d. Bot. Ges., I886, Bd. Iv,

[Kolkwitz has, however, found (Jahrb. f. wiss. Bot, I 898 , Bd. XxxiII, p. I28) that light distinctly accelerates respiration in fungi, though the increase is not more than a tenth of the previous amount.]

${ }^{3}$ Engelmann, Bot. Zeitung, I $88_{3}$, p. 5; Die Erscheinungsweise der Sauerstoffausscheidung im Licht, I 894 , Fig. 7 (Sep.-abdr. a. d. Verh. d. Amsterd. Akad.). See also Reinke, Bot. Zeitung, I $883, \mathrm{p}$. 733 .

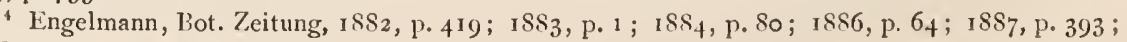
Die Erscheinungsweise, \&c., $189+$ (Sep.-abdr. a. d. Verh. d. Ansterd. Akad.). 
obtained by Engelmann for green plants in a direct sun-spectrum is shown in Fig. 5I (Ass. Green), and the assimilatory maximum corresponds to the main absorption band between B-C (cf. curve Abs. Green, and the spectra from leaves and from chlorophyll). There is a resemblance between the curves of assimilation and absorption, but the correspondence is not an exact one, and the difference would be still more marked if the frequently doubted secondary assimilatory maximum between $\mathrm{F}-\mathrm{G}$ did not exist ${ }^{1}$.

The presence of phycoerythrin in the chloroplasts of the Florideae causes the assimilatory maximum to be transposed to beyond $\mathrm{D}$, and the rays between B-C no longer exercise any specially favourable effect (Fig. 5I, Ass. Red). The alteration caused by the presence of phycocyanin (Sect. 53) in blucgreen algae is less marked (Fig. 52, Ass. Bl.-Gr.), and in both cases the curve of absorption undergoes a similar though not precisely corresponding change.

The most active assimilation is caused in purple bacteria by the infrared rays of 800 to $900 \mu \mu$ wave length (I $\mu \mu=0.00 \mathrm{I} \mu$ ), but in rays of Ioco $\mu \mu$ Engelmann could no longer detect any evolution of oxygen. Hence the heat-rays given off from a stove do not excite any assimilatory activity in these bacteria, although this power is possessed by the rays of light which pass through a solution of iodine in carbon bisulphide; in such light on the other hand ordinary green plants evolve just as much carbon dioxide as they do in darkness ${ }^{2}$. The assimilatory curve falls steadily towards the blue, where it ceases, although here the maximal absorption of light occurs. Owing to the feebleness of the evolution of oxygen Engelmann was unable to determine the assimilatory curve with precision, and hence it remains uncertain whether the visible red, where absorption is feeble, excites but little assimilation of carbon dioxide. (See also Sect. 52.)

The more deeply seated chloroplastids screened behind an outer chlorophyll-layer are exposed to light which has lost two to four times as many of the rays between $\mathrm{B}$ and $\mathrm{C}$ as of the yellow rays, and hence behind even a thin chlorophyll-layer an assimilatory minimum occurs at the point of maximal absorption (cf. Fig. 5I) ${ }^{3}$, while the assimilatory maximum is displaced to the line $\mathrm{D} \frac{1}{2} \mathrm{E}$, in the neighbourhood of the green. Engelmann found that the maximal evolution of oxygen occurred at this point when he examined the upper surface of a thick Cladophora filament illuminated from beneath, whereas on the lower surface the maximum lay between $\mathrm{B}$ and $\mathrm{C}$. For these and other reasons (such as the possibility of slight lateral diffusion, \&c.) even the bacterium method only enables an

${ }^{1}$ [That a marked secondary assimilatory maximum does exist is, however, certain. See Kohl, Ber. d. Bot. Ges., I 897 , p. III.]

${ }^{2}$ Pfeffer, Arb. d. Bot. Inst. in Wiirzburg, $x_{8} \mathrm{I}$, Bd. I, p. 4 I.

$s$ Engelmann, Bot. Zeitung, I $\$ \delta_{2}, \mathrm{p} \cdot 4^{2} \%$. 


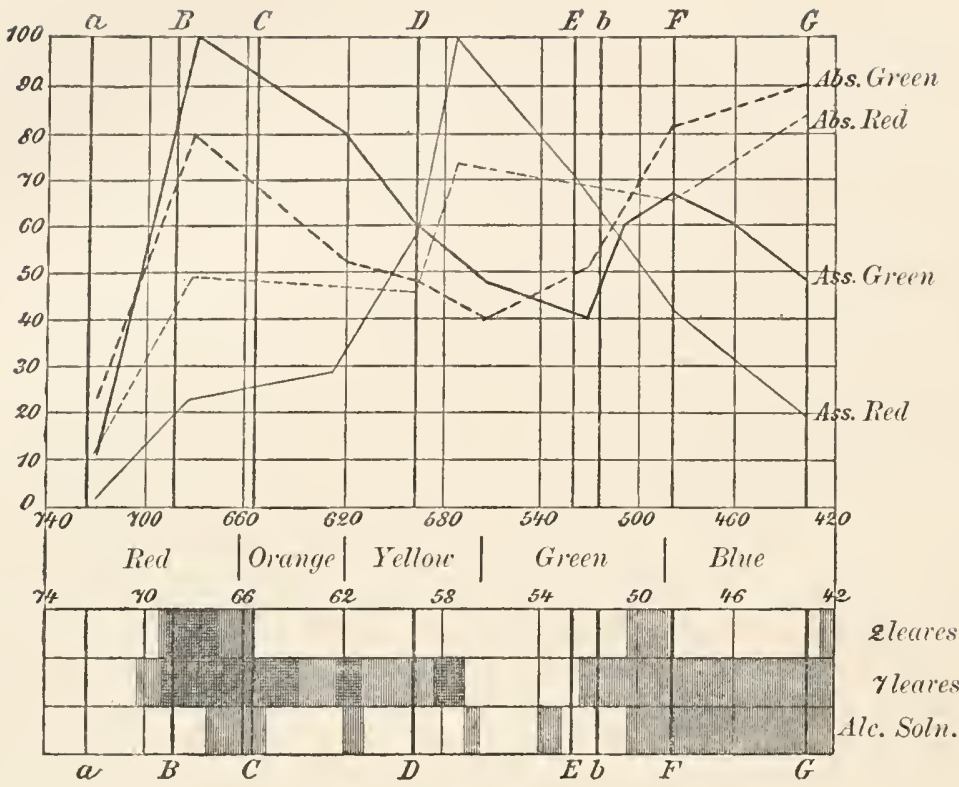

FiIG. 51 .

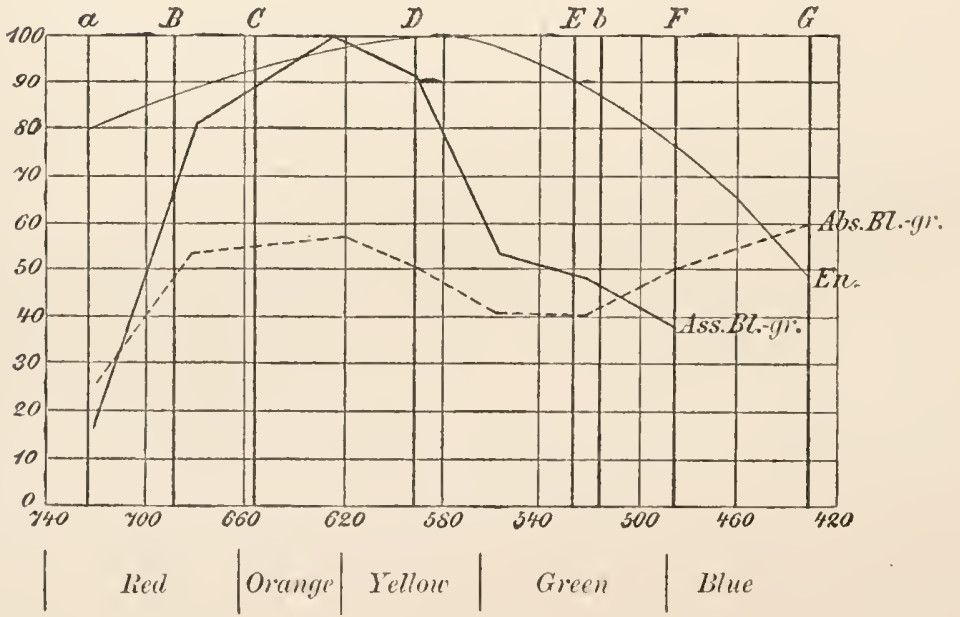

FIG. 52.

FIGS. 5I, 52. Curve or absorption (Abs.) and primary assimilation (Ass.) in sun spectrum, after Engelmann, Bot. Zeitung, 1884 , Taf, II. The ordinate for the maximal assimilation $=100$. Fig. 51 gives the curves for green chloroplastids (Ass. Green and Abs. Grecn), and for red algae (Florideac) (Abs. Red and Ass. Red), and Fig. 52 for a blue-green alga (Oscillaria) (Ass. Bl.-gr. and $A b s . B l . g r$. ). The curve En. gives the distribution of energy in the spectrum, after Langley Annal. d. chim. et 1. phys., 1882, v. sér., T. xxv, p. 212 ; cf. also Engelmann, 1. c., p. I02). The median figure is after Reinke (Bot. Zeitung, 1884, p. 59, and Pl. I, and gives the absorption spectra of two and of seven superposed leaves (2 leazies and 7 leaves) as well as of an alcoholic solution (Alc. Sol.) of leaves of Impaticns parviflora. 
approximately accurate primary curve of assimilation to be constructed, and it is easy to understand why precisely similar results may not always be obtained.

The composition of the light alters as it penetrates each successive chlorophyll-layer, and the assimilatory curve and total assimilatory effect change in a corresponding degree, the maximal point of the secondary assimilatory curve being displaced towards D. Hence various authors (Draper, Pfeffer) found that under strong illumination the maximal assimilation occurred in yellow light, so that the assimilatory curve appeared to run parallel with the visual intensity of the light ${ }^{1}$. It is easy to show that the course of assimilation and absorption is such that the total result produced by light of varying wave-length is different under different conditions, and that as the intensity of the light changes so also does the assimilatory curve alter. The variations which the secondary assimilatory curve undergoes under varying external conditions can therefore only be determined by direct experiment in each case.

The yellow rays, which penetrate more deeply, may produce a greater total assimilatory effect than the rays between $\mathrm{B}$ and $\mathrm{C}$, which are more active, but which are also more rapidly absorbed and thus act upon fewer chloroplastids than the yellow rays do; while if the red rays cause less carbon dioxide to be decomposed than the yellow rays in relation to the amount of light absorbed, as appears to be the case (cf. Ass. and Abs. Green in Fig. 5I), then the value of the latter will be still further enhanced when light penetrates a chlorophyllous tissue ${ }^{2}$. It is of the utmost importance that the light which has already passed through chloroplastids should still be able to be used in assimilation, for it is impossible in an ordinary leaf for all the chloroplastids to be exposed to direct illumination.

Both the absorption and reflection of light by chlorophyll are of importance, and the curves given in Fig. $5^{I}$ give a general idea of the former. The spectra of a green leaf and of a chlorophyll extract correspond, except for slight displacements due to the influence of the solvent media, details of which, as well as of the yellow pigments of the chloroplastids, are given in the literature quoted ${ }^{3}$. Even

${ }^{1}$ Cf. Pfeffer, Bot. Zeitung, IS72, p. 425; Arb. d. Bot. Inst. in Würzburg, I 87 I, Bd. I, p. I, and Physiol., I. Aufl., Bd. I, p. 2 I4. Reinke (Bot. Zeitung, ISS4, p. 39) obtained with Elodea, by the bubble-counting method, a curve corresponding more closely with the Ass. Green in Fig. 5 I, except that no secondary maximum was observed in the blue, for which see Kohl, Ber. d. D. Bot. Ges., 1.c. In these and the other researches quoted, no distinction has been made between the primary and secondary assimilatory curves. The curve Ass. Red in Fig. $5^{\text {I }}$ corresponds fairly well to the optical intensity of the light.

2 [Pennington (Contrib. Bot. Lab. Univ. Pennsylvania, I, IS97, p. 203) states that Spirogyra forms no starch in blue or yellow light, but this was probabiy owing to the employment of light of feeble intensity.]

3 Vierordt, Die Anwendung d. Spectralapparates zur Photometrie, IS 73 ; N. J. C. Miiller, Bot. Unters., I 876, p. 325 ; Wolkoff, Die Lichtabsorption in Chlorophylllösungen, IS 6 ; Engelmann, 
a thin semi-transparent green leaf absorbs, as a general rule, more than half of the total energy of the sunlight falling upon it, and the rays most active in assimilation are almost entirely extinguished, so that the sunlight which has passed through a green leaf is usually no longer able to induce a formation of starch or an evolution of gas bubbles ${ }^{1}$. For the absorption in the Florideae and Cyanophyceae, see Figs. $5^{1}$ and $5^{2}$, and the literature quoted.

Even in passing through colourless tissues a large amount of light is lost by reflection and absorption, and when coloured pigments are present the amount absorbed is much increased. Engelmann has shown that the red dyes frequently present in the cell-sap of chlorophyllous leaves absorb fewest of the rays which are most active in assimilation (red and orange), and most of the less active yellow rays. The total amount of assimilation is, however, decreased, and hence apparently arises the fact that copper-beeches grow more slowly than the green varieties ${ }^{2}$.

The value of either monochromatic or mixed light which reaches a chlorophyllous cell is dependent upon its concentration and the specific assimilatory energy of the component rays, and the assimilatory effect rises with an increased concentration so long as the illumination is of submaximal intensity (Sect. 59). The specific assimilatory energy remains the same for any given light-rays, whether they act alone or in combination with other rays, as is shown by the results obtained with monochromatic light, as well as by special researches ${ }^{3}$; but although assimilation may continue in monochromatic light, still in the absence of all other rays the growth and general vital activity of a plant may ultimately be injuriously affected.

The fact that the primary assimilatory curve is different in green plants to what it is in red seaweeds or purple bacteria shows that the assimilatory mechanism may exhibit specific peculiarities, and it is not impossible that organisms may be discovered in which the greatest assimilatory activity may be produced by the short ultra-violet rays, or by the rays with longest wave-length. Various rays of the spectrum can exert photo-chemical actions on dead substances, and although it is those of shorter wave-length which are especially active, still it is a fundamental error to construct a curve representing the general chemical intensity of the different rays of light based upon their action on silver salts ${ }^{4}$. Similarly, since the different rays have a definite specific assimilatory

Bot. Zeitung, I $88_{4}$, p. 87 ; I887, p. 441; I888, p. $68_{5}$; Reinke, ibid., I886, p. I61 ; Monteverde, Acta horti Petropolitani, 1893 , Bd. xiIr, p. 123; Tschirch, Ber. d. Bot. Ges., 1896, p. 76 . Other literature is given in these works.

1 Nagamatsz, Arb. d. Bot. Inst. in Würzburg, 1887, Bd. III, p. 399.

2. Jumelle, Compt. rend., I890, T. cXI, p. 380 ; Engelmann, Bot. Zeitung, I887, p. 433. Absorption, \&c. by colourless tissues : Reinke, Bot. Zeitung, I886, p. I93; Engelmann, l. c., p. 4I3.

${ }^{3}$ Pfeffer, Arb. d. Bot. Inst. in Wuirzburg, I 87 I, Bd. I, p. $45 \cdot$

t Ostwald, Lehrb. d. allgem. Chemie, Is93, Bcl. II, I, P. I024. 
value, it is impossible that the assimilatory curve can prccisely correspond to the distribution of energy in the spectrum (Fig. $52, E n$.), or to any such physical curve. The brightness of the light to the sensitive retina of the eye does not form any safe test of its photosynthetic activity; and although a general relationship exists between the optical intensity of light and its assimilatory power ${ }^{1}$, it is not the brightness of the light, but the amount of energy that can be obtained from it, which is of primary importance.

The energy for the decomposition of carbon dioxide in green chloroplastids is obtained from the rays having a wave-length of from $39 \mathrm{I} \mu \mu$ (ultra-violet) to $770 \mu \mu$ (infra-red), and a vibratory activity of 800 to 400 billion $\left(10^{12}\right.$ ) times per second, whereas in the purple bacteria it is the infra-red rays of 900 to $800 \mu \mu$ wave-length which are most active. In green plants the most active decomposition is caused by the rays of $660-680 \mu \mu$ wave-length, and further towards the red end of the spectrum the curve falls steadily (Fig. 51). A similar relationship exists for photochemical actions on dead substances; moreover, most physiological functions exhibit minimal, optimal, and maximal points, while secondary maxima may be shown similar to those which the assimilatory curve exhibits ${ }^{2}$.

A precise determination of the course of the assimilatory curve has not as yet been obtained, for even by the delicate bacterium method, assimilation is only rendered apparent by the evolution of oxygen, i.e. when the respiration is overpowered, and the same objection. and others also apply to the bubble-counting method. It is only when respiration can be estimated that the actual amount of assimilation may be calculated, and an evolution of oxygen becomes perceptible at the extreme ends of the spectrum only under light of great intensity. Since the rays of shorter wave-length undergo more marked dispersion than the red rays do, the blue end of the spectrum is spread over a larger area, and since the light is correspondingly diluted, the evolution of oxygen ceases to be perceptible sooner at this end of the spectrum than it would be if this were not the case. This error has been allowed for in constructing the curves in Figs. 51 and 52 . Just as different animals and even different people have not the same range of colour perception, so the assimilatory curve for different green plants may not always end at precisely the same region of the spectrum. Bonnier and Mangin ${ }^{3}$ have observed that in certain cases a slight assimilation of carbon dioxide occurs in the ultra-violet rays, though not sufficient to overpower respiration, whereas Pfeffer found that behind a solution P. 276 .

${ }^{1}$ Cf. Pfeffer, Bot. Zeitung, IS II $_{1}$ p. $3^{1} 9$; Sachs, Arb. d. Bot. Inst. in Würzburg, IS ${ }_{72}$, Bd. I,

${ }^{2}$ It is easy to show, by means of the bacterium method, that yellow sodium light can induce active photosynthesis, although Beyerinck supposes that it is inactive (Bot. Zeitung, ISgo, p. 743).

3 Bonnier et Mangin, Compt. rend., I $8 S 6$, T. CIr, p. I 23. 
of iodine in carbon bi-sulphide, that is in the dark infra-red rays, the same amount of carbon dioxide was produced as in darkness.

Only a small portion of the energy of the light falling upon a leaf is utilized in decomposing carbon dioxide. Thus a square metre of actively assimilating leaf surface of Nereum oleander forms $0.000535 \mathrm{grm}$. of starch in one second, by which an amount of energy equivalent to $2 \cdot 2$ caloric units is obtained, which is less than 1 per cent. of the total energy of sunlight received, according to Pouillet. by this surface area on a bright sunny day in one second, which is 333 heat units ${ }^{1}$. Similarly, Detlefsen ${ }^{2}$ found that when a leaf was prevented from assimilating by the absence of carbon dioxide, a thermopile placed behind it registered a slight rise in temperature, but only so much as to indicate that the leaf when assimilating utilized at most not more than 0.3 to $1 \cdot I$ per cent. of the total energy of the incident light.

The fact that chlorophyll continues to absorb light in non-assimilating as well as in dead leaves suffices to show that the total energy of the absorbed light is not used in assimilation, as Engelmann apparently concluded to be the case from the proportionality existing between absorption and assimilation. A similar relationship is exhibited in other photo-chemical processes in which only a portion of the absorbed energy is utilized. Nor does it follow that the energy of the light rays is directly employed in the decomposition of carbon dioxide, although in all probability this is actually the case. Nevertheless, this is merely a hypothetical assumption, for the specially active rays might merely exert a stimulating action upon the process of assimilation, the necessary energy being derived from the heat-rays directly absorbed, and perhaps also from the heat vibrations induced by the absorption of more rapidly vibrating light-rays ${ }^{3}$.

The part played by chlorophyll in absorbing and rendering available the energy of light is certainly of great importance, but assimilation is possible only when the different parts of the assimilatory mechanism all co-operate together in the proper manner, for just as a locomotive refuses to work when its machinery is out of order, however high the steam pressure may be, so also may chloroplastids, rendered entirely or partially inactive by some invisible internal alteration, be wholly or partly incapable of

1 l'feffer, liot. Zeitung, IS72, p. 429; Ostwald, Lehrb. d. allgem. Chemic, I893, 2. Aufl., 13d. 11, I, p. 1070. On radiation from the sun, see also Rubner, Centralbl. f. Physiol., I895, Bd. vili, p. 664. [One gramme of starch when burnt produces 4,100 caloric units.]

2 Detlefsen, Arb. d. Bot. Inst. in Wiirzburg, I $888, \mathrm{Bd}$. III, p. 543. The experiments are not quite faultless. N. J. C. Müller's comparative experiments (Bot. Unters., I872, Bd. 1, p. 339) with dead and living leaves are without value, owing to the changes in the absorptive powers of a leaf which are coincident with death. Cf. Rcinke, Bot. Zeitung, ISSI, p. 209.

3 Engelmann, lot. Zeitung, I88 4, p. I02; I886, p. 68; I888, p. 689; Ostwald, l.c., pp. I056, Io87. Cf. Köppen, Wärme u. Pflanzenwachsthum, i 870, p. 6.3 ; Mayer, Lehrb. d. Agriculturchemie, I87 I, p. 3I ; Pfeffer, Energctik, I8922, p. 204. 
assimilating carbon dioxide, although the absorption of energy still continues (cf. Sccts. 52, 53). These possibilities have been long neglected, and it is cvident that the amount of chlorophyll present does not afford any direct measure of the activity with which carbon dioxide may be assimilated, and that no definite relationship need necessarily exist between absorption and assimilation ${ }^{1}$. As a matter of fact, only a small percentage of the radiant energy is converted into work, and each region of the spectrum contains much more energy than is required for the most active assimilation of carbonic acid (cf. En., Fig. 52). It is even possible that colourless protoplasts may be found to exist which are capable of active photosynthetic assimilation. Ostwald has shown that iodide of silver affords an example of a substance upon which the greatest photo-chemical effect is produced by rays (at $G$ ) which have but feeble optical intensity.

Hence it is doubtful whether a general approximate coincidence exists between absorption and assimilation, as Engelmann found to be the case in the plants examined by him, and indeed very marked aberrations were frequently observed. Such observations are, however, of the utmost importance, and if used with proper caution afford important material for clearing up the mystery surrounding the process of photosynthesis. The displacement of the assimilatory curve caused by the presence of phycoerythrin in Florideae is of the utmost interest (Ass. Red., Fig. 5x), for since it appears that a chromatophore tinged only with phycoerythrin cannot assimilate, it follows that this pigment can only act indirectly as a sensitizer, enabling the chlorophyll to make better use of certain rays of light, a function which is the more remarkable since the chlorophyll and phycocrythrin do not appear to enter into physical or chemical union with one another ${ }^{2}$ (Sects. 52,53$)$.

Under certain cultural conditions no phycoerythrin is formed, and thus it may be possible to determine experimentally its precise function; and since in the presence of such sensitizing pigments a small percentage of chlorophyll may suffice for active photosynthesis, it is possible that a trace of chlorophyll may be present in the purple bacteria, for the displacement of the maximal point to the infra-red is not greater in their case than the similar displacement towards the green which can be obscrved in the case of the red algae ${ }^{3}$. The

1 This applies to Lommel's theoretical conclusion (Ann. d. Chem. u. Phys., IS7r, Bd. CxuIv, p. $5^{81}$ ) that those rays must be most active which are most markedly absorbed, and which contain the greatest amount of energy. Even before this it was attemptcd to establish a direct relation between the absorption by chlorophyll and the assimilatory activity of the different rays. Thus Dumas (Essai de statique chim. d'êtres organisées, IS24, p. 24) supposed that the blue and more refrangible rays exercised the maximal assimilatory effect, for they also are very markedly absorbed by chlorophyll.

${ }^{2}$ Cf. Abs. Red, Fig. 5 I, constructed from the absorption curves of chlorophyll and phycoerythrin given by Reinke (Bot. Zeitung, I S86, Taf. II, Figs. I I, I2).

3 [As a matter of fact, Ewart has recently shown that apparently normal chlorophyll may be extracted from purple bacteria (Sect. $\left.\left.5^{2}\right) \cdot\right]$ 
Florideae fluoresce only when they are killed, and hence phycoerythrin can hardly be of importance as an agency by which the wave-length of the light-rays may be altered. Similarly chlorophyll appears to fluoresce but little if at all in the living chloroplastid ${ }^{2}$. The solution of these and similar problems may enable us to determine whether chlorophyll acts as a sensitizer, enabling the light-rays to generate such molecular vibrations in the active parts of the chloroplastid as to redder possible the decomposition of carbon dioxide ${ }^{2}$.

History and Methods. Daubeny (1836), Draper ( 1844 ), Cloez and Gratiolet (185I), and Sachs (1864), all found that but little assimilation occurred in blue and violet light, while Draper and Pfeffer found a certain correspondence existed between the amount of assimilation and the brightness of the light. N. J. C. Müller (I872) found that the most active assimilation occurred in the yellow rays, while the exact researches of Reinke gave a maximum between $\mathrm{B}$ and $\mathrm{c}$, but did

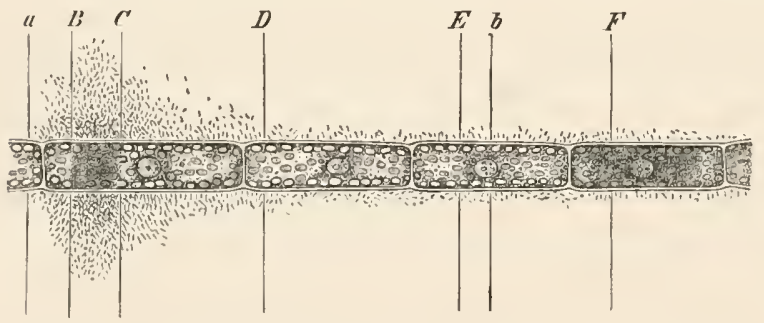

FiG. 53. Filament of Oedogonium sp. The greatest accumulation of the bacteria is between $B$ and $C$ opposite to the dark absorption band shown on the filament. Engelmann, by throwing a spectrum upon an algal filament, or on a single cell, and using the bacterium method as a test for the evolution of oxygen (Sect. 52 ). By narrowing the slit of the micro-spectroscope ${ }^{4}$ the concentration of the light may be lowered until an evolution of oxygen is made perceptible by the movement of the bacteria and their accumulation only in the most efficient regions of the spectrum (Fig. 53). In this manner it is easy to see when the illuminated side of the algal filament is examined that the most active evolution of oxygen occurs opposite B-C, and that the secondary maximum at $F$ is much less obvious. In addition to this method, Engelmann also used that of successive observations, by placing an algal filament transversely to the spectrum, and determining in each region of the latter the width of the aperture at which the movement of the bacteria first

1 Reinke, Bot. Zeitung, I 886 , p. I 79 ; Ber. d. Bot. Ges., I 883 , p. 405, and the literature there given.

${ }^{2}$ Cf. Reinke, Bot. Zeitung, I886, p. 24 I.

${ }^{3}$ Pfeffer, Arb. d. Bot. Inst. in Wüirzburg, I 87 I, Bd. I, p. I ; Bot. Zeitung, I872, p. 425. Here and by Reinke (Bot. Zeitung, I $88_{4}$, P. I) the literature is given. See also the first edition of this book, p. 216 ; Timiriaseff, Bot. Jahresb., I875, p. 779 ; Ann. d. sci. nat., I885, vii. sér., T. II, p. 99 ; and the criticism by Reinke, Ber. d. Bot. Ges., I 885 , p. 337 .

? See Engelmann, Bot. Zeitung, I882, p. 419, and Zeiss's Catalogue (Jena). 
commenced. Since the concentration of the light is directly proportional to the aperture, the width of the slit gives the relative assimilatory value of the different rays.

Engelmann generally employed a constant gas or incandescent electric light, and calculated the results obtained in terms of the solar spectrum (1. c., r 884, p. 90); the values obtained with prismatic spectra were corrected for the unequal dispersion in the different regions, and in this manner the curves given in Figs. 51 and 52 were constructed. In strong light a narrow slit may be used, giving a very pure spectrum, and it may perhaps be possible to use a grating spectrum for this purpose. Excellent as this method is, it yields only approximate values, and owing to an improper use of it Pringsheim obtained different results to those of Engelmann ${ }^{1}$.

The bubble-counting method also enables comparative estimations to be made of the total assimilatory effect produced by different rays (Pfeffer and Reinke), especially if a microscopical method is employed by means of which the diameters of the individual bubbles are measured (Kohl, Ber. d. Bot. Ges., I887, p. I I I). By these methods, as well as by gasometric means, the evolution of oxygen behind coloured media, as well as in prismatic spectra, was determined by Draper, and later by Müller, Pfeffer, Reinke, \&c. In the published works the errors due to dispersion, and to the unequal absorption of different rays in the substance of the prism, \&c., are described. The method used by Reinke is to be recommended. He blocked out regions of the spectrum determined according to Helmholtz's principle, and focussed the remainder by means of a convex lens upon the plant, the decreased evolution of oxygen representing the assimilatory value of the absent rays. Reinke also concentrated different regions of the spectrum to strips of equal size by means of cylindrical lenses, and so counteracted the unequal dispersion ${ }^{2}$.

Only an approximate estimation of the value of the different rays for photosynthetic assimilation can be obtained by experimenting under coloured media, so long as there is no means of determining the precise extent to which the different rays are absorbed. This applies even to Pfeffer's researches of $187 \mathrm{I}$. The effect upon the evolution of bubbles produced by interposing coloured solutions, or coloured plates of glass or gelatine ${ }^{3}$, may be exhibited to a large audience by means of a projection-lantern (Sect. 52). Solutions of cupric ammonium sulphate and of potassium bichromate have been most commonly employed for this purpose. After passing through both solutions sunlight retains but 8 to 20 per cent. of

${ }_{1}$ Cf. Engelmann, Bot. Zeitung, I $88_{2}$, I $88_{3}$, and $188_{7}$, pp. 100, 459; Pringsheim, Jahrb. f. wiss. Bot., I886, Bd. xv11, p. 162 ; Bot. Zeitung, I887, p. 200.

${ }^{2}$ Reinke, Bot. Zeitung, I 884 , p. 27 ; Helmholtz, Ann. d. Phys. u. Chem., 1855, p. 94; Reinke, Bot. Zeitung, $188_{5}$, p. 84 ; Ber. d. Bot. Ges., I $88_{5}^{5}$, p. 378 . Reinke (Sitzungsb. d. Berl. Akad., I 893, p. 53 I) used a grating spectrum in studying the effect of different rays on the formation of chlorophyll.

${ }^{3}$ All kinds of coloured glass plates can be obtained from Geb. Tasche in Cologne. On coloured sulutions, ef. Landolt, Ber. d. Chem. Ges., I 894 , p. $28_{72}$. See Kirschmann, Beibl. z. Ann. d. Phys. u. Chem., 1891, i3d. xv, p. +23. Coloured gelatine plates gradually become bleached. 
its original assimilatory value. The solutions may either be placed in doublewalled bell-jars, or in glass troughs with parallel sides ${ }^{1}$.

If a spectrum is projected upon a darkened leaf a formation of starch occurs in the outermost layers opposite to $\mathrm{B}-\mathrm{C}$, but the more deeply penetrating yellow rays are spread over a larger area, and have insufficient energy to induce a formation of starch at any one point, although they may excite a greater total amount of assimilation than the rapidly absorbed red rays do. Timiriazeff was able to demonstrate the primary assimilatory curve by the formation of starch in the red region of the spectrum thrown upon a leaf ${ }^{2}$.

Artificial light. The assimilatory value of artificial light depends upon its composition and concentration; thus, exposure to ordinary gaslight causes perceptible assimilation in green plants, and electric-light is still more efficient ${ }^{3}$. In both electric-light and gaslight the percentage of the more refrangible rays is relatively less than in sunlight, and hence in such light the assimilatory curve is correspondingly modified. This is especially the case when the red algae are examined, for with these the assimilatory maximum is markedly displaced towards the green * It may incidentally be remarked that polarized light also enables the plant to decompose carbon dioxide.

Absorption of light in deep zuater. The daylight which falls upon a plant is not always of the same composition, and under the shade of trees, as well as in deep water, particular parts of the spectrum become especially prominent. Thus in a clear sea with bright sunlight the different rays are reduced to about the concentration of moonlight which is insufficient for growth, at the following depths : red at 34 metres, yellow at $177 \mathrm{~m}$., green at $322 \mathrm{~m} .{ }^{5}$ Hence arises the advantage to red seaweeds of an assimilatory maximum in the yellow region of the spectrum (Fig. 5I). The distribution of red seaweeds is influenced by many other factors as well, for although they may grow at considerable depths very many are found between the tide-marks, or may grow in quite shallow water ${ }^{6}$. In turbid water the light is absorbed much more rapidly, but even under the most favourable conditions it is hardly possible that plants dependent upon the photosynthetic assimilation can grow at a depth of more than $400 \mathrm{~m}^{7}$

1 Pfeffer, Arb. d. Bot. Inst. in Wïrzburg, I 871 , Bd. I, p. 53. A marked starch formation takes place only behind solutions of potassium bichromate. Cf. Famintzin, Jahrb. f. wiss. Bot., 1867-8, Bd. v1, p. 43; G. Krraus, ibid., I869-70, Bd. v11, p. 5 I8 ; Prillieux, Compt. rend., I87o, T. Lxx, p. 46 ; Kolı, Ber. d. Bot. Ges., I897, p. II . Double-walled bell-jars were first used by Senebier (Phys.-chem. Abhandlungen, I785, Bd. I, p. 7 ), and latcr by Becquerel (La Lumière, I868, T. II, p. 278 ) and Sachs. Kreusler gives a simple mode of preparing glass troughs (Landw. Jahrb., I885, Bd. Xiv, p. 935 , footnote).

2 Timiriazeff, Compt. rend., I 890 , T. cx, p. $134^{6 .}$

${ }^{3}$ On growth and photosynthesis in elcctric light, cf. Bonnier, Rev. gén. d. Bot., I895, T. viI, p. $24 \mathrm{I}$; Bailey, Report upon Electro-IIorticulture, Ithaca, $\mathrm{I}_{922}$; Siemens, Bot. Centralbl., I88o, Bd. 1, p. 815; Kreusler, Landw. Jahrb., I $88_{5}$, Bd. XIv, p. 915 .

${ }^{4}$ Engelmann, Bot. Zeitung, $188_{3}$, p. $S$.

${ }^{5}$ Oltmanns, Jahrb. f. wiss. Bot., 1892, Bd. xxi1r, p. 419. See also Walther, Bionomie des Meeres, I 893 , Bd. I, p. 35 ; Hïfner, Archiv f. Anat. u. Physiol., IS9I, p. 68; C. Schröter u. O. Kirchner, Vegetation d. Rodensees, IS96, p. I 7 .

${ }^{6}$ Certain green algae are able to grow in deep water (cf. Drude, Pflanzengeographie, I 890, p. $2 \mathrm{I}$ ).

${ }^{7}$ Cf. Oltmanns, 1.c.; Walther, l. c.; Engelmann, Bot. Zeitung, 1872, p. $39^{6}$. 
Influence of coloured light. Plants may be unable to develop normally in monochromatic or even mixed coloured light, although energetic photosynthesis is possible so long as the chloroplastids remain healthy. Thus exposed to the less refrangible end of the spectrum (behind a screen of potassium bichromate solution) plants grow and increase in dry weight, but behave in other respects as if in deep shade owing to the absence of the blue and ultra-violet rays which prevent excessive growth and etiolation. Similarly in some cases the absence of the ultra-violet rays seems to retard or inhibit the development of flowers. The blue and ultra-violet rays are of little use for assimilation, and hence green plants grown in light passed through cuprammonia die sooner or later for want of food.

Hence the absence of certain rays may ultimately injure or even cause the death of a plant, and it is possible that monochromatic light may indirectly act in a similar manner, although no direct injurious action is exercised by the rays concerned either alone or when in mixed light. As a matter of fact certain results seem to indicate that monochromatic green light does act upon plants in some such manner, but further proof is needed ${ }^{1}$. The researches upon the growth of plants in coloured light which have been carried out by Hunt, Sachs, A. Mayer, $\mathrm{R}$. Weber, Morgen, and $\mathrm{Wollny}^{2}$, have yielded the general results already indicated. Macagno found that the greatest increase in the dry weight occurred in violet light, but this was certainly either due to the presence of other rays or to the absorption of external supplies of organic food.

\section{SECTION 6r. Theoretical.}

No precise knowledge has hitherto been obtained of the chain of processes by which the organic products of photo-synthetic assimilation are produced, but it must be clearly borne in mind that the process is a purely vital one, and hence in seeking a complete explanation we meet the same difficulties as confront us when we are dealing with any vital phenomenon. The fact that chloroplastids of unchanged external appearance may be temporarily inactive shows that these special organs are capable of assimilatory activity only when all the component parts co-operate in an appropriate manner, and that the final result is produced not by a single reaction but by the agency of a complicated and self-regulatory mechanism.

The actual primary product of photosynthetic assimilation is unknown, and we cannot say whether the first act in the assimilation of carbon dioxide takes place in red or in blue-green chloroplasts in the same manner as in green ones. The same end may often be attained by different means,

1 Bert, Rech. s. l. mouvem. d. 1. sensitive, I870, p. 28 (From Mém. d. l'Acad. d. Bordeaux, T. viI1); Compt. rend., 1870 , T. Ixx, p. 338; i 871 , 'T. LxxnI, p. 1444 ; Baudrimont, ilid., $187_{2}$, T. Lxxıv, p. 47 ; G. Kraus, Bot. Zeitung, 1876, p. 508.

${ }^{2}$ Hunt, Bot. Zeitung, 1851, p. 319 ; Sachs, ibid., 1864, p. 37 , and Arb. d. Bot. Inst. in Würzburg, 1871 , Bd. I, p. 56 ; Ad. Mayer, Versuchsst., 1867, Bd. Ix, p. 396; R. Weber, ibid., I875, Bd. xvili, p. I8; Morgen, Bot. Zeitung, I877, p. 579; Wollny, Forsch. a. d. Geb. d. Agriculturphysik, 1894 , Bd.xvir, P. 317 ; Macagno, lot. Zeitung, 1874 . p. 544 . 
for nitrate- and nitrite-bacteria are able to assimilate carbonic acid chemosynthetically and perhaps by an entirely different form of organic synthesis. It is possible, by chemical means, to produce carbohydrates from carbon dioxide by various processes of chemical synthesis, so that the photosynthesis of the latter need not always occur in the same manner. It is indeed always a fundamental error to suppose that a living organism with definite specific properties will necessarily produce a given substance in the way which seems probable to us from our present knowledge of chemistry and physics (Sect. 1).

The almost instantaneous recommencement of the evolution of oxygen in light, and the rapidity with which starch may reappear, do not necessarily indicate that the number of intermediate processes must be extremely limited; for various machines are known which form elaborated products in a very short space of time although a large number of actions intervene between commencement and completion. The end-product affords no evidence as to whether the preceding operations have all taken place between the plasmatic micellae or molecules of the chloroplastid, or whether at certain stages plasmatic compounds have been formed, whose disintegration has resulted in the production of sugar or other substances. However this may be, the chloroplast is able by utilizing the energy of sunlight to produce organic substance from carbon dioxide and water without any perceptible diminution of its own bulk. It is the task of physiology to explain as far as possible the means by which this is accomplished, and what is the precise function of the different parts, including chlorophyll. At present, however, it is not even known whether chlorophyll simply acts as a sensitizing agent, and by transferring the intercepted light-vibrations to colourless parts of the chloroplastid renders these capable of assimilating carbonic acid, or whether it is of use in other ways and perhaps takes a direct part in the decomposition of this gas (cf. Sect. 60).

The evolution of free oxygen and the formation of less highly oxidized substances from carbon dioxide and water may be distinct, though closely connected, processes, even if the intermediate changes be very limited in number. Neither physiology nor chemistry can afford any conclusive evidence as to what is the first act in the process. Light may indeed cause oxygen to be evolved from oxide of mercury, but it may also cause reduction, as, for example, in hydroquinone, and it is, moreover, capable of inducing various decompositions, or even polymerization in certain substances ${ }^{1}$. Hence it is possible that, in the different stages of assimilation, light may at one time exercise a reducing, at another an oxidizing action.

The entire process is certainly not directly connected with the presence

${ }^{1}$ See Klinger, Ber. d. Chem. Ges., 1886, p. $4^{86}$; Ostwald, Lehrb. d. allgem. Chem., 1893 , 2. Auf., Bd. I, p. 1085 . 
of light, as is indicated by the fact that starch may be formed from glucose in darkness, but those changes which lead to an evolution of oxygen seem to be directly dependent upon the action of light-energy, and the continued removal of the gas produced allows the process to continue uninterruptedly, just as an energetic decomposition of silver iodide is induced by light only when the iodine is removed as fast as it is liberated ${ }^{\text {? }}$.

Hitherto attention has almost solely been paid to the immediately perceptible green dye chlorophyll, which though an important is still simply a co-operative member in the assimilatory mechanism (Sect. 52 ). The view held by certain authors that chlorophyll is the primary assimilatory product is no longer tenable (Sect. 54 ), and Pringsheim's protective theory has also proved to be erroneous (Sect. 59). Nor has Wiesner's supposition been confirmed, according to which it is the chlorophyll which directly liberates oxygen from carbon dioxide, while since pure chlorophyll contains no iron, Horsford's suggestion that iron may exercise the necessary reducing action can no longer be admitted ${ }^{2}$.

Various theories have been put forward which are fairly plausible from a chemical point of view, but decisive evidence can only be obtained by direct observations upon the living organism, and at present these do not suffice to allow any positive conclusions to be drawn. A certain amount of probability attaches to Bayer's theory, according to which formic aldehyde is produced in the chloroplastid from carbon dioxide and water, oxygen being evolved, while from this formic aldehyde $\left(\mathrm{CH}_{2} \mathrm{O}\right)$ carbohydrates may be formed by polymerization ${ }^{3}$. The latter process is one which may be comparatively readily induced, but no evidence has as yet been brought forward to show that formic aldehyde is actually produced by the assimilation of carbonic acid. Even if plants are able to form starch when supplied with this substance it does not follow that it is the primary assimilatory product (Sect. 55), for various substances may induce a deposition of starch in chloroplastids. Moreover

1 Cf. Ostwald, l.c., p. 1080.

2 Wiesner, Sitzungsb. d. Wien. Akad., I 874, Bd. Lxix, Abth. i, p. 59 of Sep.-abdr. Cf. Pfeffer, Osmot. Unters., I 877, p. I66. Other hypotheses are given by Kraus (Flora, I875, p. 269) and by Timiarezeff (Bot. Zeitung, 1885, p. 619); Horsford, Sitzungsb. d. Wien. Akarl., 1873, Bd. LXXviI, Abth. ii, p. 436 . See also Ballo, Ber. d. Chem. Ges., I859, p 750. The fantastic hypotheses of Kisler (Jahresb. d. Chem., I 859. p. 560 ) and of Benkovich (Ann. d. Phys. u. Chem., I875, Bd. CLIv, p. 468 hardly require discusion.

3 Bayer, Ber. d. Chem. Ges., is7o, Bd. IIr, p. 66. Cf. Meyer und Jacobson, Lehrb. d. organ. Chemie, I 893 , Bd. I, p. 40 I ; also ref. in Bot. Zeitung, I886, p. 849 . The simple proof of the presence of aldehydes in the plants affords no positive evidence. See also E. Fischer, Ber. d. Chem. Ges., 1894, p. 323I. Bach (Compt. rend., I 893 , T. Cxvi, pp. 1145, 1389) states that formal aldehyde may be produced in dead masses by the action of sunlight, but these results are in urgent need of confirmation. [Curtius and Reinke (Ber. d. Chem. Ges., I 897, p. 201) find traces of aldehydes to be present in all chlorophyllous parts exposed to light, but none in Fungi, and none in seedlings grown in darkness, whether etiolated or green, although these substances soon appear in the seedlings when exposed to light.] 
glycerine might with equal justice be regarded as an intermediate assimilatory product, for the chemist can construct this substance synthetically from carbon dioxide ${ }^{1}$; similarly formic ${ }^{2}$ and oxalic acids may be synthetized with comparative ease from carbon monoxide, while carbohydrates may be produced from these by a series of chemical metamorphoses. Erlenmeyer supposed that formic acid and hydrogen peroxide are formed during the assimilation of carbon dioxide, a theory which the absence of hydrogen peroxide from assimilating plants conclusively negatives ${ }^{3}$.

Various reasons militate against the acceptance of Liebig's theory that organic acids are the primary assimilatory products, and that carbohydrates are produced from them. Urea is a substance which may be comparatively readily constructed by synthesis, and the hypothesis has already been put forward that urea is the primary assimilatory product in nitrate bacteria ${ }^{4}$.

\section{Section 62. Individual and Specific Peculiarities.}

As division of labour becomes more marked, green plants undergo successively increasing adaptive modification in order that the assimilating chloroplastids may be subjected to appropriate illumination. The enormous leaf-surface is developed for this purpose, although other factors, such as the necessity of restricting transpiration, \&c., may come into play and cither cause a reduction in the leaf-surface, or induce a marked development of cuticle (Sects. 38, 52). Very commonly a compromise is made, one function being more or less restricted in order that another may not fall below the minimum compatible with continued existence.

It is only necessary to mention a few of the salient features concerning the position, distribution, \&c. of the chloroplastids in the assimilating organs. Even in a typical leaf it may be more economical to allow the greater number of the chloroplastids to work in modified and weakened light and hence with less energy, than to waste an enormous amount of material in so increasing the surface area of the leaves that all the chloroplastids are fully exposed, for in the latter case there is a danger of overexposure, and other functions may be restricted or jeopardized. In our

1 Meyer und Jacobson, Lehrb. d. organ. Chemie, I 893 , Bd, I, P1) 579, 902.

2 Cf. Lieben, Beibl. z. d. Ann. d. Phys. u. Chem., I 895 , Bd. xix, p. $4^{6} 3$.

${ }^{3}$ Erlenmeyer, Ber. d. Chem. Ges., 1877, p. 634. Cf. Pfeffer, Oxydationsvorgänge, I889, p. 430.

4 Liebig, Die Chemie in Anwend. auf Agric., I840, Ist ed., p. $63 ; 1876$, 9th ed., p. $3^{\circ}$. Cf. Sect. 56. On the production of carbohydrates from organic acids, see Ballo, Ber. d. Chem. Ges., 1889, p. 750. Maqnenne (Chem. Centralbl., 1882, p. 329 ) regards methane as an intermediate product, Crato holds that a benzene ring is formed (Ber. d. Bot. Ges., I892, p. 250), and Putz supposes (Chem. Centralb1., I886, p. 774) that light acts by producing electrolytic currents. 
own climate most plants secm to have found it preferable to form ncw leaves each year, rather than to acquire such resistant powers as would enable them to survive over winter, but it is certainly always of importance that the new leaves both of trees and of secdlings should rapidly acquire the power of assimilating carbon dioxide, so that as little as possible of the favourable period for vegetation may be wasted. Since the functional activity of adult organs diminishes with age, it is evidently also of advantage that even in evergreens the leaves should ultimately be thrown off and replaced by new ones.

The activity with which carbon dioxide is assimilated depends not mcrely upon the illumination, or upon the position of the chloroplastids, but also upon their specific assimilatory energy, for therc is no doubt that this varies in different chloroplastids and does not bear any direct or constant relation to the amount of chlorophyll present ${ }^{1}$. Hence also whilc the number of chloroplastids present in a leaf is an important factor in determining its assimilatory power, it is not the sole one, nor does the assimilatory energy of equal leaf-areas bear any constant relation to the number of active chloroplasts in them. It is, moreover, easy to see that if the assimilatory activity is measured per unit of weight, fleshy leaves will appear to have abnormally weak assimilatory powers ${ }^{2}$. Hence in each special case the combination of factors which influence the energy of assimilation must be taken into consideration, and if the evolution of oxygen or the increase of dry weight is used as a test for the amount of assimilation, then in all cases the loss due to respiration must be calculated.

Weber grew plants under constant conditions in a greenhouse and found that after forty-eight days a plant of Phaseolus multiflorus had gained $5^{.} 8_{3} 6$ grammes in dry weight, one of Helianthus annuus 29.806 grms., so that, allowing for the loss by respiration, in ten hours a square metre of leaf-surface of Helianthus was able to assimilate 5.559 grms., of Phaseolus, 3.4I 3 grms. of carbon dioxide. Haberlandt calculated that a similar relationship existed between the number of chloroplastids present in the same area of leaf-surface (approximately 495,000 per sq. $\mathrm{m}$. in Helianthus to $28,3,000$ in Phaseolus), but such a relationship need not necessarily exist. The same criticism applies to the researches of Hansen, who found that different plants yielding 3.9 to 5.9 grms. of pure chlorophyll per sq. $\mathrm{m}$. of leaf, produced under similar conditions about I grm. of starch per $0.2 \mathrm{grm}$. of chlorophyll ${ }^{3}$.

Since in feeble light but little carbon dioxide can be assimilated, and

1 Cf. Sects. 52, 53, and 6o. Examples of chloroplastids with different specific assimilatory powers are given by Engelmann, Bot. Zeitung, I888, p. 718 .

${ }^{2}$ For examples see Aubert, Rev. gén. d. Bot., $1 \delta_{92}$, T. Iv, p. 440.

3 Weber, Arb. d. Bot. Inst. in Wiirzburg, $18 ; 9$, Bd. I1, p. 350. Cf. also Géneaud. Lamarlière, Compt. rend., I $\delta_{91}$, T. Cxill, p. 230 ; Haberlandt, Jahrb. f. wiss. Bot., Is 82 , Bd. x1II, p. 95 ; Hansen, Arb. d. Bot. Inst. in Würzburg, $188_{7}$, Bd. III, p. 428 . 
since intense light ultimately exercises an injurious influence, there must be a definite specific optimal intensity of illumination for cach plant, although if light acts directly upon other protoplastic functions as well, the general optimum may not precisely coincide with the optimum for the assimilation of carbon dioxide. Even for light-loving (photophilic) plants bright diffuse daylight seems as a general rule to be preferable to strong sunlight.

In nature plants are exposed to an illumination which varies according to the season, and to the habitat and the time of day, while the different leaves of a plant do not usually receive the same amounts of light. Thus the innermost leaves of a horse-chestnut or beech may receive only a hundredth part of the light which falls upon the fully exposed outer ones, whereas in the open foliage of the birch, all the leaves probably obtain sufficient light for active photosynthetic assimilation. In very weak light leaves and branches become feeble and die, and this tends to thin out excessively thick foliage to a certain extent ${ }^{1}$.

The feeblest intensity of light at which growth is possible varies very much in different plants, and is dependent not merely upon the assimilatory activity but also upon the amount of organic material consumed in respiration as well as upon other factors.

Rapid growth is generally accompanied by active respiration, and hence slowly developing plants are able to increase in dry weight upon a smaller production of organic material no matter whether the feeble total assimilation is due to a diminution in the amount of chlorophyll present, or to a shady habitat.

Respiration seems to be only moderately active in shade plants, while the abundance of chlorophyll ensures the utilization of as much as possible of the enfecbled light ${ }^{2}$. Many plants are able to grow indifferently either in deep shade or in strong light, but typical shade plants are unable to withstand prolonged exposure to intense illumination. On the other hand, a lessened power of growth and feebler respiratory activity are usually connected with the weaker assimilatory powers of certain photophilic plants, as is the case in fleshy plants and in crustaccous Lichens ${ }^{3}$, for the activity with which carbon dioxide is decomposed may surpass its rate of production by respiration only under strong illumination. The same is often the case in actively respiring young leaves, and Ewart has shown that the attainment of the power of evolving oxygen in the light is not

1 Wiesner, Sitzungsb. d. Wien. Akad., I 895 , Bd. crv, Abth. i, p. 605 ; Ber. d. Bot. Ges., I $\varepsilon_{94}$, p. 78 ; Wiesner, Denkschr. d. Wien. Akad., I 896 , Bd. LXIV, p. 73 .

${ }^{2}$ A. Meyer, Versuchsst., I $\delta_{92}$, Bd. XL, p. 2 I 2. Cf. Haberlandt, Jahrb. f. wiss. Bot., I $88_{2}$, bd. xiIr, p. Ifo; Spencer le Moore, Bot. Jahresb., 188s, p. 660.

3 Jumelle, Rev.gín. d. Bot., I 892 , T. Iv, p. 11 I. Cf. Ewart, Journ. Linn. Soc., I 896 , Vol. xxxi, p. $38 \mathrm{r}$. On the illumination of a room, cf. Sect. 59 . 
solely dependent upon the amount of chlorophyll present ${ }^{1}$, the maximal power of assimilating carbon dioxide being acquired when the leaves are from two-thirds to full grown.

It is not yet certain whether in shade-loving (helioplobic) plants the general cell-protoplasm may not be more sensitive to light than the chloroplastids are. In all the photophilic and heliophobic plants examined, the chloroplastids seem to be affected sooner and more markedly than the general cytoplasm, whereas in Hydra viridis it appears that the animal protoplasm is more sensitive to the action of light than are the symbiotic algal cells it contains, and it is not impossible that in many shade plants ${ }^{2}$ the general cytoplasm may be more sensitive than the chloroplastids are. The various protective adaptations against intense light, such as are due to the presence of absorptive pigments, to movements of the leaves or chloroplastids, to structural arrangements, \&c., may be of importance to protect not only the chloroplastids but also the general cell-plasma from overexposure, for as a matter of fact many colourless protoplasts are extremely sensitive to light and readily injured by intense illumination. The fact that the epidermis of terrestrial plants is usually without chlorophyll, that the chlorophyllous guard-cells of the stomata are usually on the under surface of bifacial leaves, and that the chloroplastids when subjected to intense illumination assume positions in which they are least exposed, all indicate the necessity of protecting the latter from light of excessive intensity ${ }^{3}$. The practical utility of these phenomena does not concern us, but it must be remembered that these protective adaptations need not necessarily be of importance only to the function which they more especially subserve.

Although protective adaptations exist against excessive exposure, the general arrangement and distribution of the chloroplastids is such that they receive as much light as possible. This is clearly shown in the structure and shape of all chlorophyllous organs, and the arrangements of the leaf-cells and of the chloroplastids is apparently such as to permit the penetration of a maximal amount of light to the more deeply situated green layers. It is easy to see that the more evenly the light is distributed, the more nearly may an optimal illumination be reached for the deeper layers without the peripherally situated chloroplastids being exposed to an injurious intensity of light. The presence of non-chlorophyllous areas, and the refraction and reflection which occur in every leaf, may serve to

1 Corenwinder, Ann. d. chim. et d. phys., $18_{5} 8$, iii. sér., T. LIV, p. 330 ; Ewart, 1. c., p. $45^{2}$. On the influence of the temperature, cf. Sect. 58 .

${ }^{2}$ See Ewart, 1.c., pp. 439, 573 ; Annals of Botany, $18_{97}$, Vol. xI, p. $44^{2} ; 1 \delta_{9} S$, p. $3_{7}$; and Sect. 58 .

3 The movements dependent on light will be discussed later. On pigments and their inportance, cf. Sect. S8. See also Ewart, Annals of Botany, Vol. xi, p. 475. 
secure more even distribution of the incident rays, and in the lower.palisade layers the light may be concentrated as it is in the biconvex protonema cells of Schizostega osmundacca, in which the chloroplastids collect at the points on which the incident light is focussed ${ }^{1}$.

These requirements are fulfilled in a dorsiventral leaf by the development of palisade parenchyma on the directly illuminated surface ${ }^{2}$, while a certain quantity of light may penetrate to the spongy mesophyll in which the chlorophyll is less abundant, and which also receives light reflected from beneath. The large intercellular spaces of the spongy mesophyll renders possible the rapid transference of carbon dioxide to the palisade parenchyma, and when stomata are present only on the under surface of the leaf practically the whole of the carbon dioxide absorbed by the palisade layers passes through the intercellular spaces of the mesophyll (Sect. 3I). According to the structure of the leaf and its specific properties photosynthesis may be more or less active when the under surface is turned upwards, or when the leaf is illuminated from beneath ${ }^{3}$.

It is in correspondence with the general and purposeful self-regulatory power that organs whose functional activity depends upon illumination should be markedly affected by the intensity of the light under which they develop. Thus in darkness most leaves do not turn green nor is their normal shape or structure attained, while the maximal size is usually reached in light of moderate intensity, and it is only under strong illumination that the greatest possible thickness and maximal tissue differentiation are attained. Leaves grown in the shade may be without any definite palisade layer, while two to three layers may be present in leaves which have developed in exposed situations ${ }^{4}$. Hence when grown in moderately bright light leaves very often become deeper green in colour, and contain more chloroplastids to the same area than when developed in shady situations ${ }^{5}$.

The influence of light upon growth, movement, \&c., will be discussed later. It may, however, be mentioned that changes of illumination do

1 Noll, Arb. d. Bot. Inst. in Würzburg, I 888, Bd. 111, p. 477.

${ }^{2}$ Cf. Haberlandt, Physiol. Anat, I896, 2. Auf., p. 226, where other literature is quoted. Jönsson, Zur Kenntniss d. anat. Baues d. Blattes, I 896 .

${ }^{3}$ See Kreusler, Landw. Jahrb., 1890, Bd. xix, p. 662 ; Meissner, Bot. Centralbl., I894, Bd. Lx, p. 206.

4 Chief literature: Stall, Über d. Einfl. d sonnigen u. schattigen Standorts auf Laubblätter, I $88_{3}$; Heinricher, Jahrb. f. wiss. Bot., I $88_{4}$, Bd. $x v$, p. 556 ; Johow, ibid., p. 284 ; Grosglik, Bot. Centralbl., i $88_{4}$, Bd.xx, p. 374 ; Haberlandt, Ber. d. Bot. Cies., I8S6, p. 206; Dufour, Ann. d. sci. nat., I 887 , vii. sér., T. v, p. 3 I I ; Lamarlière, Rev. gén. d. Bot., I892, T. IV, p. 48 I ; Ssurosh, Bot. Jahresb., I892, p. 95. On marine algae, ef. Berthold, Jahrb. f. wiss. Bot., I882, Bd. XIII, p. 690 .

"Lamarlière, 1.c., p. 492; Bonnier, Ann. d. sci. nat., I894, vii. sér., T. Xx, p. 337 (Alpine pllants). 
not always produce precisely the same results, for different plants may react differently, and various influences may aid in modifying the character of the response.

\section{B. Chemosy'nthetic Assimilation of Carbon Dioxide.} SECTION 63.

Our knowlcdge of the nitrate and nitrite bacteria is almost entirely due to Winogradsky's researches ${ }^{1}$. These organisms have the power of assimilating carbon dioxide chemosynthetically by means of energy derived from the oxidation of ammonia into nitrites or nitrites into nitrates. The whole of the organic food of these bacteria is obtained in this manner (Sect. 50), and hence they can develop in a fluid free from all organic substances provided the necessary inorganic salts are present. The nutrient fluid used by Winogradsky contained I gramme of potassium phosphate, 0.5 grm. magnesium phosphate, and 0.5 to 1 grm. basic magnesium carbonate to $1,000 \mathrm{grm}$. of water. To this solution 0.2 per cent. of ammonium phosphate must be added for the culture of nitrite bacteria, whereas a similar amount of potassium or ammonium nitrites must be supplied and renewed from time to time to cultivate nitrate micro-organisms.

Winogradsky has shown that nitrite-bacteria (Nitromonas, Nitrococcus) oxidize ammonia to nitrous acid, and proceed no further even when fully supplied with oxygen, whereas nitrate-bacteria (Nitrobacter) oxidize nitrites to nitrates, and thereby obtain energy for their growth and development. Both forms are always present in ordinary soil, and if well aerated no marked accumulation of nitrites ever occurs (Sect. 28). The nitro-bacteria are strongly acrobic and consume oxygen in great abundance ${ }^{2}$, while since they cannot develop in the absence of oxygen, any formation of nitrites that may occur under such conditions must be due to the reduction of preexistent nitrates by other bacteria (Sect. 102).

The nitro-bacteria are of great importance in the economy of nature by providing a continual supply of nitrates to the soil, and at the same time they obtain the energy for the chemosynthetic assimilation of carbon dioxide $^{3}$, and hence are able to grow and form organic substance when this gas affords the sole source of carbon. When carbonates are present

1 Winogradsky, I. Ann. d. l'Inst. Pasteur, I89o, T. IV, p. 2 I3 ; II. p. 257; III. p. JIo; IV. 189 I, T.v, p. $9^{2}$; V. p. 57 ; VI. Archiv. p. 1. sci. biol. à l’Inst. imp. à St.-Pétersbourg, I 892 , T. I, p. 87 ; VII. Centralbl. f. Bact., i 896 , Abth. ii, Bd. 11 , p. $4^{1} 5$.

${ }_{2}$ For measurements of the oxygen consumption, see Godlewski, Anzeiger d. Akad. d. Wiss. in Krakau, $\mathrm{I} S_{92}$ and $\mathbf{I} \delta_{95}$.

3 This energy is indirectly derived from the sun (see Sect. $5 \mathrm{I}$ ). 
the carbon dioxide may be liberated by the formation of nitrous acid from ammonia. Godlewski found however that the presence of free carbon dioxide favours the development of nitrite bacteria, and may perhaps influence the growth of pure cultures of nitrate bacteria to a still greater extent, for the oxidation of nitrites to nitrates does not necessarily involve any production of frec acid ${ }^{1}$.

Nothing certain is known as to the precise character of this process of chemosynthetic assimilation, nor as to the first organic products formed; neither has it been determined whether a portion of the synthesized carbon compounds is decomposed again with a production of carbon dioxide, nor whether in these organisms any organic material is consumed in respiration, the whole of the energy being directly derived from the oxidation of ammonia or nitrites ${ }^{2}$.

Higher plants are incapable of any such oxidation of ammonia or nitrites, whereas the performance of this process forms an essential condition for the existence of nitro-bacteria, for it has not been found possible to cultivate these organisms in the absence of suitable inorganic nitrogen compounds, and Winogradsky has shown that the results obtained by Burri and Stutzer are due to an error which readily arises during the slow growth of pure cultures ${ }^{3}$. It is possible, however, that other nitrobacteria may be found which are able to obtain energy from other substances, and it is even possible that in certain cases the energy for the chemosynthetic assimilation of carbon dioxide may be obtained by the oxidation of organic substances (Sect. 50). The growth of normally active nitro-bacteria is not hindered by the presence of organic substances, and it is possible that, in addition to the auto-assimilatory products, they may directly absorb appropriate organic food-substances when such are present, for a partial replacement of auto-assimilation is possible in green plants, although the complete inaction of the chlorophyllous organs ultimately induces pathological phenomena terminating in death.

Heraeus and Hüppe first called attention to the chemosynthetic assimilation of carbon dioxide by nitro-bacteria, but it is to Winogradsky's researches that our knowledge of these organisms is due, for he was the first to obtain pure cultures

${ }^{1}$ Godlewski, Anz. d. Akad. d. Wiss. in Krakau, Dec., I $8_{92}$, and June, 1895 ; also Centralbl. f. Bact., 1896 , Abth. ii, Bd. II, P. 45 . On the corroding action of nitrite bacteria on minerals, \&c., cf. Miintz, Compt. rend., I 891, T. Cx, p. 1370. For researches in which the access of dust, of volatile organic substances, \&c., was prevented, see Winogradsky, l. c., Nr. iii, p. $7 \mathbf{7} 7$ : Godlewski, 1.c., Dec., I 892 .

${ }^{2}$ Godlewski (1.c.) found that a certain amount of free nitrogen was liberated, but this might have been derived from the decomposition of ammonium nitrite (cf. Sect. 68).

${ }^{3}$ Burri u. Stutzer, Centralbl. f. Bact., I895, Abth. ii, Bd. I, p. 72 I. Similar erroneous results were obtained by Frankland, Biol. Centralbl., I 89 I, Bd. xI, p. 56, and Stutzer and Hartleb, Centralbl. f. Bact., I897, Abth. ii, Bd. III, pp. 8, I6r. Cf. Winogradsky, l. c., Nr. vii, and Nr. vi, p. I 29. 
and to distinguish between nitrite and nitrate bacteria ${ }^{1}$. These are all exceedingly minute forms, and owing to their slow growth they are readily suppressed by other bacteria when organic food materials are present. Each form has its own specific oxidatory power which is always extremely pronounced, so that small amounts of these organisms are able to produce large quantities of nitrites or nitrates as the case may be. A large amount of the energy obtained is consumed in the assimilation of carbon dioxide, for even under favourable conditions it requires the oxidation of 33 to 37 parts of nitrogen, in the form of ammonia or nitrous acid, to yield the energy necessary for the assimilation of one part of carbon ${ }^{2}$, and hence the accumulation of organic material takes place but slowly. Under favourable conditions Winogradsky (l. c., Nr. iv, p. 765) in sixty-five days obtained a crop containing 22.4 milligrammes of carbon which would have probably weighed when fresh 200 to 300 milligrammes.

Winogradsky suggested that urea might be formed by the polymerization of ammonium carbonate, whereas Hüppe and Loew ${ }^{3}$ suppose that formic aldehyde may be synthesized from carbon dioxide and water, but no empirical results have as yet been obtained which indicate either of these substances as the primary synthetic product. Nitrate bacteria assimilate carbon dioxide in the absence of ammonia, and non-pigmented sulphur bacteria apparently derive sufficient energy for the assimilation of this gas from the oxidation of sulphur or sulphuretted hydrogen ${ }^{4}$. The possibility of obtaining energy by the oxidation of inorganic compounds must be considered in all researches upon physiological combustion.

\section{PART III}

\section{ABSORPTION OF ORGANIC FOOD}

\section{Section 64. General.}

PLANTS which are unable to assimilate carbon dioxide must obtain all their organic food-materials from without (heterotrophic or allotrophic nutrition); by others a portion only of the organic food is drawn from

1 Heraeus, Zeitschr. f. Hygiene, I866, Bd. I, p. 210 ; Hüppe, Biol. Centralbl,, 1887, Bd. vit, p. 70I ; Chem. Centralbl., I887, p.1512; Winogradsky, l.c. Further literature is given by Burri, Centralbl. f. Bact., 1895, Abth. ii, Bd. I, p. 80. On the culture in organic nutrient solutions, and on gelatinous silicic acid, cf. Winogradsky, I.c.; on agar, l. c., Nr. vii, p. 424; Beyerinck, Centralbl. f. Bact., 1896, Bd. Xix, p. 258 . Warrington (Journ. of Chem. Soc. I878-9) and Schlösing and Muntz (Compt. rend. lxxxix. 1 879 ) were the first to conclude that the process of nitrification was due to the activity of micro-organisms.

${ }_{2}$ The heat coefficient of $\mathrm{I}$ gramme of $\mathrm{NH}_{3}$ is 90,600 calories, for I gramme $\mathrm{NHO}_{2}$ i $8,000 \mathrm{cal}$. Stohmann, Zeitschr. f. physik. Chem., 189o, Bd. vı, p. 355, and Ostwald, Lehrb. d. allgem. Chem., I 893 , 2. Aufl., Bd. II, p. 144.

${ }^{3}$ Loew, Centralbl. f. Bact., 1891, Bd. 1X, p. 691.

+ Winogradsky, l. c., Nr. ii, p. 275 ; Bot. Zeitung, $188_{7}$, p. $5+7$. Cf. Pfeffer, Energetik, 1892 , p. 208. It is uncertain whether other organisms, such as saprophytic fungi, \&c., can obtain energy by the oxidation of ferrous salts, \&c. On iron-bacteria, cf. W'inogradsky, Bot. Zeitung, 1888, p. 261 ; Molisch, Die Pflanze in Beziehung 7. Eisen, 1892, p. 64. Cf. Sects. 23 and 96. 
the external world, the rest being supplied by the imperfectly developed chlorophyll-apparatus; such may be termed 'mixotrophic' plants. Even a typical autotrophic ${ }^{1}$ plant may be artificially nourished to a certain extent, although it can obtain all the organic material it requires by photo- or chemo-synthetic assimilation. Similarly many plants live for a time parasitically, although when fully developed they may be capable of decomposing carbon dioxide, as is the case, for example, in seedlings which feed at first upon the food-materials of the seed, and also in the underground rhizomes of certain orchids which live saprophytically for a time.

Neglecting the innumerable adaptations which heterotrophic and mixotrophic plants have developed in order to obtain a sufficiency of organic food from without, it is only possible to draw a broad distinction between saprophytes and symbionts. Saprophytes obtain their nutriment from dead organic material, from the bodies of animals or plants, or from natural or artificial nutritive solutions. In those cases in which the food is obtained directly from living organisms we have examples of conjunctive symbiosis no matter whether the symbiont is epiphytic or endophytic ${ }^{2}$. Lichens form an example of reciprocal symbiosis, in which the union confers advantages upon both partners; when on the other hand the hostplant is simply preyed upon by a parasite the symbiosis is antagonistic.

These distinctions cannot, however, be rigidly adhered to, for many fungi may grow indifferently either as parasites or as saprophytes, or may regularly change from the one mode of existence to the other, while others have been successfully cultivated as saprophytes although under normal conditions they are obligate parasites ${ }^{3}$. Further it is often doubtful whether a supposed example of reciprocal symbiosis is not really a case of parasitism in which the host-plant is not injured to any great extent, but perhaps receives partial compensatory advantages, and even the most exact mutualism may be disturbed under certain circumstances, as for example when a scarcity of food material prevails, or when the conditions become abnormal in some way or other. All stages of transition between pure autotrophism and heterotrophism are exhibited among obligate or facultative mixotrophic plants. (Cf. Sect. 50.)

The balance of nature is such that various degrees of dependency exist between all forms of life, for every organic substance is directly or indirectly derived from the photosynthetic assimilation of carbon dioxide, and hence it is ultimately from this source that both saprophytic and parasitic plants and animals derive their food, while even those organisms which utilize the ultimate products of decomposition also obtain their energy indirectly

1 Frank (Lehrb. d. Bot., I 892, p. 548) has used these terms in a somewhat different sense.

${ }^{2}$ De Bary, Erscheinungen d. Symbiose, 1879 , pp. 6, 21, \&c. Cf. also Reinke, Jahrb. f. wiss. Bot., I 894 , Bd. xxvi, p. 526.

${ }^{3}$ Cf. de Bary, Fungi, Mycctozon and Bacteria, $188_{4}$, P. $3^{81}$; Zopf, Die Pilze, I89o, p. 228. 
from the sun. Each organism may therefore during its life, and also after its death, provide food for other organisms, or may create the special conditions necessary for the development of certain forms. Thus certain putrefactive bacteria form sulphuretted hydrogen, and render the development of sulphur-bacteria possible, and other organisms by absorbing oxygen provide appropriate conditions for the growth of anaerobic bacteria. A series of forms may follow one another in a putrescent fluid, each providing a suitable medium for its successor, and hence the ultimate result is by no means entirely dependent upon the original character of the nutrient fluid.

Typical conjunctive symbiosis is therefore simply a case in which a direct interchange of food-material occurs between two organisms. Two or more organisms living together, but without any direct or fixed union occurring between them, may provide the conditions necessary for their conjoint existence ${ }^{1}$. This may be termed disjunctive symbiosis, and the inter-relations existing between flowers and insects and between ants and plants are examples of it.

The different cells and organs of the same plant live together in a form of conjunctive symbiosis, and every non-chlorophyllous cell is fed like a heterotrophic plant by organic material obtained from assimilating cells. By grafting a leafy shoot of one plant upon the root-stock of another a permanent reciprocal symbiosis of similar character may be produced in certain cases". In many parasites union takes place between the corresponding tissues of the host and the parasite, as is the case in members of the Orobancheae, and in many species of Rhinanthus and Cuscuta. Active absorption is, however, possible in the absence of any such direct fusion, for a grass seedling absorbs food from the endosperm which is merely apposed to it, and the embryo of a fern as weil as the sporophyte of a moss is nourished in the same manner.

Heterotrophic plants vary much as regards the means by which they obtain nutriment. Many forms may develop under widely different conditions, owing to the marked accommodatory powers such plants frequently possess, whereas others have a very limited range or a highly specialized habitat, as is the case in the sulphur-, nitro-, and anaerobic bacteria. Many saprophytic fungi and certain parasites may be grown upon artificial nutrient solutions or solid media, and even when this is not found possible it may simply be because the experimental conditions are not properly adjusted to the plant's requirements.

The absence of any one of the essential conditions will render develop-

1 Various examples are given here, and in Chaps. viii and ix. Conjoint infections are also examples of the same phenomenon (cf. Fliigge, Mikroorganismen, 3. Aufl., IS96, Bd. I, p. 309).

${ }_{2}$ Vöchting, Transplantation, I892, p. 110 ; Daniel, Rev. gén. d. Bot., 1894, T. vi p. I. 
ment impossible, and apparently the seeds of Orobanche and Lathraca germinate only when contact with the root of a host-plant exercises a certain stimulus upon them which is probably chemical in nature. A special chemical stimulus seems also to be necessary for the development of the spores of certain fungi ${ }^{1}$. Similarly, a parasite may be unable to develop in a nutrient solution because of the non-performance of certain functions exercised when it penetrates a host, and the difficulty of cultivating many motile organisms upon solid nutrient media may be due to the fact that their inability to move exercises a certain injurious effect upon them ${ }^{2}$. As a matter of fact the enforced cessation of the assimilation of carbon dioxide induces a pathological condition in green leaves, while in Cuscuta a contact-stimulus causes a localized activity of growth leading to the formation of haustoria.

Precise data on these questions hare not as yet been obtained, nor has it been determined why Euphrasia, Rhinanthus, Thesium, \&c. can only grow when they are able to form haustorial connexions with the roots of other plants, for their own roots obtain a sufficiency of water and salts from the soil, and their chlorophyll-apparatus provides an abundant supply of carbohydrates. It is possible that such plants must obtain nitrogenous compounds or certain constituents of the ash parasitically ${ }^{3}$, and as a matter of fact the growth of carnivorous plants is favoured by a supply of nitrogenous organic substances, while other plants are unable to live unless supplied with proteid. Examples of these so-called 'peptoneorganisms' are afforded by many fungi and bacteria, while the same peculiarity is exhibited by certain algae, and especially those which may take part in the formation of lichens ${ }^{4}$. In retuin for the carbohydrate supplies received, the fungal components of many lichens seem to transfer a portion of the synthesized proteids to the alga, and the former have become so markedly adapted to this special mode of existence that they never occur free in nature, and can be cultivated only with difficulty when isolated ${ }^{5}$. Hence it is evident that we are dealing with a special

1 Koch, Entwick. d. Orobanchen, 1887, p. 3. Rhinanthus seeds germinate without any such stimulus. Koch, Jahrb. f. wiss. Bot., 18yı, Bd. xxil, p. 4. Iathraca: Heinricher, Ber. d. Bot. Ges., Generalvers., 1894, p. 126 ; Jahrb. f. wiss. Bot., 1897 , Bd. xxxI, p. 79, footnote. Fungi : cf. de Bary, Fungi, Mycetozoa and Bacteria, 1894, p. 376 . According to Benecke (Jahrb. f. wiss. Bot., ${ }_{1} 895$, Bd. xxvill, p. 501), the spores of Aspirgillus niger will not germinate in pure water.

2 An entirely fluid diet ultimately exercises an injurious effect upon man. Many motile organisms may be successfully cultivated on solid nutrient media.

${ }^{3}$ [When closely sown, the seedlings may be parasitic upon one another (Heinricher, 1. c., 1897, p. 87 ; Wettstein, Oesterr. Bot. Zeitschr., 1897 , No. 9, p. 323), a few plants surviving and reaching maturity. The seeds germinate (Rhinantheae) without contact with a host-plant.]

* Beyerinck, Bot. Zeitung, $1890, \mathrm{pp} \cdot 730,74^{6}, 766$. According to Klebs (1)ie Beclingungen d. Fortpflanzung, \&c., 1896), Scenedesmus does not require peptone, but peptone-organisms may occur more commonly among the Oscillariae, Euglenae, \&c.

"A. Möller, Die Cultur flechtenbilelencler Ascomyceten. Mïnster, 1887. 
form of mild parasitism, for the algal components can grow and develop equally well when isolated, although in the form of a lichen they are rendered more resistant to heat and to drought, and are protected against excessive insolation ${ }^{1}$. It is possible that the misletoe transfers superfluous organic material to its host-plant, but it is not known whether I iscum absorbs only water and inorganic salts from its host, or organic nitrogenous compounds as well.

All stages of transition exist between purely autotrophic and purely heterotrophic plants. Thus the saprophyte Neottic and the parasites Orobanche and Cuscuta contain a little chlorophyll and are able to produce a small and perhaps unnecessary portion of their organic food by photosynthesis, while carnivorous plants and the Scrophulariaceae previously mentioned obtain but little organic material from the external world. Moreover, every autotrophic plant is able to assimilate nitrogenous and non-nitrogenous organic substances to a certain extent, but it is not surprising that it has not as yet been found possible to cultivate autotrophic plants in a purely heterotrophic manner ${ }^{2}$.

Most green plants do not require any supply of organic food from without, and hence grow normally when supplied solely with the constituents of the ash, with water and with carbon dioxide. Liebig showed that the amount of humus might increase in cultivated ground in spite of the yearly harvest removing large quantities of organic substance, and hence correctly concluded that plants cannot obtain their organic food from the soil; and Pfeffer and others have shown that seedlings grown in humus in a receiver containing no carbon dioxide cease to develop when the reserve-materials of the seed are exhausted ${ }^{3}$. Hence humussaprophytes must have special absorptive powers, for even the easily cultivated moulds do not grow well upon humus. In correspondence with other regulatory phenomena it is probable that any reduction in the photosynthetic production of organic substance by a humus-saprophyte will tend to induce more pronounced heterotrophic nutrition.

Historical. The humus theory, according to which all plants must obtain their organic food from the external world, was a survival from the teaching of Aristotle. Van Helmont in the seventeenth century asserted that all the component

1 See Ewart, Joum. Linn. Soc. Bot., $1896, \mathrm{xxxI}$, Pp. 376,385 .

${ }^{2}$ Literature: Klebs, Unters. a. d. Bot. Inst. z. Tübingen, I 886 , Bd. II, p. $53^{8}$; Laurent, Bull. d. 1. Soc. Bot. d. Bruxelles, is8s, T. xxvi, p. 263; Nadson, Bot. Centralbl., i 890, Bd. xliI, P. 50; Acton, ibid., I89o, Bd. XLIV, p. 224 ; Bokorny, Versuchsst., 1889, Bd. xxxvi, p. 235 ; Bot. Centralbl., I896, 13d. Lxvi, 1. 304; Biol. Centralbl., I897, Bd. Xvil, p. I ; Krüger, Zouf's Beiträge, I 894 , IV, p. II 4 .

${ }^{3} \mathrm{Liebig}$, Die Chemie in Anwend. a. Agric., I84o, p. I4; Pfeffer, Monatsb. d. Berl. Akad., I873, p. $78_{4}$; Godlewshi, Bot. Zeitung, I879, p. 88 . Cf. also Cailletet, Compt. rend., IS7I, T. Lxxill, p. $1+76$. 
substances of a plant were obtained from water, but even after the production of organic material by the assimilation of carbon dioxide had been definitely proved, the absorption of organic material was regarded as necessary for the existence of all plants until Liebig gave the dogma its death-blow ${ }^{1}$. The upholders of this theory vigorously attacked ${ }^{2}$ Lielig's work, and even Th. de Saussure, the founder of the auto-assimilation theory, in his seventy-fifth year reverted to the ancient dogma. Boussingault, however, brought forward additional evidence in support of Liebig's view, and, together with Salm-Horstmar, showed that green plants can grow in soil free from humus, when supplied solely with inorganic salts (cf. Sect. 73). Liebig went so far as to deny that plants could absorb any organic substances, but Schleiden gave a fairly correct general account of plant-nutrition ".

Humus-saprophytes. Many non-chlorophyllous plants (Agaricus and other fungi, Monotropa, Lathraea, Epipogon, \&c.), and plants with but little chlorophyll (Neottia, \&c. ${ }^{4}$ ), can absorb the whole of their organic food from humus. The variable composition of humus renders it difficult to determine with certainty what substances are actually absorbed, while it is probable that the roots themselves exert a direct solvent action, for water dissolves but little material from many soils rich in humus ${ }^{5}$. The fungal mycorlizas which form symbiotic unions with the roots of most saprophytic Phanerogams (Johow, l. c., p. 50I) are probably of considerable importance in rendering the humus available for use, and at the same time they compensate for the poorly developed root-systems in such plants as Neottia Epipogon, Corallorhiza, \&c. Drude found that a young plant of Neottic surrounded by only a small portion of humus gradually became starved as development continued ${ }^{6}$.

The specific peculiarities of different phanerogamic saprophytes and parasites are mentioned in the literature given by Frank and Goebel ${ }^{7}$, while Zopf and de Bary give full details concerning fungi. In these works numerous examples are given of obligate, facultative, and temporary parasites.

Phanerogams appear to have less marked accommodatory powers, but nevertheless various stages of parasitism seem to be exhibited among the Rhinantheae ${ }^{8}$.

Details in Sachs, Geschichte d. Botanik, $1875, p \cdot 4^{81}$.

${ }^{2}$ See for example Hlubeck, Beleuchtung d. organ. Chemie d. Herrn Liebig, I $8_{4} 2$.

S Saussure, Ann. d. Chem. u. Pharm., I $8_{4^{2}}$, Bd. Xlil, p. 275 ; Boussingault, Ann. d. chim. et d. phys., I 84 I, iii. sér., T. I, p. 208 ; Schleiden, Grundziige, I 845 , 2. Aufl, Bd. 11, p. 469.

$*$ Wiesner, Pringsh. Jahrb., Bd. VIII, I872, p. 575 ; also Johow, Jahrb. f. wiss. Bot., I8s9, Bd. $\mathrm{xx}, \mathrm{p} \cdot 479$.

5 See Sachsse, Agriculturchemie, I 888, p. 122. Attempts to nourish green plants with artificial humus solutions are without value. Such experiments were performed by Hartig (Liebig, Die Chemie, \&cc., 1840, p. 192); Saussure, 1842, l.c.; Unger, Flora, 1842, p. 241 ; Wiegmann, Bot. Zeitung, I 843, p. 801 ; Trinchinetti, ibid., I 845 , p. II2. Nor is the slow diosmosis of humus solutions of marked importance. Cf. Detmer, Versuchsst., 18 $\mathbf{I}$, Bd. xiv, p. 279; Simon, ibid., 1875 , Bd. xvili, p. 470; Grandeau, Compt. rend., I872, T. Lxxiv, p. 988.

6 Drude, Biologie v. Monotropa, I873, p. 26.

7 Goebel, Entwickelungsgeschichte d. Pflanzenorgane, I883, p. 361 ; Frank, Lehrbuch, I892, p. 559 ; and of the later literature, Heinricher, Cohn's Beiträge z. Biologie, I895, Bd. vil, p. 364 (Lathraca).

${ }^{8}$ [See Heinricher, Jahrb. f. wiss. Bot., I 897, XxxI, p. 87 . Contrary to the usually accepted opinion, Monotropa is a saprophyte only (Johow, ibid., 1889, Bd. xx, p. 480 ).] 
Thus Euphrasia, Rhinanthus, Melampyrum arvense, \&c., seem to be obligate rootparasites, whereas according to Regel, Pedicularis and Bartsia, and according to Kerner, Odontites lutea, can grow even when unable to form haustoria on the roots of other plants ${ }^{1}$. Thesium seems also to be an obligate root-parasite, but it is not yet certain whether the same is the case with the indigenous species of Polygala ${ }^{2}$. Melampyrum pratense appears, however, to be a saprophyte, for according to Koch it develops haustoria only in connexion with dead parts of other plants ${ }^{3}$.

On the other hand, in Orobanche, Cuscuta, Rafflesia, and many other parasites, the vascular tissues of the host and parasite enter into intimate union similar to that taking place between a graft and graft-stock ${ }^{4}$. A close connexion of this kind is not always attained in Euphrasia or Rhinanthus ${ }^{5}$, and in Viscum only the tracheae of the wood unite, according to Peirce, indicating that Viscum obtains only water and salts from its host. These anatomical facts do not, however, form conclusive proof, for both nitrogenous and non-nitrogenous organic food-materials may be transferred in abundance and with considerable rapidity through parenchymatous tissues. Even Pitra's ${ }^{6}$ experiments, made by ringing the infested branches, do not afford decisive evidence that Viscum obtains only water and mineral constituents from the host-plant. It has already been mentioned that partial mutualism may exist between the mistletoe and its host-plant, an idea first put forward by R. Hartig (Bot. Jahresb., I875, p. 955!.

\section{SECTION 65. Special Adaptations.}

A plant can develop in a particular locality only when its structure is such that under the existing conditions it is able to obtain a sufficient supply of organic food. Hence the shape of autotrophic plants is almost entirely determined by the necessity of assigning a suitable position to the chlorophyll-apparatus, whereas in non-chlorophyllous plants which obtain their organic food from widely different sources, it is only to be expected that many remarkable peculiarities in their form and mode of life should have been developed. It must, however, suffice to give a general account of the special adaptions, or reciprocal combinations by which the end in view is reached.

It must be remembered that with the exception of Myxomycetes

1 The parasitism was first discovered by Decaisne, Ann. d. sci. nat., I $\delta_{47} 7$, iii. śr., T. virl, p. 2. Cf. Koch, Jahrb. f. wiss. Bot., I 89 I, Bd. xxıI, p. I. [On the autoparasitism of Euphrasia, \&c., see Heinricher, 1.c., and Wettstein, 1.c.] Regel, Die Schmarotzergewächse, Zürich, 1854 , p.34; Kerner, Pflanzenleben, $188_{7}$, Bd. I, p. $16_{\gamma}$. [Cf. also Heinricher, Die grinen Halbschmarotzer, Jahrb. f. wiss. Bot., IS98, Bd. XXXi1, p. 309.]

${ }^{2}$ Ficke, Abbandlungen d. naturwiss. Vereins zu Bremen, I875, Bd. 1v, p. 278.

3 Koch, Jahrb. f. wiss. Bot., I 889 , Bd. xx, p. 33.

* Koch, Entwickelung von Orobanche, IS87, p. 63. Cuscuta: Peirce, Annals of Botany, I893, Bd. VII, p. 324 .

${ }^{5}$ Koch, Jahrb. f. wiss. Bot., Bd. xx, p. 22 ; Bd. xxir, p. I8.

${ }^{6}$ Pitra, Bot. Zeitung, IS61, p. 63. 
plants can only absorb dissolved substances, and hence the solvent action of secretions is often necessary, especially in heterotrophic plants, either for the extracellular digestion of organic substances, or to render possible the penetration of dead materials or living plants. The death of a plant or animal may be caused either by these means, or by the excretion of injurious metabolic products (Bacteria, Fungi, \&c.). In the economy of nature, plants, and especially micro-organisms, are largely entrusted with the task of disintegrating and decomposing the bodies and organic remains of plants and animals, thus producing the humus necessary for the growth of humus-saprophytes, and aiding in the creation of a fertile soil (Sect. 5I). Mutual assistance and mutual interdependence are as common and as necessary in nature as is the inevitable struggle for existence, and in addition to the various disjunctive, simultaneous, or successive co-operations, there exists a still more intimate form of union in reciprocal symbiosis.

The importance of the root system to fixed plants can hardly be over-estimated; in a plant of Mucor, for example, the mycelial hyphae may attain a total bulk very much greater than that of the sub-aërial conidiophores. Organic nutriment, as well as ash constituents, may be obtained in solution from the soil, and even from dust conveyed by the air. Many so-called 'humus-collectors' possess various adaptions which enable them to retain and utilize the dust and organic débris which falls upon them, while carnivorous plants are able to capture and digest insects, and thus obtain organic nitrogenous compounds.

Freely motile organisms can seek out their food, and nutrient substances commonly act as an attractive stimulus to Bacteria, Myxomycetes, and Flagellatae. Similarly a localized accumulation of nutriment may attract growing fungal hyphae, and induce an increased branching where the food is most abundant (cf. Sect. 26). Chemotactic stimuli play an important part in the penetration of parasites, and even in reciprocal symbiosis the existence of interacting stimuli may frequently be made evident by the changed shape of one or both consorts.

Lichens are good examples of reciprocal symbiosis, for they are specific organisms formed by the union of a fungus with one or more algae, and can frequently withstand climatic conditions to which the isolated component parts succumb. The fungus, especially in those lichens which grow upon bare rocks, obtains organic food from the algac, while the fungal mycelium supplies the latter with water and salts, or even with proteids when the symbiotic algae are peptone-organisms ${ }^{1}$.

${ }^{1}$ Cf. p. 365 . Details in the text-books, and by Lindan, Lichenolog. Unters., I895, Bd. I ; Reinke, Jahrb. f. wiss. Bot., I 894 and I 895 . Warming (Lehrb. d. ökolog. Pflanzengeographie, I 896 , p. $9^{S)}$ terms this special case of symbiosis 'helotism.' 
Chlorophyllous animals. In lichens, as in grafts, the component parts are simply in close contact but remain distinct, whereas many tiny algae actually live, grow, and divide within the cells of certain animals, chiefly Protozoa, but including also Metazoa (Hydra viridis, Convoluta, \&c.). It is not, however, certain in all cases whether we are dealing with chloroplastids or with symbiotic algae, for in certain protozoa (Vorticella campamula) diffuse chlorophyll may be present, and hence it is easy to understand that definite chloroplastids may have been differentiated in higher forms ${ }^{1}$.

Leguminosac afford instructive examples of reciprocal symbiosis, for in the cells of the non-chlorophyllous root-nodules of these plants a bacterium develops, by means of which free nitrogen can be assimilated (Sect. 69).

Mycorhizas, or associations of fungal mycelia with roots, occur in almost all phanerogamic saprophytes, and also in many plants which possess an abundance of chlorophyll ${ }^{2}$. Endophytic mycorhizas, in which the fungal hyphae penetrate living cells ${ }^{3}$, are found in many of the Orchidaceae, Ericaceae, and Epacrideae, whereas the epiphytic mycorhizas of Monotropa, certain Cupuliferae, \&c., simply form an external covering to the roots. In trees the presence of a mycorhiza usually induces a marked branching of the root system, and since the mycelial hyphae often spread to a considerable distance, they may be of the utmost importance in enabling saprophytes to obtain organic nutriment from humus, especially since in such plants root-hairs are often feebly developed or

${ }_{1}$ Bütschli, Protozoen, I $88_{7}-9$, Bd. III, p. I 473 ; Dantec, Ann. d. l'Inst. Pasteur, I $89_{2}^{2}$, T. vi, p. I9o, and the literature there given. Cf. also Sect. 52. Algae often occur merely as epiphytes in the intercellular spaces, or mucilage cavities of certain plants. On the occurrence of Nostoc in Gunnera, see Jönsson, Bot. Centralbl., I $8_{94}$, Bd. Lix, p. I2. Also Möbius, Biol. Centralbl., I\$91, Bd. xI, p. 545; Schneider, Bot. Centralbl., I 894 , Bd. LIX, p. I3.

${ }^{2}$ Frank, Ber. d. Bot. Ges., I $88_{5}$, p. I 28 , and xxvir, ibid., I $88_{7}$, p. 395 ; I 888 , p. 248 ; I 891 , p. 248 ; P. E. Müller, Bot. Centralbl., I 886 , Bd. Xxvi, p. 22 ; Johow, Jahrb. f. wiss. Bot., I 889 , Bd. xx, p. 475 (Saprophytes); Schlicht, Landw. Jahrb., I889, Bd. xvıII, p. 499; Höveler, Jahrb. f. wiss. Bot., I892, Bd. Xxiv, p. 302 ; Sarauw, Beibl.z. Bot. Centralbl., I8g6, Bd. vi, p. 24; Groom, Annals of Botany, I895, Vol. Ix, p. 356; Janse, Ann. d. Jard. bot. d. Buitenzorg, I896, T. XIV, p. I8o. The other literature is quoted in these works, and in Frank's Lehrb., Bd. I, p. 274 , and Krankheiten d. Pflanzen, I895, 2. Aufl., Bd. I, p. 293.

3 Neither the injection of living organisms nor the penetration of certain parasites (cf. de Bary, Pilze, I $884, p .422)$ necessarily leads to the death of the protoplast, as is shown by the symbiotic union of algae and certain infusoria, by the fact that the nitrogen-assimilating bacteria may remain living in the cells of root-nodules, and also by the fact that certain rotifers may inhabit Vaucheria filaments. 
absent (Sect. 64). No absolute proof of this has, however, as yet been established, although, according to Frank, the growth of Pinus and of plants belonging to the Cupuliferae ${ }^{1}$ is retarded when the association of the roots with fungi is prevented by cultivation in a sterile soil.

Future researches must determine whether in all cases and under all cultural conditions this symbiotic association has the same importance. It may help to provide saprophytes with the organic food they require, but in the case of the Cupuliferae, \&c., it may be of service only in the absorption of nitrogen compounds or of mineral constituents. It has yet to be determined whether the substances required are directly transferred by the fungus, or whether it is able to elaborate certain nutrient substances for its host. (On the peptone-algae of certain lichens, cf. Sect. 64.) The fungus may be of use in the assimilation of ammonium
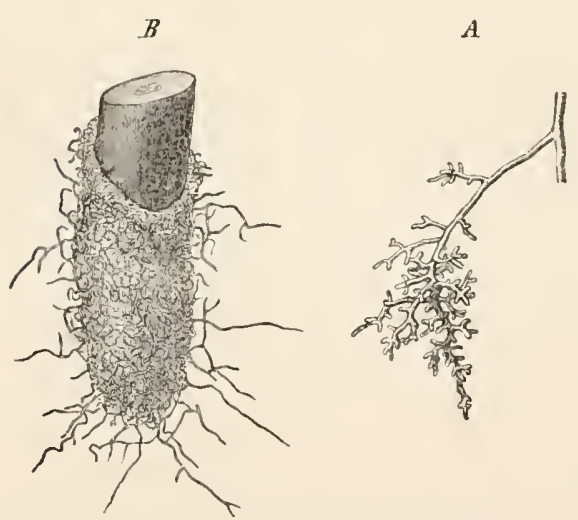

FIG. 55. Epiphytic mycorhiza of Fagus sylvatica. $(A)$ twice magnified; $(B)$ tip of root partially denuded of the investing mantle $\left(\times_{3}\right)$. salts, for although these afford a much less suitable source of nitrogen for most terrestrial plants than do nitrates, the latter are usually present only to a slight extent in forest soil ${ }^{2}$. Moreover, the increased absorptive area secured by the presence of fungal hyphae must be of importance, and in addition the latter are usually able to exercise a more energetic solvent action than root-hairs can. Frank ${ }^{3}$ supposed that the fungi may be cultivated by the roots in order to be devoured at a later period, but this has not been proved, and is highly improbable. Even when endophytic fungal hyphae, which die a natural death, are absorbed partially or entirely by the cytoplasm of the host-plant, this does not from any proof of Frank's supposition, for dead fungi or bacteria may be reassimilated in pure cultures by their own kind.

Fungi and bacteria, even without entering into conjunctive symbiosis, are of use in many ways in preparing media suitable for the development of other plants, and a correct estimation of the mutual interdependence of different organisms in the balance of nature shows that with regard to mycorhizas, whatever their use and

1 Frank, 1.c., 1888, p. 265 ; Figures in Pflanzenkrankh., 1895, p. 294. Pinus: Frank, Bot. Centralbl., i 895 , Bd. Lxxiı, p. 18.

${ }^{2}$ Baumann, Versuchsst., 1887 , Bd. xxx111, p. 302 ; Ebermayer, Ber. d. Bot. Ges., 1888, p. 217. No conclusions can be drawn from the absence of nitrates in mycorhizas (Frank, 1.c., I888, p. 249), for nitrates accumulate only when the conditions are suitable for passive secretion.

${ }^{3}$ Frank, l, c., 1891, p. 244; Lehrbuch, p. 561. On the fungus gardens of certain ants, see A. Möller, in Bot. Mitth. a. d. Tropen v. Schimper, r 893 , Heft 6. 
importance may be, the general principles of heterophic nutrition remain unaltered, although Frank denies this.

It has not yet been explained why mycorhizas are formed only on the roots of certain plants, nor has it been determined to what extent the same fungus may become associated with the roots of different plants. Epiphytic and endophytic mycorhizas are probably formed by different fungi, and hence it is possible that a variety of mycorhizal combinations with fungi may be possible. According to Frank several of the fungi which grow on humus may form mycorhiza, and it is possible that certain forms may be incapable of a separate existence.

Mycorhizas seem only to be formed in a soil rich in humus, and hence they isappear from roots which penetrate into sand, nor are any formed upon plants grown in a water-culture ${ }^{1}$. The development of a mycorhiza is accompanied by partial or complete suppression of the root-hairs, which are indeed absent in most phanerogamic saprophytes. This correlation affords an additional example of the dependence of the formation of root-hairs upon external conditions (Sect. 26).

Historical. Pfeffer was the first to suggest that the long known ${ }^{2}$ endophytic mycorhizas were examples of nutritive symbiosis, and the same view was put forward by Kamienski with regard to the epiphytic mycorhiza of Monotropa which he discovered ${ }^{3}$. The wide distribution of mycorhiza was, however, first discovered by Frank, to whom this term and most of our knowledge on this subject are due.

The penetration of parasites. Miyoshi ${ }^{4}$ has shown that the penetration of a parasite is mainly due to the action of chemotropic stimuli, for the penetration of non-parasitic fungal hyphae through stomata, or even through the epidermis, may be induced by injecting a leaf with solutions of meatextract, sugar, plant-extracts, \&c., which chemotropically attract the fungal hyphae. The same result may be obtained with widely different fungi, including normal saprophytes, and the hyphae of Penicillium glaucum and Aspergillus niger may bore through a fragment of epidermis or an artificial cellulose membrane lying between them and a nutrient solution, whereas if floated on water this does not occur.

The stimulus simply induces the fungal hypha to follow a certain path, and thus may lead it to bore through the membranes which separate it from the source of attraction. This is generally accomplished by an excretion of solvent enzymes, which aid the boring action of the apex of the elongating hypha, but although the solvent action is perhaps the most marked, still the mechanical pressure exerted by the tip of a growing hypha may suffice for the penetration of very thin gold-leaf. Radicles may

1 Frank, 1.c., 1888, p. 253. According to Johow (1. c., p. 506), mycorhizas are formed only upon the adherent surfaces of the aërial roots of tropical orchids.

${ }^{2}$ The literature is given by Wahrlich, Bot. Zeitung, I886, p. +81 ; Johow, 1.c., p. 503 .

3 Pfeffer, Landw. Jahrb., I $87 \uparrow$, Bd. vi, p. 497 ; Kamienski, Bot. Zeitung, 1881 , p. 461 .

* Miyoshi, Jahrb. f. wiss. Bot., 1895, Bd. xxviri, p. 269 ; Bot. Zeitung, 1894, p. 23. 
exert a pressure of from eight to fifteen atmospheres, and when no lateral divergence is possible, may penetrate leaves, stems, and other living organs merely by means of this mechanical pressure ${ }^{1}$. The penetration of the haustoria of Cuscuta is, however, apparently aided by the action of cellulosedissolving enzymes, and this is pcrhaps the case in all phanerogamic parasites.

In Cuscuta contact-stimuli induce the formation of haustoria, and from these the penetrating absorbent organs develop. Similarly special attaching organs are developed in many fungi (Botrytis cinerea), while whenever the growing apex exerts marked pressure in boring through a membrane, the older portion of the hypha must adhere with considerable tenacity.

A fungus which has at the outset been unable to penetrate a leaf may acquire this power when the injury of a few internal cells causes attractive juices to exude from them through the leaf. The thickness and character of the separating membranes are of course as important as are the special peculiarities of the attacking fungus, while even when penetration is possible the hyphae may not find suitable conditions for their development and may perish. In every case several factors are at work, as is shown by the restriction of certain parasites to particular hosts. No discussion of these interesting and complicated problems is however possible, for a precise determination of the different co-operating factors has not as yet been made in a single case ${ }^{2}$. All infectious diseases present similar problems for solution.

The excretion of solvent enzymes. Especially in heterotrophic plants secretory products are often used to dissolve solid materials, or to render soluble substances capable of more rapid diosmosis and absorption. In addition to acids and alkalies, enzymes or ferments are frequently employed, the latter being especially useful, since a very small amount suffices to transform a very large quantity of fermentable material. For the present we are concerned only with the importance of such secreta as a means to obtain nutriment, but ferments often take part in metabolism, and hence further mention will be made of them (Sect. 91).

In fungi and bacteria the extracellular action of enzymes is utilized in a varicty of ways, as, for example, in the penetration of cell-walls by means of cellulose-dissolving enzymes, while fungi which grow upon insects (Empusa, Entomophthora, \&c.) excrete an enzyme which dissolves chitin ${ }^{3}$.

${ }_{1}$ Pfeffer, Druck u. Arbeitsleistung, I $8_{93}$, p. $37^{2}$; Peirce, Bot. Zeitung, I 894 , p. 169.

${ }^{2}$ Cf. de Bary, Pilze, I88 4 , p. 420 , and Bot. Zeitung, I886, p. 44I ; Miyoshi, 1.c., I895; Brefeld, Unters. a. d. Gesammtgebiete d. Mykologie, $\mathrm{I}_{955}$, XI, p. 90 ; Erikson, Zeitschr. f. Pfanzenkrankheiten, $1895, \mathrm{v}, \mathrm{p}$. 8 .

${ }^{3}$ Miyoshi, Jahrb. f. wiss. Bot., I 895 , Bd. Xxix, p. 277 . Literature by de Bary, Pilze, I88 4 , p. 399 ; Zopf, ibid., IS90, p. 180. 
Similarly an excretion of acid enables certain fungal hyphae to perforate egg-shells.

Diastatic ferments are very widely distributed, and it is evident that a mould or bacterium can only grow upon starch when it is able to convert the latter into some soluble product. Similarly reserve-cellulose may be rendered available for use in seeds by means of a cellulose-dissolving enzyme, while the same occurs in the fungi which grow upon wood and in the bacteria which decompose cellulose. Moreover glucose-yeasts can grow upon cane-sugar when they are able to invert it, and many fungi also have the power of producing invertase.

Solid proteids can be absorbed only after they have been rendered soluble by means of proteolytic enzymes, and these are actually excreted by many carnivorous plants; the liquefaction of gelatine by many bacteria and certain fungi (Penicillium glaucum, Aspergillus niger) shows that they also can excrete a proteolytic ferment ${ }^{1}$.

This term, however, and that of 'diastase' as well, includes a variety of different substances; bacteria usually produce a tryptic ferment which is active in an alkaline solution, whereas in carnivorous plants and the above-mentioned fungi a peptic ferment is formed which acts only in an acid solution. Many fungi and bacteria are even able to decompose glucosides and fats.

Various fungi (Aspergillus niger, Penicillium glaucum) can perform all these fermentative decompositions, but other plants have more limited powers. Thus Drosera and Dionaea can excrete a peptic ferment only, whereas in other cases (fungi and certain embryos) not only diastase but also proteolytic enzymes are absent. The action of a particular enzyme is strictly limited, but under certain circumstances a plant may secrete several ferments simultaneously. A similar result may also be produced by the co-operation of two different organisms, as, for example, when the diastatic action of a fungus growing on starcl renders a certain amount of sugar available for other organisms.

The production and excretion of enzymes exhibit a regulatory connexion with one another, as is indeed the case in every metabolic process. Thus the secretion of an enzyme may begin only at a certain stage of development ${ }^{2}$, and may be influenced by the food supply or by other external agencies. In Dionaea pepsin is excreted only after an insect has been captured, i.e. only under the action of a chemical stimulus, and in many

1 Beyerinck states that a green alga, Scenedesmus acuuus, also liquefies gelatine (Bot. Zeitung, I 890, p. 729 ).

${ }^{2}$ For example in case of invertase, see Wasserzug, Ann. d. l'Inst. Pasteur, ISSS, T. I, p. 525; Bourquelot u. Graziani, Bot. Centralbl., I 893 , Bd. Lv, p. 326 ; in case of diastase, Duclaux, Ann. d. l'Inst. Pasteur, IS89, T. III, p. 107. 
other cases the needs of the organism exercise a marked influence upon its secretory activity, for when sugar is present in sufficient quantity the secretion of diastase may cease, and similarly the presence of peptone may inhibit the formation of proteolytic enzymes (Sect. 91). Hence it is not surprising that the same results are not always obtained in experimenting with a particular plant; moreover the action of a ferment is largely dependent upon the external conditions and especially upon the acid or alkaline nature of the medium.

Although enzymes are very widely distributed they are not essential to those heterotrophic plants which grow only upon soluble food-materials, and it appears that a few bacteria have no power of excreting enzymes. Hence it can only be determined empirically whether solvent enzymes aid in the nutrition of phanerogamic saprophytes; similarly the penetration of root-hairs, \&c. into dead leaves, masses of humus, or even into living parts of plants is not necessarily an indication of a power of excreting solvent ferments, but may simply be due to the powerful mechanical pressure which growing organs are able to exert ${ }^{1}$.

Extracellular digestion is only possible by means of excreted enzymes, but it is erroneous to suppose that every solvent action in a living cell is due to the action of ferments derived from the same cell or from neighbouring cells of the same tissue. Solvent actions may be attained by other means, as occurs, for example, in the removal of endosperm food-material, for the embryo may absorb the latter without any secretory activity of the cotyledons being necessary (Sect. I09) ${ }^{2}$.

Among sixty-two species of bacteria, Fermi ${ }^{3}$ found in twenty-four a proteolytic, in twenty a diastatic, in two an inverting enzyme, and in sixteen none. Three enzymes were found in Bacillus megatherium, and two in two other species. Among fungi, Aspergillus niger and Penicillium glaucum can produce diastase, invertase, cytase, pepsin, and fat-splitting ferments ${ }^{4}$. Aspergillus oryzae is able to secrete diastase in great abundance, whereas, according to de Bary, Peziza sclerotionum has not this power, although it is able to form a cytase or cellulose-dissolving ferment ${ }^{5}$. Hormodendron hordei produces no diastase, but secretes invertase,

1 Examples in the case of root-hairs: Drude, Biol. v. Monotropa und Neoltia, I873, p. 34; Schlicht, Landw. Jahrb., I889, Bd. xvil1, p. 499. Mosses: Haberlandt, Jahrb. f. wiss. Bot., I886, Bd. xvır, p. 476 ; Höveler, ibid., I892, Bd. xxIv, p. 293.

${ }^{2}$ For examples of the excretion of ferments by fungi and bacteria, see Zopf, Pilze, I 890, p. I 77 ; Flïgge, Mikroorganismen, I896, 3. Aufl., Bd. I, p. 197 ; Lafar, Technische Mykologie, I897; also Sect. $9 \mathrm{I}$, and the literature there given.

${ }^{3}$ Fermi, Centralbl. f. Bact., I $89^{2}$, Bd. xir, p. 715.

1 Bourquelot, Centralbl. f. Physiol., I 893 , Bd.viI, p. 660; Hansen, Flora, I889, p. 88 ; Miyoshi, 1. c. ; Schmidt, Flora, $189 \mathrm{I}$, p. 300.

5 Wehmer, Centralbl. f. Bact., Abth. ii, I895, Bd. I, pp. I52, 218 (A.oryzae); de Bary, Bot. Zeitung, 1886, p. $4^{22}$ (Peziza). 
pepsin, and a rennet ferment ${ }^{1}$. No inverting ferment has been detected in Mucor alternans and $M$. circinelloides nor in certain yeasts".

Myxomycetes exhibit all stages of transition between active and imperceptible extracellular and intracellular fermentative activities. Thus Chondrioderma and Aethalium septicum ${ }^{3}$ corrode ingested starch grains and proteids but feebly and are not always able to digest them. On the other hand Plasmodiophora Brassicae penetrates living plants, Vampyrella vorax and Leptophrys Kützingï kill and digest algae, while Monas amyli rapidly dissolves starch grains ${ }^{4}$. Vampyrella and Leptophry's kill their prey, whereas organisms ingested by Aethelium and Chondrioderma remain living, and bacteria may even increase in numbers within their vacuoles ${ }^{5}$. The latter Myxomycetes may be cultivated in sterile fluid media in which no solid food particles are present, whereas it is not known whether the same is the case with Vampyrella and Leptophrys. In these plants, and in the Flagellata and Ciliata as well, all grades of transition probably exist from organisms which absorb their food in fluid form only, to those which can digest solid food ${ }^{6}$.

Carnivora. Many plants live upon animal or vegetable food, or upon a mixture of both. Various bacteria and the fungi parastic upon insects are purely carnivorous, whereas no phanerogam can be cultivated upon an exclusively proteid diet, although the so-called 'carnivorous plants' obtain a small amount of organic food by the capture and digestion of insects.

With regard to the special peculiarities of carnivorous plants a few points only can be mentioned, and for further details reference must be made to the literature quoted beneath ${ }^{7}$. Thus in Nepenthes (Fig. 56) insects are drowned in pitchers partially filled with water, in Dionaea (Fig. 57, $A$ and $B$ ) and Aldrovanda they are captured by the sudden closure of the hinged leaf when it is mechanically irritated, while in Drosera (Fig. 58), Drosophyllum, and Pinguicula an insect may be retained by means of a sticky secretion. The contrivances used in the capture of insects are therefore such as are employed by other plants for different purposes, and so are the means of attraction by scent, colour, nectar, \&c., for these play

1 Bruhne, in Zopf's Beiträge z. Phys. u. Morph. nied. Org., 1894, I, p. 26.

${ }^{2}$ Afucor: Gayon, Ann. d. sci. nat., I882, vi. sér., T. xiv, p. 46 . Yeast: Beyerinck, Centralbl. f. Bact., I 893 , Bd. xi, p. 7 o.

${ }^{3}$ Celakovský, Flora, Erg.-bd., I $\delta_{92}$, p. $22 \zeta$, where the fact is mentioned that Krukenberg succeeded in obtaining a proteolytic enzyme from Acthalium.

${ }^{4}$ Cf. de Bary, Pilze, I8S 4 , p. $48 \mathrm{r}$; Zopf, Unters. über Monadineen, 1887, p. 24 .

${ }_{5}^{5}$ Čelakovský, 1.c., p. 224 ; Pfeffer, Aufnahme ungelöster Körper, I 89o, p. I 53.

${ }^{6}$ Sect. I9. Cf. Bütschli, Protozoen, 1883-9, Bd. II, p. 694; Bd. III, p. I797; Greenwood, Biol. Centralbl., r $\$ 94$, Bd. XIV, p. 777 .

${ }^{7}$ Darwin, Carnivorotis Plants, 1875 ; Geddes, Article in Encyc. Brit.; Goebel, Pflanzenbiol. Schilderungen, I89I-3, II, p. 53; Kerner, Natural History of Plants, 1896, Vol. I, p. II 8 . Several doubtful examples are given here. On Lathraea, see Scherffel, Bot. Zeitung, I89o, p. 4 I6 ; Heinricher, Ber. d. Bot. Ges., I893, p. 7 ; Haberlandt, Jahrb. f. wiss. Bot., I897, Bd. Xxx, p. 511 ; Goebel, Flora, I897, p. 444. On Polyporus applanatus, MacMillan, Bot. Centralbl., i893, Bd. LIII, p. 322 . 
a great part in the reciprocal relationships of plants and insects. Equally remarkable purposeful adaptations are exhibited among carnivorous fungi ${ }^{1}$,

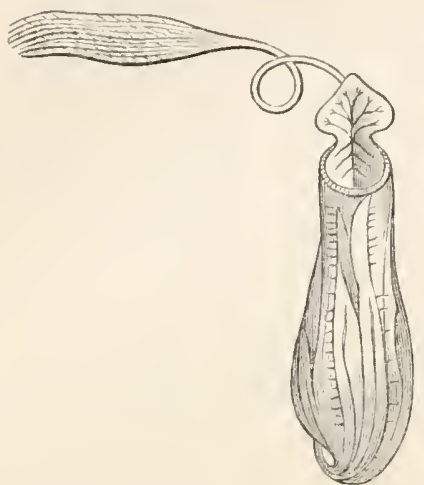

Fig. 56. Pitcher of Nepenthes, drawn to smaller scale.

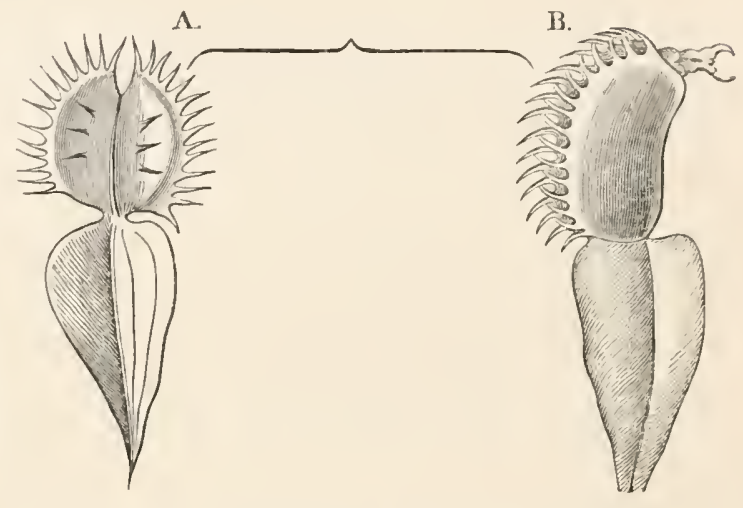

FIG. 57. Leaves of Dionaea muscipula. (A) opened, on each leaf-segment the three irritable hairs may be seen. $(B)$ an earwig is captured between the closed leaf-segments.

while freely motile plants seek out their prey as an animal does, and are frequently attracted to their food by chemotactic stimulation.

In Dionaca the absorption of the products

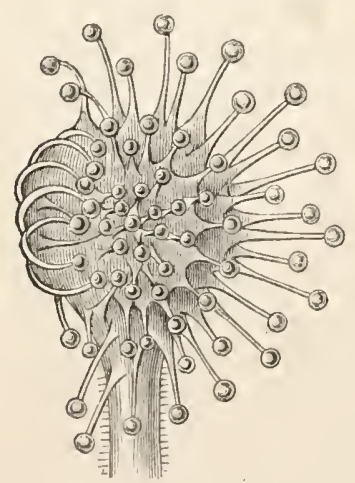

Fig. 58. Leaf of Drosera rotundifolia (magnified). Owing to stimula. tion the hairs on the left side have curved inwards. of digestion by the leaf causes it to wither and die, and certain changes ${ }^{2}$ usually occur in the absorbing glands during digestion. In this way an amount of peptone may be obtained which forms a considerable fraction of the total amount required, for frequently a large number of small insects may be digested, while in the pitchers of Nepenthes large insects may be captured ${ }^{3}$. This carnivorous habit is probably solely for the purpose of obtaining peptone, for sufficient carbon dioxide is assimilated for all requirements. It appears, however, that a supply of organic nitrogen compounds is not an absolute necessity to such plants, although it excrcises an extremely favourable influence upon growth and development, as it does also in the case of many fungi. Schenl was able to cultivate Aldrovanda vesiculosa for two years on inorganic nutrient solutions ; Nematodes.

1 Zopf (Nova Acta d. Leopold. Akad., I888, Bd. LII, p. 321) mentions a fungus which captures

${ }^{2}$ Cf. de Vries, Bot. Zeitung, I 886, p. I ; Goebel, l.c., pp. I 70 , I9\&; also the literature quoted in Sects. II and 16 , on precipitation and aggregation.

${ }^{3}$ Goebel, 1.c., p. I9I ; Haberlandt, Botanische Tropenreise, I893, p. $5^{8}$. 
Fr. Darwin observed that plants of Drosera rotundifolia fed with meat grew more strongly and produced heavier and more numerous seeds; while Büsgen obtained still more marked differences by commencing his comparative experiments with seedlings and feeding a portion of them for two years with aphides ${ }^{1}$.

In the pitchers of Sarracenia, Darlingtonia, Cephalotus, and probably also in the bladders of Utricularia, no enzyme is excreted, but nevertheless soluble nitrogenous products set free by bacterial decomposition may be absorbed. Small captured Crustacea often remain living in the bladders of Utricularia for a long time, and it is possible that a reciprocal relationship exists between the animal and the plant, the excreta of the former being utilized by the latter ${ }^{2}$.

Tischutkin ${ }^{3}$ erroneously states that only bacterial digestion occurs in carnivorous plants, for in Drosera, Drosophyllum, Dionaea, Nepenthes, \&c., there can be no doubt as to the presence of a proteolytic enzyme. This appears to be a pepsin active only in an acid solution, but the nature of the acid is unknown. De Wère has recently shown that Drosophyllum produces no formic acid ${ }^{4}$, nor has the presence of this acid been demonstrated with certainty in Dionaea and Drosera ${ }^{5}$. Further, it is uncertain whether malic and citric acids are present in the pitcher fluid of Nepenthes, either free or in the form of salts ${ }^{6}$. The enzyme and the acid are either excreted continuously or only after stimulation, and in the latter case nitrogenous and other chemical substances act as stimuli, while mere mechanical irritation may cause an increased excretion. In Dionaea a marked excretion of both acid and enzyme follows stimulation; the ferment is always present in the pitcher-fluid of Nepenthes, which becomes acid however only after the application of a chemical stimulus ${ }^{7}$.

Although the fact that certain plants captured insects was known in the

1 Fr. Darwin, Exp. on the nttrition of Drosera, I 878 , Linn. Soc. Journ., Vol. xvir ; also Kellermann u. Raumer, Bot. Zeitung, I878, p. 209 ; Buisgen, ibid., I883, p. 569 .

2 The same may occur in the ampullae of certain hepatics (Jungermannia). Cf. Goebel, 1.c., pp. 185,209 . A few green plants, such as Splachnum, Tetraplodon, only grow upon certain excrements. On Cephalotus: Goebel, 1.c., pp. 87, 17o. Utricularia: Darwin, Insectivorous Plants, I 875 , p. 395 ; Goebel, 1. c., p. I73. Biisgen observed that a supply of insects produced a favourable effect (Ber. d. Bot. Ges., i 888, p. lv).

3 Tischutkin, Bot. Centralbl., I $89^{2}$, Bd. L, p. 304, and I893, Bd. LIII, p. 322. See Goebel, 1. c., pp. I64, I 87 , I9o. On Drosophyllum, cf. de Wèvre, Anı. d. sci. nat., I895, viii. sér., T. I, p. 51. [Nepenthes: Vines, Annals of Botany, Vol. xi, I897, p. 563.]

${ }^{4}$ Goebel, 1. c., p. 193; de Wèvre, l. c., p. 39.

5 Dionaea : v. Gorup Besanez, Ber. d. Chem. Ges., 18 86, p. 673. Cf. Vines, Bot. Jahresb., I 876 , p. 935. Drosera: Will, Bot. Zeitung, 1875, p. 7 I6. According to Stein (Wunschinann, Über d. Gattung Nepenthes, $187^{2}$, p. 25$)$, citric acid is present, but according to Darwin (1. c., 18 76, p. 78 ), propionic acid or a mixture of acetic and butyric acids. Fungi can form a variety of acids (Sects. 85,86 ).

6 Völker's analyses (1849) are quoted by Balfour (Gardener's Chronicle, I875, Ir, p. 67).

${ }^{7}$ Goebel, 1.c., p. 189. On Drosera and Pinguicula, pp. 181, 197; on Drosophyllum, de Wèvre, 1. c. 
last century, Darwin was the first to study the special peculiarities of these plants more closely, and the fact that we are simply dealing with a special mode of nutrition was first clearly indicated by Pfeffer ", although the existence of carnivorous fungi should have sufficed to show that this mode of nutrition is by no means a new development.

\section{SECTION 66. Nutritive Value of Different Carbon-compounds.}

That different carbon-compounds have not the same nutritive value is well known, and this value moreover differs in different cases, so that a substance which forms a good nutritive medium for one plant, may be a poor or imperfect one for another. This is essentially a subject for experimental investigation, but no satisfactory explanation can be given even of the few facts already observed, owing to the incompleteness of our knowledge concerning the inherent nature of the vital mechanism. It is however certain that the various changes which the food-material undergoes must not only yield a supply of energy but must also produce all those substances which are cmployed in growth, for if a single part of the intricate vital mechanism fails, or if a single essential metabolic product cannot be formed, growth and development must always be partially or entirely inhibited. Thus Penicillizm glaucum and many other fungi can grow when sugar is their only source of organic food, because they arc able to construct all the essential forms of proteid from sugar and inorganic nitrogen compounds. When this power is absent development is impossible unless a suitable organic nitrogen-compound is supplied. Many fungi and bacteria require peptone and other proteids, while other species can grow when supplied with asparagin (Sect. 64).

Not only may the absence of a single essential compound finally cause a stoppage of the general metabolism, but the mere disuse of particular functions or organs may induce various disturbances which operate more or less inimically upon the plant. This is always apt to occur under abnormal nutritive conditions, as for example when a chlorophyllous plant is directly fed with sugar, or when an attempt is made to cultivate a parasite in a nutrient solution. Similarly the physiological combustion of particular carbon-compounds may be one of the conditions necessary for the existence of ccrtain organisms, and these may be unable to produce the substances in question even from otherwise suitable organic food-material.

Probably most of the lower plants require two organic food-substances 
at least, as for example is the case in Photobacterium plosphorescens and $P$. Pfiigeri. P. luminosum and P. indicum on the other hand can grow when supplied with peptone only (Beyerinck), as can Penicillium glaucum and many other fungi, the growth of which, however, is markedly favoured by a supply of carbohydrates. Various non-essential substances such as certain of the ash-constitucnts may be assimilated when metabolism is active, while many organic compounds such as oxalic acid ${ }^{1}$ may be utilized, although by themselves they do not form suitable food-material for the plants concerned.

Fungi are especially adapted for researches upon the nutritive value of different organic compounds, and such organisms as nitro-bacteria and sulphur-bacteria, which have very special requirements, may enable some idea to be obtained as to the importance and character of special partial-functions. It is possible that in a particular organism the final stages of metabolism are always of the same character whatever the organic food may be, for a given fungus retains the same characteristics and produces the same living proteids whether it is fed with sugar, asparagin, or other carbon-compounds. When a number of organic substances are at the plant's disposal, it is quite possible that one may be chiefly consumed in respiration, and another used mainly in the synthesis of proteids, but these are by no means to be regarded as specific respiratory, or synthetic material, for under different conditions either may be used for all purposes.

The nutritive necessities differ widely according to the mode of life, so that a substance highly nutritive to one plant may be of little or no use to another even though it is readily absorbed, and hence it is impossible to arrange different food-substances in a linear series of descending or ascending nutritive value which shall apply to all plants. One plant may be able to grow upon a large number of different substances, another upon a few only, but even those plants which can be fed with very simple carbon-compounds have not necessarily a wide range of possible nutrient materials. This appears to be the case, however, with Penicillium glaucum, which is almost completely omnivorous and can develop slowly on formic acid or urea ${ }^{2}$, whereas Bacillus methylicus which nourishes itself with formic acid or methyl-alcohol, and Mycoderma aceti which grows best upon acetic acid and alcohol, have a comparatively restricted range ${ }^{3}$. Similarly nitro-bacteria cannot be cultivated by supplying them

${ }^{1}$ Cf. Wehmer, Bot. Zeitung, I891, p. 533 ; Pfeffer, Jahrb. f. wiss. Bot., I 895 , Bd.xxvill, p. 2 I 2.

${ }^{2}$ Diakonow, Ber. d. Bot. Ges., I $88_{7}$, p. $38_{5}$; Wehmer, Bot. Zeitung, I 891, p. 326 ; Thiele, Die Temperaturgrenzen d. Schimmelpilze, 1896, p. 10.

${ }^{3}$ B. methylicus: Loew, Centralbl. f. Bact., 1892 , Bd. xil, p. ${ }_{4}^{62}$. M. aceti: Beyerinck, ibid., I $89 \mathrm{I}$, p. $29 \mathrm{I}$. 
with organic food, although they are able to assimilate carbon dioxide (Sect. 63), and presumably but few organic substances will enable chlorophyllous plants to continue their life and growth when the assimilation of carbon dioxide is inhibited.

The characteristics of an organism are by no means invariable constants, and it has already been mentioned (Sects. 64, 65) that the nutritive requirements may alter as development progresses. Spores of Aspergillus niger germinate but slowly on glycerine, acetic acid, or alcohol ${ }^{1}$, whereas these substances afford fairly good nutrient material for the adult fungus. Apparently in many plants a supply of proteid material including peptones is of importance or absolutely essential for embryonic development.

The following are additional examples: Bacillus perlibratus will not grow upon tartaric acid, but will upon acetic acid ${ }^{2}$, which for most mould-fungi has the lesser nutritive value. For fungi and many bacteria dextro-rotatory tartaric acid is a better food-material than laevo-rotatory, which is, however, preferred by certain bacteria, whereas other fungi and bacteria again grow equally well upon both of these stereoisomeric compounds (Pfeffer, l. c., p. 220). Fermenting organisms behave similarly; many must have a supply of sugar, whereas others primarily require tartaric or formic acids, or other substances. Both good and bad nutritive substances are found among benzole and methane derivatives, and certain fungi can grow as well upon quinic acid as upon sugar, whereas the nutritive values of stereoisomeric compounds such as tartaric, fumaric and malic acids ${ }^{3}$ may be entirely different. Although good nutritive substances are most abundant among the groups of carbohydrates and proteids, many organisms are unable to grow upon a mixture of peptone and sugar, which is the most suitable medium for most fungi.

The nutritive value and physiological importance of a substance are dependent upon properties to which no attention is paid in chemical classification and of which the constitutional formula affords no indication. The characters of the given plant are of equal importance in determining the result produced, and it is impossible to tell from the result what are the complicated and interacting factors which lead to it. There is no doubt, however, that chemical affinities largely determine the fate of a given substance and whether it shall be used in metabolism or left intact. Just as a nut may be cracked by a blow which is applied at the proper point but is ineffective elsewhere, so also may a substance be decomposed by the protoplast only when the interactions resulting from

1 Duclaux, Ann. d. l'Inst. Pasteur, I889, T. III, p. II2. This is often the case in fungi. Cf. also Bourquelot u. Graziani, Bot. Centralbl., I\$93, Bd. Lv, p. 326.

2 Beyerinck, Centralbl. f. Bact., I 893 , Bd. xIv, p. 8.34

3 Wchmer, Beiträge z. Kenntniss einheim. Pilze, I 895, II, p. 98 . Cf. also Pfeffer, 1. c., p. 228. 
their conjoint properties are such as to render molecular dissociation possible. Fischer ${ }^{1}$ has shown that even in simple hydrolytic decomposition by an enzyme more marked differences may often exist between the behaviour of the optical varieties of the same sugar, than between sugars of different chemical composition.

Nutritive value and chemical constitution. Pasteur first cultivated fungi in solutions of known composition, and this method has been largely employed by Nägeli and Reinke to determine the nutritive value of different carbon-compounds, while at the same time the requirements of certain plants have been more or less accurately indicated ${ }^{2}$. A few works chiefly upon fungi are mentioned beneath, and Flügge has obtained many interesting results with different bacteria ${ }^{3}$. Nägeli paid special attention to the requirements of a few common mould-fungi (Penicillium, Aspergillus), but more recent researches have shown that these results are by no means of general application, and that very marked specific peculiarities exist among different fungi, not only as regards the most appropriate compounds of carbon, but also those of nitrogen (Sect. 70).

Both nutritive and non-nutritive carbon-compounds are found among groups of substances widely differing in constitution and properties from one another. Thus the food-material may comprise many mono- and poly-basic organic acids (acetic, citric, benzoic, gallic acids), alcohols (ethyl alcohol, glycerin, resorcin), esters (fats, ethyl acetate), aldehydes and aldehyde alcohols (glucose, galactose), ketones and ketone acids (fructose, acetone), various nitrogen-containing compounds, amides (ethylamine, tetraethylammonium hydroxide), amido-acids (glycocoll, acetamide), ureides (allantoin, parabanic acid), nitriles (benzene nitrile, methylcyanide).

Hence Nägeli erred in supposing (l.c., p. 4oI) that compounds in which carbon and oxygen were directly associated together could not be assimilated, or that the CH-group could only be used as food when two or more atoms of carbon were united together. Certain plants grow when supplied with formic acid or methyl alcohol, both of which contain one atom of carbon only. According to Reinke the CO-radicle of parabanic acid may be used as a source of carbon, while many lower organisms can obtain carbon from oxalic acid, urea, or even from carbon dioxide by chemosynthetic assimilation. Different plants are able according to their specific properties to assimilate the most varied substances and to produce from

1 E. Fischer, Ber. d. Chem. Ges., I 894 , pp. 2992, 322S, \&c. Cf. Pfeffer, 1. c., p. 24 S.

2 Pasteur, Ann. d. chim. et d. phys., IS6o, iii. sér., T. LviII, p. $3^{2} 3$, and I862, iii. sér., T. Lxıv, p. Io6; Nägeli, Bot. Mitth., Bd. III, Über die Fettbildung niederer Pilze, I 8 ;9; Ernährung d. niederen Pilze, I879; Unters. iiber d. niederen Pilze, I882 ; Reinke, Unters. a. d. Bot. Lab. in Göttingen, ISS3, III, p. II, where a summary of the facts known up to ISS 3 is given.

${ }^{3}$ Elfving, Einwirkung d. Lichtes auf Pilze, IS9o; R. H. Schmidt, Flora, I 891, p. 300 (oil); Wehmer, Bot. Zeitung, IS9I , p. 233 ; Linossier, Centralbl. f. Bact., IS92, Bd. xII, p. I62 ; Beyerinck, ibid., IS92, XI, p. 7o, and I894, Bd. Xvi, p. 57 ; Bruhne, in Zopf's Beiträgen z. Physiol. u. Morph., I894, Heft 4, p. I ; Raciborski, Flora, IS96, Bd. Lxxxir, p. I 8 ; Thiele, Die Temperaturgrenzen d. Schimmelpilze, I 896 ; Laborde, Ann. d. l'Inst. Pasteur, I 897, T. XI, p. I ; Flügge, Mikroorganismen, ISg6, 3. Aufl. 
them similar metabolic products. At the same time a supply of energy is obtained, but this ever present necessity is by no means the sole or even the most important factor in determining the character of metabolism.

The complex organic substances which are primarily liberated by the decomposition of vegetable and animal remains afford an appropriate food supply for many heterotrophic plants, and there is probably not a single organic product of metabolism or decomposition which does not provide more or less suitable foodmaterial for some lowly organism or other. This is, however, not absolutely necessary in the balance of nature, for living plants are able to assimilate or decompose substances (such as oxalic acid, \&c.), which in themselves alone are incapable of affording an adequate food supply.

As regards the commoner mould-fungi (Penicillium glaucum, Aspergillus niger), the nutritive values of different carbon-compounds have the following order when supplied one at a time, and with ammonium nitrate as a source of nitrogen : grape and cane-sugar, peptone, albumen, quinic acid, tartaric and citric acids, asparagin, acetic acid, butyric acid, ethyl alcohol, benzoic acid, propylamine, methylamine, phenol, formic acid. The order is, however, different with other organisms, but in most fungi development is most rapid upon sugar and peptone. These results in all cases refer only to the total amount of growth upon the given solution.

The influence of varying conditions. A particular succession of nutritive values holds good only under given cultural conditions, for a change in the latter may reverse the positions of two or more substances. Thus Thiele ${ }^{1}$ has found that the maximal temperature for the growth of Penicillium glaucum on grape-sugar lies at $3 \mathrm{I}^{\mathrm{C}} \mathrm{C}$, on glycerine at $36^{\circ} \mathrm{C}$, on salts of formic acid at $35^{\circ} \mathrm{C}$. Hence at $33^{\circ} \mathrm{C}$ the feebly nutritive formic acid appears to be a better food-material than sugar. Similar changes are caused by alterations in the concentration of the nutrient solution employed, and in this respect Aspergillus niger behaves in part in a precisely opposite manner to Penicillium.

Under unfavourable conditions no development is possible, even though a supply of the most suitable carbon-compound is assured, and hence it is not always easy to say whether an apparently non-nutritious substance may not under special circumstances serve as nutrient material to a particular plant. In the absence of an appropriate source of nitrogen growth necessarily ceases, while the presence of oxygen renders the development of obligate anaerobes impossible. Similarly many fungi can withstand an acid, and in some cases a strongly acid medium, whereas a comparatively feeble acidity inhibits the development of most bacteria, for these usually require neutral or weakly alkaline media. Again, over-concentration retards growth, while strong poisons cannot be presented in sufficient amount to serve as food-material ${ }^{2}$ owing to the injurious action of even comparatively dilute

1 Thiele, Die Temperaturgrenzen d. Schimmelpilze, ı 896 , pp. Iо, 36.

${ }^{2}$ Example of limits of acidity in Sects. $8_{5}, 86$. See also Nägeli, Die niederen Pilze, I877, p. $3 \mathbf{I}$. On the limits of concentration, see Eschenhagen, Über d. Einfluss v. Lösungen versch. Concentr. auf Schimmelpilze, I891. Cf. also Thiele, 1. c., and Sect. 73. [Penicillium glaucum will grow upon I per cent, solutions of Atropin, Muscarin, and of Eserin sulphate, if supplied with inorganic salts, and also even upon 2 per cent. solutions though much more slowly.] 
solutions. Thus certain fungi develop slowly upon dilute solutions of carbolic acid, quinine, morphia, \&c., and stronger solutions might form good nutrient media were it not for the poisonous influence which they then exercise. The accumulation of excrete products and the changes produced in the nutrient fluid commonly retard further development. All these factors may influence the experimental results obtained, whereas the disturbing effects of competition may be avoided by the use of pure cultures (cf. Sect. $9^{2}$ ).

Apparently trifling circumstances have often a marked influence upon growth and development, and in many plants the commencement of the latter is due to the action of specific chemical stimuli (Sect. 64). Further, as Raulin found, a small dose of zinc or manganese produces an increased crop of fungus, and according to Richards, the same effect is given by almost any poison, although, as might be expected, with stronger doses growth is retarded '. This reaction to stimulation by accelerated growth is apparently due to an attempt on the part of the plant to counteract an injurious influence as far as possible by an increased vital activity, and similar phenomena may follow mechanical injury ${ }^{2}$.

Economic coefficient. Hitherto the nutritive value of a carbon-compound has been estimated by the rapidity of development, but this affords no criterion as to the economic coefficient, that is, the weight which is produced by a consumption of Ioo parts of the nutrient material ${ }^{3}$. This economic coefficient, like the respiratory quotient, is variable, and when the temperature is raised above the optimum for growth a larger amount of food-material is consumed in the increased respiratory activity without any equivalent growth (Sect. 95). Under otherwise similar conditions the economic coefficient is smaller for an innutritious carbon-compound involving slower growth, than for a more suitable one. If, however, when two substances are jointly supplied, one of them is consumed mainly in respiration, the economic coefficient of the other will be correspondingly increased. The value of a substance is not dependent simply upon the amount of energy which may be derived from its combustion, nor is there any direct connexion between the nutritive value of a substance and the store of potential energy which it contains.

Methods. A general account of the mode of cultivation, as well as of the precautions necessary to obtain pure cultures, is given by Hueppe ${ }^{4}$. The cultures are best performed in small flasks containing 20-200 cc. of sterilized nutrient fluid, the mouths of the flasks being closed by plugs of cotton wool. The medium must be neutral or feebly alkaline for most bacteria, and kept in this condition if necessary by the addition of sodium carbonate, or of chalk, whereas cultures of fungi should be made feebly acid by means of phosphoric acid.

If no organic nitrogen-compounds are necessary, the addition of 0.5 to 2 per cent. of one of the saline mixtures mentioned in Sect. 73 , together with 2 to 5 per cent.

1 Raulin, Ann. d. sci. nat., IS69, v. sér., T. xi, p. 252 ; Pfeffer, Jahrb. f. wiss. Bot., I895, Bd. xxvil, p. 238 ; Richards, ibid., I 897 , Bd. xxx, p. 665.

${ }^{2}$ Cf. Pfeffer, l. c., p. ${ }^{2} 3^{8}$. Cf. also Sect. 92, and on increased respiration Sect. I04.

${ }^{3}$ See Pfeffer, 1.c., p. 257 ; also Kunstmann, Über das Verhältniss zwischen Pilzernte u. verbrauchter Nahrung, $1 \mathrm{~S}_{95}$.

* Ineppe, Die Methoden d. Bacterienforschung, I89I, 5. Aufl., \&c. On anaerobes, cf. Sect. 98. PFEFFER C C 
of grape or cane-sugar yields a very suitable nutrient fluid, or the inorganic salts may perferably be supplied by dissolving $\mathrm{I}$ grm. $\mathrm{NH}_{4} \mathrm{NO}_{3}, 0.5$ grm. $\mathrm{KH}_{2} \mathrm{PO}_{4}$, $0.25 \mathrm{grm} . \mathrm{MgSO}_{4}$ in $200 \mathrm{cc}$. of water. Under certain conditions it is better to use this solution either half diluted or twice concentrated, while a trace of iron should be added, and for comparative researches always precisely the same amount. An addition of $0.2 \mathrm{grm} . \mathrm{Ca} \mathrm{Cl}_{2}$ is to be recommended though not absolutely necessary. To supply nitrogen in organic form I grm. of asparagin or I to $3 \mathrm{grm}$. peptone must replace the ammonium nitrate.

Of the different saline solutions which Nägeli employed the following may be mentioned: (I) $100 \mathrm{grm} . \mathrm{H}_{2} \mathrm{O}$, I grm. $\left(\mathrm{NH}_{4}\right)_{2} \mathrm{C}_{4} \mathrm{H}_{4} \mathrm{O}_{6}$, O. I grm. $\mathrm{K}_{2} \mathrm{HPO}_{4}, 0.02 \mathrm{grm}$. $\mathrm{MgSO}$, 0.0I grm. $\mathrm{CaCl}_{2} ;(2)$ roo grm. $\mathrm{H}_{2} \mathrm{O}$, I grm. peptone, 0.2 grm. $\mathrm{K}_{2} \mathrm{HPO}_{4}$, $0.04 \mathrm{grm} . \mathrm{Mg} \mathrm{SO}_{3}, 0.02 \mathrm{grm}$. $\mathrm{Ca} \mathrm{Cl}_{2}{ }^{1}{ }^{1}$ In place of these mixtures about $0.4 \mathrm{grm}$. of the ashes of peas or wheat grains may be used, after neutralizating with nitric acid and adding an ammonium salt.

The nutrient solution first employed by Pasteur ${ }^{2}$ contained r 00 grms. water, Io grms. cane-sugar, $0.1 \mathrm{grm}$. ammonium tartrate, and the ashes of I grm. of yeast, and frequently fresh ammonium tartrate was subsequently added. All these nutrient solutions are also adapted for the culture of bacteria, and for those which require peptone a suitable nutrient solution must contain 3 per cent. of peptone, 2 per cent. of cane or grape-sugar, and I per cent. of meat extract.

The optimal concentration of grape-sugar is reached when 5 to 15 per cent. is present, but the growth of many fungi is suppressed only when the concentration reaches as much as 50 to 60 per cent. ${ }^{3}$ The same result is produced by an isosmotic concentration of any other non-poisonous substance, and the optimal concentration is isosmotic with 5 to 15 per cent. of grape-sugar.

The works already quoted give a full account of the mode of cultivation upon gelatine, agar, gelatinous silicic acid (Sect. $6_{3}$ ) or other solid nutrient media, and the use of solid media is of especial importance for the isolation and detection of bacteria as well as for the preservation of pure cultures. Bacterial germs develop in non-nutrient gelatine, \&c., only where a drop of nutrient fluid is added and as far as it diffuses, so that by means of this auxanographic method of Beyerinck's ${ }^{4}$, the nutritive value of a given organic or inorganic substance can readily be demonstrated, and not only can the action of different substances be noticed upon the same plate, but also their conjoint influence can be observed where the diffusion zones of the different drops meet.

1 Other data are given by Nägeli, l.c.; Hueppe, l.c., p. $23^{8}$; Zopf, Die Pilze, 1890, p. 172; W'ehmer, Bot. Zeitung, I891, p. 272 ; Pfeffer, l.c., \&c.

${ }_{2}^{2}$ Pasteur, Ann, d. chim. et d. phys., i 860 , iii. sér., T. Lvili, p. 383 ; 1862, T. LXIV, p. 106.

` Eschenhagen, Über den Ëinfluss von Lösungen versch. Concentr. auf Schimmelpilze, I889, p. 55 ; Bruhne, in Zopf's Beiträgen z. Physiol. u. Morph., 189.t, Ifeft t, p. 15; Klebs, Beding. d. Fortpflanzung, 1896, p. $4^{6} 5$.

Beyerinck, Bot. Zeitung, I 890, p. 201 . 


\section{Section 67. Special Selective Power.}

Each plant has a specific preference for particular compounds of carbon, nitrogen, \&c., and hence when a varicty of carbon-compounds are presented to it, the plant consumes certain of them to a greater extent than others and may entirely neglect some. Thus when supplied with 6 per cent. of dextrose and I per cent. of glycerine, Aspergillus niger leaves the latter almost intact, whereas the greatest abundance of glycerine does not suffice to protect cven a small trace of dextrose from consumption. Similarly glycerine is totally or partially protected by peptone, and butyric acid by dextrose, whereas even when but little acetic acid and a large quantity of dextrose are present the former is consumed in relatively greater amount, although like glycerine it forms by itself a poor nutrient substance. However much acetic acid may be present, large quantities of dextrose are always consumed and the total supply is ultimately absorbed, which is a sufficient indication of the feeble nutrient value of acetic acid.

Stereoisomeric bodies may possess very unequal nutritive values: Pastcur observed that Penicillium glaucum and Aspergillus niger are able to split neutrai tartaric acid into its positive and negative optical varieties, and that more of the optically positive tartaric acid is absorbed than of the optically negative. The reverse is the case with a bacterium which develops best upon the optically negative tartaric acid, and when both varieties have the-same nutritive value for a given organism no such decomposition occurs (Asporgillus fumigatus, Bacillus subtilis, \&c.). Similar experiments upon other stereoisomeric substances are given by Pfeffer ${ }^{1}$.

It is only to be expected that the result obtained should vary according to the plant and to the nutrient mixture employed, but the causes of the differences observed can only be ascertained when a complete knowledge has been gained of all the different factors concerned. It is however possible that with a mixture of dextrose and acetic acid the latter is assimilated, because it satisfies certain of the plant's requirements more readily than does the better nutrient substance: thus certain fungi are able to oxidize oxalic acid energetically, although the energy thus obtained apparently cannot be utilized for growth. Hence the importance of a substance to a plant is not to be measured solely by the amount of growth which it induces, nor does it depend on its chemical and mechanical properties, for a given substance may, in virtue of its special qualities and relative amount,

1 Pasteur, Compt. rend., i 858 , T. XLvi, p. 617 , and i860, T. LI, p. 298; Pfeffer, Jahrb. f. wiss. Bot., 1895 , Bd. xxvil, p. 206 . Full details and a summary of the literature are given here. 
render possible vital manifestations of which the plant was previously incapable, or suppress others which are no longer necessary. Thus the presence of sufficient sugar suppresses the formation of diastase in many fungi, and it is probably owing to the inhibitory action of the other foodmaterials that under certain circumstances glycerine and both forms of tartaric acid are not assimilated although they are always absorbed.

It is by these and similar means that the whole series of processes which constitute metabolism are linked together in a regulatory manner, and in the interactions between cells and organs selective affinity and protective power play a very important part. Thus in time of abundance the stored nutricnt matcrials remain intact, but are translocated and consumed as soon as required, while starvation may cause the re-assimilation of metabolic products which under normal conditions remain permanently withdrawn from metabolism. Fungi afford eminently suitable cxperimental material for the investigation of these fundamental problems, owing to the fact that they can be grown upon a great variety of nutrient media and under widely different conditions, while at the same time the "consumption and the resulting growth can readily be determined.

\section{PART IV}

\section{THE ASSIMILATION OF NITROGEN}

\section{SECTION 68. General View.}

EVERY plant must be supplied with nitrogen in some form or other, for besides the proteid substances which constitute the main bulk of the protoplast, many other organic nitrogen compounds are present as normal plant constituents. Nitrogen, like carbon, is an essential element, and although the latter is more abundant, still more nitrogen is present than of any of the essential elements found in the ash. In the highly nitrogenous seeds of Leguminosae 4-9 per cent. of the dry weight is composed of nitrogen, in the seeds of cercals $2-3$ per cent., in bulbs, vegetables, and leaves, from $\mathrm{I} \cdot 5^{-6}$ per cent., so that in a turgid plant from 0.3 to $\mathrm{I} \cdot \mathbf{2}$ per cent. by weight of nitrogen may be present ${ }^{1}$.

1 Numerous analyses by König, Chem. Zus.-setzung. d. menschl. Nahr.- u. Genussmittel, I889, 3. Auf. Cf. also Sects. II and 79. For analyses of bacteria and fungi, see Cramer, Archiv $f$. Hygiene, I893, Bd. xvi, p. 15I ; Nischimura, ibid., 1893, Bd. xvirI, p. 318. According to Fermi (Centralbl. f. Bact., Atth. ii, I896, Bd. II, p. $5^{12}$ ), various micro-organisms, including Saccharomycetes and mould fungi, may be grown upon non-nitrogenous media, and then contain no nitrogen, so that this element is apparently non-essential in such cases (cf. Sect. II). The statement is, however, one which requires further proof. 
With the exception of those plants which are able to assimilate free nitrogen, a supply of a suitable nitrogen-compound is in all cases necessary, although the specific requirements of different plants vary widely. Thus most higher plants and many lower ones prefer inorganic compounds of nitrogen such as nitrates or ammonium salts, whereas many saprophytes and parasites either must be supplied with proteids, or develop best when nitrogen is supplied in the form of organic compounds such as peptones, amides, \&c. Many fungi, however, grow normally when ammonium nitrate affords the sole source of nitrogen, provided the nutrient fluid contains the essential inorganic salts together with sugar or glycerine. Such fungi (Ponicillium glaucum, Aspergillus, \&c.) form proteids by synthesis, and as growth progresses the total amount of proteid substance continually increases. Most chlorophyllous plants have the same power, and since the sugar is produced by photosynthetic assimilation, nothing more than a supply of inorganic salts, including nitrates, sulphates, and phosphates, is necessary to render the construction of proteid possible.

All grades of transition exist between organisms which require peptone and those which can obtain all their nitrogen in the form of inorganic salts, and hence the nutritive values of a series of nitrogen-compounds differ according to the plant examined. When a plant is fed solely with peptone or asparagin the same substance must serve as a source both of nitrogen and of carbon. A certain amount of energy can always be obtained by the oxidation of organic nitrogen-compounds, and nitrate-bacteria obtain the whole of their energy from the oxidation of ammonia or of nitrites, i. e. from compounds which at the same time afford part of the material for the synthesis of proteid (Sect. 63).

The fact that a plant requires a supply of nitrogen-compounds shows that it is unable to assimilate the free nitrogen of the air which is present in dissolved form in every living cell. Certain soil-bacteria and Leguminosae with root-nodules are, however, able to assimilate free nitrogen, and the fact that various transition forms are known, renders it possible that other plants may develop a feeble power of assimilating free nitrogen under special circumstances (Sect. 69).

The assimilation of free nitrogen by the bacteria in question is not dependent upon light or upon the presence of chlorophyll. This is here also the case of the assimilation of nitrogen-compounds and the synthesis of proteids, as is shown by the fact that fungi are able to complete their life-cycle in darkness. On the other hand, the growth of green algae in inorganic nutrient solutions exposed to light $^{1}$ shows that chlorophyllous cells can alsc synthesize proteids, and no doubt the non-chlorophyllous cells of green plants are also capable of constructing them. This is rendered 
possible by the photosynthetic production of sugar, and light appears also to exercise a direct favourable influence upon the assimilation of nitrogen compounds especially in the higher plants (Sect. 72), although it does not follow that it directly affords the energy for the synthesis of proteids. Nor is the energy required necessarily obtained by oxygen-respiration, for many anaerobic bacteria can construct proteids from inorganic nitrogen compounds or even from free nitrogen (Sect. 69).

The exact mode in which the assimilation of nitrogen or nitrogencompounds takes place is as yet unknown (Sect. 7I), but it is certain that the synthesized amides and proteids are usually immediately reassimilated, and often undergo marked disintegration. Indeed it is probable that in plants, as well as in animals, the decomposition and reconstruction of proteid continue without cessation and are necessary accompaniments of vital activity. Many fungi seem to possess a pronounced power of decomposing proteids, amides, \&c., and swen fed with albumin often produce large quantities of ammonia as the result of their metabolic activity ${ }^{1}$. Certain bacteria actually evolve free nitrogen, and many ferment-organisms may give off large quantities of this gas and of nitrous oxide ${ }^{2}$. The metabolism of such organisms as these involves a continuous loss of nitrogen, whereas in most plants, and especially in Phanerogams, the nitrogen when once assimilated is husbanded with the utmost care, so that almost the whole of it is preserved, however active metabolism and growth may be ${ }^{3}$. This is rendered possible by the fact that the nitrogenous metabolic products, like those containing sulphur and phosphorus, are such as can be drawn into metabolism again and are not aplastic ones intended to remain as permanent constituents of the plant. In spite of all these economic adaptations a certain quantity of nitrogen is lost by the unavoidable leaf-fall, but even when the natural course of metabolism involves a certain loss of nitrogen it is safe to assume that the plant works as economically as the internal and external conditions allow. Moreover in certain cases excreted nitrogenous products are of marked service to the plant in the form of enzymes or of certain odoriferous substances.

A volatile nitrogenous compound is evolved by Chenopodium vulvaria and by flowers of Crataegus oxyacantha in such abundance that a glass rod dipped

1 Cf. Wehmer, Bot. Zeitung, I S91, p. 295, and Sect. 102.

${ }^{2}$ No production of free nitrogen occurs in higher plants, for the observations of Boussingault (Ann. d. chim. et d. phys., I 881 , v. sér., T. XxII, p. 433) and others are probably due to faulty methods. Cf. Frank, Unters. über d. Emährung mit Stickstoff, 1S88, p. 25 (Sep.-abdr. a. d. Landw. Jahrb.). Of more recent works, see Aubert, Rev. gén. d. Bot., IS92, T. IV, p. 2So, and Schloesing, Compt. rend., I895, T. cxx, p. I 278.

${ }^{3}$ For examples, sce Schröder, Versuchsst., 1868, 13d. x, p. 493 ; Karsten, ibid., 1870, Bd. XIII, p. I76; Sachsse, Keimung v. Pisum, I 872 ; Detmer, Physiol.-chem. Unters. über Keimung, 1875 , p. 68; Schulze u. Urich, Landw. Jahrb., 1876, Bd. v, p. S21 (Lupinus); Frank, 1. c., I8S8, p. 25. 
in hydrochloric acid gives off fumes when introduced into a bell-jar in which the plants have been kept. This substance, according to Wicke, is Trimethylamine ${ }^{1}$ in the case of Chenopodium. Mould-fungi when grown in an alkaline solution excrete ammonia in abundance ${ }^{2}$, and the same thing commonly occurs during putrefaction, while the erolution of ammonia noticed by certain authors from germinating seeds and from agarics was apparently due to the decomposition of dead parts ${ }^{3}$. Löseke states that Agaricus oreades evolves prussic acid but apparently only after death, and the same thing occurs on the death of plants containing amygdalin, such as Pangium edule ${ }^{4}$.

Plants can continue to grow slowly for a time and produce new leaves, shoots, \&c., even when the supply of nitrogen is deficient or ceases, for the younger organs are able to withdraw a certain amount of combined nitrogen from the older ones ${ }^{5}$. Boussingault found that a plant of Hclianthus argophyllus increased its dry weight 4.6 times without being supplied with nitrogen; but a similar plant supplied with potassium nitrate acquired a dry weight I88 times greater than that of the seed. Even a small supply of nitrogen causes a marked increase in the dry weight. It is very difficult to keep a nutrient solution free from all combined nitrogen, and a fungus is able to extract every trace present in the culture fluid, and can also utilize any traces of ammonia conveyed to it by the air.

When all the other conditions are normal, but only a minimum amount of combined nitrogen is present, any addition of the latter will produce a correspondingly increased growth, and a certain proportionality has often been observed between the two ${ }^{6}$, although it is not surprising that the relationship is not always a direct one, and that in some cases none can be observed.

The circulation of nitrogen. It has already been shown how the specific peculiarities of different organisms are such as to maintain a continual circulation of nitrogen, and to ensure the maintenance of an approximate balance in the organic world between the loss and gain of combined nitrogen (cf. Sect. 51 ).

1 Wicke, Bot. Zeitung, I862, p. 393 ; Chevalier, Ann. d. sci. nat., I S24, T. 1, p. 444.

${ }^{2}$ According to Reinke (Unters, a. d. Bot. Lab. in Göttingen, I S81, Heft 2, p. 9) Aethalium septicum produces ammonia.

3 Cf. Wolff u. Zimmermann, Bot. Zeitung, i 87 I, p. 28 . Seeds: Hosaeus, Jahresb. d. Agr.Chem., I 867 , p. I00; Rauwenhoff, Linnaea, I $859-60$, Bd. xxx, p. 2 I9. M. Schultz (Journ. f. prakt. Chcm., I862, Bd. Lxxxvil, p. 129 ) states that nitrogen is also formed.

4 Agaricus: Löseke, Chem. Centralbl., x87, p. 520. Panginm: Treub, Ann. d. jard. bot. d. Buitenzorg, i 895, T. XII I, p. I.

${ }^{5}$ Cf. Hellriegel, Unters. uiber d. Stickstoffnahrung, x $888, \mathrm{pp}$. I9, I74. The same occurs when the supply of phosphorus is scanty.

6 Boussingault, Agronomie, \&c., I 860, T. 1, p. 233 ; Hellriegel, Jahresb. d. Agr.-Chem., I 868-9, p. 247, and Unters. über d. Stickstoffnahrung, i 888 , pp. 55, \&cc. ; Ritthausen, Versuchsst., I 873 , Hd. xvi, p. $3 \delta_{4}$; Fittbogen, Landw. Jahrb., I 874 , p. I 46 ; E. Wolff, Versuchsst., i 877, Bd. x. p. 395 . 
Animals and such plants as are unable to synthesize proteids from simple nitrogencompounds are dependent for their supply of combined nitrogen upon those plants which are able to assimilate such ultimate products of decomposition as nitric acid or ammonia. Both plants and animals decompose large quantities of proteids, and the organisms which grow upon animal and vegetable remains produce simpler nitrogen-compounds, including ammonia: this again may serve as a source of energy to nitrite and nitrate bacteria, which co-operate to produce nitrates in a well aërated soil ${ }^{1}$. The green plants which grow upon such soils as a rule prefer their nitrogen in the form of nitrates, and these are continually produced in small amount by the nitrifying bacteria, so that the danger of loss by the solvent action of water upon the soil is largely avoided. Most nitrates are highly soluble, and are moreover not retained by humus, and hence any large accumulation of nitrates may be almost entirely washed away by the first heavy shower of rain. Where the rainfall is slight or absent, however, the decomposition of masses of organic material may result in the production of large deposits of nitrates, as in the nitrate beds of South America.

Certain micro-organisms decompose nitrogen-compounds with a liberation of free nitrogen (Sect. 102), and the latter may also be liberated by special chemical actions. Hence a fixation of nitrogen must occur if the balance of nature is to be maintained, and certain plants are actually able to assimilate free nitrogen. This is perhaps the most important means by which nitrogen is brought into circulation again in the organic world, but other processes leading to the same end are also continually active ${ }^{2}$, such, for example, as the formation of oxides of nitrogen by electrical discharges passing through moist air during thunderstorms, or even by the passage of weak electric currents through the soil. Schönbein erroneously supposed that the evaporation of water induces the combination of nitrogen and oxygen, but it is not impossible that other natural agencies may produce this effect, for in the process of combustion or directly by the aid of heat, nitrogen may be caused to enter into combination with other elements, as takes place for example when phosphorus slowly oxidizes in contact with air and induces a production of oxides of nitrogen and ultimately of nitric acid. During volcanic outbursts, a fixation of nitrogen may occur in some such manner, and it is possible that the chemist may ultimately succeed in producing nitrogencompounds from the air with such readiness as to form a cheap source of artificial manure ${ }^{3}$.

The removal of large quantities of nitrogen from the soil by the annual harvest sooner or later causes the crops to become enfeebled, unless the loss is

${ }^{1}$ Cf. Sect. 63. Schlösing and Muntz (1877) first showed that nitrification was due to the action of micro-organisms, and all the more recent researches have confirmed their discovery. See Sachsse, Agr.-Chem., I88s, p. I39; Wortmann, Landw. Jahrb, I89I, Bd. xx, p. I75; Pitsch, Versuchsst., 1893 , Bd. xL11, p. $8_{7}$; Burri, Centralbl. f. Bact., I 895 , Abth. ii, Bd. I, p. 22.

${ }^{2}$ Literature on fixation of nitrogen : E. Schulze, Landw. Jahrb., I877, Bd.vi, p. 695 ; Baumann, Versuchsst., $188 \mathrm{~S}$, Bd. xxxv, p. 243; Frank, Unters. über d. Ernährung d. Pflanze mit Stickstoff, 188s, p. 66 (Sep.-abdr. a. Landw. Jahrb.); Ilsova, Ber. d. Chem. Ges., I 890 , ref. p. 85 ; Loew, ibid., I 890, p. I 443 ; F. v. Lepel, ibid., I 897 , p. 1027.

3 See Report Brit. Assoc. I 89 S (Presidential Address). 
made good by manuring. Boussingault calculated the annual amount removed in Alsace by a single harvest to be approximately equal to 51 kilogrammes of nitrogen per hectare ( $2 \frac{1}{2}$ acres), whereas only $2-30$ kilos. are annually brought to the soil by rain, dew, and in the form of volatile ammonia ${ }^{1}$. It appears, moreover, that the activity of the nitrogen-fixing bacteria of the soil is insufficient to compensate for this deficit. Lupins, peas, and other Leguminosae, are, however, able by means of their symbiotic union with root-tubercle bacteria to assimilate atmospheric nitrogen in such abundance as to satisfy all their nitrogenous requirements (Sect. 69), and hence by ploughing in a crop of such plants (green manuring) the percentage of combined nitrogen present in the soil may be increased. These plants attain the upper hand in soils deficient in nitrogen, and thus prepare the way for other plants which require a supply of combined nitrogen. In their absence, whether the soil loses or gains nitrogen depends upon circumstances, for the loss by drainage and by the exhalation of ammonia, \&c. may or may not be counterbalanced by the gain from rain, dew, and dust. A sterilized and protected soil loses a little nitrogen by erolving ammonia, whereas when suitable bacteria are present the amount of fixed nitrogen may increase.

\section{SECTION 69. Assimilation of free Nitrogen.}

The fixation of free nitrogen is due to the activity of certain microorganisms, some of which grow chicfly in symbiotic union with the roots of Leguminosac, while others are capable of independent existence. One of the latter forms is the Clostridium Pasteurianum discovered by Winogradsky ${ }^{2}$. It is an anaerobic bacterium, which when fed with sugar forms butyric and acetic acids as well as hydrogen and carbon dioxide, while at the same time it assimilates nitrogen in such abundance that no addition of nitrogen compounds is necessary. Clostridium Pastcurianum apparently always occurs in nature in symbiotic association with two other bacteria, from which it can only with difficulty be isolated, for all three forms can develop together in a medium free from nitrogen. When isolated, Clostridium Pastcurianum is an obligate anacrobe, but in this symbiotic union it can grow in an ordinary culture fluid in the presence of oxygen, and hence no doubt can also fix nitrogen in an ordinary aërated soil, for it appears to have the same power of assimilating free nitrogen when in symbiotic union with these accompanying bacteria, as when it is isolated. It has, however, still to be determined whether these other bacteria simply act as a protection against oxygen, or whether they exert other influences as well.

${ }^{1}$ Cf. A. Meyer, Lehrb. d. Agr.-Chem., 1895, 4. Auf., p. 193; Sachsse, Agr.-Chem., Isss, Pp. $7 S, 5^{S 6 .}$

2 Winogradsky, I, Compt. rend., I $8_{93}$, T. cxv1, P. $138_{5}$; II, ibid., I $\delta_{94}$, T. Cxv111, P. 353 ; III, Archiv, d. sci. biol. d. l'Inst. d. méd. exp., St.-Pétersbourg, I 895 , T. Il, p. 297. 
Negative results do not necessarily prove that free nitrogen cannot be assimilated in a given plant under any circumstances, for potential powers may lie dormant which are exercised only under special conditions. Isolated root-tubercle bacteria assimilate free nitrogen feebly or not at all, although under other conditions or in their normal habitat this power may be strongly developed. The root-tubercle bacteria are aerobic both when isolated and when present in the root-tubercles (Sect. 70), and are probably supplied with oxygen by the cells of the latter. It is possible morcover that aerobic forms capable of assimilating free nitrogen may exist in the soil.

It is also extremely probable that this power is possessed by different organisms in different degrees, and that we may pass by stages of transition to plants which are entirely or almost entirely devoid of this property. Winogradsky has actually isolated from soil two other bacteria, which feebly assimilate free nitrogen, but only when the presence of a small trace of a suitable nitrogen-compound renders their growth and development possible. P'uriewitsch ${ }^{1}$ finds the same to be the case with Penicillium glaucum and Aspergillus niger, but no such power has as yet been detected in any of the algac, or in any of the higher plants.

None of these plants seem to require free nitrogen, nor apparently do the root-tubercle bacteria, whereas C. Pasteurianum, according to Winogradsky, is only able to exist when supplied with free nitrogen and thus cnabled to exercise its normal nitrogen-assimilating activity. This bacterium is unable to grow in bouillon even in the presence of free nitrogen; the presence of a little ammonium sulphate does not retard its growth, indeed a portion of the nitrogen it requires may be obtained from this source. Again, an abundant supply of nitrates causes the assimilation of free nitrogen by leguminous plants to decrease to a very marked cxtent.

Winogradsky was unable to isolate any other bacterium than $C$. Pasteurianum with an active power of assimilating nitrogen, and he has shown that the bacteria isolated by Berthelot upon nutrient gelatine were incapable of any such fixation. It is, however, possible, that other nitrogen-assimilating bacteria may exist, but that they are unable to develop under the cultural conditions employed, so that C. Pastcurianum is not necessarily the only micro-organism which enriches the soil with

1 Jicr. d. Bot. Ges., $18_{95}$, p. 343 . Frank (Bot. Zeitung, $18_{93}$, p. $\mathbf{I}_{4} 6$; Landw. Jahrb., $18_{92}$, Bd. xxi, p. 6) and Berthelot (Ann. d. chim. et d. phys., 1893, vi. sér., T. xxx, p. 427) state that Penicillium and a few other fungi assimilate free nitrogen. Sestini and del Torre (Versuchsst., 1876 , Bd. XrX, p. 8) and Jodin (Compt. rend., 1862, T. LV, p. 612) obtained similar results at an earlier date, but these latter researches are inconclusive, and objections may be made even to the ones first mentioned. Winogradsky (III, $\mathbf{1} 895$, l. c., p. 350) could detect no power of fixing nitrogen in an Aspergillus he examined. 
nitrogen. Berthelot ${ }^{1}$ was the first to show that no such enrichment is possible when the soil is sterilized, and consequently that the process is due to the activity of micro-organisms. Soil containing bactcria or bacteria and algae may gain 10 or even 25 milligrammes of nitrogen per 100 grammes in a comparatively short time ${ }^{2}$, so that assuming the process continues with equal energy to a certain depth, as much as 30 kilos of nitrogen may be gained annually by each hectare $\left(2 \frac{1}{2} \text { acres }\right)^{3}$. The conditions are more favourable when small quantities of soil are experimented with than when they are performed in the open air. Direct observations show that without the help of Leguminosae no such marked gain of nitrogen is possible, although a certain amount of fixation usually occurs even when these plants are absent. It is in all cases possible, however, that nitrogenproducing bacteria may obtain the upper hand so that a loss instead of a gain of nitrogen occurs (cf. Tacke, 1. c.).

Winogradsky found that C. Pasteurianum consumed I, 000 grammes of sugar in fixing $\mathrm{I} \cdot 5$ to $3 \mathrm{mg}$. of nitrogen, but it is not impossible that other bacteria or these same forms may work more economically in the soil. It appears that a supply of some carbon-compound as food-material is always necessary, and Berthelot ${ }^{4}$ and Kossowitsch have shown that the addition of sugar causes an increased fixation of nitrogen in the soil. Under normal conditions this food is supplied by other organisms, which in sterilized sand watered with nutrient salts may consist of algae only. The algae obtain in return supplies of combined nitrogen which the soil lacks, and it is possible that in such cases the algae and bacteria live in intimate contact-symbiosis. It has yet to be determined whether the bacteria enter into similar contact-symbiosis with living roots, or whether they obtain organic food supplies simply by the disintegration of dead roots and root-tissues.

However this may be, the result is the same, the nitrogenous compounds which are produced ultimately becoming available for all plants growing in the soil in question, and in the absence of root-tubercle bacteria this is the only way in which the higher plants can obtain nitrogen when grown in a non-nitrogenous soil.

Frank observed under such conditions that many plants without roottubercles gained a certain amount of nitrogen, and erroneously concluded that all plants were able to assimilate free nitrogen. Frank did not employ a sterile

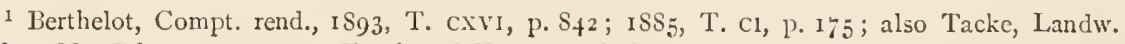
Jahrb., IS89, Bd. xi111, p. 453; Frank and Kossowitsch, 1.c.

2 Tacke, Landw. Jahrb., ISS9, Bd. XviII, p. 453, and the literature here given; Kossowitsch, Bot. Zeitung, I $S_{94}$, p. II 2.

3 Cf. Sachsse, Agr.-Chem., ISS3, p. 5 SS.

t Berthelot, Compt. rend., is 93 , 'T. Cxvi, p. $8_{4} 2$. 
soil, and frequently observed the appearance of algae in it. Petermann has, however, recently shown that barley is unable to fix any nitrogen in a sterile soil, although in carlier researches he found an energetic fixation of nitrogen occurred when the plants were grown in soil containing algae and bacteria.

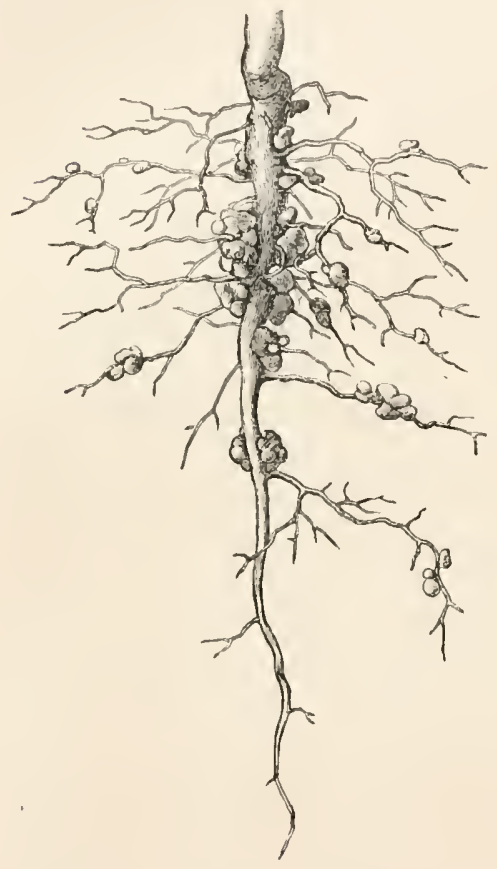

FIG. 59. Root-tubercles of Lupinus uteus ( $\frac{1}{2}$ nat. size).

According to Schlösing and Laurent the simple suppression of the algae suffices to prevent any apparent fixation of nitrogen by oats, mustard and cress, and Aeby, Pfeiffer, and Franke, all conclude that hemp plants are unable themselves to fix free nitrogen. These results all agree with Hellriegel's conclusion that only Phanerogams bearing root-tubercles are able to assimilate free nitrogen. Whether certain algae have the same power when isolated must remain at present an open question, for entirely negative results have hitherto been obtained ', and the importance of algae for the fixation of nitrogen in the soil may be simply due to their symbiotic relationship to the abovementioned bacteria ${ }^{2}$.

Leguminosac. Hellriegel's ${ }^{3}$ researches first established the fact that certain Leguminosac are able to assimilate free nitrogen, and proved that this power is due to the presence of roottubercles formed by infection witlı microorganisms from the soil. These latter are specific in nature (root-tubcrcle bacteria, Bacillus radicicola, Beyerinck; Rhizobium legunninosarum, Frank). 'Similar root-tubercles may be formed in the roots of the alder ${ }^{4}$, and, according to Nobbc ${ }^{5}$, Eleaguns and

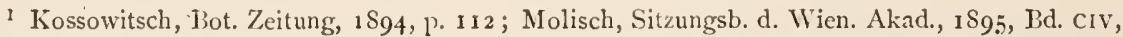
Abth. i, p. 793. Frank (Bot. Zeitung, I 893 , p. 146) supposes that algae can also fix nitrogen.

- ${ }^{2}$ Frank, I, Bot. Zeitung, 1893, p. 139; II, Unters. über d. Ernährung d. Pflanze mit Stickstoff, is88; IlI, Pilzsymbiose d. Legruminosen, I 890 ; IV, Die Assimilation des freien Stickstoffs, I 892 (Sep.-abdr. a. Landw. Jahrb.). Cf. also Frank, Lehrb. d. Bot., 1892, p. $5^{6} 3$; Petermann, Bull. d. l'Acad. roy. d. Belgique, 1893 , iii. sér., T. xxv, p. 267 ; Rech. d. chim. et d. physiol., 1894 , T. II, p. 265 ; ibid., I $88_{9}$ and 1890 ; Bouilhac, Compt. rend., I 896 , T. cxxinl, p. 828 ; Schlösing et Laurent, Ann. d. I'Inst. Pasteur, $189^{2}$, T. vi, pp. II 5 , 827 ; Aeby, Versuchsst., 1896 , Bd. XLI, p. 438 ; Pfciffer und Franke, ibid., p. 117 , and 1887 , IBd. XLvirl, p. $4 \mathrm{I} 8$, where Liebscher's view concerning mustard plants is given; Hellriegel, Unters. ïber d. Stickstoffnahrung, \&c., I 888 .

${ }^{3}$ Hellriegel, Unters. über d. Stickstoffnahrung d. Gramineen u. d. Leguminosen, IS8S; Ber. d. Bot. Ges., 1889, p. 138.

4 Hiltner, Versuchsst., I $896,13 d . x L v i$, p. 160.

${ }_{5}$ Nobbe, Versuchsst., I $\delta_{94}$, Bd. XI.v, p. 155 , and 1892, Bd. $x$ Ll, p. ${ }_{3} 8$. On the root-tubercles 
Podocarpus are also able to assimilate free nitrogen by the same means. Hellriegel's results have been confirmed by many observers, and Prazmowski, Schlösing and Laurent, Nobbe, and others have shown that in the absence of root-tubercles the Leguminosae are as little able to assimilate free nitrogen as are other Phanerogams ${ }^{1}$. Frank's contradictory results were probably duc to the presence of nitrogen-assimilating micro-organisms in the soil.

Certain Leguminosae may assimilate nitrogen in such abundance as to

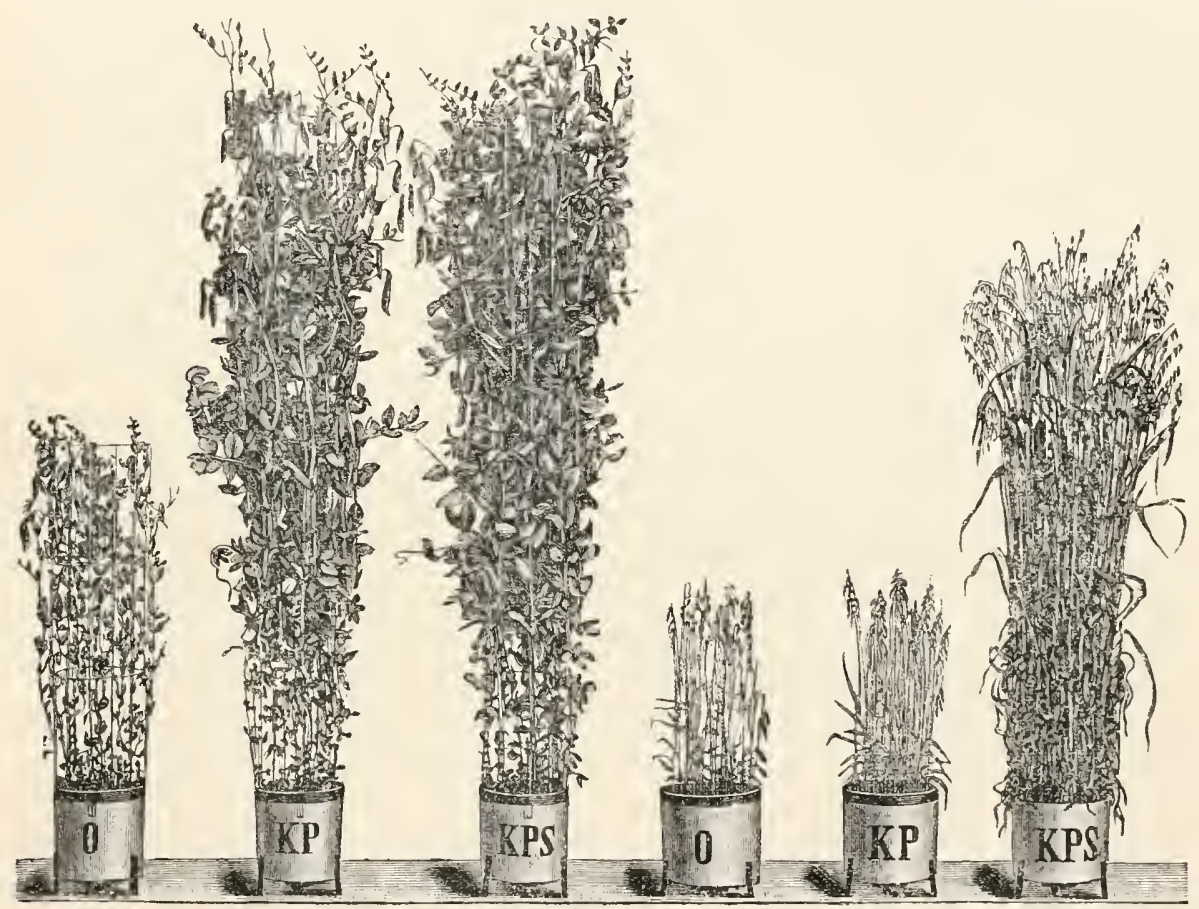

F1G. 60. The plants are grown $(O)$ in soil containing hardly any combined nitrogen, to which $(K P)$ potassium phosphate and (KPS) potassium phosphate and nitrate are added. The other ash constituents are already present, and also traces of combined nitrogen, so that feeble development is possible in $(O)$. (After Wagner, Die rationelle Düngung, 1ígI, 2. Aufl., p. I3.)

develop normally without being supplied with any nitrogen-compounds. This may be scen in Fig. 60, in which peas supplied only with ash constituents (KP) develop almost as luxuriantly as when saltpetre is present

of Fodocarpus, cf. Janse, Ann, d. Jard. bot. d. Buitenzorg, I896, T. xIv, p. 66 . It has yet to be. determined whether certain mycorhizal structures are able to assimilate free nitrogen.

${ }_{1}$ Prazmowski, Versuchsst., I89I, Bd. xxxviır, p. 5 ; Schlösing et Laurent, Ann. d. l'Inst. Pasteur, 1892 , T. vi, pp. 65 and 827 ; Nobbe, Versuchsst., r893, Bd. xLII, p. 467 ; 1894 , Bd. Xlv, p. $155 ; 1896$, Bd. xlvir, p. 266 . Further literature by Stutzer, Centralbl. f. Bact., 1895, Abth. ii, Bd. I, p. 72 . 
(KPS), whereas oats in soil containing no combined nitrogen develop but fecbly.

The following are a few of the experimental results obtained by Hellriegel and Wilfarth ' with Lufinus luteus. The plants were grown in sand almost entirely free from nitrogenous compounds, and supplied with ash constituents. Certain of the pots were infected with root-tubercle bacteria from ordinary soil; in others the formation of root-tubercles was prevented; and in $(d)$ a boiled soil extract was added to show that the trace of nitrogen thus obtained was insufficient to appreciably modify the results obtained.

Total per pot, containing two plants.

\begin{tabular}{|c|c|c|c|}
\hline ITarvested dry weight. & $N \cdot$ present. & $\begin{array}{l}\text { N. added in seed, } \\
\text { soil, and soil- } \\
\text { extract. }\end{array}$ & $\begin{array}{c}\text { Gain or loss } \\
\text { of } N \text {. }\end{array}$ \\
\hline$f(a) 38.919$ & $0.99^{8}$ & 0.022 & +0.975 \\
\hline$\{$ (b) 33.755 & $0.9^{S I}$ & 0.023 & $+0.95^{8}$ \\
\hline f (c) 0.089 & 0.016 & 0.020 & -0.004 \\
\hline$\{(d) \quad 0.828$ & 0.011 & 0.022 & -0.009 \\
\hline
\end{tabular}

In the presence of nitrates the amount of free nitrogen assimilated diminishes, and apparently when the former are abundant such assimilation may entirely or almost entirely cease ${ }^{2}$, although further research is necessary to clearly establish this fact, and also to determine whether the growth of Leguminosae and of root-tubercle bacteria is retarded by the cessation of the assimilation of free nitrogen. At present it seems that no such retardation is produced, and if so these plants possess greater accommodatory powers than Clostridium Pastcuriamum, which is unable to develop unless its normal functional activity can be exercised. It is possible that different Leguminosac exhibit various degrees of specialization in this respect, as is indicated by certain researches ${ }^{3}$, although sufficient attention may not have been paid in them to the other co-operating factors which may influence the result produced. Thus a lcssened number of root-tubercles, or the presence of a less active varicty of bacteroid will cause a plant to exhibit a lcssened power of fixing nitrogen, and hence will enable the addition of saltpetre to exercise a greater influence upon the growth of the plant than would otherwise be the case. The facts discovered by Hellriegel will retain their fundamental importance, whatever the nature of the symbiotic relationship may ultimately prove to be. It seems at present most probable that the actual fixation of nitrogen is due to the root-tubercle bacteria, for this power has as yet been discovered only in micro-organisms, and it is not surprising that the root-tubercle bacteria should only be capable of energetically assimilating nitrogen under special conditions, such as are

1 Ber. d. Bot. Ges., I 889 , p. I 4 I.

${ }^{2}$ Cf. Hellriegel, 1. c., I 888 , p. 149 ; Frank, l.c., 1892 , p. $4^{1}$; IIiltner, Versuchsst., I 896 , Pd. Xlvi, p. i6I (alder).

${ }^{3}$ Cf. Hellriegel and Frank, l. c. 
found within the living cells of the roots of a leguminous plant. As a matter of fact no power of fixing nitrogen has becn detected in pure cultures of the isolated bacteria until quite recently, and that only under special conditions ${ }^{1}$. The root-tubercles may be present in great abundance-Nobbc ${ }^{2}$ counted 4.572 upon a pca-plant-as also are the bacteria present in them, so that no such marked fixative power as is possessed by C. Pastcurianum is necessary to producc the maximal gain of nitrogen obscrved in a leguminous plant.

The fixation of nitrogen apparently only occurs in the actual root-tubercles where the bacteria are present ${ }^{3}$, and Kossowitsch's ${ }^{4}$ researches point to a localized fixation of nitrogen. The formation and behaviour of the root-tubercles, as well as the growth of the plant under normal and abnormal conditions, are in entire agreement with this conclusion ${ }^{5}$. The absorption of the bacteria as they die affords no evidence to support Frank's conclusion that they are cultivated by the plant merely in order to be devoured ${ }^{6}$.

No final decision can as yet be made, and it is not impossible that leguminous plants obtain the power of assimilating nitrogen by interaction with the symbiotic bacteria, although no evidence has been brought forward in support of this view. Frank's dictum that the presence of the bacteria operates as an accelerating stimulus is based upon the incorrect assumption that all Phanerogams have a certain power of assimilating free nitrogen. Various other conclusions have been put forward upon insufficient grounds, and it is too often forgotten that the appearance or storage of nitrogenous compounds at a given point does not afford any direct indication as to the place of their production?. Active proteid-synthesis may proceed in the green leaves of a leguminous plant, while the root-tubercles may send supplies of amides or simpler nitrogenous compounds to them.

It has yet to be determined how the nitrogen is assimilated and what is the first product of the assimilatory process, and it is possible that in this respect also specific differences may exist between different bacteria. Nobbe and Hiltner ${ }^{8}$ suppose that this property resides exclusively in the bacteroids of the root-tubercles, but they do not bring forward any satisfactory proof of this statement. The

1 Beyerinck, Bot. Zeitung, I 888 , p. 798 ; Prazmowski, I 89 I, 1. c., p. 55 ; Laurent, Ann. d. l'Inst. Pasteur, I 89 I, T. v, p. I36; Frank, l.c., IS922, p. 44 ; Berthelot, Ann. d. chim. et d. phys., I 893 , vi. sér., T. Xxx, p. 425 ; Stutzer, Centralbl. f. Bact., I $896, A$ bth. ii, Bd. II, p. 669. Mazé has recently obtained positive results under special cultural conditions (Ann. d. l'Inst. Pasteur, I897, T. XI, p. 44).

${ }^{2}$ Nobbe, Versuchsst., I 891, Bd. xxxix, p. 335. Cf. also Frank, l. c., I89o, p. 9; Kionka, Biol. Centralbl., i 89 I, Bd. XI, p. 283 .

${ }^{3}$ Frank at first supposed (1. c., I 890, pp. 26, 73) that the bacteria were distributed thronghout the entire plant. Zinsser (Jahrb. f. wiss. Bot., IS97, Bd. xxx, p. $4^{2} 3$ ) has, however, shown that this is not the case.

4 Bot. Zeitung, I 892, p. 697 .

${ }^{5}$ Cf. Prazmowski, l. c., I 89I, p. 5I ; Frank, Ber. d. Bot. Ges., IS92, 1. 27 I, and Lehrb. d. Bot.

${ }^{6}$ Frank, Ber. d. Bot. Ges., I 89 I, p. 24 S. Cf. Sect. 65.

7 Cf. Frank und Otto, Ber. d. Bot. Ges., I 889 , p. 33 I ; Frank, Bot. Zeitung, I 893 , p. I54.

${ }^{8}$ Versuchsst., I 89.3, Bd. xi.Ir, p. 459. 
aerobic root-tubercle bacteria live and are active in the presence of oxygen within the respiring root-tubercle cells ${ }^{1}$, whereas Clostridium Pasteurianum assimilates nitrogen when living anaerobically. In the latter case hydrogen is evolved, but the root-tubercles show that an evolution of hydrogen is not necessarily connected with the fixation of nitrogen ${ }^{2}$.

The conditions for the formation of root-tubercles. No further account of the morphology of the tubercles can be given, nor is it necessary to bring forward any proof that they are formed owing to the penetration of certain bacteria into the root-cells ${ }^{3}$. The infection is strictly localized, for the bacteria do not spread through the tissues, and tubercles are formed only upon those parts of the roots which are in contact with an infected soil ${ }^{4}$. Zinsser has shown that no tuberclebacteria are present either in the other parts of the root, or in the stem and leaves. Root-tubercle or other bacteria injected into sub-aërial organs die after a time without spreading or increasing in numbers ${ }^{5}$, hence the roots of seedlings and cuttings only form tubercles when infected from the soil ${ }^{6}$.

The positive results obtained with pure cultures by Prazmowski, Beyerinck, and Nobbe, show that a single species of bacterium suffices for the normal formation of root-tubercles, in which however, according to certain authors, two distinct forms may be present. It is also doubtful whether one or many species of tubercleforming bacteria exist, and whether the so-called 'bacteroids' are always simply developmental stages of the infecting bacterium, or may also include constituents of the root-tubercle cells ${ }^{7}$. If Bacillus radicicola is the only root-tubercle bacterium, it must occur in a variety of adaptive forms, for Hellriegel showed that a given soil will not infect every leguminous plant, while Nobbe found that the bacterium from the root-tubercles of Pisum sativum could infect Phaseolus, but not Robinia, Trifolium, or Serradella ${ }^{\text {. }}$

Hence it is advisable always to infect a plant from the tubercles of others of the same kind. When a particular legume has not been cultivated in a field for some time, it is now a matter of common practice to strew over it soil charged with bacteria from the root-tubercles of the plant in question, or from pure cultures. Infected soil gradually loses its power of inducing the formation of

${ }^{1}$ On the aeriferous system of the tubercles, see Frank, Ber. d. Bot. Ges., I $\$_{92}$, p. 271 .

${ }^{2}$ Cf. Winogradsky, Compt. rend., I $\delta_{94}$, T. cxviII, p. 353.

${ }^{3}$ See the works quoted on the previous page, and also Kionka, Biol. Centralbl., is 9 r, Bd. XI, p. 282.

'Cf. Hellriegel, 1.c., ISSS, p. 175 ; Laurent, Ann. d. l'Inst. Pasteur, I\$9I, T. v, p. I30; Zinsser, Jahrb. f. wiss. Bot., IS 97 , Bd. Xxx, p. 423.

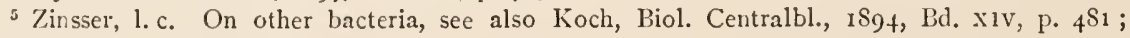
Russel, ibid., Bd. LIX, p. 375 .

${ }^{6}$ Recognized by Hellriegel. Cf. also Prazmowski, Versuchsst., I $89 \mathbf{I}$, Pd. xxxvill, p. 58; Nobbe, ibid., Bd. xxx1x, p. $35^{\circ}$; Zinsser, 1.c.

${ }^{7}$ Cf. the works already quoted, and Schneider, Ber. d. Bot. Ges., I894, p. II ; Gonnermann, Landw. Jahrb., I894, Bd. Xxil, pp. 649 , \&c. On the occurrence of different kinds of tubercles, ef. Frank, lier. d. Bot. Ges., $1 S_{92}$, pp. I 70,293 ; Möller, ibid., p. 243 ; also Morck, Über d. Formen d. Bacteroiden, $18_{9} 1$.

${ }^{8}$ Ilellriegel, l. c., 18S8, p. I 46 ; ISS9, p. I4 2 ; Nobbe, Versuchsst., IS94, Bd. XLV, p. 19; IS96, P.d. XI.viI, p. 266 . 
root-tubercles, owing to the fact that the bacteria do not remain living in the soil for longer than a certain time. The Soja plant cultivated in our gardens usually forms no root-tubercles, whereas in its normal habitat in Japan these are produced in abundance, a difference which may be due either to the influence of the rich garden soil, or to the absence of the specific bacterium form by which it is infected in Japan'.

For the formation of root-tubercles and the maintenance of the symbiosis certain specific interactions are necessary, as is indicated by the fact that the tubercles are formed only upon certain plants, and that even then certain essential external conditions must also be fulfilled. Thus Zinsser found that an injured root lost the power of forming root-tubercles until the process of regeneration was completed, that is until less heavy demands were made upon its available plastic materials, while the roots of typical terrestrial plants do not form tubercles so easily or abundantly when grown in a water-culture as they do in the soil ${ }^{2}$. The presence of large amounts of saltpetre also prevents the formation of tubercles to a more or less marked extent ${ }^{3}$.

Under optimal conditions the formation of root-tubercles occurs so rapidly that even in soil free from combined nitrogen a seedling pea or lupine experiences no starvation for want of nitrogen. All tubercles seem to have the same functional importance, and Frank's ${ }^{4}$ conclusion that those of Phaseolus and Robinia are inactive has proved to be incorrect. It is, however, not surprising that the power of forming root-tubercles should not be possessed by all leguminous plants : thus no tubercles have been observed upon the roots of Gleditschia triacanthus, nor could any be produced by artificial infection. A power of fixing nitrogen may be obtained by union with other organisms, and the possibility of the existence of simple contact-symbiosis with bacteria has already been mentioned.

History and methods. De Saussure's gasometric experiments showed, in contradiction to the opinion prevailing in his time, that green plants are unable to make use of atmospheric nitrogen, but Boussingault was the first to clearly prove that particular plants are unable to assimilate this gas ${ }^{5}$. He grew plants in bell-jars filled with air filtered through sulphuric acid, using either a non-

1 Kirchner, Cohn's Beiträge z. Biologie, 1895 , Bd. vir, p. 214.

2 Hiltner, Versuchsst., I 896 , Bd. XLVI, p. I6I. The formation of tubercles in water-cuitures was observed long ago. See Prazmowski, Versuchsst., I891, Bd. xxxviII, p. 4I.

${ }^{3}$ Nobbe u. Hiltner, Versuchsst., I 893 , Bd. xLII, p. 477; Hiltner, 1.c. Additional observations upon the infuence of the external conditions are given by Beyerinck, Bot. Zeitung, I888, p. 743; Prazmowski, Versuchsst., I 890 , Bd. xxxviI, p. I 89 ; Laurent, Ann. d. l'Inst. Pasteur, I89I, T. v, p. I33; Zinsser, l.c.

4 Cf. Prazmowski, Versuchsst., I89I, Bd. xxxvı11, p. 60; Nobbe, ibid., I89I, Bd. xxxix, Pp. $339,35^{\circ}$.

5 Saussure, Rech. chim., I804, p. 206. Saussure (Mém. d. 1. Soc. d. Phys. d. Genève, I833, T. vi, p. 570) also concluded that decomposing seeds evolved large quantities of nitrogen, although in some cases a slight fixation of nitrogen occurred. The errors of the gasometric method were at the time so great that the experiments cannot be regarded as proving the latter fact, although they suffice to firmly establish the former. Boussingault, Agron., Chim. agric. et Physiol., I 860, T. I, p. I. These works are partly contained in Ann. d. sci. nat., I 854 , iv. sér., T. I, P. 241 , and 1855 , iv. sér., T. IV, p. $3^{2}$. 
nitrogenous soil or one containing a measured quantity of saltpetre. On estimating the amount of nitrogen present in the original seeds and in the plants when harvested no perceptible gain from the free nitrogen of the air could be detected. The slight gain of nitrogen observed in the first researches in ordinary air was due to the absorption of ammonia, \&c. from the latter ${ }^{1}$. These results were obtained not only with Lepidium and with oats, but also with beans and lupins, probably because the plants were for the most part grown upon previously ignited pumicestone and watered with a solution of the necessary ash-constituents, so that no root-tubercles were formed. Lawes, Gilbert, and Pugh ${ }^{2}$ obtained similar results, using for the most part a soil composed of burnt clay. The increase in the amount of nitrogen observed in all cases by Ville ${ }^{3}$, even in cereals, was due either to faulty experimentation ${ }^{4}$ or to a fixation of nitrogen by organisms in the soil.

Practical experience has left no doubt that the Leguminosae obtain more nitrogen than is present in the soil, and it was Hellriegel who showed in I 888 that these plants assimilate this gas in the free condition, but only when provided with root-tubercles. The further progress of our knowledge has already been indicated ${ }^{5}$, as well as the reasons which led certain authors to conclude that all plants can assimilate free nitrogen. Berthelot (1885) was the first to prove that certain bacteria have this power, while Winogradsky isolated one of these bacteria and studied its special properties in great detail.

Hellriegel and most other workers, including Boussingault, estimated the increased percentage of nitrogen in the crop from sand, humus ${ }^{6}$, or watery nutrient solutions. Hellriegel placed almost non-nitrogenous sand in glass vessels of 4-8 litres capacity, and brought the cultures into the open during fine weather, so as to attain the maximal possible growth. The complete sterilization of the soil and the use of distilled water for watering sufficed to prevent any formation of root-nodules. Similarly no perceptible development of nitrogen-forming organisms occurred in sand covered with pieces of quartz on which a layer of cotton wool was superposed. The seeds were sterilized by means of $\mathrm{I}$ to 2 per cent. solution of formic aldehyde, or by washing with alcohol, ether, or a dilute solution of mercuric chloride. Beyerinck, Prazmowski, \&c., by using special vessels and adopting certain precautions, ensured the absence of all micro-organisms, but even then, as both

${ }^{1}$ Boussingault, Ann. d. chim. et d. phys., 1838 , ii. sér., T. Lxvir.

${ }^{2}$ Lawes, Gilbert and Pugh, Phil. Trans., 1862, Vol. CLI, p. 43I. The following authors obtained similar results, although their experiments were hardly performed with the same care: Mène (Compt. rend., I851, T. xxxir, p. I80); Harting (ibid., 1855, T. xLI, p. 942); Cloez et Gratiolet (Ann. d. chim. et d. phys., I851, iii. sér., T. Xxxir, p. 41) ; Bretschneider (Jahresb. d. Agr.* Chem., I 86 I-2, p. I 23).

3 Ville, Compt. rend., $185_{5}^{2}$, T. xxxv, p. $46_{4} ;$ I 854 , T. xxxvixi, pp. 703,723 ; also in Rech. exp., $\mathrm{I}_{53}$ and $18_{57}$.

${ }^{4}$ Compt. rend., 1855, T. XLI, p. 757 .

5 MacDougal gave in 1894 a summary of all the works which had appeared up to that time (Minnesota Bot. Studies, I894, Vol. 9, p. 186).

${ }^{6}$ On the effect of heating the soil, cf. Hellriegel, Ber. d. Bot. Ges., I889, p. I31 ; Richter, Versuchsst., 1896 , Bd. xLVII, p. 269. 
Hellriegel and Boussingault have shown, a small quantity of volatile nitrogen compounds may have been absorbed from the air.

Schlösing and Laurent proved in $\mathbf{I} \delta 92$ that leguminous plants absorb nitrogen from the air, whereas the amount does not diminish in vessels enclosing other plants. Frank ${ }^{1}$ supposed that all plants were able both to evolve and to fix nitrogen, both of which properties seem however to be possessed by a few special forms only. Nitrogen is apparently evolved only by certain bacteria (Sect. 68), and higher plants do not exhale any nitrogen after they have been kept for some time in an atmosphere composed of oxygen and hydrogen.

\section{SECTION 70. The Nutritive Value of Different Nitrogen Compounds.}

Most phanerogamic plants grow best when supplied with nitrates, but ammonium salts are more suitable for others, and many heterotrophic organisms either require a supply of peptone or other proteids, or attain their maximum development only when supplied with nitrogen in the form of proteids or amides. Hence it is possible to distinguish, in addition to the plants which assimilate free nitrogen, those which require (1) nitrates or ammonium salts, (2) amides, (3) peptones or other proteids ${ }^{2}$. Many plants may obtain suitable food by assimilating proteids or amides, whereas others must be supplied with carbon compounds as well (Sect. 66). No sharp line of demarcation can be drawn between any of these classes of plants, for although many are comparatively restricted in their requirements, others may be satisfied by a great variety of nitrogen-compounds, and may simply exhibit a more or less marked preference for proteids or nitrates as the case may be. It is easy to understand why proteids may not form a good source of nitrogen for those plants which normally construct their proteids from inorganic nitrogen-compounds, for the disuse of any vital activity is apt ultimately to exercise certain injurious effects : thus Clostridium Pasteurianum is unable to develop when the absence of free nitrogen prevents the exercise of its normal assimilatory activity.

The causes of the high or low nutritive value of a given nitrogencompound are not indicated by the effects which it produces upon growth and development. The nutritive value of a substance is mainly determined by the specific nature of the plant examined, although other factors may also come into play, such as solubility and absorptive power. Solid proteids are available only for those plants which excrete peptic or tryptic ferments, so that albumin only forms a good source of nitrogen

${ }_{1}$ Frank, Ber. d. Bot. Ges., I 886 , pp. 293, 380.

${ }^{2}$ Beyerinck, Bot. Zeitung, S9o, p. 73I, fontnote.

D d 2 
for the non-peptonizing yeast-plant when it is converted into peptone by the addition of pepsin ${ }^{1}$. Similarly, the difficulty of absorbing peptone tends to make it a bad food-material for Phanerogams, although in early developmental stages proteids may form their best possible food-material, as, for example, when the rescrve materials of the seed are consumed during germination. It must not, however, be forgotten that growth and development may be influenced in a variety of ways by the external conditions. Thus peptone is necessary for a few facultative anaerobes only when the supply of oxygen is deficient, while the nutritive value of a nitrogen-compound may vary in the presence of different carbon-compounds ${ }^{2}$.

Probably when several nitrogen-compounds are supplied they may support one another, or the one may protect the other in a manner similar to that observed in the case of carbon-compounds (Sects. 66, 67). Thus the presence of an abundance of nitrates lessens the amount of free nitrogen assimilated by leguminous plants, but on the other hand ammonia and nitrates do not protect one another in the case of Penicillium glaucum and Aspergillus niger, nor is ammonia protected by the presence of proteids. Similarly in phanerogams ammonium salts are always assimilated when present, however abundant nitrates may be.

Under normal conditions plants obtain almost the whole of their nitrogen from the soil or surrounding water, while if protected from rain they may still obtain a very trifling amount of nitrogen from volatile nitrogen-compounds, such as ammonia ${ }^{3}$, \&c., present in the air. The amount thus obtained may be comparatively large in plants which normally grow upon or near to manure or decaying organic matter from which volatile nitrogen compounds are evolved.

Phanerogams. Boussingault first showed that nitrates afford the most suitable source of nitrogen for most Phanerogams including Leguminosae, and this result has been frequently confirmed by means of the water-culture method ${ }^{4}$. According to Kellner ${ }^{5}$, swamp-rice, especially when young, exhibits a preference for ammonium salts, and this is probably often the case in plants which grow in swampy or badly aereated soil, where the process of nitrification takes place slowly or

1 Nägeli, Bot. Mitth., I89I, Bd. III, p. 419 .

${ }^{2}$ For yeast, cf. Chudiakow, Landw. Jahrb., 1894 , Bd. xxirl, p. 460.

${ }^{3}$ Sachs, Jahresb. d. Agr.-Chem., I 860-1, p. 78 ; Ad. Mayer, Versuchsst., I874, Bd. Xvir, p. 329 ; Schlösing, Compt. rend., 1874 , T. LxxIv, p. 700 ; also Altvater, Landw. Jahrb., I885, Bd. XIV, p. 62 I.

4 Boussingault, Agron., \&c., 1860, T. I, p. I54; also Ann. d. sci. nat., 1855, iv. sér., T. IV, p. 32 , and 1857 , iv. sér., T. VI, p. I ; Rautenberg und Kuhn, Versuchsst., I864, Bd. VI, p. 355; Lucanus, ibic., $186_{5}$, Bd. vir, p. $3^{6} 4$; Hampe, Hosaeus, Birner, Lucanus, and recently Pitsch, Versuchsst., I 895 , Bd. XLVI, p. 359 .

5 Versuchsst., I $884, \mathrm{Bd}$. Xxx, p. 18. 
not at all. In an ordinary soil traces of nitrates are continually being formed by the action of micro-organisms. Any accumulation is rapidly removed in the drainage water since the soil has hardly any power of retaining nitrates, and aquatic Phanerogams and Algae ${ }^{l}$ hence can obtain a sufficient supply of the nitrates they seem to prefer from the river or spring-water in which they grow. Neither Phanerogams nor Algae appear to have the power of forming nitrates from ammonium salts by oxidation, and the accumulation of nitrates exhibited by certain plants occurs only when an abundant external supply is provided ${ }^{2}$.

Phanerogams and Algae can also employ various organic substances as more or less valuable sources of nitrogen: urea, glycocoll, asparagin, leucin, tyrosin, guanin, kreatin, hippuric acid, uric acid, acetamide, propylamine ${ }^{3}$, but none of these is so favourable to growth as saltpetre. All these substances seem to be directly absorbed and assimilated without the aid of micro-organisms. According to Wagner (1869), hippuric acid is decomposed by plants into glycocoll and benzoic acid, the latter of which is useless. The parts of the plant where such decompositions occur are probably the same as those in which proteids are synthetized, but neither process has as yet been precisely localized. Under natural conditions Phanerogams rarely absorb organic nitrogen-compounds, whereas carnivorous plants are specially adapted to obtain supplies of peptone, and for many symbionts organic nitrogen-compounds appear to be an absolute necessity (Sect. 64).

Fungi, Bacteria, E.c. Among heterotrophic plants the nitrogen-fixing Clostridium Pasteurianum cannot be fed with organic nitrogen-compounds, and many of the common moulds as well as a whole host of bacteria grow well when supplied with ammonium nitrate, for some the nitric acid, for others the ammonium base being the preferable source of nitrogen ${ }^{4}$. The latter is especially the case with organisms which are entirely or partially anaerobic, and hence grow in media where no formation of nitrates is possible. Thus certain yeasts and bacteria can grow when supplied with ammonium salts but not when nitrates afford the sole source of nitrogen ${ }^{5}$, whereas a few strongly aerobic bacteria can make use of the latter compounds.

1 Kossowitsch, Bot. Zeitung, I 894 , p. I 09 . On the organic nitrogen compounds of the soil, see Berthelot, Compt. rend., I 891 , T. cxir, pp. I 89 , 195 . On mycorhiza in forest soils, cf. Sect. 65 .

${ }^{2}$ Molisch, Sitzungsb. d. Wien. Akad., I $88_{7}$, Bd. xcv, p. 221 ; Frank, Ber. d. Bot. Ges., I887, p. $47^{2}$; Schimper, Bot. Zeitung, I8s8, p. I21; Serno, Landw. Jahrb., IS89, Bd. xviri, p. 876; E. Schulze, Zeitschr. f. phys. Chem., I 896 , Bd. XxiI, p. 82 , and the lit. here quoted. Laurent found the same to be the case in mould.fungi (Ann. d. l'Inst. Pasteur, I889, T. III, p. 37I).

${ }^{3}$ Such experiments were performed by means of water-cultures by $\mathrm{Hampe}_{\mathrm{N}}$, Versuchsst., I $\mathrm{S}_{5}$, Bd. viI, p. 308 ; I866, Bd. viII, p. 255 ; I867, Bd. IX, p. 49 ; I868, Bd. x, p. ISo; Knop und Wolff, ibid., I $86_{5}$, Bd. vir, p. 463 , and Chem. Centralbl., I 866, p. 744; Birner und Lucanus, Versuchsst., I866, Bd. viı, p. I 28 ; Beyer, ibid., Bd. Ix, p. 480 ; Bd. xI, p. 270 ; W. Wolff, ibid., Bd. x, p. I3; P. Wagner, ibid., Bd. XI, p. $29^{2}$; Bd. XIII, p. 69 ; Bente, Bot. Jahresb., I8;4, p. 838 ; Baeseler, Versuchsst., I886, Bd. xxxiı, p. 230 ; Hansteen, Ber. d. Bot. Ges., I896, p. 362. Johnson (Versuchsst., I 666 , Bd. viII, p. 235), Ville (Compt. rend., T. Lxv, p. $3^{2}$ ), and Cameron (Jahresb. d. Agr.-Chem., I 86I-2, p. I 45) used sand as a medium. [Lutz, Compt. rend., Cxxvi, I S98, p. I 227. ]

4 See Laurent, Ann. d. l'Inst. Pasteur, I889, T. I1I, p. 368; also Nägeli, Bot. Mitth., I 88 I, Bd. III, p. 399 ; Fitz, Ber. d. Chem. Ges., 18 76 , p. 1540 ; Raulin, Ann. d. sci. nat., I869, v. sér., T. XI, p. 226 .

5 Yeast: Ad. Mayer, Unters. iiber d. alc. Gährung, I869, p. 69, and Gährungschemie, I 89 इ, 
Most fungi, including the common moulds, and such bacteria as are able to develop when supplied with nitrogen in the form of ammonium nitrate, grow better, indeed often much better, when fed with peptone, amides, or other organic nitrogen-compounds. Peptone is in fact essential for many such plants, whereas for others, amides afford a more or less suitable source of nitrogen : thus Bacillus perlibratus ${ }^{1}$ prefers asparagin to peptone, as do Rhizopus oryzae and Chlamydomucor oryzae $^{2}$, for which urea also affords an adequate source of nitrogen.

Chemical constitution and mutritive value. A great variety of nitrogen-compounds may serve as food-material for large numbers of plants or for special ones only, and the general remarks previously made in connexion with the carbon-compounds (Sect. 66) apply equally well here, for substances of similar chemical constitution may have widely different nutritive values. No doubt a detailed study of the nutrient properties of different proteids would reveal the existence of numerous specific differences. No general conclusions can be drawn from experiments made upon a few plants, for different plants have widely different specific needs. Nägeli's supposition that nitrogen cannot be assimilated when in direct combination with carbon has proved to be incorrect, for certain fungi are able to grow when supplied with nitriles ${ }^{3}$, and may even obtain their nitrogen from amygdalin or potassium cyanide (Pfeffer). According to the researches of Treub, hydrocyanic acid normally takes part in the metabolism of Pangium edule ${ }^{4}$. Free nitrogen is utilized by certain organisms, while nitrites are assimilated by nitrite bacteria, although they do not serve as a source of nitrogen for Phanerogams or mould-fungi ${ }^{5}$; it is even possible that nitrous oxide may be assimilated by certain organisms ${ }^{6}$.

According to Knop and Wolff $^{7}$, Phanerogamis are unable to assimilate nitrobenzoic acid, amido-benzoic acid, morphine, quinine, cinchonine, caffein, ferrocyanide and ferricyanide of potassium; these substances hardly seem to be suitable even for the nutrition of mould-fungi, although sufficient attention has not yet been paid to their poisonous action. Caffeine, quinine, and

4. Aufl., p. I 28 ; Nägeli, Bot. Mitth., I88 I, Bd. IlI, p. 399; Laurent, l.c., and the works already quoted. Bacteria: Nitrobacteria, Sect. 23 ; also Fermi, Centralbl. f. Bact., I89I, Bd. x, p. 405; A. Fischer, Jahrb. f. wiss. Bot., 1895, Bd. Xxv11, p. $5^{2}$.

1 Beyerinck, Centralbl. f. Bact., I 893 , Bd. x1v, p. 834 .

${ }^{2}$ Went und Prinsen Geerligs, Bcob. uiber die Hefearten d. Arrakfabrikation, 1895, p. 2 I. On the different forms of yeast, see Beyerinck, Centralbl. f. Bact., I 892 , Bd. xı, p. 68, and I 894 , Bd. xv1, p. 57, \&c.; on Bacteria, Beyerinck, Aliment photogène, I89I, pp. I 9, 5I (Sep--abdr. aus Archiv. Néerland., T. $\mathrm{xxIV}$ ); Bot. Zeitung, $\mathrm{I} 8_{9 \mathrm{I}}, \mathrm{p} .74 \mathrm{O}$, and the other literature quoted here and in Sect. 66. See also Linossier, Centralbl. f. Bact., I 892 , Bd. I1I, p. I I 2.

3 Reinke, Unters. a. d. Bot. Lab. in Göttingen, I888, IIl, p. 37 .

4 Ann. d. Jard. bot. d. Buitenzorg, I895, X111, p. I.

${ }_{5}^{5}$ Birner und Lucanus, Versuchsst., I 866, Bd. viIl, p. I 28 , for Phanerogams; Raulin, l. c., p. 229 , for fungi. On the poisonous action of nitrates, cf. Molisch, I877, l. c., p. 234. On nitrite formation, see Sect. I0з.

'Lamartina's positive conclusions (Chem. Centralbl., I 881, p. 649) are not based upon sufficiently decisive results.

7 Knop und Wolff, Versuchsst., 1865, Bd. v11, p. 308. Cf, also Bot. Centralbl., I $88_{3}$, Bd. Xvi, p. II 3 . 
many alkaloids have, however, a feeble nutrient value for certain fungi and bacteria.

\section{Section 7I. The Character of the Processes by which Nitrogen is assimilated.}

Both higher and lower plants are able to construct proteids as well as many other organic nitrogen compounds from nitrates and ammonia. Certain of these, namely, amides, may at times be present in greater abundance than the proteids, but the character of the processes by which these substances are produced forms one of the many unsolved problems of metabolism, and will remain so as long as merely the startingpoint and the end-product are known. Without doubt these synthetic processes involve a variety of complicated changes and interactions, and it is permissible to regard the simpler organic nitrogen-compounds as stages in the formation of proteids whenever the plant can produce them synthetically, and can use them at a later period-stage in the synthesis of proteids. Asparagin and other amides ${ }^{1}$ are substances of this character which are found in higher plants, and are often produced in large amount by proteid decomposition, while they may again be synthetized to form proteids. This fact, however, says but little as to the actual synthesis of proteids, which fungi are able to form from nitrates or ammonium salts, as well as from amides. The study of fungi is especially likely to afford a glimpse into these processes of constructive metabolism, owing to the fact that the external and cultural conditions can be varied to so considerable an extent. Those fungi to which amides and peptones must be supplied are organisms which have lost the power of producing amides and proteids synthetically. It may be found possible to inhibit this power entirely in such a fungus as Penicillizm, or to prevent the final stages of proteidsynthesis, and thus to obtain important data for the solution of the problem with which we are concerned. Isolated facts can only be interpreted with the utmost care, for if any conclusions are to be drawn from the fact that fungi are able to obtain their nitrogen from nitrites, the same importance would necessarily also be given to cyanides. In the case of Phanerogams, if attention were paid solely to Pangium edule, it might be concluded that hydrocyanic acid always formed a stage in the formation of proteids.

Plants can induce molecular disintegrations and reconstructions of the most diverse character. Penicillium is able to form all the substances

${ }^{1}$ Literature: Kellner, Landw. Jahrb., I879, Suppl.-Bd. viı, p. 243 ; Emmerling, Versuchsst., I8so, Bd. xxiv, p. II 3 , and 1887 , Bd. xxxiv, p. 73 ; Hornberger, ibid., I885, Bd. xxxi, p. 415 ; Serno, Landw. Jahrb., I889, Bd. xviII, p. 905 ; E. Schulze, ibid., 1888 , Bd, xvil, p. $70_{4}$. 
it requires from either methane or benzene derivatives, and it is not therefore surprising that such a plant is able to utilize a great variety of nitrogencompounds, and thus attain the same result by different means. It is not necessary that amides should always form an intermediate stage in proteidsynthesis, for the process of construction might be a direct one, the assimilated nitrogen-compounds uniting directly with certain molecules of proteids. At the same time, under different conditions, amides might be produced in abundance and used in the construction of proteid, for a living organism is always able to regulate its vital activity according to the conditions under which it exists. Nor does the existence of a benzenering in the proteid molecule necessitate that a production of benzole derivates should form one stage in the synthesis of proteid, for the aromatic substances found in plants, such as tannic acid, phloroglucin, appear to be aplastic products.

The final stages of synthetic metabolism may follow the same paths, although the starting-points differ, and when this is the case the preparatory processes must exhibit various special peculiarities according to the body with which the synthesis of proteid commences, whether it is with ammonia, nitric acid ${ }^{1}$, or nitrites, or whether methane or benzole derivatives are employed as a source of carbon. It is quite uncertain what may be the nature of the primary product in the assimilation of free nitrogen. Nitrogenous metabolism not only involves continual proteid-synthesis, but also unceasing proteid-decomposition, and when the entire supply of kinetic energy is derived from the disintegration of nitrogen-compounds, a large amount of nitrogen may be lost in the form of excreted nitrogen-compounds, or even of free nitrogen in a few cases.

Even when the chemical composition of products such as carbohydrates, asparagin, \&c., is well known, it may be impossible to say how a particular substance is produced by the plant, and this is even more markedly the case with proteids, the chemical constitution of which is still only imperfectly known (Sect. I I). According to Loew ${ }^{2}$ the hypothetical aldehyde of aspartic acid is formed, and from this the proteid molecule is derived by condensation, but these are mere speculations.

A supply of the essential ash constituents must of course always be available, and of these calcium is not necessary for fungi, and hence has no general importance in proteid-synthesis. During the assimilation of nitrates, oxalic acid is produced when necessary to combine with the liberated bases, but calcium oxalate is not always formed during proteid-synthesis, and its appearance does not localize

1 The ammonia need not necessarily be oxidized into nitric acid, or the latter reduced into ammonia, and, as a matter of fact, higher plants are unable to directly induce such changes.

"Loew, Die chem. Kraftquelle im Protopl., I882, pp. 5, \& c c.; also Ad. Mayer, Agr.-Chem., I 895,4 . Aufl., p. 163. 
the points at which such synthesis is active ${ }^{1}$. On the other hand, when ammonium chloride is assimilated, the liberated acid must be neutralized.

\section{SECTION 72. The Iocalization of Proteid-synthesis.}

Since the assimilation of nitrogen compounds and the synthesis of proteids proceed in fungi in the absence of light and chlorophyll, it can hardly be doubted that these processes take place in chlorophyllous plants also by means of chemosynthesis (cf. Sect. 68). In more highly organized plants, however, it is probable that all the cells and organs do not take the same part in the assimilation of nitrogen-compounds; indeed in the higher plants it is the leaves and chlorophyllous organs which in general seem to be of especial importance in this respect. The roots, however, seem also able to assimilate nitrates, and apparently they often form proteids from the amides which are conveyed to them. It is not yet certain whether any cells are present in the plant which have lost the power of assimilating nitrogen-compounds to such an extent that, like an obligate peptone-organism, they can live only when supplied with soluble proteids. Moreover it is still unknown whether amides or proteids are principally formed in green leaves.

It has yet to be determined what are the respective parts which the nucleus $^{2}$ and cytoplasm play in nitrogenous assimilation, and whether this process occurs in the chloroplastids as well. Even though this should prove to be the case it does not follow that the chloroplastids are organs specially adapted for this purpose, for it may also take place in nonchlorophyllous cells. Moreover, since nitrogenous assimilation continues in green cells even in darkness, it follows that the direct action of light is unnecessary ${ }^{3}$. It is true that in certain plants the formation of proteids is favoured by exposure to light, but it does not follow that the energy of the light-rays is directly used in effecting proteid-synthesis. Indeed it is much more probable that in all cases the process is a chemosynthetic one ${ }^{4}$.

Schimper, Bot. Zeitung, I888, p. 97, and Flora, 1890, p. 23I. On the relations of calcium oxalate to the formation of proteids, cf. Holzner, Flora, I867, p. 497 ; de Vries, Landw. Jahrb., I88I, Bd, x, p. 77 .

${ }^{2}$ Haberlandt ascribes on insufficient grounds a special activity to the nuclens (Function u. Lage d. Zellkernes, I 887 , p. I 6 ).

${ }^{3}$ Kosutany, Versuchsst., I896, Bd. xLvin, p. I3. Cf. Sect. 8o. The recent works of Laurent (Bull. d. l'Acad. roy. d. Belgique, I896, iii. sér., T. xxxiI, p. 8I5) and Godlewski (Anzeiger d. Akad. d. Wiss. z. Krakau, I 897 , p. I04) show that light, and especially certain rays, favour the synthesis of proteids, but leave it undetermined whether the light directly affords the necessary energy or only acts indirectly. On the other hand, Hansteen (Ber. d. Bot. Ges., I896, p. 362) has shown that Lemna can form proteids in darkness.

${ }^{4}$ It is not easy to see why Schimper (Flora, 1859 , p. 256) holds that the results obtained with fungi do not afford any criterion as to what goes on in phanerogamic plants; see also Chrapowicki, Bot. Centralbl., I889, Bd. xxxix, p. $35^{2}$. 
The fact that nitrogenous assimilation occurs mainly in the green leaves may be of advantage in many ways, especially because the products of the assimilation of carbon dioxide may be at once utilized, and hence an injurious accumulation avoided (Sects. 54 and 55). When, however, no assimilatory products are present, or when the leaf is in a pathological condition owing to the continued absence of light, it is hardly surprising to find that nitrogenous assimilation ceases (cf. Sect. 55, and for the regeneration of amides, Sect. 80).

Sachs' observation that proteid substances are continually removed from an assimilating leaf indicates that an active power of assimilating inorganic nitrogencompounds must be localized there. Schimper has since shown that the absorbed nitrates disappear from the attached as well as from the detached leaf ${ }^{1}$, and at the same time, according to Chrapowicki, the amount of proteid present increases, while Emmerling's results indicate that amides are produced in especial abundance $^{2}$. Schimper holds that the appearance of calcium oxalate favours the synthesis of proteids in the leaf. It is not yet certain to what extent nitrates may be assimilated in other organs, but an experiment by Müller-Thurgau seems to indicate that this process does actually occur in the root, and it is certain that proteids may be formed from the amides conveyed to the roots and other organs. Whether the synthesis of proteids takes place mainly in the sieve-tubes, as A. Fischer ${ }^{3}$ suggested, has yet to be proved.

\section{PART V.}

\section{THE ASH CONSTITUENTS.}

\section{SECTION 73. The Essential Elements.}

In addition to carbon, hydrogen, oxygen, and nitrogen, there are certain other essential elements which are found in the ash left behind when a plant is burnt (Sect. 50). These ash constituents form $\mathrm{I} \cdot 5$ to 5 per cent. of the dry weight in plants supplied solely with essential salts, but may reach as much as 10 or even 30 per cent. ${ }^{4}$ when large quantities of unnecessary salts are absorbed (Sect. 22). Hence it can only be found

1 Sachs, Flora, 1862, p. 298, and Bot. Zeitung, 1862, p. 372 ; Schimper, Bot. Zeitung, 1888, p. 130. From the mere distribution or accumulation of nitratcs no conclusion can be drawn; cf. Schimper, p. 120 , and the literature quoted on p. 405.

a Emmerling, Versuchsst., I580, Bd. xxiv, p. I1 3 , and I887, Bd. xxxiv, p. 73 ; Chrapowicki, Bot. Centralbl., I889, Bd. xxxix, p. $35^{2}$.

${ }^{3}$ Schimper, 1.c., p. 89; Flora, 1890, p. 257 ; Müller-Thurgau, Bot. Jahresb., 1880, 1, p. 319; A. Fischer, Ber. d. Sächs. Ges. d. Wiss. zu Leipzig, 1885 , p. 280.

* A complete series of ash analyses is given by Wolff, Aschenanalysen, 1871 and 1880 , and also by Ebermayer, Physiol. Chemie, 1882, p. 727 ; Ad. Mayer, Agr.-Chem., I895, 4. Aufl., p. 307 . See also Sect. 22. 
by direct experiment whether a particular ash constituent is essential or not. If the latter be the case the plant grows without any supply of the salt in question, whereas no development is possible in the absence of any one of the essential saline constituents.

It has bcen determined by growing plants in various nutrient solutions that potassium. magnesium, phosphorus, sulphur, and iron, are essential elements in all cases. The fungi and bacteria whose properties were investigated by Molisch, Benecke, and Loew can develop normally without calcium, whereas higher plants cannot grow unless they are abundantly supplied with this element. This is also the case with many algae (Spirogyra, Vaucheria, \&c.), while others (Microthamion, Stichococcus, Palmella) can do without calcium ${ }^{1}$.

Different plants may have different requirements, and it is quite possible that various peculiarities may exist among the lower organisms. Indeed according to Loew ${ }^{2}$ and Benccke in the case of fungi, potassium may be replaced by rubidium and perhaps by caesium also, whereas according to Molisch no such substitution is possible in algae ${ }^{3}$. All researches agree in showing that potassium cannot be replaced by sodium or by lithium, and it is very remarkable that sodium which is universally present has not found any such use in metabolism as would make it an essential element. As, however, both potassium and sodium are essential to the higher animals ${ }^{4}$, it is possible that plants also may exist which cannot live without a supply of the latter element, although this has not yet been found to be the case even in plants frequenting saline habitats. Seaweeds, hovever, have not up to the present time been cultivated in the absence of sodium chloride. The same considerations apply to silicon also, though it is possible that in its absence organisms such as diatoms may be unable to exist, for the inability to perform the functions connected with the presence of silicon may render growth and development impossible. Similarly aluminium and zinc are unnecessary, though large quantities of both elements are often accumulated, and of all the other elements chlorine is the only one which may be even of doubtful use in certain cases.

1 Molisch, Bot. Centralbl., I 894 , Bd. Lx, p. I67 ; Sitzungsb. d. Wien. Akad., I894, Bd. CIII, Abth. i, p. 566; I 995 , Bd. civ, Abth. i, p. 799 ; I896, Bd. cv, p. 633 ; Benecke, Bot. Centralbl., I894, Bd. Lx, p. 195; Jahrb. f. wiss. Bot., I895, Bd. xxvir, p. 487 ; Bot. Zeitung, r896, p. 97. Cf. also Sect. 74; Loew, Flora, I892, pp. 374, 390 ; Bot. Centralbl., 1898 , Bd. Lxx rv, p. 258 , and 1895, No. $5^{2}$.

${ }^{2}$ Loew, Landw. Versuchsst., i 879. See also Bot. Centralbl., i 898, Bd. Lxxiv, No. 7.

s According to Winogradsky (Bot. Centralbl., I $884, \mathrm{Bd} . \mathrm{xx}, \mathrm{p} . \mathrm{I} 67$ ), $\mathrm{K}$ may be replaced by $\mathrm{Rb}$, but not by $\mathrm{Cs}$, in Mycoderma vini. Nägeli holds that $\mathrm{K}$ may always be replaced by $\mathrm{Rb}$ and $\mathrm{Cs}$, but the experiments which favour this conclusion are not decisive. According to Wehmer (Beiträge zur Kenntniss einheimischer Pilze, IS95, II, p. 107), fungi can grow without $\mathrm{K}$ if $\mathrm{Na}$ is present, though they do so more slowly, but these results have been proved to be incorrect by Molisch and Benecke (l. c.). The chief literatnre is given in Sect. 74 .

1 Bunge, Physiol. Chem., r 894,3 . Aufl, p. 107. 
If potassium can actually be replaced by rubidium in fungi, this is the only case known in which two elements are completely interchangeable. The conclusion which Nägeli ${ }^{1}$ based lipon Loew's experiments, that in the case of fungi magnesium, calcium, strontium, and barium can mutually replace one another, has been proved by Molisch and Benecke to be incorrect, and it is now easy to show that fungi require magnesium, but not calcium. It is possible that in other organisms various substitutions of one element for another may be possible, and we may speculate that under totally different conditions in other worlds organisms may exist in which the place of carbon is taken by silicon.

Great caution must be used in drawing any general conclusions from results obtained with a very limited number of plants, for in different parts of the vegetable kingdom all kinds of specific peculiarities have been developed. Our knowledge of the vital processes of metabolism is insufficient to warrant our making the statement that potassium must always be essential but not calcium, or that magnesium can never be replaced by any other element. In any case calcium does not appear to take an essential part in the normal metabolism of fungi or even of certain chloro. phyllous plants. It remains to be determined whether in such cases magnesium or potassium takes on the functions performed by calcium in the higher plants, or whether the latter have developed new functions for the performance of which calcium is essential, or have become so specialized that the continuance of life is impossible in its absence. The fact that iron is necessary to all plants shows that it is not merely of importance for the formation of chlorophyll.

Full development is only possible when a certain minimal amount of each essential clement is provided, although a plant usually assimilates much more than the minimal amount when the supply is abundant. The fact that such an extra consumption is possible shows that the elements in question are used for purposes which can to a large extent continue in their absence. It is easy to understand, for cxample, that when a neutralization of organic acid is necessary, it may be immaterial whether potassium or sodium, or even calcium or magnesium, is utilized for this purpose, whether the object is simply to prevent any injurious action which the free acid might exercise, or to produce osmotically active salts. For these and similar purposes one element may be replaced by another or even by an organic compound, although the element in question may be essential for certain definite functions.

The dissimilar composition of the ashes of plants grown in different soils

1 Nägeli, Bot. Mitth., I $88_{1}$, Bd. III, p. 45 s. Benecke (1. c.) has also shown that Mg cannot be replaced by beryllium or the other metals of the zinc series. 
suggests that such substitution is possible (Sect. 22), and Wolff's ' experiments afford a direct answer to this question. Wolff grew oats in nutrient solutions containing 42.83 per cent. of potash and 7.03 per cent. soda, or I I.65 per cent. potash and $33.6 \mathrm{I}$ per cent. soda, but otherwise of similar composition, and found that in the first case the ash of the harvested plants contained 50.28 per cent. potash and 7.03 soda, in the second case 30.69 potash and 22.04 soda. Experiments in which calcium was partly replaced by magnesium gave similar results. A limited substitution of strontium for calcium seems also to be possible ${ }^{2}$. The nature of the neutralizing base is not always immaterial, for when calcium is present an insoluble oxalate is precipitated. Combination with an alkali produces a more highly osmotic salt than does combination with an alkaline earth (Sect. 24). That the fixation of organic acids is often the sole or most important function of such non-essential bases can hardly be doubted, and in the bacteria which excite lactic and butyric fermentation we have organisms which are rapidly injuriously affected as the acid which they produce accumulates (Sect. 103). It is, however, hardly the sole task of the alkalies and alkaline earths to neutralize organic acids, as Liebig supposed, being led thereby to the conclusion that they might replace one another to an unlimited extent. The diametrically opposed view of Sprengel, that all such substitution is totally impossible ${ }^{3}$, is also inaccurate.

In the absence of any one of the essential elements development is impossible, just as a watch ceases to go as soon as any of the wheels which form part of its mechanism are removed. This applies as well to iron, of which but a trace is required, as to potassium or phosphorus, of which large amounts are necessary, though not nearly as much as of carbon ${ }^{4}$. When the deficient element is presented to the plant in gradually increasing amount the rapidity of growth also increases, though by no means proportionately ${ }^{5}$, as has already been shown in the case of nitrogen (Sect. 68), while any increase above the optimum amount required must ultimately lead to a depression of the vital activity. This only occurs with a potassium salt at a high degree of concentration, whereas a poisonous action is exercised by a very dilute solution of a salt of iron. All the physiological results which may be produced by progressive changes in any of the external conditions may as a general rule be represented by a curve exhibiting minimum, optimum, and maximum points. The so-called law of minimums ${ }^{6}$ expresses the first portion of this curve, which may be constructed not only for the ash constituents,

1 O. Wolff, Versuchsst., I868, Bd. x, p. 370 ; Pellet, Ann. d. chim. et d. phys., I879, v. sér., T. Xvil, pp. 145 , \&c.

${ }^{2}$ Hasselhof, Landw. Jahrb., I 893 , Bd. xxi1, p. 851 ; Molisch, Bot. Centralbl., I896, Bd. LxviIr, p. 146 (Algae).

${ }^{3}$ Liebig, Die Chemie in ihrer Anwend. anf Agric., 1840, p. 87 ; also Mulder, Physiol. Chem., I 844-51, p. 78 ; C. Sprengel, Die Lehre vom Dünger, I 839 , p. 53 .

* Even Saussure (Rech. chim., I804, p. 26I) recognized that the amount was not all-important.

${ }^{5}$ For examples, see Wolff, Versuchsst., I 874 , Bd. xviI, p. I38; Ville, Bot. Jahresb., I 890, p. 47.

${ }^{6}$ Cf. Ad. Mayer, Agr.-Chem., I895, 4. Anfl., p. 306. 
but also for the organic food, for the action of light, and indeed for all factors or agencies which produce a perceptible effect only at a certain degree of concentration, above which their action gradually increases until the optimal effect is produced.

From the complicated interactions and correlations which exist between the different parts of the vital mechanism, it is only to be expected that the optimal amount of any given substance may within certain limits be a variable quantity. 'This is shown by O. Wolff's ${ }^{1}$ empiric results, obtained while estimating the minimal quantity of potassium or phosphorus, necessary for the full development of an oat-plant when all the other elements were present in sufficient amount. All the elements cannot however be simultaneously reduced to this lowest possible minimum. It was not found possible to develop a normal oatplant containing less than 3 to 4 per cent. of ash, whereas the latter would only amount to 2 per cent. could all the elements be reduced to their minimum amount. This may possibly be due to the fact that the place of any particular essential element may be partially taken by others when these are present in sufficient amount. Non-essential elements may also aid in this, for apparently silicon may, like calcium, help to further reduce the minimal amount of potassium or phosphorus necessary for development.

In the older organs the percentage of ash usually increases, and its composition alters. The young organs contain for the most part only what is absolutely necessary, and hence their ash retains an approximately similar composition ${ }^{2}$ in different plants. On the average 3 grammes of the ash of embryonic tissues contain the following amounts of the different elements: phosphorous pentoxide I.I g., potash I.० g., magnesia $0.35 \mathrm{~g}$., lime $0.25 \mathrm{~g}$., sulphur trioxide $0.1 \mathrm{~g}$., ferric oxide $0.03 \mathrm{~g}$., soda $0.08 \mathrm{~g}$., chlorine $0.04 \mathrm{~g}$., silica $0.05 \mathrm{~g}$.

In adult organs the calcium and silicon increase to a marked extent and may frequently form 50 per cent. of the ash, so that the harvest from a hectare ( $2 \frac{1}{2}$ acres) may remove annually 200-300 kilos of the mineral constituents of the soil ".

An essential element must always be presented in the form of appropriate chemical combinations, for upon this its nutritive value largely depends, as has already been seen in the case of nitrogen and carbon. This is not of so marked importance as regards the ash constituents, probably because the salts usually absorbed split up into their ions when they dissolve in water. Acids as well as bases can be used as food by higher plants only when highly oxidized, but fungi can assimilate sulphurous $\left(\mathrm{H}_{2} \mathrm{SO}_{3}\right)$ and hyposulphurous $\left(\mathrm{H}_{2} \mathrm{SO}_{2}\right)$ acids, while a few bacteria are able to utilize sulphur and sulphuretted hydrogen, and many fungi seem able to assimilate the lower oxides of phosphorus. It is still

1 O. Wolff, Versuchsst, 1877, Bd. xxx, p. 387 .

${ }^{2}$ Garreau, Ann. d. sci. nat., i\$60, iv. sér., T. Xirl, p. i 79. In Wolff's tables the influence of manuring is given. On fungi, see Sieber, Bact. Centralbl., $18_{9^{2}}$, Bd. $x$, p. 78 , and Zopf, Pilze, IS9o, p. 114 .

${ }^{3}$ Cf. Ebermayer, Physiol. Chem., i 882 , p. 76r 1 . 
doubtful whether certain organisms can only obtain their phosphorus, iron, and sulphur from the organic compounds which may form part or the whole of their food ${ }^{1}$. Similarly a specific difference exists between aerobes and anaerobes as regards oxygen, for the latter must obtain this gas in a combined form, whereas to the former life is only possible when a supply of free oxygen is assured.

Even when the best possible food is supplied normal vital activity is possible only when all the other essential conditions are fulfilled. Since particular organisms may have special requirements which are not at first sight apparent, a particular substance may be necessary for their growth under certain circumstances, though it is not absolutely an essential one, and may be dispensed with under different cultural conditions. Thus the presence of salt may be merely a physical condition for the development of marine algae, and it may be found possible to grow them in isosmotic solutions of other salts ${ }^{2}$. The maintenance of a suitable nutrient medium is also one of the physical conditions for growth, and for those fungi and bacteria which excrete free acid the presence of calcium carbonate or some neutralizing base is essential for continued development. The production of acid is dependent upon the cultural conditions, and these may be so altered that the nutrient fluid becomes alkaline (Sect. 86), in which case the addition of a neutralizing acid becomes necessary. These powers are possessed only to a slight extent by higher plants, but in their case also it depends partly upon the nature of the nutrient salts whether the desirable slight acidity is produced, or replaced by an alkalinity, which usually injuriously affects the roots, and hence may retard or inhibit the development of the entire plant. The presence of sodium or potassium chloride may cause the acid reaction to be maintained (Sect. 23), so that under such circumstances chlorine appears to be an essential element. In other cases also it may have a definite function to perform. There is therefore a possibility that under special cultural conditions those plants may be grown without calcium ${ }^{3}$ to which at present it seems to be an essential element.

It has already been mentioned (Sect. 64) that the seeds of Orobanche and the spores of certain fungi will germinate only when stimulated by the presence of special chemical substances, but it is very doubtful whether any of the constituents of the ash act in a similar manner. As a matter of fact a certain acceleration of growth may be caused by the

1 Cf. Sect. 64. On the necessity of organic iron compounds for animals, cf. Neumeister, Physiol. Chem, I 893 , I, p. 3 I I.

2 [Pennington (Cont. Bot. Lab. Univ. Pennsylvania, I, I 897, p. 203) finds that Spirogyra contains as much chlorine and sodium as marine algae do.]

${ }^{3}$ Dehérain and Bréal (Bot. Jahresb., I $8_{3}$, p. 40) state that seedlings do not require calcium at a high temperature, but Molisch (Sitzungsb. d. Wien. Akad., I 895 , Bd. cIv, Abth. i, p. 799) has shown the inapplicability of these observations. The temperature also affects the formation of free oxalic acid (cf. Sect. 86). 
presence of sinall amounts of cobalt, zinc, manganese, fluorine, lithium, \&c., and thus the dry weight of the crop formed in a certain time during the culture of a fungus may be much increased. It appears that the higher plants can react in the same manner, for according to Frank and Krüger a trace of a copper salt favours the growth of the potato, while in some such stimulating action may lie the explanation of Salm-Horstmar's conclusions that for the full development of summer barley the presence of fluorine and lithium is essential ${ }^{1}$.

The increased growth appears to be due to a general power of reacting against injurious influences possessed by living organisms, for similar results are produced by ether, alkaloids, \&c., not only upon growth, but also upon respiration and fermentative activity ${ }^{2}$ (Sect. IO4). Richards has shown that besides its nutritive importance, an iron salt, when present in sufficient amount to act as a feeble poison, may exercise a similar stimulating effect to that which any other poison would do. A very strong poison produces its optimal stimulating effect when extremely dilute, and growth may be retarded by doses above the optimum, while substances which act as poisons only when highly concentrated produce no perceptible result at all.

The presence of a non-essential substance may therefore be useful or even necessary under special conditions, as is the case for instance when the absorption of a nutrient material is accelerated or rendered possible by the action of an enzyme or an acid. Moreover, it must be remembered that the forced non-performance of any accessory function, even though it does not form an essential part of the actual vital mechanism, may ultimately lead to a more or less marked inhibition of the general vital activity (Sect. 64). Calcium is perhaps essential to the higher plants for some such reasons, and it is possible that in certain cases silicon may become essential in a similar manner. Plants growing wild are exposed to severe competition, and hence a very trifling cause may determine whether a given species will survive or die out (Sects. 76,92$)$. For this reason the presence of silica may be of considerable importance, in conferring a special means of protection against the depredations of animals, and against the penetration of parasitic fungi (Sect. 75).

From what has been said it is clear that it is not always easy to decide with certainty whether an element is essential or not. Moreover, if but little of a substance is required, the presence of the merest traces as impurities in

1 The stimulating action of poisonous substances was first observed by Raulin (Ann. d. sci. nat., 1869 , v. sér., T. XI, p. 252), but their general importance was established later. Cf. Richards, Jahrb. f. wiss. Bot., I 897 , Bd. xxx, p. $66_{5}$, and Pfeffer, 1895 , Bd. xxvin, p. 238 . See Sect. 66 ; Frank u. Krüger, Ber. d. Bot. Ges., I 894 , p. I ; Salm-Horstmar, Journ. f. prakt. Chemie, I 86r, Bd. Lxxxiy, p. I 4 o. On Fl, see also G. Tamman, Zeitschr. f. physiol. Chemie, I888, Bd. XII, p. 322.

${ }^{2}$ These are physiological reactions, and are quite distinct from the acceleration of a chemical reaction, which may be caused by the addition of certain substances. 
the water or salts employed, or dissolved from the walls of the glass vessel containing the culture fluid, may produce a marked effect, especially in fungi, \&c., although the traces present may be so minute that the tests employed fail to reveal them ${ }^{1}$. Even when the total amount present in the culture solution is excessively small, it is by no means negligible, for Benecke ${ }^{2}$ has shown that the presence of as little as $c .00003$ per cent. of potassium distinctly promotes the development of Aspergillus. Similarly it has not been found possible to completely suppress the development of fungi by the removal of all iron, even when the fungal power of collecting any iron present has been utilized by removing the first crop from the culture. Nor has a flowering plant ever been developed in the complete absence of silicon and sodium, a condition which, however, could only be secured by cultivation in a fluid which was not in contact with glass.

Both seeds and spores alway's contain a certain amount of the essential elements, and by means of these a bean may be able to develop as far as the formation of flowers when supplied with pure water, and may attain a dry weight two to four times greater than that of the secd ${ }^{3}$. In the absence of iron again, the first two or three leaves of maize or buckwheat seedlings become green at the expense of the iron stored up in the seed, while the subsequent ones remain chlorotic and without chlorophyll.

In a starved green plant, as rell as in a fungus, the iron and potassium may be removed from the older dying organs and transferred to the younger growing parts, so that growth may not immediately cease. Indeed continued development would be possible if, as is unfortunately not the case, the deficient element was necessary only during development of each new organ, and was then entirely available for use elsewhere.

The effect of the absence of any particular element can only be clearly distinguished by comparing the increase in weight of a plant grown under these conditions with that of a normal one, and it is best to use seeds or spores which contain but little stored nutriment. The entire absence of a non-essential substance is only possible when seeds are used which.were borne by plants grown in the absence of the element in question.

History and Methods. [Nehemiah Grew was perhaps the first to show that calcined plants yield an ash which contains various soluble salts (Anatomy of Plants, 1682, p. 258). Grew also found that different parts contain different amounts of ash, and that hardly any residual ash remains when starch is burnt.] It was some time after the atomic theory had been established that the

1 On the production of pure water, \&c., and the various precautions necessary, cf. the works of Benecke and Molisch. Glass vessels may be employed, which are covered internally with a film of paraffin.

2 Benecke, Jahrb. f. wiss. Bot., I $895, \mathrm{Bd}$. Xxvin, p. 502.

${ }^{3}$ Cf. Houssingault, Agron., Chim. agric., \&c., I 860, T. I, p. 6.4. 
ash constituents obtained from air and water were no longer regarded as the sole source of the plant's vital activity. The results obtained by Marrgraff ( 1764 ), Wiegleb (1774), Senebier, and especially de Saussure, were sufficient to convince unprejudiced observers that a plant contains only those elements which it derives from without. 'The opposite view for a time found supporters although it gradually disappeared, and was finally and decisively disproved by the exact experiments of Wiegmann and Polstorff, who showed that when plants were grown in pure sand, or fragments of platinum, the seedlings contained no more and no less ash constituents than were originally present in the seed ${ }^{1}$.

Senebier and de Saussure were probably the first to form correct ideas as to the importance of the ash constituents. In holding that the latter entered into the metabolism of the plant Senebier was considerably in advance of his time, for long afterwards the mineral elements were frequently regarded simply as stimulating agencies, or as solvents for the organic material conveyed through the plant. Sprengel, however, was the first to state that not all of the ash constituents are essential, and that the essential mineral elements have a specific importance and cannot be replaced by other elements ${ }^{2}$.

The general necessity of ash constituents was determined by the researches already mentioned, but which were the essential elements was first systematically investigated by Salm-Horstmar by means of the differential culture-method. A few experiments in this direction had previously been performed by de Cassincourt, John, Boussingault, $\& c$., but these were often inconclusive ${ }^{3}$. Both the latter authors used insoluble substrata, such as sand, quartz, and sugar-charcoal previously boiled with acid. Salm-Horstmar used similar soil saturated with a nutrient solution, and placed in tin pots lacquered with wax. Sulphur and pumice-stone have also been utilized for this purpose, but sand has been usually employed in late years, especially by Hellriegel (Sect. 69). In many cases sand-culture is preferable to water-culture, although in the latter case it is more easy to ensure the entire absence of any particular mineral constituent ${ }^{4}$.

The method of water-culture was first employed by Woodward, who showed that plants grew better in river than in rain-water, but best of all in a watery extract of soil. Woodward also used culture-solutions containing inorganic salts, but did not succeed in definitely proving the necessity of the mineral ash constituents. Duhamel simply grew plants in water, and such experiments have but little importance as compared with the previous ones by Woodward. The forgotten water-

1 Wiegmann u. Polstorff, Über d. anorg. Bestandth. d. Pflanzen, 1842. Cf. Kopp, Geschichte d. Chemie, 1845, Bd. 11I, pp. 42, 259 ; Sachs, History of Botany (Garnsey and Balfour), 1890, pp. 453, 524; John, Lrnährung d. Pflanzen, 1819 , p. 73.

2 Senebier, Physiol. végét., 1800, T. I11, pp. 28,45 ; Saussure, Rech. chim., 1804, p. 261 . Cf. Meyen, I'hysiologie, 1838 , Bd. I1, p. 120 ; C. Sprengel, Die Lehre vom Dünger, 1839 , pp. 1, 351, \&cc., and also in Bodenkunde, $1837, \mathrm{p} .4^{\mathrm{I}} 4$.

${ }^{3}$ Salm-Horstmar, Versuche u. Resultate ii. d. Emährung d. Pflanzen, $18_{5} 6$; a compilation of the works published from 1849 (Bd, XIVI) to 1855 (Bd. LXIV) in the Journ. f. prakt. Chemie; de Cassincourt, Journ. d. Pharmacie, $1818,{ }_{1}$. ${ }_{3} 81$; Boussingault in 1837 and 1838 , see ref. in Agron., Chim. agric., \&c., I860, 'T. 1, p. 3 .

* Hellriegel, Beiträge z. d. naturw. Grundlagen d. Ackerbaues, $188_{3}$, Unters. ii. d. Stickstoffnahrung, I888; Fittbogen, Versuchsst., I870, Bd. XiII, p. 8 I. 
culture method was resuscitated by Sachs, who succeeded in growing healthy plants by alternately transferring them from one solution, containing a portion of the ash constituents, to another which contained the remainder, while Knop grew plants successfully in a single solution containing all the essential mineral constituents. Numerous researches have been performed in the latter manner, and it has been found possible to obtain perfectly normal growth and development in various higher plants, when grown in watery culture - solutions, although it does not follow that all plants which grow in a soil rich in humus can be cultivated in this manner, for the root-system is under much more favourable conditions in such a soil than it is in a watery culturesolution $^{1}$ (Sec. 28 ).

In Fig. 6r the effects of the absence of iron and potassium are shown (cf. also Fig. 6o, p. 397). The glass vessel $g$ contains the nutrient fluid, and the plant is fixed in the median aperture of the porcelain lid by means of a halved cork previously soaked in parafin. Young seedlings are preferably employed and the seeds may be germi-

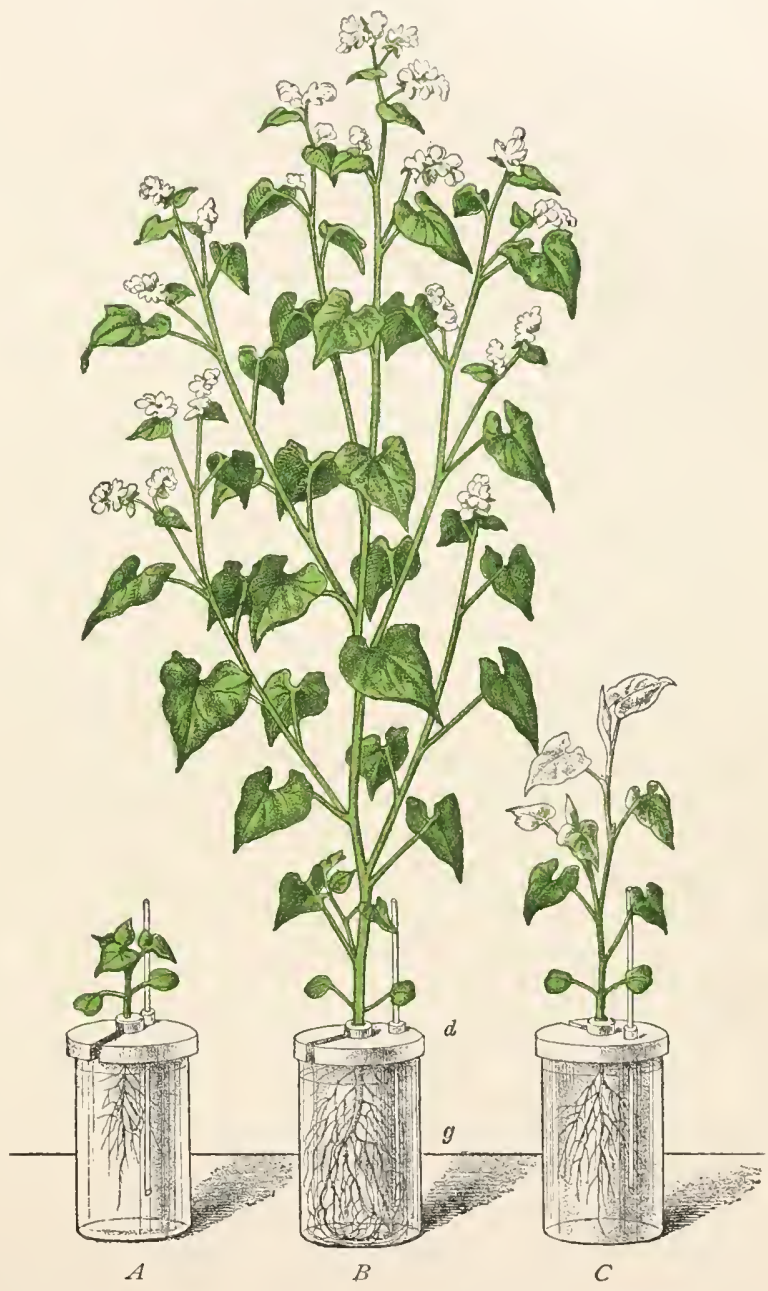

Fig. 61. Water-cultures of Buckwheat, $(A)$ without potassium, $(B)$ in normal nutrient solution, $(C)$ without iron. nated in sawdust, or between moist filter-paper, or when all absorption of mineral constituents is to be avoided from the commencement, they may be allowed to germinate on gauze netting

1 Woodward, Phil. Trans., 1699, Vol. xxi, p. 208; Duhamel, Naturgesch. d. Bäume, I765, Bd. II, p. I60 ; Sachs, Sitzungsb. d. Wien. Akad., I $8_{5} 8$, Bd. Xxvi, p. 33I ; Versuchsst., I860, Bd. II, pp. 22, 224; Knop, ibid., I86I, Bd. III, p. 295 ; Stohmann, Ann. d. Chem. u. Pharm., I862, Bd. Cxxi, p. $31_{4}$; and numerous works by Nobbe, Wolff, \&c., mentioned in Jahresb. f. Agr.Chem. for is6r and following years. 
wetted with distilled water. Roots removed from soil to a water-culture are more or less injuriously affected (cf. Sect. 26).

Good nutrient solutions are obtained by dissolving 4 grms. of calcium nitrate, I grm. potassium nitrate, I grm. magnesium sulphate, I grm. acid phosphate of potassium, $0.5 \mathrm{grm}$. potassium chloride in 7 litres of water $(=0.016$ per cent. of salt) or in 3 litres ( $=0.025$ per cent. of salt), and adding 3 to 6 drops of the medicinal solution of ferric chloride. Also $(a) 20.5$ grms. magnesium sulphate may be dissolved in $350 \mathrm{cc}$. of water and (b) 40 grms. calcium nitrate, 10 grms. potassium nitrate and 10 grms. acid phosphate of potassium in $350 \mathrm{cc}$. Then by adding $100 \mathrm{cc}$. of $(a)$ and $(b)$ to 9.8 litres of water a solution is obtained which contains 0.2 per cent. of salts, and to which only the addition of a few drops of ferric chloride, and in some cases of a little potassium chloride is necessary. Tollens gives a method by which concentrated nutrient solutions may be prepared in which no precipitate is formed ${ }^{\text {}}$.

The percentage composition of the nutrient solution may vary within comparatively wide limits, but growth is usually retarded by an excessive preponderance of sulphates or phosphates, and magnesium should be offered in less amount than calcium or potassium (cf. composition of ash constituents). The best nutrient solution for most Phanerogams contains from 0.1 to 0.5 per cent. of salts. For small plants and for demonstration experiments vessels containing 2 to 5 litres may be employed, but otherwise the use of larger vessels holding more than Io to 20 litres is to be recommended. In all cases the loss of water by evaporation, \&c. must be made good, while if small vessels are used an occasional renewal of the saline constituents will be necessary. The nutrient solution given above has an acid reaction, but the addition of a few drops of phosphoric acid is advantageous in order to prevent the fluid becoming alkaline? , though a little phosphate of iron is always precipitated. By occasionally blowing air through the culture fluid it is brought within reach of even the smallest roots, and at the same time the water is kept well aerated. Good illumination and a sufficiency of carbon dioxide are necessary for the growth of autotrophic plants, and hence the cultures should be kept in the open as much as possible ${ }^{3}$. Any over-heating of the culture fluid may be avoided by surrounding the culture vessel with sawdust, and this, at the same time, ensures that the roots are kept in darkness or exposed only to feeble light.

To secure the absence of a particular element, say potassium, sodium salts may be added in its place, or by using nitrates instead of suiphates the absence of sulphur may be assured. When a soluble salt of barium is added, and hence the absence of sulphates is necessary, the plants may be provided with sulphur in the form of isothionic acid, or taurin, or in the case of fungi by sulphur dioxide ${ }^{4}$. It must

1 Knop, Versuchsst., $188_{4}$, Bd. xxx, p. 293 ; Tollens, Bot. Jahresb., i 882, p. 36.

${ }^{2}$ Hellriegel (Unters. ii. d. Stickstoffnahrung, I $\$ 58$, p. I 40 ) states that lupines are sensitive to acid solutions. On the use of large vessels, cf. Wortmann, Bot. Zeitung, I $89^{2}$, p. 643 , and Nobbe, l. c.

3 The pots may be placed on trolleys travelling on rails, and run into a greenhouse when the weather is cold or stormy (Hellriegel, 1.c., p. I5).

* Sce Nobbe, Versuchsst., I870, Bd. X111, p. 331; Lüpke, Landw. Jahresb., I888, Bd. xv11, p. 889; Aschoff, ibid., I89o, Bd. xix, p. II 3 ; Schimper, Flora, I 890, p. 220. 
always be remembered, however, that the mere change in the composition of the nutrient solution directly affects the growth of the plant. 'The special requirements of each plant need also to be satisfied and thus many algae may be grown in still water, while others will only grow when the water is in continual motion, and for this and other reasons are not amenable to ordinary culture methods. Obligate marine algae require a saline medium, and hence will not grow in a dilute nutrient solution, but develop only under special cultural conditions ${ }^{1}$. The nutrition of fungi, and the solid and fluid nutrient media for heterotrophic plants in general, have already been dealt with (Sect. 66).

The concentration of the culture fluid is always important, for when its osmotic concentration passes a certain limit growth becomes impossible though no poisonous effect is exercised, while when the fluid is too dilute or when a single essential salt is present in insufficient amount the development of the plant is retarded. Hence there is a certain optimal concentration for every nutrient solution, and the presence of any one salt in excessive amount will lower the optinial concentration of the mixture.

The best nutrient fluid for Phanerogams contains 0.2 to 0.5 per cent. of salts ${ }^{2}$, and when the amount is increased to 2.5 per cent., many grow but little or not at all. The same result is produced by adding potassium nitrate or sodium chloride until the osmotic energy of the fluid corresponds to 2 per cent. of the former or I.7 per cent. of the latter, but in certain Phanerogams, and especially in Halophytes, growth ceases only when an osmotic concentration is reached which is equivalent to a 3 per cent. salt-solution ${ }^{3}$. Penicillium and other moulds, as well as certain bacteria, can grow in solutions which correspond in osmotic value to 20 per cent. of potassium nitrate, and certain of the lower algae have similar powers of accommodation, whereas other fresh-water algae are no more resistant than Phanerogams ${ }^{4}$.

The mininum concentration is not always extremely low, and indeed obligate marine algae can exist only in a strongly saline solution, although various forms may accommodate themselves to brackish and ultimately to fresh water ${ }^{5}$. Many plants probably exist, especially among fungi and bacteria, for which the minimal concentration is comparatively high, as according to Klebs is the case with Eurotium repens ${ }^{\circ}$.

A high minimum is not necessarily correlated with marked accommodatory

${ }^{1}$ Cf. A. Richter, Flora, $189^{2}$, p. 9 ; Klebs, Bot. Zeitung, 1891, p. 789 ; Die Bedingungen d. Fortpflanzıng, \&c., I896; Molisch, Sitzungsb. d. Wien. Akad., I 896 , Bd. cv, Atth. i, p. 634 . Algae as a general rule cannot withstand an acid solution. Oltmanns, Jahrb. f. wiss. Bot., I 892 , Bd. XxIl1, p. $28 \mathrm{I}$, and Flora, IS95, p. $5 \mathrm{I}$; Noll, Flora, I892, p. $28 \mathrm{I}$.

${ }^{2}$ Cf. Nobbe, Versuchsst., I $864, \mathrm{Bd}$. vi, pp. 40,343 , \&c. In sand-cultures a higher concentration is possible. Cf. Hellriegel, Jahresb. f. Agr.-Chem., I861-2, p. 115.

${ }^{3}$ Stange, Bot. Zeitung, 1892, p. 253 , and the literature there given.

${ }^{4}$ Eschenhagen, Einftuss d. Lösungen versch. Conc. auf Schimmelpilze, I889; Bruhne, in Zopf's Beiträge z. Physiol. u. Morph., I894, Heft 4, p. I ; A. Fischer, Jahrb. f. wiss. Bot., I 895 , Bd. xxvir, p. I51 (bacteria); Richter, Flora, I 892, p. 12; Stange, 1.c., p. 256 (algae).

${ }_{5}$ Oltmanns, Monatsl. d. Berl. Akad., I $89 \mathrm{I}$, p. 201 , and Jahrb. f. wiss. Bot., I $8_{92}$, Bd. Xxirf, p. $4^{\circ} 5$; Flora, 1895, p. $5^{1}$.

Klebs, Die Bedingungen d. Fortpflanzung. \&.c., i 896, p. 465. 
powers, for it is just those plants which can withstand the greatest concentration that can also accommodate themselves to the most dilute solutions. The question is not entirely a matter of turgidity, although an organism can accommodate itself to a concentrated solution only when it is able to increase the osmotic strength of the cell-sap to a corresponding extent. It has already been shown (Sect. 24) that this is partly attained by the penetration of the surrounding salts, and partly by a regulatory production of osmotically active substances. A rapid power of accommodation is possessed by many fungi and bacteria, as well as by those algae which are exposed to rapid changes from sea-water to fresh-water, but nevertheless a sudden difference in the concentration appears always to temporarily diminish the rate of growth ${ }^{1}$. Fungi may withstand a sudden diminution of concentration in the surrounding medium equivalent to a 5 per cent. solution of sodium chloride, but when the change is still more pronounced, mechanical ruptures may be produced owing to the enormous internal osmotic pressure coming freely into play.

In a concentrated nutrient solution many algae and fungi show a tendency to fragmentation, whereas the stem of Phanerogams usually becomes more or less condensed, and develops thicker and more fleshy leaves ${ }^{2}$. The influence of the solution is not merely due to its osmotic concentration, but the salt present appears to exercise a specific effect, for a solution of sodium chloride produces a more marked result than an isosmotic solution of potassium nitrate ${ }^{3}$.

\section{SECTION 74. The Functions of the Essential Eloments.}

The ash constituents are of importance only when they take part in metabolism, and hence the functions which any essential element, such as potassium or phosphorus, has to perform, are probably very varied in character, and cannot be determined until we know the entire series of processes in which the given element may take part from the time when it is first absorbed until it becomes of no further use to the plant. This is the ideal that physiology strives after, but our knowledge of the internal vital processes is at present extremely slight, and hence when we regard the living organism from a superficial stand-point we attach great importance to the carbon-compounds which preponderate in it, while the function of the ash constituents attracts less attention and may even be purposely neglected. So little is known of the parts played by the different mineral constituents that it is impossible to give a connected account of their importance in metabolism, and hence it must suffice to give the fragmentary information which has been gained concerning the importance of individual ash constituents and the changes they may undergo.

The fact that a particular element is an essential one gives no indication

1 True, Annals of Botany, IS95, Vol. 1X, p. 369 ; also see Eschenhagen, Stange, and Richter.

${ }^{2}$ For examples, see Eschenhagen, Stange, Kichter, Klebs; also Schimper, Indomalayische Strandfora, 1891 , p. 26 ; Dassonville, Rev. gén., I $S_{96}, 1 \mathrm{p} .28_{4}, 324$. On the influence upon starch-formation and $\mathrm{CO}_{2}$-assimilation, cf. Sects. 55 and $5^{\mathrm{S}}$; on transpiration, Sect. 39.

${ }^{3}$ Stange, 1. c., p. 366 . 
as to the part it plays in metabolism, nor does the analysis of the ash afford any evidence as to the form in which the different elements exist in the organism. The distinction between volatile and non-volatile products of combustion is of course a purely artificial one, though this, together with the widely different manner in which a green plant obtains its mineral constituents and carbon-compounds, has caused the two classes of substances to be regarded and dealt with from widely different points of view. As a matter of fact it is when they enter into combination with carbon-compounds that the ash constituents and the nitrogen become of physiological importance. Each mineral element may serve a variety of purposes, and the organic substances with which it combines may undergo such decomposition that these elements are released again in the form of inorganic compounds, while if the latter are continually reassimilated, the most active decomposition and reconstruction may not cause either an increase or a diminution in the percentage amount of a given element. The nitrogenous enzymes afford examples of substances which may induce marked changes without themselves being destroyed, and it is not impossible that certain of the ash constituents may have a similar functional importance. The frequent accumulation of potassium nitrate shows that the whole amount absorbed of an essential element does not necessarily enter into metabolism, or undergo metabolic metamorphosis; nevertheless such accumulation is possible only when the absorbed substance undergoes some change or other, the character of which is determined by the selective power which the plant possesses. Hence both the super-optimal absorption of an essential element, and the selective absorption of a non-essential one, are physiological processes due to the specific nature and vital activities of the plant under examination (Sect. 22).

It is probable that the really essential elenents take part in the composition of the protoplast. This certainly applies to sulphur, which is a constitutional element of most proteids, while several of the latter are rich in phosphorus (nuclein, \&c.), and the group of phosphorized substances known as lecithins may also be of great importance (Sect. II). Although sulphates and phosphates are found in abundance in the ash, the sulphur and phosphorus are probably present in the protoplasm in very different forms, for no sulphates or phosphates can be detected in resting seeds, and hardly any traces are present in the primary meristem, and even in the other tissues, when the plant is supplied with a minimal amount of sulphur and phosphorus ${ }^{1}$. When a seed germinates, readily translocating phospliates and sulphates are formed, while the proteids probably undergo further disintegration into amides or even ammonia.

Similarly iron occurs in the form of organic compounds, and it is

1 Schimper, Flora, 1890 , p. 223 ; Pfeffer, Jahrb. f. wiss. Bot., 1872, Bd. Vur, p. 475. 
probable that this is the case also with potassium and magnesium, for young organs rich in protoplasm as well as isolated masses of the latter contain potassium and magnesium, as well as nitrogen and phosphorus, in relatively large amount. Moreover, water does not extract from a dead cell all the potassium it contains, even though acids are present which form soluble salts of potassium, and in aleurone grains compounds of magnesium and calcium with proteids apparently occur.

The different mineral elements may be used to form various organic compounds, and hence the mineral constituents are not only present in the protoplasm, but also in the cell-sap and even in the cell-wall also. They may occur as salts of organic acids, and may also apparently combine with carbohydrates and other bodies. The soluble organic and inorganic salts of potassium and magnesium are very commonly utilized in the maintenance of turgidity (Sect. 24). although other substances are also of importance, and indeed since turgidity is only of accessory importance to the actual vital phenomena, it is not surprising to find that under certain cultural conditions sodium and calcium may partly replace potassium and magnesium, and must indeed do so in those cases in which an absorption of calcium or sodium does not cause any increase in the osmotic pressure ${ }^{\text {. }}$

It is certain that calcium may serve a variety of purposes in the plants which need it, and if, as appears probable. it may be absent from the primary meristem, it can only be of secondary importance, either to neutralize oxalic acid or to aid in the formation of the cell-wall, although when present it may enter into intimate relationship with the protoplasm *.

The results of chemical analysis afford but an approximate indication of the substances present in the living plant, for when death occurs various substances in each. cell come together which were formerly held apart. The precipitation or discolouration which may then take place is the outward sign of a few only of the various chemical reactions which are thus induced, and it is probable that the different substances which form an essential part of the protoplasm undergo more or less marked modification or dissociation as soon as life ceases (Sects. 7, I 1). In the form of organic compounds, magnesium and calcium, as well as phosphoric acid, may remain unprecipitated in a neutral or alkaline solution, and hence also in the plasma.

The function of an essential element is by no means directly indicated by the results which its absence produces, for, owing to the complex

1 Copeland (The relation of nutrieut salts to turgor, Bot. Gazette, Vol. xxiv, Dec., I897, p. 399) concludes that of the mineral constituents potassium salts play a far more important part in the maintenance of turgor than is generally supposed, but it is certainly not the case that potassium is necessarily of primary importance in all cases, viz. beet-root, onion bulbs, \&c. 7

${ }^{2}$ [Bokorny, Bot. Centralb]., Bd. Lxxiv, 1898, p. ${ }_{58} 8$. Molisch (Ber. d. Wien. Akad., I 895, Bd. CIV, p. 795) has shown that the partial absence of $\mathrm{Ca}$ causes an incomplete formation of the transverse walls in Spirogyra.] 
relationships and interactions which exist between the different vital activities, the cessation or abnormal performance of any one function may influence all the other functions as well (Sect. 4), so that the vital activity upon which the most marked external result is produced may be one in which the element in question takes no direct part. Thus, to take a concrete example, when growth is inhibited, the consumption and hence also the translocation of carbohydrates ceases, so that if the assimilation of carbon dioxide is possible the assimilatory products will accumulate in the leaves until the inhibitory limit is reached, and this result will be produced whether the stoppage of growth is due to a deficiency of potassium or phosphorus or to widely different causes. Similarly the absence of iron probably inhibits the formation of chlorophyll only in an indirect manner, for chlorophyll itself contains no iron.

Owing to the intimate correlations which exist between the different vital activities, an element essential only to a single function may exercise various stimulatory influences upon others. but it is incorrect to suppose that the ash constituents are of importance solely as stimuli to metabolic activity $^{1}$, for they undoubtedly form a constituent part of the protoplasm and influence by their respective affinities the course of various metabolic changes. The necessary supply of energy is for the most part obtained by the oxidation of carbohydrates, for the ash constituents are, as a general rule, absorbed in the form of compounds incapable of further oxidation. A few organisms, however, obtain energy from the oxidation of inorganic compounds, as is the case with the sulphur-bacteria and with nitrifying organi ms (Sect. 63).

No other element is capable of the variety of molecular combinations possible to carbon, and hence it is easy to understand why, under terrestrial conditions, carbon forms the most important and predominant element in all the compounds which build up the living organism, and which by their oxidation provide the necessary supply of energy. It is easy to understand that only those elements can have attained universal importance which have always been at the plant's disposal, but nevertheless though sodium enjoys a world-wide distribution, and is always absorbed by plants, it has not become an essential element. The same is the case with silicon, the most widely distributed of all elements, which in spite of its comparative insolubility may accumulate in large amount in certain plants.

Why the necessities of living organisms have developed as described, and why only certain of the universally present elements have become essential, remains at present a mystery, for neither from the known chemical or physical properties of these elements, such as their position in

1 This frequently-repeated idea has been recently combated by Gustarson (Bot. Jahresb., 1882, p. $3^{8}$ ). 
the periodic system, their atomic or molecular weights, their tendency to dissociation, their osmotic properties and the power of their salts to exchange ions, could the acquirement of this physiological importance have been predicted $^{1}$. The fact that none of the elements with high atomic weights are essential may simply be due to their rare occurrence and restricted distribution. Indeed if cacsium can replace potassium, it affords an example of an element with a high atomic weight acquiring important functions in the vital mechanisms of certain organisms. The atomic weights of the essential, and commonly present but non-essential elements, lie between that of iron $\left(5^{6}\right)$, and of hydrogen (1).

Iron. The absence of iron is at once perceptible in chlorophyllous plants, for they do not become green. but it seems to be of importance in many other ways, and is also essential to fungi. Moreover, since according to Molisch ${ }^{2}$ the molecule of chlorophyll contains no iron, the non-formation of chlorophyll may be merely a pathological phenomenon, for it is well known that even in the presence of iron the formation of chlorophyll may be partially or entirely suppressed when the plant is in an unhealthy condition. A small portion of the iron is apparently held by the plant in the form of organic compounds, and may thus take part in the integral structure of the plasma, and hence of the chloroplastids as well. It appears that dilute hydrochloric acid dissolves the iron from all proteids except nuclein compounds, and hence the iron present in seeds, \&c. seems to be mainly combined with nucleins. Moreover it is from plants that animals obtain the organic iron-compounds which they require ${ }^{3}$.

The necessity of iron for Aspergillus, Penicillium and other fungi was first conclusively proved by Molisch, although in spite of all precautions the development of the fungi could not be completely suppressed by its withdrawal. This is, however, readily explicable, owing to the fact that the merest trace of iron may suffice to permit growth. and hence various authors have concluded that it was not essential. When more than the optimal amount is present the growth of fungi may be accelerated owing to

1 Cf. Errera, Malpighia, I\$86; Sestini, Versuchsst., I8\$6, Bd. xxxı, p. 197 ; Benecke, Ber. d. Bot. Ges., 1894 , Generalvers., p. 115 . Nägeli's attempt (Bot Mitth., I881, Bd. IIr, p. 466) to explain the physiological difference between the alkalies and alkaline earths as due to the different relationships of the salts to water hardly requires discussion, for the ideas then curent as to the nutıitive value of the different elements were paitly erroneous, and on these Nägeli's conclusions were based.

${ }^{2}$ Molisch, Die Pfanzen in ihren Beziehungen zum Eisen, 1 892, p. S1.

s Neumeister, Physiol. Chemie, I893, I, p. $3^{11}$, and on nucleins containing iron, l. c., p. 40 ; Petit, Compt. rend., I $\$ 9^{2}$, T. CXIV, p. 246. The reagents Molisch employed caused him to erroneously believe that he could detect organic iron-compounds universally in plants, and hence it is doubtful whether the presence of organic iron-compounds in the plant has as yet been detected by microchemical means. Cf. Molisch, Ber. d. Hot. Ges., I 893, p. 73, and C. Miiller, ibid., p. 259. [Macallum's observations seem, however, to be trustworthy (sce Quar. Journ. Micr. Soc., Vol. Xxxviri, p. I75; Bot. Centralbl., 1893 , Bd. Lv, p. ${ }_{3} 8$ ), and on organic haematogen-like compounds in plants cf. Compt. rend., I $S_{9} 8$, cxxviII, p. 282.$]$ 
the stimulatory action already mentioned (Sect. 73), and the same occurs when in the presence of a minimal trace of iron a little cobalt, zinc, or manganese is added, showing that this minimal amount suffices for all requirements ${ }^{1}$. None of these metals can replace this minimal trace of iron, nor can they either in chlorophyllous plants, which do not turn green in the absence of iron although they may be supplied with salts of manganese, nickel, cobalt or aluminium².

E. Gris discovered that iron was essential to the higher plants, and it is easy to show that the formation of chlorophyll is dependent upon its presence. Thus if seeds containing but little iron, such as maize or buckwheat, are grown in a culture-solution free from iron, the first three or five leaves turn partially green, but the next one remains quite white (Fig. 6r). If a few drops of a solution of an iron-salt are added and transpiration is active these leaves begin to turn green in two to three days, the colour appearing first along the veins and thence spreading over the whole leaf. If the chlorotic leaves are painted with a dilute solution of iron they may also turn green, as was first shown by Gris. The colourless plastids which are at first formed soon undergo deformation, and when this occurs the power of recovery may be lost ${ }^{3}$.

So far as is known, a plant can make use of any compound of iron it can absorb, even potassium ferrocyanide, but since humus retains and decomposes soluble iron-salts a large quantity must be added to remove chlorosis. When growth is rapid and iron is supplied but slowly, as for example when plants in pots are forced to rapid development, the young shoots may pass through a chlorotic condition 4. During this condition the chloroplastids are functionally inactive and exhibit no power of assimilating carbon dioxide. This is not entirely due to the non-formation of chlorophyll, for the power of evolving oxygen may

1 Raulin did not therefore succeed in proving that iron was essential (Ann. d. sci. nat., I 869, v. sér., T. xI, p. 224), for he only showed that fungi grew more rapidly when the supply of iron was increased. On the other hand, Wehmer (Beiträge z. Kenntniss einheimischer Pilze, IS95, Heft 2, p. I 59) erroneously supposes that the sole action of iron is a stimulatory one. Cf. Richards, Jahrb. f. wiss. Bot., I $\$ 97$, Bd. Xxx, p. 674 .

2 Mn : Sachs, Experimentalphysiol., I $\$ 6_{5}$, p. I 44 ; also Birner und Lucanus, Versuchsst., I 866 , Bd. viII, p. I 40 ; Wagner, ibid., IS7I, Bd. xIII, p. 72 ; and Bertrand, Compt. rend., I897, T. CXXIV, p. Iо32. Ni: Risse in Sachs, Experimentalphysiol., p. 145. Al: Knop, Kreislauf d. Stoffes, I868, p. 614 .

3 E. Gris, De l'action d. composés ferrug. s. 1. végétation, $18_{43}$ and $18_{44}$, and in Compt. rend., I $8_{44-7}$. Confirmatory results by Salm-Horstmar, Vers. iiber die Ernährung d. Pflanzen, I $S_{5} 6$, pp. 8, I7; A. Gris, Ann. d. sci. nat., I 857 , iv. sér., T. VII, Pp. 201, \&c. Completely white leaves can only be obtained in Phaseolus multiflorus when the cotyledons are removed (Molisch, IS92, l.c., p. 92). Zimmermann, Beiträge z. Morph. u. Physiol., I $\$ 93$, I, p. 53. The action of Fe in aiding the development of blue flowers in Hortensia is still uncertain cf. Hoffmann, Bot. Zeitung, is 75 , p. 622 ; Unters. iber d. Variation, 1877, p. 20 ; Nolisch, Bot. Zeitung. 1897, p. 49 .

4 Sachs, Arb. d. Bot. Inst. in V'iurzburg, I 888 , Bd. I1 , p. 433. On the injurious effect of an excess of iron salts in the soil, ef. Sachsse, Agr.-Chem., 1888, p. 505; Thomson, Beibl. z. Bot. Centralbl., 1893, II I, p. 497 ; Petit, Bot. Centralbl., Is94, Bd. LIx, p. I 6 . K ${ }_{1}$ Cfy : Knop, Ber. d. Sächs. Ges. d. Wiss. 2u Leipzig, $186 y$, Bd. xxv, p. 8; Wagner, Versuchsst., Is7o, Bd x111, p. 74 . 
still be absent from partially green chloroplastids, which under other conditions are capable of photosynthesis.

Many plants, as Lemna trisulca, Trapa natans, \&., store up iron in large quantities (cf. Wolff, Ash Analyses), while an incrustation of ferric oxide is formed on the outer surface of others (Sect. 23). In these cases the accumulation of iron seems to be largely accidental, but it appears that certain bacteria may profit by it through obtaining energy by the oxidation of ferrous into ferric salts (Sect. 63 ). Ordinary plants are fully supplied with iron when 0.2 per cent. is present in the ash, so that an adult maize plant of 200 grm. dry weight and yielding 89 grm. of ash would contain $0.0 \mathrm{I} 6 \mathrm{grm}$. of iron, and the crop from a hectare $\left(2 \frac{\mathrm{I}}{2}\right.$ acres $=200 \mathrm{~kg}$. ash $)$ would remove 0.4 kilogrammes of iron from the soil.

Phosphorus is in all cases essential, for it forms a constituent of many proteids, and nuclein contains as much as 6 per cent. (Sect. II). It is not known what other functions phosphorus may discharge, and it is uncertain whether the widely distributed lecithin compounds ${ }^{1}$ are associated with proteids, or are of importance in fat formation, or have quite different functions to perform. Phosphorus is present in the form of an organic compound in lecithins, and in certain proteids, as well as in the globoids ${ }^{2}$ deposited in seeds. None of these produces any precipitate with an ammoniacal solution of magnesium sulphate, or with nitromolybdic acid. Reacting phosphates are liberated again by proteid decomposition and by metabolism in general, and they may accumulate to a marked extent in certain plants. Phosphates are usually present in a living cell in a dissolved form, but may after death be precipitated in combination with calcium, or magnesium ${ }^{3}$.

In all cases hitherto examined phosphoric acid affords the best source of phosphorus, and since pyro- and metaphosphoric acids readily change into orthophosphoric acid the former may also serve as a source of this element ${ }^{4}$. It is however possible that certain plants require or prefer their phosphorus in the form of organic compounds (Sect. 64), and fungi indeed are able to obtain all that they require from proteid compounds, while oats can partially satisfy their need for phosphorus by absorbing lecithin ${ }^{5}$. Sub-oxidized forms of phosphorus

1 On Lecithins, see Schulze und Frankfurt, Versuchsst., IS94, Bd, xLIII, p. 308 . On the tendency to asscciate with proteids, see Neumeister, Physiol. Chemie, IS93, I, p. 4I. Glycerinephosphoric acid may also be present in plants, and the frequently marked percentage of phosphorus contained in fats may be due to the presence of either of these compounds.

${ }^{2}$ Pfeffer (Jahrb. f. wiss. Bot., I $87_{2}$, Bd. VIII, p. $4^{6} 5$ ) obtained microchemical proof of the presence of $\mathrm{H}_{3} \mathrm{PO}_{4}$ as an organic compound in the globoids of seeds. Cf. also Schulze und Winterstein, Zeitschr. f. physiol. Chemie, IS96, Bd. xxII, p. 90 . On the microchemical detection of $\mathrm{H}_{3} \mathrm{PO}_{1}$ see also Zimmermann, Mikrotechnik, I892, p. 5 I ; Raciborzki, Bot. Zeitung, I893, p. 245 , and the literature here given; Polacci, Malpighia, IS94, Bd. VIII.

3 See Zimmermann, Mikrotechnik, IS92; Hansen, Flora, IS89, p. 44I ; Kohl, Kalksalze u. Kieselsäure, I889, p. II6. On calcium phosphate in living cells : Zimmermann, Beiträge z. Morph. u. Physiol., I 893 , p. 3 I 0 . On solution of calcium phosphate, see Vaudin, Ann. d. l’Inst. Pasteur, IS95, T. Ix, p. 636. On accumulation of phosphates: Schimper, Flora, I890, p. 222.

* Shown by manuring experiments by Eggerty and Nilson, Centralbl. f. Agr.-Chem., I893, p. 378 .

${ }^{5}$ Stocklasa, Bot. Centralbl., i 896, Bd. Lxvı, p. 64. 
are not assimilated by higher plants, but do not exercise any poisonous effect. Phosphorus cannot be replaced by arsenic or boron ${ }^{1}$.

A plant can absorb only those phosphates which may be rendered soluble; calcium phosphate may supply the large amount of phosphorus absorbed from a water-culture, as it is soluble in water containing carbon dioxide. If only phosphate of iron is present chlorosis is apt to occur unless the water is frequently agitated (Sect. 73). As the percentage of proteid increases so also does that of phosphorus, but the relation between nitrogen and phosphorus may vary within very wide limits ${ }^{2}$.

Sulphur forms a constituent of most proteids, and the quantity present is generally from 0.3 to 0.4 per cent., i. e. less than of phosphorus. Sulphur is also present in oil of mustard and in other similar compounds, while in seeds the greater part of the sulphur is frequently present in the form of organic compounds which give no precipitate with an acid solution of barium chloride, although during translocation they may be decomposed and form reacting sulphates. Certain bacteria produce sulphur and sulphuretted hydrogen (Sect. 102), from both of which Beggiatoa is able to obtain a supply of energy by oxidation ${ }^{3}$. Higher plants seem only able to assimilate sulphur in the form of sulphates, but fungi can make use of sulphurous and hydrosulphurous acids $\left(\mathrm{H}_{2} \mathrm{SO}_{3}, \mathrm{H}_{2} \mathrm{SO}_{2}\right)$, if they are present in sufficient dilution to be non-poisonous ${ }^{4}$. Both higher and lower plants seem able, however, to assimilate sulphur in the form of organic compounds such as taurin, or isothionic acid, or even certain sulpho-acids. Nägeli was unable, however, to nourish fungi with urea sulphonate and rhodanammonium.

The alkali metals. All research shows that in Phanerogams potassium can be replaced by no other element ${ }^{5}$, and it is still doubtful whether rubidium or caesium can be totally substituted for it in the case of fungi. Nägeli, Molisch, and Benecke all found that potassium could not be replaced by sodium, lithium or ammonium, indeed even comparatively dilute solutions of lithium salts exercise a distinctly poisonous action upon both higher and lower plants ${ }^{6}$. Sodium salts are, however,

1 Knop, in Bloneyer's Ber. v. Landw. Inst. zu Leipzig, 1881, pp. 31, 51 ; Ville, Compt. rend., 1861, T. LIII, p. 822 ; Molisch, Sitzungsb. d. Wien. Akad., I $\$ 96$, Bd. cv, Abth. i, p. 642 . Bouilhac (Compt. rend., 1894 , T. CxIx, p. 929) erroneously concluded that arsenic acid might replace phosphoric. Schleiden incorrectly supposed (Grundz. d. wiss. Bot., I 845,2 . Auf., Bd. II, p. 469) that $\mathrm{PH}_{3}$ could be directly assimilated.

${ }^{2}$ Literature by A. Mayer, Lehrb. d. Agr.-Chem., 1895 , 4. Auf., p. 264.

s Winogradsky, Bot. Zeiturg, 188 7, p. 489 . Cf. Sect. 63 . Sulphates, sic.: Schimper, Flora, I890, p. 222 ; Berthelot et André, Compt. rend., 189I, T. CXII, p. I22; Tamman, Zeitschr. f. physiol. Chemie, I885, Bd. IX, p. 4I7; E. Schulze, Landw. Jahrb., I892, Bd. xxI, p. I18. Cf. Sect. So.

* On the poisonous action of calcium sulphide on higher plants, cf. Fittbogen, Landw. Jahrb., I $88_{4}$, Bd. XIII, p. 755. Loew (Biol. Centralbl., I $89 \mathrm{I}, \mathrm{Bd}$. XI, p. $25 \%$ ) states that Spirogyra can assimilate methylsulphide. Nägeli, Bot. Mitth., 188r, Bd. III, p. 459. Phanerogams cannot obtain their supplies of sulphur from sulphites (Birner und Lucanus, Versuchsst., I $\$ 66$, Bd. viII, p. I $5^{2}$ ), and similarly Knop (1. c., I 88 I, pp. 3 I, 5I) finds that tisulphites do not afford an adequate supply of sulphur.

${ }^{5}$ Lucanus, Versuchsst., I866, Bd. viır, p. I46 ; Nobbe, ibid., I871, Bd. xiır, p. 399 ; Loew, ibid., I $8 ; 8$, Bd, Xxi, p. 389 .

${ }_{6}^{6}$ Nobbe, Versuchsst., I870, Bd. x111, p. 399 ; Gaunersdorfer, ibid., I8s 7 , Bd. xxxir, p. 175 For fungi, Benecke, Jahrb. f. wiss. Bot., I 895 , Bd. XXxirl, p. 507. On the occurrence of $\mathrm{Li}$ in 
innocuous, and may be present in the plant in greater abundance than potassium, though oats, barley, buckwheat, as well as typical saline plants, such as Salsola kali, Glaux maritima, and Psamma arenaria grow equally well in the entire absence of sodium ${ }^{1}$. Wiegmann and Polstorff reduced the amount of sodium present to the lowest possible minimum, but the complete absence of sodium can never be assured when glass vessels are used.

Potassium is presumably an integral constituent of protoplasm, and like nitrogen and phosphorus, and also magnesium, it is relatively abundant in embryonic tissues"; it is also found in combination with reserve and translocatory materials. It is possible that potassium may be intimately connected with the formation of carbohydrates, but nothing definite can be said at present, for when in the absence of potassium starch disappears from an illuminated leaf, this may simply be due to the setting up of a pathological condition. Such observations do not necessarily indicate that potassium is especially concerned in the translocation of carbohydrates as Liebig and Nobbe supposed. Bearing in mind the intimate correlation existing between different functions, it is not surprising that Nobbe found an excessive accumulation of starch in the leaves of plants of buckwheat, which grow but slowly owing to the presence of an excess of potassium sulphate and phosphate, nor that a similar result should be observed in other abnormal nutrient fluids or under special cultural conditions ${ }^{3}$.

Potassium is often present in supra-minimal amount, chiefly in the form of oxalates, nitrates, \&c. It is improbable, however, that potassium salts are indispensable for the maintenance of turgidity in primary meristem, and owing to their solubility they are very thoroughly removed from dying tissues, as also are those of sodium.

Chlorinc. A little chlorine is present in all plants, and not merely typical saline plants but many others have also a tendency to accumulate large quantities of metallic chlorides ${ }^{4}$. All plants, however, grow normally when the amount of chlorine present is very much reduced by the absence of sodium chloride, but probably no plant has as yet been grown in the entire absence of chlorine, and since its removal from a water-culture

plants, see Focke, Bot. Zeitung, 1873. p. 94. Rb has also been found in plants growing in the open. Both $\mathrm{Rb}$ and $\mathrm{Cs}$ are absorbed in large amount when present in the soil or nutrient solution.

1 Cf. Nobbe, Versuchsst., I 863, Bd. v, p. I33, and 1870 , Bd. xiII, p. 384 ; Birner u. Lucanus, ibid., I866, Bd. viII, p. $16_{5}$; G. Wolff, ibid., I868, Bd. x, p. 371; de Gassincourt, Journ. d. Pharmacie, I 8 I 8, p. 38 i ; Wiegmann u. Polstorff, Über die anorg. Bestandtheile d. Pflanzen, I $8_{4} 2$, p. 42. Cf. also Hoffmann, Bot. Zeitung, 1877 , p. 294; Weigelt, Ber. ïber d. Verh. d. Sächs. Ges. d. Wiss. zu Leipzig, I869, Bd. xxI, p. I9.

${ }^{2}$ First observed by de Saussure, Rech. chim., I804, p. 285. Cf. also Schimper, Bot. Zeitung, I8S8, p. 102, and Flora, I89o, pp. $22 \%, 26$ I.

3 Liebig, Die Chemie in ihrer Anwend. zur Agric., I876, 9. Aufl., p. 97 ; Nobbe, Versuchsst., I87o, Bd. XIII, p. 321 ; Schimper, 1.c., IS9o, p. 247. Cf. Brasch u. Rabe, Bot. Jahresb., I876, p. $88_{9}$; Knop u. Dworzak, Ber. d. Sächs. Ges. d. Wiss. zu Leipzig, I875, p. 53 ; Lüpke, Landw. Jahrb., x 888, Bd. XVII, p. 9I2.

t Cf. Wolff's Ash Analyses, 1. c.; also Mangin, Compt. rend., I88 3 , T. xCvi, p. 80 , and the literature quoted in Sect. 73 . 
has a distinctly injurious effect upon the plant ${ }^{1}$, it remains for precise researches to determine whether a minimal amount is essential, or whether chlorine simply favours growth under special cultural conditions. The latter is quite possible, for either chlorides may be specially adapted for certain metabolic processes, or the presence of chlorine may aid in the maintenance of the acidity of the nutrient solution (Sect. 23), or some accelerating action may be exercised, such as many non-essential or even poisonous substances are capable of exciting (Sect. 73). Traces of sodium chloride, though they exercise no poisonous action, may increase the aclivity of lactic fermentation. Metallic chlorides may favour the growth of fungi by diminishing the production of oxalic acid, which again exercises a retarding action only under certain cultural conditions ${ }^{2}$. Hence it is possible that the higher plants when grown in humus may dispense with chlorine, or that any favourable influence it has may be replaced by that of another element, while it has not yet been determined whether bromine and iodine may produce the same effect as chlorine. If sufficiently diluted, potassium bromide is not delcterious, nor is potassium iodide, although the latter is more apt to injure plants ${ }^{3}$.

It is still uncertain whether the action of salt upon the typical plants of the seashore is merely due to its osmotic power, or whether its influence is a specific one, and, if the latter is the case, whether the sodium or the chlorine is responsible for the changes of shape and of assimilatory activity which salt may produce, not only in strand-plants, but also in other plants as well.

The alkaline earths. Salm-Horstmar first proved the necessity of calcium and magnesium for Phanerogams, and this observation has since been confirmed by several investigators. Molisch and Benecke have recently shown that fungi and certain algae require magnesium, but not calcium. Before their researches A. Mayer had developed yeast, Raulin Aspergillus, and Winogradsky Mycoderma vini in the absence of calcium, but owing to Nägeli's erroneous assumption that in fungi the alkaline earths may replace one another, these results were capable of a different interpretation. The experiments on which Nägeli's conclusions were based must have been performed with impure salts, for when proper precautions are taken, the fact that magnesium is essential may easily be demonstrated ${ }^{4}$, and

${ }_{1}$ Nobbe, Versuchsst., IS65, Bd. viI, p. 371 , and I 870 , Bd. XIII, p. 394; Beyer, ibid., I \$69, Bd. XI, p. 262 ; Wagner, ibid., IS7I, Bd. XIII, p. I 28 ; Aschoff, Landw. Jahrb., IS9o, Bd. XIX, p. II3. According to Knop and Dworzak (Ber. d. Sächs. Ges. d. Wiss., IS75, p. 6I), maize develops normally in a nutrient solution free from chlorine.

2 Wehmer, Bot. Zeitung, IS91, p. 374. Lactic fermentation : Richet; Compt. rend., IS92, T. CXIV, p. I494.

${ }^{8}$ Dircks, Ber. d. Sächs. Ges. d. Wiss., IS69, p. 20; Knop, ibid., I885, p. 44; Loew, Flora, $189^{2}, \mathrm{p} \cdot 374$.

4 Ad. Mayer, Unters. ïber d. Alkoholgährung, I\$69, P. 44; Raulin, Ann. d. sci. nat., I 869 , v. sér., T. XI, p. 224; Winogradsky, Bot. Centralbl., ISS 4 , Bd. Xx, p. I67 ; Nägeli, Bot. Mitth., I 88 I, Bd. III, p. 46 I. 
Molisch found that it cannot be replaced by barium, strontium, beryllium, zinc, or cadmium, and none of these elements can replace calcium in those plants to which it is essential ${ }^{1}$.

The physiological difference between calcium and magnesium is shown in the higher plants, for magnesium has a distribution and importance somewhat similar to potassium, whereas but little calcium is found in young tissues, or in storage organs where the ash constituents are present in reduced amount. In adult organs however the percentage of calcium usually increases both relatively and absolutely. This calcium is for the most part not utilized further, and remains in the organ when it dies, commonly in the form of crystals of calcium oxalate, but also, though more rarely, impregnating the cell-wall either in amorphous form, or as cystoliths of calcium carbonate (Sect. 23). Calcium salts may also be present in solution in the cell-sap, and it is possible that they may unite with the organic substances which build up the protoplast ${ }^{2}$.

Calcium cannot have any general importance since fungi and certain algae may grow without it; in other plants, however, it may acquire such importance for special functions that these cease or are performed abnormally in its absence, and this may so affect the entire organism as to render further development impossible. It is uncertain whether the union of calcium salts with the constituents of the cell-wall may not exercise some influence upon the secondary growth of the latter, for the fact that calcium is not essential to certain plants simply indicates that it is not necessary for the formation and growth of the cell-wall in the primary meristem. In the absence of calcium, only the stalk of the cystolith can be formed, and according to Mangin ${ }^{3}$ the chalk in the body of this structure is derived from calcium pectate. It is probable that similar calcium-compounds may be present in most cell-walls.

Calcium may be necessary in certain plants in order to prevent a poisonous accumulation of soluble oxalates by removing oxalic acid in an insoluble form, but this can hardly be its general importance, as Schimper supposes, for in many plants calcium oxalate is either absent or present in very small amount. This may be the case even in those which produce an abundance of oxalic acid and which are injured when supplied with potassium oxalate as readily as other plants

1 Ba: Knop, Versuchsst., I\&66, Bd. vi11, p. I43. Sr and Be: Benecke, Bot. Centralbl., 1894, Bd. Lx, p. I95, and Jahrb. f. wiss. Bot., 1895, Bd. Xxv111, p. 519. Sestini erroneously supposed that Be could replace $\mathrm{Mg}$ (Bot. Jahresb., 189I, p. 27) For algae, cf. Molisch, Sitzungsb. d. Wien. Akad., 1895 , Bd. CIV, Abtì. i, p. 783. On partial replacement, cf. Sect. 73. Ba and Sr are generally absent from plants in nature, but both have been found. Cf. Forchhammer, Ann. d. Phys. u. Chem., 1855 , Bd. $x \mathrm{Cv}$, p. $S_{4}$; Boedecker u. Eckhardt, Ann. d. Chem. u. Pharm., 1856, Bd. C, p. 294; Dworzak, V'ersuchsst., 1874 , Bd. xv11, p. 398 .

2 On the distribution of $\mathrm{Ca}$, cf. Kohl, Kalksalze u. Kieselsäure, I889; also Zimmermann, Mikrotechnik, 1892, p. $5^{6}$. Hansen (Mitth. a. d. zool. Station zu Neapel, 1893 , Bd. xi, p. 258) found only traces of $\mathrm{Ca}$ and no $\mathrm{Mg}$ in the cell-sap of l'alonia, but in other cell-saps both elements are undoubtcdly present.

${ }^{3}$ Melnikoff, Unters. über d. Vorkommen d. kohlens. Kalkes, I877, p. $32 ;$ Kohl, l. c., p. I4. For further literaturc see Sect. 23. Mangin, Compt. rend., 1892, T. Cxv, p. 260; Rech. anat. s. 1. composés pectiques, 1893, p. $4^{8}$. 
are (Pfeffer) ${ }^{1}$. It is moreover probable that the plant is able to regulate the production of oxalic acid according to the amount required to decompose salts of calcium or of other metals, as is indeed actually the case in fungi ${ }^{2}$. If it were found possible to induce a formation of calcium oxalate in plants from which it is normally absent by supplying them with an excess of calcium, the precipitation of calcium oxalate would be clearly only an accidental phenomenon. In Mesembry' anthemum a formation of free oxalic acid does actually occur, but ceases before it accumulates to an injurious extent.

Whatever part calcium plays, its absence may indirectly affect one or all of the vital activities. Hence it is impossible to make any logical deductions as to its direct functional importance from the effects its absence produces upon the translocation of proteids or carbohydrates, or upon the growth of the cell-wall ${ }^{3}$; nor can any conclusion be drawn from the fact that calcium may enter in combination with proteids or carbohydrates, for the same thing may also take place in plants to which it is not essential.

If it is true that calcium may be absent from primary meristem and from young organs, then it is evident that it is not essential for vital activity in general, but only for certain special processes. Schimper was unable by micro-chemical tests to detect any calcium in young shoots of Tradescantia Selloi which had developed normally in its absence, but it is possible that a trace might have been derived by such young organs from the older parts of the plant. In other cases, however, calcium is certainly not essential, and hence Loew's hypotheses as to the part it plays in the vital mechanism no longer have any general importance ${ }^{4}$.

In nature, calcium is widely distributed, and hence the plant probably always has a sufficient supply at its disposal, so that it involves no disadvantage when, as is often the case, the seed contains less calcium than is necessary for the utilization of all the reserve material. In such cases the addition of a calcium salt may favour development ${ }^{5}$, while in others even a trifling amount may exercise a distinctly injurious effect ${ }^{n}$.

1 Schimper, Flora, 1890, p. 246 . Cf. also Groom, Annals of Botany, 1896, p. 95 ; Kohl, l. c., p. 64 .

2 Cf. Sects. 85 and 86 ; also Sect. 71 for connexion with proteid formation.

s Nobbe, Versuchsst., 1870 , Bd. xirr, p. 323 ; Raumer u. Kellermann, ibid., 1880, Bd. xxv, p. 25 ; Liebenberg, Sitzungsb. d. Wien. Akad., 1881, Bd. Lxxxrv, p. 447 ; Prianischnikow, Versuchsst., 1894, Bd. XLv, p. 274. Schimper's results (Flora, 189o, p. 247) do not prove any direct dependence of carbohydrate translocation upon the presence of $\mathrm{Ca}$.

${ }^{1}$ Schimper, Flora, 1890, p. 245 . Cf. Loew, Flora, I 892 , pp. 368,373 ; also Bot. Centralbl., 1895 , Bd. LxiIr, p. I61. [Loew, Bot. Centralbl., is98, Bd. Lxxir, p. 257.]

${ }_{5}^{5}$ Liebenberg, l.c. p. 405; Böhm, Sitzungsb. d. Wien. Akad., I 875, Bd. Lxxi, Abth. i; Stohmann, Ann. d. Chem. u. Pharm., I\&62, Bd. Cxxi, p. 319; Prianischnikow, l.c.

${ }^{6}$ See Lidforss, Jahrb. f. wiss. Bot., $189^{6}$, Bd. xxix, p. $3^{6}$ Pollen grains); Correns, Bot. Zeitung, 1896, p. 26 . 


\section{SECTION 75. The Non-Essential Ash Constituents.}

Just as plants may absorb an excess of their essential constituents, so also may non-essential mineral substances be absorbed in greater or less amount. Thus, probably, no plant grown wild is without silicon and sodium, and these elements may sometimes form the greater portion of its ash. Many plants also accumulate perceptible amounts of aluminium, manganese, and zinc, and although marine plants contain relatively but little iodine or bromine (Sect. 22), still it requires a comparatively intense absorptive power to obtain this amount from the minute traces of iodides and bromides present in sea-water. Such accumulation is an example of selective absorption, and is due to the fact that the substance absorbed is converted into an insoluble form or into a non-diosmosing compound. Whenever a diosmosing substance undergoes modification of this character, it must necessarily accumulate in the plant whether it is useful or not, and in this manner useless substances may be absorbed, which under normal circumstances the plant never encounters, such as methyl-blue and other aniline dyes. Similarly a plant may accumulate large quantities of poisonous bodies, if they are presented in such dilute form that an injurious concentration is never reached during the endosmosis through the plasma. The poisonous metallic salts are retained by humus with considerable tenacity and presented to the plant in very dilute form (Sect. 28). Zinc salts may, for example, be accumulated in large amount, although even a very dilute solution is extremely poisonous.

Selective power is always a physiological problem, for it is determined and regulated by the present or previous vital activity. Many other elements inay accumulate to a marked extent when presented to the plant, though, owing to their rarity, they are usually absent from the ash, as for example is the case with rubidium and beryllium. The fact that a substance is passively secreted in this manner does not necessarily indicate that it takes part in metabolism, for accumulation occurs whenever an insoluble compound is formed by combination with any metabolic product, while the removal of such solvents as carbon dioxide, \&c. may cause a precipitation of the penetrating substance, as silica or chalk for example. Nevertheless nonescential elements frequently become involved in metabolism, and are utilized to a certain extent, as is shown by their partial substitution for essential elements and by other facts (Sects. 73 and 74). Thus the nonessential elements, such as manganese, cobalt, or zinc, may in certain cases favour growth. It is, however, still doubtful whether chlorine is necessary or advantageous always, or only in special cases. Even sodium may be of use to the plant when the supply of potassium is reduced to the lowest possible minimum, and it has yet to be explained why a lower 
minimum of a particular essential element suffices when the others are present in abundance. Just as calcium is necessary to most plants, but not to all, so also may silicon or similar elements be essential to a few plants only. In a condition of nature, where the competition with other organisms is severe, the trifling assistance afforded by a non-essential substance may be of decisive importance, although in cultivated and protected plants no perceptible favourable influence may be exercised by the substance in question (Sects. 76, 92).

Silicon. Sachs first showed that plants could be developed in a nutrient solution containing no silica, and he harvested a maize plant which contained only 0.7 per cent. of this substance in its ash, instead of the normal 18 to 23 per cent. Knop and others have proved that silica is not essential to other grain crops, and Jodin successfully cultivated maize through four generations without it, so that the percentage originally present was reduced to the lowest possible minimum ${ }^{1}$. It has not yet been conclusively proved that silica is not essential for diatoms and for shave-grass (Equisetum hyemale) in which it is especially abundant, while siliceous cystoliths can hardly develop normally when silica ${ }^{2}$ is deficient.

Silica is usually deposited in the cell-wall, more rarely in opal-like masses within the cells, or in intercellular spaces. The tabaschir, sometimes found in the hollow internodes of the bamboo, resembles an opal in being a watery crystalline form of silica ${ }^{3}$. It is possible that organic compounds of silicon may be formed transitorily or in special cases only, but researches in this direction have yielded indecisive results ${ }^{4}$. It is still uncertain whether the silica enters into the metabolism of the plant, or whether the absorbed silicates are simply decomposed and the silica deposited. In any case if silica is directly absorbed in solution it must be deposited as soon as the solvent is removed ${ }^{5}$. The deposition of silica must be partly due to the specific properties and metabolic activity of the plant, for an abundant deposit of it is formed in submerged Diatoms, while in special cells of higher plants grains of silica may be deposited. In terrestrial plants transpiration not only aids in the absorption of silica but also in its deposition in the tissues, for the latter takes place most markedly in the epidermal walls, although the amount deposited is by no means directly proportionate to the transpiratory activity either in the same or in different plants. The silica gradually accumulates until in the ash of grasses it may form 50 to 80 per cent. of the whole, whereas in young organs the merest traces may be present.

In those plants which accumulate silica its presence may be of some use,

${ }^{1}$ Sachs, Flora, I862, p. 52 ; Knop, Versuchsst., I862, Bd. III, p. 176; also Rautenberg u. Kühn, ibid., I 864, Bd. vi, p. 359 ; Birner u. Lucanus, ibid., I866, Bd. vill, p. I 4 I ; Jodin, Ann. d. chim. et d. phys., I $88_{3}$, v. sér., T. $\mathrm{xxx}, \mathrm{p} .4{ }_{5}$.

${ }^{2}$ Kohl, Kalksalze u. Kieselsäure, I859; Zimmermann, Beiträge z. Morph. u. Physiol., I\$93, p. 306. On cystoliths of calcium carbonate, cf. Sect. 74 .

${ }^{3}$ For the distribution of silica, see Kohl, 1. c., p. 228. On siliceous bodies, see also Strasburger, Bau u. Verrichtung d. Leitungsbahnen, 1891, p. $3^{6} 7$.

+ Ladenburg, Ber. d. Chem. Ges., I8 72 , Bd. 1 , p. 568 , and Lange, ibid., I 878, Bd, xI, p. 823 .

3 Lange detected dissolved silicn in the acid sap of Equisetum. 
and it may even aid in metabolism, for Kreuzhage and Wolff ${ }^{1}$ found that oats fruited badly when grown in nutrient solutions free from silica. Similarly the presence of silica seems to economize the other ash constituents, while the hardness and brittleness which it confers may act as a protection against the ravages of animals and the penetration of fungi ${ }^{2}$. The deposition of silica does not seem to increase the strength and rigidity of the cell-walls, nor is the laying of crops after heavy rain due to the lack of silica, but rather to the partial etiolation of the basal portions of the stems of thickly-sown plants. Hence laying may be avoided by the use of the drill when the grain is sown. Cereals form haulms of normal strength when grown in the absence of silica, and Pierre found that laid crops actually contained more silica than normal ones ".

As regards the other non-essential elements, only their presence in the ash or the fact of their absorption has been determined, and since no physiological function has as yet been ascribed to any of them, it will suffice to mention a few points of general interest.

Zinc appears, according to Risse, to be present in all plants which grow upon a soil rich in this metal, as, for example, at Altenberg near Aix-la-Chapelle, where more than 20 per cent. may be present in places. In many such plants a large amount of zinc accumulates, for Risse found that the roots of Thlaspi alpestre contained in 100 parts by dry weight 0.167 parts of zinc oxide, or $\mathrm{r} .66$ per cent. of the total ash, the stem contained 0.385 or 3.28 per cent. of the ash, and the leaves 1.50 or 13.12 per cent. of the ash. Viola tricolor, Armeria vulgaris, Silene inflata, \&c., were also rich in zinc, which, like calcium, was usually very abundant in the leaves. Zinc is absent from most soils, and when but a trace is present no marked absorption seems to occur ${ }^{4}$. Zinc salts can be presented to the plant only in extreme dilution, for Baumann found that a watery solution containing more than 5 milligrammes of zinc sulphate to the litre soon acted injuriously upon Phanerogams. The zinc is probably deposited chiefly in the cell-walls and in some form which exercises no poisonous effect upon the protoplasts.

According to Hofmann ${ }^{5}$ the calamine violet (Viola lutea var. multicaulis) does not alter when grown in soil free from zinc, nor does cultivation upon soil rich in this metal produce any morphological effect upon Viola tricolor and Thlaspi alpestre. Risse observed an especially active growth in plants of Silena inflata and Armeria

1 Versuchsst., 1884, Bd. $\mathrm{xxx}$, p. I6I.

${ }^{2}$ Animals : Stahl, Yflanzen u. Schnecken, 1888, p. $7^{2}$; Kohl, 1.c., p. 304 . Fungi : Johnson, Wie die Feldfrüchte wachsen, Liebig, 1872 , p. 205.

s Pierre, Compt. rend., I 866, T. LXIII, p. 374: L. Koch, Landw. Centralbl., i 872, Bd. II, p. 202. Sorater (Bot. Jahresb., 1873, p. 521) and C. Kraus (Forsch. a. d. Geb. d. Agr.-Phys., 1890 , Bd. XIII, P. 252, and $\mathrm{x} 891, \mathrm{Bd} . \mathrm{x} I \mathrm{r}, \mathrm{p} .59$ ) have shown that laying may be induced by other causes as well.

${ }^{4}$ Cf. literature on absorption of $\mathrm{Zn}$ and its presence in plants, given by Baumann, Versuchsst., 1885 , Bd. Xxxi, p. 1. Kisse (Sachs' Lxp.-physiol., 1865, p. 153) and Jensch (Chem. Centralbl., 1894 , I, p. 281 ) observed a similar absorption of $\mathrm{Zn}$ from metallic solls in Upper Silesia.

${ }^{5}$ H. Hoffmann, Bot. Zeitung, 1875, p. 628; Unters. über Variation, 1877 , p. 36 . The calamine violet is found in other districts as well. 
growing upon the soil where zinc was most abundant, and this was probably due to the stimulatory action which small doses of poisonous substances may exert upon growth (Sect. 73).

The other metals of the zinc group, magnesium, beryllium, and cadmium, have been dealt with in the preceding section, where an account is also given of the non-essential alkaline metals and alkaline earths.

Aheminium, though universally distributed, is present only in small amount in most plants, except in Lycopodium Chamaecyparissus and L. alpinum, where it forms 22 to 27 per cent. of the ash. It is also present in abundance in Chlorangium Jusuffi ${ }^{1}$, whereas according to Church in Lycopodium phlegmaria, Selaginella, \&c. mere traces are present. It is uncertain whether the alumina is present in Lycopodium in the form of a tartrate as is stated by Arosenius.

Manganese, though usually much less abundant in the soil than aluminium, seems to accumulate in plants to a more marked extent, and small amounts seem always to be present. The ash of Trapa natans may contain 7.8 to 14.7 per cent. of red oxide of manganese, and a marked accumulation has been observed to occur in many Phanerogams and Cryptogams ${ }^{2}$. The accumulation probably proceeds in a similar manner to that by which an excess of iron may be absorbed. Manganese is also present in the incrustation of iron-oxide formed on or in certain bacteria.

Cobalt and Nickel have been detected by Forchhammer in oak wood. Nickel seems to be more poisonous than cobalt, and the stimulatory action of both metals has already been mentioned.

Copper is frequently present in minute quantities, and the ash of plants grown in soil rich in this metal may contain I per cent. The copper accumulates mainly in the older organs, and since it is extremely poisonous it can only be absorbed in minute traces at a time. Even the extremely poisonous Mercury was detected by Gorup-Besanez in plants grown on soil containing mercuric oxide, and traces of silver have been detected by Malaguti and Durocher in Fucus.

Lead is frequently present. The equally poisonous Thallium has also been found in certain cases.

The poisonous character of Chromium, Tunosten, Molybdenum, Bismuth,

1 Wolff, Aschenanalysen, I 87 I, pp. I 34, 136 ; Church, Proc. Roy. Soc. London, I888, Vol. xuIv, p. I 2 I ; Berthelot et St. André, Compt. rend,, I 895 , T. cxx, p. 288 ; Yoshida, Bot. Jahresb., I89o, p. 50 .

2 The analyses given by Wolff (1.c.) are not quoted in detail among the following references. Manganese: Ebermayer, Physiol. Chem., I 882, p. 795 ; Maumené, Bot. Jahresb., I886, p. SI ; Molisch, Die Pflanze in ihren Beziehungen zum Eisen, I892, p. 7I. Cobalt: Ann. d. Chem. u. Phys., I855, Bd. xcv, p. 86. Copper: Lehmann, Archiv f. Hygiene, I896, Bd. xxvit, p. I, and Poi. Centralbl., I896, Bd. Lxvin, p. $5^{6}$; C. Miiller, Zeitschr. f. Pflanzenkrankh., 1894, Bd. Iv, p. I42; Otto, ibid., I 893 , Bd. 111, p. 222 ; Tschirch, Das Kupfer, I $\$_{93}$. Mercury: Ann. d. Chem. u. Pharm., I86., Bd. Cxxvit, p. 24 s. Silver: quoted by Raulun, Ann. d. sci. nat., I869, v. sér., T. XI, p. 98. Lead: Tschirch, Das Kup'er, I893, p. I5; Hattensaur, Bot. Jahresb., I\$9o, p. $4^{8}$; Knop, Ber. d. Sächs. Ges. d. Wiss., IS85, p. $5^{1}$; Nobbe, Versuchsst., I8S4, Bd. xxx, p. 4I6; Phillips, Bot. Centralbl., I8s 3 , Bd. xıIl, p. $3^{6} 4$, \&.c. Thallium: Böttger, Jahresb. d. Agr.-Chemie, 1864, p. 99 ; Knop, l. c., p. 50. 
Vanadium has been shown by Knop. Zinc has been detected by Forchhanmer in certain woods, Titanium by Salm-Horstmar in cereals.

Arsenic has been frequently found in plants ${ }^{1}$. Arsenious acid is extremely poisonous, whereas many, both of the higher and of the lower plants, can withstand large doses of arsenic acid and can accumulate large quantities of arsenic when supplied to them in this form. Gosio has observed that various fungi evolve arseniuretted hydrogen in the presence of carbohydrates and arsenic compounds.

Boron is absorbed whenever present and traces are found in many plants growing in the open. Borates are rather poisonous, but according to Loew less so in the case of certain algae.

According to Knop, Telluric acid is not markedly poisonous, but selenic and selenious acids are. All three acids have been detected in Phanerogams.

Fluorine has only been found in a few cases, but traces may be frequently present in plants, for fluorides are found in bones, eggs, \&c. According to Tamman soluble metallic fluorides are comparatively poisonous.

Iodine and Bromine occur in marine plants but may be found in terrestrial plants as well, for the latter can absorb iodides and bromides. Both are probably present as soluble salts, and Golenkin's supposition that free iodine or a coloured iodine-compound may be present in the vacuoles of living cells of Derbesia Lamourouxii requires further proof.

\section{SECTION 76. The Influence of the Quality of the Soil upon the Distribution of Plants.}

Climatic conditions, especially the temperature and the amount of moisture, are the main factors in determining the geographical distribution of plants, for an autotrophic plant can obtain sufficient nutriment from any fertile soil. Nevertheless, all those plants do not develop upon a given area which might do so if protected from competition, for the latter influences the distribution of plants to a great extent, and is often responsible for the localized range of a particular species. The influence of competition is admirably shown when a garden is allowed to run wild,

1 Arsenic : Gorup Besanez, 1.c.; Daubeny, Jahresb. d. Chemie, 1861, p. 736; E. W. Davy, Jahresb. d. Agr.-Chemie, $1860-6$ I, p. 83 ; Targioni-Tozzetti, Ann. d. sci. nat., I8 46 , iii. sér., T. v, p. 177 . Arsenious acid : Nobbe, Versuchsst, I884, Bd. xxx, p. 394. Arsenic acid: Knop, 1.c., p. 49; Loew, System d. Giftwirkung, I 893. p. I 9 ; Molisch, Sitzungsb. d. Wien. Akad., I896, Bd. Cv, Abth. i, p. Io ; cf. Stoklasa, Zeitschr. f. landw. Versuchsst. in Oesterreich, I898, p. I5 ; Gosio, Jahresb. iiber Gährungsorganismen, I893, p. 83. Boron: Hotter, Versuchsst., I890, Bd. xxxviI, p. 435 ; Bechi, Bot. Jahresb., I891, p. 30 ; Brand, Bot. Centralbl., I894, Bd. LX, p. I89; Loew, Flora, 1892, p. 37. Fluorine: Salm-Horstmar, Jahresb. d. Chemie, IS6o, p. 540; Wilson, ef. Ad. Mayer, Agr.-Chemie, I895, 4. Aufl., p. 292; Tamman, Zeitschr. f. physiol. Chemie, I888, Bd. XII, p. 323. Iodine and Bromine: Dircks, Ber. d. Sächs. Ges. d. Wiss., I869, p. 20 ; Knop, ibid., I885, p. 44 ; Golenkin, Algol. Notizen, Bull. d. l. Soc. Imp. d. Naturalistes, Moscow, I894, p. I. 
or when a number of different plants are sown closely together. Various conditions decide which plants will survive, and a change in the quality or quantity of the nutriment supplied or in other cxternal factors may cause a different result to be produced. Thus in a soil kept excessively moist a particular type of plant may conquer, while the physical properties of the soil, such as its texture, consistency, depth, \&c., may exercise a decided influence upon the struggle for existence, and a very trifling difference may suffice to change the course of events and enable a plant to survive which was less adapted to the previous conditions.

It is possible indeed that a plant may be suppressed in a rich soil, whereas in a soil deficient in one or more of the essential ash constituents it may gain the upper hand, either because of its more modest requirements or because by means of a more highly developed root-system it is able to exhaust the soil, and especially the deeper layers, more completely. Further, plants which can assimilate free nitrogen are at a distinct advantage in a soil poor in combined nitrogen. Again, Glanx, Salsola, and other saline plants grow perfectly well upon soil free from salt, although in nature the competition with other plants restricts them to a saline habitat, where their growth is perhaps somewhat more luxuriant than in an ordinary soil and where most plants are unable to compete with them. The presence of a certain amount of salt seems to be an essential condition for the existence of many marine algae (Sect. 73).

Similar relationships are responsible for the specific floras of siliccous and calcareous soils, and when these are in close proximity the transition from the one flora to the other may be extremely abrupt, although ubiquitous plants may flourish in both soils. The old supposition that these differences were due to the amounts of calcium or of silicon required by the plants in question is obviously incorrect, and, as a matter of fact, the former is essential to all Phanerogams, and may be found in greatest abundance in the ash of plants which prefer a sandy soil. Moreover, almost all plants characteristic of sandy soils may be readily grown in calcareous humus and vice versa, while the various other factors which regulate distribution may bring it about that plants typical of calcareous regions may be found growing wild on sandy soils ${ }^{1}$. Hence, when the addition of chalk causes certain plants to be driven out from a given area $^{2}$, it does not follow that they are unable to grow upon the changed soil. The presence of chalk does, however, appear to act injuriously upon certain plants, and it is perhaps on this account that mosses which. grow upon siliceous rocks may disappear when continually sprinkled with chalky

1 Cf. Nägeli, Bot. Mitth., I 866, Bd. II, p. I599; Drude, Pflanzengeographie, I 890, p, 49.

${ }^{2}$ Schulz-Fleeth, Der rationelle Ackerbau, i $5_{5} 6$, p. 2 or. 
water $^{1}$. A similar result would be produced if the addition of chalk neutralized the acidity of the nutrient fluid, and thus induced conditions unfavourable to the development of plants which grow best in acid media (Sects. 73,74 ).

In nature various combinations of external conditions may be presented, and these are continually changing, so that it is difficult or impossible to determine precisely the different factors at work in producing a particular result. A single factor sometimes becomes especially prominent, as, for example, in the case of saline plants, but care must always be taken not to ascribe to a single cause a result which may be due to the combined action of several. General physiology deals only with the fundamental principles by which the organic world is governed, and hence to give an account of the problems of plant distribution is beyond our scope.

The causes influencing the distribution of heterotrophic plants are even more complicated and involved than those affecting autotrophic plants, to which the discussion has hitherto been confined, for in both antagonistic and reciprocal symbiosis a whole series of complicated relationships are necessarily introduced. Similarly saprophytes are largely restricted to particular nutrient media, according to their requirements and their powers of utilizing different mutrient substances (Sects. 64-67). Moreover the formation and excretion of certain metabolic products may ultimately lead to an accumulation of substances which inhibit further growth, either of the same or of other organisms, while at the same time a suitable medium may be provided for the development of new forms of life. This phenomenon is especially obvious in the case of fungi and bacteria, and a few remarks have already been made concerning it (Sects. 64 and 92). Thus the accumulation of butyric acid, or of alcohol, ultimately inhibits the further fermentative activity of the organisms concerned, while in a solution of sugar either fungi or bacteria inay gain the upper hand according to the acidity or alkalinity of the medium. Similarly in the process of putrefaction several different organisms follow one another, and this is not only the case in the rapid decomposition of proteids, but also in the slower disintegration of a dead tree.

As autotrophic plants gradually construct a fertile soil rich in humus from sterile sand or bare rocks, progressive series of plants follow each other at prolonged intervals (Sects. 28 and $5 \mathrm{I}$ ). With the appearance of forest trees the increasing shade renders impossible the development of lightloving plants beneath them. Again, the gradual exhaustion of a given constituent from the soil may cause the original inhabitants to be replaced by others suited to the changed conditions. It is, however, improbable

1 P'feffer, Bryogeograph. Stud. a. d. rhätisch. Alpen, I869, p. I26 (Sep.-abdr. a. d. Denkschr. d. Schweizer Naturf.-Ges.). 
that the roots of autotrophic plants are able to excrete substances which act injuriously upon the plants they displace, for the neutralizing power of humus apparently renders any such action impossible', and. as a matter of fact. carbon dioxide is almost the sole metabolic product which is excreted continually and in considerable amount, and being a gaseous product it can rarely accumulate to any marked extent. Many of the lower heterotrophic plants, however, behave quite differently, and commonly exert more or less marked injurious effects upon one another by means of certain metabolic products which they excrete.

1 This idea has been frequently put forward in earlier times. See the literature by Mohl, Vegetabilische Zelle, 1851, p. 95 , and Sect. 28. 


\section{CHAPTER VIII}

\section{CONSTRUCTIVE AND DESTRUCTIVE METABOLISM}

\section{SEction 77. General.}

METABOlism includes the whole of the chemical processes connected with vital activity, which render it possible and which it at the same time regulates and governs. The changes which the food-material may undergo in metabolism are very numerous and varied, for in this way not only are all the permanent or transitory formative materials obtained and various reserve and other products formed, but at the same time certain katabolic processes provide a supply of energy such as is essential for all vital activity. The katabolic processes involved in respiration and fermentation render kinetic energy available for various remarkable processes of synthesis involving a production of substances with higher potential energy. Every living cell exhibits unceasing katabolism and anabolism, and just as in animals, a large part, and often almost the whole, of the organic food may be utilized to provide energy (Sect. 50 ).

The simplest cell must not only be capable of respiration, or of katabolism in general, but must aiso be able to perform a variety of anabolic processes, and frequently to produce proteids, cellulose and various carbohydrates, organic acids, and many other compounds as well, either simultaneously or successively. This may be accomplished when sugar is the sole carbon-compound supplied, provided the requisite inorganic salts are present, and this sugar can be obtained by fungi only from the external world, whereas green plants manufacture their own. Metabolism may cause most profound chemical changes and molecular reconstructions, as is evidenced by the fact that certain fungi can grow equally well with either methane or benzene derivatives as their sole source of organic food. It follows that, in spite of the widely different chemical constitution of these two groups of compounds, such a fungus is able to construct from either of them all the substances it requires (cf. Sects. 50, 66). In such cases the same end is reached from widely different points of departure, and this is only possible by a series of changes, which may either follow one another in the closest and most intimate sequence, or may be separated by intervals of time or space. The food-material which is to undergo further change 
may have already becn submitted to various preparatory operations to render it available to the plant, as, for example, when nutrient substances are absorbed after undergoing extracellular digestion by the aid of an excreted enzyme. Similarly the assimilation of carbon dioxide is an example of a remarkable photosynthesis by which a supply of organic food is obtained, while nitrate bacteria can carry on a similar synthesis by means of chemical energy, and when a fungus grows upon formic or acetic acid and is supplied with inorganic salts, the chemical energy obtained by the oxidation of these acids enables the plant to synthesize from these simple materials all the substances, proteids, fats, carbohydrates, \&c. which it needs. Examples of preparatory changes are also afforded in the storage and translocation of reserve food-material, and frequently farreaching chemical modifications are induced in order to provide substances suitable for prolonged storage, while in rendering such substances available for transport to the regions where they are required, equally profound changes may be necessitated.

Even the most complete knowledge of the intimate processes of metabolism would not necessarily enable us to distinguish clearly in all cases between the preparatory stages and the final act in a metabolic process. Moreover, a substance which normally remains intact may be drawn into metabolism when the plant is starved or when the external conditions are altered in some way or other. The processes of photosynthesis and proteid-synthesis have already been dealt with in connexion with the sources of organic food, and a few general observations have been made upon the meaning and importance of metabolism in general, which may serve as an introduction to this and the following chapters (Sects. 50 and 51). Respiration and fermentation, though simply special forms of metabolism, may for didactic reasons be treated as a subject apart, and it is mainly owing to the profound decompositions which these two processes induce that the chief supply of the energy is obtained for vital activity and metabolism in general. The respiratory activity is made evident and directly measurable by means of the excretion of the products of decomposition, which must be continually removed so that an injurious or inhibitory accumulation may be avoided.

The purpose of methodical research is to investigate the exact progress of each metabolic activity and to establish its importance in the plant's economy, but at the same time attention must be paid to the interacting relationships between the different processes and their mutual dependence, for otherwise incomplete and therefore inaccurate ideas must necessarily be obtained. Thus the insufficient or abnormal performance of any functional activity must ultimately influence all the others, while the cessation of a single metabolic activity will gradually cause the rest to cease one after the other, death ultimatcly ensuing. Only when a much 
deeper insight has been obtained into the complicated series of actions and interactions which are probably at work even in the simplest metabolic activities, will it be possible to follow a molecule of a carbon or other compound in its varying fortunes from its entry into the plant until it is no longer available for metabolism. This task is made all the more difficult by the fact that a substance may be utilized in many different ways, and under certain circumstances may follow a path different from that which it does in other cases. Moreover, the same end may be attained in various ways, as is shown by the fact that a fungus may construct its organic substance from either methane or benzene derivatives (Sect. 66). Similarly amides and carbohydrates may either be formed by synthesis or by the partial or complete decomposition of proteids, and it is sufficiently evident that the substances formed in the latter case may be entirely different to those from which the proteids in question were originally constructed.

Even the limited knowledge we already possess is sufficient to indicate the very varied character of metabolism in general, although it is certain that a host of metabolic products may yet be discovered. It is indeed extremely probable that in the living protoplast many highly important but unstable proteids or proteid compounds may exist which change into stable forms as soon as cleath occurs. So little is known as to the actual composition of living protoplasm that no explanation even of the most fundamental processes which take place in it can be hoped for at present, and hence it is impossible to trace any particular vital activity to its ultimate origin from changes occurring in the living protoplast. The special dependent functions which are developed for a particular aim or purpose are more open to experimental study than are the more general functional activities which form an essential attribute of all living substance, and it is probably by means of the former that a knowledge of the working mechanism of the living protoplast will ultimately be obtained. Even when we are fully acquainted with the mode of action of an enzyme, or with the importance of a metabolic product which serves for the maintenance of turgidity or for the formation of the cell-wall, we are unable to say how the substance in question is produced or why the constructive activity of the protoplast should be manifested in this particular manner. Moreover in many partial functions, such as that involved in the production of proteid, the precise mode of synthesis is as yet unknown (Sect. 7I). The same is also the case with regard to the assimilation of carbon dioxide, although this takes place in an organ specially adapted for the purpose (Sect. 6I).

Since our linowledge of the essential character of metabolic activity is so incomplete, it is preferable to deal with the visible products and to consider their importance to the plant, for these products afford sure and certain tokens of metabolic activity, although their mode of origin is not always necessarily the same. With regard to their ultimate fate, and their 
importance to the organism, three classes of metabolic products may in general be distinguished, namely (1) building or formative substances, (2) plastic or trophic substances, and (3) aplastic or atrophic substances (Sect. 50). No sharp line of demarcation can, however, be drawn between these three classes, for substances which serve as building material may, at a later period or under changed conditions, be drawn into metabolism, as may also be the case with substances which normally remain permanently aplastic. Similarly the substances which are essential for the maintenance of a certain indispensable turgidity can take no part in metabolism, but since they are essential to the living cell, they may be regarded as building material.

Aplastic substances are purely negative in character, and hence the group includes bodies of widely different importance, and not only those which are occasionally or continually formed as unavoidable by-products of metabolism, but also others which are produced for special purposes. In the former case. unless the end-products are continually rendered available again in metabolism, they must be excreted, so that an injurious accumulation may be avoided. When exposed to light, green plants continually reassimilate the carbon dioxide they exhale, and by the synthetic processes thus induced a loss of nitrogen, phosphorus, sulphur, \&rc. is avoided, for the simple compounds of these elements produced by katabolism are continually reassimilated (Sects. 68, 73). Similar reconstruction is possible in heterotrophic plants, when they are supplied with sugar, or some other organic compound.

Those aplastic substances, which are formed only to a limited extent, probably always subserve a definite function. Thus enzymes and acids act as digestive and solvent agents, other substances act as stimuli to irritable reactions, and many metabolic products have an attractive or protective importance owing to their smell, taste, or poisonous character. In all such cases the substances in question must be permanently withdrawn from metabolism, and hence the importance or value to the plant of any product cannot cntirely be measured by its plastic or aplastic character.

If the term 'excreta' is restricted to those substances which are of 110 further use to the plant, it will include only certain aplastic products, for secretory products such as enzymes, ethereal oils, \&c. have a definite function to perform, and in the translocation of plastic material particular cells may excrete one substance and absorb another simultaneously. The terms 'assimilation' and 'dissimilation' have already been defined from a physiological standpoint (Sect. jo).

A complete knowledge of the causal relationships of the different metabolic processes can only be obtained by a thorough investigation of the powers, properties, and organic mechanism of the living plant, and although vital activity and metabolism are mutually dependent, they may partly or entirely cease when resting periods intervene in the progress of 
development, as for example occurs in winter, or when seeds ripen. This is simply an especially marked example of the self-governing porrer over all vital functions which a plant possesses, a power which is indeed absolutely essential to enable an appropriate response to be made to changed external conditions. It is owing to this self-regulatory power that the metabolic activity and formative tendencies in particular cells or in all of them alter as development progresses. Similarly a deficiency or superfluity of nutrient material or of special metabolic products may cause more or less marked acceleration or retardation of single functions or of vital activity in general, while the most various external agencies may produce similar results. Thus after the formation of the cell-wall has ceased, a new production of cellulose may be induced by plasmolyzing the protoplast, and similarly an over-accumulation of sugar causes the cessation of its production by the assimilation of carbon dioxide (Sect. 54) as well as by ferment action or decomposition, while certain plants, when fed with sugar, cease to form the diastase they previously produced in abundance. Similarly the production of many other substances, such as pigments, poisons, \&c., may be temporarily or permanently suppressed, and the white sports of blue flowers, as well as the occurrence of sweet almonds free from amygdalin, show that this loss need not involve any injury to the plant. Just as the plant does not require the universally present sodium compounds, so also may non-essential carbon-compounds be formed in metabolism, though these may, however, be of marked biological importance. Even the production of essential formative and plastic substances may not be continuous, whereas a stoppage of respiration or of katabolism must necessarily disorganize the normal vital activity.

These and other facts show clearly that under normal conditions the different functions have sub-maximal demands made upon them, and hence not only an incrcase but also a further diminution in their activity is possible. Any such change may either involve a single function almost entirely, or may affect others as well, and often simultaneously may accelerate some and retard others. It is in this manner that formative metabolism is caused gradually to diminish as growth ceases. Metabolism must be capable of a certain amount of modification in those organisms which can grow upon a variety of nutrient media, for in the main the composition of protoplasm is always the same, although the accessory products it produces may differ markedly.

There is no doubt that certain carbon-compounds take part in the maintenance of turgidity, and may also act as reserve materials or be of use in other ways. Particular carbon-compounds form an essential part of living protoplasm, but quantitative or qualitative changes may occur in the latter although this has not yet been proved to be the case. If potassium takes part in the constitution of protoplasm. then in those fungi 
in which rubidium can replace it, a corresponding change must occur in the plasma whenever such substitution is enforced on the plant. It is also probable that in respiratory and fermentative processes a certain amount of substitution is possible, for in nitro-bacteria nitrous or nitric acid forms the final excrete product of physiological combustion instead of carbon dioxide as in ordinary plants, and hence the processes of respiration can hardly be identical in the two cases.

In those fungi which can be nourished with a variety of different substances the final processes of metabolism may be precisely similar, and only the preparatory processes be different (Sect. 66), as is indicated by the fact that in the same fungus similar plastic substances are produced, whether it is fed with sugar, or with tartaric or quinolic acid. According to the food-material, however, various substances may be produced, probably as by-products of the preparatory processes, as is certainly the case when quinine is produced from arbutin, or benzoic acid from hippuric acid, for both of these substances undergo no further change. Similarly fungi when fed solely with peptone may produce large quantities of ammonia.

The whole course of metabolism can be completed in a single cell or in a unicellular organism, and mutual interchanges together with the prevention of direct contact between particular products play an important part in modifying its character. Hardly anything definite is known as regards even the most essential vital activities, and thus it has yet to be determined whether the proteid decomposition by which energy is liberated occurs mainly between comparatively stable structural elements (micellae or pangens), or whether these also undergo perpetual decomposition and reconstruction so long as life remains. A living protoplast may be compared to an extremely complex chemical manufactory in which the character and mechanical possibilities of the different departments, as well as the nature of the materials supplied, the requirements of the factory, and the demands made upon it, determine what products shall be produced and in what relative amount. The different operations may be more or less complicated, and may involve a series of processes taking place at different times in different departments, so that from the same raw material a variety of products may be formed, while the same products may be constructed from various raw materials, either by synthetic or analytic means.

A factory can continue to exist only by satisfying all its varying requirements and the demands made upon it from without, so that in addition to the stream of primary and accessory products which pass away outwardly, the manufacture of new substances must be undertaken when necessary, and that of others temporarily or permanently discontinued. The amount of expansion possible is always limited, and the different construction and powers of different factories cause each to have its own specific productive power. So long as the internal mechanism remained 
unknown, a person who controlled only the entry and exit could form no idea as to the complicated processes by means of which the different products were formed in the factory. This is preciscly our position with regard to the living plant, in which reside powers and properties of which the most complicated machines constructed by the hand of man can give but the faintest conception.

No machine or factory has ever been constructed which can automatically regulate its entire mechanism, maintain itself, and when necessary be capable of regenerating particular parts. The latter might, however, be possible in certain machines granted the necessary supply of energy and of building material. It is impossible, however, to conceive of a machine which could reproduce its kind by sexual or asexual means. In spite however of the special peculiarities of the living organism the comparison with a physical mechanism may suffice to indicate the impossibility of predicting from the nature of the visible products what are the invisible processes which led to their formation. The special powers and properties of the protoplast, along with its regulatory mechanism and its tendency to activity, primarily determine whether a particular food-substance shall enter into metabolism. If from any cause no tendency to the production of proteids exists at a given moment, no formation of the latter is possible, even though asparagin, sugar, and all the necessary building materials are present in the most appropriate form and position within the protoplast, and in direct contact with it and with one another. Moreover even in the most minute cell the different substances it contains may not be in actual contact. and hence may be incapable of reacting upon one another.

The comparative study of a number of plants is of the utmost importance in rendering possible a clear comprehension of the nature of metabolism. and since the protoplasm always retains the same general composition, the final products of constructive metabolism must in all cases be similar, or must be closely related substances. The specific differences in the metabolism of different plants are due to the fact that the same end is not always attained by the same means, so that special substances may be formed as unavoidable by-products, or as products which have definite functions to perform. The most marked deviations from normal metabolism as illustrated by the higher plants are exhibited among the lower organisms as the natural consequence of their adaption to every variety of external conditions, and certain of the lower plants have actually the power of liberating kinetic energy without the aid of free oxygen. Intermediate forms, however, exist which connect aerobic organisms with these anaerobic forms, and certain bacteria are known in which, as in the higher plants, carbon dioxide is the sole excrete product of respiration. All substances which are continually produced by katabolism must either accumulate or be excreted unless they are reassimilated as fast as they are formed. According to the 
cultural conditions many fungi and bacteria may either excrete carbon dioxide only, or in addition a variety of other metabolic products. Continual removal of the products renders possible a greater production than can occur when they are retained, and this is why a given organism may ferment so relatively large a quantity of fermentable material.

Higher plants produce quite as great a variety of substances as lower ones do, and scarcely a substance is formed by the latter which cannot also be produced by one or other of the former, although marked differences may exist between closely allied plants with regard to their metabolic products. This is especially the case with bacteria. It must be remembered, however, that the physiological importance of a substance is by no means wholly dependent upon its chemical constitution. There can be no doubt that a fundamental agreement exists between the metabolism of the higher and of the lower plants, and the same holds good for plants and animals. Even as regards the higher animals marked resemblances with plants are exhibited ${ }^{1}$, and it may with certainty be expected that the little known lower animals will be found to agree more closely with certain plants than with the higher animals as regards their metabolic products. The supposed fundamental difference between vegetable and animal metabolism was due to the failure to distinguish between the processes by which food is obtained, and those by which plastic products are produced and energy is liberated. Moreover the photosynthetic assimilation of carbon dioxide is possible only to certain plants, as is also the power of synthesizing proteids, while plants which have not this latter property must obtain fresh proteids from without in order to replace the continual loss by destructive metabolism.

Only the substances actually present in plants can be detected by analysis, but these suffice to indicate what are the products formed by metabolism from the assimilated food-material. This is especially obvious when the disappearance of one substance is accompanied by the appearance of others derived from it. Qualitative tests may suffice to render such changes evident, although sometimes quantitative determinations are necessary, but hardly any idea can be obtained by such means as to the progress or causes of such changes, or as to the regions where they occur. Nor can all the substances present in the plant be detected by analysis, for many of them may at once decompose when the plant is killed and subjected to chemical analysis.

Macrochemical analysis affords however a basis for further research, and by means of microchemical methods the source and origin of the substances discovered by macrochemistry may be more precisely determined. Many conclusions may indeed be arrived at as to the importance of the metamorphoses which particular substances may undergo, from their distribution in cells or in tissues,

1 Cf. E. Schulze, In wie weit stimmen Pflanzenkörper u. Thierkörper in ihrer Zusammensetzung überein? $1 S_{94}$ (Sep.-abdr. a. Vierteljahrsschr, d. Naturf.-Ges. z. Zïirich, BA. ххх1х). 
but (juantitative estimations are possible only by macrochemical methods, and these may enable deductions to be made for which qualitative microchemical methods afford no sure basis.

The facts obtained by these means when properly interpreted, and when full attention is paid to the special properties of the living organism, may enable a certain insight to be gained into the invisible processes which lead to the formation of visible end-products. No general methods of investigation and experimentation can be given which will be applicable to all cases, for the manner in which a given problem is attacked should be adapted to the nature of the phenomena for which an explanation is required.

An account of certain of the physiological applications of machrochemistry are given by Hoppe-Seyler, Handbuch der physiol.-chem. Analyse, r 893 ; J. König, Unters. landw. u. gewerblich wichtiger Stoffe, i 89 I ; Dragendorff, Qual. u. quant. Analyse d. Pflanzen, 1842 , and in the special works given. A general summary of microchemical methods is given by A. Zimmermann, I892, Mikrotechnik (translated by Humphreys). Physiological methods, such as when the reactive power of another organism is used as a test for the presence of a given substance, oxygen by means of bacteria, malic acid or cane-sugar by antherozooids ${ }^{1}$, are of great importance and may probably be employed to a greater extent than at present, for by these means an approximately accurate quantitative determination is possible, while at the same time the precise point at which a particular substance is evolved can be observed.

The chemical properties of the substances found in plants are described in hand-books of chemistry, and also by Husemann in Die Pflanzenstoffe, 1882-4; Ebermayer, Physiol. Chemie, I882; Tollens, Handbuch der Kohlenhydrate, I888-95. The special literature concerning the specific composition of different plants and the changes which occur under varying cultural conditions and at different stages of development have already been mentioned, but a detailed summary of the special literature upon these points, however desirable it might be, would be out of place here. In König's Chemie d. Nahrungs- und Genussmittel, I 889 , 3. Aufl., no attention is paid to physiological considerations. A few points regarding the chemical composition of fungi and bacteria are given by Zopf, Pilze, 189o, p. I I 7 ; Flügge, Mikroorganismen, 2. Aufl., I896, Bd. 1, p. 93 ; Marschall, Centralbl. f. Bact., I 897 , Bd. III, p. I 54 .

Historical. Even in the earliest times, the fact was recognized that plants are able to form substances which are not present in their food ${ }^{2}$, but it was only at a much later date that precise determinations were made of any of the metamorphoses which the absorbed substances may undergo. Thus Kollo pointed out that sugar is formed during the germination of barley, and Senebier showed that the oil and starch in seeds act as stored food-material from which various substances are produced on germination, which process he compared to fermentation".

${ }_{1}$ Pfeffer, Unters. a. d. Bot. Inst. z. Tübingen, $1 \$ 8_{4}$, Bd. I, Pp. $+^{1} 3,43^{2} ; 1 \$ 88$, Bd. 1I, p. 633.

${ }^{3}$ Cf. Sachs, IIistory of Botany (Garnsey and Balfour), i $\$ 90, \mathrm{p}$. +So.

${ }^{3}$ Rollo, Ann. d. chim., i 798 , T. xxv, p. 40; Senebier, Physiol. végét., isoo, T. IIt, P. fo6. Cf. also de Candolle, Physiol., is 33 , T. I, pl. 170, 266. 
De Saussure proved that seeds lose in weight by respiration during germination, and Proust made the first comparative analyses of germinated and dormant barleycorns, from which important conclusions as to the formation of sugar from starch were drawn ${ }^{1}$. De Saussure proved in the same manner that sugar and dextrin-like substances are produced as the starch disappears during the germination of wheat, and he showed that sugar is also formed when oily seeds germinate?

Although the analytical methods employed were faulty, still a sound basis was laid for the further researches of later authors, such as Hellriegel, i 855, Oudemans and Rauwenhoff, 1859 , \&c.

The microchemical methods now largely used were first systematically employed by Sachs, who obtained by these means a general idea as to the metamorphoses which plastic substances may undergo, and also as to their importance for translocation and for constructive purposes ${ }^{3}$.

The results thus obtained as regards non-nitrogenous bodies still hold good, although it is now known that a great variety of substances may be produced by the decomposition of proteids.

\section{SECTION 78. The Commoner Metabolic Products.}

It is more convenient to discuss the relations of different substances and groups of substances to metabolism than to study each separate metabolic activity in detail, although the latter plan would be more desirable from a physiological point of view were our knowledge not so incomplete. Hence the cconomic importance of different substances does not concern us here, nor can any complete account be given at present of those aplastic materials whose importance in metabolism is unknown. It is beyond our scope to do more than give the main facts and principles of metabolism and to illustrate them by examples, while in dealing with different groups of substances it must always be remembered that the same material may subserve a variety of purposes, and that to attain a particular end bodies of very heterogeneous chemical constitution may co-operate with one another. Hence the distinction between formative, plastic, and aplastic materials simply indicates very generalized metabolic characteristics, and does not allow any sharp line of demarcation to be drawn between different substances or groups of substances (Sect. 77).

In addition to various proteids the living protoplast contains other

${ }^{1}$ De Saussure, Kech. chim., $18_{04}$, p. I6; Proust, Ann. d. chim. et d. phys., IS I 7 , T. v, 1) $34^{2}$. bérard (ibid., 1S2I, T. XYI, pp. I52, 225) performed similar comparative analyses on ripening fruits.

${ }^{2}$ De Saussure, Mém. d. l. Soc. d. Phys. et d'Hist. Nat. d. Genève, i 833 , T. vi, p. 27 ; Bibl. univ. d. Genève, I $S_{42}$, T. XL, p. 370 .

3 Sachs, Über einige mikroskopisch-chemische Reactionsmethoden, Sitzungsb. d. Wien. Akad., I859, Bd. xxxvi, p. 5, and Keimung d. Schminkbohne, ibid., I859, Bd. xxxvir, p. 57 ; Über d. Stoffe, welche das Material zum Wachsthum d. Zellhäute liefern, Jahrb. f. wiss. Bot., I863, Bd. III, p. 183. Cf. also Rochleder, Chem. u. Phỵsiol, d. Pflanze, I85s. pP. 99, I 4. 
substances which may or may not form integral components of protoplasm. The living protoplast forms a cell-wall usually composed of cellulose but sometimes of bodies resembling chitin, and this enclosing membrane frequently undergoes subsequent chemical metamorphosis (Sect. 84). Every soluble substance present in the cell exercises its appropriate osmotic activity, but for the most part organic acids seem to be employed in the maintenance and regulation of turgor (Sect. 24). The mere presence of such bodies within the cell suffices to attain this end, whereas the protoplast is able to grow only by assimilating appropriate food-material. The materials of which the actual skeletal framework is composed are characterized by a certain stability, whereas the plastic food-substances are liable to marked and far-reaching changes, such as even the stored reserve food-substances ultimately undergo.

Experiments made with fungi show that widely different bodies may serve as plastic material, and that each plant has its own specific power of utilizing various food-substances (Sect. 66). Phanerogams appear to be confined to a more restricted range of food-materials, although owing to the complications introduced by any disturbance of the normal conditions of nutrition it is difficult to determine the actual limitations of autotrophic plants. The products formed by the plant itself do not form any criterion as to the materials which it will require as food, and fungi are even able to make use of many substances which they do not encounter in nature and which are not formed in metabolism. Omnivorous fungi appear to have in general more marked assimilative powers than higher plants, although the power of synthesizing proteids possessed by the latter is absent from certain of the former.

In autotrophic plants all carbon compounds are ultimately derived from some carbohydrate product of the assimilation of carbon dioxide, and the nitrogenous and non-nitrogenous reserve food-materials are produced by similar metabolic activity. The chief non-nitrogenous reserve-materials are various soluble and insoluble carbohydrates (sugars, starch, cellulose, \&c.), fats, and in certain cases organic acids as well. Many of these substances are widely distributed, whereas others are found only in certain plants, but none of them appear to be indispensable, for in both storage and translocation various bodies may replace one another in the same or in different plants. Thus oil is present in the seeds of many plants, starch in those of others, while oil has occasionally been observed in the seeds of certain grasses which normally contain starch ${ }^{1}$. Similarly various plants are able to form starch or sugar from oil, or vice versa, and hence it is easy to. understand why different reserve-materials may be stored up in the different

${ }^{1}$ Nägeli, Die Stärkekörner, I8 58 , p. 536; Pfeffer, Jahrb. f. wiss. Bot., I 872 , Bd. virI, p. 490. 
organs of the same plant, or even in the same cell. This applies also to the nitrogenous reserve-materials which are present mainly in the form of amides and proteids, although inorganic nitrogenous compounds may sometimes function as reserve food-material.

Food-materials are often much modified in order to render them suitable for storage: thus starch or oil are often formed when sugar is supplied, and proteids when amides are present. Even where no marked metamorphosis seems to recur a non-diosmosing compound is probably produced by some slight change or combination with another substance, so as to render further accumulation possible. Similarly the reserve-materials may be mobilized either by slight metamorphoses or by more or less complete disintegration, and during the process of translocation such changes as these may be repeated more than once. Sugar is frequently formed from starch and oil, while proteids may disintegrate into amides, or even into inorganic compounds such as ammonia, phosphoric and sulphuric acids.

These changes can easily be followed when they occur at different times and in different parts of the plant, but in all cases they involve a certain consumption of energy, and since living organisms always work as economically as possible, it is safe to conclude that whenever the processes of mobilization and translocation involve marked disintegration, the latter is either necessitated by existing circumstances or is adapted to the end in view.

The nutrient material is used in a variety of ways, and from it all formative substances required for building purposes are produced after undergoing more or less marked modification, which varies according to the similarity or dissimilarity between the food-materials and those required in constructive metabolism. Food-substances secm almost always to undergo some change, however slight, in the process of assimilation; as far as our knowledge goes the proteids which form part of the living protoplast differ somewhat from reserve-proteids, and similarly the cellwall differs slightly in composition from reserve-cellulose. The existence of such differences is, however, not necessarily essential, for since the characters and properties of an organism are determined in every case by the manner in which the different component substances are combined together, it is always possible that one or more of these substances may be directly assimilated by the protoplast without undergoing any chemical change.

Growth and vital activity are regulated and determined by the general properties of the protoplast, which maintains its specific characters in spite of growth and reproduction. In the progress of development, and under special conditions, there can be no doubt that certain alterations may occur in the physical and chemical constitution of particular parts, and hence substances may perchance be incorporated which previously were 
absent. Changes of this character do actually take place in the cell-wall, for either its original constituents may undergo chemical change, or new substances may be introduced, or both these modifications may take place simultaneously (Sect. 84).

Nitrogenous and non-nitrogenous reserve-materials have hitherto always been found associated together. This is not, however, necessarily essential, for plants can grow when sugar is the sole carbon-compound supplied to them, and when all the other essential elements, including nitrogen, are absorbed in the form of inorganic salts. Fungi may grow when proteidsubstances afford the sole source of carbon, in which case nitrogenous compounds must be excreted as one of the end-products of metabolism. In seeds, the presence of non-nitrogenous carbon-compounds renders possible a more economic consumption of those which are nitrogenous, so that a loss of nitrogen is avoided as a general rule.

The various changes which the stored substances may undergo suffice as proof that no special respiratory or cell-wall-forming substances exist, nor are particular food-materials used solely in respiration or solely in cell-wall formation, germination, \&c., although it is always possible that, when presented simultancously, one carbon compound may be mainly used in respiration, another mainly in proteid-synthesis. Owing to the preparatory changes the food may undergo when absorbed, the final stages of a particular metabolic activity may be identical whatever the food may be (Sects. 66, 77).

The use to which a formative substance is put determines its localization, and the distribution of plastic substances in the different plant-organs has already been discussed in connexion with translocation (Chap. X). With the exception of rescrve-cellulose, reserve-materials are stored within living protoplasts, partly in the vacuoles and partly in the plasma itself. In turgid cells the dissolved substances are present in solution, starch in granular form, oil either as visible drops or in the form of an emulsion so fine as to be visible only when the droplets are caused to coalesce.

Soluble carbohydrates, amides, proteids, organic acids, \&c. are presumably mainly dissolved in the cell-sap of the vacuoles, in which a large amount of oil may also be present, whereas starch-grains appear normally to remain imbedded in the protoplasm where they were formed. The stored substances present in the cell-sap or elsewhere are reassimilated by the protoplasm when necessary, and they must always pass through the latter to reach those regions where they are required. Vacuoles are important organs which are of service in many ways, for example, 
as storage reservoirs for food-substances or for excrete products. By the removal of the latter, reactions injurious to the protoplasm may be prevented (cf. Sects. 7, 19, 22, 93).

The same generalizations apply to all metabolic products, and not merely to plastic substances. Those soluble aplastic substances which remain permanently present in the living cell are apparently for the most part contained in the vacuoles. This localization is especially evident in the case of soluble pigments, which, like many other substances, may be restricted to particular vacuoles, showing that the latter organs have not always the same functional value. The importance of the excretion of such substances as tannic acid, poisons, \&c. into the vacuoles can hardly be orer-estimated, for, if present in the plasma, they might kill or fatally injure its living parts. Certain poisonous aniline dyes may, however, when slowly absorbed, accumulate in large quantities in the cell-sap without injuring the protoplasm (Sect. I6).

Any substance which is subject to metabolic change may be termed plastic, although in some cases the purpose of the metabolism may simply be to render possible the excretion of a particular waste product, such as oxalic acid, for example (Sect. 86). It is, however, always possible that under certain conditions a plastic substance may remain temporarily intact, or even permanently so, if the causes and conditions which regulate metabolism remain such as to render the substance in question unnecessary, and hence to prevent its being drawn into metabolism. Reserre-materials may be protected in this manner, whereas in a starred plant substances may be utilized which, under normal conditions, behave as aplastic material. It often happens that in case of need dormant powers may be aroused which are not exercised under normal conditions, and hence the metabolism of a plant during starvation may be abnormal in many respects.

Even a starved plant is unable to consume the whole of the plastic material which it contains: thus starch-grains frequently do not disappear from the guard-cells of the stomata, or from the the cells of the root-cap, while dead or dying cells frequently contain considerable amounts of sugar, \&c., and hence arises the fact that death may occur before turgor disappears ${ }^{1}$. Certain plastic substances must remain permanently present in order that life may be maintained, and thus living protoplasm, even

${ }^{1}$ Cf. Stange, Bot. Zeitung, $189^{2}$, p. 277 ; Copeland, Einfluss von Licht u. Temp. auf Turgor, 1896. [An irretrievable injury is not necessarily immediately followed by death, and it is indeed impossible to say precisely when the latter occurs. The profound change in the plysical properties and permeability of the protoplasm, which finally follows a fatal injury, is ustally accepted as the only certain sign of death, and in accordance with this definition it would perhaps be more correct to say that not a dead but a dying cell may continue to exhibit turgiclity.] 
if wholly composed of plastic material, can only consume a certain portion of its own substance. The proteids of which the protoplastic framework is constructed seem for the most part to be comparatively stable and aplastic, but many bacteria and fungi are able to consume the cell-walls and plasma of dead individuals of their own kind. Auto-digestion is no doubt prevented during life not merely by the chemical nature of the living substance, but also by its structural arrangements and by the proper regulation of its metabolic activity.

Even in an individual plant no hard and fast line of demarcation can be drawn between plastic and aplastic constructive materials, and a good nutrient substance for one organism may be useless to another, especially in the case of fungi and bacteria. It is, however, of the utmost importance for the maintenance of the balance of nature that almost every metabolic product of both plants and animals shall be assimilated or decomposed by some heterotrophic organism or other. This is the case with such typical aplastic products as tannin, wax, alkaloids, pigments, \&c., and even resin appears to be attacked by certain bacteria.

Many plastic materials are found in but few plants or only in small amounts, but the non-formation of a particular substance affords no sure sign that it may not be available in metabolism when presented to the plant. As might however be expected, the majority of the special products formed by a restricted number of plants, usually in limited amount, belong for the most part to the class of aplastic substances. These may function as aids to metabolism (enzymes) or may have a biological importance (pigments, ethereal oils, alkaloids, \&c.), and it is of manifest importance that a small quantity should suffice for the attainment of the required end. Why certain aplastic products, such as tannin, are commonly produced in large amount cannot as yet be satisfactorily answered.

It is always important to know whether a substance is produced as a direct means to a certain end, or only as an accidental by- or end-product (Sects. 50, 77). When an aplastic substance is formed in limited amount it is safe to assume that the former is the case, whereas continuous production and excretion characterize a waste end-product, such as carbon dioxide, or in ferment-organisms, lactic or butyric acids, alcohol, hydrogen, sulphuretted hydrogen, ammonia, \&c. The formation of such excreta can, however, be regulated and controlled, so that under different circumstances a particular waste product may cease to be formed or may even be consumed by the same organism which produced it (cf. Sects. 50, 77).

The metabolic activity must necessarily correspond to the needs of the organism and the conditions under which it exists, so that in correspondence to the adaption to different modes of life, the plastic and aplastic products of closely allied plants may differ widely in character 
and amount. This is exhibited most markedly among bacteria, owing to the great powers of adaption possessed by these organisms, but even among the flowering plants great differences may exist between the metabolic products of the members of an order, or even of a genus, while in the same plant, oil and proteids may form the reserve-materials of the seed, sugar and amides those of the rhizome. On the other hand the majority of the species of a given family or genus may produce a particular plastic substance of comparative rarity, such as mannite, dulcite, or glycogen. These peculiarities become still more obvious when attention is paid to those aplastic products which remain permanent constituents of the plant: thus quinine characterizes the genus Cinchona, while the populin of the poplar is replaced by the allied substance salicin in the willow. Even substances which occur comparatively rarely may, however. often be found in plants far removed in systematic position (indigo, cumarin, chrysophanic acid), and there is therefore no general relationship between the systematic position of plants and the chemical nature of their plastic or aplastic metabolic products, as Rochleder ${ }^{1}$ supposed.

\section{SECTION 79. Constructive and Plastic Nitrogen Compounds.}

In addition to the proteids which every plant contains, larger or smaller amounts of other nitrogenous metabolic products are always present, although their functional importance is only known in certain cases. Asparagin and glutamin, leucin and tyrosin are all products which the plant may reassimilate, whereas other amides and amido-acids (viz. phenylamine, amidovalerianic acid, arginin), as well as certain derivatives of urea (xanthin, hypoxanthin, guanin, guanidin, allantoin, adenin, caffein, thein) ${ }^{2}$ which may appear during germination or at a later stage, can only with a certain amount of probability be regarded as plastic products. The hydrocyanic acid formed in abundance by Pangizum edule and a few other plants ${ }^{3}$ appears to be used as plastic material, and the same is probably the case with amygdalin. Similarly the nitrates and ammonium salts, which are often stored in great abundance, may be drawn into metabolism again, whereas many other compounds, including alkaloids and glucosides (Sects. 87, 89),

1 Rochleder, Phytochemie, I854, p. 259.

2 A more complete enumeration with regard to higher plants is given by E. Schulze, In wie weit stimmen I'flanzen- u. Thierkörper in chem. Zusammensetzung überein? I 894 , Pp. I 4, I 9 (Sep.-abdr. a. Vierteljahrsschr. d. Naturf.-Ges. in Ziirich, Bd. xxxIx); also in Schulze's later works quoted beneath. Whether aspartic and glutamic acid may be actually present is probable but still uncertain. On bacterial products, cf. Flïgge, Mikroorganismen, I896, 3. Aufl., Bd. I, p. I68. According to Kellner (Versuchsst., I887, Bd. xxxIII, p. 378 ), thein is a plastic substance.

${ }^{3}$ Treub, Ann. d. Jard. bot. de Buitenzorg, I895, T. xil, p. I. Cf. Sect. 70. 
are for the most part aplastic products and have frequently only a biological importance.

It probably never happens that the whole of the nitrogen is present in the form of proteid compounds, which contain on the average from 50 to 90 per cent. of the nitrogen in vegetative parts. It scems moreover that the non-proteid nitrogenous compounds diminish in amount only when a deficient supply of nitrogen causes the plant to economize its resources as far as possible. Apparently these nitrogenous compounds include both plastic and aplastic material. Soluble amides and amido-acids appear as reserve-materials chiefly in rhizomes, roots, bulbs, and tubers, organs which are not normally subject to desiccation, and in such cases almost the whole of the nitrogen may be present in this form. On the other hand in seeds, and apparently in spores also, proteids predominate, so that usually only from 2 to 10 per cent. of the total amount of nitrogen is represented by non-proteid compounds ${ }^{~}$. During germination the proteids decompose into amides, \&c., and these may be formed in such abundance when leguminous seedlings are grown in darkness that 50 to 75 per cent. of their nitrogen may be present in the form of non-proteid substances.

Schulze and his pupils were the first to recognize that various amides were common products of plant-metabolism, but the formation and accumulation of asparagin was known before their researches ${ }^{2}$. By isolating the different amides, approximate quantitative determinations have been made of the total amount of nitrogen present, and an estimate of the amount of nitrogen held in the form of amides may be directly obtained by treating triturated plants with nitrous acid and hydrochloric acid, estimating the nitrogen in the form of ammonia ${ }^{3}$. This method is by no means an exact one, nor is it possible to determine with precise accuracy the amount of nitrogen present in the form of proteid.

The amount of thesc metabolic products varies in different stages of development: thus during germination the relative amount of amides increases until ro to 30 per cent. of the nitrogen may be represented by such substances, or in Lupinus, Vicia, and other Leguminosae as much as 75 per cent., mainly in the form of asparagin, although traces of glutamin, leucin, \&c. are present. In seedlings of Cucurbita the total amount of amides present is small and glutamin predominates, whereas in seedlings of Abies pectinata and other Coniferae arginin is the most abundant amide ${ }^{4}$. The relative amounts present are, however, in all cases liable to pronounced fluctuations.

${ }^{1}$ E. Schulze, Landw. Jahrb., is 88 , Bd. xviI, p. 693.

${ }^{2}$ Cf. the literature given by Pfeffer, Jahrb. f. wiss. Bot., I872, Bd. vili, p. 557. Most of Schulze's works, \&c. since 1876 are published in Landw. Jahrb., in Versuchsst., and in Zeitschr. f. physiol. Chemie.

${ }^{3}$ Cf. König, Unters, landw. wichtiger Stoffe, I89I, p. 2 It ; E. Schulze, Versuchsst., I 883 , Bd. xxix, p. 400 ; 1884 , Bd. xxx, p. 459 : I 887 , Bd. xxxill, p. 124 . To calculate the amount of proteids present by multiplying the amount of nitrogen found by 6.25 is obviously inaccurate.

+ Cf. tables, Sect. 81. Schulze (1.c., In wie weit, \&cc.) gives a summary of the literature, and 
As the reserve-materials are consumed, the amount of amides present in the regetative organs decreases until an approximate general average of from io to 20 per cent. is reached ${ }^{1}$. The amount of amides decreases in old organs, unless they are utilized as storage-receptacles. A ripe potato contains from 30 to 47 per cent. of its nitrogen in the form of amides, and similar amounts are present in the beet-root, root of Scorzonera, \&c. ${ }^{2}$ 'The potato contains asparagin in greatest abundance, along with a little tyrosin and leucin, and a trace of glutamin, whereas the latter is the most abundant amide in the beet-root, which also contains a certain quantity of betain. These different amides can replace one another just as may various proteids or carbohydrates, but asparagin appears to be the most abundant and widely distributed of them, and it has been detected even in Agaricineae and certain of the Myxomycetes ${ }^{3}$. It is possible that asparagin may be formed by a few bacteria, for in the metabolism of putrefactive and other forms a great variety of nitrogenous compounds may be produced.

Proteids form a class of substances of the highest physiological importance, and owing to the complex nature of their large molecules they offer a much greater varicty of possible combinations than carbohydrates do. There can be no doubt that many readily decomposable proteids are continually being formed by the living organism, and that even many stable proteids may exist of which we have as yet no conception. The complex nature of proteids and their multifarious chemical affinities not only render them suitable for the constitution of the living protoplast but also make them adapted to serve a variety of other purposes. Disregarding the chitinous substance which takes part in the formation of the cell-wall in fungi, many proteids may be stored or translocated as plastic material, while enzymes and tox-albumins have a special functional or biological importance, and particular proteids or proteid compounds may be employed for similar purposes.

The protoplast is apparently mainly built up of proteids, which may form not less than 40 per cent. and perhaps not more than 90 per cent. of the dry weight of the protoplasm, after the metaplasm, \&c. has been removed. The living plasma forms a certain proportion only of the cell and its relative bulk is often very small, so that the amount of proteids

also in Landw. Jahrb., I880, Bd. IX, p. I ; $188_{3}$, Bd. xir, p. 909 ; I888, Brl. Xvir, p. $68_{3}$; I 892 , Bd. XXI, p. 105 ; Versuchsst., I 895 , Bd. XLVI, p. 394 ; 1896, Bd. XlviII, p. 33 ; Zeitschr. f. physiol. Chemie, I 896 , Bd. xxII, pp. 41 I , 435. Cf. also Prianischnikow, Versuchsst., I 894, Bd. XLI, p. 247 ; I 896 , Bd. XLvi, p. 459 ; Frankfurt, ibid., I 894 , Bd. xl.II, p. I 44 ; 1895 , Bd. xLv, p. I53. Various details concerning individual substances are given by Ebermayer, Zimmermann, Mikrotechnik, \&c.

1 Kellner, Landw. Jahrb., 1879 , Bd. viri, p. 245 ; Emmerling, Versuchsst., I880, Bd. xxIv, p. I 3 ; E. Schulze, Landw. Jahrb., I88o, Bd. Ix, p. 27 ; I88 I, p. 686 ; Versuchsst., I $88_{7}$, Bd. Xxxin, p. 89. A partial summary is given by Ebermayer, Physiol. Chemie, I882, pp. $627,66_{4}$; aiso König, Nahrungs- u. Genussmittel, I889, 3. Auf., Bd. I, p. 64 I.

${ }^{2}$ Cf. Kellner, l.c.; Schulze, Versuchsit., $188_{2}$, Bd. xxirl, p. 357 ; Ebermayer, 1.c.

3 Reinke, Unters. a. d. Bot. Lab. in Göttingen, $1 \varsigma_{1}$, Heft 2, p. 166. 
in a living cell will not be more than 8 per cent. of the total dry weight, or 2 per cent. of the total weight when fresh. This corresponds with the amount of nitrogen present (Sect. 68), although a portion of the latter is always present in the form of non-proteid substances. In storage-receptacles, such as the seeds of Leguminosae, proteids may form 20 to 35 per cent. of the dry weight, and in the grains of cereals about il per cent. ${ }^{1}$ Protoplasm contains many nucleins, some rich in phosphorus and others poor in this element. Globulins and vitellins, as well as albumoses ${ }^{2}$, form the commoner forms of reserve-proteid, and traces of peptone may also be found (Sect. 9r). Nucleo-proteids and mucin often accumulate in large amount and are no doubt used along with many true proteids as plastic material. Indeed it would be surprising were not this function especially prominent among proteids in general.

It is not at present possible either to determine or to localize all the different forms of proteid present in the cell, but in general a distinction may be made between constructive and plastic proteids. The skeletal framework of the protoplasm seems to be mainly composed of proteids which are comparatively resistant to peptic digestion, while old or starved cells give no colour reaction with an alkaline solution of a copper salt, which suggests the absence of plastic proteids ${ }^{3}$. Traces may, however, still be present, since the failure of the reaction is not a sufficiently delicate test to indicate their complete absence, and it is moreover uncertain whether all plastic proteids give the same colour-reaction. Proteids may undergo marked physical modification (as regards solubility, \&c.) without any corresponding chemical change, and in this manner the same proteid may be able to subserve a variety of purposes, a plastic substance sometimes being used as building material (Sect. 78). It is moreover certain that a slight difference in chemical constitution may be correlated with a very marked difference in the nutritive value and physiological importance of allied proteids (Sect. 66). Systematic experiments on the nutrition of fungi with various proteids would undoubtedly bring this fact clearly to view, for it has already been shown that mucin, nuclein, \&c. afford suitable nutrient material for many of them. Various authors have observed that in starved plants the nuclear chromatin diminishes to a marked extent ${ }^{4}$. Hence the higher plants are able to reassimilate nucleins, and various nucleoproteids may apparently function as reserve-materials. Similarly in seedlings of Lupinus the amount of

${ }^{1}$ Cf. the analyses by König, Nahrungs- u. Genussmittel, 1889, and by Ebermayer, Physiol. Chemie, I882, p. 613. On supposed non-nitrogenous organisms, see p. 388 , footnote I.

${ }^{2}$ Palladin, Rev. gén. d. Bot., I $\$ 96$, T. vilr, p. 226, and Zeitschr. f. Biol., 1894, p. I9I, where the contradictory results of Weyl, Vines, Green, \&c., are quoted. On the storage forms of proteid, cf. Drechsel, Handwörterb. d. Chemie, IS $S_{5}, \mathrm{p} .576$, and the literature there given. The literature upon gluten substances is given by O'Brien (Annals of Botany, 1895, Vol. Ix, p. I71). Small amounts only of typical albumins are usually present in plants. Griessmayer, Die Proteide d. Getreidearten, Hiilsen friichte u. Oelsamen, 1897 .

3 Sachs, Flora, I862, p. 297.

'Cf. Zimmermann, Morph. u. Physiol. d. Zellkernes, I896, p. 79. 
proteid not digestible by pepsin decreases slightly at first, although the number of cells and protoplasts increases. The amount increases again as cellular multiplication continues, and in many cases this becomes evident at a comparatively early stage of development (Triticum vulgare, Helianthus) ${ }^{1}$.

The nutritive value for mankind decreases with an increase of the percentage of proteids difficult of digestion, and also of asparagin, whereas the latter affords a very good source of nitrogen for many plants.

In turgid plants the reserve-proteids are for the most part present in dissolved form, but in certain cases they occur as solid crystalloids ${ }^{2}$.

In seeds the proteids occur in the form of aleurone grains ${ }^{3}$ with or without crystalloids, and these may be partially soluble in saline solutions, or even in pure water if the latter dissolves some of the salts associated with them. (Pfeffer, 1. c., I 872 , p. 49 I).

\section{SECTION 80. Nitrogenous Metabolism.}

One function of metabolism is to render the absorbed food-materials of service to the plant, and the changes involved may be either constructive or destructive. In the latter case they sometimes lead to the partial or complete disorganization of proteid molecules, so that amides, amido-acids, ammonia, or in a few cases even nitrogen may be produced, especially when the plant stands in need of such substances and when they are absent from the food supplied or present in insufficient amount. This takes place when seeds of Lupinus, Vicia, \&c. germinate, for almost the whole of the nitrogen of the seed is present in the form of proteids, whereas in the seedling as much as 30 per cent. of the dry weight may consist of asparagin 4 . There can be no doubt that amides may also be formed synthetically, and hence it is not always possible to tell the precise mode of origin of the different nitrogenous compounds which may appear (Sects. 68, 71).

In a fungus fed solely with proteids and inorganic salts not only must the entire supply of energy be obtained from the partial or complete combustion or disintegration of proteids, but the cell-wall, carbohydrates, fats, and indeed every metabolic product must directly or indirectly be derived from the same source. When this is the case ammonia is a constant end-

1 Palladin, Rev. gén. d. Bot., 1896 , T. v'III, p. 228 ; also Frankfurt, Versuchsst., 1893 , Pd. XILIII. p. I 75 ; Prianischnikow, ibid., I 949 , Bd. xLv, p. 253.

2 These are probably vitellinates of alkaline earths. Schmiedeberg, Zeitschr. f. physiol. Chemie, I 877 , Bd. I, p. 205 ; Grübler, Über krystallinisches Eiweiss d. Kürbissamen, I 88 I.

3 On aleurone grains, see Pfeffer, Jahrb. f. wiss. Bot., Is $\varsigma_{2}$, Bd. VIII, p. 429: Wakker, ibid., I 888 , Bd. xIx, p. 453; Luidtke, ibid., I 889 , Bd. xxI, p. 62. All observers agree that the aleurone grains are formed in the cell-sap, but the grains possibly do not always differentiate in the same manner. Cf. Pfeffer, Aufnahme u. Ausgabe ungelöster Körper, ISgo, p. iso. (On Globoids, cf. Sect. 74 .

4 Cf. E. Schulze, I andw. Jahrb., Isৎs. Bd. Xxi, p. 694; Isso. Bd. Ix, p. I 2. 
product of metabolism ${ }^{1}$, but a supply of sugar may suffice to partially or completely inhibit the formation of this compound, or even to induce an assimilation of ammonium salts and a synthetic formation of proteids (Sects. 66, 67, 70).

It is hence casy to see why a much less marked accumulation of anides occurs during the germination of seeds which contain an abundance of starch or oil, than in that of leguminous seeds which are rich in proteids. In the latter case it is only as starch is formed that the accumulated asparagin, \&c. is regenerated into proteid substance and hence gradually diminishes ${ }^{2}$, for when the seedling is kept in air deprived of carbon dioxide the assimilating organs of Lupinus, \&c. remain loaded with asparagin until death occurs ${ }^{3}$. Under these circumstances the phosphates and sulphates set free by proteid-decomposition must remain unassimilated so long as no reconstruction of proteid is possible ${ }^{4}$.

It is apparently of considerable economic importance that amides, phosphates, \&c. should be stored up in these forms only when the quantities present are small. In the case of leguminous plants, on the other hand, more material can be condensed into a smaller space in the form of proteids, and hence a supply of nourishment is provided sufficient to cnable the scedling to develop in a non-nitrogenous soil until it is able to form root-tubercles and thus assimilate free nitrogen (Sect. 69). The stored proteids not only afford a supply of food but their decomposition renders a certain amount of energy available, no matter whether the liberated non-nitrogenous substances are used in respiration or as plastic or constructive material. The latter may occur when other non-nitrogenous reserve-materials are present, although in the case of the Leguminosae this is a point which has not yet been satisfactorily determined.

When proteids are continually assimilated and disintegrated, the nitrogenous waste products must be excreted and thus any over-accumulation prevented. In the case of Fungi this is provided for by the excretion of ammonia, and in the case of animals by that of urea. The latter is, however, formed by synthesis from ammonium carbonate ${ }^{5}$, and hence in both cases

' Nägeli, Bot. Mitth., I88I, Bd. III, p. $28_{3}$; Wehmer, Bot. Zeitung, I 89 I, p. 295 ; Marchal, Centralbl. f. Bact., I 895, Abth. ii, Bd. I, p. 75.3. The formation of ammonia during putrefaction is well known.

${ }^{2}$ Pfeffer, Jahrb. f. wiss. Bot., I872, Bd. VIII, P. 548; E. Schulze, Landw. Jahrb., 1880 , Bd. Ix, p. 44; I888, Bd. xviI, p. 691 .

${ }^{3}$ Pfeffer, Monatsb. d. Berl. Akad., I873, p. 7 80. The mode of experimentation is represented in Fig. 48 . These results have been confirmed by O. Miiller, Versuchsst., I887, Bd. xxxiII, p. 326 .

For sulphates, cf. E. Schulze, Landw. Jahrb., $18 ; 6$, Bd. r; p. 856 ; 1880 , Bd. Ix, p. 21 ; I $89^{2}$, Bd. xxi, p. 118 ; Kellncr u. Sachsse, Phytochem. Unters., I88o, I, p. $5^{8}$; Tammann, Zeitschr. f. physiol. Chemie, $188_{5}$, Bd. Ix, p. 417 . During proteid-decomposition, amides and sulphates increase concomitantly, and, as in animals, sulpho-acids are probably formed at the same time. Cf. Sect. it.

s Bunge, Physiol. Chemie, 1s94. 3. Aufl., p. 206. 
practically the same end-product is produced, the difference being that the fungus avoids any injurious accumulation or alkalinity by excreting oxalic acid or by other means (Sect. 86). It is extremely doubtful whether the lower animals produce urea, and this never seems to be formed in vegetable metabolism although various derivatives of uric acid may appear. In the higher plants the decomposition of proteids appears as a general rule only to proceed as far as the formation of amides, \&c. ${ }^{1}$, and the fact that these remain present in starved plants shows that the latter are unable to satisfy their requirements or to obtain a sufficient supply of energy by the disintegration of amides, although this power is actually possessed by certain fungi. It is always possible that exceptions may occur ; indeed the hydrocyanic acid, trimethylamin, \&c. formed by certain plants may be normal end-products of proteid metabolism, especially when the latter is artificially stimulated. Many lower organisms excrete amides and other nitrogenous substances which are produced during putrefaction.

It appears therefore that all plants are capable of inducing proteid decomposition, as is shown by the fact that a marked accumulation of asparagin and other amides takes place in cut branches of trees, herbs, mosses, \&c., or in intact plants kept in darkness or in air deprived of carbon dioxide, or in the apices only of branches, \&c. when they are kept in darkness ${ }^{2}$. Borodin ascribes this entirely to the insufficiently active regeneration of the amides produced, but apparently an increased activity of proteid-decomposition is induced at the same time, for we are dealing here only with a special case of a phenomenon common to all plants, and since an increased proteid-decomposition accompanies a deficiency of non-nitrogenous food in fungi and in animals ${ }^{3}$ it seems permissible to assume that a similar correlation exists here also. At the same time different nitrogenous substances may partially, totally, or mutually protect one another, as for example when the presence of nitrates inhibits the assimilation of free nitrogen (Sect. 70), and in many other cases as well (Sects. 67, 93). The decomposition of proteids probably continues without cessation even in adult plants which are well supplied with non-nitrogenous organic food, yielding an adequate supply of energy and sufficing for the maintenance of organs which have ceased to grow. Definite proof of this fact may possibly be obtained by a critical study of those organisms which are incapable of regenerating proteids and in whose excreta certain of the products of proteid-decomposition must therefore appear, as is the case in the higher animals.

1 The formation of traces of ammonia by seedlings is possible, but still doubtful. Cf. Hosaeus, Jahresb. d. Agr.-Chemie, 1867, p. Ioo; Sabanin u. Laskovsky, Versuchsst., I875, Bd. xvili, p. 407 ; E. Schulze, Landw. Jahrb., I 878 , Bd. vII, p. $4^{20}$; $18 S 0$, Bd. $1 \mathrm{x}$, p. 15.

2 First observed by Borodin, Bot. Zeitung, 1878 , p. Sor ; also E. Schulze, Landw. Jahrb., I 8so, Bd. IX, p. 25 ; O. Muiller, Versuchsst., I887, Bd. xxxilr, p. 327 .

3 Neumeister, Physiol. Chemie. 1893. 13d. I. p. 293. 
It is possible that the continuance of proteid-decomposition is essential to all organisms, although this cannot be postulated with absolute certainty in the case of adult cells. It may even happen that respiration need not necessarily involve any marked disintegration of proteid material (Sect. I0r). This might still be the case even though the substance consumed in respiration was associated with the protoplasm, and continually liberated only to be immediatcly oxidized.

The regeneration of the amides produced by metabolism is simply a special case of the chemosynthesis of proteids, and is indirectly influenced to a great extent by the action of light, not merely owing to the fact that by means of the latter the necessary carbohydrates may be produced, but also because light forms an essential condition for the normal performance of many vital activities. Indeed the changes in the shape and in the growth of green parts developed in darkness are necessarily accompanied by corresponding changes in the metabolic activity of the parts affected. The synthetis of proteids will probably be more or less markedly inhibited when pathological conditions of this character are induced in green organs, although respiration and proteid-decomposition may continue with the same or even greater activity. The fact that the regeneration of amides may cease in darkness even when carbohydrates are present, merely indicates that darkness may exercise some such indirect pathological action, and does not necessitate the assumption that the process of regeneration is directly due to the assimilatory action of the light absorbed, as was supposed by O. Müller ${ }^{1}$. The same regeneration is possible in roots which grow normally in darkness, and according to Monteverde a supply of sugar will partially or completely prevent the accumulation of asparagin in darkened twigs of Syringa; while Kinoshita finds that the same result is produced in seedlings of Soja grown in darkness and fed with glycerine ${ }^{2}$. It is however certain that the same effect will not be exercised upon all plants ${ }^{3}$, and the most important experiments are those in which the development in darkness is made as normal as possible, either by adopting special modes of treatment or by selecting suitable plants.

Both Müller and E. Schulze failed to recognize that metabolism is always determined and regulated by the existent vital activity, and that proteid-synthesis is possible only when all the necessary conditions are fulfilled. External conditions modify the rital activity, and this along with the regulatory mechanism brings it about that in the same cell at one time proteids may be disintegrated, at another reconstructed. In the germination of seeds of Phaseolus, Pisum, \&c., the starch and proteids are mobilized and translocated together, but it may be found possible

1 O. Miiller, Versuchsst., 1886, Bd. xxxim, p. 311.

2 Monteverde, Bot. Centralbl., 189 r, Bd. xlv, p. 379 ; Kinoshita, Bull. Coll. Agric., Tokio, 1895 , Bd. II, P. 197 .

${ }_{3}$ Prianischnikow (Versuchsst., I896, Bd. XLII, P. 458) has obtained negative results with seedlings of Vicia sativa. Hansteen (Ber. d. Bot. Ges., $18_{9} 6$, p. 322) has recently shown that Lemna is able to regenerate proteids from amides in darkness.

4 E. Schulze, Iandw, Jahrb), 1880. Bd. Ix, p. 52. 
(Sect. 93) to cause the starch to be retained intact while the amides formed by proteid-decomposition are continually removed "

The importance of asparagin as translocatory material is sufficiently evident, and it is obviously erroneous to regard it as an excrete product of metabolism, as Prianischnikow ${ }^{2}$ has recently done, thus upholding the view originally put forward by Boussingault.

\section{SECTION 8I. Nitrogenous Metabolism (continued).}

The various synthetic processes already mentioned probably all involve a more or less complicated series of operations, and indeed it is only by such means that a fungus can possibly exist when fed solely with proteids. Even when proteids are assimilated along with carbohydrates, the quantitative and qualitative differences between the nitrogenous end-products form a sufficient indication that the course of metabolism may follow widely different paths in different plants and also in the same plant under varying circumstances.

When similar products are produced from different carbohydrates it is impossible to say whether the processes become identical before this particular metabolic activity is completed or only during the very last stage, and similarly it is impossible to say whether a difference between the nitrogenous end-products is due to a change occurring immediately a proteid molecule is assimilated or only at a later stage in the ensuing chain of reactions. Moreover, it is impossible to say whether any amides that may appear have been formed directly by proteid-decomposition, or have perhaps been formed synthetically from ammonia or other disintegration-products. Amides are actually formed synthetically during the growth of many of the higher plants (Sect. 7 I), but the amides which appear during the mobilization of reserve food-materials do not seem to be produced in this manner.

It may, however, safely be concluded that owing to the reactive power and regulatory activity which every plant possesses, the mode of formation of amides may vary in the same plant under different nutritive and cultural conditions. Thus Schulze" found that glutamin usually preponderated in cucumber seedlings, but that occasionally asparagin was most abundant, while green plants of Lupinus luteus contained leucin in addition to the usual asparagin, whereas in etiolated plants amido-valerianic acid and

${ }^{1}$ For a comparison between proteid and carbohydrate metabolism, see the values given by Prianischnikow (Versuchsst., I895, Bd. XLv, p. 262) for Vicia saliva.

2 Prianischnikow, Versuchsst., 1806 , Bd. Xlvi, p. $45^{8}$.

${ }^{3}$ E. Schulze, Zeitschr. f. physiol. Chemie, I896, Bd. xxir, p. 411 ; I894, Bd. xx, p. 308 ; Versuchsst., I896, Bd. Xuvin, p. 53 . 
phenylanilin replaced the former. Purposeful experiments in this direction would undoubtedly yield many results of great importance in rendering possible a clear comprehension of the problems of metabolism. Besides the effect exercised by the lack or the abundant presence of carbohydrates, it is also probable (Sect. 93) that under certain circumstances the accumulation or continual removal of an amide, \&c. may hinder or accelerate its production. To what extent any particular plant may form substances which are not normal products of metabolism can only be answered by direct experiment, but whenever an amide does not re-enter into metabolism under the existing conditions it is evident that its appearance in small traces only is mercly due to the amount produced being small, and not to its being continually reassimilated. The regulatory production of diastase affords an example of the way in which the formation of an aplastic nitrogenous compound may be entirely suppressed.

No pepsin or trypsin has been detected in Lupinus, Vicia, and many other plants', and hence we must conclude that amides, peptones, $\&$ c. are not formed by the hydrolytic action of proteolytic enzymes. The living plasma is, however, able to bring about the most remarkable molecular rearrangements by inducing both synthetic and retrogressive metamorphoses. Hence it is possible that the retrogressive changes are from the commencement different to those induced by the action of ferments, acids, \&c. 'Sect. II'. Moreover, the numerous cases of so called 'Tautomery' show that various interactions during the actual process of decomposition may induce a different molecular rearrangement, and many facts concerning proteids render it improbable that amido-acids exist as preformed constituents of the proteid molecule ${ }^{2}$.

Hence it is impossible to agree with Schulze in postulating the same chemical and physiological decomposition of proteid in all cases as far as the formation of amides. Schulze ${ }^{3}$ has indeed recently somewhat modified this opinion, and argues that the reassimilation of amides can hardly take place always in precisely the same manner. Decisive conclusions can only be obtained empirically, and Palladin and Loew's supposition that the asparagin is formed especially by the process of respiration, the other amides by proteolytic decomposition, is an extremely improbable one ${ }^{4}$, for direct experiments show that asparagin is formed in abundance during intramolecular respiration ${ }^{5}$.

\footnotetext{
1 Neumeister, Zeitschr. f. Biol., I894, N. F., Bd. xII, p. 447. Cf. Sect. 91. [Green (Phil. Trans., IS87) has, however, detected a trypsin ferment in Lupinus hirsutus.]

${ }^{2}$ Cf. E. Schulze, I andw. Jahrb., i $\$ 92$, Bcl. xxi, p. I 2 I.

${ }^{3}$ Schulze, l. c., I892, p. I19, and Zeitschr. f. physiol. Chemie, I896, Bd. xxII, p. 434, where the earlier ideas are also given.

${ }^{4}$ Palladin, Ber. d. Bot. Ges., I888, Bd. vi, pp. 205, 296. Cf. E. Schulze, I892, l. c., pp. I 21 , I 24; also Prianischnikow, Versuchsst., I896, Bd. xuvi, p. 464; Loew, Jahresb. d. Agr.-Chem., I 889 , p. I I 3 .

${ }^{5}$ Cf. I. Schulze, 1. c., I 892 , p. I 24 ; Ziegenbein, Jahrb. f. wiss. Bot., I 893 , Bd. Xxv, p. 572 ; Clausen, Landw. Jahrb., I $\varepsilon_{9} \mathbf{I}$, Bd. xix, p. 914.
} 
Analjtical examples. The following tables constructed by Schulze show the extent to which asparagin may accumulate ", and also the increase in the amides and certain other nitrogenous substances which take place as the proteids decrease. Plants grown in darkness were harvested after eight to thirteen days; in the first case the hypocotyl was from $2-2.5 \mathrm{~cm}$. long, in the second $7-9 \mathrm{~cm}$.

\begin{tabular}{|c|c|c|c|c|c|c|}
\hline & $\begin{array}{c}1 \\
100 \text { gr. dry } \\
\text { weight of } \\
\text { ripe seeds } \\
\text { contained }\end{array}$ & $\begin{array}{c}2 \\
87 \cdot+\text { gr. re- } \\
\text { maining } \\
\text { after } 8 \text { days } \\
\text { contained }\end{array}$ & $\begin{array}{c}3 \\
\text { Difference } \\
\text { between } \\
1 \text { and } 2 .\end{array}$ & $\begin{array}{c}4 \\
81 \cdot 7 \mathrm{gr} \text {. } \\
\text { after } 13 \\
\text { days con- } \\
\text { tained }\end{array}$ & $\begin{array}{c}5 \\
\text { Difference } \\
\text { between } \\
2 \text { and } 4 .\end{array}$ & $\begin{array}{c}6 \\
\text { Difference } \\
\text { between } \\
1 \text { and } 4 .\end{array}$ \\
\hline $\begin{array}{l}\text { Conglutin } \\
\text { Albumin } \\
\text { Asparagin } \\
\text { Amides, Alkaloids, } \\
\text { and undetermined } \\
\text { substances. }\end{array}$ & $\begin{array}{l}43 \cdot 57 \\
1 \cdot 50 \\
0\end{array}$ & $\begin{array}{r}21 \cdot 40 \\
3 \cdot 53 \\
y \cdot 75\end{array}$ & $\begin{array}{r}-22.17 \\
2.03 \\
9.78\end{array}$ & $\begin{array}{r}10.25 \\
1.41 \\
18.22\end{array}$ & $\begin{array}{r}\text { II.I5 } \\
-\quad 2.12 \\
8.44\end{array}$ & $\begin{array}{r}-3.32 \\
-\quad 0.09 \\
18.22\end{array}$ \\
\hline
\end{tabular}

The following are the results obtained by Prianischnikow? with seeds of Vicia sativa germinated in darkness.

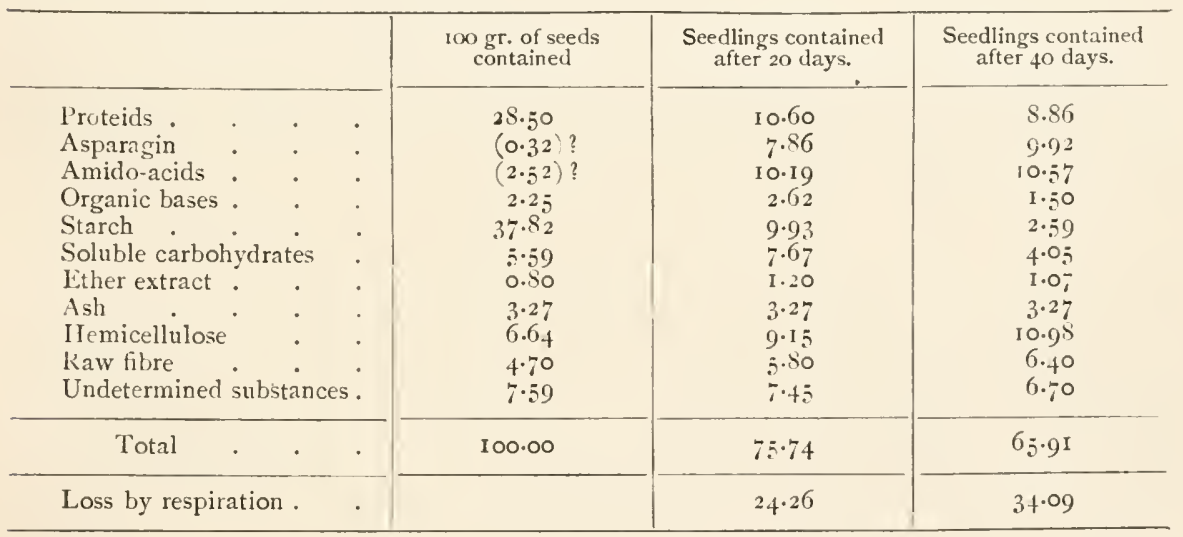

Further details concerning the methods employed, \&c. can be obtained by reference to the works quoted in Sect. 79. On microchemical methods, cf. Zimmermann, Mikrotechnik. These were first systematically employed by Pfeffer in the study of asparagin (1872). No deposition of asparagin occurs in a living cell even when the latter contains more of it than the water of the cell-sap can dissolve, but it is doubtful whether this is due to the formation of an oversaturated solution ${ }^{3}$, or to the combination of the asparagin with some other substance. Even when the total amount of water present may just be sufficient

1 E. Schulze, Landw. Jahrb., is; 6 , Bd. v, p. $s_{4} 8$. Detailed analyses of the seeds of Lupinus in Versuchsst., I 891, Bd. xxxix, p. 294.

${ }^{2}$ Prianischnilow, Versuchsst., I $89^{6}$, Bd. XLir, p. $4^{67}$.

${ }^{3}$ On over-saturated solutions, cf. Ostwald, Lehrb. d. allgem. Chemie, 2. Auf., I 89r, Bd. I, p. I039; Zeitschr. f. physik. Chemie, I897, Bd. xxır, p. 289 . 
to dissolve the whole of the asparagin, only a portion is available for solvent purposes, owing to the localized distribution of the latter. No precipitate could be detected in sections kept at $3^{\mathrm{c}} \mathrm{C}$. for a long time, even when the concentration of the cell-sap was more than doubled by plasmolysis. Other amides remained dissolved under similar circumstances, and the data given by Belzung suffice to show that the precipitation observed by him took place in dead cells ${ }^{1}$.

Historical. Before Pfeffer, in 1872 , showed that proteids may undergo very thorough disintegration in metabolism, the opinion was generally held that the plant avoided as far as possible any dissimilation of proteids when once formed ${ }^{2}$. Th. Hartig had indeed shown that crystalline nitrogenous compounds resulted from proteid decomposition, but his statements were inaccurate on several points, and the remarkable theoretical conclusions which he based upon his observations caused the latter to be disregarded ${ }^{3}$. Since 1876 , Schulze, Borodin, \&c. have brought forward more and more examples of the self-decomposition of proteids, so that the idea becomes more firmly established that an unceasing decomposition of proteids is associated with the continual liberation of kinetic energy necessary in every active protoplast ${ }^{4}$, but it is still doubtful whether such decomposition never ceases in an active cell under any circumstances, even though adult. Detmer ${ }^{5}$ supposes that this proteid-decomposition is associated with a destruction of the physiological units (Sects. 7 and 8), that is, he postulates a theoretical mode of operation for a process of which neither the universal occurrence nor the actual character have as yet been established.

\section{SECTION 82. Carbohydrates and Fats.}

All organic substances are derived from photosynthetic assimilation, and hence the carbohydrates thus produced are the commonest and most abundant organic food-materials. There is a greater production of carbohydrate than of any other substance, and every autotrophic plant consumes a large proportion of its synthesized carbohydrates in order to obtain a supply of energy and to produce directly or indirectly its different metabolic products, including all organic reserve food-materials. Carbohydrates and fats form the chief non-nitrogenous substances used for storage, other substances, such as organic acids, \&c., being usually present in small amount and only rarely predominating. It is during the early stages of

${ }^{1}$ Cf. Pfeffer, Pflanzenphysiol., I. Auf., Bd. I, p. 316, footnote; Oxydationsvorgänge in lebenden Zellen, 1889 , p. 457 ; Belzung, Ann. d. sci. nat., IS92, vii. sér., T. $x v$, p. 256 . On the insufficiency of Belzung's method, cf. E. Schulze, Zeitschr. f. physiol. Chemie, I 894, Bd. xx, p. 323.

${ }^{2}$ See Ad. Mayer, Agr.-Chem., I $S_{71}$, I. Auf., Bd. I, p. 2 I 4 ; Pfeffer, Jahrb. f. wiss. Bot., I $S_{7} 2$, Pd. vir, p. 530 , where the older literature is given; Monatsb. d. Berl. Akad., I $S_{73}$, p. 7 So.

${ }^{3}$ Th. Hartig, Entwickelungsgesch. d. Pflanzenkeimes, I 858 , p. I 26.

${ }^{4}$ Pfeffer, Landw. Jahrb., IS7S, Bd, vit, p. So7. Cf. also E. Schulze, Landw. Jahrb., isso, Bd. $x$, p. 33 .

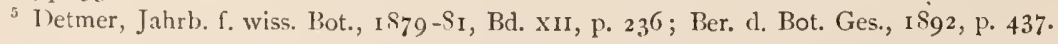


development that the largest quantities of non-nitrogenous food-materials are required, and hence, unless special adaptations come into play (Sect. 80), non-nitrogenous substances preponderate in the seed: thus in cereals, up to 80 per cent. of starch may be present, in fatty secds 70 per cent. of the dry weight may consist of oil, while a fresh beet-root may contain as much as I 6 per cent. of cane-sugar. As far as is known, carbohydrates and fats also form the main reserve food-materials in fungi, and carbohydrates may be formed by the latter when fed with other carbon-compounds (Sects. 66 and 80). Carbohydrates and fats do not function merely as food-material, for cellulose is employed in cell-wall formation, and it is questionable whether carbohydrates and fats may not form essential constituents of the protoplasm, independently of the carbohydrates which may be associated with the molecules of certain proteids (cf. Sect. I I).

The terms 'carbohydrate' and 'fat' are simply general names for large groups of substances. Carbolhydrates especially are extremely polymeric (disaccharides, polysaccharides), and readily serve for the formation of such substances as glucosides with widely different physical and chemical properties. Carbohydrate compounds of this kind are probably formed by every plant, though there is perhaps no special combination which is essential to all. Carbohydrates and fats may mutually replace one another to an extremely wide extent, and hence it arises that different organs of the same plant may contain different reserve-materials, and that not infrequently various carbohydrates and fats may be present in the same cell.

The most widely distributed plastic carbohydrates ${ }^{1}$ are starch, dextrose (glucose, grape-sugar), laevulose (fructose, fruit-sugar), and cane-sugar (saccharose). Starch is, however, absent from fungi, and of the carbohydrates with a more limited range, inulin is found abundantly in Compositae; glycogen and trehalose in many fungi. Mannite is less rare, and often occurs in fungi, as do many of the different forms of reservecellulose found in seeds. Many other carbohydrates frequently occur in small amount, or in abundance, in special plants, but these are less important, and for the most part only those with special optical properties have been detected.

As far as is known, hexoses, as well as the di- and poly-saccharides, seem to function as the chief plastic carbohydrates, whereas the pentoses which appear to be universally present ${ }^{2}$ seem for the most part not liable

1 A complete account of the chemistry and distribution of the known carbohydrates is given by Tollens, Handb. d. Kohlenhydrate, Bd. I, I 888 ; Bd. II, I895; Lippmann, Chemie d. Zuckerarten, 1895. Our knowledge of their chemical constitution is mainly due to E. Fischer.

2 Cf. Tollens, I\$95, 1. c., pp. 19\$, 240 ; Journ. f. Landw., IS96, Bd. xxIv, p. r 7 , and Beibl. $z$. Biol. Centralbl., I 896, Bd. vi, p. $33^{1}$; Goetze u. Pfeiffer, Versuchsst., r 896, Bd. xLvir, p. $5^{8 .}$ See also Sect. 54. Trioses, Nonnoses, \&c. do not seem to be present in plants, but may in certain cases serve as food for fungi, yeast, \&c. 
to further metabolism, so that usually the amount present increases as the plants grow older. Most gums, and also a few forms of reserve-cellulose, are pentosans, that is, compounds of pentose with other substances. Combined pentoses may serve as plastic material, and it is even possible that pentoses may normally, or only under special circumstances, be reassimilated. This possibility is not entirely due to the nutritive value of the substances in question, for the plant is able when necessary to protect starch, grape-sugar, \&c., from immediate consumption (Sects. 77 and 78 ).

Many pentoses (arabinose, \&c.) constitute a moderately good nutritive medium for mould-fungi, and certain forms of mucilage which may serve as reserve food-material in the tubers of Orchis, \&c. may belong to the group of pentosans. In other plants the mucilaginous substances seem to be permanently aplastic products of merely biological importance, as is the case with regard to those forms of mucilage which dissolve or swell in water. These for the most part appear to be carbohydrates. The insufficiently known pectic compounds which are present in part in the cellwall, and in part dissolved within the cell, apparently belong to the same group of substances, and probably subserve a variety of physiological uses.

The fatty oils found in plants mainly consist of glycerides of oleic, palmitic, and stearic acids, and during translocation large quantities of free fatty acid are usually present. The oil globules found in the cells of many of the Hepaticae are peculiar fatty masses, which behave as aplastic bodies ${ }^{1}$. The waxy deposits or impregnations which modify the permeability of the cell-wall are also aplastic products, although they may be uscd as food-material by certain fungi ${ }^{2}$. In some cases the waxy substances are esters of monatomic alcohols.

The different carbohydrates and fats have each their own specific nutritive value, as is shown by experiments with fungi (Sects. 66 and 67), and by the restriction of particular fermentative organisms to certain forms of sugar. The same differences probably exist as regards their nutritive value for higher plants, so far as these require external supplies of nonnitrogenous organic food ${ }^{3}$. It is evident that carbohydrates and fats exhibit a certain amount of physiological equivalence in normal metabolism, for both may serve as reserve-material in the same plant, and in certain cases inulin, starch, grape-sugar, oil, \&c., are formed in succession from the same food-substance.

These and other metamorphoses serve as means to render possible storage, translocation, mobilization, or to prevent loss by exosmosis,

${ }^{1}$ Pfeffer, Flora, I874, p. 40; W. v. Küster, Oelkörper d. Lebermoose, 1894.

${ }^{2}$ R. H. Schmidt, Flora, IS91, p. $3^{1} 5$.

3 The formation of starch grains can be induced by the presence of certain substances only (Sect. 55). 
although to attain these ends marked metamorphosis is not always necessary (Sects. 22, 78, and I08). Indeed glucose, cane-sugar, oil, \&c. seem to function either as reserve or as translocatory material, whereas starch and cellulose are capable of being translocated only when converted into a soluble form; inulin also seems to be used solely as reservematerial. Metamorphoses of this character frequently involve a loss both of encrgy and of substance, but it is easy to see, for example, that the repeated solution and regeneration of starch (Sect. Io 8 ) during its passage through chains of cells may be of considerable advantage, and this is a conversion which is repeatedly performed when twigs are alternately subjected to warmth and cold (Sect. 92). Only by considering the whole of the aims and requirements of the plant can a correct estimation of any phenomenon be obtained, and thus the formation of insoluble substances, or of soluble compounds with greater molecular weights, such as inulin and certain pulysaccharides, not only renders exosmosis more difficult, but also enables an excessive osmotic pressure to be avoided. Similarly, it is apparently advantageous that seeds which are able to withstand desiccation should contain mainly starch and oil in addition to proteids, soluble and crystallizable compounds being avoided as far as possible.

It is impossible to predicate on empirical grounds that a particular substance must always be formed during translocation in order to render a given metabolic process possible, for although reducing sugars, such as glucose, commonly appear, and perhaps are also present even when incapable of detection, it is probable that a variety of different hexoses are formed. Moreover, it has already been mentioned that similar products may be formed from different sources, although the similarity becomes evident only at a late stage in metabolism (Sects. 66 and 77).

A final decision of these and similar questions will be possible only when a more complete knowledge has been obtained of the inherent factors concerned in metabolic activity. Mobilization and translocation are, however, simply preparatory partial functions, and hence are more amenable to empirical study, as for example when some special decomposition is found to be due to the action of an enzyme. The protoplast probably dissolves the reserve-cellulose which encloses it by the aid of a ferment, and the hydrolytic decomposition of starch, inulin, cane-sugar, and other polysaccharides, and possibly of fats also, is apparently produced in the same manner (cf. Sect. 91). Enzymes are the agents employed by the protoplast when extracellular action is necessary, and they are indispensable in preparing substances for further change. There is no doubt, however, that an organism which can produce amides and carry out complicated syntheses will also be able to complete the simple changes of hydrolytic decomposition without extraneous aid. During translocation starch and various polysaccharides may be repeatedly reconstructed and decomposed, and it 
is impossible to be certain whether these simple changes may not be the result of complex decompositions and reorganizations occurring in the interior of the protoplast.

Starch always originates in special plastids or chromatophores, but no definite organs seem to be concerned in the formation of fats, which like many other products may accumulate in the cell-sap, so that the locality where fat is found affords no indication as to the place of its formation. The importance of the exchanges occurring between the cell-sap and plasma as the means by which metabolic changes may be indicated or regulated will be discussed later (Sect. 93).

As examples of non-nitrogenous metabolism, the following table giving the composition of seeds and seedlings of maize and hemp may be given. The values for maize are from Boussingault ${ }^{1}$, who germinated twenty-two seeds weighing $8.636 \mathrm{grm}$. in darkness on pumice-stone and analyzed the seedlings after fifteen days. Similar analyses were made with the same weight of grains of maize, including the integuments.

\begin{tabular}{|c|c|c|c|}
\hline & $\begin{array}{l}\text { In } 22 \text { seeds. } \\
\text { grm. }\end{array}$ & $\begin{array}{c}\text { In the seedlings. } \\
\text { grm. }\end{array}$ & Difference. \\
\hline $\begin{array}{l}\text { Starch (and Dextrin?) } \\
\text { Glucose } \cdot \\
\text { Fat }: \\
\text { Cellulose }: \\
\text { Nitrogenous material } \\
\text { Ash } \\
\text { Undetermined substances }\end{array}$ & $\begin{array}{l}6.386 \\
0 \\
0.463 \\
0.516 \\
0.880 \\
0.156 \\
0.235\end{array}$ & $\begin{array}{l}0.777 \\
0.953 \\
0.150 \\
I .316 \\
0.880 \\
0.156 \\
0.297\end{array}$ & $\begin{array}{r}-5.609 \\
0.953 \\
-0.313 \\
0.800 \\
0.000 \\
0.000 \\
0.062\end{array}$ \\
\hline - & 8.636 & $4 \cdot 5^{29}$ & $-4 \cdot 107$ \\
\hline
\end{tabular}

Detmer's ${ }^{2}$ analyses of hemp seedlings were obtained in a similar manner and are as follows :-

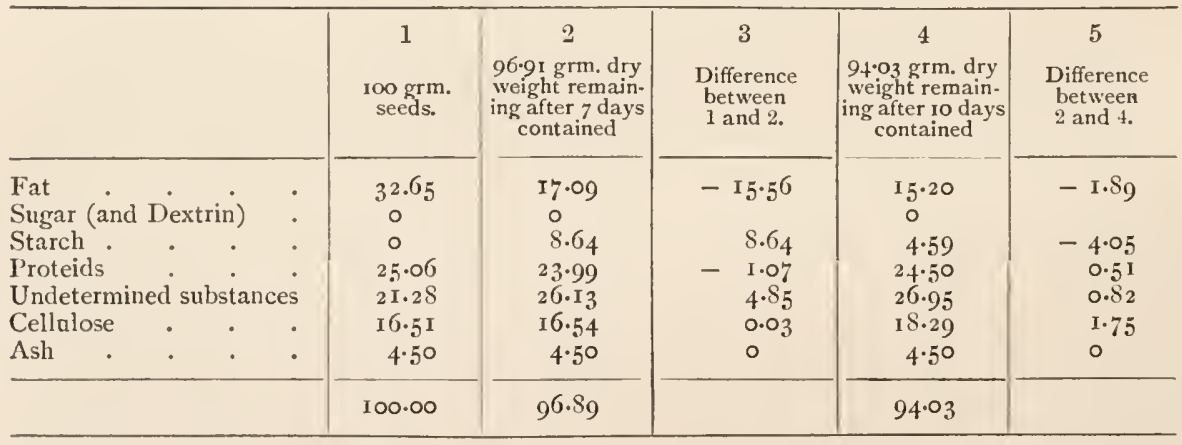

1 Boussingault, $\Lambda$ gron., Chim. agric., \&c., I868, T. IV, p. 26 I.

${ }^{2}$ Detmer, 1'hysiol.-chem. Unters. über die Keimung ölhaltiger Samen, I875, p. 40. According to Leclerc du Sablon (Rev. gén. d. Bot., I895, T. viI, p. 2 Io), a little glucose and cane-sugar are formed during germination. Cf. also Frankfurt, Versuchsst., I 894, Bd, XLIIr, p. I57. For further literature on chemistry of germination, see Sect. Io9. 
The loss by respiration rapidly becomes more and more marked, and the starch which disappears from the maize plants is used both in respiration and in the formation of sugar and cellulose, for the fat alone does not suffice to cover the loss by respiration or to produce the amounts of sugar and cellulose which appear. In hemp seedlings fat is utilized for the formation of starch and also of cellulose, the latter being possibly produced from the former, for between the seventh and tenth day a large quantity of starch is consumed and much less fat.

Experiments of this kind indicate only the beginning and the end of metabolism, but nevertheless they suffice to show that its course is by no means always the same. Thus during the germination of the oily seeds of the cucumber (Peters) and of Allium cepa (Sachs) glucose is formed abundantly, whereas hardly any appears in seedlings of Cannabis sativa. Similarly, oil is formed from glucose in the ripening seed of Ricinus (Sachs), but in the endosperm of Paeonic mainly from starch (Pfeffer). Again, a beetroot forms cane-sugar, but the tuberous roots of Dahlia and the tubers of Helianthus store inulin, from such carbohydrates as glucose and starch.

A few general remarks may be made upon the physiological relationships of the more commonly occurring non-nitrogenous substances, but for a more complete account the reader is referred to the literature already quoted. (On cellulose, cf. Sects. 83 and 84 .)

Starch grains. These remain within the chloroplastids or leucoplastids in which they were produced until they are dissolved and removed ${ }^{1}$, so that under normal conditions they are never found lying free in the protoplasm or cell-sap ${ }^{2}$. Even were the latter the case, the starch grains would not be lost, but might be rendered soluble by the action of ferments, as occurs, for example, when fungi are fed with starch. The latter moreover usually disappears from dying cells of starch-forming plants. On the action and production of diastase, see Sect. 9r. The growth of starch grains will be discussed later.

Starch grains are composed mainly or entirely of amylose, and usually turn blue with iodine; but in certain cases, as in the seed-coat of Chelidonium, Oryza, \&c., they are mainly composed of amylodextrin, and other dextrins as well, so that a red colouration is produced with iodine. These substances are formed as intermediate products of diastatic action, so that starch grains which redden with iodine may be regarded as having undergone partial conversion into sugar. Certain forms of mucilage are perhaps composed of dextrins soluble in water. Another product of diastatic action, namely maltose, apparently commonly occurs in plants ${ }^{3}$.

${ }^{1}$ Cf. Sects. 53-55. Details and literature on starch, A. Meyer, Unters. über die Stärkekörner, I 895 . Estimations of the percentage of starch present in plants by König and by Ebermayer : cereals contain from 50 to 70 per cent., potatoes $\mathrm{I}_{5}$ to 30 per cent., by dry weight.

${ }^{2}$ Pfeffer, Aufnahme u. Ausgabe ungelöster Körper, r89o, p. I77. Cf. Sect. I9. Starch may be found in living tracheae, or in those filled by tyloses (Lange, Flora, I $\$ 9 \mathrm{I}, \mathrm{p} .393$ ).

3 Detected in a few cases by Brown and Morris, Journ. of Chem. Soc., I893, p. 662; Bot. Zeitung, I $89^{2}, \mathrm{p} .4^{6} 5$. [A few forms of plant mucus (pectin componnds combined with amylose?) give a blue colouration with iodine, possibly owing to the presence of carbohydrates more or less 
Dextrose, Laevulose, Cane-sugar. These are the commonest forms of sugar, and function both as translocatory and as reserve material (cf. Sects. 16, 54, ro9). All three forms frequently occur together, but usually one preponderates. Even the beet-root contains traces of glucose along with its cane-sugar, and similarly small quantities of cane-sugar have been detected in many plants where its presence was formerly overlooked'. Of the hexoses, sometimes dextrose, at other times laevulose is more abundant, but in many cases only the total amount of reducing sugar has been determined, and in such cases it remains uncertain whether other glucoses (galactose) or reducing polysaccharides (maltose, lactose) may not also be present. Saccharose may possibly also be frequently accompanied by other nonreducing sugars ${ }^{2}$.

Cane-sugar occurs in great abundance in the beet-root and sugar-cane, in the sap of the sugar-maple, in certain fruits and inflorescences (banana, pine-apple', in the rhizomes and roots of Rubia tinctoria and of certain Labiatae, Umbelliferae, \&c., and small amounts may even be present in ripe seeds along with other sugars. The sap of the sugar-cane may contain as much as 20 per cent. of sugar, and that of wild beet-roots 6-8 per cent., and under cultivation it may rise to as high as 6 per cent. Dextrose and laevulose are usually employed for translocation, but frequently for storage also. Both are very common in pulpy fruits, and they are stored up in the bulbs of Allium cepa and of Ornithogahm arabicum, as well as in the subterranean parts of many species of Primula and Globularia ${ }^{3}$. 'The amount accumulated may often during life reach 5-10 per cent., and in grapes even 25 per cent., of the total weight. In such cases the osmotic pressure

closely ailied to starch, and soluble starch occurs dissolved in the cell-sap of the epidermal cells of certain plants (Saponaria officinalis, \&c.). Cf. Dufour, Kech. sur l'amidon soluble, Bull. d. 1. Soc. Vand. d. Sci. Nat., 'T. XxII. Similarly, the cell-sap in the epidermal cells of Arum italicum turns violet when treated with iodine, the colour disappearing on heating and returning on cooling. The substance giving this reaction escapes from the cells as soon as they are killed, and the watery extract yields on evaporation a transparent, slightly gummy residue, which turns violet or blue with a watery solution of iodine, but reddish-brown when alcoholic iocline is added, turning blue in the presence of water. After prolonged boiling a more reddish reaction is given, and also after partial digestion with diastase or ptyalin, while ultimately the colour reaction disappears, a reducing sugar being formed. This 'soluble starch' has a very much feebler osmotic value than cane-sugar or dextrose, and its molecule is presumably large and complex. Its peculiar distribution points rather to its possessing some biological function (hindrance to transpiration, protection, \&c.) than to its having any special value in nutritive metabolism. It may occur in small quantity in the cell-sap of the guard-cells of the stomata, though it seems always to be more abunclant in the surrounding cpidermal cells, and it may be still present in almost undiminished abundance after a prolonged sojourn in darkness (ten days), although no starch is then present in the mesophyll. The soluble starch soon escapes from the epidermal cells when placed in $5 \circ$ per cent. alcohol, and the same also occurs in absolute alcohol, thoug'l more slowly.]

1 Cf. Tollens, 1. c., 1888, p. $104 ; 1895$, p. 155 ; E. Schulze, Zeitschr. f. physiol. Chemie, IS95, Bd. Xx, p. 511. [C. Hoffmeister, Über d. mikrochem. Nachweis v. Kohrzucker in pflanzlichen Geweben, Jahrb. f. wiss. Bot., 1898 , Bd. xxxI, p. 688.]

2 Raffinose in the wheat embryo, accordmg to Frankfurt, Versuchsst., I 896, Bd. XLvis, p. 469. Cf. also Schulze, 1895 , 1.c., p. 534. Here and in 'Tollens' Handbook an account of Stachyose, Melezitose, \&c. is given.

${ }^{3}$ Cf. Tollens, 1.c., 1888 , pp. 32, $8_{3} ; 11_{95}$, p1). 76,126 ; G. Kraus, Bot. Zeitung, 1876, p. 604. 
in the storage-cells must be extremely high: thus 25 per cent. of glucose is equivalent to a pressure of 37 atmospheres, provided the glucoses are present in the living plant as such (Sect. 24), whereas a similar percentage of cane-sugar would only generate about half this pressure.

All three forms of sugar may be metamorphosed into one another, and may be produced from various substances; but when cane-sugar is produced after the conversion of oil or starch, it is impossible even in the latter case to be certain that the polysaccharide is formed directly by the condensation of invert-sugar. Diastase cannot aid in producing cane-sugar directly from starch, for the products of diastatic action are dextrose and maltose, whereas none of the laevulose which comes from the inversion of cane-sugar ever appears ${ }^{1}$. Dextrose and laevulose are certainly not always produced by the disintegration of cane-sugar, and hence the relative amounts present of each may vary indefinitely. Whether any preference exists for either form of sugar is uncertain, but experiments with fungi how that sometimes dextrose, at other times laevulose, is consumed with greater avidity ${ }^{2}$.

Galactose. A. Meyer ${ }^{3}$ has detected this form of sugar in various members of the Sileneae. Galactose and glucose are the products of the hydrolysis of milksugar, but it is very doubtful whether the latter ever occurs in plants.

Inulin is found especially in the Compositae, but it occurs also in many of the Campanulaceae, Lobeliaceae, and Stylidiaceae, in Drosophyllum (Penzig), and in Neomeris and a few of the Siphoneae (Cramer) ${ }^{4}$. It occurs mainly in subterranean organs, but also in small amount in the sub-aërial stems and leaves of certain plants ${ }^{5}$. Inulin apparently functions as a general rule as reservematerial, but according to G. Kraus it may also appear in the translocatory channels. Prantl found that inulin is often absent from the annual Compositae, while it commonly disappears from biennials during flowering. It is retained in dissolved form in living cells even at low temperatures or when the cell-sap is concentrated by plasmolysis, but after death granular or sphaerocrystalline precipitates are formed ${ }^{6}$. Inulin is soluble only with difficulty, and it is uncertain whether the living cell contains a super-saturated solution or whether it is held in solution by other means.

It is also unknown whether a hydrolytic enzyme always takes part in the mobilization of this variety of carbohydrate, for diastase and the invertase of yeast are

${ }^{1}$ [Gruss (Ber, d. D. Bot. Ges., XvI, 1898 , p. I 8 ) states that in barley seedlings cane sugar is formed from dextrose, and cellulose and starch fiom the former.]

2 For literature see Pfeffer, Jahrb. f. wiss. Bot., I 895 , Bd. Xxviır, p. a27.

3 A. Meyer, Bot. Zeitung, iss6, p. ro6.

4 Tollens, 1. c., IS88, p. I99; IS05, p. 233 ; Prantl, Das Inulin, 1870 ; G. Kraus, Bot. Zeitung, 1877, p. 330; Vöchting, Bibliotheca Botanica, 1887, Heft 4, p. 52 , and Sitzungsb. d. Berl. Akad., 1894 , p. 711 ; Ehrhardt, Bot. Centralbl., I894, Bd. Lx, p. 207 Leucojum); Penzig, Unters. über Drosophyllum lusitanicum, 1877 ; Cramer, Über Neomeris u. Cympolia, 1887, pp. 16, 26 (Sep.-abdr. a. Denkschr. d. Schweiz. Naturf.-Ges., Bd. xxx).

${ }^{5}$ G. Kraus; Vöchting, l. c.; Pistone de Regibus, Bot. Centralbl., I $88_{3}$, Bd́. X1II, p. $3^{6} 5$; Beauvisage, Bot. Jahresb., I888, p. 47 (in Jonidium); G. Meyer, Ber. d. Bot. Ges., I 896. p. 355 .

${ }^{6}$ Cf. Zimmermann, Mikrotechnik, 1892, p. ;6. 
without action on it. Inulin is generally accompanied in the plant by varying amounts of certain allied substances, such as Laevulin, Inulenin, Synanthrin ${ }^{\text {. }}$ Laevulin occurs in a few plants which contain no inulin, and the same is the case with Triticin, Irisin, Sinistrin, and a few other related carbohydrates ${ }^{2}$.

Glycogen. Errera ${ }^{3}$ and others have shown that glycogen occurs commonly in many fungi. It is a reserve carbohydrate allied to dextrin, and may form in beer-yeast as much as 30 per cent. of the dry weight. Clautriau has shown that in large fungi (Phallus, \&c.) it is consumed during growth. Yeast is apparently unable to ferment glycogen ${ }^{4}$, but probably, like many other non-fermentable substances, it may be used as food during aerobic existence. Certain contradictory statements may possibly be due to the existence of different varieties of glycogen, although as a matter of fact the glycogen of plants and that of animals appear to correspond very closely with one another. Both form opalescent colloidal solutions with water, and the solution diosmoses so slowly that glycogen can only be extracted from fungi or yeast after the cell-walls have been ruptured.

Mannite, Trehalose. Mannite is a fairly widely distributed hexahydric alcohol. It is found in various Phanerogams, and is especially abundant in many of the Agaricineae and in mould-fungi ${ }^{5}$.

Mannite is often associated in fungi with the polysaccharide trehalose, and in many cases the latter predominates when young, the former when older, the mannite being apparently formed from trehalose. In Agaricus campestris, however, mannite is present without trehalose, according to Müntz, so that the mode of origin of the former must in this case be different. Similarly Agaricus muscarius produces trehalose but no mannite. Again, Penicillium glaucum always forms mannite, but no trehalose, whether fed with tartaric acid, glucose, starch, or fruit-sap, whereas trehalose is regularly produced by Mucor mucedo. .

No doubt mannite is not always formed or utilized in the same manner by the higher plants: thus the mannite of young olive fruits is according to de Luca converted into oil, whereas Funaro states that mannite first appears in the fruits after the major part of the oil has already been formed ${ }^{6}$.

On the production of hydrogen during the decomposition of mannite in intramolecular respiration, cf. Sect. 99.

1 Cf. Tollens, 1. c. ; Tarnet, Compt. rend., I 893 , T. cxvir, p. 50.

${ }^{2}$ Cf. Tollens, 1. c. According to Brown and Morris (Journ. Chem. Soc., I893, p. 660), $\Lambda$. Meyer's sinistrin (Yucca filantentosa) is really inulin.

3 Errera, Le Glycogène chez 1. Basidiomycètes, I $\$ 8_{5}$ (Mém. d. l’Acad. roy. d. Belgique, T. Xxvir); Laurent, Ann. d. l'Inst. Pasteur, IS 89 , T. II, p. II6; Clautriau, Étude chim. du Glycogène, $\mathrm{I} S_{95}$. Here the remaining literature is given.

${ }^{4}$ A. Koch u. H. Hosaeus, Centralbl. f. Bact., IS94, Bd, xvi, p. 146; M. Cremer, Zeitschr. f. Biol., I 895 , Bd. xxx11, p. i.

5 Tollens, 1.c., I 888 , p. 266 ; 1895 , p. 281 ; Müntz, Ann. d. chim. ct d. phys., I 876 , v. sér., T. vir, p. 60 ; Bourquelot, Bot. Centralbl., I $\delta 9^{2}$, Bd. L, p. $7 \delta$; Winterstein, Zeitschr. f. physiol. Chemie, 1894 , Bd. xxix, p. 76 .

${ }^{6}$ De Luca, Ann. d. sci. nat., I 861 , iv. sér., T. $x v$, p. 92 ; I862, iv. sér., T. xviIr, p. 125. Cf. the literature given by A. Meyer, Bot. Zeitung, I 886 , p. I29; Funaro, Versuchsst., ISSo, Bd. $\mathrm{xxr}$, p. 55 . 
Substances allied to mannite are the Dulcite found in Melampynum, Euonymus, and many other plants, and the rarely occurring Sorbite (cf. Tollens).

Inosite, a sugar-like substance, which according to Maquenne ${ }^{1}$ is a hexahydroxyhexamethylene, disappears during the ripening of the fruit of Phaseolus as if it were a plastic product.

Mucilage. Gums and Pectin compounds form a large group of substances having similar physical properties, and include bodies of widely different chemical composition. They may take part in the formation of the cell-wall, or may be dissolved in cells or secretory receptacles, or may be excreted externally. They consist for the most part of different forms of carbohydrates, but according to Ishii ${ }^{2}$ the mucilage from the roots of Dioscorea japonica is composed of a proteid resembling animal mucin. As in animals, gluco-proteids, \&c. may often occur in plant-mucilage.

The molecules of a number of carbohydrates appear to be large and complex, and often combine with molecules of other substances ${ }^{3}$. Thus the typical gums are mainly pentosans (compounds of pentose), while dextrins are glucosans, substances whose gradual decomposition produces every variety of transition forms from gelatinous or colloid to crystalline bodies. Similarly the molecules of the partially gelatinous reserve-celluloses (hemi-cellulose) yield on hydrolysis either hexoses or both pentoses and hexoses (Sect. $8_{3}$ ).

Recent researches (Tollens, 1.c., p. 242) render it almost certain that the group of pectins are carbohydrates, which contain in their molecules hexoses and pentoses as well as other substances. Neutral pectins are complex in character, and when by hydrolysis compounds are produced which contain a carboxyl group, pectic acids result which are either soluble in water or in alkalies, but form insoluble calcium salts. Many gums show similar characteristics, and seem to be in part allied to pectin substances. Like other carbohydrates, pectins enter into the composition of the cell-wall either as dissolved substances or in solid form.

The different forms of mucilage may undoubtedly be produced in a variety of ways, and either by synthesis or by decomposition, as when the cell-wall becomes mucilaginous. Mucilage is, however, often formed in the interior of the cell without the intervention of cellulose as an intermediate product, and gelatinous coverings of comparatively firm consistency are often formed from excreted mucilage. Direct observations show that pectin substances are not usually formed by a metamorphosis of the cell-wall, an erroneous generalization formerly made by certain authors (IViesner, \&c.) ${ }^{4}$.

The retrogressive metamorphoses of the cell-wall are probably as a general

1 Maquenne, Ann. d. chim. et d. phys., I 887 , vi. sér., T. xII ; Tollens, I 888, p. 253 ; 1895 , p. 293.

${ }_{2}^{2}$ Ishï, Versuchsst., I 895 , Bd. Xlv, p. 434. On animal mucins, \&rc., cf. Neumeister, Physiol. Chemie, IS93, Bd. I, p. 35 .

${ }^{3}$ Cf. Tollens, Handb. d. Kohlenhydrate, 1895 , Bd. II, pp. 198, 240.

${ }^{4}$ Nägeli u. Schwendener, Mikroskop, I877, 2. Aufl., p. 507; Wiesner, Sitzungsb. d. Wiien. Akad., I $86_{5}$, Bd. L, Abth. ii, p. $44^{2}$, and the literature here quoted. 
rule produced by the action of enzymes, and that mucilage substances may arise in this manner is shown by the formation of dextrin during the hydrolysis of starch. Indeed gelatinous or mucilaginous substances often arise as intermediate products of the action of cellulose ferments ${ }^{3}$. The gummy modification of cell-walls is also probably caused by the action of a ferment, although none such has as yet been isolated, nor even its existence demonstrated ${ }^{2}$. Similarly 'pectase' is the problematic ferment which in the presence of calcium salts induces the decomposition of pectins with a formation of insoluble calcium pectate ${ }^{3}$.

As might be expected, the different substances mentioned are used for a variety of purposes: thus gelatinous materials take part in the formation of the cell-wall, in which pectins are perhaps always present (Sect. 83), and not only the gelatinous reserve-celluloses, but also the mucilage dissolved in the cells of orchid-tubers, rhizomes of Symphytum, \&c. ${ }^{4}$, serves as reserve food-material. Pectins may perhaps often function as plastic material, although it is doubtful whether the marked diminution in the amount of pectins during the ripening of fruits ${ }^{5}$ is due to their being converted into sugar, \&c.

The aplastic mucilaginous substances which remain permanently present in the cells or in special receptacles are probably for the most part of biological importance. Thus they may afford a certain protection against injury or the attacks of animals, while in other cases the mucilage may serve for the first attachment of seeds (Mistletoe, Linseed). It has moreover already been shown (Sect. $3^{8}$ ), that mucilage in spite of its feeble osmotic power may serve in other ways to check transpiration.

Further details will be found in the literature quoted, and the manner in which these substances may be classified will differ arcording to whether a morphological, functional, or biological standpoint is adopted. Cf. De Bary, Vergleichende Anatomie, I877, pp. 86, 150, 2 10; Tschirch, Angewandte Pflanzenanatomie, I889, p. 20.4; Walliczek, Jahrb. f. wiss. Bot., I893, Bd. xxv, p. 209 ; Schilling, Flora, I894, p. 280 ; Mangin, Bull. d. l. Soc. Bot. d. France, r 894, T. xl.I, p. xl ; Haberlandt, Physiol. Anat., I 896, 2. Auf., p. 439, and the literature quoted in these works.

Fats. Every protoplast appears to contain small anounts of fats (Sect. II), even although owing to its fine state of sub-division or to its existence in combined form it may be invisible until it is caused to coalesce and appear as little droplets ${ }^{6}$. This takes place whenever a large quantity of fat accumulates, which is a frequent occurrence. Fats serve as reserve food-material not only in numerous

${ }^{1}$ Cf. Grüss, Bibliotheca bot., I 896 , Heft 39, p. 7, and Sect. 9I.

2 Wiesner, Sitzungsb. d. Wien. Akad., Issä, Bd. xcır, p. 40. Cf. also Reinitzer, Zeitschr. f. physiol. Chemie, Is9o, Bd. xIv, p. 453 .

3 Bertrand et Mallève, Compt. rend., I895, T. cxxi, p. 726 ; Maumené, ibid., I894, T. cxix, p. 1012.

4 Frank, Jahrb. f. wiss. Bot., I $866-7$, Bd. v, pp. I\$I, 196; A. Meyer, Archiv d. Pharm., I 886.

${ }^{5}$ See Chodnew, Ann. d. Chem. u. Pharm.. 1844. Bd. I.I, p. 392, and the literature on the ripening of fruits in Sect. 1og.

${ }^{6}$ Hofmeister, Pflanzenzelle, $1 \$ 6_{7}$, p. 2. 
seeds, but also in many tubers, trees, trunks, \&c. ${ }^{1}$ Along with the neutral fats a slight amount of fatty acid may occur, or none may be present. During translocation the amount of free fatty acid increases to such an extent that as much as 10-30 per cent., or even almost the whole of the fatty acid may be liberated". In addition to oleic, palmitic, and stearic acids, a few of the higher acids may be present either singly or more usually several together, and either as such or as glycerides. As a general rule the fluid fats (oils) are more abundant, but the seeds of Cocoa and of Myristica contain fats which even in the living cells remain solid at ordinary temperatures ${ }^{3}$.

Apparently no special oil-forming plastids exist, the fats appearing directly in the protoplasm in a fine state of sub-division, and running together to form drops as the amount present increases. These drops are then excreted into the cell-sap, and the same occurs when fat is absorbed from without * In seeds the main mass of the oil present is found in the vacuoles mingled with the aleurone grains, \&c.

During germination any oil present is re-emulsified ${ }^{5}$ and absorbed anew by the protoplasm in order that it may undergo further change. The separation of neutral fats into fatty acids and glycerine may, however, take place in the cell-sap, and fungi are able to induce an extracellular decomposition of fats, while they can assimilate solid fats if these are only in a sufficiently finely divided condition. To what extent these powers are due to the formation and excretion of enzymes is unknown (Sect. 9I). Even when the liberation of fatty acids is most active no glycerine can be detected, so that it is evident the latter must be very rapidly assimilated. The absorption and translocation of fats are markedly aided by the presence of free fatty acids (Sect. I6).

On the increased absorption of oxygen necessitated when fats are being consumed, see Sect. 96. Waxy substances are mainly aplastic and usually accumulate in the substance or surface of the cell-walls ${ }^{6}$.

Lecithins are fatty substances or glycerides which contain, in addition to fatty acids, cholin and esters of phosphoric acid. Lecithin may form an essential constituent of protoplasm (Sect. I I), but whether lecithins take part in the

1 On the production of fat by $\mathrm{CO}_{2}$-assimilation, cf. Sect. 54. The aplastic waxy substances and the occurrence of aplastic fat-masses have already been mentioned.

'R. H. Schmidt, Flora, I 89 I, p. 345 ; Müntz, Ann. d. chim. et d. phys., i87 I, iv. sér., T. xxır, p. $47_{2}$, and in Boussingault's Agron., Chim. agric., \& c., I874, T. v, p. 50 ; Mesnard, Annal. d. sci. nat., I 893 , vii. sér., T. Xviri, p. 298.

${ }^{3}$ Pfeffer, Jahrb. f. wiss. Bot., i 872 , Bd. viri, p. 485 :

${ }^{4}$ Cf. R. H. Schmidt, l. c., pp. 325, 33S; Pfeffer, Aufnahme u. Ausgabe ungelöster Körper, $189^{\circ}$, p. I8o. Chromatophores are able to produce oil, and it is possible that the latter may in some cases be mainly formed by special plasmatic organs, or may accumulate in them. On Elaioplasts (oilformers), see the literature given by Zimmermann, Beih. z. Bot. Centralbl., i 894 , Bd. Iv, p. I65; v. Küster, Oelkörper d. Lebermoose, I 894 ; Schiitt, Die Peridineen, I895, p. 75. Altmann's supposition that the plasmatic granules (microsomala) are special oil-forming organs can hardly be correct.

${ }^{5}$ Pfeffer, Jahrb. f. wiss. Bot., 1872, Bd. virt, p. $5^{2} 5$.

- Cf. de Bary, Vergl. Anat., I877, p. 86; Haberlandt, Physiol. Pflanzenanat., 1896, 2. Aufl., p. 96. See also Sect. 21. 
metabolism of fats is uncertain ${ }^{1}$, for during germination the amount present undergoes irregular fluctuations. The existence of cholin in plants is probably connected with the formation and disintegration of lecithin ${ }^{2}$.

Cholesterin ${ }^{3}$ appears to be commonly present. It is an alcohol, and forms ester-like compounds with fatty acids such as the fats of hair and wool, animal products which are probably also produced by plants.

\section{Section 83. The Composition of the Cell-wall.}

The cell-wall, which forms a protective covering for the protoplasts and which subserves other functions as well, is not always composed of the same constituents, for recent researches have shown that it contains one or more anhydric compounds of different carbohydrates with which other substances may be united, so that in this way an endless varicty of modifications becomes possible. As a matter of fact mere microscopical examination suffices to detect many differences in the properties of different cell-walls, or even between the different layers of the same wall, and it is by means of the changes involved in suberization, cuticularization, gelatinization, \&c. that cell-walls become specially adapted for different purposes.

The cell-wall is a morphological term for the protective membrane which encloses the protoplast, and it may differ widely in structure and in chemical composition in different cases ${ }^{4}$. Every cell-wall has its own special chemical composition, and according to the plant examined, glucosans (dextrosans), galactans, arabans, and also gluco-mannans, galactoso-pentans, \&c. may form its constituents ${ }^{5}$.

A typical cellulose membrane when subjected to hydrolytic decomposition yields for the most part only dextrose, but in other cases mannose and other hexoses as well. Hence it is best to use the term 'cellulose' as referring not to one but to a group of substances. Schulze terms those celluloses, which are attacked and dissolved with relative ease by acids and alkalies, 'hemicelluloses.' These yield in addition to dextrose other forms of sugar, and though commonly uscd as reserve-cellulose, they sometimes take part in the formation of permanent cell-walls. The hemicelluloses form all variety of intermediate stages between typical celluloses

1 Literature: E. Schulze, Versuchsst., 1894, Bd. Xlı11, p. 307; Frankfut, ibid., I S94, Bd. XLIII, p. 177. Cf. also Neumeister, Physiol. Chemie, I 893 , Bd. I, p. 69.

${ }^{2}$ E. Schulze, l. c., 1895 , Bd. XLvi, p. 69.

${ }^{3}$ Literature: E. Schulze, Zeitschr. f. physiol. Chemie, I 890 , Bd. XIv, p. 5 I 2, and Übereinstimmung d. Stoffe im Pflanzen- u. Thierreich, 1894, p. 10; Gérard, Bot. Centralbl., 1892, Bd. L, p. 110.

4 Cf. E. Schulze, IBiol. Centralbl., I $\$ 96$, Bd. xiı, p. 849 . [E. Strasburger, Die pflanzlichen Zellhäute, Jahrb. f. wiss. Bot., ISy\&, Bd. Xxxi, p. 511.]

${ }^{5}$ Details by Tollens, Handb. d. Kohlenhydrate, I 895 , Bd. II, pp. 198,248 ; E. Schulze, Landw. Jahrb., $18_{94}$, Bd. xxır, p. 1 ; Zeitschr. f. physiol. Chemie, $18_{94}$, Bd. xix, p. 38 . Cf. also Sect. 84 . 
on the one hand and gums or mucilage on the other, so that none of these groups can be strictly marked off from the others, especially since bodies belonging to the different groups often form compounds with one another. Similarly no sharp line of demarcation can be drawn between the hemicelluloses and the insoluble pectin substances which are present in every cell-membrane and which may in some cases even predominate. Pectins are probably derived from carbohydrates by the addition of other substances to the molecule of the latter. The changes of lignification and suberization are probably produced by molecular rearrangements and by union with other substances.

The cell-walls of fungi may either be composed of cellulose, or may contain chitin; in some cases they are almost entirely composed of this gluco-proteid, which may be regarded as a carbohydrate derivative ${ }^{1}$. In various animals chitin may be associated with a little cellulose, while pure cellulose occurs in the Tunicata ${ }^{2}$, so that it is evident that no essential general difference exists between plants and animals in this respect, although while carbohydrates are mainly employed in plants for the formation of the cell-wall, chitin and various proteids subserve for the most part the same function in animals.

It is highly improbable that in both plants and animals the cellmembrane is always originally composed of the same substances; as a matter of fact fungi seem to form their chitinous membranes directly, and not by secondary modification, while the layers which serve as reserve-cellulose are deposited from the first in the form of galactans, mannans, \&c. ${ }^{3}$ Indeed it will probably be found by closer research that cell-walls or layers of cell-walls are often formed which even in the moment of their formation do not consist of cellulose. It is, however, always possible that the cell-membranes when once deposited may later undergo a variety of subsequent metamorphoses.

As our chemical knowledge increases, the distribution of the component elements of cell-membranes may be detected by microchemical means, not only throughout the plant as a whole, but also in each individual cell-wall. The present position of affairs may be seen by reference to Zimmermann's Mikrotechnik, p. I 35. By the conjoint aid of microscopical and microchemical methods

1 Winterstein, Zeitschr. f. physiol. Chem., I 894 , Bd. XIX, p. 52 I ; I 895, Bd. xxI, p. I34; Ber. d. Bot. Ges., 1895 , p. 65 ; Gilson, La Cellule, 1894 , T. xı, p. 5 ; Bot. Centralbl., 1895, Bd. LXI, p. 289 ; Tollens, l.c., p. 255 , where the remaining literature is given. On fungal cellulose, cf. de Bary, Morph. u. Biol. d. Pilze, I884, p. 9 ; Zimmermann, Mikrotechnik, I893, p. I57. On the membranes of lichens, see Escombe, Zeitschr. f. physiol. Chemie, 1896, Bd. xxiI, p. 288. The exosporium and endosporium of many spores probably contain nitrogen. [C. von Wisselingh, Mikrochem. Unters. über die Zellwände d. Fungi, Jahrb. f. wiss. Bot., I898, Bd. xxxi, p. 619.]

${ }^{2}$ Ambronn, Mitth. a. d. Zool. Station in Neapel, I89o, Bd. Ix, p. 475. On chitin, \&c., cf. Neumeister, Physiol. Chemie, i 893 , Bd. I, p. $3^{8 \text {. }}$

${ }^{3}$ Cf. Grüss, Bibliotheca botanica, 1896 , Heft 39, p. 13.

PFEFFER 
it may be possible in part to determine whether the chemical products of the decomposition of the cell-wall are derived from uniformly similar molecules or not. It must always be remembered that solvent agents may not only remove impregnating substances but may also induce decomposition, which probably occurs whenever the cellulose reaction is given only after the application of drastic modes of treatment.

Hemicelluloses frequently function as reserve-materials in seeds, and yield as the main products of metabolic disintegration mannose and galactose, and in part pentoses, such as arabinose and perhaps xylose also. Hemicelluloses are presumably complex carbohydrates, but according to microscopical observations made during the dissolution of the deposited layers, different varieties may be present ${ }^{1}$. Although reserve-cellulose is usually composed of hemicelluloses which are easily dissolved, plants are also able to reabsorb typical cellulose (viz. during conjugation, the penetration of fungi, \&c.). In algae, however, even substances which are readily soluble, and which may be extracted by means of hot water from the cell-walls, may remain intact during starvation. Since the macrochemical properties of the pectins, which are allied to hemicelluloses, are but little known, it is not possible to localize them microchemically. Hence it is uncertain whether all Mangin's ${ }^{2}$ pectin substances do actually belong to this group, although pectic substances seem to be very widely distributed in cell-walls, or in special layers of the cell-wall.

Lignification ${ }^{3}$. This probably involves molecular changes and combinations together with infiltration by gum, mineral constituents, \&c., for the reactions of lignified walls are such as to indicate that the cellulose, pentose, and aromatic substances are present in the form of more resistant compounds. It is doubtful whether ether-like compounds with lignic acid are formed, or whether there are other molecular combinations. The lignin reactions are partly due to the aromatic groups, and partly to the pentosans present. There is also positive evidence to show that cuticularization and suberization are not merely due to impregnation with waxy substances, but involve as well a formation of special compounds of cellulose from the original constituents of the cell-wall.

\section{SECTION 84. The Formation and Modification of the Cell-wall.}

The metabolic origin of the different constituents of the cell-wall is not as yet precisely known, nor is it certain in what way they are deposited, whether they occur peripherally as in the case of swarm-spores and plasmolyzed protoplasts, or in the interior of the plasma as in typical cell-division ${ }^{4}$.

${ }^{1}$ See E. Schulze, Zeitschr. f. physiol. Chemie, 1895 , Bd. xxi, p. 3922, and Ber. d. Bot. Ges., 1896, p. 66 ; also Grüss, Bibl. hot., I896, Heft 39 ; Bot. Centralbl., 1897, Bd. Lxx, p. 242 ; Elfert, Bibl. bot., I 894 , Heft 30 ; Nadelmann, Jahrb. f. wiss. Bot., I 890 , Bd. xxi, p. 609; Keiss, Landw. Jahrb., I $\mathrm{Ss}_{9}$, Bd. xvilI.

${ }_{2}^{2}$ Mangin, Rech. anat. s. 1. composés pectiques, I 893 . Cf. Zimmermann, Mikrotechnik, p. 162.

${ }^{3}$ For the literature, see Tollens, 1.c., I 895 , p. 270; I888, p. 239 ; Zimmermann, Mikrotechnik, p. 140 .

- Cellulose membranes may be formed around crystals (Wittlin, Bot. Centralbl., I $89^{6}$, 
It is doubtful whether the new wall formed around a naked protoplast is produced by a metamorphosis of the outcrmost ectoplasmic layer, or by the excretion of cellulose, or by both of these methods ${ }^{1}$. Carbohydrates arc often formed from proteids (Sect. 80), and direct researches with suitable plants such as certain fungi may possibly show that changes and decompositions resulting in the production of cellulose may be induced by the protoplast in the chitinous substances composing the cnclosing cell-wall. On the other hand, bearing in mind the marked secretory activity of which protoplasts are capable, it is equally possible that the cellulose investment may be produced by the excretion of preformed cellulose; as a matter of fact many of the Conjugatac, Flagellatae, \&c. excrete a gelatinous sheath ${ }^{2}$, which only needs condensation to become a more rigid cellulosewall. Cellulose is actually formed indeed from many mucilaginous substances when they are merely treated with acid ${ }^{3}$, protoplasts are able to induce a variety of metamorphoses in the membranes enclosing them; while there is nothing to hinder the excretion and subsequent coalcscence of solid particles of cellulose.

Nor is the mode of origin of the transverse walls in cell-division certain, for although the cell-plate appears to be formed by the production and aggregation of granules at a particular point ${ }^{4}$, it is not known whether thesc granules unite directly to form the cell-wall, or whether, as often happens, they are merely building material collected for further use, or again whether they have a widely different functional importance.

The co-operation of nucleus and cytoplasm is indispensable for the formation and growth of the cell-wall, and when this condition is fulfilled cell-membranes may be formed with considerable rapidity: thus a cell-wall appears around the egg-cell of Fucus ten minutes after the entry of an antherozooid. A cell-wall may appear around the plasmolyzed protoplasts of many plants in from fifteen minutes to a few hours ${ }^{5}$, for plasmolysis may excite to renewed activity a dormant power of producing cellulose.

Aerobes can construct cell-membranes only in the presence of free oxygen, but obligate anaerobes have this power only when free oxygen is absent; for this and other reasons it is impossible to agree with Traube ${ }^{6}$ in regarding the cell-wall

Bd. xxxvir, p. 33), and also around certain vacuoles (Berthold, Protoplasmamechanik, IS\$6, pp. 296, 304). On cellulose granules, see Pringsheim, Ber. d. Bot. Ges., 1883, p. 288.

1 No decisive experiments have as yet been made. Cf. Berthold, Protollasmamechanik, isS6, p. I 54 ; Hofmeister, Zelle, i 867 , p. 147 .

${ }_{2}$ KKlebs, Unters. a. d. Bot. Inst. z. Tübingen, is 66, Bd. II, p. 4 I I.

${ }^{3}$ Cf. Tollens, Handb. d. Kohlenhydrate, ISSS, Bd. I, P. 220.

4 Cf. Zimmermann, Morph. u. Physiol. d. Zellkernes, IS96, p. 72.

${ }^{5}$ Hofmeister, Zelle, I $8 \sigma_{7}$, p. I 51 . Townsend, Jahrb. f. wiss. Bot., $1 \delta_{97}$, Bd. xxx, p. $4{ }_{4}$; Strasburger, Studien uiber Protoplasma, 1 $\$ 76$, p. 54. [Farmer and Williams, Observations on the Cytology and Fertilization of Fucaceae, Phil. Trans. I 898, p. 625.]

"Traube, Monatsb. d. Berl. Akad., I $8_{59}$, p. $8_{3}$. 
as a direct product of the oxidation of carbohydrates. Similarly, the relations between growth and respiration are such as to render impossible the existence of any constant ratio between the amount of cellulose formed and the respiratory activity ${ }^{1}$. The manner in which calcium influences formation of the cell-wall in certain cases, has already been mentioned (Sect. 74).

The various changes which cell-walls may undergo may be either chemical in nature or due simply to impregnation with foreign substances. Both processes may occur simultaneously either during growth or after its cessation, so as to produce local or general changes in the properties of the wall. The latter may from the commencement differ from the normal type. The mucilaginous modification of the cell-wall involves marked chemical metamorphosis, such as occurs during the mobilization of reserve-cellulose, during the formation of gum in Astragalus, \&c., and of mucilage in the seed coats of linseed, quince, and sage, and in many gland-hairs. Similar changes occurring in the transverse walls of vascular elements render the formation of vessels possible, and during the copulation of plants of the Conjugatae the dividing walls of the fertilization-tubes undergo corresponding chemical metamorphosis and disintegration. Similarly the partial or complete solution of the middle lamella enables the plant to form intercellular spaces, and to complete the abscission of fruits or leaves 2 .

Although chemical changes and infiltration co-operate in producing cuticularization and suberization, it is doubtful whether the waxy substances penetrate the cell-wall as such, or are produced in it by chemical metamorphosis, and the same doubt exists with regard to wood-gum, although lignification is undoubtedly mainly due to chemical changes in the original cellulose (Sect. 83). The calcium carbonate of cystoliths is derived from an organic compound, probably calcium pectate (Sect. 74), and the character of the cell-wall may be markedly modified by the formation of compounds of this kind.

Lignification, suberization, and the mucilaginous modification are produced in living tissues only and are due to the vital activity of living protoplasts. Various chromogens, tannins, \&c. may penetrate the cellwalls after the death of the protoplast, and may thus take part in the formation of coloured duramen ${ }^{3}$.

${ }^{1}$ For such views, see Sachsse, Über chem. Vorgänge bei d. Keimung von Pisum sativum, I872, p. 40 , and Chemie d. Farbstoffe, \&c., 1877 , p. 40.

${ }^{2}$ See Sect. 83 . Further details in the works of de Bary, Zimmermann, \&c.

${ }^{3}$ On the formation of duramen, \&c., cf. Temme, Landw. Jahrb., I $8_{5}$, Bd. xIv, p. $46_{5}$; Prael, Jahrb. f. wiss. Bot., I 888 , Bd. XIx, p. 6I ; G. Kraus, Grundlinien z. Physiol. d. Gerbstoffes, ISS9; Strasburger, Leitbahnen, I 891 , p. $9^{6}$; Rathey, Über d. Auftreten v. Gummi i. d. Rebe, I896; Schellenberg, Jahrb. f. wiss. Bot., I 896, Bd. xxıx, p. 237 ; Hoppe-Seyler, Zeitschr. f. physiol. Chemie, I889, Bd. XIIt, p. 66 ; G. Lange, ibid., 1890, Bd. XIV, pp. I5, 217, 283. 
It is not surprising that the protoplast is able to modify or to dissolve its cellulose investment. The latter process is usually induced by the action of enzymes, the former by the infiltration of substances produced by the protoplast. The modifications which a cell-wall may undergo are dependent upon the external and internal conditions and upon the reactive powers of the protoplast. It is for this reason that only particular tissuc-elements lignify; that cork is produced normally in certain regions only, and is formed after an injury over all the exposed tissues; that the amount of cuticularization varies according to the external conditions; and that under certain circumstances a pathological formation of gum may occur.

None of these alterations, nor even the fact that the cell-wall is capable of growth, indicate that it is to be regarded as a living structure, nor need we regard as well-founded the supposition of Wiesner, according to which the cell-wall is permeated throughout by the protoplasm ${ }^{1}$, for neither the presence of combined nitrogen in certain cell-membranes, nor the existence of localized protoplasmic communications, suffice to prove that his conclusions have any general application.

\section{SECTION 85. Organic Acids.}

Even excluding the fatty acids, every plant produces some organic acid or other, either free or in the form of a salt. Oxalic, malic, citric, and tartaric acids occur most frequently. Formic and acetic acids are also often present, and like butyric and lactic acids may form part of the main products of particular fermentative processes (Sect. 105). Certain acids, such as fumaric acid, aconitic acid, \&c., occur only in a few plants ${ }^{2}$, and it seems that none even of the commonly occurring organic acids is of universal occurrence. The different acids belong to quite distinct series, but for the most part are derivatives of methane; the metabolic products never seem to include acids of more than tribasic value.

Organic acids may be present as neutral or acid salts, or in uncombined form: thus 6 to 7 per cent. by weight of a fresh lemon consists of citric acid, while in the Crassulaceae malic acid constitutes as much as 2 per cent. of the weight when fresh ${ }^{3}$. Similarly Citromyces ${ }^{4}$ may cause as much as 8 per cent. of citric acid to accumulate in a culture-fluid, and Bacterinm

1 Wiesner, Elementarstructur, I896; Pfeffer, Energetik, I $\$ 92$, p. $25^{2}$; Correns, Jahrb. f. wiss. Bot., I894 Bd. xxvI, p. $5^{87}$. On the so-called Dermatosomes, cf. Sect. I3.

${ }^{2}$ Cf. Husemann, Pflanzenstoffe, I $88_{2}$, and Ebermayer, Physiol. Chemie, I882. On the distribution of formic and acetic acids, see Bergmann, Bot. Zeitung, I882, p. 784 .

3 Ebermayer, l.c., p. 273; Wehmer, Bot. Zeitung, I S9I, p. 373 .

* Wehmer, Beitr. z. Kenntniss einheim. Pilze, IS93, I, p. 77 . On fungi which grow in very acid solutions, see Wehmer, l.c., I $\delta_{91}$, p. 296 , and 1895 , II, p. I43. 
aceti $\mathrm{I} 4$ per cent. of acetic acid under similar conditions (Sect. 103). Certain organisms, especially various mould-fungi, can withstand large quantities of free acid, whereas others, including most bacteria, have not this power, and hence the fermentative activity of most lactic and butyric bacteria is inhibited when the amount of acie present rises to 0.5 per cent. (Sect. I03), so that to permit of continued fermentation the acid must be neutralized as fast as it is formed. An over-accumulation of organic salts also usually inhibits vital activity, but in many Crassulaceae as much as half of the dry weight may consist of soluble salts of malic acid ${ }^{1}$, whereas the total amount of organic acid in other plants never exceeds one per cent.

With the exception of the oxalic acid present in the form of calcium oxalate, the organic acids and their salts are for the most part held in solution ${ }^{2}$. Crystals of calcium oxalate are of very common occurrence, and in a few plants this substance may form 50 per cent., in some Cactaccae even 80 per cent., of the dry weight ${ }^{3}$. Similarly during certain fermentations as much as one-half of the fermentable material may be converted into organic acid, the latter finally surpassing the total weight of the fermentative organisms ${ }^{4}$.

Organic acids are produced in many different ways and subserve a variety of purposes. Thus during certain fermentations lactic or acetic acids form end-products of metabolism which must be excreted and removed to permit of the continuance of vital activity (Sect. 77). This is, however, unnecessary in other plants such as Aspergillus and Citromyces, in which further production ceases as soon as a certain amount has accumulated. In such cases the acids may serve as plastic material, as osmotic substances, as solvent or ncutralizing agents, or as aids to the action of enzymes. Moreover certain plants may be able to suppress or kill others by means of the acids which they excrete (Sect. 92), and an acid sap or the presence of acicular crystals of calcium oxalate may protect certain plants against the depredations of animals ${ }^{5}$. Fungi are not only able to use organic acids as food-material but may also consume in part the organic acids which they themselves produce, and similarly the Crassulaceae

1 G. Kraus, Stoffwechsel bei d. Crassulacecn, 1886, p. 6 (Abh. d. Naturf.-Ges. zu Halle, 13d. XVI).

${ }^{2}$ Crystals of magnesium oxalate, calcium tartrate, and calcium citrate are occasionally, but rarely, found. Cf. Zimmermann, Mikrotechnik, I892, pp. 61, 65; Wehmer, Ber. d. Bot. Ges., I893, p. $33^{8}$.

${ }^{3}$ Literature : Kohl, Kallssalze u. Kieselsäure i. d. Pflanze, I889, p. 35 ; Zopf, Pilze, I89o, p. I93; Schimper, Bot. Zeitung, I888, p. So, and Flora, I89o, p. 237 ; Welimer, Bot. Zeitung, I 89 I, p. I 49 , and Vcrsuchsst., I892, Bd. XL, p. I13; Errera, Bull. d. l'Acad. roy. d. Belgique, I 893 , iii. sér., T. Xxvi, Nr. 7 ; G. Kraus, Flora, I 897 , p. 54 .

- Regarding mould-fungi, see Wehmer, Bot. Zeitung, I $89 \mathrm{r}, \mathrm{pp} .34^{2}, 534$, on oxalic acid, and I893, l. c., p. 4I, for citric acid. For fermentations, see Sect. I03.

s Stahl, Pflanzen u. Schnecken, I888, pp. 40, 84 . 
store up malic acid as reserve food-material ${ }^{1}$. Organic acids function as reserve material only in a few special cases, although many of the higher plants seem to have the power of reassimilating malic, citric, and even oxalic acid, while calcium oxalate, which usually remains intact, may in certain cases be dissolved and assimilated. According to $\mathrm{Kraus}^{2}{ }^{2}$ calcium oxalate disappears from rhizomes, bark, \&c. only when there is a lack of calcium, so that apparently this salt is utilized mainly for the sake of the calcium it contains. But little energy can be obtained by the consumption of oxalic acid, and experiments with fungi show that the amount of growth in a fluid culture remains almost the same, whether oxalic acid has been produced in abundance or only in minimal amount as an end-product of metabolism in the place of carbon dioxide ${ }^{3}$. Salts of organic acids are commonly employed in the maintenance and regulation of turgor. It must, however, always be remembered in this connexion that the same substance may frequently serve a variety of purposes.

\section{SECTION 86. Organic Acids (continued).}

Organic acids are usually products of katabolism, but may probably also be produced synthetically, while they may arise either during aerobic or during anaerobic existence, as when fermentation or intramolecular respiration takes place. In the former case they are apparently formed directly in the process of respiration, but nevertheless in a regulatory manner and not because only imperfect combustion is possible, for even when oxygen is present in great excess, respiration and also the production of acid proceed in the same manner both in the cases of fungi ${ }^{4}$ and of the higher plants (Sect. 56). Organic acids are not produced under all circumstances, but only when the conditions which regulate their production are satisfied, and it is simply a special instance of regulatory protection when Citromyces consumes the citric acid produced by its own metabolism as the supply of more suitable nutriment decreases. Aspergillus, Pcnicillium, and Citromyces generate a specific degree of acidity in a culture-fluid, which however in each case is less than suffices to affect injuriously the plant's own vital activity. The same is the case with regard to the cell-sap of the higher plants, for the sap only attains a certain degree of acidity when plants of the Crassulaceae are kept in darkness. Similarly the production of salts of organic acids takes place in a self-regulatory manner, for wherever, as is often the case, the same constant turgidity is maintained both

1 G. Kraus, 1. c., I 886 , p. I 5 .

${ }^{2}$ G. Kraus, Flora, 1896 , p. 54. Cf. also Kohl, l.c., p. ${ }_{4} 8$. On the solubility of calcium oxalate, see Wehmer, Versuchsst., I $892, \mathrm{Bd} . \mathrm{XL}, \mathrm{p} \cdot 45^{6}$.

3 Wehmer, Bot. Zeitung, I 891 , p. 553 ; Pfeffer, Studien z. Energetik, I 892 , p. 197.

+Wehmer, 1.c., I891, p. 537 ; I 893 , p. 50. 
during and after growth, every increase in the volume of a cell must be accompanicd by a corresponding production of organic salts or other osmotic substances ${ }^{1}$.

If the attainment of the required maximum is prevented by the continual removal of the acid as fast as it is formed, an almost unlimited amount may be produced. Wehmer has shown that Aspergillus niger and Pcnicillium glaucum produce in a sugary nutrient solution an amount of oxalic acid sufficient to render the culture medium feebly acid, whereas if the acid is continually neutralized by the addition of chalk, the fungus ultimately produces a quantity of oxalic acid several times exceeding its own body-weight.

The same is the case with Citromyces, although here the further production of citric acid ceases only when the medium becomes strongly acid, and a similar result would probably be obtained with the higher plants were it possible to continually remove the free acids or their salts from the living cells The condition of equilibrium at which further production ceases may be modified by changes in the external conditions: thus a rise of temperature to $34^{\circ} \mathrm{C}$. lowers the limit at which Aspergillus niger ceases to produce free oxalic acid ${ }^{2}$, and not only a rise of temperature, but also illumination, causes the percentage of free acid to diminish in plants of the Crassulaceae (Sect. $5^{6}$ ). Similarly the accommodation to concentrated solutions, and frequently also the performance of work against resistance ${ }^{3}$, may involve an increased turgidity, and hence also an increased production of organic acids or their salts as far as these are responsible for the regulation of turgor.

It seems that the turgor-producing salts of organic acids are aplastic products in the higher plants, so that the renewed production induced by a deficiency must ccase as soon as a condition of equilibrium is again reached. The same may also hold good for the free organic acids, though here the condition of equilibrium may simply represent the balance between production and decomposition ${ }^{4}$, for both fungi and plants of the Crassulaccae arc able to reassimilate the organic acids they themselves have produced. Carnivorous plants (Sect. 65) afford cxamples of the way in which the secretion of acid may be primarily induced by chemical stimulation, or the rate of production increased.

By means of this self-regulatory power of increasing or decreasing the production of acid, an cxcessive formation of basic or alkaline substances

1 Pfeffer, Druck u. Arbeitsleistung, I 893, p. 428 . Cf. Sect. 24.

2 Wehmer, Ber. d. Bot. Ges., I 89 I, p. I 65.

${ }^{3}$ Pfeffer, 1. c., pp. $296,4^{28}$.

${ }^{4}$ Cf. Pfeffer, Sitzungsb. d. Sächs. Ges. d. Wiss., 1891, p. 26. On the decrease of acids during the ripening of $f_{1}$ uits, cf. Sect. Iog. 
may be compensatcd for, while on the other hand a dangerous accumulation may be avoided when the neutralizing substances are produced in less than normal amount. As a matter of fact Penicillium, when fed with peptonc, would be killed by the accumulation of ammonia (Sect. 80), were it not that the amount of oxalic acid produced undergoes a corresponding increase. Similarly in the metabolism of the higher plants the quantity of bases to be neutralized must certainly vary from time to time, according to the extent to which the latter are employed in metabolism, while varying amounts of organic acids may be utilized for the gradual release of sulphuric and nitric acids from their salts by the principle of action in mass ${ }^{1}$.

Oxalic acid. The different organic acids may apparently replace one another to a certain extent, which is, however, strictly limited by the special properties of each. As regards oxalic acid, its affinities, poisonous character, feeble heat of combustion, and the insolubility of its calcium salt are all points to be taken into consideration. The immediately visible character of the calcium oxalate crystals has caused attention to be concentrated mainly or almost entirely upon oxalic acid. In the case of Phanerogams calcium oxalate is produced at certain stages of development, and various experiments indicate that here also the amount produced may be modified according to the external conditions. Oxalic acid seems to be a necessary bye-product of metabolism both in the higher and in the lower plants, while according to circumstances it may either be further decomposed or retained in combined form. This decomposition of free oxalic acid seems to be possible in the higher plants as well as in the lower ones, and when sufficiently active no neutralization of the free acid by combination with bases may be necessary. These questions may be determined empirically, and were it possible experimentally to reduce the formation of oxalic acid and prevent the production of calcium oxalate, this would suffice to prove that the production of oxalic acid can be regulated in the higher plants, and also that the main function of calcium is not to neutralize this acid (Sect. 74).

Oxalic acid can hardly have any general importance as such, when calcium oxalate accumulates, for oxalic acid itself is a very poor reserve-material, and besides calcium oxalate almost always remains intact, and it is retained by dead or dying leaves. Many plants produce hardly any calcium oxalate, whereas relatively enormous quantities may accumulate in others, either as unavoidable products of specific metabolic activities, or for the fulfilment of special purposes. It has yet to be determined why soluble oxalates are present in abundance in certain plants although they may be supplied with calcium salts, and why calcium oxalate is often deposited in particular cells or regions which are not those where the oxalic acid is formed. The shape of the crystals may enable conclusions to be made as to the conditions existing in the cell at the time of formation, for according to the

${ }^{1}$ Cf. Sects. 22, 93. C. Sprengel (Die Lehre vom Dünger, I 839, p. 6z) was perhaps the first to regard the organic acids as affording a means by which the acids of sulphates and nitrates could be gradually set free and utilized. 
conditions under which they have been formed crystals of different shape and containing two or six molecules of water may be produced ${ }^{1}$. Anatomical facts and a few theories as to the function of oxalic acid and calcium oxalate will be found in the quoted works of Zopf, Schimper, Wehmer, \&c.

Acid and alkaline reactions. The sap of plants may be either acid or alkaline, and the excretion of certain products of metabolism may create either an acidity or alkalinity in the surrounding medium, depending upon the character of the food-material supplied. Thus in the case of fungi sugar induces acidity, peptone alkalinity ${ }^{2}$. The reaction of the cell-sap in living cells may be indicated by the colour assumed by soluble pigments normally present or artificially introduced ${ }^{3}$. Thus the red colour of rose petals, beet-roots, \&c. shows that the cell-sap is acid, the blue colouration of the hyacinth, blue-bell, or cranberry (Vaccinium), \&c. that it is neutral or slightly alkaline. Treatment with dilute acid or alkali, as the case may be, may reverse the reaction without killing the cells, but after washing in water the normal reaction returns again ${ }^{4}$. Such changes may occur spontaneously, as for example when the flowers of Pulmonaria, which are at first red, become blue as they grow older. The reaction of the cell-sap is approximately the same as that of the expressed sap, and in most of the higher plants this is slightly sour and occasionally markedly so (Sect. 85 ).

The protoplasm does not normally contain any reacting pigments ${ }^{5}$, but a feeble alkalinity has been detected in certain plants by the artificial introduction of methyl-orange. By means of weak organic acids this alkalinity may, however, be converted into an acidity without affecting the vitality of the cell. An alkaline reaction may be given with litmus solution by dead tissues rich in protoplasm and by the plasmodia of Myxomycetes ${ }^{6}$, but it is possible that the protoplasm of fungi may always possess an acid reaction when growing in strongly acid solutions, especially when we consider the fact that many proteids (nucleins, \&c.) are acid compounds ${ }^{7}$. Certain protoplasts can, moreover, withstand strongly acid media, as is the case with those plants which can grow in the presence of large quantities of organic acids.

The alkaline reaction of the protoplasm is perhaps mainly due to compounds of proteids with alkalies and alkaline phosphates ${ }^{8}$, and these are probably non-

1 See Kohl, Kalksalze u. Kieselsäure, I889, p. 21.

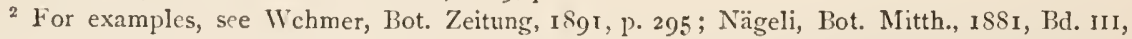
p. 283 ; Zöller, Jot. Jahresb., I874, p. 213 ; Stutzer, ibid., p. II 7 ; Pitruschky, Centralbl. f. Bact.,

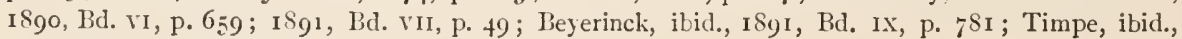
I 893, Bd. XIV: pp. $845, \&$ c.

${ }^{3}$ Pfeffer, Unters a. d. Bot. Inst z. Tüibingen, 1886, Bd. 11, p. 293 ; Čelakovský, Flora, I892, Erg.-bd., p. 233.

${ }^{4}$ I'feffer, Osmot. Unters., I 877 , p. I 40.

5 The occurrence of granules of chalk in the plasma affords no evidence one way or the other, for these are always surrounded by an investing plasmatic membrane.

- Sachs, Bot. Zeitung, I 862 , p. 257 ; F. Schwarz, Beitr. z. Biol. v. Cohn, I892, Bd. v, p. I 2 ; A. Meyer, Bot. Zeitung, I 890, p. 232 ; Krukenberg, Unters. a. d. Physiol. Inst. zu Heidelberg, Bd. II, p. 282 ; Reinke, Studien ïber Protoplasma, I 88 ז, p. 8.

7 Neumeister, Physiol. Chemie, Bd. I, p. 34.

${ }^{8}$ Cf. Pfeffer, I856, I.c. ; Fr. Schwarz, 1. c., p. $3^{2}$. 
diosmosing substances, for the alkalinity is not altered by repeated washing with water. Free acids, however, usually diosmose readily, and hence it must be determined in each separate case whether the loss of acid is prevented by the diosmotic properties of the plasmatic membranes, or by the formation of acid salts (Sects. 16, 22). A diminution in the acid reaction of the cell-sap may be produced by an increased formation of alkali, by a consumption of acid, or by union with basic substances. It has already been mentioned that the absence of certain substances from a nutrient medium may cause an acid or an alkaline reaction to be produced in it or in the cell-sap, and that a strong acid may be set free by a weak one according to the principle of action in mass ${ }^{1}$. Plants possess great capabilities in virtue of their osmotic powers, and it is even possible that an alkaline protoplast may gradually excrete large quantities of free acid, and thus render possible the digestive action of peptic ferments. Magnesium phosphate and many other substances may remain dissolved in an acid cell-sap, whereas they are are at once precipitated if it becomes alkaline.

\section{SECTION 87. Glucosides, Tannin, and Phenols.}

Probably every plant produces one or other of the numerous benzene derivatives, of which some occur in particular plants only, while others, such as tannin and phloroglucin, are widely distributed ${ }^{2}$. All these substances are often found in combination with other bodies as glucosides and similar compounds, and probably numerous unstable combinations exist in the living plant which decompose on death, and hence have not as yet been detected. The ester-like compounds of carbohydrates with aromatic radicles form substances (glucosides) ${ }^{3}$ which diosmose with difficulty, and from which the carbohydrates may subsequently be liberated, the aromatic component remaining intact in the cell or at a later stage reuniting with carbohydrates ${ }^{4}$. Certain glucosides appear to behave as aplastic products, whereas others may be entirely reassimilated. Fungi appear to be capable of decomposing almost all glucosides (viz. arbutin, salicin, phloridzin, quercitrin, glycyrrhizin, amygdalin).

Fungi can assimilate many aromatic bodies, such as tannin, resorcin,

1 Cf. Sect. 28. Animals are able to produce large quantities of free hydrochloric acid, but not plants. Bunge, Physiol. Chemie, 3. Auf., p. I43. On the sensitivity of many protoplasts to acids, cf. Pfeffer, Osmot. Unters., i 877 , p. I35.

${ }^{2}$ Waage, Ber. d. Bot. Ges., I 890, p. 250 . On the artificial production of glucosides of phloroglucin, cf. Councler, Ber. d. Chem. Ges., 1895 , p. 24 . On the microchemical tests for these substances, see Zimmermann, Mikrotechnik, i $8_{92}$, pp. $\delta_{2}, \delta_{9}$, I ro.

${ }^{3}$ Cf. E. Fischer, Die Chemie d. Kohlenhydrate, Rede, Berlin, I 894 ; Ber. d. Chem. Ges., I S94, $_{9}$ pp. 24,78 ; Tollens, Handb. d. Kohlenhydrate, II, 1895, p. 84 . It is mainly glucosides of aromatic substances that have been found in plants.

t Cf. Sect. 22. Pfeffer, Unters. a. d. Bot. Inst. z. Tübingen, I\$S6, Bd. II, p. 309; Möller, Ber. d. Bot. Ges., I $\$ \$ 6$, Generalvers., p. lxx; Mielke, Bot. Centralbl., I 894 , Bd. LIX, p. 28 I. All carbohydrates are not produced by the decomposition of glucosides, as Rochleder supposes (Phytochemie, I $85, \mathrm{p} .3^{28}$ ). 
hydroquinone, phloroglucin, \&c., but except in the case of quinic acid most of these afford very poor food-material ${ }^{1}$, and commonly exert a poisonous action even when only slightly concentrated. Similarly, owing to the liberation and accumulation of the aromatic compounds such as hydroquinone, from arbutin, \&c., an injurious effect is commonly produced when plants are fed with glucosides, although so long as a sufficient supply of sugar is present glucosides are rarely attacked. Aromatic substances do not usually seem to serve as food or as storage material, and it is still doubtful whether tannins may be used for such purposes. The latter may form as much as 6 per cent. of the total weight of a turgid plant ${ }^{2}$, and a few other benzene derivatives, including glucosides, may be produced in large amount by certain plants. Tannins, phloroglucin, and apparently all aromatic substances which accumulate to any extent, are contained in solution in the cell-sap, so that their presence does not injuriously affect the protoplast. Such substances often afford a certain protection against the ravages of animals by reason of their taste and poisonous character, and in all cases where tannins, glucosides, bitter principles, and any other aromatic substances, such as certain pigments, \&c. (Sect. 88), subserve biological functions ${ }^{3}$, they are retained as permanent aplastic products of metabolism. Many of these bodies undergo alteration when the cell dies ${ }^{4}$; this may in some cases be of importance to the plant, as, for example, in the formation of duramen, which is largely due to the changes occurring in the tannins and other substances which infiltrate the walls of the wood-vessels (Sect. 84). Similarly, the presence of aromatic derivatives may hinder the decomposition of proteids in dead internal tissues.

Tannins and glucosides are undoubtedly produced for definite purposes, and are not mere by-products produced under all circumstances. In sweet almonds the formation of amygdalin, which characterizes bitter almonds, is suppressed, and, similarly, Loew and Bokorny have observed the cessation of the production of tannin in Spirogyra, and Ashoff the same in Phaseolus multifloms when grown in a nutrient solution containing no chlorides ${ }^{5}$. Pfeffer ${ }^{6}$ has shown that certain plants are not injured when the whole of the tannin present in the living cell is precipitated by means

1 Cf. the works of Nägeli and Reinke quoted in Sect. 7o. Fungi liberate gallic acid from tannin (Muintz, Compt. rend., I877, T. Lxxxiv, p. 956), but this occurs externally, for they usually contain no gallic acid.

${ }^{2}$ G. Kraus, Grundlinien z. Physiol. d. Gerbstoffes, I889, p. 22 ; J. af Klercker, Studien über d. Gerbstoffvacuolen, Stockholm, I888.

${ }^{3}$ Stahl, Pflanzen u. Schnecken, I888, pp. $3^{2}$, \&c.; Büsgen, Beobacht. ïber das Verhalten d. Gerbstoffe i. d. Pflanzen, I $88_{9}$ (Jenaische Zeitschr. f. Naturw., Bd. xxIv); Dryer, Bot. Centralbl., 1893, pp. 53, 83; Ludwig, Biol. d. Pflanzen, 1895, p. 208.

4 Pfeffer, Oxydationsvorgänge in lebenden Zellen, r 889 , p. 49.

${ }^{5}$ Loew u. Bokorny, Biol.Centralbl., I 89 I, Bd. XI, p. 8 ; Ashoff, Landw. Jahrb., I89o, Bd. XIX, p. I 27.

${ }^{6}$ Pfeffer, Unters. a. d. Bot. Inst. z. Tüibingen, I 886, Bd. II, p. I97 ; Büsgen, 1. c., p. 8. 
of methyl-blue, and also that in the roots of Trianea, \&c., tamnin is produced only at certain stages of development, no renewed formation being induced by the precipitation of all that present in the cell. This is certainly not always the case, for the formation of callus-tissue and of galls is often associated with a marked production of tannin, which, like other benzene derivatives, may probably be produced either by synthesis or by dissociation ${ }^{2}$. During the synthesis of proteids a formation of aromatic radicles is necessary, provided none such are present in the food, but the process is not dependent upon the pre-existence of benzene derivatives.

Analogy with fungi, as well as the absence of any accumulation of aromatic bodies even during active and prolonged proteid decomposition, leaves no doubt that benzene derivatives may be reassimilated under certain circumstances in the higher plants as well as in the lower. Certain glucosides may at any rate be decomposed, and the presence of appropriate enzymes enables such decompositions to take place during life or after death. In the higher plants glucosides and their respective ferments are as a general rule deposited in different tissues, but in unicellular organisms the two substances may be present in the same cell though not in direct contact. All postmortem changes and decompositions are of course not necessarily due to the presence of enzymes in the dying cell or tissue.

Tannin. This is a technical term which has no precise chemical or physiological meaning, for the same microchemical tests with iron salts and potassium bichromate are given by various phenols and phenol compounds, but not by others such as phloroglucin, \&c., which have a similar physiological importance ${ }^{2}$. The latter can hardly, however, apply to all the aromatic substances, glucosides, and compounds of phenols with other substances, which are classed together as tannins because of their similar microchemical reactions. Moreover, since the same substance may serve several different uses, it is hardly possible to predict that the classification into plastic, aplastic, pathological and other tannins will be of any great importance ${ }^{3}$.

In spite of numerous recent researches ${ }^{4}$ but little is known as to the function of tannin. Most tannin substances seem to be aplastic, and certain contradictory statements made long ago by Wigand and Schell have not been confirmed or have become doubtful. Tannin very commonly disappears from the spot where it was produced, and accumulates at other points where it remains unaltered during life.

1 The discussion as to whether proteids or carbohydrates yield the material for the formation of tannins, \&c., is devoid of importance. Cf. G. Kraus, 1. c., ISS9, p. 45 ; Miellie, 1. c., IS94, p. 2 S I.

2 Waage, Ber. d. Bot. Ges., I S9o, p. 290 ; Nickel, Bot. Centralbl., I S9I, Bd. XLv, p. 394; Reinitzer, Lotos, IS9ı, Bd. Xı (Separatabh.); Mielke, Bot. Centralbl., IS94, Bd. Lrx, p. 280.

${ }^{3}$ Cf. Hansen, Pflanzenphysiol., I\$9o, p. I I9; Wagner, Journ. f. prakt. Chemie, i\$66, Bd. xcrx, p. 294 .

4 G. Kraus, Grundlinien z. Physiol. d. Gerbstoffs, I $S_{9}$; Büsgen, Verhalten d. Gerbstoffes in der Pflanze, ISS9 (Zeitschr. f. Naturw., Jena, Bd. xxIv); Mielke, 1. c. ; Kïstenmacher, Jahrb. f. wiss. Bot. I894, Bd. xxvi, p. I I 8 . 
This occurs in the case of the tannins produced during the metabolic changes undergone by the products of photosynthesis, according to G. Kraus and Büsgen, although on other points these authors are not in precise agreement.

Amygdalin. This glucoside is present in Amygdaleae and a few other plants, but whether it takes an active part in metabolism is uncertain ${ }^{1}$. The hydrocyanic acid found in Pangium edule, and probably also that occurring in the roots of Manihot utilissima, are produced without the intervention of amygdalin. As a general rule amygdalin is stored in the parenchyma, whereas the ferment emulsin appears to be localized in the vascular bundles, being present in sweet almonds as well as in bitter ones ${ }^{2}$. During the decomposition of amygdalin by means of emulsin prussic acid is formed, but none when invertin ${ }^{3}$ is the ferment employed, nor is any prussic acid produced when Penicillium glaucum is grown upon a one per cent. solution of amygdalin.

The potassium myronate of mustard seeds seems to be decomposed to a limited extent during germination, probably by means of the ferment myrosin which is stored in different cells ${ }^{4}$. In other cases, however, no enzymatic decomposition occurs during life, for in plants containing indican no blue colouration is produced so long as they are living, although indigo is an aplastic substance and is soon formed after death ${ }^{5}$. Similarly, the gradual accumulation of alizarin would cause the living root-cells of Rubia tinctoria to become coloured if any were formed by the decomposition of the ruberythric acid present. According to Molisch seedlings of Isatis tinctoria form no isatin in the absence of light, and in darkness any isatin that has already been formed disappears again, being metabolized without any production of indigo, although it does not follow that this substance is being continually decomposed in normal metabolism. According to Theorin both populin and salicin are consumed in spring, but for the most part even those glucosides which are present in abundance, such as hesperidin, \&c., remain intact when the plant is starved".

\section{Section 88. Pigments.}

These include substances of widely different chemical constitution and physiological importance. With the cxception of chlorophyll, ctiolin, phycophaein, phycoerythrin, bacteriopurpurin, and the pigments of certain bactcria

${ }^{1}$ Jorissen, Bot. Centralbl., I $88_{4}$, Bd. xx, p. 258 ; Portes, Compt. rend., 1877, T. Lxxxiv, p. I401; Wicke, Ann. d. Chem. u. Pharm., $18_{51}$, Bd. Lxxix, p. $79 ; 1852$, Bd. Lxxxi, p. 241 . HCN in Nanihot, Flückiger, Pharmakognosie, $188_{3}$, 2. Aufl., p. 954 ; Rochleder, Phytochemie, I $\$_{54}$, p. 54 .

2 Johannsen, Ann. d. sci. nat., I888, vii. sér., T. vi, p. II 8 ; Guignard, Compt. rend., I89o, T. cx, P. 477 ; I893, T. cxvir, pp. 493, 75I ; Green, Annals of Botany, I893, vil, p. 99.

${ }^{3}$ E. Fischer, Ber. d. Chem. Ges., I 894, p. 2990 . Cf. Sect. 91.

* Spatzier, Jahrb. f. wiss. Bot., I893, Bd. Xxv, p. 75 ; Smith, Journ. f. Physiol. Chem., I888, P. 419; Nägeli, Theorie d. Gährung, Is 79 , p. 14; Guignard, Journ. d. Bot., I $8_{94}$, pp. $67,8_{5}$.

${ }_{5}^{5}$ Molisch, Sitzungsb. d. Wien. Akad., I 893 , Abth. i, Bd. CII, p. 269. Cf, also Lookeren, Versuchsst., I894, Bd. XLIII, p. $401 ; 1895$, Bd. XLVI, p. 249.

${ }^{6}$ Pfeffer, Bot. Zeitung, I $8_{74}$, p. $4^{\delta_{1}}$; Theorin, Bot. Jahresb., Is $8_{4}$, p. $8_{7}$. 
which have the power of fixing oxygen, they have for the most part solely a biological significance, being commonly used for attractive purposes in floral organs, \&c., and in leaves and young stems scrving as a protection against excessive insolation. The absorption of certain rays necessarily produces a corresponding risc of tempcrature, by means of which the rate of transpiration may be increased and the general vital activity accelerated so long as the optimal temperature is not surpassed ${ }^{1}$. In other cases the colour is simply an accidental property of certain products of metabolism, although these have usually no marked colouration. Thus it is difficult to sce of what importance the colouration, that is, the more marked absorption of certain rays and reflection of others, can be in duramen, in fungi growing in darkness, or in those pigment-bacteria which can occlude oxygen.

The red and blue pigments dissolved in the cell-sap, such as anthocyan and erythrophyll, seem to be tannins or compounds allicd to phenols, whereas the fatty lipochrome and chlorophyll pigments, as well as the ptomainelike bacterial product pyocyanin, are of quite different chemical constitution. Aniline dyes are formed by a few bacteria, and algae possess in phycoerythrin and phycocyanin coloured and crystalloid proteid substances (Sect. 60) ${ }^{2}$. In some cases a pigment (indigo, alizarin) is formed from a pre-existent chromogen by decomposition or oxidation (Sects. 84,87), so that the last stage in the production of pigment is rendered directly evident. Tannins and other compounds allied to phenols may perhaps also serve as the precursors of pigment substances, which, however, are certainly often formed from other materials, and may often occur in plants which contain no tannin ${ }^{3}$.

In the higher plants soluble pigments are dissolved in the cell-sap for

${ }^{1}$ Cf. Ludwig, Biol. d. Pflanzen, I895, and also Sect. 62. On the importance of the warming effect, cf. Kny, Zur physiol. Bedeutung d. Anthocyans, I892 (Atti del Congresso Botanico, I892); Stahl, Ann. d. Jard. bot. de Buitenzorg, I896, T. xiri, p. I37. According to Pick, the absorption of certain inimical rays permits the translocation of starch to proceed more actively (Bot. Centralbl., I $S_{93}$, Bd. Xvi, p. 346), but this might be simply due to the heating effect. In certain tropical plants which grow in damp and shady situations, the dye appears to be mainly of importance as an aid to transpiration, especially when present only on the under surfaces of the leaves. In more exposed situations, it seems to act almost solely as a protection to young, sensitive, and especially to chlorophyllous organs, or ones in which synthetic metabolism is active (Ewart, Annals of Botany, ${ }_{\mathrm{I}} \mathrm{S}_{97}$, Vol. XI, p. 460). In darkness little or no pigment is formed, and it is interesting to notice that the absorption-spectrum of erythrophyll is to a large extent complementary to that of chlorophyll (Engelmann, Bot. Zeitung, I 887 , p. 433 ; N. J. C. Muiller, ibid., I $\$ 88$, Bd. Xx, p. $7 \&$ ).

${ }^{2}$ Cf. Hansen, Farbstoffe d. Blithen u. Friichte, I $88_{4}$; W'igand, Bot. Hefte, $188_{5}$, p. 218 ; Zimmermann, Mikrotechnik, $189^{2}$, p. 97 ; L. Müller, Vergl. Anat. d. Blumenblätter, 1893 , p. 230 ; Weigert, Bot. Centralbl., I $896, \mathrm{Bd}$. LXvi, p. 353 , and the literature here given. On bacteria and fungi : Zopf, Pilze, 1890 , p. I 43 ; Flügge, Mikroorganismen, I\$96, 3. Auf., pp. $175,4{ }_{7}$; Lafar, Technische Mykologie, I 897 , p. I25. On lichens: Bachmann, Jahrb. f. wiss. Bot., I 890 , Bd. xxi, p. I; Zopf, Ann. d. Chemie, $\mathrm{IS}_{97}$, Bd. CCxcv, pp. 222, 295.

${ }^{3}$ Cf. Wigand, 1. c. ; Pfeffer, Unters. a. d. Bot. Inst. z. Tübingen, I886, Bd. II, p. 2 IS ; G. Kraus, Grundl. z. Physiol. d. Gerbstoffes, I $88_{9}$, p. 3 I. 
the most part, while insoluble pigments are contained mainly in chromatophores. Among the numerous organic substances excreted by fungi and bacteria, pigments are often included. Different pigments may be present in a single cell either mixed together or strictly localized, as for example in a chlorophyllous cell containing red cell-sap.

Pigment substances are not formed under all circumstances, and they may appear or disappear at certain stages of development. Similarly, the existence of a plant may not be endangered by the non-formation of the normal blue, red, or other pigments of the flower, or by a change in the colouration, as is instanced by the common occurrence of racial and cultural variations.

The influence of the external conditions upon the production of coloured metabolic products is immediately perceptible, as, for example, when a change of temperature, of illumination, or of the nutritive conditions ${ }^{1}$, acts as a stimulus inducing altered activities, and either awakening or suppressing the formation of coloured metabolic products. A purely physical or chemical action may also be exercised, as, for example, when strong light bleaches and decomposes chlorophyll or other pigments (Sect. 58), or when pigment substances are produced from chromogens by means of the oxidizing agency of hydrogen-peroxide ${ }^{2}$. If when once destroyed the colouration never reappears however long the cell may remain living, it is evident that the pigment is one which is produced only at a certain stage of development. If, however, the colouration returns again, then either the destruction of the pigment must have acted as a stimulus to renewed formation, or else in the living cell fully charged with pigment, the decomposition and reconstruction are continuous and precisely counterbalance one another. Since pigments are metabolic products, it is obvious that the colouration is not produced by any such direct action of light as may be exercised upon many dead substances ${ }^{3}$.

Most plants are unable to form chlorophyll in darkness (Sect. $5^{8}$ ), and whereas red grapes, and the flowers of Tulipa Gesneriana, Pulmonaria officinalis, \&c., assume their normal colouration even in the complete absence of light, the foral

${ }^{1}$ [Ewart observed that immersion in sugar solution may induce a formation of red pigment in the cells of certain aquatic plants (The Effects of Tropical Insolation, Ann. of Bot. Vol. XI, p. 477; Journ. of Linn. Soc. Vol. xxxi, p. $56 \%$ ), an observation which has since been confirmed and extended by Overton (Jahrb. f. wiss. liot., I 899 , Bd. xxxiII, p. 1;0), who concludes that the formation of red pigment stands in genetic connexion with the presence of sugar in the cell. This may indeed frequently be one of the conditions necessary for the production of a red colouration, but in the majority of cases the action of moderately strong light is also essential. In all such cases the pigment may well have an important biological function and not be merely an accidental by-product of the metabolism of sugar, as Overton suggests.]

2 Pfeffer, Oxydationsvorgänge in lebenden Zellen.

3 Wiener, Ann. d. Physik u. Chemie, I895, N. F., Bd. Lv, p. 227. 
organs of Prunella grandiflora are abnormally shaped and imperfectly coloured when grown in darkness'. Red pigment is formed in the roots of Salix, in many rhizomes, hypocotyls, in the tentacles of Drosera, \&c. ${ }^{2}$, only when they are exposed to light, and in some cases only when the light is of considerable intensity, as in Azolla, apples, and pears, for only the surfaces exposed to sunlight become red ${ }^{3}$. Similarly, the autumnal colouration of leaves seems to be produced by the action of light as soon as the vitality of the leaf-cells sink below a certain ebb, and the change is hence frequently aided by a low temperature ${ }^{4}$, as is especially the case with regard to the brown winter colouration of Conifers. The latter is characterized by a partial or complete decomposition of the chlorophyll, and by a simultaneous appearance of a brown or red pigment (Sect. $\left.5^{8}\right)^{5}$.

In a few non-chlorophyllous organisms, such as Micrococous ochroleucus ${ }^{6}$, the pigment is formed only in the light, while in Micrococcus prodigiosus and many other bacteria its formation may be inhibited by conditions which still permit of growth, such as strong illumination, sub-maximal temperatures, a deficiency of oxygen, or the presence of poisonous substances. Spirillum rubrum, horrever, forms coloured growths only when the oxygen supply is deficient, whereas when leuco-products or chromogens are formed under such conditions, they rapidly become coloured when oxygen is admitted ${ }^{7}$. The formation of pigment substances may be suppressed most readily in bacteria, but in Phanerogams also the pigments are secretory products, and hence the amount produced may alter or become a vanishing quantity under special external conditions, which have, however, not as yet been determined with certainty ${ }^{8}$.

Schottelius, by exposing cultures to $4 \mathrm{I}^{\circ} \mathrm{C}$., obtained colourless races of Micrococcus prodigiosus which had at the same time lost the power of forming trimethylamine, and a similar result as regards the non-formation of pigment was obtained by Phisalix with Bacillus pyocyanus, and by Laurent with the red Kiel

1 Sachs, Bot. Zeitung, Beilage, $1_{8} 6_{3}$ and $186_{5}$, p. II 7 ; Askenasy, Bot. Zeitung, I876, p. I ; Vöchting, Jahrb. f. wiss. Bot., I 893 , Bd. Xxv, p. I 77 . Grapes: Laurent, Bull. d. 1. Soc. Bot. d. Belgique, I89o, T. xx1x, p. 7 I.

2 Mohl, Vermischte Schriften, I 845 , p. 390 ; Schell, Bot. Jahresb., I 876 , p. 717 ; Pick, Bot. Centralbl., 1883, Bd. Xvi, p. 315 (seedlings); de Vries, Bot. Zeitung, I886, p. 4 (Drosera); Kerner, Nat. Hist. of Plants, I 894 , Vol. I, p. $4^{8} 4$ (Rhizomes). On algae, cf. Oltmann's Jahrb. f. wiss. Bot., I $89^{2}, \mathrm{Bd}, \mathrm{xxIII}, \mathrm{p} .432$.

${ }^{3}$ Senebier, Physik.-chem. Abh., I785, iii. Th., p. $7 \mathbf{1}$; Askenasy, Bot. Zeitung, I875, p. 498. [In such cases as these, it is obvious that the red pigment has a protective function, and it can hardly induce either transpiration or a rise of temperature above that of the surrounding medium in roots of Salix, which turn red when exposed to light even though submerged in water.]

${ }^{+}$Mohl, l. c.; Schiibler, Bot. Centralbl., I886, Bd. xxviri, p. 205.

${ }^{5}$ [Schimper, 'Unters, iiber die Chlorophyllkörper,' Jahrb. f. wiss. Bot., Bd. xvil, $88_{5}$.]

6 Prove, Cohn's Beiträge z. Biol., I 887 , Bd. Iv, p. 439.

${ }^{7}$ See Fluiggre, 1.c. ; Lafar, 1. c. ; Dieudonne, Biol. Centralbl., I 895 , Bd. xv, p. Io8; Thuman, Arb. d. Bact. Inst. in Karlsruhe, I 895, Bd. I, p. 291 ; Nicolle et Bey, Ann. d. l'Inst. Pasteur, 1896, T. x, p. 669 . For details on fungi : Elfving, Einwirkung d. I.ichtes auf Pilze, I89o, p. 6; Duclaux, Ann. d. l'Inst. Pasteur, I 889 , T. II, p. III. In lichens, pigments may be formed by the co-operation of the symbionts.

${ }^{8}$ Nägeli, Sitzungsb. d. Bair. Akad., I\$ 79, p. 301 ; Hoffmann, Bot. Zeitung, I88 г, p. 379 ; Molisch, ibid., I879, p. 49. 
bacillus!. If the exposure has not been too prolonged, the power of pigment formation may gradually return in the successive generations produced under normal conditions, but by more drastic treatment this power may apparently be permanently destroyed ${ }^{2}$.

\section{Section 89. Alkaloids, Ptomaines, and other Poisons.}

Numerous plants produce one or more poisonous substances, including alkaloids, ptomaines, toxalbumins ${ }^{3}$, certain glucosides, and other chemical substances, such as hydrocyanic acid, \&c. (cf. Sect. 70).

Some of these substances are virulent poisons, and have for the most part a biological importance, forming a protection against herbivorous animals and against the penetration of parasites ${ }^{4}$. They may also cnable certain plants, especially bacteria, either to compete successfully with other organisms, or by killing the latter to provide for their own growth (Sect. 92). For the latter purpose it is obviously essential that the poisonous products should be excreted, but in many of the higher plants and fungi they are permanently retained and deposited in a manner best suited for the fulfilment of their special biological importance; as, for example, when poisons accumulate in the fluid latex which at once carries to an injured region both material for repair and a means of defence against further attacks ${ }^{5}$. Within living cells poisons are generally deposited in the cell-sap, and in this way as well as by the attainment of a certain immunity, large quantities may be accumulated without injury to the poison-producing plant. Certain fungi are capable of feeble growth on dilute solutions of morphia, \&c., but as a general rule such poisonous substances, when produced by the plant itself, do not appear to be reassimilated, as is instanced by the behaviour of the solanin of the potato during germination ${ }^{6}$. Although in this case, as well as during the germination of the seeds of Datura Stramonium and Strychnos mux vomica, a

${ }^{1}$ Schottelius, Centralbl. f. Bact., I 887 , Bd. II, p. 439. Cf. Flïgge, 1. c., p. $4^{8} 7$; Charrin et I'hisalix, Compt. rend., I892, T. cxiv, p. ${ }_{15} 6_{5}$; Laurent, Ann. d. l'Inst. Pasteur, IS94, T. XIv, p. 479 . p. 109 .

${ }_{2}^{2}$ Galeotti, Centralbl. f. Bact., I893, Bd. xrv, p. 697 ; Dieudonné, Biol. Centralbl., I 895, Bd. xv,

${ }^{3}$ Cf. Neumeister, Physiol. Chemie, IS93, p. 226; Hoppe-Seyler, Handb. d. chem. Analyse, 4. Auf., I 893, P. 94 .

${ }^{*}$ Cf. lit. in Sect. 87. Peirce has shown that the penetration of Cuscuta into a host-plant is hindered by the presence of poison in the latter, and the same is the case with fungi.

${ }^{5}$ Cf. Zimmermann, Mikrotechnik, I \$92, p. I I6; Moller, Localisation d. alcaloides d. Solanacées, Bruxelles, 1895 .

${ }^{6}$ De Vries, Landw. Jahrb., I 878 , Bd. viI, p. 243 ; Wotezal, Bot. Jahresb., I 890 , Bd. Xi.I, p. Iоo; G. Meyer, Beibl. z. Bot. Centralbl., i 896, Bd. vi, p. 6 i. 
certain diminution has been observed, the imperfection of the available methods of detection renders it doubtful whether the apparent decrease is not merely due to the distribution of the substances in question over the increasing volume of the growing plant ${ }^{1}$.

Poisons are by no means essential products of metabolism; thus in the sweet almond the power of producing amygdalin has been entirely lost, and Vogel ${ }^{2}$ was unable to detect any quinine in chinchona plants grown in European hot-houses. It is also possible for the hemlock often to contain no conine in Scotland, for the production of alkaloids is markedly dependent upon the cultural conditions ${ }^{3}$. Still more marked variations are shown by pathogenic bacteria, which may exhibit all grades of virulence according to whether the mode of treatment or culture is adapted to diminish or increase their power of producing the specific poisonous substances to which the pathogenic character of the bacterium in question is due ${ }^{4}$. Alterations of this kind may be either temporary or permanent: thus Bacillus anthracis becomes permanently non-virulent after prolonged culture at $42^{\circ} \mathrm{C}$., but after a short exposure to $50^{\circ} \mathrm{C}$. the virulence gradually returns when the microbe is cultivated under normal conditions. Even the permanently non-toxic forms may be rendered toxic by special treatment, just as sweet almonds may occasionally become bitter again. The rapidly reproducing micro-organisms are especially suitable for experiments of this kind, and it may be found possible to obtain similar results with regard to the production of enzymes or of other substances which are accessory products of metabolism.

Many poisonous substances may occur in widely different plants, and the toxalbumins which occur in the seeds of Abrus precatorius and Ricinus as well as in bacteria and in the sporophore of Amanita phalloides ${ }^{5}$, may possibly be detected in many of the higher plants. Many bacteria produce their specific poisons only when fed with proteids, others, however, also when no proteids are supplied, but nothing definite is known as to the precise metabolic origin of poisonous substances in general. Poisons are usually directly formed by the protoplast, but may also arise outside the cell by the extracellular action of certain excreta, as, for example, when poisons are formed by the peptic digestion of fibrin. Similarly after the death of a cell which contains amygdalin, prussic acid may be liberated

${ }^{1}$ Heckel, Compt. rend., I 890, T. cx, p. 88. Cf. Clautriau, Ann. d. l. Soc. Belg. d. Microscopie, I 894 , T. xviri, p. 47 .

2 Vogel, Sitzungsb. d.'Miinch. Akad., $188_{5}$, 1) 6.

${ }^{3}$ Rochleder, Phytochemie, $18_{54}$, p. $34+$. Cf. Darwin, Variation of Plants and Animals under Domestication, p. 314; Ludwig, Biol. d. Pflanzen, IS95, p. 222 ; Ad. Mayer, Versuchsst., I $8_{9}$ I, Bd. xxxvili, p. 453 (tobacco). On Agaricineac, cf. Böhm u. Kulz, Bot. Jahresb., I885, p. 2 So.

* See Fluigge, 1. c., p. 299; Lafar, 1. c., p. 27 I ; Burkmaster, Biol. Centralbl., I 95 , Bd. Xv, p. 96.

${ }^{5}$ Neumeister, Physiol. Chemie, I $_{93}$, p. 228 ; O. Loew, Giftwirkungen, I893, P. 76. 
by the aid of emulsin. In certain cases poisonous substances arise from the co-operation of two distinct organisms, as, for example, in conjoint infection (cf. Flügge, 1.c., p. 309).

\section{SECTION 90. Ethereal Oils, Resin, \&c.}

Ethereal oils, balsams, resins, india-rubber, \&c., are as a general rule not reassimilated, and even in an extremely fine state of division or in the form of an emulsion they do not afford a suitable medium for the growth of fungi. Ethereal oils serve mainly for purposes of attraction, and also affect the diathermanous character of the surrounding air, whereas balsams and latex serve to heal wounds, or by impregnating the cell-wall influence its permeability ${ }^{1}$.

These substances may be formed within the protoplast, and may appear either as an emulsion (latex, \&c.) or may coalesce to form homogenous masses of oil or balsam, the products being either permanently retained at the point of origin or excreted, internally or externally. Substances may apparently also be excreted which become converted outside the protoplast into ethereal oils or balsams, and the latter may even arise by metamorphosis of the cell-wall. The details of these processes are, however, quite unknown, in spite of the careful histological study to which the special glands, gland-hairs, secretory receptacles ${ }^{2}$, \&c. have been subjected. Many resins and forms of latex undergo certain changes when exposed to air, and it is by means of a post-mortem absorption of oxygen that the ethereal oil of the camphor-tree is converted into camphor.

Both the production and excretion of the substances mentioned may be influenced by external circumstances, as when a pathological formation of resin occurs, or when a plant becomes more odoriferous in a warm and sunny habitat. Insolation usually causes ethereal oils to evaporate more rapidly, and in Dictammus albus the air around the inflorescence may actually be capable of ignition. Many flowers are most fragrant at night, either because the production of the ethereal oils is most active at that time, or because some special causes induce more rapid exhalation ${ }^{3}$.

${ }^{1}$ Cf. Stahl, Ludwig, Kerner, \&c. The marked absorption of dark heat-rays by the vapours of ethereal oils can hardly be of any great importance as a protection against insolation.

${ }^{2}$ Cf. de Bary, Anatomie, 1877 , pp. 72,152 ; Tschirch, Pflanzenanatomie, I889, p. 460 ; Haberlandt, Physiol. Pflanzenanat., I 896,2 . Aufl., p. 432 ; Tschirch, Jahrb. f. wiss. Bot., I 893, Bd. xxv, p. 370 ; Siek, ibid., I895, Bd. Xxvir, p. 238 ; Bécheraz, Bot. Centralbl., I894, Bd. Lx, p. 20 ; Berthold, Protoplasmamechanik. I 886 , pp. I4, 27.

${ }^{3}$ Treviranus, Physiol., Bd. I. p. 93 ; de Candolle, Physiol., T. rr, p. 764 ; Regel, Bot. Centralbl., I89I, Bd. XLv, p. 343 ; Mesnard, Ann. d. sci. nat., I894, vii. sér., T. XvirI, p. 374 ; Rev. gén. d. Bot., I 894 , T. II, p. 97 , and I 896 , T. viri, p. 129. 


\section{SECTION 91. Enzymes.}

Plants frequently make use of enzymes or ferment substances capable of inducing chemical metamorphoses of altogether disproportionate magnitude relatively to the amount of ferment employed. Diastatic, inverting, proteolytic (pepsin, trypsin, papain, papayotin), glucoside-splitting, and cytasic ferments occur in many but not in all plants, and steapsins or fat-splitting ferments are probably equally widely distributed. Rennet and urea ferments have been detected in a few cases, and it is possible that many gum and pectic ferments may also exist (Sect. 82). All the known enzymes appear to be proteids ${ }^{1}$ which, like toxalbumins, have powers altogether out of proportion to their bulk. There can be no doubt that different forms of diastase, invertin, \&c., exist, and indeed each of the above terms apparently includes a group of ferments varying in detail from one another $^{2}$, for certain diastatic extracts produce maltose, and others dextrose when acting on starch. Similarly different proteolytic enzymes carry the decomposition of proteids to varying degrees of disorganization, while amygdalin may be decomposed with or without a production of prussic acid, according to the ferment employed (Sect. 87).

Each enzyme has a strictly limited sphere of action, which, however, usually includes more than one substance: thus pepsin decomposes most but not all proteids, while diastase not only hydrolyses starch but also certain forms of cellulose, and a special invertin not only acts upon most polysaccharides but also upon a few glucosides. It is possible that in many cases the extracts used may contain a mixture of two ferments, but the available experimental data suffice to prove that different varieties of invertin, diastase, \&c. do actually exist. It is not surprising to find that a given ferment may only be able to decompose one of two stereo-isomeric substances, and E. Fischer observed that when the sugar of a glucoside was replaced by its optical antithesis, a previously active ferment was unable to decompose the new glucoside.

Many of the enzymes hitherto isolated are hydrolytic in character, although a gas may be produced, as in the decomposition of urea by urase (Sect. 102). Oxidizing ferments or oxidases also exist which, in dead cells and perhaps in living ones also, may act as katalytic agents by absorbing oxygen and transferring it to other substances (Sect. IOI). Similar katalytic actions may be exercised by acids as in the inversion

1 A. Mayer, Die Lehre von den chemischen Fermenten, I882, p. I9; Ncumeister, Physiol. Chemie, I 893 , Bd. r, p. 8 I. A summary of the known ferments is given by Green, Annals of Botany, I 893 , Vol. vir, p. 83 .

${ }^{2}$ Cf. Beyerinck, Centralbl. f. Bact., I 895 , Abth. ii, Bd. r, p. 229 ; E. Fischer, Ber. d. Chem. Ges., I 894, p. $34^{81}$. 
of sugar, by finely divided platinum which acts as a vehicle for the transmission of oxygen, and by finely divided iridium which induces the decomposition of formic acid into hydrogen and carbon dioxide. Similarly the formation of ether by the aid of sulphuric acid shows that katalytic action may also induce molecular combination, and hence it is possible that certain synthetic processes may be performed by means of enzymes. It is, however, not always easy to determine whether or not a given change is proluced by a ferment, for many enzymes are extremely unstable, and hence isolation is almost or entirely impossible. Morcover certain ferments may exert a katalytic action only under the conditions presented in the living and vitally active cell, so that the power of inducing fermentation may in such cases be inhibited by anaesthetization with chloroform or ether. On the other hand the possibility of such inhibition does not prove that the alcoholic fermentation of sugar in a living yeast-cell may not be due to the action of a ferment, indeed Buchner has recently succeeded in isolating an alcohol-producing ferment from yeast ${ }^{1}$. Saccharomyces may, however, still be termed a 'ferment-organism,' since the production of ferment is due to the metabolic activity of the living yeast-cells. The term 'ferment-organism' is preferable to that of 'organized' or 'formed' ferment, and the word 'enzyme' (Kühne) or 'ferment' may be restricted to the katalytic secretion of the ferment-organism.

It is only recently that chemists have directed a proper amount of attention to the long-neglected katalytic actions. The latter may be induced by organic as well as by inorganic substances, and hence there is no reason for supposing that the remarkable activity of ferments is due to the presence of minute fragments of protoplasm. Ferments are to be regarded as stimulating or accelerating agents ${ }^{2}$, and it is the task of physical chemistry to ascertain whether ferments are vehicles for the transmission of special molecular movements, or act by means of the dissociatory power of surface-tension energy, or by generating a series of actions and reactions directed along particular channels. From a physiological point of view we are concerned with facts only, and these teach us, for example, that although sulphuric acid has theoretically an unlimited katalytic power, in actual practice the latter is found to be strictly limited. The same is the case with ferments; thus diastase is able to hydrolyze I , , oo times its own bulk of starch, while invertin can invert roo, 000 times its own volume of cane-sugar ${ }^{3}$. Since

1 E. Buchner, Ber. d. Chem. Ges., 1897, pp. 117 , I 100. [Cf. also Green, Rep. Brit. Ass., I 898 (Botany); Nature, September, 1898.$]$

2 See Ostwald, Ber. d. Sächs. Ges. d. Wiss., I 994 , p. 337. For older speculations, see Ad. Mayer, Lehre v. d. chem. Fermenten, Is 2 , p. I04; Handb. d. Chem., Bd. Iv, p. I24; Bunge, Physiol. Chemie, I 894,3 . Aufl., p. 168.

${ }^{3}$ Schleichert, Die diastat. Fermente d. Pflanzen, I $\delta_{93}$, p. $8_{5}$; Invertin, O. Sullivan and Thompson, Koch's Jahresb. d. Gährungsorg., I 890 , p. I 7 I ; A. Meyer, Unters. über die Stärkekörner, 1895, p. 67. On the alteration and wearing out of enzymes, cf. Tammann, Zeitschr. f. physiol. Chemie, I 895, Bd. Xviri, p. 427 . 
most ferments are destroyed at a comparatively low temperature, their hydrolytic action is unable to increase indefinitely as the temperature rises, although the hydrolysing power of such substances as hydrochloric acid may become perceptible only at high temperatures, and may increase until the limit of molecular stability is reached '. It is, moreover, not surprising that hydrochloric acid decomposes all carbohydrates and glucosides, but enzymes only particular forms of these substances.

Ferments serve both to digest food-substances and to render them capable of absorption, as well as to prepare them for assimilation by the protoplast. Hence the production and excretion of ferments is commoner among heterotrophic than among autotrophic plants. If the former are supplied with nutriment which can be directly assimilated, the ferments which may still be excreted are no longer of essential importance, and many plants produce ferments only at certain stages of development or not at all. In certain cases the production of ferments ceases partially or entirely under unfavourable cultural conditions, and even in an actively growing plant an abundant supply of the products of enzymatic action may partly or wholly inhibit the formation of the enzyme in question. The latter is an example of a diminution of metabolic activity induced by the action of a stimulus, whereas in carnivorous, and probably many other plants also, the secretion of ferment is excited or accelerated by special chemical stimuli. The excretion of ferment may excite fresh secretion, and the rate of secretion is subject to automatic regulation.

According to circumstances enzymes may be excreted externally, either continually or only at certain times, or may be always retained within the plant. In the latter case the ferment and fermentable material may be separated from one another, or the possibility of interaction may be prevented owing to one or the other being present in some inactive form or combination (zymogen, \&c.). The action of an enzyme may be either aided or inhibited by the presence of other substances, and moreover not only the production but also the transport of ferments is subject to regulatory modification ${ }^{2}$.

In many cases zymogens exist in the living cells, and when excreted readily decompose or change to form active enzymes ${ }^{3}$, but the metabolic

1 Tammann, Zeitschr. f. physiol. Chemie, IS92, Bd. xiI, p. 271 ; Zeitschr. f. physik. Chemie, I 895, Bd. xviri, p. 426 .

2 There do not as yet appear to be sufficient grounds for assuming that a chemical difference exists between translocatory and secretory diastase (Brown u. Morris, Bot.Zeitung, i $892, p \cdot 465$ ). Different extracts of diastases, invertins, \&c., possess unequal diosmosing powers. A. Meyer, Stärkekörner, IS 95 , p. 228 ; Griiss, Jahrb. f. wiss. Bot., I $S_{94}$, Bd. xxv'1, p. 386 ; Beyerinck, Centralbl. f. Bact., I895, Abth. ii, Bd. I, pp. 223, 228 ; Fermi (l. c. beneath).

${ }^{3}$ Cf. Green, Annals of Botany, I 893 , Vol. viI, p. I 21 ; Frankfurt, Versuchsst., IS96, Bd. Xlvir, p. 455. The proteids from which enzymes have been artificially produced may have been zymogens. See Schleichert, Die diastat. Fermente d. Pllanzen, I $\delta_{93}$, p. $\delta_{4}$. 
origin of these zymogens is entirely unknown. Both acrobic and anaerobic plants produce enzymes, and the non-formation of these substances by aerobic organisms in the absence of oxygen affords no proof that they are oxidation-products ${ }^{1}$.

Changes in the rate of production. Iiborius and Fermi ${ }^{2}$ have both shown that the production of proteolytic ferments as well as of invertin and other enzymes is dependent upon the cultural conditions, for the absence of proteids, the addition of quinine, antipyrin, \&c., or subjection or a high temperature may partially or entirely inhibit the process of their secretion. This result appears to be often induced by unfavourable cultural conditions, but in many cases no enzymes are formed although growth remains active. An excess of the products of enzymatic action may also constitute an inhibitory stimulus. Thus Katz ${ }^{3}$ found that Penicillium glaucum forms no diastase when more than $\mathrm{I}_{\frac{1}{2}}^{\frac{1}{2}}$ per cent. of cane- or grapesugar is present in the nutrient fluid. Bacterium megatherium behaves similarly, whereas Aspergillus niger produces traces of diastase even in a 30 per cent. solution of sugar. Penicillium ceases to form diastase when growing actively in sugarsolution, but not during active or feeble growth upon 3 per cent. or io per cent. solutions of quinolic acid. Hence it is evident that we are dealing with a specific stimulatory action, which is more powerful in the case of dextrose and cane-sugar, and less so in that of maltose and lactose. Aspergillus niger reacts in a similar manner but not so strongly, and similar relationships will probably hold good for other ferments as well, although a stimulus which suppresses the formation of diastase need not necessarily affect the production of other ferments ${ }^{4}$.

It is not the mere excretion of ferment which is affected by the concentration of the nutrient solution, for the diastatic activity of the excreted ferment often becomes markedly influenced only in highly concentrated sugar solutions ${ }^{5}$. Direct experiments, moreover, have shown that no diastase is present within plants of Penicillium grown on a 2 per cent. solution of sugar.

Certain fungi and bacteria, however, produce diastase even in sugar solution, although Wortmann's results are not trustworthy, since they were obtained with a mixture of bacteria ${ }^{6}$.

In the case of Phanerogams the accumulation of sugar seems only to depress

1 See Griiss, Landw. Jahrb., I 896 , Bd. xxv, p. 425 .

${ }^{2}$ Liborius, Zeitschr. f. Hygiene, I 886 , Bd. I, p. I 56 ; Fermi, Centralbl. f. Bact., I 89 I, Bd. x,

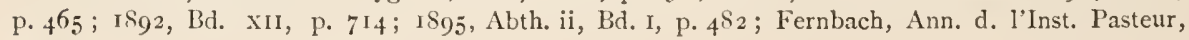
I 890 , T. IV, p. 64I. Cf. Flügge, 1. c., Bd. I, p. 209 ; Beyerinck, Centralbl. f. Bact., I895, Abth. ii, Bd. I, P. 226.

${ }^{3}$ [Katz, Die Regulatorische Bildung von Diastase durch Pilze, Jahrb. f. wiss. Bot., I $\$ 9 \$$, Bd. xxxi, p. 599.] See Sitzungsb. d. Sächs. Ges. d. Wiss., i 896, p. 5 I 3.

4 Beyerinck (Aliment photogène, I891, p. 23 , Sep.-abdr. a. Archives Néerlandaises, T. xxiv) observed that in a few luminous bacteria the secretion of diastase may be depressed by the addition of sugar but not that of proteolytic enzymes.

${ }^{5}$ For literature see Schleichert, Die diastat. Fermente, IS93, p. 45; A. Meyer, Stärkekörner, I 895, p. 66; Grïss, Landw. Jahrb., I896, Bd. xxv, p. 399; Tammann, Zeitschr. f. physik. Chemie, I889, Bd. III, p. $3^{2}$.

${ }_{6}^{6}$ Biisgen, Ber. d. Bot. Ges., $188_{5}$, p. Ixvi ; Krabbe, Jahrb. f. wiss. Bot., I 890 , Bd. xxi, p. $56_{4}$; Wortmann, Zeitschr. f. physiol. Chemie, I $S \delta_{2}$, Bd. vI, p. $3^{\mathrm{I}} 6$. 
the production of diastase to a slight extent ${ }^{1}$, and since the accumulation of the fermentative products ultimately inhibits further action, the fact that the solution of starch is hastened by the continual removal of the sugar produced, affords no evidence to show that the formation of diastase is subject to regulatory modification. Enzymatic action may be affected by the acid or alkaline reaction of the medium as well as by the presence of various substances, and hence by these means alone the plant is able to regulate the activity of ferment-substances ${ }^{2}$. Pepsin works only in an acid, trypsin only in an alkaline solution, and hence when both are mixed together one only can be active. In other cases, however, two or more enzymes may act simultaneously, and even proteolytic enzymes apparently exert only a slight destructive influence upon other enzymes ${ }^{3}$.

Temperature. Most enzymes are destroyed at a temperature lying between 60 and $70^{\circ} \mathrm{C}$., but those which are produced by thermophilous bacteria are probably more resistant ${ }^{4}$, and the character of the medium as well as the presence of certain substances may raise or lower the maximal temperature. Every enzymatic action must therefore attain an optimum at a certain temperature, beyond which the fermentative activity decreases until the point is reached at which the ferment is destroyed. Details are given in the works already quoted of Ad. Mayer, A. Meyer, Schleichert, Flügge, \&c. For an account of the inhibitory action of strong light see Green, Annals of Botany, I 893 , Vol. viI, p. 372 ; Phil. Trans., I 897 , Vol. cxliv, p. I67; Fermi, 1.c., I 894 , Bd. xvi, p. 830 ; Linz, Jahrb. f. wiss. Bot., I 896, Bd. Xxix, p. 279.

Methods. The fermentative action of an enzyme is usually employed as a test for its presence, as is also the case in Beyerinck's method of using the physiological reaction of luminous bacteria as an indication of fermentative activity. Grüss has recently employed the blue colouration given by guiacum and hydroxyl as a test for the presence of diastase, but the method requires careful control, and, according to Jacobson, various agencies may suppress the guiacum reaction without destroying the diastatic action ${ }^{5}$.

Diastases or amylohydrolytic ferments are very widely distributed, and in spite of a few negative results every plant may have the power of producing some form or other of diastase. It is, however, uncertain whether the presence and solution of starch necessarily involves a power of secreting diastase, but the latter may be detected in the most widely different plants or parts of plants either always or only at certain stages of development. Thus diastase may be present in both young

1 Literature: Brown u. Morris, Bot. Zeitung, 1892, p. 464; Griiss, Landw. Jahrb., I896, Bd. x.rv, p. $44^{2}$.

${ }_{2}$ Literature: Ad. Mayer, Die chem. Fermente, 1882 , p. 78 ; Schleichert, 1. c., p. 69 ; A. Meyer, Stärkekörner, 1895, p. 67 ; Fliigge, 1. c., p. 2 I3. According to Bertrand (Compt. rend., 1897, T. Cxxıv, p. 1032), laccase acts only in the presence of manganese.

3 Ad. Mayer, l. c., p. 97 ; Fermi, Centralbl. f. Bact., I894, Bd. xv, p. 234. On self-digestion, cf. Sect. 78 .

${ }^{4}$ [On the proteolytic enzyme of $N^{\text {eppenthes, }}$ cf. Vines, Annals of Botany, x1, Dec., I 897, p. $5^{83}$.]

${ }_{5}^{5}$ Beyerinck, Centralbl. f. Bact., 1895, Abth. ii, Bd. I, p. 222 ; Grüss, Ber. d. Bot. Ges., I 895 , p. 2 ; Landw. Jahrb., I $896, \mathrm{Bd} . \mathrm{xxv}$, p. $3^{8} 5$; Jacobson, Zeitschr. f. physiol. Chemie, I 892 , Bd. Xv', p. 340; Pawlewski, Ber. d. Chem. Ges., I 897 , p. I $33^{1} 3$. 
and old parts, and may be absent when young but appear later, or present when young and subsequently disappear. An increase usually occurs during the mobilization of reserve food-materials (Sect. I 09), but for details concerning the occurrence, distribution, and translocation of ferments the reader is referred to the works of Schleichert, A. Meyer, Flïgge, \&c. ${ }^{1}$

Since diastatic ferments may also be concerned in the solution of cellulose it is not surprising that they may occur in plants which do not produce starch, as, for example, in most fungi, and it is probably owing to the adaption to special nutritive conditions that no production of diastase can be detected in many bacteria (Sect. 65).

It does not necessarily follow that starch is only dissolved within the living protoplast by the aid of diastase, simply because the process of solution follows a similar course to that occurring externally ${ }^{2}$. No decisive evidence is afforded either by the character of the products or by the possibility of regeneration.

The different forms of diastase may be classified into a series of different types, as, for example, into glucase, maltase, and granulase ${ }^{3}$, and of these only glucase forms dextrose from starch. The other two produce maltose, with erythrodextrin as an intermediate product in the case of maltase, but isomaltose in that of granulase. All three forms occasionally occur in the same plant, but even then the embryo and the endosperm frequently contain different varieties of diastase. Similarly, fungi probably produce more than one form of diastase, and bacteria apparently also secrete those forms of diastase which act best in a weak alkaline solution. Comparative researches into the action upon different starch-grains and cell-walls will probably reveal further distinctions between the individual varieties of diastase, and in the form of compounds the diosmotic and other properties may be more or less markedly modified.

Cytohydrolytic or cellulose-dissolving enzymes are probably frequently employed to produce the dissolution or mucilaginous modification of cell-walls, as, for example, during the penetration of fungi and other parasites which may bore through resistant cellulose, or even through cuticularized or lignified walls. The fermentation of cellulose may take place externally by the agency of bacteria, while within the plant not only the hemi-celluloses deposited as reserve material, but also in certain cases when copulation or cell-fusion occurs, typical cellulose walls may be dissolved ${ }^{4}$.

No detailed study has as yet been made of the different enzymes which decompose cellulose, and it is uncertain whether all or only certain diastatic ferments

1 See also Green, Annals of Botany, 1893 , Vol. vir, p. $8_{4}$; Griiss, Jahrb. f. wiss. Bot., I 894 , Bd. xxv1, p. 379, and Landw. Jahrb., r 896 , Bd. xxv, p. 385 ; Beyerinck, Centralbl. f. Bact., I 893 , Abth. ii, Bd. 1, pp. 22 I, 265, 328 ; Linz, Jahrb. f. wiss. Bot., I 896, Bd. xxix, p. 265. Cf. also Sects. 70, 82.

${ }^{2}$ On the solution of starch by diastase, \&c., cf. Schleichert, 1. c., 1. 5I ; A. Meyer, 1. c., pp. 96, 227 ; Griiss, l. c., and Beitr. z. Bot. von Fünfstiick, $18_{95}$, Bd. I, p. 295.

${ }^{3}$ Beyerinck, 1. c., p. 268. Cf. also ref. in Ann. d. l'Inst. Pasteur, I895, T. IX, pp. 50, $121,214$.

${ }^{4}$ Cf. Sects. $6_{5}, 8_{2}, 8_{3}, 8_{4}$. On the destruction of wood by fungi, see R. Hartig, Lehrb. d. Baumkrankheiten, 2. Auf., p. 5I. Cf. also Green, 1. c., p. 93 . 
attack cell-walls. Negative results are only of value as preliminary tests, for the action may be dependent upon special circumstances, or upon special combinations of circumstances. According to Grüss those diastases which dissolve certain reservecelluloses do not appear to act upon ordinary cellulose, whereas Brown and Morris state that the diastase from barley-malt attacks ordinary cellulose but leaves reservecellulose intact ${ }^{1}$.

According to de Bary (Bot. Zeitung, 1886, pp. 4J9, 422) the enzyme of Peziza sclerotiorum which rapidly dissolves cell-walls exercises no action upon starch-paste. [Newcombe ${ }^{2}$ has shown that a cellulose-enzyme is present in seedlings of barley, lupins, and dates, as well as in Aspergillus oryzae. If extracts of equal diastatic value are prepared, those from Lupinus and Phoenix dissolve cellulose in about nine hours or so, those from Aspergillus and barley-malt in about roo hours. Newcombe concludes that distinct amylohydrolytic and cellulose-dissolving cytohydrolytic or cytase ferments are present, but the facts also coincide with the supposition that a single specific ferment is present in each extract, and that the different varieties of diastase possess varying powers of dissolving starch and cellulose.]

Invertin and glucoside-ferments. Several varieties of invertins or invertases exist, all characterized by the power of splitting soluble di- and poly-saccharides : thus Saccharomyces cerevisiae produces a readily diosmosing invertin which splits cane-sugar into dextrose and laevulose, and also a ferment which can only with difficulty be extracted, and which decomposes maltose ${ }^{3}$. Both of these leave milksugar intact, whereas the lactase of Saccharomyces Kefir decomposes both lactose and saccharose * On the other hand, emulsin decomposes lactose but not canesugar and maltose ${ }^{5}$, while inulin is decomposed by the inulase of Compositae, but not by the invertin of beer-yeast ${ }^{5}$. Fernbach has even shown that the typical saccharose-invertins of different plants exhibit certain specific peculiarities, and future comparative studies will probably reveal many other forms of invertin. Their action upon polysaccharides is not their only one, for the glucase which splits maltose, as well as the emulsin which decomposes amygdalin, are invertins. Glucosides and the ether-like compounds of carbohydrates with other substances, show in many respects a resemblance to the group of poly-saccharides, and hence

1 Grüiss, Jahrb. f. wiss. Bot., I 894 , Bd. xxvi, p. 408 ; Bibliotheca bot., I896, Heft 39 , p. I3. In a reserve-cellulose composed of different hemicelluloses, the latter may dissolve in succession. Brown u. Morris, Bot. Zeitung, I892, p. $4^{6} 4$; Reinitzer, Zeitschr. f. physiol. Chemie, I 897 , Bd. XxIIr, p. 20 .

${ }^{2}$ [Newcombe, Bot. Centralbl., Bd. Lxxıı, I 898 , No. 4 ; Annals of Botany, is89, Vol. xiII, P. 49.]

${ }^{3}$ E. Fischer, Ber. d. Chem. Ges., I 895, p. I 433 . On the distribution of invertins, cf. Flügge, Mikroorganismen, I 896, 3. Aufl., p. 202.

4 Beyerinck, Centralbl. f. Bact., I 890, Bd. vi, p. 44 ; E. Fischer, Ber. d. Chem. Ges., I894, pp. $299 \mathrm{I}, 348 \mathrm{I}$.

5 E. Fisclier, 1. c., 1894, p. $2990 ;$ I 895 , p. I 431 .

${ }^{6}$ Green, Annals of Botany, I888, Vol. I, p. 223 ; I 893 , Vol. vil, p. 89. E. Fischer (1. c., i 894 , p. 2988 ) states that invertin is inactive upon inulin. Bourquelot has shown that certain fungi are able to split inulin (Compt. rend., x 893 , T. CVI, p. II43). Cf. Sect. 82. Fernbach, Ann. d. I'Inst. Pasteur, I89o, T. IV, p. 64 I. 
glucoside-splitting enzymes may be classed as invertins. The distinction between diastases (including cellulose-splitting enzymes) and invertins is a matter of convenience only, for glucase acts as an invertin with regard to maltose, and diastases may possibly exist which are able to invert cane-sugar ${ }^{3}$. A difference in the mode of action indicates a difference in the chemical nature of the ferment substance, such as is rendered evident by the fact that the invertin of yeast decomposes amygdalin without any formation of prussic acid.

Invertins are not produced in all plants, and the invertin of cane-sugar seems to be absent in most of the higher plants even including such as contain cane-sugar ${ }^{2}$, as well as in many mould-fungi and most bacteria. This may possibly be because cane-sugar can be directly assimilated by the protoplast, whereas alcoholic fermentation is impossible without the previous inversion of the cane-sugar supplied (Sect. I03). In the absence of invertin, yeast is unable to liydrolyse cane-sugar, showing that even when the latter is used as food the living protoplast does not strive to invert it. Nor is any inversion induced by the organic acids present in the living cell, for even the strongly acid sap of the lemon contains a considerable quantity of cane-sugar ${ }^{3}$. Similarly, inulin remains unchanged in the acid cell-sap of resting tubers.

Further details concerning invertins and a few glucoside-ferments are given by Flügge and Green. Flügge (l. c., p. 21 I) also mentions the urea-ferment (urase) produced by a few bacteria (cf. Sect. I02). The decomposition of tannin by fungi has already been mentioned, as has also the hypothetical pectase enzyme.

Fat-splitting ferments (Steapsins). According to Sigmund these are widely distributed in the higher plants, and the extracellular decomposition of fats observed by Schmidt in the case of certain fungi is probably due to the excretion of such ferments ${ }^{4}$. It is, however, uncertain to what extent fat-splitting ferments aid in the metabolism of fats (Sect. 82). According to Sigmund steapsins decompose certain glucosides, while emulsin and myrosin possess a certain power of decomposing fats.

Proteolytic ferments. Trypsin is produced by many bacteria, and pepsin by many fungi and by carnivorous Phanerogams ${ }^{5}$. In such cases the importance of the ferments for the absorption of food, and also for the penetration of chitinous membranes, \&c., is at once evident, but it is uncertain what function is performed by the peptic enzyme (papain) found in some abundance in the latex of Carica

1 All diastases do not act as invert-ferments, and various fungi which are capable of active diastatic fermentation are unable to split cane-sugar. See Sect. 65 , and Flügge, l. c.

${ }^{2}$ See Baranetzsky, Die stärkeumbildenden Fermente, 1878 , p. 62 ; Green, I.c., p. 90.

${ }^{3}$ Buignet (Sachsse, Chemie d. Kohlenhydrate, 1877, p. 218 ) states that 28 per cent. of the sugar of lemons is cane-sugar.

${ }^{4}$ Sigmund, Sitzungsb. d. Wien. Akad., I 89 I, Abth. i, Bd. L, p. 328 ; I $8_{92}$, Abth. i, Bd. LI, p. 549. See also Neumeister, Physiol. Chemie, $18_{93}$, Bd. I, p. $8_{4}$. Kranch (Versuchsst., $18_{79}$, Bd. XxıI, p. I03) was unable to detect any fat-splitting ferments in plants. R. H. Schmidt, Flora, $1891, \mathrm{pp} .304,312$.

${ }^{5}$ Cf. Flügge, Mikroorganismen, 1896 , 3. Aufl., p. 207 ; Lafar, Technische Mykologie, 1897 , Bd. I, p. 268 . 
papaya, Ficus carica, Cucumis utilissimus, Ananassa sativa, \&c. ' According to Neumeister ${ }^{2}$ many of the higher plants never produce any proteolytic enzymes, and hence the latter do not seem to be essential in proteid metabolism, although a certain amount of peptone appears to be formed in those seeds (barley, poppy, beetroot, maize, \&c.) in which pepsin appears during germination. No pepsin can, however, be detected in lupins, peas, rye, oats, $\&$ c., and the slight amount of peptone which appears in these plants, and which is in some cases deposited in the seeds, must arise as a product of vital metabolism, just as amides do.

The enzyme of most bacteria is, like trypsin, active only in an alkaline or neutral solution, and this is in correlation with the fact that putrefactive bacteria commonly exist in neutral or alkaline media ${ }^{3}$. On the other hand, the enzyme of Nepenthes, Drosera, \&c. acts only in the presence of acid. The papain of latex is active chiefly in alkaline and neutral solutions, but perhaps also in faintly acid solutions ${ }^{4}$, while the proteolytic ferment of Penicillium seems to be still less influenced by the presence of acid ${ }^{5}$. Plants evidently produce several different kinds of proteolytic enzymes, but direct experiments are required to show whether vegetable-pepsins produce peptone with albumoses as intermediate products, whether vegetable-trypsins carry the digestion as far as the formation of leucin, tyrosin, \&c., and whether only the latter ferments are able to digest nucleins ${ }^{6}$.

$A$ rennet ferment that coagulates milk is produced by certain bacteria and many of the higher plants, but its importance is as yet unknown. Literature: Flügge, l. c., p. 209 ; Lafar, 1. c., p. 209; Green, Annals of Botany, I893, Vol. VII, p. I I 2 ; Bruhne, Beitr. z. Physiol. u. Morphol. v. Zopf, I894, IV, p. 27.

[Oxidase enzymes. The best known of these is the laccase first described by Yoshida ${ }^{7}$, which induces the oxidation of the sap of Rhus into black lacquer varnish when in contact with air. It also exerts a similar oxidizing action upon hydrofuinone, pyrogallol, and many polyphenols. The ferment acts best at about $20^{\circ} \mathrm{C}$. but is not destroyed by heating to $70^{\circ}$ C. ${ }^{8}$ Manganese appears to be

${ }^{1}$ Cf. Hansen, Arb. d. Bot. Inst. in Würzburg, I887, Bd. III, p. 266; Green, Annals of Botany, 1893, Vol. vir, p. I07.

2 Neumeister, Zeitschr. f. Biologie, 1894 , Bd. xxx, p. 447. The older literature is mentioned here. On the occurrence of peptones cf. also E. Schultze, Journ. f. Landw., 188I, Bd. xxix, p. 285 ; Versuchsst., I 882 , Bd. XxVII, p. $35^{8}$; Journ. f. prakt. Chemie, I885, Bd. XxxiI, p. 449 ; Frankfurt, Versuchsst., 1896, Bd. xuvir, p. 466-Reinke, Unters. a. d. Bot. Lab. in Göttingen, I88 I, Heft 2, p. 52 (Aethalium).

${ }^{3}$ Fermi, Centralbl. f. Bact., I 891 , Bd. x, p. 404. Cf. also Fligge ; Lafar, l. c. According to Hjort (Centralbl. f. Physiol., I896, Bd. x, p. 192), Agaricus ostreatus and Polyporus sulphureus contain a similar ferment.

${ }^{4}$ Cf. Neumeister, Physiol. Chemie, I893, I, p. I92; Sharp, Chem. Centralbl., I $8_{94}$, I, p. 5 I2. The enzyme of Acthalium seems to have similar properties. Cf. Čelakovský, Flora, Erg.-bd., I 892 , p. 225.

${ }^{5}$ Hansen, Flora, I889, p. 88 ; Bruhne (Zopf's Beiträge, I 894, I, p. 26) for Hormodendron.

${ }^{6}$ Cf. Neumeister, Physiol. Chemie, I, pp. I07, I 29, I87, 200. On the differences between animal pepsins, see Wroblewski, Zeitschr. f. physiol. Chemie, I895, Bd. Xxi, p. IS; Popoff, ibid., 1894, Bd. XVIII, p. 539 .

7 Yoshida, Chemistry of I acquer, Journal of the Chemical Society, ISS 3 , Vol. XLIII, p. $47^{2}$.

${ }^{8}$ Bertrand, Compt. rend., IS94, T. cxvili, p. I2 I5; 1895 , T. cxx, p. 266 ; Bull. Soc. Chim., I 894 , T. II, p. 7 I 7 ; Yoshida, l. c. 
always present, and in its absence the ferment exerts only a very feeble oxidizing power, such as is also possessed by oxide of manganese, whereas their conjoint action induces twenty to thirty times more active oxidation. Laccase has also been detected in various fungi, such as Russula, Lactarius, and Boletus. It causes the oxidation of a chromogen present in the expressed sap of these plants into blue, red, or black pigments. Bertrand ${ }^{1}$ has also isolated another oxidase 'tyrosinase' from Russula and other fungi, from the dahlia, and from beetroots. It rapidly oxidizes tyrosin into a black pigment, but exercises no action upon pyrogallol or hydroquinone. Tyrosinase is destroyed at from $50^{\circ} \mathrm{C}$. to $60^{\circ} \mathrm{C}$, and hence laccase may be separated from tyrosinase by heating to $70^{\circ} \mathrm{C}$. The action of tyrosinase is not hindered by the presence of 50 per cent. of ethyl- or methyl-alcohol. It is doubtful whether the 'oenoxydase' which frequently causes the soluble pigment of red wines to be precipitated is a special ferment or is identical with laccase ${ }^{2}$. In many plants chromogens occur whose post-mortem oxidation may or may not be due to oxidase enzymes. The hypothetical 'leptomin' which Raciborski (Ber. d. D. Bot. Ges., Aug. I 898 ) finds to be commonly present in the living elements of the conducting channels of many plants is also apparently an oxidase ferment. Its presence may be detected by the blue colouration given on testing with guiacum solution and hydrogen peroxide, but according to Grüss this reaction is due to the presence of diastase. A similar reaction may also be given by young tissues but not after immersion in alcohol, whereas the oxidase ferments included under the head of 'leptomin' retain their characteristic properties under such treatment, although the latter may be weakened. Haemoglobin and haemocyanin are bodies which have a similar power of inducing or accelerating oxidation.]

\section{SECTION 92. External Infiuences.}

Metabolism like vital activity is dependent upon the external conditions, and both its activity and character may be modificd according to existing circumstances. Detailed experiments are, however, as a general rule necessary to detect such changes, for it is seldom that the products of metabolism are so immediately evident as they are in the case of pigment substances. The amount of consumption or excretion enables the course of respiration or fermentation to be approximately determined, and similarly changes in the rate of growth, in the production of heat, in the power of movement, all afford more or less marked indications of an alteration in metabolic activity.

The different functions are, however, not always influenced to the same extent and in the same manner: thus respiration continually increases as the temperature rises (Sect. 104), whereas growth and the constructive metabolism associated with it diminish or cease beyond a certain optimal temperature. Similarly the production of pigments, poisons, enzymes, \&c.

1 Bertrand, Compt. rend., i $89^{6}$, T. cxxiII, p. $4^{6} 3$.

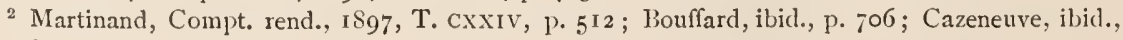
pp. 406, $7 \mathrm{Sr}$. For further literature, see Green, Science Progress, I I 9 S. Vol. VIr. P. 253. 
may be suppressed by heat, provided the maximal temperature for the formation of these substances lies below the critical point for the entire plant. Inhibition of this character can, however, be excrcised only upon those processes which are not directly essential for the maintenance of vital activity.

Since the main features of this discussion have already been given, it is only necessary to mention here a few general considerations such as have not previously been dealt with. Nutritive substances not only provide a supply of energy and material for constructive purposes, but also act as stimuli of varied and frequently extremely intricate character. Many non-nutritive substances may excite or depress either the general vital activity or special functions only. Temperature may also act in different ways, as may light also, which often constitutes a stimulus, and may either awaken a dormant activity, or provide the energy necessary for its performance.

A change in the external conditions causes the general vital activity to assume a new condition of equilibrium which is maintained so long as the internal and external conditions suffer no further change. It can, however, only be determined by direct experiment in each special case whether for any metabolic product the balance is maintained by the equality between production and consumption, or by the cessation of the former. Every compound which is continually drawn into metabolism must be as continually replaced, and hence further production must ultimately cease whenever any product remains intact in the plant. This is the case, for example, with pigments, poisons, enzymes, \&c., the formation of which may under special circumstances entirely cease, while on the other hand a plastic substance such as glycerine may under certain conditions remain completely intact (Sect. 67). The self-regulation of metabolism probably frequently involves interactions of a similar character, and it is not impossible that the starch-grains, cell-walls, \&c. of living cells, which seem to be permanent structures, are really subject to continual dissolution and regeneration. Similarly the maintenance of the acidity of the cell-sap may be due either to the balance between production and decomposition, or to the selfregulatory cessation of production as soon as a certain amount has accumulated (Sect. 86). The latter is probably the case as regards the salts of organic acids, the regulatory formation excited by a diminution maintaining a constant turgidity during growth. As regards the latter, moreover, various facts show that the potential powers of growth are by no means always fully exercised, and may indeed under certain conditions remain permanently dormant (Sect. 4).

The new condition of equilibrium produced by a marked change of temperature is apparently assumed rapidly and without causing any great disturbance. When, however, the partial pressure of oxygen is rapidly 
lowered, it requircs a ccrtain intcrval before the plant accommodates itself to the new conditions by diminishing its respiratory activity (Sect. 100), and the same would probably bc the case if the supply of nutriment was suddenly reduccd to an amount insufficient to satisfy the previous metabolic requirements. As the plant accommodated itsclf to these changed conditions it would probably not only grow more slowly but also perform all its different vital activitics in a more economical manner than when fed to excess (cf. Sect. 73). A return to the original conditions is as a general rule followed by the gradual assumption of the previous equilibrium, provided no permanent displacement of the latter has been produced.

Temperature. Since a certain temperature is one of the essential conditions for life, it follows that the action of temperature is mainly a stimulatory one, and that a change of temperature affects metabolism in a manner corresponding to the reactive power of the organism itself, and modified only to a slight extent by any direct action upon the chemical processes involved in metabolism. The physiological curve and the curve of chemical reaction hence do not coincide, for the latter usually commences at a lower temperature than the former, which again soon falls above a certain optimum, whereas the chemical curve continues to rise until a very high temperature is reached at which molecular dissociation occurs ${ }^{1}$.

A rise of temperature may affect antagonistic processes differently, as for example, when potatoes kept at $0^{\circ} \mathrm{C}$. to $6^{\circ} \mathrm{C}$. become sweet owing to the production of sugar, which above $10^{\circ} \mathrm{C}$. is again converted into starch ${ }^{2}$. The change is still more rapid in the so-called starch-trees (Tilia, Betula, \&c.), for when brought into a warm room in winter, starch appears in the cortex in a few hours, and is rapidly reconverted into sugar when returned to a low temperature ${ }^{3}$, a phenomenon which may be frequently repeated. It seems very commonly to be the case that when exposed to cold, plants contain a larger proportion of sugar, and perhaps also of other soluble substances, than when at a higher temperature 4 .

The action of different substances. Metabolism is influenced and regulated not only by the quality and quantity of the nutrient material but also by the accumulation of the metabolic products (Sect. 93). Non-nutritive substances may exercise quite as pronounced an influence, and it has already been mentioned that submaximal doses of many and perhaps all poisonous substances accelerate respiration, growth, \&c. Indeed chemical stimuli may play a much more important part in metabolism than appears possible at present.

Reciprocal and antagonistic actions. The entire organic world and all the friendly and antagonistic relationships of different organisms are primarily regulated by the necessity of obtaining food (cf. Sects. 5 1, 64, 65, 76). Fungi, bacteria, and

1 Details in text-books of physical chemistry. On changes in chemical reactions at very low temperatures, cf. R. Pictet, Compt. rend., I $89_{2}$, T. $\mathrm{cxv}, \mathrm{p} . \mathrm{SI}_{\mathbf{4}}$.

${ }^{2}$ H. Miiller-Thurgau, Landw. Jahrb., I883, Bd. XI, p. 75I ; 1885 , Bd. xiv, p. $85 \mathrm{I}$.

${ }^{3}$ A. Fischer, Jahrb. fo wiss. Bot., I89I, Bd. xxı1, p. I 2 (cf. Sect. Iog).

* For examples, see II. Miiller-Thurgau, 1. c., I $88_{3}$, p. 787 ; Copeland, Einfluss von Licht u. Temp. a. d. Turgor, I896, p. 5 ; Rosenberg, Bot. Centralbl., I 896 , Bd. Lxvi, p. 337 ; Lidforss, ibid., I 896, Bd. Lxvin, p. 33 . 
many other of the lower organisms are able to conquer and kill their adversaries by means of certain poisonous excretory products, while by means of celluloseenzymes parasitic fungi are able to penetrate a host-plant. In reciprocal symbiosis, however, the exchanges are of mutual advantage.

Even the normal excretory bye-products may injuriously affect other organisms : thus 2 to 5 per cent. of alcohol acts injuriously or fatally upon many organisms, although certain alcohol-producing species of Saccharomyces can withstand 14 per cent. (Sect. I03). Further, most bacteria and many other organisms are injured by even a slightly acid medium, and hence are suppressed and killed by fungi which produce large quantities of free acid without being themselves affected ${ }^{i}$. Thus if yeast and bacteria are sown in an acid solution of sugar the yeast attains the upper hand, whereas in an alkaline or neutral solution the bacteria rapidly develop and multiply, and the yeast is suppressed ${ }^{2}$.

The final result in all such cases depends upon the specific properties and reactive powers of the different organisms, as well upon the external conditions, that is upon several variable factors. Thus the amount of organic acid produced is liable to modification, and the power of secreting poisons may be entirely suppressed, so that both the means of attack and defence are subject to alteration. A parasite may penetrate a host only under special conditions, and it is possible to induce the penetration of many fungi which are not parasitic. The acidity of the cell-sap probably suffices to prevent the multiplication of injected bacteria and gradually to kill them ${ }^{3}$; certain metabolic products, such as those found in many varieties of latex, may also exercise a directly poisonous effect.

All plants possess more or less marked powers of accommodation, and thus a plant may gradually accustom itself to more highly concentrated media or to doses of poison which were previously fatal. Hence, whether an antagonistic symbiont excretes an injurious product rapidly or slowly, is a point of some importance, for when the latter is the case, the plant attacked has a longer time to accustom itself to the poison, or to prevent an injurious accumulation by destroying or neutralizing it. Thus in certain cases excreted oxalic acid may be decomposed or combined with bases and rendered innocuous. Similarly, although a slight amount of methyl-violet suffices to kill protoplasm, considerable amounts may slowly pass through the protoplast and acccumulate in the cell-sap in the form of a non-diosmosing and hence innocuous compound, when it is presented in extreme dilution.

A plant may also make a counter-attack upon its enemy, as, for example, by means of such secretions as weaken the energy and poisonous character of the attacking organism. Substances are frequently produced by reciprocal and antagonistic interactions, which the isolated individual plants are unable to form,

${ }^{1}$ Cf. Sect. $8_{5}$. Wehmer, Beitr. z. Kenntniss einheim. Pilze, rs 83, p. 69 ; r 895 , p. I43; Reinhardt, Jahrb. f. wiss. Bot., IS92, Bd. Xxiri, p. 5 I5.

${ }_{2}$ Nägeli, Die niederen Pilze, I8 87, p. 31 ; Pasteur, Ann. d. chim. et d. phys., I8 85 , iii. sér., T. LII, p. $4^{\mathrm{I}} 5$. The antagonistic action is due to the products, and not to any molecular action connected with fermentation.

${ }^{3}$ Cf. Zinsser, Jahrb. f. wiss. Bot., I 897 , Bd. xxx, p. 425.

PFEFFER 
as is the case with regard to certain metabolic products of lichens, or the special products formed by mixed cultures of bacteria ${ }^{1}$.

The complicated relationships involved in these organic interactions have hitherto mainly been studied in connexion with the diseases of animals ${ }^{2}$, and no doubt a close study of this general problem will reveal facts of the utmost importance. Indeed the existence of toxins and anti-toxins has already been demonstrated in the case of animals, and it has been shown that a production of substances for attack and defence may be induced in a variety of ways. Similarly, induced immunity is simply the temporary or permanent after-effect of a physiological reaction (cf. Sect. 3).

\section{SECTION 93. Self-regulation.}

Self-regulation is attained by the interactions between the different organs both of the plant and of the protoplast, and each organ, however minute, has its own specific reactive power. The most obvious example of self-regulation lies in the fact that the course of normal vital activity is such as to provide for its own continuance, while the absorption and selection of a particular nutrient substance is determined by the needs of the organism and the character of its metabolism. Similarly all the phenomena of correlation show that the different organs of the plant and of the protoplast work in harmony, and mutually influence and regulate one another (cf. Sect. 4).

A thorough comprehension of all these relationships can be gained only when a perfect knowledge of the intimate nature of the vital mechanism has been attained, but on certain points sufficient is known to render discussion profitable. Thus self-regulation is possible because potential powers are excreised in varying degrees and in some cases not at all, while any increased or diminished functional activity may more or less marlicdly influence all other directly or indirectly connected processes, as for example when a change in one or more partial functions ultimately affects metabolism as a whole.

During metabolism certain excretory products are necessarily produced which must be removed, as otherwise their gradual accumulation would $u^{1}$ timately prevent further activity. Substances destined for building purposes or for further use in metabolism are however not excreted, and as a seneral rule are produced in definite amount; so that even when the consumption of such products is inhibited, no injurious accumulation

${ }^{1}$ Cf. Nencki, Centralbl. f. Bact., $189^{2}$, Bd. Xr, p. 225 ; Burri u. Stutzer, ibid., I 895 , Abth. ii, Bd. I, p. 354 ; Lafar, Techn. Mykologie, I 896 , p. So.

$\approx$ Cf. Fligge, Mikroorganismen, IS96, 3. Auff., Bd. I, p. $27^{1}$; Buchner, Centralbl. f. Bact., I893, Bd. XIv, p. 235 . 
occurs. Since the needs of an organism may vary within wide limits, it is essential that the production of a particular substance should be regulated according to the amount present, and that the requirements of an organism should act as a stimulus exciting a sufficiently increased production to satisfy them. Nany examples of such regulatory changes have already been given (cf. Sect. 77), others are afforded by the regulation of turgor in growing organs, and by the storage of reserve-materials, for these do not accumulate beyond a certain limit however abundant the food-supply may be. Similarly the mobilization of reserve-food-materials is regulated by the amount consumed, and the direction of translocation is determined by the localization of the consuming organs (Sect. 108). Thus when a tree buds out afresh after premature defoliation, nutrient materials travel to the growing regions from far distant storage-receptacles, where they would otherwise have remained intact until the following spring. In all such cases it has to be determined whether the commencement of the mobilizing metamorphoses was clue to the removal of the traces of the diosmosing products previously present, and its continuance to their rapid consumption as fast as they are formed, or whether the consuming cells exert some special vitalistic or other influence upon those in which reserve-food is stored. Certain recent researches by Hansteen and Puriewitsch ${ }^{1}$ show that the latter is not necessarily essential, for by the continual remoral of the diosmosing sugar it was found possible to empty all the starch from the isolated endosperm of grasses, and from the cotyledons of Phascolus, as well as to remove all the glucose from single bulb-scales of Alliun ccpa. The removal of starch ceases in a 2 to 3 per cent. solution of sugar, and hence the conversion of starch soon stops when the gypsum cast in which the experimental object is partially embedded stands in only a small quantity of water. Puriewitsch found that if more sugar was added, the endosperm, cotyledons, or bulb-scales might even become refilled with starch or glucose as the case might be. Similarly the chloroplastids of many plants form starch when fed with sugar or glycerine, but temporarily lose the power of assimilating carbon dioxide when the cell is overloaded with assimilatory products (Sect. 55). This may, however, be avoided for a time by the deposition of the soluble products in the form of starch, or by their removal to neighbouring cells.

A nutrient substance may be maintained intact not only by the cessation of the functional activity by which it is consumed, but also by the formation of other substances which can replace it and can enter more readily into metabolism. This question has already been discussed (Sect. 67), and it has been shown that the phenomena of selection and

${ }^{1}$ Hansteen, Flora, I\$94, Erg.-bd., p. 419; Puriewitsch, Ber. d. Pot. Ges., I $\$_{96} 6$, p. 207; Jahrb. f. wiss. Bot., 1897, Bd. xxxi, p. I. Cf. Sect. I09. 
protection play a most important part in determining the character and course of metabolism: thus starch remains intact in the presence of an abundance of sugar, and probably every form of reserve-food-material can be protected by the presence of a suitable nutrient substance. It is, on the other hand, of the utmost importance that in case of need the plant should be able to utilize as much as possible of the nutrient material at its disposal (Sect. 78 ), so that a substance may be reassimilated under such circumstances which normally remains permanently aplastic. Similarly the fact that a substance can re-enter into metabolism does not necessarily show that its temporary permanence is due to a balance existing between the processes of disintegration and reconstruction, although it is always possible that the apparent constancy of a product may be attained by these means.

The relations between mass and chemical action are of the utmost importance in the regulation of metabolism, for by the continual removal of the products by diosmosis, metamorphosis, or combination, a feeble chemical action may be carried to completion, and hence by such means the plant is able to stop or continue a given metabolic process according to its needs ${ }^{1}$. The gradual decomposition of the passively secreted tannate of methyl-blue affords an immediately evident example of a reaction of this kind, and by similar means a salt of the strongest acid may be completely decomposed by the weakest free acid. Trifling and hardly perceptible reactions or dissociations may in this manner produce results of altogether disproportionate magnitude. The removal of the products is however due to the vital activity, and the latter frequently also creates the partial reaction or molecular dissociation which forms the necessary preliminary to any such action as mentioned above?

Indeed physiological regulation is primarily due to the direct reaction of the organism, as for example when a deficiency of a substance excites its production, and it is only by such means that any correlation of growth is possible or that the formation of diastase can be regulated in Penicillium. Similarly the conversion of starch into sugar may be inhibited by an accumulation of the latter which is insufficient to stop diastatic action, so that we are here dealing not with a direct chemical action but with a physiological reaction (Sect. 91). Moreover in the selection of foodsubstances during metabolism chemical relationship is by no means of primary importance (Sect. 67).

1 Pfeffer, Osmot. Unters., 1877 , p. $16_{3}$; Oxydationsvorgänge in lebenden Zellen, 1889 , p. 463. Cf. also Sect. 22. The relations between mass and chemical action were first clearly recognized by Berthollet. Cf. Ostwald, Grundriss d. allgem. Chemie, 1890, 2. Aufl., p. 289 .

${ }^{2}$ On the special properties of the cell, ef. Sect. 7 seq. ; diosmosis, Chap. iv; the importance of surface tension-energy, Sect. I2. The osmotic pressure exercises but little effect. Cf. Ostwald, Allgem. Chemie, IS9I, 2. Auf., Bd. I, pp. Iо I 4, I045. On reactions in confined spaces, cf. Liebreich, Zeitschr. f. physik. Chemie, I89o, Bd. v, P. 536 ; Pudde, ibid., I 89 r, Bd. vir, p. 600. 
All regulatory phenomena arise from stimulatory reactions, the nature of which is still obscure: thus when a plasmolyzed protoplast clothes itself anew with a cell-wall, it is uncertain whether the removal of the pressure against the original wall, or the change in the surface-tension, or some other cause, acts as the stimulus exciting the new activity. It is probable that changes in turgidity act directly as regulatory stimuli maintaining constant turgor ${ }^{1}$. Similarly the inhibition of the removal of the endosperm foodmaterial is not entirely due to the action of a chemical stimulus, for the same inhibition may, according to Puriewitsch ${ }^{2}$, be produced by correspondingly concentrated solutions of other substances than sugar. The chemical character of the substances in the nutrient medium is, however, of great importance in the suppression of diastase formation in Penicillium, for certain forms of sugar are especially active (Sect. 91). Chemical stimuli undoubtedly play a most important part in metabolism, and these may arise from normal products or in certain cases from specific stimulatory substances which plants may employ to attain a particular end. There is, however, no reason for Reinitzer's ${ }^{3}$ supposition that specific fatiguesubstances are produced by plants in general, for the mere accumulation of a normal product may result in the depression of a single function or of the general metabolic activity. Similarly different organisms may influence one another by means of the metabolic products they excrete, either permanently or only as the result of the action of specific stimuli.

${ }^{1}$ Pfeffer, Druck u. Arbeitsleistung, I 893, p. ${ }_{42} 8$.

${ }^{2}$ Puriewitsch, Ber. d. Bot. Ges., 1896, p. 209.

${ }^{3}$ Reinitzer, Ber, d. Bot. Ges., 1893, p. 53I ; Jäger, ibid., 1895, p. 70. 


\title{
CHAPTER IX
}

\author{
RESPIRATION AND FERMENTATION
}

\section{SECTION 94. Introductory.}

Metabolism involves processes both of construction (anabolism) and destruction (katabolism), the latter yielding the continual supply of energy necessary for vital activity. Kinetic energy may be liberated not merely by physiological combustion involving a consumption of free oxygen, but also by chemical decompositions taking place without the aid of the latter ${ }^{1}$. Energy is liberated entirely in the latter manner in certain bacteria and fungi, which are thus able to exist anacrobically. The existence of such organisms is hardly in agreement with the old dogma 'no life without respiration' unless we assume, as is here done, that respiration includes all metabolic processes which involve a liberation of energy. According to the manner in which the latter occurs, it is possible to distinguish between aerobic or oxygen-respiration and chemical, anaerobic, or intramolecular respiration.

With the exception of water, carbon dioxide is the sole continually excreted end-product of aerobic respiration in the higher plants. This limitation is purely an adaptive modification, for many aerobic fungi and bacteria may excrete such products as acetic, oxalic, or citric acid, ammonia, \&c. The higher plants have a very marked power of reconstructing proteids, and hence their metabolism is unaccompanied by any such excretion of urea as occurs in the higher animals. During anaerobic respiration, however, a variety of end-products may be formed, and the removal of these is essential for continued vital activity (Sects. 77, 92). Many acrobic and anaerobic plants are able to obtain a supply of energy by decomposing the greater part of their food into such products as alcohol, butyric or acetic acid, \&c. This process, known as fermentation, is simply a form of metabolism characterized by the special nature and disproportionate amount of the products, and it exists mainly for the purpose of obtaining a supply of energy. The fermentative activity of many aerobic organisms

1 Cf. Pleffer, Studien z. Energetik, IS92. 
is so feeble as to be hardly distinguishable from normal metabolism, and anaerobic organisms need not be necessarily capable of exciting any recognizable fermentation whatever.

According to their specific nature, obligate aerobes are only capable of existing in the presence of oxygen, obligate anaerobes in its absence, while facultative anaerobes can exist both in the presence and absence of oxygen. No sharp line of demarcation exists, however, and a facultative anaerobe which when fed in a certain manner is only capable of aerobic existence, may under other circumstances grow anaerobically. Indeed a few bacteria hitherto regarded as ob'igate anaerobes have been recently successfully cultivated as aerobes under special conditions. Certain bacteria and yeasts are temporary anacrobes, for after growing in the presence of oxygen they may increase and multiply for a time anaerobically. Even in the absence of oxygen aerobic plants continue to respire for a short time, and this intramolecular respiration is accompanied in Phanerogams by a production of carbon dioxide, alcohol, and other substances. Intramolecular respiration does not suffice in an aerobic plant to maintain life, but may enable it to survive the temporary absence of oxygen. An anaerobe exists by means of an enlarged kind of intramolecular respiration, which may or may not acquire the special features characteristic of fermentation. As will be shown later, a close genetic relationship exists between aerobic respiration, intramolecular respiration, and fermentation, while free oxygen may when present enter for a time into the metabolism of obligate anaerobes, until the disturbance of the normal vital activity induced by its presence becomes so pronounced as to cause death. Even aerobic organisms die in a similar manner when the partial pressure of the surrounding oxygen is raised to a sufficient extent.

The actual course of respiration within the protoplast is quite obscure, and the visible end-products afford an index only to the activity of the process and to any changes in its character, without giving any indication as to the way in which these are produced. Respiration is of importance only as a factor in metabolism as a whole, and it is not always possible to distinguish clearly between the products of respiration and those which are destined for constructive purposes, although usually the two are clearly defined from one another.

\section{SECTION 95. Aerobic respiration.}

Except in the case of anaerobic plants aerobic respiration is as necessary for plants as for animals, oxygen being absorbed and carbon dioxide exhaled by all aerobic plants in darkness. Even in resting organs such as bulbs, tubers, \&c., respiration still continues, and it is absent from dry but living mosses, lichens, or seeds only so long as they remain in a desiccated 
condition. In all other cases the cessation of respiration is an infallible sign of death. (On post-mortem production of carbon dioxide, cf. Sect. 10I.) The cessation of oxygen-respiration ultimately causes death in acrobes, and as a gencral rule is accompanied by a rapid or immediate cessation of growth, plasma-streaming, and many other irritable movements. The existence of any one of these activities forms one of the visible tokens of respiratory activity, and the latter is no doubt an essential characteristic of every living plasmatic organ, or particle of plasma, for in any isolated mass of protoplasm streaming ceases on the removal of oxygen, and recommences again in its presence ${ }^{1}$.

Growth and streaming movements may continue in green cells exposed to light in the absence of all external supply of oxygen. This is simply because the processes of respiration and assimilation counterbalance one another, the power of assimilating carbon dioxide possessed by the chloroplastids being more than sufficient to decompose the amount of this gas produced by the whole of the rest of the protoplasm. The gaseous exchanges connected with respiration become fully manifest in green plants at night or when the chloroplastids have been rendered inactive ${ }^{2}$. Respiration is but little influcnced by light, whereas the assimilatory activity of a highly chlorophyllous organ may be twenty to forty times greater in bright light than its respiratory activity, so that during the day the surrounding air becomes poorer in carbon dioxide and correspondingly richer in oxygen. Hence during the daytime a green leaf usually reassimilates the whole of the carbon dioxide produced by respiration, although sometimes minute traces may escape ${ }^{3}$. Even when the subaërial parts are assimilating most actively, carbon dioxide may be excreted in abundance from the roots, flowers, or fruits ${ }^{4}$, but in a confined space the whole of this will be reassimilated during the daytime, and as long as the plant remains living this circulation continues without the dry weight undergoing any increase or decrease. De Saussure ${ }^{5}$ first correctly interpreted this phenomenon and showed that death cnsucs sooner when the carbon dioxide evolved is absorbed by means of potash.

${ }^{1}$ Pfeffer, Zur Kenntniss'd. Plasmahaut u. Vacuolen, I8go, p. 279. Cf. Sect. 9. [Provided the protoplasm is strictly aerobic, for in certain cases intramolecular respiration may provide sufficient energy to permit of the continuance of streaming for days or even weeks in the absence of free oxygen (Nitella, \&c.).]

${ }^{2}$ Cf. Sect. $5^{8}$, and Bonnier et Mangin, Ann. d. sci. nat., I 886 , vii. sér., T. III, p. $4^{2}$.

${ }^{3}$ Garreau, Ann. d. sci. nat., I850, iii. sér., T. xv, p. 5 ; I $85 \mathrm{I}$, T. Xvı, p. $27 \mathrm{I}$; Blackman, Annals of Botany, I 895 , Vol. Ix, p. 164. [By means of the decolorization of a faintly alkaline drop of phenolphthalein, it can be readily shown that traces of $\mathrm{CO}_{3}$ escape from a green cell or algal filament exposed to light in an atmosphcre of pure hydrogen.]

${ }^{4}$ Knop, Ann. d. Chcm. u. Pharm., 1864 , Bd. Cxxix, p. 287 ; Corenwinder, Ann. d. sci. nat., r 86 \&, v. sér., T. Ix, p. 63 ; Dchérain et Vesque, Compt. rend., I877, T. Lxxxrv, p.959. Cf. Sect. 57 .

s De Saussure, Rech. chim., $1804, \mathrm{pp} .60,194$. On the importance of auto-assimilation in preserving the functional activity of chloroplastids, ef. Sect. 55 . 
In parts containing but little chlorophyll respiration may always be more active than photosynthetic assimilation, but even the slight amount of chlorophyll present in Neottia nidus-avis suffices to cause a feeble evolution of oxygen in bright light ${ }^{1}$. Similarly many fruits evolve less carbon dioxide during the daytime, until as they ripen the chlorophyll is gradually decomposed and the power of photosynthesis lost ${ }^{2}$. Similarly respiration is at first more active than photosynthetic assimilation in buds, seedlings, \&c., although the latter soon surpasses the former and increases until the plant becomes adult ${ }^{\circ}$. As the temperature rises the respiratory activity continually increases, whereas beyond a certain optimum the activity with which carbon dioxide can be assimilated decreases. Hence at a certain point the curves cross, and even in strong light more oxygen is consumed by respiration than is produced by the assimilation of carbon dioxide.

Respiration is markedly influenced by changes of temperature, but under similar conditions it is most active in the more vigorous parts and organs: thus resting tubers, bulbs, buds, \&c. cxhibit a feebler respiratory activity than growing shoots, in which again respiration is more active than in adult leaves and branches. No definite and constant relationship exists between growth and respiratory activity, for a varicty of other factors influence the former. Indeed many adult organs respire energetically, while in the spadix of aroids respiration and the production of heat attain a simultaneous maximum which does not coincide with the period of most active growth. Moreover beyond a certain optimal temperature growth is retarded, whereas respiration increases until death ensues.

Active embryonic cells are usually rich in protoplasm, and hence it is often the case, as Garreau observed, that a certain correspondence exists between richness in proteid and respiratory activity. The energy of respiration is, however, not directly dependent, as Palladin supposes, upon the quantity of proteids present ${ }^{4}$, for in spite of the abundance of the latter

1 Drude, Biol. v. Monotropa u. Neottia, IS73, p. IS. On other Phanerogams containing but little or no chlorophyll, cf. Lory, Ann. d. sci. nat., I847, iii. sér., T. virr, p. I60; Bonnier et Mangin, ibid., I884, vi. sér., T. XvirI, p. 332 ; also Sect. 64 .

${ }_{2}^{2}$ Ingenhousz, Versuche mit Pflanzen, I 786, Bd. I, p. 72 ; Bd. II, p. 223 ; de Saussure, Rech. chim., I 804 , pp. 57, I 29 ; Ann. d. chim. et d. phys., I821, T. XIx, p. I 58 ; Bérard, ibid., IS2I, T. Lxxvi, pp. I 52, 225 ; Frémy, Compt. rend., I 864 , T. LviII, p. 656 ; Cahours, ibid., pp. 495,653 . Cf. Sect. Iog. A weak evolution of oxygen may actually be detected, by means of the bacterium method, from the chlorophyllous cells of certain young green fruits when exposed to light (Ewart, Journ. of Linn. Soc., I 896 , Vol. xxxi, p. 437).

3 Ingenhousz, 1.c., Bd. I, pp. II 2, 355 ; Garreau, Ann. d. sci. nat., I $8_{5}$ I, iii. sér., T. XVI, p. $27^{2}$; Corenwinder, Ann. d. chin. et d. phys., I $8_{5} 8$, iii. sér., T. LIv, p. 326 , and 1878 , v. sér., T. XIv, p. IIS ; Ann. d. sci. nat., IS64, v. sér., T. I, ppv 297, \&c. For the stage of development at which a power of evolving oxygen is attained by the leaves of the commoner trees, \&c., see Ewart, Journ. of Linn. Soc., I 896 , Vol. xxxi, p. $45^{2}$.

${ }^{4}$ Garreau, Ann. d. sci. nat., I $85^{1}$, iii. sér., T. xv, p. $3^{6}$; T. xvi, p. 292 ; Palladin, Rev. gén. d. Bot., 1893 , T. v, p. 472 . 
the respiratory activity is lessened in buds and tubers during the resting period, and similarly respiration sinks to a minimum in the cells of resting cambium or of dormant primary meristem however richly they may be charged with food-materia!s.

A supply of oxygen is essential for the existence of all acrobes, and at the same time the excreted carbon dioxide must be continually removed. Special air-passages and respiratory movernents are not always necessary for this purpose, and as a matter of fact do not commonly occur either in the lower plants or in the lower animals. Air may be transferred in the higher plants by means of aeriferous channels to decp-seated tissucs (Sects. 29-32), and certain bacteria have even the power of producing pigments which, like haemoglobin, enter into loose combination with oxygen, and thus act as vehicles for the transference of oxygen to the bacterial protoplasm ${ }^{1}$. Physiological combustion ahways produces a certain amount of heat, but plants are devoid of special adaptations for maintaining a constant body temperature, and hence the latter, as in cold-blooded animals, rises and falls with that of the external medium.

Under favourable conditions respiration may be even more active in plants than in warm-blooded animals: thus in man the carbon dioxide produced in twenty-four hours forms 1.2 per cent. of the body weight, but in mould-fungi it amounts to more than 6 per cent., while bulk for bulk, very active bacteria may consume oxygen 200 times more rapidly than man ${ }^{2}$. Such bacteria and fungi in virtue of their intense disintegratory powers play a most important part in the economy of nature (Sect. $5 \mathrm{I}$ ). The relative respiratory energy is as great in many seedlings as it is in man, provided that they are maintained at the temperature of the human body. As the seedlings grow older, however, more and more cells die or become less active, and hence the respiratory activity of the entire plant or even of single branches is considerably diminished, although the growing embryonic tissuc respires as strongly as in the seedling. Similarly old bacterial cultures respire less actively than young ones in which all the cells are rapidly growing and multiplying. Many bacteria, fungi, and lichens exist, however, in which growth is slower and respiration less active, while in certain phanerogams the respiratory activity even under favourable conditions is not more than a fraction of the values given above.

The greater part, and in adult organs almost the whole, of the nutriment is sacrificed in order to obtain a supply of energy, and it is owing to this necessity that seeds germinated in darkness ultimately lose half

\footnotetext{
1 Ewart, Journ. of Linn. Soc., I $8_{97}$, Vol. xxxin, p. I 23 (Evolution of Oxygen from coloured Bacteria).

2 Vignal, Rev. gén. d. Bot., I89o, T. II, p. 5 Io; Pfeffer, Oxydationsvorgänge in lebenden Zellen, I $889, \mathrm{p} .475$.
} 
their dry weight. Owing to its active respiration the spadix of Anum italicum loses in a few hours during the period of maximum heat-production one-fourth of its dry weight ${ }^{1}$, while the dry weight of a crop of fungus even under most favourable conditions does not form more than one-third to one-tenth of the organic food consumed, and this economic coefficient is still further lessened by cultivation at a high temperature ${ }^{2}$.

Methods. The apparatus in Fig. 62 is suited to demonstrate the evolution of carbon dioxide from plants. The object is placed under the air-tight bell-jar $g$, which is covered with black cloth when a green plant is used. Air is drawn through the apparatus by means of an aspirator attached to $c$, and before entering $g$ it passes first over pumice-stone moistened with potash in the U-tube $k$, and then through baryta-water, which should remain clear, showing that all the carbon dioxide in the air has been absorbed. After a time the taps or pinch-cocks at $h$ and $h^{\prime}$ may be opened and the current resumed, when a white precipitate of barium carbonate is formed in the vessel $a$ containing baryta-water. In Fig. 63 , the cylinder, $b$,

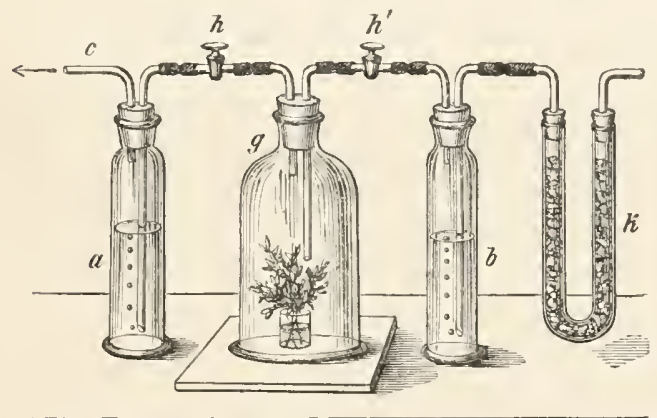

FIG. 62.

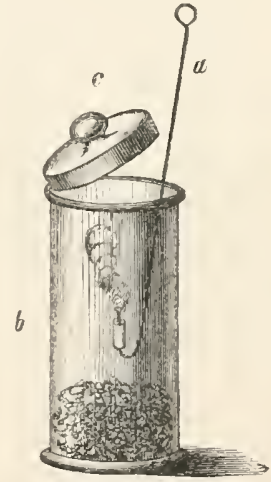

FIG. 63.

contains flowers, germinating seeds, \&c., and when after a time the cover is raised and a lighted taper or candle introduced, the flame is extinguished owing to the large amount of carbon dioxide which has accumulated.

For quantitative determinations the following method has been frequently enployed. The carbon dioxide is absorbed by titrated baryta-water in Pettenkofer's tubes through which a constant stream of air is drawn by means of a Stammer's drop-aspirator and regulated if necessary by an Elster's gas-meter ${ }^{3}$. In a confined

1 G. Krans, Über d. Blüthenwärme bei Amu italicum, r $S_{4}$, pp. 9, 67 (Abh. d. Naturf.-Ges. zu Halle, Bd. xvi). Cf. also G. Kraus, Ann. du Jard. bot. de Buitenzorg, i 896 , T. XIII, p. 27 I.

2 Pfeffer, Jahrb. f. wiss. Bot., IS95, Bd. Xxrir, p. 257 ; Kunstmann, Über d. Verhältniss zwischen Pilzernte u. verbrauchter Nahrung, Leipziger Dissert., I $\$ 95$; Fligge, Milkroorganismen, IS96, 3. Auf., Bd. I, p. I52. Cf. Sect. 66.

3 For details concerning apparatus and methods, Pfeffer, Unters. a. d. Bot. Inst. z. Tübingen, I8S5, Bd. I, p. 637 ; Johannsen, ibid., p. 688; Elfving, Einwirkung des Lichtes auf I'ilze, IS90, 
space containing a vessel filled with potash the diminution of volume may serve as an index for the consumption of oxygen and hence for the production of carbon dioxide. The apparatus in Fig. 42 (p. 3 I o) may serve for purposes of demonstration if a little potash is introduced '. In Fig. 64 a large volume of air may be enclosed, the decrease in the rolume giving the amount of oxygen absorbed, while by titrating the potash in $k$ the amount of carbon dioxide exhaled can be determined ${ }^{2}$. Or the change in the percentage composition of the enclosed air may be determined by direct analysis ${ }^{3}$, while the loss of carbon can be estimated by comparative analyses of seeds and seedlings, and provided that no organic food is absorbed, and that no carbon has been excreted except in the form of carbon dioxide, this will enable the respiratory activity to be calculated.

Specific respiratory activity. The respiratory curve for every plant and every organ attains a maximum at a certain period of development, and beyond this it diminishes, although secondary maxima and minima may be exhibited, for even

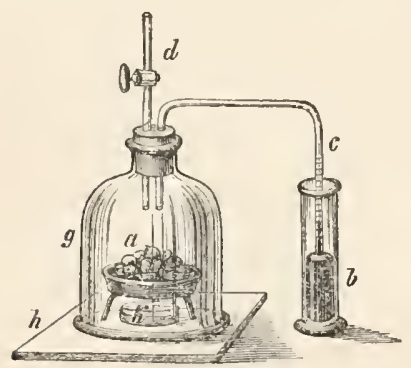

Fig. 64. $(g)$ air-tight bell-jar, fixed to the plate $(h) ;(a)$ experimental object (k) ressel with potash; (b) vessel containing mercury; (c) graduated tube; (d) glass tap. The entire apparatus should be placed under water. when the external conditions are constant the course of the curve for the entire plant is dependent upon the amount of stored food as well as upon the rate of growth, and is moreover merely the resultant of the different activities of the individual cells and organs.

It has long been known that the production of carbon dioxide commences soon after dry seeds have imbibed water ${ }^{4}$, and the path of the respiratory curve in developing seedlings has been studied by Ad. Mayer, Borodin, Rischavi, Godlewski, Bonnier, and Mangin ${ }^{5}$. Mayer found that the respiratory activity of wheat seedlings attained a maximum after fifteen to sixteen days at a mean temperature of $\mathrm{I} \cdot \mathrm{S}^{\circ} \mathrm{C}$., and after eight days at $23 \cdot 8^{\circ} \mathrm{C}$, after which the curve fell rapidly, whereas according to Rischavi in Vicia faba it remained almost parallel with the abscissa-axis for the next

pp. $68, \&$ c. In certain cases it is preferable to use soda-lime for the absorption of the exhaled carbon dioxide. Cf. Chudiakow, Landw. Jahrb., 1894 , Bd. XxiII, p. 400, and plate ii ; Kreusler, ibid., I $88_{5}$, Bd. XIV, p. 916.

1 'This method was first employed by Garreau (Ann. d. sci. nat., I $\delta_{5} \mathbf{I}$, iii. sér., T. $x v$, p. 8 ), and later by Wolkoff and A. Mayer (Landw. Jahrb., I874, Bd. III, p. 489).

${ }^{2}$ A similar apparatus was employed by Godlewski, Jahrb. f. wiss. Bot., IS\&2, Bd. XIII, p. 493, and Bot. Zeitung, I8S2, p. 804 , and by Stich, Flora, IS91, p. 8.

${ }^{3}$ See Sect. 52. On a mode of automatic registration, cf. Regnard, Compt. rend., ISS2, T. xcv, p. 77 .

* Huber, Mém. s. l'influence d. l’air dans la germination, iSor, p. I Io; de Saussure, Mém. d. l. Soc. Phys. d. Genève, I833, T. vi, p. 557 ; Fleury, Ann. d. chim. et d. phys., I $86_{5}$, iv. sér., T. Iv, p. 44; Wiesner, Versuchsst., IS 72 , Bd. Xv, P. 135 ; Ewart, Trans. Liverpool Biol. Soc., I S94, Vol.viII, p. 234 .

${ }^{5}$ Ad. Mayer, Versuchsst., I 875 , Bd. Xvili, p. 245 ; Borodin, Sur la respiration d. Plantes, 1875 ; Rischavi, Versuchsst., I876, Bd. xIx, p. 321 ; Godlewski, Jahrb.f. wiss. Bot., I882, Bd. XIII, p. 49 I ; Bonnier et Mangin, $\Lambda$ nn. d. sci. nat., I $88_{4}$, vi. sér., T. xvin, p. 369 ; I886, vii. sér., T. II, p. $3^{6} 3$. 
twenty days. Further details are given in the works mentioned. Similar curves have been obtained with cultures of mould-fungi.

Similarly the respiratory activity attains a maximum during the expansion of the buds; and not only becomes relatively feebler in the adult leaves but gradually diminishes as they grow older ${ }^{1}$. Owing to the rapid development and short duration of flowers, their respiratory curves exhibit a rapid rise and fall, the maximum usually coinciding with the opening of the flower ${ }^{2}$. A mass of flowers occupying the space of one cubic centimetre may consume in one hour during the period of most active respiration $\frac{1}{2}-\mathrm{I}$ C.cm of oxygen; the flowering spadix of Arum italicum may use as much as thirty times its own volume per hour, whereas before and after opening it consumes less than one-third its volume per hour ${ }^{3}$. It has frequently been shown that the respiratory activity decreases during the ripening of fruits, tubers, bulbs, \&c. ${ }^{4}$, and a similar diminution occurs in bulbs, tubers and trees during winter, although respiration does not entirely cease until the temperature falls below $-\mathrm{Io}$ to $-\mathrm{I} 2{ }^{\circ} \mathrm{C}$.

The tables given by Garreau and Aubert, contain further examples of specific respiratory activities ${ }^{5}$. According to Aubert, I gramme weight of living leaves and stems consumes at $\mathrm{r} 2-\mathrm{r} 3^{\circ} \mathrm{C}$. of oxygen per hour:-Cereus macrogonus 3 , Picea excelsa 44, Faba vulgaris 97, Triticum sativum 29r cc. Shade plants respire in general comparatively feebly, probably in adaption to their habitat (Sect. 62), and according to Freyberg ${ }^{6}$ the same is the case in swamp- and water-plants.

Historical. After Malpighi had shown the necessity of air for germination, Scheele proved that during this process oxygen (fire-air) was consumed and carbon dioxide (fixed-air) produced, as in the respiration of animals. Further researches were made in connexion with the assimilation of carbon dioxide by Ingenhousz and

${ }_{1}$ De Saussure, Rech. chim., I804, p. Ioo; Garreau, Ann. d. sci. nat., I851, iii. sér., T. xvi, p. 279 ; Dehérain et Moissan, ibid., I 874 , v. sér., T. xix, p. 327 ; Borodin, Bot. Jahresb., I $\delta_{7} 6$, p. 922 , and Bot. Centralb]., IS94, Bd. Lvili, p. 374; Mangin, Bull. d. l. Soc. Bot. d. France, 1886 , p. $1 S_{5} ;$ Aubert, Rev. gén. d. Bot., I $S_{92}$, T. IV, p. 352.

${ }_{2}$ De Saussure, Ann. d. chim. et d. phys., IS22, T. xxi, p. 292 ; Lory, Ann. d. sci. nat., is 47 , iii. sér., T. VIII, p. I61 ; Cahours, Compt. rend., IS64, T. LVIII, p. I206; Bonnier et Mangin, Ann. d. sci. nat., ISS 4 , vi. sér., T. XVIII, p. $35^{\circ}$.

${ }^{3}$ Garreau, Ann. d. sci. nat., I $8_{5}$ I, iii. sér., T. Xvi, p. 254 . Cf. also G. Krans, Über die Bliithenwärme von Arum italicum, ISS 4 (Abh. d. Naturf.-Ges. z. Halle, Bd. Xir).

${ }^{4}$ Fruits: de Saussure, Ann. d. chim. et d. phys., I821, T. xix, p. I63, 33S; Cahours, Compt. rend., I864, T. xxi, p. 496; Laskovsky, Versuchsst., I878, Bd. Xxi, p. 195. Tubers: Nobbe, Versuchsst., I $\delta_{5}$, Bd. viI, p. $45^{1}$; Heintz, Jahresb., IS 73 , p. $35^{8}$; Müller-Thurgau, Landw. Jahrb., I 885 , Bd. XIV, p. 857 ; Stich, Flora, IS9I, p. I5; Richards, Annals of Botany, I896, Vol. x, p. 531 .

' Garreau, Ann. d. sci. nat., I851, iii. sér., T. xr, p. 33; Aubert, Rev. gén. d. Bot., IS92, T. IV, p. 375 .

${ }^{6}$ Freyberg, Versuchsst., IS79, p. 463. Further examples of specific respiratory activity are given in the following works: Jönsson, Compt. rend., $\mathrm{x} \mathrm{S}_{94}$, T. crx, p. 440 (Mosses); Jumelle, Rev. gén. de Bot., IS92, T. IV, p. II 2 (Lichens); Diakonow, Ber. d. Bot. Ges., ISS6, p. 3 ; Bonnier et Mangin, Ann. d. sei. nat., I 884 , vi. ser., T. XviI, p. 209 (Fungi); Hesse, Zeitschr. f. Hygiene, I 893 , Bd. xv, p. IS3 (Bacteria); Mangin, Bull. d. l. Soc. Bot. d. France, I $\$ 86$ (Pollen-tubes); Palladin, Rev. gén. d. Bot., r $\$_{93}$, T. v, p. 368 (Normal and Etiolated Plants). 
de Saussure ${ }^{1}$. The former showed that all living tissues exhale carbon dioxide in darkness and that non-chlorophyllous parts do the same even when illuminated. De Saussure proved that both water and carbon dioxide are produced during respiration, and was probably aware that respiration continued in green parts exposed to light, although he did not definitely establish the simultaneity of respiration or 'inspiration ' and photosynthetic assimilation or 'expiration.' It had probably long been known that the exhalation of carbon dioxide was due to a vital process analogous to that occurring in animals, when Liebig disproved the view that it was absorbed as such from the ground and was not a metabolic product ${ }^{2}$. Meyen was probably the first to clearly show that the respiration and assimilation of carbon dioxide are two distinct and independent processes, and he also correctly enunciated their respective values to the plant. Similarly Dutrochet, and especially Garreau and Mohl, held correct views with regard to the relationship between respiration and photosynthetic assimilation. Both processes were, however, unfortunately termed respiration, which was supposed to differ in the daytime from what it was at night, until Sachs in 1865 pointed out that only the processes concerned in the production of carbon dioxide can be rightly termed respiration or breathing ${ }^{3}$.

\section{SECTION 96. The Products of Aerobic Respiration.}

Even in adult organs the excretion of respiratory and other metabolic products is difficult or impossible, except in the cases of carbon dioxide and water. It seems probable that even the last act of physiological combustion may differ somewhat in different plants, the substances produced and consumed not bcing always precisely the same: thus not only carbon dioxide and water but also organic acids may be derived from the combustion of the organic carbon-compounds to which most plants are restricted, and sometimes large quantities of organic acids may appear. Moreover in certain bacteria energy is obtained not from the combustion of carbon-compounds but by the oxidation of sulphuretted hydrogen to sulphur and sulphuric acid (sulphur-bacteria), ammonia to nitrous and nitric acid (nitro-bacteria), and even ferrous into ferric oxide (iron-bacteria) ${ }^{4}$. Begriatoa is actually able to oxidize from two to four times its own weight of sulphur in one to

1 Malpighi, Opera omnia, I $86_{\gamma}, \mathrm{I}, \mathrm{p} .108$; Scheele, Chem. Abh. von d. Luft, iibers. v. Bergmann, 1777, p. 125; Ingenhousz, Versuche mit Pflanzen, I786; Saussure, Rech. chim., 1804, and later works previously quoted.

${ }^{2}$ Liebig, Die org. Chemie in ihrer Anwendung auf Agric. u. Physiol., I $S_{4} \mathrm{O}$, p. 30.

${ }^{3}$ Meyen, Pflanzenphysiol., I $\&_{3} \&$, Bd. II, p. I62 ; Dutrochet, Mémoires, Bruxelles, I $\&_{37}$, pp. I $6_{9}$, I85; Garreau, Ann. d. sci. nat., I8 51 , iii. sér., T. Xvi, p. 290 ; Mohl, Grundzuige d. Anat. u. Physiol., I85 I, P. 86 .

4 II inogradsky, Bot. Zeitung, IS88, p. 261 ; Molisch, Die Pflanze in ihren Beziehungen z. Eisen, I $89^{2}$, p. 6o. Cf. Sects. 23,63 ; also Pfeffer, Energetik, IS92, p. 208. 
two days ${ }^{1}$, so that the process may be even more active than normal respiration. It is possible that eertain micro-organisms may even obtain energy by the physiological combustion of hydrogen ${ }^{2}$.

According to Winogradsky, nitro- and sulphur-bacteria produce little or no carbon dioxide, but it is still doubtful whether any organic material at all is oxidized by these organisms, or whether under certain eircumstances organic substances may be consumed in respiration.

The physiological combustion of carbon-compounds, either normally or under special conditions, may frequently only proceed as far as the formation of organic acids, and certain vinegar-bacteria may evolve no carbon dioxide when oxidizing an abundanee of alcohol to acetie aeid (Sect. 103). Similarly the Crassulaceae exhale no carbon dioxide when first placed in darkness, in spite of an active absorption of oxygen, and normal respiration commences only when a large amount of malic or other aeid has accumulated. Hence if the aeid were removed as fast as it was formed, the excretion of carbon dioxide might be permanently suppressed in these plants (Sects. 56, 86). In fungi, however, carbon dioxide is always exhaled even when the production of oxalic, citric or other acids is greatest. A decreased formation of oxalic or other organic acids is accompanied by an increase in the ratio of carbon dioxide exhaled to oxygen absorbed, and hence it is evident that the organic acids are dircet products of physiological combustion ${ }^{3}$. Organic acids, though imperfectly oxidized compounds, are not produced beeause of any defieiency in the supply of oxygen but beeause the metabolic activity assumes this charaeter for the time being. Similarly, nitrite-bacteria are permanently incapable of oxidizing the nitrous acid they produce into nitric acid. Citronyces when deprived of sugar is, however, able to oxidize citric aeid still further into carbon dioxide and water, and Bacterium aceti ean do the same with acetic acid when alcohol is deficient, so that the eourse of metabolism is here liable to regulatory modification adapted to the prevailing conditions (Sect. 93). It does not however follow that respiration always necessarily involves two or more such stages of successive oxidation, for the combustion may proceed direetly without the intervention of any intermediate products such as oxalic acid, \&c. Similarly oil may either be directly consumed in respiration, or may merely partially combine with oxygen ultimately producing sugar, as for example when oily

1 Winogradsky, Bot. Zeitung, I $88_{7}$, pp. 547, 590. Cf. Sect. 63, and Pfeffer, Energetik, I $8_{92}$, p. 207. There is no reason to give special names to these different varieties of respiration, as Detmer proposes (Detmer, Jahrb. f. wiss. Bot., I $S_{79}-S_{I}$, Bd. XII, p. 245). On the structure of sulphurbacteria, cf. A. Fischer, Unters. ïber Cyanophyc. u. Bact., I 897, p. S8.

2 According to Immendorf (Landw. Jahrb., I $8_{92}$, Bd. xxI, p. 323), certain micro-organisms are able to cause the gradual combination of a mixture of hydrogen and oxygen.

${ }^{3}$ Cf. Sect. S6; also Wehmer, Bot. Zeitung, I891, p. 535 ; Kunstmann, Ủber das Verhältniss zwischen Pilzernte u. verbrauchter Nahrung, I 895. 
seeds germinate and assimilate large" quantitics of free oxygen ${ }^{1}$. The respiratory products may therefore vary qualitatively and quantitatively according to the external conditions and the substances consumed. IVhen, however, a fungus fed with peptone produces an abundance of ammonia, the latter probably arises not in the actual process of respiration but during the preparatory changes which provide the material for combustion, and energy may be obtained by the combustion of substances which take no part whatever in constructive metabolism.

Similarly the ratio of carbon dioxide exhaled to oxygen absorbed differs, as might be expected, according to the respiratory activity and the material consumed: thus it usually becomes smaller in fungi fed with carbon compounds poor in oxygen, but may be greater than unity when the food contains a high percentage of combined oxygen. Similarly any formation of organic acid must also decrease the respiratory quotient, and as a matter of fact the evolution of carbon dioxide by the Crassulaceae may almost or entirely cease when the former process is active. In other cases the quotient is such as to indicate that the substances consumed are completely oxidized into carben dioxide and water, and in the case of carbohydrates the respiratory quotient is then unity. This usually occurs in adult organs, whereas in growing plants, owing to the production of organic acids and other substances, the quotient is usually less than unity even when carbohydrates are consumed. Neither the materials consumed nor the products afford any indication as to the actual course of physiological combustion, and the constancy of the quotient during changes of temperature simply indicates that the character of the respiratory processes is not altered by their increased or diminished activity. In members of the Crassulaceae and in Aspergillus, however, a rise of temperature may cause an increased combustion of organic acid and hence a transitory increase in the amount of carbon dioxide evolved, until equilibrium is again attained (Sects. 56, 86).

In most plants and in all the higher plants carbon dioxide is the sole gaseous product of respiration, if we neglect water-vapour ${ }^{2}$. Many lower organisms may however produce other gases even when living acrobically, but it is not quite certain whether this is due to a deficiency of oxygen (Sects. 98, 102) or to other causes. Thus the ammonia evolved by fungi fed with peptone does not seem to be a respiratory product, and similarly it is doubtful whether the nitrogen liberated by certain aerobic nitro-bacteria, and a few

${ }_{1}$ Hellriegel, Journ. f. prakt. Chemie, I 855 , Bd. Lxiv, p. Io2 ; Laskovsky, Versuchsst., I 874 , Bd. Xvir, Pp. 235, 240 ; Detmer, Unters. iiber die Keimung ölhaltiger Samen, 1875, p. 30 ; Godlewski, Jahrb. f. wiss. Bot., I $88_{2}$, Bd. Xiır, p. 507.

2 Confirmed by the researches of Bonnier et Mangin, Ann. d. sci. nat., I $88_{4}$, vi. sér., T. Xvir, p. 265 ; ibid., T. Xviri, p. $3{ }_{4} 4$; T. XIx, p. 228 ; Miintz, Ann. d. chim. et d. phys., I 876 , v. sér., T. vin, 1. 67 ; Sachsse, Keimung von Pisum, I 872, p. I9. The older contradictory results obtained by Fleury, Vogel, \&c., Were probably due to the lack of oxygen or to the presence of putrefactive bacteria. 
other bacteria as well, is actually liberated by the process of respiration (Sect. 102).

Respiratory ratio. De Saussure was the first to observe that the respiratory ratio $\left(\mathrm{CO}_{2}: \mathrm{O}_{2}\right)$ varied according to the nature of the plant, the stage of development, \&c., and that it is usually less than unity during the germination of oily seeds ${ }^{1}$. Numerous researches have confirmed and extended these results". "Thus Godlewski, and Bonnier and Mangin, have shown that as soon as the oil of an oily seed is nearly all replaced by starch the respiratory ratio approaches unity, and that this quotient, which in higher plants lies between 0.3 and $\mathbf{I} \cdot 2$, is in most cases not markedly modified by changes of temperature or of the oxygen partial-pressure. Diakonow found that the respiratory quotient for Penicillium glaucum when fed with sugar was I ; with tartaric acid 2.9 ; with ethylamine 0.67 . Similar results are obtained by comparative analyses of seeds and seedlings germinated in pure water, for these lose almost nothing but carbon dioxide ${ }^{3}$.

Formation of zeater. De Saussure showed that the loss of dry weight is greater than can be accounted for by the amount of carbon dioxide evolved, the difference representing the amount of water formed during respiration ${ }^{4}$. Laskovsky has directly proved that water is produced by respiration by determining the amounts present before and after germination ${ }^{5}$.

Errors due to absorption. Owing to the ready solubility of carbon dioxide it requires a certain interval to saturate a tissue previously free from it and thus render possible the exhalation of the gas produced by respiration. Similarly the transference from an atmosphere rich in carbon dioxide to one poor in it is sufficient to cause a marked temporary increase in the evolution of this gas ${ }^{6}$. On the other hand, it is owing to the production of organic acid that, as de Saussure found, a cactus stem placed in darkness commenced to evolve carbon dioxide only after having absorbed $\mathbf{r} \frac{\mathbf{l}}{\mathbf{4}}$ times its own rolume of oxygen. The accumulation of carbon dioxide in tissues may be aided by combination with sodium phosphate, \&c., by the

1 De Saussure, Mém. d. 1. Soc. d. Phys. d. Genève, 1833 , T. vi, Pp. 547,554 ; Bibl. univers. d. Genèvc, 1842 , T. xL, p. $3^{68}$.

2 Of the more recent literature may be mentioned: Godlewski, Jahrb. f. wiss. Bot., 1882, Bd. xI1I, p. 491 ; Bonnier et Mangin, Ann.d. sci.nat., 1884, vi. sér., T. xvir, p. 209; T. xviri, p. 293 ; T. XIX, p. 218 ; 1886 , vii. sér., T. II, pp. 315,365 ; T. III, p. 5 ; Dehérain et Maquenne, Compt. rend., 1885 , 'T. cI, p. 887 ; Palladin, Ber. d. Bot. Ges., 1886, p. 327 ; Diakonow, ibid., I887, p. 115 (Fungi); Aubert, Rev. gen. d. Bot., I 892 , T. 1v, p. 330 (Crassulaceae); Jumelle, ibid., p. I I 2 (Lichens); Jönsson, Compt. rend., 1894, T. cix, p. $44^{\circ}$ (Mosses); Purjewicz, Bot. Centralbl., 1894, Bd. LviII, P. $37^{2}$; Hesse, Zeitschr. f. Hygiene, IS93, Bd. xv, p. 17 (Bacteria); Mesnard, Ann. d. sci. nat., 1894 , vii. sér., T. XviIr, p. 295 ; Richards, Annals of Botany, 1896, Vol. x, p. 577 ; Gerber, Compt. rend., 1897, T. cxxiv, pp. 1106, 1109 (Fungi and Fruits). The older literature is in part quoted by Bonnier et Mangin, 1.c., T. xvil, p. 217.

${ }^{3}$ Cf. Bonssingault, Ann. d. sci. nat., 1838 , ii. sér., T. x, p. 257 ; Fleury, Ann. d. chim. et d. phys., 1865 , iv. sér., T. 1 , p. 47 ; Sachssc, Keimung v. Pisum, 1872, P. 30 ; Detmer, Keimung ölhaltiger Samen, 1875 , p. 70 .

t De Saussure, Rech. chim., ISo, p. 17.

${ }^{5}$ Laskorsky, Versuchsst., 1874, 13d. xvil, p. 231.

${ }^{6}$ De Saussure, 1. c. Pp. 79.111 ; Bororlin, Sur I. respiration, 1.75. p. 6.

PEEFFER

M $\mathrm{m}$ 
necessity of diosmosing through the cell-membranes before escape is possible, or by other means ${ }^{1}$.

Oxygen being less soluble usually accumulates to a much less marked extent, for the power of entering into loose combination with oxygen is apparently restricted to certain pigment-producing bacteria (Sect. Ior). A trace of free nitrogen is normally present in every cell, but apparently merely in dissolved form?

\section{SECTION 97. Respiratory Katabolism in Anaerobes.}

Many bacteria are obligate anaerobes, whereas facultative anaerobes can exist in the absence of oxygen only when supplied with suitable food. At a sufficiently high partial-pressure of oxygen all organisms cease to exist, and a descending series of plants exhibiting all grades of specific sensitivity to oxygen may be compiled, which terminates in those obligate anaerobes to which the presence of only a trace of free oxygen is fatal (Sect. 100). Temporary anaerobes in which growth or even division may continue for a time in the absence of oxygen form a transition stage between permanent anaerobes and aerobes, and many organisms which are termed anaerobes, owing to the continuance of growth in the absence of oxygen, may be really only temporarily such ${ }^{3}$. On the other hand, there can be no doubt that permanent anaerobes actually exist, for when a few germs such as those which induce butyric fermentation are sown in a hermetically sealed flask from which all free oxygen has been removed, countless generations succeed one another, and further multiplication ceases only when the food-material is consumed and when injurious waste-products accumulate to an inhibitory extent ${ }^{4}$.

Saccharomyces cerevisiae is only a temporary anaerobe even under the best conditions, although it may reproduce itself twenty or thirty times in the absence of oxygen ${ }^{5}$. Mrucor racemosus possesses a somewhat less marked power of temporary anaerobiosis although it can excite very active fermentation, while $M I$. mucedo and $M$. stolonifer do not grow in the absence

1 Cf. Chap. v. Observations on the absorbed gases of turnips by Heintz, Bot. Jahresb., I 873 , p. 360 . On cucumber seedlings by Laskovsky, Versuchsst., 1874, Bd. XviI, p. 223. Absorption by cell-walls and dead plants, Böhm, Ann. d. Chemie, i 877 , Bd. CLxxxv, p. 257 ; Bot. Zeitung, $188_{3}$, p. 559 .

2 A few details by Dehérain et Landrin, Ann. d. sci. nat., I 874 , v. sér., T. xix, p. I65; Compt. rend., i875, T. Lxxxi, p. 198 ; Leclerc, ibid., 1875, T. Lxxx, p. 26 ; Bonnier et Mangin, 1.c.

3 These are Beyerinck's terms (Über Butylalkoholgährung, i $\$_{93}, \mathrm{p} .46$ ).

4 Beyerinck, 1.c., pp. 33, 47 (Sep.-abdr. a. Verh. d. Akad. zu Amsterdam). Beyerinck has recently doubted the existence of permanent anaerobes (Centralbl. f. Bact., Abth. ii, I897, Bd. II, p. 4i).

${ }_{5}$ This was first observed by Pasteur. Beyerinck, I893, 1. c., pp. 33, 47. 
of oxygen in spite of the fecble fermentative power which they possess ${ }^{1}$. Hitherto permanent anaerobes have been found only among bacteria, but it is not as yet certain in all cases whether the anaerobiosis is temporary or permanent $^{2}$.

Many pathogenic bacteria have acquired the power of anaerobiosis in adaptation to the special conditions under which they exist, and it is of great importance in the economy of nature that the processes of decomposition may be able to continue in the interior of organic masses where no free oxygen may be present. As soon as the putrefactive processes consume the whole of the available oxygen, the facultative anaerobes commence to live anacrobically as far as the nature of the food-material will allow, while the dormant anaerobic spores germinate and multiply. As the disintegration is completed, oxygen finds more and more ready access to the interior and the anaerobic germs cither die or pass into a resting condition. It is possible that for certain organisms the continual recurrence of aerobic and anaerobic modes of existence may be essential, and that such organisms are unable to develop either as permanent aerobes or as permanent anaerobes, but this is a question which is still open to discussion. There can be no doubt, however, that a change in the external conditions must always exercise a certain stimulating effect upon an organism, and hence it is possible that in some cases the alternation between two modes of existence may become an essential condition for continued existence.

Anaerobic germs are nearly always present in mud, soil, \&c., and form colonies when inoculated upon oxygenless media. Of these some develop only in the absence of oxygen, but others also when it is present. But little is known of the species thus obtained ${ }^{3}$.

Obligate anaerobes are: various butyric bacteria (Gramulobacter saccharobutyricum, G. butyricum; a few lactic bacteria (Sect. 103), Spirillum desulfuricans ${ }^{4}$, Bacillus denitrificans", Clostridium foetidum, Bacillus polypiformis, B. tetani, $B$. oedematis maligni (Flügge, l. c.). The anaerobic Clostridium pasteuriamum can live aerobically in symbiotic union with certain other bacteria ${ }^{6}$, and the bacillus of cattle-plague, Bacillus carbonis, Mig., may under special conditions develop as an aerobe ${ }^{7}$.

1 Brefeld, Landw. Jahrb., IS76, Bd. v, pp. 293, 313 ; Diakonow, Ber. d. Bot. Ges., IS96, p. 2 ; Beyerinck, IS93, 1. c., p. 47.

2 On certain temporarily anaerobic worms, cf. Bunge, Zeitschr. f. physiol. Chemie, iss 3 , Bd. vil I, p. $4^{S} ; \mathbf{r} 888$, Bd. xil, p. 565 .

${ }^{3}$ See the literature, and Fliigge, Mikroorganismen, I896, 3. Aufl., Bd. I, p. I27; Liborins, Zeitschr. f. Hygiene, 1896, Bd. I, p. I69.

4 lieyerinck, Centralbl. f. Bact., I 895 , Abth. ii, Bd. I, P. 59.

5 Burri u. Stutzer, ibid., p. 43 r.

${ }^{6}$ Cf. Sect. 69. Kedrowski (Zeitschr. f. Hygiene, 1895 , Bd. xx, Heft 3 ) mentions other examples.

7 Kitt, Centralbl. f. Bact., I 895, Bd. xvi1, p. 168. 
Facultative anaerobes include: certain butyric bacteria (Gramulobacter lactobutyricum) and lactic bacteria (Sect. I03); a few nitrogen-producing bacteria (Burri and Stutzer, 1.c.); certain thermophile bacteria ${ }^{1}$, Bacillus phosphorescens ${ }^{2}$, B. prodigiosus, B. typhi-abdominalis, Spirillum cholerae asiaticae, Proteus vulgaris (Flügge, l.c.). Granulobacter polymyxa and a certain form of Bacterium termo are temporary anaerobes ${ }^{3}$, as are species of Saccharomyces.

Obligate aerobes: among micro-organisms are Bacillus subtilis, B. cyanogenus, B. aerophilus, Photobacterium luminosum, P. indicum (Beyerinck, I8S9, 1. c.), Sarcina lutea. A trace of oxygen is required by sulphur-bacteria, and Saccharomyces mycoderma ${ }^{4}$ and red yeast are also obligate aerobes.

\section{SECTION 9S. The Sources of Energy in Anaerobes (Respiratory Katabolism continued).}

In anaerobes as in aerobes the relation between the amount of material consumed and the crop produced varies according to the specific nature of each organism and the prevailing external conditions, certain organisms working more economically than others (Sect. 66). Thus some anaerobes are able to exist upon a small quantity of food-material without exhibiting any marked fermentative activity, whereas in the case of yeast and the bacteria of lactic and butyric fermentation, growth is necessarily accompanied by fermentative activity. Many, and perhaps most anacrobes, are able to grow without exciting fermentation and in many cases are apparently entirely devoid of this power (Bacillus cholerae asiaticae, B.typhi, B. polypiformis, \&c.) ${ }^{5}$. Bacillus prodigiosus can also develop without exciting fermentation, although in the presence of sugar this power becomes manifest, and similarly most anaerobes can be grown upon suitable media without any fermentative activity being exhibited.

The rate of growth bears no direct relationship to the amount of kinetic energy liberated: thus in the absence of oxygen yeast finally ceases to grow in spite of its continued fermentative activity and the marked production of lieat. Indeed growth may be inhibited with comparative readiness although acrobic or anaerobic respiration may remain active (Sect. 104). This may occur when an obligate anaerobe is exposed to a trace of oxygen, while a facultative anacrobe may under special conditions grow more actively in the presence of oxygen than in its absence,

1 Rabinowitsch, Zeitschr. f. Hygiene, I $\$ 95$, bd. xx, p. 154.

2 Beyerinck, Le Bact. lumin., I 889, p. I (Sep.-abdr, a. Archiv. Néerland., T. xxıII).

${ }^{3}$ Beyerinck, Centralbl. f. Bact., I $8_{97}$, Abth. ii, Bd. III, p. 4 I.

'Beyerinck, ibid., I\$95, Abtlı. i, I3d. I, p. 74.

${ }^{6}$ Cf. Lilorius, Zeitschr. f. Hygiene, I886, Bd. I, p. 172; Fliigge, Mikroorganismen, IS96, 3. Aufl., Bd. I, p. I $2 \%$. 
and also more economically with regard to the amount of material consumed ${ }^{1}$.

As in the case of aerobes it is the specific character of an anaerobic organism which determines whether a particular substance can be assimilated, and to what uses it may be put, although at the same time the products formed may vary to a certain extent according to the external conditions, the stage of development, \&c. It is, however, not surprising that a facultative anaerobe may assimilate a variety of substances during aerobic existence, but only a few when living as an anaerobe: thus yeasts are able to ferment and live anaerobically upon particular forms of sugar, whereas in the presence of oxygen they can grow upon a variety of substances without exhibiting any fermentative activity ${ }^{2}$. Similarly many bacteria require particular forms of sugar when living anaerobically, while other anaerobic bacteria may be nourished with various substances: thus certain butyric bacteria grow upon invert-sugar, mannite, or glycerine, others upon lactic acid (Sect. 103), while for a few bacteria tartaric ${ }^{3}$, malic, or citric acid may serve as the sole organic food. Peptone, either alone or in combination with other substances, may suffice for many anaerobes, and especially those which do not induce fermentation ${ }^{4}$. It is possible that many organisms may be discovered which can exist both in the presence and absence of oxygen without exhibiting any fermentative activity, as is indeed the case when Bacillus prodigiosus is grown in the absence of sugar ${ }^{5}$.

The products of anaerobic metabolism are many and various: thus ethyl-alcohol is the chief product of the fermentation of sugar by beer-yeast, whereas butyl-alcohol, lactic acid, or butyric acid are produced by certain bacteria. When successfully nourished by a variety of different substances the fermentative products remain in general the same. All substances which are continually produced and excreted are to be regarded as direct or indirect products of metabolic activity, and the whole host of fermentative products comes under this category. The anaerobic fermentative products include alcohols (ethyl-, butyl-, amyl-alcohol), acids (butyric, lactic, propionic, oxalic, citric, valerianic, formic), while from proteid decomposition result ammonia, fatty amido-acids, leucin, tyrosin, skatol and similar aromatic

1 According to Rabinowitsch (Zeitschr. f. Hygiene, I $\$ 95$, Bd. xx, p. I59), thermophile bacteria grow better at low temperatures in the absence of oxygen.

${ }^{2}$ Cf. Laurent, Ann. d. l'Inst. Pasteur, I889, T. III, p. II 4 ; Beyerinck, Centralbl. f. Bact., I892, Bd. XI, p. 70; Bokony, Pfliger's Archiv f. Physiol., I897, Bd. Lxvi, p. II 4

3 Pasteur, Étude s. 1. bière, I 876 , p. 279 .

4 Liborius, 1.c., p. I68; Beyerinck, Bot. Zeitung, I891, p. 475. Cf. also Sect. 66, and Flïgge, 1.c., p. 219 . The experiments have for the nost part been made with ferment-organisms.

${ }_{5}^{5}$ Liborius, l.c., p. 168 ; Liideritz, Zeitschr. f. Hygiene, I889, Bd. v, p. I5+; Smith, Centralbl. f. Bact., I 893 , Bd. Xrv, p. $86_{5}$; I 895 , Bd. xvir, p. I. Smith's supposition that only sugar can be fermented is incorrect. 
derivatives, as well as sulphuretted hydrogen, methylmercaptan, and other malodorous gases. Carbon dioxide is usually produced, also hydrogen commonly, and more rarely methane, carbon monoxide, and nitrogen ${ }^{1}$.

Several of these products may be simultaneously produced by the same organism, but in the fermentation of sugar by ccrtain bacteria alcohol and butyric acid are the two chicf products, while about 5 to 6 per cent. of the sugar is employed in the construction of various other substances. A portion of the excrete products may arise during the preparatory modifications which the food-material may undergo, or secondary reactions may take place between them. Certain experimental results point indeed in this direction, but the actual course of metabolism is even more difficult to determine in the case of anaerobes than it is in that of aerobes (cf. Sect. 77). Probably no one product of anacrobic respiration is produced by a single organism only; ethyl-alcohol is formed not only by yeasts but also by different bacteria, and by the higher plants when insufficiently supplied with oxygen. On the other hand not one of the products is of universal occurrence, and even carbon dioxide appears to be absent in certain cases, as in the lactic fermentation and in the denitrifying fermentation of saltpetre ${ }^{2}$. Pammel describes certain fermentations in which no gaseous products are evolved ${ }^{3}$, but in all cases continued activity is possible only when the waste-products are excreted or removed. It is possible on the other hand to conceive of anacrobic decompositions which may yield a supply of energy and result in the formation of gaseous products only, as is the case when formic acid decomposes into carbon dioxide and hydrogen, or when nitroglycerine undergoes complete combustion.

Historical. Pasteur ${ }^{4}$ was the first to show that yeast and certain bacteria can grow in the absence of oxygen. The objections raised against Pasteur's experiments were not justified by the facts, for any traces of oxygen originally present are rapidly consumed by the micro-organisms, which continue to increase for weeks in a nutritive fluid containing no free oxygen. Moreover since the original germs contained the merest trace of oxygen it is obvious that anaerobic existence is not rendered possible by means of oxygen stored in these germs. Pasteur indeed showed that in the absence of oxygen no growth is possible upon food-materials which only permit aerobic existence. Growth continues only so long as a supply of energy is assured, and Pasteur recognized that in the case of yeast the anaerobism is strictly limited. It is still uncertain whether certain organisms are permanent or temporary anaerobes, but that the former do actually exist has been shown by the

1 Cf. Fluigge, 1. c., Bd. I, pp. 168, 219 , where the chief literature is given. Cf. also Sect. 103.

2 Burri u. Stutzer, Centralbl. f. Bact., I $\$_{95}$, I, Bd. I, p. $4{ }^{2} 7$; Ad. Mayer, ibid., IS92, Bd. XII, p. 99. Kayser (Ann. d. I'Inst. Pasteur, $18_{94}$, 'T. vill, p. 743) found that another lactic bacterium evolves carbon dioxide.

3 Pammel, Centralbl. f. Bact., i 896 , II, Bd. II, p. 633.

- Pasteur, Jahresb. d. Chem., I S6I, p. 724 ; Étude s. 1. bière, 1876, p. 257. 
researches of Traube (1874), Hüfner (1876), Nägeli (1879), Nencki (1880), liborius (1886), and others ${ }^{3}$.

Methods. The following arrangement may be employed to demonstrate the existence of anaerobic organisms. A flask of about $300 \mathrm{cc}$. capacity is two-thirds filled with a slightly alkaline nutrient solution containing 5 per cent. sugar, I per cent. meat-extract and I per cent. peptone, and coloured blue by indigo-carmine. After introducing a trace of a suitable putrefying fluid, hydrogen is passed through, $a$ and $b$ are closed, and the whole apparatus is placed in a warm room. The indigo is soon reduced as the developing bacteria absorb the last traces of oxygen, and the increasing turbidity is obviously due to the development of anaerobes. At the end of the experiment oxygen may be readmitted, when the reduced indigo-carmine at once turns blue 2 .

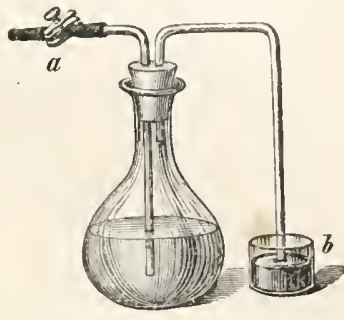

FIG. 65. (a) pinchcock; (b) mercury.

The air may also be removed from an inoculated flask by means of an airpump. The enclosed fluid is then caused to boil for some time at about $30^{\circ} \mathrm{C}$. until about one-third of the contents have evaporated, when the tapering neck of the flask may be drawn out and sealed in a Bunsen flame. When gelatine cultures are desired, hydrogen may either be passed through the gelatine while still fluid, or through special chambers into which the gelatine has been previously poured. The most sensitive anaerobes are able to develop upon Petri plates or in test-tubes placed in a chamber containing no oxygen, as in Fig. 66. The bell-jar is repeatedly exhausted and then re-filled with hydrogen, and if the taps are air-tight and the hydrogen pure this method suffices for all requirements. To attain the latter end the acid in the Kipp's generating apparatus should be covered with a layer of liquid parafin and the hydrogen passed through pyrogallol and potash in addition to the usual purifying tubes. (Figure in Unters. a. d. Bot. Inst. z. Tübingen, I885, Bd. I, p. 637.)

The usual methods of isolation and cultivation are applicable here also (cf. Sect. 66). The development may be followed microscopically by the use of gaschambers $^{3}$, in which any given pressure

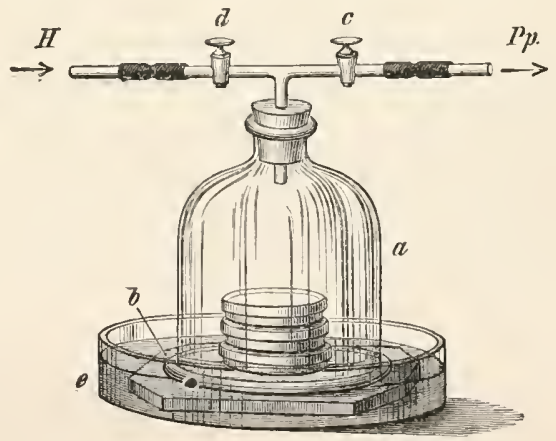

FIG. 66. (e) vessel containing water. of oxygen may be maintained by partial evacuation or by the introduction of

1 For literature, see Hiippe, Methoden d. Bakterienforschung, 189 I, 5. Auf., p. 354 ; Flïgge, l.c., p. 125 .

${ }^{3}$ For an account of the different methods employed in the culture of anaerobes, cf. Hiippe, Methoden d. Bakterienforschung, I89 I, 5. Aufl., p. 35t; Novy, Centralbl. f. Bact., I893, Bd. Xiv, Pp. $5^{66,} 5^{81}$; Beyerinck, Butylferment, 1893, pp. 17, \&c.

${ }^{3}$ Clark, Ber. d. Bot. Ges., i $\$ 88$, p. 273. Cf. Bot. Zeitung, i $\$ 87$, p. 3 I. 
varying mixtures of air and hydrogen. The gases evolved may be determined by the usual methods of gas analysis.

\section{SECTION 99. The Intramolecular Respiration of Aerobes.}

Even in an atmosphere of hydrogen aerobes continue to produce carbon dioxide until death ensues. Anaerobes bchave similarly when the food supplied is not such as to permit of anaerobic existence, and it is probable that several metabolic products, and not always the same ones, are produced by the intramolecular respiration of obligate and facultative anaerobes. In all other plants, including both phanerogams and fungi ${ }^{1}$, the main products appear to be alcohol and carbon dioxide, although, as is shown by the taste or smell, larger or smaller quantities of other substances may be formed, which do not appear when oxygen is present. Hydrogen scems also to be produced by a few of the higher and lower plants, and especially by those which contain mannite ${ }^{2}$.

The intensity and cliaracter of intramolccular respiration is dependent upon the specific nature of the plant and upon the quality and quantity of the available food-matcrials. In the absence of fermentable sugar, ycast produces but little carbon dioxide when all free oxygen is removed, and soon dies, even when fed with quinic acid and peptone, a mixture which forms a suitable nutrient medium in the presence of oxygen ${ }^{3}$. The aerobic Mucor stolonifer, Penicillium glaucum, and Aspergillus niger which, ferment sugar slowly, produce much less carbon dioxide in the absence of oxygen than when living aerobically ${ }^{4}$, although more when fed with sugar than with quinic acid, which affords almost equally suitable nutrient material for the aerobic existence of these fungi (Sect. 66). Penicillium and Aspergillus are killed or severely injured by the withdrawal of oxygen for a single hour, but both live a little longer when provided with sugar, than when the latter is replaced by quinic acid.

1 Müntz (Ann. d. chim. et d. phys., 1876, v. sér., T. VıI, p. 86) was mable to detect any alcohol only in the case of Polyporus destrucior.

${ }^{2}$ Muintz, 1. c., p. 67, and in Boussingault's Agron., Chim. agric. et physiol., i 878, T. vi, p. 2 I I (Agaricineae); De Luca, Ann. d. sci. nat., 1878, vi. sér., T. vi, p. 292 (Olives, Ligustrum, \&c.). Diakonow (Ber. d. Bot. Ges., I 886, Bd. IV, p. 4) states that Penicillium glaucum, which contains mannite, forms no hydrogen, but this may be due to the feebleness of its intramolecular respiration. Selmi (Bot. Jahresb., i 876 , p. I 6 ) has detected an evolution of hydrogen from mould-fungi. The older statements by Humboldt and Marcet as to the evolution of hydrogen by fungi probably arose from experiments conducted in the absence or deficiency of oxygen.

${ }^{3}$ Chudiakow, Landw. Jahrb., I894, Bd. Xxin, p. 489; Pfeffer, Unters, a. d. Bot. Inst. zil Tïbingen, $188_{5}, \mathrm{Bd}$. I, p. $6_{55}$.

'Diakonow, Ber. d. Bot. Ges., 1886, p. 2 ; Bot. Centralbl., I894, Bd. L1x, p. 132. Cf. Pfeffer, l. c., p. 659 . 
The extent to which intramolecular respiration suffices to support vital activity depends again upon the specific nature of the particular plant and upon the kind of food supplied. As a general rule aerobic organs in which metabolism is active will be the first to suffer from the lack of oxygen, and hence life can be longer maintained in the absence of oxygen when the temperature is low. Thus seedlings of Zea mays die and cease to evolve carbon dioxide in twelve hours at $40^{\circ} \mathrm{C}$, in twenty-four hours at $18^{\circ} \mathrm{C}$, and may live a few days at still lower temperatures, dying after approximately the same amount of carbon dioxide has been produced in each case? Apples and pears may remain living for months at moderate temperatures in an atmosphere of hydrogen or nitrogen ; Lechartier and Bellamy ${ }^{2}$ found that two pears weighing $282 \mathrm{grms}$. produced $1762 \mathrm{mg}$. of carbon dioxide in five months. On the other hand, fungi and strongly aerobic bacteria soon die owing to their high metabolic activity ${ }^{3}$. Thus Spirillum undula dies in about an hour after the removal of all free oxygen, and so short a period as this does not allow the disturbing influence of starvation or of the accumulation of waste-products to become manifest.

The value of intramolecular respiration to the plant is not solely dependent upon the amount of decomposition induced, for this varies in different cases. As a general rule the metabolic activity is reduced in acrobes during the absence of oxygen, but in certain phanerogamic seedlings, according to Palladin, it increases. Cotyledons of Vicia faba and of Pisum sationm produce a greater amount of carbon dioxide during intramolecular respiration than during oxygen-respiration, whereas seedlings of Vicia faba and Ricinus produce the same amount ${ }^{4}$.

Since the metabolic products in Phanerogams are similar to those produced by Saccharomyces and aerobic fungi, it is probable that in them also sugars are mainly consumed in intramolecular respiration, and even although traces only of sugars may be present they may be continually formed from other substances ${ }^{5}$. In the case of mould-fungi and Saccharo-

Chudiakow, 1. c., p. 360. Cf. also Palladin, Rev. gén. d. Bot., I894, T. vr, p. 201. Brefeld (Landw. Jahrb., 1876, Bd. v, p. 327 ) states that seedlings may remain living in the absence of oxygen for weeks or even months, but this requires confirmation, and besides, very young seedlings of peas, \&c. may reassume the dormant condition characteristic of the seed.

2 Compt. rend., IS72, T. Lxxv, p. I 204 ; I874, T. Lxxix, pp. 949, I006; Kny, Ber. d. Bot. Ges., I889, p. $16+$ (I'otato-tubers).

${ }^{3}$ On intramolecular respiration of aerobic bacteria, cf. Hesse, Zeitschr. f. Hygiene u. Infect., I 893, Bd. xv, Heft I ; Smith, Centralbl. f. Bact., I\$95, Bd. XviII, p. 4 .

* Palladin, Bot. Centralbl., ISSS, Bd. Xxxiı, p. Ioz; Pfeffer, Unters. a. d. Bot. Inst. z. Tiibingen, I $88_{5}$, Bd. I, p. 657 ; Diakonow, Ber. d. Bot. Ges., I8S6, p. 4I 2.

5 According to Palladin (Rev. gén. d. Bot., IS94, T. vI, p. 209), a supply of sugar favours the intramolecular respiration of etiolated seedlings. The consumption of carbohydrates during the intramolecular respiration of fruits has been proved by Lechartier and Bellamy, Compt. rend., i 869 , T. Lxix, p. 466 ; Pasteur, Étude s. 1. bière, 1876, p. 260 ; de Luca, Ann. d. sci. nat., i 878 , vi. sér., T. vI, p. 302. 
myces, proteids, even in combination with quinic acid, \&c., do not serve to maintain intramolccular respiration for any length of time, whereas nutrient media of this kind satisfy all the requirements of ecrtain anaerobic bacteria. It secms probable therefore that intramolecular respiration is not directly connected with the decomposition of proteid substances.

The continued exhalation of carbon dioxide in the absence of oxygen was first observed a century ago', and the formation of alcohol in fruits kept in a chamber devoid of oxygen has been mentioned by several authors ${ }^{2}$, but in these observations the complete absence of oxygen and of micro-organisms was not assured. The latter objection applies to certain of the researches of Lechartier and Bellamy, Pasteur, and Traube ${ }^{3}$, which however along with the experiments by Brefeld and Müntz render it certain that carbon dioxide and alcohol are produced during the intramolecular respiration of the most widely different plants.

Wortmann supposed that the same amount of carbon dioxide is produced whether oxygen is present or not, but IVilson has shown that the ratio between the respective amounts of carbon dioxide produced by intramolecular and aerobic respiration differs in different parts of the same plants and lies between the limits of 0.2 and unity in different Phanerogams and Agaricineae ${ }^{4}$. Pfeffer then pointed out that the phenomenon was a vital one connected with the normal processes of respiration, and not one of death as Nägeli supposed ${ }^{5}$. Hence in the absence of oxygen intramolecular respiration immediately commences, while as soon as the supply of frec oxygen is restored aerobic respiration is resumed. It is only when the plant has been injuriously affected by prolonged exposure that a latent period of recovery intervenes before the oxygen-respiration acquires its full normal activity. Chudiakow has also shown that the ratio between the amounts of carbon dioxide evolved during intramolecular and aerobic respiration remains comparatively constant at different temperature, both forms of respiration being correspondingly affected by a rise or fall of temperature (Sect. 104).

Alcohol is formed in variable quantities. According to Brefeld (l. c., p. 237), not more than $\frac{1}{2}$ per cent. of the weight when moist accumulates in leaves and flowers,

${ }^{1}$ Rollo, Anm. d. chim., I798, T. xxv, p. $4^{2}$; Saussure, Rech. chim., I 804 , p. 201 ; Bérard, Ann. d. chim. et d. phys., I $\delta 2 \mathrm{I}$, T. Xvi, p. 7 I 4 . More rccently, Broughton, Bot. Zeitung, i 870 , p. 647 ; Pfeffer, Arb. d. Bot. Inst. in Würzburg, I $\delta_{71}$, Bd. I, p. 34.

${ }_{2}$ Dumont, Neues Journ. f. Pharmacie, I 8 I9, Bd. III, p. 568 ; Döbereiner, Gilbert's Ann. d. Physik, I $\delta_{22}$, Bd. Lxx1I, p. 430. See also Döpping u. Struve, Journ. f. prakt. Chemie, I $\delta_{4} 7$, Bd. Xi.I, p. $27 \mathrm{I}$.

${ }^{3}$ Pasteur, Compt. rend., I 872 , T. Lxxv, p. 1056, and 1. c.; Traube, Ber. d. Chem. Ges., i $\delta_{74}$, p. 885 .

4 Wortmann, Arb. d. Bot. Inst. in Wiirzburg, I $\delta 80$, Bd. II, p. 500 ; Wilson, Flora, I 882 , p. 93 .

${ }^{5}$ Nägeli, Thcorie d. Gährung, I 879 , p. 43 ; Pfeffer, Unters. a. d. Bot. Inst. z. Tüibingen, i $88_{5}$, Bcl. I, p. 637 , where the apparatus used is described. The same method is still usually employed of removing all oxygen by a current of hydrogen, and collecting the $\mathrm{CO}_{2}$ evolved in titrated barytawater. These results have been confirmed by Möller, Ber. d. Bot. Ges., I884, p. 307 ; Jentys, Bot. Jahresb., I884, p. 89 ; Stich, Flora, I891, p. 21 ; Amm, Jahrb. f. wiss. Bot., I893, Bd. xxv, p. 1; Chudialkow, IS94, I. c. 
but cherries may contain $\mathbf{I} \cdot 8$ to 2.5 per cent., and seedling peas as much as 5 per cent. Brefeld's observations upon the formation of alcohol by Penicillium were probably not made upon $P$. glaucum, for according to I)iakonow the latter rapidly dies in the absence of oxygen, and Elfving ${ }^{1}$ did not observe any formation of alcohol by Penicillium glaucum when grown upon readily fermentable media. In addition to alcohol, other substances, including organic acids, are probably produced, and hence no constant relationship necessarily exists between the amount of carbon dioxide and of alcohol ${ }^{2}$.

\section{SECTION 100. The Mechanism and Causes of Physiological Combustion.}

Respiration is a vital function regulated and maintained by the living organism, which draws neutral oxygen into its metabolism, and by inducing the physiological oxidation of certain substances provides the cnergy necessary for further metabolic activity and for the continuance of life. The consumption of oxygen is regulated by the requirements of the organism, and when these are satisfied the presence of a surplus produces within certain limits hardly any perceptible effect (cf. Sect. 22). As a matter of fact the respiratory activity of many plants is not markedly modified when the percentage of oxygen is reduced to one-half its normal amount, or when its partial pressure is five to ten times increased. In the latter case an excess of free oxygen collects in the cell, but under normal circumstances it is present both in the protoplasm and cell-sap, as is shown by the existence of living animals within Vaucleria, and by the continued activity of organisms enclosed in the plasmodia of Myxomycetes ${ }^{3}$. Hence all theories fall to the ground which postulate the abscnce of free oxygen from the intcrior of the cell, and thus restrict physiological combustion to the outermost layer of the cyto-plasm ${ }^{4}$.

The protoplast can absorb the last traces of oxygen, but bclow a certain dilution the amount absorbed is insufficient to supply all requirements. In the higher plants this point is reached only when the percentage of oxygen falls below 5 to 8 per cent. ${ }^{5}$, and hence they are able to respire normally on the summits of the highest mountains. If, however, the absorption is rendered more difficult, as, for example, by smearing them with fat, the

1 Elfving, Einwirkung d. Lichts auf Pilze, I $\$ 90$, p. 125.

2 Godlewski, Anzeig. d. Akad. d. Wiss. zu Krakau, Juli, IS97.

3 Pfeffer, Unters. a. d. Bot. Inst. z. Tübingen, I $88_{5}$, Bd. I, p. $68_{4}$; Oxydationsvorgänge, I $\$ S_{9}$, pp. 449, 500 ; Čelakovský, Flora, Erg.-bd., IS92, pp. I9t, $209,226$.

\& Reinke, Bot. Zeitung, $18 S_{3}$, p. 95 ; l’ringsheim, Sitzungsb. d. Berl. Akad., 1887, p. 77.

${ }^{5}$ Cf. Stich, Flora, I 89 , p. I, and the literature there quoted. 
tissues may be insufficiently supplied with oxygen even in ordinary air (cf. Sects. 29-32). The respiratory exchanges are especially active in bacteria, and sulphur-bacteria are able to obtain the large amounts of oxygen they require in exceedingly dilute form ${ }^{1}$. When a seedling is suddenly placed in air containing only 3 to 4 per cent. of oxygen intramolecular respiration commences, and a volumc of carbon dioxide is evolved which greatly exceeds the amount of oxygen absorbed. After a time, however, the plant accommodates itself to the changed conditions, and in twenty-four hours the same supply of oxygen may suffice for the diminished respiratory activity ${ }^{2}$.

Similarly when the partial-pressure of oxygen is increased to an excessive extent death ultimately ensues, and respiration perforce ceases. Many plants, espccially among Phanerogams, continue at first to respire and grow at almost the normal rate under a pressure of twenty to thirty atmospheres, but in only a few hours both functions gradually diminish and ultimately cease at the onset of death, which occurs after several hours or days ${ }^{3}$. Under such circumstances seeds and spores are unable to germinate. Many of both the higher and the lower plants develop normally in pure oxygen or in air under a pressure of five atmospheres ${ }^{4}$. There can be no doubt that all grades of sensitiveness to oxygen are exhibited throughout the vegetable kingdom, connecting these highly resistant forms with those to which the presence of a trace of oxygen is fatal. Thus sulphur-bacteria are strongly aerobic but require and indeed prefer the presence of traces of oxygen only, and although a very small amount of this gas is fatal to certain obligate anaerobes (Bacillus polypiformis, B. ocdcmatis maligmi, Spirillum desulfuricans), others may continue to grow even in the presence of a little of it (Clostridium foetidum, Gramulobacter butylicum) ${ }^{5}$. According to Beyerinck the last-named organism grows best when supplied with a little oxygen if cultivated upon starch-paste and peptone, and $\mathrm{K}_{\text {itt }}{ }^{6}$ has found it possible to cultivate Bacillus carbonis upon bouillon in air, although Migula

1 Winogradsky, lot. Zeitung, I 887 , p. 539; Beitr. z. Morph. u. Physiol. d. Bact., I888, I, p. 50. Red sulphur-bacteria can obtain oxygen by $\mathrm{CO}_{2}$-assimilation, and hence remain living for weeks if exposed to light in closed oxygenless tubes containing a little $\mathrm{H}_{2} \mathrm{~S}$ and a trace of reduced indigocarmine. Cf. Engelmann, Die Purpurbakterien, Bot. Zeitung, ISS8, p. 66 г ; Ewart, Journ. of Linn. Soc., 1897 , Vol. xxxiı, p. I53.

${ }^{2}$ Stich, l. c., p. I3 ; Godlewski, Jahrb. f. wiss. Bot., I8S2, Bd. xIIr, p. $5^{22}$.

3 Johannsen, Unters. a. d. Bot. Inst. z. Tübingen, I $\delta_{5}$, Bd. I, p. 7 I 6 ; Jentys, ibid., I $8 s 8$, Bd. I, p. 457. On bacteria, cf. Wossnesenski, Compt. rend., I $\$ 8_{4}$, T. XCVIII, P. $3 \mathrm{I} 4$.

${ }^{4}$ Cf. Johannsen u. Jentys, 1. c. ; also Jaccard, Rev. gén. d. Bot., I $\delta_{93}, v$, p. $3^{8} 3$, and the literature here quoted. On bacteria, Fränkel, Centralbl. f. Bact., I $88_{9}, \mathrm{Bd} . \mathrm{v}, \mathrm{p} .2 \mathrm{Ic}$.

${ }_{5}^{5}$ Cf. Liborius, Zeitschr. f. Hygiene, i 886, Bd. I, pp. I68, г 70 ; Beyerinck, Centralbl. f. Bact., I 895 , Abth. ii, Bd. I, p. I09; Über die Butylalkoholgährung, 1893, p. 45.

${ }^{6} \mathrm{Kitt}$, Centralbl. f. Bact., I $S_{95}$, Bd. xviI, p. I68. On the influence of different substances upon anaerobiosis, cf. also Kitasato u. Weyl, Zeitschr. f. Hygiene, I 890 , Bd. viII, p. $4^{\mathrm{I}}$; Bd. IX, p. I 7 . 
supposed it to be an obligate anacrobe. Various anzerobes grow in the presence of oxygen when associated with other micro-organisms, but since even then the former must come more or less into direct contact with the gas, it is obvious that they must be influenced in some special manner by the symbiotic association ${ }^{1}$.

It is possible that both the minimum and maximum pressures of oxygen for a particular plant may vary to a certain extent according to circumstances, and their precise determination is made still more difficult by the fact that an oxygen-pressure which the plant can withstand even for a prolonged time may ultimately cause death. This is the case not only with aerobes but also with anaerobes, for the latter may continue to move for a long time in the prescnce of a super-maximal amount of oxygen. Certain plants possess hardly any accommodatory powcr, and sulphurbacteria can withstand only slight variations from the optimal percentage of oxygen, whereas many facultative anaerobes are able to live at as great an oxygen-pressure as higher plants.

The minutest traces of free oxygen are absorbed even by anaerobes. Growth may be inhibited either by a sufficient decrease or increase in the oxygen-pressure, both in Phanerogams and in facultative anaerobes. There can be no doubt, moreover, that the growth even of the most sensitive obligate anaerobes must be possible in the presence of a minimal trace of oxygen, and hence no sharp line of demarcation can be drawn between those anaerobes which continue to grow and those whose growth ceases in the presence of oxygen ${ }^{2}$.

Each organism is able to withstand a specific density of oxygen, but as is shown by facultative anaerobes the power of anaerobism is not necessarily connected with an excessive sensitiveness to this gas, such as characterizes the obligate anacrobes. The injurious action is peculiar to frec oxygen, for this clement is present in combined form in all anacrobes, while in the processes of metabolism highly oxidized end-products, such as carbon dioxide, \&c., may be produced. The over-concentration of oxygen does not act injuriously by either decreasing or increasing the activity of physiological combustion ${ }^{3}$, for as a matter of fact, growth, respiration, and indeed all vital functions, ${ }^{4}$, are at first but little altered.

${ }^{1}$ Kedrowski, Zeitschr. f. Hygiene, ${ }^{8} 8_{95}$, Bd. xx, Heft 3 . On Clostridium Pasturrianum, cf. Sect. 69 .

${ }^{2}$ Beyerinck, Centralbl. f. Bact., i 895, Abth. ii, Bd. I, p. Iog, footnote.

3 Both views have been put forward (cf. Jentys, Unters. a. d. Bot. Inst. z. Tübingen, I SSS, Bd. II, p. $45^{8}$ ). The non-oxidation of phosphorus in compressed air affords no criterion as to the action upon a living organism, and in most cases combustion is more active in pure oxygen than in air. Cf, van 't Hoff, Chem. Centralbl., is 95, I, p. 676 .

4 As, for example, phosphorescence and heat-production. Cf. de Vrolik et de Vriese, Ann. d. sci. nat., $18_{39}$, ii. sér., T. xi, P. 77 . 
Respiration does, indeed, increase slightly in some plants as the pressure of oxygen rises ${ }^{1}$, but the much more marked increase produced by a high temperature does not exercise any injurious effect. Moreover the presence of a superfluity of a nutrient substance often induces an increased consumption, and in certain cases small doses of poison may produce more active growth, respiration, and production of heat (Sect. 104). Beyond a certain limit of temperature, and beyond a particular concentration either of poison or of food-material, the plant is injuriously affected and ultimately killed. The action of an increased density of oxygen is of similar character, and, as Bert showed, pure oxygen at atmospheric pressure exercises the same influence as air under a pressure of five atmospheres. The mechanical effect of an increased pressure is but slight, and it is more marked in the case of tissues than with isolated cells or cellular filaments ${ }^{2}$.

An increased pressure of oxygen acts in a similar manner upon obligate anaerobes, for the movement of butyric bacteria ceases more or less rapidly according to the partial-pressure of oxygen to which they are exposed, death cnsuing in a few hours or a few days ${ }^{3}$. The metabolism of aerobes is not markedly altered by an injuriously high partialpressure of oxygen, and hence the latter may possibly exercise some poisonous effect or other. In anaerobes the metabolic disturbances induced by the presence of oxygen may suffice to produce the injurious effects observed, and, as is always the case when the conditions are unfavourable to continued vital activity, death must ultimately ensue unless a dormant resting condition can be assumed.

P. Bert ${ }^{4}$ first studied the effects produced by a rise in the pressure of oxygen, Johannsen observed the influence upon respiration, and Jentys that upon growth. In their works the older researches are mentioned and a description given of the apparatus employed. In Sect. 98, and in the works of Wieler and Stich, the methods are given for experimenting in rarefied air. Cultures of anaerobic bacteria in gelatine tubes exposed to air form colonies only in the deeper layers of the gelatine, and the closeness with which they approach the surface affords a direct indication as to their sensitiveness to oxygen.

1 Johannsen, 1.c., p. 714, for further literature. Jentys, 1.c., p. 457. In pure oxygen the respiratory quotient remains the same. Godlewski, Jahrb. f. wiss. Bot., I $88_{2}$, Bd. XIII, p. 522 ; Bonnier et Mangin, $A n n$. d. sci. nat., I $\$ 8_{4}$, vi. sér., T. Xvill, p. 364 ; I886, vii. sér., T. II, p. 370.

${ }^{2}$ Cf. Jentys, l. c., pp. 455,463 ; Jaccard, Rev. gén. d. Bot., I $\$ 93$, T. v, p. $3^{\delta_{3}}$.

${ }^{3}$ Lideritz, Zeitschr. f. Hygiene, I889, Bd. v, p. I57. Cf. also Pasteur, Compt. rend., I861, T. LII, p. 340, and Étude s. l. bière, is 76, p. 293 ; Grossmann u. Mayershausen, Archiv f. Physiol., I 877 , Bd. xv, p. 245 ; leyerinck, Butylferment, I893, p. 6; Centralbl. f. Bact., I895, Abth. ii, Bd. I, p. I I 2 .

1 P. Bert, Compt. rend, i 873 , T. Lxxvil, p. $53^{1}$; Ann. d. chim. et d. phys., 1876 , v. sér., T. VII, p. I46; La pression barométrique, 1878, p. $8_{5} 6$; Wicler, Unters. a. d. Bot. Inst. z. Tiibingen, $188_{3}$, Bd. I, p. 189 ; Stich, Flora, I89ז, p. I. 
An optimal oxygen-pressure exists for every organism, but the course of this curve is not necessarily coincident with that for growth. As the result of a change of oxygen-pressure certain after effects may ensue such as have already been mentioned as following the removal of free oxygen. Thus Johannsen found that after remaining for a time under a high partial-pressure of oxygen, respiration was temporarily more than normally active when the plant was returned to ordinary air. The action of sudden changes has not yet been determined, and hence it is uncertain whether an anaerobe is injured more rapidly by a sudden supply of oxygen than by one which gradually increases to the same level.

\section{Section ioi. The Causes and Mechanism of Physiological Combustion (continnted).}

A clear comprehension of the phenomena of physiological combustion has not yet been obtained, and the genetic relationship between aerobic and anaerobic respiration simply shows that the oxidation is a metabolic process regulated by the vital activity of the protoplast. Neutral oxygen is drawn into metabolism by means of the affinities which the latter creates, and without being oxonized, for no such oxidizing effects are produced upon pigments or chromogens present within normal living protoplasts, as are induced by simple treatment with a weak oxidizing agent such as peroxide of hydrogen. Protoplasm does not act therefore as a general oxidizing agent, although almost any substances which enter into metabolism may undergo oxidation when necessary. Thus certain nitro-bacteria oxidize ammonia into nitrites, while others which are unable to oxidize ammonia complete the process of oxidation into nitrates. Similarly in the process of respiration, organic acids and other substances which are capable of still further oxidation may be produced in spite of the presence of an abundance of oxygen (Sects. 85 and 86).

Those organisms in which energy is obtained partially or entirely by the oxidation of nitrites, sulphur, \&c., are of the utmost importance in the study of the general characteristics of physiological combustion (Sect. 96), for in them the means by which oxidation is induced may be revealed. It is, however, possible that the process of oxidation is different in different organisms and according to the substance oxidized, for facultative anaerobes afford examples to show that energy need not always be obtained in precisely the same manner. It is, moreover, uncertain whether oxidizable substances are produced by metabolism and that these combine with oxygen as rapidly as they are formed, or whether the protoplasm itself induces the oxidation of compounds upon which oxygen exercises no direct effect. In the latter case the oxidizing action 
might be due to substances resembling ferments, to molecular vibrations generated by the plasma, or to the continual decomposition and regeneration of certain compounds. Special interactions may occur between the protoplasm, oxygen, and the compounds consumed in respiration, while at the same time the protoplasm may combine loosely with oxygen and transfer it to the substances oxidized. The absorption of oxygen and the exhalation of carbon dioxide are closely connected processes, for the latter undergoes an immediate and very marked decrease when the former ceases ${ }^{1}$. Moreover, the fact that methyl-blue remains intact within the protoplasts even in the absence of all free oxygen, is sufficient to show that the induced affinities for oxygen are not such as to render the organism capable of obtaining it from any reducible substance².

It is therefore impossible to say whether a continual decomposition of proteids is the unavoidable accompaniment of all metabolism involving a liberation of energy, for although many observations point to this conclusion, the mere fact that such decomposition has been shown to occur in certain cases does not warrant such an assumption, and moreover the oxidation of nitrites, sulphuretted hydrogen, \&c., may not involve any proteid decomposition whatever. No proteid decomposition can occur if the power of regeneration is absent, and it is not impossible that energy may be obtained by the oxidation of substances which do not enter into direct union with the protoplasm. Moreover it is worthy of notice in this connexion that Saccharomyces and certain bacteria are incapable of anaerobic existence when fed with proteids (Sect. 99).

Continual proteid-decomposition need not necessarily accompany vital activity, for the energy necessary for the maintenance of the latter may be obtained by the aerobic or anaerobic decomposition of other substances, provided this decomposition actually takes place in the protoplasm or between its component parts, and even when these substances enter into simple association with proteids, as probably occurs during fermentation, no proteid-decomposition is necessarily involved. It is of course essential that the specific constructive elements of the protoplasm, to which it owes its hereditary character, must not be irreparably disorganized however active respiration may be (Sect. 7).

Metabolic processes liberating energy must take place in all protoplasmic organs, and it has been observed indeed that isolated fragments of protoplasm continue to respire as long as they remain living. It is, however, also possible that extracellular oxidations which are of service to the plant may be carried out by katalytic action on the surface of the protoplast,

1 See Pfeffer, Unters. a. d. Bot. Inst. z. Tuibingen, $18 s_{z}$, Bd. I, PI. $67_{22}, 677$; Oxydationsvorgänge in lebenden Zellen, $\mathrm{s} 88^{\circ}, \mathrm{p}, 490$.

"Pfeffer, Issg, l. c., p. 5I3. Cf. also Sect. Io2. 
or by means of enzymatic or other secretory products. This has not yet been proved to be the case in living cells, for even in highly energetic mouldfungi no power of inducing extracellular oxidation can be detected even by the most delicate tests (indigo-carmin, methyl-blue, potassium iodide-starch, and iron) ${ }^{1}$, and it has yet to be determined whether any such power is exercised by acetic acid bacteria, or by other micro-organisms.

The reactions given by dead cells, or by the expressed sap, form no sure indication as to the conditions existing in the living cell, for in the latter, substances may be kept apart which react when in contact, as for example when a glucoside and a glucoside enzyme are present in the same cell. Various post-mortem oxidations may occur after death, as for cxample when the sap of Monotropa, Vicia faba, \&c., turns brown ${ }^{2}$. These appear to be produced by the action of certain substances to which the provisional name of 'oxydases' may be given ${ }^{3}$, and from facts already mentioned it is not improbable that substances may be produced which are intended to act only after the death of the cell containing them. Schonbein ${ }^{4}$ has shown that expressed sap has a slight power of inducing ozonization, but it is erroneous to draw any conclusions from this fact as to what occurs in the living cell, for as a matter of fact no ozone is present in the latter. No discussion is therefore necessary of those theories which are based upon this false assumption, nor can any reasons be given for the production of ozone which is frequently induced by autoxidation ${ }^{5}$.

In many cases marked changes due to oxidation take place after death, and these may or may not be accompanied by an evolution of carbon dioxide, which latter, however, in other plants ceases at ordinary temperatures as soon as death ensues ${ }^{6}$. Brenstein's contradictory results were due to experimental error. On the evolution of carbon dioxide at $70^{\circ}-100^{\circ} \mathrm{C}$., cf. Schlösing, Compt. rend., I888, T. cri, p. I 293; I889, T. cviII, p. 527 ; Berthelot et André, i 894, T. cxviII, pp. 45, I 04.

The permanent absence of ozone and of hydrogen peroxide from the living cell is shown by the fact that certain oxidations are not carried out which are at once

1 Pfeffer, Oxydationsvorgänge, т $8 S_{9}, \mathrm{p} \cdot 47 \mathrm{r}$.

2 Pfeffer, 1. c., pp. 430, 447 .

${ }^{3}$ Cf. Lafar, Technische Mykologie, I897, Bd. I, p. 357. Bertrand (Compt. rend., I895, T. cxx, p. 266 ; I 896 , T. cxxis, p. I2I5) calls the oxidizing ferment of the lac tree 'Lakkase' and states (1. c., I 897 , T. CXXIr, p. I032) that it acts only in the presence of Mn. Bourquelot (Compt. rend., I $896, T$. CxxiI , pp. $260,3^{I} 5,4^{6} 3$ ) has detected oxidizing ferments in fungi. Cf. also Gruiss, Landw. Jahrb., I $\$ 96$, Bd. $x x v$, p. $3^{S S}$ (potato). Cf. also Sect. 91. [Raciborski (Ber. d. Bot. Ges., IS99 Bd. XvI, pp. 53, II9) has found that a substance capable of inducing the oxidation of hydroxylguiacum solution is widely distributed in many tropical plants, but whether one or more oxidases are concerned in producing the blue reaction used as a test is quite uncertain, and hence the special term 'leptomin' is without justification. Cf. Grïss, Ber. d. Bot. Ges., I $\delta_{9} \delta$, Bd. xv r, p. I 29.]

* The literature is in part given by Pfeffer, Is $\$ 9,1$. c., p. 466 .

${ }^{5}$ Cf. Pfeffer, 1. c., pp. $444,49^{S}$; also Nasse, Pfliger's Archiv f. Physiol., IS $S_{7}$, Bd. xLI, p. $3^{S o .}$

6 See the literature mentioned by Pfeffer (Oxydationsvorg., I $\$ 8_{9}$, p. 50I), and also Kreusler (Landw. Jahrb., I89o, p. $66_{4}$; Clausen, ibid., IS9I, p. 2 I). 
produced by treatment with $0.00 \mathrm{I}$ to 2 per cent. solutions of hydrogen peroxide. In such dilution the latter is innocuous, but it causes the cell-sap in the roots of Vicia faba to turn brown, and decolourizes the purple pigment in the cell-sap of the staminal hairs of Tradescantia. Such changes are permanent when once induced, and hence the feeblest oxidatory action in the cell-sap would ultimately become perceptible. Similarly no active oxygen is present in the protoplasm, for when permeated with cyanin, the latter undergoes no oxidation, which however at once ensues when a little hydrogen peroxide is added '.

The transference of oxygen from an absorbent substance to one which is oxidized does not involve any ozonization, nor does this occur in those pigmentbacteria which Ewart's researches have shown to be capable of entering into a loose combination with oxygen similar to that formed by haemoglobin ${ }^{2}$. The oxygen absorbed apparently unites with the excrete bacterial pigment which remains in close association with the bacterial colonies, and hence the pigment not only plays a most important part in the transference of oxygen to the living and aerobic bacteria, but also conveys oxygen to the interior of the colony. The oxygen thus stored up does not, however, suffice for more than a few hours' respiration. In ordinary plants no oxygen is stored in this manner as a compound which slowly dissociates when the partial-pressure is reduced, for when all free oxygen is removed rotation soon ceases, although it can continue when the oxygen partial-pressure is extremely low (Clark, Ber. d. Bot. Ges., 1888, p. 273). In certain cases streaming movements persist for a longer time, but this may simply be a manifestation of the energy derived from intramolecular respiration and does not necessarily prove that any oxygen is held by such cells in occluded form. [Cf. Ewart, l. c., p. I45, and I 896, Vol. xxxı, p. 420 . Kühne (Zeitschr. f. Biol., Bd. xxxv, I897, p. 43 ; I898, p. I) states that streaming may continue in Nitella as long as fifty days in darkness, and concludes on insufficient grounds that a store of oxygen must be present in the living cells. The explanation seems to be, however, that Nitella is a partial anaerobe. In any case the results are in urgent need of confirmation, for a very minute trace of oxygen apparently suffices to maintain rotation in Chara for an almost indefinite length of time. Cf. Ewart, l.c.]

\section{SECTION 102. The Relationship between Aerobic and Anaerobic Respiration.}

The withdrawal of oxygen causes metabolism to assume a different character, and may thus lead to the formation of products which did not previously appear, either owing to the awakening of dormant powers, or, as is more probably the case, because under the new conditions the

1 Pfeffer, Oxydationsvorgänge, 1889 . On the poisonous action of $\mathrm{H}_{2} \mathrm{O}_{2}$, cf. Kny, Ber. d. Bot. Ges., $188_{9}$, p. $16_{5}$; Fligge, Mikroorganismen, 1876, 3. Aufl., Bd. I, p. 461 . Ozone is much more poisonous. Cf. Pfeffer, 1. c., p 427 ; Flügge, 1. c., p. 46 I.

${ }^{2}$ Ewart, Journ. of Linn. Soc. Bot., I 897 , Vol. Xxxi1, p. 123; Abstract by Pfeffer in Ber. d. Sächs. Ges. d. Wiss., I $\$ 96$, p. 379 . 
same primary causes act in a somewhat different manner ${ }^{1}$. The action may be in part direct, the absence of oxygen influencing the molecular constitution of certain of the substances formed under the changed conditions, but there can be no doubt that a stimulating influence is also exercised, modifying the normal chemical or formative activity, or even inducing new processes (Sect. 93). It is therefore possible that the formation of alcohol in the intramolecular respiration of aerobes may arise from a series of processes which are quite foreign to the normal respiratory activity, and indeed various facts point to this conclusion. It is also evident that carbon dioxide cannot be derived from the combustion of alcohol in those cases in which the same quantity is produced in the presence as in the absence of oxygen.

In adaptation to their varying habitats, the anaerobic metabolism of different organisms exhibits widely different characteristics, and a series of modifications which are accompanied by a gradual widening of metabolic activity, lead finally to those aerobes and anaerobes which possess marked fermentative powers. Certain of the new properties thus obtained may attain a more or less inherent character, as for example in those organisms in which intramolecular respiration and fermentative activity are not suppressed by the presence of free oxygen. This is the case in Saccharomyces cerevisiae, \&c. (Sect. 103), whereas Mucor racemosus (Sect. 98) and Saccharomyces mycoderma ${ }^{2}$ excite alcoholic fermentation in the presence of a little oxygen, but not when the latter is abundant. Similarly the fermentative activity of Bacillus Fitsianus ${ }^{3}$ (ethyl-alcohol from glycerine) is not suppressed when oxygen is supplied, and the same is the case with Bacillus prodigiosus ${ }^{4}$ when sugar is present. An abundance of oxygen, however, inhibits fermentation in the case of Granulobacter polymyxa ${ }^{5}$ (butyl-alcohol), and of Bacillus phosphorescens and B. Pfiugeri ${ }^{6}$, although these require a certain limited supply of oxygen. The formation of hydrogen and sulphuretted hydrogen during putrefaction is largely inhibited by the presence of an abundance of oxygen, either owing to the suppression of intramolecular respiration, or to the direct or indirect oxidation of the products of the latter.

Free oxygen is always drawn into metabolism to a greater or less extent when present, and hence it must necessarily exercise some influence upon the vital processes ${ }^{7}$, as is the case when yeast consumes more sugar

${ }^{1}$ Cf. Pfeffer, Unters. a. d. Bot. Inst. z. Tübingen, I $88_{5}$, Bd. I, p. $66_{2}$; Oxydationsvorgänge, $188_{9}$, PT. $497,510$.

2 Beycrinck, Centralbl. f. Bact., I 895 , Abth. ii, Bd. I, p. 74 .

${ }^{3}$ Fitz, Ber. d. Chem. Ges., IS 76 ; Buchner, Zeitschr. f. physiol. Chem., IS85, Bd. IX, p. 393.

- Liborius, Zeitschr. f. Hygiene, I886, Bd. I, p. I72. A few other examples also.

5 Beyerinck, Über d. Butylalkoholgährung, I 893 : p. 9 .

${ }^{6}$ Beyerinck, Aliment photogène, IS9I, p. I3 (Sep.-abdr. a. Archiv. Néerland., T. xxiv).

${ }^{7}$ Nägeli (Theorie d. Gährung, 1879 , p. I16) supposes that ferment-organisms are in general less capable of inducing complete oxidation, but this is still doubtful. 
in the presence of oxygen for the same production of alcohol. On the other hand, free oxygen depresses the metabolic activity of obligate anacrobes, and the character of the food exercises an important influence upon the result produced, as when Saccharomyces oxidizes alcohol in the absence of sugar, and Bacterinum aceti vinegar in the absence of alcohol. In certain cases the maximal fermentative activity is attained in the presence of a trace of oxygen, and many plants in which aerobic and anaerobic respiration can proceed simultaneously, may find optimal conditions for growth and development only when both are possible.

It is not the case that in the absence of free oxygen aerobic respiration continues by means of oxygen drawn from reducible substances present in the cell, but rather that, as in normal aerobic metabolism, changes and decompositions are induced in one or more substances, which in general lead to a liberation of energy and to a simultaneous production both of reduced and of oxidized products. Carbon dioxide is the most commonly excreted oxidized product, while among the products of reduction are hydrogen, sulphuretted hydrogen, alcohol, \&c. The heat of combustion of alcohol is greater than that of sugar for the same number of carbon atoms, or for the same weight. During the anacrobic metabolisin of carboncompounds, not only may various carbon affinitics be satisfied, and compounds of carbon and hydrogen and carbon and carbon decomposed, but also combinations and dissociations may occur similar to those involved in aerobic metabolism. In neither case is the formation of carbon dioxide an absolute necessity (Sect. 98), and it is not impossible that certain anaerobic organisms may be able to obtain all the energy they require from chemical actions between inorganic compounds, such as sulphur and potassium nitrate, for example. It depends entirely upon the specific character of the organism itself whether it has or has not the power of utilizing to a sufficient extent the energy latent in a given substance, and hence it is always possible that organisms may be discovered which obtain energy from totally different sources to those with which we are at present familiar ${ }^{1}$. Energy can be obtained from a greater variety of substances by aerobic than by anaerobic respiration, and hence usually only one or a few substances are suitable for the fermentative activity or respiratory metabolism of an anaerobe.

The products of anaerobic respiration may induce further changes; thus sulphuretted hydrogen is a powerful reducing agent, as is also nascent hydrogen. Hence it is often difficult or impossible to say whether a given substance is an actual product of respiratory metabolism, or results from

${ }^{1}$ Fermentative changes need not necessarily always follow precisely the plan given by HoppeSeyler, Pflüger's Archiv f. Physiol., I875, Bd. X1I, p. I; Physiol. Chem., I877, p. II6. See also Ad. Mayer, Gährungschemie, 1895 , 4. Aufl, p. 200, and Frankland, Centralbl. f. Bact., I 894 , Bd. xv, p. 103. 
subsequent secondary reactions (Sects. 77 and 98). Moreover in certain cases the action of free oxygen may be mainly confined to inhibiting these secondary reactions, and thus a marked apparent result may be produced, although the actual anaerobic respiration (or fermentation) continues unchecked. The pronounced general reducing action is probably for the most part, if not entirely, due to certain products of anaerobic metabolism, and is not directly caused by the induction of those affinities, which in aerobic life cause free oxygen to enter into molecular combination with the protoplasm. Most plants, including many ferment-organisms, have no such reducing power, and no such action is exercised upon reagents imbibed by the plasma even when all free oxygen is removed. It is, however, possible that the protoplast in certain cases withdraws oxygen from substances with which the latter is loosely combined, so that certain anacrobes may obtain oxygen from reducible substances ${ }^{1}$.

The reduction of solutions of indigo, litmus, and other substances which do not penetrate the protoplast is undoubtedly due to the action of special metabolic products, for in coloured gelatine the reduction may occur at a certain distance from the bacterial colonies ${ }^{2}$. The sulphuretted hydrogen which is frequently evolved suffices to produce this result, which, however, in other cases is due to a different product, while in others again, no reducing action is exhibited. Saccharomyces is able to evolve a slight amount of this gas under certain conditions, and hence to exercise a corresponding power of reduction ${ }^{3}$.

Nitrates may in part be reduced to nitrites by the action of excretory products, but this does not occur in all cases where indigo is reduced ${ }^{4}$. Nascent hydrogen may exercise very marked reducing powers within the cell and may perhaps be even capable of producing sulphuretted hydrogen from sulphates. This, however, must occur under special conditions only, for usually no reduction of sulphates is induced in spite of the formation of hydrogen, nor does the latter always cause nitrates to be reduced to nitrites ${ }^{5}$.

1 The growth of certain anaerobic bacteria is favoured by the presence of reducible substances (cf. Kitasato u. Weyl, Zeitschr. f. Hygiene, IS9o, Bd. vili, p. 4I, and Bd. IX, p. 96).

${ }^{2}$ Cf. Fliigge, Mikroorganismen, I 896 , Bd. I, p. I69; Huippe, Methode d. Bakterienforschung, I 89 I, 5. Aufl., p. 257 ; Pfeffer, Oxydationsrorgänge, I889, p. 510 , and the literature here quoted; also Smith, Centralbl. f. Bact., i $896, \mathrm{Bd}$. xix, p. I 87 .

${ }^{3}$ Rubner, Archiv f. Hygiene, Bd. xıx, p. I 74 ; Beyerinck, Centralbl. f. Bact., I 895 , Abth. ii, Bd. I, p. 5 ; Nastukoff, Compt. rend., I 895 , T. CXxi, p. 535, and Ann. d. l'Inst. Pasteur, I895, T. $\mathrm{Ix}, \mathrm{p} .766$.

4 The literature on reducing bacteria is given by Burri u. Stutzer, Centralbl. f. Bact., I 895 , Abth. ii, Bd. I, p. 259. Cf. also Rubner, Koch's Jahresb. d. Gährungsorganismen, I $8_{93}$, p. 93 ; Leyerinck, Centralbl. f. Bact., I 895 , Bd. I, p. $5^{8}$ (Methods). According to Laurent (Ann. d. l'Inst. Pasteur, I 890 , T. IV, p. 742), a few mould-fungi exercise a reducing action, which power Beyerinck states is absent from yeast (Butylalkoholgährung, I $89.3, \mathrm{p} .4^{8}$ ). No reduction of nitrates to nitrites occurs in higher plants (Molisch, Sitzungsb. d. Wien. Akad., I887, Bd. XCv, Abth. i, p. 242).

${ }^{5}$ Outside of the cell nascent hydrogen reduces nitrates, but not sulphates (Fitz, Ber. d. Chem. Ges., I876, p. I349; I879, p. $4^{80}$; Rubner, 1. c., I893, p. 94). 
The same metabolic products may frequently arise in a variety of ways: thus nitrites may also be formed by the oxidation of ammonia (Sect. 63) and sulphuretted hydrogen may not only be produced by proteid-decomposition, but also by the reduction of sulphates, and perhaps by the formation and decomposition of sulphides in plants fed with precipitated sulphur ${ }^{1}$. The manner in which sulphates are reduced is doubtful, and it is possible that in this case the sulphuretted hydrogen results from the decomposition of an intermediate product. Certain bacteria always produce this gas under normal cultural conditions, but in other cases it is formed, as in Saccharomyces, only under very special nutritive conditions.

The liberation of nitrogen (sometimes accompanied by a little nitrous oxide) from nitrates by certain bacteria (Bacillus denitrificans, scc.) is a phenomenon of reduction, and the greater part or almost the whole of the nitrogen of potassium nitrate may be liberated with such rapidity as to cause a foam to appear on the surface of the fermenting fluid ${ }^{2}$. The process is apparently inhibited by the presence of an abundance of oxygen, and hence it is possible that these organisms, which are facultative anaerobes either alone or when associated with other bacteria, may utilize the oxygen of nitrates in the absence of atmospheric oxygen. Organisms may exist which can make similar use of nitrous oxide, although Phanerogams, \&c. have not this power ${ }^{3}$. The liberation of nitrogen by the bacteria mentioned takes place in the absence of ammonia, but in other cases it may be liberated by the decomposition of ammonium nitrite ${ }^{4}$, and this may be the reason for the slight production of nitrogen which usually accompanies the oxidation of ammonia to nitrites by nitro-bacteria ${ }^{5}$. (On the circulation of nitrogen, cf. Sect. 68.)

The reducing and enzymatic actions which may result from vital activity do not form essential factors in respiratory metabolism, and it is indeed not impossible that all fermentative activity is only the result of metabolism, in so far as the latter produces the enzyme responsible for the changes in question. Even when fermentation ceases coincidently with the death of the ferment-organism, this may simply be because the exciting enzyme at once decomposes, or is only able to act under the conditions existing in the living cell. Indeed, Miquel ${ }^{6}$ isolated a urea ferment (urase) only after many futile attempts, and this ferment acts in precisely the same manner as the bacteria which decompose urea. It is possible also that an

${ }^{1}$ Cf. Flïgge, 1. c., p. 17o, and the literature here given; Beyerinck, Centralbl. f. Bact., 1895, I, Bd. I, p. I.

${ }^{2}$ Giltay et Aberson, Archiv. Néerland., I $89 \mathrm{I}, \mathrm{T}$. xxv, p. $34 \mathrm{I}$; Burri u. Stutzer, Centralbl. f. Bact., I 895 , Bd. I, p. 257 ; г 896, Bd. II, p. 473.

3 The supposition that aerobic respiration could be maintained by $\mathrm{N}_{2} \mathrm{O}$ has been disproved by Detmer (Landw. Jahrb., 1S82, Bd. XI, p. 213 ; Möller, Ber. d. Bot. Ges., 18S4, p. 25). Cf. also Correns, Flora, is 92 , p. I 5 o.

- Cf. Loew, Hiol. Centralbl., isqo, Bd. x, p. $5^{89}$.

5 Godlewski, Centralbl. f. Bact., I\$96, Abth. ii, Bd. II, p. $45^{8}$. Cf. Sect. 63.

- Cf. literature by Flügge, Mikroorganismen, 1896, 3. Auf., Bd. 1, p. 21 , and Herfeldt, Centralbl. f. Bact., 1895 , Abth. ii, Bd. I, p. 114. 
enzyme may be obtained from Saccharomyces, which is capable of decomposing sugar into alcohol and carbon dioxide ${ }^{1}$. However this may be, it is quite possible that the energy liberated by an enzymatic decomposition occurring in the plasma may be of direct scrvice to the organism. The lactic fermentation of sugar involves only a very slight liberation of encrgy, and in each case it must be determined whether the fermentative activity is for the purpose of providing a supply of energy, or fulfils quite different functions, whose performance is accompanied by a more or less marked liberation of energy as an accidental corollary. In the case of Saccharomyecs alcoholic fermentation may possibly become unnecessary in the presence of oxygen, provided the whole of the energy required can be obtained by acrobic respiration. Many katalytic actions are indeed known, including such simple hydrolytic decompositions as those induced by diastase, \&c. (cf. Sect. 91), and formic acid may be decomposed into carbon dioxide and hydrogen by fincly divided Iridium, just as in the bacterial fermentation of this acid $^{2}$.

Various marked decompositions may be induced outside of the protoplast by means of enzymes, or cven perhaps by the transference of molecular vibrations, \&c.; indeed Nägeli has suggested that both oxidatory and disintegratory fermentations are extracellular processes. In many cases, however, there is no doubt that the fermentative products are the direct result of metabolic activity; fungi may produce relatively enormous quantities of partially oxidized organic acids, and thus act as ferment-organisms (Sects. 86 and 95). Moreover, in the intramolecular respiration of aerobes alcohol and carbon dioxide are certainly formed directly by the protoplast, and hence it is possible that the alcoholic fermentation in Saccliaromyces is also intracellular. If such fermentative activity is directly connected with metabolism, a knowledge of the causes which regulate the former can only be obtained when a thorough comprehension of the latter has been gained. It is, however, sufficiently clear that the fermentative decomposition of sugar, for example, is not the direct result of the withdrawal of oxygen, and still less can this be the case in those fermentative activities which persist when air is admitted.

Our knowledge of the inherent protoplastic mechanism is too incomplete to afford a sound basis for any theory concerning the phenomena of respiration, and hence also of fermentation. It is possible that the combination of oxygen with the substances consumed in respiration may be induced by specific molecular vibrations transferred to these substances by the living protoplasm, and Nägcli ${ }^{3}$ built up

1 E. Buchner states that the expressed sap of yeast has this power (Ber. d. Chem. Ges., I $\delta_{97}$, pp. 117 , IIIO), and his results have been confirmed from several sources. Cf. Sect. 9 I.

2 IIoppe-Seyler, Zeitschr. f. physiol. Chem., IS87, Bd. XI, p. 566. Cf. Meyer u. Jacobson, Lehrb. d. org. Chem., I $S_{93}$, Ld. I, p. 3 I9

3 Theorie d. Gährung, is 79 , p. 29 
a theory of fermentation upon this assumption, and postulated the possibility of an extracellular extension of these vibrations, and hence of vitalistic fermentation also. Nägeli cites the extracellular reduction of litmus as an argument in support of this theory, but this reduction is not, however, performed by pure cultures of actively fermenting yeast, and Chudiakow ${ }^{1}$ has shown that Nägeli's other arguments do not coincide with the facts observed. The small size, and hence relatively enormous surface-area, of ferment-organisms render possible more rapid exchanges than are required by the most active intracellular fermentations. (Nägeli, 1. c., p. 37.)

Historical. After Pflüger had indicated the decomposition continuing in the absence of oxygen as being the direct cause of oxygen-respiration in animals, Pfeffer pointed out the genetic relationship between intra-molecular- and oxygen-respiration in plants ${ }^{2}$, and subsequently put forward the conclusions here expounded ${ }^{3}$. The same studies definitely established the relationship between aerobic and anaerobic respiration which had been denied by Godlewski, Bowdin, Reinke, \&c., and proved the incorrectness of Nägeli's supposition that intra-molecular respiration is a meaningless physiological phenomenon. It was also proved that molecular oxygen is drawn into metabolism without being ozonized (Sect. Ior), and that in the absence of oxygen no such reducing influences are generated in the protoplast as would withdraw oxygen from easily reducible substances (Sect. 102).

Pasteur assumed the existence of a certain connexion between intra-molecular respiration and anaerobic fermentation, and Pfeffer showed that the latter was simply a specially adapted modification of the former, and in certain cases acquires so inherent a character that the fermentative activity of anaerobic metabolism may continue or even be accelerated in the presence of free oxygen. This latter fact does not coincide with Pasteur's view ${ }^{*}$ that in the absence of free oxygen this gas is withdrawn from other compounds and thus directly excites fermentative activity, but at a time when oxygen-respiration was regarded as an absolute essential for life, this theory served to render the phenomenon of anaerobiosis discovered by Pasteur more readily comprehensible. At a later date Pasteur ${ }^{5}$ apparently laid no especial importance upon this theory, but simply regarded the process of fermentation as the source of the necessary supply of energy. The same general view applies also to those cases of anaerobiosis which are not characterized by any special fermentative activity, for the utilization of the liberated energy is not dependent upon the quantity of material decomposed, and in aerobes the necessary energy may be obtained with or without the aid of fermentation. Since katabolism continues in adult organs, Pasteur was in error

${ }^{1}$ Chudiakow, Landw. Jahrb., IS94, Bd. xxiı, p. 454. Cf. also Ad. Mayer, Zeitschr. f. Biol., I $88_{2}$, Bd. Xvili, p. 523 ; Lafar, Techn. Mykologie, I 997 , Bd. I, p. 20 (here the different theories of fermentation are mentioned).

${ }^{2}$ Pfliuger, Archiv f. Physiol., I 875 , Bd. x, p. 25 I ; Pfeffer, Landw. Jahrb., I 878 , Bd. vil, p. So5.

${ }^{3}$ Pfeffer, Unters. a. d. Bot. Inst. z. Tübingen, I $88_{5}$, Bd. I, p. $6_{3} 6$; Oxydationsvorgänge, I 889 , p. 480 .

${ }^{4}$ Pasteur, Compt. rend., IS6I, T. LII, p. I260.

${ }^{8}$ Ibid., I $S_{75}$, T. Lxxx, p. $45^{2}$; Etudes s. 1. bière, I $S_{7} 6$, p. ${ }_{25} \delta$. 
in supposing that growth was a necessary accompaniment of fermentative activity. I icbig ${ }^{1}$ recognized the distinction between the two processes but regarded fermentation almost solely from a chemical point of view, and failed to recognize its physiological importance.

In every case it depends upon the specific properties and capabilities of the organism itself whether a given substance can be assimilated and used as a source of energy, and hence the fermentative activity of a living organism is a problem of metabolism. Even Nägeli's theory of fermentation fails to recognize with sufficient clearness the intimate connexion existing between the two. Fermentation was first clearly indicated as a physiological process owing to the works of Schwann, Schröder, Dusch, and Pasteur ${ }^{2}$.

\section{Section I03. General View of Certain Fermentative Activities.}

The power of exciting fermentation and the relation of the latter to growth varies in different ferment-organisms ${ }^{3}$, which are in some cases able to ferment many different substances. but in others only a few or a single one. The latter is more commonly the case in disintegratory fermentation, such as is induced by beer-yeast, than in typical oxidatory fermentation, such as the oxalic fermentation which certain omnivorous fungi can bring about in a variety of nutrient media. In many fermentations only one or two products are formed, but in other cases these are numerous; indeed every one of the substances which may result from respiratory metabolism (Sect. $9^{8}$ ) will probably be found as a product of some fermentative activity or other.

Fermentative activity is only exercised when the whole or a portion of the energy must be obtained by its means. The growth of Penicillium or Aspergillus remains about the same whether an abundant supply of sugar is fermented to oxalic acid, or whether it undergoes complete physiological combustion ${ }^{4}$. According to Liborius ${ }^{5}$ Bacillus prodigiosus grows as a fermentative anaerobe in the presence of sugar, but under other conditions does not exhibit any fermentative activity. Fermentation is not a necessary accompaniment of anaerobic life, and hence it may be possible, as in the case of pigment-production, to suppress permanently or temporarily the fermentative activity, and thus to obtain races which may or may not recover this power. Fitz ${ }^{6}$ states, indeed, that the latter occurs in Bacillus

${ }^{1}$ Liebig, Ann. d. Chem. u. Pharm., I87o, Bd. CliII, p. I.

${ }^{2}$ Cf. Flügge, Mikroorganismen, I 896 , 3. Auf., Bd. I, pp. 6, 266 ; Lafar, Techn. Mykologie, $18_{97}$, Bd. I, p. 6 ; Ad. Mayer, Die Gährungschemie, $18_{95}$, 4. Aufl, pp. 20 , \&c.

${ }^{3} \mathrm{Cf}$. the text-books mentioned above, as well as P. Frankland, Centralbl. f. Bact., I 894, Bd. xv, p. 103 .

"Wehmer, Bot. Zeitung, 1891, p. 553. Cf. Sect. 85 .

3 Liborius, Zeitschr. f. Hygiene, i 886 , Bd. I, p. 172.

${ }^{6}$ Fitz, Ber. d. Chem. Ges:, 1882, p. 867. According to Wehmer (Centralbl. f. Bact., 1897 , 
butylicus as the result of prolonged cultivation at a high temperature, or owing to the presence of an abundance of oxygen. Grimbert ${ }^{1}$ has shown that Bacillus orthobutylicus, after previous cultivation on dextrose, ferments inulin with a copious production of butyl-alcohol, and there is other evidence to show that the power of exciting fermentation may undergo more or less marked modification.

It is possible that in certain cases the fermentative activity is simply the result of an increase in the essential respiratory katabolism (Sect. 102), or is due to special processes which accompany and run parallel with the latter. It has already been mentioned that during the aerobic existence of yeast the fermentation of alcohol may no longer be essential, or may cease to be of use in respiratory metabolism although it still persists. Hence in such cases it may be possible to distinguish clearly between accidental processes of fermentation and those which form an essential feature of respiratory metabolism. During aerobic existence the greatest amount of energy is obtained by complete combustion into carbon dioxide and water, but it often happens that the regulatory production of acids becomes of great importance (Sect. 85), and indeed the accumulation of acids, alcohol, \&c. may be of great use in the competition with other organisms provided that the plant is relatively resistant to the injurious effect exercised by its own products. From a general economic point of view the partial decompositions induced by anacrobes, as well as by certain aerobes, are of the highest importance, and many lower organisms work quite as economically as do certain fungi, although the latter induce the complete combustion of enormous masses of organic material (Sect. 95), and would certainly be termed ferment-organisms if other products than volatile carbon dioxide and water resulted from their intense disintegratory activity. In every plant only a certain portion, and often a very small fraction, of the food is used for plastic and formative purposes (Sects. 50 and 77), and in alcoholic and lactic fermentations 95 per cent. of the sugar supplied may be fermented, and only 5 per eent. permanently assimilated.

A production of secretory and excretory products is a constant accompaniment of all metabolism, and since the extent to which the respiratory materials undergo total combustion varies within wide limits, it is evident that no sharp line of demarcation can be drawn between fermentation and respiration. Thus according to circumstances Aspergillus may produce much or little oxalic acid from sugar, Mucor raccmosus much alcohol or none at all according as oxygen is absent or present. It is, however, permissible to use the popular term fermentation to include all

Abth. ii, Iid. III, p. 102), certain varieties of Aspergillus niger have not the power of exciting oxalic fermentation.

1 Ann. d. l'Inst. Pasteur, I 893 , T. vir, p. 40 I. 
those cases of marked physiological decomposition which are not the result of complete physiological combustion. Minute lower plants are especially capable of excreting non-volatile products, and a very large number of hetcrotrophic fungi and bacteria possess the power of exciting fermentation (cf. Sect. 94). A marked evolution of gas, to which Beyerinck ${ }^{1}$ attaches primary importance, is not a necessary accompaniment of fermentation, and indeed appears never to be set up by certain anaerobes (Sect. 98). When it occurs, it may serve to ensure continual agitation and admixture, as well as to carry acrobic ferment-organisms to the surface where a supply of oxygen may be secured. The term ferment-organisms is, therefore, mercly a conventional one of somewhat vague application, and even although an alcoholic enzyme has been isolated the plant which produces it still remains a ferment-organism (Sect. IO2).

For a fuller discussion of the better known fermentations than is possible here, reference may be made to the works by Lafar, Flügge, Ad. Mayer, Schützenberger, \&c., which have been already mentioned.

Alcoholic fermentation ${ }^{2}$. The power of fermenting sugar into ethylalcohol and carbon dioxide $\left(\mathrm{C}_{6} \mathrm{H}_{12} \mathrm{O}_{6}=2 \mathrm{C}_{2} \mathrm{H}_{6} \mathrm{O}+2 \mathrm{CO}_{2}\right)$ is not possessed by all plants, although in certain species of Mucor it may become almost as pronounced as in the case of the more active yeasts, which are able to carry on a prolonged anaerobic existence by means of their fermentative activity (Sect. 98).

The different forms of yeast are able to ferment only certain hexoses, as well as a few artificially produced trioses and nonnoses, but not tetroses, pentoses $^{3}$, heptoses, and octoses. Moreover, polysaccharides can be fermented only when they are first converted into monosaccharides, and this takes place by the agency of an enzyme, which has ultimately been detected in all cases, and which appears usually but not always to be excreted (cf. Sect. 91). The latter is the casc in Monilia candida, which was formerly regarded as being directly able to ferment cane-sugar. Saccharomyces octosporus can neither invert nor ferment a polysaccharide, though it is capable of splitting and fermenting maltose. Saccharomyces Marxianus has properties which are precisely the opposite of those possessed by S. octosporus. An enzyme which decomposes milk-sugar has been isolated from those yeasts which ferment lactose ${ }^{4}$, and various negative

1 Beyerinck, Centralbl. f. Bact., I $\delta_{92}$, Bd. XI, p. 73 ; Butylalkoholgährung, I $\delta 93$, pp. 44, $5 \mathrm{I}$. Cf. also Flïgge, Mikroorganismen, IS96, Bd. I, p. 219 ; Ad. Mayer, 1.c., p. 19; Lafar, l. c., p. 23; Wehmer, Centralbl. f. Bact., I 894 , Bd. xv, p. 544 .

2 In addition to the works of Flügge, Lafar, and Ad. Mayer, cf. Jörgensen, Mikroorganismen d. Gährungsindustrie, $1 S_{92}$; Hansen, Unters. a. d. Praxis d. Gährungsindustrie, $1 \delta_{90}$, and Meddelelser f. Carlsberg Laboratoriet, I 888 , Bd. II, p. I43; Lintner, Handb. d. landw. Gewerbe, I 893 , \&c.

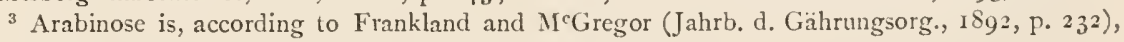
fermented by certain bacteria into ethyl-alcohol and acetic acid.

4 Details by Tollens, Handb. d. Kohlenhydrate, I 595, Bd. Ir, p. 4 . Of the newer works, cf. 
results show that cane-sugar cannot be inverted by the protoplasm without the intervention of an enzyme (Sect. 91). Hence it may be concluded that reserve and plastic carbohydrate materials are utilized for fermentation only in so far as they can be converted into monosaccharides.

The fermentability of different hexoses varies widely: thus yeasts ferment dextrose and laevulose, but not their optical counterparts, although it is uncertain whether or not the latter may be used as food by the aid of oxygen-respiration. It is, however, well known that two stereo-isomeric substances may have a widely different nutritive value (Sect. 66), and of two structurally different compounds, such as dextro-rotatory glucose (an aldose) and dextro-rotatory fructose (a ketose), certain yeasts preferably ferment the former, others the latter. Bacillus butylicus is, however, able to carry on alcoholic fermentation when supplied with glycerine ${ }^{1}$, and there is no reason to suppose that the glycerine is previously converted into sugar.

Nägeli and other authors ${ }^{2}$ have shown that the fermentative activity of normally cultivated yeast is not suppressed even in the presence of an abundance of oxygen, but on the other hand the same amount of alcohol is produced as before. In Giltay and Aberson's experiments, however, about 2 I per cent. of the sugar was consumed in oxygen-respiration, and hence relatively less alcohol was produced in relation to the amount of sugar consumed, while since aëration caused more rapid multiplication it is evident that the fermentative activity of the individual yeast-cells must have been correspondingly reduced. Hence in the absence of oxygen the fermentation of sugar is more perfect and economical, while in its presence the aid of oxygen-respiration enables the plant to employ the sugar consumed to better purpose in growth and reproduction. In the absence of oxygen, yeast remains living and capable of exciting fermentation for a long time, but it is impossible to say whether under prolonged periods of experimentation the conjoint maximum of growth and of fermentative activity would occur with a continuous deficiency of oxygen or when air was only intermittently supplied.

Chudiakow found that in the presence of peptone or malt-extract aëration does not diminish the fermentative activity of yeast, whereas if ammonium salts provide the sole source of nitrogen the evolution of carbon dioxide gradually

E. Fischer, Ber. d. Chem. Ges., I 895 , pp. 984, 3024, 3034, and Beyerinck, Centralbl. f. Bact., I895, Abth. ii, Bd. I, p. 224.

1 Buchner, Zeitschr. f. physiol. Chem., I $88_{5}$, Bd. Ix, p. 393 .

${ }^{2}$ Nägeli, Theorie d. Gährung, I 879 , p. I 7 ; Giltay u. Aberson, Jahrb. f. wiss. Bot., 1894, Bd. xxvi, p. 543 ; Chudiakow, Landw. Jahrb., I 894 , Bd. xxir, p. 428; Iwanowsky, Jahresb. d. Gährungsorg., I 894 , p. I 6 . In these works the other researches are given. It is not necessary to discuss the correct usage of the term 'fermentative power.' Cf. Giltay u. Aberson, 1.c., and Jahrb. f. wiss. Bot., I 896 , Bd. xxx, p. 7 I ; A. Brown, Centralbl. f. Bact., 1897 , Bd. IIr, p. 33. None of the above designations are clearly defined constants. 
decreases. Chudiakow supposes that in the latter case the change in the nutritive conditions operates injuriously and thus inhibits fermentation, but since growth continues it is possible that the stoppage is produced in the same manner as in the case of Hucor. It is well known that certain bacteria grow or die in the presence of air according to the cultural conditions. Rapp ${ }^{1}$ was unable to confirm Chudiakow's results; he also observed that strong agitation diminishes the fermentative activity of yeast. Hence Chudiakow's results can only be explained by supposing that the sensitiveness of yeast to agitation, such as is caused by the passage of streams of air-bubbles, varies according to the culture-medium.

The activity of fermentation depends upon the supply of sugar, the multiplication of the yeast-cells, the accumulation of the products, the temperature, \&c. (Sect. IO4). The most actively fermenting yeasts withstand percentages of alcohol which are fatal to most plants, for alcohol may accumulate in a fermenting liquid to the extent of It per cent. by volume, although the growth of the yeast is checked when 12 per cent. is reached $^{2}$. Hansen found that with Mucor ercctus and $M$. raccmosus as much as 8 per cent. might accumulate, but in the case of Mucor mucedo the culture fluid only contained 3 per cent. of alcohol after three months activity $^{3}$. Hence many seedlings and fruits exhibit a more marked production of alcohol in the absence of oxygen than Mucor mucedo does (Sect. 99).

Even the best yeasts use 5 to 6 per cent. of the sugar in producing glycerine, succinic acid, acetic acid, and small quantities of other substances, both the absolute and relative amounts of which vary during the progress of fermentation and according to the external conditions. Many plants form in addition to alcohol large quantities of other substances, and in certain bacterial fermentations ethyl-alcohol appears only as a by-product. As a general rule the amount of by-products produced by yeast increases under unfavourable conditions, but at $30^{\circ} \mathrm{C}$. Nägeli ${ }^{4}$ found that beer-yeast was able to ferment forty times its own dry weight of cane-sugar in twenty-four hours, and that almost the whole of this sugar underwent alcoholic fermentation.

A similar fermentative activity does not indicate any systematic relationship between plants. This statement applies to the yeast-like growths formed by certain fungi under conditions which excite fermentative activity ${ }^{5}$.

1 Rapp, Ber. d. Chem. Ges., I 896 , p. I 983 .

2 Cf. Kayser, Jahresb. d. Gährungsorg., I S92, p. I I 4 ; Wortmann, Landw. Jahrb., I S94, Bd. xxili, pp. $54^{8}, 555$.

${ }_{3}$ Hansen, Meddelelser f. Carlsberg Laboratoriet, I882, Bd. II, p. I60; Brefeld, Landw. Jahrb., I 876 , Bd. v, p. 305 ; Pasteur, Étude s. 1. bière, I 876 , p. I33; Lesage, Ann. d. sci. nat., I 897 , vii. sér., T. III, p. I 5 I.

* Nägeli, l. c., p. 32. See also Giltay u. Aberson, l. c., I 894 , p. 560.

${ }_{5}$ Cf. Klebs, Die Bedingungen d. Fortpflanzung, \&c., I896, p. 509; Schostakowitsch, Flora, Erg.-bd., I 895, pp. 37I, 39I. 
Various authors, among whom Jörgensen may be mentioned, have attempted to show that Saccharomyces is simply a growth-form of other fungi, but hitherto without success ${ }^{1}$. Hansen has shown that cultural varieties may be produced from several of the species of Saccharomyces.

Butyric fermontation. Different authors ${ }^{2}$ have observed that butyric acid, which commonly appcars during putrefaction, forms a main or byproduct of the metabolism of many bacteria. Certain of these are obligate acrobes, but most are obligate or facultative anacrobes, among which the nitrogen-assimilating Clostridium Pasteurianum is included (Sect. 69). All the species examined ferment dextrose and certain other substances as well, while the anaerobic Clostrillium foctidum of Liborius and Hüppe's aerobic Bacillus butylicus energetically decompose proteids with a production of malodorous gases. Hydrogen and carbon dioxide are the only gases which are formed during the butyric fermentation of carbohydrates, but other products may also appear. The relative amounts of the different products vary according to the food-supply and the progress of fermentation, and are also dependent upon the external conditions, as has been shown by Fitz, Perdrix, Grimbert, Beyerinck, Duclaux, \&c. Different species decompose the same food-material in different ways, and although lactic acid may appcar as an intermediate product in some cases, the butyric fermentation of sugar certainly does not in all cases follow this course, for Botkin ${ }^{3}$ found that a certain butyric bacterium is quite unable to ferment lactates.

According to Grimbert the anaerobic Bacillus orthobutylicus ferments dextrose, cane-sugar, maltose, milk-sugar, starch, dextrin, inulin, mannite, arabinose, and glycerine, but does not attack gum-arabic, erythrite, glycol, calcium lactate and tartrate. During the fermentation of sugar an abundance of butyric acid is formed as well as large quantities of butyl-alcohol, acetic acid, hydrogen, and carbon dioxide. The hydrogen diminishes in relation to the carbon dioxide evolved as fermentation progresses, while at the same time the amount of butyric and acetic acids produced decreases, but that of butyl-alcohol increases. Apparently the accumulation of free acid exercises a certain inhibitory action, for the addition of chalk causes the production of acid to increase again. The properties of these and other bacteria are subject to transitory or permanent modification, and this makes

${ }^{1}$ Klöcker u. Schiönning, Centralbl. f. Bact., 1896 , Abth. ii, Bd. II, p. I85; Meddelelser f. Carlsberg Laboratoriet, 1896 , Bd. v, p. 66 ; Hansen, Centralbl. f. Bact., i $8_{95}$, Abth. ii, Bd. I, p. 859 .

2 Thus: Prazmowski, Unters. ï. Fermentwirk. einiger Bacterien, ISSo; Fitz, Ber. d. Chem. Ges., $188_{2}$, p. $867 ; 188_{4}$, p. 188 ; Hiippe, Mitth. a. d. k. Gesundheitsamt, $188_{4}$, Bd. II, p. 319 ; Perdrix, Ann. d. l'Inst. Pasteur, 1891 , T. v, p. 287 ; Grimbert, ibid., 1893 , Bd. vIl, p. 353; Beyerinck, Butylalkoholgährung, 1893 (Sep.-abdr. a. d. Verh. d. K. Akad. d. Wetenschappen, Amsterdam);

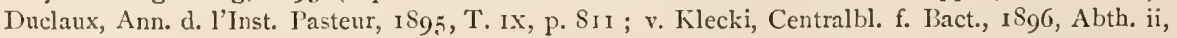
Bd. II, p. 288 . Summary by E. Baier, Centralbl. f. Bact., I 895 , Abth. ii, Bd. 1, p. 17 ; Fligge, l.c., p. 236 ; Lafar, l. c., p. 164 .

s Jotkin, Zeitschr. f. Hygiene, I 892 . Hd. XI, P. 432. 
the identification of the species employed by different authors correspondingly difficult. Hence it is uncertain how far the following species studied by Beyerinck are identical with those of other authors. According to Beyerinck, (r) Granulobacter saccharobutyricum (anaerobe) is the common butyric bacterium which ferments sugar producing butyric acid and also carbon dioxide, hydrogen, and a little butyl-alcohol. It may be identical with Fitz's Bacillus butylicus, and allied to $B$. orthobutylicus. (2) Granulobacter butylicum is also an anaerobe and forms in addition to carbon dioxide and hydrogen, mainly butyl-alcohol, and a little butyric acid when fermenting dextrose, but none from maltose. (3) Granulobacter lactobutylicum is able as an anaerobic Clostridium form to ferment calcium lactate, forming calcium butyrate, carbon dioxide, hydrogen, and a few by-products, but when exposed to air the power of fermentation is lost and the calcium lactate is converted into carbonate without any formation of butyric acid. When cultivated as an anaerobe this species perishes after exciting a slight amount of fermentation, and hence it is possibly an example of an organism in which an alternation from aerobiosis to anaerobiosis is necessary for continued existence (Sect. 97).

Lactic formentation. As the result of the fermentation of various sugars by different aerobic and anaerobic bacteria, lactic acid appears as a main or by-product. Kayser ${ }^{1}$ experimented with a large number of lactic bacteria and found that under favourable circumstances 95 per cent. of the sugar was converted into lactic acid $\left(\mathrm{C}_{6} \mathrm{H}_{12} \mathrm{O}_{6}={ }_{2} \mathrm{C}_{3} \mathrm{H}_{6} \mathrm{O}_{3}\right)$, while at the same time carbon dioxide and traces of acetic, formic acids, \&c. were produced. In very many cases but little carbon dioxide is formed, and indeed it occasionally happens that no gaseous products at all appear (Sect. 98). But little cnergy is derived from the direct lactic fermentation of sugar, and hence the process may not be of primary importance in the respiratory metabolism of lactic bacteria. Owing to the sensitiveness of these to free acid the fermentative activity soon ceases unless chalk is added, but the amount of acid which may accumulate varies according to the species and the cultural conditions, both of which influence the ratio between the main and by-products. A bacterium which normally produces an optically inactive form of lactic acid may under different conditions form an optically positive or optically negative variety of lactic acid 2 .

Acetic fermentation. A small quantity of acetic acid is commonly produced in both oxidative and disintegratory fermentations, and in the oxidation of ethyl-alcohol by certain bacteria acetic acid is formed in abundance. According to Lafar a certain form of yeast has the same power ${ }^{3}$. These acetic bacteria, of which Hansen's researches have made Bacterium

${ }^{1}$ Kayser, Ann. d. l'Inst. Pasteur, $18_{94}$, T. viII, p. 737. Cf. also Fliigge, 1.c., p. 232 ; Lafar, 1. c., p. 200 , and the literature here given.

2 Cf. Pfeffer, Jahrb. f. wiss. Bot., I 895 , Bd. xxvili, p. 226.

3 Lafar, Centralbl. f. Bact., I893, Bd. xin, p. $68_{4}$. Cf. Flügge, 1. c., p. 248 ; Lafar, 1.c., p. 343 ; Ad. Mayer, 1.c., p. 170; Henneberg, Centralbl. f. Bact, $1 \$_{97}$, Abth. ii, Bd. III, p. 223 . 
aceti and $B$. Pastcurianum ${ }^{1}$ especially well known, obtain their energy by this process of oxidation, and so long as alcohol is present no carbon dioxide appears to be formed (Sect. 96), although it is produced in abundance when in the absence of alcohol the acetic acid is still further oxidized. Here alcohol protects acetic acid, but this is not the case with Mycoderma aceti $^{2}$. Similarly, acetic acid is consumed by Penicillium even when the most suitable organic food is supplied (Sect. 67). The process is a vital one and ceases at death ${ }^{3}$, although it is not certain whether it is solely intracellular or in part extracellular (Sect. IO). An apparently similar oxidation may be induced by finely divided platinum, but this affords no conclusive evidence as to what occurs in the living cell. According to A. J. Brown ${ }^{+}$ acetic bacteria are able to oxidize propyl-alcohol to propionic acid, but they do not attack butyl-, amyl-, or methyl-alcohol. It is not yet known whether these bacteria will grow upon glycerine, sugar, \&c., and what products are then formed. Acetic fermentation is most active betwcen $20^{\circ} \mathrm{C}$. and $30^{\circ} \mathrm{C}$., and ceases to be provoked by B. Pastcurianum at $4{ }^{\circ} \mathrm{C}$, but continues to be set up slowly by $B$. aceti at this temperature ${ }^{5}$. Under favourable conditions acetic acid may accumulate to the extent of even I4 per cent. before further activity ceases ${ }^{6}$. (On oxalic and citric fermentations, cf. Sects. 85 and 86 ; on other oxidations, Sect. 96.)

Conjoint actions of different organisms may induce productive activities of which the individual organisms are incapable (Sects. 51, 64, 92). This is seen when a bacterium prepares a particular substance for assimilation by other plants to which it was previously inaccessible, or when cellulose is converted into fermentable sugar ${ }^{7}$. Putrefaction is due to the action of several bacteria, and differs according to the putrefying substances and other conditions. Proteid putrefaction yiclds an especially large number of products, including many malodorous gases. When well aërated these and many other products do not appear, owing to the fact that the activity of the obligate anaerobes is suppressed and that of facultative anaerobes modified (Sect. I02), while obligate or facultative aerobes attain the upper hand. The latter may oxidize any sulphuretted hydrogen

${ }^{1}$ Hansen, Med. f. Carlsberg Lab., I $8_{94}$, Bd. III, p. I $\delta_{2}$.

${ }^{2}$ Cf. Beyerinck, Centralbl. f. Bact., I $8_{92}$, Bd. xı, p. 7 I.

${ }^{3}$ Kinerim u. Ad. Mayer, Versuchsst., I $8_{73}$, Ld. xvi, p. 305 .

* A. J. Brown, Journ. Chem. Soc., i 896 , Vol. Xlix, pp. 172, 432; $188_{7}$, p. 638 ; Seifert, Centralbl. f. Bact., I $8_{97}$, II, Bd. III, p. 336. Nägeli supposed that they.could decompose methylalcohol.

${ }^{5}$ Lafar, Centralbl. f. Bact., I 895 , Abth. ii, Bd. I, p. I 45 .

${ }^{6}$ Lafar, l. c., p. I 39 ; Steinmetz, Jahresb. d. Gährungsorg., I 892, p. 243 .

${ }^{7}$ Certain organisms can not only dissolve cellulose, but also ferment the sugar produced. On cellulose fermentation, cf. Fliigge, l. c., p. $24 \mathrm{I}$; Herfeldt, Centralbl. f. Bact., I 895 , Abth. ii, Bd. I, p. I 6 ; Omelianski, Compt. rend., I 895 , Nov. On mixcd cultures, cf. Sects. 64,92 . On the gingerbeer plant, ef. Marshall Ward, Phil. Trans., I 892 , p. I 25. On Kefir, cf. Flïgge, l.c., p. 262 ; Freudenreich, Centralbl. f. Bact., $1 \$_{97}$, Abth. ii, Bd. IIr, p. 87 . 
present and thus prevent any accumulation even if such products are still formed. For an account of the processes of putrefaction, cf. Flügge, 1. c., p. 254; Lafar, 1. c., p. 260; Herfeldt, Centralbl. f. Bact., I 895, Abth. ii, Bd. I, p. 74 .

\section{SECTION 104. The Influence of the External Conditions.}

Any change in the external conditions will as a general rule exercise some effect or other upon respiratory metabolism, and hence any induced modification of growth or movement will be accompanied by a certain alteration in the respiratory activity. This has not been proved in all cases, but all the experimental evidence seems to show that at a constant temperature growth and movement are accompanied by increased respiration, although the reverse does not necessarily follow. Respiration rapidly accommodates itself to changed conditions, but when any injurious effect is produced a return to the original activity may be gradual, and may only be completed some time after the original conditions have been restored. A change in the respiratory activity may not directly result from the altered conditions, but may be due to a regulatory reaction leading to the assumption of a new condition of equilibrium. Many mechanical and chemical stimuli which are not sufficiently powerful to depress vital activity may induce a transitory or permanent increase in the respiratory activity, and this may in some cases be accompanied by more rapid growth.

Temperature. Respiration ceases in living turgid plants only at comparatively low temperatures, being still feebly active at -2 to $-4^{\circ} \mathrm{C}$., and perceptible even at $-10^{\circ} \mathrm{C}$. in the case of conifers and lichens ${ }^{1}$. It continually increases as the temperature rises, until the injurious action of the high temperature becomes manifested by a decrease in the respiratory activity at once or after the lapse of a certain latent period. Hence respiration exhibits no distinct optimum lying within limits of temperature which can be endured for prolonged periods (cf. Fig. 50), whereas growth and assimilation exhibit optimal points beyond which the activity decreases without any permanent injury being necessarily produced. It is possible that plants are unabie to regulate their respiratory activity, and hence at high temperatures necessarily consume a larger amount of food-substances ${ }^{2}$, while at the same time the increased production of heat tends to lower the maximal temperature which the plant can withstand.

1 Jumelle, Rev. gén. d. Bot., I $\$_{93}$, T. Xxv, p. 599. Cf. also Kreusler, Landw. Jahrb., I $\$ 88$, Bd. xwir, p. I 72 ; Clausen, ibid., I $\$_{90}$, p. $8_{97}$; Ziegenbein, Jahrb. f. wiss. Bot., I $8_{93}$, Bd. xxv, p. 599. The older literature is here qtoted (Ad. Mayer, Rischavi, Pedersen).

2 On the alteration of the economic coefficient according to temperature, cf. Sects. 66 and 95 . On the relationship between $\mathrm{CO}_{2} \cdot$ assimilation and respiration, Sect. 58 . 
Both the older and later researches ${ }^{1}$ have shown that the respiratory curve possesses the character indicated, while the existence of an optimum is still uncertain and the latter must in any case be near the critical temperature. In Clausen's and Ziegenbein's experiments the plants were apparently temporarily injured and did not immediately assume their original respiratory activity when returned to the previous temperature, as should properly be the case. Hence the curve constructed by these authors was a secondary one resulting from prolonged exposure, and was not due to the direct and immediate action of the rising temperature. Respiration may be twenty to forty times more active at the maximum temperature than it is at $\circ^{\circ} \mathrm{C}$., and the activity frequently increases in geometric proportion as the temperature rises to the maximum, while the respiratory quotient $\mathrm{CO}_{2}: \mathrm{O}_{2}$ usually but not always remains constant.

Owing to the influence of temperature upon growth and hence ultimately upon the mass of respiring protoplasm, a certain permanent optimal temperature exists for growing plants at which the total amount of respiration is greatest, while above this point the higher respiratory activity becomes ultimately unable to counterbalance the diminished bulk due to the decreased growth. This optimum lies between 25 to $30^{\circ} \mathrm{C}$. in the case of alcohol-producing bacteria, and a similar optimum has been observed in other bacterial fermentations in which the fermentative activity increases as the bacteria increase in numbers. According to Chudiakow ${ }^{2}$ the curves of oxygen- and intramolecular-respiration follow a parallel course as the temperature alters, and similarly when the growth of Saccharomyces is inhibited, the fermentative activity continually increases up to the maximum temperature. It is not however permissible to make any generalization from this fact, for fermentative activity may be a special process which is only indirectly connected with anaerobic respiration (Sect. 102), and in deciding this question it would be of the utmost importance if at different temperatures it were found possible to distinguish between these two functions. The production of oxalic acid by Aspergillus is markedly diminished at high temperatures, owing to the increased oxidatory activity, and other oxidizing fermentations may be modified in a similar manner by changes of temperature.

Light. All critical experiments have shown that as a general rule respiration is not markedly influenced by changes in the illumination. Bonnier and Mangin ${ }^{3}$ state that light conmonly induces a slight diminution

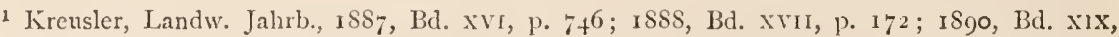
p. 663 ; Clausen, 1.c., I 890, p. $89+$; Ziegenbein, 1.c., I 893 ; Chudiakow, Landw. Jahrb., I894, 13d. XxiıI, p. 349 ; Bonnier et Mangin, Ann. d. sci. nat., $188_{4}$, vi. sér., T. XVII, p. 271 ; I $88_{4}$, T. xvir, p. 359 .

${ }^{2}$ Chudiakow (Landw. Jahrb., I 894 , Bd. Xxili, p. 350) has shown that Amm's contradictory conclusions (Jahrb. f. wiss. 13ot., $\mathbf{r} 893$, Bd. xxv, p. 27) are not justifiable.

${ }^{3}$ Ionnier et Mangin, Ann. d. sci. nat., I $88_{4}$, vi. sér., T. XviI, p. 28 ; ; T. xvili, p. 353 ; also Elfving, Einwirkung d. Lichtes auf Pilze, ISgo, p. $9^{\S}$; Puricwitsch, Bot. Centralbl., iS91, Bd. XLvil, 
in the respiratory activity, and that this is mainly due to the less refrangible rays. The same authors have also directly shown (1.c., 1886, vii. sér., T. III, p. 14), by rendering the chloroplastids inactive with ether, that green plants behave similarly to non-chlorophyllous plants or organs, and it is evident that Pringsheim's hypothesis that light increases respiratory activity arose in connexion with his theory of the action of chlorophyll and was not founded on fact ${ }^{1}$.

The prolonged absence of light must ultimately affect the respiratory activity of all such plants as grow abnormally in darkness ${ }^{2}$, while in autotrophic plants owing to the cessation of carbon dioxide assimilation the plant is gradually starved, and hence its respiratory activity will as a general rule decreasc, as was observed by Borodin ${ }^{3}$ when twigs were kept in continuous darkiness. Hence the respiratory activity of the roots and rhizomes of green plants may decrease slightly during the night when photosynthetic assimilation is feeble, whereas Areboe ${ }^{4}$ could detect no such diminution when assimilation was active. Whenever light injuriously affects the plant and depresses its vital activity, respiration must also diminish, and many bacteria are killed by direct sunlight, or even by bright diffuse daylight, in a comparatively short time. On the other hand, by inducing a more rapid decompssition of organic acids, light may temporarily accelerate the evolution of carbon dioxide in the case of fleshy plants and of fungi (Sects. $5^{6}$ and 86).

Nutritive and poisonous substances. When food is supplied to a starved plant the general activity is increased and hence that of respiration also, while an excessive supply will ultimately retard both. Respiration is, however, by no means directly dependent upon the food supply, and the awakening of growth and respiratory activity occurs in an onion only when the winter rest is over, in spite of the constant presence of a rich store of glucose. It is however possible that the increased respiratory activity cxhibited when frozen potatoes which have become sweet are returned to an ordinary temperature, may be mainly due to the accumulation of sugar ${ }^{5}$, although the latter is not always the cause of the more active respiration which usually follows a sojourn under abnormal conditions ${ }^{6}$,

p. I30; Areboe, Forsch. a. d. Geb. d. Agr.-Phys., I893, Bd. xvr, p. 450. On intramolecular respiration, Wilson, Flora, Iss2, p. $9^{6 .}$

${ }^{1}$ [Kolkwitz has recently found (Jahrb. f. Wiss. Bot. I 898 , Bd. xxxır, p. I 28 ) that light distinctly accelerates respiration in the case of certain fungi, but that this increase does not amount to more than ten per cent. of the previous values. It is however possible that the apparent increase is due to the direct action of light upon the organic acids which these fungi produce. See infra.]

${ }^{2}$ On the respiration of green and etiolated leaves, cf. Palladin, Rev. gén. d. Bot., I 893 , T. v, p. 369 . On that of mosses grown under different conditions, Jönsson, Compt. rend., I 890 , T. CIX, P. $44^{2}$. On the influence of light on potatoes, Ziegenbein, Jahrb. f. wiss. Bot., I893, Bd. xxv, p. $59^{2}$.

${ }^{3}$ Borodin, Bot. Jahresb., i8 76, p. 920.

4 Areboe, 1.c., p. 459. This daily pcriodicity was first observed by Saikewicz, Bot. Jahresb., I $877, \mathrm{p} \cdot \mathrm{723}^{2}$.

5 Miiller-Thurgau, Landw. Jahrb., I\$93, Bd. XI, p. 794; IS85, Bd. Xrv, p. E6o.

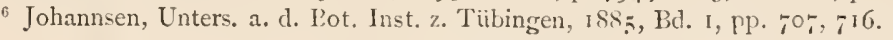

0 O 2 
as for cxample at high temperatures, in compressed air, or in oxygenless atmosphercs ${ }^{1}$.

Small doses of poison may cause a temporary increase in the rapidity of growth, and hence also in that of respiration, although it has yet to be determined whether more sugar is consumed than before in relation to the crop produced. Similarly it is doubtful, whether the accelcration of fermentative activity by traces of poisonous substance ${ }^{2}$ (cobalt, fluorine, chloroform, \&c.) is due to more rapid growth and reproduction or to the greater activity of the individual cells. Adult parts of higher plants react in a somewhat similar manner, for Elfving and Laurén ${ }^{3}$ frequently observed that respiration was more active after treatment with ether or chloroform, provided such treatment was not carried so far as to permanently injure the plant. This reaction is unaccompanied by growth, but when etherization awakens a plant from its winter rest ${ }^{4}$, the resumption of growth permanently increases the total respiration. It has, however, not been found possible to inhibit respiration entirely in turgid plants without death ensuing, and hence it is all the more important to determine the accuracy of Cl. Bernard's statement that alcoholic fermentation can be temporarily inhibited by means of chloroform ${ }^{5}$, and if this be so, whether aerobic or anaerobic respiration can be caused to undergo a similar temporary suspension.

Both growth and respiration are injuriously affected by any excessive increase of respiratory or fermentative products (Sects. 77, 78). Higher plants are usually injured by air containing 4 to I 5 per cent. of carbon dioxide (Sect. 57), and a still higher percentage exerts a depressing influence upon many mould-fungi and bacteria ${ }^{6}$, whereas Saccharomyces and those bacteria which normally grow in the presence of an abundance of carbon dioxide are much more resistant. Fermenting ycast may generate a pressure of twenty-five atmospheres in a closed vessel before the further production of carbon dioxide is inhibited ${ }^{7}$, and those bacteria which may cause tins

1 Maquenne, Compt. rend., I 894 , T. cxix, p. Ioo.

${ }^{2}$ First observed by Schulz, Bot. Zeitung, IS58, p. 610. See also Lintner, Handb. d. Landw. Gewerbe, I893, p. 238 ; Effrant, Compt. rend., I 894 , T. cxix, pp. 254, \&c. On the accommodation

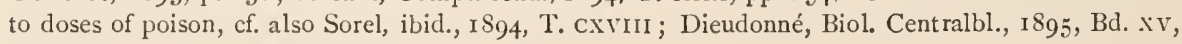
p. 109.

${ }^{3}$ Elfving, Oefversigt af Finska Vctensk. Soc. Forh., ISS6, Bd. xxvir ; Laurén, Bot. Jahresb., IS92, p. 92. Detmer detected no such reaction (Landw. Jahrb., ISS2, Bd. XI, p. 227); nor did Bonnier ct Mangin (Ann. d. sci. nat., I\$86, vii. sér., T'. III, p. I6).

4 Johannsen, Bot. Ccntralbl., I 896, Bd. Lxviı , p. 337.

${ }^{5} \mathrm{Cl}$. Bernard, Leçons s. 1. phénom. d. l. vie, I 879 , T. I, p. 276.

${ }^{6}$ Fränkel, Zeitschr. f. Hygiene, I $\$ 8_{9}$, Bd. v, p. $33^{2}$; Frankland, ibid., I\$9o, Bd. vi, p. I 3 . Additional literature by Lopriore, Jahrb. f. wiss. Bot., I 895 , Bd. Xxvin, p. 531 ; Fligge, Mikroorganismen, I 896,3 . Aufl., Bd. I, p. 445 .

"Melsens, Compt. rend., I87o, T. Lxx, p. 632. According to Lechartier and Bellamy (ibid., T. Lxxv, P. I 203) and de Luca (ibid., I 876 , T. LxxxıI, p. 5I 2 ), fruits may generate a pressure of 
of conserves to explode probably possess a similar power. Sabrazès and Bazin ${ }^{1}$ observed that certain bacteria remained living for days in carbon dioxide at a pressure of fifty atmospheres, whereas in other cases d'Arsonval found that death ensued under these circumstances. The action of the increased pressure of carbon dioxide is apparently a physiological one, for a very much higher pressure would be necessary to direct.y prevent the liberation of this gas. (For the inhibiting action of other fermentative products, cf. Sects. $86,92,103$.)

Percentage of water ${ }^{2}$. Thoroughly dried seeds ${ }^{3}$, mosses and lichens do not respire, although respiration commences when but little water is present and rapidly increases as more is absorbed. On the other hand, the injection of the stomata or intercellular spaces with water renders gaseous interchanges more difficult and thus diminishes the respiratory activity. Hence Kreusler found that leaves respire most actively when in the normal turgid condition. Aubert found this to be the case in plants of the Crassulaceae and Jumelle in lichens.

Injuries and wounds. Owing to the plant's reactive power, a wound becomes covered by callus or wound-cork, and at the same time more or less markedly increased respiratory activity is usually excited. This was first shown by certain isolated observations made by Böhm and by Stich, while Richards has recently studied the phenomenon more in detail and has established its general importance ${ }^{4}$.

Tubers and other resting organs react most markedly: thus 300 grms. of small potatoes evolved at the laboratory-temperature $\mathrm{I} \cdot 2$ to $2 \mathrm{mgrm} . \mathrm{CO}_{2}$ per hour, but after being cut into four pieces $9 \mathrm{mgrm}$. were evolved during the 2 nd hour, I 4.4 during the $5^{\text {th }}, \mathrm{I} 6.8$ during the $9^{\text {th, }} \mathrm{I} 8.6$ during the 28 th, I 3.6 during the 5 Ist, 3.2 per hour after four days, and $\mathrm{I} \cdot 6 \mathrm{mg}$. per hour after six days. In other cases also the reaction is a transitory one, the maximum being attained in leaves usually in a few hours, and these commonly do not even attain a doubled production of carbon dioxide in spite of numerous incisions having been made.

two atmospheres, owing to the carbon dioxide evolved by intramolecular-respiration, but it is possible that micro-organisms may have aided in producing this result.

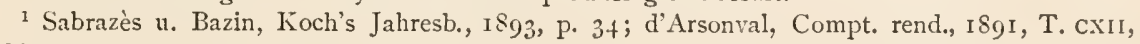
p. 667 .

2 Seeds : Detmer, Landw. Jahrb., I882, Bd. xi, p. 229 ; Krensler, ibid., I885, Bd. Xr, p. 95 I ; I887, Bd. xvi, p. 748. Mosses and lichens: Bastit, Rev. gén. d. Bot., IS9I, T. III, p. 476; Aubert, ibid., I892, T. Iv, p. 379; Jumelle, ibid., I892, T. Iv, p. I69; Lund, ibid., I894, T. vi, p. 353; Jönsson, Compt. rend., I 894, T. Cix, p. 44 I.

${ }^{3}$ [Living seeds always retain a trace of imbibed water, even when dried in a desiccator, and this may amount to as much as I or 2 per cent., or in the case of certain licliens the minimal water percentage consistent with the preservation of vitality may be more than 5 per cent. Cf. Ewart, Trans. Liverpool Biol. Soc., I 897 , XI, p. I 5 ; Schıöder, Unters. a. d. Bot. Inst. z. Tiibingen, I886, Bd. I I, Heft I, p. 5.]

4 Böhm, Bot. Zeitung, IS\$7, p. 686 ; Stich, Flora, 1891 , p. I5; Richards, Annals of Botany, 1896, Vol. x, p. 531. (Mbstract by Pfeffer in Ber. d. Sächs. Ges. d. Wiss., I 896, p. $3^{8} 4$.) 
The traumatic stimulus extends only to a certain distance from the incision, and hence the greatest total effect is produced when a potato or.leaf is cut into a number of pieces, for the respiratory activity subsequently attains the highest possible maximum. A rise of temperature accompanies the increased respiratory activity, and by means of thermoelectric measurements Richards has shown that this ceases to be perceptible in a potato at about 2 centimetres from the injured surface. The rise of temperature is, however, only slightly less marked at this distance in the bulb of Allim cepa, and other facts show that the reaction due to the wound spreads over the entire bulb.

In the absence of oxygen no such reaction is possible in acrobic plants, but an increased intramolecular-respiration is exhibited if the air is replaced by hydrogen after the reaction has commenced. When the primary reaction due to the injury has passed away, the production of carbon dioxide may fall below its original value, owing to the depressed vital activity of the separated parts; this was observed to be the case by Ad. Mayer and Wolkoff ${ }^{1}$ in isolated portions of seedlings, and by Borodin ${ }^{2}$ in cut branches. On the other hand, respiration may undergo a permanent increase when an injury leads to the formation of new shoots or roots. Böhm ${ }^{3}$ has shown that a potato attacked by Phytophthora respires more actively, and it is probable that similar phenomena accompany the formation of galls by fungi or insects.

\section{SECTION 105. The Importance of Respiration.}

A continual supply of energy is necessary for the maintenance of vital activity (Sects. 77 and 94), and hence the possibility of aerobic or anacrobic respiration is a primary essential for all vital processes, including those which do not involve any direct consumption of kinetic energy. Functions of this kind are more open to investigation than the fundamental vital processes which proceed in intimate connexion with acrobic or anaerobic respiration. It is still uncertain whether the katabolic processes by which energy is liberated take place outside the living protoplasmic elements, or involve a continual destruction and regeneration of the latter (Sect. IOI). Still less is known as to the manner in which the energy thus liberated is made available. for the maintenance of vital activity,

1 Landw. Jahrb., IS 74 , Bd. III, pp. 501, 523 .

${ }^{2}$ Borodin, Bot. Jahresb., I $\$ 76, p \cdot 9^{22}$. Miiller-Thurgau observed a decrease in the respiration

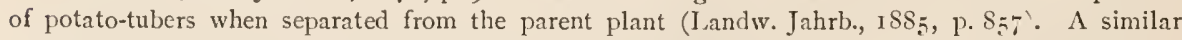
example of the same phenomenon was also observed by Ewart (On Assim. Inhib., Journ. of Linn. Soc., 1896, Vol. xxxin, p. 392) on thawing branches injured by frost.

${ }^{3}$ Böhm, Verh. d. Zool.-Bot. Ges. in Wien, $189^{2}$, Bd. $x \mathrm{xl} 1$, p. 47 . [This increase must not be confounded with that due to the presence of the living and respiring fungus, and any agency which suffices to kill or remove the fungus may act as a stimulus exciting an increased respiratory activity in the potato.] 
and whether it is directly or only indirectly utilized in the performance of the manifold synthetic processes of which the plant is capable.

Energy is obtained in anaerobes in a similar manner to that liberated by the explosion of gunpowder or dynamite, whereas aerobic respiration may be compared with ordinary combustion in the presence of free oxygen (Sect. 94). A production of heat accompanies both aerobic and anaerobic respiration, and the chemical decompositions which accompany fermentative activity usually induce a marked rise of temperature ${ }^{1}$. This heat-production appears, however, to be simply the unavoidable accompaniment of katabolism, for plants do not attempt to maintain a constant body-temperature, but allow the latter to rise and fall with that of the surrounding medium. Hence they have necessarily acquired much greater resistant powers to extremes of temperature than are possessed by warm-blooded animals, whose body-temperature may vary only within comparatively narrow limits. Under favourable conditions the respiratory activity and production of heat is unit for unit more marked in certain plants than it is in the higher animals, but owing to the relatively enormous radiating surface area the temperature of even energetically respiring mould-fungi hardly rises above that of the surrounding medium even when transpiration is suppressed.

Many marked manifestations of energy are not directly the result of respiration; this is the case for example with regard to the osmotic energy by means of which comparatively enormous rcsistances may be overcome, as when a root which grows into a crevice ultimatey bursts a rock asunder. Such external actions may be measured, but it is impossible to determine the actual value of the internal manifestations of energy, and it is still uncertain whother the protoplasm directly utilizes the chemical energy of decomposition, or whether the latter becomes available in some indirect manner (Pfeffer, 1.c., p. 177). Even although only a fractional percentage of the total manifestations of energy directly result from that liberated by protoplasmic respiration, and even though the latter acts mainly in a preparatory or stimulatory manner or simply by providing a supply of energy, it still remains the first essential for the maintenance of vital activity.

Only a portion, and probably only a small portion of the encrgy liberated by respiration is converted into chemical or mechanical work, for according to Rodewald ${ }^{2}$ almost the whole of the energy actually liberated appears in the form of heat. Even when the production of

${ }^{1}$ Cf. Pfeffer, Studien z. Linergetik, I 892 , P. 1 \%o.

${ }^{2}$ Rodewald, Jahrb. f. wiss. Bot., I 888 , Bd. Xix, p. 29I, and 1887 , Bd. xirir, p. 342. Cf. Pfeffer, I.c., p. 20I. Bouffard (Compt. rend., I 895 , T. cxxi, P. 536) found that in the alcoholic fermentation of $180 \mathrm{grm}$. sugar, 23.5 heat-calories were erolved instead of the theoretically-estimated 32.1. The deficiency may, however, be due to a variety of factors. 
heat is less than might be expected from the respiratory activity, it does not follow that the difference represents the amount of chemical energy which has been directly converted into work, for the consumption of heat in transpiration may lower the plant's temperature below that of the surrounding air, and when heat is converted into work by an osmotic manifestation, this is an endothermic process which would be produced equally well by a supply of energy derived from the external world, and without any need of the assistance of the heat evolved by respiration.

The amount of the respiratory energy converted into work probably differs in different plants and varies in the same plant according to circumstances: thus as the temperature rises respiration continually increases, whereas growth and movement diminish beyond a certain optimum. Similarly when rotation is inhibited by the action of ether, the undiminished respiratory activity is no longer required to supply energy for this purpose, and this energy is therefore manifested in the form of heat. Rapid growth is usually but not always accompanied by active respiration, but certain plants undoubtedly work more economically than others. Even when the respiratory activity is apparently altogether out of proportion to the needs of the organism, the latter may nevertheless possess a certain special importance in the economy of nature in virtue of this seemingly superfluous activity. This may be the case as regards many fermentations in which disproportionately large amounts of material are decomposed and energy liberated. It is, however, uncertain whether other anaerobes exhibit a less marked production of heat, and hence work in a more economical manner. Obligate aerobes produce much less heat in the absence of oxygen than in its presence, but this is simply because intramolecular respiration does not afford a supply of energy sufficient to satisfy the normal requirements. To obtain the same amount of energy by anaerobic metabolism requires a greater consumption of a given substance than when it undergoes perfect acrobic combustion.

The chemical energy latent in a given substance is not necessarily decisive as to its nutritive and respiratory value (Sect. 66), for certain bodies, such as sulphuretted hydrogen, \&c., can be utilized by a few organisms only, and others cannot be assimilated in any form whatever, as is the case for example with regard to the amorphous or crystalline varities of carbon, \&c. Moreover, substances may be utilized in respiration which by themselves alone are unable to yicld an adequate supply of energy. Complete isodynamic decomposition does not appear to be of general occurrence in plants, but in animals it probably happens to most of the actual nutrient materials, for here the production of heat by katabolism is of especial importance ${ }^{\text {. }}$. 
An insufficient supply of energy or unfavourable external conditions usually leads at first to the stoppage of single characteristic functions, such as growth, movement, \&c., and only after a longer or shorter period of such inactivity does death ensue. This result is produced by chloroform, high temperatures, an excessive oxygen-pressure, \&c., and in aerobes by the absence of oxygen also. In the latter case all the reactions and properties which are indissolubly connected with the power of exhibiting vital activity are retained so long as intramolecular respiration continues. Moreover the physical and plastic properties of the protoplasm remain the same, and hence the phenomena of turgidity, diosmosis, passive secretion, aggregation, \&c., are unmodified ${ }^{1}$. Similarly all processes due to the action of enzymes or other preformed substances continue in the absence of oxygen, and even when vital activity is totally suppressed.

On the other hand, a number of activities may be temporarily suppressed in the absence of oxygen: thus aerobes cease to grow, the power of movement is lost, the streaming of cytoplasm slows and stops. Facultative temporary or permanent anaerobes may continue the above functions in the absence of oxygen for a very short or a longer time, or even permanently if appropriately nourished, and these organisms exhibit all stages of transition to typical aerobes, among which certain plants are able to perform special processes of growth and movement for a short time in the complete absence of free oxygen. Thus certain obligately aerobic bacteria may continue to move for from five to sixty minutes. Correns found that the tentacles of Drosera responded to mechanical and chemical stimuli for a short time after all free oxygen had been withdrawn, and, according to Demoor, the division of the nucleus, when once commenced, proceeds to a certain point in the absence of this gas ${ }^{2}$. Similarly a frog's muscle retains the power of contractility for a long time in an oxygenless atmosphere, and an hour's deprivation of oxygen does not take from many chloroplastids the power of immediately resuming the assimilation of carbon dioxide when exposed to light, although after several hours' exposure a latent period of half an hour or longer may intervene, during which the power of photosynthetic assimilation is in abeyance ${ }^{3}$.

It requires a little time to remove all free oxygen from the plant, and hence even when a function rapidly ceases it may possibly have continued for a time in the complete absence of the gas. This is certainly

${ }^{1}$ Pfeffer, Unters. a. d. Bot. Inst. z. Tüibingen, I 886, Bd. II, p. 28 4 ; Osmot. Unters., I $8_{\text {氵 }}$, p. I 33 . Cf. Chap. iv. The protoplasmic aggregation induced by induction-shocks was first observed by Kühne, Unters, über d. Protoplasma, I 864 , p. Io6. Cf. Klemm, Jahrb. f. wiss. Bot., I 895 , Bd. XxviII, p. 627 .

${ }^{2}$ Correns, Flora, I 892 , p. I 44 ; Demoor, Contrib. à l'étude d. 1. physiol. d. l. cellule, I 894, p. 76 (Sep.-abdr. a. d. Archiv d. Biol., Bd. xilı). Cf. Sect. 9.

${ }^{3}$ [A simple method suited for class-demonstration is mentioned by Darwin, Proc. Camb. Phil. Soc., Vol. Ix, p. 33S.] 
often the case, and seedlings of Helianthus annuns may perhaps continue to grow for a short period without free oxygen, for Wieler ${ }^{1}$ observed distinct growth in an atmosphere containing at most 0.0003 per cent. by volume of it. In such an atmosphere the oxygen-respiration is reduced to a minimum or entirely suppressed, and intramolecular respiration only is active. In the case of other seedlings growth ceases often even when the oxygen percentage is not lower than 0.5 per cent. Correns found that geotropic curvature is usually possible in the presence of 0.5 per cent. of this gas, whereas heliotropic curvature is no longer produced in the case of Sinapis seedlings in 6 per cent., and in that of sunflower seedlings in I per cent., of oxygen. Similarly, streaming movements cease in some cases only when the amount of this gas is reduced to a minimum, and in others when it exists at a comparatively high partial-pressure, while it is possible that in Chara rotation may continue for a time in the complete absence of free oxygen ${ }^{2}$. Since the cessation of such movements may also be induced by chloroform, high temperatures, \&c., although respiration persists or its activity increases, it is evident that the stoppage in the absence of oxygen by no means shows that these functions are directly dependent upon oxygen-respiration for the necessary supply of energy, and this supposition is conclusively negatived when the functions are performed for a short time under anaerobic conditions ${ }^{3}$.

Normal respiration may continue for a limited period in the case of certain pigment-bacteria by means of a store of occluded oxygen, but other plants do not appcar to possess any such store ${ }^{4}$, as is shown by the rapid cessation of various movements which are able to continue for days or hours in the presence of a trace of oxygen, and by the immediate commencement of intramolecular-respiration when all free external oxygen is removed. It is possible that facultative anacrobes may continue to respire normally for a time in the absence of free oxygen by means of such a store, or even by utilizing the combined oxygen of highly oxidized substances. Even in obligate acrobes single functions may not immediately cease when aerobic respiration is no longer possible, and the fact that certain phosphorescent bacteria continue to shine for a short time in the absence of oxygen does not prove that these organisms possess a special

1 Wieler, Unters. a. d. Bot. Inst, z. Tüubingen, I $8 \varepsilon_{3}$, Bd. I, pp. 200, 223.

2 Cf. Lopriore, Jahrb. f. wiss. Bot., I 895 , Bd. xxvir, P. 576 ; Lwart, Journ. of Linn. Soc., isg6, Vol. xxxi, p. 420 ; Farmer, Annals of Botany, 1896, Vol. x, p. 288 . [Kühne states (Zeitschr. f. Biol., i 898 , Bd. xxxvi, p. 1) that streaming may continue for fifty days in Nitella in darkness and absence of oxygen, a fact which requires further confirmation, since a minute trace of oxygen may suffice for the continuance of rotation (cf. Ewart, l.c.).]

${ }^{3}$ Palladin (Ber. d. Bot. Ges., IS\$6, p. 322) supposes that respiration influences growth only by producing osmotic sulsstances, but growth is not solely dependent upon the osmotic pressure, and, moreover, turgidity is at first unaltered when all free oxygen is removed.

* Cf. Ewart, Journ. of Linn. Soc., 1897, Vol. xxxıI, p. 123. 
reserve store of it, as Beyerinck supposes ${ }^{1}$. When oxygen is admitted to a culture containing reduced indigo, phosphorescence commences before the latter turns blue, and this is because at first the bacteria absorb all the available oxygen, either directly or from the indigo solution, before the latter has time to become fully oxidized.

Historical. After Malpighi had rccognized the necessity of air for the germination of seeds, Senebier, de Saussure, and others proved the dependence of growth upon the presence of oxygen. The lowest oxygen pressures which aerobic plants can successfully withstand for prolonged periods were determined by Bert and by Wieler $^{2}$ (on anaerobes, cf. Sect. 98). From the time of Dutrochet it was recognized that oxygen was essential for the movements of aerobic plants, and a few contradictory results by Kabsch were probably due to the incomplete removal of the free gas. Correns' researches have established the limited anaerobic continuance of certain movements, and have determined the lowest percentages of oxygen in which particular movements are still possible ${ }^{3}$. After Kühne had clearly established the dependence of the protoplasmic movements of aerobes upon a supply of oxygen, Clark determined the lowest limits at which streaming, ciliary, and amoeboid movements were possible ${ }^{4}$.

As a general rule the gradual cessation of a given activity indicates that under these conditions it cannot be aroused; indeed Bert observed that germination did not occur in rarefied air in which previously active growth continues for a longer or shorter time. Any diminished activity thus induced must finally act injuriously, and hence plants are unable to grow luxuriantly in the presence of an oxygen percentage which may at first render growth more active ${ }^{5}$. On the optimal pressure for growth and development, cf. Sect. Ioo.

1 Archiv. Néerlandaises, I $\$ 89$, T. Xxiri, p. $4^{20}$.

2 Bert, La pression barométrique, I 878 ; Compt. rend., I 873 , T. Lxxvi, pp. 443, I 276 ; Wieler, 1. c., p. I 89 , and there the other literature is quoted.

${ }_{3}$ Dutrochet, Mém. p. servir à l'hist. d. vég. et anim., Bruxelles, I 837 , pp. I 86,259 ; Kabsch, Bot. Zeitung, I 862 , p. $34 \mathrm{I}$; Correns, Flora, I $\$ 92$, p. 87 .

${ }^{4}$ Clark, 1. c., I 888 , p. 273 ; Kühne, Unters. iiber d. Protoplasma, i $86+$, pp. 89 , I0.4.

${ }^{5}$ Cf. Wieler, 1. c.; Lucas, Bot. Centralbl., I886, Bd. xxvin, p. 29 S; Jaccard, Rev. gén. d. Bot., x 893, T. v, p. $28_{9}$. 


\section{CHAPTER N}

TRANSLOCATION

\section{Section 106. Translocation of Organic Food-substances.}

Whenever food-materials are, as is often the case, utilized or consumed in parts far removed from those where they are produced or absorbed, a transference from the one region to the other becomes necessary. Thus the ash constituents absorbed by the roots of a tree from the soil must be transferred to the foliage-leaves even when these are situated at its summit many yards from the ground, whereas the products of photosynthetic assimilation pass downwards to the roots, which are provided in this manner with organic food. Similarly the nutriment absorbed by a fungus mycelium from the substratum must be transferred in sufficient abundance to nourish the erect sporangiferous or sterile sub-aërial hyphae, no matter whether these are multi- or uni-cellular filaments. Indeed the metabolism of every individual cell is unavoidably associated with an absorption and excretion of nutritive substances and metabolic products, which are transferred from one region of the cell to another after their absorption or before their excretion.

We must therefore deal with the translocatory channels, the nature of the translocatory substances, and the means by which the transference of material from one part to another is induced, rendered possible, performed and regulated. The main principles involved have already been discussed (Chap. IV, cf. also Sect. 93), and it only requires a simultaneous consideration of the properties, structural relationships, and vital activities of the parts involved either to render possible a clear comprehension of the manifold phenomena of translocation, or to provide a preliminary explanation where our knowledge is as yet incomplete. It has already been mentioned why various metamorphoses commonly accompany translocation, and the importance of these changes has been previously discussed, while it has also been shown how and why translocation is regulated by the consumption, demand and supply. Hence the processes of translocation are modified during the progress of development, and are also influcnced by the external conditions. Thus reserve materials accumulate at certain periods, 
and are at a later stage mobilized and transferred to the consuming organs, while the removal of the translocatory products by artificial means may induce premature depletion. Translocation is regulated to a very marked extent by consumption, and hence a substance present in sufficient quantity may undergo very rapid translocation until the plant's needs are satisfied. Different substances may be transferred in opposite directions at the same time and along the same channels; indeed this occurs in every plant, for the excretion of carbon dioxide and other substances proceeds simultaneously with the absorption of nutriment and of oxygen.

When a seed germinates, the reserve food is conveyed from it to the elongating root and shoot. Water and oxygen, as well as such inorganic ash constituents as may be present in the soil, are absorbed and transferred to the different parts of the plant from the commencement of germination onwards. As soon as green leaves are formed these begin the photosynthetic production of organic food, and before long the whole of the organic nutriment may be provided in this manner. In an annual plant growth and the formation of new shoots, leaves, and roots, decrease towards the end of the vegetative period, while large quantities of foodmaterial are consumed in the production of the fruit and are stored up in the seed. The translocation of food-materials for this purpose becomes more and more marked, while the activity of photosynthetic assimilation gradually decreases, and ceases before the chlorophyllous organs are completely dead. In addition to organic substances large quantities of ash constituents are stored up in the fruits, and the percentage of ash undergoes but little increase in the rest of the plant subsequently to the period of most active absorption and growth.

In perennial plants a large portion of the assimilated material is conveyed to the permanent organs, such as the subterranean roots or rhizomes of perennials, and to the stems of trees, while in spring a reverse current conveys food to the developing buds.

Water and dissolved food-materials necessarily follow the same channel in a fungal hypha, whereas the tissue-differentiation in higher plants is accompanied by a more or less marked division of labour, the xylem conveying water and salts, the phloem plastic substances. The phloem contains conducting tissue-elements which are as efficient and important as those of the xylem, and when rapid translocation to far distant parts is necessary, the food-materials pass almost solely through the phloem, for transference takes place but slowly through the cortex and pith. Hence as a general rule substances are translocated as far as possible by means of the phloem, and pass through relatively few parenchyma cells to reach their destination, for it is only when the distance to be traversed is small that plastic substances can be transferred through parenchyma with sufficient rapidity to satisfy the needs of an organ 
in which growth or metabolism is active. The vessels of the wood and phloem are surrounded by tissue-elements which act as intermediaries between the conducting channels and the external tissues, and the branching and intercommunicating system of vascular bundles provides for the transport of water and food-materials to all parts of the plant.

The division of labour is always relative in character and can never be absolute, for every living cell and every tissue has necessarily the power of absorbing and excreting nutritive substances and metabolic products along with water, while the water-channels convey a certain amount of foodmaterial in the shape of the ash constituents drawn from the soil. Sugar and other soluble nutrient substances derived from the reserves in the root, stem, tubers, \&c., may be conveyed in a similar manner if they penetrate the water-conducting tracheae, and in places where the sap is rich in sugar, \&c., as in the birch (Sect. 43), large quantities of plastic substances may be transferred by means of the wood-vessels. This is not, however, possible in other cases, for both the sap exuded from a wound and that obtained directly from the wood may contain hardly any organic material whatever, as in the vine, \&c. Similarly in herbaceous plants the water-channels seem only to be utilized to a slight extent for the conveyance of organic food (Sect. I09), and moreover transference through the xylem is practically possible only in one direction, for the ascending stream of water carries the dissolved substances with it and almost entirely prevents any downward diffusion. It was in order to secure the possibility of transference in all directions that a certain division of labour became necessary, and a special conducting-tissue has been evolved for organic food-materials, while, as might be expected, the conducting elements as well as the groundtissue of the phloem have acquired no special importance in the rapid transference of water through the plant.

Not only proteids but apparently all plastic substances are preferably conducted through the phloem, for even when the bark of a tree is removed down to the latter, carbohydrates are still transferred over as wide distances as before ${ }^{1}$. During translocatory activity the living elements of the phloem, and especially the sieve-tubes, contain an abundance of proteids, carbohydrates, fats, amides and phosphates, and the results of ringing experiments are such as to indicate that all these substances and others also are translocated mainly by means of this tissue. Czapeli ${ }^{2}$ has shown that the cortical parenchyma of woody plants is unable to transfer carbohydrates in sufficient amount even for a short distance.

${ }^{2}$ Lecomte, Ann. d. sci. nat., ISS9, vii. sér., T. x, p. 300; Strasburger, Bau u. Verrichtung d. Leitungsbahnen, i $S_{9}$ I, p. 9 I 6.

${ }^{2}$ Czapek, Ber. d. Bot. Ges., I\$97, p. 124 ; Sitzungsh. đ. Wien. Akad., $1 \$_{97}$, Bd. Cri, Abth. i, P. 155 . 
Every tissue has, however, a certain power of translocation, and no doubt different parenchymatous tissues exhibit varying degrees of functional activity and differentiation. Thus large quantities of reserve-materials are rapidly transferred to the developing embryo through parenchymatous endosperm, and in young seedlings the further transference probably takes place mainly through the cortical and medullary parenchyma. This is all the more probable because the distances to be traversed are small, and the relatively large sectional areas of the parenchymatous tissues compensate for the slower rate of transference. The extent to which plastic substances are, or may be, conveyed through the cortex, medulla, \&c., has in no case been precisely determined, but in the stems of seedlings, and in shoots, branches, \&c., whenever reserve-materials are being consumed or stored, chains of parenchyma cells in the intermediate regions of the cortex, and of the medulla as well, are usually found filled with starch, sugar, oil, asparagin, \&c. It is, however, impossible to say whether these tissues serve as direct channels or simply as temporary storage reservoirs, in which any excess is stored for a time and later returned to the phloem for further transference. Exchanges of this kind are of general occurrence between the conducting channels and the storage tissues. Thus in trees the assimilatory products from the leaves are conveyed to the main trunk by the phloem and there distributed horizontally by the medullary rays, whence they may again be transferred to the conducting phloem tissues. Similarly it is in the parenchyma immediately surrounding the phloem that the translocated substances first appear, and when the amounts translocated are small a perceptible accumulation may only occur at this point. In such cases the transference takes place in the transverse direction through the parenchyma, but there is no reason why longitudinal conduction should not also be possible. Indeed translocation is in general more rapid when the parenchyma cells are elongated in a direction parallel to the length of the stem, and hence also to the phloem elements.

It is probable therefore that in seedlings, and frequently also where the distance to be traversed is small, the cortical and medullary parenchyma may be concerned to a greater or less extent in the transportation of all or of single nutritive or excretory substances. Schimper ${ }^{1}$ indeed has found that after the removal of the vascular bundles from the leaves of Plantago the bundle-sheaths are still able to convey the assimilatory products to the stem, though somewhat less rapidly. An accumulation of starch, sugar, \&c. observed in a tissue during translocation does not necessarily show that the tissue in question forms an active conducting channel, for accumulation involves retention, whereas in the actual channels the transference may be so rapid that no perceptible quantity of the trans-

${ }^{1}$ Schimper, Bot. Zeitung, IS85, P. 756. . Cf. also Czapek, 1. c., p. I 26. 
locating substance can be detected in them at any particular moment. For all these reasons it is not surprising to find that, in contradiction to what was formerly supposcd, the occurrence of starch in the bundle-sheath or pericycle does not indicate that these layers possess any special importance or significance in translocation ${ }^{1}$.

All substances are not translocated and accumulated with the same readiness, and hence a partial separation of the different plastic substances is possible; thus in seedlings, starch, sugar and oil are mainly present in the cortical and medullary parenchyma, while the proteids occur for the most part in the phloem and especially in the sieve-tubes ${ }^{2}$, which are especially well adapted by means of their open intercommunications for the transference of these slowly diosmosing bodies. In general the amount of proteid translocated is small, and hence the channels mentioned suffice for all requirements, for whenever reserve-proteids are mobilized they usually undergo marked decomposition and a great part of the nitrogen is translocated in the form of asparagin and other amides, which along with sugar and starch arc found in the fundamental parenchyma, although proteids are probably subject to direct translocation in seedlings ${ }^{3}$. This separation betwcen translocating substances which mect again at their final destination is never complete, and is by no means a general necessity, for in sieve-tubes, endosperm tissue, fungal hyphae, \&c., proteids and carbohydrates travel together.

The component elements of the phloem are, like those of the xylem, of uncqual functional value. The sieve-tubes seem to be the most active conductory channels for all substances, whereas the cambiform cells are of less value in translocation, and a subordinate importance only is attached to the companion cells, and still less to the phloem parenchyma. The elongated tissue-elements both of the wood and of the phloem form the most important conducting channcls, and special importance attaches to the vessels or long tubes formed by the perforation of the transverse walls of contiguous cells. The water-conducting tracheae are however usually dcad, whercas for the most part living cells and cell-fusions serve for the conveyance of plastic matcrials. Hence the sieve-tubes lose their conducting power as soon as they die, which occurs after a limited period of activity in plants which exhibit secondary growth and form new sievetubes from year to year.

The mobilized products may undergo transitory deposition in the process of translocation, and thus the occurrence of starch in the conducting channels by no means indicates that carbohydrates are transferred in this form. Other substances, however, including colloidal proteids, may

\footnotetext{
1 Heine, Versuchsst., r 888, Bd. xxxv, p. I6I ; Schimper, 1. c., p. 757 ; Strasburger, 1. c., p. 487.

2 Sachs, Flora, I $\$ 62$, p. $297 ;$ i $\$ 63$, p. 38.

3 Pfeffer, Jahrb. f. wiss. Bot., I 872 , Bd. virI, p. $5.3^{8}$, and Sect. So.
} 
diosmose through the protoplasts and hence be transferred without undergoing any marked change, while oil may be absorbed or translocated in the form of a fine emulsion. All the typical reserve-materials may be transitorily deposited in the translocatory channels, and the appearance of such storage substance in the latter seems to bear some indirect relation to the activity of translocation. Glucose and starch are the most commonly occurring non-nitrogenous products, while in many cases canesugar appears and may along with other polysaccharides take a more prominent part in translocation than is superficially apparent. Mannite seems to serve as translocatory material in the Olive, glycogen in Fungi. Fatty oils commonly subserve the same purpose, and according to Kraus ${ }^{1}$ this is also the case with malic acid in the Crassulaceae. Nitrogen is usually translocated in the form of asparagin or other amides and of various proteids, while in Pangium edule hydrocyanic acid acquires a similar importance. The ash constituents are frequently stored up in the form of organic compounds and may undergo direct translocation as such, but usually decomposition precedes mobilization, so that a portion of the phosphorus, sulphur, \&c. travels in the form of inorganic compounds. The ash constituents are usually absorbed from the soil as inorganic salts and are transferred in this condition to all parts before they enter into metabolism.

The aplastic metabolic products as well as the non-essential ash constituents undergo similar translocation. Thus resins are transferred to certain regions by means of cells or special canals, while carbon dioxide often travels a long distance before it can be excreted. Hence when calcium oxalate (Sect. 86) or tannins (Sect. 87) undergo translocation, it does not follow that they are used in metabolism as nutritive material. The mobilized products frequently do not accumulate to any extent, and in certain cases no soluble carbohydrates can be detected during the active translocation of starch. The small amount of these readily diosmosing compounds present at any one time is of considerable importance, for in this way not only is the danger of loss very much minimized but also any concentration is avoided which might be injurious to the tissues. Not only does no accumulatin occur when consumption is active, but also when the consuming cells have no power of passively secreting the food-materials supplied to them. The latter is usually the reason why starch and sugar are absent from the primary meristem of the root and stem, for no accumulation occurs when the consumption is very much lessened by the stoppage of growth due to imbedding in a plaster-of-paris cast. Soluble proteids which give the biuret reaction with copper are commonly present in considerable amount in primary meristem cells, but disappear as the latter elongate. An accumulation of starch, sugar, proteids, \&c., as a preparation for subse- 
quent rapid growth or active metabolism is a phenomenon of frequent occurrence and obvious utility.

Our knowledge of the processes of translocation have largely been obtained by means of the micro-chemical methods first systematically employed by Sachs, who paid attention to the source, destination and character of the translocatory products as well as to the nature of the conducting tissues (cf. Sect. 77). The facts gained by means of ringing experiments are also of importance, although results thus obtained need to be interpreted with the utmost care. Similarly a careful consideration of the internal structural relationships will also be of aid in comprehending translocatory phenomena, for the possibility of

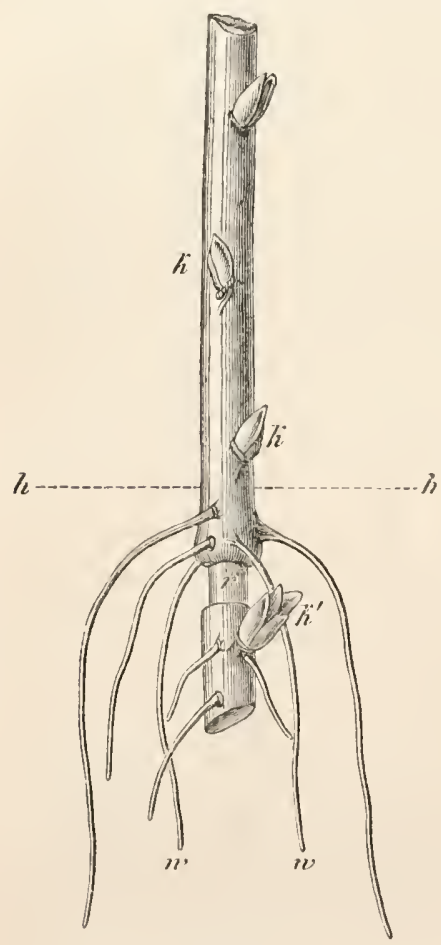

FiG. 67. translocation necessarily involves the existence of purposeful adaptions directed to this end (cf. Sect. 35). A knowledge of the latter is however assumed here ${ }^{1}$, and it is sufficient to mention that conductive power in a particular direction is increased by the elongation of the conducting elements and by the removal of all obstructing resistances or hindrances, while by means of the continuity and branching of the special conducting channels, and by their connexion with the surrounding tissues, the conveyance of foodmaterials to and from all parts of the plant is assured. The most marked division of labour is exhibited among Phanerogams, and the remarks hitherto made have mainly reference to these.

Ringing experiments ${ }^{2}$. If a ring of bark is removed from a branch of Salix, Ligustrum \&c., at $r$, and the latter then placed in water or earth up to $h-h$, after a few weeks only a few feeble roots develop from the smaller lower end $\left(k^{\prime}\right)$, whereas above the point ringed plenty of strong roots are produced and continue to elongate. If, however, only the cortex is removed and the phloem left intact, roots develop equally well and abundantly from the lower end. This is the case also when the experiment is performed with Monocotyledons, or Dicotyledons with bicollateral bundles (Solanaceae, Cucurbitaceae, Asclepiadaceae, Apocynaceae, Cichoraceae ${ }^{3}$ ) or with medullary bundles. Similar results are also obtained with

${ }^{1}$ See Haberlandt, Physiol. Pflanzenanat., 1896 , 2. Aufl., p 263; Strasburger, Leitungsbahnen, I $99 \mathrm{I}$; de Bary, Anat., $18_{77}$, and the works here quoted.

${ }^{2}$ Hansteen (Jahrb. f. wiss. Bot., I860, Bd. II, p. 392) gives numerous experiments, and also mentions the older literature. Cf. also Vöchting, Organbildung im Pflanzenreich, 1878; I884, p. I I4; Bot. Zeitung, I895, p. 84 ; A. Fischer, Jahrb. f. wiss. Bot., I891, lid. xxir, p. I32 ; Strasburger, Leitungsbahnen, IS91, p. 877 ; Jost, Bot. Zeitung, I893, pp. I 20, \&c.

3 The three last-named families possess a latex system. 
Salix if a bridge of phloem is left connecting $k$ and $k^{\prime}$ (Fig. 67), and the fact that the roots in $k^{\prime}$ are formed mainly beneath this bridge shows that the nutrient materials are transferred rapidly through the phloem longitudinally, but diffuse horizontally only slowly. When a spiral strip of phloem is removed from a plant in which the sieve-tubes do not anastomose or when two partial ringings are made on opposite sides of the stem at a little distance from one another, according to the structure of the plant, a more or less markedly decreased production of roots is observed on the lower piece of stem, as was first shown by Hales, Cotta and Knight.

The phloem is hence the best conducting tissue for food-materials, but it is not, as Sachs supposed (Flora, I863, p. 23), specifically adapted for the special transference of proteid, for non-nitrogenous materials also travel most actively in the phloem, and are, according to Czapek, transferred too slowly through the cortex or bundle-sheaths to satisfy the needs of tissues at any distance. When an abundance of stored carbohydrates is present, the diminished growth after ringing must naturally be due to the lessened supply of proteids, and it is probably seldom the case that the conducting powers of the phloem are normally exercised to their full limits.

No downwardly directed transference of plastic substances normally occurs in the wood, but an upward conduction with the water-current is always possible. Thus Th. Hartig ${ }^{1}$ observed the total depletion of starch from the roots of trees (oak, \&c.) although a broad ring of bark had been removed from the base of the tree, and showed that buds beneath which the continuity of the phloem had been broken still continued to develop. The experiments were not always successful, probably owing to operative injuries or differences in constitution. Hanstein obtained negative results with herbaceous plants, whereas Strasburger (1. c., p. 900) observed that in some Umbelliferae fruit-formation continued after the continuity of the phloem had been broken. Inflorescences and buds are, however, only capable of a slight amount of development by means of their own reserve-materials, and hence not only non-nitrogenous but also nitrogenous food-materials must have been conveyed upwards along with the transpiration-current. This is also indicated by certain experiments by Th. Hartig and other authors, and as a matter of fact the exuded sap of many plants contains a certain amount of proteids, amides, \&c. The power of utilizing the transpiration-current for this purpose probably varies under different circumstances and according to the plant examined, and hence it is not surprising that A. Fischer was unable to detect any such utilization of the water-current in translocation in any of the herbaceous plants he examined. In woody plants usually only small amounts are conveyed in this manner from the roots and the main stem, whereas the amount transferred becomes very large when an increased consumption is caused by the renewed bud-formation

1 Bot. Zeitung, 1858 , p. $33^{8}$; Sachs, Flora, 1863 , p. 66 ; R. Hartig, Bot. Zeitung, 1888, p. 837 ; Holz d. Rothbuche, I8s8, p. 38 ; Anat. u. Physiol., I89I, p. 235 ; F. Müller, Bot. Centralbl., I 889 , Bd. xxxix, p. $3^{1}$; A. Fischer, Jahrb. f. wiss. Bot., I891, Bd. xxil, p. I33 ; Strasburger, Leitungsbahnen, i891, pp. 879 , 915 ; Mer, Lot. Centralbl., i892, Bd. LIt, p. 188. 
and development induced by the removal of the leaves. Similarly after flowering commences large quantities of reserve-materials are conveyed to the ripening fruits.

The upward conduction probably takes place to a large extent in the phloem also, and the conclusion arrived at by certain authors ${ }^{\mathrm{I}}$ that the phloem of trees is incapable of upward translocation is hardly supported by a careful consideration of the available experimental evidence. Indeed the converse appears to be true, for when a branch is planted upside down the assimilatory products are still conveyed from the leaves to the root by the sieve-tubes, that is in the opposite direction to the normal one.

The diminution of growth must be proved to be due to a deficiency of nutriment before it can be used as an indication that the continuity of the conducting channels has been interrupted, for operative injuries may directly induce diminished growth. Hence arose the incorrect supposition that the phloem is incapable of upward translocation, from the fact that the stem of a tree ceases to grow in thickness below the point ringed. As a matter of fact food-materials are conveyed in this direction when growth is excited by an injury, or when buds commence to develop and consume food-material, while in some cases reserve-materials are even deposited in the non-growing portion of the stem. Growth in thickness also ceases in the stems of Periploca and Tecoma below the injury, although after being ringed the continuity of the phloem is maintained by their medullary phloem strands ${ }^{2}$. Again, the most abundant supply of food is unable to induce growth where no tendency to growth exists. Similarly when a flower is not fertilized, the peduncle does not develop further, although otherwise as the fruit-stalk it would have functioned as an extremely active translocatory channel ${ }^{3}$. Tittmann ${ }^{4}$ has shown also that according to circumstances the formation of callus tissue may be induced either upon the upper or under cut surface of a twig.

Sieve-tubes ${ }^{5}$. These are especially active translocatory channels and contain an abundance of both nitrogenous and non-nitrogenous substances. Thus G. Kraus ${ }^{6}$ found in the sap escaping from the sieve-tubes of a cucumber 7 to 10 per cent. of solid or disssolved substances, of which 20 per cent. were proteids, $3 \circ$ per cent. amides, and $3^{8}$ per cent. soluble carbohydrates, while among the ash constituents compounds of potassium and phosphoric acid were most abundant, and those of magnesium next. This coincides with Schimper's micro-chemical studies (Flora, I 890 , pp. 228, 260), according to which the phosphoric acid occurs mainly in the

${ }^{1}$ Cf. Th. Hartig, Bot. Zeitung, 1888, p. 339 ; Sachs, 1.c.; Strasburger, 1. c., p. 891 ; A. Fischer, l. c., p. 150.

${ }^{2}$ Jost, Bot. Zeitung, I $S 93$, p. I 20, and the literature here given.

s On similar actions of this kind, cf. de Vries, Jahrb. f. wiss. Bot., IS9I, Bd. xxIr, p. 50; Busch, Ber. d. Bot. Ges., i $\$ \delta_{9}$, Generalvers., p. 29; Jost, Bot. Zeitung, I $\$ 9$ I, p. 530; I $\$ 93$, p. I3 I.

4 Tittmann, Jahrb. f. wiss. Bot., I 895, Bd. xxv1I, p. 193.

${ }^{3}$ Literature: Haberlandt, Physiol. Anat., i $\$ 96,2$. Aufl, p. 286 ; Strasburger, Leitungsbahnen, I891, pp. 476, 918; A. Fischer, Unters. ii. d. Seitenröhrensystem d. Cucurbitaceae, I $88_{4}$; Sitzungsb. d. Sächs. Ges. d. Wiss., I $88_{5}$, p. $245 ;$ i $\$ 86$, p. 291.

${ }^{6}$ G. Kraus, Siebröhreninhalt von Cucurbita, Sitzungsb. d. Naturf.-Ges. z. Halle, Feb., I 894. 
form of organic compounds. The sieve-tubes become partially empty during winter, but fill again in spring as the reserve-materials are mobilized, and this is sufficient to show that they serve as translocatory channels and not as storage reservoirs as Blass erroneously supposed '. It is moreover still doubtful whether Fischer's suggestion that the sieve-tubes are especially active in the synthesis of proteids is correct.

It has yet to be determined whether the cambiform and companion cells function mainly as conducting channels, or simply as intermediaries between the sieve-tubes and surrounding tissues and as temporary storage reservoirs, while the same doubt attaches to the function of the phloem-parenchyma and associated tissues. The presence or absence of fine inter-protoplasmic communications has no importance for the question at issue, for the value of these in translocation is still doubtful (Sect. 20). On the other hand, both non-diosmosing and undissolved substances may pass through the coarse pores in the sieve-plate, although the starch grains often present in sieve-tubes are usually larger than any of the pores (cf. Strasburger, l. c., p. 478 ). As the result of this continuity fluid escapes from the sieve-tube when it is cut open, and this usually causes the remaining contents to accumulate in each segment on one side of the sieve-plate. Such currents in mass probably often aid in the transport of materials through the living plant, but it is uncertain whether they are passively produced by accidental pressure, swaying movements, \&c., or are due to the activity of the living non-nucleated contents of the sieve-tubes, which are maintained alive and active by means of the nuclear influences radiating from the contiguous cells ${ }^{2}$. In many cases the sievetubes become closed in winter by means of a callus covering each sieve-plate, and this callus is dissolved and disappears in spring; in other plants, however, the sievetubes remain open so long as they are living ${ }^{3}$. In most Gymnosperms no coarse sieve-pores are present, while certain mosses and algae possess more or less rudimentary sieve-tubes (Haberlandt, l. c., pp. 308, 334).

Latex is of comparatively limited distribution, and it is still doubtful to what extent the laticiferous or lactiferous ${ }^{4}$ tubes (latex-vessels, latex-cells or intercellular spaces) function under normal conditions as channels for the transport of plastic substances. The latex of many plants contains perceptible amounts of proteids, sugars ( 5 to ro per cent. in Morus according to Faivre), fats, and in certain cases starch also, but these are in all cases accompanied by aplastic products, and in other plants the latter are almost entirely pre-eminent. Thus as far as is known (Sect. 87-90', india-rubber, resin, ethereal oils, alkaloids, \&c. are all incapable of further metabolism, and this is also true in most cases of the tannins which

1 Blass, Jahrb. f. wiss. Bot., Isy, Bd. xxil, p. 2yo. Cf. also Haberlandt, Physiol. Anat., 2. Aufl., p. $2 S S$.

2 Cf. Pfeffer, Sitzungsb. d. Sächs. Ges. d. Wiss., r 896, p. 509.

${ }^{3}$ Anatomical details by Haberlandt, 1.c., p. 29 ; Strasburger, 1. c., p. 927 ; de Bary, Comparative Anatomy, I8S2, pp. I91, 210, 455. On fungi, de Bary, Morph. u. Biol. d. Pilze, 18S4, 2. Aufl.; Istvánffi, Jahrb. f. wiss. Bot., I $896, \mathrm{Bd} . \mathrm{x} \times 1 \times \mathbf{x}, \mathrm{p} .405$.

4 [Nehemiah Grew (Anatomy of Plants, I682, p. 86) was the first to clearly distinguish between lactiferous vessels and sap-vessels (wood-tracheae).] 
occur in abundance in certain varieties of latex ${ }^{1}$. These substances do not seem to be merely unavoidable by-products, but appear to have a certain economic value, for certain constituents of latex give it the power of coagulating when exposed to air, and hence enable it to protect a wounded or exposed surface. Similarly its poisonous properties act as a protection against insects or herbivorous animal $^{2}$, and it is possible that herein lies the importance of the latex and lactiferous tubes in many plants, although in other cases the latex system may serve to a certain, or even to a marked extent for the conveyance of plastic materials.

In certain cases at least the plastic substances diminish at certain stages of development, or during starvation, as Schullerus ${ }^{3}$ found to be the case with the starch in the latex of Euphorbia, and sugar in that of Morus. A certain amount of the emulsified substances may also be re-assimilated, for Faivre found the latex in seedlings of Tragopogon porrifolius became clearer in darkness, or in an atmosphere free from carbon dioxide, but quite milky again when photo-synthetic assimilation was allowed to recommence. Faivre also concluded on similar grounds that the latex of Morus alba has a nutritive value and contains a large amount of stored food during winter, and his ringing experiments on various species of Ficus seem to indicate that the latex system is of marked importance for the transference of nutrient and plastic substances ${ }^{4}$. The milkiness of the latex is a very unsafe index to employ, and Hanstein ${ }^{5}$ has in part obtained contradictory results to Faivre. The structure and arrangement of the lactiferous tubes renders them markedly adapted for translocation, and various causes may induce streaming currents of latex to move from one part to another in intact plants ${ }^{6}$. These characteristics are, however, of similar importance for the conveyance of latex to a wound, and the anatomical arrangements are equally adapted for the performance of the protective and biological functions already mentioned, so that a definite decision as to the functional value of the latex system is not at present possible (cf. Schimper, Bot. Zeitung, I 885 , p. 77 I).

Historical. Malpiglii's conclusions as to the circulation of food-materials in the plant were altogether remarkable considering the epoch at which they were made, but as the result therefrom a one-sided and incomplete theory of sap

${ }^{1}$ For analy'ses of latex, cf. de Bary, 1.c., p. I94; Wiesner, Die Rohstoffe d. Pflanzenreiches, 1873 ; Boussingatlt, Agron., \&c., I 894, T. vil, p. 64 (Cow-tree); Chimani, Bot. Centralbl., I895, Bd. LXI, p. 385. Microchemical methods, Schimper, Flora, 1890, p. 228.

${ }^{2}$ De Vries, Landw. Jahrb., I881, p. 687 ; Ludwig, Biol. d. Pfl, 1895, p. 231 ; Zander, Bibl. Bot., 1896 , Heft 37 , p. 37; B. H. Biffen, Annals of Botany, I897, Vol. xı, p. 334. The importance of the peptic cnzymes occurring in certain forms of latex is not yet certain.

${ }^{3}$ Schullerus, Die phys. Bedeut. d. Milchsaftes v. Euphorbia, I882, p. $9^{2}$ (Sep.-abdr. a. d. Abh. d. Bot. Vereins Brandenburg, Bd. XxIv); Treub, Ann. d. Jard. bot. d. Buitenzorg, I882, T. III, p. 37. Cf. Schimper, Bot. Zeitung, $188_{5}$, p. 774 .

${ }^{4}$ Faivre, Compt. rend., I 879 , T. LxxxvinI, p. 369 ; Ann. d. sci. nat., I 866 , v. sér., T. vı, p. 33 ; I 869 , T. x, p. 97 .

${ }_{5}$ Hanstein, Die Milchsaftgefässe, 1864 , p. 54. On aggregations in latex, cf. Schwendener, Monatsb. d. Berl. Akad., I $88_{5}$, p. 335 .

${ }^{6}$ Cf. Schwendcner, 1. c., p. 326 . Usually no latex escapes from withered plants or from the older parts when cut. 
circulation was developed (cf. Sachs, History of Botany, I882, p. 494). 'The ascent of 'crude sap' in the interior of the plant and the descent of 'formed sap' in the bark after elaboration in the leaves is simply one special example of translocation and is by no means so all important as was subsequently supposed. Hence it arose that in spite of the accumulation of new facts and observations no satisfactory generalization had been made up to $\mathbf{I} 85^{\prime}$. This was, however, finally obtained by Sachs' researches ${ }^{2}$, in which not only were the different processes of translocation taken into account, but also a conscious attempt was made to determine the nature of the different translocatory substances, and the means by which their mobilization and translocation is procured, whereas hitherto it had been customary to speak of a general 'formative sap' the detailed characteristics of which were comparatively unknown. Sachs also showed that the different translocatory substances might undergo a certain amount of separation from one another in the translocatory channels. The main fundamental principles thus established still hold good, although the sieve-tubes, in opposition to Sachs' view, also function as the most active conductory channels for non-nitrogenous substances, and although, as Pfeffer ${ }^{3}$ has shown, proteids are translocated in a similar manner to carbohydrates, after undergoing decomposition into amides, \&c. Mohl's conclusion that the phloem, and especially the sieve-tubes and elongated phloem elements, were of primary importance for translocation had previously been established by Hanstein's researches ${ }^{4}$.

\section{Section 107. The Translocation of the Ash Constituents.}

These undergo translocation either in the form of inorganic salts or in combination with organic substances, and although in many cases neither the conducting channels nor the translocatory materials have been ascertained, there is no doubt that in general the ash constituents follow the same path as organic substances do. The greater part of the ash constituents are drawn from the soil in the form of an exceedingly dilute solution, conveyed upwards by the transpiration-current to the living tissues and there utilized. Certain of the elements thus obtained are soon permanently deposited as aplastic materials, whereas many others remain available in some form for further metabolism, or are removed and deposited in storage receptacles, in which all the essential ash constituents must necessarily be contained. In many cases, especially in seeds,

1 Cf. Mohl, Veg. Zelle, I851, p. 71 ; Unger, Anat. u. Physiol., 1855, p. 329; Th. Hartig, Pflanzenkeim, 1858 , p. 69 ; Bot. Zeitung, 1862, p. 82 .

${ }^{2}$ Sachs, Sitzungsb. d. Wien. Akad., I859, Bd. xxxvir, p. 57 ; Jahrb. f. wiss. Bot., I863. Bd. I1I, p. 183 ; Flora, 1863 , p. 32 .

${ }^{3}$ Pfeffer, Jahrb. f. wiss. Bot., i 872 , Bd. viri, p. $53^{8}$.

4 Mohl, Bot. Zeitung, I 885 , p. 897 ; Hanstein, Jahrb. f. wiss. Bot., I860, Bd. 11, p. 392. The older literature is here quoted. 
a large proportion of the phosphorus and sulphur, and perhaps of potassium also, is stored in the form of organic compounds, but in tubers, roots, \&c., the former accumulate mainly as sulphates and phosphates (cf. Sects. $74,77,80$ ). When mobilized, a portion of the organic compounds which contain sulphur and phosphorus decomposes, liberating phosphoric and sulphuric acids, and these are translocated, along with organic compounds of phosphorus and sulphur and other ash constituents, to the regions where metabolism is active. Both the organic and inorganic compounds apparently pass through the phloem and its associated tissues, for during translocation to and from the storage receptacles large quantities of organic and inorganic compounds of sulphur and phosphorus, along with a smaller amount of those of magnesium and still less of those of calcium, are found in the sieve-tubes ${ }^{1}$. A portion of the phosphorus and sulphur is probably translocated in the form of proteids, although both may appear in the cortex without any accompanying accumulation of the latter. Just as in the case of sugar, it is uncertain whether inorganic salts can be translocated sufficiently actively over wide distances through the cortex alone, or whether in all cases the aid of the phloem is necessary. The fact that many parenchyma cells are able to passively secrete sulphates, phosphates, nitrates, \&c., would tend to render direct translocation through such tissues more difficult, for before any transference could occur through the tissue all the absorbing cells would need to be completely saturated.

Every element may be found undergoing translocation in the plant, even including such as calcium or silicon which are for the most part deposited in the form of aplastic compounds, but the readiness with which different substances are mobilized and translocated varies very much. Thus Schröder ${ }^{2}$ found that the shrivelling cotyledons of Phaseolus seedlings only lost one-half of their calcium, whereas not more than a quarter of the phosphorus, and one-third of the potassium, magnesium, nitrogen, and sodium remained. A complete removal of all the essential elements is never possible, for even in a starved plant certain essential structural constituents cannot be mobilized or consumed. After the death of a cell, however, additional materials may be extracted from it, but these usually consist only of the more readily diosmosing substances, to which compounds of calcium do not for the most part belong. An extraction of this kind always occurs when a dead cell is surrounded by living ones ${ }^{3}$, whereas

${ }^{1}$ Schimper, Flora, 1890, pp. $241,260$.

2 Schröder, Versuchsst., i 868 , Bd. x, p. 468 . Other examples by Homberger, Jahresb. d. Agr.Chem., 1 $\$ 82$, p. 159; Schimper, 1. c., p. 241, and certain of the works on seedlings, tubers, \&c., quoted in Sect. 109. The same occurs during the opening of the bud (Schröder, Forstchem. u. pfl--phys. Unters., $1878, \mathrm{I}, \mathrm{p} .77$ ).

3 Examples in the case of the xylem: Hartig, Holz d. Rothbuche, r888, p. 177 ; Daube, Bot. Jahresb., i $88_{3}$, p. 44 . 
the shrivelling of the leaf or its fall prevents many substances from being conveyed to the parent plant, which are liberated only at death.

In those organs which are not used for storage, the accumulated calcium remains for the most part permanently deposited. This is the case for example in foliage-leaves in which the absolute amount of calcium, and of silicon also, continually increases until the death of the leaf, whereas when the latter is adult the absolute amounts of phosphorus, potassiun, magnesium, and nitrogen, remains comparatively constant, although a certain decrease often occurs when the vitality of the leaf declines, or even before this, owing to the economic transference of a portion of these elements to the parent plant ${ }^{1}$. In such cases a portion of the living protoplasm may be decomposed and translocated, or even directly consumed. The constancy of the percentages of phosphorus, potassium, \&c. in the adult leaf can only be the result of unceasing exchanges, for the transpiration-current continually carries fresh supplies to it. These elements are probably conveyed to the stem along with the assimilatory products and probably partly in the form of proteid substances, while, as might be expected, no constant relationship exists between the percentages of phosphorus, nitrogen, \&c. (Sect. 74).

The period during which the production (or absorption) of organic substance is most active, does not necessarily precisely coincide with the period of maximal absorption of ash constituents, but nevertheless a certain indirect relationship exists between the accumulation of organic and of inorganic materials. Translocation may, however, be active without involving a marked increase in the dry weight, and this is always the case when the production is equalized by the consumption, while even in dying plants or in isolated parts or organs nutritive materials may still be conveyed from one region to another. Thus the fruits of cereals withdrat all the available materials from the surrounding parts of the inflorescence even after the ear has been removed from the parent plant ${ }^{2}$. Cereals under normal conditions appear to have accumulated a sufficient supply to complete their development before flowering is over, and hence if plants grown in a nutrient solution are then removed to pure water, normal fertile

1 Wehmer, Landw. Jahrb., I 892 , Bd. Xxi, p. 5133, and Ber. d. Bot. Ges., 1892, p. 152; Zöller, Versuchsst., I864, Bd. VI, p. 231 ; Rissmuiller, ibid., I874, Bd. Xvir, p. I7; Dulk, ibid., 1875 , Bd. XviII, p. I88; Flêche et Grandeau, Ann. d. chem. et d. phys., I876, v. sér., T. vili, p. $4^{56}$; Pässler, Chem. Centralbl., I 893 , II, p. 654 . On the leaves of evergreens, cf. Briosi, Bot. Jahresb., I 888 , p. 23. On the behaviour during premature leaf-fall cansed by summer drought, see G. Kraus, Bot. Zeitung, I 873, p. 4 oI, and the criticisms of Wehmer, l. c.

${ }^{2}$ Literature : de Candolle, Pflanzenphysiol., Bd. II, P. I 82 ; Lucanus, Versuchsst., I862, Bd. IV, p. I 47 ; Siegert, ibid., I864, Bd. vI, p. I34; Heinrich, Ann. d. Landw., I87I, Bd. LvII, p. 31 ; Nowacki, Unters. über das Reifen d. Getreides, I870; Nobbe, Versuchsst., I874, Bd. xvir, p. 277 ; Balland, Bot. Jahresb., 1888 , P. $4^{2}$; Holfert, Flora, I 890 , p. 281 ; Hotter, Versuchsst., I 892 , Bd. XL, p. 356 . 
fruits and seeds may be formed. Ash constituents are, however, usually absorbed from the soil up to the last stages of ripening, although finally only in very minute amounts. Cases have been observed, however, in which towards the end of ripening the total percentage of ash underwent no further increase or even decreased. The latter may also occur in certain organs from which ash constituents are withdrawn for storage, and since the different inorganic salts are unequally concerned in the various processes of constructive metabolism, it is only to be expected that the amounts of each salt absorbed from without should undergo marked fluctuations during the progress of development.

Both the concentration and the composition of the nutrient solution are of great importance, and any deficiency of such elements as phosphorus or potassium, \&c., causes the growing parts to withdraw them from the older organs and hence induces the premature death of the latter (Sect. 93). An abundant supply, however, not only prevents such exhaustion, but also suppresses to a certain extent the removal of the substances in question from the storage receptacles (Sect. 93), while at the same time its consumption rises slightly above the normal. Moreover when the roots are supplied with concentrated solutions of inorganic salts, the transpiration current may produce so marked an accumulation of these in the leaves that saline efflorescences form upon them (Sect. 23), and in this way a portion of the superfluous salts is removed. Similarly an excretion of saline substances is exhibited when a root-system is removed from a normal or concentrated nutrient solution to a very dilute one.

Micro-chemical methods have been employed, more especially by Schimper (Bot. Zeitung, I885, p. $75^{6}$; Flora, I 890 , p. 2 I I), for the detection of ash constituents, but Pfeffer (I. Aufl., Bd. I, p. 330) was perhaps the first to show that a salt might be traced by such means. Macro-chemical researches have for the most part been made in connexion with studies upon the accumulation and distribution of organic substances, and such are quoted in Sect. I09. A large part of the literature is mentioned in E. Wolff's Aschenanalysen, I8 7 I and i 880 . In addition, the following works may be mentioned: Arendt, Wachsthum d. Haferpflanze, I859; Fittbogen, Versuchsst., 1864 , Bd. vi, p. 474, and I870, Bd. xiII, p. I00; Pierre, Rech. s. 1. dév. du blé, ז 866 ; Knop u. Dworzak, Ber. d. Sächs. Ges. d. Wiss., I875, p. 76 ; Kreusler, Landw. Jahrb., I878, Bd. vir, p. 548 ; Pott, Versuchsst., I880, Bd. xxv, p. 95 ; Weiss, ibid., I880, Bd. xxvı, p. I9r ; Berthelot et St. André, Ann. d. chim. et d. phys., I883, vi. sér., T. v, p. 385 ; Compt. rend., I89I, T. cxiI, pp. I22, \&c. 
SECTION I08. The Mechanism and Causes of Translocation.

Any diosmosing substance will continue to penetrate a cell until a condition of equilibrium is reached, and hence the consumption of the substance in question will by disturbing this equilibrium cause further supplies to flow inwards. It is in this way that consumption regulates absorption and translocation, while by the conversion of the diosmosing compounds into other substances, large quantities of reserve-materials may be accumulated in the cells of storage receptacles. The selective power of a plant as well as of each individual cell is determined by its inherent properties and by the special character of its metabolism, and hence it arises that one cell may absorb much, another little or none of a given substance, and that in time very large quantities of a dissolved salt may be absorbed from an exceedingly dilute solution. On the other hand, any reconversion into diosmosing substances results in an outward flow away from the regions of higher concentration (Sect. 22). Translocation is induced and regulated by these means, and when the terminal cell of a hair, or the tip of a fungal hypha, consumes nutriment, a current is induced which supplies the deficiency by means of materials drawn from the productive organs or storage receptacles. The translocatory substances probably pass mainly through the cavities of the cells, but may diffuse to a certain extent through the imbibed water of the cell-walls when no penetration through the protoplasts is possible. Substances may be translocated in the form of excessively dilute solutions, so that frequently none can be detected in the translocatory channels, although large amounts may be conveyed to the consuming tissues, or may gradually accumulate in terminal storage tissucs. Starch, sugar, asparagin, may be passively secreted by the cells forming the translocatory channels, and the cell-walls may even be permeated by a very dilute solution of sugar or other soluble foodmaterials. All the substances contained within the protoplasts are retained when a translocatory tissue is placed in water, and hence for the transit from cell to cell a reconversion of the transitorily stored compounds into diosmosing ones is necessary. Consequently mere traces of the actual translocatory substances are present at any given moment, and the storage and mobilization must be repeated as many times as there are cells to be passed. This cannot be directly seen when the translocatory channels are filled with asparagin or sugar, but can readily be followed in the case of starch, starch grains being continually deposited and redissolved. It is obvious that these repeated reconversions must involve a considerable expenditure of energy, but this is more than counterbalanced by the decreased danger of loss and by the avoidance of concentrations injurious to the protoplast. 
By these means different substances may be scparated from one another, and the translocating materials restricted to a given path, for the tendency to irregular diffusion after diosmosis through the protoplast is counteracted by the absorbent activity of the cells capable of passive secretion. The seedling draws reserve-materials from the seed in the same manner, and similarly in a starved plant the young growing organs may withdraw the food-materials they require from the less retentive older leaves, and so induce premature leaf-fall ${ }^{1}$. A similar antagonism exists between the phloem and the neighbouring tissues, and hence it is only when the former is completely saturated that the latter receive and passively secrete the superfluous translocatory materials, although even then an accumulation is possible only where a power of passive secretion exists. It is for the latter reason that no starch or glucose can be detected in the epithelial cells of the scutellum of Triticum vulgare, although they are active agents in the transference of food-materials from the endosperm to the seedling ${ }^{2}$. This forms what is known as an interrupted translocatory channel ${ }^{3}$. In the primary meristem the non-accumulation of starch or sugar is frequently due merely to the absence of any power of passive secretion, but in other cases is owing to the rapidity of consumption.

Living protoplasts have apparently the power of modifying their permeability from time to time in various ways, and it is possible that not only may solid particles and oil-drops be ingested, but that dissolved non-diosmosing substances may be passed through the protoplast in a similar manner. The power of diosmosis is moreover not solely dependent upon the size of the molecules of the diosmosing substance, for many colloids are readily absorbed and excreted (cf. Chap. iv). Within the tissues a direct transference from cell to cell is possible by means of the fine interprotoplasmic connexions, but it is improbable that these are utilized to any marked extent in the transport of nutrient materials, for the passage from cell to cell is probably sufficiently rapid for all requirements if the cell-walls are normally permeable. Indeed, the large quantities of food-material conveyed to the seedling from the endosperm pass from cell to cell in this manner, and the transference still remains active even if direct contact between the scutellum and endosperm of a maize seed is interrupted by the interposition of a piece of filter paper or a thin film of gypsum. The coarser plasmatic connexions of the

1 Cf. Sects. 93, 106. The withdrawal of ash constituents from the older organs was proved by C. Sprengel, Die Lehre rom Diinger, 1839, p. 47 . A similar transference occurs during the repeated tuber-formation of potatoes grown in darkness. Cf. Schacht, Ber. iiber d. Kartoffelpflanze, 1856 , p. 6 ; Hanstein, Sitzungsb. d. Niederrh. Ges., Feb. 3, i 87 I, \&c. On transference of water, cf. Sect. 34 .

2 Sachs, Jahrb. f. wiss. Bot., $186_{3}$, Bd. 1II, p. 248 .

s Several such observations are mentioned by Sachs, l. c. Cf. also de Vries, Landw. Jahrb., I 879 , Bd. viri, p. 444 . 
sieve-tubes seem, however, to be used to a marked extent for direct translocation from one sieve-tube segment to another, and a very slight pressure suffices to cause currents to flow in a given direction through a sieve-tube (cf. Sect. 20).

Since diffusion takes place with great slowness, currents in mass and mechanical admixture are of the utmost importance in ensuring rapid transference, for if a substance becomes rapidly distributed throughout a cell immediately after its entry, the transit to the next cell requires only a comparatively short time, no matter whether the passage from one cell to the other is effected diosmotically or by means of protoplasmic connexions. Hence granted similar osmotic powers, longitudinal transference will be much more rapid through prosenchyma than through parenchyma, where the number of partition-walls to be passed is much greater. It is perhaps mainly for this reason that translocation is most rapid in the phloem, and the coarse perforations of the sieve-plates render the sieve-tubes especially adapted for this purpose (Sect. I06).

A sufficiently rapid admixture is attained within the cell by mechanical swaying or bending movements of the entire plant, by changes of temperature, by variations of turgidity or of tissue-tensions, \&c.. while streaming movements of the plasma are especially important, although these do not occur in many cases under normal conditions, and are usually not exhibited by the sieve-tubes or conducting tissue of the phloem. Hence sufficiently active translocation is possible without the aid of any marked streaming movements in the plasma, which de Vries ${ }^{1}$ erroneously regarded as of universal and indeed of decisive importance in this respect. Whenever streaming movements are of normal occurrence, they are primarily of importance in securing the rapid distribution of substances within the cell, for apparently the plasma of the connecting threads is stationary, or at any rate not in such active movement as would be necessary to convey large quantities of substance from cell to cell ${ }^{2}$. Even marked unilateral pressure is insufficient to induce any streaming movement in mass through the minute canaliculi occupied by the connecting plasmatic threads, whereas this readily happens through the coarse pores of the sieve-plate, as is shown by the fact that when the sieve-tubes are opened

${ }^{1}$ De Vries, Bot. Zeitung, I $88_{5}$, p. I. Cf. Pfeffer, Landw. Jahrb., i 876 , Bd. v, p. I I ; Fnergetik, 189 I, p. 269, and Sects. 20 and 22. De Vries supposed that plasma-streaming was of general normal occurrence, from observations made on sections, whereas Halptfleisch has shown (Jahrb. $f_{0}$ wiss. Bot., 1892 , Bd. Xxiv, p. I73) that streaming frequently commences in response to the stimulating effect of injury, or is brought into prominence by the action of special external stimuli. On the absence of plasma-streaming in sieve-tubes, cf. Strasburger, Leitungsbahnen, I 89 I, p. $3^{6} 3$.

${ }^{2}$ Cf. Pfeffer, Energetik, I 89 I, p. 272 . Czapek, Ber. d. Bot. Ges., I 897 , p. I 28 (Sitzungsb. d. Wien. Akad., ${ }_{1} 8_{97}$, Bd. Cvi, Abth. i, p. I 55). Kienitz-Gerloff's discussions (Bot. Zeitung, I 893 , p. $\left.3^{6}\right)$ do not alter the matter as here represented. 
their contents escape. In the intact plant, the direction of the currents through the sieve-plates probably varies according to the point at which pressure is applied, and such irregular movements along with the admixing movements in companion cells, \&c. are probably of great importance in accelerating the transference of non-diosmosing and diosmosing substances in both directions. This is the main function of the sieve-tubes and phloem, and the existence of a constant upward or downward stream of water would hinder or prevent translocation in the opposite direction, and hence would be a great disadvantage to the plant.

The exchanges to and from a living cell are primarily determined by its own powers and vital activity, and these are influenced in various ways by the surrounding cells and by the other external conditions. The complicated relationships and interactions involved have already been dealt with as far as possible in Sect. 9.3, where it has been mentioned that isolated endosperms, cotyledons, pieces of rhizomes or of bulbs, become completely depleted when in contact with sufficient water, especially if the latter is renewed from time to time, for a slight accumulation of the mobilized products apparently suffices to inhibit the further production of diosmosing substances. The diosmotic propertics of the protoplast are, however, capable of modification, and hence it is impossible to say in what manner a stoppage of depletion is produced by the action of chloroform or cther, or by the absence of oxygen ${ }^{1}$. Similarly Czapek's observation that translocation ceases in a chloroformed leaf-stalk may have several meanings, and no definite conclusion can be drawn from the fact that the absorption and accumulation of aniline dyes by living cells is not prevented by anaesthetization or by the removal of oxygen ${ }^{2}$. The continuation of translocation in partially plasmolyzed conducting channcls simply shows that turgidity is not necessary for the performance of this function.

Living tissues influence one another not only by the removal of particular substances and by the excretion of others, but also by direct stimulatory actions. Thus enzymes and solvent substances are frequently employed not only to obtain nutriment from dead masses, but even from other organisms or from other organs of the same plant (Sects. 65, 91). The haustorial organs of many seedlings (grasses, \&c.) act upon the endosperm of the seed in this manner, but even when absorption is most active, plastic substances may escape in the opposite direction from the endosperm when it is in direct contact with water. The depleted storage receptacles may be more or less completely filled again in the case of rhizomes, bulbs, cotyledons, \&c., whereas the endosperm of grasses when

1 Puriewitsch, Ber. d. Bot. Ges., I 896, p. 2 Io.

${ }^{2}$ Pfeffer, Unters. a. d. Bot. Inst. z. Tuibingen, 1896 , Bd. II, p. 284 . Cf. Sects. 16, 17. 
once cmptied appears to have fulfilled its purpose and to have lost the power of renewed storage (Puriewitsch, l.c., p. 2I I). The structure of the conductory channels and their connexions with the surrounding tissue are of the utmost importance in translocation, and the point of union of the leaf-trace-bundle will partially determine the length of the course through which the plastic products from the leaf must travel in order to reach the growing apex. The conducting channels may be more or less completely isolated by being surrounded by air spaces or less permeable tissues. Similar means are adopted by the plant to lessen the intercourse with the external world, and the suberized endodermis and similar layers are probably of importance for insulating purposes and to prevent irregular diffusion ${ }^{1}$.

Corky and cuticular coverings seem to prevent the extraction of diosmosing food-materials from parts in contact with water, and this is of great importance in the case of beetroots, turnips, bulbs and rhizomes. Puriewitsch found that a bulb-scale of an onion lost no perceptible quantity of sugar while its cuticularized surface was in contact with water, but that the whole of the stored sugar was removed when a cut surface was bathed with renewed supplies of liquid. Cork and cuticle are absent from the young root where a power of rapid exchange is necessary, and sugar is actually excreted from the root of a seedling imbedded in gypsum, in which case plastic materials accumulate to excess owing to the enforced cessation of growth. Over-accumulation is normally prevented by energetic consumption, by translocation, and by temporary storage in the phloem and neighbouring tissues, while in semi-aquatic plants the upwardly directed water-current aids in preventing any loss through the roots. Other factors may also enter into play, and it is possible that mere contact with water is insufficient to withdraw any of the transitorily stored substances from the intact root-apex. Some such specific peculiarity is essential in the case of algae to prevent the escape of the stored materials, for if the thallus of these plants behaved similarly to endosperm-tissues no accumulation of sugar or starch would be possible so long as it was in contact with water, whereas as a matter of fact starch is often present in considerable abundance. Methyl-blue is permanently retained by living cells when once absorbed, and it is only when traces of diosmosing products are continually formed and removed that a non-diosmosing substance gradually disappears from a cell (Pfeffer, 1.c., p. 296). Both the outer and inner walls of algal filaments are readily permeable, and hence the diosmotic tranference of sugar from one cell to another would apparently not be possible without great waste. It has yet to be determined by what means this loss is prevented, or whether protoplasmic connexions become in this case of 
especial importance in translocation by transfering the plastic materials from cell to cell mainly in the form of non-diosmosing substances.

The above account of the phenomena of translocation is based mainly upon the researches of Pfeffer (Landw. Jahrb., $1876, \mathrm{Bd} . \mathrm{v}$, p. III). It is at present impossible to distinguish clearly between the actual translocatory substances and those which undergo transitory storage along the conducting channels, for it is still doubtful whether sugar, asparagin, \&c. are stored up in the form of non-diosmosing compounds, or whether it is the protoplasmic activity which determines their retention within the cell or their transference in either direction. It is, however, certain that glucose, cane-sugar, asparagin, and certain proteids are able to penetrate the cell-walls and living protoplasts, and hence may be directly absorbed or excreted as such, while living cells may also absorb finely emulsified fatty oils. All such substances are therefore capable of diosmotic transference, but it does not follow that they function as translocatory compounds in all plants, for in addition to the existence of specific peculiarities, different plants frequently utilize the same materials in a variety of ways. Thus in certain plants the stored fatty oils are partially translocated in the form of glucose, but in other cases, where the conducting channels become filled with oil, the latter may undergo direct translocation (seedlings of Linum usitatissimum, Camnabis sativa, Papaver somniferum, Allium cepa ${ }^{1}$ ). Cane-sugar is also capable of diosmotic transference, but in the beetroot it is not carried in this form to the growing stems and leaves. Similarly proteids may be directly translocated, although usually they undergo previous decomposition into amides, \&c., which in Pangium edule is accompanied by a formation of hydrocyanic acid. It is quite possible that substances capable of direct translocation may accumulate in the storage receptacles, or even in the cells of the conductory channels, side by side with others, such as starch, in which a preparatory modification must precede translocation. Many substances, however, mainly occur in the storage receptacles, whereas others are found wherever translocation is active.

\section{SECTION IC9. Special Examples of Translocation.}

Only a few of the commoner instances of storage and translocation can be mentioned, and for further details reference must be made to the quoted literature. No essential difference exists between the food-substances which are intended for immediate consumption and those which are retained during long resting periods, for the latter may be artificially shortened or suppressed, while substances which normally are only transitorily accumulated may remain intact for long periods of time when vital activity is depressed to a minimum. Seedlings and isolated parts, which are able to nourish themselves only after a certain stage of development is reached,

' Sachs, Bot. Zeitung, 1863, p. 57 ; Jahrb. f. wiss. Bot., 1863, Bd. 1II, pp. 213, 25I ; R II. Schmidt. Flora, is9 1, p. 34 2; Leclerc du Sablon, Rev. gén. d. Bot., is95, T. viı, p. I 49. 
must be furnished at the outset with a sufficient store of nutriment to enable the seedlings to form at least one root and one assimilating leaf. Similarly it is by means of their stored reserve-materials that trees form new leaves in spring. Usually more food-material is accumulated in seeds than is necessary for the formation of an independent seedling. and hence unripe seeds may germinate and develop although they contain but little food-material ${ }^{1}$. Similarly when the endosperm of a maize fruit, or the cotyledons of Vicia, Phaseolns, and Helianthus, are partially removed, the maimed embryo may finally develop to maturity, although when too much is taken away the growing embryo soon dies of starvation ${ }^{2}$. Similar results are obtained when buds or the eyes of potatoes are removed together with small portions of the neighbouring tissues and are allowed to develop. In all such cases the usually slow and feeble growth shows how important a rich supply of food-material is for strong and healthy development, and plants favoured in this manner at the commencement may retain an advantage during the entire summer, and would assuredly suppress weaker plants if free to compete with them.

As a general rule the reserve-materials of the seed suffice for the development of several leaves, so that the production of plastic products by photosynthesis becomes sufficiently active before the stored food is exhausted. Frequently, however, especially in seeds with but little reservefood, the latter is nearly exhausted at a certain stage of development before photosynthetic assimilation is active, and a retardation of growth ensues. No such transitory starvation occurs normally during the development of shoots from trees, bulbs or tubers, and trees contain sufficient food to form a second crop of leaves after the first has been removed. Similarly potatoes contain a large quantity of food-material after the sub-aërial shoots have been formed, and this food is transferred to the young developing tubers ${ }^{3}$.

The different food-materials of the seed are not always present in corresponding amounts, but instead the percentage composition may alter as development progresses, and if all supplies from without are cut off large quantities of certain substances may be left untouched. Thus the

${ }^{1}$ See Nobbe, Samenkunde, 1876, p. 339; also Sagot, Bot. Jahresb., I 874 , p. 831. [Many grass seeds, however, contain barely sufficient food-materials for germination, and hence the young seedlings rapidly perish in darkness or when sown at some depth.]

2 Such researches were performed by Malpighi, Opera omnia, 1687, I, p. 109, and Opera posthuma, 1698 , p. 86. Cf. also Treviranus, Physiol., 1838 , Bd. II, p. 594. More recent researches: Sachs, Keimung d. Schminkbohne (Sitzungsb. d. Wien. Akad., 1859, Bd. xxxrir, p. 84, and Bot. 7eitung, I862, p. 148); van Tieghem, Ann. d. sci. nat., I8 73, v. sér., T. Xvir, p. 206 ; Blocisrewski, Landw. Jahrb., 1876 , Bd. vi, p. I46; G. Haberlandt, Die Schutzeinrichtungen d. Keimplanze, 1877 , p. 28 ; Brown und Morris, Bot. Zeitung, $189^{2}$, p. $4^{62}$ (see also Journ. Chem. Soc. for $189^{2}$ ); Mesnard, Ann. d. sci. nat., I 893 , vii. sér., T. Xvir I, p. $29^{6}$; Hansteen, Flora, Erg.-bd., pp. $4^{28}$, \&cc. ; Ewart, Journ. of Linn. Soc., Vol. xxxi, I 896, p. 560 influence upon development of power of photosynthesis).

${ }^{3}$ De Vries, Landw. Jahrb., is 77 . Bd. vi, p. 510. 
seeds of the Leguminosae contain relatively too little non-nitrogenous material, for asparagin accumulates to a marked extent in the seedling when no photosynthetic production of organic (non-nitrogenous) substance is possible. Moreover Godlewski ${ }^{1}$ found that seedlings of Raphanus, germinated in darkness or in air free from carbon dioxide, develop to a further extent on an inorganic nutrient solution than they do on pure water. This is because the seed is relatively deficient in ash constituents. In several other seeds calcium is present in insufficient amount (Sect. 74). De Vries found, however, that clover seeds contain the proper relative amount of ash constituents ${ }^{2}$. Although certain plants are able to grow when supplied with organic food solely in the form of proteids, it is usually the case that the reserve-materials include both non-nitrogenous and nitrogenous substances, and indeed the former are generally more abundant (Sect. 79). The commoner reserve-materials have already been mentioned, as also the fact that no particular reserve-substance is essential for all plants. Starch, dextrose, laevulose, fatty oil and cane-sugar are the commoner non-nitrogenous reserve-materials, and they may either occur singly or several together in the same plant. Inulin is less generally distributed, but often forms the main reserve-substance in the underground parts of perennial Compositae. Many other substances are restricted to certain groups of plants or are localized in special organs; thus glycogen is present in many fungi, while reserve-cellulose occurs mainly in certain seeds (Sect. 83). Pectins and such forms of carbohydrates as mannite, trehalose, gums or mucilage function as reserve food-materials in a few plants (Sects. $82,8_{3}$ ), and organic acids acquire a similar importance in the Crassulaceae (Sect. 85). Nitrogen is usually stored in the form of proteids and amides, such as asparagin, leucin, glutamin, \&c. The proteids of seeds contain almost the whole of the nitrogen present, whereas in tubers, bulbs, and rhizomes, frequently from 40 to 70 per cent. of the nitrogen occurs either in the form of amides, or of other compounds, among which ammonium salts or nitrates are sometimes especially abundant (Sect. 79). The ash constituents are deposited partly as salts, and partly as organic compounds. Almost the whole of the phosphorus, sulphur and iron is present in the seed in organic combination, so that the reactions of the free ions are not given, whereas in bulbs, tubers, \&c., only a portion of these elements is retained in this form (Sect. 74).

With the exception of reserve-cellulose the nutrient substances usually accumulate within the living protoplast, and starch is actually imbedded in the plasma, whereas fatty oils when abundant are excreted into the central vacuole. The cell-sap also contains the greater part of the soluble foodmaterials and the reserve-proteids, which when present in excess may

${ }_{1}$ Godlewski, Bot. Zeitung, 1879, p. 99. Cf. also Prantl, ibid., 18S1, p. 77 I (Prothalli).

${ }^{2}$ De Vries, Landw. Jahrb., 1877 , Bd. VI, p. 510 . 
separate out as amorphous masses or as regular crystalloids (Sect. 78 ). In the form of insoluble or colloid bodies very marked accumulation is possible without a dangerously high osmotic pressure being produced (Sect. 24). The condensation of monosaccharides to polysaccharides is of similar importance, although in certain cases, as for example in the onion, glucose may accumulate to a marked extent without undergoing any condensation, and without any conversion into non-diosmosing carbohydrates being necessary. In organs which are normally subject to desiccation only small quantities of soluble crystalloids appear to be present, and this may be of importance in avoiding the injurious effect of over-concentration during drying. Seeds are organs of this character, and usually contain in addition to proteids mainly starch or oil, or both together. Fatty oil seems in general to be preferable, for according to Nägeli ${ }^{1}$ nine-tenths of all Phanerogams possess oily seeds. The oil fills up the spaces between the aleurone grains, which may contain crystalline or amorphous masses of proteid, and in addition globoids composed of an organic compound of magnesium and calcium rich in phosphoric acid.

Fatty oils are very widely distributed and may even be deposited in succulent organs, such as the tubers of Cyperzis esculcntus, but the preferable employment of oil as reserve-material in seeds, spores, \&c. must be due to its possessing some special advantages. It is possible that the presence of oil increases the power of resistance to desiccation ${ }^{2}$, while a fact of the utmost importance is that in the form of oil a greater supply of energy can be stored up in a certain space than in the form of carbohydrates. Hence for this reason alone oil is preferable wherever economy of storage is necessary, or when seeds or spores disseminated by the wind require to be as light as possible.

In all phenomena of this kind many factors enter into play, and when we consider that different food-substances may mutually replace one another in the various organs of the same plant, it is obviously erroneous to conclude from any of the facts mentioned that a substance such as oil affords under all conditions the most suitable nutrient or reserve-material. Development may be either slow or rapid no matter whether the organic food is supplied in the form of sugar, starch, oil or reserve-cellulose, and as a matter of fact a supply of nutriment simply renders possible the

1 Die Stärkekörner, 1858, p. 536 . Globoid : Pfeffer, Jahrb. f. wiss. Bot., 1872, Bd. viri, p. 429. Aleurone grains : cf. Sect. 79. Phosphoric acid: Sect. 74. On other crystalloids, cf. Zimmernann, Beitr. z. Morph. u. Physiol., 1893, p. 54.

${ }^{2}$ [Organs containing oil do not appear on this account to be more resistant to desiccation: thus Dicranum (moss), rich in oil is less resistant than Ortholrichum or Bryum (Assim. Inhib., Journ. of Linn. Soc., 1896, xxxi, p. $3^{68}$ ), hemp and willow seeds (oily), haricots, kidney beans, \&c. (starchy), are readily killed by desiccation, whereas peas, barley (starchy), and linseed (oily) are extremely resistant, and the seeds of Helianthus and Cucurbita occupy an intermediate position (cf. Ewart, Trans. of Liverpool Biol. Soc., Vol. viri, 1894, p. 207, and Vol. xI, 1897).]

$\mathrm{Q}$ q 2 
development of the characteristic vital activity. Hence beetroots, onions, \&c., in spite of their great store of sugar, are just as capable of a winter resting-period as are starch-laden trees or potatoes, and the rapidity with which the reserve-materials are consumed in spring is entirely dependent upon the inherent character of the plants in question and their activity of consumption.

It has already been mentioned (Sect. 78 ) that plastic and reservesubstances in general differ somewhat from the actual constructive materials of which the plant is composed, and that any given cell may according to circumstances store certain substances temporarily or for prolonged periods. Hence the differentiation of special secretory cells and tissues is not necessarily essential, although for purposes of maintenance and reproduction the formation of special storage receptacles, such as seeds, tubers, bulbs, \&c., has become of almost essential importance ${ }^{1}$. Similarly in trees the restriction of the reserve-materials to particular tissues has arisen by secondary adaptation in correspondence with economic necessities.

Germination of seeds ${ }^{2}$. The reserve-materials are either stored up in the cotyledons of the embryo, or to a greater or less extent in accessory tissues as endosperm or perisperm. The cotyledons may subsequently function as green leaves, or simply as storage receptacles, or may act as haustoria by means of which food-materials are absorbed from the albumen-tissue. The same natural order may contain plants with cotyledons which become capable of photosynthesis (Lupinus), and others whose cotyledons do not acquire this power (Pisum). The former come above ground, but those of Pisum usually remain buried, while the cotyledons of Phaseolus if exposed to light may turn green and exhibit a feeble power of photosynthetic assimilation before they shrivel ${ }^{3}$.

The nutrient materials are always absorbed from the accessory tissues of the seed through or by means of the cotyledons, and these in Mirabilis jalapa surround the endosperm (c, Fig. 69) and when the latter is exhausted unfold as green foliage-leaves. Similarly the cotyledons of Ricinus escape from the emptied sac-like endosperm, and they may become partially green while still enclosed by it. In Allium cepa the cotyledon also functions as a foliage-leaf and withdraws its apex from the endosperm after the whole of the food-material has been transferred to the developing embryo. In grasses the cotyledon forms the scutellum, functions solely as an absorbent organ ( $s$, Fig. 70 ), and does not increase in size, whereas the

1 Cf. Haberlandt, Physiol. Anat., i 896,2 . Aufl., p. 345 .

${ }^{2}$ On the morphology and biology of the process, cf. Klebs, Unters. a. d. Bot. Inst. z. Tübingen, I $88_{5}$, Bd. I, p. $53^{6}$; Lubbock, Contrib. to Knowledge of Seedlings, IS92; H. Haberlandt, Schutzeinrichtungen d. Keimpflanze, 1877 ; Nobbe, Samenkunde, 1875 .

${ }^{3}$ Eren though green, cotyledons assimilate but little or not at all while still loaded with reservematerials (cf. Ewart, Journ. of Linn. Soc., I 897 , Vol. xxxi, p. 557). 
tip of the cotyledon of the date grows to such an extent as absorption progresses that it finally almost entirely fills the cavity of the seed ${ }^{1}$.

The extent to which the endosperm and the embryo are respectively concerned in the depletion of the former can only be determined by direct observation in each case, bearing in mind the fact that a very slight accumulation of the translocatory products suffices to inhibit further mobiliza-

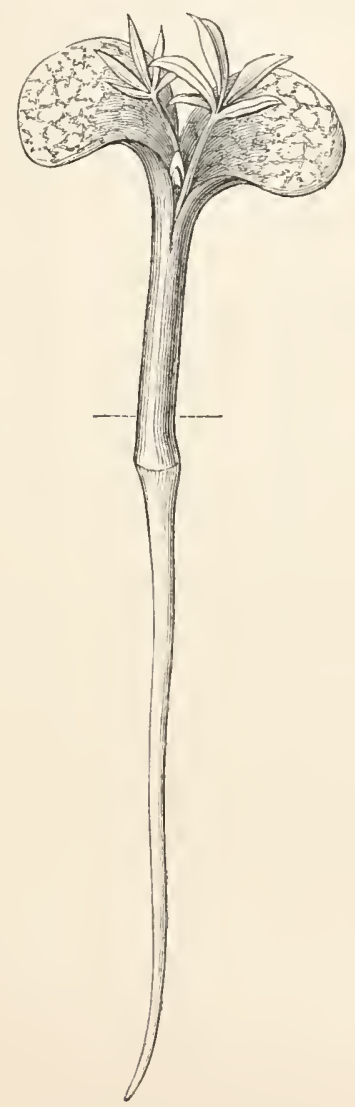

FIG. 68. Seedling of Lupinus lutens. The dotted line indicates the surface of the soil.

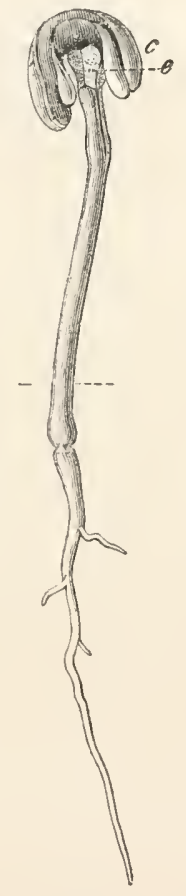

FIG. 69. Seedling of Mirabilis Jalapa.

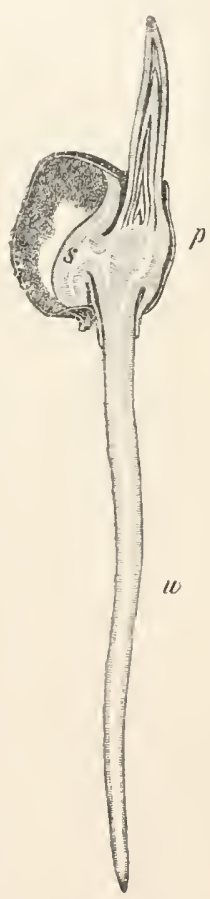

F1G. 70. Young seedling of Zea Mays in median longitudinal section.

tion, which therefore soon ceases in isolated endosperm or in isolated cotyledons. Hence van Tieghem erroneously concluded that the endosperm of grasses, Phoenix, \&c. played an entirely passive part, whereas Hansteen was able to completely or partially empty these supposed inactive storage tissues by continually removing the mobilized products as fast as they

${ }^{1}$ For details, see Haberlandt, Physiol. Anat., IS96, p. 210 ; Ann. d. Jard. bot. d. Buitenzorg, ${ }_{1893}$, T. XII, p. 111 ; Tschirch, ibid., 1891, p. 17y; Hirsch, Bot. Jahresb., 189o, p. 660; Klebs, l.c.. p. 561 ; Ebeling, Flora, 1885 , p. 19s ; Schlickum, Bibl, bot., 1896 , Heft 35. 
were formed ${ }^{1}$. The endosperm of Ricinus grows to a certain extent ${ }^{2}$ even when isolated, and this is accompanied by marked metamorphosis of the reserve-materials. The latter may also occur in the absence of any growth if the products do not at once exert an inhibitory restraining influence, or if they undergo further changes, or are deposited again in some other form. The potential powers which isolated organs exhibit may not be called into play in the intact plant, or at least may not be exercised to the full possible extent (Sect. 4).

Hansteen was able to completely empty the non-growing and apparently inactive endosperms of Hordeum anlgare and Zea Mays, by attaching the surface normally in contact with the scutellum to a little column of gypsum standing with its base in water (cf. Sect. 93). The same result is obtained by direct contact with water if the remainder of the endosperm is exposed to air and hence sufficiently supplied with oxygen ${ }^{3}$, while since the entire absence of micro-organisms was assured in these experiments, it is obvious that the mobilization and depletion are due to the activity of the endosperm itself. The process ceases as soon as the products accumulate to only a slight extent, and hence it soon stops if a small quantity of water is used, while no conversion of starch is produced if the endosperm is placed in direct contact with a solution of sugar.

Different varieties of the same species possess this power in varying degrees, and thus Puriewitsch found the endosperm of certain kinds of maize might be completely emptied, whereas other varieties only underwent partial depletion even when kept in contact with large quantities of water. In such cases as these the aid of the secretory activity of the scutellum is apparently necessary for complete depletion, and as a matter of fact the scutellum is able to excrete large quantities of diastase and thus to exercise a marked solvent action upon dead endosperm or upon starch paste $^{4}$. Diastase is apparently excreted into the endosperm by the scutellum even when full depletion may take place without such assistance, but it is always possible that the production and excretion of this accessory

1 Van Tieghem, Ann. d. sci. nat., I 876 , vi. sér., T. IV, p. I83. Cf. also Green, Annals of Botany, IS90-9I, Vol. IV, p. 383; Hansteen, Flora, I894, Erg.-bd., p. 419 ; Pfeffer, Ber. d. Sächs. Ges. d. Wiss., I 893 , P. 422 ; also Puriewitsch, Ber. d. Bot. Ges., 1896, p. 207 ; Jahrb. f. wiss. Bot., IS97, Bd. xxxı, p. 1; Grüss, ibid., I $8_{95}$, p. 10; Landw. Jahrb., I896, Bd. xxv, p. $38_{5}$; Linz, Jahrb. f. wiss. Bot., I 896 , Bd. XXIX, p. 265. That the reserve-materials are deposited in living tissues has been shown by Bredow, Jahrb. f. wiss. Bot., I 891 , Bd. Xxir, p. 349 ; Zimmermann, Bcitr. z. Bot. Centralbl., I 893 , Bd. III, p. 430 ; Zacharias, Flora, I 895 , Erg.-bd., p. 228.

${ }^{2}$ Mohl, Bot. Zeitung, i 861, p. $25 \%$. Cf. also van Tieghem, 1. c.; Leclerc du Sablon, Rev. gén. d. Bot., I 895 , T. VII, pp. 162, 269 ; Zacharias, Flora, I895, Erg.-bd., p. 234 .

3 Puriewitsch, l. c., p. 209. Grüss (Ber. d. Bot. Ges., I S95, P. I) attempted to show that the phenomenon was due to the action of the gypsum, but has since altered this opinion to onc more in accordance with the facts.

"Hanstecn, 1. c. Cf. also the literature given by IIansteen (1. c., p. 426) and Griiss (Landw. Jahrb., I 896 , Bd. xxv, p. 429); also Linz, l. c., and Grüss, Jahrb. f. wiss. Bot., I 897, Bd. xxx, p. 645. 
diastase may be regulated according to the needs of the plant. The disappearance of starch from the scutellum inwards affords no certain proof that the latter excretes diastase, for the same phenomenon is shown when the isolated endosperm is emptied by contact with water ${ }^{1}$, and if the back of the endosperm is exposed to contact with water the disappearance of starch progresses from this surface inwards. The fact that fragments of living endosperm may be partially or entirely emptied in a similar manner shows that all cells of the endosperm possess more or less marked mobilizing powers, and also disproves Haberlandt's ${ }^{2}$ supposition that the alcurone layer is responsible for the production of diastase. The changes produced can hardly be due to diastase alone, for not only reducing sugar but also a non-reducing sugar (probably canc-sugar) appear as the products of mobilization. No enzymatic action is necessary in the seeds of grasses for the removal of their nitrogenous reserves, for according to Puriewitsch these appear in the surrounding fluid mainly in the form of proteids. It is however possible that in certain cases the aid of proteolytic ferments may be invoked.

The absorbent parts of the cotyledons seem to have a more or less marked power of producing and excreting diastatic and cytasic or cellulosedissolving ferments. This is especially the case when insoluble carbohydrates are stored in the seed, for a cellulose ferment has commonly been detected in seeds which contain reserve-cellulose. The latter may, however, also be mobilized by the storage-cells themselves, for Hansteen found that in time the isolated mucilaginous endosperm of Tetragonolobus furpurcus might be almost entirely emptied by continually removing the sugar produced, while Puriewitsch has shown that the same occurs in the isolated endosperm of Phoenix dactylifera when kept in contact with a continually renewed supply of water ${ }^{3}$.

Similarly the starch disappears from the isolated cotyledons of Pisum sationu, Vicia faba, Phascolus multiflorus (Puriewitsch), if the sugar produced is continlially removed, and oil disappears from the isolated cotyledons of Lupiuus albus (Puriewitsch) and Helianthus aumuns (Hansteen) in the same manner when they are kept in contact with water. Moreover

1 The progress of the metamorphosis may be different when the mobilized products exercise no marked inhibitory action. Van Tieghem (1. c., p. I S6) states that the changes commence peripherally, and rapidly spread over the entire albumen. Sachs (Sitzungsb. d. Wien. Akad., I 859, Bd. Xxxvil, p. 90) observed that in the scarlet-runner the conversion of starch begins at the stalk of the cotyledon and travels outwards, whereas Baranetzsky (Die stärkeumbildenden Fermente, is $8 \delta, p .5^{8}$ ) found that in other cases the solution of starch progressed centripetally.

${ }^{2}$ Haberlandt, Ber. d. Bot. Ges., i S9o, p. ${ }_{4}^{6 .}$

${ }^{3}$ On the different forms of reserve-cellulose, cf. Sect. $8_{3}$. On the mode of deposition and the process of solution: Grüss, Landw. Jahrb., IS96, Bd. xxv, p. 386 ; Bibl. bot., I 896 , Heft 39 ; E. Schulze, Ber. d. Bot. Ges., I896, p. 66 ; Elfert, Bibl. bot., I\$94, Heft 30 ; Nadelmann, Jahrb. f. wiss. Bot., 1890 , Bd. XxI, p. 6o9; Reiss, Landw. Jahrb., I $\$ 8_{9}$, Bd. xix; Leclcrc dn Sablon, Rev. gén. d. Bot., I895, T. vir, p. 401 ; Coley, Bot. Ccutralbl., I897, Pd. Lxx, p. $20_{4}$. 
the nitrogenous reserve-materials may be removed by the same means, asparagin escaping from the cotyledons of Lupims, but mainly soluble proteids from the endosperm of grasses and of Phoenix, although the proteid decomposition which is especially marked during the germination of the Leguminosae ${ }^{1}$ probably occurs to a certain extent in all cases ${ }^{2}$.

Translocation of the photosynthetic products. The paths which these follow have already been mentioned in Sects. 55 and $106^{3}$. All translocation from the leaf soon ceases when its assimilatory activity is suspended by darkness or by the alterations which occur during autumn. In the latter case the disorganization of the chloroplastids and other plasmatic organs may render available a certain amount of food-material ${ }^{4}$, although as a general rule little or no phosphorus or nitrogen is removed from the leaf before it falls. Similarly the fallen leaves may in some cases contain a by no means inconsiderable amount of glucose, and usually starch is present to the last in the guard-cells and occasionally in other cells also ${ }^{5}$.

${ }^{2}$ Large quantities of asparagin are formed during the germination of Mimosa pudica and Acacia lophanta (Pfeffer, Monatsb. d. Berl. Akad., 1873 , p. 788 ), but only a slight amount in Tropacolum majus, \&c. Jahrb. f. wiss. Bot., I8 72 , Bd. viII, p. 561).

${ }^{2}$ Details are given in the works already quoted, as well as in the following ones. Mlainly microcliemical works are: Sachs, Sitzungsb. d. Wien. Akad., 1859, Bd. xxxril, p. 57 (scarlet-runner); Bot. Zeitung, I 859 , p. $17 \%$ (oily seeds); ibid., 1862, p. I 45 (grasses); ibid., I862, p. 241 (date); ibid., $186_{3}$, p. 57 Allium cepa) ; Summary in Jahrb. f. wiss. Bot., I863, Bd. III, p. I83; Hofmann, Jahresb. d. Agr.-chem., I865, p. 133 (wheat and clover); Roestel, ibid., I868-69, p. 229 (rye); I'feffer, l. c. (Leguminosae); Gressner, Bot. Zeitung, I $8_{7 .+}$, p. SoI (Cyclamen); De Vries, Landw. Jahrb., 1877, Bd. vi, p. 466 (clover), and ibid., I878, Bd. vir, p. I9 (potato); Jörissen, Les phén. chim. d. l. germ., I $88_{5}$, and Bot. Centralbl., $188_{7}$, Bd. Xxx, p. 5 ; Schmidt, Flora, 189I, p. $33^{5}$; Belzung, Ann. d. sci. nat., I892, vii. sér., T. xv, p. 203; Mesnard, ibid., I893, T. Xvirl, p. 270; Leclerc du Sablon, Rev. gén. d. But., i 895 , T. viI, p. I62; is97, T. 1x, pp. 5, 313. The works of Hanstein, Griiss, Reiss, \&c., have already been mentioned. The following are the principal macrochemical researches: Hellriegel (rape), Journ. f. prakt. Chcm., I855, Bd. Lxiv, p. 94; Oudemanns u. Rauwenhoff (peas and buckwheat), Linnaea, Is $59-60, \mathrm{Bd} . \times \mathrm{xx}, \mathrm{p} .224$; v. Planta (maize), Ann. d. Chem. u. Pharm., I860, Bd. c.xv, p. 332 ; Peters (cucumber), Versuchsst., I86 I, Bd. III, p. I ; Flcury (hicinus, Euthorbia, rape, and ahmond), Ann. d. chim. et d. phys., 1865, iv. sér., T. 1r, p. 47; A. Beyer (Lupinus', Versuchsst., $186_{7}$, Bd. IX, p. I6s; H. Karsten (bean), ibid., I870, Bd. XIII, 1. I 76 ; Sachssc, Über chem. Vorgänge bei lieimung $x$. Pisum, IS $\boldsymbol{\tau}_{2}^{2}$; Laskorsky cucumber), Versuchsst., $18_{7+}$, Bd. Xvir, p. 239; Mïntz in Boussingault's Agron., \&c., i $\delta_{7+}$, T. r, p. 50; Detmer, l'hysiol.-chem. Unters. über Keimung ölhaltiger Samen, I875; E. Schulze, numerous works already quoted from 1876 onwards (Sects. 79-8I); Brown and Morris, Journ. Chem. Soc. I892 (Ref. in Bot. Zeitung, I\$92, p. $4^{62}$ ); Frankfurt, Versuchsst., I 893 , Bd. XLIII, p. I 42 ; Prianischnikow, ibid., I 894 , Iil. XLY, p. 253; Jessen-IIansen, Meddelelser fra Carlsberg Lab., I896. Bd. v, p. 69; Merlis, Versuchsst., I 897 , Id. NLVIr, p. fis; also the works of Jörissen, Schmidt, Leclerc du Sablon, quoted above. Additional litcrature in Chaps. viii and ix. $\Lambda$ few numerical examples are given in Sects. $S_{1}$ and $S_{2}$.

${ }^{3}$ Cf. Sachs, Flura, 1862 , pp. 316,362 ; Schimper, Bot. Zeitung, $188_{5}$, p. 762 ; Haberlandt, l'hysiol. Anat., Isg6, 2. Aufl., p. 24 I.

${ }^{4}$ Sachs, Flora, IS63, p. 200 ; Zacharias, Bot. Zeitung, I883, p. 214 ; Busch, Ber. d. Bot. Ges., 1889. Generalvers., p. 25; Kywosch, ibid., I897, p. I95. Cf. Sect. 107; Pässler, Chem. Centralbl., I 892, II, p. 654 .

${ }^{5}$ A. Fischer, Jahrb. f. wiss. Bot., I891, Bd. xxir, p. 90 (glucose); Sachs, l. c.; Lidforss, Bot. Centrabbl, I8g6, Bd. x.хvil, p. 33. [G. F. Kiohi (Über Plasmaverbindungen d. Schliesszellen, \&c., But. Centralbl., I 877, Bd. LXXII, p. 257) states that starch disappcars from the guard-cells of very 
Similar phenomena are observed when old evergreen leaves die, but these apparently do not function to any marked extent as storage receptacles during winter ${ }^{1}$. Owing to the rapidity with which the assimilatory products are translocated, leaves prematurely killed by summer-drought do not contain an unusually large amount of organic material?.

Fruits and seeds. The large quantities of organic food necessary for the formation of the fruit and seed are either entirely or for the most part conveyed from the assimilating organs. Even in the most highly chlorophyllous fruits the amount directly assimilated is comparatively small and is moreover not absolutely necessary, for grapes attain their full development when the inflorescence is kept in darkness after the flowers have been fertilized ".

The food-materials are conveyed to the developing ovule mainly or entirely through the funicle, but the growing embryo probably often absorbs food through its entire periphery, and in many cases displaces and consumes the preformed endosperm tissue. The development both of the fruit and seed follows as the direct consequence of fertilization, and the resulting translocatory activity usually continues until the ripening is complete and may not immediately cease when an unripe inflorescence is removed from the parent plant ${ }^{4}$. Marked metamorphoses may occur during the final stages of development, and thus when unripe seeds of Paconia are removed from the carpel, the accumulated starch is replaced by fatty oil, while apples picked when unripe still undergo metamorphoses characterized by changes of colour and taste ${ }^{5}$, the latter being partly due to a diminished acidity and partly to an increased percentage of sugar. It is, however, doubtful whether the acids are neutralized or decomposed, both of which are possible. Indeed according to Portele ${ }^{6}$

many plants before the fall of the leaf, but it does not necessarily follow that the sugar produced is also removed.]

1 E. Schulz, Flora, 1888, p. 223 ; Lidforss, l.c.

${ }^{2}$ Cf. G. Kraus, Bot. Zeitung, i 873 , p. 401. On translocation in perianth leaves, cf. L. Miuller, Vergl. Anat. d. Blumenblätter, I 893 , p. 296.

${ }_{3}$ Muiller-Thurgau, Bot. Jahresb., I 877 , p. 8 I 5 . Cf. also Sachs, Bot. Zeitung, I865, p. I I 7 . On the decomposition of carbonic acid by fruits, and the gradual decrease during ripening, cf. Ingenhousz, Versuche mit Pflanzen, 1786, Bd. I, p. 72; Bd. II, p. 233 ; Saussure, Rech. chim., 1804, pp. 57, I29, and Ann. d. chim. et d. phys., I 821 , T. XIX, p. I58; Bérard, ibid., I 821 , T. LXXvi, pp. I52, 225 ; Fremy, Compt. rend., I864, T. Lvili, p. 656 ; Cahours, ibid., pp. 495,653 , \&c.

* Cf. Sect. Io7 for the after-ripening of cereals. [Also Schmid, Bau u. Function d. Grannen unserer Getreidearten, Bot. Centralbl., Bd. Lxxvi, i 898 , p. I.]

${ }^{5}$ On the decomposition of chlorophyll in fruits, cf. G. Kraus, Jahrb. f. wiss. Bot., I8 72 , Bd. vili, p. I31; Millardet, Bot. Zeitung, I 876 , p. 733 ; Schimper, Jahrb. f. wiss. Bot., I885, Bd. xvi, p. I. l'aeonia, Pfeffer, ibid., I872, Bd. vili, p. 510.

${ }^{6}$ I'ortele, Bot. Jahresb., 1879, p. 290 ; Erlenmeyer, quoted by Liebig, Die Chemie in Anwend. auf Agric., \&c., I876, 9. Aufl., p. 30, footnote. [C. Gerber (Ann. d. sci. nat., IN, I897, pp. I-280) states that during the ripening of fleshy fruits citric and tartaric acids decompose at $30^{\circ} \mathrm{C}$., malic at $\left.15^{\circ} \mathrm{C}.\right]$ 
the tartaric acid of grapes is neutralized but the malic acid decomposed, and Erlenmeyer states that the disappearance of the glycolic acid present in unripe grapes is brought about in the same manner. Mach and Haas found that the percentage of tannin decreases during ripening ${ }^{1}$. The marked accumulation of organic substances in the flesh of fruits is as a general rule of bionomical importance, and not as in the seed for the purpose of storing up nutrient reserve-materials.

Sugars and starch often occur in the translocatory channels leading to fruits, and in the case of olives mannite may perhaps take part in translocation, and synanthrose in cereals ${ }^{2}$. Amides seem always to be present in flowers and young fruits, and nitrogenous materiąls probably reach the fruits partly in the form of proteid and partly as amides, for certain obscrvations of Borodin's ${ }^{3}$ indicate that asparagin is transferred to the fruits and seeds of Prumus padus, Comus sanguinea, Sambucus raccmosa, \&c. ${ }^{4}$

Trees. The plastic assimilatory products from the leaves accumulate mainly in the roots, and then spread upwards to the permanent parts of the stem, while sufficient food-materials are stored in the winter buds for the first stages of their development. A certain provision of nutrient materials is always present in the younger branches, and hence when removed these are able to form new roots and shoots to a limited extent in the absence of any photosynthetic assimilation ${ }^{5}$.

As a general rule the reserve-materials are stored up in the living cells of the wood, cortex, and in some cases of the medulla also. The medullary rays are especially adapted for the transverse distribution of the plastic products brought by the phloem ", and Gris ${ }^{i}$ has observed that

${ }^{1}$ Mach, Bot. Jahresb., I 877, p. 716 ; Haas, Chem. Centralbl., i 878, p. 700. Buignet apparently observed a similar diminution in many fruits (Ann. d. chim. et d. phys., i 86 I, iii. sér., T. LXI, p. 281 ).

${ }^{2}$ Miintz, Ann. d. sci. nat., i 886 , vii. sér., T. III, p. 61. Cf. Sachs, Jahrb. f. wiss. Bot., I863, I3d. III, p. 230 ; Ililger, Vcrsuchsst., I $8_{74}$, Bd. Xvir, p. 245 (grapes); Dahmen, Jahrb. f. wiss. Bot., 1892 , Jd. xxirr, pp. $45.3,460$; Mcsnard, Ann. d. sci. nat., I 893, vii. sér., T. xvin, p. 316.

3 Bot. Zeitung, I8 8 8, p. 812 ; also Dahmen, 1. c. ; Portes, Compt. rend., I 876 , T. Lxxxiri, p. $922 ;$ i 877 , T. Lxxxiv, p. I 401 .

${ }^{4}$ Macro-chemical researches on fleshy fruits in different stages of derelopment are :-Apples and pears: O. Pfeiffer, Chen. Unters. über d. Reifen d. Kernobstes, i876; Pfeil, Bot. Jahresb., i 880 , p. 869 ; Kulisch, ibid., I 892 , p. 437 ; Lindet, Compt. rend., I 893 , T. cxvi1, p. 696 . Cherries: Amthor, Zeitschr. f. physiol. Chem., I $88_{3}$, Bd. VII, p. I 97 . Grapes: Hilger-Gross, Versuchsst., I $88_{7}$,

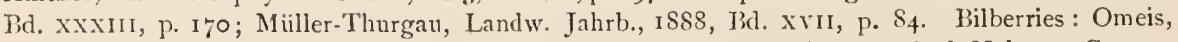
liot. Ccntralbl., I 890, Bd. XuıII, p. 8 . Additional literature by König, Chemie d. Nahr.- u. Genussmittel, i 889, p. $† 69$.

5 Sachs, Flora, I $\$ 62$, p. 331 ; J. Schröder, Jahrb. f. wiss. Bot., IS69-70, Bd. VII, p. 305 ; A. Fischer, ibid., I $\delta_{91}$, I3d. xxis, p. I 25. On the reserve-materials in the buds of Abies, cf. Busse, Flora, I893, p. I57; in bud-scales, Schaar, Jot. Jahresb., I 890, p. 666.

${ }^{6}$ For anatomical details, cf, de Bary, Comp. Anat. of Phanerogams and Ferns; Haberlandt, Physiol. Anat., I 896, 2. Auf., pp. 263, 345, 490; Strasburger, Leitungsbahncn, I 891.

${ }^{7}$ Gris, Compt. rend., I 866 , T. Lxx, p. 603 . Cf. de Bary, 1. c., p. 526 ; Gris, Ann. d. sci. nat.,

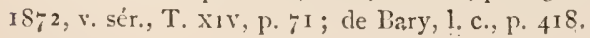


starch is deposited in the thirty year's' old wood of the ash and oak, and that the pith of Betula, Qucrcus, Fraximus, \&c. may contain starch even when twenty years old.

A large portion of the nitrogenous reserves are apparently stored up in the form of proteids, for in buds and young tissues more especially proteids are present which turn violet when treated with an alkaline solution of copper ${ }^{1}$. Amides are also of general occurrence, asparagin being especially abundant in the roots of Robinia pscudacacia.

Non-nitrogenous materials are preferably stored in the form of starch, and this may frequently be partially or entirely replaced during winter by sugar or fat. Fischer ${ }^{2}$ has shown that in the oak, beech, and most trees with hard wood, the main mass of the starch in the wood and medulla remains unchanged during winter (starch-trees), whereas in the cortex the starch may be entirely converted into soluble carbohydrates. In fatforming trees on the other hand the whole of the starch in the wood, cortex, and pith is replaced by fatty oil, along with a little sugar. A rise of temperature during winter or at the commencement of spring causes the opposite change to occur, and this phenomenon may be repeated more than once. Hence a secondary accumulation of starch is usually exhibited in spring, and the amount present rapidly diminishes as growth is resumed and consumption becomes active. In the meantime the young foliage commences to assimilate and a maximum of starch is again reached in autumn. The course of this periodic annual change is dependent upon a variety of circumstances, and hence it differs much in different plants. Th. Hartig ${ }^{3}$ observed that the deposition of reserve starch began again during the middle of May in the maple, in the larch in June, the oak in July, and the pine in September. The starch is deposited first in the lower parts of the stem and then higher and higher up, the deposition reaching the young twigs of the maple at the beginning of August, those of the larch at the beginning of October, of the oak in the middle of September, and the pine in the middle of October. Fischer, however, has observed the recommencement of the deposition of starch as early as May in certain cases.

As is indicated by the power of bleeding and by the growth in thickness, metabolism becomes active in spring before the buds have opened, and as soon as this takes place large quantities of reserve-materials are carried to the developing shoots, the starch in the stem disappearing

1 Sachs, Florn, I862, p. 33I ; Schröder, Jahrb. f. wiss. Bot., I 869-7o, Bd. v11, p. $31+$.

2 A. Fischer, Jahrb. f. wiss. Bot., I $8_{91}$, Bd. Xxir, p. $8_{7}$. The literature is quoted here. Con-

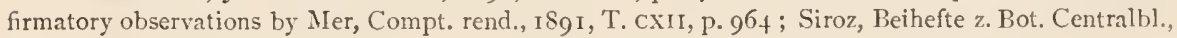
I 891 , Bd. I, p. $34^{2}$; Fünfstiick, Beitr. z. wiss. Bot., I 895 , Bd. I, p. 77. According to Rosenberg (Bot. Centralbl., I 896, Bd. Lxvi, p. 337), similar changes occur in rhizomes.

3 Th. Hartig, Bot. Zeitung, I \&58, p. 332 . 
from above downwards ${ }^{1}$. The growth in thickness appears to cause a disappearance of starch extending under favourable circumstances to the innermost margin of the first or second annual ring ${ }^{2}$, but no further, for by this time the unfolded leaves are able to provide for all requirements. The removal of the leaves causes a much more complete depletion of the reserve-materials of the wood and bark ${ }^{3}$, which also occurs when seed-production is especially active (R. Hartig, l.c.). Both the deposited and the newly-formed reserve-materials may be utilized in a variety of ways, and Th. Hartig ${ }^{4}$ is certainly in error in supposing that growth in thickness always occurs at the expense of the reserve-materials. Nor is Schröder's supposition correct, according to which the starch deposited in the bark is used in bud-formation and development, that in the wood for growth in thickness.

Bulbs, Tubers, Rlizomes. It must suffice to mention the literature ${ }^{5}$ in which a general account is given of the reserve-materials and of their utilization and translocation. In cold weather potatoes turn sweet owing to the starch being partially converted into sugar, and according to Rosenberg similar conversions to those occurring in trees are produced in rhizomes.

1 Th. Hartig, 1. c.; Schröder, 1. c. ; Reichardt, Versuchsst., IS8I, Bd. XIV, p. 329 ; Russow, Sitzungsb. d. Dorpat. Naturf.-Ges., I8s 4 ; Baranetzky, Bot. Centralbl., IS84, Bd. xviII, p. I57; R. Hartig. Bot. Zeitung, IS88, p. 837 ; Bot. Centralbl., I893, Bd. Lvi, p. 57 ; Wotczal, ibid., I89o, Bd. XLI, p. 99; A. Fischer, IS9I, l. c., p. Io6.

${ }^{2}$ R. Hartig, Bot. Zeitung, I 888, p. 837 ; A. Fischer, 1. c., p. I Io.

${ }^{3}$ Cf. Lutz, Ber. d. Bot. Ges., 1895 , p. I 8 -

1 Th. Hartig, Bot. Zeitung, 1858 , p. 330 ; 1862, p. 75 . Cf. Sect. 92.

${ }^{5}$ Literature: Sachs, Jahrb. fo wiss. Bot., I 863 , Bd. III, p. 219 . On potatoes: De Vries, Landw. Jahrb., 1878, Bd. vir, p. 216 ; Müller-Thurgan, ibid., I885, Bd. xiv, p. 864 ; Kreusler, ibid., I886, Bd. xv, p. 309; Hungerbiihler, Versuchsst., I 886, Bd. xxxiI, p. 38 I ; Prunet, Rev. gén. d. Bot., I883, T. v, p. 49; Detmer, Ber. d. Bot. Ges., IS93, p. I 49. Beetroot: De Vries, Landw. Jahrb., IS79, Bd. vin, p. 4Ió. Slachys tuberifera: Planta, Versuchsst., ISS8, Bd. xxxv, p. 473; IS92, Bd. XL, p. 277. Helianthus tuherosus and other Compositae: Prantl, Das Inulin, I870; Vöchting, Sitzungsb. d. Berl. Akad., I894, p. 7 II ; Tauret, Compt. rend., I893, T. cxvir, p. 50. Different rhizomes: A. Fischer, Jahrb. f. wiss. Bot., IS91, Bd. XxiI, p. So; Behrens, Flora, I894, p. $36_{4}$ (hops); A. Meyer, Stärkekörner, 1895, p. 249 (Adoxa). Cf. also König, Chemie d. Nahr.- u. Genussmittel, I $889, \mathrm{p} .64 \mathrm{I}$. A few notices also in the following morphological and other works: Vöchting, Bíbl. bot., I 887 , Heft 4 ; Rothert, Vergl. Unters. iiber Knollen u. Rhizome, I885; Seignette, Rev. gén. d. Bot., I8 89 , T. I, P. 415 ; Drobnig, Bot. Centralbl., I893, Bd. Lvi, p. 89 . On the reservematerials of pollen-grains, see Planta, Versuchsst., IS95, lid. Xxxı, p. 214 ; Molisch, Sitzungsb. d. W'ien. Akad., Bd. chI, Abth. i, p. +43 ; Green, I'hil. Trans., I 894, Vol. clxxxv, p. 385. On Fungi : de Bary, Fungi, Mycetozoa, and Bacteria, I888, p. 8 ; Zopf, Pilze, I89o, p. I74 ; Errera, Glycogène chez 1 . Basidiomycètes, 1885 ; Bourquelot, Bot. Centralbl., I 892 , Bd. L, p. 78 ; Cérard, ibid., p. I Io ; Brommer, Sclérotes et cordons mycéliens, I894; Istvánffii, Jahrb. f. wiss. Bot., 1896, Bd. xxix, p. 405. Lichens : Zukal, Bot. Zeitung, 1886, p. 762 ; Fünfstiick, Beitr. z. wiss. Bot., 1895, 1, p. 219. Algae : cf. Haberlandt, Physiol. Anat., I8g6, 2. Auf., pp. 334, 372. Rhizomes: Rosenberg, Bot. Centralbl., I8g6, Bd. LxVI, p. 337 ; cf. Sect. 92. 


\section{N D E X}

Abies pectinata, nitrogenous compounds in, p. 458.

Abrus precatorius, alkaloids of, 499.

Absorption and excretion, IOI.

- and retention of salts by soil, 166.

-, colouration and precipitation tests for, 98; hygroscopic, I6 1 ; mechanism of, 86; influence of stomata on abs. of carbon dioxide, 330; of fats, I00; of free nitrogen, 402 ; methods, 403 ; of dyes by living cells, 96 ; of light in deep water, 352 ; of water, 209 ; relation of, to transpiration, $227, \mathrm{sec} .37$; - apparatus for, 232 ; influence of temperature on, 2 Io; of neutral and poisonous gases on, 230 ; by subaerial organs, 158.

Acaciar, 238; A. lophanta, formation of asparagin by, 600 .

Accommodation, phenomena of, $2 \mathrm{I}$; to concentrated nutrient solutions, I 39 .

Acer platanoides, 262, 265; A. pseudoplatanus, 282 ; A. saccharinum, bleeding of, 212.

Acetamide, 383 .

Acetic fermentation, 559 ; influence of temperature on, 560 .

Acetic acid, nutritive value of, for fungi, 384 .

Acid reactions of sap and of protoplasm, 490.

Acidity of tissues, 328 .

Acorus calamus, I 56, I9I.

Acqua, formation of nucleus, 52, 53 .

Acrylcolloid, 70.

Active albumin, 67-69.

Acton, on nectar, 284 ; synthesis of carbohydrates, 325,326 ; absorption of organic food, 367 .

Adie, osmotic apparatus, I 44.

Adler, 202.

Aeby, 396.

Aeriferous system, in aquatic and terrestrial plants, I8I ; in swamp plants, I8o; openings of, I88; pressure of air in, I 82.

Aerobes, 539, 540 .
Aerobic respiration, products of, 526; relation to anaerobic, 547 .

Aesculus hippocastanum, 202, 324.

Aethalium, I I ; A. septicum, 377 ; analyses of, 67 ; corrosion of marble plates by, I73; production of ammonia by, 391 .

Agaricineae, asparagus in, 459.

Agaricus, $368 ; A$. campestris, mannite in, 476; A. muscarius, trehalose in, 476 ; $A$. oreades, evolution of prussic acid by, 39I ; A. ostreatus, ferment of, 509. Agarie, 257.

Aggregation, 95.

Aimé, 206.

Albizza saponaria, 238 .

Albumen, 64-66; nutritive value of, for fungi, 384 .

Albuminoids, 65, 66.

Alburnum, conductivity of, for water, 2 I 3 , 2 I 4.

Alcohol, production of, 538 .

Alcoholic fermentation, $555-7$.

Aldrovanda, 377-9.

Alessandri, 240, $25 \mathrm{I}$.

Aleurone grains, 46I, 595 .

- layer, 599.

Algae, maximal depth for, 352; reserve materials of, 604 .

Alisma plantago, $\mathbf{1 9}$ I.

Alkali-chlorophyll, 316.

Alkaline reactions of cell-sap and protoplasm, 490 ; tests for, and influence of peptone on, 490.

- metals, in plants, 429 .

- earths, in plants, 431 .

Alkaloids, 498 ; function of, 499.

Allantoin, 383 .

Allium, 334; A. cepa, 319, 325, 473, 474, 600 ; glucose in, 142,515 ; oil in, 592 ; rise of temperature in, 566 ; germination of, 596.

Allotrophic nutrition, 363 .

Alnus, I91 ; A. glutinosa, 267.

Altmann, 479. 
Altvater, 404 .

Aluminium, 437.

Amanita phalloides, 499.

Amaryllis formosissime, stomata of, I9I, I92, 194.

Ambronn, 481; on optical properties, 82, 83.

Amici, I90, 196.

Amides, accumulation of, 462 ; consumption of, 457,459 ; estimation of, $45^{8}$; formation of, 46I; over-saturated solution of, 467,468 .

Amido-valerianic acid, in etiolated seedlings, 465.

Amm, 538, 562 .

Ammonia, production of by fungi, 391 ; by seedlings, 463 .

Ammonium salts, preference of swamp plants for, 404 .

Ampelopsis, response to contact, $13 ; A$. quinquefolia, 265.

Ampullae, as traps for insects, 379.

Amthor, on ripening of cherries, 602 .

Amygdalin, 494.

Amylodextrin, 78, 473.

Amylose, 78,473 .

Anaerobes, cultivation of, 535 ; maximal percentage of oxygen for; 541, 542; reducing action of, 549 ; sources of energy in, 532 ; oxygen in, 549.

Anaerobic metabolism, 533, 534 .

Anaerobiosis, history of, 534 ; methods of demonstration, 535, Fig. 65, 66.

Anaesthetics, influence of, on alcoholic fermentation, 502, 564; - on bleeding, 264 ; - on photosynthesis, 336, 339 ; - on respiration, 564; - on resting tubers, 564 ; - on streaming of protoplasm, 569; - on translocation, 590 ; - on vital activity, 569 .

Ananassa sativa, papain in, 509.

Anderson, 242.

Anisotropy, 82, 83.

Anthurium Hiigelii, I 59.

Antitoxins, 514.

Aplastic substances, 289, 445 .

Apocynaceac, ringing experiments on, 578 .

Apples, ripening of, 601 .

Arbutin, 49r.

Areboe, 563 .

Arendt, 277,586 ; capillary ascent of water, 277.

Aristotle, 9, 367 .

Armeria a'ulgaris, 436.

Arnold, 308 .

Arsenic, 438.

Arsenious acid, 438 .

d'Arsonval, influence of carbon dioxide on bacteria, 565 .

Arum inaculatum, 197 ; A. italicum, 245, 474 ; consumption of oxygen by, 525 ; production of heat by, 523 .

Ascent of water, 222, 225 .
Aschoff, 420, 43I : influence of chlorides on production of tannin, 492 .

Ascophyllum, I82, 320 .

Ash constituents, non-essential, 434 ; removal of from leaf, 585 ; translocation of, 583 .

Askenasy, on ascent of water, 223, 224, 226: on pigments, 497.

Asparagin, 457-9, 465; nutritive value of, for fungi, 384 .

Aspartic aldehyde, 65.

Aspergillus, 382-4, 389, 417, 426, 431, 528 ; production of organic acid by, 486-8 ; influence of temperature on, 562 ; growth and fermentative activity of, 553; A. fumigatus, $387 ; A$. niger, $366,373,375,376,404,504,554$; accommodation to concentrated solutions, I39, 140 ; assimilation of nitrogen by, 394 ; selective power of, 387 ; temporary anaerobiosis of, $536 ; A$. oryzue, 507.

Asphyxie, 336, 339.

Aspidium, 337.

Asplenium nidus-avis, 159: A. serratum, I 59.

Assimilation, 290; of carbon dioxide, changes of volume due to, 321,322 ; composition of gas evolved, 322 ; by aquatic plants, 33I; Englemann's bacterium method, 350, Fig. 53; equation for, $32 \mathrm{I}$; history and methods, 350 ; influence of temperature on, 337,338 , Fig. 50; of light, 339; of different rays, 342 ; of polarized and artificial light, 352 ; of coloured sap on, 346 ; of percentage of water, 338 ; of chloroform and ether, 339; of oxygen, 339; of accessory pigments, 343,344 , Fig. 5 I ; inhibition of, $335-7$; products of, 317 , $320-6$; rays effective in, 346,347 ; ratio of, to respiration, 339; to carbohydrates formed, 321 ; to light absorbed, 348 ; secondary curve of, 343 ; theories of, 353 ; values for, 332 .

Astragalus, 484 .

Atropa belladonna, 244.

Atrophic substances, 445 .

Aubert, I 82, 206, 209, 238, 239, 244, 250, $327-9,357,390,529$; action of light on Crassulaceae, 206 ; specific respiratory activity, 525 ; influence of moisture on respiration, 565 .

Auto-assimilatory products, influence on growth, 324, 325 .

Automorphic plants, 29.

Automorphosis, 24.

Autoparasitism, 366, 369.

Autotroplic plants, 29, 364 .

Auxanographic method of Beyerinck, 386.

Avédissian, 235.

Avena sativa, influence of injury on heliotropism of, I9; stomata of, I93, Fig. 2 I. 
Avogadro, I44.

Azolla, 124, 497 ; absorption of methyl-blue by, 94 .

Bach, 355 .

Bachmann; corrosion by lichens, 173 ; pigments of lichens, 495.

Bacillus anthracis, non-virulent varieties of, 499 ; B. butylicus, 558, 559; - alcoholic fermentation of glycerine by, 556 ; - suppression of fermentative power in, 553; B. carbonis, 531; B. cholerae asiaticae, 532; $B$. denitrificans, $53 \mathrm{I}$, $550 ;$ B. Fitzianus, 547 ; B. liquefaciens, v. vulgaris, 31 I, Fig. 43 ; B. megatherium, 376; $B$. methylicus, 381; $B$. orthobutylicus, 558, 559; fermentation of inulin by, 554; $B$. perlibratus, 382 , 406 ; B. Pfliggeri, $547 ; B$. phosphorescens, 532, 547; B. polypiformis, 540, 531, 532; B. prodigiosits, 532, 533, 547,$553 ; B$. pyocyanus, 497 ; B. oedematis maligni, $53 \mathrm{I}$; B. radicicola, 396, $400 ; B$. subtilis, 387, 532 ; B. tetani, $531 ;$ B. typhi, 532 .

Bacteria, induced variations in, 33 .

Bacterio-purpurin, 343, 349.

Bacterium aceti, 485,527, 548, 559, 560; B. megatherium, 504; B. Pasteurianum, 560 ; $B$. photometricum, 306, 307; B. termo, 3II, 340, 532.

Bacteroids of root tubercles, 399-40r.

Baeseler, 405.

Baier, 558.

Bailey, 352 .

Baisse, de la, I 5 I, 217.

Balfour, 379.

Balland, 585 .

Ballo, 355, 356 .

Ballota nigra, 278.

Baranetzsky, 240, 247, 248, 250, 257, 258, $261,266,267,508,604$; progress of depletion in seeds, 599 .

Barthélemy, I 82 , I 83 , I 85 , I $87,201,205$, 250; closure of stomata under negative pressure, 198.

Bartsia, 369 .

Barus and Schneider, $7 \mathrm{I}$.

Bary, de, 40, 7I, I I 2, I I 8 , I3I, I90, I9I, 195, 196, 216, 278, 284, 364, 366, 368, $37 \mathrm{I}, 374,376,478,479,578,581,582$, 602,604 ; on fungal cellulose, 481 ; on lenticels, I 89 .

Bastit, 322, 338, 565 .

Batalin, 333 .

Baudrimont, 206, 353 .

Baumann, 372, 392, 436; silver reduction test, 68.

Bayer, theory of photosynthesis, 355 .

Beauvisage, 475 .

Bechi, 438.

Becquerel, I 72, 327, 352 .

Beet-root, percentage of amides in, 459 .
Beggiatoa, 526.

Begonia, 243; B.manicata, I45; B. hydro. cotylifolia, I 82 .

Behrens, 604.

Belajeff, 54 .

Belzung, 3I4, 468, 600 .

Bemmelen, van, 72, 169 .

Benecke, 366, 4II, 4I 7, 426, 432 ; importance of subsidiary guard-cells, I92.

Benkovich, 355 .

Bente, 405.

Benzoic acid, nutritive value of, for fungi, 384.

Bérard, 538, 60 I.

Berger, 262.

Bergmann, 485 .

Bernard, 564 .

Bert, 352, 353, $57 \mathrm{I}$; influence of compressed. air and of oxygen, 542 .

Berthelot, 394, 395, 399, 402, 405 .

- et St. André, 429, 437, 545, 586.

Berthold, $182,360,482$; on calcareous incrustations, 133 .

Bertrand, 427, 509; on laccase, 505, 545; on tyrosinase, 5 Io.

- et Mallève, 478.

Beta, 326, 334 .

Betula, 262 ; influence of heat on formation of starch in, 512 ; starch in pith of, 603 ; $B$. alba, 259, 265; B. lenta, exudationpressure of, 259 .

Beyer, I69, 405, 431, 600.

Beyerinck, 305-7, 3II, 31 2, 347, 363, 366, $375-7,382,383,399,401-6,490,501-7$, $53 \mathrm{I}-3,547-50,560$; on anaerobes, 530,535 ; auxanographic method of, 386 ; butyric bacteria, 558,559 ; evolution of gas by fermentation, 555 ; by green plants, 339 ; influence of oxygen on respiration, $538-42$; of food on anaerobiosis, 540; photobacteria, 581 ; reserve-oxygen, $57 \mathrm{I}$.

Bicollateral bundles, influence of, on ringing experiments, 578 .

Biedermann, on differential absorption, I29, I 32 .

Biffen, 582 .

Bineau, 389 .

Biology, 9.

Bionomy, 9.

Biot, 262.

Birner and Lucanus, 405, 406, 427-30, 435 .

Bischoff, 206, 290; on calcareous excreta, I33.

Bismuth, occurrence of, 437 .

Blackman, I 88, 330, 340, 520 ; on diosmosis of gases through cuticle, I86; on influence of stomata on absorption of carbon dioxide, 196.

Blass, 581.

Bleeding, methods of demonstration, 256, Fig. 33, 257, Fig. 34; daily periodicity in, 266 ; - yearly, 264, 265; - causes 
of, 267 ; causes of, 269-72; of injured plants, 253; from branches, 254, 255 ; influence of external conditions on, 263 ; - temperature, 263 ; - light, gravity, oxygen, chloroform, 264; - atmospheric pressure, $26 \mathrm{I}$; instances of active bleeding, 258 ; quality of escaping sap. 262.

Bleeding pressure, 254 .

Blocisrewski, 593.

Boedecker u. Eckhardt, 432 .

Bogdanoff, 75 .

Böhm, 20I, 2I I, 256, 258, 3I 4, 319, 325 , $326,33 \mathrm{I}, 332,338,433,530$; ascent of water, 220,226 . Influence of injuries on respiration, 565,566 .

Böhm u. Kulz, 499.

Bokorny, 219, 325, 326, 367, 424, 533 .

Boletus, 5 Io.

Bonnet, 243,308 .

Bonnier, I96, 201, 283-6, 305, 33I, 35I, 360 ; nectar as food-reserve, 284 ; percentage of cane-sugar in, 285 .

- et Mangin, 239, 3I I, 322, 520-4, $528,542,564$ : on photosynthesis in ulta-violet, 346 ; influence of light on respiration, 562,563 ; - on transpiration, 247 ; - of ether on photosynthesis, 339 ; respiration in oily seeds, 529 .

Borodin, 320, 463, 468, 524, 525, 529, 566 ; accumulation of amides in cut twigs, 463 ; - in seeds and fruits, 602 ; respiration of cut branches, 563 .

Boron, occurrence of, 438 .

Botkin, 558 .

Botridium, 37 .

Botrytis cinerea, 374 .

Böttger, 437.

Bouffard, 5 Io ; on heat of fermentation, 567.

Bouilhac, 396, 429.

Bourquelot, 376, 476, 507, 545, 604.

- et Graziani, 375,382 .

Boussingault, 100, I I 8, I 6 I, I 72, I 86-8, $232-4,243,250,286,303,308,3$ I I, $321,322,327-32,336-40,368,390,393$, $4 \mathrm{OI}-4,417,418,465,472,529,582$; on absorption by leaves, I 60 ; alkalinity of sand cultures, I 32 ; asphyxie, 338; growth in absence of nitrogen, 391 ; on transpiration, 233; - influence of smearing with fat on, 244 .

Boveri, on centrosomes, 47; on hybrid Echinoderms, 54; physiology of cell division, 55 .

Bovin, 4I.

Bracounot, 285 .

Brand, 438.

Brasch u. Rabe, 430 .

Brass, 48.

Bredow, 598.

Brefeld, 276, 374, 531, 53S. 539, 557 ; resistance of seedlings to absence of oxygen, 537.
Brenstein, 545 .

Bretschneider, I 70, 402.

Briosi, 320,585 .

Bromine in plants, 438 .

Brommer, 604 .

l3rosig, 257, 263, 266, 267 .

Broughton, 538 .

Brown, A. J., I7.

- John, 560.

- and Morris, 320, 322, 476, 503, 505, $507,556,593,600$; on occurrence of maltose, 473 .

Brücke, 227, 254, 258, 260, 266; on glycogen, 7I ; on myelin forms, I07.

Bruden's spring manometer, 257.

Bruhne, I 40, 377, 383, 386, 421 ; on rennet, 509.

Brunner and Chuard, 320.

Bruns, 320.

Bryonia, $2 \mathrm{I} 9$.

Bryophyllum, 329: B. calycinum, 328.

Bryum, 337, 339, 595.

l3uchner, 5 I 4, 547, $55 \mathrm{I}$, 556 ; yeast ferment, 522.

Buckwheat, water-cultures of, 4I9, Fig. 6I.

Buignet, 508, 602 .

Bulbs, 604 .

Bunge, 64, 101, 41 I, 462.

Bunsen, I8, I87.

Burgerstein, I 8 , 160, 229, 234-9, 243-6, $249,277,281$; guttation, 253; influence of salts on transpiration, 249 ; - of low temperatures, 246.

Burkmaster, 499.

Burri, 392.

- and Stutzer, 362, 514, 534, 549, 550.

Busch, 580,600 .

Büsgen, II $\delta, 2 \delta_{3}, 286,504$; influence of carnivorous diet on Drosera, 379; on tannin, 492-4.

Busse, 602.

Butomus, 149.

Bütschli, I o8, 306, 308, 37 I, 377 ; structure of protoplasm, 44,45 ; - of organized bodies, 72 .

Butylalcohol, production of, $55^{8}$.

Butyric fermentation, 558, 559; acid, nutritive value of, for fungi, 384 .

Buxus, 336.

Cacalia suaveolens, 326.

Cactaceae, 333; percentage of calcium oxalate in, 486 ; production of malic acid by, 327 .

Caesalpinia pluriosa, 286 .

Cahours, 521, 525, 601.

Cailletet, 367 .

Calamine violet, 436 .

Calcium, distribution of, 432,433 ; essential character of, for Phanerogams, 43I ; influence of deficiency of, on cell-wall formation, 424; organic compounds of, 432. 
Calcium oxalate; importance of, 489 ; as plastic product, 487 ; as protection, 486 .

- pectate, 432 .

Calla ethiopica, 197; bleeding of, 256.

Calliandra haematocephala, 238 ; C.Saman, 286.

Calluna v'ulgaris, mycorhiza of, 371, Fig. 54 .

Callus, 580 .

Calvert et Ferrand, 206.

Cameron, 405.

Campbell, on changes on death, 66 ; staining of living nucleus, 94 .

Candolle, de, 26, 127, I3I, 152, 171, 172, 2 I $2,226,244,275,306,309,331,500$, 585 .

Cane-sugar, occurrence of, 474 .

Cannabis sativa, 473, 592 .

Canstein, 258.

Carbohydrates, 469; distribution of, 471 ; origin of, 472 ; nature of, 469,470 ; as products of photosynthesis, 319 .

Carbon, nutritive compounds of, 380 , sec. 66 ; sources of, in heterotrophic plants, 383,384 .

- dioxide, absorption of, from water and bicarbonates, 33I ; assimilation of, 302 (ch. vii. pt. ii); evolution of, by green plants, 520; growth in absence of, 318, Fig. 48 ; influence on growth of nitrate bacteria, 362 ; methods, 523 , 524, Figs.62,63,64; optimal percentage of, 329,332 ; sources of, 329 (sec. 57 ).

Carbon-assimilation, 302.

Carbonyl compounds, 328 .

Carica papaya, 508.

Carnivorous plants, 377 ; absorption by, 378 ; mode of digestion in, 379 .

Carotin, 316.

Carpinus, 332.

Cassia montana, 238; C. neglecta, 283, 285.

Cassincourt, de, 418 .

Catharinea, $339 ;$ C. undulata, 161 .

Caulerpa, 39, 60, 61, 84, 148.

Cauvet, 130.

Cazeneuve, 510.

Čelakovský, 111, 313,377, 490, 509; on fusion of plasmodia, 10y.

Cell, definition of, 6o, 61 ; diosmotic properties of, 90 (sec. 16); uni- and multinucleate, 58.

- wall, composition of, 480 ; formation and modification of, 482 (sec. 84 ).

Cellulose, fermentation of, 560 ; formation of, around crystals and vacuoles, 482 , 483 ; granules of, 483 .

Celtis, 133 .

Centrosomes, 40, 55, 56.

Cephalotus, 275, 379 .

Ceratophyllum, i 88, 204, 205, 310.

Cereals, after-ripening of, 585 , 60 I ; percentage of proteids in, 460 .

PFEFFER
Cereus macrosonus, respiratory activity of, 525.

Cetraria islandica, 84 .

Chamberlain, 254, 261 .

Chara, 61, 133, I86, 334, 337, 341; rotation in absence of oxygen, 570 .

Charrin et Phisalix, 498.

Chelidonium, 473 .

Chemical constitution and nutritive value, 406.

- tests for absorption, 98.

Chemistry of protoplast, 6I (sec. II).

Chemomorphosis, 25.

Chemosynthesis, definition of, 290 ; influence of light, sugar, and glycerine on, 464 .

Chemosynthetic assimilation, 361 (sec. 63).

Chenopodium vulvaria, 390.

Chevalier, 391.

Chimani, 582 .

Chimielewsky, 161, 314 .

Chitin, 64.

Chlamydomucor oryzae, 406.

Chlorangium Jusuffii, 437.

Chlorine, importance of, 431 ; occurrence of, 430 .

Chlorophyll, composition of, 315,316 ; influence of ; intluence of light on formation and decomposition of, 333,334 ; of different rays, 334 ; - of temperature, oxygen, and food-supply, 335 ; occurrence of, in animals, 37 I ; Pringsheim's protective theory of, $34 \mathrm{I}$; product of photosynthesis, 320 .

Chlorophyllan, 316, 320 .

Chloroplastid, conditions for development of, 333 ; division of labour in, 314,315 ; pigments of, 314-6, Fig. 47 ; structure and properties of, 312, Figs. 44-46.

Chlorovaporization, 247 .

Chodnew, 478 .

Cholin, 479, 480 .

Cholesterin, 63,480 .

Chondrin, 65,66 .

Chondrioderma, 377 ; C.difforme, 11 1, Fig.7.

Chrapowicki, 319, 4 10.

Chromatium okenit, 308.

Chromatophores, 3 I 2.

Chromium, occurrence of, 437 .

Chromogen. 495 ; oxidation of, 496 .

Chrysophanic acid, 457.

Chudiakow, 404, 524, 536-8; on influence of temperature on fermentation, 562 ; -- of peptone and ammonium salts, 557 , $55^{8}$; objections to Nägeli theory of fermentation, 552 .

Church, 437 ; calcareous deposits, I33.

Cicer arictinum, 275 .

(inchona, 457; non-formation of quinine by, 498 .

Circaea lutetiana, 275 .

Citric acid, nutritive value of, for fungi, $3 s_{4}$.

Citromyces, 527 ; production of citric acid by, 485 ; regulation of, $486-8$. 
Cladonia rangiferina, 158.

Cladophora, 59, 61, 3+3.

Clark, I 53, 258, 259, 262, 535, 57 I ; minimal percentage of oxygen for protoplasmic movements, 546 .

Clathrocystis roseo-persicina, 307 .

Clausen, 466,545 ; influence of temperature on respiration, 561,562 .

Clautriau, 499; glycogen in fungi, 476.

Claviceps purpurea, 276.

Clifford, 12.

Cloez, 309.

- et Gratiolet, 322, 331, 350, 402.

Clostridium foetidum, 53I, 540; butyric fermentation by, 558; C.Pasteurianum, $393-5,398,400,403,405,531$; butyric fermentation by, 558 .

Cobalt, occurrence of, 437 .

- method, 241, 243, Fig. 3 I.

Cocoa, fat in seeds of, 479 .

Codium, 6 r.

Coelobogyne, parthenogenesis of, 35 .

Coenocyte, 60.

Cohn, 173, 213 ; on proteids, 65 .

- Jonas, changes in boiled collenchyma, 84.

Coley, 599.

Colocasia, 201 ; C.esculenta, 280 ; C. antiquorum, $28 \mathrm{I}$.

Coloured light, influence of, 352 .

- solutions, experiments with, 217.

Colutea, 205.

Comes, 248.

Compositae, inulin in, 475, 594.

Composition of intercellular air, 205.

Concentration, influence on vapour tension, 162.

Conductivity of vascular tissue, 219 ; influence of temperature on, 230.

Conglutin, 66.

Coniferae, 333 ; arganin in, 458; winter colouration of, 497 .

Coniin, 498.

Conjoint actions, 560 .

Conjugatae, 483 .

Conocephalus, 258, 278, 279.

Constantin, 216.

Constellation, protoplasmic, 62 .

Convoluta, 378 .

Copeland, 424,455 ; on turgidity of starved cells, I $39 ;-$ at low temperatures, 512 .

Copper, occurrence of, 437 ; influence of, on growth, 416.

Coprinus, 276.

Corallina, 133 .

Corallorhiza, 368.

Corenwinder, 309, 322, 330, 359, 520, 52 I.

Cork, permeability of, I 6 (sec. 21 ), I 86.

Cornu, 28I.

Comus sanguinea, 602 .

Correlation, phenomena of, 12, 26.

Correns, $68,433,485,550,571$; on dermatosomes, 81 ; influence of oxygen on ir- ritability, 569, 570; ninimal percentage of oxygen for development of chlorophyll, 234 ; optical properties of organized bodies, $82 ;$ - of superposed lamellae, 78 .

Corrosion, by roots, 172 ; by fungi, lichens, \&c., 173 .

Corsinia marchantioides, 276.

Costerus, 318, 323 .

Cotta, 212.

Cotyledons, auto-assimilation by, 596; as absorbent organs, 596; as storage organs, 593-6.

Councler, 491 .

Coupin, 75 .

Cracovi, 332.

Cramer, 388 ; on calcareous incrustations, I 32 .

Crassulaceae, 594; influence of darkness on acidity of sap, $487 ;-$ on evolution of gas by, 527 ; malic acid in, 141, 577 ; — isomalic, 328 ; malates in, 486 ; used as food-material, 487 .

Crataegus oxyacantha, 390.

Crato, 356 ; on physodes, 48.

Credner, 133, 297.

Crocus, 218.

Crystalloids, 8o, 595.

Cuboni, 322.

Cucurbita, I17, 219, 334, 595 ; percentage of amides in, $458 ; C$. melo, $263 ; C$. pepo, 156, 259, 324; C. utilissimus, papain in, 509.

Cucurbitaceae, ringing experiments on, 578 .

Cumarin, 457.

Cupuliferae, $37 \mathrm{I}$.

Culture media, $42 \mathrm{I}$; influence of concentration, 422 .

- methods, for fungi and bacteria, 385 ; for phanerogams, 422-33.

Curcuma mbricaulis, 145 .

Curtel, 340.

Curtius u. Reinke, 355 .

Cuscuta, 335, 365-9; penetration of, 374, 498 ; response to contact, $13 ; C$. cephalanti, $306 ;$ C. europaea, 306.

Cuticle, diosmotic properties of, I I6 (sec. $21)$; influence on transpiration, 243.

Cuticularization, 482 .

Cyan-alcohol, 65 .

Cyanophycin, 320.

Cynareae, 270.

Cynosurus echinatus, non-closing stomata of, 191 .

Cyperus esculentus, oil in tubers of, 595 .

Cystopteris, 337 .

Cytase, 506.

Czapek, 102, I13, I26, I73, 174, 275, 589 ; on acid excretions of roots, I00, 168; - etchings produced by, 172 ; influence of chloroform on translocation, 590; translocation in cortex, 574 .

Czech, 190, 194. 
Dallia, 473; exosmosis of albumin from tubers of, 95 ; D. variabilis, bleeding of, 256 .

Dahmen, 602 .

Dalbergia linga, 238 .

Dalibard, 233 .

Daniel, 365 .

Dantec, II $1,306$.

Darlingtonia, 379 .

Darwin, 26, 97, 175, 194, 285, 377, 380, 499 ; on excretion of water by Lathraed, 276 ; on pangens, 49,57 .

- (F.), 569 ; influence of carnivorous diet on Drosera, 379.

- and Pertz, 330; D. and Phillips, 22.

Dassen, 254.

Dassonville, 422.

Datura Stramonium, 498.

Daube, 584 .

Daubeny, 306, 331, 350, -438 ; infl. of light on transp., 247.

Daucus carota, 206.

Davy, 332, 438 .

Decaisne, 369.

Dehérain, IOI, 248 ; turgidity of seedlings, 142 ; D. et Bréal, 4I 5 ; D. et Landrin, 530 ; D. et Maquenne, 529; D. et Moissan, 525 ; D. et Vesque, 520.

Dehnecke, 306.

Delage, 49,57 .

Demoor, division of nucleus after death of cytoplasm, 52, 54; - in absence of oxygen, 569 .

Derbesia Lamourouxii, 438 .

Dermatosomes, 81,485 .

Detlefsen, distribution of light in a windowed room, 340 ; lessened absorption of light by non-assimilating leaves, 348 .

Detmer, 100, 162, 163, 173, 230, 261, 266, $27 \mathrm{I}, 333,368,390,468,472,527,550$, $564,600,604$; absence of respiration in seeds, $565 ;$ minimal temperature for bleeding, 263 ; swelling of see $\mathrm{ds}$, .

Deutoplasm, 46.

Devaux, I79, I 87, I88, I98, 204-6 ; circulation of intercellular gases, $196 ;-$ composition of, 206 ; - diosmosis of, I 85 ; - pressure of, 201.

Diakanow, 381, 531, 536, 538; influence of food on respiratory ratio, 529 ; respiration in fungi, 525 .

Diaphragms of aquatic plants, $18 \mathrm{I}$.

Diastase, hydrolyzing power of, $522 ;$ tests for, 505 ; properties of, 505, 506 ; forms of, 506 .

Diathermanicity, 500.

Dicramm, 337, 595; D. scoparium, 158.

Dictammus albus, 500 .

Dieudonné, 302, 497, 498, 564.

Diffusion, 125 .

Dimitrievicz, 230.

Dionaed, 275, 375-9, Fig. 57 ; D. muscifula, 270, $27 \mathrm{I}$.
Diosiorea jafionica, 477.

Diosmosis, I05; of gases through membranes, I88; - Exner's formula for, I 87 ; history of, I10, I 43.

Dipsacus, 160.

Dircks, 431, 438.

Dischidia rufflesiana, 160.

Dissimilation, 290.

Dixon, 225, 240.

Dixon and Joly, 162, 225, 226; on passage of water through tracheae at low temperatures, 230 ; theory of ascent of water, 222-4.

Döbereiner, 538 .

Döpping u. Struve, 538.

Draba verna, root-hair of, I 5 I, Fig. II.

Dragendorff, 450 .

Draper, 350; rays active in photo-synthesis, 345 .

Drechsel, $64,65,460$; artificial formation of crystalloids, 80 .

Drobnig, 604 .

Drosera, II8, 375, 377, 379, 497 ; enzyme of, 509; excretion of water from, 271 ; localization of irritability in, 15: response to stimuli in absence of oxygen, 569.

Drosophyllum, 377-9, 475 .

Drude, $183,306,352,368,376,439,521$.

Dryer, 492.

Duchartre, I6I, 280, 281 .

Duclaux, $375,3^{8} 3,497$; on butyric fermentation, 555 .

Dufour, $182,222,360,474$; on thermodiffusion, 204 .

Duhamel, 212, 213, 230-3, 257, 418, 419; elongation of roots in water, 155 ; oblique conduction of water, 217 .

Dulcite, 325, 477 .

Dulk, 585 .

Dumont, 538 .

Duramen, conductivity of, 213 ; formation and colouration of, 484 .

Dusch, 553.

Dutrochet, 1 79, 1 96, 197, 206, 254, 271, 309, $310,526,571$; composition of intercellular air, 206; use of plasmolytic solutions, I 43 .

Dworrak, 432 ; D. u. Knop, I 32.

Dyer, 286.

Ebeling, 597 .

Eberdt, 229, 240, 242, 245, 247, 248: automatic variations in transpiration, 250.

Ebermajer, I 7 I , 209, 235, 298, 372, 4 IO, 414 , $437,450,459,460,485$; annual consumption of carbon dioxide, 298.

Ebner, on anisotropy, $S_{2}$.

Echinocactus, 238.

Eckstein, on galls, 56 .

Economic coefficient, 385 .

Ectoplasinic membrane, 91. 
Eder, I I 8, 237, 243, 250 ; automatic register of transpiration, 242 .

Effrant, 564.

Eggerty u. Nilson, 428 .

Ehrhardt, 475 .

Elaioplasts, 479 .

Eleagnus, root-tubercles of, 396.

Electrosynthesis, 292.

Elfert, 482, 599.

Elfving, 221, 308, 335, 383, 497, 523, 539 ; action of light on fungi, 562 ; of ether on respiration, 564; of light and low temperatures on etiolin corpuscles, 335 .

Elodea, 94, 96, 188, 204, 205, 310, 333, $337,341,345$.

Emissaria, 253.

Emmerling, I 73, 407, 410, 459.

Empusa, 374 .

Endoplasmic membrane, $8 \mathbf{I}$.

Endosperm, depletion of, 597-9; formation of enzymes by, 598, 599.

Energid, 60 .

Engelmann, 302, 304, 307, 308, 31 I-I 5, $335,336,341,345,352,358,540$; on assimilation by etiolated plants, 304 ; bacterium method of, 350 ; gas vacuoles, 109, 137; purple bacteria, 307 ; curves of photosynthesis and absorption, 342, 343, Figs. 51, 52 ; - proportionality between, 348 ; - aberrations from, 349 ; spectrum of erythrophyll, 495 .

Entomophthora, 374 .

Enzymes, 64, 501 ; excretion of, by parasites, $374 ;-$ by roots, I74; functions of, 503 ; changes in rate of production, 504 ; influence of external conditions on action of, 505 ; - of temperature on, 505 .

Epacrideae, 371 .

Epilobium hirsutum, 275.

Epipogon, 368.

Equisetum, I 80, 435.

Eranthis hiemalis, 285 .

Erdmann, 206.

Ericaceae, 371 .

Erikson, 374 .

Erlenmeyer, 356, 601 ; ripening of grapes, 602.

Erodium gruinum, $16 \mathbf{1}$.

Errera, 36, 221, 426, 486, 604; on glycogen, 71 ; - in fungi, 476 ; on karyokinetic and magnetic figures, 56 .

Erythrophyll, influence of sugar on production of, 486 ; - of light, $496,497$.

Eschenhagen, $384,386,421$; accommodation to concentrated solutions, 140.

Escombe, $48 \mathrm{I}$.

Essential elements, 410 (sec. 73) ; amounts of present in ash, 414; influence on turgidity, 424 ; definition of, 296 ; functions of, 422 (sec. 74 ); methods of investigation, 417-9; present as organic compounds, 423,424 .
Etard, 316.

Ethereal oils, 500 .

Ethyl-alcohol, nutritive value of, for fungi, 384 ; production of, by yeast and bacteria, 555-7; by fruits, 557 .

Ethylamine, 383 .

Etiolin, 304, 305, 316.

Etiolin corpuscles, power of photosynthesis in, 333 .

Eucladium verticillatum, 132.

Euglena, 320.

Euphorbia, starch in latex of, $582 ; E$. cyparissias, 25.

Euphrasia, 366, 369; E. officinalis, 306.

Euonymus, dulcite in, 477 .

Eurotium repens, $42 \mathrm{I}$.

Evaporation, from leaf and from water, 240.

Evolution of oxygen, 310, Figs. 41, 42.

Ewart, I86, 195, 238, 298, 304-7, 312, 313 , $319-24,330,333-6,34 \mathrm{I}, 349,35^{8}, 524$, 570 ; chlorophyll in purple bacteria, 308; conditions for formation of root hairs, 156; etiolin as an assimilatory pigment, 303; evolution of oxygen from coloured bacteria, 307 ; - from green fruits, 52I; - through cuticle, II 7 ; function of erythrophyll, 495; growth of red bacteria in absence of oxygen, 540; influence of accumulation of the products on photosynthesis, 322 ; - of anaesthetics, 339 ; - of age, 359; - of starvation, 593; - of temperature, 327 ; - of oil on resistance to desiccation, 595 ; - of sugar on production of erythrophyll, 496; on methods, 340 ; minimal temperature for photosynthesis, 338 ; percentage of water in seeds, 565; photosynthesis in isolated chloroplastids, 5 I ; occlusion of oxygen by pigment-bacteria, 546 ; resistance to desiccation, 84 ; - rapidity of revival from, I 58 ; respiration in pigment bacteria, 522 ; - in plants injured by frost, 566.

Excreta, definition of, 445 .

Excretion, of nectar, influence of external conditions on, 285 ; causes and me. chanism of, I $29,282,283$; of water, influence of chemical stimuli on, 270, 27 I ; from uninjured plants, 272 (sec. 47); from water-pores, 279; causes of, 280.

Exner, I 87.

Exosmosis, 99.

Expiration, 526 .

Exudation of water, 268 (sec. 46), Fig. 36 .

- pressure, intensity of, 259; definition of, 254 ; influence of injuries on, 260 , $26 \mathrm{I}$.

Faba vulgaris, 525 .

Facultative anaerobes, 532 .

Fagus sylvatica, mycorhiza on roots of, 372 , Fig. 55 . 
Faivre, on latex, 581, 582 .

- et Dupré, composition of air in vessels, 206.

Famintzin, 334, 341, 352.

Farmer, 570.

- and Williams, 483 .

Fatigue substances, 517 .

Fats, 468 (sec. 82) ; distribution of, 470, 471 ; origin of, 472 ; metabolism of, 479 .

Fauncopret, 338 .

Feddersen, on thermo-diffusion, 204 .

Feist, 96.

Ferment-organism, 502, 555 .

Fermentation, 518 (chap. ix).

Fermi, 388, 406, 504, 505, 509; on ferments of bacteria, 376 .

Fernbach, 504, 507 .

Ferric oxide, incrustations of, I34, 427.

Ficke, 369.

Ficus, 278 ; latex system of, $582 ; F$. carica, papain in, 509; $F$. elastica, 243; absence of lateral translocation in wood of, 217 .

Figdor, 256.

Filtration under pressure, 226.

Fischer (A.), 67, 145, 262, 263, 308, 313, $322,406,410,421,527,578-80,600$, 604 ; on bacteria, 43 ; - cilia of, 47,51 ; - influence of concentrated media on, I 40 ; - permeability of, 99 ; on starchtrees, 512,603 .

- (E.), 355, 49I, 494; fermentative capability of stereoisomers, 501 ; - of optical varieties of sugar, 383 .

Fittbogen, 235, 391, 418, 429, 586.

Fitz, 405, 547-9; butyric fermentation, $55^{8}$, 559; suppression of fermentative power, 553.

Flêche et Grandeau, 585 ; influence of calcareous soil on ash, 129.

Fleck, gases in soil, $17 \mathbf{I}$.

Fleischmann, 239.

Fleurent, 64 .

Fleury, 529, 600 .

Flïckiger, 1 19, 494.

Flügge, $365,376,383,450,457,495,505$, $506,508,509,514,523,531-5,549$, $550,555,564$; on putrefaction, 561 ; rennet, 509 .

Fluorescence, in Florideae, 350 ; in chlorophyll, 350 .

Fluorine, occurrence of, 438 ; influence on growth of barley, 416 .

Focke, 430.

Food-materials, circulation of, 296 ; statistics of, $297,298$.

Forchhammer, I28, 432, 437 .

Formal aldehyde, as product of photosynthesis, 355 .

Formic acid, nutritive value of, on bleeding, 264.

Fraas, 153.
Frank, 1 53-5, 334, 364-8, 371, 372, 390$400,403-5,478$; accumulation of nitrates, 98 ; - influence of, on development of root-system; root-tubercles, 393-403.

- u. Kringer, 416.

- u. Otto, 399.

Franke, 396.

Fränkel, 540; influence of carbon dioxide on fungi and bacteria, 564 .

Frankfurt, 459, 461, 472, 503, 509, 600 .

Frankland, 362,548 ; influence of carbon dioxide on bacteria, 564 .

- and McGregor, fermentation of arabinose, 555 .

Fraximus, 284, 603; F. excelsior, 259.

Frémy, 521, 601.

Freudenreich, 560 .

Freyberg, respiration in aquatic plants, 525 .

Fritillaria, 286; F. imperiulis, 282, 283.

Fruits and seeds, 601; changes during ripening of, 601,602 .

Fuchsia, 278; F. hybrida, 280, Fig. 39; F. globosa, 275 .

Fucosan, 320 .

Fucus, 437; influence of drying on cellwall of, 84 .

Funaro, 476.

Fünfstück, 103, 603, 604; on corrosion of rocks by lichens, 173 .

Fungi, excretion of ammonia by, 462 ; glycogen in, 476 ; reserve-materials of, 604 .

Funkia, 197; parthenogenesis of, 35 .

Gain, 75, I 53, 235, 263.

Galactose, $325,383,475$.

Galanthus, 326.

Galeotti, 498.

Galls, 55 .

Ganong, I6I.

Gardiner, 206, 275-8, 334; on protoplasmic continuity, 59 .

Garreau, I80, I86, I 88, 243, 340, 414, 520, $521-6$; on specific respiratory activity, 525 .

Gaseous currents, 205 ; - exchange, mechanisin of, 176 .

Gassincourt, de, 430.

Gaudichand, 227.

Gaunersdorffer, $219,429$.

Gayon, 377 ; G. et Dubourg, 105.

Geddes, 377 .

Gelatinous membranes, protection afforded by, 117 .

Geleznow, 233.

Gérard, 480, 604.

Gerasimoff, reversal of mitosis by cold, 56 .

Gerber, 328, 529, 601.

Gerland, 320 .

Germination of seeds, 597 .

Giesenhagen, I 58 ; on calcium pectate in cell-wall, 133 .

Gilson, 119,481 . 
Giltay, 235 ; G. u. Aberson, on denitrifying bacteria, 550; influence of oxygen on fermentative activity, 556.

Gingerbeer-plant, 560.

Gingko biloba, 333 .

Glaux, 439; G. maritima, 430.

Gleditschia triacanthus, 401.

Globoids, 428, 461, 595.

Globulariu, 474 .

Glucose, 506.

Gilucoside-ferments, 507 .

Glucosides, 65, 66, 3I9, 49I (sec. 87); functions of, 492.

Glutamin, 457-9, 465 .

Gluten, 460.

Glycerine, power of inducing starch formation, 326 .

Glycocoll, 383 .

Glycogen, 476 .

Glycyrrhizin, 491.

Gnentzsch, 216.

Godlewski, 222, 318, 319, 322, 332, 36I-7, $409,524-8,539,540-2$, 550; drop experiment with porous wood, 227 ; influence of mineral food on germination, 594; respiration in oily seeds, 529; theory of ascent of water, $217,226$.

Goebel, 26, 37, 39, 1 58, I 59, 172, I 80, I $81,228,275,276,280,368,377-9$; negative pressure in intercellular air, 201.

Goedechens, analyses of ash of algae, I27.

Goetze u. Pfeiffer, 409.

Golenkin, $320,438$.

Gonnermann, 400.

Goppelsröder, 219.

Göppert, I75.

v. Gorup Besanez, 379, 437; ash analyses of, 128 .

Gosio, 438 .

Graham, I87; on osmosis, 92.

Grandeau, 368.

Gramulobacter butylicum, 509, 540; lactobutylicum, 509; lacto-butyricum, 532 ; polymyxa, 547; saccharo-butyricum, 559.

Granuloplasm, 48.

Granulose, 506.

Grapes, disappearance of glycolic acid from, 602 ; ripening of in darkness, 601 .

Grassmann, 284.

Grating spectrum, use of, 350 .

Gravity, influence of injuries on response to, I9; influence of, on bleeding, 264.

Green, Reynolds, 460-6, 494, 502-7, 5 Io, 598,604 ; on ferments, 501 ; on rennet, 509 ; zymogen, 503.

Greenwood, 377 .

Gressner, 600.

Grew, Nehemiah, 31 5, 417 ; ascent of water, 225 ; - theory of, 226 ; lactiferous vessels, 581 ; spongioles, I 52.

Griessmayer, 460 .
Grihaut, 75 .

Grimbert, on butyric fermentation, 558 ; influence of cultivation on fermentative activity, 554 .

Gris, 427 ; deposition of starch in wood, 602,603 .

Grischow, 33I.

Groom, 160, 276, 371, 433 .

Grosglik, 360.

Grossmann u. Mayershausen, 542.

Groth, on molecular character of crystals, 79.

Growth, influence of temperature on, 562 ; - of cessation of on other vital activities, 425 .

Growth and development, causal relationship of, 23.

Griibler, 46I ; formation of crystalloids, So.

Gruiss, 475-8, 48I, 503-7, 5 Iо, 545, 598 ; test for diastase, 505 ; reserve-cellulose, 482 .

Guettard, 237, 24I ; importance of diastomatic transpiration, 243 ; - in summer, 250.

Guignard, 494.

Guillemin, 334 .

Gum, 477 .

Gunnera, 371 ; G. scabra, potassium chloride in petiole of, $14 \mathrm{I}$.

Gustavson, 425.

Guttation, 253.

Gymnoplast, 88 .

Haacke, 55 ; electric currents in living cells, I 04 .

Haas, 602.

Haberlandt, 52, 59, 159, I8o, I89, 191-8, $216,217,228,235,242-4,276-9$, $28 \mathrm{I}-7,307,313,322,334,357,358$, $360,376-8,409,478-80$, 59I, 596, 597. 600- 4 ; absorption by water-pores, I6I ; hydathodes, 253; internal negative pressure in mosses, 203 ; production of diastase by aleurone layer, 599 ; sievetubes of mosses and algae, 581 ; waterglands, 278 .

- (F.), 250, 25 I ; (G.) 250, 334, 593.

Haeckel, 9.

Haematochrome, 316.

Haematogen compounds, 426 .

Haemoglobin, as test for oxygen, 3 I I.

Hales, 198, 21 2, 227, 237, 242, 250, 259, $261,263,266$; absorption by leaves, I60; - by evergreens in winter, 230 ; amount of transpiration, 220; - relation to absorption, 232 ; ascent of water, 225 ; - causes of, 211 ; bleeding, 254 ; blocking of tracheae, 23I ; diminished transpiration in moist air, 245 ; existence of rarified air in plants, 20I, Fig. 2 I ; expansive power of swelling seeds, 75 ; exudation-pressure at different levels, 26o; oblique transference of 
water, 21 $7 ;-$ conducting channels for ; ringing experiments, 579; Halophytes, 421 .

Hamburger, I45.

Hammarsten, 64, 65.

Hampe, 405.

Hansen, 219, 308, 314-7, 357, 376, 432, $493-5,509,555-8,560$; analysis of cell-sap, I4I; lactic bacteria, 559: production of alcohol by fungi, 557 .

Hansteen, 320, 405, 409, 593-8; depletion of endosperm, 515,590 ; - self-depletion of, 597-9; regeneration of proteids in darkness, 464.

Hanstein, 579, 583-8; exosmosis of sugar from endosperm, 95 ; incrustations, chalky, I33; - of iron, I 34 ; latex, 582 ; metaplasm, 46 ; monoplasts and symplasts, 60 ; plastids, 43 ; ringing experiments, 578 .

Hartig, 201, 213,216, 217, 220, 237, 250, 260, 26I, 368, 369, 402, 584 ; alburnum, 225 ; exudation of water, 257 ; gaspressure theory, 226; hygroscopic absorption, I6I; negative pressure in transpiring trees, 2II; percentage of water in wood, 233.

- (R.), 579, 6c4.

- (Th.), 273, 468, 580-3, 604; deposition of starch in trees, 603; removal of, from roots of ringed trees, 579 .

Hassak, 33I ; absorption of $\mathrm{CO}_{2}$ from $\mathrm{Na} \mathrm{HCO}_{3}, \quad$ I 32 ; calcareous incrustations, I33.

Hasselhof, 4I 3 .

Hattensaur, 437.

Hauptfleisch, 5I ; on plasma streaming, I 26,589 .

Haustoria, 374 .

Heat, essential stimulating action of, for life, 17.

Heckel, 499.

Hedera, $243 ; H$. helix, activity of transpiration of, 244 .

Hedin, 65.

Hegler, on mitosis, 56 ; Shizophytes, 43.

Heidenhain, 96.

Heine, 67, 576 .

Heinrich, 229, 338, 585 .

Heinricher, 360, 366, 377, 368 .

Heintz, 530.

Helianthus, 334, 46I, 473, 595; importance of cotyledons of, 593; H. annuus, 26I, 324 ; disappearance of oil from isolated cotyledons of, 599 ; growth of, in absence of oxygen, 570; photosynthetic activity of, $357 ; H$. argophyllus, growth of, in absence of nitrogen, 391; H. tuberosus, 604 ; potassium nitrate in, I4I.

Heliophobic plants, 359.

Helleborus, 190, I91, Fig. 20, 286.

Hellriegel, I 53, 247, 391-8, too-3, 418, +20, $421,451,528,600$.
Helmholtz, 350 .

Helmont, Van, 367.

Helotism, 370.

Hemerocallis fulra, 319.

Hemicelluloses, 480 ; as reserve-material, 482.

Hempel, $31 \mathrm{I}$.

Henle and Pfeufer, 74.

Henneberg, 559.

Hepaticae, elaioplasts of, 470 .

Heraeus, 362, 363 .

Herbst, 24-26, I96; heterogenetic induction, 21 .

Heredity, $3 \mathbf{I}$ (sec. 5).

Herfeldt, 550, 560, 561 .

Hermann, 96, I0I ; on gas vacuoles, I09.

Hert, I 29.

Hertwig, 49, 57 ; on Altmann's theory, $4 \mathrm{I}^{-}$ 44 ; centrosomes, 47 ; composition of proteids, 66,67 ; influence of nucleus on movement, 5 I- 55 .

Hesperidin, 494.

Hesse, 529, 537 ; on respiration of bacteria, 525 .

Heteromorphosis, 24.

Heterotrophic nutrition, 363 .

Hexahydroxyhexamethyline, 477.

Heyne, 328.

Hilger, 602.

Hilger-Gross, 602 .

Hiltner, 396, 398, 399, 40 I, 597.

Hippuric acid, as source of nitrogen, 405 .

Hjort, 509.

Hlubeck, 368 .

Hochreutiner, 260.

Höfer, movements in non-nucleated $A$ moebae, $5 \mathrm{I}-53$.

Hoffmann, 27I, 427, 430-6, 497 ; on germination, 600 .

Hofmeister, 67, 7 I, I I 8, 233, 258, 259, 260-6, $271,454,474,478,483$; on bleeding of herbaceous plants, 254 ; - influence of tissue tensions on, 264 .

Höhnel, Von, I93, $211,227,232,239,241-3$, 250,251 ; negative pressure in tracheae, 202, 203; transference of air through intercellular spaces, I 97.

Hohnfeldt, I 89 .

Holfert, 585 .

Holle, $320,322$.

Holzner, 409.

Homberger, 584 .

Honey-dew, 286.

Hoppe-Seyler, 64, 3I 2, 450, 484, 548, 55 I.

Hordeum vulgare, root-system of, I 57 , Fig. I4; self-depletion of endosperm of, 598 .

Hormodendron, 509; H. hordei, 376.

Hornberger, 263, 407.

Horsford, 355 .

Hortensia, influence of iron on colour of flowers, 427 .

Horvath, 259-6I. 
Hoseaus, 391, 463 .

Hotter, 438,585 .

Hövcler, 153-5, 174, 371, 376 .

Hoya carnosa, 285; H. fraterna, 320.

Huber, 523.

Hüfner, 187,352 .

Huie, 55,56 .

Humboldt, I75, 258, 536; exudation of water from Agave, 257.

Humus collectors, 370 .

- saprophytes, 368.

- theory, 367.

Hungerbuihler, 604.

Hunt, 353.

Hiippe, $362,363,385,535,549$; on Bacillus butylicus, $55^{8}$; on character of stimulating actions, 18.

Husemann, 450, 485 .

Hyacinthus orientalis, 174 .

Hyaloplasm, 48.

Hybrids, 54 .

Hydathodes, 253, 278, 28I.

Hydra viridis, $359,37 \mathrm{I}$.

Hydrocharis morsus-ranae, I08, Fig. 6.

Hydrogen peroxide, changes of colour induced by, 98,545 ; poisonous action of, 546 .

Hymenophyllum, iss.

Hypochlorin, 320 .

Hypoxylon carpophilum, 276.

Idioplasm, 57 .

llex, I $18,335,336$.

Ilsova, 392 .

Imbibition, crystals capable of, 8o ; energy of, manifested as heat, 76 ; force of, 70.

Immendorff, 527.

Immortality of bacteria, 40 .

Impatiens, 209, 278; I. parviflora, 232, Fig. 27; chorophyll spectrum of, 344 , Fig. $51 ;$ I. sultani, bleeding of, 256 .

Indigo, 457 .

Indigo-carmin, as test for oxygen, 3 I 2.

Induction, isogenetic and heterogenetic, $2 \mathrm{I}$.

Ingenhousz, 206, 308, 52 I, 525, 601.

Ingestion (and excretion) of solid bodies, 88, 11 I (sec. I9).

Injection of fluids, influence on conductivity of wood, $22 \mathrm{I}$.

Inosite, 477 .

Inspiration, 526.

Interprotoplasmic communication, $3 \mathrm{r}$.

Intramolecular respiration, 536 ; influence of temperature on, 537 .

Inulenin, 476.

Inulin, 475 .

Invertase, 375 .

Invertin, 507; fermentative power of, 502.

Iodine, occurrence of, 438 .

Iridium, katalytic action of, $55 \mathrm{I}$.

Iris, $218,326$.

Irisin, 476 .

Iron, 413 ; influence on formation of chloro- phyll, 427; - on colours of flowers, 427 ; importance of, in photosynthesis, 355; necessity of, for Phanerogams, 426 ; - for fungi, 427 ; organic compounds of, 426 ; sources of, 427 ; stimulating action of, 416 .

Iron-bacteria, 363 .

Irritability, definition of, I 2, I 3 ; localization of, I 5 ; nature of, Io.

Isathionic acid, as source of sulphur, 429.

Isatis tinctoria, 494.

Ishii, 477.

Isotropy, 82 ; of protoplasm, 78 .

Israel, I 2 I.

Istvánffi, 581, 604.

I wanowsky, 556 .

Jaccard, 540-2, $57 \mathrm{I}$.

Jacobson, on Grüss' test for diastase, 505 .

Jäger, 517 .

Jahn, 216.

Jamin, energy of imbibition in non-swelling bodies, 75 .

Jamin's chain, 203, 222-4.

Janse, 99, 226, 37 I, 397; permeability of protoplasm, I 39 .

Jauret, 604 .

Jensch, 436 .

Jentys, $332,538,540$; influence of oxygen on growth, 542 .

Jessen-Hansen, 600.

Jodin, 305, 394, 435 .

Johannsen, 494, 523, 540, 563, 564 ; aftereffect of high pressure of oxygen, 543 ;

John, 4 I 8.

- influence of, on respiration, 542 .

Johnson, 405, 436 .

Johow, I 89, 360, 368, $37 \mathrm{I}-3$.

Jonidium, 475 .

Jönsson, I82, 360, 37 I, 529, 563-5 ; respiration in lichens, 525 ; on Saccharomyces, 558.

Jörissen, 494, 600 .

Jost, I $80,216,324,325,578,580$.

Joulin, 206.

Jumelle, $248,322,336-8,346,358,529$; influence of water on respiration, 565 ; respiration in lichens, 525,561 .

Jungner, I 58.

Jungermannia, 379 .

Just, 240, 244, 326.

Kabsch, 571 .

Kamerling, I 58, I 83, 200-2, 223.

Kamienski, 373 .

Karsten, I $80,390,600$.

Karyoids, 48.

Karyoplasm, 43.

Katalytic action, 502.

$\mathrm{Katz}$, on regulation of diastase-production, 504 .

Karulfussia, I9I. 
Kayser, 185, 534, 557 ; on lactic fermentation, 559 .

Kedrowski, 531, 541.

Keeble, i 58.

Kefir, 560 .

Kekuli, 72 ; on molecular structure of colloids, 79 .

Kellermann u. Raumer, 379.

Kellner, 404-9, 457.

- u. Sachsse, 462.

Kern, 241.

Kerner, 162, 190, 237, 284, 369, 377, 497.

Ketone, 383 .

Kienitz-Gerloff, 589 ; on protoplasmic connexions, I 13 , I 15 .

Kihlmann, 237 ; xerophily in swamp plants, 231.

Kinerim u. Nayer, 560.

Kinoplasm, 48.

Kinoshita, influence of glycerine on accumulation of asparagin, 464 .

Kionka, 399, 400.

Kirchner, 401 .

Kirschmann, 351 .

Kitasato u. Weyl, 540 ; influence of reducible substances on growth of anaerobes, 549.

Kitt, 531 ; aerobiosis of Bacillus carbonis, 540, 541 .

Klebahn, air passages in periderm, 189 , 198 ; on gas vacuoles, 48 , 109, 137.

Klebs, 51, 307, 314, 320, 323-5, 336, 366, $367,386,421,483,557,596,597$; nutritive importance of tartrate of iron, 105 ; permeability of protoplasm, 99 ; alkaline excretions, 132 .

Klecki, Von, 558 .

Klemm, 68, 569 ; foamy structure of protoplasm, 44-46; phloroglucin in cell-sap, 97, 98 .

Klercker, $5 \mathrm{I}$; on precipitates in living cells, 97,98 ; on tannin, 492.

Klinger, 354 .

Klöcker u. Schionning, 558 .

Knight, 155, 213, 231, 262 ; ringing experiments, 579 .

Knop, 157, 240, 420, 427-9, 43I-7, 520 ; on alkalinity of water-cultures, 132; backward diffusion in transpiring plants, I30; differential absorption, 132.

- u. Biedermann, on differential absorption, 129.

- u. Dworzak, 430, 586 ; on development of maize in absence of chlorine, $43 \mathrm{I}$.

- u. Wolff, 157, 405, 406 .

Knuth, 285 .

Kny, $51,160,237,307,537,546$; on anthocyan, 495.

Koch, 366, 369, 400, 436.

- u. Hoseaus, 476 .

Kohl, 117, 189, 190, 191, 216, 235, 239, $242-8,310,345,352,428,432-6,486$; 600,601 ; bubble-counting method, 350 ; calcium pectate in cell-wall, 133 ; calcareous incrustations, I3I ; development of cuticle, 117 ; forms of calcium oxalate, 490 ; influence of light on stomata, 195; - of temperature on absorption, 229 ; -.- of pressure on transpiration current, 222; interprotoplasmic communication, 59; secondary maximum of photosynthesis in blue, 343 .

Kolkwitz, 72 ; influence of light on respiration, 563 .

König, 127, 209, 388, 450, 458, 460, 602-4.

- u. Ebermayer, 473 .

—u. Haselhoff, 174 .

Koorders, 28I.

Köppen, I 19, 347.

Korthals, 280.

Kosaroff, absorption of water at low temperatures, 230, 23I.

Kossel, on cholesterin, 63 ; on proteids, 65 ; nuclein in leucocytes, 67.

Kossowitsch, 156, 395, 396, 399, 405.

Kosutany, 409.

Kowalevsky, absorption of aniline dyes by animals, 96.

Krabbe, 504 ; optical properties of superposed lamellae, 78 ; osmotic pressure, I 38 .

Krakatoa, return of vegetation to, 175 .

Kranch, 508.

Kraus (C.), 259, 262, 279.

- (G.), 1 56, 245, 315-10, 327-9, 352-3, $436,474,475,484,493-5,523,585$, 601 ; bleeding from isolated stems, 251 ; calcium oxalate as plastic product, 487 ; contents of sieve-tubes, 580; malic acid as translocatory material, 577 ; in Crassulaceae, 141 ; rapidity of starch production, 321 ; on tannin, 492-4.

Kreusler, 31 I, 323, 332-5, 352, 360, 524, 545, $562,565,586,604$; on activity of photosynthesis, $324 ;$ - influence of temperature on, 337, 338, Fig. 50; - of light on, $34 \mathrm{I} ;-$ optimal percentage of $\mathrm{CO}_{2}$ for, 332 ; influence of water on respiration, $565 ;$ - of temperature, 561.

Kreuzhage u. Wolff; influence of absence of silica on oats, 436 .

Krüger, 367.

Krukenberg, 377, 490.

Kruticki, 206; composition of air in vessels, 206.

Krutizsky, 242.

Kühne, I 86, 569-7 1 ; on plasma-streaming in absence of oxygen, 546.

Kulisch, 602 .

Kundt, 204.

Kunstmann, 523, 527 .

Kupfer, on paraplasm, 46.

Küstenmacher, on tannin, 493.

Küster, 71, 470, 479 .

Labiatae, occurrence of cane-sugar in, 474 . 
Laborde, 383 .

Laccase, 545 ; influence of mangancse on, 509 .

Lactarius, laccase in, 510.

Lactase, 507.

Lactic acid, production of, 559 .

- fermentation, 559.

Lactiferous tubes, 581,582 .

Ladenburg, 435; apparatus for estimation of osmotic pressure, I 44.

Laevulin, 476 .

Laevulose, 474.

Lafar, $376,495,508,514,545,555,560,561$; lactic fermentation by yeast, 559 ; on rennet, 509; theories of fermentation, 552.

Lamarlière, de, $240,357,360$.

Lamartina, 406.

Laminuria, 75, 161.

Landolt, $35 \mathrm{I}$.

Lange, 9, 1o, 435, 484; on living vessels, 202.

Langendorff, an automatic potometer, 242 .

Langley, distribution of energy in solar spectrum, 344, Fig. 52.

Larix, 333.

Laskovsky, 525, 528, 530, 600; production of water during respiration, 529 .

Laspeyres, 244.

Latex, 581, 582 .

Latex-system, influence of, on ringing experiments, 578 .

Lathom, 65.

Lathraea, 133, 365, 368, 377.

Lathyrus sylvestris, root-system of, I 53.

Laticiferous tubes, 581 .

Laurent, 314, 325, 326, 367, 399, 401-9, $476,497,498,533,549$; concentration of sap necessary for starch-formation, 326.

Lauterborn, staining of living nucleus, 94.

Law of minimums, 413 .

Lawes, Gilbert and Pugh, 402.

Laying of crops, 436.

Lead, occurrence of, 437 .

Leaf, premature fall of, 246 ; removal of ash constituents from, 585 .

Lechartier, 204, 205.

- et Bellamy, 538,564 ; on intramolecular respiration, 537 .

Lecithin, $63,428,479$.

Leclerc du Sablon, 472, 530, 592, 598-600.

Lecointe, 258,574 .

Lecoq, 204 .

Legumin, 66.

Leguminosae, 600 ; percentage of amides in, 458 ; - of protcids in, 460 ; reservematerials of seed, 594; symbiosis of, with bacteria, $37 \mathrm{I}$.

Lehmann, 97, II $8,219,437$; heat of imbibition, 76: micellar constitution of crystals, 79, 80; molecular structure of colloids. 72 .
Leitgeb, 159, 189-91, 276; on chalk in ccllwalls, 133; closure of stomata by induction shocks, 194; - opening of, in darkness, 194.

Lemna, 409; regeneration of proteids in darkness, $464 ;$ l. minor, I20, 124 ; absorption of methyl-bluc by, 94, 96; L. trisulca, 428 .

Lenticels, passage of gases through, 198.

Leonumis cardiaca, 278 .

Lepel, Von, 392.

Lepidium, 402.

Leptomin, 5 Io.

Leptophrys Kiitzingii, 377.

Lesage, 323, 557 .

Leucin, 457-9.

Leucojum, 475 .

Lewith, influence of desiccation on coagulation, $8_{5}$.

Liborius, 504, 531-3, 540, 547 ; on Clostridium foetidum, 558 ; influence of sugar on fermentation, 553 .

Lichens, reciprocal symbiosis in, 370 ; reserve-materials of, 604 .

Lidforss, 433, 600 ; on bursting of pollengrains in distilled water, 134 ; increased percentage of sugar at low temperatures, 512.

Lieben, 356.

Liebenberg, 433 .

Liebig, I 27, 128, I 69, 17 I-4, 329, 367, 368, 413 , $430,526,553$; force of imbibition, 75 ; supposed function of organic acids, 356 .

Liebreich, 516.

Liebscher, 396.

Lietzmann, I 86-8; influence of drying on permeability to gas, 183 .

Life, nature of, 5 ; general characteristics of, $\mathrm{I}-3$.

Light, influence of, on bleeding, 264; - on photosynthesis, 340-56, 358-60; - on acidity of issues, 327 ; - on pigment formation, 496, 497; - on respiration, 562 ; - on secretion of nectar, 285 ; - of different rays of, 353 .

Lignification, 482 .

Ligustrum, 578 .

Lilienfeld, 65.

Lilium, 2 I8.

Lind, $173,315$.

Lindau, 370.

Lindet, 602 .

Linossier, $383,406$.

Linseed, attachment of sceds of, 478 .

Lintner, 555, 564 .

Lintz, 506 .

Linum usitatissimum, translocation of oil in, 592.

Linz, 505, 598.

Lippmann, 469.

Lithium, influence of, on growth of barley, 416 ; occurrence of, in plants, 429 ; use of, to demonstrate water-current, 219. 
Lobelic erimus, 285.

Lobeliaceae, inulin in, 475 .

Loew, 363, 38I, 392, 4I 1, 429, 43 I, 438, 466, 499,550 ; hypothesis of proteid formation, 405; importance of calcium, 433.

- and Bokorny, 67-69; influence of chlorides on production of tannin, 492 ; precipitations in cell-sap, 97.

Lommel, 349.

Lookeren, 494 .

Lopriore, 332, 564, 570 .

Lory, 521,525 .

Löseke, 391.

Lotze, I9.

Lubbock, 596.

Luca, de, 476, 536, 537, 564, 571 .

Lucanus, 404, 429, 585 .

Luck, 306.

Lüderitz, 533, 542.

Liidtke, $46 r$.

Ludwig, $75,495,499,582$; on differential absorption, 74 ; on tannin, 492.

Lund, 565 .

Lupinus, 458, 460, 462; absence of pepsin and trypsin from, 466 ; exosmosis of asparagin from living cotyledons of, 95, 600; autotrophic cotyledons of, 596 ; enzymes of, 507 ; formation of amides during germination, $46 \mathrm{I}$; sensitivity to acid solutions, 420 ; L.albus, disappearance of oil from isolated cotyledons, 599; L. hirsutus, presence of trypsin in, 466; L. luteus, 398 ; analyses of seedlings, 467; leucin in, 465; root tubercles of, 396, Fig. 59; seedling of, 597, Fig. 68 .

Lüpke, $420,430$.

Lutz, 405, 604.

Lycopodium alpinum, 437 ; L. chamaccyparissus, 437 ; L. phlegmaria, 437.

Maby, 173.

Macagno, 353 .

Macaire, I 38 .

Macallum, 426.

Macdougal, 325, 402.

Mach u. Haas, decrease of tannin during ripening of fruits, 602 .

Macmillan, 377.

Mac Nab, on use of lithium salts, 2 I 9.

Magnesium, distribution of, 343 ; functions of, 431 .

Magnol, 217.

Magnolia, 256.

Maize, development of, in absence of chlorine, $43 \mathrm{I}$.

Malaguti et Durocher, 437; influence of soil on composition of ash, 129.

Malfatti, on proteids, 65,66 .

Malpighi, 225, 525, 571, 582, 593 .

Maltase, 506 .

Mali'a arborea, 247.
Nanganese, occurrence of, 437 .

Mangin, I80, I 85, I $88,327,332,430-2$, 478,525 ; on calcium pectate in cellwalls, 133 ; on extracellular protoplasm, 59 ; influence of temperature on diosmosis, 187 ; on pectins, 482 ; respiration of pollen-tubes, 525 .

Manihot utilissima, 494.

Mannite, 325, 476 .

Maquenne, $356,477,564$.

Narcet, 536 .

Marchantia, 28, 191, 275; induction of dorsiventrality in, $20 ; M$. polymortha, rhizoid of, I 5 I, Fig. I I.

Marchlewski, on chlorophyll, carotin, \&c., $315,316$.

Marey, 242.

Mariotte, 225,241 ; law of, I44 ; on absorption by leaves, 160 .

Marloth, I62; on saline incrustations, 131.

Marrgraff, 418.

Marschall, 450, 462.

Marshall Ward, 37 ; on gingerbeer-plant, 560.

Martin, 206.

Martinand, 5 ro.

Matteuci, 264.

Maumené, 437, 478 .

Maxwell, I62.

Mayer, 174, 328, 348, 353, 404-8, 4 IoI3, 431, 499, 505, 524, 534, 548, 555 , 559 ; decreased respiration in cut seedlings, 566 ; on fermentation, 552 .

Mazé, 399.

Mechanomorphosis, 24 .

Medium, influence of, on growth, 4 I 5.

Meinecke, I 59.

Meissner, 339, 360.

Meister, absorptive capacity of soils, 169.

Melampyrum, 369 ; dulcite in, 477.

Melezitose, 474.

Melnikoff, 432 ; on calcium pectate in cellwall, 133 .

Melobesia, I33.

Melsens, 564 .

Mène, 402.

Menze, 321.

Mer, 323, 539, 603.

Mercury, 437.

Merget, 179, 180, 242 ; on currents of air in Nelumbium, 205.

Merkel u. Bonnet, 290.

Merlis, 600.

Merulius lacrymans, 276.

Mesembryanthemum, 433; oxalic acid in, 328 .

Mesnard, 479, 500, 529, 593, 600-2.

IIesocarpus, 342 .

Metabolic products, $45 \mathrm{I}$, sec. 78 ; localization of, 454 ; consumption of, 456 ; plastic and aplastic, 457 .

Metabolism, analytical examples of, nitrogenous, 461 , sec. 80,465 , sec. 81 ; - 
non-nitrugenous, 472 ; historical account of, 450 ; influence of external conditions on, 510 , sec. 92 ; of temperature, 512 ; of special substances, 512 ; of antagonistic and reciprocal symbiosis, 51-53; similar character of, in plants and animals, 449 .

Metaplasm, 46.

Methyl-alcohol, formation of starch from, 326.

Methylal, formation of starch from, 326.

Methylamine, nutritive value of, for fungi, $3 S_{4}$.

Metzgeria, 337.

Meyen, 151, 226, 257, 418, 526.

Meyer (A.), I3I, I63, 168-70, 201, 298, 314-2I, 325-7, 358, 393, 468, 475-8, $490,501-5,604$; on calcareous incrustations, I33; on carbohydrates, 8I ; composition of starch grains, 78 ; determination of osmotic pressure, I 44 ; galactose, 475; hypochlorin, 316; inter-protoplasmic communication, 59 ; sphaero-crystals, 70 ; swelling of organized bodies, 72,73 ; trichites, 80 .

-u. Jacobson, 355, 550 .

- (G.), 475, 498 .

- (L.), I 25 .

Micellae, 77; anisotropous character of, 78 .

Microchemical methods, use of, 586 .

Micrococcus ochroleucus, influence of light on pigment formation in, $497 ; M$. prodigiosus, 497.

Microsomata, 43 .

Microthammion, $4 \mathrm{I}$ I.

Mielke, 492, 493.

Migula, 54I.

Mikosch u. Stöhr, 333 .

Millardet, 3I 5, 60I.

Milroy, 65 .

Mimosa, 238, 270, 337 ; 1. pulica, 331 ; formation of asparagin by, 600 ; transmission of stimuli in, 13-17, 20.

Miquel, 247 ; on urase, 550.

Mirabilis jalapa, cotyledons of, 596 ; seedling of, 597, Fig. 69.

Mistletoe, attachment of seeds of, 478 .

Mixotrophic plants, 364 .

Miyoshi, 374,375 ; on penetration of parasites, 373 .

Mnium, 188 ; M. cuspidatum, 193.

Möbius, 371 .

Mohl, 174, 192, $217,226,240,309,333,335$, $44 \mathrm{I}, 497,526,583,598$; influence of light on stomata, I94; - mode of closure of, 190, I91.

Moldenhawer, I 72.

Molecular structure of cell-walls, 81 ; changes of, due to swelling, 83 ; hypotheses of, 76.

Molisch, 174, I90, 256-8, 275, 31 5-7, 333, $360,396,405,406,41 \mathrm{I}-5,42 \mathrm{I}, 424$, $427,429,431,432,437,438,497,526$,
604 ; on absence of reducing action in higher plants, 549; influence of light on production of isatin, 494 ; incrustations of iron, I34; necessity of iron for fungi, 426.

Moll, 182, 242, 277, 278, 330 ; on emissaria, 253 ; on injection with water, 277.

Mölier, 366, 372, 491, 498, 538, 550.

Molybdenum, occurlence of, 437 .

Momordica, 185 .

Monas amyli, 377; M. okenii, $306 ;$ M. vinosa, 307.

Monilia candida, 555 .

Monoplasts, 60 .

Monotropa, 368 ; epiphytic mycorliza in, 371 ; post-mortem oxidation of, 545 .

Montemartini, 332 .

Monteverde, $315,316,333,346$; influence of sugar on accumulation of asparagin, 464.

Morck, 400

Morgen, 318, 353.

Morus, 206 ; latex of, $58 \mathrm{I}$; - sugar in, 582 ; M. albus, 259.

Movements of enclosed air, I99.

Mucilage, 477.

Mucin, 65, 66 .

Mucor, 37, 61, 148, 370, 555; M. alternans, $377 ; M$.circinelloides, $377 ; M$. erectus, $557 ; M$. mucerlo, production of alcohol by, 557 ; trehalose in, 476 ; as a fermentative aerobe, 530 ; M. racemosus, alcoholic fermentation by, 557 ; - influence of oxygen on, 547 ; temporary anaerobiosis of, 530; $M$. stolonifer, growth of, in absence of oxygen, 536.

Mucorineae, 58.

Muilder, I 27, 4 I 3 .

Müller, I 53, I 83-5, I $88,198,330,426$; influence of induction shocks on stomata, I94.

Miiller (A.), 327.

- (C.), 228,437 .

- (F.), 539 .

- (Johannes), 16, I7.

- (L.), 495, 60I.

- (N. J. C.), 197, 204, 239, 260, 332, 341, $34^{8}-50,462-4,495$.

- (O.), I 40.

- (P. E.), $37 \mathrm{I}$.

Müller-Thurgau, 323, 410, 525, 563-6,6oI-4; sweetening of potatoes by cold, 512 .

Multinucleatae, 58 .

Multinucleate cells, 58, sec. 10.

Müntz, 362, 476, 479, 528, 536-8, 600-2 ; liberation of gallic acid from tannin by fungi, 492.

Musa, 319, 320.

Musanga, bleeding of, 25 S.

Muscari moschatum, 319.

Musset, 2 I I 286.

Mustel, $23 \mathrm{I}$.

Mycetozoa, 58 . 
I/ycoderma aceti, $38 \mathrm{I}, 560 ; M$. aini, 4I I, 431.

Mycorhiza, 368, 371; conditions for development of, 372 ; characters of, 373 ; endophytic, 37 I, Fig. 54 ; epiphytic, 372 , Fig. 55 ; importance for absorption, 156. Myriophyllum, 204.

Myristica, 478 .

Myxomycetes, alkalinity of, 490 ; asparagin in, 459 .

Nadelmann, 482, 599 .

Nadson, 319, 323-6, 367; on Shizophytes, 43. Nagamatsz, 33I, 346.

Nägreli, I Io, $212,240-4,290,309,38_{3}-6$, $404-6,412,426,429,439,452,462,490$, $497,513,538,547,560$; arrangement of micellae in starch grain, $8 \mathbf{I}$; copper in distilled water, I2I ; diosmotic properties of cell, I 43 ; fermentation, $551-3$; - continuance of, in presence of oxygen, 556 ; excretion of albumin by yeastcells, I05; hypotheses of molecular structure, $76-82 ;-$ of colloids, 72 ; idioplasmatic elements of, 49,57 ; oil as reserve-material, 594 ; optical characters of organized structures, 8I ; spongioplasm, 48 ; substitution theory, 431 ; swelling and water of organized bodies, 71,73 .

- u. Schwendener, 77, 197, 227; on optical properties, 82 ; resistant powers of cells with small radii, 140 ; rigidity of stretched bodies, I 34 .

Nasse, 545.

Nastukoff, 549 .

Nathusius, 153.

Naumann, 204.

Nectar, composition of, 284 ; excretion of, $28 \mathrm{I}$, sec. 49.

Negative pressure, in intercellular spaces, 201 ; in vessels, 202, 203.

Nelumbium, 158, 201 ; N. speciosum, 197, 205.

Nematoplasts, 48 .

Nencki, 3I6, 5 I4.

Neomeris, 61; occurrence of inulin in, 475.

Neottia, 367,368 ; N. nidus avis, 306 ; evolution of oxygen by, $52 \mathrm{I}$.

Nepenthes, 269, 275, 280, 281, 377, 378, Fig. 56; acids in pitcher of, 379; enzyme of, $379,505,509$.

Nereum oleander, I86, 348.

Nestler, 279.

Neubauer, 262.

Neumeister, 64, 4I 5, 426-8, 463-6, 490, 498, 499 , 501 ; on chitin, 481 ; constitution of proteids, 65 .

Newcombe, 507.

Nickel, occurrence of, 437, 493.

Nicolle et Bey, 497.

Nicotiand, $325 ;$ N. tabacum, 256.

Nilsson, 23I.
Nischimura, 388 .

Nitella, 1 86 ; streaming of plasma in absence of oxygen, 546,570 .

Nitrate bacteria, 29I, 292, 36I-3.

beds, 392 .

Nitrates, importance of, for Phanerogams, 40.4 .

Nitrification, 392.

Nitriles, 383 ; as source of carbon, 406.

Nitrite bacteria, 36I-3.

Nitrobacter, $36 \mathbf{I}$.

Nitrococcus, $36 \mathrm{I}$.

Nitrogen, 388 ; amount removed by crops, 393 ; assimilation of, 393, sects. 68 and 69 ; - by Leguminosae, 396 ; circulation of, 391 ; influence of sugar on assimilation of, 395 ; evolution of, 390 , 550 ; fixation of, 392 ; mode of assimilation of, 407, sec. $7 \mathrm{I}$; percentage of, in seeds, \&c., 388 ; supposed absence of, in certain micro-organisms, 388 .

Nitrogen compounds, nutritive value of, 403 , sec. 70 ; - for Phanerogams, 404; for fungi and bacteria, 405; plastic, 457 , sec. 79 .

Nitromonas, $36 \mathrm{I}$.

Nobbe, I 53, I 56, 396-9, 400, 420, 42 I, 429, $430-3,437,585,593,596$; on ash analyses, 129; development of rootsystem, I 53; - optimal concentration for, 155 ; influence of potassium on accumulation of starch, 430 ; respiration in tubers, 525 ; swelling of seeds, 75 .

- u. Siegert, I20; on saline incrustations, I 30.

Noll, 237, 31 5, 360, 421 ; on heterogenetic induction, $2 \mathrm{I}$.

Nonnoses, 469 .

Nostoc, 37 I.

Nostocaceae, 71 .

Novy, on cultivation of anaerobes, 535 .

Nowacki, 585 .

Nuclein, 64-66.

Nucleo albumin, 66.

Nucleo-plasm, 43.

Nucleo-proteids, as plastic materials, 460.

Nucleus and cytoplasm, relations between, 50,55 , sec. 9 .

Nuphar luteum, 206.

Nutrient saline solutions, 386; - solid media, 386 .

Nutrition, 287 , sec. 50 ; influence of temperature on, $384 ;$ - of external conditions on, 384,385 .

Nyctalis, 276.

Nymphaea, I 49, I 58, I 80, I 97, 201 ; N. alba, 198.

Obligate anaerobes, 53I, 532.

O'Brien, 460 .

Odontites lutea, 369.

Oedogonium, 350, Fig. 53.

Oenoxydase, 5 Io. 
Ohlert, 152.

Oil, as product of photosynthesis, 320; as reserve food, 595 ; translocation of, 592.

Olive, mannite in, 577 .

Oltmanns, 352, 42I, 497 ; osmotic value of cell-sap in marine plants, 140.

Omeis, 602.

Omelianski, 560 .

Oncidium altissimum, 159.

Optical characters of organized structures, $8 \mathrm{I}$.

Orchidaceae, 371 .

Orchis, 470.

Organic acids, 485 , sec. 85,487 , sec. 86 ; decomposition of, by light, 327,328 ; - by heat, 328 ; accumulation and production of, 328 ; formation of starch from, $327-9$; as food-material, 594; influence of neutralization on production of, 488 ; - of alkalinity, 489 ; - of temperature, 488 ; Liebig's view of, 329.

Organic food, absorption of, 363 .

Ornithogalum arabicum, 474 .

Orobanche, 155, 365-9.

Orthonitrophenylpropiolic acid, as test for grape sugar in nectaries, 285 .

Orthotrichum, 337, 339, 595.

Oryza, 473 .

Oscillaria, curve of photosynthesis in, 344, Fig. 51 .

Osmotic pressure in the cell, 134, sec. 24 ; influence of chemical combination on, $141 ;$ - of dissociation on, $142 ;$ - of curvature of limiting membrane on, 136 ; - of temperature on, 138 ; estimations of, 145 ; physics of, 144 ; values for, $137,146,147$.

- systems, 135, 136 .

Ostwald, $67,75,76,82,92,105,125,126$, $145,327,346-50,354,363,516$; on characters of crystals, 79 ; influence of temperature on osmotic pressure, 138 ; nature of irritable reactions, 10 ; osmotic pressure of mixed solutions, 142 ; physics of, 144 .

Otto, 437.

Oudemans u. Rauwenhoff, 172, 451, 600 .

Overton, 99, 103, 104; on absorption of aniline dyes, 96; erythrophyll, 496.

Oxalic acid, influence of temperature and light on production of, 488 ; production and decomposition of, 488, 489 .

Oxalis, 337 ; oxalic acid in, 141 .

Oxidase enzymes, 509, 510.

Oxygen, effects of absence of, 569 ; influence of, on fermentative activity, 547,554 ; on development of chloroplastids, 335 ; on photosynthesis, 339: minimal and maximal percentages of, for aerobes and anaerobes, 539-42; occlusion of, 570: presence of, in cell, in neutral form, 321 .

Ozone, absence of, from living cell, 545 . l'acht, ior.

Padina paronia, 128.

Paeonia, 473; ripening of, 6or.

Palla, 320 ; influence of nucleus on cell-wall formation, 53; on karyoids, 48; Schizophytes, 43 ; staining of living nucleus, 94.

Palladin, 64, 335, 460, 461, 466, 529, 537, 570 ; influence of oxygen on metabolism, 537; respiration in etiolated plants, 525 ; - in leaves, 563 ; ratio between respiration and percentage of proteids, 521 .

Palmella, 4II.

Pammel, on fermentation without gas-production, 534 .

Pangens, 49, 50.

Pangium edule, 391, 406, 407, 457, 494; hydrocyanic acid in, 577 ; - translucation of, 592.

Papain, 508, 509.

Papaver sominiferum, 592.

Pappenheim, 182, 202; negative pressure in tracheides, 201.

Paraheliotropic movements, importance of, 238.

Paramylon, 320.

Paraplasm, 46.

Parasites, penetration of, 373 .

Parmelia, 337.

Passage of gases through cells and cellwalls, 183 , sec. 30 .

Pässler, 585,600 .

Pasteur, 306, $383,397,533,537,538,542$, 557 ; on anaerobiosis, 530,534 ; on fermentation and intramolecular respiration, 552; nutrient solution of, 386.

Pawlewski, 505.

Payen, calcareous excretions, 133.

Peckolt, 262.

Pectin compounds, 477 ; distribution of, $4 \delta 2$.

Pedicularis, 369 .

Peirce, $216,336,369,374$; on penetration of Cuscuta, 498.

Pellet, 413 .

Pellionia, 304 ; P. daveauana, 3r4, Fig. 47.

Pencillium, 210, 275, 407, 408, 426, 516 , 517,560 ; growth of, in concentrated media, 421 ; influence of acid on proteolytic ferment of, 509; production of alcohol by, 539; regulation of production of acid, 487-9; relation between growth and fermentative activity of, 553.

- glaucum, 373-6, 380-4, 387-9, 404, 494; accommodation of, to concentrated solutions, 139; assimilation of nitrogen by, 394; influence of sugar on production of diastase by, .504; mannite in, 476 ; respiratory ratio of, 529 ; temporary anaerobiosis of, 536.

Pennington, 345,415 . 
Pentoses, 320 ; nutritive value of, 470 .

Penzig, 475.

Peperomia, water-storage tissue in, 228.

Pepsin, 508, 509.

Peptone, nutritive value of, for fungi, 384 .

Perdrix, on butyric fermentation, 558 .

Peridinium tripos, 306.

Periodicity, induction of, 25.

Periploca, 580.

Perrey, 320.

Perseke, I 58 .

Peters, 170, 600.

Petermann, 396.

Petit, 426, 427.

Peyroul, 206.

Peziza, 276; P. sclerotionum, 376; enzyme of, 507 .

Pfaundler, on viscous solids, 45 .

Pfeffer, 139, 174, 175, 181, 213, 217, 222, 260, 269-7I, 284, 29I-3, 298, 306, 312-8, 32I-3, 33I, 336, 343, 349, 350, $352,355,356,363,367,373,374,377$, 380, 381, 385-7, 423, 428, 440, 450-2, $458,46 \mathrm{I}, 462,468,470-9,485,490,49 \mathrm{I}$, $494,495,516,520-3,536-9,546,549$, $552,559,567-9,576,581,589-9 \mathrm{I}$, 598 ; absence of reducing action in living cells, 544; absorption of aniline dyes, 93-6, 120-6 ; - by root-system, 156 ; absorption and diosmosis, 10III I; accommodation to concentrated solutions, 99; active albumin, 70-73; bubble-counting method, $35 \mathrm{I}$; changes on death, 66-68; dermatosomes, 81 ; detection of salts by microchemistry, 586; diosmotic properties of cell, 9I ; energy liberated by oxidation of sugar into organic acids, I4I ; hyaloplasm, 48; hygroscopic absorption; I6I, I62; formation of asparagin in seeds, 600 ; - permeability of Mycetozoa to, 99; formation of vacuoles, 47 ; injection and excretion of solids, 45,46 ; irritability, $2 \mathrm{I}-3$; osmotic pressure, influence of curvature of limiting membrane on, I 36 ; - of temperature on, 138 ; - in mixed solutions, I 42 ; - physics of, I 44 ; - restoration of, I43; - values for, I 45, I 46 ; oxidation of chromogens by $\mathrm{H}_{2} \mathrm{O}_{2}, 496$; oxidatory changes, 545; pangens, 49; photosynthesis, absence of, in ultra-red rays, 347,348 ; secondary maximum of, in yellow rays, 345 ; proteids in living cells, 63 ; resistant powers of cells with small radii, I 40 ; slow diffusion of colloids, 138 ; sources of energy, IO, $2 \mathrm{I}-23$; swelling of organized bodies, 74-7; tagma, 77-9; tannin, 492 ; translocation, 592 ; - of proteids, 583 ; - through plasmatic connexions, I 15 ; turgidity, readjustment of, 142 ; of guard and epidermal cells, I94; importance of, 142.
Pfeiffer, 356; on ripening, 602 .

Pfeil, 602.

Pfitzer, 219, 220; water-storage tissues, 228.

Pflüger, 552.

Phallus, 476.

Phaseolus, I7 I, 324, 400, 40I, 464; autotrophism of cotyledon of, $596 ;$ - depletion of, 515; - importance of, 593; - removal of ash constituents from, 584; I. multiflorus, 427; photosynthetic activity of, 357 ; self-depletion of cotyledons of, 599 .

Phenol, nutritive value of, for fungi, 384 .

Phenylanilin, replacement of leucin by, in etiolated seedlings, 465 .

Phillips, 437.

Phisalix, 497.

Phloem, importance of, in translocation, 574,575 .

Phloroglucin, 491-3 ; in cell-sap, 97.

Phoenix, 597 ; enzymes of, 507 ; escape of proteids from endosperm of, 95, 600 ; $P$. dactylifera, depletion of isolated endosperm of, 599 .

Phosphates, 428.

Phosphorus, source and importance of, 428 .

Photobacterium, 381 ; P. luminosum, 532.

Photomorphosis, 25.

Photophilic plants, 358.

Photosyntax, 302.

Photosynthesis, definition of, 292 ; of other carbon compounds, 326 ; inhibition of, 336 ; optimal percentage of carbon dioxide for, 332; theory of, 352; specific powers of, in different chloroplastids, 357 .

Photosynthetic assimilation, definition of, 302 ; influence of temperature on, 337 ; of anaesthethics and oxygen, 339 .

Phycocyanin, 317,495 ; influence on photosynthesis, 343, 344, Fig. 51 .

Phycoerithrin, 316, 495; influence of, on photosynthesis, $342,3+3.348-35$ I.

Phycophaein, 317.

Physiological combustion, mechanism and causes of, 539, sec. $100 ; 543$, sec. 101 .

Physiology, aim of, 5, 8; definition of, 3 .

Pliysodes, 320.

Phytolacia decandra, 279.

Phytophthora, 566.

Picea excelsa, respiratory activity of, 525 .

Pick, 495-7.

Pickering, 70.

Pictet, influence of temperature on chemical reactions, 512 .

Pierre, 436, 586 .

Pigments, 494, sec. 88 ; nature of, 495 ; influence of external conditions on formation of, 496,497 .

Pilobolus, 275, Fig. 37 .

Pinguicula, 378, 379; P. alpina, 285 .

Pinus, 149, 372;P. abies, root-system of, I 53: P.piced, 333; P. sylzestris, 259; 
bleeding of branches of, 254 ; green cotyledons of, 333 ; root-system of, 153 . Pisonia alba, 334 .

Pistia texensis, I 82.

Pistone de Regibus, 475.

Pisum, I7I, I 81, 206, 464; cotyledons of, 596 ; P. sationm, 400 ; intramolecular respiration of, 537 ; self-depletion of cotyledons of, 599 .

Pitra, 258, 259,266,369; bleeding of isolated stems, 254.

Pitruschky, 490.

Pitsch, 392, 404.

Plant organs, structure and function of, 37 .

Planta, Von, 284, 600, 604 .

Plantago, translocation in bundle-sheaths of, 575 .

Plasmatic membranes, importance of, in diosmosis, 92, 93; characters of, 107-9.

- organs, origin of, 46 , sec. 8 .

Plasmodiophora, i 12 ; P. brassicae, 377.

Plasmolysis, naturally produced, 143 ; theoretical importance of, 135 ; origin of term, I 43 .

Plasmolytic tests, for permeability, 98 .

Plastic substances, 445 .

Plastids, 43 .

Plastin, $65,66$.

Pleon, 77.

Pneumathodes, I 89.

Poa anmua, root-hair of, I 51 , Fig. II.

Jodocarpus, assimilation of free nitrogen by, 397 .

Poiret, 306.

Poisons, 498, sec. 89 ; function of, 499 ; influence of, on respiration, 563 ; suppression of formation of, $49 \mathrm{I}$.

Polacci, 428.

Pollen grains, reserve-materials of, 604 .

Polstorff, 418.

Polygala, 369 .

Polyporus, 276; P. applanatus, $377 ; P$. destructor, 536; P. sulphureus, enzyme of, 509 .

Polytrichum juniperinum, rhizoid of, I5I, Fig. I I.

Ponterieria, 205.

Popoff, 66, 509 .

l'opulin, 457, 494.

Populus, $243 ; P$. comadensis, 265.

I'orous cork, igs.

I'orteau, 227.

Portele, 6oI.

Portes, 494, 602.

P'ositive pressure of intercellular air, causes of, 203, 204; demonstration of, 204 , Fig. 25.

J'otamogeton, 132, 309.

Potassium, essential character of, 4I I, 429; functions of, 430 .

- myronate, 494.

- salts, importance of, in maintenance of turgor, 424 .
Jotato, percentage of amides in, 459.

I'otometer, 242, Fig. 30 .

Pott, 586.

Pouillet, radiant energy of sunlight, 347 .

Poulsen, 284 .

Prael, 484.

Prantl, 473, 594, 604 .

Prazmowski, 397-402, 558 .

Precipitates in living cells, 96.

Precipitation membranes, I06; modifications in permeability of, ro6.

Preyer, 36.

Prianischnikow, 338, 409, 433, 46I, 464-6, 600.

Priestley, 308 .

Prillieux, 352.

Primordial utricle, $9 \mathbf{I}$.

Primula, 474; P. sinensis, 218.

Pringsheim, 305-8, 320, 32I, 334-6, 539 ; incrustation on Chara, I33; cellulose granules, 483 ; erroneous hypothesis of, 563; hypochlorin, 3I7; improper use of bacterium method by, 351 ; influence of anaesthetics on photosynthesis, 339; permeability of precipitation membranes, 106; protective theory of chlorophyll, $34 \mathrm{r}, 342,355$.

Prismatic spectrum, use of, $35 \mathrm{I}$.

Propionic acid, production of, by acetic bacteria, 560 .

Propyl-alcohol, fermentation of, 560 .

Propylamine, nutritive value of, for fungi, 384 .

Protea mellifera, 285.

Proteid, living, changes in on death, 66; decomposition of, 463 ; - in respiration, 544; - historical account of, 468 ; percentage composition of, 67 ; synthesis of, 409 .

Proteolytic ferments, 508.

Proteus vulgaris, 532.

Protochlorophyll, 3 I6.

Protoplasm, proteids of, 460 ; structure of, 42 , sec. 7 .

Protoplasmic continuity, I I $3-5$.

Proust, 45I.

Prove, 497.

Prunclla grandiflora, influence of darkness on development of flower of, 497 .

Prunet, 604 .

Prumus, 335; P. cerasus, 259 ; bleeding of branches of, $254 ; P$. laurocerasus, I 18, I 86, 282, 283, 286; P.padus, 602 .

Prussic acid, evolution of, 391.

Psamma arenaria, 430.

Ptomaines, 498, sec. 89.

Pulmonaria, changes of colour in cell-sap of, 98 ; - in flower of, 490 .

Puriewitsch, 98, I02; depletion of endosperm, 5 I 5, 590, 591, 598 ; - of cotyledons, $599 ;$ - influence of concentration on, 5 I 7 ; - exosmosis of albumin during, 95 ; foamy structure of protoplasm, 44; 
influence of light on respiration, 562 ; nitrogen-assimilating organisms, 394 .

Purjewicz, 327, 529.

Purple-bacteria, 540.

Putz, 356.

Pyrenoids, 48, 3 I 4 .

Pyrrol, 3 I6.

Pjores, 243.

Quercitrin, 49I.

Quercus, starch in old pith of, 603; Q. robur, bleeding of leafy branches, 254 .

Quincke, IOI ; on plasmatic membrane, I Io. Quinic acid, 447 ; nutritive value of, for fungi, 384 .

Quinine, 457 .

Rabinowitsch, 532 ; influence of oxygen on thermophile bacteria, 533 .

Raciborski, 383, 428, 5 10; on leptomin, 545 .

Raffenau-Delile, 196, 197; currents of air in Nelumbium, 205.

Raffinose, 474 .

Raflesia, 369.

Ranunculus, 198.

Raoul, I03.

Raphamus, influence of mineral food on, 594 .

Rapp, 557.

- Raspail, on calcareous incrustations, 133 .

Rathey, on duramen, 484 .

Raulin, 405, 406, 416, 427, 43I; influence of zinc and manganese on growth of fungi, 385 .

Raumer u. Kellermann, 433.

Rautenberg u. Kuhn, 404, 435; influence of chlorides on acidity of cultures, 132.

Ravizza, 263, 269.

Rawenhoff, 217, 232, 391 .

Ray, on bleeding of plants, 254 .

Reaumuria hirtella, saline incrustation of, 162.

Reducing action, absence of, in respiring protoplasts, 544 .

Regel, 369, 500.

Régnard, 524 ; on swelling of seeds, 75 .

Regnault, 305.

Regulatory mechanism, I 2.

Reichardt, 604.

Reinhardt, 5 I 3 .

Reinitzer, 478, 493, 507; on fatigue substances, 517 .

Reinke, 67, 284, 314-7, 326, 33I-5, 342, $345-50,364,370,383,391,406,459$, $490,509,539$; on bubble-counting method, 35I; force of imbibition, 75, 76 ; influence of different rays on formation of chlorophyll, 334; maximal intensity of light for photosynthesis, 340, 34I ; swelling of organized bodies, $73 ;$ - at low temperatures, 230.

Reinsch, I 59.

Reiss, $187,482,599$.

Rendle, 319 .
Rennet-ferment, 509 .

Resa, I 53.

Reserve-cellulose, 599.

Reserve-materials of seeds, 593, seq.

Resin, 500, sec. 90.

Resorcin, 383, 491.

Respiration, 518 , chap. ix ; activity of, 522 ; aerobic, 519 , sec. 95 ; - in photophobic plants, 358,$525 ;-$ in aquatic plants, 525 ; anaerobic, 530, sec. 97 ; - extravagance of, 568 ; errors due to absorption, 529; formation of water by, 529 ; historical account of, 552,571; importance of, 566 , sec. 105; influence of external conditions on, $56 \mathrm{I}$, sec. 104 ; - temperature, 56I ; - light, 520, 562 ; - nutritive and poisonous substances, 563; - injuries, 565, 566; - percentage of water, 565 ; - ether, 564 ; relation between evolution of $\mathrm{CO}_{2}$ and absorption of oxygen, 544 ; - in green plants, $520,52 \mathrm{I}$.

Respiratory ratio, 529; influence of temperature on, 562 .

Rhamnus frangula, I13, Fig. 8.

Rheum, oxalic acid in, I4I.

Rhinanthus, 365, 366, 369 .

Rhizobium leguminosarum, 396.

Rhizoids, solvent action of, I 7 I.

Rhizomes, reserve-materials of, 604 .

Rhizopus oryzae, 406.

Richardia ethiopica, 268.

Richards, 311, 427, 525, 529; influence of wounds on respiration, 565 ; - of poisons on growth, $385 ;$ - of iron salts on fungi, 4 I 6 .

Richardson, 327.

Richet, $43 \mathrm{I}$.

Richter, I40, 323, 336, 402, 421 .

Ricinus, $149,261-3,473,499$; behariour of cotyledons of, during germination, 596; growth of isolated endosperm of, 598 ; intramolecular respiration of, 537 ; Ricinus communis, 259 ; bleeding of, 256.

Ringing experiments, 574, 578, Fig. 67; effects of different modes of, 213 ; influence of, on secondary growtl, 580 .

Rischavi, 524 .

Risler, 243, 355.

Risse, 436.

Rissmuller, 585 .

Ritthausen, 39I ; on artificial formation of crystalloids, So.

Robinia, 238, 400, 401 ; R. pseudacatia, asparagin in roots of, 603 .

Rochleder, 127, 451, 491, 494, 499; relationship between metabolism and systematic position, 457 .

Rodewald, on force of imbibition, 75 ; phenomena of swelling, 73 ; heat produced by swelling starch, 76 ; work done and heat produced by respiraticn. 567 . 
Roestel, on germination, 600 .

Rollo, 450, 538.

Root-hairs, I64, I65; importance of, 150 ; characters of, 151 ; influence of external conditions on development of, 156.

Root-parasites, 368, 369 .

Root-pressure, definition of, 254 .

Root-system, importance of, 149, sec. 26 ; influence of external conditions on, 1 53-7 ; in potted plants, I 54 ; power of adaption of, 157,158 ; types of, 152 .

Root-tubercules, of Leguminosae, 396-402; of other plants, 396, 397; conditions for formation of, 400 ; influence of medium and of saltpetre on, 401 ; number of, 399.

Roots, absence of root-cap from, 152 ; etchings produced by, I6I, I62; solvent action of, 171.

Rosa, changes of colour in cell-sap of, 98 .

Rosanoff, 315.

Rosen, 41, 67; corrosion of marble by Aethalium, 173 .

Rosenberg, 231, 242, 243, 512, 603 .

Rothert, 604.

Rotondi et Ghizzoni, 262.

Roux, 290.

Ruberythric acid, 494.

Rubia tinctoria, 474, 494.

Rubidium, 4II ; occurrence of, in plants, 430 ; power of replacing potassium in fungi, 429 .

Rubner, $348,549$.

Rubus, 332; R. fruticosus, 324 .

Rumex, 197.

Ruscus, 237.

Russel, 400.

Russow, 196, 222, 604.

Russula, occurrence of laccase in, 510.

Rywosch, 600.

Sabanin u. Laskovsky, 463.

Sabrazès et Bazin, resistance of bacteria to carbon dioxide, 565 .

Saccharomyces, $537,53^{8}, 544,548,550,551$; enzyme of, 502 ; evolution of $\mathrm{H}_{2} \mathrm{~S}$ by, 549 ; influence of temperature on fermentative activity of, 562 ; resistance to alcohol, 513; - to carbon dioxide, 564; continuance of fermentation in presence of oxygen, 547 ; invertin of, 507 ; temporary anaerobiosis of, 530 ; S. glutinis, $308 ; S$. Kefir, lactase of, $507 ;$ S. mycoderma, 532 ; influence of oxygen on fermentative activity of, 547 ; S. Marxianus, 555; S. octosporus, 555.

Sachs, 25, 37, 40, 107, 1 57, I \&2, 196, 197, 201, 2 I 7-9, 227-9, 232, 233, 24I , 256, 26 I, 27 I, 28I , 290, 308-10, 31 8, 31 9, 322-4, 333-5, $340,350,352-3,368,404,410,418,419$, $427,435,450,451$, 460, 490, 497, 526, 576 , $580,588,592,593,601-4$; absorption and retention of water by soils, 168,169 ; - by walls of wood tracheides, 7I, 161 ; energids, 60; etchings produced by roots, 1 1 1, 172; germination of seeds, 600 ; imbibition theory of, 226, 222 ; influence of acids and alkalies on transpiration, 249; mechanomorphosis, 24; method of estimating heat-production, 246 ; minimal temperature for development of chlorophyll, 335 ; presence of air in wood, 225 ; progress of depletion in seeds, 599 ; on translocation, 583 ; transport of water in Laminaria, 210 ; use of microchemical methods by, 578 . Sachsse, 163, 169, 240, 25 I, 320, 330, 368, $390-3,395,427,484,528,529,600$.

Sagot, 593 .

St. Pierre et Magnien, 206.

Salicin, 457, 491, 494.

Saline plants, growth of, in absence of sodium chloride, 430 .

Salix, 191, 278, 497; ringing experiments on, 578,579 .

Salkowski, I73.

Saln-Horstmar, 368, 416, 4I $8,427,431$, 438.

Salsola, 439; S. kali, 430.

Sambucus, 284; S. racemosa, 602.

Saponaria officinalis, 474.

Saposchnikoff, 314, 319, 321-6; percentage of carbohydrates formed to $\mathrm{CO}_{2}$ assimi . lated, 320 .

Sap-pressure, 254.

Sarauw, 37 .

Sarcina lutea, 532.

Sarrabat, 264.

Sarracenia, 160, 275, 379 .

Saussure, de, IoI, 128, 206, 306, 309, 322, $327-9,332,368,401,413,418,451$, $520,521-6,538,571,601$; law of, 129 ; respiration in Cactaceae, 529 ; respiratory ratio, 529 .

Sauvageau, 59, 260.

Savart, 83 .

Saxifragaceae, $1 \hat{3} 3$.

Scenedesmus, $366 ;$ S. acutus, 375.

Schaar, 602.

Schacht, 588 .

Schäfer, I91, 193.

Scheele, 525.

Scheit, 202, 22 I, 226; on bleeding in vacuo, 266.

Schell, 497.

Schellenberg, 484 .

Schenck, 180, 181, 216, 378 .

Scherffel, 377; calcareous deposits on Lathraea, 133 .

Schilling, 1 17, 478.

Schimper, 227, 283-5, 313-5, 319,320, 322-6, $333^{-6}, 405,409,410,420-3,428,429$, $58 \mathrm{I}-6,600-\mathrm{I}$; accumulation of salts and sugar in living cells, 98 ; on calcium oxalate, 486 ; concentration necessary for 
formation of starch, 326 ; functions of latex, 582 ; importance of calcium, 433 ; influence of light on excretion of nectar, 285; mode of absorption in Tillandsia usneoides, 159 ; translocation in bundlesheaths, 575 ; trichites of starch grains, so.

Schindler, 75.

Schizostega osmundacer, 360.

Schleichert, $502,504$.

Schleiden, 290, 368, 429.

Schlicht, 37 I, 376.

Schlickum, 597.

Schlösing, 322, 390, 404, 454 ; influence of humidity on percentage of ash, 235.

- et Laurent, 396, 397, 403.

- et Muntz, 392.

Schlossberger, 129.

Schmid, 601.

Schmidt, $217,280,333,376,600$; on absorption of fats, IOO; on steapsins, 508 ; swelling of seeds, 75 .

Schmidt (R. H.), 383, 470, 479, 508, 592.

Schniedeberg, 401.

Schmitz, 52, 276, 3 I 5, $318,320$.

Schneider, 37 I, 400.

Schniewind-Thies, 284 .

Schönbein, 392 ; ozonization by cell-sap, 545 .

Schostakowitsch, 557 .

Schottelius, 498 ; on suppression of power of pigment-formation, 497 .

Schröder, 1 58, 262, 390, 553, 565, 602, 603 ; removal of ash from cotyledons, 584 ; on reserve-starch in wood, 604 ; resistance to desiccation, 84 .

Schröter, 302 ; Schröter u. Kirchner, I73, 351 .

Schrötter-Kristelli, 3 I6.

Schübler, 497.

Schullerus, on plastic materials of latex, 582.

Schulz, influence of poisons on fermentative activity, 564 .

Schulz-Fleeth, 127, I65, 439.

Schulze, 64, 392, 405-7, 429, 449, 457, 46I, $624,468,474,482,509,599,600,601$; on cholesterin, 63 ; criticism of, 463,464 ; glutamin in seedlings, 465 ; hemicelluloses, 480 ; hydrolysis of proteids, 65 ; hypothetical decomposition of, 466 .

- u. Frankfurt, 285,428 .

- Ulrich, 390.

- Umlauft, Ioo.

- - Winterstein, 428 .

Schumacher, I 53.

Schunck u. Marchlewski, 3 I6.

Schusöger, 327.

Schütt, 3I7, 479; on extra-cellular protoplasm, 59 ; on plastids, 43,48 .

Schutzenberger, 332 .

- u. Quinquand, 338 .

Schwann, 553.
Schwarz, 64, 66, I 55-8, 204, 315, 490 ; changes of size in nucleus, 55 ; influence of anaesthetics on photosynthesis, 339.

Schwendener, I I 8, I90-4, 2 II, 2 I 4, 217 , $220-7,258,260,582$; antagonistic action of guard and epidermal cells, I93; apparatus for demonstrating exudationpressure, 256; molecular structure, 79; - of cell-walls, 81 ; pressure of air in tracheae, 203; swelling of organized bodies, 72 .

Scorzonera, 459 .

Scott and Sargent, I6o.

Scrophulariaceae, parasitism in, 366-9.

Scutellum, excretion of diastase by, 599 .

Secretion, mechanism of, 129.

Sedum Fabaria, I6o.

Seeliger, 54 .

Seifert, 560 .

Seignette, 604 .

Selaginella, 334, 337, 437; formation of roots on rhizophores of, 154 ; S.lepidophylla, 200.

Selection of ash constituents, I27.

Selective absorption, historical account of, 127.

- power, I I9, sec. $22 ; 387$, sec. 67.

Selenious acid, 438 .

Self-regulation, 514 , sec. 93.

Selmi, 536.

Sempervivum, $212,228,237$.

Senebier, I27, 15 I, 306-9, 35 I, 41 8, 450, 497, 571.

Sergent, on division of nucleus, 56 .

Serno, 405-7.

Serradella, 400.

Sestini, 432; Sestini et del Torre, 394.

Sharp, 509.

Sieber, 414.

Siedler, 2 I 6.

Siegert, 585 .

Siemens, 352 .

Sieve-pores, $58 \mathrm{I}$.

Sieve-tubes, 580, 581 ; importance of, in translocation, 576, 589, 590 .

Sigmund, on steapsin, 508 .

Sigwart, 12.

Silene infiata, 436.

Sileneae, occurrence of galactose in, 475 .

Silica, importance of, 416 ; percentage of, in ash of grasses, 435 .

Silicon, 435.

Simon, 368.

Sinapis, 570; S. alba, seedling of, I 5 I, Fig. I 2 .

Sinistrin, 319, 476 .

Siphoneae, 58.

Siroz, 603.

Smith, 494, 533, 537, 549.

Sodium oxymethylsulphonate, formation of starch from, 326.

Soil, absorption by, I69; decomposition and 
disintegration of, 174,175 ; importance and properties of, 163 ; influence of, on distribution of plants, 438 , sec. 76 ; nutritive solution in, 170 ; retention of water by, 168 .

Soja, 401, 464 .

Solanaceae, ringing experiments on, 578 .

Sorauer, 436.

Sorbite, 477.

Sorel, 564 .

Spanjer, 279.

Sparmannia, 243.

Spartium junceum, 247.

Spatzier, 494.

Specific photosynthetic activity, 357 ; - respiratory activity, 524 .

Spencer, 9; physiological units of, 49, 57 .

- le Noore, 358 .

Sphaerocrystals, 73 .

Spirillum, as test for oxygen, $312 ; S$. cholerae-asiaticae, $532 ; S$. desulfuricans, 531, 540; S. rubrum, 318 ; influence of oxygen on pigment-formation in, 497; S. undula, sensitiveness of, to absence of oxygen, 537 .

Spirogyra, 307, 312, Fig. 45, 321, 325, $342,345,411,415,429$; changes of turgidity in, 143 ; influence of chlorides on production of tannin by, 492 ; - of deficiency of calcium on, 424 ; passive secretion of methyl-blue by, 94 ; sénsitiveness to copper salts, 121 .

Splachnum, 379.

Spongioles, I 52.

Spongioplasm, 48 .

Sprengel, I72, 413, 418; importance of organic acids, 489; withdrawal of ash from old organs, 588 .

Ssurosh, 360.

Stachyose, 474.

Stachys tuberifera, 604 .

Stadler, 284 .

Stahl, I 58, I81, I 82, I 88, 191-4, 198, 234, 235, $239,240,243,247,275,277,323,330$, $360,436,495$; on acid secretions, I 30 ; closure of stomata in winter, I90; cobalt method, 242; calcareous deposits, 133 ; protective function of calcium oxalate, 486 ; on tannin, 492.

Stange, $323,421,455$; examples of turgid pressure, 139; influence of concentrated media, 140.

Starch, conditions for formation of, 318, 319 , Figs. 48, 49; percentage of, present in plants, 473 ; rapidity of production of, 320 ; soluble form of, 474 .

- formation, substances capable of inducing, 326; concentration necessary, 326.

- grains, 473; swelling and molecular constitution of, 80 .

Starch-trees, 512, 603.

Steapsin, 508 .
Stein, 379.

Steinbrinck, 72 ; ' ijeberquellung,' 81 .

Steinmetz, 560 .

Stenström, 231, 240.

Stephan, on rapidity of diffusion, I 26.

Stereoisomers, nutritive value of, 387 .

Stich, 310, 524, 525, 538, 539, 542; influence of injuries on respiration, 565 .

Stichococcus, 4 II.

Stimuli, autonomous and inductive, II ; conjoint action of, 19; nature of, I3; trophic, I I.

Stipa pennata, hygroscopic awns of, I6I.

Stohmann, 155, 363, 419, 433 .

Stoklasa, 428, 438 ; on lecithin, 63 .

Stomata, abundance of, 195 ; occlusion of, 190 ; communications of, 196 ; experiments with, 197, Figs. 22, 23 ; distribution of, I 89 ; closure of, I90; influence of epidermal cells on movements of, I9I ; - of light, I94; - of induction shocks, I 94 ; mechanism of movements of, I92; non-closing, 191 .

Storage receptacles, depletion of, 590, 59 I. Strasburger, 40, 41, I 83, 196-8, 2 I I-7, 222 -7, $232,256,258,260,272,273,435,480$, $539,574,578,581,591$; absence of streaming in sieve-tubes, 126 ; centrosomes, 47; duramen, 484; granuloplasm, 48; importance of anatomical arrangement of xylem, 217 ; influence of injection on conductivity of dead wood, 221 ; kinoplasm, 48; mitosis, $55,56,58$; multi-nucleate cells, 60 ; negative pressure in vessels, 202, 203 ; molecular structure, 79 ; transference of water through dead stems, 220 ; trophoplasm, 48 ; use of eosin, 219.

Stratification, bearing of, on molecular structure, 78 .

Strelitzia, 320 .

Striation, bearing of, on molecular structure, 78.

Strychnos mux vomica, 498.

Stutzer, 327, 397-9, 490; alkalinity in sandcultures, I 32 .

- u. Hartleb, 362

Suberization, 482 .

Substitution of essential elements, 4I I-3.

Sugar, nutritive value of, for fungi, 384 .

Sullivan and Thompson, on invertin, 502.

Sulphur, importance and occurrence of, 429 ; oxidation of, 429 ; sources of, $414,429$.

Surface tension energy, importance of, in imbibition, 73 .

Symbiosis, conjunctive, 364 ; disjunctive, 365 ; intracellular, 32 .

Symphytum, 478 .

Symplasts, 60 .

Synanthrin, 476

Syringa, 464; S. vulgaris, transpiratory activity of, 244 . 
Tabaschir, 435 .

Tacke, 395 .

Tagma, 77 .

Tamarix mannifera, saline incrustations of, 162.

Tamman, I03, 4I6, 429, 438, 462, 502; on dissociation and osmotic pressure, 142 .

Tannin, 320, 491, sec. 87; function of, 492, 493; occurrence in cell-sap, 97.

Targioni-Tozzetti, 438 .

Tartaric acid, nutritive value of, for fungi, 384 .

Taurin, as source of sulphur, 429.

Tautomery, 466.

Taxus baccata, 246.

Tecoma, 580.

Telluric acid, 438 .

Temme, on duramen, 484 .

Temperature, influence of, on bleeding, 263 ; on fermentation, 560 ; on development of chloroplastids, 336; on metabolism, 512 ; - on nutrition of fungi, 384 ; on vapour tension, 162,163 ; - on transpiration, 246; on secretion of nectar, 286 ; optima and minima of, for photosynthesis, 337,338 ; -.. for respiration, $56 \mathrm{I}, 562$.

Tetraethylammonium hydroxide, 383 .

Tetragonolobus purpureus, depletion of isolated endosperm of, 599.

Tetraplodon, 379 .

Thallium, occurrence of, 437.

Tharander, 264.

Thein, aplastic character of, 457 .

Theorin, 494.

Thermo-diffusion, 204 .

Thermophile bacteria, 64 .

Thermosynthesis, definition of, 292.

Thesium, 366, 369.

Thiel, 1 53-5, 381-4.

Thlaspi alpestre, 436 .

Thomas, I9o.

Thomson, 427.

Thuja, 333; reversal of bilaterality in, 20 .

Thuman, 497.

Tieghem, van, 204, 247, 341, 593; on chlorovaporization, 247 ; inactivity of endosperm, 597 ; mobilization of reservematerials, 598, 599.

- et Bonnier, IOO.

Tilia, 9; influence of temperature on starch production in, 512 .

- europaea, transpiratory activity of, 244 .

Tillandsia usneoides, I 59.

Timiriaseff, $350,352,355$.

Tischutkin, 379 .

Tissues, division of labour in, 574 .

Titanium, 438 .

Tittmann, 239; on formation of callus, 580 ; regulatory development of cuticle, 117 .

Tollens, 420, 450, 469, 474-7, 48I 483,555 ; on acrylcolloid, 70.

Townsend, influence of nucleus on formation of cell-wall, 53; ... distance of transmission of, 58; rapidity of cellwall formation, 483 .

Toxalbumins, 64 .

Toxins, 514 .

Trachcae, air-bubbles and water-columns in, 223 .

Tradescantia, 52, 185; changes in colour of cell-sap of, 98, 118; - induced by hydrogen peroxide, 546; T. discolor. I 45, I $95 ; T$. Selloi, 433.

Trugopogon porrifolizs, latex of, 582.

Translocation, 573; of organic food, 573 . sec. 106 ; in bulbs, tubers, rhizomes, 604 ; - trees, 602 ; - fruits and seeds, 601 ; - leaves, 600; - algae, 591; from cell to cell, I I 2 ; of ash constituents, 583 , sec. 107 ; causes of, 587 , sec. 108 ; historical account of, 582 ; infuence of mechanical movements on, 589 ; - of oxygen on, 590 ; - of plasma streaming, 589; - of plasmolysis, 590 ; - of protoplasmic connexions, 588; - of vital activity, 590 ; inhibition of, by chloroform, 590; materials employed in, 577 ; mechanism of, 87,587 , sec. 108 ; of oil, 592 ; of photosynthetic products, 600 ; of sugar, 592.

Translocatory channels, direct, 587-9; interrupted, 588 .

Transpiration, cuticular and diastomatic, 236 ; - relative amounts of, 243 ; estimations of, 247, 250, 25I; Garreau's apparatus for, 244, Fig. 32 ; influence of concentration of sap on, 238 ; - of ethereal oils on, 238 ; - of hairs, 238 ; of waxy coverings, 244 ; - of tannin, 239; of external conditions, $245 \mathrm{sec} .39$; - of humidity of air, 245 ; - of temperature of plant and of air, 246 ; - of visible rays of spectrum, 246 ; - of aircurrents and mechanical vibrations, 248 ; - of dissolved substances, 249 ; - of light, on green plants, 246 ; - on fungi, 247 ; - of specific peculiarities, 234 ; under normal conditions, 249 , sec. 40 ; influence of, on percentage of ash, 235 ; - on development of wood, 216: methods of demonstration, 241 ; protections against, 228 , secs. 38,40 , 236-9; from twigs and branches, 237 ; - influence of cork formation on, 237 ; weighing balance for, 241 .

- and absorption of water, relation between, 227, sec. 37 ; - apparatus for demonstration of, 232.

- current, reversal of, 212 .

Trapa natans, 428, 437; analyses of ash of, 128.

Traube, 110,538 ; on cell-wall as an oxidation product, 483 ; on precipitation membranes, 106, 107.

Trees, translocation in, 602 .

Trehalose, 476 . 
Treub, 160, 406, 582; hydrocyanic acid in Pangium edule, 457 .

Treviranus, 151, 212, $217,225,226,231,241$, 262, 500, 593 .

Trianea, temporary production of tannin by, 493 ; T. Bogotensis, 59, 194 ; accumulation of methyl-blue in cell-sap of, 94 ; of methyl violet and cyanin in plasma of, 94 .

Trichites, 80.

Trichostomum tophaceum, calcareous incrustation of, 132 .

Trifolium, 400 .

Trimethylamine, 132; evolution of, by Chenopodium, 391 .

Trinchinetti, IOI, 368; absoption from dilute solutions, 129.

Trioses, 469.

Trioxymethyl, 326.

Triticin, 476.

Triticum, 151; root-system of, 152, Fig. I 3 ; respiratory activity of, $525 ; T$. vulgare, 461 ; absence of carbohydrates from epithelium of scutellum. 588 .

Tropaeolum, 243, 278, 331 ; T. majus, 340; formation of asparagin during germination of, 600 .

Trophic substances, 445 ; definition of, 289. Trophoplasm, 48 .

True, 422.

Trypsin, 508, 509 .

Tschaplowitz, 235 .

Tschirch, I1 8, 189, 190, 315,316, 437, 478, 500,597 ; on chlorophyllan, 316 .

Tubers, 604; respiration of, 525 .

Tulipa Gesneriana, production of coloured flowers in darkness, 496.

Tumas, 349.

Tungsten, 437 .

Tunicata, cellulose in, 481 .

Turgid cell, model of, 135 .

Turgid pressure, of terrestrial, aquatic and starved plants, 139.

Turgidity, existence of, in dying cells, 455 ; importance of, 142 .

Turgor, 134 .

Typha, 180.

Tyrosin, 459 .

Tyrosinase, 510.

Ulbricht, 262.

Umbelliferae, occurrence of sugar in, 474 .

Unger, I 30, I 56, I 61, I 82, 191 , I 94-7, 211 1, 212 , $217,227,231,241-5,250,258,260-2$, $269,280-2,286,309,368,583$; on calcareous incrustations on Saxifrages, I3I; - on Eucladium and Trichostomum, I32 ; evaporation from leaf and free surface of water, 240 .

Uninucleate cells, 58 , sec. IO.

Urase, 508.

Urea, as product of chemosynthesis in nitrate bacteria, 356 .
Urtica, 185; U. urens, 258; U. dioica, 259. 278.

Utricularia, 379.

Vaccinizm, 490.

Vacuolar membrane, 91.

Vacuoles, function of, 42 .

Vaizey, 216.

Vallisneria spiralis, 311, Fig. 44.

Valonia, absence of magnesium from cell-sap of, 432.

Vampyrella vorax, 377 .

Vanadium, 438.

Van Beneden, on deutoplasm, 46.

Van 't Hoff, 71 ; physics of osmotic pressure, 144 .

Vanilla aromatica, i 56.

Variation, 31, sec. 5 .

Vascular systems, in mosses, climbers and aquatic plants, 216.

Vaucheria, 42, 58, 61, 4II ; endophytic rotifers of, $37 \mathrm{I}, 539 ; V$. sessilis, 320 .

Vaudin, 428.

Verschaffeldt, 248 .

Verworn, effects of absence of nucleus, $51,52,55$.

Vesque, 221, 232, 239; automatic registration of transpiration, 242 ; influence of temperature on absorption of water, 229.

Vessels, composition of air in, 206.

Vicia, 458, 461, 466; removal of cotyledons of, $593 ; V \cdot f a b a, 181,285$; respiration in, 524 ; - intramolecular, 537 ; postmortem oxidation in, 545 , 546; self-depletion of cotyledons of, $599 ; V$ sativa, 464,465 ; analyses of seedlings, 467 .

Vierordt, 345 .

Vignal, 522.

Ville, 402, 405, 413, 429.

Vines (S. H.), $211,257,324,379,460$.

Viola lutea var. multicaulis, 436 ; V.tricolor, 436.

$V$ is a tergo, 226.

Viscum, 367, 369 .

Vitis, 206, 278; V. vinifera, 265 ; exudation-pressure of, 259.

Vöchting, 324, 365, 475, 497, 578, 604.

Vogel, 499; non-formation of quinine in Europe, 499.

Voigtländer, on diffusion, 92.

Völcker, 281, 379.

Volkens, $190,203,228,278,280$; on saline incristations, $131,162$.

Vorticella campanula, 306.

Vries, de, 108, 196, 232, 327, 332, 378, 409, $497,498,580,582,588,593,600,604$; examples of turgid pressure, 139; - due to different substances, I4I ; importance of plasma-streaming in translocation, I26, 589 ; osmotic pressure of substances in cell-sap, I42; osmotic values, I45, I46 ; permeability 
of protoplasm, 99; plasmolysis, I43; reserve-materials of clover seeds, 594 . Vrolik, de, et de Vries, 541 .

W'aage, 152, 49I ; on phloroglucin, 493.

Wagner, I 89, 405, 427, 43I, 493; on assimilation of free nitrogen, 397, Fig. 60.

Wahrlich, I I 3, 373 .

Wakker, I 55, 320, $46 \mathrm{I}$.

Walden, I03, I44; experiments with precipitation membranes, 94 .

Waldeyer, $4 \mathrm{I}$.

Walliczek, I I 7, 478 .

Walser, I 30.

Walther, 352.

Walz, 320.

Warburg, $327,328$.

Warming, 370 .

Wasserzug, 375 .

Water, amount exuded, 257, 258 ; conducting channels for, 2I3; excretion of, 252; conveyance of, in transpiring plants, 210 , sec. 34 ; influence of external conditions on percentage of, 229 ; internal circulation of, 260; general account of movements of, 207 ; oblique transference of, 217 ; percentage of, in different parts, 209 ; proofs of transference in tracheal cavities, 221 ; reyersed current of, 212 ; storage of, 228 ; transference of, through dead stems, 221.

Vater-columns, cohesion of, 224.

Water-culture, 4I8-20; nutrient solutions for, 420 .

Water-current, experiments with coloured solutions, 2 I 4 ; influence of ringing on, 2 I 5 ; rapidity of, 2 I 9.

Water-pores, 278-8o, Figs. 3S, 39.

Water-transport, mechanism of, 220 .

Water-vapour, excretion of, 234 .

Way, I69.

Weber, 352, 353, 357 ; influence of illumination on composition of ash, I29; law of, 19.

Wehmer, 327, 376, 38 I-3, 386, 390, 4I I, 427, $431,462,513,527,553,585$; on calcium oxalate, 486 ; on Citromyces, 485 ; influence of sugar on acidity of sap, 490 .

Weigelt, 430 .

Weigert, 495.

Weismann, biophores of, 49; theory of heredity, 57.

Weiss, I I 8, I 95,586 .

Weisse, 189.

Went, 156, I59; on vacuoles and osmotic pressure, 137 .

- u. Prinsen Geerligs, 406.

Westermaier, 182, $210,226,228,319$.

- u. Ambronn, 216.

Wettstein, $284,366$.

Wèvre, de, 379 .

Weyl, 331, 339.
Wicke, 391, 494 .

Wiedemann u. Luideking, 76 .

Wiegleb, 418.

Wiegmann, 368, 418.

- u. Polstorff, I 30, 418, 430.

Wieler, I 55, I 58, I 89, 2 I 3, 2 I 6-9, 22 I , 227 , $255,256,260,26 \mathrm{I}, 266,267,270,276-8$, 542,571 ; on absorption of aniline dyes, 96 ; on bleeding, from isolated stem and branches, 254; - induction of. during winter rest, 265 ; - influence of cortex on bleeding of roots, 268 ; - of oxygen and chloroform, 264; of concentrated nutrient solutions. 263 ; - minimal temperatures for, 263 ; permeability of protoplasm, 99 ; pressure of exudation, 258,259 ; growth in absence of oxygen, 570 .

Wiener, 496.

Wiesner, 184-8, I98, 2 I 7, 235, 247, 248, 306, $313,316,333-5,368,477,478,524,582$ : on dermatosomes, $8 \mathbf{I}$; absorption by leaves, I6o ; impermeability of dry cellwalls to gases, I83; influence of different rays on formation of chlorophyll, 334 ; - of feeble light, 358 ; molecular structure, 79 ; permeation of cell-wall by protoplasm, 485 ; plasomes, 49,57 ; plastids, 43 ; theory of function of chlorophyll, 355 .

- u. Molisch, I 16, I 86 ; gaseous diffusion in capillaries, $\mathbf{I} 88$.

- u. Pacher, 237; transpiration at low temperatures, 246.

Wigand, 495.

- u. Schell, on tannin as plastic product, 493 .

Wilfarth, 398 .

Wilhelm, I62, I90.

Wille, i i 8, 160, 206, 379.

IVilson, $282,285,438$; excretion of nectar. $282,284,285$; - influence of light on, 285 ; respiratory ratio, 538 .

Winkelmann, 73, 76, I 05, I 26, I 44, I 85-7 ; on diffusion through membranes, 92.

Winkler, $314,335$.

IVinogradsky, 308, 400, 402, 4I I, 429, 43I, 540; on iron-bacteria, I 34 ; on nitrate and nitrite bacteria, $36 \mathrm{I}-3$; nitrogenassimilating organisms, 393, 394; nonformation of carbon dioxide by sulphurand nitro-bacteria, 526, 527 .

Winterstein, 476, 48I.

IV isselingh, v., I I 9 ; on fungal cellulose, $4 \delta$ I.

Wittlin, cellulose membranes of crystals, 482 .

Wladimiroff, I 45.

Wolff, 230, 39I, 405, 4IO, 4I 3, 4I 4, 430, 437 : on accumulation of nitrates, 98 ; ash analyses, 127-9; backward diffusion in transpiring plants, I 30.

- (E.), 586.

- (G.), 430 .

- 1 . Zimmermanı, 391. 
Wolkoff, 566 ; maximal intensity of light for photosynthesis, 340, 34I.

Wollny, 175, 235, 251-3.

Woodward, 127, 225, 241, 419; water-culture method of, 418 .

Wortmann, 158, 323, 392, 504, 538, 557 .

- u. Stilling, on aniline dyes as antiseptics, 96.

Wossnesenski, 540 .

Wotezal, 49, 604 .

Wroblewski, 509.

Wundt, 9 .

Wunschmann, 275, 280, 281.

Xenomorphosis, 24.

Xerophily in swamp-plants, 231 .

Xerophytes, 207, 236, 237 ; influence of soil on, 231 .

Yeast, production of alcohol by, 553-561; ferment of, 502 .

Yellow pigments, 316.

Yoshida, 437 ; on lacćase, 509.

Yucca, 320; Y. flamentosa, 319, 476.

Zacharias, 55, 118, 187, 314, 598, 600; absence of nuclei in sieve-tubes, 59; behaviour of nucleus during division, 56 .

Zander, 582.

Zea Mays, 91, I1 4, I34, 156, 247, 334; bleeding of, 256 ; exosmosis of nutri- ment from endosperm of, 95 ; influence of temperature on intramolecular respiration of, 537 ; seedling of, 597 , Fig. 70 ; self-depletion of endospern of, 598.

Ziegenbein, 466, 563; influence of temperature on respiration, 561,562 .

Zimmermann, 41, 51, 53, 66, 68, I18, 306, $312-9,427,428,435,450,460,475$, $479,48 \mathrm{I}-3,495,598$; on absorption of nuclear membrane, 56; calcium pectate in cell-walls, 133 ; cilia, 47 ; honeycomb structure of protoplasm, 44 ; nematoplasts, 48; nucleus, 47; optical properties, influence of mechanical strains on, $82 ;$ - of cell-walls and starch grains, 78; phloroglucin in cell-sap, 97; plasmatic threads, 59,60; - origin of, I I 3-5; plastids, 43; Schizophytes, 43. Zinc, occurrence of, in plants, 436 ; - in wood, 438 ; influence of, on growth, 436 . Zinsser, 513 ; on root-tubercle bacteria, 399401 .

Zöller, 172, 490, 585 .

Zoochloranthellae, 306.

Zopf, 173, 276, 316, 368, 376-8, 386, 414, 450,604 ; on pigments of fungi, 495 . Zukal, 604 .

Zygnema cruciatum, 312, Fig. 46.

Zymogen, 503. 



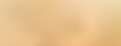

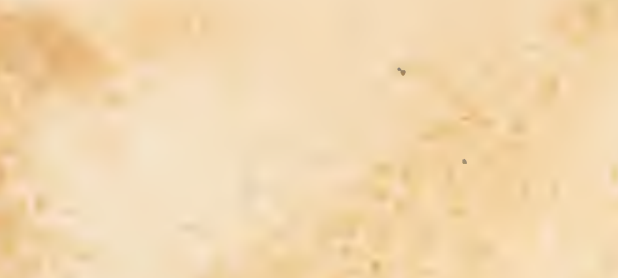

.

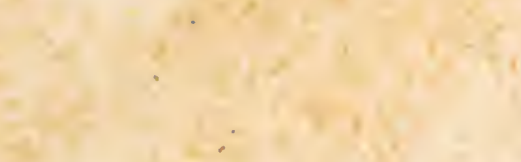
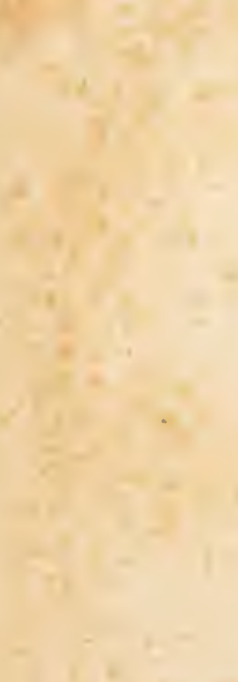

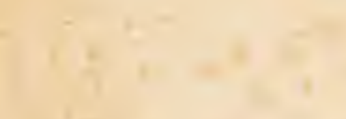

$+\frac{10}{2+2}$

axit thas

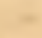

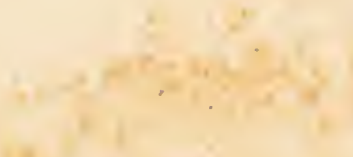

$3 x^{2}+4$

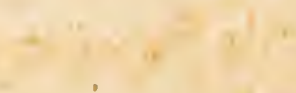

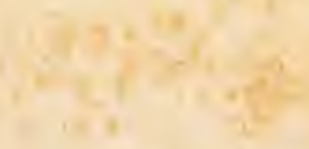

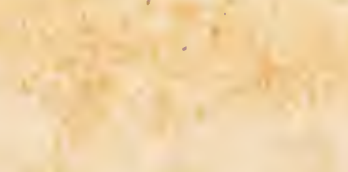
$a^{2}=$

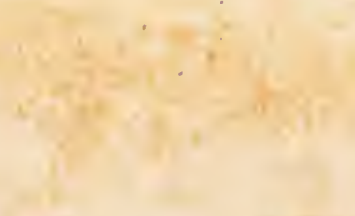

$+$

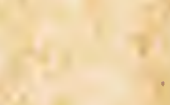

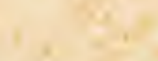

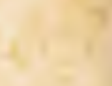

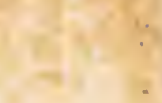




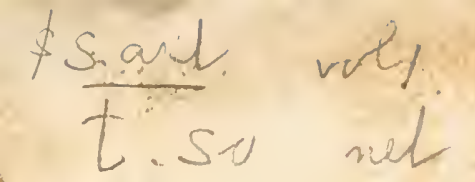


